NUREG / CR -4551

SAND $86-1309$

Volume 1

Printed February 1987

\section{x.}

DRAFT FOR COMMENT

MAR 171987.

\title{
Evaluation of Severe Accident Risks and the Potential for Risk Reduction: Surry Power Station, Unit 1
}

A. S. Benjamin, G. J. Boyd, D. M. Kunsman, W. B. Murfin, D. C. Williams

Prepared by

Sandia National Laboratories

Albuquerque New Mexico 87185 and Livermore Calıforna 94550

for the United States Department of Energy

under Contract DE AC04 760P00789

Prepared for 


\section{NOTICE}

This report was prepared as an account of work sponsored by an agency of the United States Government Neither the United States Government nor any agency thereof, or any of their employees, makes any warranty, expressed or implied, or assumes any legal liability or responsibility for any third party's use, or the results of such use, of any information, apparatus product or process disclosed in this report, or represents that its use by such third party would not infringe privately owned rights

Avarlable from

Superintendent of Documents

U S Government Printing Office

Post Office Box 37082

Washington, D C 20013-7082

and

National Technical Information Service

Springfield, VA 22161 


\section{DISCLAIMER}

This report was prepared as an account of work sponsored by an agency of the United States Government. Neither the United States Government nor any agency Thereof, nor any of their employees, makes any warranty, express or implied, or assumes any legal liability or responsibility for the accuracy, completeness, or usefulness of any information, apparatus, product, or process disclosed, or represents that its use would not infringe privately owned rights. Reference herein to any specific commercial product, process, or service by trade name, trademark, manufacturer, or otherwise does not necessarily constitute or imply its endorsement, recommendation, or favoring by the United States Government or any agency thereof. The views and opinions of authors expressed herein do not necessarily state or reflect those of the United States Government or any agency thereof. 


\section{DISCLAIMER}

Portions of this document may be illegible in electronic image products. Images are produced from the best available original document. 
NUREG/CR-4551

TI87 006468

SAND86-1309

Volume 1

\section{EVALUATION OF SEVERE ACCIDENT RISKS AND THE POTENTIAL FOR RISK REDUCTION: \\ SURRY POWER STATION, UNIT 1}
A. S. Benjamin
G. J. Boyd*
D. M. Kunsman
W. B. Murfin**
D. C. Williams

Printed February 1987
Sandia National Laboratories
Albuquerque, NM 87185
Operated by
Sandia Corporation
for the
U. S. Department of Energy

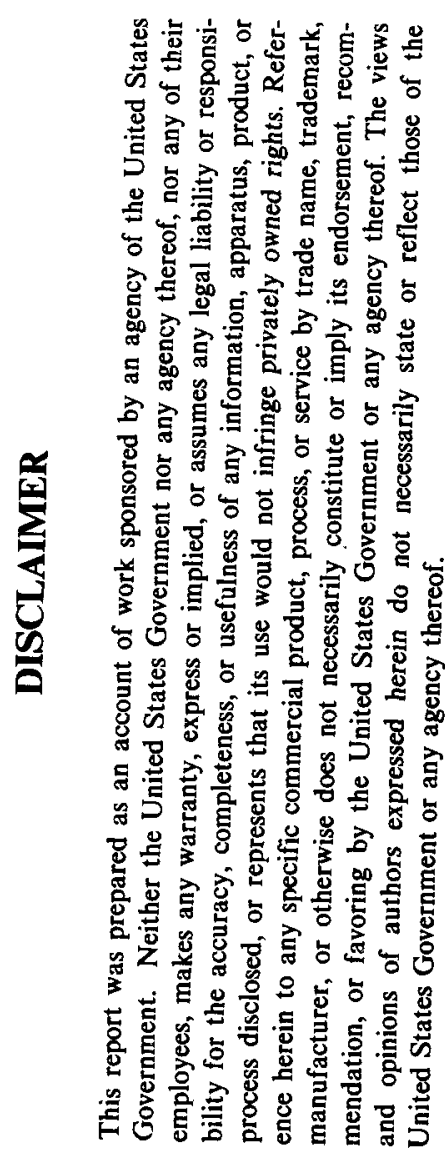

Prepared for

Division of Reactor System Safety

Office of Nuclear Regulatory Research

U. S. Nuclear Regulatory Commission

Washington, DC 20555

Under Memorandum of Understanding DOE 40-550-75

NRC FIN No. A1322

*Safety and Reliability Optimization Services, Inc., Knoxville, TN

**Technadyne Engineering Consultants, Inc., Albuquerque, NM 


\section{PREFACE}

Because of the time constraints imposed on this work to meet the Nuclear Regulatory Commission's schedule for publication of NUREG-1150, this draft report has not yet received the full level of peer and management review customarily accorded to reports issued by Sandia National Laboratories. The reviews will be completed and corrections made, if necessary, prior to final publication. 


\section{R.EOUEST FOR COMMENT}

This report, NUREG/CR-4551, "Evaluation of Severe Accident Risks and Potential for Risk Reduction," was prepared for the U.S. Nuclear Regulatory Commission by the Sandia National Laboratories and its subcontractors.

The methods and results set forth in this report are being used by the NRC to support the development of the Reactor Risk Reference Document (NUREG-1150) and will be used in areas of broad public interest such as probabilistic risk analyses, emergency response planning, sitina, NR.C safety goal applications, and cost/risk/benefit analyses--indeed, wherever risks to public health need to be considered in regulatorv applications. Thus, it is considered imperative that an opportunity for public comment on the results as presented in the report be provided. Comments should be sent to the U.S. Nuclear Regulatory Commission, Washington, D.C. 2.0555, Attention: Joseph Murphy, Division of Reactor System Safety. These comments will be most useful to the staff if they are received by June 1, 1987.

of particular interest to us is the receipt of comments on the methodology, and results, related to uncertainty analysis. One criticism voiced with respect to the Reactor Safety Study was its lack of an uncertainty analysis. We have included an uncertainty analysis, but we are sure that its nature will be the subject of lively debate. We welcome this, and solicit constructive advice and criticism.

The NRC hereby expresses its great appreciation to all participants in this study for their considerable efforts, as well as to all who will take the time and effort to provide it with comments on this report.

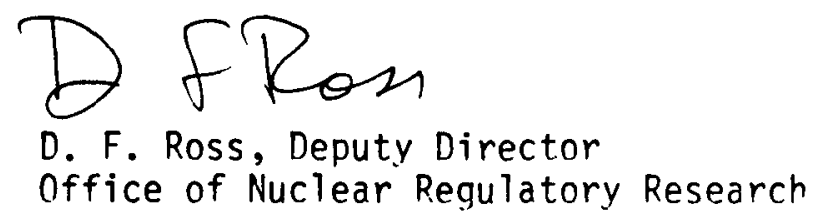




\begin{abstract}
The Severe Accident Risk Reduction Program (SARRP) has completed a rebaselining of the risks to the public from a particular pressurized water reactor with a subatmospheric containment (Surry, Unit 1). Emphasis was placed on determining the magnitude and character of the uncertainties, rather than focusing on a point estimate. The risk-reduction potential of a set of proposed safety option backfits was also studied, and their costs and benefits were also evaluated. It was found that the risks from internal events are generally lower than previously evaluated in the Reactor Safety Study (RSS). However, certain unresolved issues (such as direct containment heating) caused the top of the uncertainty band to appear at a level that is comparable with the RSS point estimate. None of the postulated safety options appears to be cost effective for the Surry power plant. This work supports the Nuclear Regulatory Commission's assessment of severe accidents in NUREG-1150.
\end{abstract}




\section{CONTENTS}

Section $\quad \underline{\text { Page }}$

1. INTRODUCTION............................................................

1.1 Background and Objectives..............................................

1.2 Scope of Analysis.......................................................

1.3 Overview of the Surry Plant...............................................

1.4 Organization of the Report................................................ $1-7$

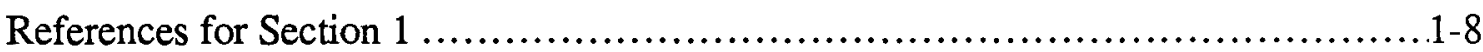

2. METHODOLOGY FOR REBASELINING OF RISK $\ldots \ldots \ldots \ldots \ldots \ldots \ldots \ldots \ldots \ldots . \ldots \ldots \ldots$

2.1 Overview of Risk Integration and Review Activities.......................... $2-1$

2.1.1 Integration of Project Activities.................................... 2-1

2.1.2 Review and Quality Assurance....................................... $2-5$

2.2 Approaches to the Evaluation of Uncertainties............................... 2-8

2.2.1 Overview of Uncertainty Treatment....................................2-8

2.2.2 The LLH Approach.................................................... 2-12

2.2.3 The OCP Approach............................................ 2-24

2.3 Reassessment of Dominant Core-Damage Sequences..........................2-26

2.4 Evaluation of Containment Release-Mode Probabilities...................... 2-29

2.4.1 Development and Quantification of the Containment Event Tree.........2-29

2.4.2 Plant Features Important to Containment Response.....................2-34

2.4.3 Definition of Containment-Release Modes.............................2-36

2.5 Assessment of the Radiological Source Term................................2-38

2.5.1 Integration With Other Risk Assessment Tasks..................... 2-38

2.5.2 Source Term Code Package Results............................... 2-42

2.5.3 Derivation of Source Terms for Other Bins.......................... 2-45

2.5.4 Derivation of Source Terms for Uncertainty Analysis..................2-48

2.6 Offsite Consequence Analysis......................................... 2-53

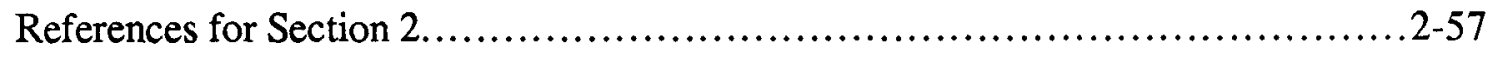

3. METHODOLOGY FOR THE ASSESSMENT OF RISK-REDUCTION OPTIONS... 3-1

3.1 Identification of Safety-Improvement Options............................. 3-1

3.1.1 Generic Risk-Reduction Options........................................ $3-1$

3.1.2 Surry-Specific Risk-Reduction Options................................ $3-2$

3.2 Evaluation of Costs and Other Impacts.................................... $3-8$

3.3 Evaluation of Effects on Risk.............................................. 3-9

3.4 Value/Impact Assessment.............................................. 3-10

3.5 Treatment of Uncertainties............................................ $3-12$

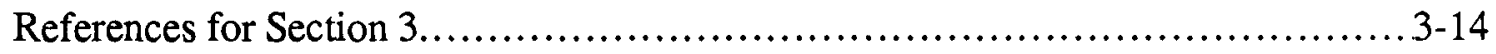




\section{CONTENTS (CONTINUED)}

Section

4. EVALUATION OF KEY UNCERTAINTY ISSUES..............................4-1

4.1 Sequence Frequency Issues........................................... 4-2

4.1.1 Reactor Coolant Pump Seal LOCA Model.................................4-2

4.1.2 Use of the Gas Turbine Generator for Offsite Power Recovery........... 4-3

4.1.3 Uncertainty in the Common Cause Evaluation Methodology...............4-3

4.1.4 Modeling and Data Uncertainties in the V Sequence......................4-3

4.1.5 Uncertainties Concerning Core Melt Due to Containment Failure.......... 4-4

4.2 Containment Loading and Performance Issues............................4-4

4.2.1 Probability and Location of Induced Failure in the RCS..................4-5

4.2.2 Mode of Reactor Vessel Breach.........................................4-7

4.2.3 Pressure at Vessel Breach Due to Direct Heating and Steam Spike.........4-8

4.2.4 Magnitude of Hydrogen Burn Pressure Increment at Vessel Breach....... 4-9

4.2.5 Containment Failure Pressure...........................................4-10

4.2.6 Containment Failure Size...........................................4-11

4.2.7 Probability of Containment Spray Failure..............................4-12

4.2.8 Probability of Submergence of the V Sequence Failure Site..............4-12

4.3 Radiological Source Term Issues........................................4-13

4.3.1 In-Vessel Release from the Fuel....................................4-14

4.3.2 Amount of CsI Decomposition...................................... 4-14

4.3.3 Retention in the Reactor Coolant System...........................4-15

4.3.4 Decontamination Factor for the V Sequence with Release Submerged... 4-17

4.3.5 Release of RCS Species from Containment...........................4-18

4.3.6 Magnitude of Core-Concrete Interaction Releases.......................4-18

4.3.7 Release of Core-Concrete Interaction Species from Containment........ 4-20

4.3.8 Decontamination Factors for Containment Sprays....................... 4-20

4.3.9 Aerosol Agglomeration Uncertainties.................................4-21

4.3.10 Late Iodine Releases From Containment............................ 4-23

4.3.11 Late Revolatilization from the RCS.................................. 4-23

4.3.12 Releases Associated with High Pressure Ejection.........................4-24

4.3.13 Releases Associated with Direct Heating............................ 4-25

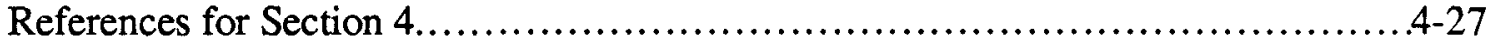

5. RESULTS OF RISK REBASELINING..................................... $5-1$

5.1 Core-Damage Frequency Results..................................... $5-1$

5.1 .1 Sequence and Damage State Frequencies............................. $5-1$

5.1 .2 Uncertainty Representation.......................................5 5

5.1.3 Observations Concerning the Core-Damage Frequency................ 5-10

5.1.4 Comparison to the Reactor Safety Study ............................5-11

5.2 Containment Analysis Results............................................5-12

5.2.1 LLH Containment Analysis Results................................. 5-12

5.2.2 OCP Containment Analysis Results.................................. 5-17 


\section{CONTENTS (CONTINUED)}

Section Page

5.3 Radiological Source Term Analysis Results..............................5-22

5.3.1 LLH Source Term Results........................................... 5-22

5.3.2 OCP Source Term Results........................................... 5-27

5.4 Offsite Consequence Results.............................................. 5-30

5.5 Risk Results........................................................5

5.5.1 Early and Latent Cancer Fatality Risk Measures.....................5-37

5.5.2 Other Risk Measures...................................................5-57

5.5.3 Observations Concerning the Risk Results........................5-60

5.5.4 Limitations..................................................... $5-62$

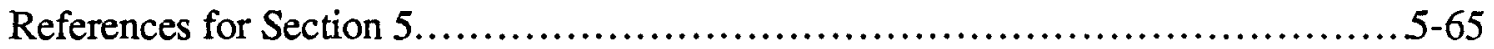

6. RESULTS OF RISK REDUCTION ANALYSIS..............................6-1

6.1 Prevention Option Effects on Core-Damage Frequency.........................6-1

6.2 Mitigation Option Effects on Containment/Source Terms.......................6-3

6.3 Benefit of Risk-Reduction Options: Averted Risk........................... 6-7

6.4 Costs of Risk-Reduction Options........................................ 6-10

6.5 Costs Versus Benefits................................................. 6-10

6.6 Observations Concerning Risk-Reduction Analysis......................... 6-12

7. INSIGHTS AND CONCLUSIONS.......................................... $7-1$

7.1 Insights and Conclusions from the Rebaselining of Risk....................... $7-1$

7.2 Risk-Reduction Insights and Conclusions.................................. $7-4$

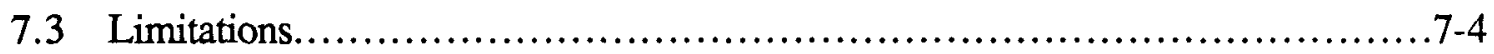




$$
\text { CONTENTS (CONTINUED) }
$$

Section

\section{APPENDICES}

A. REASSESSMENT OF THE CORE-MELT FREQUENCY FOR SURRY...........A-1

B. CONTAINMENT ANALYSIS UNCERTAINTY ISSUES FOR THE LIMITED LATIN HYPERCUBE STUDY OF SURRY $\ldots \ldots \ldots \ldots \ldots \ldots \ldots \ldots \ldots \ldots \ldots \ldots \ldots . . . \ldots \ldots$

C. SUPPORTING ANALYSIS FOR THE SURRY SOURCE TERM UNCERTAINTY ASSESSMENT. ............................................................. 1

D. DETAILED LISTINGS OF RISK RESULTS.................................

E. ASSESSMENT OF RISK REDUCTION MEASURES.............................

F. SUMMARY OF REVIEW TEAM COMMENTS CONCERNING THE LIMITED LATIN HYPERCUBE ANALYSIS AS IMPLEMENTED IN SARRP ...............F-1

G. DATA BASE FOR ESTIMATION OF COST AND PERSONNEL DOSE FOR

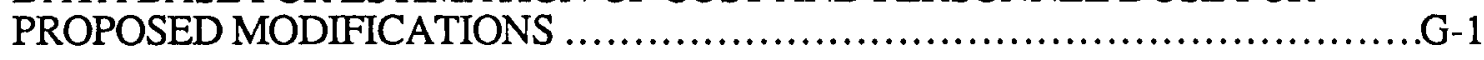




\section{LIST OF TABLES}

Table Page

2-1 Participants in SARRP Review Tasks for the Surry Analysis......................2-7

2-2 Uncertainty Characteristics of Different Analysis Activities.........................2-11

2-3 Summary of Top Events for Surry Containment Event Tree......................2-30

2-4 Use of Information Sources Addressing the Issues in the Containment Event Tree. .2-33

2-5 Key Characteristics of the Surry Source-Term Bins..............................2-41

3-1 PWR Accident-Prevention Options......................................... 3-3

3-2 PWR Accident Mitigation Options......................................... 3-4

5-1 Dominant Core-Damage Sequences for Surry ............................... 5-2

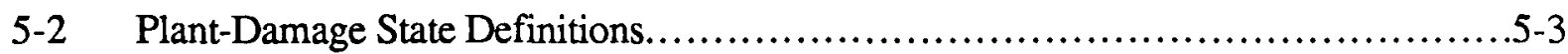

5-3 Plant-Damage States and Contributing Sequences............................. 5-4

5-4 Sensitivity Studies for Core-Damage Analysis................................5-7

5-5 Likelihood of Containment Release Modes by Plant Damage State (Central) ....... 5-19

5-6 Likelihood of Containment Release Modes by Plant Damage State (Optimistic) ..... 5-20

5-7 Likelihood of Containment Release Modes by Plant Damage State (Pessimistic) ....5-21

5-8 Release Fractions for Surry LLH Clusters …...............................

5-9 Central Estimate Release Fractions for Surry ............................... 5-28

5-10 CRAC2 Estimates of Mean Consequences for Surry Source Term Clusters ......... 5-31

5-11 MACCS Estimate of Mean Consequences for Surry Source Term Clusters .........5-32

5-12 CRAC2 Estimate of Mean Consequences for Surry Source Term Bins............. 5-35

5-13 MACCS Estimate of Mean Consequences for Surry Source Term Bins..............5-36

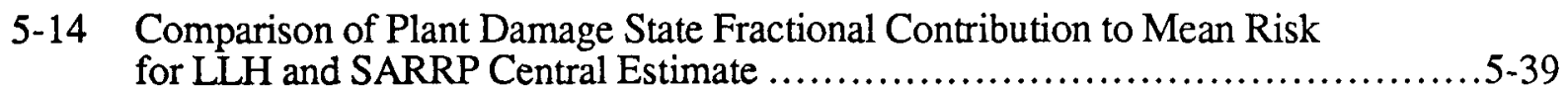

5-15 Comparison of OCP Central and LLH Mean Source Term Bin Fractional Contribution to Risk ........................................................ 51

5-16 Fractional Contribution of Important LLH Clusters to Risk......................5-42

5-17 Results of Chi-Squared Test as Calculated with CRAC2 ........................5-51

6-1 Reduction in Core-Damage Frequency Associated with Preventive Safety Options... 6-3

6-2 Relative Contribution of Source Term Bins to Latent Fatality Risk for Mitigation Options .

6-3 Estimated Present Value Total Cost for Risk-Reduction Measures..................6-11

6-4 Value of Reduction of Risk to Zero as Calculated with CRAC2 ...................6-12 


\section{LIST OF FIGURES}

Eigure $\quad$ Page

1-1 Relationships of Research Programs to SARRP Tasks..............................1-4

2-1 Overview of SARRP Methodology..........................................2

2-2 Integration of SARRP Tasks............................................... $2-3$

2-3 Overview of the LLH Process..............................................2 14

2-4 Example of LLH Output for Risk Measures..................................2-18

2-5 Example of LLH Rank Regression Analysis...............................2-18

2-7 Example of CCDF Risk Display........................................2 $2-19$

2-6 Example of the OCP Process for One CET Question...............................2-25

3-1 Example of LLH Cost/Benefit Display....................................3 13

4-1 Outcomes and Weighting Factors for Location and Size of Induced LOCA.......... 4-6

4-2 Outcomes and Weighting Factors for Mode of Reactor Vessel Breach..............4-7

4-3 Outcomes and Weighting Factors for Magnitude of Pressure Loading at Vessel Breach Due to Direct Heating and Steam Spike ..................................4-9

4-4 Outcomes and Weighting Factors for Magnitude of Hydrogen Burn Pressure Increment at Vessel Breach

4-5 Outcomes and Weighting Factors for Containment Failure Pressure...............4-11

4-6 Outcomes and Weighting Factors for Containment Failure Size...................4-12

4-7 Outcomes and Weighting Factors for In-Vessel Release from the Fuel.............4-15

4-8 Outcomes and Weighting Factors for CsI Decomposition........................4-16

4-9 Outcomes and Weighting Factors for In-Vessel Retention.......................4-17

4-10 Outcomes and Weighting Factors for Decontamination Factor for the V Sequence When the Release Occurs Under Water ........................................4-18

4-11 Outcomes and Weighting Factors for Magnitude of Core-Concrete Interaction Releases ..................................................................4-19

4-12 Outcomes and Weighting Factors for release of Core-Concrete Interaction Species

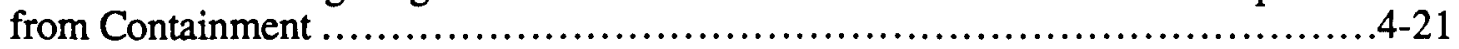

4-13 Outcomes and Weighting Factors for Decontamination Factor for the Containment

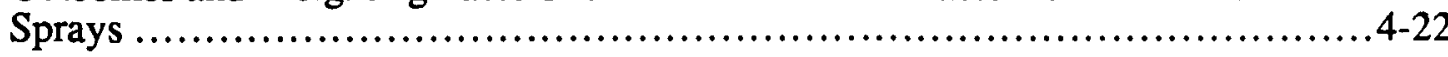

4-14 Outcomes and Weighting Factors for Late Iodine Releases from Containment...... 4-23

4-15 Outcomes and Weighting Factors for Late Release for the RCS..................4-24

4-16 Outcomes and Weighting Factors for Releases During High Pressure Ejection......4-25

4-17 Outcomes and Weighting Factors for Releases Associated with Direct Heating..... 4-25

5-1 "Box-and-Whisker" Display of Uncertainties for Total Core-Damage Frequency.... 5-8

5-2 Box-and-Whisker Plots for Damage States with Mean Frequencies $>1.0 \times 10^{-7} \ldots . .5-8$

5-3 Conditional Probability of Early Containment Failure: All Sequences Included.....5-13 


\section{LIST OF FIGURES (CONTINUED)}

Figure

Page

5-4 Conditional Probability of Early Containment Failure: Damage State TNNN........5-14

5-5 Rank Regression Analysis of Early Containment Failure for All Sequences.........5-15

5-6 Comparison of Base Case and Sensitivity Study: Early Containment Failure Probability for All Sequences

5-7 Rank Regression Analysis of Early Containment Failure for All Sequences: No Direct Heating Sensitivity Case .

5-8 Sampled Surry Source Term estimates from SURSOR for Group 2...............5-23

5-9 Sampled Surry Source Term estimates from SURSOR for Group 3................5-23

5-10 Sampled Surry Source Term estimates from SURSOR for Group 4................5-24

5-11 Sampled Surry Source Term estimates from SURSOR for Group 5.............5-24

5-12 Sampled Surry Source Term estimates from SURSOR for Group 6...............5-25

5-13 Sampled Surry Source Term estimates from SURSOR for Group 7..............5-25

5-14 Comparison of SARRP and RSS Source Terms............................5-29

5-15 Risk of Latent Cancer Fatalities...............................................

5-16 Risk of Early Fatalities......................................................

5-17 CCDF for Risk of Latent Cancer Fatalities (MACCS) ............................. 5-44

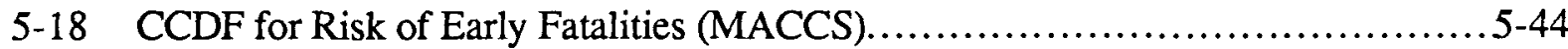

5-19 Rank Regression Analysis for Risk of Latent Cancer Fatalities...................5-45

5-20 Rank Regression Analysis for Risk of Early Fatalities........................5-46

5-21 Comparison of Risk Results for Latent Cancer Fatalities With and Without Direct Heating as an LLH Issue.......................................... 5-47

5-22 Comparison of Risk Results for Early Fatalities With and Without Direct Heating as an LLH Issue....................................................5-47

5-23 Rank Regression Analysis for Risk of Latent Cancer Deaths: No Direct Heating... 5-48

5-24 Rank Regression Analysis for Risk of Early Fatalities: No Direct Heating..........5-48

5-25 Comparison of LLH and OCP Results: Latent Cancer Fatalities..................5-55

5-26 Comparison of LLH and OCP Results: Early Fatalities........................5-55

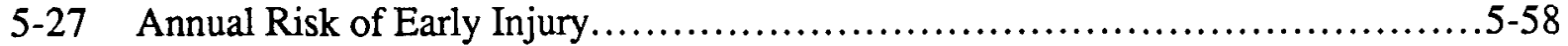

$5-28$ Individual Risk of Death...................................................58

5-29 Results for Offsite Cost Risk Measure...................................5-59

5-30 Results for Risk Measure of Population Dose Per Year........................5-59

6-1 Reduction of Latent Fatality Risk for Prevention Options........................6-8

6-2 Reduction of Early Fatality Risk for Prevention Options........................6-8

6-3 Reduction of Latent Fatality Risk for Mitigation Options.............................6-9 
NUREG/CR-4551,VOL.1: DRAFT REPORT FOR COMMENT (SEPTEMBER, 1986)

\section{LIST OF FIGURES (CONTINUED)}

Figure Page

6-4 Reduction of Early Fatality Risk for Mitigation Options.............................6-9

6-5 Cost/Benefit Comparison for Prevention Options (CRAC2).....................6-13

6-6 Cost/Benefit Comparison for Mitigation Options (CRAC2).......................6-14

6-7 Cost/Benefit Comparison for Prevention Options (MACCS)......................6-15

6-8 Cost/Benefit Comparison for Mitigation Options (MACCS).......................6-16 


\section{LIST OF ACRONYMS AND ABBREVIATIONS}

$\mathrm{AE}$

AFW

ASEP

ATWS

BCL

$\mathrm{BNL}$

$\mathrm{CCDF}$

$\mathrm{CCI}$

CDB

CET

CF

CLWG

CPWG

DF

$\mathrm{DH}, \mathrm{DCH}$

ECCS

HPI

IDCOR

IREP

LLH

LOCA

LPI

NRC

NSSS

OCP

PORV

PRA

PRUEP

PWR

QC

QUEST

RCP

RAP

RCS

RSS

RWST

SARRP

SASA

SAUNA

SCG

SGTR

STCP

USI
Architect-Engineer

Auxiliary feedwater

Accident Sequence Evaluation Program

Anticipated transient without scram

Battelle Columbus Laboratories

Brookhaven National Laboratory

Complementary cumulative distribution function

Core-concrete interactions

Coolable debris bed

Containment-event tree

Containment failure

Containment Loads Working Group

Containment Performance Working Group

Decontamination factor

Direct (containment) heating

Emergency-core cooling system

High pressure injection system

Industry Degraded Core Program

Interim Reliability Evaluation Program

Limited Latin hypercube (uncertainty analysis method)

Loss-of-coolant accident

Low pressure injection system

U. S. Nuclear Regulatory Commission

Nuclear steam supply system

Optimistic/central/pessimistic (method for uncertainty analysis)

Power-operated relief valve

Probabilistic risk assessment

Probabilistic Risk Uncertainty Evaluation Program

Pressurized water reactor

Quality control (review group)

Quantitative Uncertainty Estimation of the Source Term Program

Reactor-coolant pump

Regulatory Analysis Program

Reactor-coolant system

Reactor Safety Study

Refueling water storage tank

Severe Accident Risk Reduction Program

Severe Accident Sequence Analysis Program

Severe Accident Uncertainty Analysis Program

Senior consultant group

Steam generator tube rupture

Source-Term Code Package

Unresolved safety issue 


\section{Section 1 INTRODUCTION}

This report summarizes the efforts and results of the Severe Accident Risk Reduction Program (SARRP), which was conducted by Sandia National Laboratories with the support of several other contractors to the U. S. Nuclear Regulatory Commission (NRC). The goal of SARRP was to put into a risk perspective the insights that have been generated as a result of recent research into systems behavior and physical phenomena under severe accident conditions in nuclear power plants. In pursuing this goal, these insights have been applied to obtaining a new estimate of risk for several operating plants and to evaluating the potential benefits and costs of measures intended to enhance safety. This report will serve as a principal input to the development of the report NUREG-1150, which will summarize the technical position of the NRC on the significance of severe nuclear power plant accidents.

This report is one of a series of five, each concerning one of the NUREG-1150 reference plants. The plant under consideration in this report is Virginia Electric Power Company's Surry Unit 1, a Westinghouse pressurized water reactor with a dry, subatmospheric containment. The goal of understanding the changes in estimated risk due to advances in the technology is therefore particularly appropriate for this study because Surry was one of the two plants analyzed in the Reactor Safety Study (RSS) [1].

\subsection{BACKGROUND AND OBJECTIVES}

A number of research activities are currently engaged in extensive efforts focused on better characterizing the hazards associated with the operation of nuclear power plants. These efforts typically focus on one or more of the following areas of reactor safety:

- The likelihood and nature of accidents severe enough to liberate substantial quantities of fission products from the reactor fuel;

- The challenges such accidents would present to the integrity of the reactor containment;

- The quantities and physical properties of fission products that could be released to the environment; and

- The ultimate consequences of these accidents, both in terms of their effects on public health, and their associated costs to society and to the plant owners.

The first attempt to incorporate the state-of-the-art in all of these areas into an integrated perspective on the risk associated with the operation of nuclear power plants culminated in the 
Reactor Safety Study, which was published in 1975. Although that study produced the first comprehensive assessment of plant risk, it also focused attention on a number of gaps in the understanding of accident processes.

Since that time, many risk assessments of specific plants have been performed. In general, each of these has progressively reflected at least some of the advances that have been made in the ability to predict accident behavior. In order to investigate the significance of more recent developments in a comprehensive fashion, it was concluded that the current efforts of research programs being sponsored by the NRC should be coalesced to produce an updated representation of risk; this led to the formulation of the current SARRP objectives.

The results of the specific research programs, including their significance with respect to various questions concerning reactor safety, are, of course, documented individually. It is the objective of SARRP to integrate these results and to cast them in the context of their implications with respect to risk. This includes not only an estimate of risk as measured by a number of health effects and financial consequences, but also a thorough understanding of the important sensitivities and uncertainties associated with the state-of-the-art in reactor safety assessment. Many of the critical reviews of former risk studies, particularly the RSS, concluded that the uncertainties in the results had not been fully examined or displayed. A major focus of the SARRP program has been the characterization of these uncertainties, and this emphasis is one of the features that distinguishes this study from many other risk assessment programs.

This risk context also provides a mechanism to investigate the degree to which safety might be improved as a result of various modifications to existing plants. Comparisons of the risk reduction that could be achieved and the associated costs incurred as a consequence of such modifications can provide additional information useful to the NRC's decision-making process.

\subsection{SCOPE OF THE ANALYSIS}

The re-evaluation of the frequency of potential core-damage accidents at Surry was accomplished under the auspices of the Accident Sequence Evaluation Program (ASEP) and is reported in Reference 2. The SARRP study included interaction with the accident sequence evaluation to ensure appropriate integration with the additional analyses needed to calculate the risk of the core-damage accidents. The program embodied in SARRP involved five major activities: 
- The formulation of a means to provide a comprehensive delineation of the possible types of containment response to severe core-damage accidents and the estimation of their relative frequencies;

- Development of fission product source terms for the spectrum of accident sequences and resultant containment response end states;

- Calculation of the offsite consequences of the accidents considered;

- Integrated calculation of risk using these accident sequences, containment and source term analyses, and offsite consequence calculations; and

- Investigation of the benefits and costs of measures intended to reduce risk.

All of these activities involved a central estimate and an evaluation of key sensitivities and uncertainties. The relationships of these tasks and the other research and analysis programs are illustrated in Figure 1-1.

The first SARRP activity led to the development of a containment event tree specific to the evaluation of the Surry containment. The details of the containment analysis are reported under separate cover [3], although the principal inputs and results are repeated in this report. The containment event tree is similar in nature to those used in recent plant-specific risk assessments, but it is generally much more detailed. It provides a mechanism for tracing the accident progression for the different types of core-damage events, including various phenomena that could affect the likelihood and severity of containment failure, and the performance of the containment safety features. As illustrated in Figure 1-1, this task involved original analysis as well as integration of the results of many other activities. These sources provided information relating to the nature of severe-accident phenomena, the severity of various challenges to containment integrity, and the capacity of the containment to withstand these challenges. The level of detail in the containment event tree was dictated by the desire to display the possible outcomes for a variety of core-damage accidents (recognizing that uncertainties regarding some severe-accident phenomena prevent precise prediction of the containment response), and the need to provide appropriate discrimination of the conditions associated with the release of fission products to the environment to allow an assessment of the accident consequences.

The second major activity also involved drawing from a number of other research and analysis activities (see Figure 1-1). The central estimate source term evaluation for the accident sequences most important to risk was performed by Battelle Columbus Laboratories (BCL). Battelle's calculations were performed with the Source Term Code Package (STCP), which is basically an integration of the codes used in the analyses previously reported in BMI-2104, 


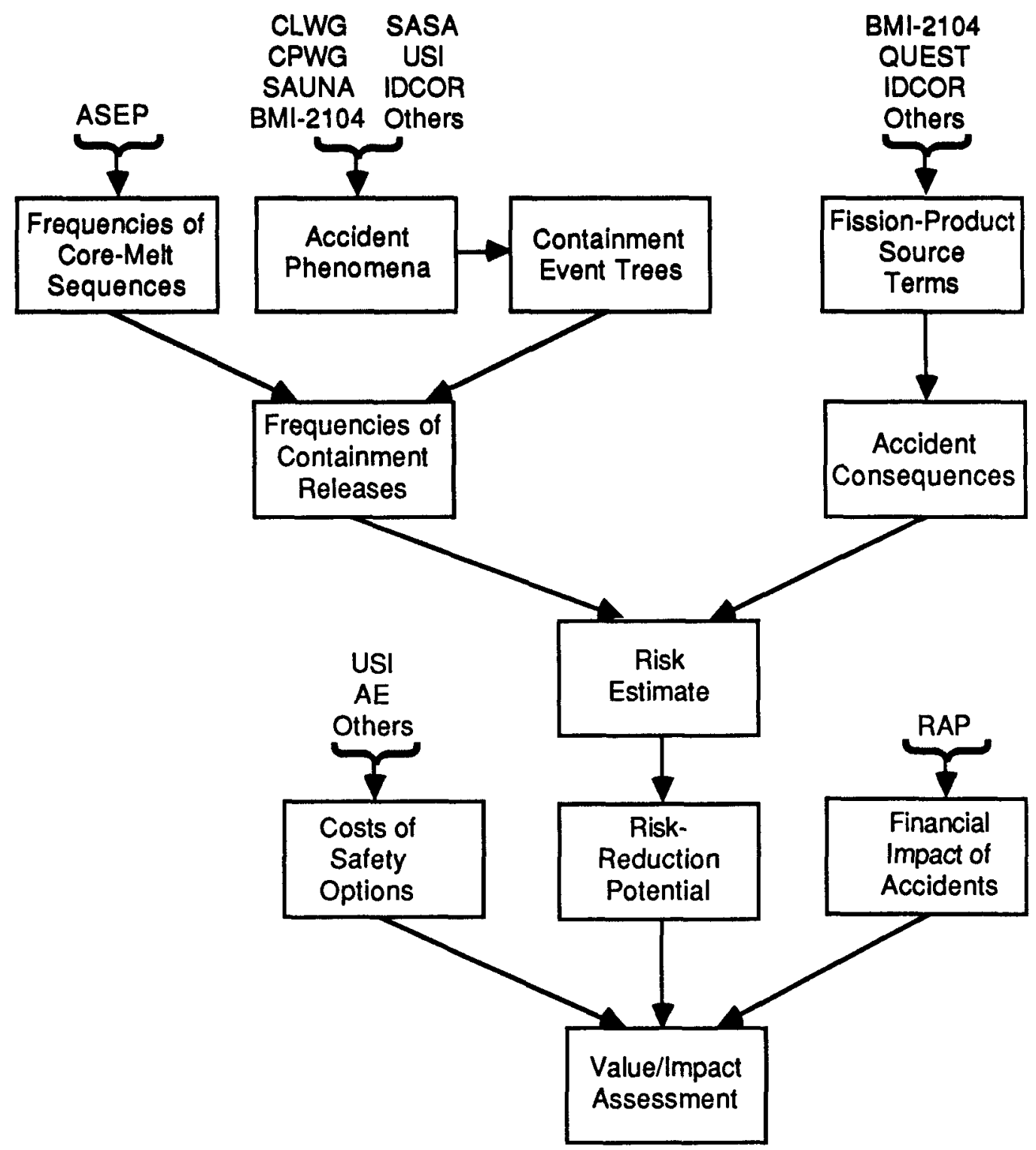

Key

ASEP Accident Sequence Evaluation Program

CLWG Containment Loads Working Group

CPWG Containment Performance Working Group

SAUNA Severe Accident Uncertainty Analysis

BMI-2104 Battelle assessment of radiological source term

SASA Severe Accident Sequence Analysis

USI Studies of unresolved safety issues

IDCOR Industry Degraded Core Program

QUEST Quantitative Uncertainty Estimation of the Source Term

AE Architect-engineer studies

RAP Regulatory Analysis Program

Figure 1-1. Relationships of Research Programs to SARRP Tasks 
Radionuclide Release Under Specific Accident Conditions [4]. ${ }^{*}$ Radiological source terms for accident scenarios of secondary significance were generally derived at Sandia from the results of STCP analyses with similar characteristics and from the results of other programs. The uncertainty analysis involved the solicitation of input from experts that was used to modify the STCP results to reflect the range of uncertainty about important phenomena or parameters. This process included adjustment of the results to account for phenomena believed to be important but not included in current STCP models.

The offsite consequences of the severe accidents were calculated at Sandia using both the standard code for this task, CRAC2 [5], as well as the newer code recently developed for this purpose, MACCS [6]. The consequence measures considered included offsite health and economic effects. The final activity described above involved the integration of these analyses into a comprehensive evaluation of risk. This process actually proceeded concurrently with the other tasks to ensure efficient transfer of information at the interfaces among the different types of analyses.

As already noted, all of the activities in SARRP included an evaluation of the principal sources of uncertainty. For the Surry plant this task was completed using two different approaches:

- A limited application of Latin hypercube sampling techniques for key uncertainty issues in each area of the program, termed the limited Latin hypercube (LLH) approach; and

- A method involving three walkthroughs of the entire analysis process using three different sets of inputs and assumptions, corresponding to the range of opinions in the reactor safety community about the key processes involved. This analysis is called the optimistic, central and pessimistic (OCP) approach.

The results of both of these uncertainty representations are discussed in this report, as are the insights derived from a comparison of the outcomes.

Following the reassessment of risk, the effects of a number of potential modifications aimed at improving safety were investigated. For each modification, the elements of the risk calculation were repeated, providing an estimate of the change in risk, or benefit, for each modification. The costs and other impacts associated with each modification were also

"In reality, only a very few of the calculations here were performed with the STCP. Most of the Surry analysis was performed using the codes that preceded and were eventually integrated into the STCP. The term STCP is therefore used somewhat loosely in this report, but calculations reported here that would differ depending on exactly which version of the code was used are noted wherever possible. 
estimated. Together, these parameters provided a relatively straightforward comparison of the benefits and costs of the modifications.

The primary thrust of this report is the presentation of the methodology and results of these analyses and the identification of the conclusions that can be drawn from them. For some of the elements of the analyses, the detailed calculations are provided in other reports: Analysis of Core Damage Frequency From Internal Events: Surry, Unit 1 [2] and Containment Event Analysis for Postulated Severe Accidents: Surry Power Station, Unit 1 [3]. Details are summarized in this report only to the extent that they provide a needed understanding and perspective for the results. Areas of analysis not covered in other reports are described in detail in the appendices to this report.

\subsection{OVERVIEW OF THE SURRY PLANT}

As already noted, the subject of this analysis was Virginia Electric Power Company's Surry Unit 1. It is a Westinghouse-designed pressurized water reactor ( $2441 \mathrm{MWt})$ built by Stone and Webster. The feature of the plant most different from other facilities is the subatmospheric containment, designed as a reinforced concrete cylinder with a steel liner. The facility went into commercial operation in December of 1972.

In order to familiarize the reader with the plant, some of the key system design features are summarized below:

- Three diesel generators for two units, and two main battery sets per unit.

- Three-loop reactor coolant system with U-tube steam generators.

- Two-train, three-pump auxiliary feedwater system with two electric-driven pumps and one turbine-driven pump.

- Decay heat removal system located inside the containment.

- Three high pressure injection pumps, with one normally operating as the charging pump.

- Three code safety valves and two power-operated relief valves for primary system overpressure protection.

- Three principal cooling water systems which affect accident sequence progression: the charging pump cooling system for the high pressure injection system, the service water system which cools the spray recirculation heat exchangers, and the component cooling system which cools the reactor coolant pump thermal barriers.

There are additional containment features important to remember when considering the results and conclusions of this study: 
- As already mentioned, the containment is maintained at a subatmospheric pressure ( $\sim 10 \mathrm{psia})$ to provide additional margin in the case of an accident that results in containment pressurization.

- There are separate containment spray injection and recirculation pumps, and two sets of two recirculation pumps for the sprays, one set inside containment and the other outside. There are no containment fan coolers for removal of post-accident heat.

- The reactor cavity does not communicate directly with the sump, and the cavity only has significant water in it after an accident if the sprays have functioned.

The details of the Surry design important to the analysis and results are discussed where appropriate in the report.

\subsection{ORGANIZATION OF THE REPORT}

The main report summarizes the methods used for all of the reference plants in the rebaselining of risk (in Section 2) and the investigation of risk-reduction measures (in Section 3). Due to the importance of a thorough understanding of the uncertainty issues considered in this program, Section 4 presents a definition of the issues considered in the LLH study and also includes a brief description of the expert review group input for each issue. The results for each major step of the analyses, including the overall risk estimates, are presented in Section 5, while the effectiveness of potential risk-reduction measures and the comparisons of costs and benefits are outlined in Section 6. The conclusions that can be drawn from the program regarding the significance and usefulness of the results are provided in Section 7.

The details of the risk-rebaselining and risk-reduction calculations and results are provided in a separate appendix for each area of the analysis: Appendices $A$ through $C$ discuss the sequence, containment and source term analyses, respectively. Additional detail concerning the results of the risk calculation is included (in Appendix D), and similar detail on risk reduction is provided in Appendix $\mathrm{E}$. Appendix $\mathrm{F}$ is a discussion of the limitations of the LLH uncertainty approach which is provided to allow additional perspective on the insights of the overall study. Finally, Appendix $G$ outlines the methods and sources of data used to estimate the costs for the modifications considered. 


\section{References for Section 1}

1. Reactor Safety Study--An Assessment of Accident Risks in U.S. Commercial Nuclear Power Plants. U.S. Nuclear Regulatory Commission Report WASH-1400 (NUREG75/014), Washington, DC: 1975.

2. Bertucio, R. C., et al. Analysis of Core Damage Frequency From Internal Initiating Events: Surry, Unit 1. U.S. Nuclear Regulatory Commission Report NUREG/CR4550, Volume 3 (Draft Report), Sandia National Laboratories, Albuquerque, NM: April, 1986.

3. Benjamin, A. S., et al. Containment Event Analysis for Postulated Severe Accidents: Surry Power Station, Unit 1. U.S. Nuclear Regulatory Commission Report NUREG/CR-4700, Volume 1, Sandia National Laboratories, Albuquerque, NM: February, 1987.

4. Gieseke, J. A., et al. Radionuclide Release Under Specific Accident Conditions. Battelle Columbus Laboratories Report BMI-2104, Columbus, OH: 1984.

5. Ritchie, L. T., et al. CRAC2 Model Description. U.S. Nuclear Regulatory Commission Report NUREG/CR 2326, Sandia National Laboratories, Albuquerque, NM: 1984.

6. Alpert, D. J., et al, "The MELCOR Accident Consequence Code System." Proceedings of the CEC Workshop on Methods of Assessing the Off-Site Radiological Consequences of Nuclear Accidents. (to be published). 


\section{Section 2 METHODOLOGY FOR REBASELINING OF RISK}

The overall approach taken in performing the reassessment of the risk of the Surry plant was similar to that employed in performing a plant-specific probabilistic risk assessment (PRA). This section describes that process as it was implemented for SARRP. The major elements of the SARRP methodology were outlined in Section 1.2 and are displayed in Figure 2-1 in terms of the types of input and output required for each area. The methodologies used in the analysis for each major task are discussed in more detail in this section: Sections 2.3 through 2.6 describe the analysis methods for the activities illustrated in the figure. Additional detail on each of the tasks is provided in the appendices to this report. Sections 2.1 and 2.2 below address two topics that were critical to the SARRP study of Surry: (1) project integration and review, and (2) uncertainty analysis methods.

\subsection{OVERVIEW OF RISK INTEGRATION AND REVIEW ACTIVITIES}

The accomplishment of the objectives of SARRP required a great deal of analysis by many individuals with differing areas of expertise and associated with a number of different organizations. The process used to effectively integrate all of these activities was therefore critical to the success of the program. The basic integration steps employed in SARRP are described in Section 2.1.1. An equally important activity was the assurance of technical quality through effective review as discussed in Section 2.1.2.

\subsubsection{Integration of Project Activities}

As illustrated in Figure 2-1, the analysis in each of the principal task areas generated a substantial volume and variety of information. In order to provide the necessary coordination among the various portions of the analysis, as well as to develop a manageable number of accidents for which consequences would be estimated, the intermediate results at each step of the analysis were sorted into groups, which in turn served as the inputs to the next step. Although this consolidation into groups involves some loss of resolution, the results and insights of each task drew on all of the outputs, and the detail has been maintained in the results sections of this report. The key transition points are illustrated in Figure 2-2; the means by which the integration steps were performed include the following:

- Plant-damage states. The core-damage sequences were grouped into plantdamage states according to the conditions that could affect the response through the containment event tree. These conditions include the pressure 


\section{INPUT}

PROCESS

OUTPUT

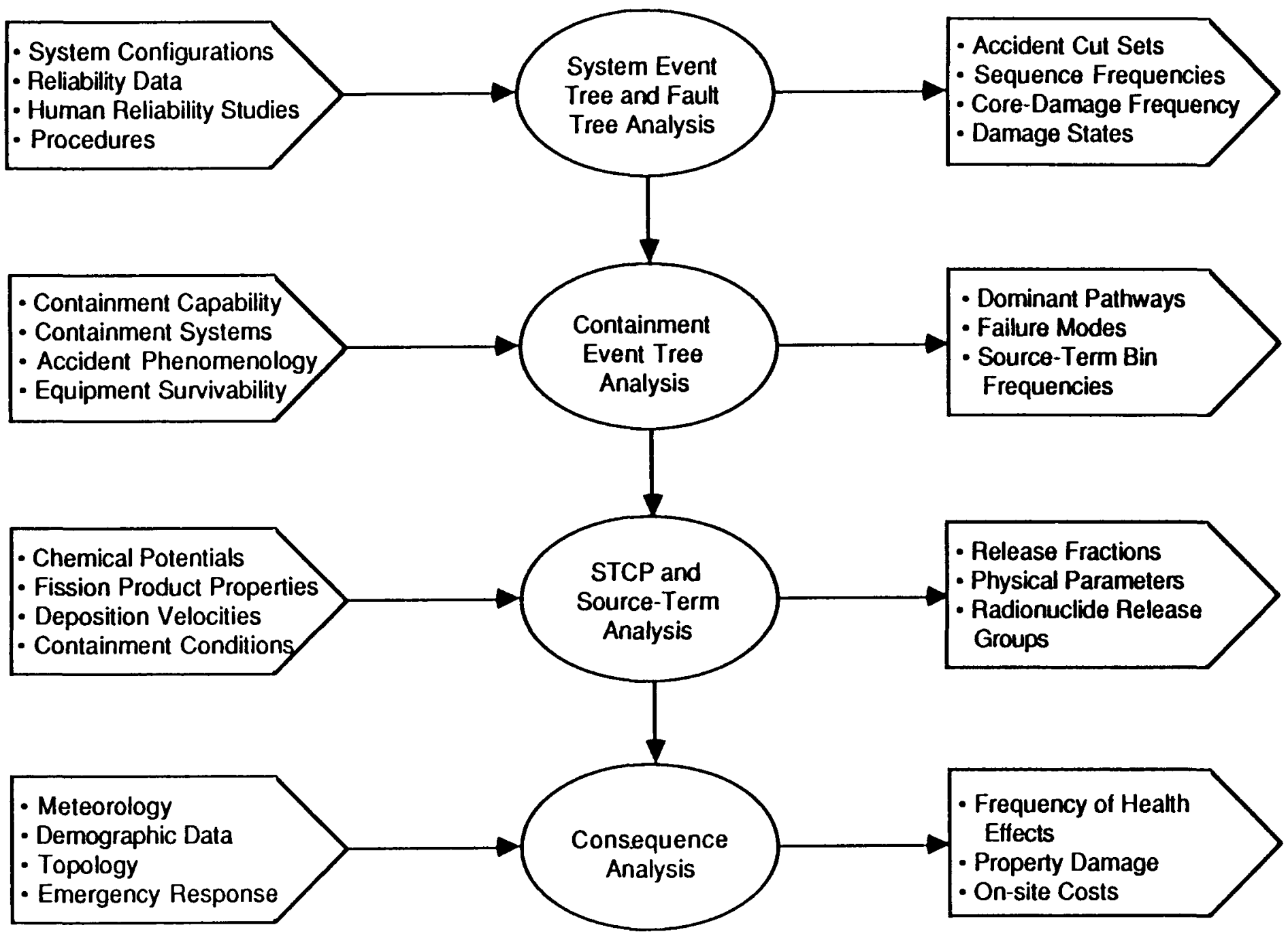

Figure 2-1. Overview of SARRP Methodology 
of and leakage rates from the reactor-coolant system, the status of containment safety features and other systems, and the timing of the event progression.

- Source-term bins. These bins collected the outcomes of the containment event tree into categories according to the features that have a unique impact on the variables important to the radionuclide release; these features include mode and timing of containment failure and status of the containment spray system. Every event-tree outcome assigned to the same bin was assumed to have the same source term (including such release characteristics as timing and energy of release).

- Radionuclide release groups. The fission products were grouped according to similarities in physical and chemical properties that could affect the manner in which they are transported and released. Every radionuclide in a particular release group was assumed to have the same release fraction.

- Source-term clusters. The sampling performed in the LLH uncertainty analysis described in Section 2.2 produced a very large number of source terms, such that it was not feasible to perform consequence calculations for all of them. Therefore, clusters were used to group source terms that would be expected to produce similar consequences. All of the source terms assigned to a given cluster were assumed to have the same offsite consequences, although they might arise from very different containment event-tree outcomes.

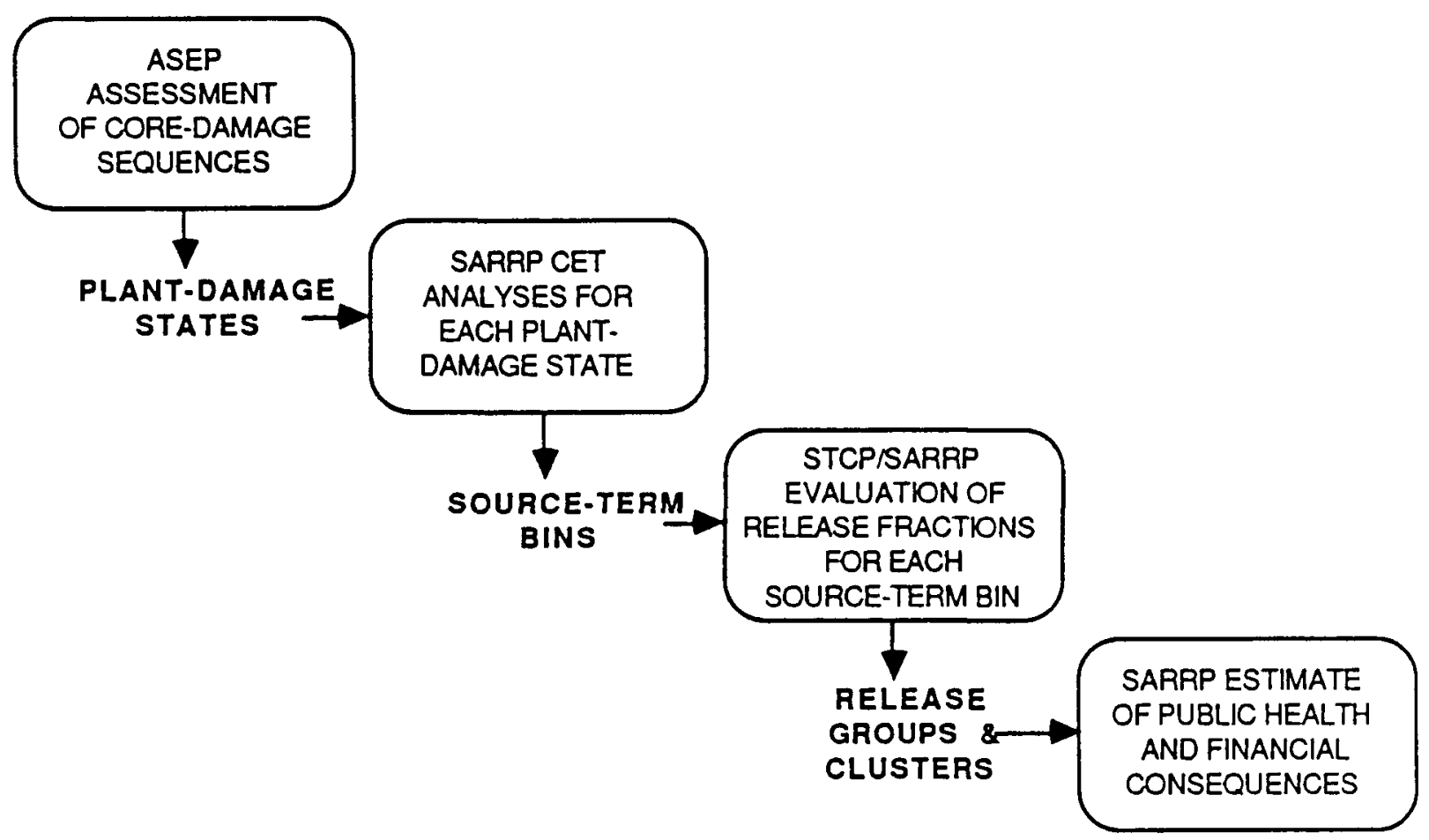

Figure 2-2. Integration of SARRP Tasks 
The key accident sequence boundary conditions that have a significant impact on the assessed phenomenology in the containment event tree (CET) were identified by the CET analysts. The ASEP accident sequence analysts and the CET analysts then interacted to identify the degree to which the sequences needed to be discriminated to preserve the boundary conditions important to the CET analysis, and also to provide preliminary indication of the relative importance of different sequence types. A definition of a limited number of damage states was then developed; for Surry this resulted in about 12 damage states that had nonnegligible frequency. The damage states serve one other purpose: they accommodate change or update without necessarily involving a great amount of new containment analysis that would be required if every sequence were analyzed separately.

The containment analysis in SARRP was made possible through the development of a new computer code (EVNTRE) to manipulate all of the data and automatically trace and quantify the pathways [1]. The code was reviewed by the CET review group and is currently in use in several other programs.

The CET developed in this program was very large, and millions of individual pathways through the tree for each damage state were calculated. This number of outcomes could obviously not be propagated through the source term and risk study. The CET and source term analysts therefore defined the radiological source term bins that collected the pathways into discrete categories with similar characteristics relative to the source term study. Due to the size of the basic tree developed initially, most of the criteria important to the source term could be discriminated directly, although in some cases the interaction at this interface resulted in some changes to the CET to better represent the source term study. The task of defining source term bins also involved external review as described in the following section. The CET code (EVNTRE) was used to automatically sort the outcome pathways according to the definitions of the bins.

The investigation of radionuclide releases led to the development of source-term bins, which were defined in the context of the parameters important to the offsite consequence analysis, including physical conditions (i.e., timing, duration, and energy of release) and release fractions for the radionuclide groups. The SARRP analysts and the source-term review group were heavily involved in the definition of the source-term bins.

Due to the very large data manipulation requirements, the integration of all of the areas of the study into final risk results was accomplished using a computer code developed 
specifically for that purpose. This code, called the Risk Integration, Sensitivity and Quantitative Uncertainty Evaluation code, or RISQUE*, requires the following input:

- Frequencies of plant-damage states;

- Containment event tree outcomes in terms of frequencies of source term bins for each plant damage state; and

- Magnitudes of public health consequences and cost impacts for each source term bin.

The magnitudes of the release fractions for each source term bin are not included in the RISQUE code input because they are used directly as the input data for the consequence analyses using the CRAC2 or MACCS codes. The RISQUE code also manipulates the information associated with uncertainty analysis and the risk-reduction evaluation. These other uses are discussed in Sections 2.2 and 2.3.

\subsubsection{Review and Ouality Assurance}

There were a number of internal review activities associated with the task performance in SARRP. These activities included independent checks of code input and output, review for reasonableness and consistency, thorough documentation, and peer and management review of results and documentation.

In addition to the diligence and internal review processes that help to ensure a quality product, all important aspects of the study were subject to review by experts not directly involved in the project analysis. These external reviews covered methodology as well as specific application of the methods.

The ASEP evaluation of accident sequences was reviewed by two separate groups: (1) a senior consultant group (SCG) which focused on the the basic methods and their manner of implementation, and (2) a quality control (QC) team which considered the details of the analysis of the Surry plant. The members of the SCG and QC teams for Surry are listed in Table 2-1.

The containment event trees (CETs) were reviewed in considerable detail by the containment event tree review group consisting of representatives from other national

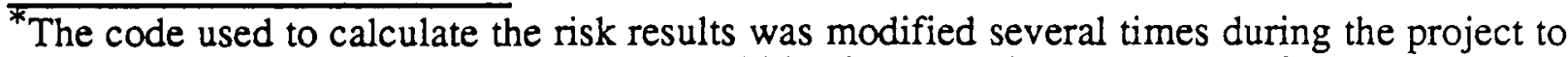
improve ease of use and to expand its capability for analyzing the results. Some of the final risk calculations were completed with a version of the code called RISKEE. The details of the code manipulation will be found in a forthcoming report [2].
} 
laboratories, a university and a reactor safety consulting firm (see Table 2-1). In evaluating the CETs, the review group set out to answer the following questions:

- Is the CET reasonable?

- Are the questions in the tree scrutable, complete, and consistent?

- Is the level of detail appropriate?

- Is the CET quantification reasonable?

- Are the branch point probabilities traceable?

- Given traceability, are the referenced sources documented, reviewed and complete?

- Is there an underlying methodology for the estimates (i.e., consistency of the approach)?

The CET review group provided a number of recommendations which have been published in a separate report [3]. Where practical within the schedule constraints of SARRP, the project team attempted to implement these recommendations.

The radiological source term review activities were more interactive in nature. Review meetings attended by representatives of Sandia, Battelle Columbus Laboratories, Brookhaven National Laboratories, and the NRC were held periodically to consider the selection of STCP input for the accident sequences. The methods used to derive source terms for all sequences were reviewed by a separate review group whose makeup is also listed in Table 2-1. The STCP itself has been the subject of an extensive review by several major NRC contractors [4].

All task areas had the benefit of one additional level of review associated with the uncertainty tasks discussed in the next section. The members of the expert review groups that provided the LLH input required a fairly detailed knowledge of task performance in order to assess the weighting factors. This process therefore offered another level of review which did lead to some improvements in the models and data. 
Table 2-1

PARTICIPANTS IN SARRP REVIEW TASKS FOR THE SURRY ANALYSIS

\begin{tabular}{|c|c|c|}
\hline Review Activity & Participant & Affiliation \\
\hline $\begin{array}{l}\text { ASEP Senior Consultant } \\
\text { Group }\end{array}$ & $\begin{array}{l}\text { D. Bley } \\
\text { M. Bohn } \\
\text { J. Murphy } \\
\text { W. Vesely }\end{array}$ & $\begin{array}{l}\text { Pickard, Lowe and Garrick, Inc. } \\
\text { Sandia National Laboratories } \\
\text { U. S. Nuclear Regulatory Commission } \\
\text { Science Applications International Corp. }\end{array}$ \\
\hline $\begin{array}{l}\text { ASEP Quality Control } \\
\text { Group }\end{array}$ & $\begin{array}{l}\text { B. Bell } \\
\text { G. Kolb } \\
\text { E. Krantz } \\
\text { G. Parry } \\
\text { A. Payne }\end{array}$ & $\begin{array}{l}\text { Battelle Columbus Laboratories } \\
\text { Sandia National Laboratories } \\
\text { Idaho National Engineering Laboratory } \\
\text { NUS Corporation } \\
\text { Sandia National Laboratories }\end{array}$ \\
\hline $\begin{array}{l}\text { SARRP Containment } \\
\text { Event Tree Review } \\
\text { Group--Surry }\end{array}$ & $\begin{array}{l}\text { M. Corradini } \\
\text { R. Denning } \\
\text { S. Hodge } \\
\text { T. Pratt } \\
\text { A. Torri }\end{array}$ & $\begin{array}{l}\text { University of Wisconsin } \\
\text { Battelle Columbus Laboratories } \\
\text { Oak Ridge National Laboratory } \\
\text { Brookhaven National Laboratory } \\
\text { Pickard, Lowe and Garrick, Inc. }\end{array}$ \\
\hline $\begin{array}{l}\text { SARRP Containment } \\
\text { LLH Issues Review } \\
\text { Group--Surry }\end{array}$ & $\begin{array}{l}\text { K. Bergeron } \\
\text { M. Berman } \\
\text { M. Corradini } \\
\text { R. Denning } \\
\text { G. Greene } \\
\text { K. Perkins } \\
\text { A. Torri } \\
\text { W. Von Riesemann }\end{array}$ & $\begin{array}{l}\text { Sandia National Laboratories } \\
\text { Sandia National Laboratories } \\
\text { University of Wisconsin } \\
\text { Battelle Columbus Laboratories } \\
\text { Brookhaven National Laboratory } \\
\text { Brookhaven National Laboratory } \\
\text { Pickard, Lowe and Garrick, Inc. } \\
\text { Sandia National Laboratories }\end{array}$ \\
\hline $\begin{array}{l}\text { SARRP Source } \\
\text { Term Binning } \\
\text { Group--Surry }\end{array}$ & $\begin{array}{l}\text { A. Benjamin } \\
\text { P. Cybulskis } \\
\text { R. Denning } \\
\text { F. Haskin } \\
\text { J. Mitchell } \\
\text { D. Williams }\end{array}$ & $\begin{array}{l}\text { Sandia National Laboratories } \\
\text { Battelle Columbus Laboratories } \\
\text { Battelle Columbus Laboratories } \\
\text { Sandia National Laboratories } \\
\text { U. S. Nuclear Regulatory Commission } \\
\text { Sandia National Laboratories }\end{array}$ \\
\hline $\begin{array}{l}\text { SARRP Source Term } \\
\text { LLH Issues } \\
\text { Review Group }\end{array}$ & $\begin{array}{l}\text { R. Denning } \\
\text { J. Gieseke } \\
\text { T. Kress } \\
\text { D. Powers } \\
\text { T. Pratt }\end{array}$ & $\begin{array}{l}\text { Battelle Columbus Laboratories } \\
\text { Battelle Columbus Laboratories } \\
\text { Oak Ridge National Laboratory } \\
\text { Sandia National Laboratories } \\
\text { Brookhaven National Laboratory }\end{array}$ \\
\hline
\end{tabular}




\subsection{APPROACHES TO THE EVALUATION OF UNCERTAINTIES}

The treatment of uncertainty within this program is defined in this section. Sections 2.2.2 and 2.2.3 describe the two methods used in the study of Surry, while the first subsection provides the background which explains the selection of the methods.

\subsubsection{Qverview of Uncertainty Treatment}

Although there have been significant advances in all areas of risk-assessment technology, there remains significant uncertainty in each of the analysis tasks. The appropriate means by which to characterize this uncertainty remains a topic of substantial debate within the technical community, and there was no attempt in this program to resolve these differences by developing the "correct" representation of uncertainty. The most important results of the analyses reported in this document are engineering and scientific insights that become evident after the completion and integration of each of the steps in the program and thorough review of the results. However, the significance of many of these insights can often be better understood within a quantitative framework. It is therefore essential that a clear presentation be made of the elements considered to be uncertain, and of the potential effects of these uncertainties on the results. The formulation of the uncertainty presentation for the SARRP results therefore had the following objectives:

- Provide decision-makers with engineering and/or scientifically based information that allows them to understand the analysts' treatment of important issues as well as the impact on the analysis of the range of viewpoints that experts in the field hold for these issues.

- Provide a quantitative estimate of uncertainty that reflects a credible and realistic range in which the analysts have a reasonable confidence that the correct answer lies.

- Identify as completely as possible the key sensitivities and sources of uncertainty for each portion of the analysis.

- Discuss qualitatively the uncertainty and sensitivities that have the most significant impact on calculated risk measures.

- Estimate, for each part of the analysis, a quantitative impact on the results for the uncertainties that were identified.

- Evaluate the quantitative impact on the total risk measures of the uncertainty in each part of the analysis and the combinations of the uncertainties and sensitivities for the different portions of the analysis.

Because many portions of the analysis rely heavily on expert opinion and only limited data from actual experience, it was judged that a direct statistical treatment of all uncertainties was neither practical nor necessarily meaningful. In addition, another NRC-sponsored 
program, the Probabilistic Risk Uncertainty Evaluation Program (PRUEP), is investigating a more comprehensive evaluation of uncertainty [5].

The statement of the SARRP goals relative to uncertainty relies on language that is highly subjective. The question of "reasonable" assessment is certainly of a subjective nature, and each analyst involved would have different interpretations of its meaning. It was intended that the general trend in the analysis be such that the uncertainty presentation represents a belief on the part of the analysts that there is roughly a $90 \%$ probability that the correct result would lie within the range. Clearly, this is difficult to assess, impossible to test, and analyst dependent. In addition, several of the participants in the uncertainty analysis task feel very strongly that the methods of uncertainty input collection and manipulation rule out any probabilistic interpretation such as a confidence interval. This is discussed further at the end of this section, in the limitations discussions accompanying the results and in Appendix F. Another problem is semantics, particularly associated with the use of the terms "uncertainty" and "sensitivity". The basic method chosen for the uncertainty representation in this study does have some of the features of a sensitivity study, but the results are defined here to represent a measure of uncertainty. There was considerable discussion within the expert review groups about the use of terminology, and individual interpretations are contained in Appendix F. Once again, since universal definitions are not available, these terms are defined here only as they apply to SARRP. The terminology associated with the two uncertainty treatments is defined with the basic methodology discussion below.

For all portions of the analysis, qualitative discussions of the important contributors to uncertainty are provided. This is especially useful for some of the areas in which it was not possible to generate a meaningful quantitative estimate of uncertainty.

\section{Sources and Types of Uncertainty}

There are many ways to categorize the sources of uncertainty in a risk assessment. One characterization involving three types of uncertainty is presented below:

- Data

- Modeling

- Construction
Uncertainties arising from incomplete or inconsistent data pertaining primarily to equipment failure rates.

Uncertainties arising from incomplete or incorrect modeling of either phenomenological processes or engineered systems.

Uncertainties arising from variability in plant construction or configuration from that designed and studied, due to both errors and variability in physical properties. 
The different analysis activities involve varying degrees of each type of uncertainty. The types of uncertainty associated with each analysis task are summarized in Table 2-2.

Because of time and resource limitations, not all of the uncertainties listed in Table 2-2 were treated in the SARRP analyses, and those that were included were not necessarily considered in the same way. The primary focus of the SARRP uncertainty evaluation was on data and modeling uncertainties. Data uncertainties were evaluated only for the ASEP coredamage frequency study. Modeling uncertainties were considered for the sequence, containment and source term analyses, but not for the consequence study. The modeling uncertainties for the various tasks were combined together to give a composite uncertainty due to modeling. These modeling uncertainties were not, however, combined with the data uncertainties which are always displayed separately. Construction uncertainties were considered only with respect to containment structural capability (i.e., failure pressure), and this evaluation was limited in scope in that it did not involve a new, plant-specific evaluation of containment capability and uncertainty.

Once stated, the means of satisfying the uncertainty analysis objectives listed above were not immediately obvious. The program analysts and the Nuclear Regulatory Commission sponsors initially agreed to one method of uncertainty treatment which was later determined to meet the objectives less fully than desired. Another method of evaluation was then developed and used. For the Surry plant, both methods were implemented, affording a unique opportunity for comparison and derivation of additional insights over either alone.

One element was common to both approaches--the evaluation of data uncertainties for the ASEP estimate of core-damage sequence frequencies. The data uncertainty was calculated as a statistical parameter using standard Monte Carlo techniques that have been applied in many other risk assessments [6,7]. The probability distributions for individual events were propagated through the model to obtain a $5 / 95 \%$ range of uncertainty about the central estimate.

The principal method of analysis of modeling uncertainty in SARRP is the limited Latin hypercube (LLH) approach, limited because not all uncertainties are addressed. Particular issues selected by the SARRP analysts and an expert review group were investigated by determining the range of outcomes that might be expected and sampling the outcomes based on the assignment of weighting factors by the experts. This method is described in Section 2.2.2.

The other method for modeling uncertainties, completed only for Surry, is called the "optimistic, central and pessimistic" (OCP) approach. This involved three separate walkthroughs of the analysis using assumptions and data considered, within the total spectrum 
Table 2-2

UNCERTAINTY CHARACTERISTICS OF DIFFERENT ANALYSIS ACTIVITIES

\begin{tabular}{lll}
\hline Analysis Task & Uncertainty & \multicolumn{1}{c}{ Comments } \\
\hline Data & $\begin{array}{l}\text { Uncertainty in quantification of fault and event } \\
\text { tree models. }\end{array}$ \\
$\begin{array}{c}\text { ASEP Core-Damage } \\
\text { Sequence Analysis }\end{array}$ & Modeling & $\begin{array}{l}\text { Potential for incomplete or incorrect models: } \\
\text { system success criteria, or phenomenological } \\
\text { (e g. pump seal response). }\end{array}$ \\
Construction & $\begin{array}{l}\text { Possibility of system configurations or capabilities } \\
\text { different from design. }\end{array}$
\end{tabular}

Data

Containment Event Modeling Tree Analysis

Radiological Source

Term Analysis

Data

Modeling
Some system data uncertainties and some interpretation of applicable research data.

Potential for incomplete or incorrect models of phenomenology or plant response or errors in implementation of models. Some phenomena have very different models proposed by different experts.

Construction Principal concern is in the containment response relative to predicted failure characteristics.

Collection and interpretation of applicable research data.

Potential for incomplete or incorrect models of phenomenology or a lack of any model at all. The modeling uncertainties in this task extend to a very fine level of detail concerning the behavior of individual species in a variety of circumstances. Errors in coding or input data are also possible.

Construction Not of primary concern for this task.

Data

Consequence Analysis Modeling

Construction Not applicable.
Some uncertainty in demographics, weather, etc. although impacts are rather limited

Potential for incomplete or incorrect models of health effects, physical phenomena or emergency response. 
of the reactor safety community, to be optimistic, central, or pessimistic. The method is useful because it considers uncertainty for nearly every input, but suffers from a lack of ability to assess the degree of optimism or pessimism in the results. The methodology is summarized in Section 2.2.3.

As already stated, the construction uncertainty was limited to a consideration of variability in containment failure pressure. The pressure at which containment fails was taken to be a normally distributed variable having a standard deviation about a mean. The standard deviation was used to characterize the construction uncertainties (i.e., departures from design or material properties variability). In addition, the failure pressure was characterized as having a modeling uncertainty component (i.e., variability resulting from the use of different computational algorithms or input failure criteria). This component was treated as part of the LLH and $O C P$ approaches by allowing the distribution itself, both mean and standard deviation, to be a variable. For a given containment pressure load, the likelihood of failure was evaluated by comparing the load to the failure distribution. As noted, this study was limited in scope and did not involve a new, detailed evaluation of the Surry containment. Further illustration of this aspect of the uncertainty analysis is given in Section 2.5.2.

\subsubsection{The LLH Approach}

The limited Latin hypercube (LLH) approach was selected as the principal means of modeling uncertainty analysis and representation as it best met the objectives stated previously, within the other constraints such as schedule and budget. Latin hypercube sampling techniques have been investigated in other programs and found to have very good sampling characteristics when compared to other methods [8]. A full description of Latin hypercube sampling may be found in other sources such as Reference 9. The Latin hypercube approach is similar to the better known Monte Carlo techniques, except that the sampling scheme is constrained or stratified. The application of the method and the constraints on the sampling are best explained in terms of the specific process used in SARRP.

The basic interest here is the uncertainty in risk due to the uncertainty in the input information. Risk can be defined by the equation given below:

$$
\begin{aligned}
& \operatorname{RISK}_{\mathrm{k}}=\Sigma_{\mathrm{i}} \Sigma_{\mathrm{j}} \text { FREQ }_{\mathrm{i}} * \mathrm{CRMP}_{\mathrm{i}, \mathrm{j}} * \mathrm{CONS}_{\mathrm{k}}\left(\mathrm{FP}_{\mathrm{i}, \mathrm{j}}\right) \\
& \begin{aligned}
\text { where } \\
\text { RISK }_{\mathrm{k}}=\text { the risk associated with consequence measure } \mathrm{k} \\
\mathrm{FREQ}_{\mathrm{i}}=\text { the frequency of accident sequence } \mathrm{i}
\end{aligned}
\end{aligned}
$$


NUREG/CR-4551, VOL.1: DRAFT REPORT FOR COMMENT (SEPTEMBER, 1986)

$$
\begin{array}{ll}
\mathrm{CRMP}_{\mathrm{i}, \mathrm{j}}= & \begin{array}{l}
\text { probability of containment release mode } \mathrm{j} \text {, given } \\
\text { accident sequence } \mathrm{i}
\end{array} \\
\mathrm{FP}_{\mathrm{i}, \mathrm{j}} & =\begin{array}{l}
\text { fission product source term for containment } \\
\text { release mode } \mathrm{j} \text { of accident sequence } \mathrm{i}
\end{array} \\
\mathrm{CONS}_{\mathbf{k}} & =\begin{array}{l}
\text { mean magnitude of consequence } \mathrm{k}, \text { given fission } \\
\text { product source term (FP) for sequence } \mathrm{i}, \mathrm{j}
\end{array}
\end{array}
$$

There are identifiable uncertainties in each of the inputs to this risk equation. As already described above, a full uncertainty analysis would involve sampling from the range of potential outcomes for every detailed element of every input to the risk equation. The goal here was to identify the major uncertainties in each of the inputs and then determine the effect of the uncertainty on the risk measures due to a reasonable variation of those inputs.

The limited Latin hypercube was the method selected. The entire LLH process used in this study is represented schematically in Figure 2-3. The first feature which must be recognized when using the results of the study is that the uncertainty analysis was not complete. The word "limited" in the title refers to the number of issues selected--only a few major uncertainties were addressed (approximately 25). These uncertainties are defined in terms of "issues" which are high-level representations of uncertainties in the input. For example one issue is the magnitude of pressure increase due to direct heating. This obviously involves many other uncertainties (heat generation rates, thermal-hydraulic uncertainties, amount of debris involvement, physical structure effects on debris transport, chemical reaction uncertainties, etc.) which were not considered individually but rather in terms of their overall effect--the pressure rise due to direct heating. The source term uncertainties involve even broader issues; for example, one fission product issue is concerned with all uncertainties affecting the release of all radionuclides from the fuel during the in-vessel phase of the accident. It was the intent of the LLH study to include all of the most important uncertainties. The issues were initially selected by the SARRP team but were then reviewed by the groups of experts in each area of the analysis. It is anticipated that most of the major uncertainties are addressed, but only more thorough review and comment will verify the appropriateness of the issue set selected. Hence, the first limitation in use of the results is the recognition that the results are limited and not all uncertainties have been addressed.

The second step of the analysis was the generation of a range of outcomes for each individual issue. This was intended to represent a reasonable variation of the outcome (e.g. pressure rise) considering all of the uncertainties in the input parameters of the modeling. A second limitation therefore arises from the subjectivity involved in the selection of 


\section{Steps In LLH Process}

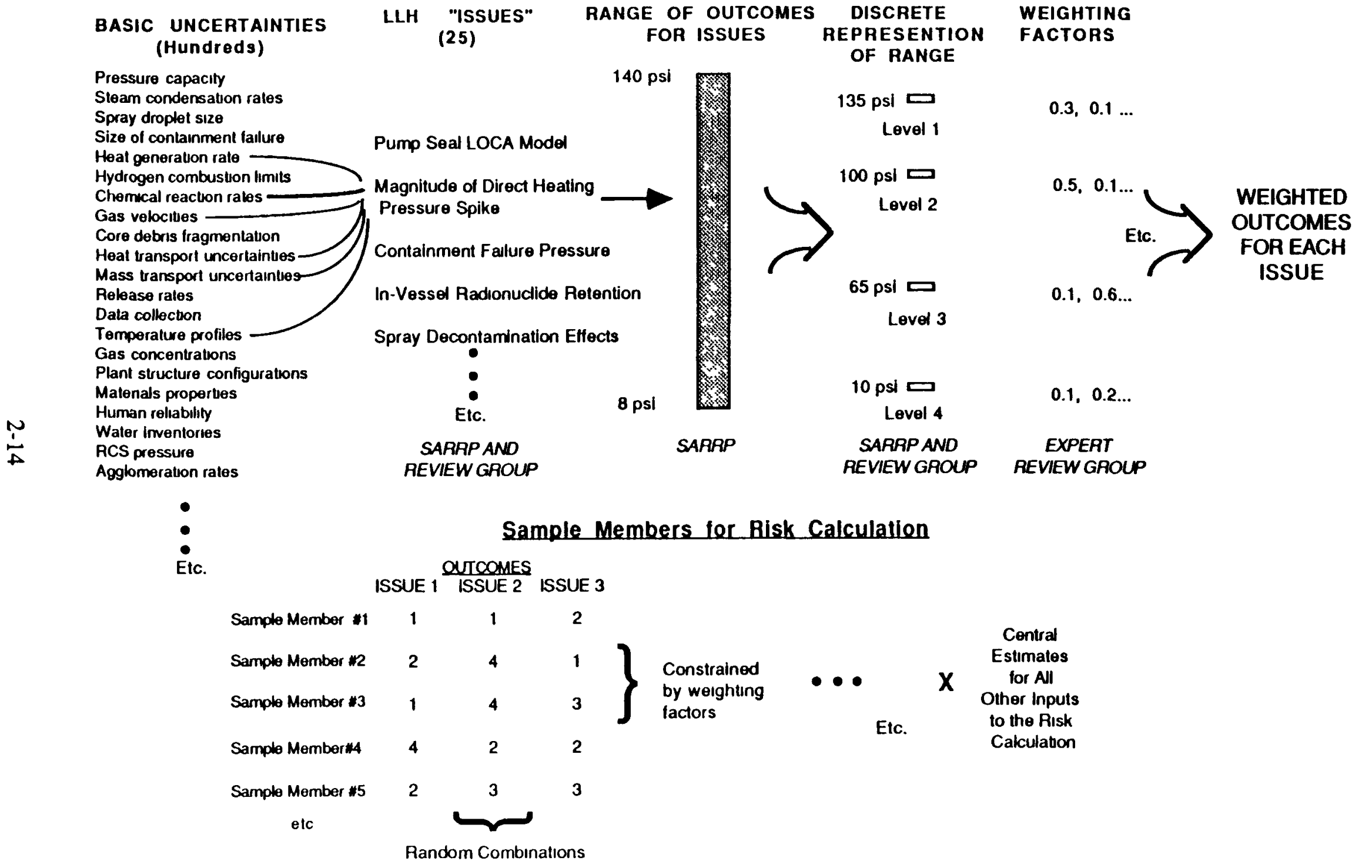

Figure 2-3. Overview of the LLH Process 
"reasonable" range. The ranges were developed by examining the applicable information developed by the reactor safety community. In order to allow efficient manipulation of the information, and to allow expert input in a consistent manner, the ranges were then discretized and represented by specific outcomes (called levels), usually three to five per issue.* Where possible, the levels represented the results of particular analyses performed by various organizations within the reactor safety community. The levels were originally proposed by the SARRP analysts, but the experts provided additional input and changed the levels where appropriate.

The next step in the process was completed by the expert review groups. This activity was more than a review--a group of experts in each area of the analysis was selected to provide their input as to the selection of the range and the reasonableness of the individual outcomes. This input then formed the entire basis for the subsequent sampling process. In addition to the quantitative input, the reviewers were asked to provide information as to the physical processes they thought governed the uncertainty. This qualitative information is perhaps more important than the quantitative weighting factors and considerable effort was expended collecting and reporting this information (see Section 4 and Appendices B and C of this report). The input was derived separately for the three principal areas covered (systems, containment and source term) and is described below after the description of the general methodology.

For each level of an issue, each individual reviewer supplied weighting factors to express his opinion of the relative likelihoods of the outcomes represented by that level. Essentially the reviewers provided their "degree of belief" for each level, with the constraint that the total weighting factors sum to one for the issue. There is a great deal of subjectivity involved not only in the input but also in the individual interpretations of the process:

- Reviewers had to judge "reasonable" ranges in terms of their own experience and views on the uncertainties in the issues, aided by their awareness of prior analyses.

- The expression of "degrees of belief" when dealing with a range that has been discretized into a limited number of levels also involves interpretation.

- There could be differing views of the scope of individual issues.

The process used to elicit the weighting factors from the review team was designed to minimize the potential problems associated with individual interpretation. The SARRP project team issued to each individual a package that described the background, reference material, and

*This discretization can lead to further limitations in statistical interpretation of the results as discussed in Section 5. 
role of the issue in the analysis, and suggested the levels to represent the range of outcomes. A meeting was then scheduled that was attended by all reviewers. At the meeting the SARRP team provided some explanation of the issue to ensure that all individuals were considering the same scope of problem and a common issue definition. The reviewers were able to then discuss their preliminary opinions and to question each other as well as the SARRP analysts. This interactive process, which is documented in the appendices to this report, helped to identify the principal physical processes contributing to the uncertainty in the issues and to clarify the individual expert's views. There are both advantages and piffalls to this interactive solicitation of input which are discussed at the end of this section, but schedule constraints did not allow a more rigorous approach to be undertaken. At these meetings the reviewers provided their weighting factors as well as any adjustments to issue and level definitions (in some cases the reviewers provided some final values after the meeting).

After collection of all of the input, the groups' weighting factors were combined into composites to represent the issue in the LLH sample. The process of creating composites to represent the group depended on the type of input for the issue. Some of the issues involved specification of discrete distributions to approximate a continuous variable (e.g., pressure rise from direct heating). For these issues, each reviewer gave his view of the distribution by providing weighting factors on parameter values. The composite represented a distribution comprised of the average of the individual responses. Other issues required probabilistic input for a branch point (e.g., probability of ignition of hydrogen for a given sequence), and each reviewer was asked to provide a single probability estimate. Two methods were used to represent such issues. In the first method, each reviewer's probability was weighted equally and assigned as an issue outcome for the sampling process; thus the number of outcomes equaled the number of responses. In the second method, the probability space was discretized into two outcomes (zero and one), and the reviewers' responses were averaged to obtain composite weighting factors for the two outcomes. In the final results, the first method was used for all probabilistic issues except the issue involving likelihood of induced LOCA (as described in Section 4.2.1).*

As illustrated in Figure 2-3, this process resulted in the reduction of the hundreds of individual uncertainties with wide ranges of possible outcomes into a limited number of weighted outcomes for the whole uncertainty analysis (about four levels each for twenty-five

*The first method of treatment could understate the range of uncertainty for issues involving a probabilistic outcome, whereas the second method might overstate the uncertainty. Sensitivity studies on Surry indicated that the use of one method versus the other would not change the results significantly. 
issues). The key to the LLH method is that the individual issue levels are constrained by the weighting factors--the fraction of sample members having a given outcome for a given issue is equal to the weighting factor. For example, if the weighting factor for the highest pressure increment associated with direct heating is 0.1 , then this outcome will be in $10 \%$ of the sample members. In addition, the method did allow for correlations between issues or issue levels. These correlations were minimized to those essential for a correct characterization because of the increased complexity of the input and to maintain some separation among the issues so that their individual effects on the results could be identified. All correlations were decided by a consensus of the review group. The combination of issue outcomes for the different issues is not constrained; it is a random sampling (except for the intended correlations). Accidental correlations that can occur in any random sampling were limited by performing the random sampling several times and selecting the input with the least accidental correlation as measured by statistical tests. The sample for Surry consisted of 100 sample members. Each sample member has one of the outcome levels for each of the issues, while all other inputs not associated with the LLH issues are maintained at a central, or best estimate value determined by the SARRP analysts.

A typical result is illustrated in Figure 2-4. All of the sample members are calculated and plotted. The specific combinations of issue outcomes associated with each sample member are also available to enhance understanding of particular sample members. For display and discussion purposes, the plot of the results includes a bar that illustrates the 5th and 95th percentile of the sample. It must be recognized that this bar does not have statistical meaning concerning the uncertainty in risk, it merely applies to the distribution of the sample members. The results of this task are limited to a representation of the uncertainty in the risk measures attributable to the limited number of specific issues selected, and are further limited to the input of those providing the weighting factors. It is only an approximation to the uncertainty in risk, and a different group of experts could well have established weighting factors with different results. It is hoped that the selection of the review group allowed the representation of the industry, but it is known (post-completion) that the review group did not provide ranges for some issues as wide as those that would be indicated by literature on the subject. Nevertheless, it was the intent to provide the NRC with an analysis of reasonable uncertainty in the results; the degree of success will be judged by review of the input and results by other experts.

Figure 2-5 illustrates another output of the LLH analysis. Rank regression techniques were used to evaluate the relative importance of specific issues. The regression calculated the 


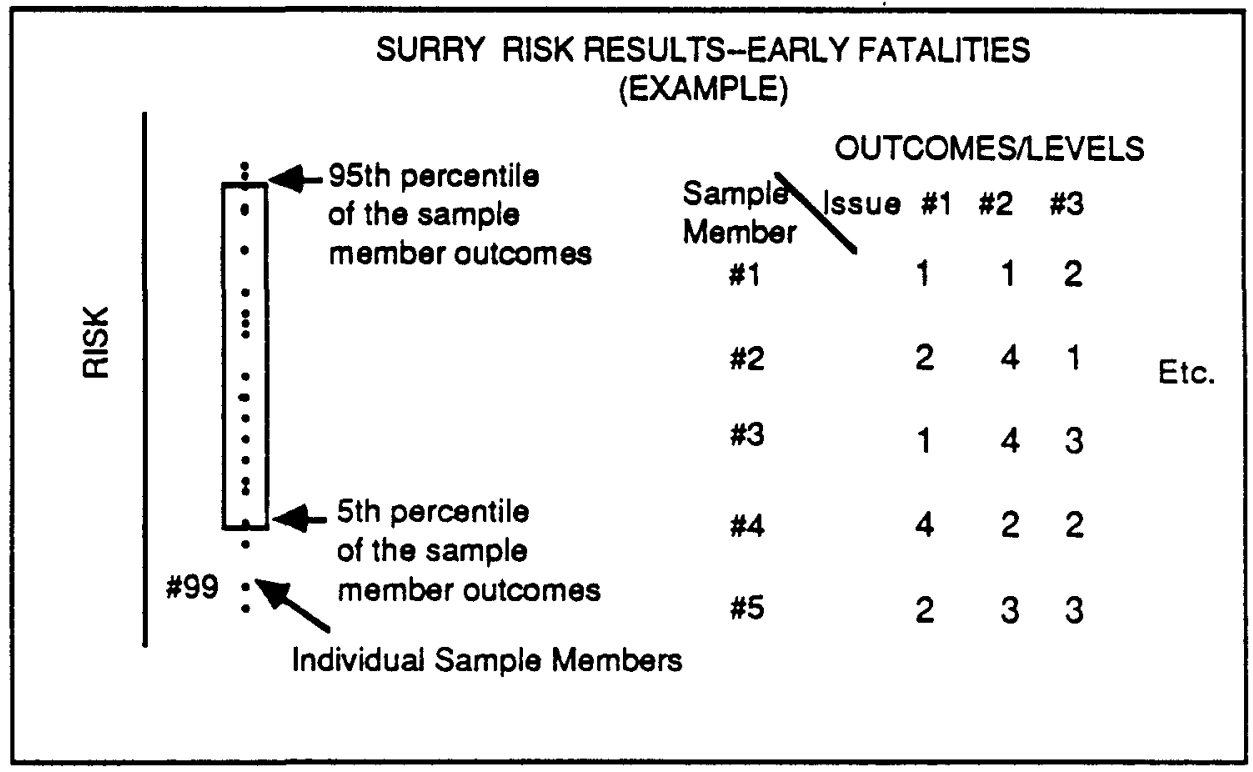

Figure 2-4. Example of LLH Output for Risk Measures

fraction of the total variance explained by the individual issue. " These techniques are a good supplement to detailed review of the results; however, the statistics of the sample sizes used for SARRP do not always allow for meaningful regression analyses. These importance calculations are completed where meaningful, and any limitations associated with the method of calculation are explained. In all cases, the insights generated by a thorough review of the results by the SARRP team are also included.

Additional statistical tests are also included. The $\chi^{2}$ test for "goodness of fit" was performed to help determine the probability of deviation from expected outcomes. Unfortunately, this test requires an expectation of five members in each category, and with the number of issues and levels in the Surry sample only two categories could be defined: below the median and at or above the median. Thus this test results in a probability that any clustering of sample members with specific issue outcomes either below or above the median has occurred as a result of chance alone. This test is done for each issue level and for each issue as a whole, although the latter would rarely be expected to be significant since it would require all of the levels to be clustered either below or above the median (the middle levels for the issue

\footnotetext{
${ }^{*}$ If all of the uncertainties could be accurately modeled, the fractional variances would sum to unity. However the correlations in the sample cause some issues to explain variance due to a correlation with another issue, and the total variance fraction sometimes exceeds one.
} 


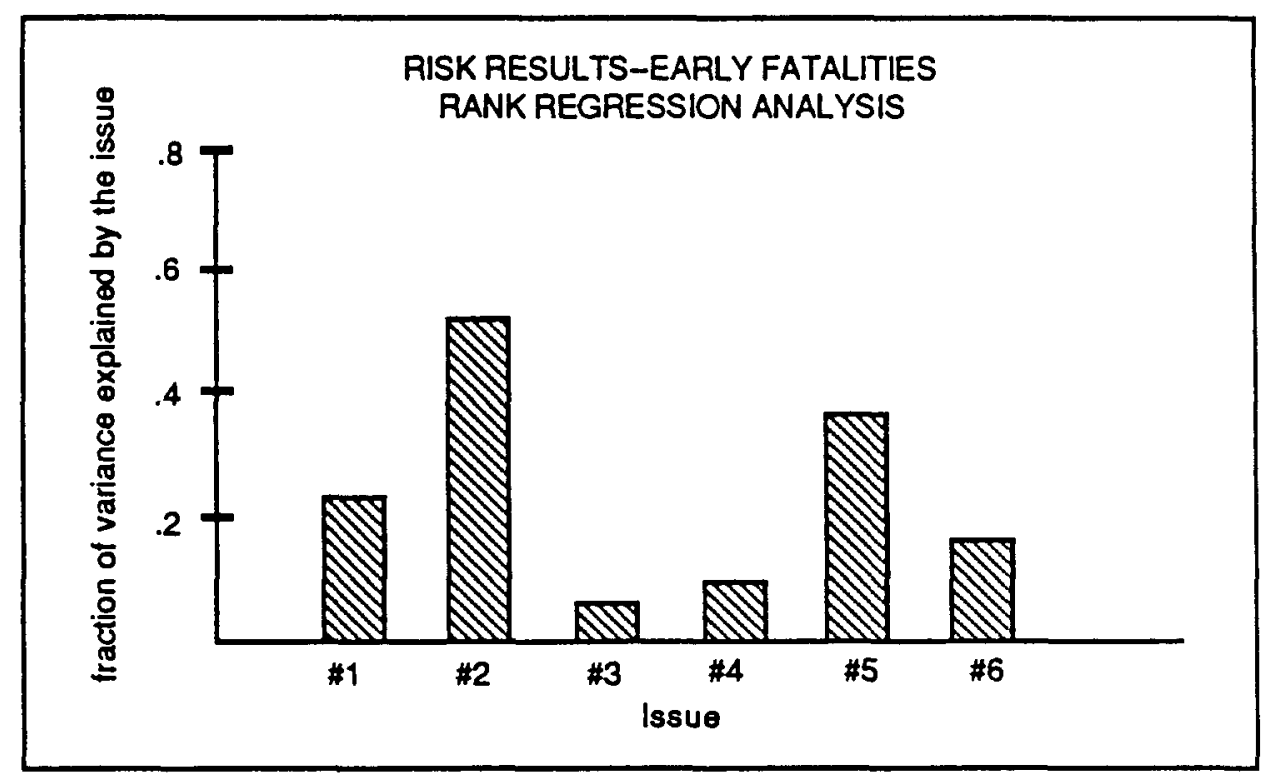

Figure 2-5. Example of LLH Rank Regression Analysis

are very likely near the middle of the outcomes by the way the problem was set up). The $\chi^{2}$ test can be used to corroborate other tests of issue importance.

The final statistical test of the data included in the analysis package is a STEP multiple regression which fits all issues to risk (rather than rank of risk) [10]. The poorest fitting issues are dropped, one at a time, until a specified significance level is reached. The issues remaining give the best fit to the risk data within that significance. This test sometimes helps to further identify the issues driving the results for a particular risk measure, but the setup of the LLH is not particularly well suited to this regression and the results are only useful as a diagnostic tool.

In the course of implementing the LLH analysis, a number of observations were made that provide additional perspective in attempting to understand the process and the results obtained. First, one of the factors that led to the selection of the LLH analysis was the desire to pursue an approach that would culminate in the representation of uncertainty in the risk results by bounds representing "reasonable" uncertainty. It was hoped that the analysts participating could say that they had roughly $90 \%$ confidence that the correct result would lie in the range calculated. This was viewed as a weakness of the OCP approach, since it was not possible to interpret the optimistic and pessimistic values as the bounds of any particular confidence interval. However, because the LLH uncertainty analysis is limited (in that only a relatively small number of areas assumed to be most important were considered) and because it relies 
completely on the assignment of subjective weights for the individual issues, it is inappropriate to interpret the $L L H$ results in the context of a probability distribution, either. Instead, the $L L H$ results can be considered to represent a range within which the "true" value of risk could reasonably be expected to lie, with no statistical interpretation of "reasonable". This statement, however, is subject to the caveat that the analysis was limited in scope; for example, external initiating events and operator errors of commission (as distinguished from errors of omission) were not considered." Subject to these limitations (which are described further in Section 5), it is believed that the most significant uncertainty issues were treated and that the limited number of issues results in a range that is not significantly narrower than that which would result from consideration of more issues.

It was the initial intent that the selection of the review-group members provide for representation of the broad spectrum of views within the nuclear-safety community, but in practice the review groups were comprised primarily of persons engaged in NRC-sponsored research. In particular, the IDCOR program and other industry viewpoints were not well represented. A preferable approach, had it been practical within the constraints of the program, would have been to compile a large list of qualified reviewers, and to assemble the review group based on a random selection from that list. As in the previous discussion, one aspect of the makeup of the groups that provided input on the LLH issues would be a tendency for the ranges to be narrower. However, because the levels for specific issues tended to cover a broad range, it is reasonable to assume that a different composition of the review group would result in a shift in weighting factors, but not necessarily in the levels considered. This would imply that the range of LLH outcomes might not be appreciably changed, particularly given the methodology used to calculate composites--arithmetic averaging. Other methods of manipulating input from a wider group of experts might have resulted in somewhat different uncertainty presentations, as demonstrated by some of the OCP results presented in Section 5.

Furthermore, although the interactions among the group members have been identified as a positive attribute of the process due to the resultant sharing of information and the assurance that all of the reviewers had an equivalent understanding of the issue they were being asked to evaluate, these interactions also raised a concern. Frequently, the interactions appeared to lead to a shift in the positions of some of the reviewers toward consensus. This is primarily of importance relative to the treatment of certain containment issues that were characterized as probabilities, with each reviewer's input treated as a level. Consensus in these instances could lead to a definite narrowing of the uncertainty range. A more appropriate

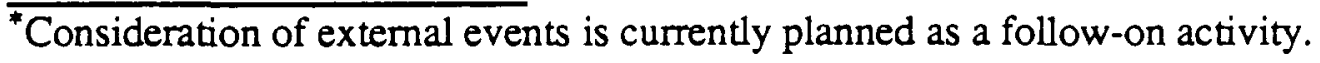


approach might have been to propose levels corresponding to a range of probabilities, and to allow the reviewers to assign weights to the levels.

The application of the LLH method to each area of the analysis and the types of results displayed are described below. Section 4 of the main report discusses the actual issues considered in the analysis of Surry.

\section{Core-Damage Frequency Study}

The uncertainty analysis for the core damage sequences was completed by the systems analysts in consultation with other reviewers and did not involve the same process concerning review meetings described above for the other inputs. Two quantitative measures of uncertainty were calculated: (1) sensitivity of the results to important phenomenological, data collection or interpretation, or modeling uncertainties, and (2) uncertainty due to data variability. The latter was done independent of the LLH study and is provided directly for the results of the core-damage frequency analysis. The outcomes of the sensitivity studies were used in the LLH approach. From the twelve sensitivity studies calculated by ASEP, five issues were defined. The sensitivity studies defined the upward and downward sensitivity to the issues relative to the central estimate. The ASEP analysts also provided weighting factors for the outcomes associated with the sensitivity studies which were then used directly in the LLH input.

\section{Analysis of Containment Response}

The containment event tree provided the framework for quantification of the likelihood of various containment failure modes. The branch points corresponding to the LLH issue outcomes selected for inclusion were restructured in terms of the level definitions representing the range of outcomes. The dependencies in the CET were also checked to ensure that the correlations provided by the review group were appropriately modeled. All other branch points were quantified using the central estimate of the CET study [11]. The actual issues are defined in Section 4 and the experts providing the input were listed previously in Table 2-1.

The results of the LLH study were also decomposed to allow examination of the uncertainties relative to measures other than risk. The containment parameter of most interest with respect to risk is the probability and timing of containment failure given a core-damage accident. Therefore the type of display represented in Figure 2-4 for risk measures was also created for lower level results, e.g., the probability of early containment failure for all 
sequences, and for selected individual sequences where useful. The results of other studies and the OCP method described below are also included for comparison.

\section{Source-Term Uncertainties}

The central estimates of the radiological source terms were obtained directly from STCP runs or derived from the most applicable STCP results. The source term issues were used to modify the central estimate source terms according to the prescriptions agreed to by the review team. The levels were correlated as appropriate and used to establish new source terms for the various combinations of issue levels. Because the combinations of LLH issue outcomes result in hundreds of different source terms (1900 for Surry), some means was required to reduce the explicit source terms in order to limit the time-consuming calculation of consequences for individual source term combinations. A method was developed to cluster the source terms, resulting in a more limited number of clusters which could represent all of the source terms without introducing great inaccuracy. The method is described in Appendix C. As described previously, each cluster has a unique set of release fractions (and hence consequences) that are defined based on numerical comparison to the full range of outcomes. The clusters are a calculational convenience and cannot be directly related back to specific outcomes of the containment analysis.

Insights concerning the role of source term phenomena in risk and risk uncertainty can be derived from careful examination of the detailed risk results. These insights are presented in Section 5.3.

\section{Uncertainty in the Consequence Analysis}

No LLH issues were defined for the offsite consequence analysis. Stochastic uncertainty associated with plant-specific meteorology is reflected in the complementary cumulative distribution functions (CCDFs). The comparison of CRAC2 and MACCS results also allows some consideration of modeling differences which provide additional insight, particularly with respect to differences in the modeling of health effects from radiation doses.

The mean risk displays illustrated in Figure 2-4 are supplemented by the CCDFs as illustrated in Figure 2-6. These CCDFs illustrate the probabiiity per year (on the ordinate) that a given magnitude of consequence (on the abscissa) will be exceeded. As with the displays of mean risk, the results for each sample member are plotted, as are curves representing the 5 th and 95 th percentile of the sample. It must be reiterated that these curves do not have statistical meaning concerning uncertainty in risk, they merely apply to the distribution of sample 


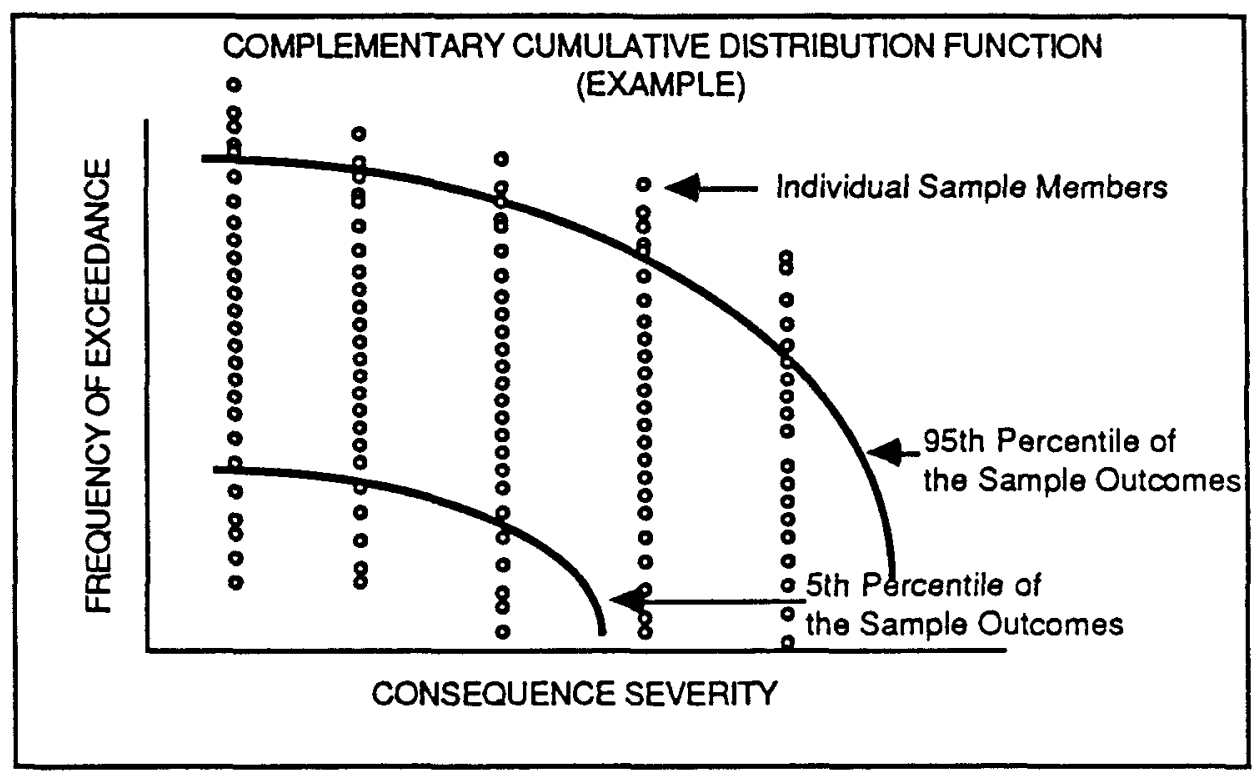

Figure 2-6. Example of CCDF Risk Display

members. Because CRAC2 does not provide CCDFs for the evacuation assumptions used in the SARRP analysis, all CCDFs in this report correspond to MACCS results.

\section{Presentation of LLH Risk Besults}

The RISQUE (and RISKEE) codes include provisions for calculating a great deal of information concerning the risk results. Due to sheer volume, not all of the results are printed or even calculated; rather the risk results can be investigated through careful selection of more detailed calculations to support particular uses. The most important measures and breakdowns of the risk results are summarized in Section 5 and provided in detail in Appendix D. The full complement of available output for any given risk measure includes the following:

- Risk conditional on core-melt frequency,

- Risk for any given individual or set of plant-damage states (groups of coredamage sequences),

- Fractional contribution of each plant-damage state to mean risk,

- Fractional contribution of each source term bin to mean risk,

- Fractional contribution of each cluster to mean risk,

- Rank regression for all LLH issue outcomes,

- Step multiple regression for all LLH issue outcomes,

- $\chi^{2}$ tests of uniformity of risk for all issue outcomes, and

- Detailed analysis of individual sample members, including fractional contributions of damage states, source term bins and clusters. 
The Surry output includes only a subset of this information since many of the tests and breakdowns were developed as the program progressed after Surry risk had already been calculated. In any case, a carefully thought out smaller set of output can generally be used to gain all important insights.

\subsubsection{The OCP Approach}

As already described, the OCP approach involved three separate walkthroughs of the analysis using three sets of data. The inputs to the walkthroughs for each of the chief analysis activities are discussed below.

\section{Core-Damage Erequency Study}

The same uncertainty analyses for the assessment of core-damage sequences served the LLH approach and the OCP approach. Two quantitative measures were employed: (1) sensitivity of the results to important phenomenological (or modeling) uncertainties, and (2) uncertainty due to data inputs. The details are discussed above in Section 2.2.2. The outcomes of the sensitivity studies were used in the $\mathrm{OCP}$ approach depending on their impact, e.g., the sensitivities resulting in lower frequencies were assigned to the optimistic case.

\section{Analysis of Containment Response}

The containment event tree provided the framework for quantification of the likelihood of various containment failure modes. The approach taken to the quantification of the branchpoint probabilities varied according to the type of event; consequently, the uncertainty assessment was also a function of the type of branch point. Branch points corresponding to system unavailabilities (e.g., failure of containment isolation) were assessed using available reliability data. For the events that serve as switches to track the plant-damage states through the containment event tree, there was of course no uncertainty estimate.

The OCP assessment was an integral part of the estimation of branch-point probabilities for the events reflecting accident phenomenology. For each of these branch points, a range of potential branch-point probabilities was represented by three discrete values, designated as pessimistic, central, and optimistic. In general, the pessimistic and optimistic estimates correspond to the range of opinions of experts in the appropriate fields. The estimates are not intended to reflect specific parameters of a probability distribution. An example of the OCP input for one branch point is illustrated in Figure 2-7.

The overall OCP assessment for this task was performed by doing three complete quantifications, or walkthroughs, of the containment event tree, one each for the complete set 
of central, optimistic, and pessimistic branch-point probabilities. It should be noted that there was some correlation of the branch-point probabilities to ensure a consistent treatment, since the connotation of optimistic and pessimistic could vary depending on the context of the prior event-tree events. The OCP containment event tree quantifications are discussed in Section 2.4 and Reference 11.

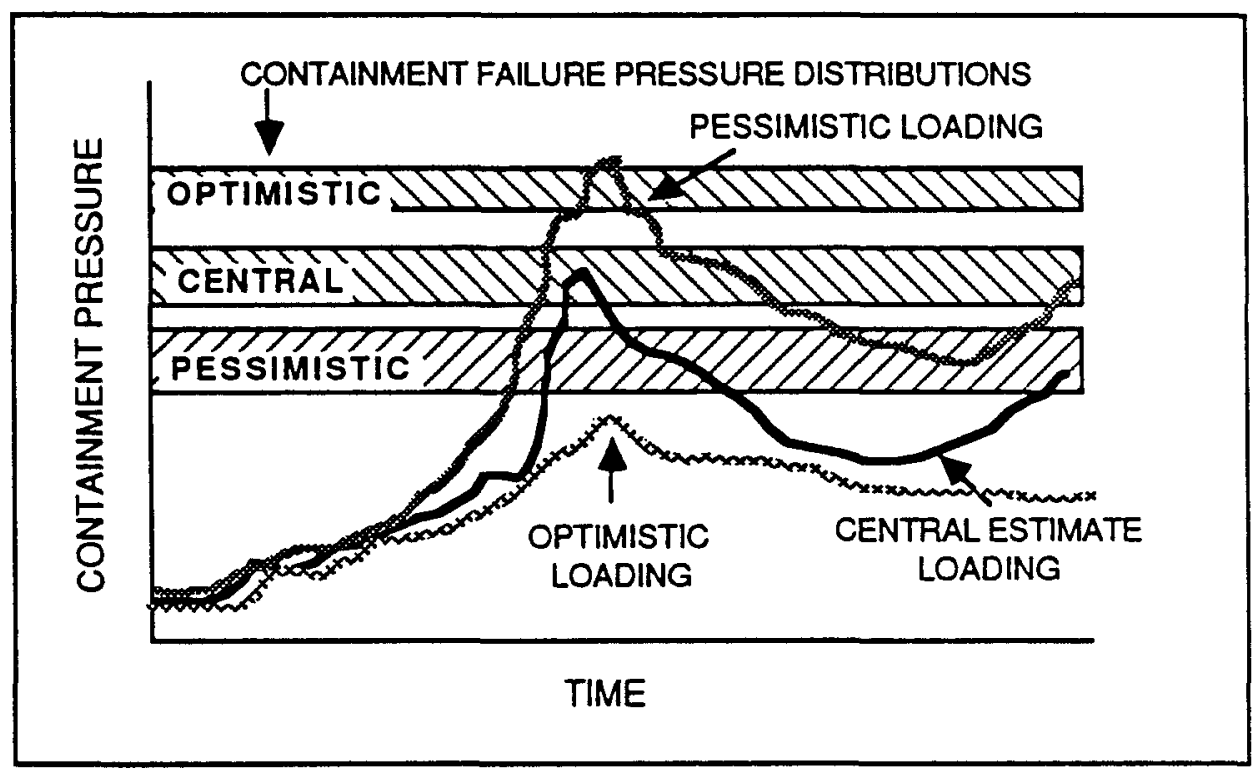

Figure 2-7. Example of OCP Analysis for One CET Question

\section{Source-Term Uncertainties}

Three sets of source terms were developed for the OCP study. The central estimate source terms were developed for the central estimate of the containment event tree analysis. The central estimates were obtained directly from STCP runs or derivations from the mostnearly applicable STCP results. The optimistic and pessimistic source terms reflect changes in the outcomes that would result if different models or data had been used. These estimates were developed to be relatively independent of the containment event tree walkthrough, although insofar as dependencies do exist, the optimistic source terms generally correspond to the optimistic containment event tree walkthrough, and the pessimistic source terms likewise correspond to the pessimistic containment event tree walkthrough. Typical sources of large uncertainty include the release rates for the fuel, core thermal history, aerosol science, effects of decontamination mechanisms, uncertainties in physical parameters, and many others. The uncertainty estimates for the source terms were intended to represent the range that might be seen within the NRC research community and were generated by analyst judgment based on 
available information. Due to time limitations, this task relied very heavily on the results of the QUEST program [12]; indeed most of the optimistic and pessimistic release fractions were derived from information provided in that study. The OCP source terms are discussed in Section 2.5 and Appendix C.

\section{Uncertainty in the Consequence Analysis}

No OCP parameters were developed for the offsite consequence analysis. Some uncertainty insights are available from a comparison of the CRAC2 and MACCS results.

\section{Presentation of OCP Uncertainty in_Risk_Results}

While the uncertainty measures provide useful information concerning the individual portions of the risk assessment, the relevance of this uncertainty to the overall measures of risk is of primary importance in SARRP. Therefore, the risk results reflect each of the uncertainty contributors described above. The impact on the risk results of each of the following contributors to uncertainty, considered one at a time (with all other inputs as central estimates or means as appropriate), is displayed:

- Data uncertainties in the core-damage accident sequences reflected by 5 th and 95 th percentiles;

- Phenomenological (or modeling) uncertainties associated with the coredamage sequences, presented as sensitivity (variation in the mean coredamage frequency) to a range of assumptions for key issues one at a time and in logical combinations;

- Uncertainty in the containment analysis as represented by the three walkthroughs using optimistic, central, and pessimistic branch-point probabilities; and

- Optimistic, central, and pessimistic source terms.

In addition to these displays of the results, the uncertainty (impact on risk measures) due to combinations of two of the uncertainties above are displayed. A breakdown of the results by fractional contribution of plant-damage states and source term bins to risk is also provided as illustrated in Appendix D.

\subsection{REASSESSMENT OF DOMINANT CORE-DAMAGE SEQUENCES}

The updating process performed by ASEP focused on changes made to the plants since the original PRA and improved understanding of plant behavior, and incorporated additional plant-specific reliability data and improved methods for systems analysis. The methods used to 
perform these updates are summarized here; additional detail is provided in Appendix A, and the complete ASEP report is documented in a separate report [13]. ${ }^{*}$

Each plant-specific analysis began with a review of the existing PRA and a visit to the reference plant. These plant visits encompassed several man-weeks of effort, and were valuable in developing in a relatively short time information important to a number of areas. Following the plant visits, new system event trees were constructed to delineate the coredamage sequences. An important aspect of the definition of the end states for these event trees was the coordination with the containment analyses. In order to provide the required degree of integration, a set of plant-damage states identifying the types of sequences that could have different impacts on the likelihood of containment failure and severity of resulting releases was developed. These plant-damage states reflected the parameters important to determining the progression of the accident, including the following:

- The rate of leakage from the reactor-coolant system (RCS) and corresponding pressure at the time the reactor vessel fails;

- The timing of the failure of systems needed to provide core heat removal;

- The operability of the containment safety features;

- The amount of water at various locations in the reactor containment; and

- The potential for the accidents to result directly in a bypass of containment.

System fault-tree models were then developed for each of the events in the core-damage event trees. The fault trees were constructed only to the level of detail necessary to account properly for dependencies among different systems (for example on electric power or other supporting services) and to provide for the efficient application of available reliability data. Together with the event trees, these fault trees provided an integrated model of plant behavior.

The data base used to quantify the events in the system fault trees was developed from both generic and plant-specific sources. A generic data base [14] was derived primarily from that originally produced by the Interim Reliability Evaluation Program (IREP) [15], with data from other studies also incorporated. For each individual plant assessment, this generic data base was augmented by a search of maintenance records that generated additional, plantspecific data for key failure modes. The assessment of human reliability, including the

${ }^{*}$ The results in this draft for comment are all based on the draft ASEP report for Surry. Some changes in sequence/damage state frequency were incorporated between the draft and final Surry ASEP report, but the recalculation of all risk results presented in this report was not possible in the available time frame. The changes are relatively small, and they have been reviewed in terms of risk impact, with the conclusion that none of the results presented here would change appreciably. 
estimation of the likelihood of various actions taken to recover lost safety functions during an accident, was performed using a combination of available methods and engineering judgment on the part of the systems analysts and human reliability specialists.

The models and data were combined to quantify the sequences by obtaining sequencelevel minimal cut sets. These cut sets are minimum combinations of initiating events (i.e., various LOCAs or transients, such as loss of main feedwater) and hardware faults or human actions leading to failure of the systems needed to prevent core damage. At this level of detail, the sequence results can be reviewed carefully for the specific aspects of plant operation that contribute most to core-damage frequency and risk, which in turn allows for the definition of potential safety improvements that are most likely to be cost effective. The final assessment of accident sequence cut sets included the consideration of appropriate recovery events that would likely be attempted by the plant staff. Thus the final sequence cut sets are intended to represent a realistic best estimate of plant accident sequences and their frequency. The results of the sequence analysis were therefore generated on three levels:

(1) The makeup and frequencies of the specific accident cut sets contributing most to risk,

(2) The frequency of each of the core-damage sequences, and

(3) The frequencies of the plant-damage states that serve as the entry points to the containment event trees. 


\subsection{EVALUATION OF CONTAINMENT RELEASE-MODE PROBABILITIES}

A major focus in SARRP was the development of a containment event tree for each of the plant types represented by the reference plants. These event trees delineated the pathways that could lead to releases of fission products as a consequence of core-damage accidents. The approach taken to the development and application of the containment event tree for Surry is summarized in this section. The results of the containment event analysis are provided in Section 5, and additional detail is provided in Appendix B. The entire analysis is documented in a separate report [11].

\subsubsection{Development and Quantification of the Containment Event Tree}

The containment event tree for Surry was developed by identifying the types of containment responses that might be expected to impact risk and the various events and conditions that could affect those responses, at a level of detail that could reasonably be supported by the information currently available. This led to the construction of an event tree that is significantly expanded beyond those previously used in PRAs; the Surry containment event tree contains 59 questions, many of which have more than two branching options. The event tree is comprised of the following types of questions:

(1) Entry states. What are the conditions in the reactor coolant system and containment prior to melting of the core that could influence the accident progression?

(2) Phenomenological events. What are the phenomena that could affect the progression of severe accidents, at what points during the accident timeline do they occur, and what are their subsequent effects on the accident development?

(3) Reactor coolant system failure modes. What are the size and location of the reactor coolant system breach and the corresponding pressure during core meltdown?

(4) Survivability of containment systems. Do the containment spray systems survive the conditions occurring during severe accidents that exceed their design bases?

(5) Containment failure modes. What are the loads that challenge containment, does containment survive these loads, what is the nature of the failure (approximate size and location), and what is the subsequent pathway for release of fission products to the environment?

Because of the number of possible branching points, the event tree is too large to depict graphically. The questions are listed in Table 2-3. 
Table 2-3

SUMMARY OF TOP EVENTS FOR SURRY CONTAINMENT EVENT TREE

Question Posed by the Event Tree

Dependencies on

Prior Questions

1. Is ac power available after the initiating event?

None

2. Does emergency core cooling fail prior to overpressurization of containment?

None

3. What is the level of pre-existing containment leakage or isolation failure?

1

4. Where is the initial reactor coolant system break?

None

5. What is the size of the initial reactor coolant system break?

None

6. Is the containment initially bypassed?

4

7. Are the steam generators wet or dry?

None

8. Do the fan coolers fail to actuate before core degradation

N/A*

9. Do the containment sprays fail to actuate in the injection mode before core degradation?

$1,3,5,6$

10. Do the containment sprays fail to actuate in the recirculation mode before core degradation?

9

11. To what degree, if any, is the auxiliary building initially bypassed?

$3,4,7$

12. Where, if at all, is there a temperature-induced failure of the reactor coolant system during the period of core degradation?

13. What is the size of the temperature-induced reactor coolant system failure?

14. What is the primary system pressure during core degradation? Also, what would be the containment pressure increment from a primary system blowdown?

15. At what level, if any, is containment bypassed during core degradation?

16. What is the rate of blowdown to containment during core degradation?

17. Do the containment sprays fail to actuate during the period of core degradation?

$5,6,13,15$

18. Is there containment heat removal during core degradation?

$1,3,10,16$

8,17

19. At what level, if any, does containment fail due to steam pressurization before vessel breach?

20. What is the containment pressure before vessel breach?

$2,3,15,18$

21. Is there a hydrogen burn before vessel breach? Also, what is the pressure increment from such a burn?

22. Does containment fail because of a hydrogen burn before vessel breach? Also, what is the associated failure pressure and standard deviation?

23. To what degree, if any, is the auxiliary building bypassed before vessel breach?

$3,16,18,19$

$3,16,18$

20,21

24. Do the fan coolers fail after the early hydrogen burn?

$7,11,12,19,22$

N/A*

25. Do the containment sprays fail after the early hydrogen burn?

$1,17,21,22$

24,25

14

27. What is the mode of vessel breach?

$14,17,27$

28. Does direct heating of the containment atmosphere occur just after vessel breach? Also, what is the pressure increment from a steam spike alone and from a steam spike plus direct heating?

*The event tree was developed to be generally applicable to plants with large, dry containments. Since Surry does not have fan coolers for emergency operation, these questions are not applicable. 
Table 2-3

SUMMARY OF TOP EVENTS FOR SURRY CONTAINMENT EVENT TREE (Continued)

Question Posed by the Event Tree

Dependencies on Prior Questions

29. Is there a hydrogen burn just after vessel breach? Also, what is the pressure increment from such a burn?

30. Does containment fail due to a steam spike, direct heating, and/or hydrogen burn just after vessel breach?

31. What is the mode of containment failure, if any, just after vessel breach?

$3,16,21,26$

$14,20,28,29$

32. To what degree, if any, is the auxiliary building bypassed just after vessel breach?

$3,19,22,27,28,29,30$

33. Do the fan coolers fail after vessel breach?

23,31

N/A*

34. Do the containment sprays fail after vessel breach?

$1,25,31$

35. Is there an oxidation release?

27,28

36. Is ac power restored after vessel breach?

1

37. Do the fan coolers fail late in the accident?

N/A*

38. Do the containment sprays fail late in the accident?

34,36

39. Is there containment heat removal late in the accident?

37,38

40. Has the inventory of the refueling water storage tank been injected into containment?

$9,17,25,34,38$

41. What is the amount of water injected into containment?

42. Do significant core-concrete interactions occur after vessel breach?

$2,14,16,40$

43. Is ac power recovered late in the accident?

$14,18,27,34,41$

44. What is the containment pressure late in the accident?

1

45. Has a large leak or gross failure occurred late in the accident?

$26,39,40,42$

46. Does a late hydrogen burn occur?

47. What pressure rise would occur if combustible gases were to burn late in the accident?

$3,15,19,22,31$

$21,29,39,44,45$

$28,42,43,46$

48. Would containment fail due to a late hydrogen burn or steam production?

44,47

49. In what way, if at all, does containment fail due to gradual pressurization from boiloff of water or non-condensible gases or hydrogen burning (leakage or gross rupture)?

50. To what degree, if any, is the auxiliary building bypassed late in the accident?

$45,46,48$

32,49

N/A*

52 Do the containment sprays fail after the late hydrogen burn?

$38,46,49$

53. Is there containment heat removal very late in the accident?

51,52

54. Is there a vaporization release?

$17,25,34,42$

55. Does containment meltthrough occur, given no prior containment failure

$26,38,39,42,45$

$48,53,54,55$

56. To what degree, if any, does containment leakage occur due to high temperatures late in in the accident?

41,45

48,53

57. Does containment depressurize after basemat meltthrough?

$26,39,53,56$

58. What is the ultimate containment failure mode, if any, resulting from core-concrete interactions?

$42,45,48,53$, $55,56,57$

59. To what degree, if any, is the auxiliary building bypassed very late in the accident?

$50,55,57$ 
The questions are posed in ways that require the answers to be expressed in terms of likelihoods. The estimation of these likelihoods is often a highly uncertain process, and requires that information from a large number of sources be examined and incorporated in order to reflect the current level of understanding most appropriately. Table 2-4 lists many of the key issues represented by the questions in the event tree, and identifies some of the major research and analysis programs from which information was drawn to evaluate the corresponding questions. Among the sources of information used in the analysis are the following:

(1) Containment Loads Working Group (CLWG) [16,17];

(2) Containment Performance Working Group (CPWG);

(3) Battelle calculations for the Accident Source Term Project Office [18];

(4) Quantitative Uncertainty Estimation for the Source Term (QUEST) program [12];

(5) Industry Degraded Core (IDCOR) program [19];

(6) Severe Accident Sequence Analysis (SASA) program [20-24];

(7) Severe Accident Uncertainty Analysis (SAUNA) Program [25];

(8) Accident Sequence Evaluation Program (ASEP) [13];

(9) SARRP Phenomena Assessment Task Force [26];

(10) Steam Explosion Review Group [27];

(11) High-pressure ejection test series [28];

(12) Available probabilistic risk assessments [29-34];

(13) The Final Safety Analysis Report [35];

(14) Architect-engineer and other estimates of containment failure pressure [36.37];

(15) Others [38-46].

The NRC provided general guidance with respect to the priorities to place upon the use of this variety of sources in evaluating the events in the containment event trees. To the extent possible, the information developed by the CLWG, CPWG and other analyses performed or sponsored by NRC were to be used. To provide a more complete treatment of issues raised in the containment event tree, other references, including analyses sponsored by the utility industry, were to be considered.

Because the information provided by these sources often either did not provide a direct means to quantify particular branch points, or provided divergent indications of the appropriate probabilities, as noted in Section 2.2 three separate estimates of the probabilities for each 
USE OF INFORMATION SOURCES IN ADDRESSING THE ISSUES IN THE SURRY CONTAINMENT EVENT TREE

\begin{tabular}{|c|c|c|c|c|c|c|c|c|c|c|c|c|c|c|}
\hline & \multirow[b]{2}{*}{ Issue } & \multirow[b]{2}{*}{ CLWG } & \multirow[b]{2}{*}{ CPWG } & \multirow[b]{2}{*}{ BMI-2104 } & \multirow[b]{2}{*}{ QUEST } & \multirow[b]{2}{*}{ IDCOR } & \multicolumn{3}{|c|}{ Informatuon Sources* } & \multirow[b]{2}{*}{ SERG } & \multirow[b]{2}{*}{ HIPS } & \multirow[b]{2}{*}{ PRA } & \multirow[b]{2}{*}{$\mathbf{A E}$} & \multirow[b]{2}{*}{ Others } \\
\hline & & & & & & & SASA & SAUNA & PATF & & & & & \\
\hline 1 & Size of Preexisting Containment Leakage & -- & $\mathbf{x}$ & - & -- & $\mathbf{x}$ & -- & -- & -- & -- & -- & $\mathbf{x}$ & -- & $\mathbf{x}$ \\
\hline 2 & $\begin{array}{l}\text { Size and Location of the Primary System Break } \\
\text { During the Melt Release (e g . Hot Leg vs Cold Leg) }\end{array}$ & -- & -- & - & - & - & - & - & $\cdot-$ & -- & - & - & -- & -- \\
\hline 3. & $\begin{array}{l}\text { Timing of Accumulator Discharge Relative to } \\
\text { Timng of Reactor Vessel Breach }\end{array}$ & -- & - & $\mathbf{x}$ & - & $\mathbf{X}$ & $\mathbf{x}$ & - & - & -- & -- & $\mathbf{X}$ & -- & $\mathbf{X}$ \\
\hline 4 & $\begin{array}{l}\text { Occurrence of In-Vessel Steam Explosson Large } \\
\text { Enough to Fail the Reactor Vessel }\end{array}$ & -- & -- & - & - & -- & -- & $\mathbf{X}$ & -- & -- & -- & - & $\cdots$ & $\mathbf{X}$ \\
\hline 5 & $\begin{array}{l}\text { Occurrence of In-Vessel Steam Explosion Large } \\
\text { Enough to Fail the Containment. }\end{array}$ & $-\cdot$ & -- & - & - & $\mathbf{X}$ & -- & $\mathbf{X}$ & -- & $\mathbf{X}$ & -- & $\mathbf{X}$ & - & $\mathbf{X}$ \\
\hline 6 & Timng and Magnitude of Early Hydrogen Burns & $\mathbf{x}$ & -- & $\mathbf{X}$ & -- & $\mathbf{X}$ & $\mathbf{X}$ & $\mathbf{X}$ & $\mathbf{x}$ & -- & -- & $\mathbf{x}$ & -- & $\mathbf{X}$ \\
\hline 7 & Magnitude of the Ex-Vessel Steam Spike & $\mathbf{x}$ & -- & $\mathbf{x}$ & $\mathbf{X}$ & $\mathbf{x}$ & $\mathbf{x}$ & $\mathbf{x}$ & $\mathbf{x}$ & -- & $\mathbf{X}$ & $\mathbf{X}$ & -- & $\mathbf{X}$ \\
\hline 8 & $\begin{array}{l}\text { Extent of Direct Heating of the Atmosphere } \\
\text { Following Vessel Breach }\end{array}$ & $\mathbf{X}$ & -- & - & -- & $\mathbf{X}$ & -- & - & -- & -- & $\mathbf{X}$ & - & -- & - \\
\hline 9 & Containment Structural Fallure Pressure & -- & - & - & -- & $\mathbf{x}$ & -- & - & $\mathbf{X}$ & -- & - & $\mathbf{X}$ & $\mathbf{X}$ & - \\
\hline 10 & $\begin{array}{l}\text { Size of Contaunment Leakage Induced by } \\
\text { Temperature or Pressure }\end{array}$ & -- & $\mathbf{X}$ & - & - & $\mathbf{X}$ & - & - & $-\cdot$ & -- & -- & - & -- & - \\
\hline 11 & $\begin{array}{l}\text { Survivability of Containment Sprays } \\
\text { at Vanous Times During the Accident. }\end{array}$ & - & -- & - & - & $\mathbf{X}$ & -- & - & -- & -- & -- & $\mathbf{X}$ & -- & $\mathbf{X}$ \\
\hline 12 & Extent of Core-Concrete Interaction & $\mathbf{x}$ & -- & $\mathbf{x}$ & - & $\mathbf{X}$ & $\mathbf{X}$ & $\mathbf{x}$ & - & -- & -- & $\mathbf{X}$ & - & $\mathbf{X}$ \\
\hline 13 & Timing and Magnitude of Late Hydrogen Burns. & $\mathbf{x}$ & - & $\mathbf{x}$ & -- & $\mathbf{x}$ & $\mathbf{X}$ & - & -- & -. & -- & - & -- & $\mathbf{X}$ \\
\hline 14 & $\begin{array}{l}\text { Potentual for Drect Core Debrns Attack on } \\
\text { Containment Structures }\end{array}$ & $\mathbf{X}$ & -- & - & -- & - & -- & - & -- & - & -- & - & -- & - \\
\hline 15 & $\begin{array}{l}\text { Potentral for Core Debris to Melt Through } \\
\text { the Basemat. }\end{array}$ & - & - & $\mathbf{x}$ & -- & $\mathbf{X}$ & -- & $\mathbf{X}$ & -- & -- & -- & $\mathbf{X}$ & -- & $\mathbf{X}$ \\
\hline 16 & $\begin{array}{l}\text { Potential for Basemat Meltthrough to Cause a } \\
\text { Depressurization of Containment. }\end{array}$ & -- & - & $x$ & -- & - & - & $\mathbf{X}$ & -- & -- & -- & $\mathbf{X}$ & -- & -- \\
\hline 17 & $\begin{array}{l}\text { Potental for Effluent to Pass Through Adjacent } \\
\text { Structures, Such as the Auxiliary Building }\end{array}$ & -- & - & $\mathbf{X}$ & -- & $\mathbf{X}$ & -- & - & -- & $\cdots$ & -- & - & -- & -- \\
\hline
\end{tabular}


question were propagated through the tree. These estimates were termed optimistic, central, and pessimistic. The one labeled "pessimistic" tends to provide higher probabilities for the pathways that lead to higher source terms and lower probabilities for lower source-term pathways. The ones labeled "central" and "optimistic" are analogously interpreted. The estimates are presented as a reflection of the information that is available from the reactor safety community.

In quantifying the event tree, the branch points were often assessed first in subjective, qualitative terms (e.g., an event might be "likely" or "remotely possible"). Values were then assigned to these verbal descriptors. The sensitivity of the release-mode probabilities to the selection of these values was examined, and it was found that the variances were generally small relative to the differences among those for the optimistic, central, and pessimistic walkthroughs.

\subsubsection{Plant Features Important to Containment Response}

The branch points and probabilities for the Surry containment event tree reflected consideration of a number of plant-specific features that could have important effects on the progression of a core-damage accident. These plant features include those described below.

Maintenance of containment at subatmospheric pressure. The Surry containment is normally maintained at subatmospheric pressure (approximately $10 \mathrm{psia}$ ) in order to provide an additional margin to accommodate the steam pressures that could be realized under accident conditions. This aspect also helps to ensure that there are no large, pre-existing leaks at the time of the accident, since it would be difficult to maintain the appropriate level of vacuum. This situation was reflected in the event-tree analysis by the assumption that pre-existing leaks larger than the makeup capacity of the vacuum pumps were impossible.

Containment pressure capacity. The pressure at which failure would be expected to occur is a key determinant in the likelihood and timing of containment failure during pressure transients that result from the generation of steam or noncondensibles, direct heating and the combustion of hydrogen. The pressure at containment failure can also influence the dispersion of fission products released to the environment. The Surry containment is a cylindrical, domed, steel-reinforced concrete building with a steel liner. The pressure capacity was estimated from analyses performed for the Reactor Safety Study [22] and by Stone and Webster [36]. The results of these evaluations were combined to obtain three normal distributions (for the optimistic, central, and pessimistic walkthroughs) representing estimates of containment failure pressure for Surry. For each pathway passing through a node in the 
containment-event tree at which the possibility of overpressure failure of containment was considered, the estimated pressure associated with the conditions of the pathway (e.g., due to a hydrogen burn or steam spike) was compared to the appropriate distribution for the walkthrough. This produced a probability that the containment capacity would be exceeded for that pathway.

Containment Heat Removal. Containment heat removal at Surry is accomplished by a containment spray system consisting of six trains, two of which are capable of injecting water from the RWST into containment. Both of the injection trains are actuated when containment pressure reaches approximately 10 psig. The other four trains are actuated after spray injection is initiated; they draw suction directly from the containment sump and recirculate flow back to the spray headers in containment. The pumps for two of these recirculation trains are located outside containment, and all of the equipment for the other two trains is inside. Each of the four recirculation trains has a heat exchanger, providing for rejection of containment heat to a service water system. If the spray systems are not operable, it is much more likely that pressures generated by various phenomena during the accident will be sufficient to overpressurize containment. On the other hand, with the spray systems not operating, it is also more likely that the containment atmosphere will be filled with steam, providing inerting against the combustion of hydrogen.

The spray recirculation systems take on added significance since they are the principal means of removing decay heat following a LOCA or other event in which the containment serves as the heat sink for emergency core cooling systems (unlike most PWRs, the lowpressure recirculation system is not equipped with heat exchangers). Therefore, there is the possibility for failure of containment heat removal to lead to both a loss of core cooling and the potential for overpressurization of the containment.

Configuration of Reactor Cavity. The design of the reactor cavity can have an important impact on accident progression due primarily to three design aspects: (1) the degree to which the core debris is inhibited from being dispersed following ejection from the reactor vessel, (2) the ability of water in containment to reach the core debris, and (3) the capability to transfer heat from the cavity to the containment atmosphere. At Surry, the reactor cavity does not communicate directly with the containment sump. Thus, if the containment spray systems have not operated, the cavity will be relatively dry (for accidents proceeding at high pressures, the cold-leg accumulators may discharge following breach of the reactor vessel, providing some water to the cavity). It is unlikely that the amount of water in the cavity will be sufficient to preclude the possibility for the direct heating phenomenon to occur. 


\subsubsection{Definition of Containment-Release Modes}

The outcomes of the containment event tree form a set of discriminated accident pathways. Since the number of such pathways was quite large, it was necessary to group them into a smaller set of bins that could be used for source-term analysis. The information reflected by these bins includes the modes by which the containment fails, as well as other details that could influence the source term, such as whether or not core-concrete interactions sufficient to generate a vaporization release have occurred. For Surry, the containment-release modes include the following:

(1) The containment ruptures before or at the time of reactor-vessel failure, with the reactor coolant system at high pressure prior to vessel breach and with the containment spray system failing to operate through the period of core-concrete interactions. The failure modes include early burning of hydrogen generated during the core degradation process; failure at the time of vessel breach due to the steam spike generated when the core debris is discharged into the water in the cavity or due to an in-vessel steam explosion; or failure due to the burning of the hydrogen released at the time of vessel failure. Failures from direct heating are treated separately below.

(2) Early failure of containment, as in (1), but with the containment spray system operating through the period of core-concrete interactions.

(3) The containment ruptures before or at the time of vessel breach, with the reactor coolant system not at high pressure and with the containment spray system not operating through the period of core-concrete interactions. The failure causes are similar to those in (1) above.

(4) Early failure of containment, as in (3), but with the containment spray system operating through the period of core-concrete interactions.

(5) Failure of containment prior to core damage, due to failure to remove heat from the containment during operation of the emergency core cooling systems; failure of core cooling is a consequence of the containment failure.

(6) Large pre-existing leakage or failure of containment isolation, with the spray system not operating.

(7) Large pre-existing leakage or failure of containment isolation, with the spray system operating.

(8) Rupture of the containment late in the accident due to the generation of steam or the burning of hydrogen and other flammable gases. Either the spray system is operable, or the sprays fail but the debris bed is nevertheless permanently coolable.

(9) Late rupture of the containment, as in (8), but the spray system is not available.

(10) Long-term overpressurization of containment due to the generation of steam and non-condensible gases as a consequence of core-concrete 
interactions, resulting in leakage sufficient to prevent further pressurization but insufficient to produce rapid depressurization.

(11) Containment is bypassed, with the point of release at a location that would be submerged by a significant quantity of water.

(12) Containment bypass, but with little or no submergence at the point of release.

(13) The core debris penetrates the containment basemat, with a high driving pressure due to the unavailability of containment sprays.

(14) Penetration of the basemat occurs with the sprays available.

(15) The containment remains intact throughout the accident, with the release equivalent to the design basis leakage.

(16) The containment fails at the time of vessel breach due to direct heating, with the reactor coolant system previously at high pressure. The spray system fails before or at the time of containment failure.

(17) The containment fails due to direct heating, as in (16), but the sprays continue to operate after vessel breach.

(18) The containment fails due to direct heating at vessel breach, with the reactor coolant system at moderate pressure and with the sprays failing at the time of vessel breach.

(19) The containment fails due to direct heating at vessel breach, with the reactor coolant system at moderate pressure and with the sprays continuing to operate after vessel breach.

(20) The containment fails due to direct heating at vessel breach, with the reactor coolant system at moderate pressure and with the sprays failed from the outset of the accident.

The likelihood of these release modes was calculated for each of the plant-damage states assessed by ASEP to have non-negligible frequencies. This generated the frequencies for each of the source-term bins, for integration into the risk calculation. 


\subsection{ASSESSMENT OF THE RADIOLOGICAL SOURCE TERM}

The next step in the assessment of the public risk associated with Surry was the estimation of the radiological release to the environment. The science of fission product release and transport has been the subject of considerable research in recent years, resulting in increased understanding and new estimates of accident consequences. The understanding is unfortunately not to the point at which definitive results agreed to by the whole of the research community are available, but the analyses summarized in this report include the advances that have been made to date and are intended to allow an interim perspective and an improved understanding over the previous comprehensive evaluation of public risk in the Reactor Safety Study [29]. The second objective of this analysis is to act as a catalyst for the scientific interaction required to achieve the goal of developing a definitive understanding of the radiological effects of severe nuclear power plant accidents.

This report summarizes the methods and results of an effort that integrated a number of research activities. The goal of this summary is to afford the reader sufficient understanding to review the integrated risk analysis and to allow efficient reference to the more detailed research. More specific information on the source term task is included in Appendix C.

It was the intent of this program to estimate the plant-specific source terms using the state-of-the-art methods embodied in the Source Term Code Package (STCP) [47]. The STCP was a coordinated effort by the NRC and its contractors to upgrade the agency's analytical procedures for, and understanding of, reactor accident radiological releases. The NRC staff's review of the STCP and their conclusions concerning its viability are presented in NUREG0956 [46]. The NRC report is based in part on results and insights described in Radionuclide Release Under Specific LWR Accident Conditions (referred to hereafter as BMI-2104) [18]. Since the publication of BMI-2104, there has been additional work to refine various portions of the suite of codes that make up the STCP, as well as extension of the plant-specific analyses to both additional accident scenarios and additional reference plants [48,49].

The next two subsections describe the origins of the source terms for the central estimate for all source term bins. The last subsection briefly describes the source term used in the uncertainty analyses performed in SARRP.

\subsubsection{Integration With the Other Risk Assessment Tasks}

The goal of the source-term analysis was the estimation, for each unique combination of core and containment conditions following core melt, of the amount of each radioactive 
species that would be released to the environment and would therefore be of concern for public health risk. Due to the large number of both potential accident conditions and different fission products, a method was needed to make the calculation tractable. Two principal simplifications (although there are many other more specific simplifications) form the basis of the overall source-term methodology:

(1) Core and containment conditions and ultimate post core-melt conditions were grouped into categories, called source-term bins. The scenarios assigned to a bin each produce similar response in terms of release of the radioactive species to the environment. The list of containment release modes described in Section 2.4.3 constitutes the source term bins used for Surry.

(2) The radioactive species were grouped into classes that behave similarly.

A third grouping was required for the LLH uncertainty analysis: source term clustering. This is discussed in Section 2.5.4.

These simplifications were necessitated by limitations in the practical application of the current technology; is not possible to assess fully the error that is introduced through their use. What are believed to be the most critical sensitivities and uncertainties are outlined in Section 4 and Appendix $C$, and summarized in the conclusions of this report.

\section{Source-Term Binning}

The first step in the analysis was the development of categories, called source-term bins, that could be used to group the different combinations of core and containment end states into categories that could be assessed together in terms of the source term. These categories were developed for Surry by delineating the critical parameters that are the determinants of the radioactive release. The bins developed in this process reflect the understanding of what was expected to be important to each bin. Several key characteristics considered in the bin development are listed below:

- Primary system status, which determines RCS vessel release timing and pressure characteristics;

- Containment failure mode, which identifies timing and release path;

- Timing of the accident process;

- Availability of water in the reactor cavity, which affects core-concrete interactions and the potential for developing a permanently coolable debris bed;

- Containment safeguards status, including the spray system for its effect on both pressure and radioactive release; and 
- Core-melt characteristics, which impact event timing.

Through the process of iteration, the source terms bins were made equivalent to the results of the containment event tree analysis. The previous section described the 20 containment release modes which are equivalent to the source term bins used in this study. For reference purposes, the bins are delineated by characteristics in Table 2-5. Additional discussion of the bins is included in the next two sections along with the description of the representative calculations used to determine the source terms.

\section{Grouping of Radionuclides}

The second simplification was the grouping of radionuclides that have similar properties such that their release and deposition phenomena can be treated together. The definitions of the radionuclide groups are the same as those used in the Reactor Safety Study (RSS). These groups were retained as a basis for the SARRP radionuclide group definition both because they continue to provide a fairly effective means of grouping the radioactive species and because they are the standard for the consequence analysis and comparison to other studies. The release groups used for Surry in SARRP are listed below.

\begin{tabular}{|c|c|c|}
\hline Group & Key Radionuclides & Release Type \\
\hline 1 & $\mathrm{Xe}, \mathrm{Kr}$ & Noble gas release \\
\hline 2 & $\mathrm{I}, \mathrm{Br}$ & $\begin{array}{l}\text { Halogens (the SARRP bin includes } \\
\text { organic iodide) }\end{array}$ \\
\hline 3 & Cs, Rb & Alkali metals \\
\hline 4 & $\mathrm{Te}$ & Tellurium, also includes $\mathrm{Se}$ and $\mathrm{Sb}$ \\
\hline 5 & $\mathrm{Sr}, \mathrm{Ba}$ & Strontium and Barium \\
\hline 6 & $\mathrm{Ru}$ & $\begin{array}{l}\text { Noble metals, also includes } \mathrm{Mo}, \mathrm{Pd} \text {, } \\
\text { Rh, and Tc }\end{array}$ \\
\hline 7 & $\mathrm{La}$ & $\begin{array}{l}\text { Lanthanum group, also including } \mathrm{Ce} \text {, } \\
\mathrm{Nd}, \mathrm{Np}, \mathrm{Nb}, \mathrm{Pu}, \mathrm{Eu}, \mathrm{Y}, \mathrm{Pr}, \mathrm{Pm} \text {, } \\
\mathrm{Sm} \text {, and } \mathrm{Zr} \text {. }\end{array}$ \\
\hline
\end{tabular}

It should be noted that there was some further discrimination of two of the groups, 5 and 7, in the analysis of the other reference plants. Many of the STCP calculations for this plant also included these refinements, but the SARRP process for Surry was not changed to include the new groups. 
Table 2-5

KEY CHARACTERISTICS OF SURRY SOURCE-TERM BINS

\begin{tabular}{|c|c|c|c|c|c|c|c|c|c|c|c|c|c|c|c|c|c|c|c|c|}
\hline $\begin{array}{c}\text { Sequence and Containment } \\
\text { SLatus }\end{array}$ & 1 & 2 & 3 & 4 & 5 & 6 & 7 & $\begin{array}{l}\text { Sour } \\
8\end{array}$ & & $\begin{array}{c}\text { Term } \\
10\end{array}$ & $\begin{array}{l}\text { Bins } \\
11\end{array}$ & 12 & 13 & 14 & 15 & 16 & 17 & 18 & 19 & 20 \\
\hline \multicolumn{21}{|l|}{ Containment Ealure } \\
\hline Rupture Before Core Melt & & & & & $\sqrt{ }$ & & & & & & & & & & & & & & & \\
\hline Early Overpressure & $\sqrt{ }$ & $\checkmark$ & $\sqrt{ }$ & $\checkmark$ & & & & & & & & & & & & $\checkmark$ & $\sqrt{ }$ & $\sqrt{ }$ & $\sqrt{ }$ & $\sqrt{ }$ \\
\hline Lale Overpressure-Rupture & & & & & & & & $\sqrt{ }$ & $\checkmark$ & & & & & & & & & & & \\
\hline Late Overpressure-Leakage & & & & & & & & & & $\checkmark$ & & & & & & & & & & \\
\hline Meltthrough & & & & & & & & & & & & & $\checkmark$ & $\checkmark$ & & & & & & \\
\hline Leak or Isolation Fallure & & & & & & $\sqrt{ }$ & $\sqrt{ }$ & & & & & & & & & & & & & \\
\hline Containment Bypass-Wet & & & & & & & & & & & $\sqrt{ }$ & & & & & & & & & \\
\hline Containment Bypass-Diy & & & & & & & & & & & & $\checkmark$ & & & & & & & & \\
\hline No Falure & & & & & & & & & & & & & & & $\checkmark$ & & & & & \\
\hline \multicolumn{21}{|l|}{ Contaument Spray System ${ }^{1}$} \\
\hline Operates Throughout Aceident & & 0 & & 0 & & & $\sqrt{ }$ & 0 & & & & & & $\sqrt{ }$ & & & 0 & & 0 & \\
\hline Operates at Least Up to $C F^{2}$ & & & $\sqrt{ }$ & $\sqrt{ }$ & & & & $\sqrt{ }$ & & & & & & & & & $\checkmark$ & $\checkmark$ & $\checkmark$ & \\
\hline Unavalable & $\checkmark$ & & & & & $\checkmark$ & & & $\checkmark$ & & & & $\checkmark$ & & & $\checkmark$ & & & & $\checkmark$ \\
\hline \multicolumn{21}{|l|}{ Pnmary System Pressure } \\
\hline High & $\checkmark$ & $\checkmark$ & & & & & & & & & & & & & & $\checkmark$ & $\sqrt{ }$ & & & \\
\hline Moderate & & & $\checkmark$ & $\checkmark$ & & & & & & & & & & & & & & $\checkmark$ & $\checkmark$ & $\sqrt{ }$ \\
\hline Low & & & & & $\checkmark$ & & & & & & & & & & & & & & & \\
\hline \multicolumn{21}{|l|}{ Conlaunmenu Pressure ${ }^{3}$} \\
\hline High & & & & & & & & & & & & & $\sqrt{ }$ & & & & & & & \\
\hline Low & & & & & & & & & & & & & & $\checkmark$ & & & & & & \\
\hline \multicolumn{21}{|l|}{ Core Coolabuluy } \\
\hline Permanenuy Coolable & & 0 & & 0 & & & & 0 & & & & & & & & & 0 & & 0 & \\
\hline Non-colable & $\checkmark$ & & v & & $\checkmark$ & $\checkmark$ & $\checkmark$ & & & & & & & & & $\checkmark$ & & $\checkmark$ & & $\checkmark$ \\
\hline \multicolumn{21}{|l|}{ Diregl Heaung Effect } \\
\hline None & $\checkmark$ & $\checkmark$ & $\checkmark$ & $\checkmark$ & & & & & & & & & & & & & & & & \\
\hline Significant & & & & & & & & & & & & & & & & $\checkmark$ & $\checkmark$ & $\checkmark$ & $\checkmark$ & $\checkmark$ \\
\hline
\end{tabular}

$\checkmark$ - The charactensuc is requred for the bin Charaensucs oo marked are not determinant of the bin and any combination may apply-see the actual bin definiuons

0 - Esther of the charactenstics noted by this symbol apply to the bia in combination with the others marked $V$

${ }^{1}$ The spray quesuon is dependent on uming as noced The bin definitoons in the text should be referenced for further explanation ${ }^{2} \mathrm{CF}=$ containment fallure

${ }^{3}$ This is only used as a discriminator for bins 13 and 14 Obviously contanment pressure is high in many oher bins 
NUREG/CR-4551,VOL.1: DRAFT REPORT FOR COMMENT (SEPTEMBER, 1986)

\subsubsection{Source Term Code Package Calculations}

The selection of appropriate STCP runs to apply to the source-term bins as used in SARRP was based on an assessment of the critical parameters for each bin and the availability of results indicating the effects of those parameters. The primary procedure used to determine these effects was a meeting of the source-term binning group, listed previously in Table 2-1. In this meeting, the sequence analysts identified the anticipated important core-damage states, the containment analysts outlined the critical containment issues, and the source-term analysts delineated the information important to their assessment. The result of the meeting was a preliminary list of computer code runs needed and a preliminary definition of how the results apply to other situations that were not selected for detailed study.

The STCP analyses generally were developed for individual accident sequences that were used as surrogates for particular source-term bins. In addition, there were some runs used to determine the impact of conditions that might also be associated with the other accident sequences within the same source-term bin. For the bins determined to be of less risk significance, another STCP run, or a composite of several runs, was used as a base, and the results were adjusted to account for the particular conditions associated with the bin.

\section{STCP Updates Since BMI-2104}

There have been a number of changes to the STCP since the publication of BMI-2104. Separate documentation on the current code package will soon be issued [47]. Most of the changes involve the logistics of code usage and are merely improvements in the interfaces. There have, however, been several improvements in individual models which are summarized below and described more fully in Reference 49.

- A new version of the CORSOR code, CORSOR-M, has been incorporated into MARCH. It allows a more consistent treatment of the release of fission products and the transport of sources of decay heat from the fuel. In addition, the release rates of silver and indium from the control rods has been changed to reflect recent research.

- The STCP has been updated to include CORCON-Mod2 to improve calculation of the thermal-hydraulic loads on containment associated with core-concrete interactions and as input to the VANESA code which calculates the releases from these interactions. The BMI-2104 results include different codes that were not entirely compatible.

- The coupling of the MERGE and TRAP-MELT has been improved since BMI-2104, with potentially significant impact. Any comparison of this study with BMI-2104 must consider the following:

- The decay heat contribution to the thermal-hydraulics is now considered; 
NUREG/CR-4551,VOL.1: DRAFT REPORT FOR COMMENT (SEPTEMBER, 1986)

- The nodalization in the fission product transport calculations was improved;

- Gas properties in TRAP now account for hydrogen, and the heat transport coefficients have also been changed in TRAP;

- Aerosol modeling has been changed somewhat; and

- The treatment of chemisorption on walls was improved with potential effects on the highly reactive species (Te).

While there have been several improvements to the codes, the STCP can not at this time be considered verified and error free. An on-going review by Brookhaven National Laboratories is intended to provide this assurance.

\section{STCP Runs for Individual Bins}

As stated previously, it was the intent of the program to have STCP runs for all dominant accidents. This was achieved for the most part, although there was one major exception: the release associated with direct heating is not modeled in the STCP, and these bins were calculated using other information as indicated in the next section. Source terms for some other bins of little risk significance were also derived rather than modeled directly.

The STCP requires a specific accident sequence designation to establish all of the boundary conditions for the code input. It was therefore necessary to establish a representative sequence for each bin, with the resulting source term to apply for all sequences in a bin. This task was carried out through interaction of the Battelle analysts with the Sandia SARRP analysts, followed by additional review and advice from the source term binning group. This process also involved iteration and refinement of the source term bin definition to ensure that all sequences within a bin were similar enough to the sequence represented in the calculation. Some sensitivity studies were performed with the STCP to assist in this effort [48,49].

Most of the analyses used directly in SARRP were calculated as updated versions of sequences previously analyzed in BMI-2104. These calculations were performed to provide additional background for the NRC publication NUREG-0956 or directly in support of SARRP. Most of the calculations are reported in Reference 49, and any calculations included in this section that are not from that report are referenced separately. The code calculations used for each sequence are defined below.

Bin 1 corresponds to an early containment failure with the containment sprays unavailable. This release is used for core melts with high primary system pressure and with a rupture failure of containment at the time of reactor vessel failure. The core debris is uncoolable and there is no direct heating. The source 
term was calculated directly by Battelle as Surry sequence TMLB'- $\delta$ with no steam condensation.*

$\operatorname{Bin} 2$ is used for sequences with conditions similar to bin 1 , with the exception that the sprays operate or the core forms a permanently coolable debris bed. The source term is a composite: the in-vessel release species are taken directly from bin 1, while the core/concrete interaction species are taken from bin 4, defined below. No credit is taken for spray action on the RCS release because the high reactor system pressure is assumed to lead to a puff release, possibly precluding spray effectiveness. (This assumption was later found to be too conservative, as discussed in Appendix C.)

Bin 3 applies to sequences with containment failure at the time of vessel failure and intermediate pressure in the primary prior to vessel failure. The containment sprays operate up until vessel breach and then fail at that time. Once again, the debris bed is not coolable. This bin was calculated as $S_{2} D-\gamma$ without sprays.

Bin 4 accounts for the action of the containment sprays following containment failure or the elimination of the core-concrete interaction release associated with a coolable debris bed. The other conditions are the same as bin 3. This was calculated by Battelle as a variation of the $S_{2} D-\gamma$ sequence discussed in bin 3 , with changes to account for the sprays.

Bin 5 was developed to represent the sequences involving containment failure prior to core melt. A particular set of the ASEP sequences involved the loss of all containment cooling which could induce a core melt due to system failures at the time of containment failure. This was modeled as the sequence AG, and this bin is therefore appropriate to low pressure conditions in the RCS and is too conservative for sequences with the same scenario but with higher RCS pressure.

Bin 6 applies to releases from containment due to a large, pre-existing leak or an isolation failure, without the operation of containment sprays. The source term was taken directly from recent (July 1984) Battelle calculations for the sequence TMLB'- $\beta$ [48].

Bin 7 is used for scenarios involving large, pre-existing leakage or isolation failure with the operation of sprays. The releases were taken from Battelle calculations for $\mathrm{S}_{2} \mathrm{D}-\beta$.

Bin 8 is used for scenarios involving late containment ruptures in which the sprays operate for at least a very short time immediately prior to rupture, and either the sprays continue to operate after rupture or else the debris bed is permanently coolable. Bin 8 source terms were derived from Battelle calculations of the TMLB'- $\gamma$ sequence, including the delayed operation of containment sprays.

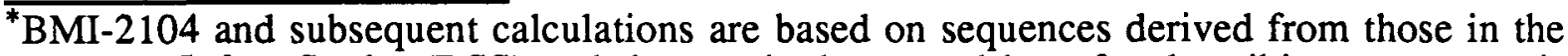
Reactor Safety Study (RSS) and the terminology used here for describing sequences is therefore the same as that used in the RSS. 
Bin 9 applies to the same containment failure mode as bin 8, late rupture; however, in this case the sprays either do not operate before the containment failure, or they fail later in the sequence with an uncoolable debris bed. Bin 9 was assumed to correspond to the BMI-2104 source term for the TMLB'- $\varepsilon$ sequence with an atmospheric release [18].

Bin 10 was applied for all cases in which the containment was predicted to have a late leakage that was large enough to preclude rupture but too small to depressurize the containment. Its release fractions were taken from the TMLB' late-leakage calculations reported in Reference 48.

Bin 11 models containment bypass sequences that are direct to the auxiliary building with scrubbing by water in the release path. The sequence was run directly by Battelle for SARRP, correcting an error in the earlier version of the $\mathrm{V}$ sequence reported in BMI-2104."

Bin 12 is the analogous bin to bin 11 for a bypass sequence but there was no water in the release path through the auxiliary building. The updated $\mathrm{V}$ sequence was run without water scrubbing for this source term.

The remaining source terms, for Bins 13-20, were not based directly on STCP calculations. The methods used to derive source terms for these other bins are described in the following section.

\subsubsection{Derivation of Source Terms for Other Bins}

There were two types of sequences which required source terms that were not calculated using the STCP: 1) containment leakage scenarios and 2) direct heating scenarios. The specific calculations for each bin are defined in the subsections below.

\section{Model for Estimation of Releases Due to Late Containment Leakage}

The results of BMI-2104 were focused on the more significant consequences associated with reactor accidents, those involving containment failure. In order to accomplish SARRP's goal of a comprehensive risk assessment, it was therefore necessary to estimate the smaller releases associated with design basis leakage that would be the only source if there was no containment failure. A simple model was used in SARRP to generate source terms for these less critical conditions. The method described below also applies to the cases in which a small containment isolation failure exists, termed "small pre-existing leaks" in the containment event tree.

\footnotetext{
"Recent calculations with the actual STCP (rather than the precursor codes used here) show substantially higher $\mathrm{Cs}$ and I release fractions and lower refractory releases due to improvements in the model for this type of sequence.
} 
For design-basis and other small leaks, it is appropriate to assume that the residence time for fission products in containment is long with respect to the mean aerosol residence time. Therefore the aerosol species that are released from the fuel when the vessel fails and after the debris enters containment are reduced as the aerosols leave the containment atmosphere. The release to the environment from the containment for aerosol species may be approximated by:

$$
\mathrm{Ft}(\mathrm{i})=[\mathrm{FRCS}(\mathrm{i})+\mathrm{FCCI}(\mathrm{i})] * \operatorname{Tr}(\mathrm{i}) * \mathrm{Rlk}
$$

where $\operatorname{Tr}(i)$ is the mean residence time for a species $i(10,000$ seconds was used as the general value) and Rlk is the fractional release rate for gases from the containment. For the noble gases and organic iodine, the release from containment is simply a function of the amount available, the gas leakage from containment, and the time period of interest:

$$
\mathrm{Ft}(\mathrm{i})=\mathrm{Qa}(\mathrm{i}) * \mathrm{Rlk} * \mathrm{Tst}
$$

where $\mathrm{Qa}(\mathrm{i})$ is assumed to be the total core inventory for noble gases and $0.5 \%$ of total iodine inventory for the organic iodine release fraction. The total time over which the releases are integrated (Tst) depended on the specific bin. The releases for different levels of assumed containment leakage are calculated by changing the gas leak rate (Rlk). The specific calculations used to represent the bins are described below.

Bin 13 was used for cases in which basemat meltthrough is the only containment failure, with the additional condition that the containment pressure remains high. It was derived by adding $100 \%$ of the noble gases to the design basis leakage case represented in bin 15 . Organic iodine fractions were calculated as in the base case but integrated over 30 days rather than 5 days. This bin has such small releases that differences resulting from different accident phenomenology are unimportant and one bin is appropriate.

Bin 14 was subdivided from bin 13 to account for basemat meltthrough with little containment driving pressure. The noble gas release was calculated for a 30 day interval. Organic iodine fractions were calculated as in bin 13, for a 30 day period.

Bin 15, the design basis leakage source term, applies to all accidents which do not lead to containment failure. Design leakage was assumed to be $.1 \%$ per day, but this bin is also applicable to larger leakage by direct scaling. The noble gas and organic iodine releases were integrated over 5 days, roughly the order of the longest half-life for the noble gas species of concern. Once again, variations due to aerosol phenomenology are negligible due to the small releases associated with this bin. 


\section{Direct Heating and High Pressure Ejection Releases}

The SARRP containment analysis of Surry identified a significant fraction of scenarios involving either high pressure ejection or direct heating. High pressure ejection involves the forced ejection of the molten core material at the time of vessel breach, resulting in significant aerosol formation. Direct heating is always preceded by high pressure ejection, but further involves substantial heating of the containment atmosphere (with a resultant increase in the release of radioactive species). It was therefore necessary to establish bins and source terms for direct heating scenarios. As described below, all releases except for the direct heating or high pressure ejection component were based on other bins with similar conditions. The incremental releases due to these other processes were calculated after review of available information and consultation with the review groups. The details of the derivations of these releases are included in Appendix C. In summary, the amount of core participating was assumed to release $100 \%$ of the remaining $\mathrm{Te}$ and $50 \%$ of the $\mathrm{Ru} ; 1 \%$ of all other constituents were also assumed to be aerosolized (only significant for Group 7). The amount of core participating in the ejection and direct heating process was a required input that was calculated from the results of the central walkthrough of the containment event tree. The treatment of specific bins is defined below:

Bin 16 is appropriate for accident conditions similar to those of bin 1, with the exception that the direct heating phenomenon contributes directly to the containment failure at the time of vessel failure. The same STCP run was used for all releases except direct heating (TMLB'- $\delta$ with no steam condensation) except that a correction was needed to reduce the core-concrete aerosols to avoid double counting of some species.

Bin 17 was calculated the same as above, but the base release and accident conditions are the same as for bin 2--a high pressure early containment failure with spray operation. The direct heating was therefore reduced by a factor of two to account for the amount of direct heating assumed in the CET.

Bin 18 applies to the same conditions as bin 3, except that direct heating is a predominant phenomenon contributing to the containment failure. The same STCP run was used-- $S_{2} D-\gamma$ with sprays failing at the time of containment failure. The direct heating release of bin 16 was reduced by a factor of five to account for the lesser amount of material involved. This factor of five was backed out of the central walkthrough of the CET.

Bin 19 is analogous to bin 18, except that the accident scenario is that of bin 4 . The same STCP run was used-- $S_{2} D-\gamma$ with sprays. The direct heating release of bin 16 was reduced by a factor of five to account for the amount of direct heating estimated in the CET.

Bin 20 was a bin that was defined late in the analysis to better represent one of the particular outcomes of the analysis--containment failure at vessel breach, 
intermediate pressure in the primary system at vessel breach, with failure of the sprays from the onset of the accident (similar to bin 18 but with sprays unavailable).

\subsubsection{Derivation of Source Terms for Uncertainty Analyses}

The discussion above refers to the central estimates of the source terms. The uncertainty analyses described in Section 2.2, OCP and LLH, required additional source terms to represent the uncertainty in this part of the analysis. The actual calculation of the expected ranges of uncertainty about the central estimate is very complex. The selection of issues for the LLH study is discussed in Section 4 and Appendix C. A brief discussion of the estimates of optimistic and pessimistic source terms is included below, details are included in Appendix C.

\section{Source Terms for the LLH Uncertainty Analysis}

The input of the expert review group was the basis for the creation of source terms that provide some measure of uncertainty in this portion of the analysis. The source term uncertainty was considered in terms of issues which describe phenomena that are both uncertain and that could significantly impact the total source term, such as decontamination factors for the sprays or the amount of CsI decomposition. Several of the issues considered phenomena that are not included in the STCP but that are thought to be important, while nearly all issues involve ranges that are beyond what the STCP would predict." It was therefore necessary to generate source terms that could account for all the combinations of issue outcomes proposed by the review group. This involved the development of a simple parametric model of the release, separating the components of the releases to a level of detail appropriate to the issue definition. The method for the development of source terms for the LLH is described below, but it should be noted that there many subtleties in the application of this method. The details of this process are described fully in Appendix C.

In defining issues and issue levels, the results of actual calculations were usually relied upon whenever possible. In all cases, three to four levels have been specified for each issue. For most issues, one level is based upon the results of a Battelle code calculation. Exceptions arise because, in a few cases, the review group made changes to the level definitions in such a way that no level corresponds exactly to the result of a STCP or BMI-2104 calculation, or else because the issue addressed is not even modeled in the STCP. The highest and lowest levels are based upon QUEST results insofar as possible. In many of these cases, substantial

"Once again, the term STCP is used loosely here to refer also to the precursor codes that were actually used in this study. 
extrapolations have been required, and in other cases nearly no evidence was available to support the assignment of ranges, and analyst judgment is the only justification.

Since the possible values of the issue outcomes form a continuum, it might seem more appropriate to define the outcomes in terms of a continuous range of values, rather than a set of discrete values. However, the present LLH study employed sets of discrete values; changing this feature would have presented difficulties not justifiable in terms of any possible improvement in the results. Since it is the discrete values which actually are used in the calculation, it is these same values that were presented to the reviewers for the assignments of weighting factors.

The results presented here are discussed in the context of the following simple equation for the release fractions of the various radionuclide species:

$\mathrm{Ft}(\mathrm{i})=\mathrm{FCOR}(\mathrm{i}) * \mathrm{FVES}(\mathrm{i}) * \mathrm{FCONV}(\mathrm{i})+\mathrm{FCCI}(\mathrm{i}) * \mathrm{FCONC}(\mathrm{i})$

The following notation and terminology are used:

$$
\begin{aligned}
& \mathrm{Ft}(\mathrm{i})=\text { fraction of the initial core inventory of species } i \text { that is ultimately } \\
& \text { FCOR(i) = fraction of initial core inventory of species } \mathrm{i} \text { released from the }
\end{aligned}
$$

It must be emphasized that the actual construction of a set of source terms from a set of issue level specifications requires consideration of many features not represented explicitly in the equation above, which should be thought of more as providing a conceptual framework for discussion than as an actual algorithm for calculation, except when suitably modified and/or restricted. For example, the STCP assumes that iodine will be present as CsI while recent experimental data indicate that, in the presence of steam and radiation, CsI tends to decompose to elemental iodine (or some other highly volatile form) which may be released from the primary system with zero retention, although the cesium derived from the CsI may still be retained. In order to allow for this possibility, the equation is modified for iodine as follows:

$$
\mathrm{Ft}(\mathrm{I})=\mathrm{FCOR}(\mathrm{I}) *[\mathrm{FI} 2+(1-\mathrm{FI} 2) * \mathrm{FVES}(\mathrm{I})] * \mathrm{FCONV}(\mathrm{I})+\mathrm{FCCI}(\mathrm{I}) * \mathrm{FCONC}(\mathrm{I})
$$


NUREG/CR-4551,VOL.1: DRAFT REPORT FOR COMMENT (SEPTEMBER, 1986)

Here, FI2 is the fraction of the iodine released from the fuel which is converted to the more volatile form; no in-vessel retention is credited for this more volatile form.

It also must be emphasized that the approach being used to construct source terms from issue specifications is a purely parametric one. No mechanistic or phenomenological modeling is used; the possible effects one issue may have upon another, or possible restrictions as to when a given result is applicable, are taken into account only if explicit provision is made for them.

In general, it is not possible to meaningfully express the effect of a source term "issue" upon the source term directly, but the possible effects of an issue can be expressed in terms of its effect upon one or more of the quantities appearing in the parametric equation, at least as a first approximation. The possible outcomes and associated sample weights are therefore discussed in terms of these quantities (in Appendix C). To keep the number of issues manageable and yet also provide reasonable coverage of the myriads of phenomena that are actually involved, the definition of "issues" often must be very broad.

The actual manipulation of this parametric model was carried out with a simple code written for this purpose called SURSOR. This code accounts for all of the issues and issue levels and calculates the release fractions for each release group for a specified set of issue levels. The sample member defines the issue level combinations to be considered. Thus a source term for each of the source term bins can be calculated for each LLH sample member. This process results in the calculation of 1900 source terms (100 sample members each with 19 bins).

It is not practical to calculate consequences for each of the 1900 source terms so generated. Thus an additional simplification was required for the LLH: source-term clustering. This involves the grouping of the 1900 source terms into a lesser number of clusters that can approximately characterize many of the individual source terms. Consequences are then calculated for the clusters only, in this case reducing the required consequence evaluations from 1900 to 25 . The clustering process itself is described in Appendix C, Section C.3. The clustering is based on two characteristics of the release that are the principal determinants of the consequences: magnitude and timing of the releases. The clustering process leads to some loss of resolution since the clusters do not have meaning relative to the accident sequence or containment response, but a review of the results suggests that the loss of resolution does not lead to an unacceptable distortion of the uncertainty results. Some discussion of the impact of clustering is provided in Section 5 and Appendix C. 


\section{Optimistic and Pessimistic Source Terms}

The characterization of uncertainty in the source-term assessment for SARRP using the OCP method reflects a number of resources beyond the STCP methodology and results. The key source of information was the QUEST program which treated specific sequences in some detail [12]. This is the most comprehensive assessment of uncertainties in the phenomenology of accident source terms currently available. Other sources [25,50] contain applicable information on several of the key uncertainties in the analysis. These sources were used in that the degree of scientific disagreement on several important issues was reflected in the SARRP analyses. However, these other sources could not be used as directly as the QUEST results owing to the inherent differences in overall approach which precluded effective quantitative comparisons. It is hoped that the study described in this report will help to focus these areas of contention and direct future interactions of researchers.

Two other sets of source terms in addition to the central estimates were derived for the OCP study. ${ }^{*}$ These source term sets, optimistic and pessimistic, are intended to represent a range of uncertainty that is a reasonable portrayal of the results that would be obtained if the important sensitivities were varied. These estimates are not bounding and were not developed in a statistical sense, hence their meaning is subject to strict limitations. The discussion of uncertainty here cannot include the details of the estimates that were made for each of the release bins. Let it suffice to say that the optimistic and pessimistic parameters were developed in a manner that attempts to be internally consistent, represents the subjective opinions of the analyst responsible for the Surry source-term work, and accounts for the key sensitivities and most viewpoints of the technical community. The uncertainty parameters were developed with insight from a variety of STCP and QUEST results, but it should be recognized that the quantitative estimates are a product of SARRP and include significant departures from both the STCP and QUEST results. The overall approach was to vary the inputs in two directions about the central estimate: the optimistic removes conservatism and accounts for phenomenologies that tend to reduce the source terms, and the pessimistic takes the conservative result when knowledge is incomplete.

As already stated, the subject of source-term uncertainty is too complex to permit a useful summary here of the individual calculations affecting each bin. Most of the issues important to the uncertainty are discussed the Section 4 because the OCP uncertainty issues include all of the LLH issues. Some of the issues of concern are listed below:

${ }^{*}$ Appendix $\mathrm{C}$ describes in detail the development of the optimistic and central source terms. 
RCS Pressurization. The pressure history of the RCS throughout the core-melt process is critical to the release fractions associated with the accident.

Zirconium Oxidation. As with the RCS pressure history, the amount of $\mathrm{Zr}$ released is important to the containment response as well as the radionuclide releases.

In-Vessel Fuel Release. The processes that result in the in-vessel release of fission products are complex and highly dependent on the exact conditions. Current modeling capabilities are limited by practicality, basic understanding, and experimental evidence.

RCS Deposition. In-vessel deposition of radionuclide vapors can greatly reduce the source term, and there is great uncertainty in this phenomenon.

Iodine Phenomena Uncertainties. The are a number of uncertainties involving the phenomenology of iodine releases that could lead to significant uncertainty in the total iodine release to the environment.

Core-Concrete Interaction Releases. Although there are codes for predicting core-concrete interaction releases, there are enormous uncertainties concerning the effects of type of concrete, temperature, zirconium content of the melt and other physical parameters.

Aerosol Agglomerations. The actual behavior of aerosol species in the containment is not fully known.

Containment Sprays. Recent research of containment-spray effectiveness in reducing radioactive release has indicated that its impact is more complex than previously thought, and that the decontamination factors are dependent on many physical parameters.

Direct Heating. The uncertainty in the potential for the occurrence of this process is coupled with an uncertainty in the estimation of the release that might be expected.

Bressure Effects on Core/Concrete Interaction. It has been suggested that the pressurization of the containment could lead to substantial reduction in the rate of release during a core/concrete interaction.

Debris Coolability and Reactor Cavity Water. The amount of water in the cavity, as well as the timing of its presence, can greatly influence the release of radionuclides during the ex-vessel processes.

Source-Term Binning. This process can introduce uncertainty if the bins are too coarse to correctly discriminate the important characteristics or if the dominant (probabilistically) sequences in the bins are not the same as the assumed "average" bin characteristics.

The generation of quantitative uncertainty parameters due to these effects in a straightforward manner is not possible, since combined effects of many different uncertainty issues are often difficult to predict. For Surry, there were two attempts to assess the importance of these uncertainties, and the results discussions in Section 5 and Appendix $C$ describe the insights developed through this analysis. 


\subsection{OFFSITE CONSEQUENCE ANALYSIS}

The estimation of offsite consequences due to reactor accidents for the assessment of Surry was performed using the CRAC2 computer code [55]. CRAC2 is an improved version of the CRAC code, which was developed for and used to estimate the offsite consequences reported in the Reactor Safety Study [31].

Some SARRP analyses for Surry were also performed using a new consequenceanalysis package, the MELCOR Accident Consequence Code System (MACCS) [56]. This new code is a much improved version of CRAC2, so much improved that it was deliberately not added to the series and called CRAC3. The principal improvements are (1) an improved architecture that facilitates development of intermediate results and the performance of uncertainty studies, (2) a multi-plume dispersion model that includes a multi-step crosswind concentration profile, (3) a dry deposition velocity that depends on particle size, (4) an improved wet deposition model that does not overpredict ground concentrations produced by rainout, and (5) expanded sets of environmental transport, dosimetry, health effects, and economic cost models, all supported by critical reviews of input data. Not all of the models have yet been verified by thorough peer review.

The consequence analysis requires the following input:

- A source term-release fractions for radionuclide groups, along with timing of release as well as sensible heat associated with the release;

- The inventory at reactor scram of all isotopes important to offsite consequences;

- The population distribution around the reactor;

- Emergency response parameters and assumptions; and

- Weather, land use data, and economic data for the region around the reactor site.

Given these inputs, consequence codes such as CRAC2 and MACCS predict the following:

- The downwind transport, dispersion, and deposition of the radioactive materials released from the failed containment;

- The radiation doses received by the exposed populations via direct (cloudshine, inhalation, groundshine) and indirect (ingestion, inhalation) pathways;

- The mitigation of those doses by emergency response actions (evacuation, sheltering, and relocation of people), interdiction of milk and crops, and decontamination or interdiction of land and buildings; 
- The fatalities and injuries expected to occur within one year of the accident, and the latent cancer fatalities expected to occur over the lifetime of the exposed individuals;

- The total population dose received by the people living within some distance (50 miles) of the plant and the early fatality risk to persons living near the plant (within one mile); and

- The offsite costs of emergency response actions, and of the interdiction and decontamination of land, buildings, milk and crops.

By performing calculations for all possible combinations of representative sets of source terms, weather sequences and exposed populations, statistical distributions of consequence measures are developed that depict the range and probability of consequences. If desired, the uncertainties associated with offsite consequence estimates can be developed by a variation of input parameter values using structured Monte Carlo sampling techniques [57], but this was not performed as part of this study.

Site-specific offsite consequence calculations require as input a population distribution for the region around the reactor and a one year file of hourly meteorological data that is characteristic of the region. The Surry population distribution is constructed from 1980 census data on a polar grid, which has 16 angular sectors and 18 radial intervals that extend outward to 500 miles. (The radial intervals used were $1,2,3,4,5,10,20,30,40,50,60,70,85,100$, $150,200,350$, and 500 miles.) The census data was also corrected for average daily transient population.

A representative set of weather sequences was constructed from a file of meteorological data as follows. First, the 8760 (the number of hours in a year) weather sequences in the meteorology file were sorted into 29 bins using wind speed, atmospheric stability, and precipitation as sorting criteria. Then four sequences were randomly selected from each bin that contained sequences (28), which produced a stratified sample of 112 representative weather sequences. The Surry analysis was based on one year of meteorological data gathered from the Shearon Harris site.

The land usage and economic data were collected for the 15 states within 500 miles of the Surry site. Each of the 228 elements of the 16 sector, 18 radial interval polar coordinate grid was then associated with one of the 15 states, or the ocean if applicable. The habitable land fraction of each element was estimated by inspection of maps. State average values were used for the fraction of land in each element that is devoted to farming, the identity of the crops grown, and their worth [58]. National average data (see Reference 58) was used for the worth 
of public and private urban land, buildings, equipment, inventories and possessions (expressed as total worth of tangible public and private assets per person).

The following emergency response assumptions were used in the Surry CRAC2 calculations:

- Of the population located within 10 miles of the site, $95 \%$ are evacuated radially at a speed of $1.2 \mathrm{~m} / \mathrm{sec}$. The evacuation begins one hour after the warning time and once the evacuees reach a radial distance of 15 miles they are assumed not to receive additional dose.

- People who do not evacuate continue their normal activities (they are not directed to shelter). The non-evacuees who are projected to receive a groundshine dose to bone marrow within seven days of the accident that equals or exceeds $25 \mathrm{rem}$ are relocated at the end of one day. All other nonevacuees are relocated in seven days if interdiction and decontamination can not reduce projected groundshine dose to bone marrow to less than 25 rem in 30 years.

- Radiation shielding factors for evacuees and for non-evacuees as follows:

\section{Non-Evacuees Evacuees}

$\begin{array}{lll}\text { Cloud dose } & 0.75 & 1.0 \\ \text { Ground dose } & 0.33 & 0.5 \\ \text { Inhalation dose } & 0.75 & 1.0\end{array}$

Finally, the Surry CRAC2 calculations used a dry deposition velocity of $0.01 \mathrm{~m} / \mathrm{sec}$ for airborne particles and assumed that the washout coefficient (lambda) in the rain model equals 0.0001 times the rainfall rate.

The source term information was an output of the previous task. However, some of the releases are not well characterized by a uniform release rate; rather a hot, short duration puff followed by a cooler, longer duration tail provides a better description. Since CRAC2 cannot treat multipuff releases and because the variation with time of individual release groups was not known, two CRAC2 calculations were performed for the seven source terms best described as a multipuff. For the first calculation, the release was assumed to be uniform over the full duration. For the second calculation, the entire release was compressed into the puff portion of the source term and the sensible heat release rate during the puff portion of the release was substituted for the average release of sensible heat over the full duration. The second calculation was used for all cases where a multipuff model would better fit the accident.

Finally, the clustering step for source terms in the LLH analysis has been defined in previous sections. The source terms were clustered as described in Appendix $C$, and the other determinants of the source term were calculated as weighted sums of the central source term values of those parameters. This process was carried out on the warning times, the sensible 
heats, the release times, durations, and elevations. For example, the release duration $\Delta \mathrm{t}_{\mathrm{i}}$ and the rate of release of sensible heat $E_{\mathfrak{i}}$ for cluster $i$ were calculated with the following equations,

$$
\Delta t_{i}=\begin{gathered}
\Sigma_{j} n_{j} P_{j} \Delta t_{j} \\
\sum_{j} n_{j} P_{j}
\end{gathered} \quad E_{i} \Delta_{i}=\frac{\sum_{j j} n_{j} P_{j} E_{j} \Delta t_{j}}{\Sigma_{j} n_{j} P_{j}}
$$

where $\Delta t_{j}, E_{j}$ and $P_{j}$ are, respectively, the release duration, the release rate of sensible heat and the relative probability of occurrence of central estimate source term $j$, and $n_{j}$ is the number of variants of central source term $j$ that contributed to cluster source term $i$.

The actual source terms used in the consequence evaluation and the results of the CRAC2 and MACCS consequences are provided in Section 5. 


\section{References for Section 2}

1. Griesmeyer, J. M. Users' Guide for the EVNTRE Computer Code. Sandia National Laboratories, Albuquerque, NM: (to be published).

2. Murfin, W. B. Users' Guide for the RISKEE Computer Code. Sandia National Laboratories, Albuquerque, NM: (to be published).

3. Corradini, M. L., et al. A Review of the SARRP Containment Event Trees, U.S. Nuclear Regulatory Commission Report NUREG/CR-4569, University of Wisconsin, Madison, WI: May, 1986.

4. Review of the Source-Term Code Package. Brookhaven National Laboratories, Upton, NY: (to be published).

5. Probabilistic Risk Uncertainty Evaluation Program (PRUEP) . U.S. Nuclear Regulatory Commission Report NUREG/CR-XXXX, Sandia National Laboratories, Albuquerque, NM: (to be published).

6. PRA Procedures Guide. U.S. Nuclear Regulatory Commission Report NUREG/CR2300, Washington, DC: 1983.

7. Probabilistic Risk Assessment Reference Document. U.S. Nuclear Regulatory Commission Report NUREG-1050, Washington, DC: 1984.

8. Iman, R. L. and J. C. Helton. A Comparison of Uncertainty and Sensitivity Analysis Techniques for Computer Models. U.S. Nuclear Regulatory Commission Report NUREG/CR-3904, Sandia National Laboratories, Albuquerque, NM: March, 1985.

9. Iman, R. L. and W. J. Conover. Sensitivity Analysis Techniques: Self-Teaching Curriculum. U.S. Nuclear Regulatory Commission Report NUREG/CR-2350, Sandia National Laboratories, Albuquerque, NM: June, 1982.

10. Iman, R. L. et al. Stepwise Regression with PRESS and Rank Regression.. Sandia National Laboratories Report SAND79-1472, Albuquerque, NM: 1980.

11. Benjamin, A. S., et al. Containment Event Analysis for Postulated Severe Accidents: Surry Power Station, Unit 1. U.S. Nuclear Regulatory Commission Report NUREG/CR-4700, Volume 1, Sandia National Laboratories, Albuquerque, NM: February, 1987.

12. Lipinski, R. J., et al. Uncertainty in Radionuclide Release Under Specific LWR Accident Conditions. Sandia National Laboratories Report SAND84-0410, Albuquerque, NM: 1985.

13. Bertucio, R. C., et al. Analysis of Core Damage Frequency From Internal Initiating Events: Surry, Unit 1. U.S. Nuclear Regulatory Commission Report NUREG/CR4550, Volume 3 (Draft Report), Sandia National Laboratories, Albuquerque, NM: April, 1986.

14. Harper, F. T. "ASEP Data Reevaluation." Memorandum to Senior Consultant Group, et al., Sandia National Laboratories, Albuquerque, NM: March 15, 1985.

15. Carlson, D. D. Interim Reliability Evaluation Program Procedures Guide. U.S. Nuclear Regulatory Commission Report NUREG/CR-2728, Sandia National Laboratories, Albuquerque, NM: 1983.

16. Estimates of Early Containment Loads from Core Melt Accidents, Report of the Containment Loads Working Group. U.S. Nuclear Regulatory Commission Report NUREG-1079 (Draft for Comment), Washington, DC: December, 1985. 
17. Containment Loads Working Group. "Consensus Summaries for Standard Problems 1 through 6." Letter Reports to J. Telford, U.S. Nuclear Regulatory Commission, MayJune, 1984.

18. Gieseke, J. A., el al. Radionuclide Release Under Specific Accident Conditions. Battelle Columbus Laboratories Report BMI-2104, Columbus, OH: 1984.

19. IDCOR Task 23.1: Integrated Containment Analysis. Technology for Energy Corporation, Knoxville, TN: 1984.

20. Haskin, F. E., et al. Analysis of Hypothetical Severe Core Damage Accidents for the Zion Pressurized Water Reactor. U.S. Nuclear Regulatory Commission Report NUREG/CR-1989, Sandia National Laboratories, Albuquerque, NM: 1982.

21. Camp, A. L., et al. MARCH-HECTR Analysis of Selected Accidents in an IceCondenser Containment. U.S. Nuclear Regulatory Commission Report NUREG/CR3912, Sandia National Laboratories, Albuquerque, NM: December, 1984.

22. Gasser, R. D., et al. Analysis of Station Blackout Accidents for the Bellefonte Pressurized Water Reactor, U.S. Nuclear Regulatory Commission Report NUREG/CR4563, Sandia National Laboratories, Albuquerque, NM: September, 1986.

23. King, D. B., et al. Gas Transport Calculations for a Large, Dry PWR Containment (Bellefonte) for Arrested Sequences U.S. Nuclear Regulatory Commission Report NUREG/CR-4599, Sandia National Laboratories, Albuquerque, NM: (to be published).

24. Haskin, F. E., et al. "Combustion-Induced Loads in Large Dry PWR Containments." Proceedings of the Second Containment Integrity Workshop, U.S. Nuclear Regulatory Commission Report NUREG/CP-0056, Washington, DC: August, 1984.

25. Rivard, J. B., et al. Identification of Severe Accident Uncertainties. U.S. Nuclear Regulatory Commission Report NUREG/CR-3440, Sandia National Laboratories, Albuquerque, NM: September, 1984.

26. Benjamin, A. S., et al, "SARRP - Risk Rebaselining and Risk Reduction Analysis." Proceedings of the 11th Water Reactor Safety Research Information Meeting. U.S. Nuclear Regulatory Commission Report NUREG/CP-0048, Vol. 3, Washington, DC: January, 1984.

27. Steam Explosion Review Group. A Review of the Current Understanding of the Potential for Containment Failure Arising from In-Vessel Steam Explosions. U.S. Nuclear Regulatory Commission Report NUREG-1116, Washington, DC: February, 1985.

28. Tarbell, W., et al. High-Pressure Melt Streaming (HIPS) Program Plan. U.S. Nuclear Regulatory Commission Report NUREG/CR-3025. Sandia National Laboratories, Albuquerque, NM: August, 1984.

29. Reactor Safety Study--An Assesșment of Accident Risks in U.S. Commercial Nuclear Power Plants. U.S. Nuclear Regulatory Commission Report WASH-1400 (NUREG75/014), Washington, DC: 1975.

30. Reactor Safety Study Methodology Applications Program. U.S. Nuclear Regulatory Commission Report NUREG/CR-1659, Volumes 1 through 4, Sandia National Laboratories, Albuquerque, NM: 1981-1982.

31. Commonwealth Edison Company. Zion Probabilistic Risk Assessment. Chicago, Illinois: 1980. 
32. Sizewell B Probabilistic Safety Study. Westinghouse Electric Co. Report WCAP-9991, Monroeville, PA: 1982.

33. Seabrook Plant Probabilistic Safety Study. Yankee Atomic Electric Co., Seabrook, NH: December, 1983.

34. GESSAR-II, BWR/6 Standard Plant Probabilistic Risk Assessment. General Electric Co., San Jose, CA: 1982.

35. Final Safety Analysis Report for Surry Unit 1. Virginia Electric Power Company, Richmond, VA: 1971.

36. Drozd, A., et al. "Parametric Study of Aerosol Behavior Following AB and TMLB Accidents," Proceedings of the American Nuclear Society 1984 Annual Meeting . TP8454, Stone and Webster Engineering Corp.: 1984.

37. Greismann, L., et al. Final Report, Containment Analysis Techniques, a State-of-the-Art Summary. U.S. Nuclear Regulatory Commission Report NUREG/CR-3653, Ames Laboratories, Ames, IA: March, 1984.

38. Weinstein, M. B., et al. "Primary Containment Leakage Integrity: Availability and Review of Failure Experience," Nuclear Safety: 21, 1980.

39. Pratt, W. T., et al. Containment Response During Degraded Core Accidents Initiated by Transients and Small Breack LOCA in the Zion/Indian Point Reactor Plants. U.S. Nuclear Regulatory Commission Report NUREG/CR-2228, Brookhaven National Laboratories, Upton, NY: July, 1981.

40. Lyons, W. G, "Presentation at NRC/IDCOR Meeting on Containment Loads and Fisssion Product Behavior." U.S. Nuclear Regulatory Commission: May, 1984.

41. Murfin, W. B., et al. Report of the Zion/Indian Point Study. U.S. Nuclear Regulatory Commission Report NUREG/CR-1410, Sandia National Laboratories, Albuquerque, NM: August, 1980.

42. Camp, A. L., et al. Light Water Reactor Hydrogen Manual. U.S. Nuclear Regulatory Commission Report NUREG/CR-2726, Sandia National Laboratories, Albuquerque, NM: August, 1983.

43. Berman, M., et al. An Uncertainty Study of PWR Steam Explosions. U.S. Nuclear Regulatory Commission Report NUREG/CR-3369, Sandia National Laboratories, Albuquerque, NM: May, 1984.

44. Niemczyk, S. J., et al. The Consequences from Liquid Pathways After a Meltdown Accident. U.S. Nuclear Regulatory Commission Report NUREG/CR-1596, Sandia National Laboratories, Albuquerque, NM: June, 1981.

45. McClymont, A. S. and B. W. Poehlman. Loss of Off-Site Power at Nuclear Power Plants: Data and Analysis. Electric Power Research Institute Report NP-2301 (Interim Report), Palo Alto, CA: March, 1982.

46. Reassessment of the Technical Bases for Estimating Source Terms. U.S. Nuclear Regulatory Commission Report NUREG-0956, Washington, DC: August, 1985.

47. Gieseke, J. A., et al. Source Term Code Package: A User's Guide (Mod1). U.S. Nuclear Regulatory Commission Report NUREG/CR-4587, Battelle Columbus Laboratories, Columbus, OH: July, 1986. 
48. Gieseke, J. A., et al. Informal Report on Source Term Predictions for Various Containment Failure Assumptions. Battelle Columbus Laboratories, Columbus, OH: August, 1984.

49. Denning, R. S., et al. Report on Radionuclide Release Calculations for Selected Severe Accident Scenarios: Volume III, PWR Subatmospheric Containment Design, U.S. Nuclear Regulatory Commission Report NUREG/CR-4624, Volume 3, Battelle Columbus Laboratories, Columbus, $\mathrm{OH}$ : July, 1986.

50. Industry Degraded Core Rulemaking Program (IDCOR): Technical Summary Report. Technology for Energy Corporation, Knoxville, TN: 1984.

51. Ritchie, L. T., et al. CRAC2 Model Description. U. S. Nuclear Regulatory Commission Report NUREG/CR-2326, Sandia National Laboratories, Albuquerque, NM: 1984.

52. Alpert, D. J., et al, "The MELCOR Accident Consequence Code System." Proceedings of the CEC Workshop on Methods of Assessing the Off-Site Radiological Consequences of Nuclear Accidents. (to be published).

53. Alpert, D. J. and J. C. Helton, "Uncertainty and Sensitivity Analysis for Reactor Accident Consequence Models." Proceedings of the CEC Workshop on Methods of Assessing the Off-Site Radiological Consequences of Nuclear Accidents. (to be published).

54. Aldrich, D. C., et al. Technical Guidance for Siting Criteria Development.. U. S. Nuclear Regulatory Commission Report NUREG/CR-2239, Sandia National Laboratories, Albuquerque, NM: December, 1982. 


\section{Section 3 METHODOLOGY FOR THE ASSESSMENT OF RISK-REDUCTION OPTIONS}

The second principal goal of SARRP was the assessment of the values and impacts of potential plant changes that could reduce risk. Risk-reduction measures that have been proposed in previous research efforts, and new options that were based on the specific results of the risk rebaselining for Surry were included in the scope of this task. These measures include modifications to plant systems, addition of new equipment, changes to procedures, and very specific changes aimed at the dominant, plant-specific risk contributors. Risk reduction was considered for all consequence measures, including health effects and costs, and the measures considered therefore included options that would reduce the assessed core-damage frequency (preventive options) as well as the consequences of an accident (mitigative features). The assessment of the relative merit of these options included an examination of both positive and negative impacts, the latter including the total costs of implementation and a very brief review of the potential for the risk for some types of sequences to be increased by the change. The following sections describe the approach taken in performing this task. Details of the actual assessment are provided in Appendix E, while the results are provided in Section 6 of the main report.

\subsection{IDENTIFICATION OF SAFETY-IMPROVEMENT OPTIONS}

There were two types of safety-improvement options that were included in this value/impact study: (1) a generic list developed from options proposed in other studies or from insights gained in the performance of previous risk assessments, and (2) a list of changes derived from the specific results for each of the reference plant. Each of these is described below.

\subsubsection{Generic Risk-Reduction Options}

Risk-reduction options have been proposed over the last few years as a result of safety insights gained through various other research activities. Some of these suggestions have been in response to particular safety issues that have arisen relative to operating plants, while others reflect key uncertainties or sensitivities that appear to have significant risk implications. Other programs have also been completed or are currently underway to assess some of these options, and SARRP drew from these programs to evaluate the options for the reference plants. 
Specific engineering designs were not developed for these generic options. The changes are discussed as functional improvements that would change a plant's vulnerability to classes of accidents. For purposes of the value/impact assessment, the individual plant analyses assumed a basic engineering design, as discussed in the appropriate appendix.

Table 3-1 summarizes the generic safety options for PWRs that would serve to reduce core-damage frequency. Table 3-2 delineates the mitigation options that would reduce the risk impact of potential core-damage accidents.

One option not listed in the tables, but suggested in a number of studies as a riskreduction measure, is the overall improvement of plant operations through the initiation of reliability enhancement programs. While this is certainly a desirable goal, it is not possible to perform a meaningful value/impact assessment of an improvement of this nature. However, as discussed in the results, it is possible to identify the value of such improvements, including an assessment of the averted costs for some measure of reliability increase. These conclusions were derived directly from the results and no detailed assessment was performed.

\subsubsection{Surry-Specific Risk-Reduction Options}

The generic safety options discussed above were considered in the context of the Surry plant and either defined more specifically or eliminated. Some of the generic options were not included in the analysis for Surry because they would clearly have a limited effect, or because they are not applicable to the Surry design. For example, one involved the improvement of procedures for effecting recirculation of emergency core cooling after depletion of the refueling water storage tank (RWST). Because an automatic system for accomplishing the switchover task has already been implemented at Surry, it was judged that such an option would have no potential to be cost-effective.

In addition to the generic options, the Surry results were reviewed to define more specific changes that would have a significant impact on any of the risk measures. For the offsite consequences, the dominant contributors to risk from any portion of the analysis were fully examined to estimate a risk impact for potential changes. Thus the improvements could reduce risk by (1) decreasing the frequency of the sequences, (2) reducing the potential for containment failure modes that result in more severe consequences, or (3) improving the potential for conditions which reduce the radioactive release. This process helps to identify effective risk-reduction options because it results from an integrated assessment; a single, carefully selected modification could result in changes to all three of the areas noted above. For the onsite costs, a reduction in the total core-melt frequency results in a corresponding 
Table 3-1

PWR ACCIDENT-PREVENTION OPTIONS

\begin{tabular}{|c|c|c|}
\hline Proposed Option & Impact & Reason for Inclusion \\
\hline $\begin{array}{l}\text { Dedicated Feed and Bleed } \\
\text { or High Pressure Injection }\end{array}$ & $\begin{array}{l}\text { Increased potential for successful } \\
\text { feed and bleed cooling }\end{array}$ & $\begin{array}{l}\text { - Importance of high pressure sequences in PRAs } \\
\text { - Reduces impact of station blackout } \\
\text { - Some plants have limited high pressure pumping } \\
\text { capability } \\
\text { - Increased frequency of small LOCAs }\end{array}$ \\
\hline $\begin{array}{l}\text { Additional Independent } \\
\text { Auxiliary Feedwater }^{1}\end{array}$ & $\begin{array}{l}\text { Increased reliability for secondary- } \\
\text { side cooling and potential reduction } \\
\text { in induced LOCA frequency }\end{array}$ & $\begin{array}{l}\text { Reliability problems as evidenced by industry } \\
\text { experience; particular concern about common- } \\
\text { cause failures }\end{array}$ \\
\hline $\begin{array}{l}\text { Dedicated Reactor Coolant } \\
\text { Pump (RCP) Seal Cooling } 1,2\end{array}$ & $\begin{array}{l}\text { Reduced frequency RCP seal } \\
\text { LOCAs }\end{array}$ & $\begin{array}{l}\text { - Uncertainty about seal integrity without cooling } \\
\text { - Importance to the station blackout scenario }\end{array}$ \\
\hline Improved Ac Power & $\begin{array}{l}\text { Increased reliability of onsite ac } \\
\text { power, with resultant impact on } \\
\text { prevention and mitigation }\end{array}$ & $\begin{array}{l}\text { - Importance of station blackout sequences in PRAs } \\
\text { - Concerns about diesel generator reliability }\end{array}$ \\
\hline Improved De Power & $\begin{array}{l}\text { Increased reliability of batteries, } \\
\text { including long-term operation }\end{array}$ & $\begin{array}{l}\text { - Importance of station blackout sequences in PRAs } \\
\text { - Uncertainty about life of batteries during blackout }\end{array}$ \\
\hline $\begin{array}{l}\text { Rapid Primary System } \\
\text { Depressurization } 1,3\end{array}$ & $\begin{array}{l}\text { Enables use of low pressure systems } \\
\text { for high pressure accident sequences }\end{array}$ & $\begin{array}{l}\text { - Importance of high pressure sequences in PRAs } \\
\text { - Reduces impact of station blackout } \\
\text { - Some plants have limited high pressure pumping } \\
\text { capability } \\
\text { - Increased frequency of small LOCAs }\end{array}$ \\
\hline $\begin{array}{l}\text { Rapid Secondary System } \\
\text { Depressurization } 1\end{array}$ & $\begin{array}{l}\text { Enables use of low pressure systems } \\
\text { for high pressure accident sequences, } \\
\text { including secondary makeup } \\
\text { systems such as condensate pumps }\end{array}$ & $\begin{array}{l}\text { - All of above for primary system } \\
\text { Optimizes use of installed secondary makeup } \\
\text { capacity }\end{array}$ \\
\hline $\begin{array}{l}\text { Dedicated Long-term } \\
\text { Heat Removal }\end{array}$ & $\begin{array}{l}\text { Increased reliability of cooling of } \\
\text { core or containment }\end{array}$ & $\begin{array}{l}\text { - Importance of sequences involving loss of } \\
\text { service water or other heat rejection systems }\end{array}$ \\
\hline $\begin{array}{l}\text { Improved Testing of High/ } \\
\text { Low Pressure RCS Interfaces }\end{array}$ & $\begin{array}{l}\text { Reduces frequency of LOCAs that } \\
\text { bypass containment }\end{array}$ & $\begin{array}{l}\text { - Risk significance of containment bypass } \\
\text { sequences }\end{array}$ \\
\hline $\begin{array}{l}\text { Improved Capability for } \\
\text { Replacement of Water that } \\
\text { Serves as the Injection Source }\end{array}$ & $\begin{array}{l}\text { Extends time for initiating } \\
\text { recirculation and provides additional } \\
\text { options for tube ruppure }\end{array}$ & $\begin{array}{l}\text { - Importance of recirculation failure } \\
\text { sequences }\end{array}$ \\
\hline $\begin{array}{l}\text { Backup Reactor Protection } \\
\text { System }\end{array}$ & Reduces probability of scram failure & - Importance of ATWS in some PRAs \\
\hline Upgraded Boration System & Reduces severity of ATWS sequence & - Importance of ATWS in some PRAs \\
\hline
\end{tabular}


Table 3-2

PWR ACCIDENT-MITIGATION OPTIONS

\begin{tabular}{|c|c|c|}
\hline Proposed Option & Impact & Reason for Inclusion \\
\hline $\begin{array}{l}\text { Dedicated Containment } \\
\text { Spray Injection }{ }^{1}\end{array}$ & $\begin{array}{l}\text { Increased reliability } \\
\text { of spray, decreased } \\
\text { source term and less } \\
\text { containment loading }\end{array}$ & $\begin{array}{l}\text { - Importance of sprays for early } \\
\text { containment phenomenology } \\
\text { - Significance of spray to } \\
\text { radionuclide release }\end{array}$ \\
\hline $\begin{array}{l}\text { Dedicated or Improved } \\
\text { Spray with Recirculation } \\
\text { and Heat Removal Capability } 1\end{array}$ & $\begin{array}{l}\text { Same as above } \\
\text { but also improves late } \\
\text { heat removal } \\
\text { capability }\end{array}$ & $\begin{array}{l}\text { - Importance of sprays for early } \\
\text { containment phenomenology } \\
\text { and for release mitigation } \\
\text { - Importance of late heat removal } \\
\text { failure sequences in many PRAs }\end{array}$ \\
\hline $\begin{array}{l}\text { Additional Containment } \\
\text { Cooling } 1\end{array}$ & $\begin{array}{l}\text { Same as above } \\
\text { without impact of } \\
\text { spray on release }\end{array}$ & $\begin{array}{l}\text { - Importance of heat removal } \\
\text { failure sequences in PRAs }\end{array}$ \\
\hline $\begin{array}{l}\text { Filtered-Venting Capability } \\
\text { Early and Late }\end{array}$ & $\begin{array}{l}\text { Change in containment } \\
\text { overpressure failure } \\
\text { with resultant reduction } \\
\text { in total release }\end{array}$ & $\begin{array}{l}\text { - Recognition of the desirability } \\
\text { of preventing uncontrolled } \\
\text { overpressure }\end{array}$ \\
\hline $\begin{array}{l}\text { Hydrogen Burn } \\
\text { Prevention } 1\end{array}$ & $\begin{array}{l}\text { Eliminates potential } \\
\text { impact on containment } \\
\text { of large hydrogen burns }\end{array}$ & $\begin{array}{l}\text { - Importance of hydrogen as a } \\
\text { contributor to containment failure } \\
\text { in several other studies }\end{array}$ \\
\hline Hydrogen Ignition ${ }^{1}$ & $\begin{array}{l}\text { Reduces potential for } \\
\text { impact on containment } \\
\text { from large hydrogen } \\
\text { burns and eliminates } \\
\text { as a long-term problem }\end{array}$ & $\begin{array}{l}\text { - Importance of hydrogen as a } \\
\text { contributor to containment failure } \\
\text { in several other studies }\end{array}$ \\
\hline Reactor Cavity Flooding 1 & $\begin{array}{l}\text { Ensures water in } \\
\text { cavity increasing core } \\
\text { coolability potential } \\
\text { and provides scrubbing } \\
\text { for any ex-vessel } \\
\text { radionuclide releases }\end{array}$ & $\begin{array}{l}\text { - Uncertainties concerning } \\
\text { availability of water for specific } \\
\text { plants } \\
\text { - Has a mitigative effect on direct } \\
\text { heating }\end{array}$ \\
\hline Missile Shield & $\begin{array}{l}\text { Prevents containment } \\
\text { failure if steam } \\
\text { explosion is possible }\end{array}$ & $\begin{array}{l}\text { - Remaining uncertainty } \\
\text { in steam explosion potential }\end{array}$ \\
\hline
\end{tabular}

It is assumed that these modifications are independent of existing ac power sources. 
reduction in onsite cost impact. The final options considered for Surry are discussed below. The Surry results did not indicate many outliers that would be amenable to simple changes with a large impact. The service water system was found to have a particular weakness and a new service water system was therefore considered. The importance of the direct heating phenomenon suggested another option, which was a system to flood the cavity with water prior to vessel failure. The actual options considered are described below.

\section{Description of Accident-Prevention Options}

The options intended to reduce risk through prevention of accident sequences are described below. The details of their design and implementation are described in Appendix G.

Option P1: Improved Testing of Interfacing Check Valves. The purpose of this first option would be to reduce the likelihood of a LOCA that occurred outside containment due to failure of isolation between the high-pressure reactor-coolant system and systems at lower design pressures (event V). One option that could significantly reduce the frequency of these failure combinations would be to provide for testing that would allow for positive determination of the status of the valves at more frequent intervals.

Option P2: Improved Onsite Ac Power System. Because of the importance of sequences involving the total loss of ac power, the second option considered was the improvement of the emergency ac power system. Although there are several options for accomplishing this improvement, a specific proposal is required for value/impact analysis. For this study, the addition of a diesel generator is proposed with other changes to allow crossties between Surry units.

Qption P3: Extension of Battery Life. The evaluation of the Surry plant assumed that the station batteries have a seven-hour life following the loss of all ac power. This is one of the limiting features for station blackout sequences; although the turbine-driven auxiliary feedwater (AFW) pump could continue to operate after depletion of the batteries, it was assumed that it would be difficult to ensure continued long-term cooling with no instrumentation to maintain control. Thus, the third option considered was the improvement of the dc power system. A significant improvement would be estimated if the batteries could supply power reliably for greater than about $12 \mathrm{hr}$, since additional time available for restoring ac power provides a lower value for non-recovery. For purposes of this analysis, the addition of new batteries was selected as representative of this option.

Option P4: Addition of an Independent High-Pressure Injection System. A number of the important sequences for Surry included the failure of highpressure injection and/or seal injection for the reactor-coolant pumps (RCPs). The fourth option considered consists of an independent means of providing high-pressure primary inventory makeup and RCP seal injection. In developing this option, it was assumed that a single-train of high-pressure injection (HPI) with dedicated power and other support systems would be added to the current design. The system was assumed to have sufficiently high shut-off head to provide feed-and-bleed cooling using the pressurizer safety relief valves. 
Manual initiation from the control room was also assumed. This option also has a mitigative effect, in that it changes the probability of a temperatureinduced RCS failure during core degradation.

Qption P5: Addition of an Independent Train of Auxiliary Feedwater. This option involves the addition of a train of auxiliary feedwater, assumed to be independent of all current plant support systems, with the possible exception that it would share a suction source with the current AFW system. It was assumed that the system would be capable of delivering feedwater for a relatively long time period (at least 12 hours).

Option P6: New Low-Pressure Cooling Capability. Because of the risk significance of sequences involving the loss of core cooling as a consequence of failure of containment heat removal, the provision for an independent train of low-pressure cooling was considered. This option would encompass the capability to circulate either primary coolant or containment sump water through a heat exchanger and back to the RCS.

Option P7: New Service Water System for Containment Cooling. The Surry ASEP analysis indicated that the service water system designed to remove heat from containment systems is potentially subject to common-cause failures that could defeat the containment heat removal function. The sequences resulting from this scenario could result in particularly severe consequences, since the containment would already be in a failed state at the time of core melt. This option would require careful design consideration to ensure that it was not subject to the same common-cause problem associated with biofouling of the valves or heat exchangers that affects the assessed reliability of the existing system. Although the same effect could be obtained by preventing future occurrences of the biofouling failure mode, it is not clear what changes, if any, would be adequate, and a new, independent system was therefore selected to represent this option for the value/impact analysis.

Qption P8: RCS Depressurization System. Some of the sequences contributing to the risk for Surry involved failure of the high-pressure injection system, or of the high-pressure recirculation system after successful injection, in the event of a small LOCA or total loss of feedwater. If the RCS pressure could be reduced sufficiently, core-heat removal could be sustained by lowpressure injection or recirculation, as appropriate. Therefore, an option was considered that would facilitate the operators' ability to blow down the RCS to a lower pressure. It should be noted that this option also has a mitigative effect. It can reduce the potential for direct heating by changing the accident from one involving high RCS pressure to one involving low pressure.

\section{Description of Options Intended to Mitigate Accidents}

A number of safety options intended to reduce the likelihood of containment failure and/or the severity of releases following a core melt were identified. Several of the options which were initially proposed for their mitigative effects also had an impact on the frequency of the core-damage sequences which was also included in the calculation of benefits.

Qption M1: Missile Shield. The provision of a shield to preclude the possibility of containment failure as a consequence of a missile generated due to 
an in-vessel steam explosion (the failure mode designated $\alpha$ ) was considered. Because the risk reduction associated with this option was expected to be small, details of the design were not considered. Instead, the risk was recalculated by eliminating all $\alpha$-mode failures.

Option M2: Hydrogen Ignition System. The containment event tree for Surry provided the opportunity for hydrogen combustion whenever the containment atmosphere reached potentially flammable conditions. An ignition system would be intended to bum off hydrogen at lower concentrations to prevent buildup and combustion on a scale large enough to lead to containment failure. The impact of this option on risk was assessed by forcing ignition to occur at each node in the containment event tree at which hydrogen burning would be possible. The system was assumed to be independent of existing ac power supplies.

Option M3: Prevention of Hydrogen Combustion. As an alternative to option $\mathrm{M} 2$, the provision for preventing the combustion of hydrogen (e.g., through inerting of the containment atmosphere) was considered. The effectiveness of such an option was investigated by precluding the possibility of combustion at each of the associated nodes in the containment event tree.

Qption M4: Early Containment Venting. Venting of the containment atmosphere through filters to prevent overpressurization and a more serious release was investigated. The option was assumed to be designed with independent power supplies. This option considered the potential for venting early in the accident to preventive most overpressure failures.

Option M5: Late Containment Venting. This system would be the same as option M4, but the venting strategy would be different. The value of venting the containment later in the accident progression, following breach of the reactor vessel, was evaluated, thus eliminating all late containment failures due to late overpressure.

Option M6: Additional Containment Cooling--Containment Spray. Because of the significance to risk of sequences involving overpressurization due to failure of containment cooling, the addition of an altemative containment spray system was considered. The system evaluated was assumed to be independent of the current support systems, and includes the capability for recirculation and heat rejection through a new service water system.

Qption M7: Additional Containment Cooling--Fan Coolers. This option is similar in effect to the option described above, but fan coolers are used for the heat removal. This option does not have the same effect as the previous one, however, since the sprays have a significant radiological decontamination effect. For this study, the fan coolers were not assumed to prevent containment failure following a large LOCA in the absence of other containment heat removal.

Qption M8: Containment Flooding System. This option is designed to provide a means of introducing a large quantity of water into the reactor cavity prior to vessel failure. The option was designed specifically to mitigate direct heating scenarios. 
One of the preventive options, the RCS depressurization system (option P8), also has a mitigative effect relative to direct heating that was considered in the analysis.

\subsection{EVALUATION OF COSTS AND OTHER IMPACTS}

An impact assessment was performed for all options identified for each of the reference plants. The following impacts were considered:

- Installation costs,

- Continuing maintenance costs,

- Radiation exposure during installation, and

- Radiation exposure due to continuing maintenance.

The scope of the SARRP risk-reduction task did not include detailed design development for all of the proposed modifications. Some of the key mitigation features were the subject of a detailed design and cost estimation effort specifically for SARRP [1], while several others have been the subject of previous investigations [2,3]. The scope of SARRP did not allow for a detailed investigation of the remaining safety improvements, and the impacts for these options were estimated based on comparisons to available data. The information used for this estimation is presented in Appendix G.

For each reference plant, each proposed risk-reduction option was described in terms of its specific application for the plant. This includes a description of design parameters and delineation of the major equipment needs, if any. The data in Appendix $G$ was then used to generate the estimates of the impact measures from the most applicable source. While this process is not ideal, it does allow a basic comparison to the expected benefits, and any options that appear to have the potential to be cost-effective can be more fully evaluated.

There is one additional impact that could be the dominant cost for some of the proposed modifications--replacement power needed during the implementation of the option. This is a difficult parameter to estimate because the cost is dependent on a number of factors: (1) the specific schedule for implementation, (2) the status of other projects in progress at the utility, (3) the financing arrangement of the utility, (4) the availability of power at the time of the modification, and (5) a whole host of political considerations. Several previous studies have attempted to include replacement power costs by considering the total amount of work and dividing it to determine if it could be performed in successive shutdowns without causing an increase in the shutdown period. The basic assumption in these analyses is that there is no regulatory pressure driving the implementation schedule, and that very large work crews could 
be employed. These assumptions are probably not realistic for some of the larger options, and past experience indicates that unexpected delays in implementation due to a variety of causes has been common.

Due to the large variability that could be realized if replacement power costs were included, the basic value/impact ratios were calculated without this factor. This allowed a formulation of preliminary conclusions and enabled a paring of options to those that had a potential for sufficient net benefit to merit further consideration. Conclusions were then generated that included some consideration of replacement power. To support this activity, insights concerning replacement power costs are included in Appendix E.

\subsection{EVALUATION OF EFFECTS ON RISK}

The value of each option was assessed by recalculating all of the risk measures accounting for the total effect of the change. For safety improvements that resulted only in a change in sequence frequencies, this involved a straightforward requantification of the risk parameters. For options that had an impact on containment or source-term analyses, appropriate model changes and requantification was performed, and the pieces of the analysis were integrated to result in new estimates of risk.

The evaluation of the specific effects of each option involved a number of assumptions that are documented in Appendix E. These assumptions include estimates of reliability of the option once implemented, and the capability of the system in terms of ability to prevent or mitigate accident conditions. Important sensitivities to these assumptions are discussed in the results of the risk-reduction analysis.

Any plant change that is directed at reduction of risk or core-melt frequency also has the potential for competing risks that could result in an overall adverse impact. These competing risks can be difficult to recognize because even a reduction in core-melt frequency can increase risk if there is a redistribution of frequency into more severe damage states, and because very subtle interactions could be introduced in the actual implementation of the design. The scope of this study did not allow for a full evaluation of competing risks; as already noted, detailed designs needed for such an assessment were not developed. However, in order to appropriately evaluate a plant change, each was reviewed for competing risks and the description of each alternative was amended to include restrictions intended to reduce the potential for undue competing risks. The model changes associated with a safety option were integrated with the rest of the model, and in some cases negative impacts were important. 
Similar to the procedure used for replacement power costs, all safety options that appeared to have a net positive impact were more thoroughly reviewed and are discussed in Section 6.

\subsection{VALUE/IMPACT ASSESSMENT}

Procedures and assumptions used in value/impact analysis can vary widely with both the analyst and the purpose of the study. The NRC has already reviewed this issue and generated specific features that are needed for regulatory use of value/impact analysis [4]. While it was not the intent of this program to perform the regulatory analysis to determine specific rulemaking requirements, the procedure adopted for the value/impact analysis in SARRP is consistent with NRC regulatory uses and therefore facilitates review and comparison. In general, the SARRP results presented in this report and its appendices include more information and some additional value/impact ratios that serve to provide additional insight; that is the ultimate goal of this study.

The methods used for the value/impact assessment also drew upon the Handbook for Value Impact Assessment [5]. In the terminology of that report, the analysis in SARRP is a "limited effort value/impact" assessment. However, in some cases there is additional information presented that is not outlined in the handbook. The two references above do not specify all aspects of the analysis, and other studies were reviewed for particular parameters $[2,6]$.

The value/impact assessment was performed through direct comparison of the recalculated risk measures expressed as averted accident costs with the total impact measured as a one-time cost. These cost/benefit comparisons were generated for different measures of averted costs due to differing interpretations and assumptions required for value/impact analysis. The comparisons were calculated separately for the each of the various cost elements: (1) the total offsite cost as determined by the consequence code, (2) the total of both the onsite and offsite costs, (3) total offsite cost calculated by assuming a cost impact of $\$ 1000$ per person-rem for exposure out to fifty miles, and (4) the sum of offsite costs, calculated as in item (3), and onsite costs. The offsite costs calculated by the consequence code include the costs of health effects as well as relocation expenses, lost wages, decontamination costs, lost public and private property, and the value of interdicted land and crops.

The estimated onsite impacts of the accidents reflected consideration of the costs associated with lost capital, cleanup and decommissioning, and lost power generating capacity. The cost estimates were made using the methods reported in NUREG/CR-3673 [6]. The replacement power required as a consequence of the accident was assumed to be supplied by 
already-installed spare capacity. It was assumed that the equivalent of the rated power of the plant, adjusted for a $65 \%$ capacity factor, required replacement. A discount rate of $4 \%$ was applied, and because the spare capacity would almost certainly be supplied by a fossil plant, an escalation of $6 \%$ was also used in the calculation. It was assumed that replacement power would be required for 10 years, after which a new plant would supply power. A correction was included to account for the replacement power if the accident occurred in the last 10 years of plant life.

The capital cost of the loss of the utility's investment was calculated, with the present worth being derived with a $4 \%$ discount rate. This calculation assumed a 40 year plant life (accounting for the actual remaining life of the facility at the time of core damage), and also assumed a uniform depreciation over the 40 years. The 1985 cost of construction was used as a base ( $\$ 3000 / \mathrm{kwe}$ installed). The remaining onsite costs were taken from NUREG/CR-3673 [6]: cleanup cost was assumed to be $\$ 1.7 \times 10^{9}$; decommissioning cost was taken as $\$ 1.0 \times 10^{8}$; and onsite health costs were estimated to total $\$ 6.5 \times 10^{6}$.

In the NRC regulatory analysis, the value is calculated as a reduction in the total population dose out to fifty miles, evaluated at $\$ 1000$ per person-rem. Other values for averted cost calculated in SARRP are based on the reduction in consequence measure as determined by the consequence code. For these calculated costs, offsite property damage costs were used directly, while averted health effects were valued at one million dollars per early fatality, one hundred thousand dollars per latent cancer fatality and one hundred thousand dollars per early illness. These values were obtained from an investigation of available statistics pertaining to the amount which society has been willing to expend to avert fatalities and injuries [7]. The specific numbers selected are somewhat unimportant because offsite costs are almost always dominated by property damage rather than health effects. All costs in the value/impact analysis were treated in the same manner as the costs for the rebaselined risk; all values were discounted $(4 \%)$ to obtain present worth.

As described above, the SARRP value/impact analysis resulted in the generation of a number of different measures for assessing the overall value of the proposed safety options. These results allowed a detailed review of contributing factors and resulted in additional conclusions that might not be possible if one ratio using a particular formula had been used. Conclusions regarding the merit of the option or the need for additional information to fully assess the option were derived from these results. 
NUREG/CR-4551,VOL.1: DRAFT REPORT FOR COMMENT (SEPTEMBER, 1986)

\subsection{TREATMENT OF UNCERTAINTIES}

The two methods of uncertainty analysis discussed in Section 2.2 (LLH and OCP) that were used in the risk-rebaselining task were used in the risk-reduction task as well. Most of the inputs that were needed for the value/impact analysis (construction costs, maintenance costs, etc.) were estimated using a three-point uncertainty range of high, central, and low values based on a review of the available data. These values were then used in the value/impact analysis of each option, for both of the uncertainty methods of risk calculation. Because of the large amount of output generated by the safety options, not all of the results of the riskreduction study are included in Section 6, which describes the results; detailed computer output is included in Appendix E.

The impact of the preventive options was calculated by determining the factors of reduction in frequency for each accident sequence. The risk was then recalculated and the results are displayed in Section 6. It should be noted that the preventive options were not calculated separately for outcomes that would be obtained for all of the LLH sample members. It was determined that the frequency reduction factor for the central estimate results would be applicable to all but two core-damage LLH issue outcomes, and that only a small error would be introduced by assuming the same reduction for all sequences. The evaluation of mitigative options was somewhat more complex. The containment event tree code was used to determine the impact of the mitigation options for each of the combinations of issue levels determined in the LLH sampling method. The new risk results were calculated from the new containment event tree outcomes using a portion of the RISQUE code developed specifically for this purpose.

As with the basic risk results, the RISQUE (and RISKEE) codes include provisions for calculating a great deal of information concerning the risk-reduction results. The most important measures and breakdowns of the risk-reduction results are summarized in Section 6 and provided in detail in Appendix E. The full complement of available output for any given risk measure includes the following:

- Risk-reduction for any given individual or set of plant-damage states (groups of core-damage sequences),

- Fractional contribution of each plant-damage state to mean risk including the new risk for safety options,

- Fractional contribution of each source term bin to mean risk,

- Fractional contribution of each cluster to mean risk, and

- Detailed analysis of individual sample members to risk, including fractional contributions of damage states, source term bins and clusters. 
The new risk calculated for each safety option is plotted along with the base case risk in the results. The format is the same as for the base case: a box is drawn around the middle 95 sample members. It should be noted that for all risk reduction calculations, the sample members are reordered based on risk, and therefore the effect on individual sample members can only be understood through detailed review of the individual sample member results.

Figure 3-1 illustrates the value/impact analysis which is also performed as part of the risk-reduction task. The averted costs are plotted with the cost of the option. The figure is simplified because the value of the option (averted cost) is always calculated and plotted for all four costs measures described in the previous section. As already described, the averted costs are determined for each LLH sample member by considering the reduction in health effects and property damage in monetary terms, with the option costs represented by a three-point uncertainty range. The sample members are reordered and plotted in terms of total averted cost, with a bar used to represent the range between the 5th and 95th sample member. A bar is also used to represent the range of costs for the option. Areas where the bars overlap indicate potential cost effectiveness. Given the potential cost effectiveness, the option is subject to further investigation of competing risks and other cost impacts not included in the initial comparison (replacement power, etc.). The results illustrated in the figure were obtained for the OCP method as well, but only the central estimate results are included in Section 6 (full results are reported in Appendix E).

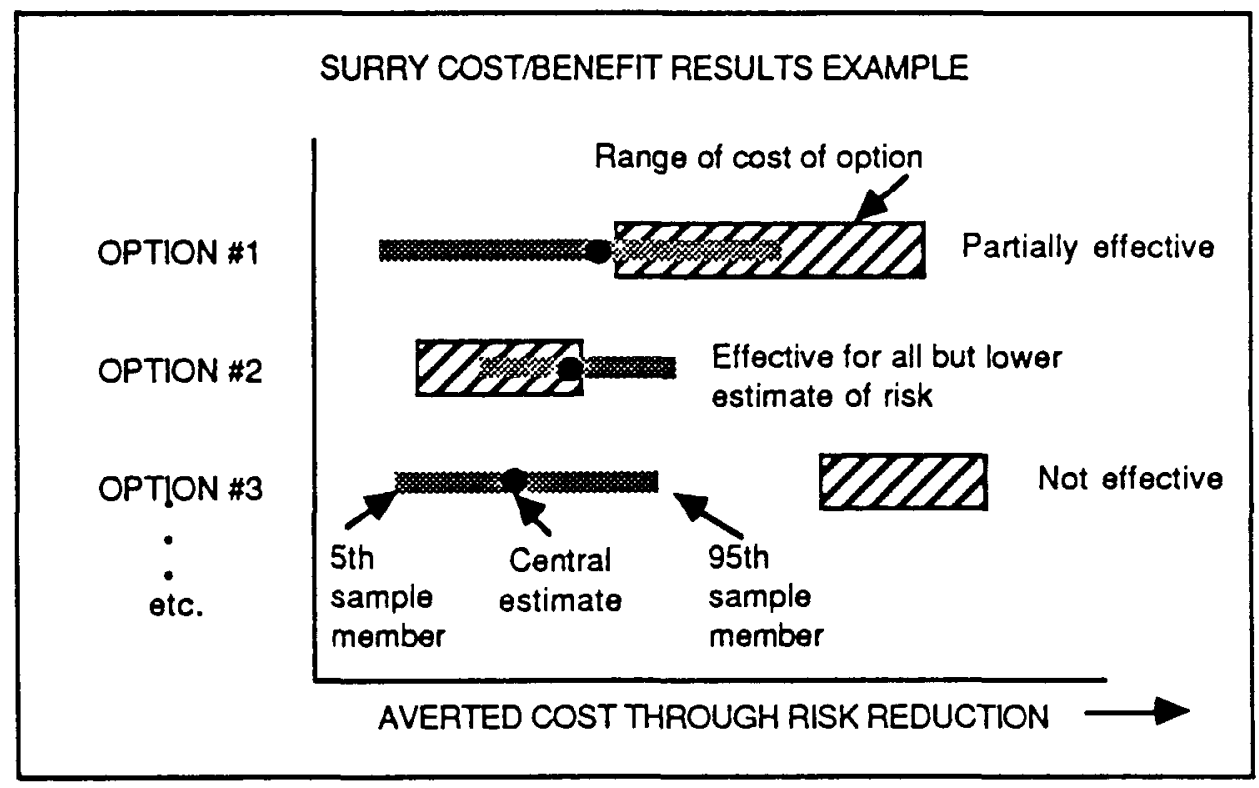

Figure 3-1. Example of LLH Cost/Benefit Display 


\section{References for Section 3}

1. Cherdack, R, et al Study of Severe Accident Mitigation Systems, U.S. Nuclear Regulatory Commission Report NUREG/CR-4781, Sandia National Laboratories, Albuquerque, NM: February, 1987.

2. Berry, D. L., and G. A. Sanders. Study of the Value/Impact of Alternative Decay Heat Removal Concepts for Light Water Reactors. U.S. Nuclear Regulatory Commission Report NUREG/CR-2883, Sandia National Laboratories, Albuquerque, NM: 1983.

3. Benjamin, A. S., and F. T. Harper. Value Impact Investigation of Filtered-Vented Containment Systems and Other Safety Options for a BWR Mark I Containment. Sandia National Laboratories, Albuquerque, NM: 1984.

4. Denton, H. R. (Memorandum). "NRR Office Letter No. 16, Revision 2--Regulatory Analysis Guidelines." U.S. Nuclear Regulatory Commission, Washington, DC: October 30, 1984.

5. Heaberlin, S. W, Handbook for Value-Impact Assessment. U.S. Nuclear Regulatory Commission Report NUREG/CR-3568, Pacific Northwest Laboratory, Richland, WA: 1983.

6. Burke, R. P., and D. C. Aldrich. Economic Risks of Nuclear Power Accidents. U.S. Nuclear Regulatory Commission Report NUREG/CR-3673, Sandia National Laboratories, Albuquerque, NM: 1984.

7. Strip, D. R., Estimates of the Financial Consequences of Nuclear Power Reactor Accidents. U.S. Nuclear Regulatory Commission Report NUREG/CR-2723, Sandia National Laboratories, Albuquerque, NM: September, 1982. 


\section{Section 4 \\ EVALUATION OF KEY UNCERTAINTY ISSUES}

As described in Section 2.2, the limited Latin hypercube uncertainty approach relied on the selection of key uncertainty issues that had a significant impact on the estimated risk of the Surry plant. These issues are defined in this section, along with a discussion of the range of uncertainty associated with each. The issues were considered in three categories:

(1) Sequence frequency issues,

(2) Containment loading and performance issues, and

(3) Radiological source term issues.

For the first category, sequence frequency issues, the systems analysts reviewed the initial core-damage sequence results and the basic plant models to define the key issues known both to have large uncertainties and a significant impact on the results. The analysts then developed a range of possible outcomes for those issues based on the current body of knowledge. For the latter two categories, similar criteria were used to establish the list of issues, but the issues often involved greater uncertainties due to lack of any available research or large disagreement within the reactor safety community as to both the methods of evaluation and the potential outcomes. It was decided that for these issues, mostly phenomenological in nature, the best representation of the range of uncertainty would be obtained through the input of an expert review group, as opposed to just the analysts' input used in the sequence issue uncertainty evaluation. This review group broadened the base of information for both selection and evaluation of the issues. As described in Section 2.2, the experts were called on to provide their individual views on a range of distinct outcomes for each issue by selecting weighting factors for each outcome. These weighting factors determined the relative importance of each outcome in the LLH sampling technique.

The purpose of this section is to provide the reader with an introduction to all of the uncertainty issues included in the LLH sampling. The details of the evaluation of these issues are included in the appendices of this report, and their significance in terms of risk is discussed in Section 5. The ranges of uncertainty provided by the review group are also provided in this section, although the actual use of this input in the LLH study sometimes required alteration due to limitations of the methodology. The actual implementation is also described in the appropriate appendix for each area of the analysis. 


\subsection{SEQUENCE FREQUENCY ISSUES}

Five issues affecting only the assessment of the core-damage sequence frequencies were included in the LLH study. These issues address the key sensitivities associated with the dominant accident sequences. It should be noted that uncertainties due to the reliability data were also considered in the ASEP study, and these uncertainties are also addressed in the results provided in Section 5 of this report. The LLH issues focused on key sensitivities affecting the accident sequence results and include uncertainties in both modeling assumptions and application of data when information is very limited. Each issue is defined separately below. Details concerning these issues are available in Reference 1 .

\subsubsection{Reactor Coolant Pump SeaL LOCA Model}

There is a general lack of information concerning the response of the reactor coolant pump (RCP) seals to a loss of all cooling, both seal injection and thermal barrier cooling. This is particularly important to the Surry results due to the dominance of the sequences involving a loss of all ac power (which results in a loss of all seal cooling). Due to the large uncertainty, the central estimate of the seal cooling response was obtained by establishing a distribution of seal LOCA timing, with 1 and 10 hours corresponding to the 5\% and $95 \%$ bounds of the distribution for the occurrence of a seal LOCA. These bounds corresponded to values used in several other risk assessments. This distribution was then used to obtain the central estimate of a 0.65 probability of a seal LOCA for a loss of seal cooling greater than 1 hour and less than 6 hours. It was also assumed that uncovering of the core would occur one-half hour after the seal LOCA. A loss of cooling services for less than 1 hour was assumed not to result in a seal LOCA.

Since the central estimate study already considered some aspects of the uncertainty in this issue (through the use of a distribution), it was decided that the LLH input would include consideration of seal response outside the bounds of the distribution used for the central estimate. Two other cases were considered, based on deterministic rather than probabilistic models:

(1) The occurrence of a large seal LOCA one-half hour after the loss of all cooling, uncovering the core one-half hour later; and

(2) Assured integrity of the seals, i.e., no seal LOCAs due to loss of cooling.

Each of these cases was assigned a 0.05 weighting factor to be consistent with the central estimate model, which accounts for the 5 to $95 \%$ bounds. The first case is based on the model used in the Zion Probabilistic Safety Study [2], while the second corresponds to the opinions 
of some in the reactor safety community that no significant seal LOCAs would develop due just to a loss of cooling.

\subsubsection{Use of the Gas Turbine Generator for Offsite Power Recovery}

The central estimate did not consider the use of the non-safety grade gas turbine generator at the Surry site. The generator is currently used for peaking power, but it was initially intended for black-start capability. This was assessed by allowing an additional recovery of power for the sequences involving a loss of all normal and emergency ac power. It was assumed that use of the generator would only apply after one hour due to the manual actions associated with its start and lineup, and additionally that the system would have a 0.35 unavailability. The systems analysts assigned a 0.5 weighting factor to this case and the central estimate alike.

\subsubsection{Uncertainty in the Common-Cause Evaluation Methodology}

The basic ASEP methodology called for the use of the beta-factor method to assess the importance of common-cause failures not due to explicit dependencies. There is considerable uncertainty in both the application of this method and in the use of data to support the method. The central estimate assumed that the beta factors provided in EPRI NP-3967 [3] were upper bounds of a distribution with an error factor of 10 . The uncertainty in this issue was assessed by considering two alternative models for the common-cause failures:

(1) Use of the values from EPRI -NP-3967 as mean values with an error factor of 10 , and

(2) Elimination of all beta factors.

The systems analysts assigned weighting factors to these cases of 0.34 and 0.01 , respectively. This reflected their view that some model was needed to account for these common-cause failures that are not otherwise modeled, but that there was considerable uncertainty in the values currently available.

\subsubsection{Modeling and Data Uncertainties in the $\mathrm{V}$ Sequence}

Although the interfacing systems LOCA (V sequence) did not have a high frequency, it was found to be a key contributor to the central estimate of risk. Recognizing the uncertainties in the assessment of this sequence, it was included in the LLH study. The base case model for this sequence is described in Reference 1. Once again, two alternative evaluations were considered: 
(1) Requantification using lower frequencies for check valve ruptures and leakage. The values in the central estimate are known to include events of differing severities but the $\mathrm{V}$ sequence would only result from severe failures. The failure rates were reduced by a factor of 5 to account for potential conservatism.

(2) Requantification using higher frequencies for check valve ruptures (the $95 \%$ value of the generic data distribution).

The first case was assigned a weighting factor of 0.4 , and the second case 0.1 , reflecting concern about possible conservatism in the central estimate.

\subsubsection{Uncertainties Concerning Core Melt Due to Containment Failure}

This issue is actually both a sequence frequency issue and a containment response issue. A fairly important class of accident sequences was found to involve a loss of all containment cooling, eventually leading to overpressurization and containment failure. The impact of containment failure on the operability of the emergency coolant recirculation pumps is uncertain. The most conservative assumption (used in most previous PRAs, including the RSS [4]) is that the containment failure leads directly to failure of all core cooling pumps, and therefore to core melt. While the pumps could be vulnerable to failure as a result of containment failure, a more realistic assessment would indicate that pump failure is not assured. These accident sequences were further analyzed considering the potential pump failure modes induced by containment failure (see Appendix A). This evaluation included input from the containment expert review group, which is represented by two cases other than the base case. For this issue, the reviewer input resulted in very close agreement, and the two cases involved only an increase or decrease of a factor of two over the central estimate (a probability of 0.02 for a core vulnerable sequence proceeding to core damage). Both the two uncertainty levels and the central estimate were weighted equally in the LLH study. Upon further consideration after completion of the LLH analysis, it was realized that the method used for collecting and manipulating the uncertainty data for this issue did not allow a very wide spread in the result. It is therefore likely that the uncertainty illustrated in the result is significantly understated, and any conclusions regarding the importance of this issue must be carefully qualified.

\subsection{CONTAINMENT LOADING AND PERFORMANCE ISSUES}

The following containment loading and response issues were included in the LLH study for Surry:

(1) Probability and location of induced failure of the RCS pressure boundary, 
(2) Mode of reactor vessel breach,

(3) Magnitude of pressure loading at vessel breach due to direct heating and steam spike,

(4) Magnitude of hydrogen burn pressure increment at vessel breach,

(5) Containment failure pressure,

(6) Size of containment failure,

(7) Probability of late containment spray system failure given earlier operation, and

(8) Probability of submergence of the interfacing systems LOCA failure location.

Each of these issues is defined in the following sections. It should be noted that the consideration of several of the issues involves a level of detail considerably greater than that presented here, and that Appendix B defines the actual implementation of the LLH input for the containment analysis. There are certainly other containment issues that involve considerable uncertainty, but the consensus of the SARRP analysts and reviewers was that the issues listed above were the most significant relative to the assessment of the Surry plant. For this plant, additional insight is also available from the output of the OCP uncertainty approach, which included uncertainty for every containment event tree question. These insights are discussed in Section 5.

\subsubsection{Probability and Location of Induced Failure in the RCS}

Recent analyses have indicated the potential for very high gas temperatures in the primary system for core damage sequences involving high pressure in the RCS prior to vessel breach [5]. These results have prompted the consideration of induced failures of the pressure boundary that would lead to a change in the scenario phenomenology due to RCS depressurization and could also lead to a partial containment bypass if the induced failure were a steam generator tube rupture. These failures have not been included in previous studies, but were found to be of sufficient significance that they were included in both the SARRP central estimate and the LLH study. Six potential LOCA outcomes (and one outcome for no induced LOCA) were considered based on location and size of the failure:

- Large hot-leg LOCA

- Large RCP seal LOCA

- Large steam generator tube rupture

- No induced failure
- Small hot-leg LOCA

- Small RCP seal LOCA

- Small steam generator tube rupture 
For the LLH sampling, the composite of the reviewers' weights was used to determine which of the possible outcomes would apply for each sample member. In the final analysis, the large and small hot leg LOCA categories were combined.

The expert review group assigned a composite weighting factor to the "no LOCA" outcome of 0.4 , indicating an overall opinion that an induced failure was fairly likely. ${ }^{*}$ Figure 4-1 illustrates both the composite weights and the range of responses for the individual reviewers for each outcome for a particular set of boundary conditions. As the figure illustrates, there was good agreement within the review group on the relative likelihood of particular locations, although the overall assignments of weighting factors tends to indicate wide uncertainty since most locations were weighted relatively equally. For the boundary conditions considered, a seal LOCA was considered most likely. The range of reviewer input on the probability of any LOCA occurring was wider, but within an order of magnitude. This issue is potentially important because it impacts the phenomenology of the high pressure core melt sequences and it is therefore correlated with the most significant risk uncertainty at Surry, which is that associated with direct heating (see Sections 4.2.3 and 4.3.3).

\section{Preconditions: Reactor coolant pump seal cooling not available, steam generators dry, operator does not depressurize reactor coolant system.}

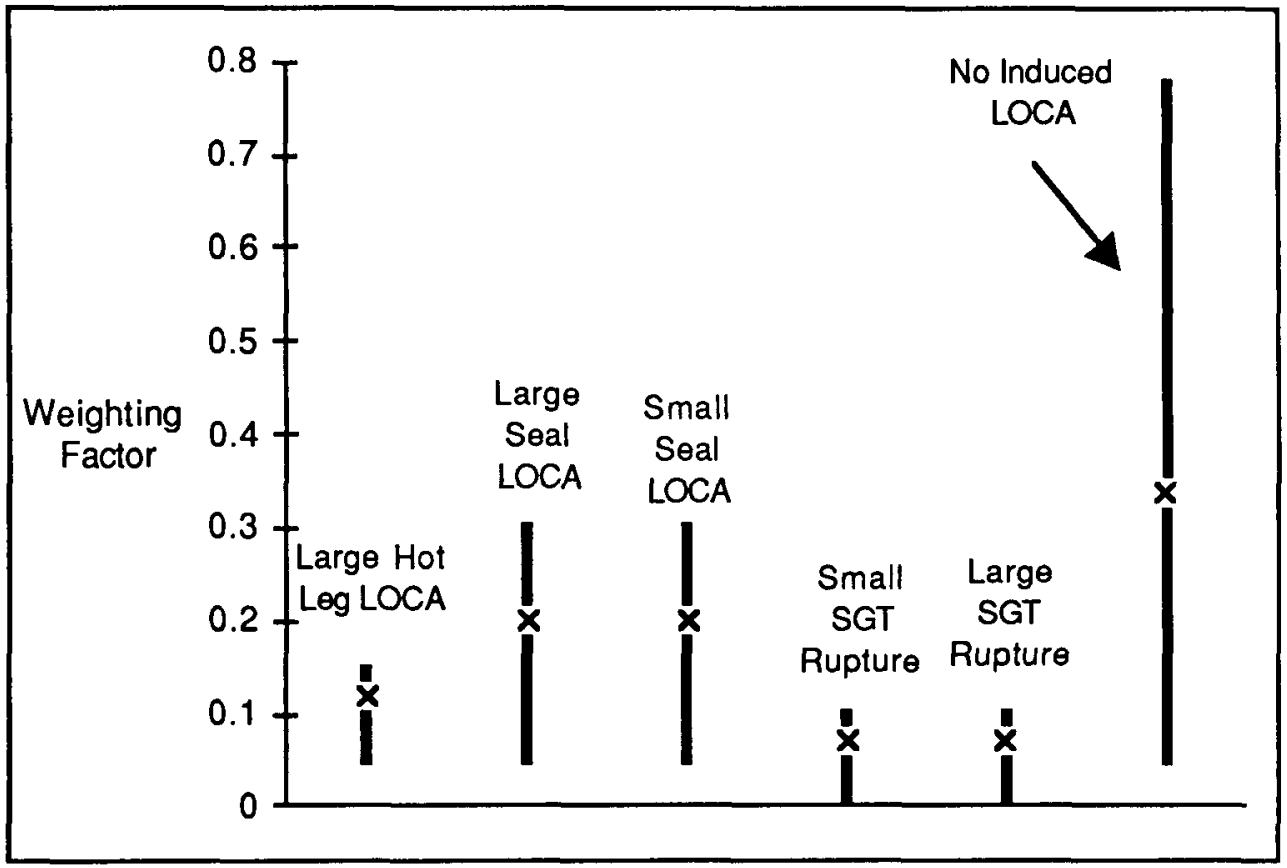

Figure 4-1. Outcomes and Weighting Factors for Location and Size of Induced LOCA

"All graphs in this section include the composite review group result as an " $\mathrm{X}$ " on the plot. 


\subsubsection{Mode of Reactor Vessel Breach}

The mode of vessel breach for both high and low pressure core-damage sequences was also considered in the Surry LLH study. This issue includes the rather controversial containment failure mode of direct containment failure by a steam explosion-induced missile (the $\alpha$ mode). The issue also addresses the potential for high pressure ejection of the core material, gravity flow after vessel breach, and in-vessel steam explosions leading to vessel failure but not containment failure. Figure 4-2 illustrates the expert review input for this issue. ${ }^{*}$ It should be noted that only ranges are illustrated because composites were not used for this issue. In this case, each reviewer's input was treated as a separate level. The in-vessel steam explosion leading to containment failure had a wide range of weighting factors (two orders of magnitude), but most of the reviewers thought that this failure mode had a probability of $10^{-3}$ or less. For high-pressure scenarios, the reviewers assessed a very high likelihood of high pressure ejection and gave no credence to a gravity flow. Most reviewers thought that a high pressure ejection was not possible in low pressure scenarios and all thought a gravity pour was most likely.

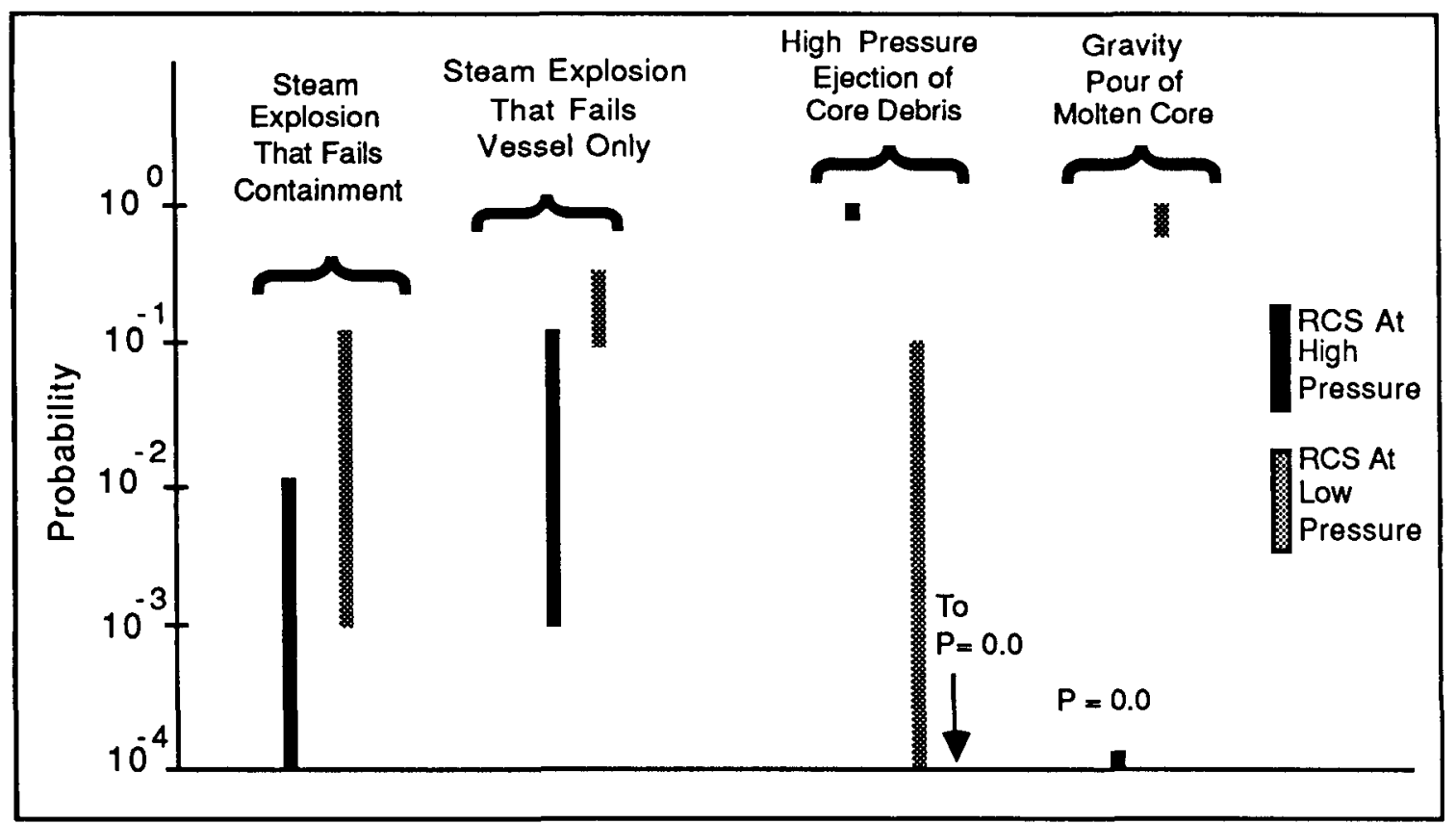

Figure 4-2. Outcomes and Weighting Factors for Mode of Reactor Vessel Breach

\footnotetext{
${ }^{*}$ The results are plotted on a $\log$ plot so that they would all fit on one graph, but this does tend to distort the actual ranges of uncertainty assigned. Any detailed conclusions concerning this issue should be based on the actual data which is provided in Appendix B.
} 
This issue is important because of the possibility of direct containment failure (the $\alpha$ mode) and because the high pressure ejection scenario is correlated to the potential for direct heating.

\subsubsection{Pressure at Vessel_Breach Due to Direct_Heating_and Steam_Spike}

This issue deals primarily with the phenomenon of direct heating, which is the addition of substantial heat directly to the containment atmosphere caused by the dispersal throughout containment of finely fragmented core material following vessel breach. To limit the detail considered in the LLH study, the issue was defined in terms of the pressure loading of containment at vessel breach due to the generation of steam and the possibility of direct heating. Reviewers were asked to incorporate all their considerations about the uncertainties in these phenomena into the final pressure increment. Thus the issue includes uncertainties in several phenomena, such as the following:

- Amount of core initially ejected

- Efficiency of energy transfer

- Completeness of the metal oxidation reactions
- Amount of core exiting the cavity

- Probability of the phenomenon itself

The input of the review group for the most significant case, a high pressure sequence with no water in the reactor cavity, is illustrated in Figure 4-3. As described in Appendix B, the reviewers based their input on previous discussions of this issue by the Containment Loads Working Group (CLWG) [6] and recent experimental evidence [7]. The first level illustrated, an $11 \mathrm{psi}$ increment, refers to the no direct heating outcome. The reviewers assigned a composite weight to this outcome of approximately 0.2 . Thus there was a 0.8 weighting factor assigned to the occurrence of the direct heating phenomenon. As the figure illustrates, there was fairly wide uncertainty indicated by the inputs of the individual expert reviewers, but the overall conclusion was that direct heating was likely to produce very large pressure loadings at vessel breach; one-half of the LLH samples for these conditions have pressure increments of $75 \mathrm{psi}$ or greater at vessel breach, not including any hydrogen burn contribution.

The second interesting input of the review group was that the phenomenon of direct heating would be almost as severe for intermediate pressure sequences as for high pressure; the pressure increments assigned were, on the average, $75 \%$ of the values used in the high pressure scenarios. For the Surry plant, the reviewers also thought that a reactor cavity full of water would have a relatively small impact on the resulting pressure increments due to direct 
heating (a $25 \%$ reduction in the peak pressures on the average). No reviewers thought that the phenomenon was possible at low pressure.
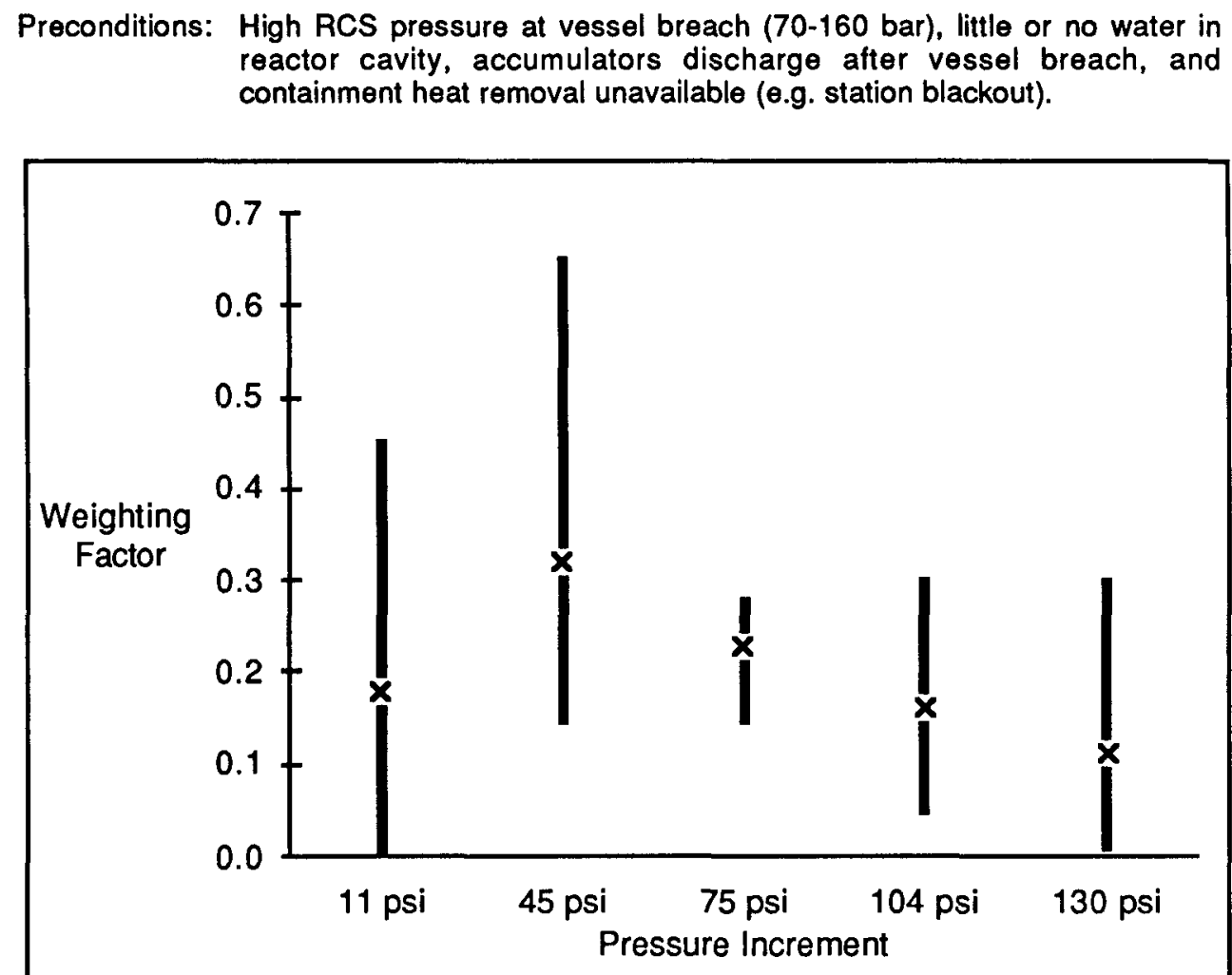

Figure 4-3. Outcomes and Weighting Factors for Magnitude of Pressure Loading at Vessel Breach Due to Direct Heating and Steam Spike

\subsubsection{Magnitude of Hydrogen Burn Pressure Increment at Vessel Breach}

All of the uncertainties relative to hydrogen generation and burning at vessel breach were included in this issue, in which the reviewers were asked to weight pressure increments due to hydrogen burning at vessel breach. The most significant uncertainty in the issue is the amount of hydrogen generated up to the time of vessel breach, although there is also uncertainty in the amount left in the vessel at vessel breach as well as the ignition characteristics of the hydrogen steam mixture. This issue required a considerable number of cases to account for important boundary conditions: steam concentration, the occurrence of direct heating, and the magnitude of the steam spike.

One case is illustrated in Figure 4-4. The pressure increments corresponding to $50 \%$ or less equivalent $\mathrm{Zr}$ oxidation were weighted most heavily, but the range of pressure increments with weighting factors greater than 0.1 was still quite large: 0 to $70 \mathrm{psi}$. One other important 
factor was determined by the review group input for other cases: the potential for significant pressure increments associated with hydrogen when direct heating occurs, even when the containment is steam inert relative to large scale burning. These increments were attributed to recombination reactions of hydrogen and oxygen in the local vicinity of the hot fuel particles dispersed in containment, or to the possibility that normal inerting criteria do not apply when the atmosphere is very highly heated.

Preconditions: No prior burn, maximum steam spike (1.9 bar), direct heating occurs, and containment heat removal not operating (atmosphere globally steam inert).

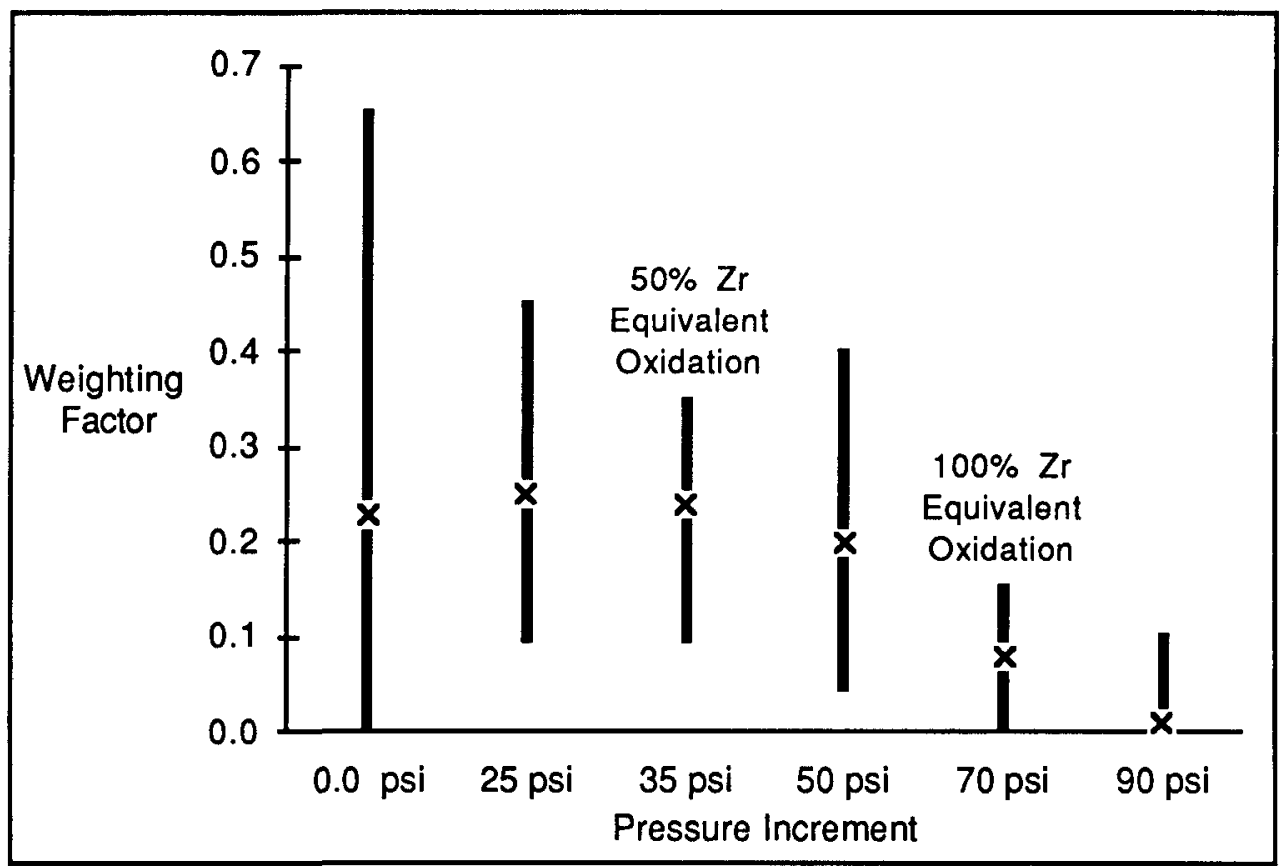

Figure 4-4. Outcomes and Weighting Factors for Magnitude of Hydrogen Burn Pressure Increment at Vessel Breach

\subsubsection{Containment Failure Pressure}

For Surry, two previous studies of containment structural capability were directly applicable: the RSS [4] and a Stone and Webster Study [8]. In addition, the experts cited other studies of similar containments that provide insight into the expected capacity of the Surry containment. The input of the review team is illustrated in Figure 4-5. As indicated, the Stone and Webster value was weighted most heavily, but there was also significant weight attached to the next highest pressure capacity because the Stone and Webster evaluation only considered yield strength of materials, rather than ultimate strength. It should be noted that the values indicated are only the mean values of a distribution; the uncertainty in materials parameters was also included through the selection of standard deviations of the failure pressure distribution. 


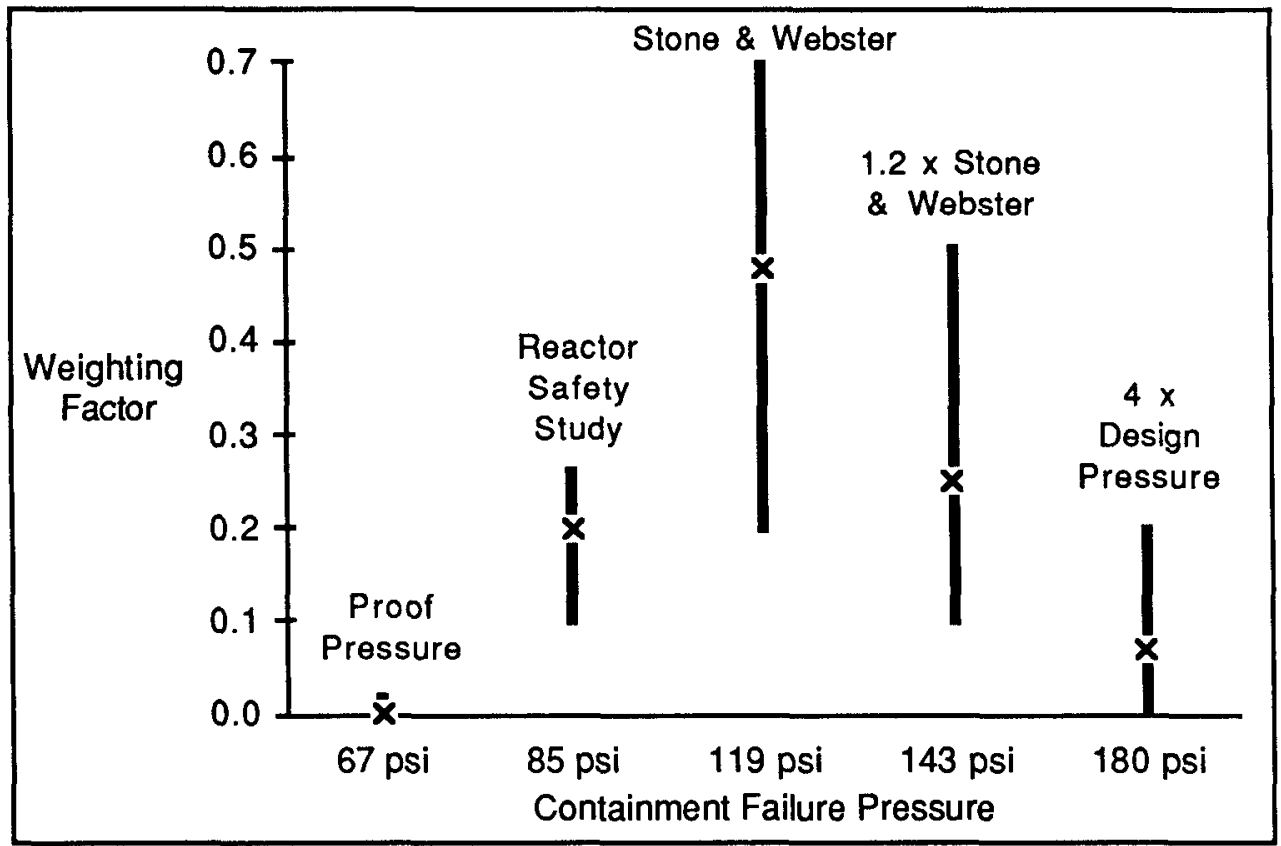

Figure 4-5. Outcomes and Weighting Factors for Containment Failure Pressure

\subsubsection{Containment Failure Size}

The LLH study also included consideration of the size of the containment failure opening. The reviewers thought that there were two principal correlations associated with this issue: (1) the size would be correlated with the the ultimate pressure capacity assumed, and (2) the size would also be dependent on the level of pressurization relative to the mean failure pressure. This latter correlation could not be accommodated within the constraints of the schedule, and the reviewer input had to be redefined in terms of only two failure sizes for the same reason. The original input is illustrated in Figure 4-6 for two cases of containment failure pressure. As illustrated, for the low pressure capacity the reviewers assigned a very high weighting factor to small openings based on a belief that a low pressure capacity would be due to an anomaly that would result in a local failure. The second case for a higher ultimate capacity illustrates a shift to larger openings based on the view that the higher the capacity, the more likely a gross rupture would be to occur since the shell is strained to a high value and the resulting crack would likely grow very rapidly rather than self-arresting. 
(a) 85 psig Failure Pressure (RSS)

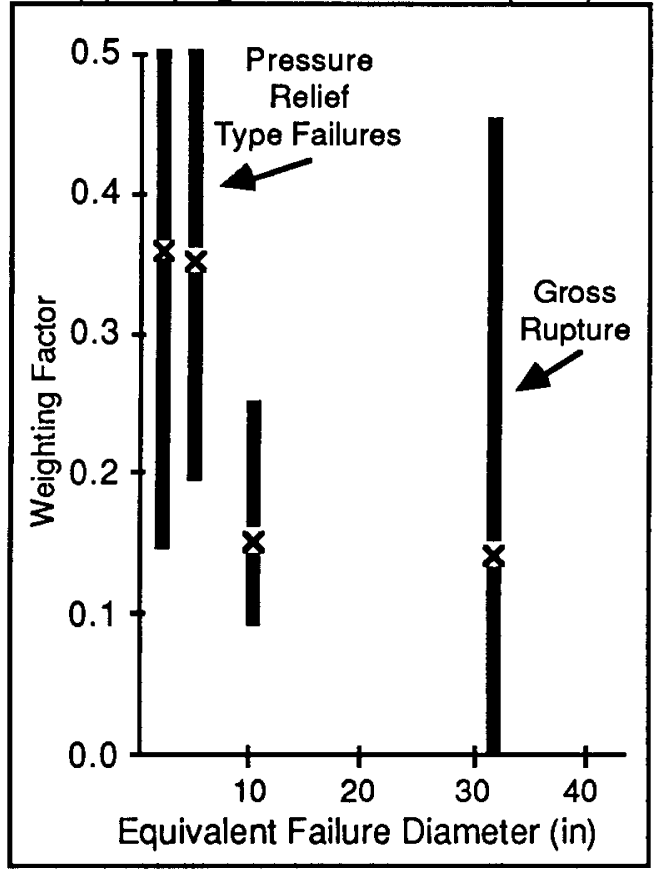

(b) 119 psig Failure Pressure (S \& W)

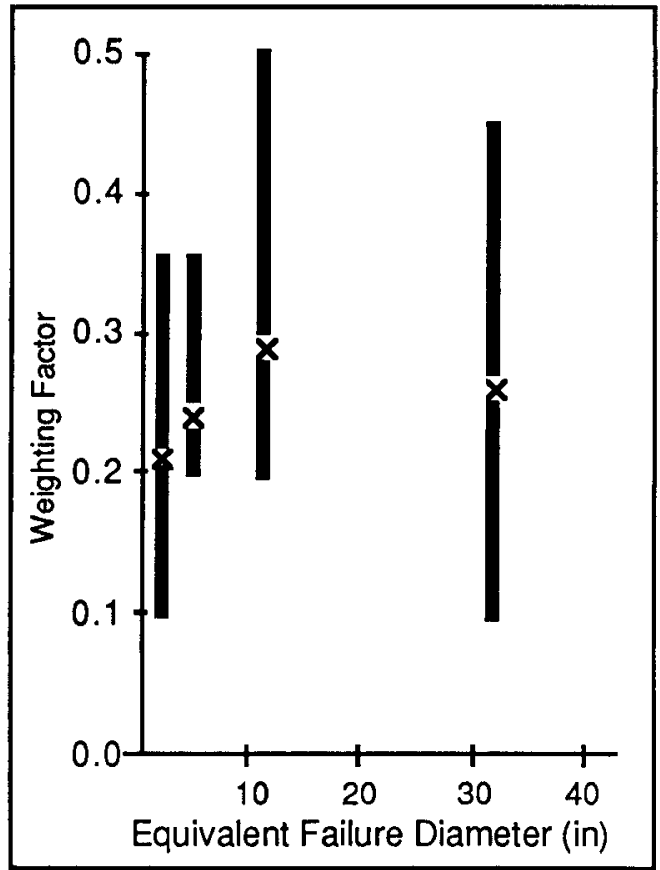

Figure 4-6. Outcomes and Weighting Factors for Containment Failure Size

\subsubsection{Probability of Containment Sprav Failure}

This issue deals with the late failure of the containment sprays due to phenomena in the containment. The review group was asked to provide weighting factors for three containment states. The first case was for no containment failure and was intended to account for failures due to debris in the sump or possible damage due to hydrogen burns. All of the reviewers except one thought that there would be no increase in failure rate over the basic spray failure unreliability for the case when there was no severe environment, and the other reviewer thought that the failure probability would be 0.05 . The results for the case of a containment failure with a small failure opening were similar. The reviewers did assess a significant failure probability ( 0.25 average) for the case of containment failure by gross rupture.

\subsubsection{Probability of Submergence of the $V$ Sequence Failure Site}

The interfacing systems LOCA, or V sequence, was determined early in the analysis to be a very significant risk contributor. There are a number of uncertainties associated with this sequence, and this issue deals with the physical conditions associated with the failure in the auxiliary building: whether or not the failure site is covered by water after the initial blowdown of the primary system. This issue is actually important to the assessment of the radiological 
source term, but the input was more appropriate to the containment performance experts than the source term experts. Based on several analyses of the situation $[2,10]$ the reviewers assigned a probability of submergence. There was limited variation in the input, and the average submergence probability was approximately 0.8 .

\subsection{RADIOLOGICAL SOURCE TERM ISSUES}

The analysis of the radiological source term was accomplished using the STCP as the principal tool, but not all of the sequences could be analyzed due to time and budget constraints. In addition, the models of the STCP are known to involve both considerable uncertainty and limitations relative to modeling some specific phenomena. The SARRP analyst and the expert review group established a list of issues that appeared to be most significant to the assessment of the risk of the Surry plant and then provided weighting factors for discrete levels of outcome, similar to the containment issues described above. The uncertainties in source term assessment were more difficult to evaluate within the constraints of the LLH study due to the level of detail needed to properly consider individual effects. For example, the method did not allow for detailed consideration of the effects of individual sequences. The "issues" are therefore defined more broadly and include many sub-issues which are described more fully in Appendix C. Practical constraints forced some compromises and some loss of resolution of the advances made in understanding of detailed source term science. Wherever possible these details have been documented in the appropriate sections of this report (particularly Appendix C), and all expert review group input, even at a lower level of detail, was documented. The discussion of the results in Section 5 also includes insights concerning these analyses.

The following radiological source term issues were included in the LLH analysis of Surry:

(1) Magnitude of in-vessel release from the fuel,

(2) Amount of CsI decomposition in the RCS,

(3) Amount of RCS retention,

(4) Decontamination factor for $V$ sequence release under water,

(5) Fraction of species released from the RCS that are subsequently released from containment,

(6) Magnitude of core-concrete interaction release,

(7) Fraction of core-concrete interaction species that is subsequently released from containment, 
NUREG/CR-4551,VOL.1: DRAFT REPORT FOR COMMENT (SEPTEMBER, 1986)

(8) Decontamination factor for the containment sprays,

(9) Aerosol agglomeration uncertainties,

(10) Amount of iodine released late in the accident from containment sources,

(11) Revolatilization of cesium and iodine from the RCS after vessel breach,

(12) Release associated with the high pressure ejection vessel failure mode, and

(13) Release associated with direct heating.

Each of these issues is defined briefly below. The input of the expert review group is also provided for most of the issues to illustrate the range of inputs assessed in the LLH study. For most issues, one of the distinct levels, or sets of outcomes, was constrained to be that which would be obtained using the STCP, if available. Thus the weighting factors help to illustrate the view of the experts concerning the uncertainty in the STCP in modeling the particular issues.

\subsubsection{In-Vessel Release from the Fuel}

There are a number of uncertainties associated with the estimated releases from the fuel during the melting of the core in the reactor vessel. The models of the actual radionuclide releases are known to be uncertain due to a lack of experimental evidence and data. The factors most affecting the release such as core thermal history also cannot be assessed with great accuracy. The levels and the assigned weighting factors are illustrated in Figure 4-7. As noted above, the levels include discrete outcomes for each of the source term groups. The reviewers weighted the STCP result most heavily (labeled base case on the figure) but also assigned significant and equal weighting factors for the levels on either side of the base case. Most reviewers thought that the very high releases associated with the highest level of outcomes were rather unlikely.

\subsubsection{Amount of CsL Decomposition}

This issue is a more specific concern with the iodine releases from the vessel. The STCP assumes that iodine will always be present as CsI. More recent experimental data indicates that, in the presence of steam and radiation (as in the primary system), CsI tends to decompose to more volatile forms such as elemental iodine. The more volatile forms could then be released directly without retention in the primary system, with a potentially significant impact on public health risk measures. At this time, the evidence is not conclusive, and four outcomes representing a uniform spacing between 0.0 and 1.0 as the fraction of iodine that is 
I. OUTCOMES

II. WEIGHTS

Fission Product
Elements
$\mathrm{Xe}, \mathrm{Kr}$
$\mathrm{C}$
$\mathrm{Cs}, \mathrm{Rb}$
$\mathrm{Te}$
$\mathrm{Sr}, \mathrm{Ba}$
$\mathrm{Ru}$ Group
La Group

\begin{tabular}{cccc}
\multicolumn{5}{c}{ Fraction Released From Fuel } \\
Low & Base & High & High-High \\
0.5 & 1.0 & 1.0 & 1.0 \\
0.5 & 1.0 & 1.0 & 1.0 \\
0.5 & 1.0 & 1.0 & 1.0 \\
0.03 & 0.3 & 0.9 & 1.0 \\
0.01 & 0.1 & 0.5 & 1.0 \\
0.008 & 0.04 & 0.1 & 0.5 \\
$1 \times 10^{-5}$ & $3 \times 10^{-4}$ & $8 \times 10^{-3}$ & $7 \times 10^{-2}$
\end{tabular}

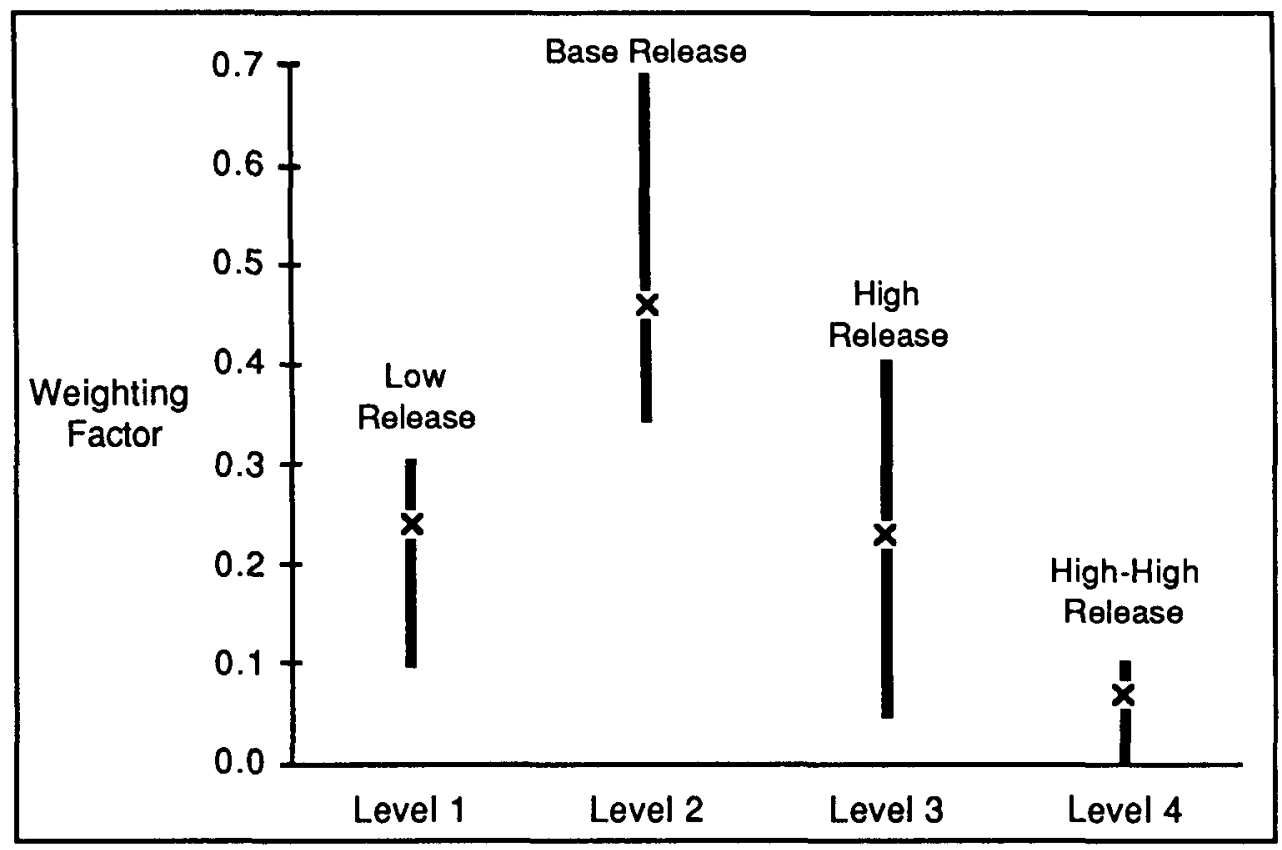

Figure 4-7. Outcomes and Weighting Factors for In-Vessel Release from the Fuel

released directly without retention due to conversion to a more volatile state were used in the LLH study. It should be noted that this issue does not include the rather large uncertainties associated with the retention of the iodine that is in the form of CsI; this is part of issue 3 .

The review group thought that there was great uncertainty in this issue, as illustrated by their composite weighting factors shown in Figure 4-8. The individual inputs indicated a high level of agreement among the reviewers, although several thought that either extreme was highly unlikely, while others provided a more uniform distribution.

\subsubsection{Retention in the Reactor Coolant System}

It is believed that there is significant uncertainty in the predicted radionuclide retention levels for the primary system. There are uncertainties in our understanding of the physical 


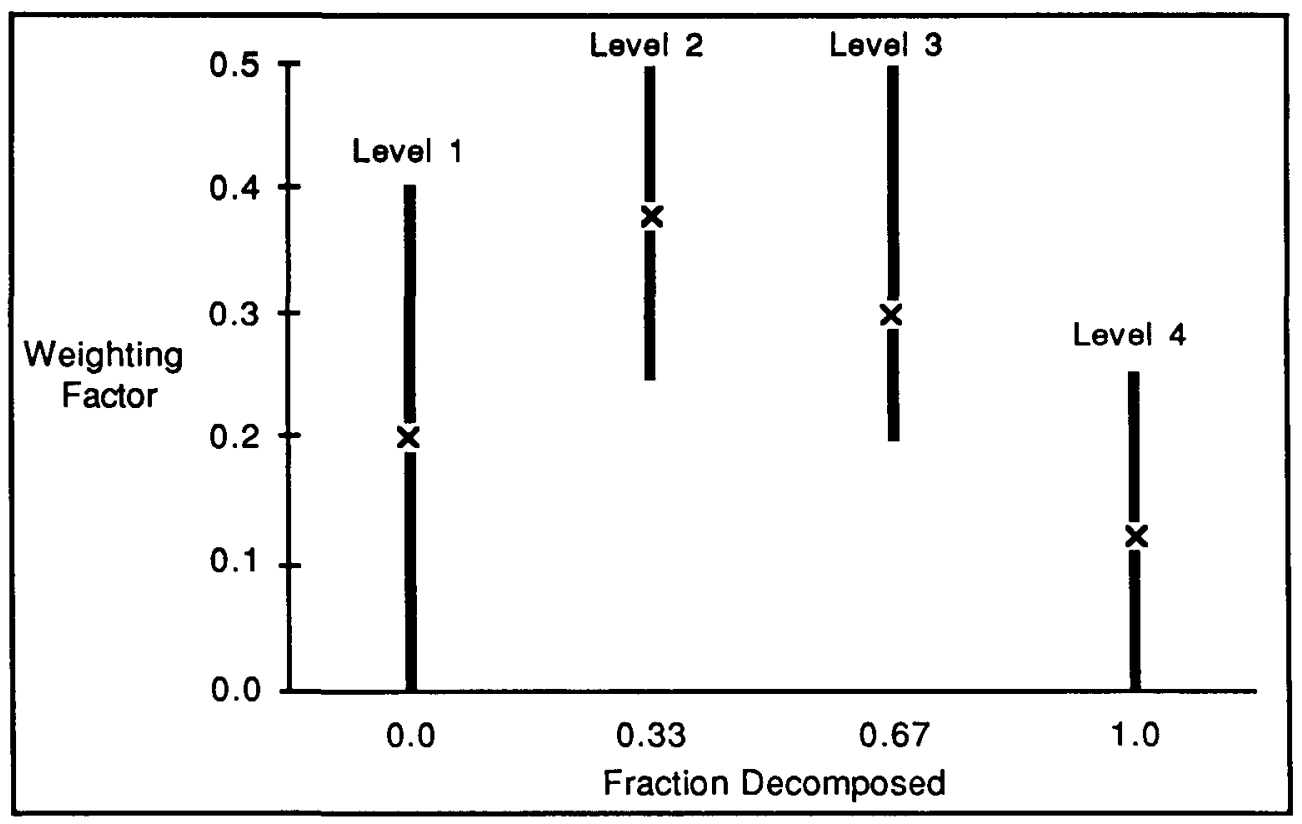

Figure 4-8. Outcomes and Weighting Factors for CsI Decomposition

processes as well as limitations in the current modeling capability which may introduce very significant uncertainty in the results. Representation of this issue as an uncertainty parameter is very complex because it would be desirable to acknowledge the dependencies on the accident sequence, the individual fission product group, and the fuel release, while further recognizing that the resulting correlations could be complex and unique for individual species. The sequence boundary conditions were treated by defining the levels specifically for individual types of sequences, although the assignment of weighting factors must necessarily apply to all levels as defined for all sequences.

The most significant code limitation that affects this issue is the fact that the current models do not account for heat and mass transport due to natural convection, whereas natural convection may completely dominate steam-driven transport. The impact of this has been studied in QUEST [11] and considered carefully within the SARRP program. The outcomes for two of the sequence groups are illustrated in Figure 4-9, as are the ranges of weighting factors assigned by the reviewers. The weighting factors indicated that the reviewers thought that the base case or lower levels of deposition were most likely. Thus the results indicate that the reviewers believe there is a significant chance that the STCP overpredicts the amount of retention, due partly to the lack of consideration of natural convection. 
I. OUTCOMES

Fission

Product

Elements

I, Cs

Te

$\mathrm{Sr}, \mathrm{Ru}, \mathrm{Ba}$

II. WEIGHTS
(A) High RCS Pressure Fraction Deposited in RCS*

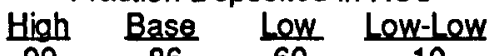

$\begin{array}{llll}.99 & .86 & .60 & .10\end{array}$

$\begin{array}{llll}.99 & .85 & .70 & .10\end{array}$

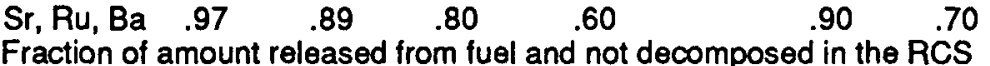

(B) Low RCS Pressure Fraction Deposited in RCS*

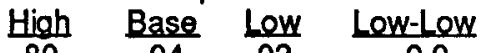

$\begin{array}{llll}.80 & .04 & .02 & 0.0\end{array}$

$.90 \quad .70 \quad .50 \quad .10$

$\begin{array}{llll}.90 & .70 & .30 & .10\end{array}$

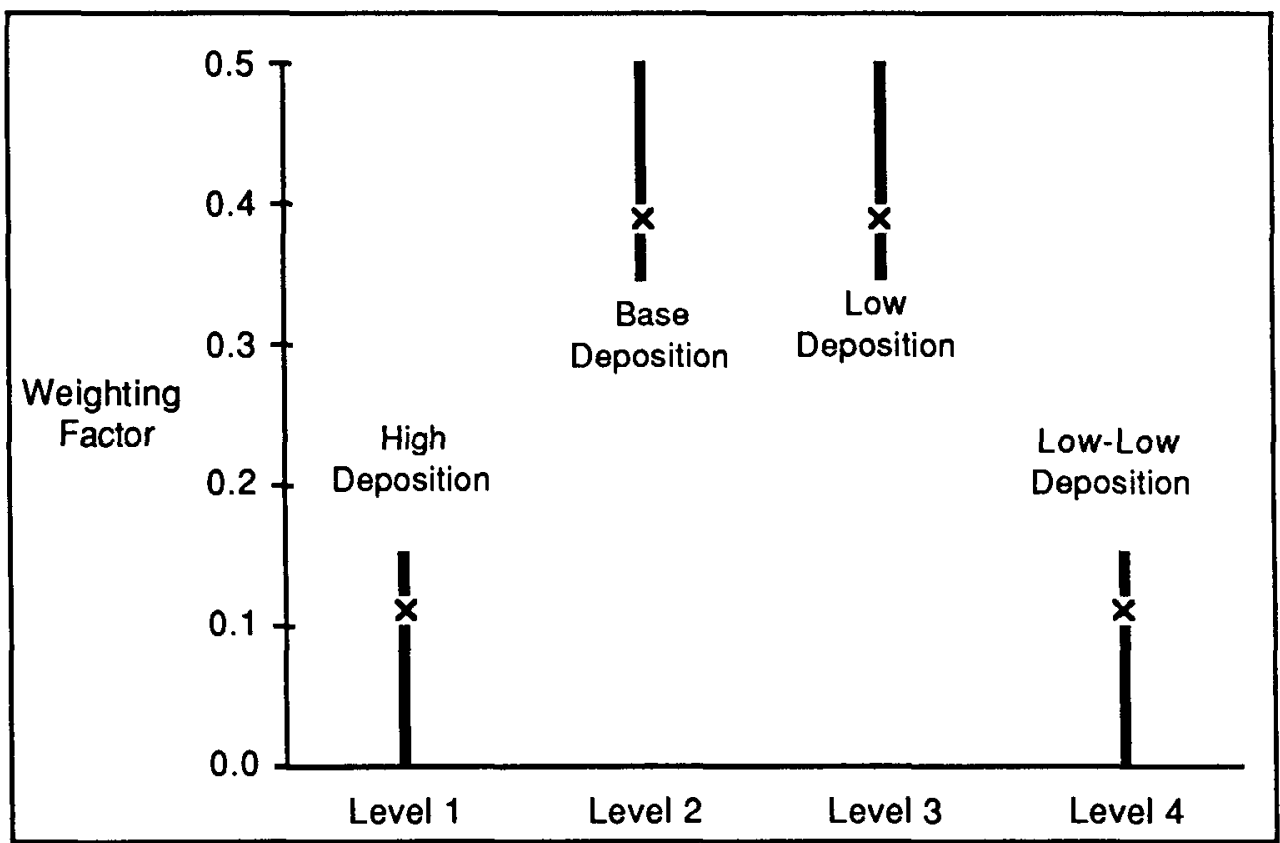

Figure 4-9. Outcomes and Weighting Factors for In-Vessel Retention

\subsubsection{Decontamination Factor for the $V$ Sequence with Release Submerged}

This issue is specific to the interfacing systems LOCA (or V sequence), which is important to the public health risk for Surry. There is considerable uncertainty associated with nearly all aspects of the $\mathrm{V}$ sequence, and this issue is intended to treat the more specific case of an interfacing systems LOCA in such a location that the primary system inventory and some portion of the injection water results in local flooding of the region around the pipe break. In this case, the release of fission products from the primary system following the resultant core melt would still bypass containment, but only after passing through the overlying water. The phenomenology of this circumstance is uncertain since there could be varying levels of scrubbing, physical displacement of the water by pressure from the primary, and re-release of fission products after initial scrubbing. Four levels of decontamination were weighted, spanning the range of reasonable decontamination factors associated with water scrubbing. 
The input of the review group is illustrated in Figure 4-10. Once again, a wide uncertainty is exhibited, although the very large decontamination factor was weighted less heavily.

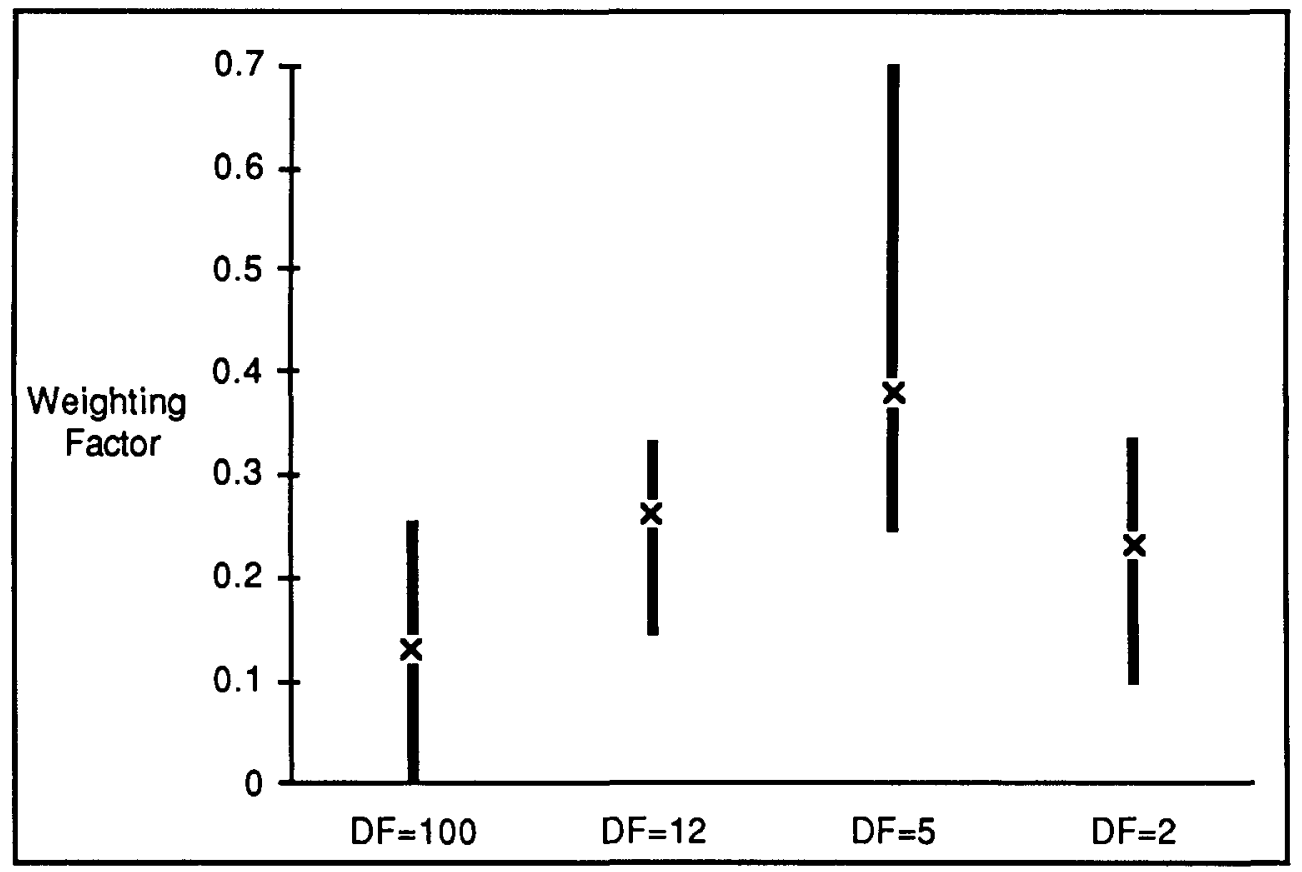

Figure 4-10. Outcomes and Weighting Factors for Decontamination Factor for the V Sequence When the Release Occurs Under Water

\subsubsection{Release of RCS Species from Containment}

This issue was defined to deal with a specific anomaly in the STCP which resulted in underprediction of the containment release fraction. The expert review group suggested a direct adjustment of the STCP results rather than treatment as an uncertainty issue. This issue was retained in the computer model, but it only has one outcome and is therefore not an uncertainty issue. The issue is included here only to maintain consistency throughout the report in terms of numbering and ordering of the LLH issues.

\subsubsection{Magnitude of Core-Concrete Interaction Releases}

There are also uncertainties in the estimated releases that occur during the core-concrete interactions. The levels of release and the ranges of weighting factors are illustrated in Figure 4-11. The low level results reflect considerably lower values than those predicted by the STCP. These lower values account for decontamination associated with the potential for crusting of the core due to the uncertainties associated with heat generation. The results of 
recent German studies were also thought to have applicability to the Surry plant, and these studies indicate the potential for the smaller releases associated with level 1. The strongest influence on these results is the very high level of silica in the Surry concrete. The uncertainties in the meltdown heat transfer coupled with the Surry concrete could lead to a low internal heat source and a crusting that would act as a decontamination mechanism. The values for the high release fractions, level 3, result from the chemical uncertainties associated with the core-concrete interactions.

As illustrated, the reviewers weighted the base case STCP most heavily $(0.57$ composite), but also allowed significant possibility of the releases associated with the other two levels. However, the range of uncertainty is really represented the significant weight on level 1 , which has very much lower release fractions for all species.

I. OUTCOMES

II. WEIGHTS

Fission Product
Elements
I, Cs, Rb
$\mathrm{Te}$
$\mathrm{Sr}, \mathrm{Ba}$
$\mathrm{Ru}$ Group
$\mathrm{La} \mathrm{Group}$

$\begin{gathered}\text { Fraction of Inventory Remaining in Fuel } \\ \text { That is Released During CCl } \\ \text { Level } 2\end{gathered}$
$\begin{array}{ccc}\text { Level } 1 & 1.0 & \frac{1.0}{1.0} \\ .02 & .4 & .8 \\ .008 & .05 & .2 \\ .002 & 3 \times 10^{-5} & .07 \\ 1 \times 10^{-6} & 8 \times 10^{-4} & .02 \\ 2 \times 10^{-5} & \end{array}$

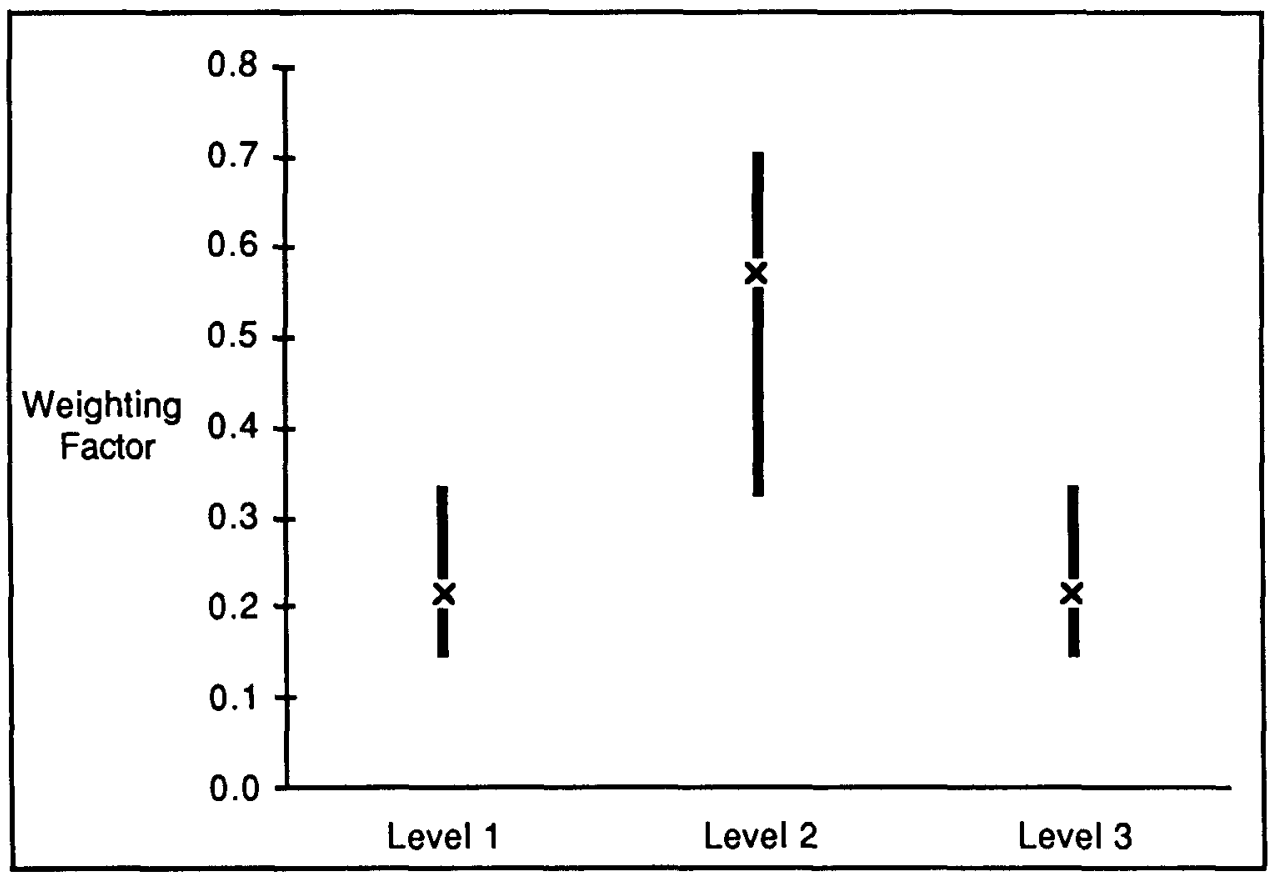

Figure 4-11. Outcomes and Weighting Factors for Magnitude of Core-Concrete Interaction Releases 


\subsubsection{Release of Core-Concrete Interaction Species from Containment}

This issue refers to the uncertainty in the physical processes taking place in containment that involve the inventory of species that have been released from the core-concrete interactions. It should be noted that this issue does not include the uncertainty in the effect of the containment sprays or in aerosol agglomeration, both of which are separate issues. The early containment failure scenarios were determined to be sufficiently understood so that treatment as an uncertainty issue was not warranted, and this issue was therefore limited to late failure scenarios. For these scenarios, clearly the driving factor in the uncertainty is the timing of the containment failure. It was generally concluded that the late failure scenarios could involve time periods as short as several hours to as long as several days.

Levels of outcomes were estimated for the fraction of species that would be released from containment given that they were released into the containment. These are represented in Figure 4-12, as are the ranges of the expert review group weighting factors. Level 3 represents the STCP results. It is recognized that the levels for this issue represented mostly analyst judgment due to a lack of applicable data. The weighting factors indicate the review group's opinion that the results predicted by the code tend to overpredict the release. This was based on the fact that there could be many reasons suggested that would lengthen the timing of the release, but very few scenarios involving less time could be postulated.

\subsubsection{Decontamination Factors for Containment Sprays}

The uncertainties in the decontamination factors expected for the the action of the containment sprays were also included in the LLH study. There are uncertainties in the models, in the distribution of droplet sizes, in temperature effects, and several other physical parameters. Three cases were defined to allow representation of the different boundary conditions: (1) effect on RCS species released in high pressure sequences concurrent with containment failure, (2) effect on RCS releases for all other sequences, and (3) effect on the core-concrete interaction releases. For the first case, it was assumed that the RCS release would be in a puff and that no scrubbing would apply; this assumption was maintained throughout the study. ${ }^{*}$ The three levels of decontamination for the latter two cases are listed on Figure 4-13. The uncertainty represented by these outcomes is again very large. The

\footnotetext{
"This assumption involves potentially significant conservatism for some accident sequences. This limitation is discussed further in Section 5.3 and Appendix C.
} 
1. OUTCOMES

II. WEIGHTS

Fission Product
Elements
I, Cs, Rb
$\mathrm{Te}$
$\mathrm{Sr}, \mathrm{Ba}$
$\mathrm{Ru}$ Group
La Group

\begin{tabular}{cccc}
$\begin{array}{c}\text { Fraction of CCl Release Released From } \\
\text { Level 1 }\end{array}$ & $\begin{array}{c}\text { Containment } \\
\text { Level 2 }\end{array}$ & $\begin{array}{c}\text { Level 3 } \\
\text { Level 4 }\end{array}$ \\
\hline .001 & .01 & .1 & .3 \\
.015 & .15 & .37 & .4 \\
.003 & .03 & .16 & .3 \\
.015 & .15 & .37 & .4 \\
.003 & .03 & .16 & .3
\end{tabular}

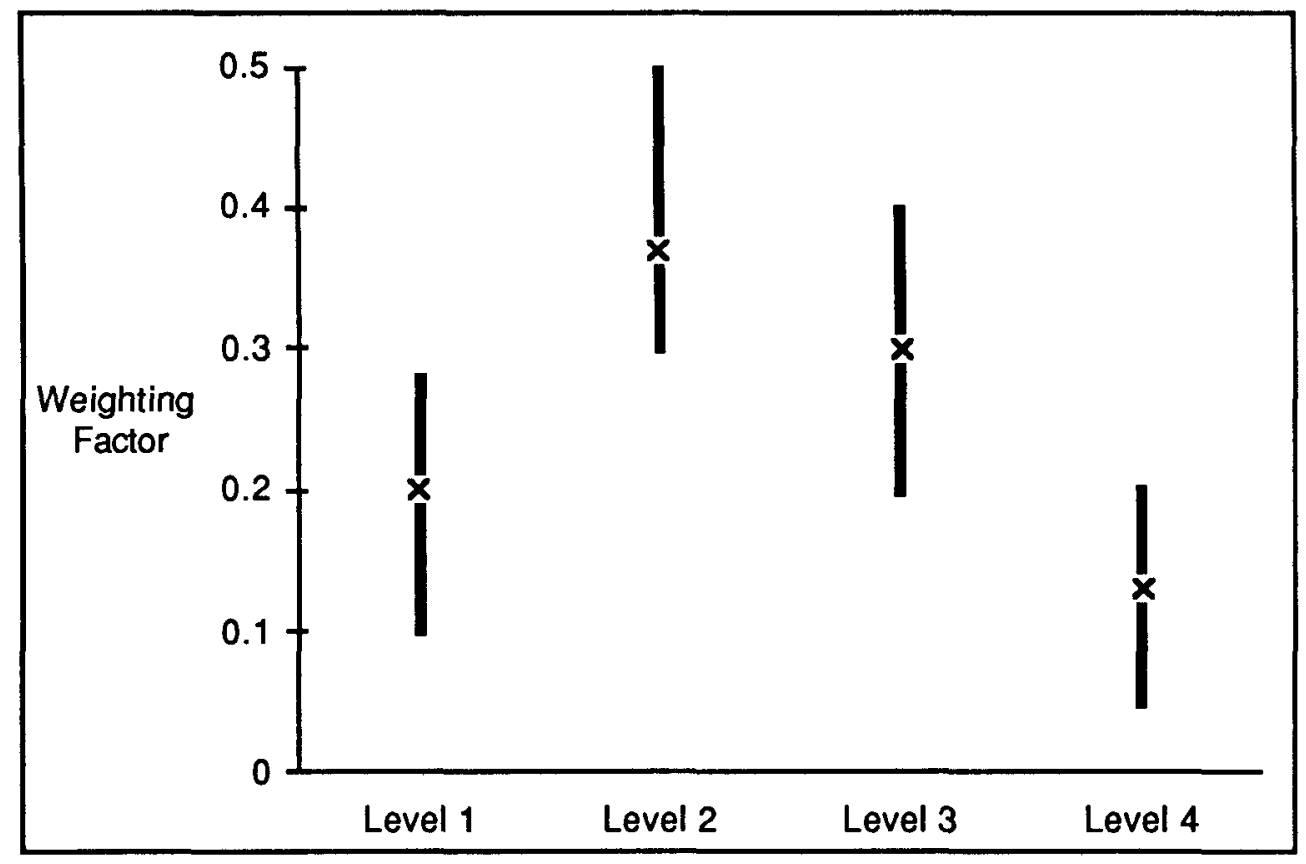

Figure 4-12. Outcomes and Weighting Factors for Release of Core-Concrete Interaction Species from Containment

reviewers assigned an approximate 0.5 weight to the base case represented by level 2 , and the rest of the weight was assigned approximately equally to the other levels of decontamination.

\subsubsection{Aerosol_Agglomeration Uncertainties}

The QUEST study indicated that the current STCP modeling of the aerosol species in containment possibly resulted in higher releases from containment than might be realistically expected due to the effects of aerosol agglomeration. The details of the effects of aerosol shape factors and turbulent agglomeration are outlined more thoroughly in QUEST [11]. This uncertainty has been represented here as a decontamination factor that is applied to the aerosol species in containment. The decontamination associated with this is only applied to scenarios involving failure of the containment sprays, and not for any sequence involving an early 
NUREG/CR-4551,VOL.1: DRAFT REPORT FOR COMMENT (SEPTEMBER, 1986)
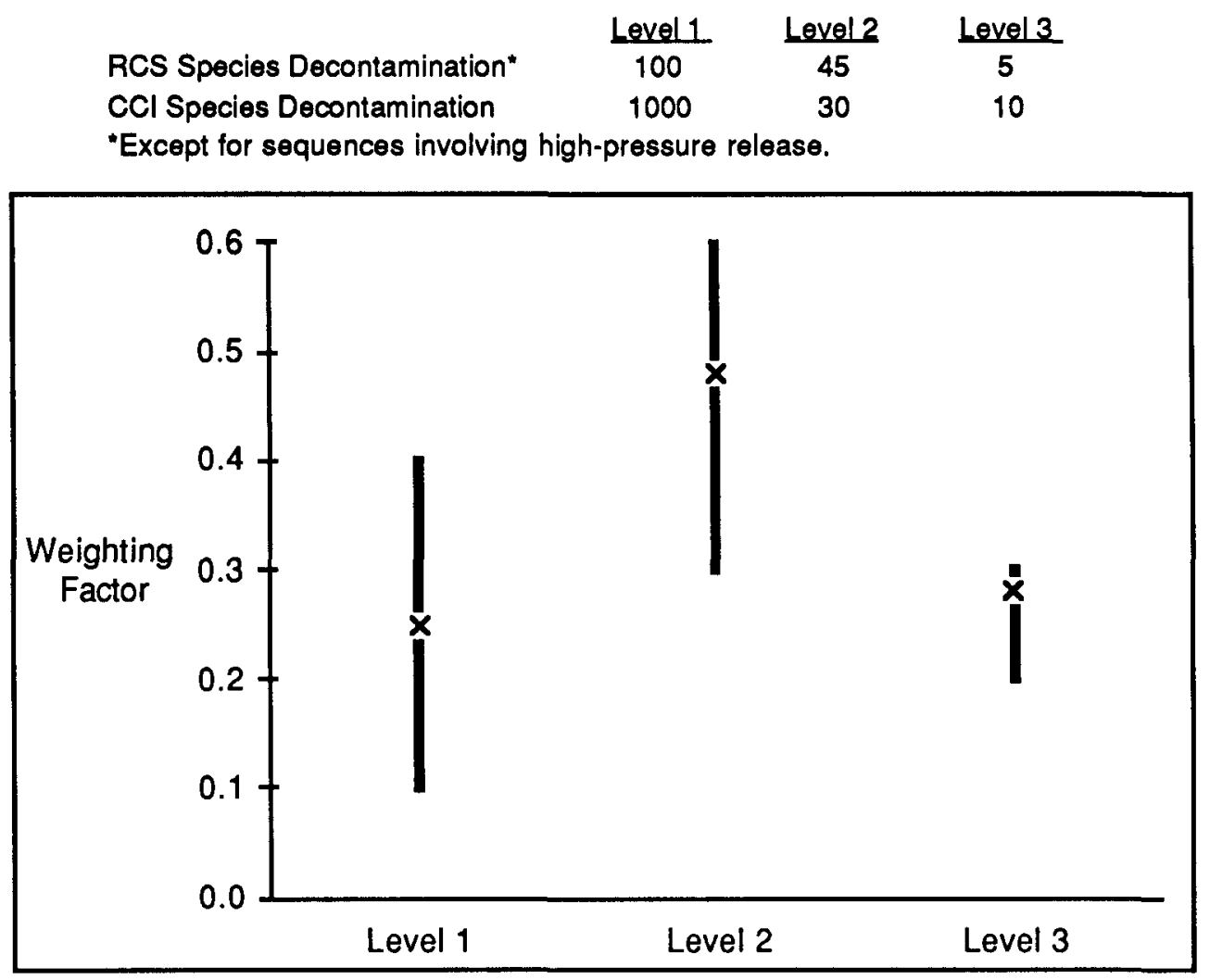

Figure 4-13. Outcomes and Weighting Factors for Decontamination Factors for Containment Sprays

containment failure following a high-pressure vessel breach (because of the limited time for the phenomenon to act in a puff-type release).

Based on the referenced QUEST result, a range of outcomes for decontamination factors to account for this feature were developed and are listed below. There was nearly uniform agreement at the review meeting, and therefore a graphical representation of this uncertainty is not necessary. The composite is listed below.
Decontamination factor
10 3 1
Composite weighting factors
0.23
0.49
0.38

The results indicate an order of magnitude uncertainty in this effect. As described in Appendix $\mathrm{C}$, there was an alternative set of smaller decontamination factors used for sequences in which agglomeration would not be expected to play a major role, such as the $\mathrm{V}$ sequence. 


\subsubsection{Late Iodine Releases from Containment}

As already discussed, this new issue treats late releases of iodine from the sources in containment. The issue specifically dealt with organic iodine sources and late releases from water pools. No decontamination or mitigating factors apply since this issue is limited to gaseous sources.

Additions to the iodine source term that could result from processes which are not included in the current code calculations were defined and are repeated on Figure 4-14. For this issue, the outcomes refer to the fraction of iodine that is in containment at late time periods that is ultimately released to the environment. ${ }^{*}$ As illustrated, there is large uncertainty in this issue: approximately two orders of magnitude. Overall, the reviewers felt that this was a potentially important source of iodine release.

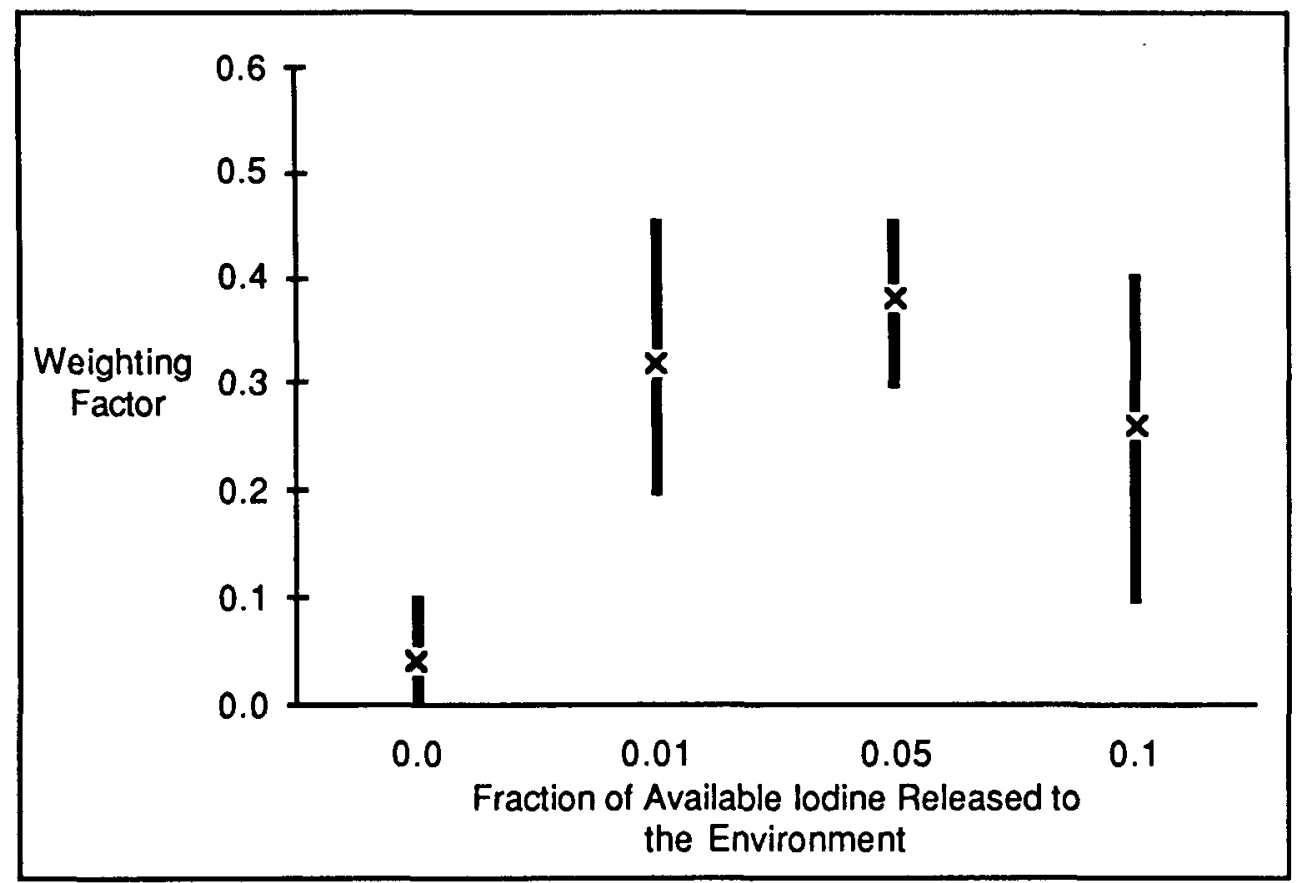

Figure 4-14. Outcomes and Weighting Factors for Late Iodine Releases from Containment

\subsubsection{Late Revolatilization from the RCS}

This issue refers to the phenomena that could result in releases of volatile species from the RCS that are not modeled in the source term code package. This issue was limited to Cs

\footnotetext{
*Due to confusion in the exact definition of this issue, there was a factor of two increase in the releases over that which was intended by the review group; the factors in Figure 4-14 were applied to all iodine, not just the fraction in containment.
} 
and I only. If it is assumed that a substantial amount of these species are retained in the primary system, decay heating of the primary could eventually result in their revolatilization and addition to the source term in containment at later times. The phenomenology of this revolatilization, including both the degree and the timing, is very uncertain. Outcome levels were derived for this issue to span this uncertainty and represent the case where no addition to the source term results, to very high releases that reflect very strong revolatilization.

The range of weighting factors provided by the reviewers are illustrated in Figure 4-15. Once again there were significant weighting factors assigned to all the levels indicating a large uncertainty for this issue which is not included in the STCP.

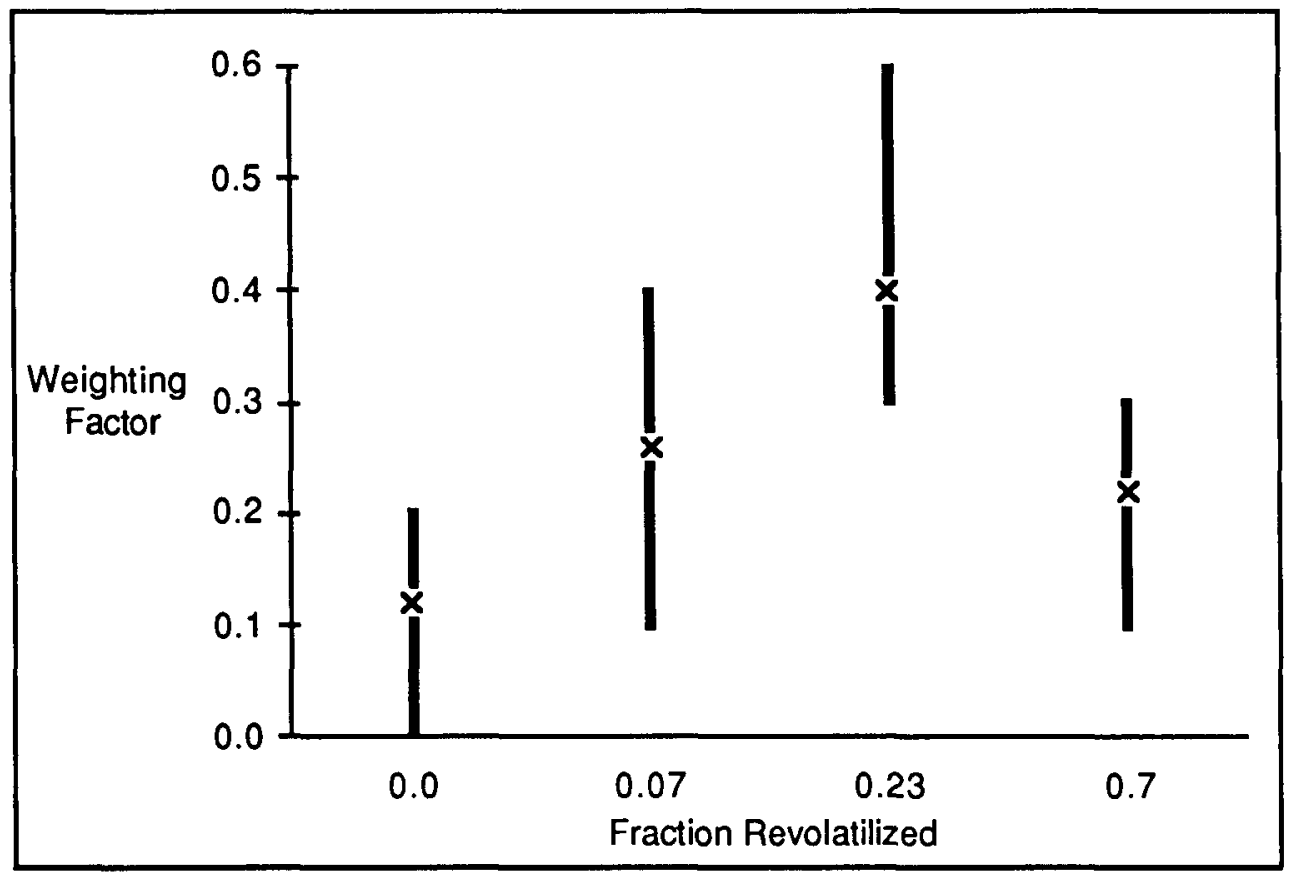

Figure 4-15. Outcomes and Weighting Factors for Late Releases from the RCS

\subsubsection{Beleases Associated with High Pressure Ejection}

The review group determined that the physical processes associated with the ejection of a significant quantity of core material for scenarios involving vessel breach at high pressure would also likely lead to enhanced radionuclide release. This phenomenon is not treated in the STCP. Experimental evidence indicates very significant aerosol formation for this vessel failure mode scenario [7]. The evidence supporting the levels for this issue is quite detailed and involves correlation with the amount of core ejected at high pressure (see Appendix C). The release fractions and the reviewer weighting factor assignments are listed in Figure 4-16. 
The fractions listed indicate the release to containment of species remaining in the melt at the time of vessel breach. The values listed are for $100 \%$ core participation and must be adjusted to the individual scenario; the average amount of core involved in high pressure ejection was estimated to be $40 \%$, although it varies with the outcomes of containment issues.

I. OUTCOMES

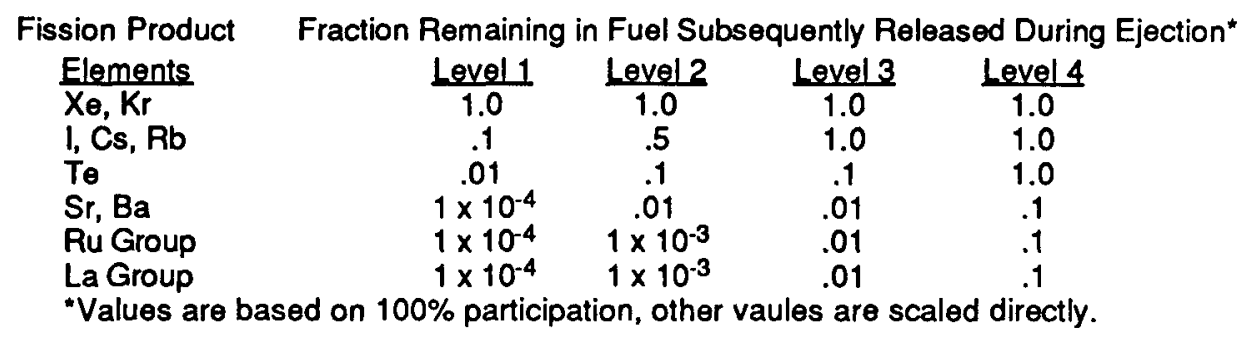

II. WEIGHTS

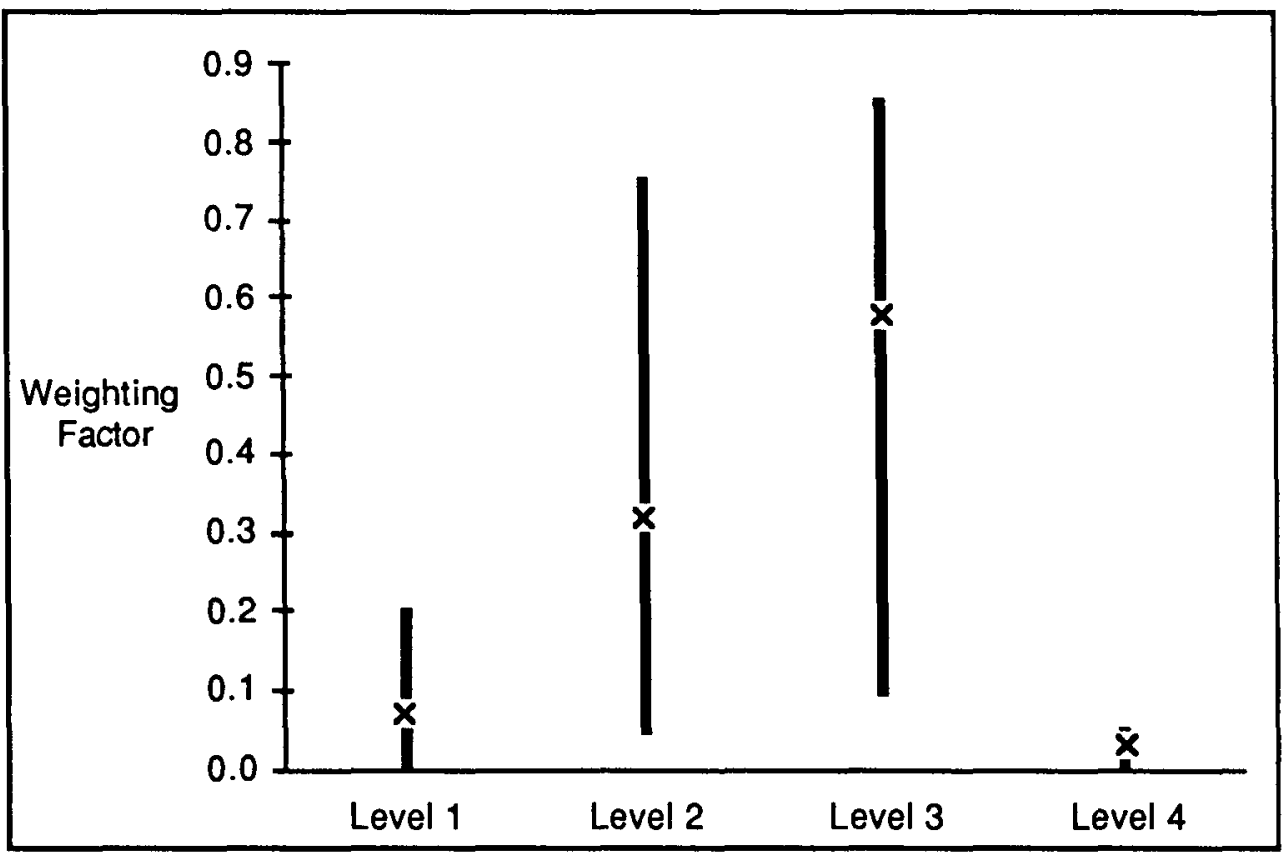

Figure 4-16. Outcomes and Weighting Factors for Releases During High Pressure Ejection

\subsubsection{Releases Associated with Direct Heating}

This issue accounts for an additional release associated with direct heating, over and above the release for high pressure ejection described in the previous issue. This release is anticipated to occur as a result of additional aerosol formation and radionuclide release associated with the oxidation of the core material in the atmosphere. This is also not treated in the STCP. The outcome levels refer to the fraction of radionuclides remaining in the fuel after 
high pressure ejection that are then released to the containment in the direct heating process. The justification for the levels is described in Appendix C.

The levels and reviewers inputs are depicted in Figure 4-17. As illustrated, the direct heating phenomenon is postulated to result in additional releases of radionuclides (in addition to the very strong pressure loading effect).

I. OUTCOMES

II. WEIGHTS
Fraction Remaining in Fuel After High Pressure Ejection that is Subsequntly Released During Direct Heating

\begin{tabular}{cccc} 
Level 1 & Level2 & Level 3 & Level 4 \\
\hline 1.0 & 1.0 & 1.0 & 1.0 \\
.5 & 1.0 & 1.0 & 1.0 \\
$1 \times 10^{-4}$ & .01 & .2 & .2 \\
$1 \times 10^{-6}$ & .05 & .2 & .9 \\
$1 \times 10^{-6}$ & .01 & .05 & .1
\end{tabular}

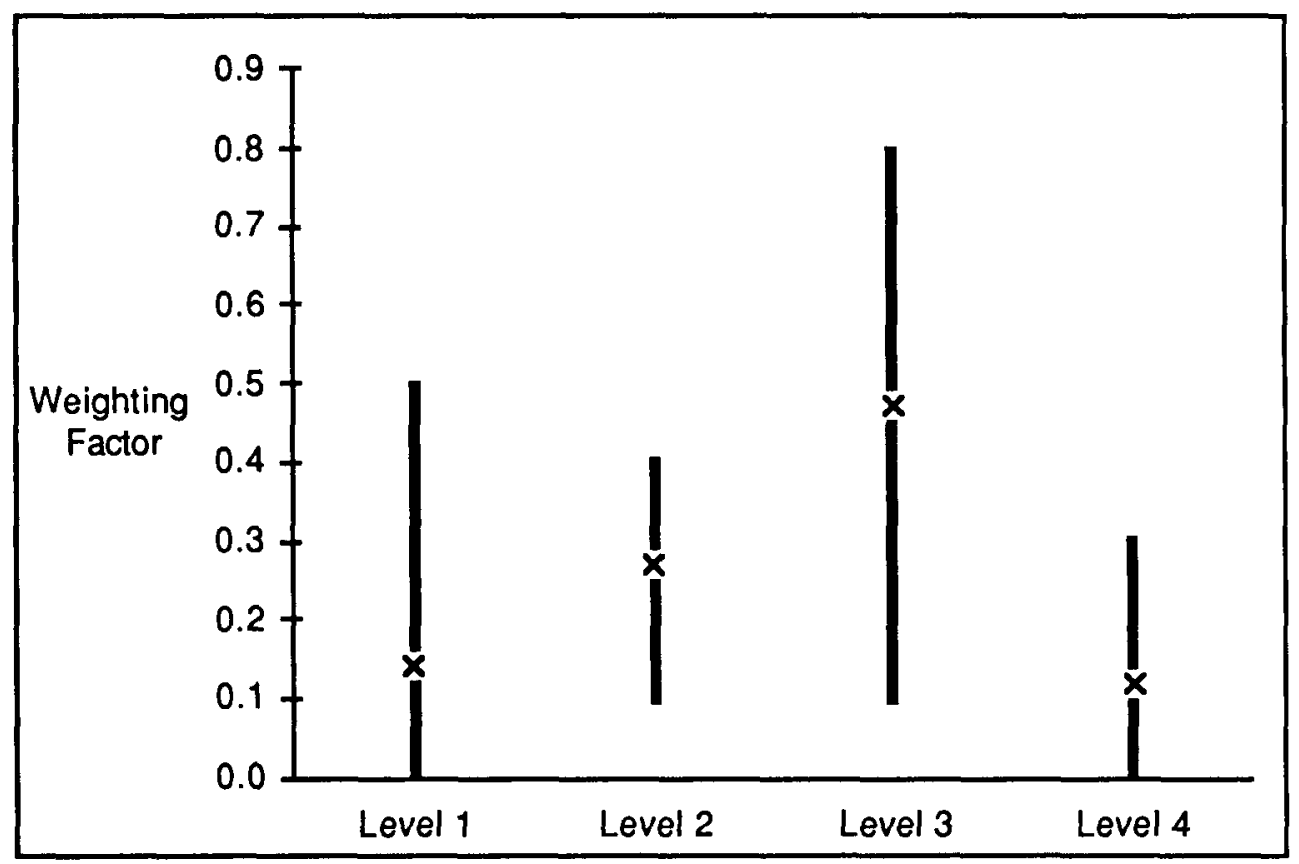

Figure 4-17. Outcomes and Weighting Factors for Releases Associated with Direct Heating 


\section{References for Section 4}

1. Bertucio, R. C., et al. Analysis of Core Damage Frequency From Internal Initiating Events: Surry, Unit 1. U.S. Nuclear Regulatory Commission Report NUREG/CR4550, Volume 3 (Draft Report), Sandia National Laboratories, Albuquerque, NM: April, 1986.

2. Commonwealth Edison Company. Zion Probabilistic Risk Assessment. Chicago, IL: 1980.

3. Fleming, K. N., et al. Classification and Analysis of Reactor Operating Experience Involving Dependent Events. Electric Power Research Institute Report NP-3967, Palo Alto, CA: June, 1985.

4. Reactor Safety Study--An Assessment of Accident Risks in U.S. Commercial Nuclear Power Plants. U.S. Nuclear Regulatory Commission Report WASH-1400 (NUREG75/014), Washington, DC: 1975.

5. Lyons, W. G, Presentation at NRC/IDCOR Meeting on Containment Loads and Fission Product Behavior. U.S. Nuclear Regulatory Commission, Washington, DC: May 15$17,1984$.

6. Containment Loads Working Group, "Consensus Summaries for Standard Problems 1 through 6." Letter Reports to J. Telford, U. S. Nuclear Regulatory Commission, Washington, DC: May-June 1984.

7. Tarbell, W., et al. High-Pressure Melt Streaming (HIPS) Program Plan. U.S. Nuclear Regulatory Commission Report NUREG/CR-3025, Sandia National Laboratories, Albuquerque, NM: August, 1984.

8. Drozd, A., et al. "Parametric Study of Aerosol Behavior Following AB and TMLB Accidents," Proceedings of the American Nuclear Society 1984 Annual Meeting. TP84-54, Stone and Webster Engineering Corp.: 1984.

9. Drozd, A., et al. "The V Sequence: an Engineering Viewpoint," Proceedings of the American Nuclear Society Topical Meeting on Fission Product Behavior and Source Term Research. Snowbird, Utah: July 15-19,1984.

10. Ritzman, R. L., et al. Surry Source Term and Consequence Analysis. Electric Power Research Institute Report NP-4096, Palo Alto, CA: June, 1985.

11. Lipinski, R. J., et al. Uncertainty in Radionuclide Release Under Specific LWR Accident Conditions. Sandia National Laboratories Report SAND84-0410, Albuquerque, NM: 1985. 


\section{Section 5 \\ RESULTS OF RISK REBASELINING}

The results of the SARRP study of Surry risk are discussed in this section. Brief summaries of the results of each analysis area (core-damage frequency, containment response, radionuclide release, and consequences) are included in sections 5.1 through 5.4, respectively. Section 5.5 contains the integrated calculation of risk. The results of the risk-reduction evaluation are discussed in Section 6.

\subsection{CORE-DAMAGE FREQUENCY RESULTS}

\subsubsection{Sequence and Damage State Frequencies}

The ASEP study of the Surry plant resulted in a new estimate of the total frequency of core damage and the identification of the individual accident sequences contributing to that total [1]. The total mean core-damage frequency reported by ASEP was $2.6 \times 10^{-5}$ per year. It should be noted that this total includes one type of accident sequence considered to result in conditions which may or may not result in core damage (termed "core vulnerable"). This class of sequences results from an initiating event which is followed by a loss of all containment cooling, eventually leading to a containment failure due to overpressurization. The response of systems to the overpressure failure is not fully known, and it was assumed in ASEP that the core could be vulnerable to damage due to system faults following the containment failure. Phenomenological analyses were carried out for SARRP which concluded that the conditional probability of core damage given a core-vulnerable sequence was 0.02 . The mean estimate frequency of core damage after accounting for this conditional probability is approximately $2.1 \times 10^{-5}$.

The dominant accident sequences are listed in Table 5-1. As illustrated, there are about 15 sequences that contribute to make up $95 \%$ of the total frequency. There were no single sequences found to dominate the result. The table also illustrates the importance of the initiating event involving loss of offsite power. This initiator is involved in sequences

\footnotetext{
*The results reported here are based on the ASEP draft report for Surry which was released in April 1986. The Surry results have since been revised and are available, but the risk results reported here could not be updated prior to the publication date for this report. The changes have been reviewed and none are expected to have a major impact on the risk results or conclusions presented here. In order to keep this report internally consistent, none of the ASEP material in this report is updated to reflect changes since the April draft.
} 
NUREG/CR-4551, VOL.1: DRAFT REPORT FOR COMMENT (SEPTEMBER, 1986)

Table 5-1

DOMINANT CORE-DAMAGE SEQUENCES FOR SURRY

\begin{tabular}{|c|c|c|}
\hline Sequence & Description & Frequency ${ }^{*}$ \\
\hline $\mathrm{T}_{1} \mathrm{SL}-\mathrm{D}_{1} \mathrm{CF}_{1}$ & $\begin{array}{l}\text { Loss of offsite power, resulting in a reactor-coolant pump } \\
\text { (RCP) seal LOCA due to the failure of all seal cooling. } \\
\text { High-pressure injection, containment spray injection, } \\
\text { and spray recirculation are all unavailable (all caused by the } \\
\text { extended unavailability of all electric power). }\end{array}$ & $5.6 \times 10^{-6}$ \\
\hline $\mathrm{T}_{4 \mathrm{~J}} \mathrm{QH}_{1}$ & $\begin{array}{l}\text { Loss of } 4 \mathrm{kv} \text { bus } \mathrm{J} \text {, followed by failure of a pressurizer } \\
\text { relief valve to reclose, and failure of low-pressure recirculation. }\end{array}$ & $1.5 \times 10^{-6}$ \\
\hline $\mathrm{T}_{4 \mathrm{H}} \mathrm{QH}_{1}$ & $\begin{array}{l}\text { Loss of } 4 \mathrm{kv} \text { bus } \mathrm{H} \text {, followed by failure of a pressurizer } \\
\text { relief valve to reclose, and failure of low-pressure recirculation. }\end{array}$ & $1.3 \times 10^{-6}$ \\
\hline $\mathrm{T}_{1} \mathrm{LP}$ & $\begin{array}{l}\text { Loss of offsite power, followed by failure of auxiliary } \\
\text { feedwater and failure of the power-operated relief valves } \\
\text { to be opened to permit feed-and-bleed cooling. }\end{array}$ & $1.2 \times 10^{-6}$ \\
\hline $\mathrm{T}_{1} \mathrm{~L}(\mathrm{LT}) \mathrm{D}_{1} \mathrm{CF}_{1}$ & $\begin{array}{l}\text { Loss of offsite power and failure of auxiliary feedwater } \\
\text { in the long term due to the extended unavailability of electric } \\
\text { power and eventual depletion of the batteries. The unavail- } \\
\text { ability of power also results in unavailability of high-pressure } \\
\text { injection and containment sprays. }\end{array}$ & $1.1 \times 10^{-6}$ \\
\hline $\mathrm{T}_{1} \mathrm{~L}(\mathrm{ST}) \mathrm{D}_{1} \mathrm{CF}_{1}$ & $\begin{array}{l}\text { Loss of offsite power and failure of auxiliary feedwater } \\
\text { in the short term due to failure of the turbine-drive pump. } \\
\text { The unavailability of power also results in failure of high- } \\
\text { pressure injection and containment sprays. }\end{array}$ & $1.1 \times 10^{-6}$ \\
\hline $\mathrm{T}_{4 \mathrm{~J}} \mathrm{QH}_{2}$ & $\begin{array}{l}\text { Loss of } 4 \mathrm{kv} \text { bus } \mathrm{J} \text {, followed by failure of a pressurizer } \\
\text { relief valve to reclose, and failure of high-pressure } \\
\text { recirculation. }\end{array}$ & $1.1 \times 10^{-6}$ \\
\hline $\mathrm{TKRD}_{4}$ & $\begin{array}{l}\text { Transient followed by failure to scram and failure of high- } \\
\text { pressure injection for emergency boration. }\end{array}$ & $9.8 \times 10^{-7}$ \\
\hline $\mathrm{T}_{4 \mathrm{H}} \mathrm{QH}_{2}$ & $\begin{array}{l}\text { Loss of } 4 \mathrm{kv} \text { bus } \mathrm{H} \text {, followed by failure of a pressurizer } \\
\text { relief valve to reclose, and failure of } \\
\text { high-pressure recirculation. }\end{array}$ & $9.2 \times 10^{-7}$ \\
\hline $\mathrm{V}$ & Interfacing-systems LOCA. & $9.0 \times 10^{-7}$ \\
\hline$S_{2} D_{1}$ & Small LOCA with failure of high-pressure injection. & $7.7 \times 10^{-7}$ \\
\hline$S_{1} D_{1}$ & Intermediate LOCA with failure of high-pressure injection. & $7.7 \times 10^{-7}$ \\
\hline $\mathrm{S}_{2} \mathrm{H}_{1}$ & Small LOCA followed by failure of low-pressure recirculation. & $7.2 \times 10^{-7}$ \\
\hline $\mathrm{S}_{2} \mathrm{H}_{2}$ & Small LOCA followed by failure of high-pressure recirculation. & $5.5 \times 10^{-7}$ \\
\hline $\mathrm{S}_{1} \mathrm{H}_{1}$ & $\begin{array}{l}\text { Intermediate LOCA with failure of low-pressure recirculation. } \\
\text { Total (95\% of frequency) }\end{array}$ & $\frac{5.1 \times 10^{-7}}{1.9 \times 10^{-5}}$ \\
\hline
\end{tabular}

* Point estimate values derived by propagating mean values of individual events. 
contributing one-half of the total. Other initiating events involving failure of individual power busses contribute another $25 \%$ of the total frequency. The accident sequences describe the individual contributors, but the plant damage-states served as the transition from the coredamage analysis to the containment analysis. For reference, Table 5-2 repeats the damage state definitions. The plant-damage states with non-negligible frequency are listed in Table 5-3.

Table 5-2

\section{PLANT-DAMAGE STATE DEFINITIONS}

\section{EXAMPLE PLANT-DAMAGE STATE: TYYBD}

First Letter-The integrity of the reactor-coolant system (RCS) pressure boundary

A Large or intermediate LOCA leading to low pressure in the RCS prior to breaching of the reactor pressure vessel

S Small LOCA resulting in intermediate pressure in the RCS prior to breaching of the reactor pressure vessel

T No initial LOCA or a very small LOCA with the RCS at high pressure prior to the vessel breach (the pressurizer relief valves may be cycling)

V LOCA that bypasses the containment boundary

Second Lefter--Whether or not water from the refueling water storage tank (RWST) has been discharged into containment

Y The RWST inventory has been discharged to containment, either by the emergency injection systems or by the containment spray injection system

$\mathrm{N}$ The RWST inventory has not been discharged to the containment

Third Letter--Whether or not containment heat removal is available

Y Containment heat removal is available

N Containment heat removal is not available

Eourth Letter--The status of the containment spray systems

B Both the containment spray injection and the containment spray recirculation systems are available

I The containment spray injection system is available, but the recirculation system has failed

R Containment spray recirculation is available, but the injection system failed (possible in Surry because of the separate, in-containment spray recirculation system)

$\mathrm{N}$ Neither injection nor recirculation flow is available

Eifth Letter--Whether or not steam generator heat removal is available

(only needed for some damage state definitions)

D The steam generators are dry

N The steam generators are wet. 


\begin{tabular}{|c|c|c|c|}
\hline SYYB & $\begin{array}{l}\mathrm{S}_{2} \mathrm{H}_{1} \\
\mathrm{~S}_{2} \mathrm{H}_{2} \\
\mathrm{~S}_{2} \mathrm{D}_{1} \\
\mathrm{TKRZ} \\
\mathrm{T}_{4 J \mathrm{JD} 1}\end{array}$ & $\begin{array}{l}\mathrm{T}_{4 \mathrm{H}} \mathrm{QD}_{1} \\
\mathrm{~T}_{4 \mathrm{~J}} \mathrm{QH}_{1} \\
\mathrm{~T}_{4 \mathrm{H}} \mathrm{QH}_{1} \\
\mathrm{~T}_{4 \mathrm{~J}} \mathrm{QH}_{2} \\
\mathrm{~T}_{4 \mathrm{H}} \mathrm{QH}_{2}\end{array}$ & $7.1 \times 10^{-6}$ \\
\hline SNNN & $\mathrm{T}_{1} \mathrm{SL}-\mathrm{D}_{1} \mathrm{CF}_{1}$ & $\mathrm{~T}_{1} \mathrm{QD}_{1} \mathrm{CF}_{1}$ & $5.7 \times 10^{-6}$ \\
\hline TNNN & $\mathrm{T}_{1} \mathrm{~L}(\mathrm{LT}) \mathrm{D}_{1} \mathrm{CF}_{1}$ & $\mathrm{~T}_{1} \mathrm{~L}(\mathrm{ST}) \mathrm{D}_{1} \mathrm{CF}_{1}$ & $1.9 \times 10^{-6}$ \\
\hline AYYB & $\begin{array}{l}\mathrm{AH}_{1} \\
\mathrm{~S}_{1} \mathrm{H}_{1}\end{array}$ & $\begin{array}{l}\mathrm{S}_{1} \mathrm{D}_{1} \\
\mathrm{AD}_{5}\end{array}$ & $1.8 \times 10^{-6}$ \\
\hline TYYBD & $\mathrm{T}_{1} \mathrm{LP}$ & & $1.1 \times 10^{-6}$ \\
\hline TYYBW & $\mathrm{TKRD}_{4}$ & $S_{3} D_{1}$ & $1.0 \times 10^{-6}$ \\
\hline V & & & $9.0 \times 10^{-7}$ \\
\hline AYNI & $\mathrm{S}_{1} \mathrm{~F}_{1} \mathrm{~F}_{2}$ & $\mathrm{AF}_{1} \mathrm{~F}_{2}$ & $9.8 \times 10^{-8}$ \\
\hline
\end{tabular}

Key

AYYB LOCA leading to low pressure in the RCS prior to vessel breach, with RWST inventory discharged to containment; containment heat removal, spray injection, and spray recirculation are all available.

SYYB Small LOCA with intermediate pressure in the RCS prior to vessel breach and RWST inventory discharged to containment; containment heat removal, spray injection, and spray recirculation are all available.

SNNN Small LOCA with intermediate pressure in the RCS prior to vessel breach; no RWST inventory is discharged to containment and the containment spray systems are not available. Note that this implies that there will be very limited water available in the reactor cavity.

TNNN Intact RCS prior to vessel breach, with to RWST inventory discharged to containment and the containment spray systems not available. Note that this implies that there will be very limited water available in the reactor cavity.

TYYBW Intact RCS prior to vessel breach, with RWST inventory discharged to containment; containment heat removal, spray injection and recirculation are available. The steam generators have water inventory.

TYYBD Intact RCS prior to vessel breach, with RWST inventory discharged to containment; containment heat removal, spray injection, and spray recirculation are all available. The steam generators are dry.

$\mathrm{V}$ Interfacing-systems LOCA.

AYNI LOCA leading to low pressure in the RCS prior to vessel breach, with RWST inventory discharged to containment; containment spray injection is available, but containment heat removal and recirculation are not functioning. 
It should be noted that one of the damage states in the ASEP analysis, TYYB, required further discrimination later in the analysis process. It was divided into the damage states TYYBD and TYYBW, differing only by the status of secondary side heat removal; the former referred to sequences in which the steam generators were dry at the onset of core degradation, and the latter included sequences involving wet steam generators.

\subsubsection{Uncertainty Representation}

The results discussed above are mean or point estimate frequencies. It was also a goal of the ASEP re-evaluation to estimate the important uncertainties. As described in Section 2.2, there were two types of uncertainty representation. The first uncertainty is that due to variation in the data used for the basic event frequencies and probabilities. Considering the uncertainty in the results due just to this data uncertainty, the results of ASEP can be represented by a distribution with the following parameters:

$\begin{array}{ll}\text { Upper 95\% confidence bound } & 6.1 \times 10^{-5} \\ \text { Mean value } & 2.6 \times 10^{-5} \\ \text { Median value } & 1.7 \times 10^{-5} \\ \text { Lower 5\% confidence bound } & 6.1 \times 10^{-6}\end{array}$

These uncertainty parameters include the total frequency contribution of the core-vulnerable states discussed above. The adjusted mean frequency after further considering the corevulnerable scenarios is approximately $2.1 \times 10^{-5}$. As illustrated above, there is about an order of magnitude uncertainty in the core-damage frequency associated with the input data. This degree of uncertainty, of course, applies to the total core damage frequency. The data uncertainty associated with individual sequences could be greater or less (for example, the data uncertainty associated with the $\mathrm{V}$ sequence is about two orders of magnitude).

The second uncertainty characterization was carried out in ASEP as a sensitivity study. Some key uncertainties in modeling were examined through sensitivity studies which looked at the impact on the results if different assumptions had been made. These issues represent uncertainties in the phenomena that could lead to core melt, alternative uses of reliability data, and credit for equipment not considered in the base analysis. The specific issues were the following:

- Loss of cooling for reactor-coolant pump (RCP) seals. The likelihood and severity of serious leakage from the RCP seals following the loss of seal cooling is not well known. 
- Recovery of electric power following station blackout. In part because of the first issue, the potential for recovery of electric power is a very important consideration.

- Likelihood of common-cause failures. Because of the redundancy in many of the important systems, failures of components due to common causes can have a strong effect on the frequency of core-damage sequences. However, there is a very limited data base available from which to derive estimates of the probabilities of such failures.

- Check-valve failure rates. The interfacing-systems LOCA involves failure of two check valves in series and, because it results directly in an early bypass of containment, has the potential to be a dominant contributor to risk. The frequency of this event is strongly dependent upon the failure models and rates used for the check valves. However, the data base for such occurrences is not well developed, largely because such failures are relatively rare and difficult to detect.

- ECCS operability after containment failure. The basic assumption in ASEP was that the ECCS systems would fail following a loss of containment integrity. This sensitivity case examined the importance of that assumption by setting the conditional probability of failure to 0.01 . After consideration of the phenomenology during the SARRP evaluation, this sensitivity case came closer to representing the central estimate.

Based on these issues, a set of twelve sensitivity cases aimed at investigating their significance was developed as indicated in Table 5-4. The impact on total core-damage frequency (point estimates based on the propagation of mean values for the primary events) is also noted. None of the issues makes difference to the total core-damage frequency greater than a factor of two. Note that this does not necessarily imply a lack of significance when considering risk, since several of the uncertainties are associated with plant-damage states that have high consequences.

The second step in the uncertainty analysis was the propagation of the probability distributions for the individual events in the sequence cut sets to obtain an overall estimate of uncertainty in the frequencies of core melt and of the plant-damage states. This was done by the performance of a Monte Carlo simulation using the SEP computer code [2]. The propagation was performed both for the base-case analysis and for each of the ten sensitivity cases. The result was the development of a "box-and-whisker" display for the uncertainties in the core-damage frequencies, as illustrated by Figures 5-1 and 5-2. The bounds of the box represent the highest and lowest mean values from the sensitivity studies. The figure also includes the mean, 5th percentile, and 95th percentile values for the base-case analysis, and the

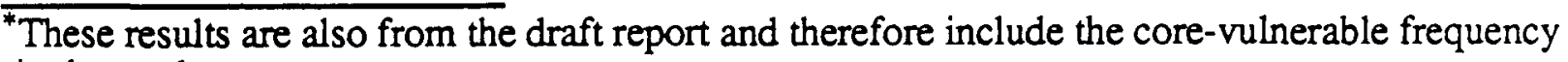
in the total. 
Table 5-4

SENSITIVITY STUDIES FOR CORE-DAMAGE ANALYSIS

\begin{tabular}{|c|c|c|c|}
\hline No. & Issue & Description of Sensitivity Study & $\begin{array}{l}\text { Total Core- } \\
\text { Damage } \\
\text { Frequency* }\end{array}$ \\
\hline-- & & Core-damage frequency for the base case. & $2.6 \times 10^{-5}$ \\
\hline 1. & RCP Seal LOCA & $\begin{array}{l}\text { Assume that seal LOCA occurs } 1 \mathrm{hr} \text { after loss of all seal } \\
\text { cooling, and that ac power must be restored within } 1 / 2 \mathrm{hr} \\
\text { after seal LOCA to prevent core damage. }\end{array}$ & $3.7 \times 10^{-5}$ \\
\hline 2. & RCP Seal LOCA & Assume seal LOCA does not $\propto c c u r$ after loss of cooling. & $2.2 \times 10^{-5}$ \\
\hline 3. & Recovery of power & $\begin{array}{l}\text { Use altemative (more optimistic) set of data for experience in } \\
\text { recovery of offsite electric power. }\end{array}$ & $1.9 \times 10^{-5}$ \\
\hline 4. & Recovery of power & $\begin{array}{l}\text { Permit credit for use of gas turbine-generator as a backup means } \\
\text { of restoring electric power. }\end{array}$ & $2.2 \times 10^{-5}$ \\
\hline 5. & $\begin{array}{l}\text { Common-cause } \\
\text { failure rates }\end{array}$ & $\begin{array}{l}\text { Use alternative value for probability of recovery of the } \\
\text { common-cause failure of service water valves due to bio-fouling. }\end{array}$ & $3.5 \times 10^{-5}$ \\
\hline 6. & $\begin{array}{l}\text { Common-cause } \\
\text { failure rates }\end{array}$ & $\begin{array}{l}\text { Use alternative (more pessimistic) interpretation of beta factors } \\
\text { for common-cause failure rates. }\end{array}$ & $3.3 \times 10^{-5}$ \\
\hline 7. & $\begin{array}{l}\text { Common-cause } \\
\text { failure rates }\end{array}$ & Eliminate use of beta factors. & $2.2 \times 10^{-5}$ \\
\hline 8. & $\begin{array}{l}\text { Check-valve } \\
\text { failure rates }\end{array}$ & $\begin{array}{l}\text { Apply beta factors for motor-operated valves to check valves for } \\
\text { interfacing-systems LOCA calculation. }\end{array}$ & $3.4 \times 10^{-5}$ \\
\hline 9. & $\begin{array}{l}\text { Check-valve } \\
\text { failure rates }\end{array}$ & Use more optimistic distribution for failure rates for check valves. & $2.4 \times 10^{-5}$ \\
\hline 10. & $\begin{array}{l}\text { Check-valve } \\
\text { failure rates }\end{array}$ & Use more pessimistic distribution for failure rates for check valves. & $3.0 \times 10^{-5}$ \\
\hline 11. & ECCS Operability & $\begin{array}{l}\text { Assignment of a conditional probability of ECCS failure of } 0.01 \\
\text { for all sequences involving containment failure before core damage. }\end{array}$ & $2.1 \times 10^{-5}$ \\
\hline 12. & $\begin{array}{l}\text { Combination of } \\
\# 3,4 \text {, and } 6\end{array}$ & Sensitivity to combinations of issues. & $2.2 \times 10^{-5}$ \\
\hline
\end{tabular}


NUREG/CR-4551,VOL.1: DRAFT REPORT FOR COMMENT (SEPTEMBER, 1986)

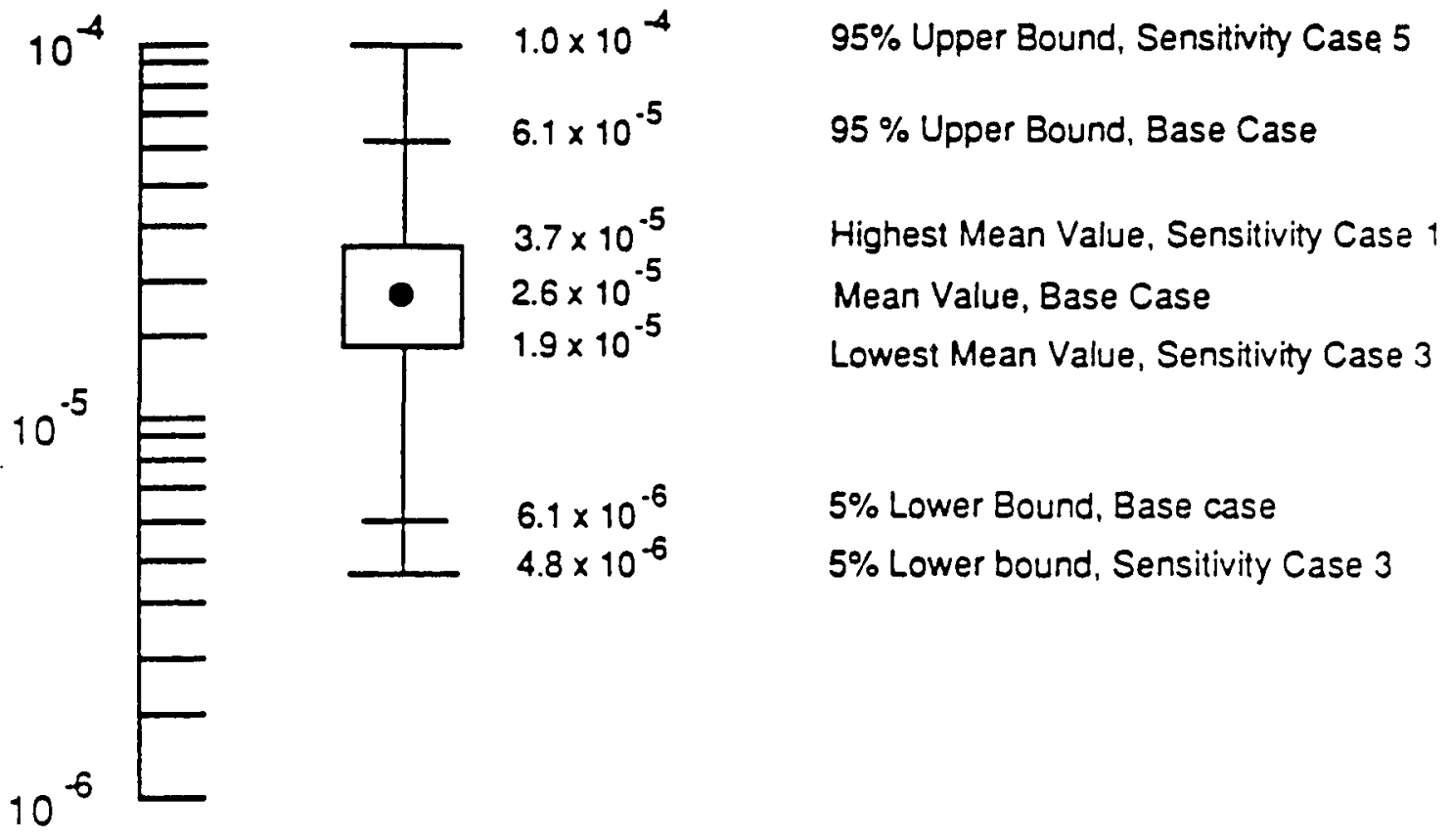

Figure 5-1. "Box-and-Whisker" Display of Uncertainties for Total Core-Damage Frequency

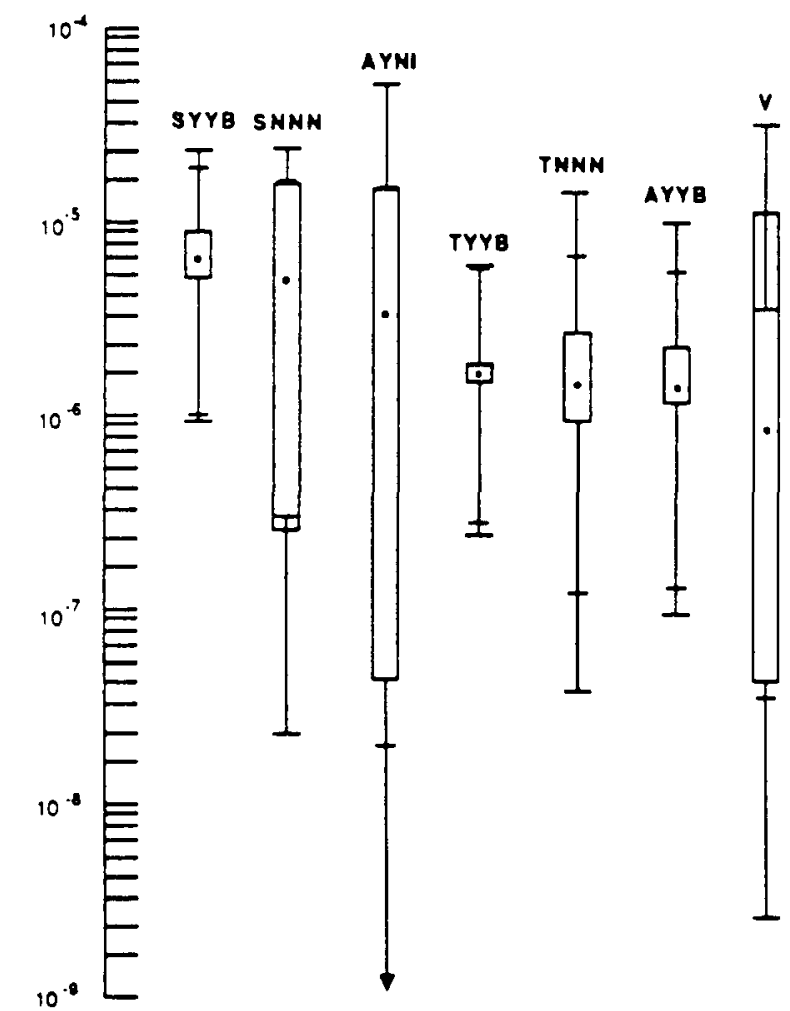

Figure 5-2. Box and Whisker Plots for Surry Plant Damage States with Mean Frequencies $>1.0 \times 10^{-7}$ 
highest 95th-percentile and lowest 5 th percentile values from the sensitivity cases. As illustrated, the variation in mean frequency of core damage associated with all of the individual sensitivity cases is very limited (less than a factor of two). However, the uncertainty associated with combinations of sensitivity cases was only considered for one combination in the ASEP analysis. The 5th and 95th percentiles of the base case resulting from data uncertainties account for an order of magnitude uncertainty. Combining the worst combinations of uncertainties from the sensitivity and data uncertainty analyses yields a total uncertainty range of approximately one and one-half orders of magnitude.

The results of the base case and uncertainty representations (sensitivity studies and data) are illustrated in Figure 5-2 for the most frequent bins. The impact of the sensitivity issues on individual bins can be seen more clearly in this figure. For example, sensitivity cases 9 and 10 cause a wide variation in the damage state $V$, since those sensitivity cases specifically addressed the sequence in that damage state. A comparison of Figures 5-1 and 5-2 yields the conclusion that the uncertainty in given damage states may be very large (several orders of magnitude) but that that the total uncertainty is more limited since no one damage state dominates the frequency and a combination of sensitivities are therefore needed to have a large impact on the total.

In keeping with the LLH methodology described in Section 2, the ASEP analysts were asked to provide weighting factors for the issues represented in the sensitivity cases. These weighting factors were used with the outcomes of the sensitivity cases as issues and levels similar to those developed expressly for SARRP for the containment and source term analysis. The following LLH input was developed:

Issue

1. RCP Seal Loca Model

2. Offsite Power Recovery

3. Common-Cause Model

4. V Sequence Model

5. ECCS Operability (treated as the probability of core damage given containment failure)
Qutcome

Sens. Case 1

Sens. Case 2

Base Case

Sens. Case 4

Base Case

Sens. Case 6

Sens. Case 7

Base Case

Sens. Case 9

Sens. Case 10

Base Case

Probability $=0.01$

Probability $=0.02$

Probability $=0.04$
Weighting Factor

0.05

0.05

0.90

0.5

0.5

0.34

0.01

0.65

0.4

0.1

0.5

0.33

0.33

0.33 
As illustrated by the weighting factors, the ASEP analysts thought that sensitivity cases 4,6 , and 9 had a fairly high probability of representing the true situation. The input for the last LLH issue, the probability of ECCS failure given containment failure, was solicited from the containment loading experts who provided the containment issue weighting factors. As noted in Section 4, the uncertainty in this issue is quite likely understated, and conclusions concerning its importance are probably not appropriate without further consideration. Note also that the lowest probability estimate for this issue corresponds to ASEP's sensitivity case 11. The results of the LLH computation of core-damage frequency are listed below:

$\begin{array}{ll}\text { 95th percentile } & 1.5 \times 10^{-5} \\ \text { Mean } & 2.0 \times 10^{-5} \\ \text { Median } & 2.0 \times 10^{-5} \\ \text { 5th Percentile } & 3.1 \times 10^{-5}\end{array}$

The issues sampled resulted in a spread of approximately a factor of two as indicated above, which was a result consistent with the ASEP sensitivity studies. This result again arises due to the fact that no single accident type dominates the core-damage frequency profile. The risk significance of the sequence frequency issues is considered in Section 5.5.

\subsubsection{Qbservations Concerning the Core-Damage Frequency}

There were a large number of contributors to the overall core-damage frequency at Surry; hence, there were no outliers that could be eliminated to result in a significant reduction in total frequency. As already stated, the sequences involving loss of power initiating events were most significant, with the most important of these being the station blackout scenarios (i.e., loss of offsite power followed by failure of onsite emergency power). Another plantspecific contributor found to be important to the ASEP results was the common-mode failure of the service water system leading to failure of containment cooling. This was found to be important to risk in the central estimate but was not important in the LLH study due to the low probability (and uncertainty) assigned for the core-vulnerable sequences to proceed to core damage. Additional insights concerning the core-damage analysis are derived from a comparison to the RSS, as described in the next section, and from the examination of the risk results in Section 5.5. 


\subsubsection{Comparison to the Reactor Safety Study}

The Reactor Safety Study estimated a core-melt frequency for Surry of $4.4 \times 10^{-5}$ [3], compared to the updated ASEP value of $2.1 \times 10^{-5}$ (after accounting for the reduction in the core-vulnerable sequences). The difference in these two values is not particularly significant, although it should be noted that the estimate from the original study was a point estimate calculated by propagating median values (a process which would generally yield a value lower than the mean), while the updated frequency is represented as a mean value. However, there were important differences in the sequences and features that were assessed to contribute most to core-damage frequency in these two investigations, including the following:

- A number of changes have been made to the Surry plant since completion of the original study more than a decade ago;

- Improved understanding of accident phenomenology has allowed the definition of new success criteria for several systems under different accident conditions;

- The ASEP updated analysis included substantially more consideration of operator actions that could be taken to recover lost functions prior to melting of the core;

- In the update, more powerful computer tools were used to quantify the sequence frequencies by obtaining sequence cut sets, enabling enhanced tracking of dependencies among systems, and allowing for the detailed consideration of recovery actions in a realistic framework;

- Newer methods and data for the assessment of common-cause failures were used in the ASEP study; and

- The updated study incorporated newer data bases than were available at the time of the original, including some plant-specific data.

These factors were the principal contributors to differences in the two studies. Comparing the dominant sequences for both studies yielded the following insights:

- The potential for RCP seal LOCAs as a consequence of a loss of seal cooling played a major role in the ASEP study, but was not considered in the original analysis.

- The likelihood of loss of all containment heat removal was assessed to be higher in the update to account for plant-specific experience involving common-cause failures in the service-water system.

- Sequences involving station blackout and a loss of all auxiliary feedwater were assessed to have similar frequencies in the two studies.

- The ASEP update considered sequences initiated by individual electrical bus failures, and these appeared to be significant; the transient initiators were not considered at that level of detail in the prior study. 
- There were substantial changes in the sequences involving failure to scram; these changes arose due to improved understanding of plant behavior under these conditions, an updated assessment of the likelihood of scram failure, and differences in the treatment of operator actions.

- The frequencies of interfacing-systems LOCAs have been reduced by the institution of periodic testing to verify the status of the isolating check valves.

- The frequencies of sequences initiated by LOCAs and followed by failure of emergency core cooling are generally lower in the update. In this case, the higher likelihood of common-cause failures is offset by further consideration of recovery actions.

- In light of improved understanding of accident phenomena, two sequences were eliminated: a small LOCA with failure of containment spray injection (sequence $S_{2} \mathrm{C}$ ), and a transient followed by total loss of feedwater (sequence TML). In the case of the latter sequence, the update credited the use of feed-and-bleed cooling as an alternative means of core heat removal.

\subsection{CONTAINMENT ANALYSIS RESULTS}

A brief review of the intermediate results of the analyses is presented below to provide a perspective on the evaluation of containment response. Section 5.2.1 describes some of the $\mathrm{LLH}$ results, although the presentation is limited because it would require a great deal of detail to back out only results of the containment analysis portion from the integrated LLH risk calculation. Section 5.2.2 includes the results of the containment event tree analysis for the OCP method. It should be noted that additional perspective on the importance of containment phenomena to the risk measures is provided in Section 5.5 of this report.

\subsubsection{Results for the LLH Analysis of Containment Resoonse}

It is not practical to examine the results of the containment portion of the analysis alone for all of the 100 risk samples. Therefore, it was decided to provide additional insight through the key determinant of importance of the containment response to the risk results, which is the probability of early containment failure. Early containment failure is taken here to include all cases for which significant radionuclide releases to the environment occur before or during the time of reactor vessel breach. For example, the $\mathrm{V}$ sequence is included in the evaluation of early containment failure, since the breach of containment occurs at the onset of the accident. Figure 5-3 illustrates the probability of early containment failure when considering all accident sequences (the sequences are weighted by their frequencies). As indicated, the LLH results for probability of early containment failure range from $10^{-2}$ to nearly unity. For the LLH sample, the median value was 0.1 and the mean value was 0.2 . These results are dominated by 
containment failures occurring from direct heating during station blackout sequences (with some contribution from concurrent hydrogen buming pressure increments). Also shown on the figure is the central value for the $O C P$ method, $5 \times 10^{-2}$. The central estimate was primarily influenced by the $\mathrm{V}$ sequence. In general, the $\mathrm{LLH}$ input from the expert reviewers pointed to more severe containment conditions during direct heating scenarios than predicted by the central estimate, which had been obtained prior to the LLH process. The mean probability from the Reactor Safery Study is also indicated for comparison; it is nearly identical to the $\mathrm{LLH}$ mean. Although there is no IDCOR study of Surry, the study of Zion allows comparison to a large, dry PWR containment. The comparable $\mathrm{DCOR}$ value is approximately $5 \times 10^{-3}$, dominated by containment isolation failure rather than containment structural failure. In general, the steam and hydrogen bum loadings are predicted to be less in IDCOR, and direct heating is not considered to be a significant phenomenon.

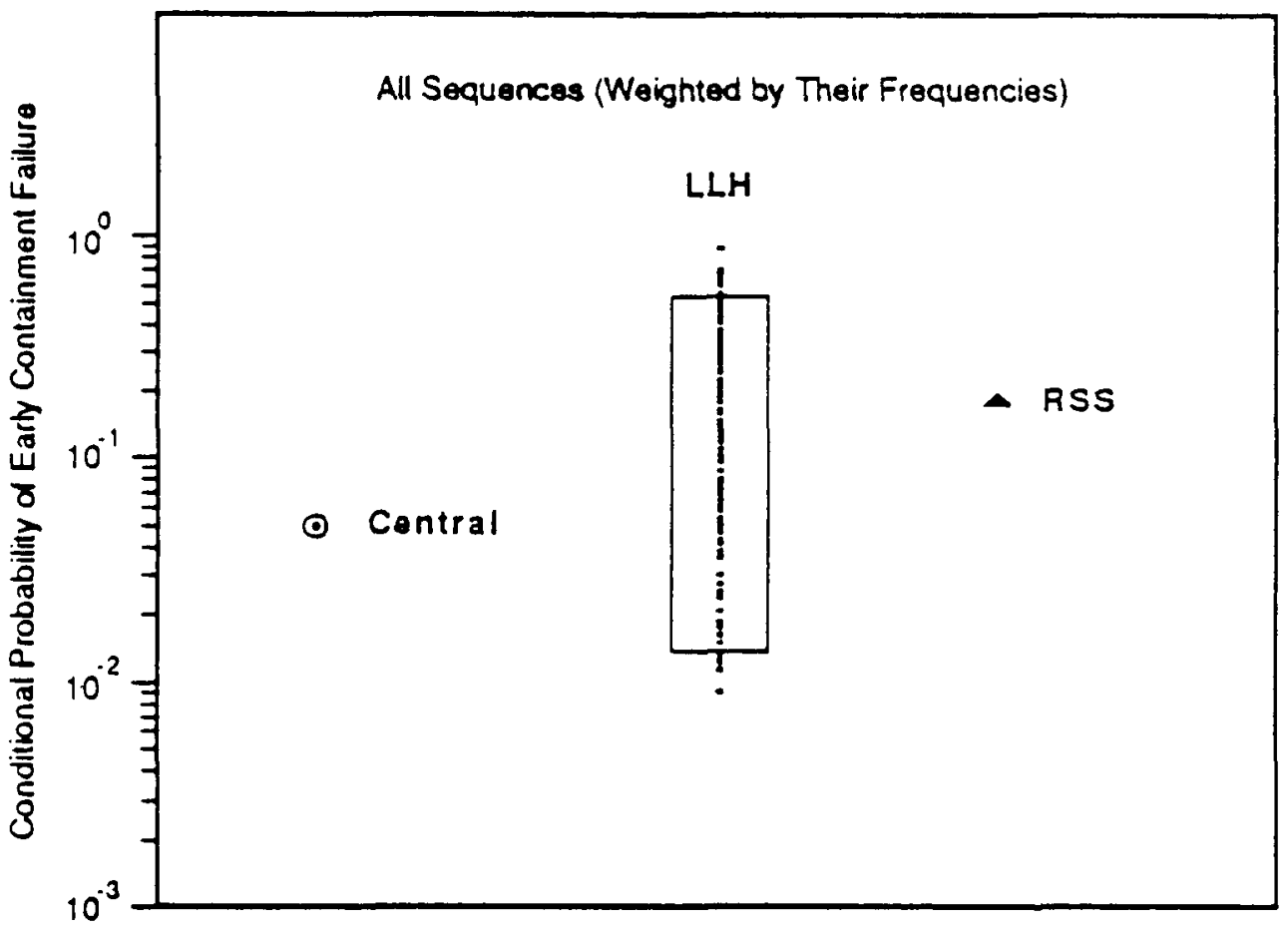

Figure 5-3. Conditional Probability of Early Containment Failure: All Sequences Included

Figure 5-4 provides the same results for an individual core-damage state (TNNN) that corresponds to a loss of all ac power with no failure of the RCS pump seals (hence the primary system is at high pressure prior to core damage)." The LLH range for early containment failure probability spans from $3 \times 10^{-3}$ to nearly unity. For this damage state, the OCP results

"Optimistic and pessimistic results of the OCP study are also illustrated. The OCP is discussed further in Section 5.5.2. 
indicated a wider spread than the LLH results, but the difference is associated with the lower bound where containment isolation failures and steam generator tube ruptures become important in the LLH study, essentially defining the lower bound conditional probability of early containment failure. These failure modes had negligible influence in the OCP optimistic estimate. Thus in the optimistic walkthrough, there was virtually no chance for early containment failure, but it was nearly certain to occur in the pessimistic walkthrough. The central estimate was $5 \times 10^{-3}$. Again, the key difference between the LLH and central estimates is the review groups' viewpoint regarding direct heating scenarios. The RSS result is also illustrated; as in the results shown for all sequences in Figure 5-3, the RSS result is again near the upper end of the $L L H$ range. The $I D C O R$ value for this type of sequence at the Zion plant is approximately $5 \times 10^{-3}$, once again dominated by isolation failures. The results for other damage states were also examined and found to produce trends similar to those depicted in Figure 5-4.

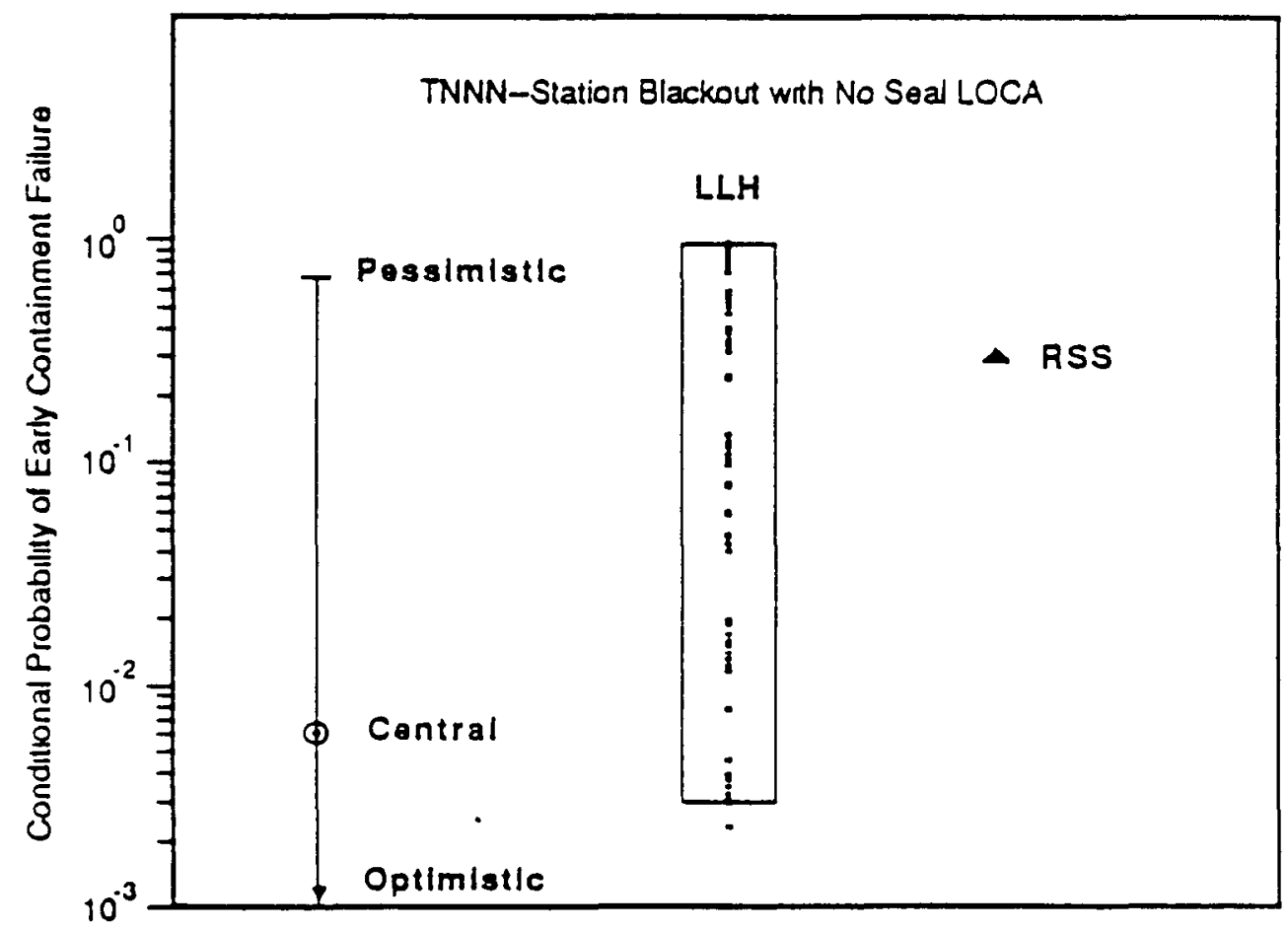

Figure 5-4. Conditional Probability of Early Containment Failure: Damage State TNNN--Station Blackout Without a LOCA

The contribution of individual issues to the overall uncertainty range was examined through a rank regression analysis of the probability of early containment failure. This was done both for all sequences and for individual damage states, and the results were very similar. 
The rank regression for all sequences is illustrated in Figure 5-5.* The sequence and containment issues were considered, and although all are plotted, only those with fractions greater than about $10 \%$ should be considered significant. Examining the relative contributions of the individual issues, the regression shows that the direct heating phenomenon is the most important uncertainty affecting the LLH range of results. A more detailed review of the individual sample member results suggests that containment failure pressure is also important; nearly all of the samples with high probability of early failure have one of the two lowest levels of containment capacity, while most samples with low conditional probabilities of containment failure have one of the two higher levels of containment pressure capacity. The rank regression figure also illustrates that containment failure size and spray failure are important issues, but this is due to the direct correlation of the input levels for these issues with each other and with those for containment failure pressure, and is therefore not a real effect. One other issue, hydrogen burn pressure, indicates marginal importance in the rank regression.

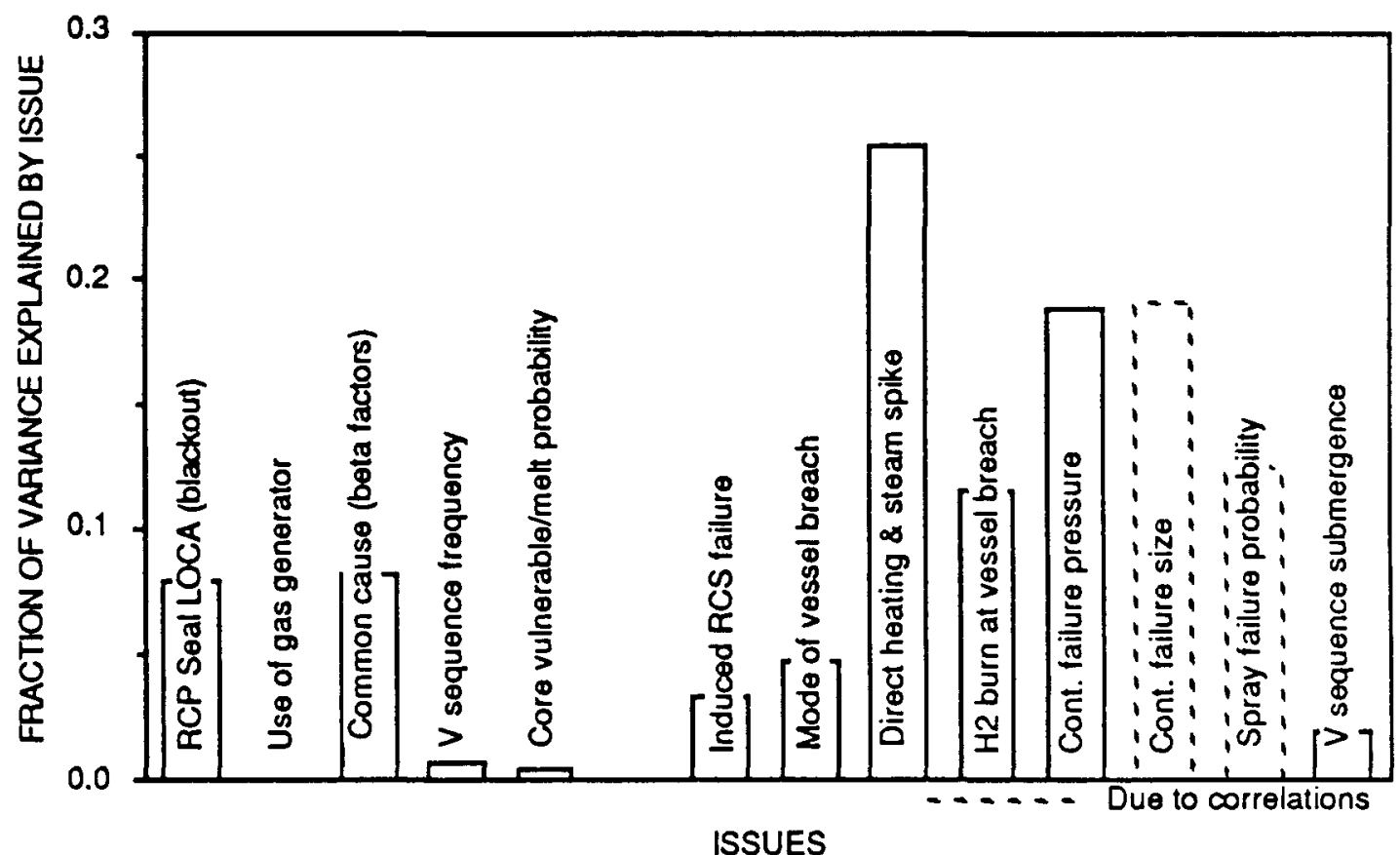

Figure 5-5. Rank Regression Analysis of Early Containment Failure for All Sequences

To further analyze the sensitivity of the results to the direct heating issue, the probability of early containment failure for all sequences was recalculated with direct heating

* The rank regression analysis calculates the fraction of the variance explained by the variation in individual issues. If issues are correlated, the fraction explained will include that due to the issue itself as well as that due to the issue with which it is correlated. The total fraction of variance explained can therefore exceed unity. 
removed completely from the analysis. As illustrated in Figure 5-6, the band of uncertainty does not change for this sensitivity, although the distribution of sample members is shifted to somewhat lower values within the range. (The mean of the sample is $3 \times 10^{-1}$ for the base case with direct heating, while for the sensitivity case without direct heating, the mean is reduced to $1 \times 10^{-1}$.) The center of the band is driven by the $V$ sequence, while the high end of the profile appears to be driven by combinations of containment failure pressure levels with issue levels for hydrogen bum and steam loadings that lead to failure of containment. These observations are corroborated by the rank regression results, which are presented in Figure 5-7. (Again, the apparent significance of containment failure size results from its correlation in the $\mathrm{LLH}$ process with containment failure pressure.) Another issue appearing as modestly important in the regression is that of RCS failure induced by high temperatures and pressures during core degradation. The significance of this issue emanates from the possibility of induced steam generator tube ruptures, which lead to containment bypass. The important point is that the removal of direct heating as an issue does not significantly affect the overall uncertainty in early containment failure due to combinations of other issues that are important. Uncertainties are discussed in more detail in Section 5.5.

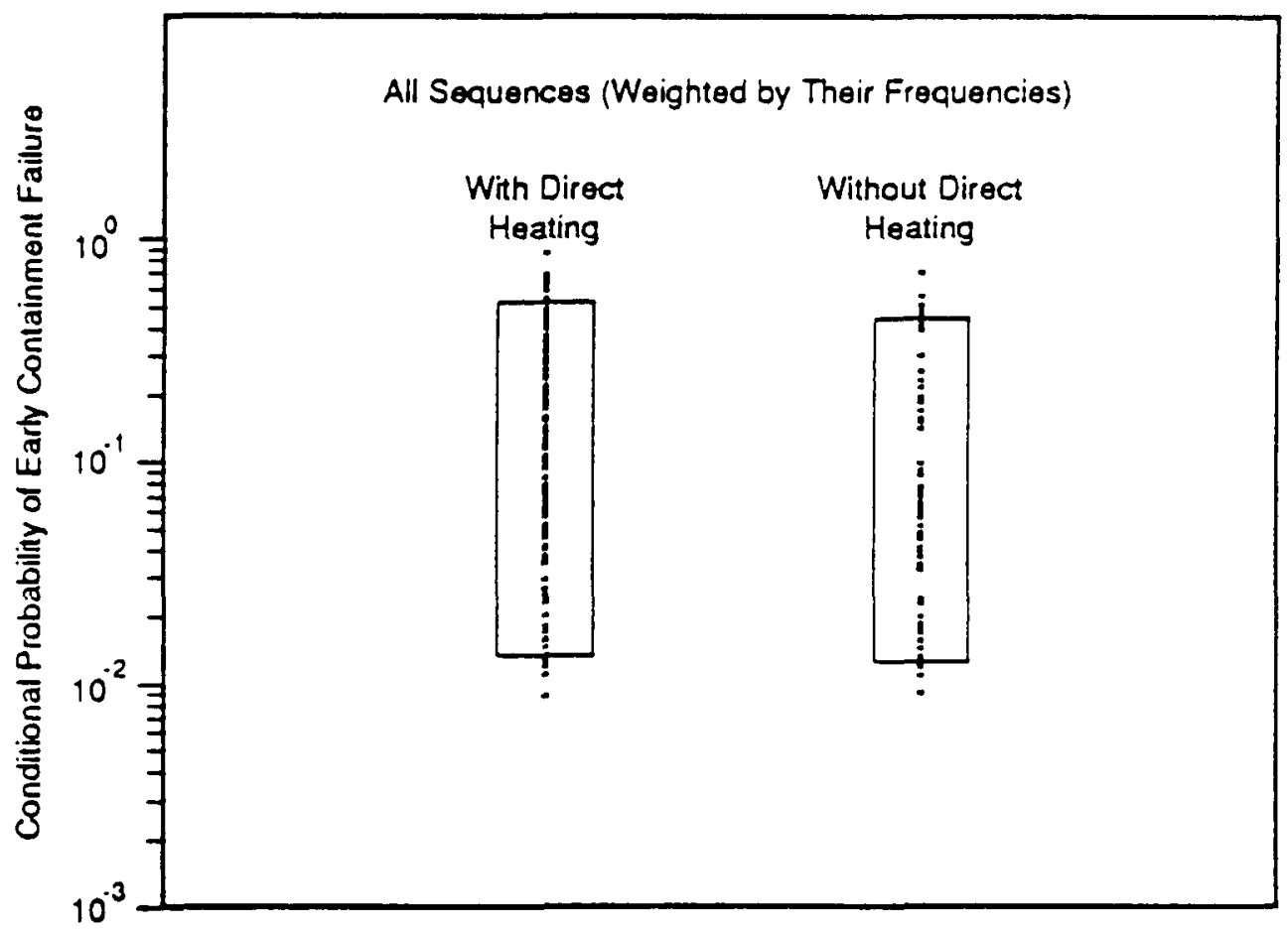

Figure 5-6. Comparison of Base Case and Sensitivity Study: Early Containment Failure Probability for All Sequences 
NUREG/CR-4551,VOL.1: DRAFT REPORT FOR COMMENT (SEPTEMBER, 1986)

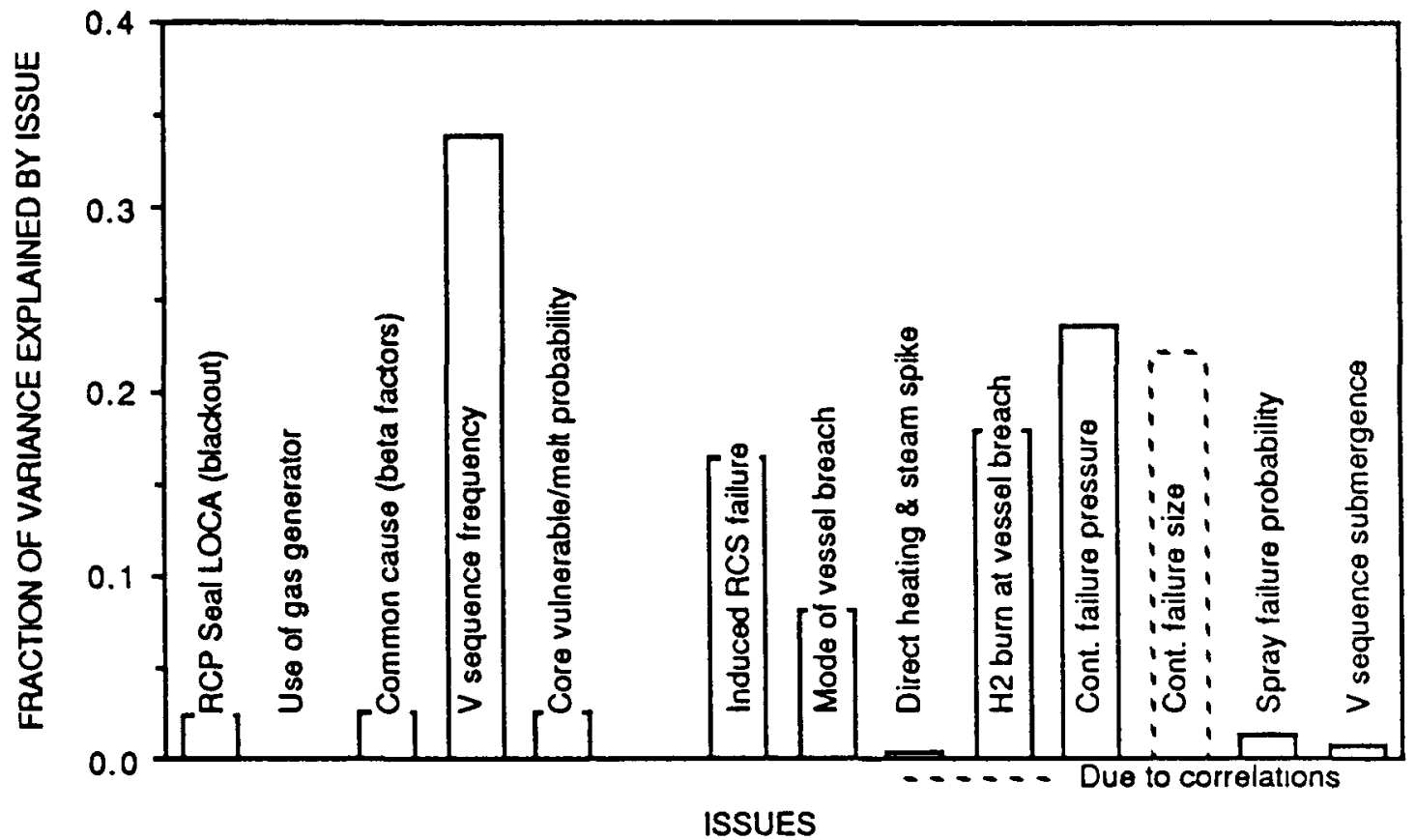

Figure 5-7. Rank Regression Analysis of Early Containment Failure for All Sequences: No Direct Heating Sensitivity Case

\subsubsection{Besults of the OCP Analysis of Containment Resnonse}

The OCP results of the containment analysis are documented in a separate report [4]. As already seen in the previous section, the range of OCP results was very large for this issue. Tables 5-5 through 5-7 delineate the results of the containment event tree analysis. Several damage states which are important to the results but which were examined outside the containment event tree are not included in the table: damage states AYNI, AYNN, SYNN, and TYNI all involve loss of containment heat removal and failure of the containment (probability of one for all three walkthroughs) prior to core damage. The $\mathrm{V}$ sequence is also not illustrated since it always involves early containment bypass, although the probability of a submerged release was varied in the walkthroughs. The probability of submergence was $1.0,0.75$, and 0.5 for the optimistic, central, and pessimistic walkthroughs, respectively.

A review of the entries in the tables shows the wide variation in the OCP results. For example, $90 \%$ of the optimistic cases involve no containment failure at all, while only $1 \%$ of the pessimistic cases involve no containment failure. The central estimate suggests a $50 \%$ chance of no containment failure. Although the OCP analysis differed substantially from the LLH analysis in that the former considered uncertainty at each of the 59 containment event tree questions, the factors driving the results are similar. In the pessimistic evaluation, as in the 
upper region of the LLH band, early containment failure can occur either from direct heating or from the combination of steam spike and hydrogen burn pressure loadings. (It is significant, however, that the LLH reviewers allowed for the possibility of hydrogen burning in situations where the containment would be considered classically steam-inerted, whereas the SARRP analysts did not consider this possibility even in the pessimistic case.) In the optimistic evaluation, as in the lower regime of the LLH distribution, direct heating, steam spikes and hydrogen burns are not sufficient to fail containment. In this case, the events that lead to containment bypass are important in the LLH, but are still considered negligible in the optimistic calculation. The SARRP central evaluation predicts a value for early containment failure lower than either the mean or median of the LLH sample distribution, implying that the review group assigned higher probabilities and/or greater severities to hydrogen burn and direct heating scenarios. 
I duble 55

LKELHOOD OF CONTAINMENT RELEASE MODES BY PLANT DAMAGE STATE (CENTRAL WALKTHROUGH)

\begin{tabular}{|c|c|c|c|c|c|c|c|c|c|}
\hline \multirow{2}{*}{\multicolumn{2}{|c|}{ Contanment Release Mode }} & \multirow{3}{*}{$\begin{array}{r}\text { AYYB } \\
\left.\begin{array}{lll}(18 & 8\end{array}\right) \\
51\end{array}$} & \multirow{3}{*}{$\begin{array}{l}\text { ANNN } \\
\left(\begin{array}{lll}3 & 2 & 8\end{array}\right)\end{array}$} & \multirow{3}{*}{$\begin{array}{l}\text { SYYB } \\
\left(\begin{array}{l}7 \\
0\end{array} 6\right) \\
51\end{array}$} & \multicolumn{3}{|c|}{ Plant Damage State (Frequency) } & \multirow[b]{2}{*}{$\begin{array}{l}\text { TYYBW } \\
\left(\begin{array}{lll}1 & 1 & 6\end{array}\right)\end{array}$} & \multirow[b]{2}{*}{$\begin{array}{l}\text { TYYBD } \\
\left(\begin{array}{lll}1 & 2 & 6\end{array}\right)\end{array}$} \\
\hline & & & & & $\begin{array}{l}\text { SYNI } \\
(438)\end{array}$ & $\begin{array}{l}\text { SNNN } \\
(576)\end{array}$ & $\begin{array}{l}\text { TNNN } \\
(196)\end{array}$ & & \\
\hline 1 & No containment fatlure & & & & 51 & 51 & 62 & 64 & 64 \\
\hline 2 & Basemat meltthrough, with containment sprays operatıng & 30 & -. & 30 & -- & 29 & 17 & 17 & 17 \\
\hline 3 & Basemal melthrough, with containment sprays inoperable & 001 & 20 & 001 & 32 & 03 & 02 & 001 & 001 \\
\hline 4. & $\begin{array}{l}\text { Long term overpressurization due to steam and non-condensible gases, } \\
\text { resulung in leakage sufficient to preclude further pressurization }\end{array}$ & 02 & 06 & 02 & 02 & 02 & 02 & 02 & 02 \\
\hline 5 & $\begin{array}{l}\text { Late overpressurization due to hydrogen bum or steam generation with } \\
\text { the sprays operatung through the period of core-concrele interactions }\end{array}$ & 05 & 005 & 05 & -- & 05 & 09 & 10 & 10 \\
\hline 6 & $\begin{array}{l}\text { Late overpressunzation due to hydrogen bum or steam generation with } \\
\text { the sprays not operaung through the penod of core-concrete interactions }\end{array}$ & 12 & 73 & 12 & 16 & 11 & 07 & 07 & 07 \\
\hline 7 & $\begin{array}{l}\text { Fallure before or at vessel breach (with the RCS at high pressure) } \\
\text { duc to hydrogen bum, steam spike at vessel breach, or in vessel } \\
\text { steam explosion Sprays operate through core concrete interactions }\end{array}$ & - & - & -- & -- & - & - & -. & - \\
\hline 8 & Same as 7 , but sprays fail to operate through core-concrete interactions & - & - & -- & -- & - & - & -- & -- \\
\hline 9 & $\begin{array}{l}\text { Fallure at or before vessel breach, as in } 7 \text {, but RCS not at high } \\
\text { pressure Sprays operate through core-concrete interactions }\end{array}$ & - & - & -- & -- & - & - & -- & -- \\
\hline 10 & $\begin{array}{l}\text { Same as } 9 \text {, but sprays fall to operate through the penod of core- } \\
\text { concrete interactions }\end{array}$ & - & - & -- & -- & - & - & -- & -- \\
\hline 11. & $\begin{array}{l}\text { Falure at vessel breach due to direct heaung, with the RCS at high } \\
\text { pressure and with the spray system avalable after vessel breach }\end{array}$ & - & - & -- & -- & - & 003 & 001 & 001 \\
\hline 12 & $\begin{array}{l}\text { Falure due to direct heating, with the RCS at high pressure but with the } \\
\text { spray system failed before or at the ume of containment fallure }\end{array}$ & - & - & - & -. & - & 001 & -- & -- \\
\hline 13 & $\begin{array}{l}\text { Failure due to direct heatung with the RCS at moderate pressure, and } \\
\text { with the sprays contunuing to operate following vessel breach }\end{array}$ & - & - & .. & -- & -- & - & -- & -- \\
\hline 14 & $\begin{array}{l}\text { Falure due to direct heating with the RCS at moderate pressure, but with } \\
\text { the sprays failed pror to or at the tume of containment fallure }\end{array}$ & - & - & - & -- & - & - & -- & -- \\
\hline 15 & $\begin{array}{l}\text { Falure due to direct heating with the RCS at moderate pressure and with the } \\
\text { containment spray system unavalable from the outsct of the accident. }\end{array}$ & - & - & -- & & - & - & -- & $\cdots$ \\
\hline 16 & $\begin{array}{l}\text { Large pre-existung leakage or fallure of containment isolation, with } \\
\text { the containment spray system operaung }\end{array}$ & 002 & - & 002 & - & 002 & 002 & 002 & 002 \\
\hline 17 & $\begin{array}{l}\text { Large pre-existung leakage or fallure of containment isolation, with } \\
\text { the spray system not operating }\end{array}$ & & 002 & - & 002 & - & - & & -- \\
\hline
\end{tabular}

Large pre-existung leakage or fallure of containment isolation, with 
Table 56

UKELJHOOD OF CONTAINMENT RELEASE MODES BY PLANT DAMAGE STATE (OPTIMISTIC WALKTHROUGH)

\begin{tabular}{|c|c|c|c|c|c|c|c|c|c|}
\hline & Containment Release Mode & $\begin{array}{l}\text { AYYB } \\
(186)\end{array}$ & $\begin{array}{l}\text { ANNN } \\
\left(\begin{array}{l}3 \\
2-8\end{array}\right)\end{array}$ & $\begin{array}{l}\text { SYYB } \\
(70-6)\end{array}$ & 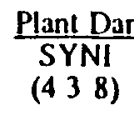 & $\begin{array}{r}\text { age Stale } \\
\text { SNNN } \\
(576)\end{array}$ & $\begin{array}{l}\text { requency) } \\
\text { TNNN } \\
\left(\begin{array}{lll}1 & 9 & 6\end{array}\right)\end{array}$ & $\begin{array}{l}\text { TYYBW } \\
\left(\begin{array}{lll}1 & 1 & 6\end{array}\right)\end{array}$ & $\begin{array}{l}\text { TYYBD } \\
(126)\end{array}$ \\
\hline 1 & No containment failure & 86 & 05 & 90 & 90 & 90 & 89 & 90 & 90 \\
\hline 2 & Basemat meluhrough, with containment sprays opcrating & 05 & - & 009 & - & 009 & 02 & 01 & 01 \\
\hline 3 & Basemat melt through, with contanment sprays inoperable & 005 & 46 & 001 & 01 & 001 & 002 & 001 & .001 \\
\hline 4 & $\begin{array}{l}\text { Long-term overpressurization due to steam and non-condensible gases, } \\
\text { resultung in leakage sufficient to preclude further pressurization }\end{array}$ & 08 & 44 & 08 & 08 & 08 & 08 & 08 & 08 \\
\hline 5. & $\begin{array}{l}\text { Late overpressunzation due to hydrogen bum or steam generation with } \\
\text { the sprays operatung through the penod of core-concrete interactions }\end{array}$ & 005 & - & 009 & -- & 009 & 008 & 009 & 009 \\
\hline 6 & $\begin{array}{l}\text { Late overpressurzation due to hydrogen burn or steam gencration with } \\
\text { the sprays not operaung through the penod of core-concrete interactions }\end{array}$ & 005 & 06 & 001 & 01 & 001 & 002 & 001 & 001 \\
\hline 7 & $\begin{array}{l}\text { Fallure before or at vessel breach (with the RCS at high pressure) } \\
\text { due to hydrogen bum, steam spike at vessel breach, or in vessel } \\
\text { steam explosion Sprays operate through core concrete interactions }\end{array}$ & - & - & -- & -- & - & - & -- & -. \\
\hline 8 & Same as 7 , but sprays fall to operate through core-concrete interactions & - & - & -- & -- & - & - & -- & -- \\
\hline 9 & $\begin{array}{l}\text { Fallure at or before vessel breach, as in } 7 \text {, but RCS not at high } \\
\text { pressure Sprays operate through core-concrete interactions }\end{array}$ & -- & - & -- & -- & - & - & -- & -- \\
\hline 10 & $\begin{array}{l}\text { Same as } 9 \text {, but sprays fall to operate through the penod of core } \\
\text { concrete interactions }\end{array}$ & - & -- &.- & -- & - & - & -- & -. \\
\hline 11 & $\begin{array}{l}\text { Fallure at vessel breach due to diroct heating, with the RCS at high } \\
\text { pressure and with the spray system avalable after vessel breach }\end{array}$ & - & - & -. & -- & -- & - & -- & -- \\
\hline 12 & $\begin{array}{l}\text { Fallure due to durect heating, with the RCS at high pressure but with the } \\
\text { spray system falled before or at the tume of containment falure }\end{array}$ & - & - & -- & -. & - & - & -- & -- \\
\hline 13 & $\begin{array}{l}\text { Fallure due to diroct heatung with the RCS at moderate pressure, and } \\
\text { with the sprays contunuing to operate following vessel breach }\end{array}$ & - & - & -- & -- & - & - & -. & -. \\
\hline 14 & $\begin{array}{l}\text { Fallure due to direct heatung with the RCS at moderate pressure, but with } \\
\text { the sprays failed proor to or at the time of containment failure }\end{array}$ & - & - & -. & -- & -- & - & - & - \\
\hline 15 & $\begin{array}{l}\text { Falure due to direct heating with the RCS at moderate pressure and with the } \\
\text { containment spray system unavallable from the outset of the accident. }\end{array}$ & - & - & & - & - & - & -- & -. \\
\hline 16 & $\begin{array}{l}\text { Large pre-existung leakage or fallure of containment isolation, with } \\
\text { the containment spray system operating }\end{array}$ & - & - & -- & & - & - & - & -- \\
\hline 17 & $\begin{array}{l}\text { Large pre-existing leakage or fallure of contuinment isolation, with } \\
\text { the spray system not operaling }\end{array}$ & & - & & - & - & - & -- & -- \\
\hline
\end{tabular}


Table 57

LIKELHOOD OF CONTAINMENT RELEASE MODES BY PLANT DAMAGE STATE (PESSIMISTIC WALKTHROUGH)

Contanment Release Mode

\section{No containment faslure}

Basemat meltthrough, with containment sprays operating

Basemat melthrough, with contanment sprays inoperable

4 Long-term overpressurization due to steam and non condensible gases, resulung in leakage sufficient to preclude further pressurization

5 Late overpressurization due to hydrogen burn or stcam gencration with the sprays operating through the period of core-concrele interactions

6 Late overpressunzation due to hydrogen burn or steam generation with the sprays not operaung through the penod of core-concrele interactions

7 Fallure before or at vessel breach (with the RCS at high pressure) due to hydrogen bum, steam spike at vessel breach, or in vesscl steam explosion Sprays operate through core concrete interactions

8 Same as 7 , but sprays fall to operate through core-concrete interactions

9 Fallure at or before vessel breach, as in 7 , but RCS not at high pressure Sprays operate through core-concrete interactions

10. Same as 9., but sprays fall to operate through the penod of coreconcrete interactions

11 Falure at vessel breach due to direct healing, with the RCS at high pressure and with the spray system avalable after vessel breach

12 Fallure due to direct healing, with the RCS at high pressure but with the spray system fatled before or at the tume of containment falure

13 Faslure due to direct heating with the RCS at moderate pressure, and with the sprays conunuing to operate following vessel breach

14 Failure due to durect heatung with the RCS at moderate pressure, but with the sprays failed proor to or at the tume of containment failure

15 Falure due to diroct heatung with the RCS at moderate pressure and with the contanment spray system unavaldable from the outset of the accident

16 Large pre-exisung leakage or failure of containment isolation, with the contasnment spray system operating

17 Large pre-exisung leakage or fallure of containment isolation, with the sprdy systcm not opcrating

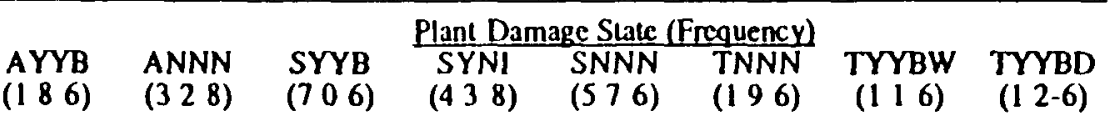

\begin{tabular}{|c|c|c|c|c|c|c|c|}
\hline 02 & - & 007 & 01 & 01 & 01 & 006 & 006 \\
\hline 04 & - & 007 & -. & - & - & 003 & 003 \\
\hline- & 003 & -. & 002 & 002 & 001 & -- & -- \\
\hline- & - & -- & -- & - & - & -- & -- \\
\hline 06 & - & 04 & - & 27 & 19 & 04 & 04 \\
\hline 16 & - & 06 & 75 & 48 & 25 & 03 & 03 \\
\hline- & - & - & -. & - & 004 & 16 & .16 \\
\hline -. & - & - & -- & - & 11 & 25 & 25 \\
\hline 31 & 90 & $\cdots$ & -- & - & - & -- & - \\
\hline 41 & 10 & - & -- & - & - & -. & -- \\
\hline- & - & $\cdots$ & -- & - & 05 & 21 & .21 \\
\hline- & - & - & - & - & 35 & .21 & 21 \\
\hline- & - & 39 & -- & 004 & - & 04 & 04 \\
\hline- & - & 49 & 24 & - & 03 & .05 & 05 \\
\hline- & - & -- & -- & 24 & - & - & -- \\
\hline 001 & - & 001 & -- & 001 & 001 & -. & -- \\
\hline 001 & 002 & 001 & 002 & 001 & - & -. & -- \\
\hline
\end{tabular}




\subsection{RESULTS OF THE RADIOLOGICAL SOURCE TERM ANALYSIS}

Similar to the containment analysis results presented in the previous section, some of the source term results are discussed in this section to provide additional insight into the overall risk results. Sections 5.3.1 and 5.3.2 describe aspects of the LLH and OCP analyses respectively. Later, in Section 5.5, the contributions of both individual source term bins and LLH uncertainty issues to the risk measures are described.

\subsubsection{LLH_Source Term_Results}

Due to the interdependencies and level of detail, it is difficult to derive partial results for the LLH source term analysis. Most of the insights relative to the source term analysis are best derived from an examination of the overall risk results as described in Section 5.5. Details concerning the source term study are provided in Appendix C.

Figures 5-8 through 5-13 show the range of release fractions for release groups 2 through 7 , plotted by release bin for each sample member considered in the LLH evaluation. These release fractions are calculated by the SURSOR code as described in Appendix C. The central estimates for each source term are also plotted. The large variation in estimated source terms among and within bins and the overlap of the source term ranges for different categories led to the development of the clusters.

The use of clusters, as described in Section 2.5, reduced the number of separate consequence analyses required to characterize the risk from approximately 1900 (19 for each LLH outcome) to 25 . One type of source term result is the actual magnitude of the clusters and the frequency with which each cluster is used. Table 5-8 delineates the source terms for each cluster. These provide some perspective for the reader familiar with typical source term magnitudes. ${ }^{*}$ The second column in the table includes the mean frequency of the cluster usage (i.e., the conditional probability of each of the clusters given the occurrence of a core-melt accident). As illustrated, the two lowest source term clusters are used most frequently (71\% of the time), although the risk is mostly due to the high magnitude clusters that are associated with early containment failure. Most of the other clusters are used 1 to $2 \%$ of the time.

\footnotetext{
${ }^{*}$ The central estimates of source terms for each source term bin are presented in the next section. These central estimates are also useful to the understanding of the LLH analysis since they provided some of the basis for the LLH source terms.
} 
NUREG/CR-4551,VOL.1: DRAFT REPORT FOR COMMENT (SEPTEMBER, 1986)

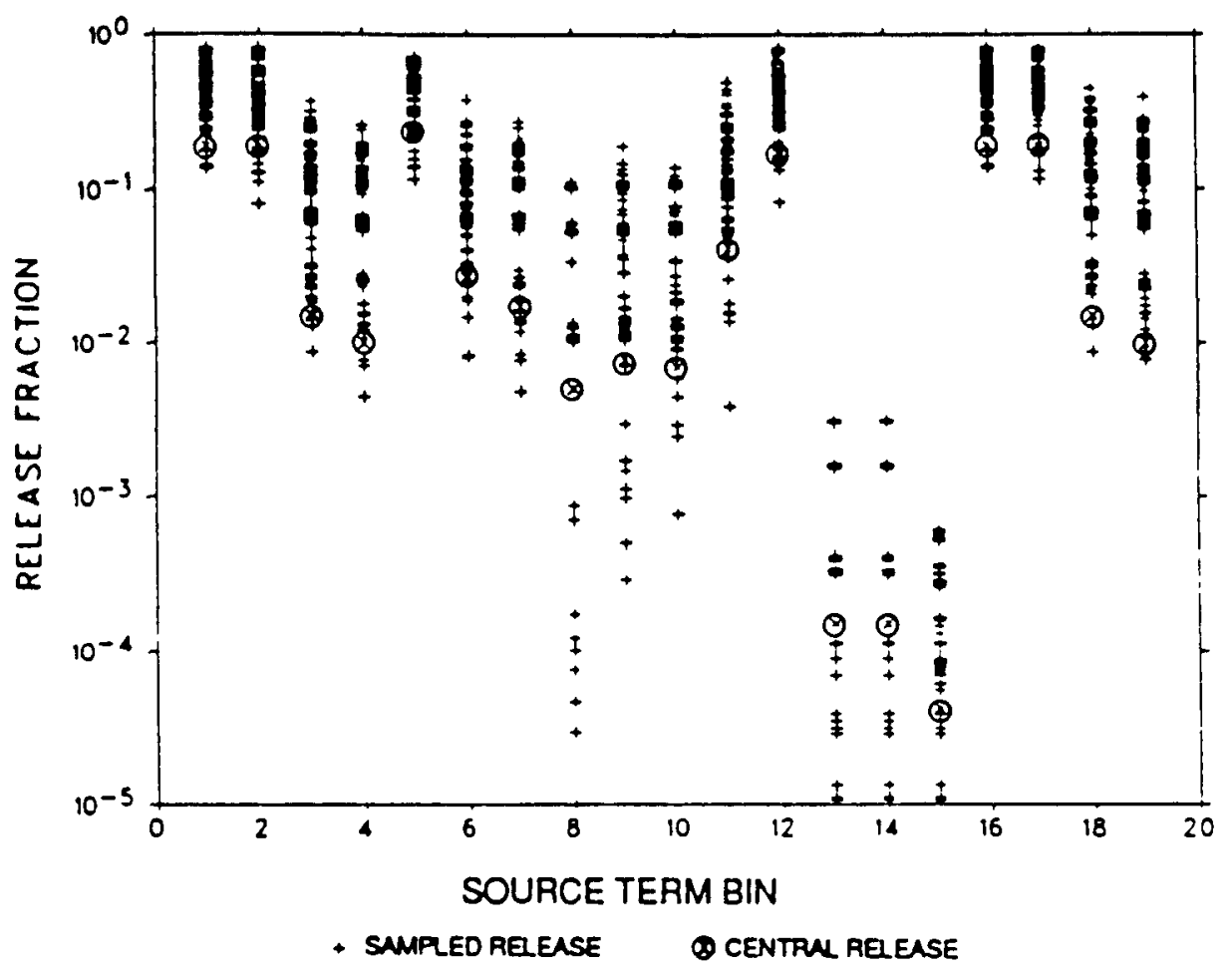

Figure 5-8. Sampled Surry Source Term Estimates from SURSOR for Group 2 (I, Br)

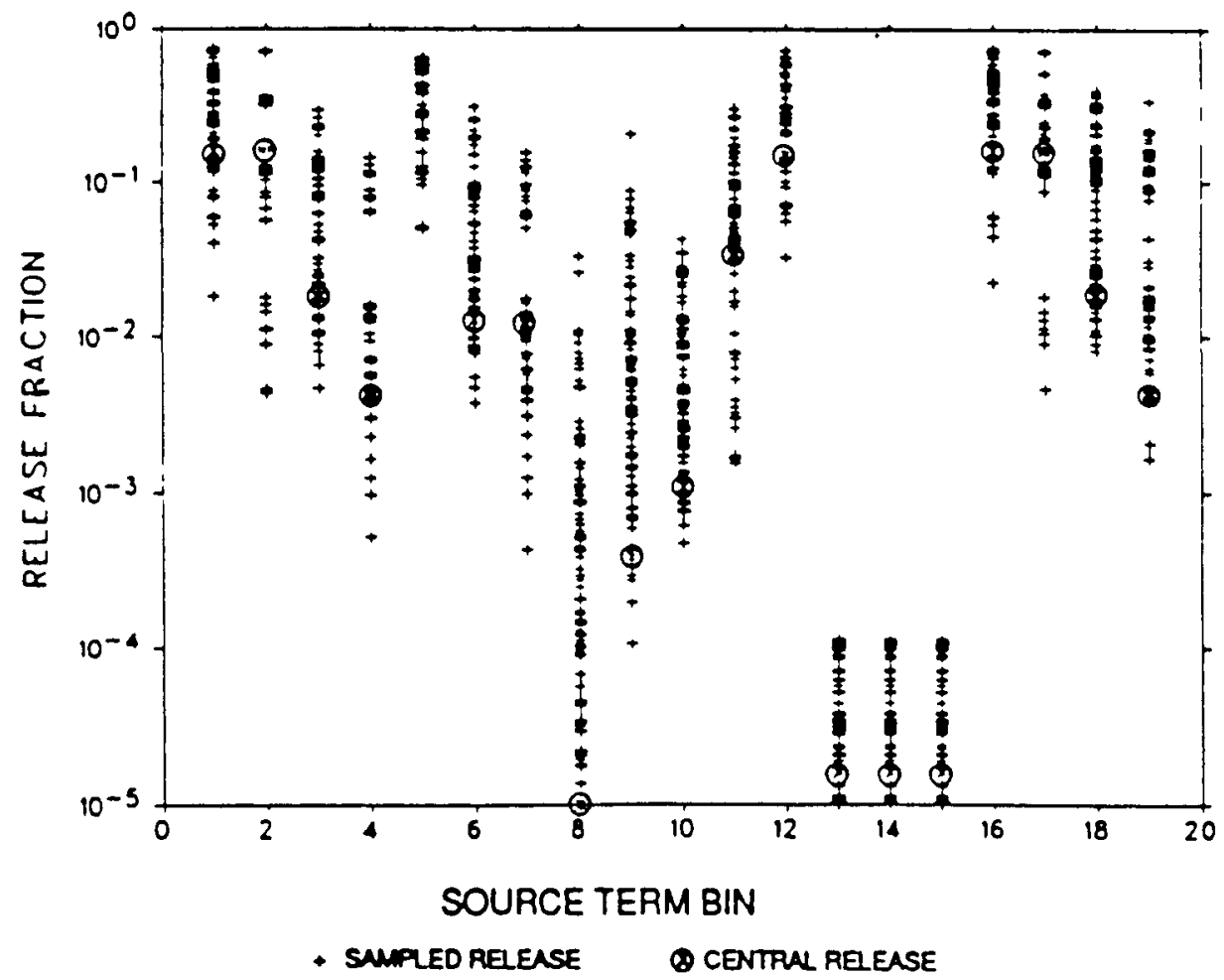

Figure 5-9. Sampled Surry Source Term Estimates from SURSOR for Group 3 (Cs, Rb) 
NUREG/CR-4551, VOL1: DRAFT REPORT FOR COMMENT (SEPTEMBER, 1986)

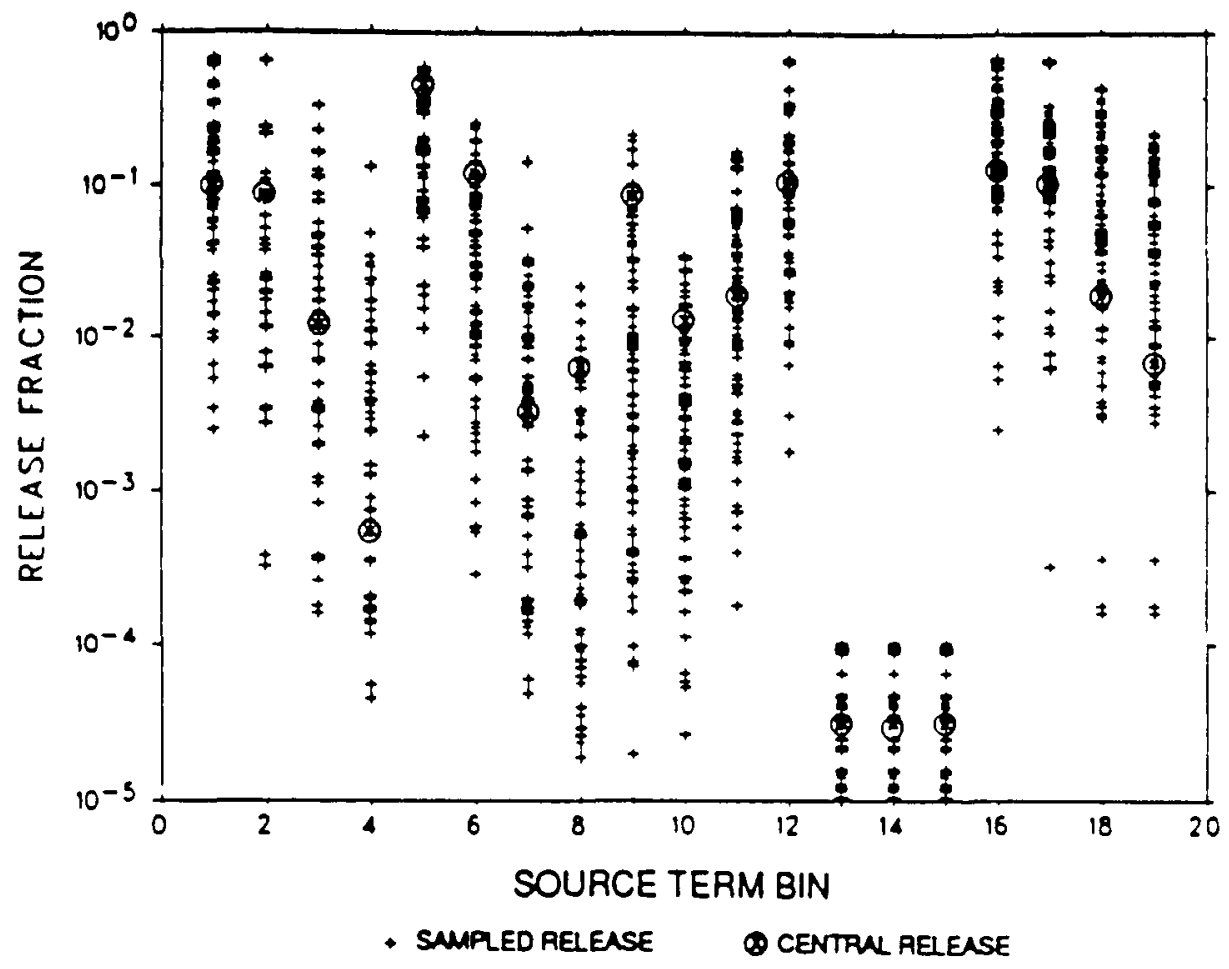

Figure 5-10. Sampled Surry Source Term Estimates from SURSOR for Group 4 (Te, Se, Sb)

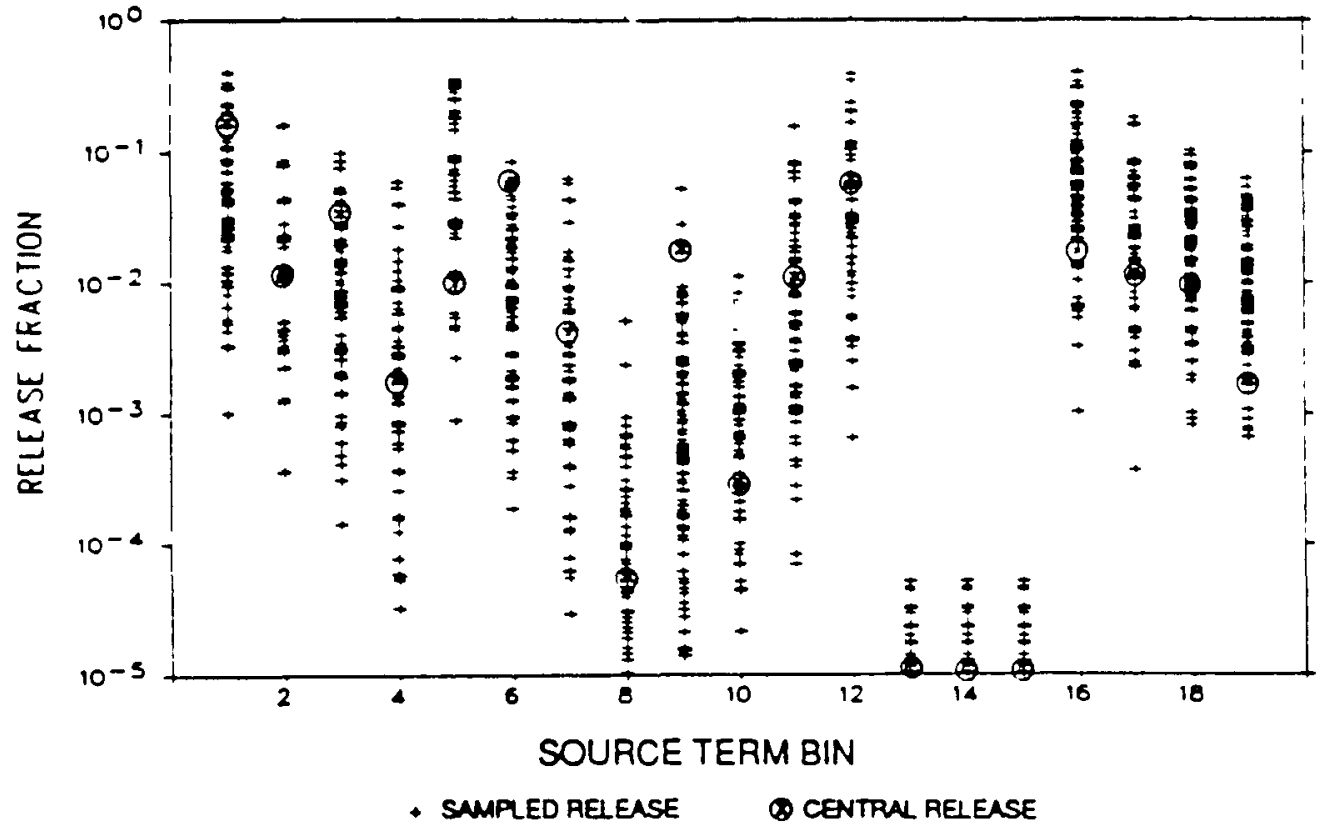

Figure 5-11. Sampled Surry Source Term Estimates from SURSOR for Group 5 ( $\mathrm{Sr}, \mathrm{Ba}$ ) 
NUREG/CR-4551,VOL.1: DRAFT REPORT FOR COMMENT (SEPTEMBER, 1986)

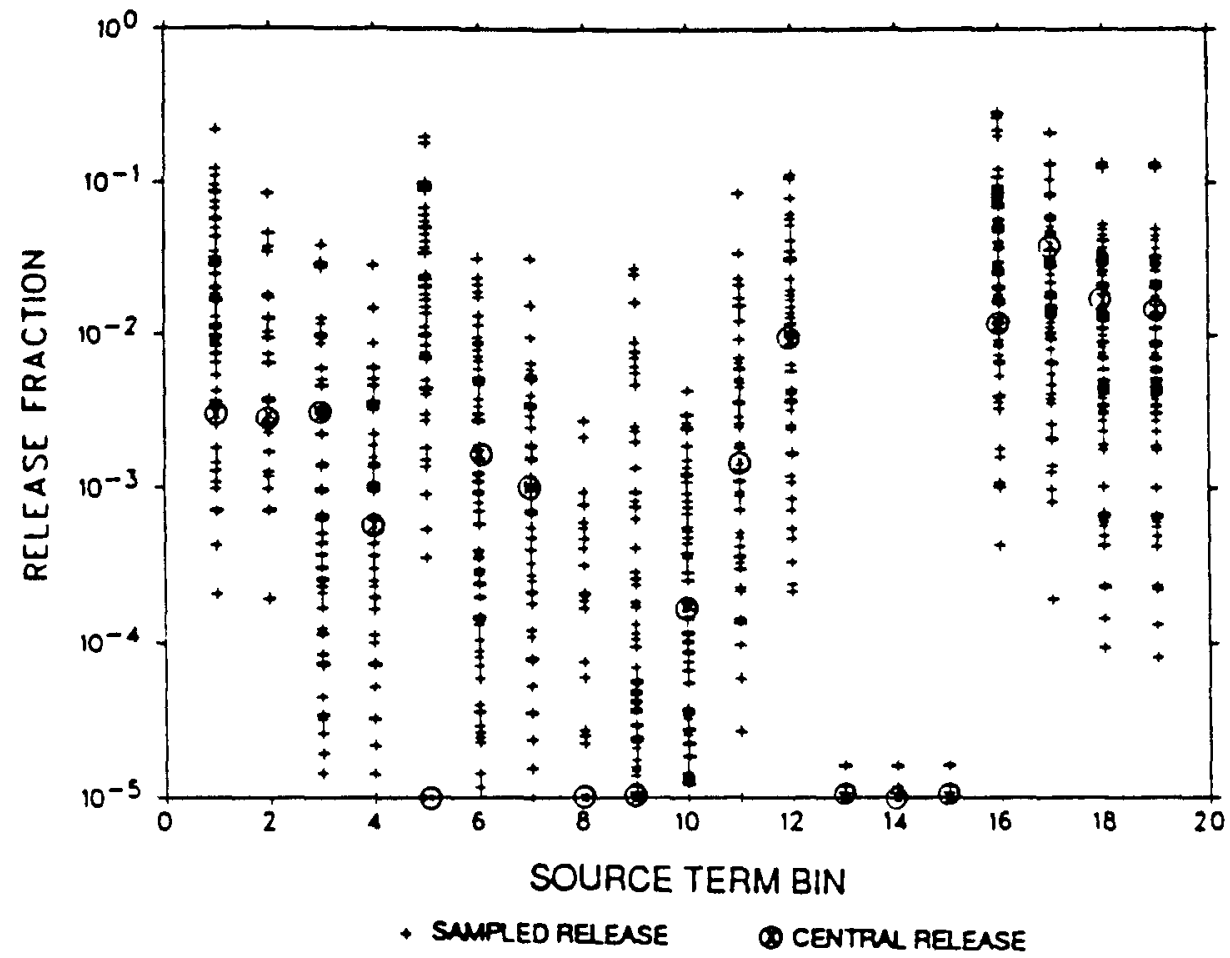

Figure 5-12. Sampled Source Term Estimates from SURSOR for Group 6 (Ru, Mo, etc.)

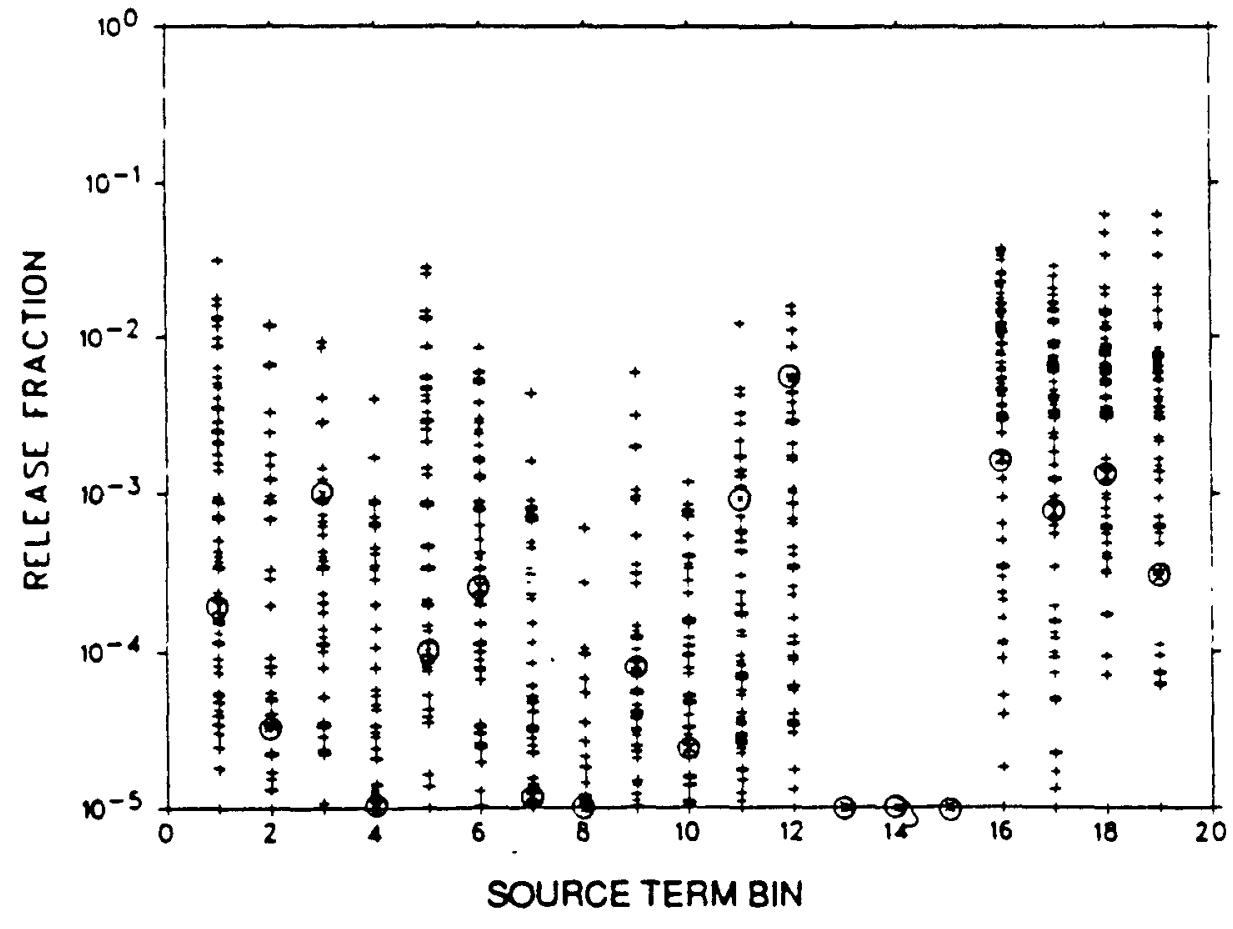

- suarled release o central release

Figure 5-13. Sampled Source Term Estimates from SURSOR for Group 7 (La, Ce, etc.) 
NUREG/CR-4551,VOL.1: DRAFT REPORT FOR COMMENT (SEPTEMBER, 1986)

Table 5-8

RELEASE FRACTIONS FOR SURRY LLH CLUSTERS

\begin{tabular}{|c|c|c|c|c|c|c|c|c|c|c|c|c|}
\hline \multirow{2}{*}{$\begin{array}{l}\text { Cond }^{2} \\
\text { Prob. } \\
\text { Clus. }\end{array}$} & \multirow{2}{*}{$\begin{array}{r}\mathrm{Rel}^{3} \\
\text { Time } \\
\text { (hr) }\end{array}$} & \multicolumn{3}{|c|}{ Warn ${ }^{3}$} & \multicolumn{8}{|c|}{ Release Fraction By Group ${ }^{1}$} \\
\hline & & $\begin{array}{l}\text { Dur } \\
\text { (hr) }\end{array}$ & $\begin{array}{l}\text { Per. } \\
\text { (hr) }\end{array}$ & $\begin{array}{l}\text { Elev } \\
(\mathrm{m})\end{array}$ & $\begin{array}{l}\text { Heat } \\
(\mathrm{cal} / \mathrm{s})\end{array}$ & $\begin{array}{c}1 \\
\mathrm{Kr}-\mathrm{Xe}\end{array}$ & $\begin{array}{l}2 \\
\mathrm{I}\end{array}$ & $\begin{array}{c}3 \\
\text { Cs-Rb }\end{array}$ & $\begin{array}{c}4 \\
\mathrm{Te}\end{array}$ & $\begin{array}{c}5 \\
\text { Sr-Ba }\end{array}$ & $\begin{array}{c}6 \\
\mathrm{Ru}\end{array}$ & $\begin{array}{c}7 \\
\mathrm{La}\end{array}$ \\
\hline $1 \quad .02$ & 2.5 & 0.2 & 0.5 & 9 & $5.4+6^{4}$ & 1.0 & $6.9-1$ & $6.2-1$ & $5.6-1$ & $3.2-1$ & $1.4-1$ & $2.0-2$ \\
\hline $2 \quad .02$ & 2.9 & 0.2 & 0.6 & 10 & $5.3+6$ & 1.0 & $7.0-1$ & $6.4-1$ & $5.3-1$ & 2.3-1 & $8.1-2$ & $9.3-3$ \\
\hline $3 \quad .02$ & 2.6 & 0.2 & 0.6 & 9 & $5.2+6$ & 1.0 & $6.9-1$ & $6.1-1$ & $3.1-1$ & $1.0-1$ & $4.2-2$ & $5.3-3$ \\
\hline $4 \quad .03$ & 2.7 & 0.2 & 0.6 & 9 & $4.5+6$ & 1.0 & $5.3-1$ & $4.3-1$ & $2.6-1$ & $1.1-1$ & $5.0-2$ & $8.2-3$ \\
\hline $5 \quad .02$ & 2.5 & 0.3 & 0.6 & 8 & $3.3+6$ & 1.0 & $4.9-1$ & $3.6-1$ & $2.1-1$ & $6.2-2$ & $2.9-2$ & $7.2-3$ \\
\hline $\begin{array}{ll}6 & .02\end{array}$ & 2.4 & 0.3 & 0.6 & 9 & $3.9+6$ & 1.0 & $5.1-1$ & $3.0-1$ & $1.5-1$ & $4.8-2$ & $2.2-2$ & $4.0-3$ \\
\hline $7 \quad .02$ & 2.2 & 0.3 & 0.6 & 8 & $3.6+6$ & 1.0 & $3.8-1$ & $2.5-1$ & $1.2-1$ & $3.8-2$ & $2.2-2$ & $3.8-3$ \\
\hline $\begin{array}{ll}8 & .02\end{array}$ & 2.3 & 0.4 & 0.7 & 6 & $1.7+6$ & 1.0 & $2.9-1$ & $2.1-1$ & $1.0-1$ & $3.1-2$ & $1.1-2$ & $2.0-3$ \\
\hline $9<.01$ & 12 & 1.0 & 10 & 10 & $7.8+4$ & 1.0 & $9.7-2$ & $7.8-2$ & $1.7-1$ & $2.7-2$ & $1.7-2$ & $2.8-3$ \\
\hline $10<.01$ & 2.2 & 0.2 & 0.5 & 9 & $4.7+6$ & 1.0 & $4.9-1$ & $1.2-1$ & $8.6-2$ & $3.1-2$ & $1.1-2$ & $2.0-3$ \\
\hline 11.02 & 1.9 & 0.5 & 0.7 & 5 & $1.3+6$ & 1.0 & $2.4-1$ & $1.5-1$ & $6.9-2$ & $2.4-2$ & $1.1-2$ & $2.8-3$ \\
\hline $12<.01$ & 2.2 & 0.2 & 0.5 & 9 & $5.4+6$ & 1.0 & $4.4-1$ & $3.4-2$ & $2.4-2$ & $1.0-2$ & $4.2-3$ & $1.2-3$ \\
\hline 13.02 & 1.6 & 1.0 & 0.7 & 3 & $5.5+5$ & 1.0 & $1.7-1$ & $1.2-1$ & $5.1-2$ & $2.0-2$ & $7.3-3$ & $1.6-3$ \\
\hline $14<.01$ & 10.2 & 2.7 & 8.2 & 10 & $3.3+4$ & 1.0 & $9.7-2$ & $4.0-2$ & $5.0-2$ & $6.3-3$ & $2.3-3$ & $4.5-4$ \\
\hline $15 \quad .01$ & 2.0 & 0.8 & 0.7 & 4 & $6.4+5$ & 1.0 & $1.5-1$ & $8.1-2$ & $3.6-2$ & $1.3-2$ & $5.5-3$ & $1.4-3$ \\
\hline 16.01 & 7.2 & 5.4 & 5.2 & 10 & $2.0+4$ & 1.0 & $8.7-2$ & $9.1-3$ & $1.2-2$ & $1.8-3$ & $6.6-4$ & $1.3-4$ \\
\hline $17 \quad .01$ & 1.5 & 0.6 & 0.7 & 4 & $6.9+5$ & 1.0 & $1.5-1$ & $4.7-2$ & $2.9-2$ & $7.8-3$ & $3.6-3$ & $1.2-3$ \\
\hline $18 \quad .01$ & 7.3 & 5.1 & 5.3 & 10 & $2.0+4$ & 1.0 & $5.1-2$ & $3.6-3$ & $3.6-3$ & $6.2-4$ & $1.3-4$ & $2.5-5$ \\
\hline 19.01 & 1.4 & 0.9 & 0.8 & 2 & $2.7+5$ & 1.0 & $8.5-2$ & $3.0-2$ & $2.0-2$ & $6.4-3$ & $2.6-3$ & $8.9-4$ \\
\hline $20<.01$ & 6.5 & 6.1 & 4.5 & 4.5 & $1.8+4$ & 1.0 & $1.4-2$ & $8.2-3$ & $6.8-3$ & $1.4-3$ & $4.0-4$ & $6.5-5$ \\
\hline $21 \quad .01$ & 1.4 & 1.5 & 0.7 & 3 & $2.2+5$ & 1.0 & $7.4-2$ & $1.4-2$ & $7.7-3$ & $3.0-3$ & $8.0-4$ & $1.8-4$ \\
\hline $22<.01$ & 11.4 & 3.7 & 9.4 & 8 & $2.1+4$ & 1.0 & $3.6-3$ & $4.6-4$ & $6.1-4$ & $8.2-5$ & $3.4-5$ & $5.8-6$ \\
\hline 23.01 & 1.2 & 1.7 & 0.7 & 2 & $6.5+4$ & 1.0 & $3.2-2$ & $7.2-3$ & $3.0-3$ & $1.3-3$ & $3.4-4$ & $6.4-5$ \\
\hline $24 \quad .27$ & 24 & 1 & 22 & 6 & 0.0 & $1.8-2$ & $8.3-4$ & $4.4-5$ & $2.8-5$ & $1.0-5$ & $2.8-6$ & $4.4-7$ \\
\hline 25.45 & 24 & 1.0 & 22 & 0 & 0.0 & $2.5-3$ & $4.2-5$ & $1.6-5$ & $3.1-5$ & $1.2-5$ & $3.7-7$ & $6.5-8$ \\
\hline
\end{tabular}

${ }_{1}$ A 25 th cluster was added to more properly characterize the lower range of the sample results. This cluster was defined by inspection.

${ }^{2}$ The mean conditional probability of the cluster given a core damage accident.

${ }^{3}$ Release time is the time between reactor shutdown and release, duration is the time over which the release takes place, and warning period is the time between notification and the release.

$4_{5.4+6}=5.4 \times 10^{6}$. 


\subsubsection{QCP Source Term Results}

The bin definitions in Section 2.5 described the characteristics of the source term bins as well as the technique used to derive the release fractions for the central estimate. The results of the calculations for the central estimate in terms of the fraction of initial core inventory that is released are presented in Table 5-9. The central estimates were used in the OCP analysis and were also the general basis for the LLH analysis, although specific LLH issues were then considered that altered most of the source terms, based on the expert review group input. Table 5-9 also lists the relative frequency of use for each source term bin (i.e., the conditional probability of each of the source term bins given a core damage accident). The results are presented in the second column; the lowest magnitude bins, 14 and 15, have the highest conditional probabilities.

The OCP methodology for uncertainty representation required the development of optimistic and pessimistic source terms. The actual source terms obatined and the information used in their development are discussed in Appendix $C$. The ranges of source terms for several bins are discussed in this section, along with a comparison to those used in the Reactor Safety Study.

Figure 5-14 illustrates the ranges of release fractions for some of the source term bins. The central estimates for each release group are represented by dots (the actual values for the central estimate were presented in Table 5-9). The solid bar represents the range of release fractions (i. e., each end of the line corresponds to the optimistic or pessimistic release fraction for that release group and bin). As illustrated, the range of uncertainty in the OCP analysis is two orders of magnitude for most release fractions. As already outlined in Sections 2.5 and 4.3 , there are areas of significant uncertainty in many areas of source term science. When these issues are considered in the $\mathrm{OCP}$ context, the results calculated assuming optimistic assumptions are quite different from those obtained considering pessimistic assumptions, supporting the general conclusion that the source term uncertainties are very significant. No one uncertainty issue drives these results, and each release fraction for each group may have a unique set of driving factors; these are discussed in Appendix C.

Since one of the goals of SARRP was to update the previous estimates of risk, the source term results for this assessment are also compared to the RSS results in Figure 5-14. The RSS bin release fractions are plotted with the analogous SARRP bins. Not all comparisons are direct; for example, the first comparison is for a direct heating SARRP bin versus a steam explosion bin for RSS. It was determined that the increased releases associated with these processes would provide the best comparison. The second graph illustrates the 
NUREG/CR-4551,VOL.1: DRAFT REPORT FOR COMMENT (SEPTEMBER, 1986)

Table 5-9

CENTRAL ESTIMATE RELEASE FRACTIONS FOR SURRY

\begin{tabular}{|c|c|c|c|c|c|c|c|c|c|c|c|c|c|}
\hline Bin & $\begin{array}{l}\text { Cond }{ }^{3} \\
\text { Prob. }\end{array}$ & $\begin{array}{c}\text { Rel }^{4} \\
\text { Time } \\
\text { (hr) }\end{array}$ & $\begin{array}{l}\text { Dur } \\
\text { (hr) }\end{array}$ & $\begin{array}{l}\text { Warn } \\
\text { Per. } \\
\text { (hr) }\end{array}$ & $\begin{array}{l}\text { Elev } \\
(\mathrm{m})\end{array}$ & $\begin{array}{l}\text { Energy } \\
(\mathrm{cal} / \mathrm{s})\end{array}$ & $\begin{array}{c}1 \\
\mathrm{Kr}-\mathrm{Xe}\end{array}$ & $2^{2}$ & $\begin{array}{c}3 \\
C s-R b\end{array}$ & $\begin{array}{c}\text { ion } B y \\
4 \\
T e\end{array}$ & $\frac{5}{5}$ & $\begin{array}{c}6 \\
\mathrm{Ru}\end{array}$ & $\begin{array}{c}7 \\
\mathrm{La}\end{array}$ \\
\hline 1 & 0.0 & 2.5 & 10 & 0.5 & 10 & $1.4+5^{5}$ & 1.0 & $1.9-1$ & $1.6-1$ & $1.0-1$ & $1.7-2$ & $3.0-3$ & $1.9-4$ \\
\hline 2 & 0.0 & 1.5 & 1.0 & 0.5 & 10 & $1.4+5$ & 1.0 & $1.9-1$ & $1.6-1$ & $8.7-2$ & $1.2-2$ & $3.0-3$ & 3.4 . \\
\hline 3 & 0.0 & 2.5 & 3.0 & 0.5 & 10 & $7.7+5$ & 1.0 & $1.5-2$ & $1.8-2$ & $1.2-2$ & $3.4-2$ & $3.0-3$ & 9.9 \\
\hline 4 & $<.01$ & 2.5 & 1.0 & 0.5 & 10 & $4.8+4$ & 1.0 & $1.0-2$ & 4.4-3 & $5.4-4$ & $1.8-3$ & 584 & $3.9-$ \\
\hline 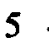 & $<.01$ & 12 & 10 & 2.0 & 10 & $1.4+5$ & 1.0 & $5.8-1$ & $5.7-1$ & $4.7-1$ & $1.0-2$ & $8.0-7$ & $1.0-$ \\
\hline 6 & $<.01$ & 2.0 & 10 & 0.0 & 10 & $1.4+4$ & 1.0 & $2.7-2$ & $1.3-2$ & $1.2-1$ & $6.2-2$ & $1.6-3$ & 2.6 \\
\hline 7 & $<.01$ & 1.0 & 10 & 0.0 & 10 & 0 & 1.0 & $1.7-2$ & $1.0-2$ & $2.2-3$ & $4.2-3$ & $1.0-3$ & 1.2 \\
\hline 0 & .04 & 8.0 & 1.0 & 6.0 & 10 & $4.8+4$ & 1.0 & $5.0-3$ & $2.5-9$ & $6.4-3$ & $5.5-5$ & $2.0-7$ & $1.2-$ \\
\hline 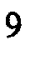 & .1 & 1 & 1.0 & 10 & 10 & $7.8+4$ & 1.0 & $7.8-3$ & 3.9 & 85 & 1.8 & 3 & $8.1-5$ \\
\hline 10 & .02 & 2.5 & 10 & 0.5 & 10 & $1.4+4$ & 1.0 & $6.9-3$ & $1.1-3$ & $1.3-2$ & $5.8-3$ & $1.7-4$ & 2.4 \\
\hline 11 & .03 & 1.0 & 2.0 & 0.8 & 0.0 & $2.4+5$ & 1.0 & $4.1-2$ & 3.5 & $2.0-2$ & 1.1 & 1. & 9.5 \\
\hline 12 & .01 & 1.0 & 2.0 & 0.8 & 0.0 & $2.4+5$ & 1.0 & $1.7-1$ & $1.5-1$ & $1.1-1$ & $6.2-2$ & $9.0-3$ & 5.5 \\
\hline 13 & .01 & 24 & 1.0 & 22 & 0.0 & 0 & 1.0 & $1.5-4$ & $1.6-5$ & $3.1-5$ & $1.2-5$ & $3.7-7$ & 6.5 \\
\hline 14 & .26 & 24 & 1.0 & 22 & 0.0 & 0 & $3.0-2$ & $1.5-4$ & $1.6-5$ & $3.1-5$ & $1.2-5$ & 277 & 6.5 \\
\hline 15 & .51 & 24 & 1.0 & 22 & 10 & 0 & $2.5-3$ & $4.2-5$ & $1.6-5$ & $3.1-5$ & $1.2-5$ & $3.7-7$ & 6.5 \\
\hline & $<.01$ & 1.5 & 10 & 0.5 & 10 & $1.4+5$ & 1.0 & $1.9-1$ & $1.6-1$ & $1 . J-1$ & $1.7-2$ & $1.5-2$ & 1.7 \\
\hline & $<.01$ & 1.5 & 1.0 & 0.5 & 10 & $1.4+5$ & 1.0 & $1.9-1$ & $1,6-1$ & $1.0-1$ & $1.2-2$ & $3.9-2$ & 7.8 \\
\hline 18 & 0.0 & 2.5 & 3.0 & 0.5 & 10 & $7.7+5$ & 1.0 & $1.5-2$ & $1.8-2$ & $1.9-2$ & $3.4-2$ & $1.7-2$ & 1.3 \\
\hline 19 & 0.0 & 2.5 & 1.0 & 0.5 & 10 & $4.8+4$ & 1.0 & $1.0-2$ & $4.4-3$ & $7.1-3$ & $1.8-3$ & $1.5-2$ & 3.0 \\
\hline 20 & 0.0 & 1.5 & 10 & 0.5 & 10 & $1.4+5$ & 1.0 & $1.9-1$ & $1.6-1$ & $1.1-1$ & $1.7-2$ & $3.9-2$ & 9.4 \\
\hline
\end{tabular}

The groups are defined as in the Reactor Safety Study, except that Group 7 does not have any contribution from $\mathrm{Zr}$ or $\mathrm{Nb}$.

2 This group includes CsI and an organic iodine contribution of $5.0 \times 10^{-3}$ in Bins 1-12 and 16-20, $1.4 \times 10^{-4}$ in Bins 13 and 14, and $2.5 \times 10^{-5}$ in Bin 15 .

${ }^{3}$ The mean conditional probability of the bin given a core damage accident

${ }^{4}$ Release time is the time between reactor shutdown and release, duration is the time over which the release takes place, and warning period is the time between notification and the release.

$51.4+5=1.4 \times 10^{5}$ 
NUREG/CR-4551,VOL.1: DRAFT REPORT FOR COMMENT (SEPTEMBER, 1986)
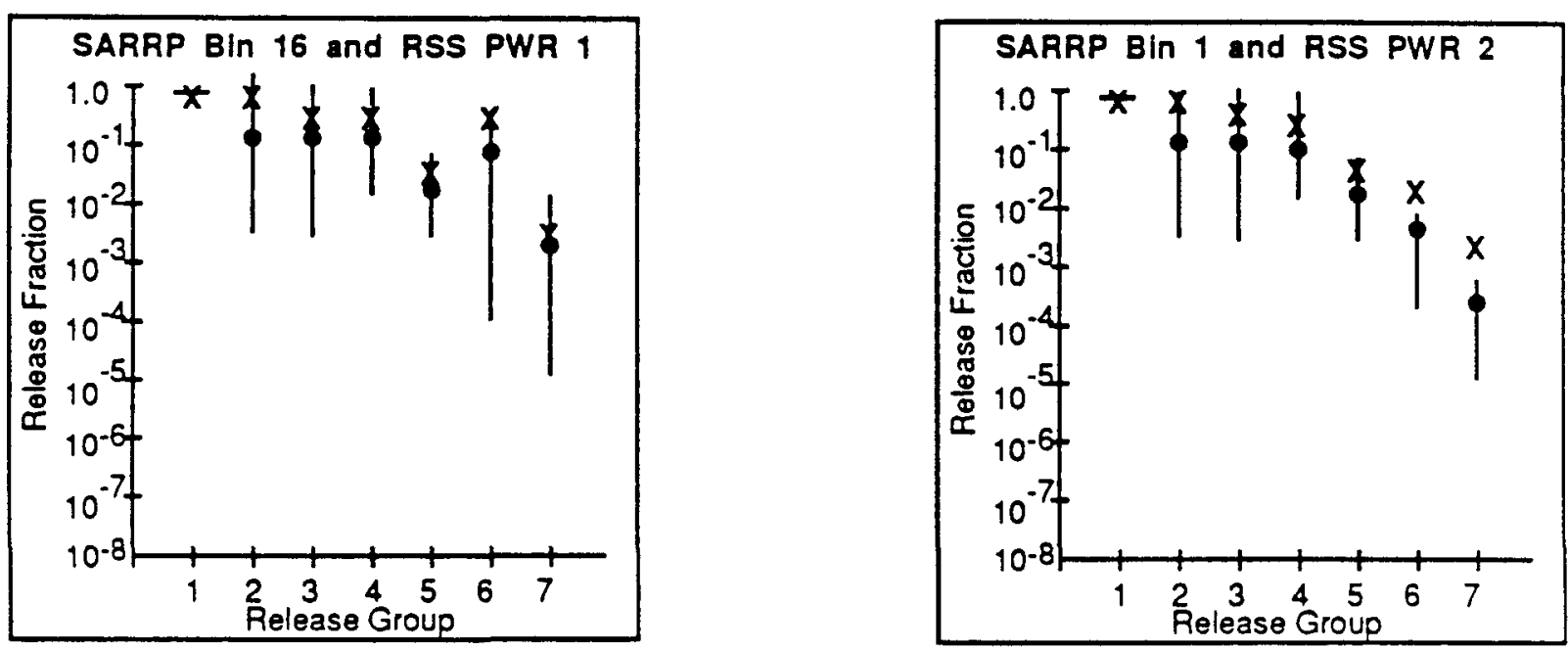

X RSS Median

- sarRp Central
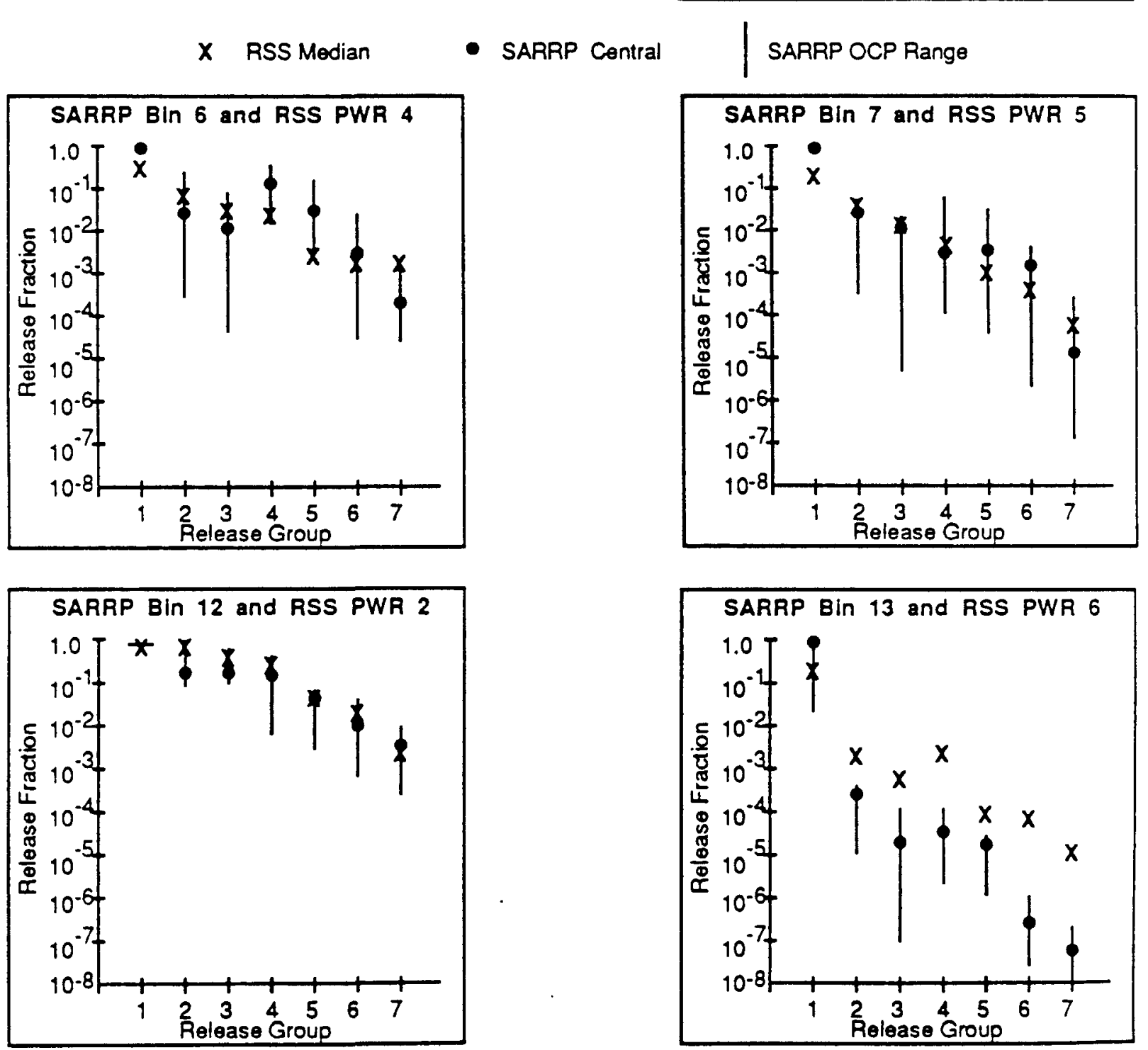

Figure 5-14. Comparison of SARRP and RSS Source Terms 
SARRP bin 1 compared to RSS PWR 2; both are early containment failure sequences without sprays. SARRP Bins 6 and 7 are analogous to RSS categories PWR 4 and 5, respectively. These correspond to accidents involving containment isolation failures (the exact definitions of failure size vary, and comparison is therefore of limited value), with and without the action of the containment sprays. The fifth chart illustrates the releases assigned in the two studies for a $V$ sequence that does not occur under water, while the final graph depicts late releases due to a meltthrough of the containment basemat.

There is generally a reduction in predicted source terms for the SARRP central estimate compared to the RSS median for most of the important species (the noble gases are unchanged). As illustrated, the difference between the SARRP central and RSS value is generally a factor of 2 to 10 . This reduction is due to a conglomeration of changes to the modeling of each of the sequences. BMI-2104 outlines the advances in the technology, including improvements in modeling capability, understanding of some of the thermalhydraulic aspects, and in the incorporation of more experimental evidence. The figures also illustrate that while the general trend is a reduction in predicted source term, some individual release groups are estimated to have higher values than previously assessed; this too is due to a combination of modeling changes that have been incorporated into the STCP.

Figure 5-14 also includes the OCP uncertainty range compared to the RSS. The RSS value is often near the top of the OCP range (the pessimistic values). The optimistic values are consistently lower than the RSS estimates, often by several orders of magnitude. This results from newer evidence perceived by some to indicate much smaller releases than previously estimated by the generally more conservative approach taken in the RSS. Overall, these comparisons indicate the general trend of prediction of lower source terms due to advances in the methods since the RSS. The fact that the upper ends of the OCP uncertainty bands generally equal or exceed the RSS values indicates that there are some outstanding issues or combinations of issues that have the potential for larger source terms than predicted in the past.

\subsection{OFFSITE CONSEQUENCE RESULTS}

For each of the source terms described in the previous section (the 25 clusters for the LLH and the 20 bins for the OCP cases), offsite consequences were calculated using both the CRAC2 and the MACCS codes. Mean results for five consequence measures (early fatalities, early injuries, latent cancer fatalities, population dose within 50 miles of the site, and total offsite costs) for the LLH clusters are presented in Tables 5-10 for CRAC2 and Table 5-11 
NUREG/CR-4551,VOL.1: DRAFT REPORT FOR COMMENT (SEPTEMBER, 1986)

Table 5-10

CRAC2 ESTIMATES OF MEAN CONSEQUENCES FOR SURRY SOURCE TERM CLUSTERS

\begin{tabular}{|c|c|c|c|c|c|}
\hline Cluster & $\begin{array}{c}\text { Early } \\
\text { Fatalities }\end{array}$ & $\begin{array}{c}\text { Early } \\
\text { Injuries }\end{array}$ & $\begin{array}{l}\text { Latent } \\
\text { Cancer } \\
\text { Fatalities }\end{array}$ & $\begin{array}{l}\text { Offsite } \\
\text { Costs } \\
(\$)\end{array}$ & $\begin{array}{l}\text { Population } \\
\text { Dose to } 50 \text { Miles } \\
\text { (person-rem) }\end{array}$ \\
\hline 1 & 28 & 891 & 1770 & $1.1 \times 10^{9}$ & $4.4 \times 10^{6}$ \\
\hline 2 & 16 & 675 & 1540 & $1.1 \times 10^{9}$ & $3.9 \times 10^{6}$ \\
\hline 3 & 5 & 352 & 1300 & $1.0 \times 10^{9}$ & $3.4 \times 10^{6}$ \\
\hline 4 & 2.6 & 216 & 1100 & $7.8 \times 10^{8}$ & $3.4 \times 10^{6}$ \\
\hline 5 & 1.9 & 128 & 921 & $6.7 \times 10^{8}$ & $3.2 \times 10^{6}$ \\
\hline 6 & 1.3 & 101 & 806 & $5.8 \times 10^{8}$ & $3.0 \times 10^{6}$ \\
\hline 7 & .5 & 40 & 706 & $5.1 \times 10^{8}$ & $2.9 \times 10^{6}$ \\
\hline 8 & .2 & 18 & 558 & $4.1 \times 10^{8}$ & $2.7 \times 10^{6}$ \\
\hline 9 & .1 & 1.5 & 276 & $1.5 \times 10^{8}$ & $2.0 \times 10^{6}$ \\
\hline 10 & .6 & 52 & 473 & $2.9 \times 10^{8}$ & $2.6 \times 10^{6}$ \\
\hline 11 & .1 & 12 & 433 & $2.9 \times 10^{8}$ & $2.5 \times 10^{6}$ \\
\hline 12 & .2 & 17 & 232 & $9.1 \times 10^{7}$ & $1.7 \times 10^{6}$ \\
\hline 13 & .1 & 4.5 & 356 & $2.2 \times 10^{8}$ & $2.3 \times 10^{6}$ \\
\hline 14 & 0.0 & .2 & 159 & $5.4 \times 10^{7}$ & $1.5 \times 10^{6}$ \\
\hline 15 & 0.0 & 2.9 & 281 & $1.7 \times 10^{8}$ & $2.0 \times 10^{6}$ \\
\hline 16 & 0.0 & 0.0 & 55 & $2.7 \times 10^{7}$ & $5.9 \times 10^{5}$ \\
\hline 17 & 0.0 & 3 & 201 & $1.0 \times 10^{8}$ & $1.6 \times 10^{6}$ \\
\hline 18 & 0.0 & 0.0 & 26 & $1.9 \times 10^{7}$ & $3.1 \times 10^{5}$ \\
\hline 19 & 0.0 & .6 & 135 & $5.1 \times 10^{7}$ & $1.2 \times 10^{6}$ \\
\hline 20 & 0.0 & 0.0 & 45 & $2.3 \times 10^{7}$ & $4.9 \times 10^{5}$ \\
\hline 21 & 0.0 & .27 & 81 & $2.6 \times 10^{7}$ & $7.8 \times 10^{5}$ \\
\hline 22 & 0.0 & 0.0 & 5 & $1.4 \times 10^{7}$ & $5.3 \times 10^{4}$ \\
\hline 23 & 0.0 & 0.0 & 43 & $1.4 \times 10^{7}$ & $4.4 \times 10^{5}$ \\
\hline 24 & 0.0 & 0.0 & .4 & $4.0 \times 10^{6}$ & $4.4 \times 10^{3}$ \\
\hline $25^{*}$ & 0.0 & 0.0 & .1 & $4.0 \times 10^{6}$ & $1.6 \times 10^{3}$ \\
\hline
\end{tabular}

*Defined by inspection to better represent the low magnitude source terms. 
NUREG/CR-4551,VOL.1: DRAFT REPORT FOR COMMENT (SEPTEMBER, 1986)

Table 5-11

MACCS ESTIMATE OF MEAN CONSEQUENCES FOR SURRY SOURCE TERM CLUSTERS

\begin{tabular}{|c|c|c|c|c|c|}
\hline Cluster & $\begin{array}{c}\text { Early } \\
\text { Fatalities }\end{array}$ & $\underset{\text { Injuries }}{\text { Early }}$ & $\begin{array}{l}\text { Latent } \\
\text { Cancer } \\
\text { Fatalities }\end{array}$ & $\begin{array}{l}\text { Offsite } \\
\text { Costs } \\
(\$)\end{array}$ & $\begin{array}{l}\text { Population } \\
\text { Dose to } 50 \text { Miles } \\
\text { (person-rem) }\end{array}$ \\
\hline 1 & 27 & 194 & 8080 & $2.9 \times 10^{9}$ & $1.6 \times 10^{7}$ \\
\hline 2 & 8.0 & 93 & 7240 & $2.5 \times 10^{9}$ & $1.4 \times 10^{7}$ \\
\hline 3 & 15 & 78 & 6560 & $2.4 \times 10^{9}$ & $1.2 \times 10^{7}$ \\
\hline 4 & 6.2 & 34 & 5900 & $1.8 \times 10^{9}$ & $1.2 \times 10^{7}$ \\
\hline 5 & 1.6 & 21 & 5200 & $1.5 \times 10^{9}$ & $1.0 \times 10^{7}$ \\
\hline 6 & 2.1 & 26 & 4910 & $1.4 \times 10^{9}$ & $8.7 \times 10^{6}$ \\
\hline 7 & 0.76 & 9.1 & 4420 & $1.0 \times 10^{9}$ & $8.2 \times 10^{6}$ \\
\hline 8 & 0.18 & 2.0 & 3890 & $8.1 \times 10^{8}$ & $7.7 \times 10^{6}$ \\
\hline 9 & 0.13 & .84 & 1620 & $4.4 \times 10^{8}$ & $4.9 \times 10^{6}$ \\
\hline 10 & 2.5 & 23 & 3320 & $7.5 \times 10^{8}$ & $6.6 \times 10^{6}$ \\
\hline 11 & 1.8 & 1.3 & 2840 & $6.0 \times 10^{8}$ & $6.3 \times 10^{6}$ \\
\hline 12 & 0.15 & 17 & 1570 & $3.6 \times 10^{8}$ & $3.5 \times 10^{6}$ \\
\hline 13 & 0.07 & 0.68 & 2390 & $5.0 \times 10^{9}$ & $5.2 \times 10^{6}$ \\
\hline 14 & 0.004 & 0.05 & 1030 & $1.6 \times 10^{8}$ & $2.9 \times 10^{6}$ \\
\hline 15 & 0.05 & 0.37 & 1820 & $3.7 \times 10^{8}$ & $4.4 \times 10^{6}$ \\
\hline 16 & 0.0006 & 0.01 & 362 & $8.7 \times 10^{7}$ & $1.4 \times 10^{6}$ \\
\hline 17 & 0.05 & 0.44 & 1270 & $2.6 \times 10^{8}$ & $3.3 \times 10^{6}$ \\
\hline 18 & 0.0 & 0.003 & 183 & $5.0 \times 10^{7}$ & $7.5 \times 10^{5}$ \\
\hline 19 & 0.017 & 0.15 & 794 & $1.8 \times 10^{8}$ & $2.3 \times 10^{6}$ \\
\hline 20 & 0.0 & 0.001 & 334 & $2.9 \times 10^{7}$ & $1.3 \times 10^{6}$ \\
\hline 21 & 0.01 & 0.11 & 408 & $1.1 \times 10^{8}$ & $1.4 \times 10^{6}$ \\
\hline 22 & 0.0 & 0.0 & 34 & $6.6 \times 10^{6}$ & $1.6 \times 10^{5}$ \\
\hline 23 & 0.006 & 0.06 & 233 & $4.4 \times 10^{7}$ & $9.1 \times 10^{5}$ \\
\hline $24 *$ & 0.0 & 0.0 & 4.0 & $2.2 \times 10^{6}$ & $2.1 \times 10^{4}$ \\
\hline
\end{tabular}

*Cluster 25 was not defined for the MACCS calculations. 
for MACCS. (The consequences for the central estimate bins are provided later in this section.) Inspection of the results presented in the Tables 5-10 and 11 shows the following:

(1) The predicted numbers of mean early fatalities differ significantly from zero only for the large magnitude clusters;

(2) For the larger source terms, MACCS predicts slightly more mean early fatalities than CRAC2, and CRAC2 three to four more times early injuries than MACCS;

(3) MACCS predicts about five times more latent cancer fatalities than CRAC2

(4) MACCS estimates of population dose within 50 miles of the site exceed those of CRAC2 by factors of 2 to 3 ; and

(5) Offsite costs predicted by MACCS are usually twice those predicted by CRAC2.

There are a number of reasons for the differences in the results. These are explained below by individual topic.

Dose Thresholds. Because early fatalities and injuries both have dose thresholds, their occurrence is very sensitive to meteorological conditions and emergency response effectiveness. If, at the time of containment failure, unstable (turbulent) atmospheric conditions prevail, dilution of the released plume rapidly causes doses to fall below threshold values. Even when threshold doses would be exceeded within some distance downwind from the site, effective evacuation or sheltering can prevent the occurrence of any early fatalities or injuries. Consequently, when a mean result is calculated for many weather scenarios, because most produce no early effects, the value of the mean result is very sensitive to the magnitudes of the few non-zero results. Therefore, mean early fatality and injury values are quite uncertain, which suggests that the CRAC2 and MACCS results are not very different.

Early Injuries. The MACCS mean early injuries are the sum of prodromal vomiting and lung impairment, which as calculated by MACCS occur in nearly equal numbers. The CRAC2 mean early injuries are the sum of prodromal vomiting, lung impairment, and gastrointestinal tract injuries, which as calculated by CRAC2 are almost entirely prodromal vomiting. CRAC2 predicts few cases of lung impairment because, as mentioned above, the CRAC2 lung threshold is much higher than that used in MACCS. Differences in crosswind plume profiles and in the sets of weather sequences selected by MACCS and CRAC2 cause CRAC2 to predict that many more people will receive doses that will cause prodromal vomiting than are predicted by MACCS.

Population Dose and Latent Cancer Fatalities. The CRAC2 code assumes that crop pathways produce societal population exposures two to three times larger than the exposures produced by the milk pathway. Recent data incorporated into MACCS suggest that the crop pathways are actually 11 to 15 times more important than the milk pathway. In MACCS, both the population dose and latent cancer fatalities are dominated by the crop pathway. In CRAC2, the crop pathway is less important, latent cancer fatalities being caused principally by groundshine dose, especially groundshine dose to the breast. In fact, with root 
uptake suppressed, CRAC2 predicts more latent cancer fatalities than MACCS, primarily because CRAC2 predicts such a high incidence of breast cancer.

The net effect of all these differences is that MACCS predictions of latent cancer fatalities exceed those of CRAC2 by about a factor of five. Unfortunately, it is difficult to examine all of the competing factors to compare the codes, and the features discussed above are possible explanations for the differences. CRAC2 and MACCS comparisons are continuing to be made, and detailed explanations for any differences should soon be available.

Total Costs. The MACCS estimates of total offsite costs exceed those of CRAC2 by about a factor of two principally because decontamination is assumed to be more costly in MACCS than in CRAC2, and because MACCS calculates emergency response costs while CRAC2 does not.

Thus there are several causes for the differences in CRAC2 and MACCS results. In general, the results calculated with the different codes do not change the overall conclusions with regard to the significance of various risk contributors.

The mean consequence as predicted by both codes for the central estimate source term bins are provided in Tables 5-12 and 5-13. These tables are useful for diagnostics concerning the central estimate, particularly in the light of the fact that the source term bins have physical meaning relative to containment response, whereas the clusters do not. 
NUREG/CR-4551,VOL.1: DRAFT REPORT FOR COMMENT (SEPTEMBER, 1986)

Table 5-12

CRAC2 ESTIMATE OF MEAN CONSEQUENCES FOR SURRY CENTRAL ESTIMATE SOURCE TERM BINS

\begin{tabular}{lrrrll}
\hline Bin & $\begin{array}{c}\text { Early } \\
\text { Fatalities }\end{array}$ & $\begin{array}{c}\text { Early } \\
\text { Injuries }\end{array}$ & $\begin{array}{c}\text { Latent } \\
\text { Cancer } \\
\text { Fatalities }\end{array}$ & $\begin{array}{l}\text { Offsite } \\
\text { Costs } \\
(\$)\end{array}$ & $\begin{array}{c}\text { Population } \\
\text { Dose to 50 Miles } \\
\text { (person-rem) }\end{array}$ \\
\hline $1^{*}$ & 0.05 & 4.9 & 507 & $3.6 \times 10^{8}$ & $2.3 \times 10^{6}$ \\
$2^{*}$ & 0.05 & 4.6 & 495 & $3.6 \times 10^{8}$ & $2.3 \times 10^{6}$ \\
3 & 0.0 & 0.007 & 147 & $3.8 \times 10^{7}$ & $1.4 \times 10^{6}$ \\
4 & 0.0 & 0.04 & 29 & $9.4 \times 10^{6}$ & $3.0 \times 10^{5}$ \\
$5^{*}$ & 1.6 & 31 & 857 & $6.9 \times 10^{8}$ & $3.1 \times 10^{6}$ \\
6 & 0.04 & 0.26 & 119 & $3.4 \times 10^{7}$ & $1.3 \times 10^{6}$ \\
7 & 0.0 & 0.03 & 55 & $2.3 \times 10^{7}$ & $5.5 \times 10^{5}$ \\
8 & 0.0 & 0.003 & 3.1 & $4.2 \times 10^{6}$ & $2.9 \times 10^{4}$ \\
9 & 0.013 & 0.17 & 29 & $5.6 \times 10^{6}$ & $3.2 \times 10^{5}$ \\
10 & 0.0 & 0.0 & 16 & $1.5 \times 10^{7}$ & $1.8 \times 10^{5}$ \\
$11^{*}$ & 0.002 & 0.32 & 118 & $3.9 \times 10^{7}$ & $1.0 \times 10^{6}$ \\
$12^{*}$ & 0.56 & 1.3 & 448 & $2.8 \times 10^{8}$ & $2.6 \times 10^{6}$ \\
13 & 0.0 & 0.0 & 1.5 & $4.0 \times 10^{6}$ & $1.0 \times 10^{3}$ \\
14 & 0.0 & 0.0 & 0.16 & $4.0 \times 10^{6}$ & $1.9 \times 10^{3}$ \\
15 & 0.0 & 0.0 & 0.12 & $4.0 \times 10^{6}$ & $1.5 \times 10^{3}$ \\
$16^{*}$ & .15 & 9.4 & 4360 & $1.3 \times 10^{9}$ & $6.1 \times 10^{6}$ \\
$17^{*}$ & 0.08 & 6.5 & 650 & $4.0 \times 10^{8}$ & $2.5 \times 10^{6}$ \\
18 & 0.0 & 1.2 & 565 & $3.8 \times 10^{8}$ & $2.4 \times 10^{6}$ \\
19 & 0.0001 & 0.07 & 57 & $1.4 \times 10^{7}$ & $4.8 \times 10^{5}$ \\
20 & 0.02 & 3.9 & 566 & $3.3 \times 10^{8}$ & $3.6 \times 10^{6}$ \\
\hline
\end{tabular}

*The consequences for these bins were assumed to be the puff releases, calculated as defined in Section 2. 
NUREG/CR-4551,VOL.1: DRAFT REPORT FOR COMMENT (SEPTEMBER, 1986)

Table 5-13

MACCS ESTIMATE OF MEAN CONSEQUENCES FOR SURRY CENTRAL ESTTMATE SOURCE TERM BINS

\begin{tabular}{|c|c|c|c|c|c|}
\hline Bin & $\begin{array}{c}\text { Early } \\
\text { Fatalities }\end{array}$ & $\begin{array}{l}\text { Early } \\
\text { Injuries }\end{array}$ & $\begin{array}{c}\text { Latent } \\
\text { Cancer } \\
\text { Fatalities }\end{array}$ & $\begin{array}{l}\text { Offsite } \\
\text { Costs } \\
(\$)\end{array}$ & $\begin{array}{c}\text { Population } \\
\text { Dose to } 50 \text { Miles } \\
\text { (person-rem) }\end{array}$ \\
\hline 1 & 0.16 & 2.8 & 3890 & $9.8 \times 10^{8}$ & $6.2 \times 10^{6}$ \\
\hline 2 & 0.42 & 6.8 & 4030 & $9.6 \times 10^{8}$ & $4.5 \times 10^{6}$ \\
\hline 3 & 0.008 & 0.18 & 1070 & $2.2 \times 10^{8}$ & $5.0 \times 10^{6}$ \\
\hline 4 & 0.09 & 0.56 & 174 & $2.9 \times 10^{7}$ & $8.1 \times 10^{5}$ \\
\hline 5 & 0.04 & 0.50 & 8460 & $1.8 \times 10^{9}$ & $1.1 \times 10^{7}$ \\
\hline 6 & 0.04 & 0.40 & 841 & $1.4 \times 10^{8}$ & $6.7 \times 10^{6}$ \\
\hline 7 & 0.03 & 0.63 & 351 & $4.6 \times 10^{7}$ & $1.6 \times 10^{6}$ \\
\hline 8 & 0.0 & 0.001 & 6.6 & $8.2 \times 10^{6}$ & $3.8 \times 10^{4}$ \\
\hline 9 & 0.013 & 0.01 & 228 & $4.0 \times 10^{7}$ & $2.8 \times 10^{6}$ \\
\hline 10 & 0.002 & 0.04 & 128 & $1.6 \times 10^{7}$ & $1.5 \times 10^{6}$ \\
\hline 11 & 0.04 & 0.40 & 800 & $1.3 \times 10^{8}$ & $2.8 \times 10^{6}$ \\
\hline 12 & 0.57 & 6.7 & 2830 & $7.2 \times 10^{8}$ & $7.4 \times 10^{6}$ \\
\hline 13 & 0.0 & 0.0 & 2.4 & $1.2 \times 10^{6}$ & $1.3 \times 10^{4}$ \\
\hline 14 & 0.0 & 0.0 & 1.7 & $1.2 \times 10^{6}$ & $1.2 \times 10^{4}$ \\
\hline 15 & 0.0 & 0.0 & 1.7 & $1.1 \times 10^{6}$ & $1.3 \times 10^{4}$ \\
\hline 16 & .98 & 1.3 & 4360 & $1.3 \times 10^{9}$ & $6.1 \times 10^{6}$ \\
\hline 17 & 1.0 & 1.4 & 4020 & $1.1 \times 10^{9}$ & $5.4 \times 10^{6}$ \\
\hline 18 & 0.19 & 1.2 & 880 & $3.5 \times 10^{8}$ & $5.0 \times 10^{6}$ \\
\hline 19 & 0.18 & 1.0 & 235 & $1.8 \times 10^{8}$ & $8.1 \times 10^{5}$ \\
\hline 20 & 1.3 & 9.0 & 2980 & $8.3 \times 10^{8}$ & $5.6 \times 10^{6}$ \\
\hline
\end{tabular}




\subsection{RISK RESULTS}

The risk results for the Surry plant are presented in this section. Six risk measures are included: early fatalities, early injuries, latent cancer fatalities, individual risk of early death, offsite costs (in dollars), and population dose (in person-rems). All risk results reported are mean values calculated using the CRAC2 and MACCS codes as indicated, and all are computed per year of reactor operation. Early and latent cancer fatalities are discussed in detail first, followed by the results of the other risk measures. It should be noted that the full results included many other, more detailed presentations of risk contributors, and these are included in Appendix D.

\subsubsection{Early and Latent Cancer Eatality Risk_Measures}

The early and latent cancer fatality risk results are presented in this section. A discussion of the important sensitivities and uncertainties is also included.

\section{Risk Results}

The results for latent cancer fatalities are presented in Figure 5-15. The central estimate of latent cancer fatality risk is approximately $3 \times 10^{-4}$ per year. The data uncertainty about the central estimate is about an order of magnitude, more in the downward direction than the upward relative to the central estimate." The modeling uncertainty as measured by the LLH analysis is about two orders of magnitude, and mainly upward with respect to the central estimate. The results obtained with MACCS are somewhat higher than those obtained with CRAC2. As discussed in Section 5.4, it is believed that these differences are due to the new models in MACCS which predict a higher dose from the crop pathway.

Since it was the goal of the SARRP program to address the change in perception of risk since the previous risk assessment, the RSS point estimate is also indicated." The RSS value for latent cancer fatalities is about two orders of magnitude higher than the SARRP central estimate, and above the highest sample member for the CRAC2 LLH result. The reasons for the differences are discussed in Section 5.5.3.

\footnotetext{
The LLH uncertainty displays do not include these data uncertainties. Any estimate of total uncertainty should include consideration of the range exhibited by the LLH plus the range associated with uncertainty in the input data

**For latent cancer fatalities, the RSS reported the average yearly rate assuming a 30 year latency. To convert to total latent cancer fatalities, the RSS value was multiplied by a factor of 30 .
} 
NUREG/CR-4551,VOL1: DRAFT REPORT FOR COMMENT (SEPTEMBER, 1986)

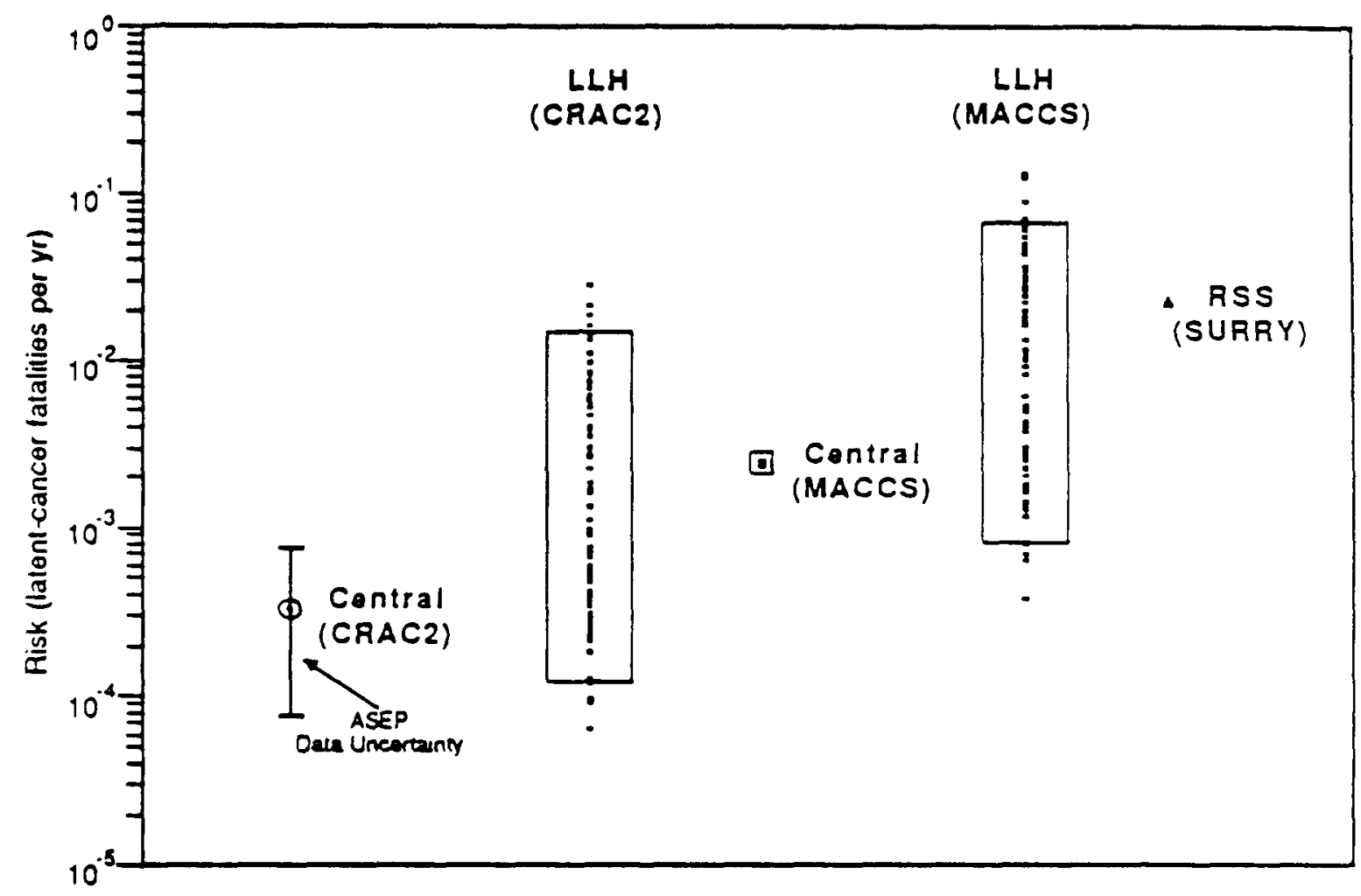

Figure 5-15. Risk of Latent Cancer Fatalities

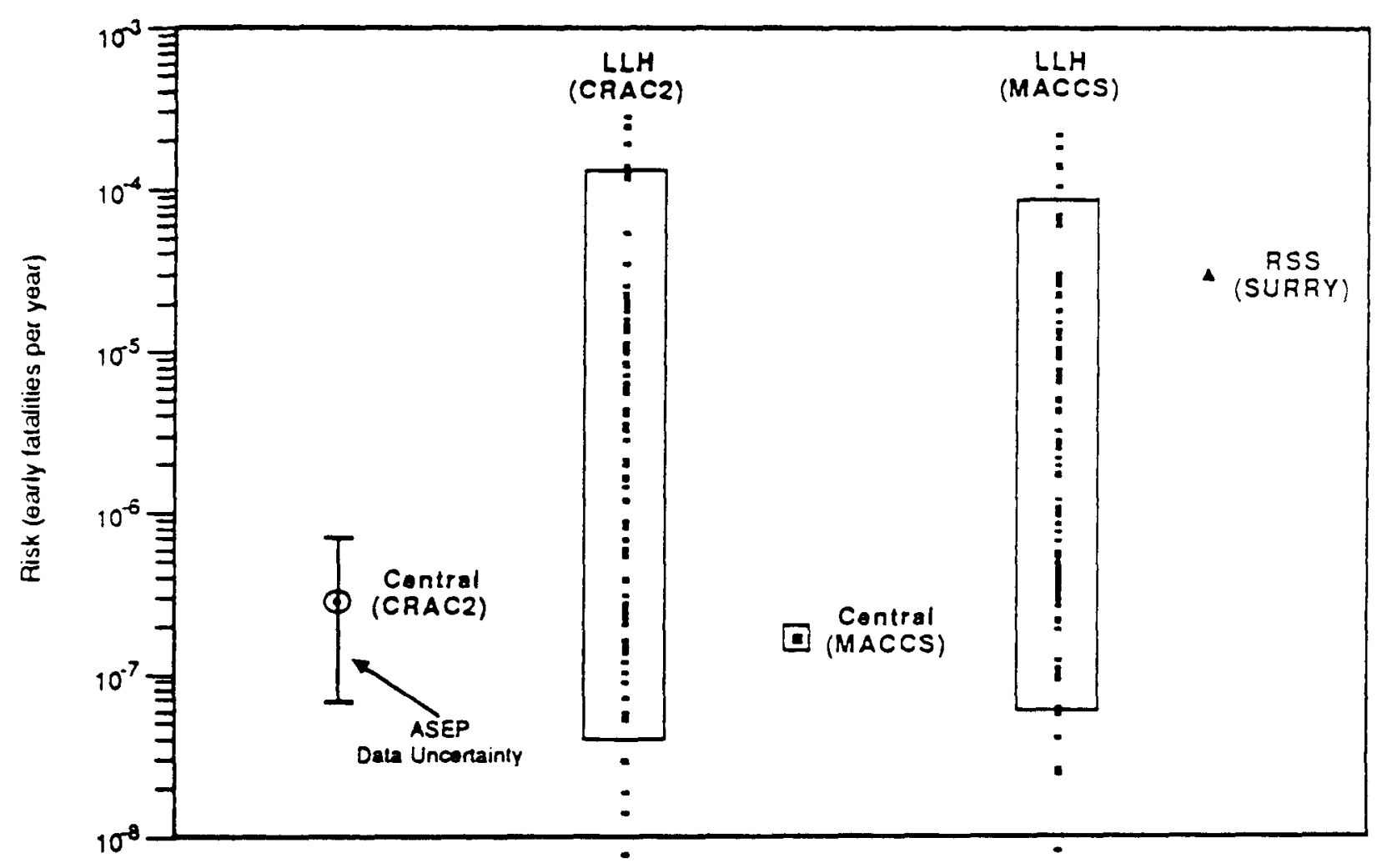

Figure 5-16. Risk of Early Fatalities 
The basic risk results for early fatalities are displayed in Figure 5-16. As illustrated, the central estimate is about $3 \times 10^{-7}$ early fatalities per year. Uncertainty about the central estimate associated with equipment failure data is about one order of magnitude; once again the greater uncertainty is in the downward direction. The uncertainty associated with modeling issues included in the LLH approach is also illustrated. The total range is greater than three orders of magnitude, with the uncertainty relative to the SARRP central estimate mostly in the upward direction (increasing risk). The RSS point estimate is about two orders of magnitude above the SARRP central, but half an order of magnitude lower than the top of the LLH range. Finally, the CRAC2 and MACCS results are similar for this consequence measure, although MACCS does exhibit a smaller spread in the results.

Table 5-14

COMPARISON OF PLANT DAMAGE STATE FRACTIONAL CONTRIBUTION TO MEAN RISK FOR LLH AND SARRP CENTRAL ESTTMATE

\begin{tabular}{|c|c|c|c|c|c|c|c|c|}
\hline \multirow{3}{*}{$\begin{array}{c}\text { Plant } \\
\text { Damage State }\end{array}$} & \multicolumn{4}{|c|}{$\begin{array}{l}\text { Contribution to } \\
\text { Early Fatalities }\end{array}$} & \multicolumn{4}{|c|}{$\begin{array}{l}\text { Contribution to } \\
\text { Latent Cancer Fatalities }\end{array}$} \\
\hline & \multicolumn{2}{|c|}{$\mathrm{CRAC2}$} & \multicolumn{2}{|c|}{ MACCS } & \multicolumn{2}{|c|}{ CRAC2 } & \multicolumn{2}{|c|}{ MACCS } \\
\hline & $L \mathrm{LH}^{*}$ & Central & LLH & Central & $\mathrm{LLH}$ & Central & $\mathrm{LLH}$ & Central \\
\hline SYYB & $2 \%$ & $3 \%$ & $2 \%$ & $6.5 \%$ & $13.5 \%$ & $9.4 \%$ & $13.4 \%$ & $9.6 \%$ \\
\hline SYNT & $\varepsilon$ & $\varepsilon$ & $\varepsilon$ & $\varepsilon$ & $\varepsilon$ & $\varepsilon$ & $\varepsilon$ & $\varepsilon$ \\
\hline SNNN & $67 \%$ & $2 \%$ & $64 \%$ & $4.6 \%$ & $48 \%$ & $6.7 \%$ & $46 \%$ & $\varepsilon$ \\
\hline SYNN & $\varepsilon$ & $1.4 \%$ & $\varepsilon$ & $\varepsilon$ & $\varepsilon$ & $.6 \%$ & $\varepsilon$ & $1 \%$ \\
\hline ANNN & $0 \%$ & $\varepsilon$ & $0 \%$ & $\varepsilon$ & $0 \%$ & $\varepsilon$ & $\varepsilon$ & $\varepsilon$ \\
\hline AYNN & $.8 \%$ & $.3 \%$ & $.7 \%$ & $\varepsilon$ & $.9 \%$ & $.1 \%$ & $.8 \%$ & $.2 \%$ \\
\hline AYNI & $2 \%$ & $45 \%$ & $1.7 \%$ & $1.4 \%$ & $2 \%$ & $22 \%$ & $2 \%$ & $29 \%$ \\
\hline AYYB & $.8 \%$ & $.8 \%$ & $\varepsilon$ & $1.7 \%$ & $.7 \%$ & $2.4 \%$ & $.9 \%$ & $2.5 \%$ \\
\hline TNNN & $15 \%$ & $.7 \%$ & $18 \%$ & $4.9 \%$ & $14.5 \%$ & $2.9 \%$ & $16 \%$ & $2.8 \%$ \\
\hline TYYBW & $5.3 \%$ & $3 \%$ & $5 \%$ & $1.4 \%$ & $6.2 \%$ & $1.2 \%$ & $6 \%$ & $1.1 \%$ \\
\hline TYYBD & $5.6 \%$ & $3 \%$ & $5.9 \%$ & $1.5 \%$ & $6.8 \%$ & $1.3 \%$ & $6.7 \%$ & $1.3 \%$ \\
\hline TYNI & $\varepsilon$ & $.5 \%$ & $\varepsilon$ & $\varepsilon$ & $\varepsilon \%$ & $.3 \%$ & $\varepsilon$ & $.3 \%$ \\
\hline V & $2 \%$ & $46 \%$ & $2 \%$ & $77 \%$ & $7.7 \%$ & $53 \%$ & $7.7 \%$ & $46 \%$ \\
\hline
\end{tabular}

*The fractional contribution is difficult to illustrate in the LLH, since each of the 100 sample members has a different outcome. It is possible to calculate the mean result of the LLH, and that is the value illustrated in the table, but there are strict limits on the interpretation of these values. They are provided here to illustrate differences between the central and the LLH, but the mean of the LLH is only used as a representative result. Some of the experts who provided input were very clear in their desire not to attach probabilistic significance to the weighting factors, and therefore the mean must not be considered as a statistical parameter in this sense. 
Several methods of decomposing the risk results were utilized to help understand the various contributors to risk. The contribution of each plant damage state to risk is listed in Table 5-14 to provide a perspective on the important accident sequences. For the central estimate, the $\mathrm{V}$ and $\mathrm{AYNI}$ plant damage states dominate the risk. These damage states are comprised of sequences $\mathrm{V}, A F_{1} F_{2}$, and $S_{1} F_{1} F_{2}$ (listed in Table 5-3), which involve either containment failure prior to core damage or containment bypass. The core-vulnerable sequences $\left(F_{1} F_{2}\right)$ were treated the same in the central estimate as in the LLH result, with an average reduction in frequency of 0.02 to account for the probability of the core-vulnerable sequence leading to a core-damage sequence. The difference in risk between the $O C P$ and LLH is therefore not due to sequence frequency for this damage state. On the other hand, for the LLH analysis, damage state SNNN clearly dominates the risk of both consequence measures, with state TNNN contributing most of the balance. A review of the accident sequences presented in Section 5.1 indicates that both of these damage states are dominated by station blackout sequences (i.e., complete losses of ac power). The states differ only in integrity of the primary system; SNNN involves a seal or relief valve LOCA. These damage states are important to risk because the failure of power disables all containment safeguards, and because the reactor cavity is dry in these accidents leading to the potential for very significant direct heating.

A similar breakdown is provided in Table 5-15 for the source-term bins. For the central estimate, bins 5 and 12 are the most important contributors for early fatalities, with bin 11 also contributing substantially for latent cancer fatalities. As identified in Section 2.5.1, bin 5 specifically applies to containment failures prior to core melt while bins 11 and 12 apply to containment bypass, or the $\mathrm{V}$ sequence. For the LLH study, the large majority of the risk of both early and latent fatalities is associated with bin 16 . Bin 16 refers to a high pressure ejection failure of the vessel with a large amount of direct heating leading to containment failure soon after vessel breach. No containment safeguards are available since power is unavailable. This bin was used for all direct heating failures associated with damage states SNNN and TNNN, which were already shown to dominate risk in Table 5-14.

The fractional contribution to risk is also provided for the source-term clusters in Table 5-16. This is a useful feature for detailed study of the LLH results, but the clusters do not have physical meaning relative to the containment response and there are limited insights to be gained directly from the information provided in this table. As might be expected, the higher magnitude clusters contribute most to the risk. 
Table 5-15

COMPARISON OF SARRP CENTRAL ESTIMATE AND LLH MEAN SOURCE TERM BIN FRACTIONAL CONTRIBUTION TO RISK

\begin{tabular}{|c|c|c|c|c|c|c|}
\hline \multirow{3}{*}{$\begin{array}{c}\text { Source } \\
\text { Term Bin }\end{array}$} & \multicolumn{3}{|c|}{$\begin{array}{l}\text { Contribution to } \\
\text { Early Fatalities }\end{array}$} & \multicolumn{3}{|c|}{$\begin{array}{l}\text { Contribution to } \\
\text { Latent Cancer Fatalities }\end{array}$} \\
\hline & \multicolumn{2}{|c|}{ CRAC2 } & \multirow{2}{*}{$\frac{\operatorname{MACCS}^{1}}{L L H}$} & \multicolumn{2}{|c|}{$\mathrm{CRAC2}$} & \multirow{2}{*}{$\frac{\text { MACCS }}{\text { LLH }}$} \\
\hline & $\mathrm{LLH}^{2}$ & Central & & LLH & Central & \\
\hline 1 & $1 \%$ & $0 \%$ & $3.7 \%$ & $1 \%$ & $0 \%$ & $3 \%$ \\
\hline 2 & $\varepsilon$ & $0 \%$ & $\varepsilon$ & $\varepsilon$ & $0 \%$ & $\varepsilon$ \\
\hline 3 & $\varepsilon$ & $0 \%$ & $\varepsilon$ & $.7 \%$ & $\varepsilon$ & $.7 \%$ \\
\hline 4 & $\varepsilon$ & $0 \%$ & $\varepsilon$ & $\varepsilon$ & $\varepsilon$ & $.3 \%$ \\
\hline 5 & $2.6 \%$ & $47 \%$ & $2.5 \%$ & $3 \%$ & $23 \%$ & $3 \%$ \\
\hline 6 & $0 \%$ & $\varepsilon$ & $0 \%$ & $0 \%$ & $\varepsilon$ & $0 \%$ \\
\hline 7 & $0 \%$ & $0 \%$ & $0 \%$ & $\varepsilon$ & $.5 \%$ & $.1 \%$ \\
\hline 8 & $0 \%$ & $0 \%$ & $0 \%$ & $\varepsilon$ & $1 \%$ & $.7 \%$ \\
\hline 9 & $\varepsilon$ & $7 \%$ & $\varepsilon$ & $.7 \%$ & $18 \%$ & $.7 \%$ \\
\hline 10 & $0 \%$ & $0 \%$ & $0 \%$ & $\varepsilon$ & $1.6 \%$ & $.3 \%$ \\
\hline 11 & $\varepsilon$ & $5 \%$ & $0 \%$ & $4.4 \%$ & $23 \%$ & $5 \%$ \\
\hline 12 & $3 \%$ & $41 \%$ & $3.9 \%$ & $7.6 \%$ & $29 \%$ & $8 \%$ \\
\hline 13 & $0 \%$ & $0 \%$ & $0 \%$ & $0 \%$ & $\varepsilon$ & $0 \%$ \\
\hline 14 & $0 \%$ & $0 \%$ & $0 \%$ & $\varepsilon$ & $\varepsilon$ & $.1 \%$ \\
\hline 15 & $0 \%$ & $0 \%$ & $0 \%$ & $\varepsilon$ & $\varepsilon$ & $\varepsilon$ \\
\hline $16^{3}$ & $86 \%$ & $\varepsilon$ & $81 \%$ & $61 \%$ & $\varepsilon$ & $58 \%$ \\
\hline 17 & $5.6 \%$ & $\varepsilon$ & $5.4 \%$ & $7.6 \%$ & $1.5 \%$ & $7.6 \%$ \\
\hline 18 & $.8 \%$ & $0 \%$ & $.1 \%$ & $3.6 \%$ & $0 \%$ & $3.6 \%$ \\
\hline 19 & $.8 \%$ & $0 \%$ & $1 \%$ & $8.9 \%$ & $0 \%$ & $8.9 \%$ \\
\hline
\end{tabular}

${ }_{1}^{1}$ This parameter was not available for the central MACCS result, but it would be very similar to the CRAC2 result.

${ }^{2}$ The fractional contribution is difficult to illustrate in the LLH since each of the 100 sample members has a different outcome. The mean of the LLH is possible to calculate, but there are strict limits on the interpretation of these values. They are provided here to illustrate differences between the central and the LLH, but the mean of the LLH is only used as a representative result and must not be considered as a statistical parameter of risk uncertainty.

${ }^{3} \mathrm{Bin} 20$ was defined too late in the process to be included in the risk results. Unfortunately, bin 20 is specifically defined for damage state SNNN, which is the dominant contributor. Some portion of the large contribution of bin 16 would be shifted to bin 20 had it been included. 
Table 5-16

FRACTIONAL CONTRIBUTION OF IMPORTANT

LLH CLUSTERS TO RISK*

\begin{tabular}{|c|c|c|c|c|}
\hline \multirow{2}{*}{$\begin{array}{c}\text { Source } \\
\text { Term Cluster }\end{array}$} & \multicolumn{2}{|c|}{$\begin{array}{l}\text { Contribution } \\
\text { to Early Fatalities }\end{array}$} & \multicolumn{2}{|c|}{$\begin{array}{c}\text { Contribution to } \\
\text { Latent Cancer Fatalities }\end{array}$} \\
\hline & CRAC2 & MACCS & CRAC2 & MACCS \\
\hline 1 & $44 \%$ & $43 \%$ & $14 \%$ & $12 \%$ \\
\hline 2 & $31 \%$ & $20 \%$ & $15 \%$ & $13 \%$ \\
\hline 3 & $9 \%$ & $15 \%$ & $11 \%$ & $11 \%$ \\
\hline 4 & $7 \%$ & $8 \%$ & $15 \%$ & $15 \%$ \\
\hline 5 & $3 \%$ & $3.7 \%$ & $8.5 \%$ & $8.7 \%$ \\
\hline 6 & $3 \%$ & $6.8 \%$ & $9.9 \%$ & $11 \%$ \\
\hline 7 & $.9 \%$ & $1.5 \%$ & $6.7 \%$ & $6.4 \%$ \\
\hline 8 & $.4 \%$ & $.5 \%$ & $5.6 \%$ & $7.3 \%$ \\
\hline 9 & $\varepsilon$ & $\varepsilon$ & $.2 \%$ & $.1 \%$ \\
\hline 10 & $.2 \%$ & $.8 \%$ & $1 \%$ & $1.2 \%$ \\
\hline 11 & $.3 \%$ & $.3 \%$ & $4 \%$ & $5.2 \%$ \\
\hline 12 & $\varepsilon$ & $.4 \%$ & $.1 \%$ & $.3 \%$ \\
\hline 13 & $.2 \%$ & $.1 \%$ & $2.5 \%$ & $3.2 \%$ \\
\hline 14 & $\varepsilon$ & $0 \%$ & $.3 \%$ & $.2 \%$ \\
\hline 15 & $\varepsilon$ & $\varepsilon$ & $1.4 \%$ & $1.5 \%$ \\
\hline 16 & $0 \%$ & $0 \%$ & $.2 \%$ & $.3 \%$ \\
\hline 17 & $\mathcal{\varepsilon}$ & $\varepsilon$ & $.8 \%$ & $1.6 \%$ \\
\hline 18 & $0 \%$ & $0 \%$ & $.1 \%$ & $.2 \%$ \\
\hline 19 & $\varepsilon$ & $\varepsilon$ & $.3 \%$ & $.9 \%$ \\
\hline 20 & $0 \%$ & $0 \%$ & $\varepsilon$ & $.1 \%$ \\
\hline 21 & $0 \%$ & $\varepsilon$ & $.3 \%$ & $.3 \%$ \\
\hline 22 & $0 \%$ & $0 \%$ & $\varepsilon$ & $\varepsilon$ \\
\hline 23 & $0 \%$ & $\varepsilon$ & $.2 \%$ & $.1 \%$ \\
\hline 24 & $0 \%$ & $0 \%$ & $\varepsilon$ & $.1 \%$ \\
\hline 25 & $0 \%$ & $0 \%$ & $\varepsilon$ & $\varepsilon$ \\
\hline
\end{tabular}

*The fractional contribution is difficult to illustrate in the LLH since each of the 050 sample members has a different outcome. The mean of the LLH is illustrated in the table, but there are strict limits on the interpretation of these values. They are provided here to illustrate differences between the central and the LLH, but the mean of the LLH is only used as a representative result and must not be considered as a statistical parameter of risk uncertainty. 


\section{Complementary Cumulative Distribution Functions (CCDFs)}

CCDFs corresponding to the risk results of Figures 5-15 and 5-16 are shown in Figures 5-17 and 5-18 for the MACCS results. (Only MACCS results are shown because CRAC2 does not provide CCDFs for the evacuation assumptions used in SARRP.) As discussed in Section 2.2.2, these represent the probability per year (on the ordinate) that a given number of fatalities (on the abscissa) will be exceeded. The LLH risk displays shown in the previous two figures represent the integral of the data shown on Figures 5-17 and 5-18, evaluated separately for each sample member. Symbolically, this may be represented as follows:

$$
R=\int_{0}^{\infty} P\left(C>C_{0}\right) d C_{0}
$$

where $R$ is the mean risk, $P$ is the probability of exceedance, and $C$ is the magnitude of the consequence. Thus the CCDFs separate risk into its two constituents, probability and consequence.

Also shown are the CCDFs for Surry obtained in the RSS.* The following observations apply:

- The RSS CCDF for latent cancer fatalities falls near the top of the LLH band at low consequence levels, and near the middle of the band at high consequence levels. These differences are probably due to the effects of the differing source term magnitudes, the single versus multipuff model (the SARRP results are all MACCS while the RSS result is based on CRAC) and the plant specific population distribution; but the relative importance of these potential differences cannot be determined without additional, detailed study.

- For early fatalities the opposite trend occurs. It is believed that the RSS curve is above the SARRP curve at high consequences due to improvements in the rainout model that are incorporated in MACCS. The CRAC plumes essentially had no length, while the MACCS plumes have lengths defined by the release durations. This leads to less concentrated plumes in the area of a rainout and the peak consequences are predicted to be lower.

- The latent cancer fatality curve at the low end (the 5th of the LLH sample distribution) has a different slope than the high end. This is a result of some feature of the risk results rather than uncertainty in the consequence result. The exact cause of the shape of this curve is difficult to establish; however, it is known that the low consequence regime is dominated by late containment failures and small source terms while the large consequence regime is dominated by early containment failure and large source terms.

\footnotetext{
*Again, the RSS-reported value had to be multiplied by a factor of 30 to convert from the yearly rate of incidence to total fatalities.
} 


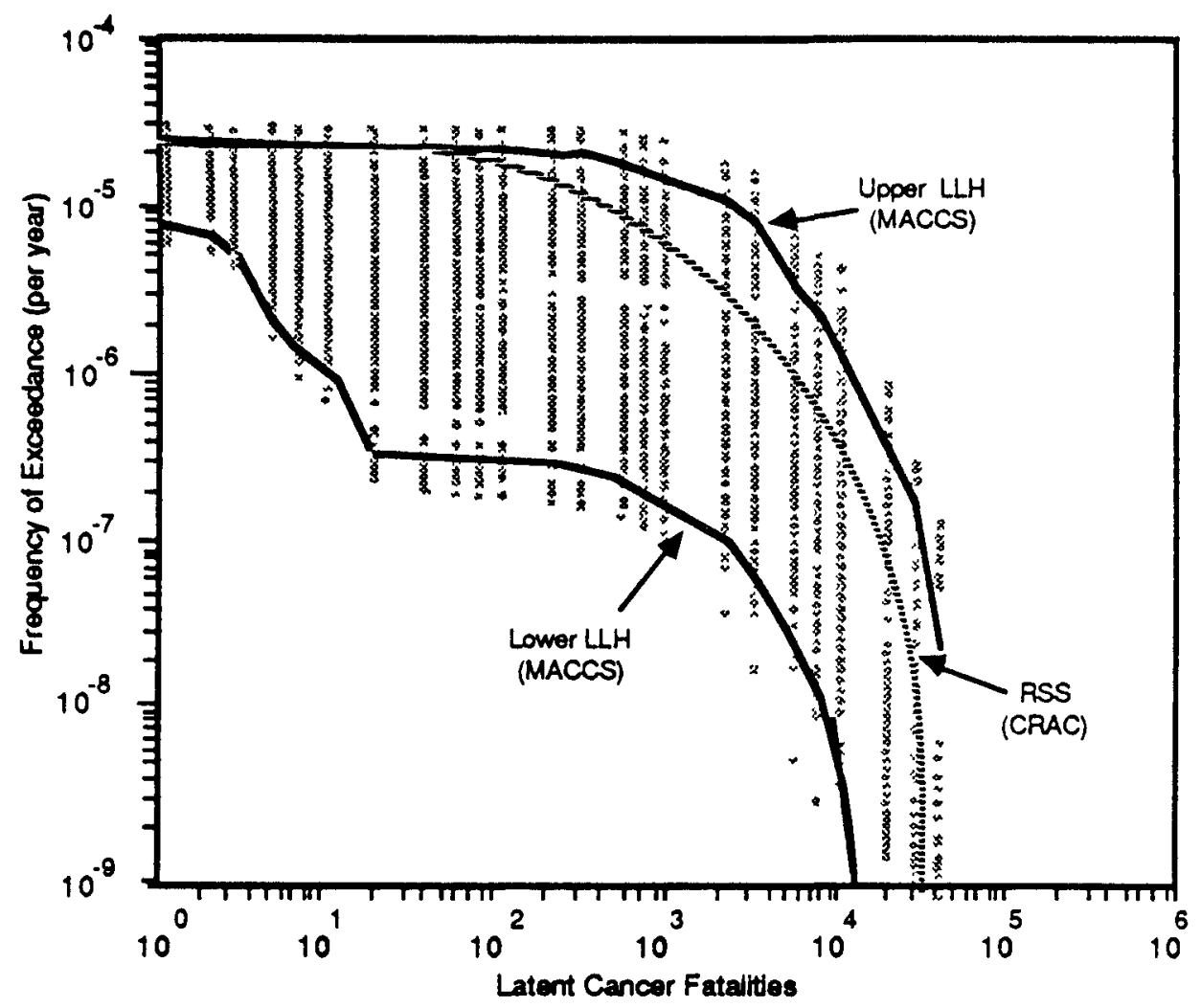

Figure 5-17. CCDF for Latent Cancer Fatalities (MACCS)

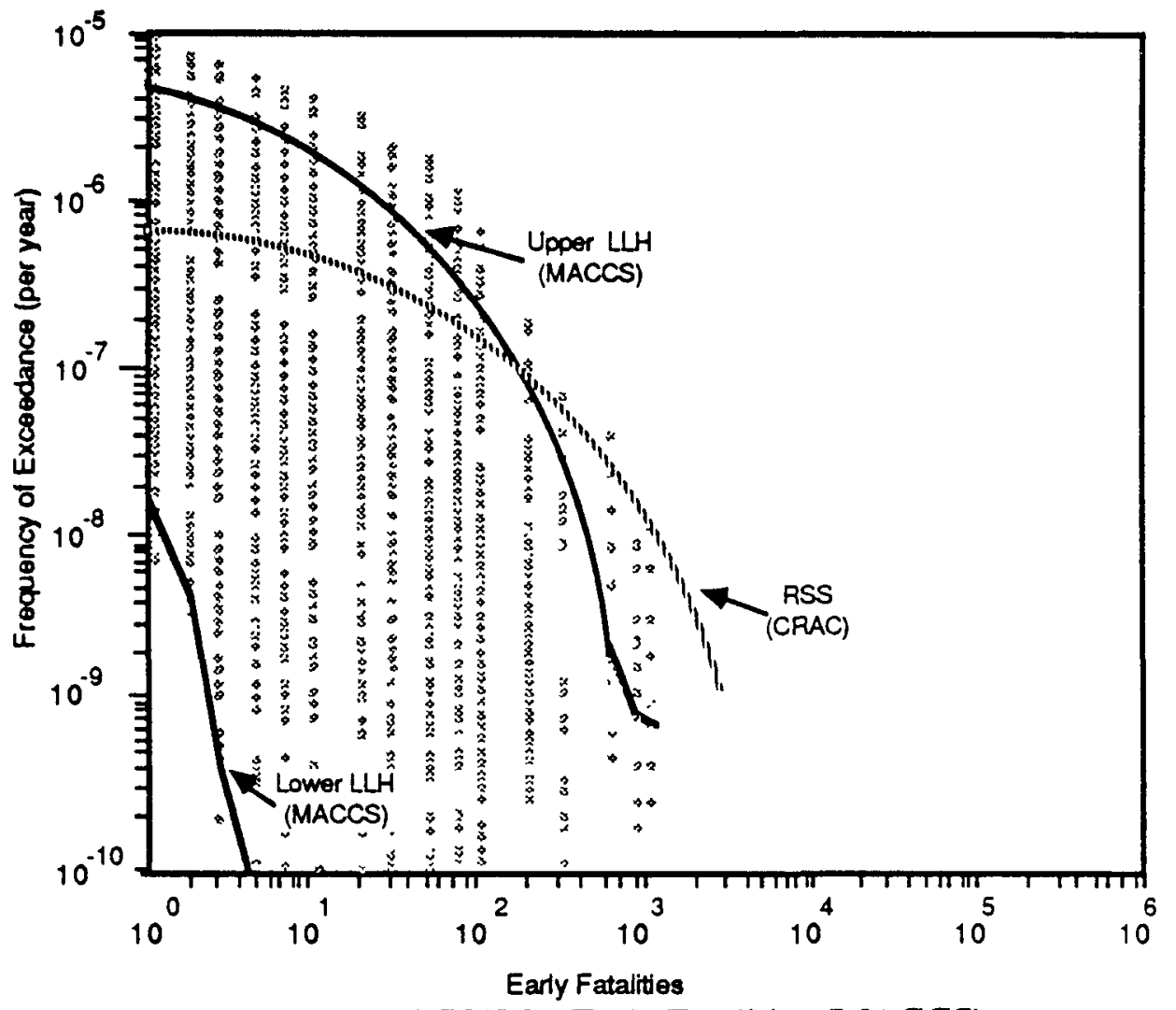

Figure 5-18. CCDF for Early Fatalities (MACCS) 


\section{LLH Uncertainty_Results and Insights}

The LLH uncertainty ranges have been illustrated in the previous sections. The reasons for the ranges and the importance of the various issues are discussed in this section. The first method used to examine the relative importance of the LLH issues was a rank regression analysis of the results, in which the contribution of each issue to the overall variance is calculated (including contribution due to correlation with other issues). The results of these regressions are plotted in Figures 5-19 and 5-20. Unfortunately, the statistical sample (which involved 100 sample members) is too small for fractional contributions less than about $10 \%$ to be significant. From both of the figures it is obvious that the direct heating issue is the most important issue suggested by the rank regression. It should be noted that the issue of importance is the occurrence of and the pressure loading from the direct heating, not the issue regarding the potential source term increase due to this phenomenon. Figure 5-20 also indicates a significant contribution from the issue of aerosol agglomeration. The sample used had a chance correlation between input levels for direct heating and for aerosol agglomeration. Some of the apparent importance of aerosol agglomeration may be a result of this chance correlation, and it is not possible to determine from this particular result whether aerosol agglomeration is or is not an important issue.

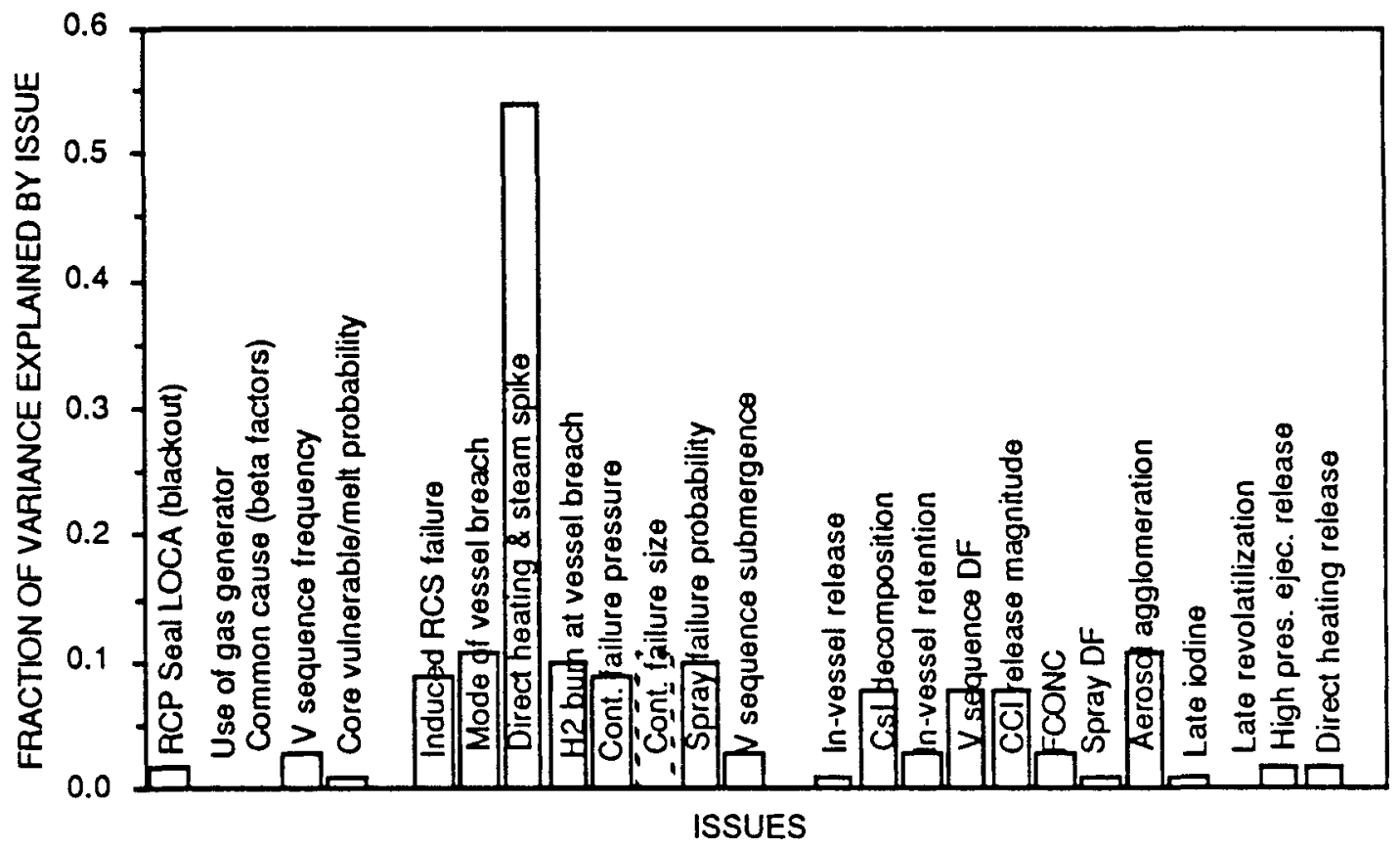

Figure 5-19. Rank Regression Analysis for Risk of Latent Cancer Fatalities 
NUREG/CR-4551,VOL.1: DRAFT REPORT FOR COMMENT (SEPTEMBER, 1986)

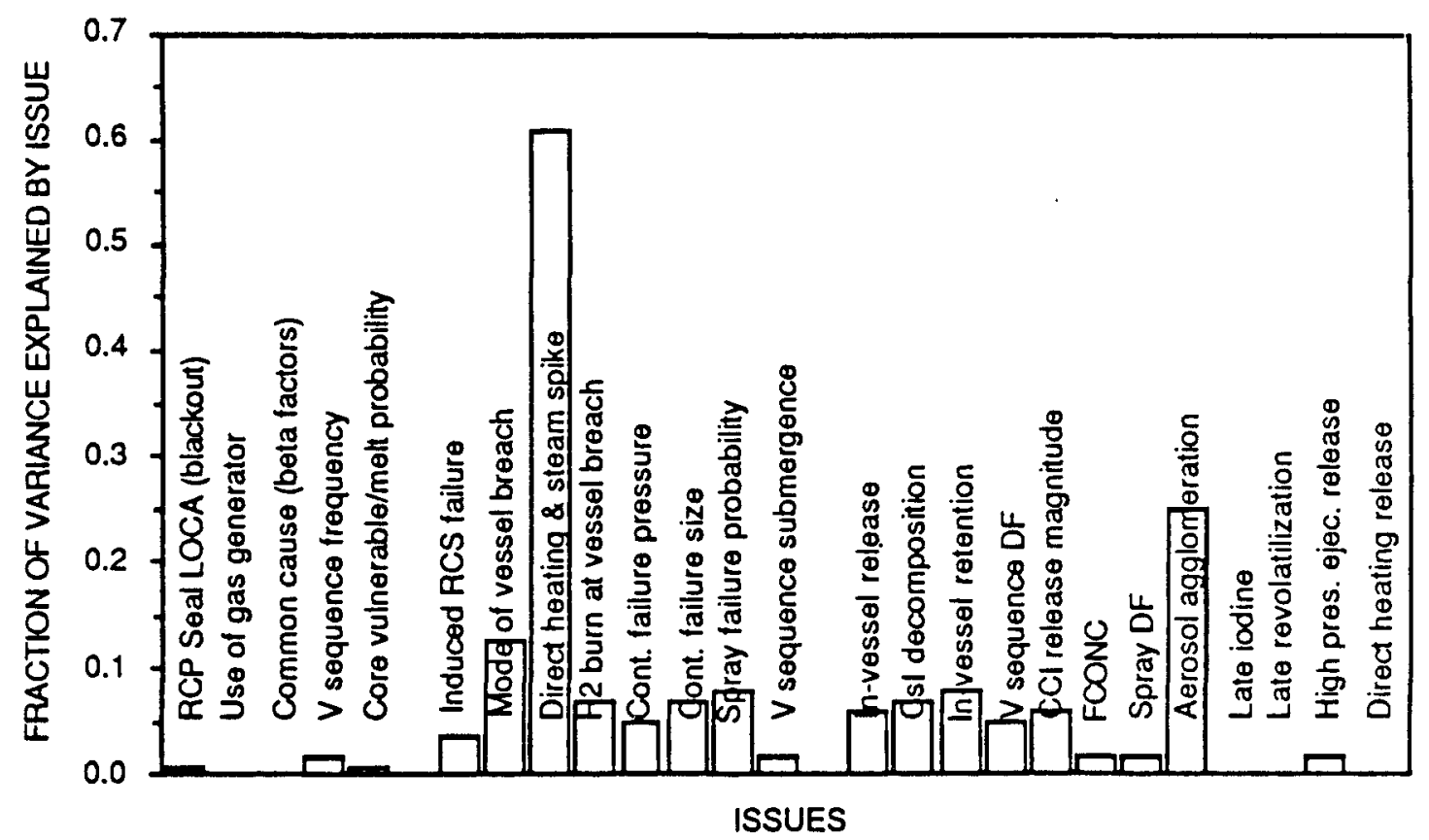

Figure 5-20. Rank Regression Analysis for Risk of Early Fatalities

The importance of direct heating was further investigated through a sensitivity study in which risk was recalculated with the direct heating phenomenon completely removed from all aspects of the study. These results are compared to the risk with direct heating in Figures 5-21 and 5-22, for latent and early fatalities respectively. The comparisons in these figures do illustrate an impact of direct heating, but not as substantial as might have been suggested by the apparent importance of the issue in the rank regression study. The overall reduction in the uncertainty band was less than a factor of five, with a general shift toward lower risk in the mean of the LLH sample members of approximately a factor of five.

Figures 5-23 and 5-24 present the rank regression results for the case without direct heating. For latent cancer fatalities, Figure 5-23, the regression indicates that several issues become more important when direct heating is eliminated: the frequency of the $\mathrm{V}$ sequence, the likelihood of induced RCS LOCAs, the magnitude of hydrogen burn pressure increment at vessel breach, and the containment failure pressure. The apparent influence of containment failure size is due to its correlation with failure pressure and is therefore not an indication of importance of the issue itself. These results reflect the fact that the reviewers provided significant weights for large hydrogen burn pressures that can be quite important for low containment capacity levels. For early fatalities, Figure 5-24, the driving features appear to be source term issues: aerosol agglomeration, the amount of in-vessel retention, and the size of 


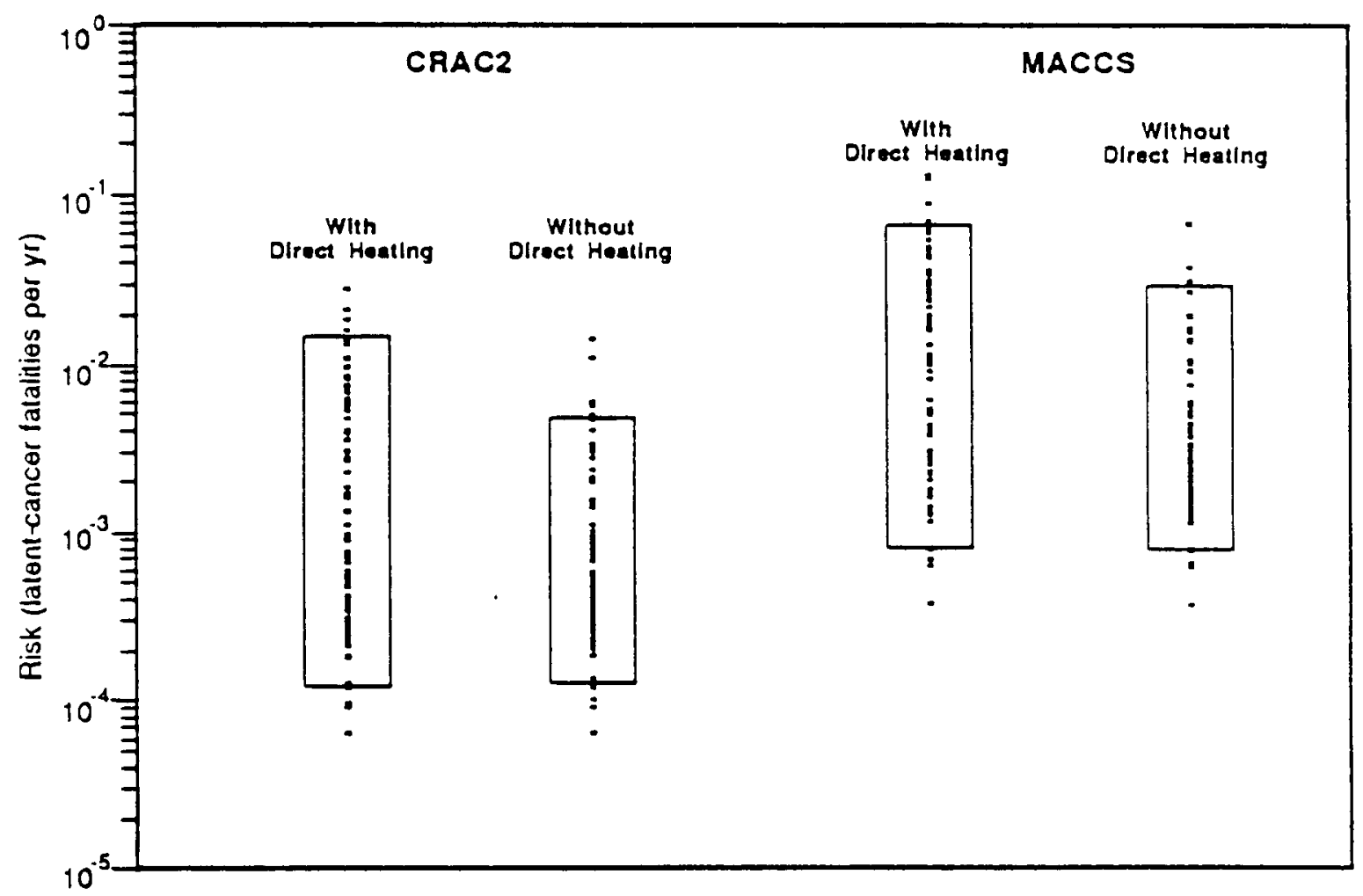

Figure 5-21. Comparison of Results for Latent Fatalities With and Without Direct Heating

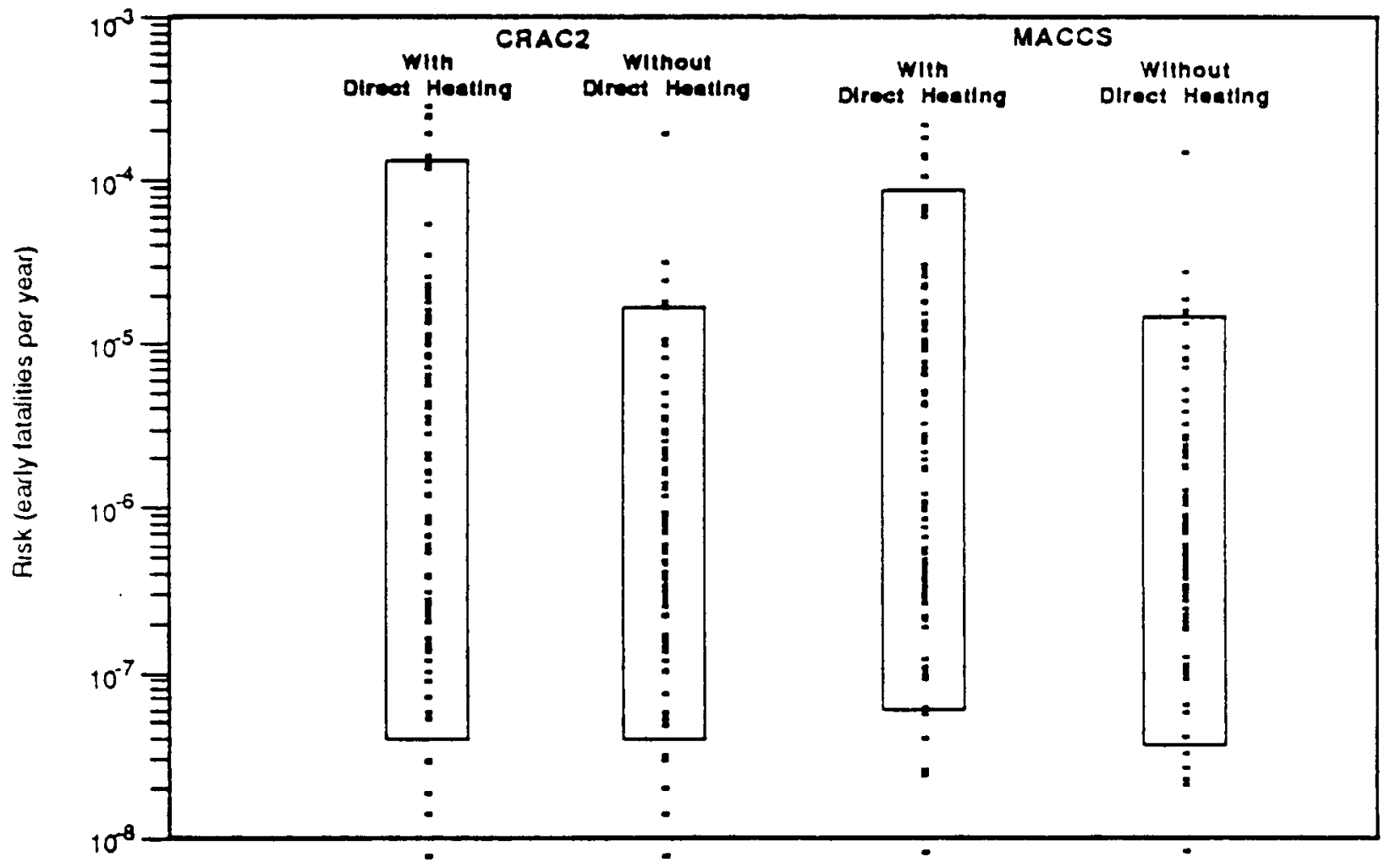

Figure 5-22. Comparison of Early Fatality Risk Results With and Without Direct Heating 
NUREG/CR-4551,VOL.1: DRAFT REPORT FOR COMMENT (SEPTEMBER, 1986)

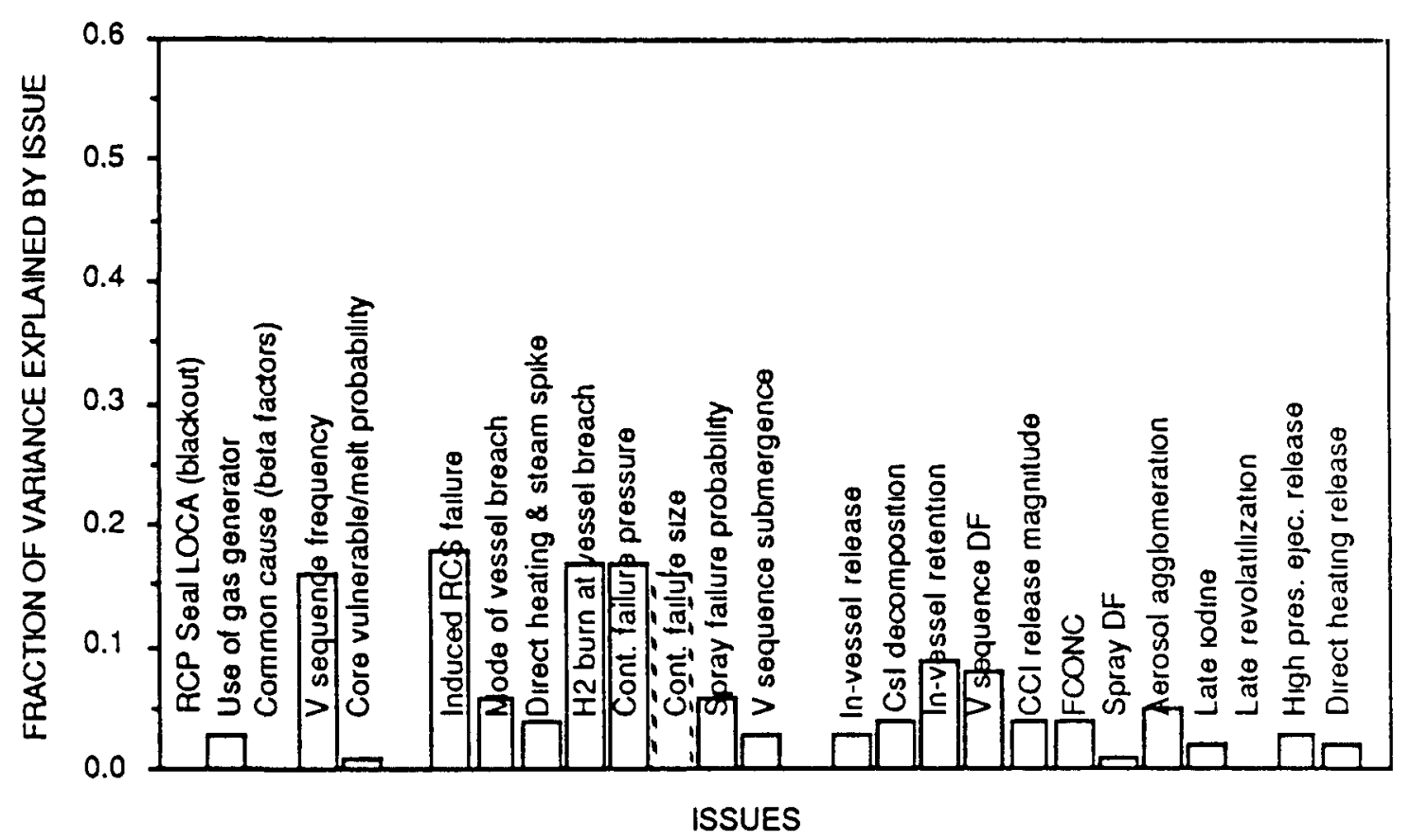

Figure 5-23. Rank Regression Analysis for Risk of Latent Cancer Deaths: No Direct Heating

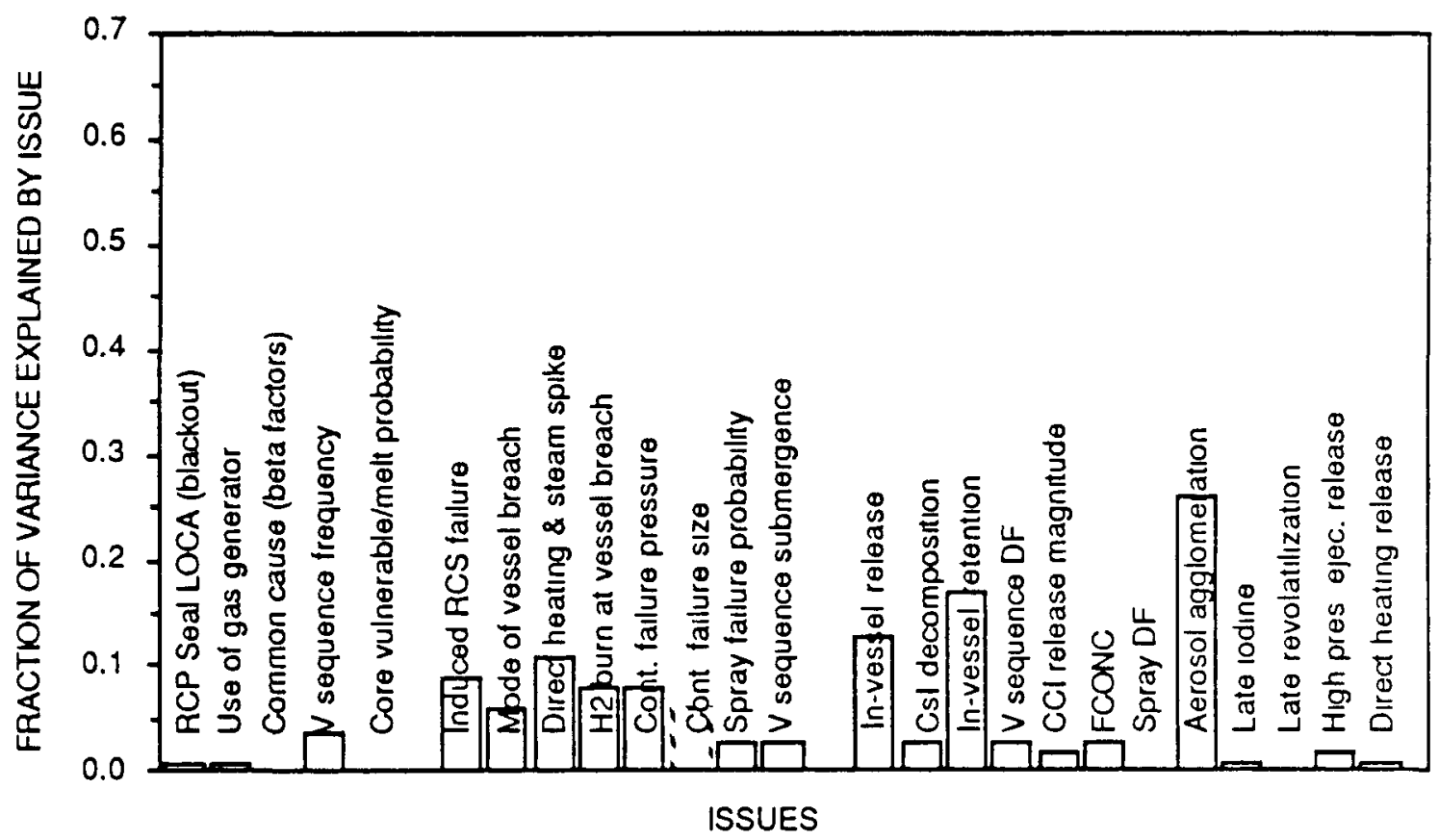

Figure 5-24. Rank Regression Analysis for Risk of Early Fatalities: No Direct Heatung 
the in-vessel release from the fuel. These are issues which have the potential for decreasing the source term below the threshold in the dose effects model for early fatalities. Note also that the inadvertent correlation between aerosol agglomeration and direct heating results in the appearance of direct heating in the rank regression even though the effects of direct heating are completely removed for this sensitivity case.

As described in Section 2, a chi-squared test for goodness of fit was also performed on the LLH results. This test was used to examine the number of samples of a given level of a given issue above and below the median, and to compare these values with what would be expected due to chance alone. Thus, if any level of an issue is present predominantly at high or low risk, this test should highlight that effect. One advantage of this approach is the ability it provides to examine the effects of individual levels of a particular issue. The parameter calculated, the significance, is the probability that the effect of the issue is due to random causes. For example, if the significance calculated in the test is 0.12 for a given level of a given issue, then there is a $12 \%$ chance that the stratification of results with this issue level occurred by chance, or conversely, an $88 \%$ chance that the effect is due to the meaning of the level relative to risk.

The chi-squared test results are listed in Table 5-17. When direct heating is included, direct heating and aerosol agglomeration illustrate the strongest effect, although agglomeration is most important to early fatalities. There are several other individual issue levels which appear to be significant if one considers significance values of $10 \%$ or less. The results for the base case (with direct heating) also suggest the following significant issues:

- The mode of vessel breach is significant, probably because of the correlation between vessel failure mode and direct heating;

- The lowest level of the issue involving hydrogen burn pressures at vessel breach is significant for latent cancer fatalities;

- Containment failure pressure is quite significant for the risk of latent cancer fatality;

- CsI decomposition is important ;

- The decontamination factor for the $\mathrm{V}$ sequence appears to play a role in the uncertainty;

- The in-vessel release magnitude (level 3) and the in-vessel retention (level 1) issues appear to be important to the early fatality risk measure;

- The release magnitudes associated with core-concrete interactions are important to the risk of latent cancer fatalities; and

- Some levels of the issue pertaining to failure of the containment sprays are important. 
The importance of the hydrogen burn pressure issue is from the introduction of a subissue which came to light during the meetings with the expert reviewers. Prior to the expert input, hydrogen burns were not allowed for station blackout scenarios, which are the most important accidents in the core-damage profile. Owing to the failure of containment heat removal, these scenarios were thought to produce a steam-inerted containment, precluding the possibility of hydrogen burning. The review group assigned a fairly high likelihood of significant pressure increments due to hydrogen burns in direct heating scenarios, regardless of the steam concentration. Possible phenomena cited were the local burning or recombination of hydrogen in the vicinity of the hot fuel particles or a change in inerting criteria at very high pressures; in any case the resultant pressure rise was estimated to be large, up to that of a global deflagration. For many of the accidents, it appears that this additional pressure increment may be one of the causes for exceeding the containment pressure capacity. Direct heating alone does not fully account for the early containment failure probabilities seen in the LLH result. Of course, both hydrogen burning and direct heating are intimately coupled with the containment failure pressure.

As illustrated in Table 5-17, the chi-squared test was also performed for the sensitivity study in which all direct heating was removed. The first noticeable feature is that aerosol agglomeration appears as the most significant issue, and this is not due to any correlation with direct heating in this case. A number of other issues also appear to be significant:

- The $V$ sequence frequency (level 3 ) is significant relative to the latent cancer fatality risk measure;

- One level of the issue involving the mode of induced RCS failure (induced large LOCA) also shows significance when direct heating is not included;

- The hydrogen burn pressure increment (level 1) is significant for latent cancer fatalities; and

- Containment failure pressure is important for both risk measures.

Regarding the issue of induced RCS failure, the level involving steam generator tube ruptures could not be evaluated with the chi-squared method because of too few data points. However, visual observation of the individual sample members indicated that all steam generator tube ruptures appeared in the upper regimes of the risk results.

The results of this review confirm the significance of the direct heating and aerosol agglomeration issues, but also point out the fact that the question of an issue's significance relative to uncertainty is quite complex, due to the interactions among the issues. Thus an issue or level that appears to be significant might not be significant if a sample were constructed from 
an alternative set of issues or levels, as illustrated by the results with and without direct heating. The statistical tests cannot really consider all of the combinations of issue levels that might be important, and conclusions regarding the importance of single issues must be carefully qualified.

Table 5-17

RESULTS OF CHI-SQUARED TEST AS CALCULATED WITH CRAC2

\begin{tabular}{|c|c|c|c|c|c|}
\hline \multirow{3}{*}{$\begin{array}{l}\text { Issue \& } \\
\text { Outcome }\end{array}$} & \multicolumn{4}{|c|}{ Chi $^{2}$ Significance } & \multirow[b]{3}{*}{ Comments } \\
\hline & \multicolumn{2}{|c|}{ Early Fatalities } & \multicolumn{2}{|c|}{ Latent Cancer Fatalities } & \\
\hline & With DH & Without $\mathrm{DH}$ & With DH & Without DH & \\
\hline Issue 1 & \multicolumn{5}{|c|}{ SeaLLOCA Probability } \\
\hline $\begin{array}{l}\text { Level } 1 \\
\text { Level } 2 \\
\text { Level } 3\end{array}$ & $\begin{array}{l}\text { NA } \\
1.0 \\
\text { NA }\end{array}$ & $\begin{array}{l}\text { NA } \\
0.8 \\
\text { NA }\end{array}$ & $\begin{array}{l}\text { NA } \\
1.0 \\
\text { NA }\end{array}$ & $\begin{array}{l}\text { NA* } \\
0.8 \\
\text { NA }\end{array}$ & $\begin{array}{l}\text { This issue appears to have almost no } \\
\text { impact on the uncertainty range, but the } \\
\text { sample is too small for statistical tests. }\end{array}$ \\
\hline Issue 2 & \multicolumn{5}{|c|}{ Use of Gas Generator } \\
\hline $\begin{array}{l}\text { Level } 1 \\
\text { Level } 2\end{array}$ & $\begin{array}{l}0.4 \\
0.6\end{array}$ & $\begin{array}{l}0.8 \\
0.6\end{array}$ & $\begin{array}{l}0.8 \\
1.0\end{array}$ & $\begin{array}{l}0.3 \\
0.2\end{array}$ & This appears to be of little importance. \\
\hline Issue 3 & \multicolumn{5}{|c|}{ Beta_Eactor Usage } \\
\hline $\begin{array}{l}\text { Level } 1 \\
\text { Level } 2 \\
\text { Level } 3\end{array}$ & $\begin{array}{l}\text { NA } \\
0.8 \\
0.7\end{array}$ & $\begin{array}{l}\text { NA } \\
0.8 \\
1.0\end{array}$ & $\begin{array}{l}\text { NA } \\
0.8 \\
0.7\end{array}$ & $\begin{array}{l}\text { NA } \\
0.5 \\
0.5\end{array}$ & $\begin{array}{l}\text { Probably of little importance to total } \\
\text { uncertainty. }\end{array}$ \\
\hline Issue 4 & \multicolumn{5}{|c|}{ V Sequence Frequency } \\
\hline $\begin{array}{l}\text { Level } 1 \\
\text { Level } 2 \\
\text { Level } 3\end{array}$ & $\begin{array}{l}0.3 \\
0.4 \\
0.5\end{array}$ & $\begin{array}{l}0.6 \\
0.8 \\
0.2\end{array}$ & $\begin{array}{l}1.0 \\
0.4 \\
0.01\end{array}$ & $\begin{array}{l}0.1 \\
0.6 \\
0.01\end{array}$ & $\begin{array}{l}\text { Level } 3 \text { is significant relative to latent } \\
\text { cancer fatalities. }\end{array}$ \\
\hline Issue 5 & \multicolumn{5}{|c|}{ Containment Eailure Effect on ECCS } \\
\hline $\begin{array}{l}\text { Level } 1 \\
\text { Level } 2 \\
\text { Level } 3\end{array}$ & $\begin{array}{l}0.3 \\
0.5 \\
0.7\end{array}$ & $\begin{array}{l}0.8 \\
0.5 \\
0.3\end{array}$ & $\begin{array}{l}0.7 \\
0.7 \\
1.0\end{array}$ & $\begin{array}{l}0.5 \\
1.0 \\
0.5\end{array}$ & No significance. \\
\hline Issue 6 & \multicolumn{5}{|c|}{ Location and Size of Induced RCS Failure } \\
\hline $\begin{array}{l}\text { Level } 1 \\
\text { Level } 2 \\
\text { Level } 3 \\
\text { Level } 4 \\
\text { Level } 5 \\
\text { Level } 6\end{array}$ & $\begin{array}{l}0.6 \\
0.7 \\
0.7 \\
\text { NA } \\
\text { NA } \\
1.0\end{array}$ & $\begin{array}{l}0.3 \\
1.0 \\
0.7 \\
\text { NA } \\
\text { NA } \\
0.7\end{array}$ & $\begin{array}{l}0.6 \\
0.2 \\
1.0 \\
\text { NA } \\
\text { NA } \\
1.0\end{array}$ & $\begin{array}{l}0.6 \\
0.07 \\
0.7 \\
\text { NA } \\
\text { NA } \\
0.3\end{array}$ & $\begin{array}{l}\text { Difficult to establish significance due to } \\
\text { the numerous levels, although a large } \\
\text { induced LOCA (level 2) does appear } \\
\text { important to latent cancers for the no } \\
\text { direct heating case. }\end{array}$ \\
\hline Issue ] & \multicolumn{5}{|c|}{ Mode of Vessel Breach } \\
\hline $\begin{array}{l}\text { Level } 1 \\
\text { Level } 2 \\
\text { Level } 3 \\
\text { Level } 4 \\
\text { Level } 5 \\
\text { Level } 6 \\
\text { Level } 7 \\
\text { Level } 8 \\
\text { Level } 9\end{array}$ & $\begin{array}{l}0.5 \\
0.2 \\
0.06 \\
0.2 \\
0.2 \\
1.0 \\
0.08 \\
0.5 \\
1.0\end{array}$ & $\begin{array}{l}0.2 \\
0.6 \\
0.6 \\
0.6 \\
0.2 \\
0.2 \\
0.6 \\
1.0 \\
1.0\end{array}$ & $\begin{array}{l}0.5 \\
0.06 \\
0.3 \\
0.08 \\
0.07 \\
1.0 \\
1.0 \\
1.0 \\
1.0\end{array}$ & $\begin{array}{l}0.6 \\
0.2 \\
0.2 \\
0.2 \\
0.5 \\
1.0 \\
0.6 \\
0.2 \\
0.6\end{array}$ & $\begin{array}{l}\text { The mode of vessel breach is only } \\
\text { important when direct heating is an issue } \\
\text { since direct heating only occurs for a } \\
\text { pressurized ejection. }\end{array}$ \\
\hline
\end{tabular}

*NA implies that there were too few sample members with this outcome to permit a chi-squared test. 
Table 5-17 (Continued)

RESULTS OF CHI-SQUARED TEST AS CALCULATED WITH CRAC2

\begin{tabular}{|c|c|c|c|c|c|}
\hline \multirow{3}{*}{$\begin{array}{l}\text { Issue \& } \\
\text { Outcome }\end{array}$} & \multicolumn{4}{|c|}{$\mathrm{Chi}^{2}$ Significance } & \multirow[b]{3}{*}{ Comments } \\
\hline & \multicolumn{2}{|c|}{ Early Eatalities } & \multicolumn{2}{|c|}{ Latent Cancer Fatalities } & \\
\hline & With DH & Without $\mathrm{DH}$ & With DH & Without $\mathrm{DH}$ & \\
\hline Issue 8 & \multicolumn{5}{|c|}{ Direct Heating } \\
\hline Level 1 & 0.05 & - & 0.005 & - & \multirow{5}{*}{$\begin{array}{l}\text { As with the other methods of review, } \\
\text { the Chi-squared test points to direct } \\
\text { heating as a very important issue. }\end{array}$} \\
\hline Level 2 & 0.0 & - & 0.0 & - & \\
\hline Level 3 & 0.04 & - & 0.1 & - & \\
\hline Level 4 & 0.003 & - & 0.0 & - & \\
\hline Level 5 & 0.002 & - & 0.002 & - & \\
\hline Issue 9 & \multicolumn{5}{|c|}{ Hydrogen Bum at Vessel Breach } \\
\hline Level 1 & 0.1 & 0.3 & 0.02 & 0.05 & \multirow{6}{*}{$\begin{array}{l}\text { Level } 1 \text { is of some importance for } \\
\text { the latent cancer risk measure. }\end{array}$} \\
\hline Level 2 & 1.0 & 0.2 & 1.0 & 0.2 & \\
\hline Level 3 & 0.1 & 0.1 & 0.2 & 0.2 & \\
\hline Level 4 & 1.0 & 0.7 & 0.7 & 0.7 & \\
\hline Level 5 & 0.2 & 0.2 & 1.0 & 1.0 & \\
\hline Level 6 & NA & NA & NA & NA & \\
\hline Issue 10 & \multicolumn{5}{|c|}{ Containment Eailure Pressure } \\
\hline Level 1 & 0.2 & 0.07 & 0.007 & 0.007 & \multirow{4}{*}{$\begin{array}{l}\text { The lowest level of containment } \\
\text { capability (level 1) is significant, } \\
\text { particularly when direct heating is } \\
\text { removed. }\end{array}$} \\
\hline Level 2 & 0.8 & 0.8 & 0.6 & 0.8 & \\
\hline Level 3 & 0.7 & 0.2 & 0.7 & 0.05 & \\
\hline Level 4 & NA & NA & NA & NA & \\
\hline Issue 11 & \multicolumn{5}{|c|}{ Centainment Eailure Size } \\
\hline Level 1 & 0.1 & 0.3 & 0.04 & 0.1 & \multirow{6}{*}{$\begin{array}{l}\text { This issue is generally not significant } \\
\text { except perhaps for level } 6 \text { for latent } \\
\text { cancers. }\end{array}$} \\
\hline Level 2 & NA & NA & $\mathrm{NA}$ & NA & \\
\hline Level 3 & 0.4 & 1.0 & 0.4 & 0.7 & \\
\hline Level 4 & 0.1 & 0.5 & 0.3 & 1.0 & \\
\hline Level 5 & 0.6 & 1.0 & 0.1 & 0.3 & \\
\hline Level 6 & 1.0 & 0.3 & 0.02 & 0.02 & \\
\hline Issue 12 & \multicolumn{5}{|c|}{ Spray Eailure Probability } \\
\hline Level 1 & 1.0 & 0.7 & 0.4 & 0.7 & \multirow{5}{*}{$\begin{array}{l}\text { The sprays have some effect on the } \\
\text { release, but are mainly important } \\
\text { for the direct heating case. }\end{array}$} \\
\hline Level 2 & 0.07 & 0.4 & 0.03 & 0.03 & \\
\hline Level 3 & 0.4 & 1.0 & 0.07 & 0.2 & \\
\hline Level 4 & 0.07 & 0.2 & 0.07 & 1.0 & \\
\hline Level 54 & 0.2 & 0.7 & 0.4 & 1.0 & \\
\hline Issue 13 & \multicolumn{5}{|c|}{ Brobability of V Sequence Submergence } \\
\hline Level 1 & 0.5 & 0.2 & 0.5 & 1.0 & \multirow{5}{*}{$\begin{array}{l}\text { This issue did not illustrate significance } \\
\text { at any level. }\end{array}$} \\
\hline Level 2 & 0.3 & 0.5 & 0.1 & 0.5 & \\
\hline Level 3 & 0.5 & 0.5 & 0.1 & 0.5 & \\
\hline Level 4 & 0.5 & 0.2 & 1.0 & 1.0 & \\
\hline Level 5 & 1.0 & 0.6 & 0.5 & 0.5 & \\
\hline Issue 14 & \multicolumn{5}{|c|}{ In-Vessel Release } \\
\hline Level 1 & 0.4 & 0.04 & 0.7 & 0.4 & \multirow{4}{*}{$\begin{array}{l}\text { This issue appears to be of some } \\
\text { importance relative to early fatalities, } \\
\text { but it depends on whether or not direct } \\
\text { heating has occurred. }\end{array}$} \\
\hline Level 2 & 0.2 & 1.0 & 1.0 & 0.6 & \\
\hline Level 3 & 0.04 & 0.9 & 0.7 & 1.0 & \\
\hline Level 4 & NA & NA & NA & NA & \\
\hline Issue 15 & \multicolumn{5}{|c|}{ CsLDecomposition } \\
\hline Level 1 & 0.07 & 0.7 & 0.007 & 0.4 & \multirow{4}{*}{$\begin{array}{l}\text { This issue (level 1) appears to be } \\
\text { significant for early fatalities, but } \\
\text { only when direct heating has occurred. }\end{array}$} \\
\hline Level 2 & 0.07 & 0.7 & 0.8 & 0.8 & \\
\hline Level 3 & 0.3 & 0.5 & 0.1 & 0.7 & \\
\hline Level 4 & 0.6 & 0.6 & 1.0 & 0.6 & \\
\hline
\end{tabular}


NUREG/CR-4551,VOL.1: DRAFT REPORT FOR COMMENT (SEPTEMBER, 1986)

Table 5-17 (Continued)

RESULTS OF CHI-SQUARED TEST AS CALCULATED WITH CRAC2

\begin{tabular}{|c|c|c|c|c|c|}
\hline \multirow{3}{*}{$\begin{array}{l}\text { Issue \& } \\
\text { Outcome }\end{array}$} & \multicolumn{4}{|c|}{$\mathrm{Chi}^{2}$ Significance } & \multirow[b]{3}{*}{ Comments } \\
\hline & \multicolumn{2}{|c|}{ Early Eatalities } & \multicolumn{2}{|c|}{ Latent Cancer Fatalities } & \\
\hline & With DH & Without $\mathrm{DH}$ & With DH & Without $\mathrm{DH}$ & \\
\hline Issue 16 & In-Vessel & etention & & & \multirow[b]{2}{*}{$\begin{array}{l}\text { This issue appears to be of some } \\
\text { significance relative to early fatalities, } \\
\text { with an increased effect when direct } \\
\text { heating is turned off. }\end{array}$} \\
\hline $\begin{array}{l}\text { Level } 1 \\
\text { Level } 2 \\
\text { Level } 3 \\
\text { Level } 4\end{array}$ & $\begin{array}{l}0.01 \\
1.0 \\
0.3 \\
1.0\end{array}$ & $\begin{array}{l}0.02 \\
0.3 \\
0.3 \\
0.07\end{array}$ & $\begin{array}{l}0.2 \\
1.0 \\
0.7 \\
1.0\end{array}$ & $\begin{array}{l}0.6 \\
0.1 \\
0.3 \\
0.2\end{array}$ & \\
\hline Issue 17 & \multicolumn{5}{|c|}{ V Sequence Decontamination } \\
\hline $\begin{array}{l}\text { Level } 1 \\
\text { Level } 2 \\
\text { Level } 3 \\
\text { Level } 4\end{array}$ & $\begin{array}{l}0.09 \\
0.4 \\
0.2 \\
0.7\end{array}$ & $\begin{array}{l}0.1 \\
1.0 \\
0.3 \\
1.0\end{array}$ & $\begin{array}{l}0.03 \\
1.0 \\
0.2 \\
1.0\end{array}$ & $\begin{array}{l}0.1 \\
0.1 \\
0.2 \\
0.2\end{array}$ & $\begin{array}{l}\text { Level } 1 \text { tests significant for early } \\
\text { fatalities when direct heating is } \\
\text { included. }\end{array}$ \\
\hline Issue 19 & \multicolumn{5}{|c|}{ Magnitude of CCL Releases } \\
\hline $\begin{array}{l}\text { Level } 1 \\
\text { Level } 2 \\
\text { Level } 3\end{array}$ & $\begin{array}{l}1.0 \\
0.3 \\
0.03\end{array}$ & $\begin{array}{l}1.0 \\
0.8 \\
1.0\end{array}$ & $\begin{array}{l}0.4 \\
0.06 \\
0.01\end{array}$ & $\begin{array}{l}0.4 \\
0.6 \\
0.3\end{array}$ & $\begin{array}{l}\text { The test does not show this as a } \\
\text { significant issue. }\end{array}$ \\
\hline Issue 20 & \multicolumn{5}{|c|}{ CCLReleases from Containment } \\
\hline $\begin{array}{l}\text { Level } 1 \\
\text { Level } 2 \\
\text { Level } 3 \\
\text { Level } 4\end{array}$ & $\begin{array}{l}1.0 \\
1.0 \\
0.7 \\
0.6\end{array}$ & $\begin{array}{l}0.7 \\
1.0 \\
0.7 \\
0.3\end{array}$ & $\begin{array}{l}1.0 \\
1.0 \\
0.5 \\
0.3\end{array}$ & $\begin{array}{l}1.0 \\
0.7 \\
0.3 \\
0.3\end{array}$ & $\begin{array}{l}\text { This issue does not appear to be } \\
\text { significant. }\end{array}$ \\
\hline Issue 21 & \multicolumn{5}{|c|}{ Spray Decontamination } \\
\hline $\begin{array}{l}\text { Level } 1 \\
\text { Level } 2 \\
\text { Level } 3\end{array}$ & $\begin{array}{l}0.7 \\
0.4 \\
0.4\end{array}$ & $\begin{array}{l}1.0 \\
0.8 \\
0.7\end{array}$ & $\begin{array}{l}0.4 \\
0.4 \\
0.7\end{array}$ & $\begin{array}{l}0.4 \\
1.0 \\
0.4\end{array}$ & $\begin{array}{l}\text { The value for the DF due to sprays } \\
\text { does not lead to major uncertainty in the } \\
\text { result }\end{array}$ \\
\hline Issue 22 & \multicolumn{5}{|c|}{ Aerosol Agglomeration } \\
\hline $\begin{array}{l}\text { Level } 1 \\
\text { Level } 2 \\
\text { Level } 3\end{array}$ & $\begin{array}{l}0.003 \\
0.5 \\
0.004\end{array}$ & $\begin{array}{l}0.003 \\
0.3 \\
0.001\end{array}$ & $\begin{array}{l}0.09 \\
0.3 \\
0.02\end{array}$ & $\begin{array}{l}0.4 \\
0.3 \\
0.1\end{array}$ & $\begin{array}{l}\text { This issue appears to be quite significant } \\
\text { for early fatalities. See discussion of } \\
\text { this point in the main text. }\end{array}$ \\
\hline Issue 23 & \multicolumn{5}{|c|}{ Late lodine Releases } \\
\hline $\begin{array}{l}\text { Level } 1 \\
\text { Level } 2 \\
\text { Level } 3 \\
\text { Level } 4\end{array}$ & $\begin{array}{l}0.2 \\
0.4 \\
0.8 \\
1.0\end{array}$ & $\begin{array}{l}0.6 \\
0.3 \\
0.8 \\
0.4\end{array}$ & $\begin{array}{l}0.3 \\
0.7 \\
1.0 \\
0.4\end{array}$ & $\begin{array}{l}0.6 \\
0.4 \\
0.8 \\
0.2\end{array}$ & $\begin{array}{l}\text { This issue does not illustrate } \\
\text { significance by this test. }\end{array}$ \\
\hline Issue 24 & \multicolumn{5}{|c|}{ Late Revolatilization } \\
\hline $\begin{array}{l}\text { Level } 1 \\
\text { Level } 2 \\
\text { Level } 3 \\
\text { Level } 4\end{array}$ & $\begin{array}{l}\text { NA } \\
0.3 \\
0.5 \\
1.0\end{array}$ & $\begin{array}{l}\text { NA } \\
0.8 \\
0.8 \\
0.7\end{array}$ & $\begin{array}{l}\text { NA } \\
0.6 \\
1.0 \\
0.7\end{array}$ & $\begin{array}{l}\text { NA } \\
1.0 \\
0.2 \\
0.03\end{array}$ & $\begin{array}{l}\text { This issue does not illustrate } \\
\text { significance by this test. }\end{array}$ \\
\hline Lssue 25 & \multicolumn{5}{|c|}{ High Pressure Ejection Release } \\
\hline $\begin{array}{l}\text { Level } 1 \\
\text { Level } 2 \\
\text { Level } 3 \\
\text { Level } 4\end{array}$ & $\begin{array}{l}\text { NA } \\
0.3 \\
0.8 \\
\text { NA }\end{array}$ & $\begin{array}{l}\text { NA } \\
0.7 \\
0.6 \\
\text { NA }\end{array}$ & $\begin{array}{l}\text { NA } \\
0.2 \\
0.3 \\
\text { NA }\end{array}$ & $\begin{array}{l}\mathrm{NA} \\
0.3 \\
0.4 \\
\mathrm{NA}\end{array}$ & $\begin{array}{l}\text { This issue does not illustrate } \\
\text { significance by this test. }\end{array}$ \\
\hline Issue 26 & \multicolumn{5}{|c|}{ Direct Heating Release } \\
\hline $\begin{array}{l}\text { Level } 1 \\
\text { Level } 2 \\
\text { Level } 3 \\
\text { Level } 4\end{array}$ & $\begin{array}{l}\text { NA } \\
0.7 \\
0.5 \\
0.6\end{array}$ & $\begin{array}{l}0.3 \\
0.4 \\
0.8 \\
0.6\end{array}$ & $\begin{array}{l}0.6 \\
0.7 \\
1.0 \\
1.0\end{array}$ & $\begin{array}{l}0.6 \\
0.4 \\
0.4 \\
1.0\end{array}$ & $\begin{array}{l}\text { This issue does not illustrate } \\
\text { significance by this test. }\end{array}$ \\
\hline
\end{tabular}


In a further attempt to help identify important uncertainties, the sample members at either end of the range were reviewed in detail. Of the $100 \mathrm{LLH}$ sample members, the top 20 and bottom 20 contributors to early and latent fatalities were examined individually and compared to each other. The results of that examination are included in Appendix D. In general, the detailed review of the top and bottom twenty sample members corroborated the chi-squared analysis results.

\section{Comparison to the OCP Results}

As described earlier, one of the goals of the Surry analysis was to compare the results of the two uncertainty representations, LLH and OCP. This section describes that comparison and discusses the rather large differences in some results as calculated by the two methods. Figures 5-25 and 5-26 portray this comparison for the overall risk measures of early and latent cancer fatalities. Before discussion of the comparison, it is useful to note the uncertainty associated with data in the ASEP analysis of core-damage frequency. As illustrated, the uncertainty is approximately one order of magnitude. None of the other uncertainty representations include this uncertainty, a fact that must be emphasized when drawing conclusions concerning the range of uncertainty in the results.

The results also illustrate a breakdown of the LLH issues. The system issues alone account for an order of magnitude uncertainty, while the system and containment issues bring about a shift upward in risk and a factor of three to five greater spread. The most interesting result is for the combination of all issues for the early fatality risk measures. The addition of the source term issues (to the risk calculated for the system and containment issues) brings about a factor of 20 increase in the mean and greater than an order of magnitude increase in the uncertainty range. This result appears to be anomalous at first glance, since the regression analysis and other reviews of top and bottom sample members identified containment issues (particularly direct heating) to be the highest contributors to the overall LLH variance. However, the result is explained when one recognizes two factors. First, it is the combination of containment and source term issues that produces the high LLH risk results for many sample members. The second reason is that containment issue results are more heavily dominated by one issue (direct heating), while the contribution of the source term issues is more or less equally spread among several issues, any of which can independently produce relatively high source terms. The latent cancer fatality results do not illustrate this point as strongly, and the containment issues are the principal cause of the large uncertainty range. The chi-squared results also point to the significance of source term issues to early fatalities. 


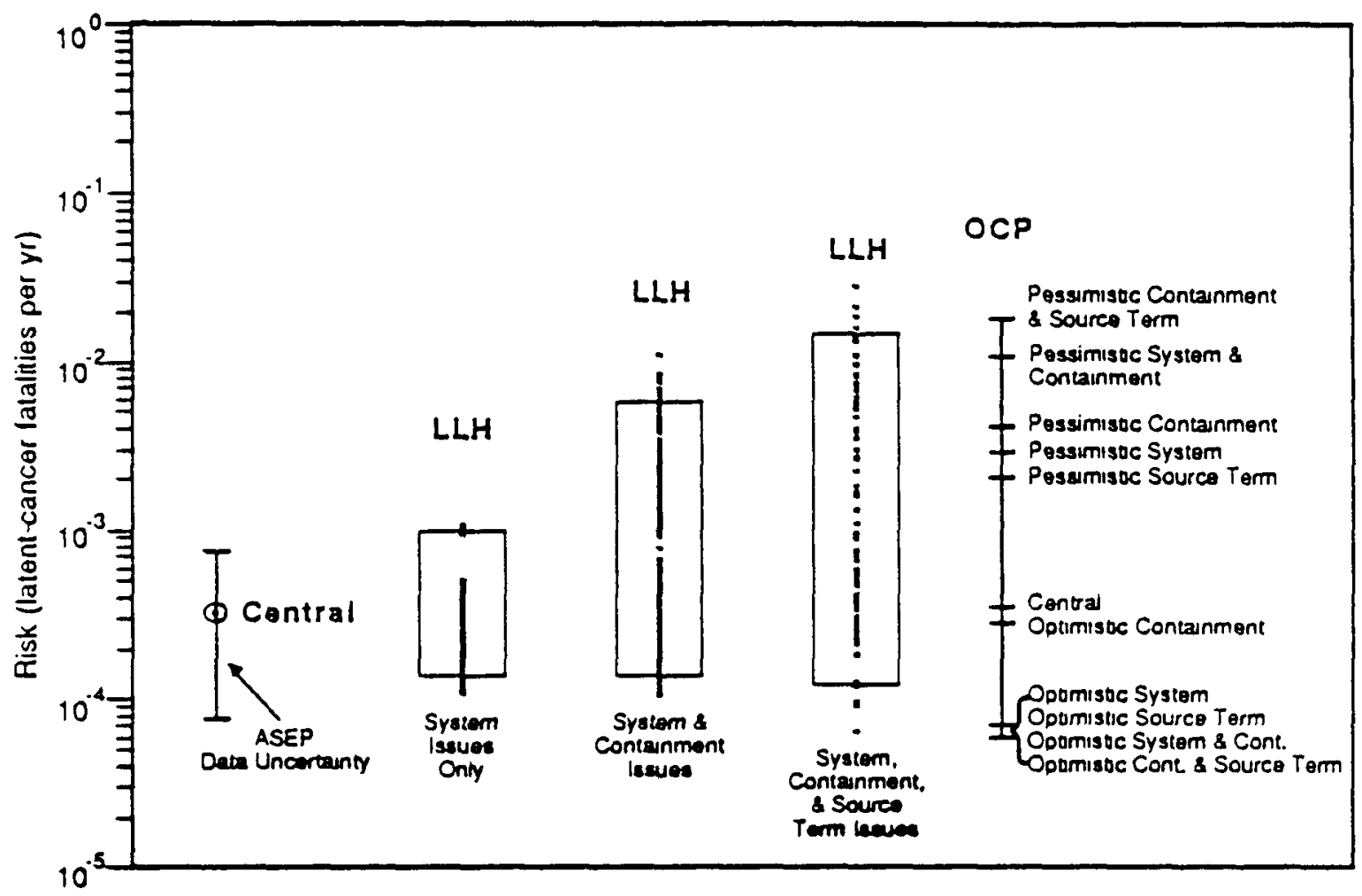

Figure 5-25. Comparison of LLH and OCP Results: Latent Cancer Fatalities

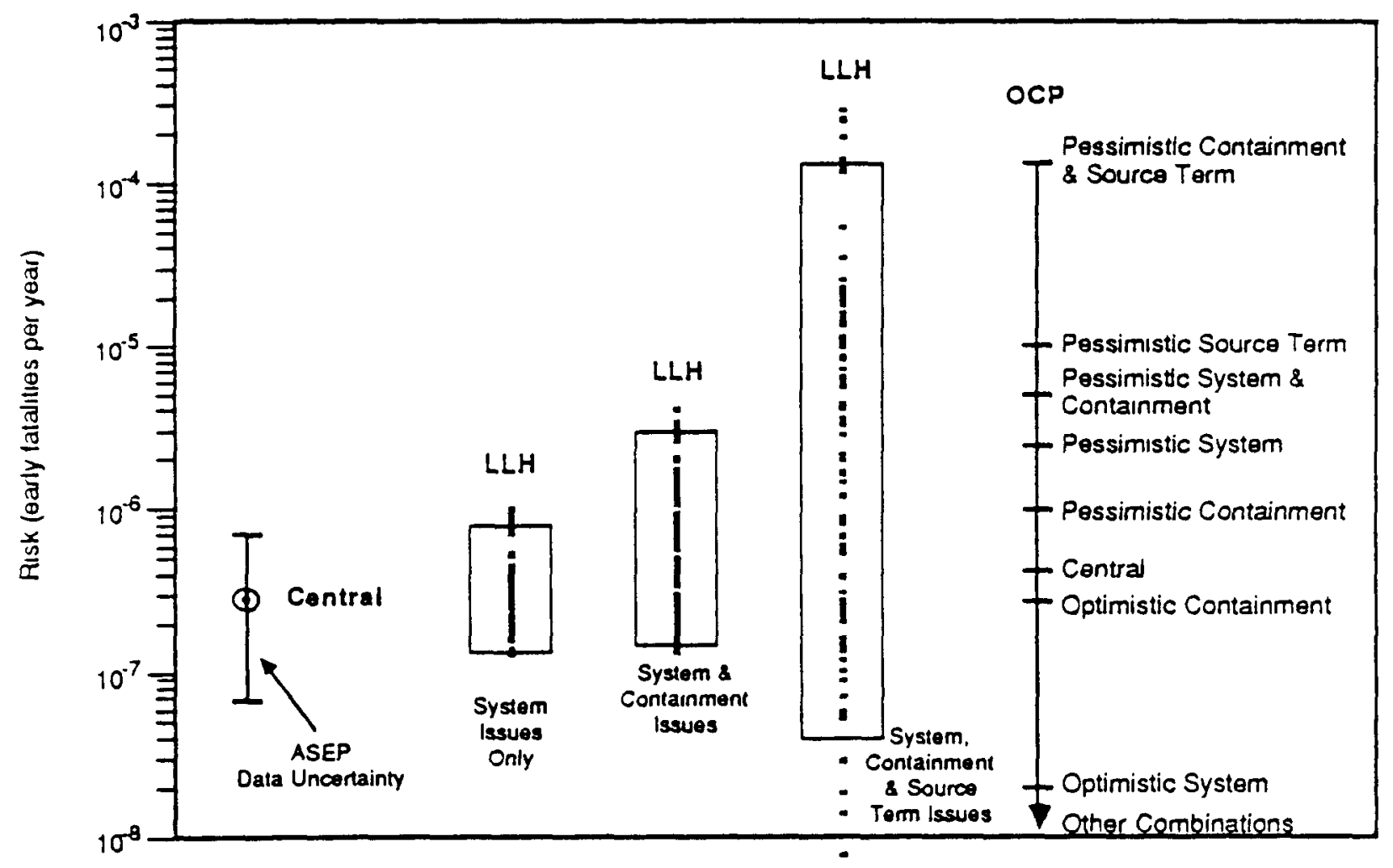

Figure 5-26. Comparison of LLH and OCP Results: Early Fatalities 
Some of the OCP results are also plotted. The comparison for the latent cancer fatality risk measure is illustrated in Figure 5-25. The OCP range, considering up to two combinations of optimistic or pessimistic results, nearly matches the LLH range. The OCP central estimate is a factor of four lower than the LLH median and eight times lower than the LLH mean.

For early fatalities, the central estimate is approximately eight times lower than the $\mathrm{LLH}$ median and 50 times times lower than the $\mathrm{LLH}$ mean. The combination of double pessimistic uncertainties of containment and source term analysis coincides with the 95th percentile of the LLH samples. The lower range of uncertainty associated with optimistic source terms or any double combinations of source terms is significantly lower $\left(<10^{-10}\right)$ than the bottom of the LLH distribution. Thus, the OCP results for early fatalities indicate an uncertainty of four orders of magnitude, just considering optimistic and pessimistic source terms with all other inputs at their central values. The OCP results therefore seem to point to a significantly larger uncertainty in the downward direction (less risk) than the LLH results. It should be noted, however, that the results for early fatality risk at the low end of the LLH range are highly influenced by threshold effects in the dose-health effect relationship, and that in both cases the low end of the risk uncertainty is so low as to constitute a negligible risk. With all of this information in hand, it is straightforward to state several factors contributing to the differences between the OCP central estimates and the LLH results:

- The direct heating phenomenon was not significant in the central estimate of the OCP but was very significant in most of the LLH samples;

- The hydrogen bum pressures were less in the OCP than for the average LLH input, and hydrogen burning was never allowed in a classically steaminert environment in the OCP study;

- The central estimate had one containment pressure capability distribution while the LLH sampled other distributions with lower failure pressures;

- Several source term LLH issues raised the magnitude of the source term above the central estimate; for example, CsI decomposition and late revolatilization from the RCS were not included in the central estimate; and

- The central estimate had very few early containment failures, and the damage states with containment bypass or failure prior to loss of core cooling therefore dominated.

In summary, the OCP and LLH studies did yield some different insights which help to identify the actual uncertainties in the process, since the difference seem to further demonstrate the uncertainties in some issues which dominated the results. To a certain extent, these differences are not surprising, since the two analyses represent the views of two different groups of analysts: the SARRP analysts and the expert reviewers for the LLH process. A comparison of the ranges of the OCP and LLH results seems to indicate that the LLH review 
group tended toward what was called the pessimistic result in the OCP study for a number of very important issues; further, the limited makeup of the group providing the LLH input may not have allowed for the broader interpretation of several issues that would have been obtained if larger representation of the safety community could have been included.

\subsubsection{Other Risk_Measures}

The other risk measures (early injuries, individual risk of death, offsite costs, ano population dose) are presented in this section. The results are presented with only limited discussion, since the insights in the previous section apply to these results, and no new insights were discovered through the calculation of these additional measures. Some additional detail is provided in Appendix $\mathrm{D}$ for the interested reader. The risk presentations in this section are less detailed than those presented previously. Each graph illustrates the range of LLH outcomes, the OCP central estimate, and the ASEP uncertainty for data as an additional perspective on an uncertainty not included in the LLH technique. The rank regression analyses for the other risk measures indicate the same issues as discussed previously and are therefore not presented here.

Figure 5-27 illustrates the results for the early injuries. The CRAC2 results for this risk measure are greater than MACCS due to the calculation of prodromal vomiting in the CRAC2 code, as discussed in Section 5.4.

The results for individual risk of death are presented in Figure 5-28. The results indicate similar trends to the risk measures already presented, with the difference between CRAC2 and MACCS resulting from the modeling differences discussed in Section 5.4. The offsite costs and population dose shown in Figures 5-29 and 5-30 are similar to the latent cancer results, since all three measures are driven by the total source term released offsite. MACCS predicts cost about twice as high as illustrated on the figure, while the population dose is higher for MACCS for the same reasons as the differences in the latent cancer consequences predicted by the two codes. 
NUREG/CR-4551,VOL1: DRAFT REPORT FOR COMMENT (SEPTEMBER, 1986)

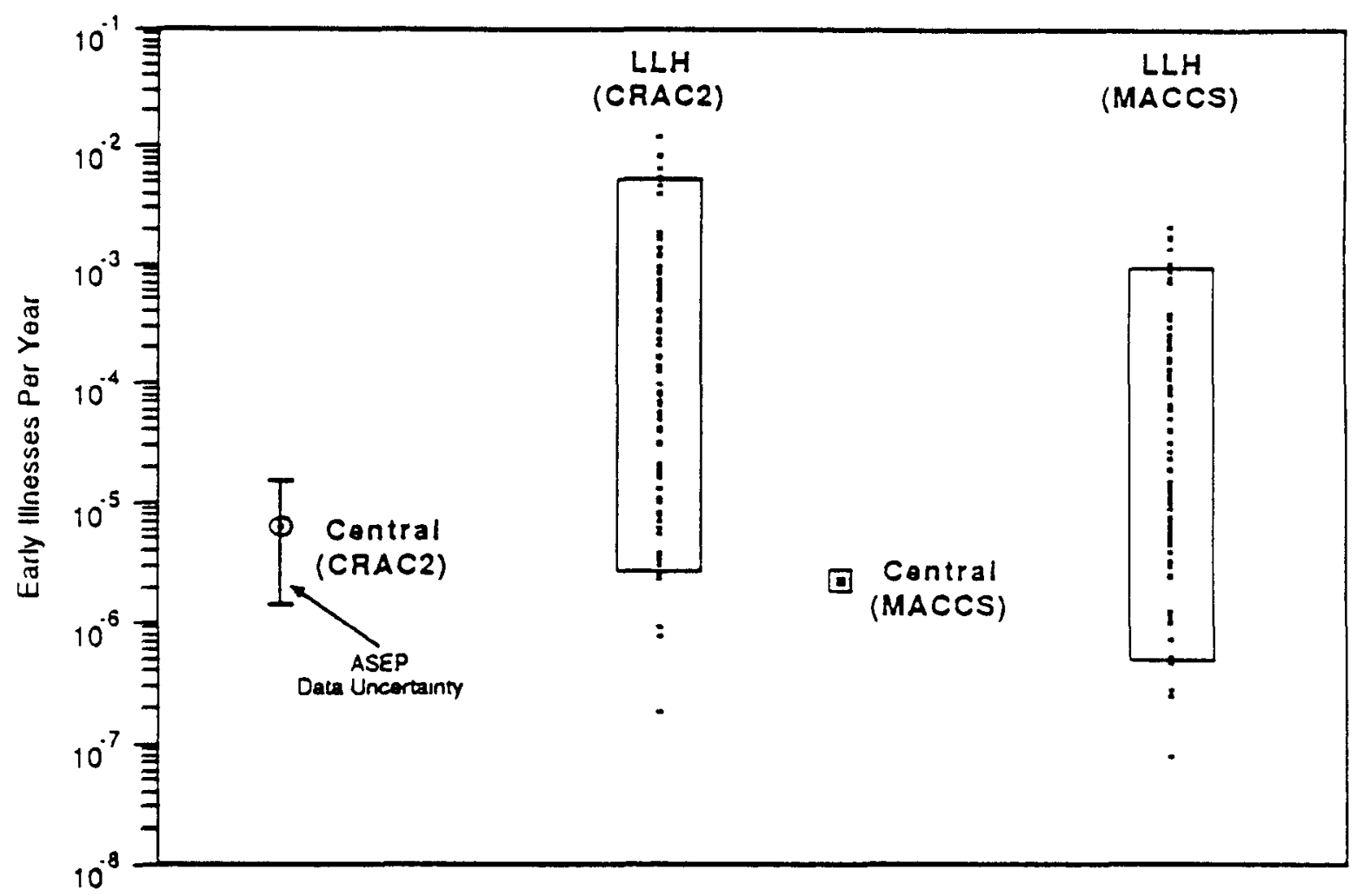

Figure 5-27. Annual Risk of Early Injury

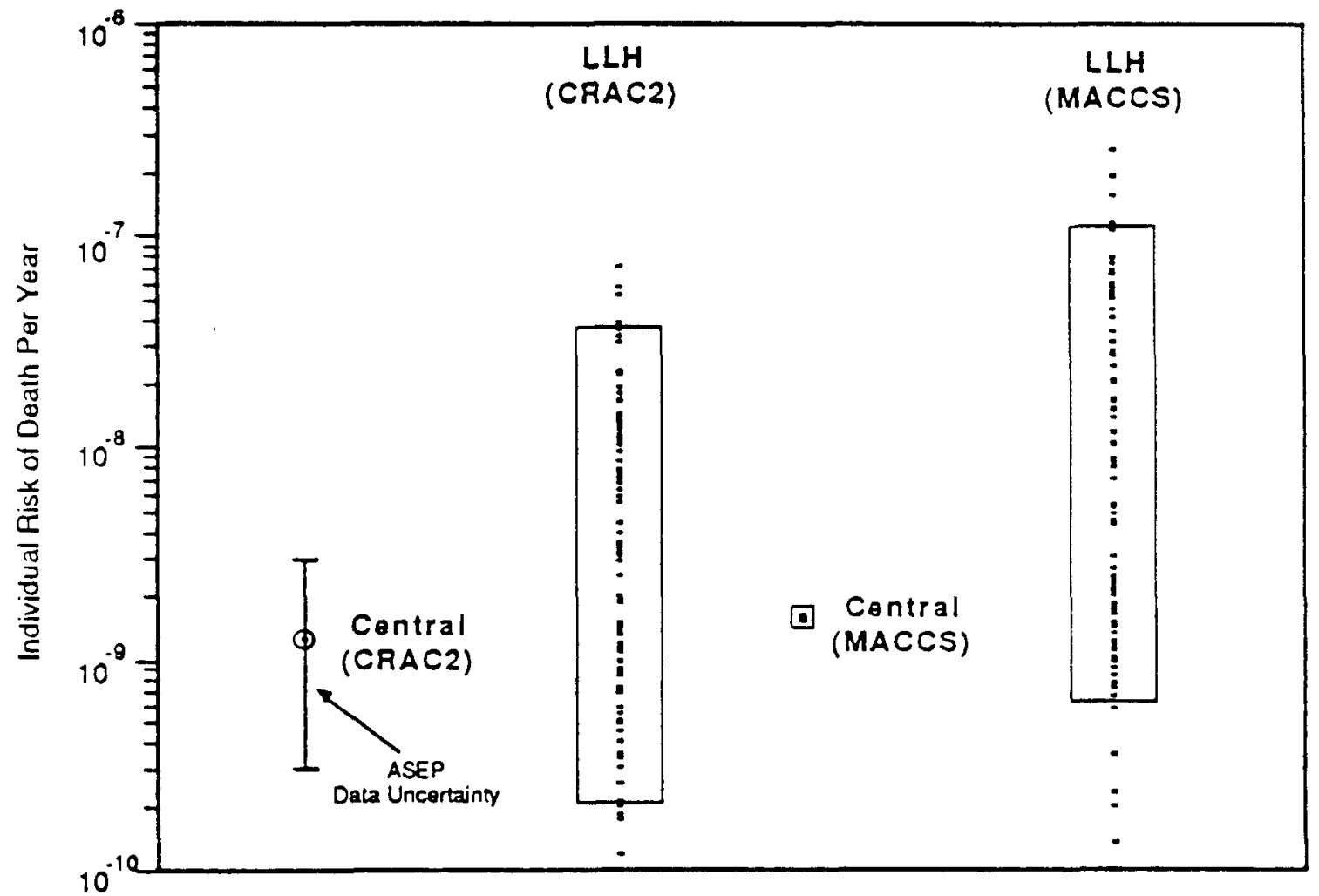

Figure 5-28. Individual Risk of Death 
NUREG/CR-4551, VOL1: DRAFT REPORT FOR COMMENT (SEPTEMBER, 1986)

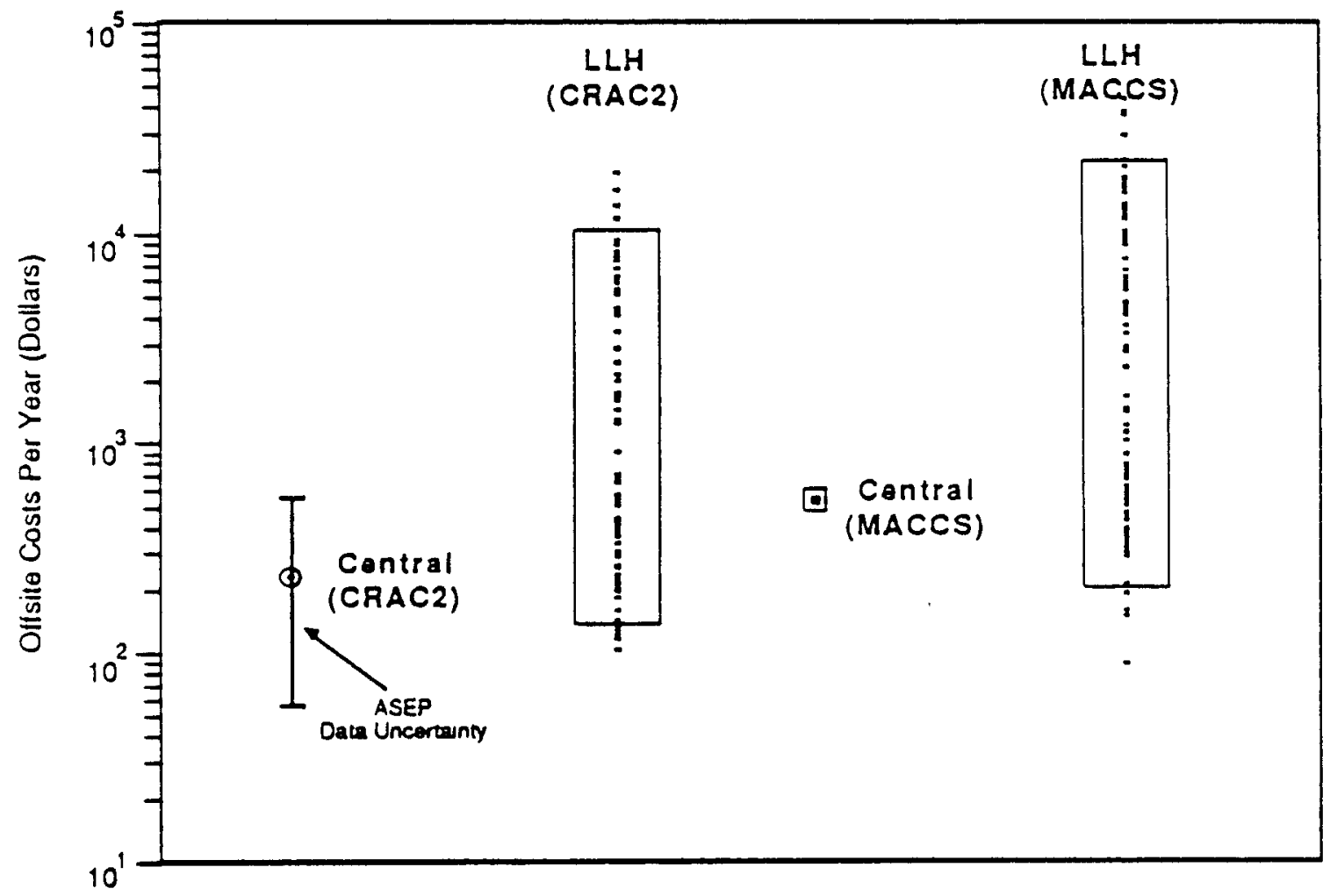

Figure 5-29. Results for Offsite Cost Risk Measure

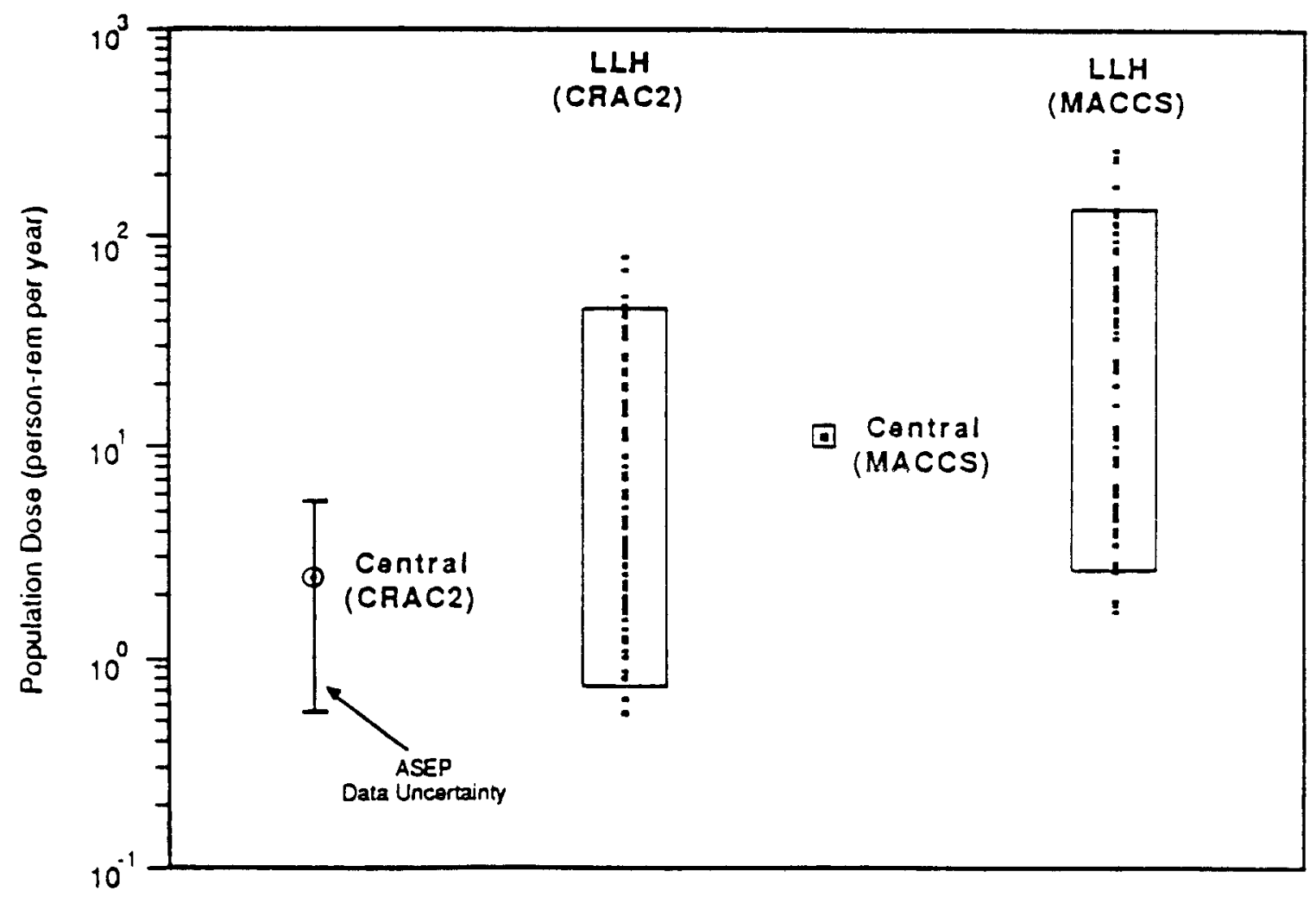

Figure 5-30. Results for Risk Measure of Population Dose per Year 


\subsubsection{Obseryations Concerning the Risk_Results}

The results presented in this section suggest several interesting insights into current perceptions of risk, and the change in that perception since the Reactor Safety Study. In general, the results of this study (SARRP central estimate) indicate lower risk than that calculated in RSS.

Two types of uncertainty were treated in the analysis, data and modeling. The data uncertainty is approximately an order of magnitude, with about a factor of two more uncertainty in the downward direction than the upward, whereas modeling uncertainty appears to be mostly in the upward direction relative to the SARRP central estimate. The top of the LLH uncertainty band is at levels comparable to the RSS point estimate for most risk measures. It should also be noted that comparisons are subject to limitations because the studies involve other differences that are not immediately obvious. For example, the RSS risk was calculated for a generic site with different evacuation assumptions: the RSS model assumed 100\% evacuation $70 \%$ of the time and no evacuation $30 \%$ of the time, whereas the current model assumed that $95 \%$ of the population would evacuate during any severe accident. Notwithstanding these differences, there are a number of key factors which explain the changes that have occurred between the RSS and the current assessment:

- The frequency of the $V$ sequence has decreased due to specific actions taken to reduce its frequency following the RSS report, and the consequences are also predicted to be less because of the consideration of the potential for scrubbing by overlying water.

- The accident sequence S2C was critical to the RSS result, but further analysis in SARRP indicated that this was not a core-damage sequence since containment would not be likely to fail. A similar sequence (similar in that containment heat removal and containment itself fail before core damage) was important to the ASEP result, but the LLH study showed this to be of limited risk potential.

- There have been a number of advances in source term analysis, many of which result in the prediction of lower source terms. Many of the advances are incorporated directly in the Source Term Code Package models. Deposition of radioactive species in the primary system was not previously considered, and deposition in containment is now predicted to be larger than previously thought. On the other hand, many of the source term issues considered in the LLH analysis could lead to higher source terms than predicted by the STCP. The sections describing the source term study discuss these changes in detail.

- Our understanding of the important core-damage sequences has changed due to plant changes and better understanding of accident thermal-hydraulics and plant systems; for example, the feed-and-bleed mode of core cooling was not considered in the Reactor Safety Study, but neither was the 
potential for RCP seal LOCAs which dominate the current estimate of coredamage frequency.

- The current understanding of containment phenomenology is different. Both the direct heating and hydrogen burning phenomenology illustrate this change in our understanding. There is also a much wider uncertainty associated with the potential outcomes of the containment scenarios. The in-vessel steam explosion that fails containment is also considered less likely and, if it does occur, to have lower source terms.

It was a particular goal of this study to provide perspective on the risk uncertainties. The LLH approach was selected as the primary method. It should be emphasized that the objective was to supply "reasonable" ranges of uncertainty in the results, not rigorous statistical bounds. As discussed in the next section, it must be recognized that the limited Latin hypercube approach is just that-limited. Not all uncertainties are considered, but rather those thought by a group of experts to be most significant were investigated. In addition, the input as to the range of uncertainty and "degree of belief" of the reviewers for the outcomes of the issue was limited by the selection of the review group. For this plant, the OCP uncertainty representation was also completed, yielding additional insights. Overall the comparison resulted in the conclusion that the LLH method appears to have resulted in a range of uncertainty that is probably appropriate in its portrayal of the upper end of uncertainty, but that, when considering the whole of the reactor safety community, the lower range might extend lower than the LLH result would indicate for some risk measures. Both uncertainty representations provided information concerning important uncertainty issues. Unfortunately, it is not simple to determine the total impact of a given uncertainty issue, but detailed examination of the results presented in this section have illustrated some important insights:

- Direct heating is an important uncertainty, but elimination of just the direct heating uncertainty only narrows the LLH range by less than a factor of 5 .

- Aerosol agglomeration was identified as an important uncertainty, a result that is not entirely intuitive. The issue is most important to the early fatality risk measure, and threshold effects could be playing a role in the apparent significance of this issue. A review of the other source term issues indicates that the source term can be significantly increased by any one of a number of issues if it is at a high level. It is therefore possible that the agglomeration issue is one of the few processes that could act across other issues to reduce the source term.

- The difference between significant issues in the base case and the sensitivity case without direct heating suggests that the significance of an individual issue or level is quite interdependent on all of the other issues. For example, the spray failure issue is more important to the base case but not to the sensitivity case with direct heating removed. The statistical tests on the results do not account for complex correlations that cause issues to be important only when other issues are at certain levels. It is therefore not 
possible to make very many absolute statements conceming the significance of uncertainty sources, since any conclusions are highly dependent on the exact risk profile created by all of the other inputs, and changes in sequences, frequencies, or other issues could change any conclusions concerning the important issues.

- These results suggest that other issues play a role in the uncertainty of this current risk profile. For late effects, the $V$ sequence frequency, the possibility of induced RCS failure, the hydrogen burn pressure increment, and the containment failure pressure all appear to contribute to the uncertainty. For early effects, the amount of in-vessel radionuclide release and the in-vessel retention levels were found to be significant relative to uncertainty. Other issues play a small role also, including the amount of CsI decomposition and the failure probability for the containment sprays.

- The source term uncertainties appear to have a substantial impact on overall uncertainty, but the statistical results do not point to many of the issues individually. It is suspected that many of the issues have similar overall impact, but that none dominates, other than the aerosol agglomeration issue discussed above.

- A comparison of the OCP and LLH results suggests that the optimistic result can be changed significantly if any one of a number of source term and containment issues is at a level that is not the most optimistic. Thus the LLH tends to illustrate higher lower bounds due to the low probability that all uncertainties would be at their lowest values relative to risk.

The details of the uncertainty analysis are discussed throughout this report and are summarized in the Conclusions, Section 7.

\subsubsection{Limitations}

The limitations associated with probabilistic risk assessment have been discussed in many forums $[5,6]$, and a reiteration of the entire subject would not be useful here. There are some specific limitations that should be recognized when interpreting the results of this study. The individual discussions of the principal tasks in the main report and the appendices include a summary of important limitations. Some of the limitations are outlined here for emphasis.

- The results apply to the Surry plant only and must not be inferred as being generally applicable to other plants.

- The results and conclusions are based on internal initiating events only, since treatment of external events was out of scope.

- The consideration of operator actions was limited by the groundrules to actions associated with written procedures. Some additional recoveries could be expected, and the whole question of operator action to limit the consequences was not really considered. For example, the containment event tree had very limited consideration of operator action. On the other hand, errors of commission are not really included either, and the 
techniques for human reliability assessment suffer from the same limitations that affect all risk assessments.

- The study was also limited in that not all uncertainties are included in the results. The uncertainties due to data and those due to modeling are not combined in the current results. Consequence methodology uncertainties were not addressed, except that some indication of modeling uncertainties is available from the CRAC2/MACCS comparisons.

- The appropriateness and interpretation of the limited Latin hypercube methodology remains the subject of considerable discussion among analysts familiar with the process and with most of the participants (see Appendix F for a brief review of this topic). The largest area of concern is the interpretation of the result; many of the participants did not think that a probabilistic meaning should be applied to the weighting factors. Rather, the process should just be used to obtain ranges of uncertainty. Therefore, any of the information provided based on the mean of the LLH should be used with caution, since that mean should not be considered a statistical parameter of risk.

- The clustering methodology that allows a practical approach to the calculation of consequences has not been fully investigated in terms of its impact on the overall result. Clustering does lead to some loss of resolution and makes the review of the results somewhat more difficult. Some clustering calculations (see Appendix C) indicate that the loss of resolution is small compared to the uncertainty in the issues. However, the examination of the role of clustering is continuing.

- Any conclusions concerning the importance of individual issues must be made carefully since the interrelationships among issues can be quite complex. In addition, the statistical sample used here has limitations based on its size, and the techniques for analyzing the results are not good for identifying the effects of multiple combinations of issues.

- The ASEP study of core-damage frequency was not intended to be as comprehensive as some risk assessments, and it was completed with less resources than a full-scope PRA. Although there was excellent interaction with the plant staff, there was no direct utility involvement.

- Accident sequences resulting in core damage were assumed to proceed to core melting and subsequent breaching of the reactor vessel. It is anticipated that a significant portion of those sequences might be arrested prior to vessel breach.

- Much of the containment analysis relied on other studies rather than direct calculations for Surry. For example, pressures associated with some phenomena were scaled from other studies or from input of reviewers based on simple calculations.

- The containment event tree, while very detailed, still includes a great many simplifications, and there are numerous branch point split fractions which are highly uncertain.

- The source term analysis is based on the STCP (or its precursor codes) for the central estimates, although the LLH input required significant departures from the STCP results. The STCP also has limitations such as lack of inclusion of some phenomenology such as revaporization after vessel 
failure, CsI decomposition, in-vessel flow recirculation, buoyancy-driven containment flow, and direct heating. The treatment of these issues is discussed in the detailed sections concerning the SARRP source term analysis.

- The source term analysis also involved many assumptions and simplifications to make the problem tractable, and the effect of these assumptions is not fully known. For example, the releases are collected first into bins, whereas specific accident scenarios could have subtle phenomena differentiating them from others within the bin. These assumptions were controlled through careful review, but the topic is too complex to ensure the completeness of this review.

- The consequences were calculated with weather data from a site other than Surry, although the resultant limitation is probably not too great compared to many of the others, since the sites would be expected to have similar weather patterns. The results are also based on $95 \%$ evacuation with no detailed consideration of the uncertainty in this assumption.

- The LLH results are limited to the views of the participants in the process and are limited in the number of issues considered.

While many of these limitations appear to be severe in the abstract, most are not too different from the limitations associated with any risk assessment. The methods selected were based on the input of many in the reactor safety community. Some reflect the bounds of the state of the art, while others reflect restrictions in schedule or budget. The limitations in the uncertainty analysis reflect the current situation in the PRA industry in that there are no generally accepted techniques for fully characterizing the uncertainty. The value of the study is manifested in the engineering and scientific insights which are derived through the process itself and the examination of the results. 


\section{References for Section 5}

1. Bertucio, R. C., et al. Analysis of Core Damage Frequency From Internal Initiating Events: Surry, Unit 1. U.S. Nuclear Regulatory Commission Report NUREG/CR4550, Vol. 1 of 5 (Draft Report), Sandia National Laboratories, Albuquerque, NM: April, 1986.

2. Olman, M. D. Quantitative Fault Tree Analysis Using the Set Evaluation Program (SEP). U.S. Nuclear Regulatory Commission Report NUREG/CR-1965, Sandia National Laboratories, Albuquerque, NM: September, 1982.

3. Reactor Safety Study--An Assessment of Accident Risks in U.S. Commercial Nuclear Power Plants. U.S. Nuclear Regulatory Commission Report WASH-1400 (NUREG75/014), Washington, DC: 1975.

4. Benjamin, A. S., et al. Containment Event Analysis for Postulated Severe Accidents: Surry Power Station, Unit 1. U.S. Nuclear Regulatory Commission Report NUREG/CR-4700, Volume 1, Sandia National Laboratories, Albuquerque, NM: February, 1987.

5. The PRA Procedures Guide. U.S. Nuclear Regulatory Commission Report NUREG/CR-2300, Washington, DC: January, 1983.

6. Probabilistic Risk Assessment (PRA) Reference Document. U.S. Nuclear Regulatory Commission Report NUREG-1050, Washington, DC: September, 1984. 


\section{Section 6}

\section{RESULTS OF RISK-REDUCTION ANALYSIS}

The results of the SARRP study of risk-reduction measures for Surry are discussed in this section. These results draw heavily on more detailed descriptions of the inputs to this analysis described in the appendices to this report.

The options considered are intended to be examples of changes that could be made that could impact some of the major risk contributors. To perform the cost/benefit analysis it was necessary to assume a conceptual design, but there could be many other ways of achieving the same goal. Because of this fact, and because development of a detailed design prior to understanding the potential impacts would be inefficient, the safety options are not analyzed in this study to as significant a level of detail as would be required before implementation could be considered. This analysis is intended as a screening step to allow further, more detailed consideration of modifications that appear to be of potential net benefit.

Each risk-reduction option considered in this assessment was assumed to have a demand failure rate characteristic of systems of that type. Aside from this consideration, the risk-reduction potential of the option was calculated by assuming "best effects". That is to say, each option was assumed to be properly designed to minimize competing risks and inadvertent actuations which could reduce its overall risk-reduction effectiveness. For example, it was assumed that there would not be an inadvertent actuation or failure that would cause an accident that would not otherwise have occurred. Achievement of a high quality system that minimizes competing risks requires careful design and implementation, which could increase the cost of an option.

On the other hand, the possibility was considered that a well designed system could have negative effects by changing the progression of an accident in a way that increases risk. For example, a mitigation system designed to prevent one type of containment failure could cause a different failure to occur with a higher consequence result. Such cases are discussed when they appear. In this section, the individual effects of the options are first summarized, followed by a discussion of the overall impact on important risk measures.

\subsection{PREVENTIVE OPTION EFFECTS ON CORE-DAMAGE FREQUENCY}

The methods and reasons for the selection of the accident prevention options for Surry were described in Section 3.1. Once again, the prevention options considered are as follows: 
P1: Improved Testing of Interfacing Check Valves

P2: Improved Onsite Ac Power System

P3: Extension of Battery Life

P4: Addition of an Independent High-Pressure Injection System

P5: Addition of an Independent Train of Auxiliary Feedwater

P6: New Low-Pressure Cooling Capability

P7: New Service Water System for Containment Cooling

P8: RCS Depressurization System

The impact of these options on the core-damage frequency is delineated in Table 6-1.* Because of the large number of essentially equal contributors to the overall core-damage frequency, the effect of any single option is limited. The maximum reduction in frequency of core damage is associated with the addition of a new train of high pressure injection (independent of current power supplies), with a nearly equal reduction for the addition of a diesel generator. These options have a large effect because they impact the accident sequences involving loss of all ac power, which was noted in Section 5.1 to be the largest contributor to core-damage frequency. The high pressure injection system also affects other sequences involving the loss of highpressure ECCS, and it therefore has a larger impact. It should be noted that the preventive options were considered with regard to the risk measures also, and a large reduction in total core-damage frequency is not necessary for an option to be effective. For example, the $\mathrm{V}$ sequence was found to be a significant contributor to the risk of health effects in the OCP analysis, and an option aimed at reduction of the frequency of this one sequence was therefore included, although its effect on the overall frequency of core damage was known to be negligible. One option was aimed specifically at a particular problem identified at Surry, the unreliability of the containment service water system. The sequences resulting from this failure were the core-vulnerable accidents in which containment failure is predicted but core-damage does not necessarily occur. The effect of option P7 is therefore limited because of the low frequency of core damage for core-vulnerable sequences as assessed in SARRP. Option P8 also has very significant mitigative effects. The prevention aspect is achieved through the reduction of system pressure, allowing the use of low pressure systems for sequences in which high pressure systems might be unavailable. The mitigative effect is manifested through a

"One option which was grouped with the mitigation options, additional containment sprays, also had a preventative effect in further reducing the frequency of the core-vulnerable sequences which involve containment failure before core damage. 
lower frequency of direct heating, since that phenomenon can only occur if the RCS is at elevated pressure prior to vessel breach.

Table 6-1

\section{REDUCTION IN CORE-DAMAGE FREQUENCY ASSOCIATED WITH PREVENTIVE SAFETY OPTIONS}

\begin{tabular}{|c|c|c|c|c|c|c|c|c|c|}
\hline \multirow{2}{*}{$\begin{array}{l}\text { Damage } \\
\text { State }\end{array}$} & \multirow[b]{2}{*}{ Frequency } & \multicolumn{8}{|c|}{ Erequency With Option Divided by Erequency Without ${ }^{1}$} \\
\hline & & P1 & P2 & P3 & P4 & P5 & P6 & P7 & P8 \\
\hline SYYB & $7.1 \times 10^{-6}$ & - & - & - & .9 & - & .5 & - & .6 \\
\hline SNNN & $5.7 \times 10^{-6}$ & - & .05 & - & .05 & - & - & - & - \\
\hline TNNN & $1.9 \times 10^{-6}$ & - & .05 & .65 & .05 & .03 & & - & - \\
\hline AYYB & $1.8 \times 10^{-6}$ & - & - & - & .58 & - & .6 & - & - \\
\hline TYYBD & $1.1 \times 10^{-6}$ & - & - & - & .02 & .03 & - & - & .01 \\
\hline TYYBW & $1.0 \times 10^{-6}$ & - & - & - & .02 & - & - & - & .01 \\
\hline v & $9.0 \times 10^{-7}$ & .06 & - & - & - & - & - & - & - \\
\hline AYNI & $8.2 \times 10^{-8}$ & - & - & - & - & - & .05 & .05 & - \\
\hline TYNI & $8.0 \times 10^{-8}$ & - & - & - & - & .03 & - & .05 & - \\
\hline SYNN & $\varepsilon$ & - & - & - & - & - & .05 & - & - \\
\hline AYNN & $\boldsymbol{\varepsilon}$ & - & - & - & - & - & .05 & - & - \\
\hline $\begin{array}{l}\text { Total } \\
\text { Frequenc }\end{array}$ & $2.1 \times 10^{-5}$ & $2.1 \times 10^{-}$ & $\times 1$ & $\times 14$ & 10 & 80 & $\times 1$ & $\times 1$ & $6 \times 10^{-5}$ \\
\hline
\end{tabular}

The ratio is only shown for damage states that changed.

\subsection{MITIGATION OPTION EFFECTS ON CONTAINMENT/SOURCE TERMS}

The mitigation options were aimed at specific phenomena thought to be important to risk. The effects on accident progression are discussed below.

Qption M1: Missile Shield. The provision of a shield to preclude the possibility of containment failure as a consequence of a missile generated due to an in-vessel steam explosion (the failure mode designated $\alpha$ ) was considered.

Ontion M2: Hydrogen Ignition System. An ignition system would be intended to burn off hydrogen at lower concentrations to prevent buildup and combustion on a scale large enough to lead to containment failure. The impact of this option on risk was assessed by forcing ignition to occur at each node in the containment event tree at which hydrogen burning would be possible. The 
option therefore prevents the buildup of hydrogen that could bum and lead to higher pressure loadings. The system is assumed to be independent of the existing power sources.

Option M3: Prevention of Hydrogen Combustion. As an alternative to option M2, the provision for preventing the combustion of hydrogen (e.g., through inerting of the containment atmosphere) was considered. The system was assumed to be similar to the containment inerting currently in use at some BWRs. The effectiveness of such an option was investigated by precluding the possibility of combustion at each of the associated nodes in the containment event tree. (This option should have included some effect on direct heating since there would be limited oxygen for chemical reactions in the containment atmosphere, but this effect was not included in the results presented here.)

Eption M4: Early Containment Venting. Venting of the containment atmosphere through filters to prevent overpressurization and a more serious release was investigated. The effects of venting early in the accident were simulated by setting the probability of a moderate-sized (less than one square foot) failure of containment isolation to 1.0, and assigning the resulting release to the bin corresponding to meltthrough of the containment basemat at high pressure. The system was assumed to have independent power capability to allow its operation regardless of the damage state.

Option M5: Late Containment Venting. The value of venting the containment later in the accident progression, following breach of the reactor vessel, was evaluated in a manner similar to option M4. The resulting release was again assessed as a high-pressure meltthrough of the basemat, corresponding to a filter of moderate effectiveness.

Option M6: Additional Containment Cooling--Containment Spray. Because of the significance to risk of sequences involving overpressurization due to failure of containment cooling, followed by the consequential failure of long-term core cooling, the addition of an alternative containment spray system was considered. The containment event tree was reevaluated considering the impact of increased spray availability for each branch point involving spray operation or containment cooling, including power loss sequences since the system was assumed to have separate power sources. This modification was also assumed to reduce the frequency of the core-vulnerable sequences in which the loss of containment cooling was the principal system fault (e.g., the sequences $S_{1} F_{1} F_{2}$ and $A F_{1} F_{2}$ ).

Option M7: Additional Containment Cooling--Fan Coolers. This option is similar in effect to the option described above, and the containment event tree questions regarding fan coolers which were included in the tree development so that the logic would have wider applicability were changed to reflect the addition of fan coolers for Surry. This option was not assumed to eliminate containment failure before core damage (core-vulnerable accidents). As with the sprays, an unavailability of 0.05 was used in the calculations, and an independent power supply was assumed.

Option M8: Containment Flooding System. The containment flooding system was selected to reduce the risk associated with direct heating by always assuring a water filled cavity, thereby reducing direct heating pressure increments. This option also affected questions concerning coolability of the debris in the cavity. 
NUREG/CR-4551,VOL.1: DRAFT REPORT FOR COMMENT (SEPTEMBER, 1986)

The option was assumed to be used in accidents where the cavity is not flooded by other sources, and was assumed to be independent of existing power.

Because the LLH technique does not allow for a breakdown of containment failure modes without considering the detail of all 100 sample members, it is not possible to illustrate all of the quantitative effects on containment failure modes. One good measure of effectiveness can, however, be extrapolated from an examination of the relative contribution of source term bins to the risk as displayed in Table 6-2. The source term bins correspond to the major containment phenomena and the impact of the issues can be seen more fully than simply through the descriptions above. For example, the first option is intended to eliminate the steam explosion-induced failure of containment, and the relative contribution of bin 1 decreased; however, the effect was limited because the failure mode was not very important to the base case. Option M2, the hydrogen igniters, had little impact on the mean risk of latent cancer fatalities. Option M3 did cause a reduction in hydrogen burn failure modes, but more importantly, it reduced the contribution of direct heating. This resulted from a feature discussed in Section 5. The direct heating failure mode is affected by the hydrogen burn pressure increment that the LLH reviewers assigned for this case, including a hydrogen increment in a steam-inert environment. (Once again, the effect on direct heating of this option due to elimination of oxygen was not included in this analysis.)

The early containment venting option (option M4) decreased the probability of early containment failure, including that associated with direct heating, since the base containment pressure is reduced. The late filtered venting option had almost no impact since early failures dominate the risk. The most effective option is M6, the new train of containment spray. This option increases the availability of containment cooling for nearly all scenarios, while at the same time providing a high assurance of a flooded cavity, which has a significant impact on the direct heating pressure increments. Therefore, the relative contributions of most early failures is less, with the result that many sequences contribute approximately equally to the risk. The fan coolers, option M7, are not as effective as sprays since they do not impact direct heating; the flooding of the cavity associated with option M6 does not happen for option M7. The final option was intended to reduce the direct heating contribution, but the effect was somewhat limited because the review group assigned fairly high pressure even with a full cavity, and the hydrogen burn pressure increments are still possible. One option that has preventative and mitigative effects is also included in table, option P8, RCS depressurization. As indicated, the 
option had a large impact on the direct heating scenarios, essentially eliminating them. The risk reduction was greatest for this option.

It should be noted that the results above apply to the LLH sample. The OCP results were somewhat different, due principally to the reduced importance of the direct heating phenomenon. The mitigation options had less impact for the OCP central estimate since no single phenomenon dominated the containment response. The addition of containment sprays had the most significant impact on containment response.

Table 6-2

RELATTVE CONTRIBUTION OF SOURCE TERM BINS TO LATENT CANCER FATALTTY RISK FOR MITIGATION OPTIONS ${ }^{1,2}$

\begin{tabular}{|c|c|c|c|c|c|c|c|c|c|c|c|}
\hline \multirow[b]{2}{*}{ Bin } & \multirow{2}{*}{$\begin{array}{l}\text { Distinguishing } \\
\text { Feature }\end{array}$} & \multicolumn{10}{|c|}{ Eraction of Base Case Risk Contributed by Each Bin for Each Option } \\
\hline & & Base & M1 & M2 & M3 & M4 & M5 & M6 & M7 & M8 & P8 \\
\hline 1 & Early rupture including $\alpha$ & $3.6 \%$ & $2.5 \%$ & $3.5 \%$ & $1 \%$ & $2.5 \%$ & $3.5 \%$ & $.2 \%$ & $2 \%$ & $2.7 \%$. & $9.5 \%$ \\
\hline 2 & Same as 1 with sprays & - & - & - & - & - & - & - & - & - & -- \\
\hline 3 & As 1, with lower RCS pres & $.2 \%$ & $.2 \%$ & $.5 \%$ & $.3 \%$ & $.2 \%$ & $.5 \%$ & $.6 \%$ & $.5 \%$ & $.3 \%$ & $.8 \%$ \\
\hline 4 & As 2 with lower RCS pres & $.2 \%$ & $.2 \%$ & $.2 \%$ & - & $.1 \%$ & $.2 \%$ & $.2 \%$ & $.2 \%$ & $.1 \%$ & $.2 \%$ \\
\hline 5 & Failure before core melt & $6 \%$ & $6 \%$ & $6 \%$ & $6 \%$ & $4 \%$ & $6 \%$ & $6 \%$ & $6 \%$ & $3 \%$ & $6 \%$ \\
\hline 6 & Isolation, no sprays & - & - & - & - & - & - & - & - & -- & -- \\
\hline 7 & Isolation, sprays & $.1 \%$ & $.1 \%$ & $.1 \%$ & $.1 \%$ & - & $.1 \%$ & $.1 \%$ & $.1 \%$ & $.1 \%$ & $.1 \%$ \\
\hline 8 & Late rupture, with spray & - & - & - & - & - & - & - & - & - & - \\
\hline & Late rupture, do spray & $.6 \%$ & $.6 \%$ & $.6 \%$ & $.6 \%$ & - & - & $.7 \%$ & $.6 \%$ & $.4 \%$ & $.5 \%$ \\
\hline & Late overpressure & - & - & - & - & - & - & - & - & -- & -- \\
\hline & $V$ sequence, wet & $4.7 \%$ & $4.7 \%$ & $4.7 \%$ & $4.7 \%$ & $4.7 \%$ & $4.7 \%$ & $4.7 \%$ & $4.7 \%$ & $2.5 \%$ & $4.7 \%$ \\
\hline & $V$ sequence, dry & $7.3 \%$ & $7.3 \%$ & $7.3 \%$ & $7.3 \%$ & $7.3 \%$ & $7.3 \%$ & $4.6 \%$ & $7.3 \%$ & $4 \%$ & $3 \%$ \\
\hline \multicolumn{3}{|c|}{13 Basemat meltthrough, high pres } & - & - & - & - & - & - & - & - & - \\
\hline \multicolumn{3}{|c|}{14 Basemat meltthrough, low pres } & - & - & - & - & - & - & - & - & -- \\
\hline & Design Leakage & - & - & - & - & - & - & - & - & - & -- \\
\hline & Direct Heating, high pres & $72 \%$ & $71 \%$ & $71 \%$ & $38 \%$ & $51 \%$ & $71 \%$ & $4.2 \%$ & $56 \%$ & $29 \%$ & $1 \%$ \\
\hline & As 16 , with sprays & $3.3 \%$ & $3.3 \%$ & $3.3 \%$ & $2.7 \%$ & $8.8 \%$ & $3.3 \%$ & $3.3 \%$ & $9.5 \%$ & $2 \%$ & - \\
\hline & Direct Heating, lower pres & $.7 \%$ & $.6 \%$ & $.7 \%$ & $.2 \%$ & - & $.6 \%$ & $1 \%$ & $.6 \%$ & $.4 \%$ & -- \\
\hline & As 18 with sprays. & $1.7 \%$ & $1.7 \%$ & $1.7 \%$ & $5 \%$ & $10 \%$ & $1.7 \%$ & $2.8 \%$ & $\underline{2.8 \%}$ & $1 \%$ & $=$ \\
\hline & Total & $100 \%$ & $99 \%$ & $100 \%$ & $61 \%$ & $89 \%$ & $99 \%$ & $29 \%$ & $91 \%$ & $46 \%$ & $26 \%$ \\
\hline
\end{tabular}

${ }^{1}$ Preventive option P8, RCS depressurization, has a mitigative effect which is listed in this table.

${ }^{2}$ These values were obtained from the LLH mean. 


\subsection{BENEFIT OF RISK-REDUCTION OPTIONS: AVERTED RISK}

The risk was recalculated for all of the safety options by changing the analysis to reflect the improvements in core-damage frequency or containment response. The results are illustrated for all of the options (and some combinations of the options) in Figures 6-1 through 6-4. Only the early and latent cancer fatality risk measures for CRAC2 are illustrated; the others risk measures and the MACCS results show similar trends. Note that the figures include both the LLH range and the OCP central estimate. For the risk of latent cancer fatalities in the central estimate, options P1 (V sequence procedures), P4 (high pressure injection), P6 (new RHR), P7 (new service water) and M6 (additional sprays) have the most impact (although the total impact is rather limited), principally because they reduce the frequency of the highest risk contributors (see Section 5, Table 5-11). The early fatality results show similar trends, although options P1 and P6 have the largest impact due to the dominance of sequences affected by these options to the early fatality risk.

For latent cancer fatalities as calculated by the LLH method, the following options have the most reduction:

$\begin{array}{ll}\text { P2 } & \text { New diesel generator; } \\ \text { P4 } & \text { New, independent HPI; } \\ \text { P8 } & \text { RCS depressurization system; } \\ \text { P6 and P8 } & \text { New low pressure RHR system and RCS depressurization; } \\ \text { M6 } & \text { New containment sprays; and } \\ \text { M4 and P2 } & \text { Early filtered venting and new diesel generator. }\end{array}$

The combination of options P6 and P8 has the largest reduction, over a factor of 20 for the mean of the LLH for latent cancer fatalities. The early fatality risk shows approximately the same behavior. Many of the options had little effect on overall risk because they were aimed at reducing specific sequences or phenomena and other contributors were equally or more important.

For some of the LLH sample members and for some of the extremes of the OCP results, slight risk increases were calculated. For example, the early filtered vent option increases risk at the low end on the LLH range, as does the hydrogen igniter option for the OCP optimistic result. These results are easily understood since these options have competing effects; the former could lead to an increased release over scenarios with the potential for no failure of containment, and the latter could increase the ignition probability for some paths in the containment event tree. None of the risk increases calculated were significant relative to the estimated uncertainty in risk. 


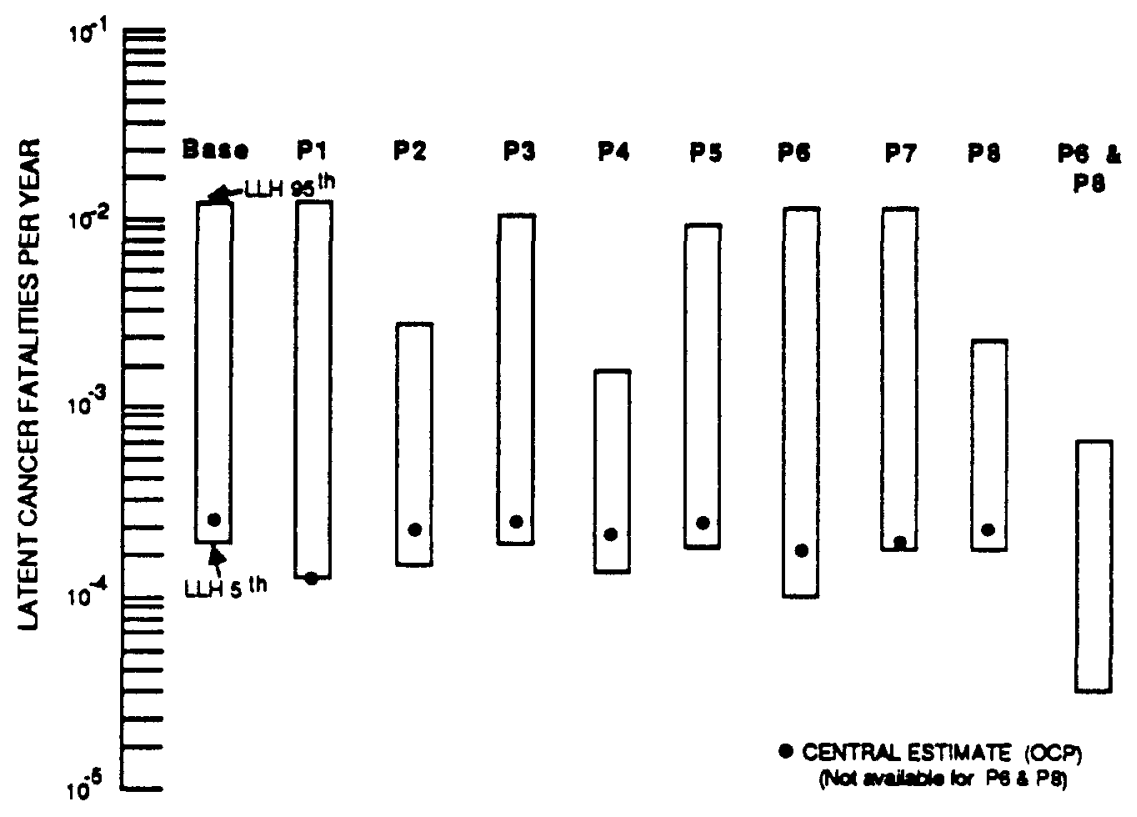

Figure 6-1. Reduction of Latent Fatality Risk for Prevention Options (CRAC2)

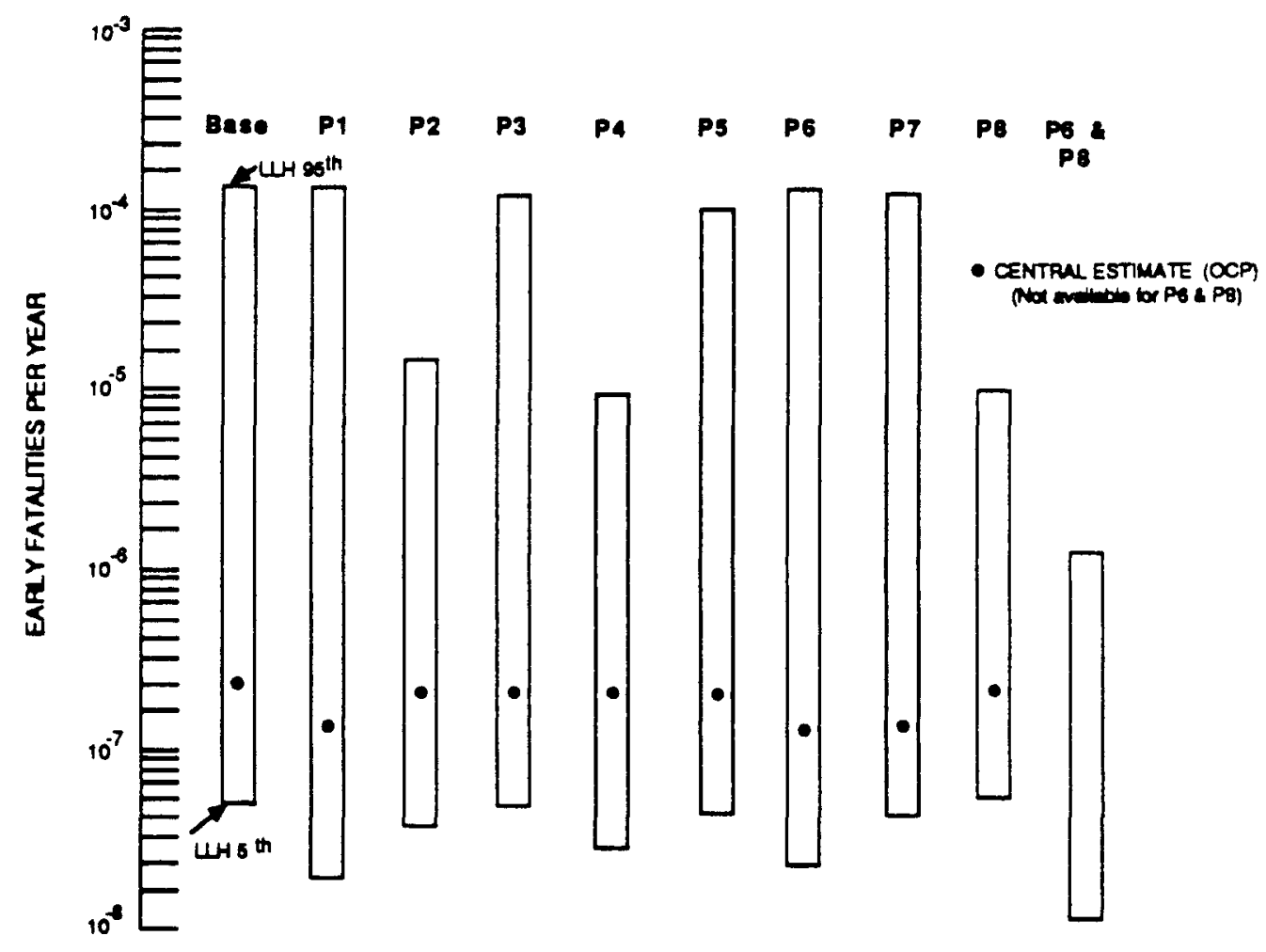

Figure 6-2. Reduction of Early Fatality Risk for Prevention Options (CRAC2) 


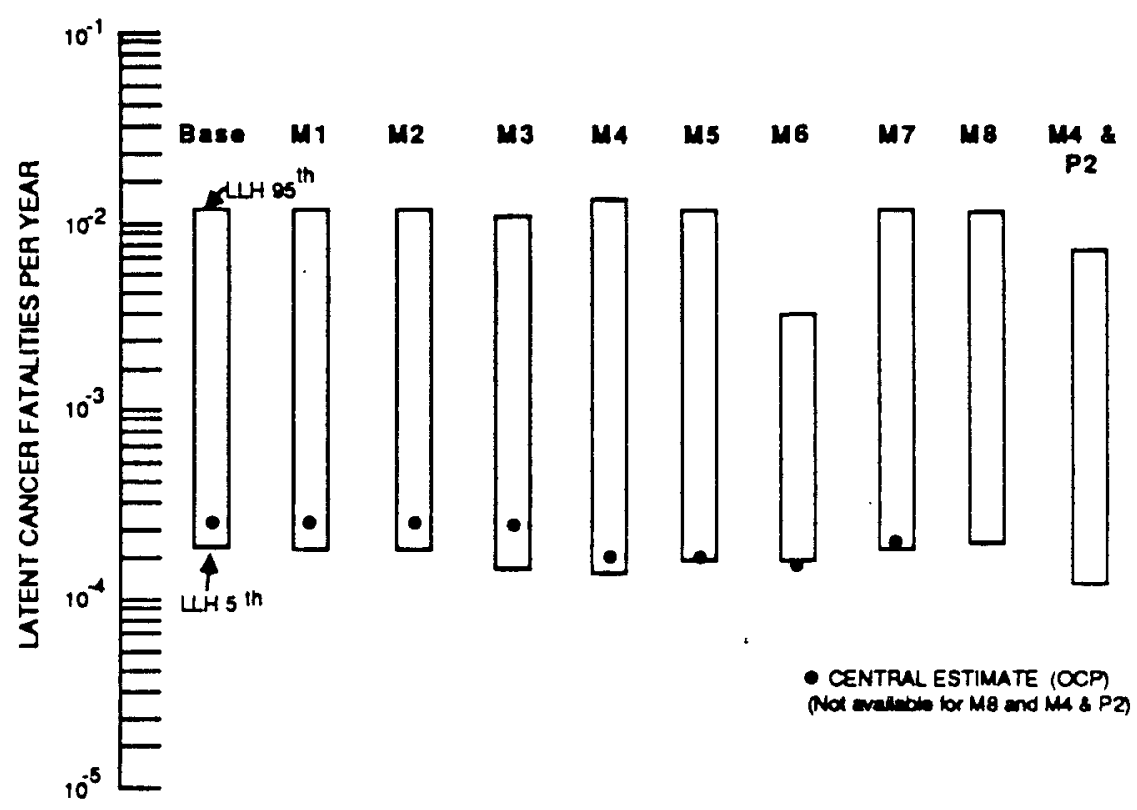

Figure 6-3. Reduction of Latent Fatality Risk for Mitigation Options (CRAC2)

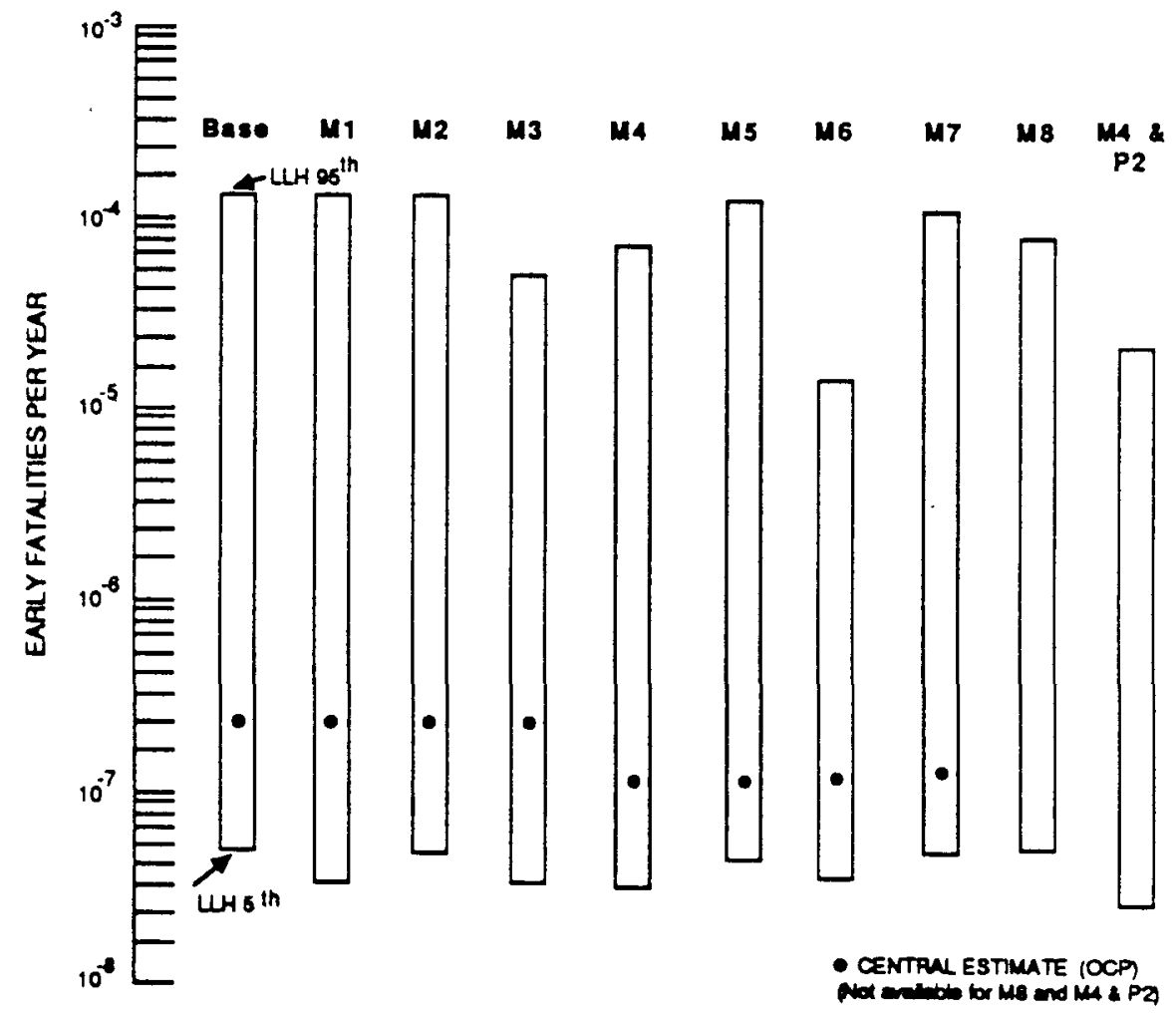

Figure 6-4. Reduction of Early Fatality Risk for Mitigation Options (CRAC2) 
Greater risk reduction can sometimes be obtained when safety options are taken in well chosen combinations. Two pairs of options were considered here. It is not clear that synergistic effects are obtainable with the options in this study, and the reduction is actually a sum of the individual option effects.

\subsection{COSTS OF RISK-REDUCTION OPTIONS}

The investigation of the merit of the proposed risk-reduction measures requires a thorough evaluation of impact that would result from their implementation. As discussed in Section 3 of the main report, there are three principal types of impact considered in the evaluation of each option:

(1) The total cost associated with installation of the proposed modification.

(2) The ongoing costs to the utility (maintenance, increased plant downtime, etc.) that arise from the modification.

(3) The personnel dose associated with installation and maintenance of the option.

At this time, replacement power costs are not included under the assumption that all options could be completed within shutdown periods. For several of the options this assumption is probably not particularly good, but as illustrated later, the detailed consideration of this point was not needed to establish conclusions as to cost effectiveness of the options.

The costs for the modifications considered for Surry are delineated in detail in Appendix E. All of these estimates are based on the impact information included in Appendix G. Although the cost of replacement power is not included in the basic cost estimates, Appendix $E$ includes a brief discussion of this potential impact. The results of the cost evaluation are listed in Table 6-3. The costs in the table are the present value of total cost of the option, including all maintenance and operation costs over the life of the plant.

\subsection{COSTS VERSUS BENEFITS}

The benefits were calculated in terms of averted accident costs to allow comparison to the cost of implementing the options. Due to the differing opinions within the community on the appropriate way to calculate the averted accident costs, four calculations were performed:

- Averted actual offsite costs, consisting of property damage costs (as calculated by CRAC2 or MACCS) and health effects costs (see Section 3);

- Averted offsite costs calculated as reduction in person rems (as estimated by CRAC2 or MACCS) times $\$ 1000$ per person-rem averted; 
Table 6-3

\section{ESTIMATED PRESENT VALUE TOTAL COST FOR RISK-REDUCTION MEASURES}

\begin{tabular}{|c|c|c|c|c|}
\hline & Option & Central & $\begin{array}{l}\text { Total Cost } \\
\text { Upper }\end{array}$ & Lower \\
\hline P1. & $\begin{array}{l}\text { New test procedure for } \\
\text { interfacing check valves }\end{array}$ & $\$ 930,000$ & $\$ 1,800,000$ & $\$ 360,000$ \\
\hline P2. & $\begin{array}{l}\text { Improved ac power through the } \\
\text { addition of a diesel generator }\end{array}$ & $\$ 19,000,000$ & $\$ 38,600,000$ & $\$ 13,000,000$ \\
\hline P3. & $\begin{array}{l}\text { Improved de power system } \\
\text { through extension of battery life }\end{array}$ & $\$ 1,300,000$ & $\$ 2,600,000$ & $\$ 680,000$ \\
\hline P4. & $\begin{array}{l}\text { Additional, independent train } \\
\text { of high-pressure injection }\end{array}$ & $\$ 29,000,000$ & $\$ 58,600,000$ & $\$ 20,000,000$ \\
\hline P5. & $\begin{array}{l}\text { Additional, independent train } \\
\text { of auxiliary feedwater }\end{array}$ & $\$ 8,200,000$ & $\$ 16,400,000$ & $\$ 5,800,000$ \\
\hline P6. & $\begin{array}{l}\text { Long-term residual heat removal } \\
\text { low-pressure recirculation }\end{array}$ & $\$ 27,000,000$ & $\$ 55,000,000$ & $\$ 18,800,000$ \\
\hline P7. & $\begin{array}{l}\text { New service water system } \\
\text { for containment }\end{array}$ & $\$ 6,200,000$ & $\$ 8,300,000$ & $\$ 16,000,000$ \\
\hline P8. & $\begin{array}{l}\text { Rapid primary system } \\
\text { depressurization }\end{array}$ & $\$ 3,700,000$ & $\$ 6,000,000$ & $\$ 2,400,000$ \\
\hline M1. & Missile shield & $\$ 10,000,000$ & $\$ 30,000,000$ & $\$ 7,000,000$ \\
\hline M2. & Hydrogen ignition system & $\$ 8,400,000$ & $\$ 16,800,000$ & $\$ 2,600,000$ \\
\hline M3. & $\begin{array}{l}\text { Prevention of hydrogen } \\
\text { combustion }\end{array}$ & $\$ 8,000,000$ & $\$ 17,000,000$ & $\$ 4,900,000$ \\
\hline M4. & Early containment venting & $\$ 15,100,000$ & $\$ 30,300,000$ & $\$ 10,500,000$ \\
\hline M5. & Late containment venting & $\$ 15,200,000$ & $\$ 30,300,000$ & $\$ 10,100,000$ \\
\hline M6. & $\begin{array}{l}\text { Additional containment } \\
\text { cooling-sprays }\end{array}$ & $\$ 9,500,000$ & $\$ 19,000,000$ & $\$ 6,600,000$ \\
\hline M7. & $\begin{array}{l}\text { Additional containment } \\
\text { cooling--fans }\end{array}$ & $\$ 4,900,000$ & $\$ 9,100,000$ & $\$ 3,400,000$ \\
\hline M8. & $\begin{array}{l}\text { Containment flooding } \\
\text { system }\end{array}$ & $\$ 1,200,000$ & $\$ 2,500,000$ & $\$ 750,000$ \\
\hline
\end{tabular}

- Averted actual offsite costs (as in the first method) plus averted onsite costs (calculated as described in Section 3); and

- Averted offsite costs at $\$ 1000$ per person-rem plus averted onsite costs.

The results are illustrated in Figures 6-5 through 6-8. It is fairly straightforward to see that none of the options appears cost effective. The bars in the figures represent the 5 th to 95 th percentiles of the LLH sample result for averted risk, and none approaches the cost of the applicable option. The MACCS results show slightly higher values, because of the fact that MACCS predicts accident costs about two times greater than CRAC2. It should be noted that 
there are some sample members outside the LLH range illustrated which could show cost effectiveness, but the conclusions should be drawn from the reasonable ranges presented. The figures illustrate the $\mathrm{OCP}$ central as well, and a similar conclusion holds. Only option P1, the improved $\mathrm{V}$ testing, approaches cost effectiveness, and only for lowest costs compared to highest averted costs.

\subsection{OBSERVATIONS CONCERNING RISK-REDUCTION ANALYSIS}

As illustrated in the previous section, none of the options appeared to be cost effective. One reason for this is that Surry was not assessed to have one or two risk outliers that could be reduced resulting in a large effect; rather there are many risk contributors. In addition, there were no subtle, simple fixes discovered, and all of the options would involve significant cost.

Another way to view this result is to consider the amount of money that could be allocated to eliminate the public risk due to Surry. This was calculated the same way as for the other options discussed previously by suggesting a hypothetical option that eliminated all of the core-damage accidents. The result is listed in Table 6-4 below. As illustrated, the highest value of complete elimination of risk is $\$ 2$ million, based on the use of CRAC2. Obviously, this does not allow for any significant hardware changes, and only other options to improve safety through better operations offer any chance of being cost effective. The MACCS code does predict higher costs, but only by approximately a factor of two.

Table 6-4

VALUE OF REDUCTION OF RISK TO ZERO AS CALCULATED WITH CRAC2

\begin{tabular}{lccc}
\hline \multicolumn{1}{c}{ Option } & \multicolumn{2}{c}{ TOTAL VALUE OF AVERTED RISK } \\
& LLH Median & LLH High & LLH Low \\
\hline TOTAL AVERTED OFFSITE COSTS & $\$ 16,000$ & $\$ 186,000$ & $\$ 3,700$ \\
TOTAL AVERTED OFFSITE COSTS & $\$ 110,000$ & $\$ 787,000$ & $\$ 25,000$ \\
COMPUTED AT \$1000/PERSON-REM & $\$ 940,000$ & $\$ 1,400,000$ & $\$ 450,000$ \\
TOTAL AVERTED OFFSITE COSTS & $\$ 1,000,000$ & $\$ 2,000,000$ & $\$ 480,000$ \\
PLUS ONSITE COSTS & & & \\
TOTAL AVERTED OFFSITE COSTS & & & \\
COMPUTED AT \$1000/PERSON-REM & & & \\
PLUS ONSITE COSTS & & & \\
\hline
\end{tabular}




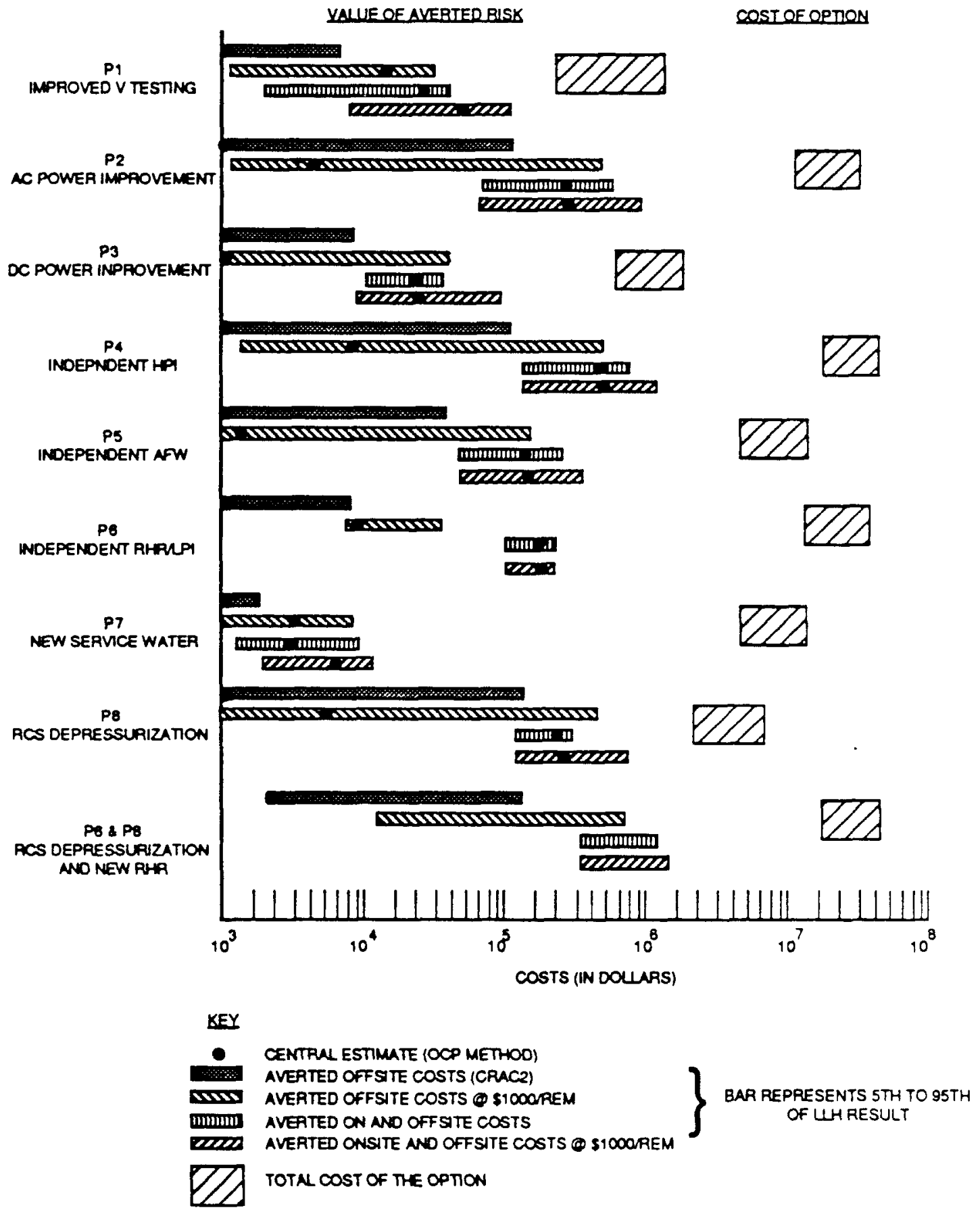

Figure 6-5. Cost/Benefit Comparison for Prevention Options (CRAC2) 


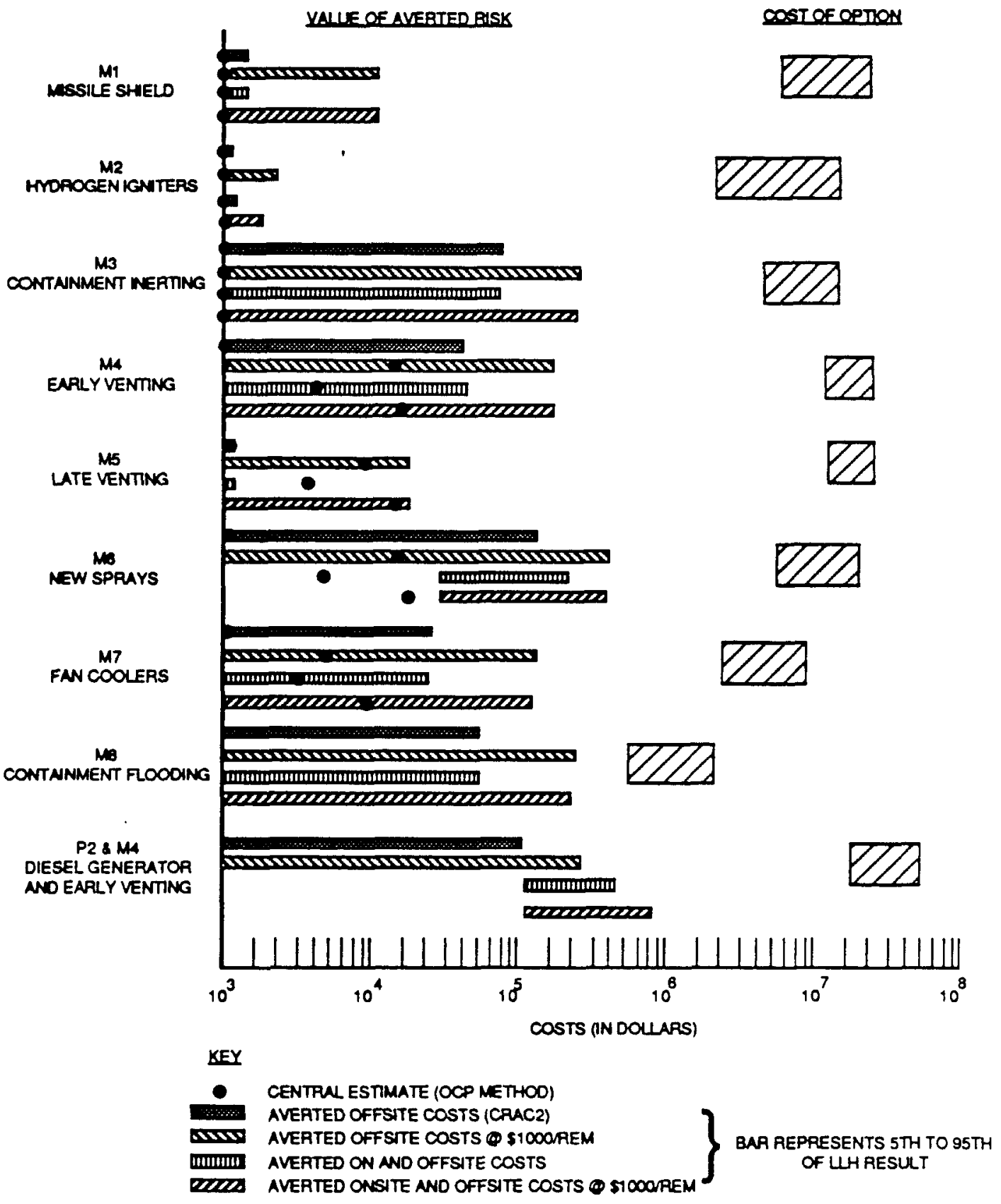

Figure 6-6. Cost/Benefit Comparison for Mitigation Options (CRAC2) 

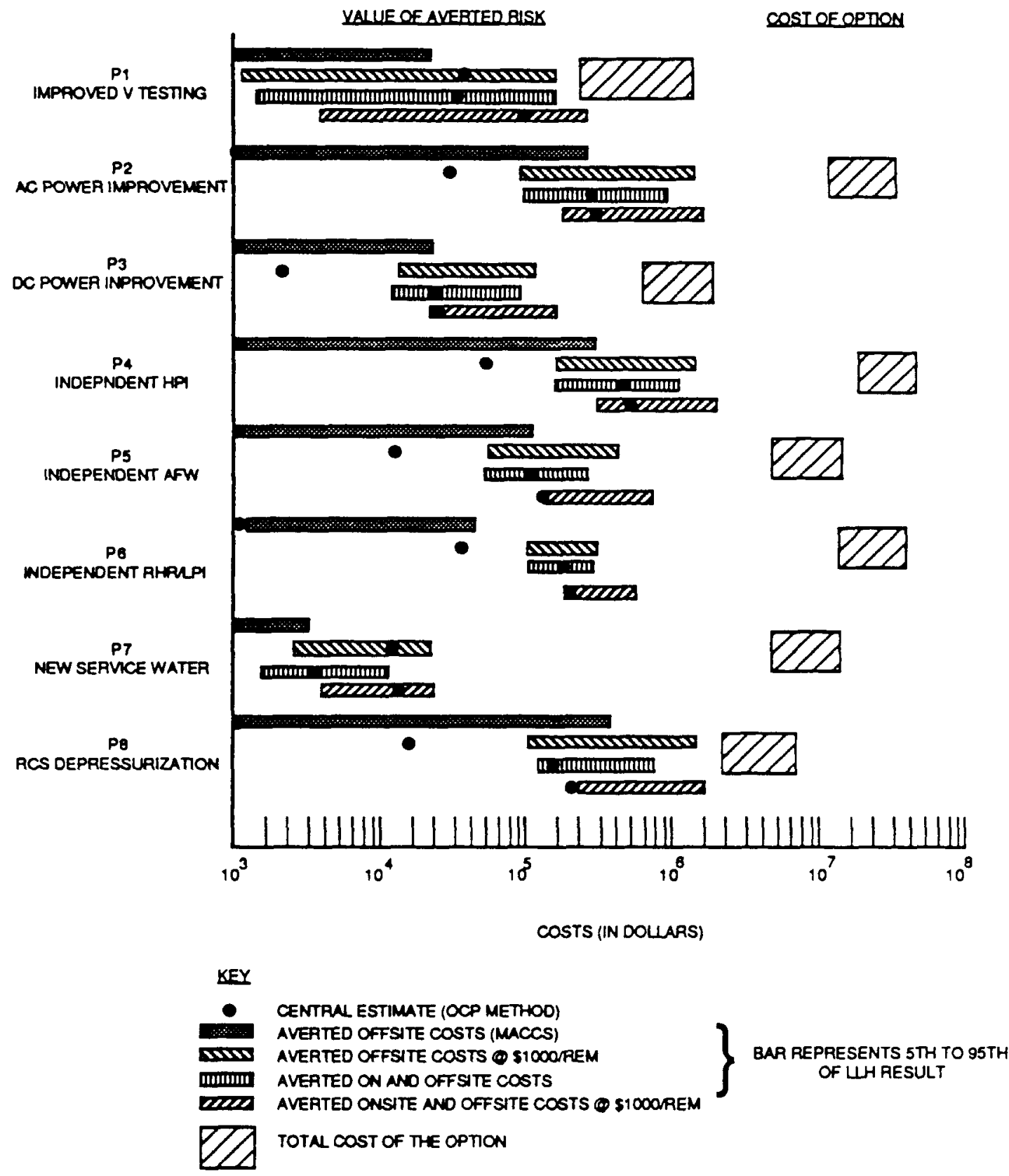

Figure 6-7. Cost/Benefit Comparison for Prevention Options (MACCS) 


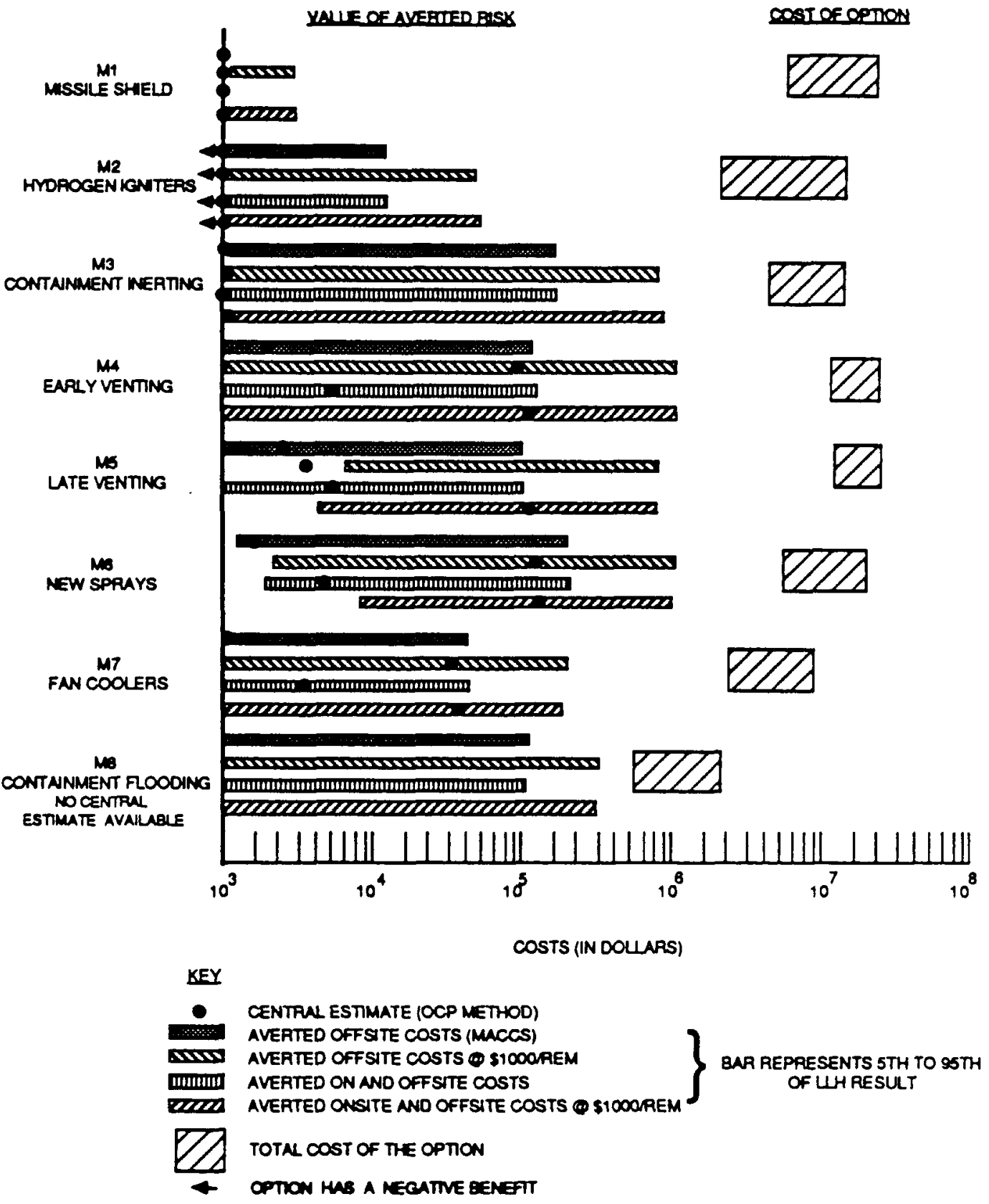

Figure 6-8. Cost/Benefit Comparison for Mitigation Options (MACCS) 
As already stated, the risk reduction measures were calculated using both the $\mathrm{OCP}$ and LLH methods. Several of the options had quite different effects between the two, due to the different contributors to risk (presented in Section 5.5). For example, option P1 appeared attractive for some combinations of costs versus estimated risk reduction in the OCP due to the fact that the $V$ sequence was more important, whereas RCS depressurization was most important to the LLH due to the importance of direct heating. The list of dominant contributors, either to sequence frequency or phenomenology, is critical to the identification of effective options. The comparison of the methods illustrates considerable uncertainty in the dominant contributors, and establishing options with clear effectiveness is difficult. 


\section{Section 7}

\section{INSIGHTS AND CONCLUSIONS}

The results of the SARRP study of risk and risk-reduction for Surry have been outlined in the previous two sections, along with a description of the insights derived from those results. Several of the more important insights are summarized in this section relative to the objectives of the program: (1) to reassess the risk of the Surry facility with current technology and to understand the changes in the perception of risk, (2) to provide insights as to issues most important to the uncertainty in the estimates of risk, and (3) to examine the potential for reduction in risk in a cost/benefit framework. These objectives have been satisfied in the program with the generation of a significant quantity of information regarding reactor safety. Many of the insights that were developed are applicable to certain areas of risk assessment technology and are too detailed for inclusion in this summary, but such insights have been included in the appropriate sections of the main report and appendices.

\subsection{INSIGHTS AND CONCLUSIONS FROM THE RISK REBASELINING}

Several of the more important insights and results of the Surry risk rebaselining are listed briefly below. These are given first with respect to the core-damage frequency, then, in order, for the containment, source term, consequence, and finally the risk analyses. Comparisons to the Reactor Safety Study (RSS) are included where appropriate.

- The core-damage frequency reported in this document (based upon the draft ASEP study of Surry) has a central value of $2.1 \times 10^{-5}$. Uncertainties associated with reliability data could cause the core-damage frequency to be a factor of three higher or lower. In addition, the uncertainty associated with models in the systems analyses could lead to higher or lower frequencies by about a factor of four in either direction.

- The central estimate is about a factor of two less than the RSS and well within the range of results in other current risk studies. It should also be noted that the dominant contributors for the RSS and the ASEP study are quite different. The reasons for the change in assessed frequency and relative contributors include the following: (1) plant modifications, (2) changes in understanding of plant response, and (3) additional evidence from industry experience that has been incorporated into PRA data and methods.

- The SARRP central estimate of containment response in this study indicates that the average probability of early containment failure (i.e., the probability of failure occurring before or at the time of vessel breach) given a core damage sequence is approximately 0.05 , down a factor of four from the RSS results. However, the uncertainty analysis based on the LLH method 
indicated that the value could be as high as 0.8 or as low as 0.02 , with considerably more sample members having results near the top of this range.

- The containment phenomenon of direct heating (ejection of finely fragmented core material and dispersal throughout containment with an efficient heating effect) was found to be an important uncertainty relative to containment failure probability, but a sensitivity study showed that the LLH range for probability of early containment failure did not really change if direct heating was removed. Combinations of other issues such as hydrogen burn pressures and containment failure pressure result in similar uncertainty.

- The source term results for the central estimate are based on the Source Term Code Package (actually most Surry analysis was done with the precursor codes, before they were fully integrated into the STCP), and the predicted releases are generally smaller than those estimated in the RSS. The uncertainty in the source term analysis is very large, with order of magnitude uncertainties in release fractions typical. The LLH uncertainty study identified several uncertainty issues resulting in both larger and smaller releases than the STCP, and several of those issues involving increases were responsible for higher estimates of source terms for the LLH result. These uncertainty issues also account for phenomena not included in the STCP.

- The principal results reported herein have been calculated with the CRAC2 and MACCS consequence codes. The models are similar to those in the RSS, but there have been several improvements. In addition, the evacuation assumptions are different. There were some significant differences between the MACCS results and those calculated using CRAC2. In general, the MACCS code predicts higher consequences by factors of two to five, except that CRAC2 predicts a higher incidence of early injuries. The consequence uncertainties were not evaluated in this study, except for the insights from the CRAC2 and MACCS comparison provided in Section 5.

- Risk was recalculated with methods including many improvements in each area of analysis. The resulting SARRP central estimate of public health risk was found to be low, generally two orders of magnitude lower than the values cited in the RSS. The uncertainty analysis indicated that the RSS point estimate lies near the top of the reasonable range of uncertainty. That is to say, there is a reasonable likelihood that the risk could be as high as the RSS estimate, but a greater likelihood that it is considerably less. The changes in risk are due to fairly complex combinations of the changes in each input to the risk equation, and identifying the importance of individual changes is not practical, but the information presented in the detailed discussions of each analysis area describe the important changes in data and methods.

- The uncertainty was represented by three separate calculations in this study: (1) variability in reliability data, (2) the LLH method, and (3) the OCP method. The first of these is the standard calculation of uncertainty due to reliability data, and it leads to one and one-half orders of magnitude uncertainty about the central estimate of risk. The second and third methods attempt to characterize a different source of uncertainty, namely that 
associated with modeling of phenomena that affect systems, containment and source term analyses. (Uncertainties in consequence modeling were not included due to time and budget constraints.) The LLH method, which was the primary uncertainty analysis method in the study, was based on the input of a group of experts. The experts selected a limited number of the uncertainties thought to be most significant and provided their views of the uncertainty in each. The results of the LLH study indicate about two orders of magnitude uncertainty for the risk of latent cancer fatalities and three and one-half orders of magnitude for early fatality risk, and the uncertainty is generally in the upward direction relative to the SARRP central estimate.

- The OCP method attempts to characterize the uncertainty by calculating three risk values, corresponding to results that might be obtained if the calculation had been performed by three distinct groups within the reactor safety community with differing (and opposing) views on most of the uncertainties. The results of the OCP study help to confirm that the upper values of the LLH uncertainty range were reasonable, but indicate that the lower values of the LLH range might not represent the full uncertainty because they tend to be higher than the OCP lower limit.

- The uncertainty analyses indicated that the identification of individual contributors to uncertainty is not simple because of the complex interrelationships of all the uncertainties relative to the total risk. Direct heating was found to be an important, but not overwhelming, source of uncertainty. Aerosol agglomeration was indicated as a large uncertainty, but the consideration of source term uncertainties is complex, and the agglomeration effect may not be as important as it appears in the statistical tests of the results.

- After direct heating and aerosol agglomeration, a number of other issues were found to be important. For late effects, the $\mathrm{V}$ sequence frequency, the possibility of an induced RCS LOCA, the pressure increment from hydrogen burns and the containment failure pressure appeared as significant uncertainties. For early effects, the amount of in-vessel release from the fuel and the amount of in-vessel retention were significant issues. The containment spray failure probability uncertainty was somewhat important to both measures.

The overall conclusion is that the risks calculated in this study are generally lower than the previous estimate obtained in the RSS. There are significant uncertainties, but the uncertainty in the upper estimate of risk still leads to a relatively low total risk compared to levels of risk typified by the RSS. The downward uncertainty is also quite large, and although the LLH technique may not have fully characterized the lower end of the risk profile, the exact uncertainty in lower risk may not be of great significance since the values are low enough that absolute precision is not required or even practical. 


\subsection{RISK-REDUCTION INSIGHTS AND CONCLUSIONS}

The conclusion stated above, that the risk is generally low, impacts the risk-reduction study. No options were found to be cost effective in the current analysis for Surry because (1) there were no outliers discovered that would be amenable to simple (and inexpensive) fixes, and (2) the low risk levels limited the dollar value associated with any averted risk. The analysis described in Section 6 indicates that the total benefit that could be obtained for averting all of the risk associated with the facility would be limited to several million dollars. This limits any potential changes to improvements in operating practices and procedures that would improve equipment and operator reliability, or minor equipment modifications.

\subsection{LIMITATIONS}

There are several limitations noted in each discussion of the task activities associated with SARRP. It should first be recognized that this study is subject to the same uncertainties as any risk assessment concerning the potential for inaccuracies or incompleteness. The results and conclusions are also Surry-specific, and no extensions of the insights reported herein to other facilities is appropriate without further analysis. Extemal initiating events are not considered, and the consideration of operator action is limited, primarily to those actions called for in procedures. Thus both innovative actions outside procedures and errors of commission are not generally included. The budget and schedule limitations lead to some simplifications which are described in the appropriate task descriptions and summarized in Section 5.5. The uncertainty analysis is not rigorous in a statistical sense, and is intended to represent reasonable ranges of outcomes with insights as to the most important issues. As described in Section 5 , the uncertainty approaches used yielded a number of observations regarding the importance of individual issues, but the complex interrelationships could not be fully evaluated. 
NUREG/CR-4551, VOL. 1: DRAFT REPORT FOR COMMENT (SEPTEMBER, 1986)

\section{Appendix A \\ REASSESSMENT OF CORE-MELT FREQUENCY FOR SURRY}


•

•

-

-

- 


\section{CONTENTS}

Section

A.1 DOMINANT CORE-DAMAGE SEQUENCES A-1

A.2 INTEGRATION OF SEQUENCE AND CONTAINMENT ANALYSIS.

A.2.1 Definition of Plant-Damage States.

A.2.2 Core-Vulnerable Sequences..............................................

A.2.3 Review of Sequence Cut Sets........................................ 13

A.3 CHARACTERIZATION OF UNCERTAINTIES AND SENSITIVITIES..........A-14

A.4 FREQUENCIES OF THE PLANT-DAMAGE STATES.......................A-16

A.5 COMPARISON TO RESULTS FROM THE REACTOR SAFETY STUDY........A-19

References for Appendix A ...........................................................

\section{LIST OF TABLES}

Table

$\underline{\text { Page }}$

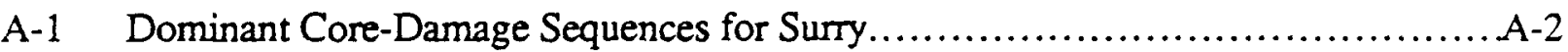

A-2 Definition of Plant Damage States........................................

A-3 Effect of Containment Failure on ECCS Pump Operability ......................A-10

A-4 Effect of Containment Failure on Injection Piping.......................... A-10

A-5 Reviewer Input For Mean Containment Failure Pressure....................... A-10

A-6 Containment Failure Given Slow Pressurization.............................. 11

A-7 Overall Probabilities of ECCS Failure........................................

A-8 Sensitivity Studies for Core Damage Analysis............................... 15

A-9 Plant Damage States and Contributing Sequences..............................

\section{LIST OF FIGURES}

Figure

A-1 Bridge Event Tree for Evaluation of Core-Vulnerable Sequences..................A-9

A-2 Example Box and Whisker Display of Uncertainties............................

A-3 Plant Damage State Frequencies With Uncertainties............................A-18

A-4 Box and Whisker Display of Uncertainties for Total Core-Damage Frequency..... A-19 
-

-

-

-

- 


\section{Appendix A \\ REASSESSMENT OF CORE-MELT FREQUENCY FOR SURRY}

Under the Accident Sequence Evaluation Program (ASEP), a relatively detailed reassessment of the sequences dominating the frequency of core damage for Surry has been performed. The details of that analysis are documented in a separate report [1]. The results that are important to the estimation of risk for Surry are provided in this Section. ${ }^{*}$

\section{A.1 DOMINANT CORE-DAMAGE SEQUENCES}

The core-damage analysis for Surry was performed by developing an event tree for each of several categories of initiating events. For each of the system failures represented as a top event in the event trees, a fault tree was constructed. In order to quantify a particular sequence, as defined by the success or failure of systems appearing as events in the event tree, the appropriate fault trees were first combined and then boolean-reduced. The result of this reduction was a list of minimal cut sets defining the sequences in terms of basic component unavailabilities and human errors. These cut sets were reviewed to identify those that contained failures that could be corrected or compensated for by operator action to terminate the sequence prior to core damage--these actions are referred to as recovery events.

The revised listings of cut sets were then used to obtain the frequencies for the sequences leading to core damage. As described in section A.3, there were two approaches to quantifying the uncertainty in the result. One approach involved the propagation of uncertainties in the reliability data while the other involved sensitivity studies for principal issues. The overall core-damage results (including core-vulnerable), considering only the uncertainties in data, are represented by a distribution with the following parameters:

$\begin{array}{ll}\text { Upper 95\% confidence bound } & 6.1 \times 10^{-5} \\ \text { Mean value } & 2.6 \times 10^{-5} \\ \text { Median value } & 1.7 \times 10^{-5} \\ \text { Lower 5\% confidence bound } & 6.1 \times 10^{-6}\end{array}$

The sequences assessed to dominate the frequency of core damage for Surry in the updated baseline analysis are summarized in Table A-1. The frequencies reported are the pointestimate values obtained by propagating the mean values for the primary events comprising the sequences.

${ }^{*}$ These results are based on the May, 1986 ASEP draft report for Surry, which has since been updated. There was no time to update the risk results prior to the publication of this draft, and the draft ASEP results are reported here to maintain internal consistancy in this report. The changes have been reviewed and their impact in terms of the risk result is minimal. 


\begin{tabular}{|c|c|c|}
\hline Sequence & Description & Frequency* \\
\hline$\overline{T_{1} S L D_{1} C F_{1}}$ & $\begin{array}{l}\text { Loss of offsite power, resulting in a reactor-coolant pump (RCP) seal LOCA due } \\
\text { to the failure of all seal cooling. High-pressure injection, containment spray } \\
\text { injection, and spray recirculation are all unavailable (all caused by the } \\
\text { extended unavailability of all electric power). }\end{array}$ & $5.6 \times 10^{-6}$ \\
\hline $\mathrm{AF}_{1} \mathrm{~F}_{2}$ & $\begin{array}{l}\text { Medium LOCA with failure of containment spray recirculation (which also results } \\
\text { in failure of containment and possibly core heat removal. [Core-vulnerable sequence] }\end{array}$ & $3.3 \times 10^{-6}$ \\
\hline $\mathrm{AF}_{1} \mathrm{~F}_{2}$ & $\begin{array}{l}\text { Large LOCA with failure of containment spray recirculation (which aiso results } \\
\text { in failure of containment and possibly core heat removal. [Core-vulnerable sequence] }\end{array}$ & $1.6 \times 10^{-6}$ \\
\hline $\mathrm{T}_{4 \mathrm{~J}} \mathrm{QH}_{1}$ & $\begin{array}{l}\text { Loss of } 4 \mathrm{kv} \text { bus } \mathrm{J} \text {, followed by failure of a pressurizer relief valve to reclose, } \\
\text { and failure of low-pressure recirculation. }\end{array}$ & $1.5 \times 10^{-6}$ \\
\hline $\mathrm{T}_{4 \mathrm{H}} \mathrm{QH}_{1}$ & $\begin{array}{l}\text { Loss of } 4 \mathrm{kv} \text { bus } \mathrm{H} \text {, followed by failure of a pressurizer relief valve to reclose, } \\
\text { and failure of low-pressure recirculation. }\end{array}$ & $1.3 \times 10^{-6}$ \\
\hline$T_{1} L P$ & $\begin{array}{l}\text { Loss of offsite power, followed by failure of auxiliary feedwater and failure of the } \\
\text { power-operated relief valves to be opened to permit feed-and-bleed cooling. }\end{array}$ & $1.2 \times 10^{-6}$ \\
\hline $\mathrm{T}_{1} \mathrm{~L}(\mathrm{LT}) \mathrm{D}_{1} \mathrm{C}$ & $\begin{array}{l}1 \text { Loss of offsite power and failure of auxiliary feedwater a the long term due to the } \\
\text { extended unavailability of electric power and eventual depletion of the batteries. } \\
\text { The unavailability of power also results in unavailability of high-pressure } \\
\text { injection and containment sprays. }\end{array}$ & $1.1 \times 10^{-6}$ \\
\hline$T_{1} L(S T) D_{1} C F$ & $\begin{array}{l}1 \text { Loss of offsite power and failure of auxiliary feedwater in the short term due to } \\
\text { failure of the turbine-drive pump. The unavailability of power also results in } \\
\text { failure of high pressure injection and containment sprays. }\end{array}$ & $1.1 \times 10^{-6}$ \\
\hline $\mathrm{T}_{4 \mathrm{~J}} \mathrm{QH}_{2}$ & $\begin{array}{l}\text { Loss of } 4 \mathrm{kv} \text { bus } \mathrm{J} \text {, followed by failure of a pressurizer relief valve to reclose, } \\
\text { relief valve to reclose, and failure of high-pressure recirculation. }\end{array}$ & $1.1 \times 10^{-6}$ \\
\hline $\mathrm{TKRD}_{4}$ & $\begin{array}{l}\text { Transient followed by failure to scram and failure of high pressure injection for } \\
\text { emergency boration. }\end{array}$ & $9.8 \times 10^{-7}$ \\
\hline $\mathrm{T}_{4 \mathrm{H}} \mathrm{QH}_{2}$ & $\begin{array}{l}\text { Loss of } 4 \mathrm{kv} \text { bus } \mathrm{H} \text {, followed by failure of a pressurizer relief valve to reclose, } \\
\text { and failure of high-pressure recirculation. }\end{array}$ & $9.2 \times 10^{-7}$ \\
\hline $\mathrm{V}$ & Interfacing-systems LOCA. & $9.0 \times 10^{-7}$ \\
\hline$S_{2} D_{1}$ & Smail LOCA with failure of high-pressure injection. & $7.7 \times 10^{-7}$ \\
\hline $\mathrm{S}_{1} \mathrm{D}_{1}$ & Intermediate LOCA with failure of high-pressure injection. & $7.7 \times 10^{-7}$ \\
\hline $\mathrm{S}_{2} \mathrm{H}_{1}$ & Small LOCA followed by failure of low-pressure recirculation. & $7.2 \times 10^{-7}$ \\
\hline $\mathrm{S}_{2} \mathrm{H}_{2}$ & Small LOCA followed by failure of high-pressure recirculation. & $5.5 \times 10^{-7}$ \\
\hline $\mathrm{S}_{1} \mathrm{H}_{1}$ & Small LOCA followed by failure of low-pressure recirculation. & $5.1 \times 10-7$ \\
\hline TKRZ & $\begin{array}{l}\text { Transient followed by failure to scram during a period when the moderator } \\
\text { temperature coefficient is unfavorable leading to a pressure increase in excess } \\
\text { of RCS capacity }\end{array}$ & $4.8 \times 10^{-7}$ \\
\hline $\mathrm{AD}_{5}$ & Large LOCA with failure of the accumulators to inject. & $4.0 \times 10^{-7}$ \\
\hline$T_{1} Q-D_{1} C F_{1}$ & $\begin{array}{l}\text { Loss of offsite power and failure of emergency power, station blackout, with a } \\
\text { stuck open pressurizer relief valve. The unavailability of power also results in } \\
\text { unavailability of high-pressure injection and containment sprays. }\end{array}$ & $2.7 \times 10^{-7}$ \\
\hline
\end{tabular}


Table A-1 (continued)

DOMINANT CORE-DAMAGE SEQUENCES FOR SURRY

\begin{tabular}{|c|c|c|}
\hline Sequence & Description & Frequency ${ }^{*}$ \\
\hline $\mathrm{AH}_{1}$ & Large LOCA with failure of low pressure recirculation. & $2.5 \times 10^{-7}$ \\
\hline$S_{3} D_{1}$ & Very small LOCA with failure of high-pressure injection. & $1.5 \times 10^{-7}$ \\
\hline $\mathrm{T}_{4 \mathrm{~J}} \mathrm{Q}-\mathrm{CF}_{1}$ & $\begin{array}{l}\text { Loss of } 4 \mathrm{kv} \text { bus } \mathrm{J} \text {, followed by failure of a pressurizer relief valve to reclose, } \\
\text { relief valve to reclose, and failure of containment sprays. [Core-vulnerable sequence.] }\end{array}$ & $7.7 \times 10^{-8}$ \\
\hline $\mathrm{T}_{4 \mathrm{H}} \mathrm{Q}-\mathrm{CF}_{1}$ & $\begin{array}{l}\text { Loss of } 4 \mathrm{kv} \text { bus } \mathrm{H} \text {, followed by failure of a pressurizer relief valve to reclose, } \\
\text { relief valve to reclose, and failure of containment sprays. [Core-vulnerable sequence.] }\end{array}$ & $5.3 \times 10^{-8}$ \\
\hline $\mathrm{S}_{2} \mathrm{H}_{1} \mathrm{~F}_{1} \mathrm{~F}_{2}$ & $\begin{array}{l}\text { Small LOCA followed by failure of high-pressure recirculation and containment } \\
\text { spray recirculation }\end{array}$ & $4.8 \times 10^{-8}$ \\
\hline $\mathrm{T}_{1} \mathrm{LF}_{1} \mathrm{~F}_{2}$ & $\begin{array}{l}\text { Loss of offsite power with failure of auxiliary feedwater and failure of containment } \\
\text { heat removal. [Core-vulnerable sequence.] }\end{array}$ & $5.0 \times 10^{-8}$ \\
\hline $\mathrm{S}_{1} \mathrm{CF} F_{1}$ & $\begin{array}{l}\text { Intermediate LOCA, followed by failure of containment spray injection and } \\
\text { recirculation. [Core-vulnerable sequence.] }\end{array}$ & $9.7 \times 10^{-9}$ \\
\hline $\mathrm{T}_{2} \mathrm{LF}_{1} \mathrm{~F}_{2}$ & $\begin{array}{l}\text { Loss of the power conversion system with failure of auxiliary feedwater and failure } \\
\text { of containment heat removal. [Core-vulnerable sequence.] }\end{array}$ & $5.4 \times 10^{-9}$ \\
\hline $\mathrm{ACF}_{1}$ & $\begin{array}{l}\text { Large LOCA, followed by failure of containment spray injection and } \\
\text { recirculation. [Core-vulnerable sequence.] }\end{array}$ & $4.9 \times 10^{-9}$ \\
\hline$S_{1} D_{6} D_{1} C$ & $\begin{array}{l}\text { Small LOCA followed by failure of the high and low pressure injection systems } \\
\text { and the containment spray injection system. }\end{array}$ & $2.7 \times 10^{-9}$ \\
\hline \multirow[t]{2}{*}{$\mathrm{ACD}_{6}$} & $\begin{array}{l}\text { Large LOCA, followed by failure of low pressure recirculation and containment } \\
\text { spray injection. }\end{array}$ & $1.4 \times 10^{-9}$ \\
\hline & Core Damage Total (including core-vulnerable sequences) & $2.6 \times 10^{-5}$ \\
\hline
\end{tabular}

* Point estimate values derived by propagating mean values of individual events.

\section{A.2 INTEGRATION OF SEQUENCE AND CONTAINMENT ANALYSIS}

In order to ensure both that the core-damage sequences include sufficient discrimination to allow appropriate treatment in the containment event tree and that the containment analysis correctly reflects the nature of the sequences, the two areas of the analysis must be carefully coordinated. For Surry, this integration was accomplished primarily through the binning of sequences into plant-damage states. The plant-damage states and other aspects of the integration process are described in this section.

\section{A.2.1 Definition of Plant-Damage States}

The plant-damage states reflect the sequence effects that can impact the likelihood, timing, and severity of resulting releases. This binning process has three important attributes: 
- It helps to ensure that the core-damage sequences reflect the system states that could influence the containment response;

- Because the plant-damage states are defined according to the needs of the containment analysis, it provides for a more systematic treatment of the sequences in the walkthroughs for the containment event trees; and

- It allows for the expedient grouping of sequences with similar characteristics, reducing the potential for unnecessary repetition of containment analyses.

The plant-damage states for Surry were initially developed in a working session attended by representatives of both SARRP and ASEP, and were later refined through an iterative process. A number of parameters were identified that could conceivably have an impact on the progression of a core-damage accident. This list was reduced to the following set of parameters considered to be most important:

- The size and location of the breach in the reactor-coolant system,

- Whether or not there is water in the reactor cavity at the time of the breach of the reactor vessel following core melt,

- The status of the containment spray and heat removal systems,

- The status of the secondary side heat removal systems when there is no LOCA,

- Whether the sequence involved failure of the containment prior to melting of the core, and

- Whether or not the accident involved a bypass of containment.

From this list, a set of five parameter categories was developed. Four of the categories are applicable for all cases, while one is only needed as a discriminator for accidents initiated by transients with no LOCAs. The possible states for each of these categories were assigned a letter, leading to the definition of four-element designators for each plant-damage state. The parameter categories and their states are as follows:

1. The integrity of the reactor-coolant system (RCS) pressure boundary:

A Large or intermediate LOCA leading to low pressure in the RCS prior to breaching of the reactor pressure vessel,

$S$ Small LOCA resulting in intermediate pressure in the RCS prior to breaching of the reactor pressure vessel,

T No initial LOCA or a very small LOCA with the RCS at high pressure prior to the vessel breach (the pressurizer relief valves may be cycling), and

$\mathrm{V}$ LOCA that bypasses the containment boundary. 
2. Whether or not water from the refueling water storage tank (RWST) has been discharged into containment:

Y The RWST inventory has been discharged to containment, either by the emergency injection systems or by the containment spray injection system, and

$\mathrm{N}$ The RWST inventory has not been discharged to the containment.

3. Whether or not containment heat removal is available:

Y Containment heat removal is available, and

$\mathrm{N}$ Containment heat removal is not available.

4. The status of the containment spray systems:

B Both the containment spray injection and the containment spray recirculation systems are available,

I The containment spray injection system is available, but the recirculation system has failed,

$\mathbf{R}$ Containment spray recirculation is available, but the injection system failed (possible in Surry because of the separate, incontainment spray recirculation system), and

$\mathrm{N}$ Neither injection nor recirculation flow is available.

5. Whether or not steam generator heat removal is available:

D The steam generators are dry, and

W The steam generators are wet.

From this list, a total of 65 plant-damage states is possible. However, many of the possible combinations are not of interest (for example, for a bypass of containment, the status of the containment spray system is not very important) or are not physically meaningful (e.g., if the RWST inventory has not been discharged to containment, it is not possible for containment spray injection to have functioned. Thus, of the possible states, there are 20 of interest. They are identified and described in Table A-2.

\section{A.2.2 Core-Vulnerable Sequences}

One type of sequence is not addressed specifically by this set of plant-damage states. This type of sequence involves conditions in which the core is being cooled by high- or lowpressure recirculation, with decay heat rejected to the containment through a break or through the pressurizer relief valves. Under these circumstances, heat removal must be provided by the containment spray recirculation systems and their heat exchangers. Because of the mass and energy discharged to containment, failure of the containment heat removal system is likely to 
Table A-2

DEFINITION OF PLANT-DAMAGE STATES

\begin{tabular}{ll}
\hline Designator & Description \\
\hline $\mathrm{V}$ & Interfacing systems LOCA \\
AYYB & $\begin{array}{l}\text { LOCA leading to low pressure in the RCS pror to vessel breach, with the RWST inventory } \\
\text { discharged to containment; containment heat removal, spray injection, and spray recirculation are } \\
\text { all available. }\end{array}$
\end{tabular}

AYNI LOCA leading to low pressure in the RCS pnor to vessel breach, with the RWST inventory discharged to containment; containment spray injection is avallable, but containment heat removal and recurculation are not functioning.

AYYR LOCA leading to low pressure in the RCS pnor to vessel breach, with the RWST inventory discharged to containment; contanment spray injectuon has faled, but recirculation and heat removal are avalable.

AYNN LOCA leading to low pressure in the RCS prior to vessel breach, with the RWST inventory discharged to containment. The containment spray are not avalable. Nole that this implies that there may be limited water in the reactor cavity.

ANNN LOCA leading to low pressure in the RCS prior to vessel breach, with no RWST inventory discharged to contannment. The contanment spray are not avaulable. Note that this implies that there may be very limuted water in the reactor cavity.

SYYB Small LOCA leading to intermediate pressure in the RCS prior to vessel breach, with the RWST inventory discharged to contanment; containment heat removal, spray injection, and spray recurculation are all avalable.

SYNI Small LOCA leading to intermediate pressure in the RCS pnor to vessel breach, with the RWST inveatory discharged to containment; containmeat spray injection is available, but containment heat removal and recurculation are not functioning.

SYYR Small LOCA leading to intermediate pressure th the RCS prior to vessel breach, with the RWST inventory discharged to containment; contanment spray injection has failed, but recirculation and heat removal are avaulable.

SYNN Small LOCA leading to intermediate pressure in the RCS pnor to vessel breach, with the RWST inventory discharged to contanment. The contannment spray are not avalable. Note that this implies that there may be limited water in the reactor cavity.

SNNN Small LOCA leading to intermediate pressure in the RCS pnor to vessel breach, with no RWST inventory discharged to contanment. The containment spray are not avalable Note that this implies that there may be very limited water in the reactor cavity.

TYYBD Transient or very small LOCA (high RCS pressure prior to vessel breach), with the RWST inventory discharged to containmeat; containment heat removal, spray injection, and spray recirculation are all avalable. The steam generators have no water inventory.

TYYBW As TYYBD with water in the steam generators.

TYNID Transient or very small LOCA (high RCS pressure pnor to vessel breach), with the RWST inventory discharged to containment; containment spray injection is avallable, but containment heat removal and recirculation are not functioning. The steam generators have no water inventory

TYNIW As TYNIW with water in the steam generators.

TYYRD Transient or very small LOCA (high RCS pressure prior to vessel breach), with the RWST inventory discharged to containment; containment spray injection has falled, but recirculation and heat removal are avallable. The steam generators have no water inventory

TYYRW As TYYRD with water in the steam generators. 
Table A-2 (continued)

DEFINITION OF PLANT-DAMAGE STATES

\begin{tabular}{ll}
\hline Designator & Description \\
\hline TYNND & $\begin{array}{l}\text { Transient or very small LOCA (high RCS pressure prior to vessel breach), with the RWST inventory } \\
\text { discharged to containment. The containment spray are not available. Note that this implies that } \\
\text { there may be limited water in the reactor cavity. The steam generators have no water inventory. }\end{array}$ \\
TYNNW & $\begin{array}{l}\text { As TYNND with water in the steam generators. } \\
\text { TNNND }\end{array}$ \\
$\begin{array}{l}\text { Transient or very small LOCA (high RCS pressure prior to vessel breach), with no RWST inventory } \\
\text { discharged to containment. The containment spray are not available. Note that this implies that } \\
\text { there may be very limited water in the reactor cavity. There are also no systems supplying water to } \\
\text { the steam generators. }\end{array}$
\end{tabular}

lead eventually to overpressurization of the containment. These sequences may or may not result in core melt, and were therefore designated by ASEP as "core-vulnerable" sequences.

Several of these scenarios were examined by Battelle to ascertain whether or not containment failure would occur, and if so, when the failure would be expected. In consultation with the ASEP analysts, the SARRP team constructed a bridge event tree which identifies phenomenological events, system failures, and operator actions that might determine whether a core-vulnerable sequence proceeds to a core-damage sequence. Assessing the probabilities of the individual pathways resulted in a fraction that determines the percent of core-vulnerable sequences expected to result in core melt as a result of the initial containment failure.

\section{Affected_Sequences}

For small LOCAs and transients, it was determined that the removal of decay heat via the steam generators would prevent overpressurization of containment, provided feedwater were available [2]. Even if feedwater were unavailable, the time it would take for containment to be overpressurized would be very long, although this case was retained for further analysis using the bridge event tree. The thermal hydraulic analysis of containment cooling modeled the specific Surry conditions and procedures as closely as possible.

For large and intermediate LOCAs however, the Battelle analysis showed that containment would be likely to fail at 51 hours. This result may well be conservative for two reasons. First, the Source Term Code Package does not adequately model containment heat sinks and the loss of heat to the environment is probably greater than the value calculated. Second, although the non-emergency fan coolers at Surry are at least partially submerged when 
the RWST is injected, and are therefore unavailable during these accidents, the operations staff could continue to supply cooling water to the fans cooling units, achieving some degree of heat removal.

\section{Bridge Event Tree}

It is recognized that the containment failure does not automatically lead to core damage. First of all, the core cooling systems could continue to operate after the containment failure. Second, if the systems did fail, other systems could potentially be used to perform the cooling function. Third, because of the duration of the accident, the operators possibly could act to prevent overpressurization altogether, by a controlled depressurization. The ASEP sequence frequency already accounted for recovery of the heat removal systems in the time frames presented by these accidents.

In consultation with ASEP analysts, the bridge event tree illustrated in Figure A-1 was constructed to determine the probability of core-vulnerable sequences resulting in core damage. The events and phenomena that could lead to a consequential failure of emergency core systems are described below:

- Containment pressure relief. Pressure could be maintained within the containment capacity if operator action were taken to accomplish venting. The operations staff, and support personnel would have two days to take this type of corrective action.

- Iniection piping not damaged. If the containment were to fail structurally, there is a potential that the injection piping itself could be damaged by the failure.

- Net-positive suction head (NPSH) for the low-pressure injection pumps maintained. As the containment depressurizes after failure, some of the water in the containment sump will flash to steam; sump water will also continue to evaporate in the ensuing period. If sufficient subcooling of the sump water is lost, the low-pressure injection pumps may fail due to a lack of adequate NPSH.

- Low-pressure injection pump in standby. In the long period following the early phases of the accident, it is very possible that the operators will secure equipment that is not needed for the immediate preservation of core cooling. If a low-pressure injection pump has been returned to a standby condition, it may be possible to maintain core cooling even if the operating pump fails due to loss of NPSH. It was judged to be likely that, in this situation, the operators would start the standby pump and take measures to protect it from failure. For example, because this situation would only arise many hours into the accident, it would be possible to stop the pump periodically if it were to begin to overheat due to NPSH problems. 


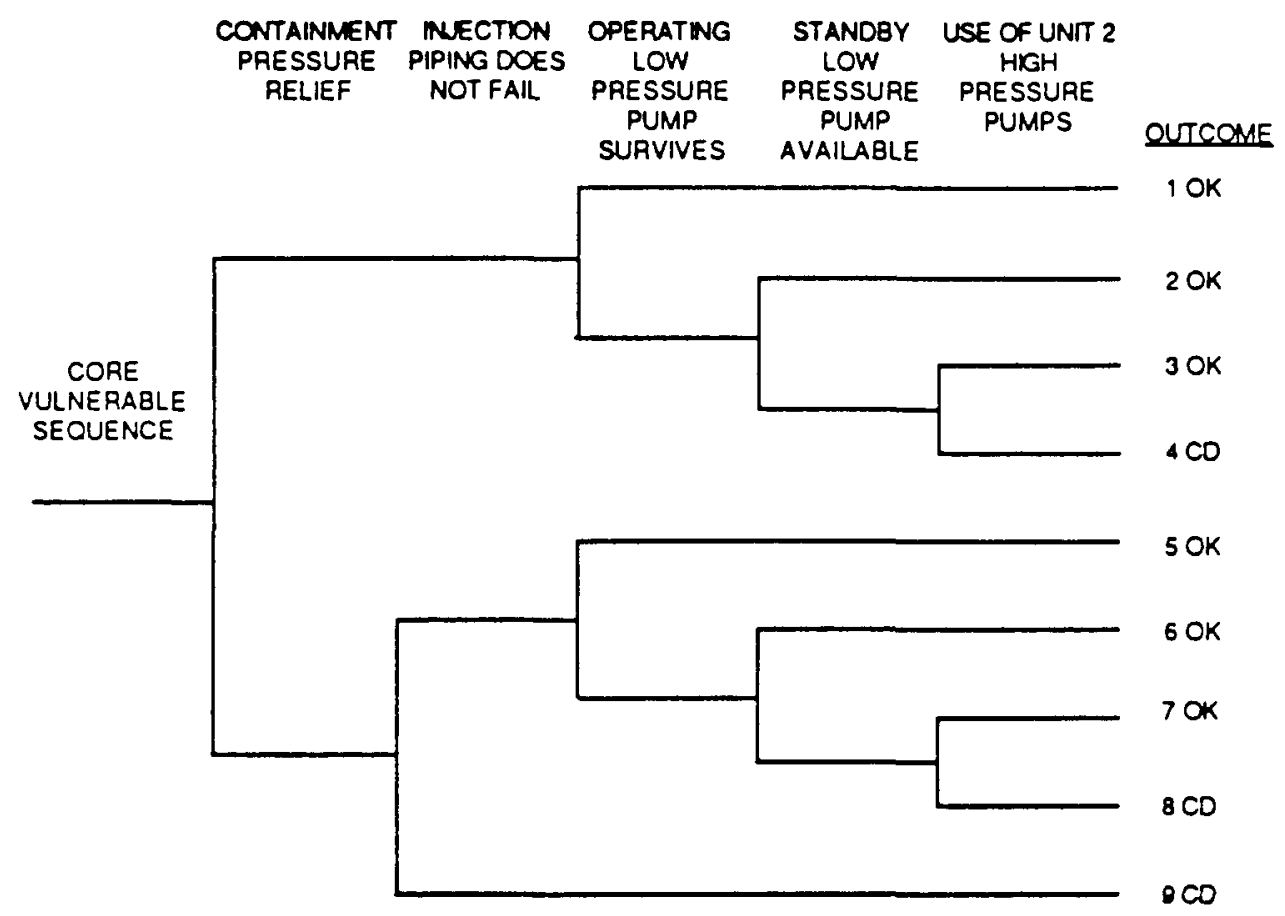

Figure A-1. Bridge Event Tree for Evaluation of Core-Vulnerable Sequences

- Use High Pressure Pump from Other Unit or Refill RWST and Use HighPressure Injection. The high pressure systems are Surry are cross connected between units, and the RWST inventory of Unit 2 is part of the technical specification of Unit 1. The high pressure pumps can be used to inject into the hot legs to avoid any problems with the buildup of boron. A final possibility considered in the event tree was the restoration of a level in the RWST adequate to provide suction to the high-pressure injection pumps. Again, it was judged to be likely that the operators could establish such a pathway for core cooling. Because of the reduced requirements for decay-heat removal at this point, the flow could be throttled back to prevent runout of the high-pressure injection pumps, while still providing adequate core cooling.

Of the five events, the second and third were referred to the containment review experts for consideration. The other three and the actual tree constructions were examined jointly by SARRP and ASEP.

\section{Expert Review Input}

Tables A-3 and A-4 illustrate the inputs of the reviewers for the probability of ECCS pump and piping failure as a function of containment failure size. Table A-5 shows the 
probability estimates of these same reviewers for containment failure pressure and Table A-6 illustrates their estimates of containment hole size as a function of containment failure pressure. Only three of the reviewers responded to all four questions as indicated. The inputs for containment failure pressures of 67 and 180 psig were added to those of 85 and 143 psig, respectively, for the purposes of this analysis.

These inputs were combined into composites as illustrated in Table A-7. The composite results of each reviewer were used for three distinct walkthroughs of the bridge tree.

TABLE A-3

EFFECT OF CONTAINMENT FAILURE ON ECCS PUMP OPERABILITY

\begin{tabular}{|c|c|c|c|}
\hline \multirow{2}{*}{$\begin{array}{c}\text { OUTCOME } \\
\text { CORRELATED } \\
\text { TO FAILURE SIZE }\end{array}$} & \multicolumn{3}{|c|}{$\begin{array}{l}\text { WEIGHTING FACTORS } \\
\text { Reviewer }\end{array}$} \\
\hline & 1 & 2 & 3 \\
\hline 1. 4 sq. in. & 0.0 & 0.0 & 0.0 \\
\hline 2. $.1 \mathrm{sq} . \mathrm{ft}$ & 0.0 & 0.0 & 0.0 \\
\hline 3. $.7 \mathrm{sq} . \mathrm{ft}$. & .2 & .2 & .1 \\
\hline 4. 7 sq. ft. & .3 & .4 & .3 \\
\hline
\end{tabular}

TABLE A-4

EFFECT OF CONTAINMENT FAILURE ON INJECTION AND SUCTION PIPING

\begin{tabular}{llcl}
\hline $\begin{array}{c}\text { OUTCOME } \\
\text { CORRELATED } \\
\text { TO FAIIURE SIZE }\end{array}$ & 1 & $\begin{array}{c}\text { WEIGHTING FACTORS } \\
\text { Reviewer }\end{array}$ & 3 \\
\hline 1.4 sq. in. & 0.0 & 0.0 & 0.0 \\
$2 . \quad .1$ sq. ft. & 0.0 & 0.0 & 0.0 \\
3.7 sq.f. & 0.0 & 0.0 & 0.0 \\
4.7 sq. ft. & .1 & .01 & .1 \\
\hline
\end{tabular}

TABLE A-5

REVIEWER INPUT FOR MEAN CONTAINMENT FAILURE PRESSURE

\begin{tabular}{lllcl}
\hline & $\begin{array}{c}\text { MEAN } \\
\text { FAIIURE } \\
\text { PRESSURE }\end{array}$ & 1 & $\begin{array}{c}\text { WEIGHTING FACTORS } \\
\text { Reviewer }\end{array}$ & 3 \\
\hline 1. & $67 \mathrm{psi}$ & 0.0 & 0.0 & 0.0 \\
2. & $85 \mathrm{psi}$ & .25 & .2 & .1 \\
3. & $119 \mathrm{psi}$ & .5 & .5 & .3 \\
4. & $143 \mathrm{psi}$ & .2 & .2 & .5 \\
5. & $180 \mathrm{psi}$ & .05 & .1 & .05 \\
\hline
\end{tabular}


NUREG/CR-4551, VOL. 1: DRAFT REPORT FOR COMMENT (SEPTEMBER, 1986)

TABLE A-6

CONTAINMENT FAILURE SIZE GIVEN SLOW PRESSURIZATION

\begin{tabular}{|c|c|c|c|}
\hline \multirow[t]{2}{*}{$\begin{array}{l}\text { OUTCOME } \\
\text { Size of Opening }\end{array}$} & \multicolumn{3}{|c|}{$\begin{array}{c}\text { WEIGHTING FACTORS } \\
\text { Reviewer }\end{array}$} \\
\hline & 1 & 2 & 3 \\
\hline \multicolumn{4}{|c|}{ For Containment Failure Pressure 119 psig } \\
\hline 1. 4 sq. in. & .15 & .35 & .2 \\
\hline 2. $.1 \mathrm{sq} . \mathrm{ft}$. & .2 & .35 & .2 \\
\hline 3. .7 sq.ft. & .2 & .2 & .3 \\
\hline 4. 7 sq. $\mathrm{ft}$. & .45 & .1 & .3 \\
\hline \multicolumn{4}{|c|}{ For Containment Failure Pressure 85 osig } \\
\hline 1. 4 sq. in. & .15 & .5 & .4 \\
\hline 2. .1 sq. ft. & .2 & .4 & .5 \\
\hline 3. .7 sq.ft. & .2 & .1 & .1 \\
\hline 4. 7 sq. ft. & .45 & 0.0 & 0.0 \\
\hline \multicolumn{4}{|c|}{ For Containment Failure Pressure 143 or 180 psig } \\
\hline 1. 4 sq. in. & .15 & .3 & 0.0 \\
\hline 2. .1 sq. $\mathrm{ft}$. & .2 & .3 & 0.0 \\
\hline 3. .7 sq.ft. & .2 & .2 & .4 \\
\hline 4. 7 sq. $\mathrm{ft}$. & .45 & .2 & .6 \\
\hline
\end{tabular}

TABLE A-7

OVERALL PROBABILITIES OF ECCS FAILURE

\begin{tabular}{llcc}
\hline $\begin{array}{c}\text { OUTCOME } \\
\text { CORRELATED } \\
\text { TO FAILURE SIZE }\end{array}$ & 1 & $\begin{array}{c}\text { WEIGHTING FACTORS } \\
\text { Reviewer }\end{array}$ & 3 \\
\hline $\begin{array}{l}\text { PUMP FAILURE } \\
\text { PROBABIITY }\end{array}$ & .08 & .17 & .1 \\
$\begin{array}{l}\text { PIPING FAIIURE } \\
\text { PROBABIITY }\end{array}$ & .0014 & .03 & .019 \\
\hline
\end{tabular}


NUREG/CR-4551, VOL. 1: DRAFT REPORT FOR COMMENT (SEPTEMBER, 1986)

\section{Event Tree Sequence Description}

The sequences of the bridge event tree are described briefly below. The quantification of the other events is also discussed.

Sequence 1. No core or containment damage results from this sequence. The containment pressure has been relieved and the operating pumps have not failed. The pressure relief is assumed to be controlled such that cavitation is not a problem with the operating pumps.

Sequence 2. Once again there is no core damage. The operating pump does fail, but the operator then uses the pump he previously placed in standby.

Sequence 3 . This is similar to sequence 2 , but the operator uses the high pressure injection pumps from Unit 2.

Sequence 4. Core damage results if the depressurization is large enough to cavitate the pumps and the operations staff does not perform any recovery action.

Sequence 5. The containment fails, but the core is maintained by the operating pumps which are not damaged by the depressurization.

Sequence 6. This is analagous to sequence 2 , except that the containment does fail. The core is in a stable, cooled state.

Sequence 7. This is analagous to sequence 3, except that the containment does fail. The core is in a stable, cooled state.

Sequence 8. Core damage results because depressurization is large enough to cavitate the pumps and the operations staff does not perform any recovery action.

Sequence 9. Core damage results because the containment failure causes the injection piping to fail directly.

For the first event in the tree, containment pressure relief, a probability of 0.5 was assigned. This value was chosen because the analysts are nearly completely uncertain considering the likelihood of this event. There is certainly the time and means to affect containment venting, but to our knowledge, there are no procedures to do so. Also, both the ASEP and SARRP analyses have assumed that the instrumentation will continue to operate. If it does not, the operations staff may be very reluctant to create a containment opening without good knowledge of core and containment conditions. In addition, state and federal authorities could intervene, how, we cannot speculate.

For the fourth and fifth events, it was assessed "likely" that the staff will succeed if the operator has successfully recognized the situation and vented the containment. In keeping with 
the philosophy of verbal descriptor probabilities used in the containment analysis, a value of 0.9 for success of each of these events was assigned

If venting did not occur, the fourth and fifth events are believed to be "uncertain", and a probability of 0.5 was assigned. At this point in time it is outside the scope to assess all of the reasons for failure to vent, but it is this event which is closely coupled with the other operator recoveries.

\section{Results of the Bridge Tree Assessment}

Three separate walkthroughs of the tree were quantified. All three used the split fractions of the first, fourth and fifth events discussed above. Each walkthrough differed in the second and third events by using the separate estimates of the three individual reviewers.

The probabilities of core vulnerable sequences resulting in core damage were calculated as $0.01,0.02$, and 0.04 . These were used as the optimistic, central and pessimistic in the OCP analysis, respectively. In the limited Latin hypercube analysis, each result was weighted equally. ${ }^{*}$

\section{A.2.3 Review of Sequence Cut Sets}

After the cut sets for the core-damage sequences were obtained, the final step in the integration process was a review to assure that they fit the plant-damage states to which the sequences had been assigned. For cases in which the timing or other aspects of failures represented by the cut set implied a different plant-damage state, it was reassigned.

This process was also important in identifying other conditions that could affect the likelihood of events in the containment event tree. One such event of particular importance is the availability of electric power, a condition that is also reflected in the first event in the containment event tree. It was necessary to flag sequences that were dominated by or had a strong contribution from station blackout (loss of all ac power) in order for them to be treated properly in the containment analysis. It was also necessary to specify what, if any, credit had been given to recovery of electric power prior to core damage. Later recovery is considered in the containment event tree, since it can allow for the restoration of the containment spray systems long after core damage has begun. The likelihood of the later recovery is, of course, dependent upon the credit given for recovery earlier in the sequence.

\footnotetext{
${ }^{*}$ The resulting spread in the results is limited, perhaps more than if the uncertainty in this issue had been addressed by a larger group, or if their input had been manipulated differently.
} 
Finally, as described previously, the probability of core damage conditional on a total loss of containment heat removal following a large or intermediate LOCA was estimated to be 0.02. The frequencies of the core-vulnerable sequences were therefore adjusted by this factor.

\section{A.3 CHARACTERIZATION OF UNCERTAINTIES AND SENSITIVITIES}

The characterization of uncertainties in the estimated frequency of core melt was performed in two steps. First, the core-melt analysts developed a set of issues they considered to be most capable of affecting the assessed frequency of core melt. These issues represent uncertainties in the phenomena that could lead to core melt, alternative uses of reliability data, and credit for equipment not considered in the base analysis. These issues were the following:

- Loss of cooling for reactor-coolant pump (RCP) seals. The likelihood and severity of serious leakage from the RCP seals following the loss of seal cooling is not well known. Because a station-blackout event could result both in the loss of seal cooling (possibly leading to a LOCA), and unavailability of emergency core cooling, this is a significant source of uncertainty.

- Recovery of electric power following station blackout. In part because of the first issue, the potential for recovery of electric power is a very important consideration.

- Likelihood of common-cause failures. Because of the redundancy in many of the important systems, failures of components due to common causes can have a strong effect on the frequency of core-melt sequences. However, there is a very limited data base available from which to derive estimates of the probabilities of such failures.

- Check-valve failure rates. The interfacing-systems LOCA involves failure two check valves in series and, because it results directly in an early failure of containment, has the potential to be a dominant contributor to risk. The frequency of this event is strongly dependent upon the failure models and rates used for the check valves. However, the data for such occurrences is not well developed, largely because many such failures are difficult to detect.

Based on these issues, a set of ten sensitivity cases aimed at investigating their significance was developed. These ten cases are described briefly in Table A-8, and their impact on total core-damage damage frequency (point estimates based on the propagation of mean values for the primary events) is also noted. The core-vulnerable sequences are included in these totals without accounting for the reduction in frequency outlined above. 
Table A-8

SENSITIVITY STUDIES FOR CORE-DAMAGE ANALYSIS

\begin{tabular}{|c|c|c|c|}
\hline No. & Issue & Description of Sensitivity Study & $\begin{array}{l}\text { Total Core- } \\
\text { Damage } \\
\text { Frequency }\end{array}$ \\
\hline -- & & Core-damage frequency for the base case. & $2.7 \times 10^{-5}$ \\
\hline 1. & RCP Seal LOCA & $\begin{array}{l}\text { Assume that seal LOCA occurs } 1 / 2 \mathrm{hr} \text { after loss of all seal } \\
\text { cooling, and that ac power must be restored within } 1 / 2 \mathrm{hr} \\
\text { after seal LOCA to prevent core damage. }\end{array}$ & $3.7 \times 10^{-5}$ \\
\hline 2. & RCP Seal LOCA & Assume seal LOCA does not $\propto c c u r$ after loss of cooling. & $2.2 \times 10^{-5}$ \\
\hline 3. & Recovery of power & $\begin{array}{l}\text { Use altemative (more optimistic) set of data for experience in } \\
\text { recovery of offsite electric power. }\end{array}$ & $1.9 \times 10^{-5}$ \\
\hline 4. & Recovery of power & $\begin{array}{l}\text { Permit credit for use of gas turbine-generator as a backup means } \\
\text { of restoring electric power. }\end{array}$ & $2.2 \times 10^{-5}$ \\
\hline 5. & $\begin{array}{l}\text { Common-cause } \\
\text { failure rates }\end{array}$ & $\begin{array}{l}\text { Use alternative (more optimistic) distribution for probability } \\
\text { of common-cause failure of service water valves due to bio-fouling. }\end{array}$ & $3.5 \times 10^{-5}$ \\
\hline 6. & $\begin{array}{l}\text { Common-cause } \\
\text { failure rates }\end{array}$ & $\begin{array}{l}\text { Use alternative (more pessimistic) interpretation of beta factors } \\
\text { for common-cause failure rates. }\end{array}$ & $3.3 \times 10^{-5}$ \\
\hline 7. & $\begin{array}{l}\text { Common-cause } \\
\text { failure rates }\end{array}$ & Eliminate use of beta factors. & $2.2 \times 10^{-5}$ \\
\hline 8. & $\begin{array}{l}\text { Check-valve } \\
\text { failure rates }\end{array}$ & $\begin{array}{l}\text { Apply beta factors for motor-operated valves to check valves for } \\
\text { interfacing-systems LOCA calculation. }\end{array}$ & $3.4 \times 10^{-5}$ \\
\hline 9. & $\begin{array}{l}\text { Check-valve } \\
\text { failure rates }\end{array}$ & Use more optimistic distribution for failure rates for check valves. & $2.4 \times 10^{-5}$ \\
\hline 10. & $\begin{array}{l}\text { Check-valve } \\
\text { failure rates }\end{array}$ & Use more pessimistic distribution for failure rates for check valves. & $3.0 \times 10^{-5}$ \\
\hline
\end{tabular}

The second step in the uncertainty analysis was the propagation of the probability distributions for the individual events in the sequence cut sets to obtain an overall estimate of uncertainty in the frequencies of core melt and of the plant-damage states. This was done by the performance of a Monte Carlo simulation using the SEP computer code [3]. The propagation was performed both for the base-case analysis and for each of the ten sensitivity cases. The result was the development of a "box-and-whisker" display for the uncertainties in the core-damage frequencies, as illustrated by Figure A-2. The bounds of the box represent the highest and lowest mean values from the sensitivity studies. The figure also includes the mean, 5th-percentile, and 95th-percentile values for the base-case analysis, and the highest 95th-percentile and lowest 5 th percentile values from the sensitivity cases. 


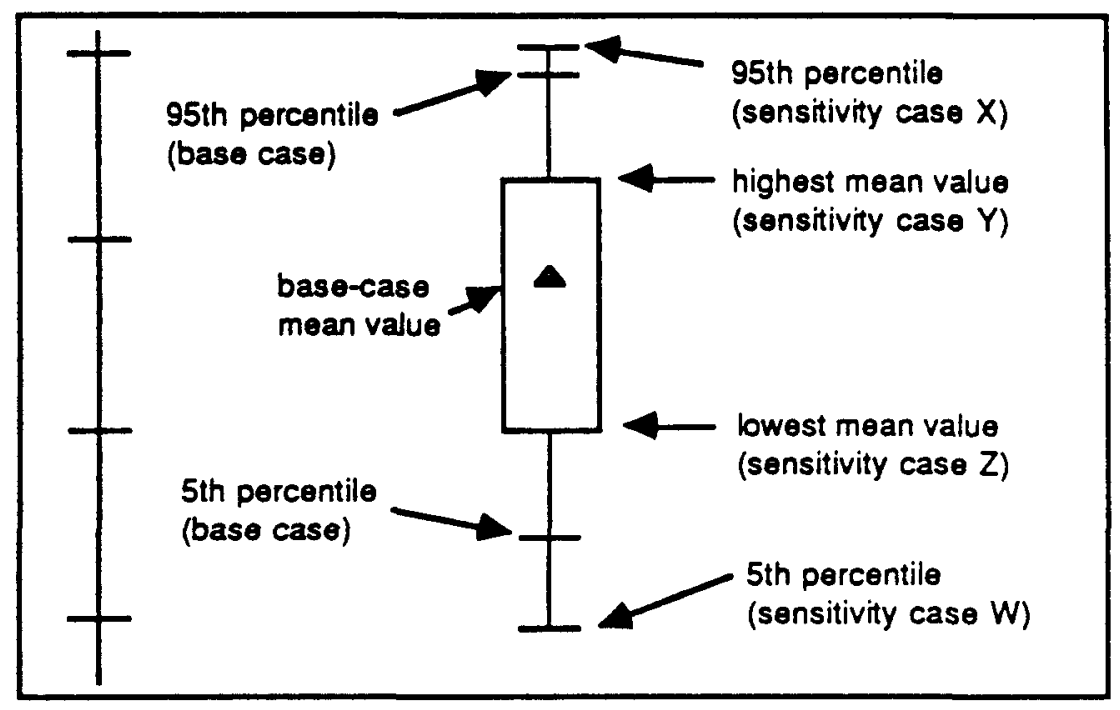

Figure A-2. Example "Box-and-Whisker" Display of Uncertainties

It should be noted that the final uncertainty representation selected for SARRP was somewhat different than the approach described above. The sensitivity studies did provide the basis for the LLH issues affecting core-damage frequency. Additional information was required for the LLH study, mainly the assignment of weighting factors for the sensitivity studies. The details of the SARRP uncertainty assessment are included in Sections 2.1 and 5.1 of the main report.

The following section presents the results for each of the plant-damage states in this format illustrated in Figure A-2.

\section{A.4 FREQUENCIES OF THE PLANT-DAMAGE STATES}

The plant-damage states assessed to have non-negligible frequencies the sequences contributing to their frequencies are summarized in Table A-9, along with the mean frequencies for each plant-damage state for the base case. The "box-and-whisker" presentations for each of the plant-damage states and for the overall core-melt frequency and are provided in Figures A-3 and $A-4$. 
NUREG/CR-4551, VOL. 1: DRAFT REPORT FOR COMMENT (SEPTEMBER, 1986)

Table A-9

PLANT-DAMAGE STATES AND CONTRIBUTING SEQUENCES

\begin{tabular}{|c|c|c|c|}
\hline \multirow{2}{*}{$\frac{\text { Plant-Damage State }}{\text { SYYB }}$} & \multicolumn{2}{|c|}{ Contributing Sequences } & \multirow{2}{*}{$\begin{array}{c}\begin{array}{r}\text { Mean Damage- } \\
\text { State Frequency* }\end{array} \\
7.1 \times 10^{-6}\end{array}$} \\
\hline & $\begin{array}{l}\mathrm{S}_{2} \mathrm{H}_{1} \\
\mathrm{~S}_{2} \mathrm{H}_{2} \\
\mathrm{~S}_{2} \mathrm{D}_{1} \\
\mathrm{TKRZ} \\
\mathrm{T}_{4 \mathrm{~J}} \mathrm{QD} 1 \\
\mathrm{~S}_{3} \mathrm{D}_{1}\end{array}$ & $\begin{array}{l}\mathrm{T}_{4 \mathrm{H}} \mathrm{QD}_{1} \\
\mathrm{~T}_{4 \mathrm{~J}} \mathrm{QH}_{1} \\
\mathrm{~T}_{4 \mathrm{H}} \mathrm{QH}_{1} \\
\mathrm{~T}_{4 \mathrm{~J}} \mathrm{QH}_{2} \\
\mathrm{~T}_{4 \mathrm{H}} \mathrm{QH}_{2}\end{array}$ & \\
\hline SNNN & $\mathrm{T}_{1} \mathrm{SL}_{-} \mathrm{D}_{1} \mathrm{CF}_{1}$ & $\mathrm{~T}_{1} \mathrm{QD}_{1} \mathrm{CF}_{1}$ & $5.7 \times 10^{-6}$ \\
\hline TYYB & $\mathrm{TKRD}_{4}$ & $T_{1} L P$ & $2.1 \times 10^{-6}$ \\
\hline TNNN & $\mathrm{T}_{1} \mathrm{~L}(\mathrm{LT}) \mathrm{D}_{1} \mathrm{CF}_{1}$ & $\mathrm{~T}_{1} \mathrm{~L}(\mathrm{ST}) \mathrm{D}_{1} \mathrm{CF}_{1}$ & $1.9 \times 10^{-6}$ \\
\hline AYYB & $\begin{array}{l}\mathrm{AH}_{1} \\
\mathrm{~S}_{1} \mathrm{H}_{1}\end{array}$ & $\begin{array}{l}S_{1} D_{1} \\
\mathrm{AD}_{5}\end{array}$ & $1.8 \times 10^{-6}$ \\
\hline $\mathrm{V}$ & & & $8.6 \times 10^{-7}$ \\
\hline AYNI & $S_{1} F_{1} F_{2}$ & $A F_{1} F_{2}$ & $8.2 \times 10^{-8}$ \\
\hline SYNI & $\mathrm{S}_{2} \mathrm{HF}_{1} \mathrm{~F}_{2}$ & & $5.0 \times 10^{-8}$ \\
\hline ANNN & $\mathrm{S}_{1} \mathrm{CD}_{6} \mathrm{D}_{1} \mathrm{C}$ & $\mathrm{ACD}_{6} \mathrm{D}_{1}$ & $3.9 \times 10^{-9}$ \\
\hline SYNN & $\mathrm{T}_{4 \mathrm{H}} \mathrm{Q}-\mathrm{CF}_{1}$ & $\mathrm{~T}_{4 \mathrm{H}} \mathrm{Q}-\mathrm{CF}_{1}$ & $2.6 \times 10^{-9}$ \\
\hline TYNI & $\mathrm{T}_{1} \mathrm{LF}_{1} \mathrm{~F}_{2}$ & $\mathrm{~T}_{2} \mathrm{LF}_{1} \mathrm{~F}_{2}$ & $1.1 \times 10^{-9}$ \\
\hline
\end{tabular}




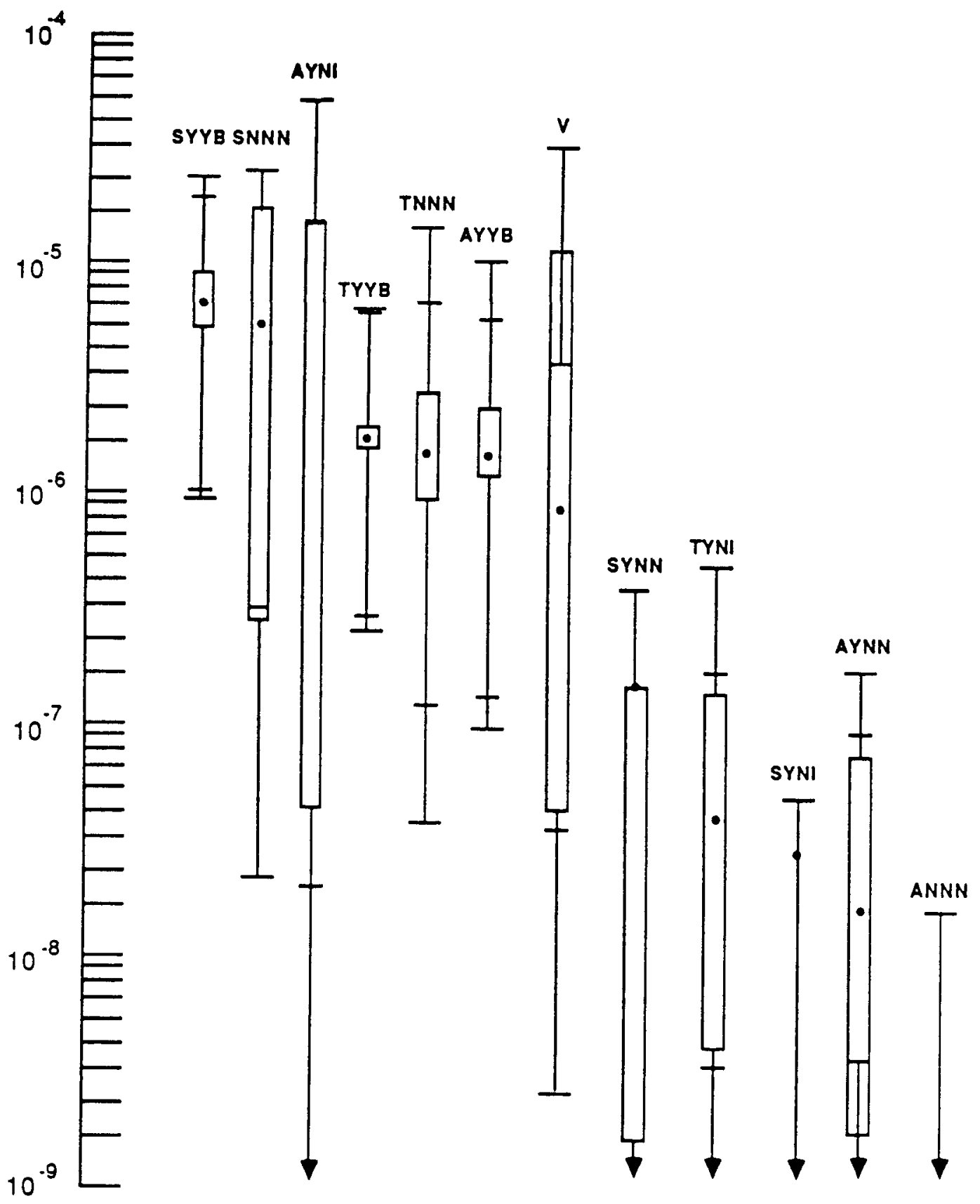

Figure A-3. Plant-Damage State Frequencies with Uncertainties (Core-Vulnerable Sequences not Adjusted to Core Damage) 


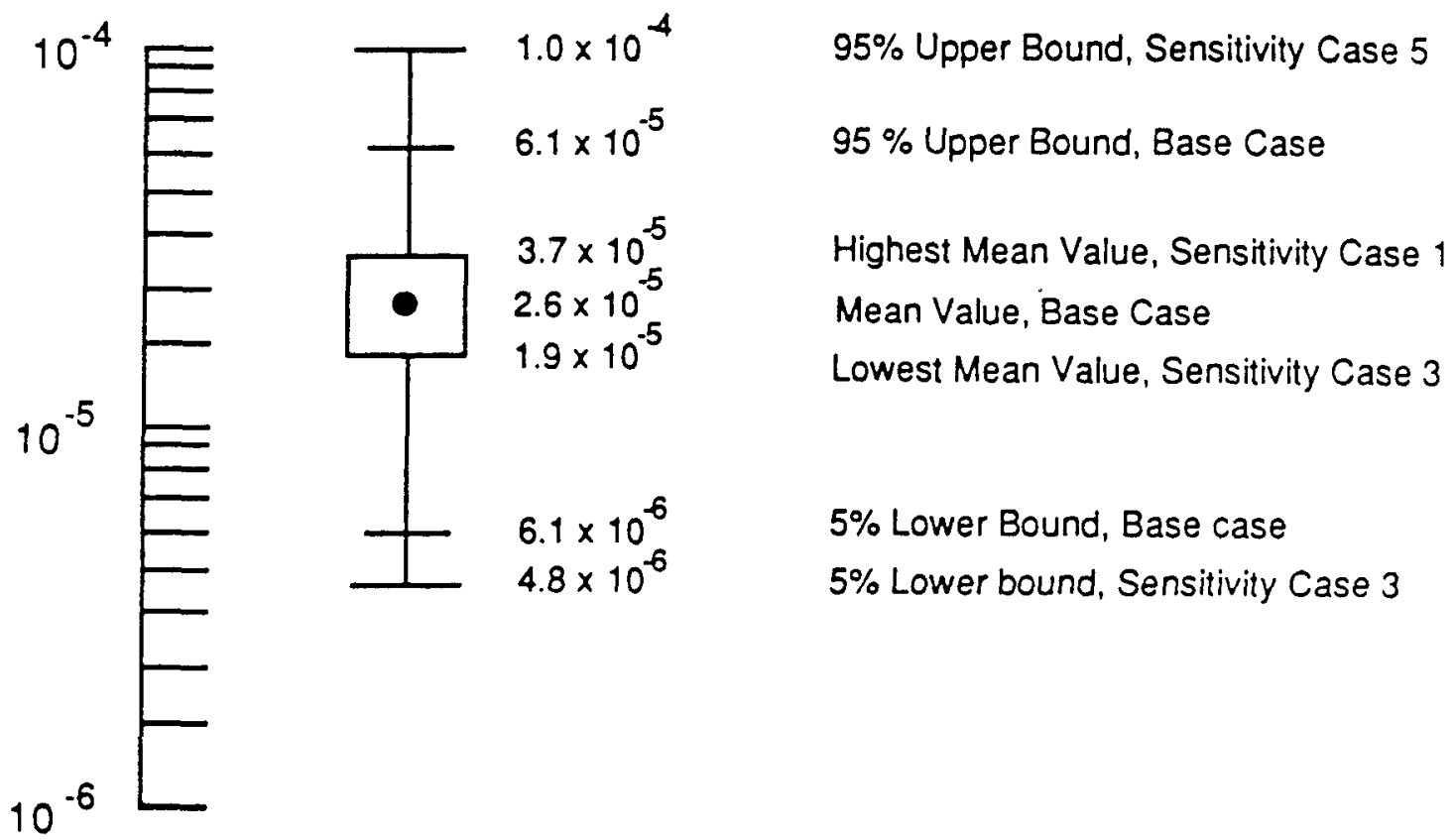

Figure A-4. "Box-and-Whisker" Display of Uncertainties for Total Core-Damage Frequency (Core-Vulnerable Sequences Not Adjusted to Core-Damage)

\section{A.5 COMPARISON TO RESULTS FROM THE REACTOR SAFETY STUDY}

The Reactor Safety Study estimated a core-damage frequency for Surry of $4.4 \times 10^{-5}$ [4], compared to the updated value of $2.6 \times 10^{-5}$, or $2.1 \times 10^{-5}$ after adjusting the corevulnerable sequences. The difference in these two values is not particularly significant, although it should be noted that the estimate from the original study was a point estimate calculated by propagating median values (a process which would generally yield a value lower than the mean), while the updated frequency is represented as a mean value. However, there were important differences in the sequences and features that were assessed to contribute most to core-melt frequency in these two investigations, including the following:

- A number of changes have been made to the Surry plant since completion of the original study more than a decade ago;

- Improved understanding of accident phenomenology has allowed the definition of new success criteria for several systems under different accident conditions;

- The ASEP updated analysis included substantially more consideration of operator actions that could be taken to recover lost functions prior to melting of the core; 
- In the update, more powerful computer tools were used to quantify the sequence frequencies by obtaining sequence cut sets, enabling enhanced tracking of dependencies among systems, and allowing for the detailed consideration of recovery actions in a realistic framework;

- Newer methods and data for the assessment of common-cause failures were used in the ASEP study; and

- The updated study incorporated newer data bases than were available at the time of the original, including some plant-specific data.

These factors were the principal contributors to differences in the two studies. Comparing the dominant sequences for both studies yielded the following insights:

- The potential for RCP seal LOCAs as a consequence of a loss of seal cooling played a major role in the ASEP study, but was not considered in the original analysis.

- The likelihood of loss of all containment heat removal was assessed to be higher in the update to account for plant-specific experience involving common-cause failures in the service-water system.

- Sequences involving station blackout and a loss of all auxiliary feedwater were assessed similar frequencies in the two studies.

- The ASEP update considered sequences initiated by individual electrical bus failures, and these appeared to be significant; the transient initiators were not considered at that level of detail in the prior study.

- There were substantial changes in the sequences involving failure to scram; these changes arose due to improved understanding of plant behavior under these conditions, an updated assessment of the likelihood of scram failure, and differences in the treatment of operator actions.

- The frequencies of interfacing-systems LOCAs have been reduced by the institution of period testing to verify the status of the isolating check valves.

- The frequencies of sequences initiated by LOCAs and followed by failure of emergency core cooling are generally lower in the update. In this case, the higher likelihood of common-cause failures is off-set by further consideration of recovery actions.

- In light of improved understanding of accident phenomena, two sequences were eliminated: a small LOCA with failure of containment spray injection (sequence $\mathrm{S}_{2} \mathrm{C}$ ), and a transient followed by total loss of feedwater (sequence TML). In the case of the latter sequence, the update credited the use of feed-and-bleed cooling as an alternative means of core heat removal. 


\section{References for Appendix A}

1. Bertucio, R. C., et al. Analysis of Core Damage Frequency From Internal Initiating Events: Surry, Unit 1. U.S. Nuclear Regulatory Commission Report NUREG/CR4550, Volume 3 (Draft Report), Sandia National Laboratories, Albuquerque, NM: April, 1986.

2. Denning, R. S., et al. Report on Radionuclide Release Calculations for Selected Severe Accident Scenarios: Volume III, PWR Subatmospheric Containment Design (Draft Report). Battelle Columbus Laboratories, Columbus, OH: January, 1986.

3. Olman, M. D. Quantitative Fault Tree Analysis Using the Set Evaluation Program (SEP). U.S. Nuclear Regulatory Commission Report NUREC/CR-1935, Sandia National Laboratories, Albuquerque, NM: September, 1982.

4. Reactor Safety Study--An Assessment of Accident Risks in U.S. Commercial Nuclear Power Plants. U.S. Nuclear Regulatory Commission Report WASH-1400 (NUREG75/014), Washington, DC: 1975. 
NUREG/CR-4551, VOL. 1: DRAFT REPORT FOR COMMENT (SEPTEMBER, 1986)

Appendix B

CONTAINMENT ANALYSIS UNCERTAINTY ISSUES FOR THE LIMITED

LATIN HYPERCUBE STUDY OF SURRY

$B-i / i i$ 
○

-

-

-

- 


\section{CONTENTS}

Section

B.1 SELECTION OF UNCERTAINTY ISSUES..................................

B.2 ISSUE 1: LOCATION AND SIZE OF INDUCED LOCA......................... B-4

B.3 ISSUE 2: MODE OF REACTOR-VESSEL BREACH.........................8

B.4 ISSUE 3: MAGNITUDE OF DIRECT HEATING AND STEAM SPIKE.......... B-12

B.5 ISSUE 4: MAGNTUDE OF LOADNG DUE TO HYDROGEN BURN AT VESSEL BREACH................................................ $\mathrm{B}-18$

B.6 ISSUE 5: CONTAINMENT FAILURE PRESSURE ......................... B-26

B.7 ISSUE 6: CONTAINMENT FAILURE SIZE.............................

B.8 ISSUE 7: EFFECT OF CONTAINMENT FAILURE ON SPRAY PUMP

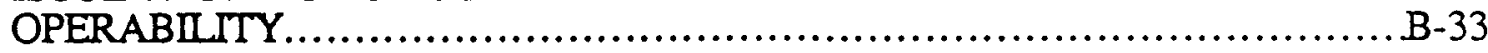

B.9 ISSUE 8: V SEQUENCE SUBMERGENCE.............................. B-34

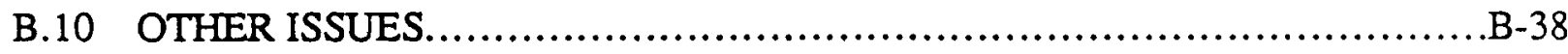

B.10.1 Issue 9: Effect of Containment Failure on ECCS Operability............. B-38

B.10.2 Size of V Sequence Break........................................ 40

B.10.3 Containment Failure by Detonation or Accelerated Burning ............... B-40

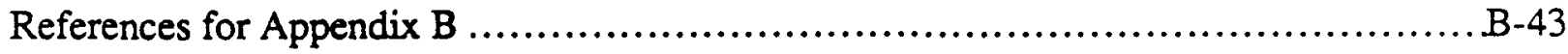




\section{LIST OF TABLES}

Table Page

B-1 Uncertainty Issues Relevant to Containment Loading and Response for Surry........B-3

B-2 Outcomes and Weighting Factors for Issue 1: Induced RCS Failure................ B-6

B-3 Outcomes and Weighting Factors for Issue 1 for Different Boundary Conditions.... B-9

B-4 Outcomes and Weighting Factors for Issue 2: Mode of Reactor Vessel Breach......B-11

B-5 Outcomes and Weighting Factors for Issue 3: Magnitude of Steam Spike and Direct Heating Pressure Increments at Vessel Breach.............................. B-15

B-6 Factors for Issue 3 Boundary Conditions................................ 18

B-7 Outcomes and Weighting Factors for Issue 4: Hydrogen Bum at Vessel Breach-Steam Concentration $>56 \%$ and Direct Heating Occurs........................... 21

B-8 Outcomes and Weighting Factors for Issue 4: Hydrogen Burn at Vessel Breach-Minimum Steam Spike and Steam Concentration < 56\% ........................ B-22

B-9 Outcomes and Weighting Factors for Issue 4: Hydrogen Burn at Vessel Breach-Maximum Steam Spike, Steam Concentration $<56 \%$ and No Direct Heating........ B-23

B-10 Outcomes and Weighting Factors for Issue 4: Hydrogen Burn at Vessel Breach-Maximum Steam Spike, Steam Concentration < 56\% With Direct Heating.......... B-23

B-11 Outcomes and Weighting Factors for Issue 4: Hydrogen Burn at Vessel Breach-Steam Concentration $>56 \%$ and No Direct Heating............................. 24

B-12 Equivalent Hydrogen Burn Levels for the Base Case Weighting Factors........... B-26

B-13 Weighting Factors and Outcomes for Issue 5: Containment Failure Pressure....... B-24

B-14 Weighting Factors for Issue 6: Containment Failure Size Given a Slow Pressurization to a Total Pressure Less Than the Median of the Containment Capacity Distribution....................................................... 29

B-15 Weighting Factors for Issue 6: Containment Failure Size Given a Rapid Pressurization to a Total Pressure Greater Than the Median of the Containment Capacity Distribution.......................................................... 30

B-16 Reviewers' Input Contingent on Containment Pressurization.................... B-31

B-17 Reviewers' Input Contingent on Containment Pressurization.................... B-32

B-18 Noncontingent Weighting Factors for Size of Containment Failure................ B-32

B-19 Outcomes and Weighting Factors for Issue 7: Effect of Containment Failure on Spray Pump Operability.........................................................

B-20 Probabilities for Issue 8: Submergence of the V Sequence Failure Site............. B-37

B-21 Outcomes and Weighting Factors for Issue 8.1: Effect of Containment Failure on ECCS Pump Operability....................................................

B-22 Outcomes and Weighting Factors for Issue 8.2: Effect of Containment Failure on on Injection and Suction Piping.............................................. B-39 
NUREG/CR-4551, VOL. 1: DRAFT REPORT FOR COMMENT (SEPTEMBER, 1986)

\section{LIST OF FIGURES}

Eigure

Page

B-1 Discrete and Continuous Weighting Functions for the Base Case Hydrogen Burn.. B-25

B-2 Constructed Cumulative Distribution Function for Two Boundary Conditions...... B-25

B-3 Probability of a Small Opening as a Function of Mean Failure Pressure............ B-31 


\section{Appendix B}

\section{CONTAINMENT ANALYSIS UNCERTAINTY ISSUES FOR THE LIMITED LATIN HYPERCUBE (LLH) STUDY OF SURRY}

The issues included in the limited Latin hypercube (LLH) study of Surry were summarized in Section 4.0 of the main report. The details of the input provided by the review group are provided in this appendix. The uncertainty issues chosen for consideration were selected based on the interim results of the $\mathrm{OCP}$ analysis as well as through interactions with experts in the field. This initial list of issues was then ranked in importance, as guidance to the SARRP team on which issues should be considered first. This step was required due to the practical constraints on the $\mathrm{LLH}$ approach being used in the program. Then a range of potential values was selected for each issue that was intended to represent the uncertainty in the outcomes associated with the issue. This range was initially selected by the SARRP analysts and was then adjusted by the review group as necessary. Finally, each reviewer provided weighting factors for each discrete level of the range, and these were combined into a composite. The inputs of the expert reviewers for the containment are all described in this appendix.

A number of experts were chosen to provide input in terms of weighting factors, although it should be recognized that the sources of information for the study were not limited to the individuals below, and many other experts were consulted. Due to the different types of issues involved, two different panels were formed, one for containment loading phenomena and the other for containment response issues. The participants in the LLH review task for the Surry containment analysis are listed below:

\section{Containment Loading}

- W. Murfin

- G. Greene

- S. Hodge

- A. Torri

- M. Berman

- M. Corradini

- R. Denning

- K. Perkins

- K. Bergeron
Technadyne (Principal SARRP Analyst)

Brookhaven National Laboratory

Oak Ridge National Laboratories

Pickard, Lowe, and Garrick

Sandia National Laboratories

University of Wisconsin

Battelle Columbus Laboratories

Brookhaven National Laboratory

Sandia National Laboratories

${ }^{*}$ The reviewers are identified by number only in the discussion that follows. The order of participants below is not the same as the order of reviewers identified in the tables. 
NUREG/CR-4551, VOL. 1: DRAFT REPORT FOR COMMENT (SEPTEMBER, 1986)

\section{Containment Response}

- W. Murfin Technadyne (Principal SARRP analyst)

- R. Denning Battelle Columbus Laboratories

- K. Perkins Brookhaven National Laboratory

- A. Torri Pickard, Lowe, and Garrick

- W. Von Reisemann Sandia National Laboratories

\section{B.1 SELECTION OF UNCERTAINTY ISSUES}

The SARRP analysts developed a list of candidate issues prior to the review meeting with the two groups described above. This list was based on the insights from the OCP containment analysis for Surry which had already been completed at the time of this process. Thus considerable research on the uncertainties had already been performed, a number of very detailed review cycles had already elicited the major uncertainties, and the completed OCP results provided an integrated perspective including the dominant sequences as well as the risk significance of each of the outcomes. The process used to develop the uncertainty ranges for this task was therefore well along prior to the review meeting. The SARRP analyst provided to the review team: 1) a list of issues and background concerning the state of the art on the issues, 2) a proposed set of discrete issue outcomes (levels), and 3) a SARRP estimate of weighting factors. (The report issued prior to the LLH meeting is referred to as the "strawman".) All of the same data is provided in this report also, along with the additional information supplied by the groups of experts.

The list of issues is illustrated in Table B-1, as are the individual priority rankings of the reviewers. The priorities were assigned on a three point scale, with a " 1 " being most important; i.e., the uncertainty in the issue would likely have a significant impact on the risk measures for Surry. These rankings were requested to allow the SARRP analysts to deal with the most important issues first if the full list of uncertainties was not practical using the LLH methods. The reasons behind the rankings of the issues are discussed in the sections that follow, along with a detailed description of the issues and the uncertainty associated with each. In some cases the issues are not independent and the group developed weighting factors based on simple correlations that could be included in the LLH model. 
Table B-1

UNCERTAINTY ISSUES RELEVANT TO CONTAINMENT LOADING AND RESPONSE FOR SURRY

\begin{tabular}{|c|c|c|c|c|c|c|c|c|c|c|}
\hline \multirow{2}{*}{\multicolumn{2}{|c|}{ ISSUE }} & \multicolumn{9}{|c|}{$\begin{array}{l}\text { PRIORITY } \\
\text { Reviewer }\end{array}$} \\
\hline & & SARRP & 1 & 2 & 3 & 4 & 5 & 6 & 7 & Composite \\
\hline 1. & $\begin{array}{l}\text { Location and Size of Induced } \\
\text { LOCA }\end{array}$ & 2 & 2 & 2 & 2 & 2 & 2 & 1 & 2 & 2 \\
\hline 2. & Mode of Vessel Breach & -- & 2 & 1 & -- & -- & 1 & 1 & 1 & 1 \\
\hline & Magnitude of Direct Heating and & & & & & & & & & \\
\hline & Steam Spike at Vessel Breach & 1 & 1 & 1 & 1 & 1 & 1 & 1 & 1 & 1 \\
\hline & at Vessel Breach & 1 & 1 & 1 & 1 & 1 & 1 & 1 & 1 & 1 \\
\hline 5. & Containment Failure Pressure & 1 & 1 & 1 & 1 & 1 & 1 & 1 & 1 & 1 \\
\hline 6. & $\begin{array}{l}\text { Size of Containment Failure } \\
\text { Opening }\end{array}$ & 2 & 1 & 1 & 1 & 1 & 1 & 1 & 1 & 1 \\
\hline & $\begin{array}{l}\text { Effect of Severe Environment on } \\
\text { Spray Operability }\end{array}$ & 2 & 2 & 2 & 2 & 2 & 2 & 2 & 2 & 2 \\
\hline & $\begin{array}{l}\text { Submergence of Release for } \\
\text { the V Sequence }\end{array}$ & 1 & 1 & 1 & 1 & 2 & a & 1 & 1 & \\
\hline & $\begin{array}{l}\text { Effect of Containment Failure } \\
\text { on ECCS Operability }\end{array}$ & 1 & 1 & 1 & 1 & 1 & 1 & 1 & 1 & 1 \\
\hline $\begin{array}{l}10 . \\
11\end{array}$ & $\begin{array}{l}\text { Size of V Sequence Break } \\
\text { Containment Failure by Local } \\
\text { Detonation or Accelerated H2 }\end{array}$ & - & 1 & -- & $1^{b}$ & -- & a & 1 & $1^{b}$ & \\
\hline & Burning & - & -- & 2 & 3 & 2 & 1 & 2 & 2 & \\
\hline
\end{tabular}

a The reviewer thought this should be a source term issue.

b The reviewer thought the issue should be combined with Issue 8

The final step in the $L L H$ process was the translation of the reviewer input into a format that could be processed in the SARRP analysis. In some cases this involved some changes to the reviewer input, while in other cases it meant additional analysis to supplement that provided in the strawman, with the resultant change to some level definitions. The LLH input required some sort of composite view of the expert reviewers' inputs. Whenever a single set of weighting factors was assigned, the composite was calculated as the arithmatic mean of the individual experts inputs as well as the SARRP weighting factor. For some of the other issues where, for example, the reviewers supplied their estimates of probabilities of given events, each reviewer's input was treated as a separate level that were each weighted equally. Finally, sometimes there was little distinction between some levels, or some levels were assigned weighting factors too small to be sampled in the small sample size being used (approximately 
100 samples for all issues in all analysis areas). In these cases the SARRP analyst sometimes adjusted the input, retaining the original intent of the LLH reviewers.

The expert input on each issue is supplied below. The discussion also includes a description of how the input was actually implemented in SARRP, and any limitations resulting from the method of implementation.

\section{B.2 ISSUE 1: LOCATION AND SIZE OF INDUCED RCS FAILURE}

Recent analyses have indicated a potential for very high temperature gases associated with core-melt accidents that occur with high pressure in the primary system [1]. The temperatures are high enough that induced failure of the pressure boundary is a possibility, and this has not generally been considered in previous risk assessments. Reference 1 reports temperatures of $2000^{\circ} \mathrm{F}$ in the vicinity of the hot leg nozzles for a station-blackout sequence at a PWR and similar results would be expected for any high-pressure sequence with failure of the injection systems. Based on these results, the Containment Loads Working Group (CLWG) [2] and other experts have raised the possibility of a temperature-induced failure of the primary

system. A reactor coolant pump seal LOCA has been suggested as one strong possibility by IDCOR and others [3,4], particularly for sequences involving coincident failure of the seal cooling function (such as the station blackout sequences). A seal LOCA could have a range of sizes, but generally it would be expected to change a very small LOCA scenario into a small LOCA. The temperature profiles that have been postulated could also result in a large failure of the primary system boundary, probably at the hot leg nozzle welds, resulting in a large LOCA. Other connections in the hot leg could be failure sights, although the temperature effects are greatest near the vessel. One additional consequence of the high gas temperatures that has been included in the Surry containment event tree is the induced failure of one or more steam generator tubes. There have been several different opinions on the seriousness of this potential, for example the Zion and Indian Point studies [4,5] included the possibility, but IDCOR considered tube ruptures to be insignificant risk contributors [3].

The result of an induced failure in terms of risk impact is somewhat difficult to establish. Induced failure can be beneficial for Surry since the reduction in primary system pressure prior to vessel failure reduces the potential for the direct heating phenomenon (Issue 3 ) which is of primary importance. On the other hand, induced failures of the RCS also affect the source term phenomenology, particularly by reducing the amount of radionuclides retained in the vessel. The tube rupture phenomenon obviously has potentially significant impact, although the issue has not been studied extensively and both the thermalhydraulics and source 
term aspects of a tube rupture during core melt are extremely uncertain. The OCP methodology required that the phenomenon be established as either optimistic or pessimistic. For that study, induced failures were selected as optimistic because of the overriding importance of the direct heating phenomenon assessed for the Surry plant. The LLH does not require a constraint to predetermine the effect of the failure: the range of possibilities was varied in accordance with the weighting factors, and risk was determined for specific combinations of this event with all other uncertainty issues.

In order to limit the potential outcomes to a manageable number, four locations were selected to represent the range of induced LOCA that could be possible. These four locations, coupled with size differences, also differ in effect on the progression of the accident following the failure, as outlined in the previous discussion. The outcomes offered to the review team for application of weighting factors were: 1) no induced failures, 2) large LOCA hot-leg failure, 3) cold-leg failure at the reactor coolant pump seals, either large or small in size, and 4) steam generator tube ruptures, large and small. For the third case, different weighting factors were requested for the sequence boundary condition--with or without seal cooling availability. For the fourth case, tube rupture, it was assumed that the availability of steam generator cooling would eliminate the tube rupture potential. These cases are delineated in Table B-2, along with the weighting factors provided by the review team.

There was considerable discussion conceming the issues and the reviewers' reasons for selecting their weighting factors. The pertinant comments by the review team are described below, as they best summarize the uncertainty in the issue and the status of the research for resolving the issue. One of the reviewers abstained on this issue. As illustrated in the table, there were two additional outcomes that were put forth by Reviewer \#1. The first allows for the induced LOCA in the hot leg to be something less than the large LOCA considered as the first option. After discussion, the other reviewers generally concluded that although a smaller LOCA was possible, the important effects of the induced LOCA would be distinguished by LOCA or no LOCA, and that additional distinction between the two cases would not provide additional insight.

The second new consideration is concerned with operator action associated with this type of accident sequence. Several of the reviewers felt that this was a critical issue that must be considered in the development of the outcomes for this issue. Given, as the sequence implies, that there would be no options for cooling the core at high pressure, what would be the likelihood of the operations staff depressurizing the primary by any means available in order to at least attempt cooling though available low pressure systems? While it was pointed 
NUREG/CR-4551, VOL. 1: DRAFT REPORT FOR COMMENT (SEPTEMBER, 1986)

Table B-2

OUTCOMES AND WEIGHTING FACTORS FOR ISSUE 1 : INDUCED RCS FAILURE -NO SEAL COOLING AND NO STEAM GENERATOR COOLING-

\begin{tabular}{|c|c|c|c|c|c|c|c|c|c|}
\hline \multirow[t]{2}{*}{ OUTCOME } & \multicolumn{9}{|c|}{ WEIGHTING FACTORS } \\
\hline & SARRP & 1 & 2 & 3 & $\frac{\text { Revie }}{4}$ & $\frac{1 e r}{5}$ & 6 & 7 & Comp. \\
\hline 1. Large Hot-Leg LOCA & .15 & .05 & .1 & .1 & .1 & $--a$ & .15 & .15 & .12 \\
\hline 2. Large Seal LOCA & .2 & .3 & .1 & .05 & .1 & -- & .3 & .3 & .2 \\
\hline 3. Small Seal LOCA & .2 & .3 & .1 & .05 & .2 & -- & .3 & .3 & .2 \\
\hline 4. Small Steam Generator Tube & & & & & & & & & \\
\hline Rupture & .1 & .05 & .09 & .01 & .05 & -- & .1 & .1 & $.07^{\mathrm{c}}$ \\
\hline $\begin{array}{l}\text { 5. Large Steam Generator Tube } \\
\text { Rupture }\end{array}$ & .1 & .05 & .01 & .01 & .05 & -- & .1 & .1 & .07 \\
\hline 6. No Induced LOCA & .25 & .2 & .5 & .78 & .5 & -- & .05 & .05 & .34 \\
\hline 7. Small Hot-Leg LOCA & 0.0 & .05 & 0.0 & 0.0 & 0.0 & -- & 0.0 & 0.0 & 0.0 \\
\hline Probability tha & & & & & & & & & \\
\hline Depressurize the Primary & .8 & .8 & b & .6 & -- & -- & .8 & -- & \\
\hline
\end{tabular}

aReviewer abstained on this issue.

bThe reviewer felt that the factor was inappropriate.

cThe small and large tube rupture outcomes were coalesced into one category for the final composite and the composite value is therefore 0.15 .

out that the sequence frequency does, in some cases include consideration of this option, the action could occur here after the the core damage had already started--something outside the scope of the front-end core-damage frequency study. There was considerable discussion of this issue that dealt primarily with: 1) the procedural guidance for this event, and the fact that at this time the various response teams would be on site and the decision would likely be strongly influenced by advice from many others; ${ }^{*}$ ) the availability of methods to depressurize and of low pressure cooling methods--ultimately pointing out the sequence specific nature of the event; and 3) the cases where no action was possible due to loss of support services that should not be adjusted for this types of operator action. It was finally concluded that if the plant had the ability to perform the action then the probabilities in the table for depressurization should be considered before the induced LOCA question. This part of the reviewers' input was not implemented in the SARRP analysis due to schedule constraints. This could be a somewhat

\footnotetext{
${ }^{*}$ There was also some discussion of the groundrules for the ASEP program which essentially limit consideration of non-procedural actions--although it was pointed out that these groundrules should not prevent the proper uncertainty characterization.
} 
important omission since direct heating dominates the results and it is affected by the pressure of the primary, although most of the important accidents at Surry would involve limited availability of low pressure equipment, reducing the probability that the operations staff would lower the pressure. Further consideration of the induced failure uncertainty should include additional investigation of the depressurization issue.

Most of the reviewers did not specifically discuss their weighting factors, but Reviewer \#6 did give specific rationale for his selections. His first basic premise was that the temperatures would be hottest in the vessel and would decrease throughout the rest of the RCS, and this would have the strongest influence over the structural aspect of this problem. His second consideration dealt with the response of the various locations to the overheating-essentially a physical properties question with the order of fragility being the seals, the pressure vessel and nozzles, and finally the tubes. He then considered the intersection of these two factors which resulted in his opinion that the seals would be the dominant concern, as illustrated in his weighting factors. This reviewer had two concerns on this issue: 1) there was no structural analysis performed and this might not be so uncertain if the proper experts were to review the structural problems and 2) the issue is likely to be very sequence dependent and the weighting factors will not include this dependence.

Others commented on this rationale, noting that there were still many assumptions and that the problem was not all structural since even the temperature profiles were very uncertain. For example, there would most likely be water in the pipe before the gases could reach the seals and their temperature profile might therefore be entirely different. During this discussion it was also pointed out that the sequence frequency included the probability of a seal LOCA up to the time of the start of core melt for the sequences where no other LOCAs are involved or where seal cooling had failed--principally the station blackout sequences. The seals are not included in the front-end model after core melt initiation or for sequences already involving a LOCA. It was pointed out that this seal failure probability was also very uncertain, and based on extremely limited data.

Reviewer \#1 also provided rationale for his weighting factors. He assigned a high likelihood to a seal LOCA (when seal cooling is unavailable) based on the evidence of all past PRAs on PWRs. Given that there is little evidence on size of the seal LOCA and there are proponents of both large and small rupture he assigned equal weight to Levels 2 and 3 . Given that a seal LOCA did not occur, Reviewer \#1 weighted the "no LOCA" outcome with a 0.5 and equal weight to all other outcomes. The justification for this distribution of weighting factors is that there is no evidence or mechanistic calculations to support any outcome. 
The treatment of this issue in the LLH sample was unique due to some limitations associated with the source term analysis. In most issues for which reviewers selected probabilities, each reviewer's input was treated as a level and assigned an equal weight in the sampling scheme. The source term calculation required explicit recognition of the RCS status relative to induced LOCAs, and for this issue only, the mean split fractions were used as the composite. For example, $12 \%$ of the samples will have large induced LOCAs. In this manner, each sample had one unique induced LOCA outcome and the source term could be calculated directly for each sample. There is a disadvantage here in that the uncertainty in this issue is somewhat understated because the range of reviewer input is lessened by the selection of a composite, but no other alternatives were available. Also, the distinction between large and small steam generator tube ruptures was not significant and it was dropped for the composite.

The weighting factors provided by the review group applied to the boundary conditions of no seal cooling and no secondary side heat removal. The reviewers stated that it was their intention that the tube rupture outcome have no weighting factor if steam generator cooling was available, and that seal LOCAs would be prevented by the availability of seal cooling; with all other weighting factors renormalized. Once again, the limitations on the $L L H$ process did not allow for sequence-specific consideration of all weighting factors, and the intent of the reviewers was implemented through some changes to the containment event tree used in the LLH sampling. In any case, the effective composite weighting factors for other boundary conditions are indicated in Table B-3. The small hot leg LOCA outcome was not weighted significantly enough to be sampled and it was dropped with the weighting factors re-distributed to other outcomes.

\section{B.3 ISSUE 2: MODE OF REACTOR-VESSEL BREACH}

The mode of failure of the reactor vessel can have important effects on subsequent challenges to containment integrity. In the Reactor Safety Study (RSS), it was assumed that the reactor vessel would fail during core meltdown either as a result of a large steam explosion or by meltthrough of the vessel skirt, causing the hemispherical bottom head to detach [6]. The Sandia Zion/Indian Point review pointed out that other failure modes might be at least as likely as these [7]. The Zion Probabilistic Safety Study (ZPSS) [4] and IDCOR [3] argued that the most probable point of failure would be a local failure at a core instrumentation tube, and that the core debris would emerge as a jet in high-pressure sequences or would dribble out in lowpressure sequences. 
Table B-3

OUTCOMES AND WEIGHTING FACTORS FOR ISSUE 1 FOR DIFFERENT BOUNDARY CONDITIONS

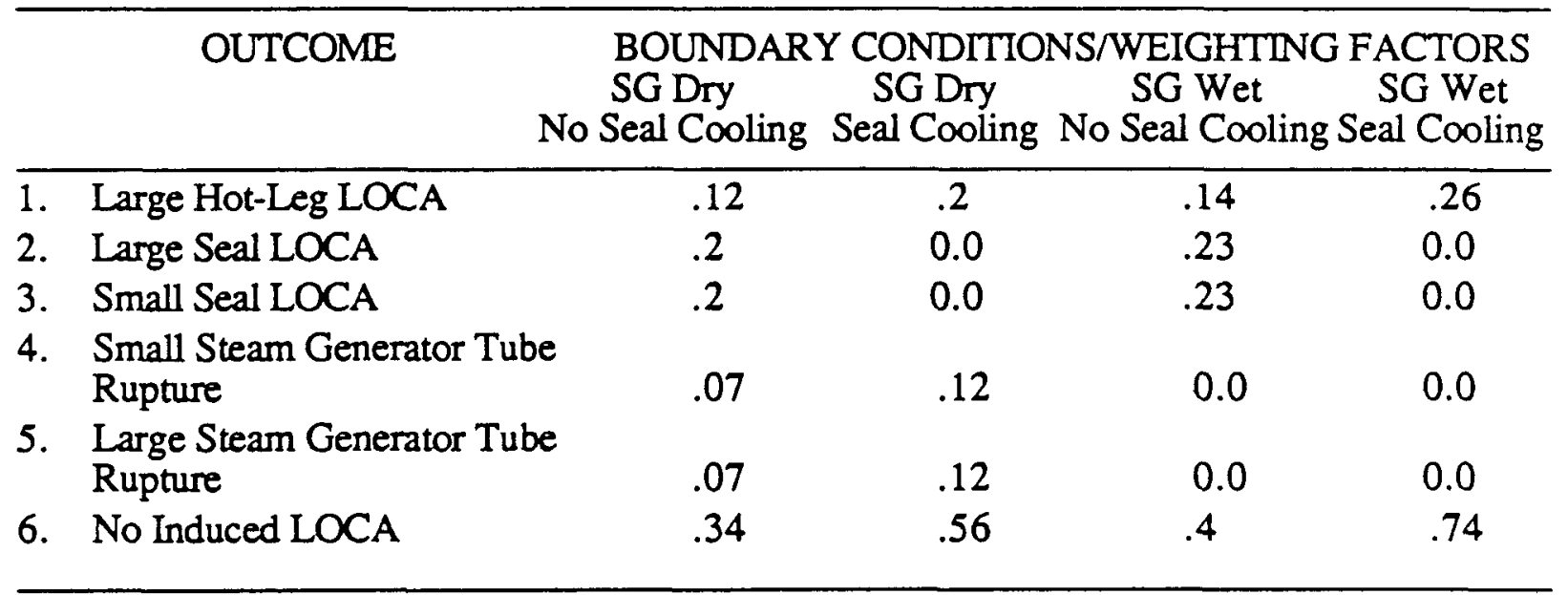

The containment event tree included five possible modes of vessel breach:

(1) A steam explosion which fails the upper vessel head and generates a missile that fails containment;

(2) A steam explosion that fails the upper head only (not the containment);

(3) A steam explosion that fails the bottom head;

(4) A pressurized ejection of the debris; and

(5) A gravity-driven pouring of the melt from the pressure vessel.

The second and third outcomes do not lead to containment failure and are treated in this issue as one outcome, primarily with the characteristics of a bottom head failure.

The possibility of an in-vessel steam explosion capable of causing direct failure of containment (referred to as the $\alpha$-mode) is the subject of a wide range of opinions. The RSS estimated the likelihood of this failure mode to be 0.01 . More recent estimates (for example, the ZPSS and IDCOR) have disputed this estimate as being unrealistic, or as being physically impossible at high pressures. The SAUNA program reported that triggering a steam explosion at high pressures might be more difficult, but that the probability that an adequate trigger would be available was not known [8]. They cited experimental evidence that, while it might be more difficult to trigger a high-pressure steam explosion, this effect is counteracted by the fact that the conversion ratio tends to be higher. 
Berman, et al., conducted a Monte Carlo study of the probabilities of steam explosions causing failure of the reactor vessel, and for those cases in which upper head failure occurred, the subsequent probability of a large missile with sufficient velocity to fail containment [2]. Several geometric and physical parameters used in the study were uncertain and were given uniform probability distributions which were sampled by the Monte Carlo procedure. To assess the sensitivity of the results to the assumed distributions, three different distributions were assumed for each parameter. These were labeled "low," "middle," and "high." For example, the three distributions for conversion ratio from thermal to mechanical energy were 0 $1.7 \%, 1.7-3.3 \%$, and $3.3-5.0 \%$. Out of 10,000 samples, it was determined that 2,126 resulted in a failure of the bottom head, while only one led to a failure of the upper head.

The majority of the members of the Steam Explosion Review Group (SERG) selected "best-estimate" values of 0.0001 within a factor of three either way [10]. One member of the SERG selected 0.1 as his upper value, and another selected 0.04 . The RSS also cited an upper value of 0.1 in conjunction with its best estimate of 0.01 . Berman, et al., also obtained a value of approximately 0.04 when the conversion ratio was assigned its high distribution while the others were kept at their middle distributions. In addition, in a recent experiment, they estimated the conversion to be much higher than the 3.3-5.0\% "high" distribution quoted above.

The outcomes are listed in Table B-4, along with the weighting factors of the individual reviewers. The issue was divided into two cases to allow consideration of the evidence summarized above that indicated a different response in different pressure regimes. The first case, for high pressure sequences, involves only three outcomes: steam explosion that fails containment, steam explosion that fails either the upper or lower head but not containment, or pressurized ejection. This final outcome does not imply anything concerning the amount of direct heating that might be associated with the ejection, that is treated within the next issue. The reviewers all had relatively low probabilities for the steam explosion that fails containment based on their interpretation of the research summarized above. Several of the reviewers stated that it was their opinion that the experimental evidence suggested that steam explosions that could fail containment are not possible, and assigned a $1.0 \times 10^{-3}$ probability as their interpretation of impossible or negligible. Most reviewers pointed to the evidence summarized above that suggests a decrease in the triggerability of a steam explosion at high pressure. The reviewer who assigned the largest probability to this event cited uncertainty in the applicabilitry of the experimental evidence. He thought that the experiments pointed to a strong pressure dependence but that all aspects had not been fully investigated yet, and he was also concerned 
NUREG/CR-4551, VOL. 1: DRAFT REPORT FOR COMMENT (SEPTEMBER, 1986)

Table B-4

OUTCOMES AND WEIGHTING FACTORS FOR ISSUE 2: MODE OF REACTOR-VESSEL BREACH

\begin{tabular}{llllllllll}
\hline & \multicolumn{1}{c}{ WEIGHTING FACTORS } \\
LEVELS & SARRP & 1 & 2 & 3 & $\frac{\text { Reviewer }}{4}$ & 5 & 6 & 7 & 8 \\
\hline
\end{tabular}

Case 1: High Bressure Prior to Vessel Breach ( $>500$ psig)

1. In-vessel steam explosion $\quad \begin{array}{lllllllll}.01 & .001 & .001 & .0001 & .01 & .001 & .001 & .001 & .01\end{array}$ that fails containment

2. Steam explosion that fails the $\begin{array}{lllllllll}.1 & .05 & .05 & .01 & .05 & .02 & .01 & .1 & .1\end{array}$ vessel but not containment

$\begin{array}{llllllllll}\text { 3. High pressure ejection of } & .89 & .949 & .949 & .9899 & .94 & .979 & .989 & .899 & .89\end{array}$ molten debris

Case 2: Low Pressure Prior to Vessel Breach $(-100$ psig $)$

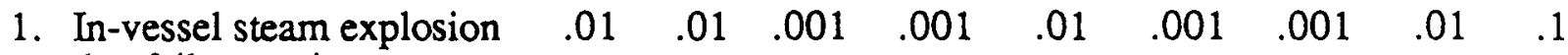
that fails containment

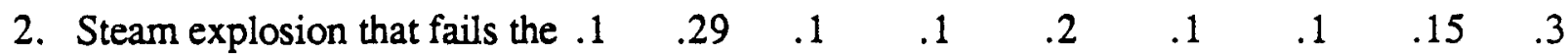
vessel but not containment

3. High pressure ejection of $\begin{array}{llllllllll}0.0 & 0.0 & .1 & 0.0 & 0.0 & .001 & .1 & 0.0 & 0.0\end{array}$ significant molten debris*

$\begin{array}{llllllllll}\text { 4. RCS depressurizes and melt } & .89 & .7 & .799 & .899 & .79 & .898 & .799 & .84 & .6\end{array}$ flows out by gravity

*Not to be applied to large LOCA leak sizes.

that some of the experimental evidence might not scale in size up to the type of mass involved in a core meltdown accident. He assigned a probability of $1.0 \times 10^{-2}$ to allow for all of these uncertainties. The second outcome allows for the possibility of vessel failure through a steam explosion type of interaction, and the reviewer's estimates for this event ranged from $1 \%$ to 10\%. Most reviewers thought that an ejection of the melt was the most likely outcome. This outcome allows direct heating as a possibility, but it does not imply the amount of direct heating that might be involved.

For the low pressure case, most reviewers thought that a steam explosion that fails containment would still be very unlikely. About one-half of the reviewers increased their prrobabilities for this outcome by a factor of ten over the high pressure case to account for the greater likelihood of steam explosions at lower RCS pressures, while others thought that the chance of failing comntainment by this mechansim did not change greatly with pressure. The 
second outcome, steam explosion that fails the vessel only, was assigned a 10-20\% chance by most reviewers, with one estimate as high as $30 \%$. The latter estimate was derived directly from the studies of Berman discussed above, while the other reviewers cited the experimental evidence but assigned a somewhat lower probability. Once again, the increase in trigger likelihood resulted in the increase in the probability assigned to this outcome for the low pressure case over the high pressure case. This low pressure case involved an additional outcome over the high pressure case: the last outcome is defined as vessel failure followed by a gravity-driven pour of the core material. All reviewers thought that this last outcome was most likely for this case. The controversy on this issue involved the third outcome: a pressure-driven ejection of core material. Most of the reviewers thought that this was impossible, simply by the definition of the case. Three reviewers did assign a probability as high as 0.1 to this case to account for any mechanism that would result in a temporary refreezing of veesel penetrations followed by a repressurization, or any other sequence of events in which the core material would be available for release at a time coincident with a repressurization event. It was a consensus that this outcome would not apply to an accident involving a large LOCA leakage rate from the primary.

\section{B.4 ISSUE 3: MAGNITUDE OF DIRECT HEATING AND STEAM SPIKE}

This issue represents the uncertainty in the phenomenon of containment heating associated with the dispersal of finely divided core material throughout the containment atmosphere at the time of vessel breach, for a high or intermediate pressure core melt sequence-referred to as direct heating [11,12]. Due to the intimate coupling of this with the other phenomenon, the issue was defined as the uncertainty in the pressure loading on containment at vessel breach from both the direct heating and steam generation. It should be noted that this was selected as one of the most critical uncertainties because of both the magnitude of the uncertainty and the significant impact on risk due to the potential for early containment failure. The OCP results for Surry indicated that this event was important for the pessimistic analysis, and played some role in the central walkthrough. For the LLH analysis, an additional outcome was added to provide better resolution on the potential outcomes that might be expected.

The steam spike associated with vessel failure has been investigated in several forums. Both the CLWG and IDCOR agree that some fraction of the core would be involved in the rapid transfer of heat to water and the generation of a steam spike. Accident sequences involving spray operation at Surry would include large quantities of water in the reactor cavity and on the containment floor. The accumulators would also provide additional water after vessel breach for any sequences at high or intermediate pressure. The plant-damage states 
involving low pressure core melts without the operation of the sprays would be the only scenarios with very limited water in the cavity, since the accumulators would have discharged previously. Thus for all damage states except the latter, there would be some steam spike associated with vessel breach. There are various sources available for estimation of this steam spike. IDCOR calculated a spike of $15 \mathrm{psi}$ for the $\mathrm{S}_{2} \mathrm{D}$ sequence, assuming that $50 \%$ of the core would be quenched [3]. The CLWG predicted similar pressures that were totally dependent on the assumption of the fraction of the core involved, with 26 psi corresponding to a $100 \%$ quench with no containment cooling [11]. The STCP result reported in BMI-2104 concluded that there would be the possibility that the containment cooling systems would not have time to reduce the pressure, particularly if the fuel was highly fragmented. These sources therefore provide the range of pressure outcomes suggested to the review group for the steam spike portion of the total pressure.

For this issue, the direct heating pressure increments are to be added to the steam spike increments discussed above. As already noted, this issue is highly uncertain, with IDCOR discounting it as a viable containment heating phenomenon, and with other sources projecting as much as $50 \%$ of the core distributed throughout the containment atmosphere. The CLWG was divided on this issue, with one group predicting only $2 \%$ of the core participating in direct heating with $80 \%$ being quenched and producing a steam spike, while the other group predicted a $50 \%$ participation in direct heating with an equal part contributing to the steam spike [12].

There have been a limited number of experiments at Sandia (HIPS) that provide useful insights into the direct heating issue [13]. Eighty $\mathrm{kg}$ of thermitic melt were discharged under pressure into one-tenth scale cavities with and without water. The dry cavity cases yielded a highly aerosolized plume of ejecta, a large fraction of which was thoroughly dispersed throughout the contained atmosphere. Based on these results, it has been projected that the energy involved in a full scale phenomenon would be enough to disperse the material throughout the containment atmosphere.

The cases with water in the cavity had a somewhat different result. The core material first caused a relatively coherent ejection of the cavity water as a slug which became finely divided after leaving the cavity. This was followed by an ejection of the hot debris, which appeared to become intimately mixed with the previously ejected water. ${ }^{*}$ A white fog then appeared, indicating that perhaps much of the heat from the core material had been transferred

\footnotetext{
${ }^{*}$ These results involve considerable judgment, because the visual results are somewhat difficult to fully interpret, given the fog-type conditions created by the results.
} 
to the initially ejected water droplets, leading to steam heating rather than direct containment heating. The amount of this energy transfer is mostly conjecture.

Additional energy transfer mechanisms have also been proposed as a part of direct heating. For example, there is the potential for additional metal-water reactions that would add to the pressure loading [12,14]. Local reactions of high temperature material with hydrogen and oxygen have also been proposed, as described in more detail in the description of the weighting factors to follow.

There is little more experimental evidence to calculate the pressure increment associated with direct heating. The SARRP analysts developed a simple model of the pressure increment considering the types of energy transfer possible, and varying the amount of the core material participating in each type of transfer. The variations included:

- The amount of core mass ejected at the time of vessel breach, from 50-100\%

- The percent of the core quenched in water, from 50-80\%

- The percent of the core quench interacting with steam, from 0-50\%

- The amount of the core quenched thermally, from 2-50\%

- The amount of the thermally quenched portion that reacts with steam

- The amount of the atmospherically dispersed material that reacts with oxygen

Discrete variations in the parameters above were used to generate pressure increments for each of the energy transfers, and these were used to generate the pressure increments used in the weighting factor assignment. The base case considered was a scenario involving high primary system pressure, with a dry cavity. The four pressure increments presented to the review group, including the contribution from the steam spike were: 11, 45, 75, and 104 psi.

As already noted, there are a number of boundary conditions that would impact the results. The most important of these is the presence of water in the cavity, which clearly changes the associated phenomenon, although the effect on pressure loading is highly uncertain. In addition, it might be expected that the degree of primary system pressurization at the time of core melt is a significant factor. These boundary condition issues were also presented to the review group, in the form of multiplicative factors on the pressure increments for the high-pressure, dry-cavity case.

The weighting factors selected by the review group for the base case are presented in Table B-5. There was considerable discussion about this issue, which is summarized below. Perhaps the most obvious result of the interaction is the addition of another discrete value to the high end of the pressure increment range. This value, $130 \mathrm{psi}$, was selected to indicate the 
Table B-5

OUTCOMES AND WEIGHTING FACTORS FOR ISSUE 3: MAGNITUDE OF STEAM SPIKE AND DIRECT HEATING PRESSURE INCREMENTS AT VESSEL BREACH

\begin{tabular}{lcccccccccc}
\hline \multicolumn{1}{c}{$\begin{array}{c}\text { OUTCOME } \\
\text { Pressure Increment }\end{array}$} & \multicolumn{1}{c}{ WEIGHTING FACTORS } \\
& SARRP & 1 & 2 & 3 & 4 & 5 & 6 & 7 & 8 & Comp. \\
\hline 1. $11 \mathrm{psi}$ (no direct heating) & .25 & .12 & .01 & .2 & .25 & 0.0 & .45 & .1 & .2 & .18 \\
2. 45 psi (no direct heating) & .25 & .32 & .65 & .3 & .4 & .15 & .34 & .25 & .2 & .32 \\
3. 75 psi & .25 & .27 & .28 & .3 & .15 & .25 & .15 & .25 & .2 & .23 \\
4. $104 \mathrm{psi}$ & .25 & .17 & .05 & .1 & .15 & .3 & .05 & .2 & .2 & .16 \\
5. $130 \mathrm{psi}$ & -- & .12 & .01 & .1 & .05 & .3 & .01 & .2 & .2 & .11
\end{tabular}

opinion of several of the reviewers that the range offered to the review group was too narrow. All participants then voted on this new value, and as indicated in the table, there was sharp division within the group on the possibility of having a pressure loading that high. It should also be noted that the OCP central value was essentially Level 2 while the mean of the LLH is closer to Level 3. The individual rationale for selecting weighting factors are discussed below, after a summary of more general comments on these issues.

The first major concern of the reviewers was with the calculation of the pressure increments presented by the SARRP team. This included the four cases of distinct variation of each of the energy transfer mechanisms outlined above. The majority of reviewers had major disagreements with the calculations, and it was a conclusion that the reviewers did not want to imply any endorsement of the method used to derive the pressure ranges selected for the analysis. On the other hand, the outcomes of the calculation did provide, in the reviewers' opinion, a relatively good range of discrete pressure outcomes that might be expected. One reviewer then pointed out that the maximum pressure as calculated by the CLWG would be significantly higher, such as $150 \mathrm{psi}$. Simple hand calculations for Surry were performed for a case of $100 \%$ oxidation and $100 \%$ heat transfer for $40 \%$ of the core--resulting in a value of 130 psi. This was suggested as a reasonable value for the high end of the range. It was then decided that the reviewers would vote on the pressure increments alone (including the new value of $130 \mathrm{psi}$ ) with no selection of specific, and detailed pressure contributions to final value. 
One reviewer expressed concern that for this most important issue, final values were being generated by guesses, rather than actual detailed calculation of the inputs. In the discussion that followed, it was pointed out that the modeling of the phenomenon is very complex--so complex that voting on final pressure is the only available approach at this time. In addition, the experimental evidence is limited, such that even if the phenomenon could be modeled directly, the some important parameters would remain unknown. There was considerable discussion of the very limited state of knowledge on this issue, yet it was the final conclusion that even crude calculations of the pressure increments would provide the perspective on the uncertainty that is needed for useful input to the decision-makers.

Reviewer \#5 had the most detailed comments concerning the phenomenon. As illustrated in the table, he weighted the high pressure increments rather high due to a number of factors. In addition, he had available a crude, computerized model of the process which he exercised to obtain insights into the effect of various parameters. One important criteria for the Surry plant pertained to geometry. Testing has shown that escape paths of geometry similar to those that are present around the top of the vessel at Surry could lead to a very significant transfer of material out of the cavity. It was his opinion that the competition between paths would result in the transfer of $25 \%$ of the material directly to the upper containment, while the rest of the material would follow the more traditionally examined pathways at the bottom of the cavity. Thus he was less swayed by arguments that the material would be stopped in the pathways prior to reaching the containment. Another factor which he derived from the test, was that the mode of vessel breach may not play as big of role in the ejection from the cavity. The energy transfer due to a gravitational drop as illustrated in the test was very efficient, with droplets of core material actually exploding after complete oxidation and very significant energy transfer. This energy would lead to ejection of some material from the cavity. The second driving factor for his numbers was his view that the type of energy transfer from the core debris was moot, whether additional steam generation or direct chemical reaction, the thermodynamic outcome would be the same.

Finally, another potentially significant phenomenon that was discussed was the recombination of hydrogen and oxygen in the atmosphere. This would not have to be a bum, since the reaction would be a chemical recombination in the close proximity of the finely divide particles of the fuel. Thus even if the atmosphere was steam inert with respect to hydrogen 
NUREG/CR-4551, VOL. 1: DRAFT REPORT FOR COMMENT (SEPTEMBER, 1986)

burning, the same energy transfer could be accomplished by direct heating, all within the same time periods as the other phenomenon. ${ }^{*}$

Most of the other reviewers indicated that their most important concern was uncertainty in how much material would get out of the cavity. The pathways are not straightforward, and wide dispersal in containment would be less likely. Once out, most believed that there were enough energy transfer mechanisms to assure relatively high pressure increments. The amount of transfer would depend on complex interactions, ultimately determining the residence time in the atmosphere. These phenomenon affecting the ultimate disposition of the core material would be very difficult to predict.

Reviewer \#6 indicated that his driving criterion was the amount of core discharged, with $50 \%$ as his selected value based on a lack of any applicable evidence. His pressure increments weighting factors were based on the SARRP methodology that had been provided, and once again, several of the reviewers stated their opinion that $50 \%$ ejection did not imply the low pressure calculated using that method. In any case, this reviewer calculated pressure rises from the SARRP model by varying the amount of core ejected, the amount of ejected core quenched and the amount of remaining core participating in direct heating. He then created a probability distribution, discretized it and provided weighting factors based on these calculations.

Finally, one other issue that relevant to this issue was the impact of operator actions on the final outcome. Of interest here was containment flooding. The SARRP analysts concluded that the best way to handle the event at this time would be through the safety options. The flooding of containment would then change the values represented in the table for direct heating.

The other input requested of the review team was their weighting factors on the factors used to account for different boundary conditions that the base case. Table B-6 illustrates the combinations of boundary conditions of interest: pressure in the primary and water in the cavity.

The SARRP entries were based on a review of the experimental evidence and consideration of the other factors already discussed. The wet cavity cases could result in lower pressure due to the action of the water as a heat sink through its latent heat, as well as in the potential for a quicker cooldown of the material leading to less transfer of chemical energy.

"The next issue includes hydrogen burning pressure increments as a separate issue, and this issue was considered there--correlated to the direct heating issue. 
NUREG/CR-4551, VOL. 1: DRAFT REPORT FOR COMMENT (SEPTEMBER, 1986)

Table B-6

FACTORS FOR ISSUE 3 BOUNDARY CONDITIONS

\begin{tabular}{|c|c|c|c|c|c|c|c|c|c|c|c|}
\hline \multicolumn{3}{|c|}{ BOUNDARY CONDITION } & \multicolumn{9}{|c|}{ FACTORS TO APPLY TO BASE CASE } \\
\hline RCS Pressure & Cavity & SARRP & 1 & 2 & 3 & $4^{\underline{K}}$ & $\frac{\text { lewer }}{5}$ & 6 & 7 & 8 & Comp. \\
\hline High & Fulla & .6 & .7 & .8 & .8 & 1.0 & 1.0 & .6 & .55 & .8 & .76 \\
\hline Intermediate & Dry & .6 & .7 & 1.0 & .5 & .95 & 1.0 & .6 & .55 & .7 & .76 \\
\hline Intermediate & Full & .35 & .5 & .6 & .3 & .6 & 1.0 & .35 & .35 & .5 & .5 \\
\hline Low & Dry & 0.0 & 0.0 & 0.0 & 0.0 & .1 & 0.0 & 0.0 & 0.0 & .1 & 0.0 \\
\hline Low & Full & & & & & m spił & only & & & & \\
\hline
\end{tabular}

a Full means water up to the bottom of the vessel or higher.

The cases involving lower RCS pressure would result less energy for the ejection of the material from the cavity, the driving force for this phenomenon. Several of the reviewers sided nearly directly with the values proposed by SARRP--generally citing the lack of any applicable information to suggest changes. Other reviewers did not agree. Based on the available experimental evidence, Reviewer 5 maintained that the base case would apply for any cases except those with the RCS at very low pressure. His rationale was that there was enough energy available to transfer the material efficiently enough to the atmosphere, particularly considering that the Surry geometry would allow direct paths to the upper atmosphere. Reviewer 8 had a different opinion, that there would be significantly less pressure involved because of the transfer of energy to the water droplets that would accompany the core debris into the atmosphere. The values in the table were combined and a composite value was used to modify all the pressure values (that involved direct heating) presented in the previous table.

\section{B.5 ISSUE 4: MAGNITUDE OF LOADING DUE TO HYDROGEN BURN AT VESSEL BREACH}

There have been numerous studies on the issue of hydrogen generation and burning following the onset of core damage or melt. These studies illustrate the uncertainty in this issue in that there are widely varying estimates of the magnitude of the effect. The dominant uncertainty is in the amount of hydrogen generation, principally from metal water reactions involving zirconium, although other sources also play a role. The results of some of the studies are listed below as an indication of the uncertainty range: 


\begin{abstract}
Source
WASH-1400 [6]

CLWG [12] and

SASA
\end{abstract}

IDCOR [3]

CLWG [25]

BMI-2104 [16]

SARRP Task Force [17]

Sandia HYDR [18]
Amount of Zirconium Oxidation

$50-100 \%$

$35-100 \%$

$25 \%$

25-100\%

60-90\%

$10-100 \%$

Additional 50\% equivalent
Comments

Surry Calculation

Sequoyah Calculation

Sequoyah Calculation

Includes Subatmospheric Containment

Surry S2D Calculation

Surry TMLB'

Steel Oxidation

Each of the studies also showed some variation in predicted oxidation for different sequences, but the variation was small compared to the overall uncertainty ranges. In order to fully cover the range of outcomes for the Surry LLH study, metal-water reaction values of $25 \%, 50 \%, 75 \%, 100 \%$, and $130 \%$ of the total zirconium inventory were considered, the last value greater than $100 \%$ to account for steel oxidation. The pressure increments associated with these hydrogen generation values were calculated from the results presented by the CLWG [11], which included a 70 psi increment for $100 \%$ oxidation for Surry, 35 psi for $50 \%$ zirconium equivalent, and 25 psi for $25 \%$ reaction. Although the pressure increments are not truly linear, the variation due to initial pressure and other factors is small compared to the other uncertainties involved in this issue, and the pressure increment for the other cases were simply extrapolated. One other case was added, $0.0 \mathrm{psi}$, to account for the possibility that the hydrogen would not be ignited. Reference 11 also concluded that the containment sprays would have little effect on the pressure increments for burn times predicted to be typical (and supported by the bum time evidence at TMI-2--8 seconds).

The containment event tree was used to generate the amount of hydrogen available for burn at vessel breach, since this is the only critical time that a burn contributes to the early containment failure frequency for Surry. Thus the tree allows for escape of hydrogen and bum at earlier times depending on the sequence boundary conditions. On the basis of MARCH calculations $[16,19]$, the following values were selected for the percent of hydrogen produced that is released to the containment before vessel breach:

$\begin{array}{ll}\text { Large LOCA } & 75 \% \\ \text { S1 LOCA } & 50 \% \\ \text { S2 or S3 LOCAs } & 25 \%\end{array}$


The containment event tree accounts for any hydrogen burn before vessel breach, precluding later burns if an early burn occurred, because of oxygen depletion and the lower hydrogen concentration in containment. Steam inerting (steam concentration greater than $56 \%$ precludes large-scale hydrogen deflagration) was also considered by including the status of the containment heat removal systems in the boundary conditions for this question.

There is also some uncertainty associated with the source of ignition and the timing thereof. Many experts $[12,14,15,12]$ consider immediate ignition on vessel breach likely due to the hot core debris. Others have cited the potential for local steam inerting at the time of vessel breach due to the copious quantities of steam generated at the same time [3].

One final area of uncertainty was already raised with respect to the previous issue pressure loading due to hydrogen recombination associated with direct heating. Once agail, this proposed phenomenon would result from potential for local recombination of hydrogen and oxygen in the vicinity of the hot particles of core debris throughout containment. This phenomenon could potentially occur even in a steam inerted containment, since the scale of the reaction is local and the energy transfer is through recombination rather than bulk deflagration. There is considerable uncertainty associated with this, and even more uncertainty in the timing of the phenomenon, since, in order to be risk significant, the pressure increment due to this effect would have to be simultaneous with the direct heating increment as well as the steam spike.

All of these uncertainties and boundary conditions were grouped into cases that include the conditions relevant to the Surry calculations. The base case is illustrated in Table B-7--the pressure increment for the conditions of no prior burn, minimum steam spike, and non-inert containment (steam concentration less than 56\%). The other cases that include the important boundary condition combinations of steam spike, direct heating, and steam concentration are illustrated in Tables B-8 through B-11. In each case, the reviewers were asked to weight the six discrete values for the final pressure increment associated with the burn.

There was little discussion of the base case and the factors described above influenced each reviewer somewhat differently. The weighting factors reflect each reviewer's opinion of the importance of each of the various factors based on his professional experience. Essentially the entire issue can be reduced to uncertainty over the amount of zirconium oxidized, and, as illustrated above, different analysts can have widely varying results for this problem. The results in the table below for the base case illustrate that the heaviest weightings were associated with pressure values of 35 and $50 \mathrm{psi}$. 
Table B-7

WEIGHTING FACTORS FOR ISSUE 4: HYDROGEN BURN AT VESSEL BREACH --MINIMUM STEAM SPIKE AND STEAM CONCENTRATION LESS THAN 56\%--

\begin{tabular}{|c|c|c|c|c|c|c|c|c|c|}
\hline \multirow[t]{2}{*}{ OUTCOME } & \multicolumn{9}{|c|}{ WEIGHTING FACTORS } \\
\hline & SARRP & 1 & 2 & 3 & $\frac{k e}{4}$ & $\frac{\text { dewer }}{5}$ & 6 & 7 & Comp. \\
\hline 1. $0.0 \mathrm{psi}$ & .25 & .05 & 0.0 & 0.0 & .25 & .15 & .1 & 0.0 & .17 \\
\hline 2. $25 \mathrm{psi}$ & .15 & .15 & .25 & .2 & .25 & .25 & .35 & .25 & .2 \\
\hline 3. $35 \mathrm{psi}$ & .25 & .25 & .35 & .5 & .25 & .25 & .25 & .25 & .27 \\
\hline 4. $50 \mathrm{psi}$ & .2 & .3 & .25 & .25 & .1 & .25 & .25 & .25 & .2 \\
\hline 5. $70 \mathrm{psi}$ & .1 & .15 & .15 & .05 & .1 & .1 & .15 & .15 & .11 \\
\hline 6. $90 \mathrm{psi}$ & .05 & .1 & 0.0 & 0.0 & .05 & 0.0 & .1 & .1 & .05 \\
\hline
\end{tabular}

One reviewer (\#6) did describe a method for arriving at his weighting factors. He first weighted the amount of $\mathrm{Zr}$ produced, ranging from 25 to $100 \%$, the latter value including $25 \%$ equivalent steel oxidation. This reviewer did not believe that core oxidation of more than $75 \%$ was credible. He calculated pressure increments using his own code which accounts for the temperature dependence of specific heats and for incomplete burn efficiencies when the hydrogen is in the range of four to six volume percent. He then calculated the pressure increments for the different boundary conditions considered by SARRP, and translated the results into the discrete pressure distributions.

There was some additional discussion concerning the case illustrated in Table B-8. For this case, the containment atmosphere is steam inert relative to mass hydrogen burning, but direct heating occurs as discussed in the previous issue. It was pointed out that recombination of hydrogen and oxygen could occur as a catalysis event, caused by the high temperature around the finely divided fuel particles. The chemistry of this reaction is very complex and would involve temperatures, gas densities, and many other factors. Several reviewers indicated that it would be a realistic event, but that the amount of such recombination was conjecture. Reviewer \#7 indicated that the problem was even more complex because of competing chemical reactions. For example, much of the hydrogen would go directly to metallic surfaces, and oxygen would also be consumed due to interaction with the fuel and other debris. The profile of gases around the particles is therefore difficult to predict, and the resultant pressure loading is unknown. There is no simple way to predict which reaction is 
preferred because the local chemistry and boundary conditions are to complex. The question of oxygen depletion to the point where the recombination could not happen was examined in some detail through a review of reaction tables and results of previous studies. It was generally concluded that the direct heating oxygen reactions would only consume about a third of the oxygen, and there would oxygen available for substantial reaction with hydrogen or other oxygen reactions.

The discussion also considered the possibility of correlations with the previous event. Unfortunately some reviewers would predict a positive correlation while other would see a negative one. One one hand, large direct heating contributions would imply significant energy throughout containment and many local sites for recombination, yet high oxidative direct heating pressures could also imply that there was significant unoxidized zirconium prior to the event and the hydrogen concentrations might be different that expected. Oxygen depletion could also be postulated for high direct heating cases. It was the opinion of several reviewers that correlations were too complex to propose, and that establishing even a simple correlation would imply that there was more knowledge available than truly existed. The result are indicated in Table B-8. The pressures in the table are intended to be added to the direct heating pressure associated with the previous issue.

The pressures expected for other preconditions were also provided by the reviewers (these weighting factors were provided after the meeting and not all of the reviewers participated). These other weighting factors were provided without comment. As described below, the constraints of the evaluation method required some adjustment to the reviewer input to arrive at a workable $\mathrm{LLH}$ input.

Table B-8

WEIGHTING FACTORS FOR ISSUE 4: HYDROGEN BURN AT VESSEL BREACH --STEAM CONCENTRATION GREATER THAN 56\% WITH DIRECT HEATING --

\begin{tabular}{|c|c|c|c|c|c|c|c|c|c|}
\hline \multirow{2}{*}{ OUTCOME } & \multirow[b]{2}{*}{ SARRP } & \multicolumn{8}{|c|}{ WEIGHTING FACTORS } \\
\hline & & 1 & 2 & 3 & $\frac{R}{4}$ & $\frac{\text { iewer }}{5}$ & 6 & $7^{a}$ & Comp. \\
\hline 1. $0.0 \mathrm{psi}$ & .40 & .05 & .2 & .3 & .2 & .05 & .65 & 0.0 & .23 \\
\hline 2. $25 \mathrm{psi}$ & .15 & .15 & .35 & .45 & .25 & .3 & .1 & .25 & .25 \\
\hline 3. $35 \mathrm{psi}$ & .15 & .35 & .3 & .2 & .25 & .3 & .1 & .25 & .24 \\
\hline 4. $50 \mathrm{psi}$ & .2 & .4 & .1 & .05 & .2 & .3 & .1 & .25 & .2 \\
\hline 5. $70 \mathrm{psi}$ & .1 & .05 & .05 & 0.0 & .1 & .15 & .05 & .15 & .08 \\
\hline 6. $90 \mathrm{psi}$ & 0.0 & 0.0 & 0.0 & 0.0 & 0.0 & .1 & 0.0 & .01 & .01 \\
\hline
\end{tabular}

a The reviewer concluded that steam concentration would play no role in direct heating cases. 
Table B-9

WEIGHTING FACTORS FOR ISSUE 4: HYDROGEN BURN AT VESSEL BREACH -MAXIMUM STEAM SPIKE, STEAM CONCENTRATION

LESS THAN 56\% AND NO DIRECT HEATING--

\begin{tabular}{llllccr}
\hline OUTCOME & & \multicolumn{5}{c}{ WEIGHTING FACTORS } \\
& SARRP & $2^{\mathrm{a}}$ & 5 & 6 & 7 & Composite \\
\hline 1. $0.0 \mathrm{psi}$ & .45 & .2 & .25 & .55 & 0.0 & .32 \\
2. $25 \mathrm{psi}$ & .15 & .25 & .3 & .15 & .25 & .21 \\
3. $35 \mathrm{psi}$ & .2 & .2 & .25 & .15 & .25 & .21 \\
4. $50 \mathrm{psi}$ & .15 & .15 & .15 & .1 & .25 & .16 \\
5. $70 \mathrm{psi}$ & .05 & .1 & .05 & .05 & .15 & .08 \\
6. $90 \mathrm{psi}$ & 0.0 & 0.0 & 0.0 & 0.0 & .1 & .02 \\
\hline
\end{tabular}

a The reviewer assumed that the steam concentration below $56 \%$ would be a local condition. The values for high pressure were reduced from the base case steam could suppress the burn.

Table B-10

WEIGHTING FACTORS FOR ISSUE 4: HYDROGEN BURN AT VESSEL BREACH -MAXIMUM STEAM SPIKE, STEAM CONCENTRATION LESS THAN 56\% WITH DIRECT HEATING--

\begin{tabular}{lcccccc}
\hline OUTCOME & & \multicolumn{5}{c}{ WEIGHTING FACTORS } \\
& SARRP & $2^{\mathrm{a}}$ & $5^{\mathrm{a}}$ & $\frac{\text { Reviewer }}{6}$ & $7^{\mathrm{b}}$ & Composite \\
\hline 1. $0.0 \mathrm{psi}$ & .35 & .1 & .05 & .1 & 0.0 & .15 \\
2. $25 \mathrm{psi}$ & .2 & .3 & .2 & .35 & .25 & .24 \\
3. $35 \mathrm{psi}$ & .2 & .35 & .35 & .25 & .25 & .27 \\
4. $50 \mathrm{psi}$ & .2 & .2 & .3 & .2 & .25 & .23 \\
$5.70 \mathrm{psi}$ & .05 & .05 & .1 & .1 & .15 & .08 \\
6. $90 \mathrm{psi}$ & 0.0 & 0.0 & 0.0 & 0.0 & .1 & .03 \\
\hline
\end{tabular}

a The direct heating would, in this reviewers opinion, almost guarantee widespread ignition that consumes nearly all the available hydrogen. The direct heating would not limit the amount of oxygen available. The higher pressure cases are therefore weighted higher.

$b$ The reviewer agrees that the direct heating would lead to an efficient hydrogen burn. 
Table B-11

WEIGHTING FACTORS FOR ISSUE 4: HYDROGEN BURN AT VESSEL BREACH --STEAM CONCENTRATION GREATER THAN 56\% AND NO DIRECT HEATING--

\begin{tabular}{lccccccc}
\hline OUTCOME & & \multicolumn{5}{c}{ WEIGHTING FACTORS } \\
& SARRP & 2 & $5^{\mathrm{a}}$ & $\frac{6}{6}$ & $7^{\mathrm{b}}$ & Comp. \\
\hline 1. $0.0 \mathrm{psi}$ & 1.0 & .6 & .8 & 1.0 & 1.0 & .88 \\
2. $25 \mathrm{psi}$ & 0.0 & .2 & .1 & 0.0 & 0.0 & .06 \\
3. $35 \mathrm{psi}$ & 0.0 & .1 & .1 & 0.0 & 0.0 & .04 \\
4. $50 \mathrm{psi}$ & 0.0 & .05 & 0.0 & 0.0 & 0.0 & .01 \\
5. $70 \mathrm{psi}$ & 0.0 & .05 & 0.0 & 0.0 & 0.0 & .01 \\
6. $90 \mathrm{psi}$ & 0.0 & 0.0 & 0.0 & 0.0 & 0.0 & 0.0 \\
\hline
\end{tabular}

a Ignition is highly improbable, but there is only weighting to a small burn due to the uncertainty in the inerting concentration and the uniformity of that concentration in containment.

As illustrated in the previous tables, the reviewers assigned weighting factors for each pressure level. The SARRP containment event trees contain parameter values such as pressure increment due to hydrogen burn, as well as branching ratios for each set of boundary conditions. It is therefore possible to enter distinct levels of pressure for each set of boundary conditions, but it is not possible to enter distinct weighting factors for each boundary conditions. The implementation of the reviewers' inputs therefore required the development of pressure levels consistent with a set of weighting factors.

Although the reviewers voted on distinct pressure increments, the reviewers were for the most part thinking of continuous or piecewise continuous distributions. The simplest representation of the reviewers' inputs would be a set of uniform distributions spaced to give the same cumulative function as the discrete representation for the base case (see Figure B-1). This continuous probability distribution function would result in the cumulative distribution function represented as the solid curve in Figure B-2. The dashed curve in Figure B-2 illustrates the same function for a case of maximum steam spike with no direct heating. The horizontal lines on the figure represent the base case levels, which cross the solid curve at the base case pressure increments. The point of intersection of these horizontal lines representing the levels with the other, dashed curve represent equivalent pressures which can then be used in the containment event tree for the boundary conditions associated with that curve. This 
allows the use of the appropriate weighting factors with the containment event tree pressure adjusted to the particular boundary conditions.

The results of the process above for all boundary conditions are listed in Table B-12. The operation of the containment heat removal system is used as a proxy for low initial steam concentration, whereas the presence of water in the sump or accumulator discharge at vessel breach is used as a proxy for steam spike. It may be noted that the Level 3 of the base case corresponds to the OCP central, but the pressures for the other walkthroughs do not correspond to the $\mathrm{OCP}$ values.

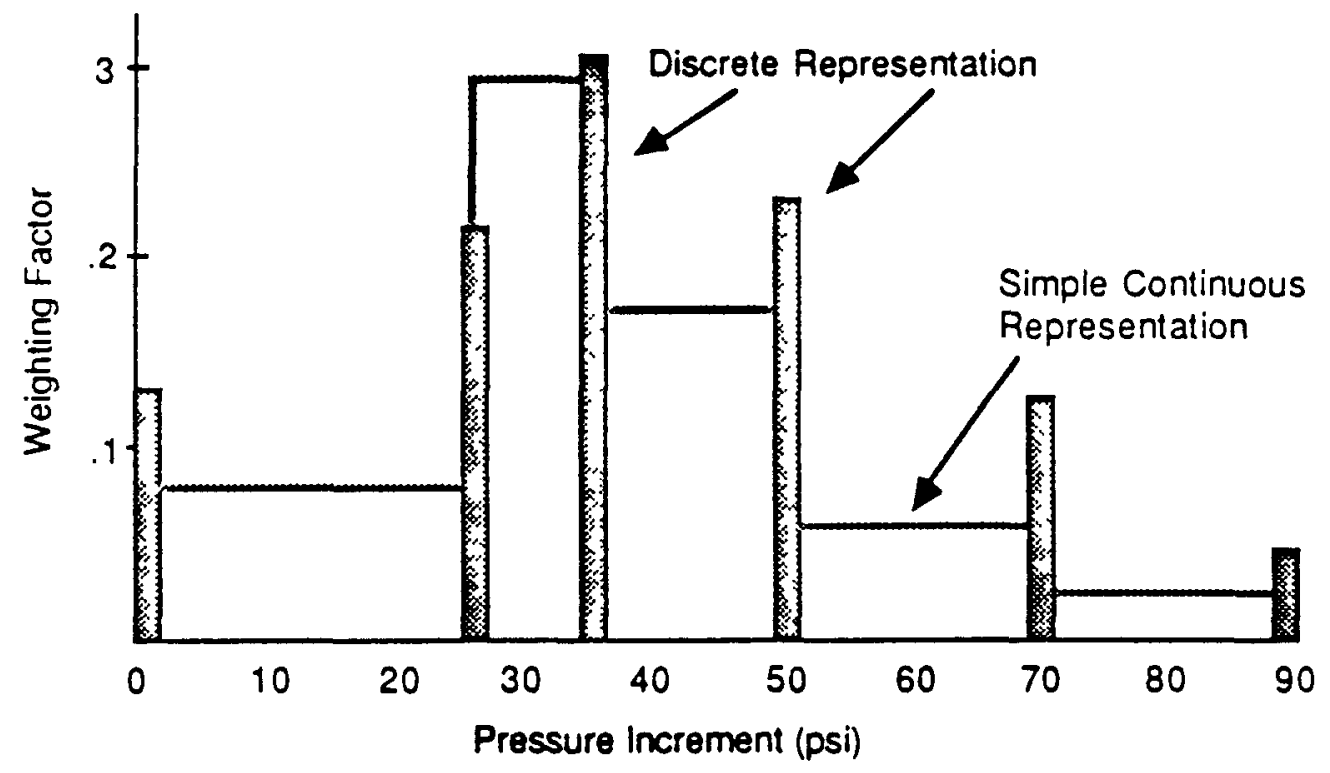

Figure B-1. Discrete and Continuous Weighting Functions for the Base Case Hydrogen Burn

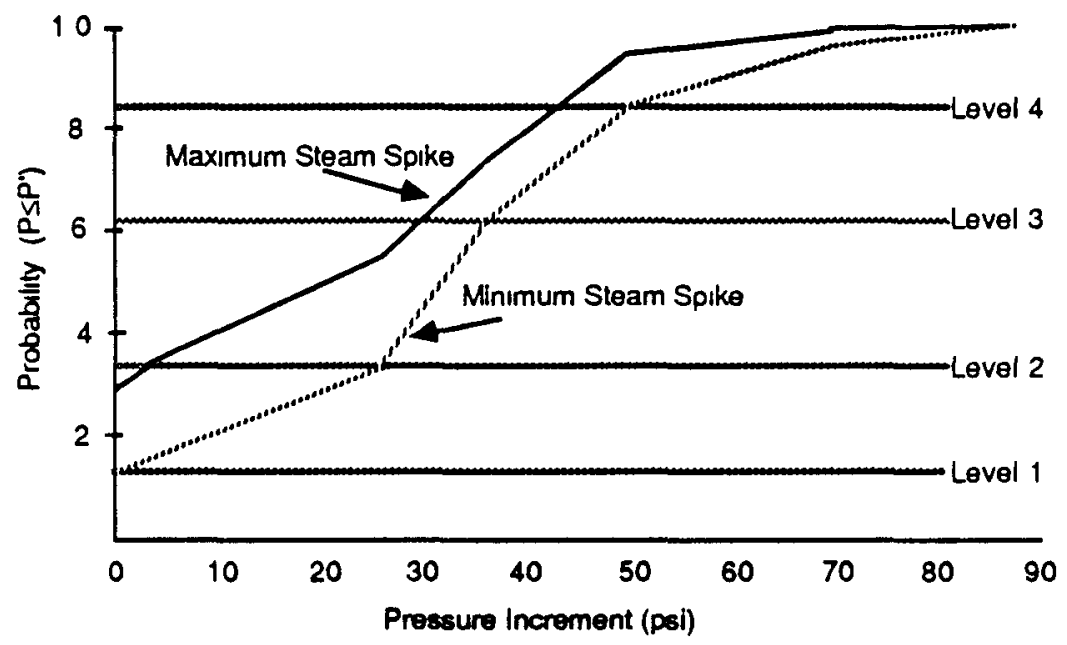

Figure B-2. Constucted Cumulative Distribution Function for Two Boundary Conditions 
NUREG/CR-4551, VOL. 1: DRAFT REPORT FOR COMMENT (SEPTEMBER, 1986)

Table B-12

EQUIVALENT HYDROGEN BURN LEVELS FOR THE BASE CASE WEIGHTING FACTORS

\begin{tabular}{|c|c|c|c|c|c|c|c|}
\hline \multirow[b]{2}{*}{ LEVEL } & \multirow[b]{2}{*}{$\begin{array}{l}\text { WEIGHTING } \\
\text { FACTOR }\end{array}$} & \multirow[b]{2}{*}{$\begin{array}{l}\text { STEAM SPIKE } \\
\text { DIRECT HEATING } \\
\text { STEAM CONC. }\end{array}$} & \multicolumn{5}{|c|}{ CONDITIONS } \\
\hline & & & $\begin{array}{r}\mathrm{Min} \\
\mathrm{No} \\
<56 \%\end{array}$ & $\begin{array}{r}\operatorname{Max} \\
N o \\
<56 \%\end{array}$ & $\begin{array}{r}\text { Max } \\
\text { Yes } \\
<56 \% \\
\text { SSSUR }\end{array}$ & $\begin{array}{l}\text { Yes } \\
>56 \% \\
\text { (osi) }\end{array}$ & $\begin{array}{r}-- \\
\text { No } \\
>56 \%\end{array}$ \\
\hline 1. & .17 & & 0 & 0 & 0 & 0 & 0 \\
\hline 2. & .2 & & 25 & 6 & 23 & 14 & 0 \\
\hline 3. & .27 & & 35 & 31 & 35 & 32 & 0 \\
\hline 4. & .2 & & 50 & 46 & 48 & 46 & 0 \\
\hline 5. & .11 & & 70 & 83 & 70 & 63 & 10 \\
\hline 6. & .05 & & 90 & 86 & 90 & 78 & 32 \\
\hline
\end{tabular}

\section{B.6 ISSUE 5: CONTAINMENT FAILURE PRESSURE}

Another source of uncertainty is the containment failure pressure. The design pressure of Surry is 45 psi with a proof pressure (accounting for a 1.5 safety factor) of 67 psi. There are several sources that have information applicable to the Surry plant, but no detailed calculations were performed for this program. There was however a simplified analysis performed by Stone and Webster which is the most applicable and recent of available analyses [20]. The median failure pressure calculated in this document, based on general yielding of the reinforcement, was 119 psi. The Reactor Safety Study used a mean failure pressure of 85 psi based on the assumption that failure would likely occur due to yielding of the reinforcement and shear failure of the concrete in the upper portion of the shell or in the dome [6]. Detailed studies on other plants that have considered actual strength in detail and/or have accounted for some pressure capacity beyond yield have generally resulted in ultimate pressures 4 or 5 times failure pressure[21,22,23]. In order to reflect the uncertainty in this parameter, the reviewers were asked to weight possible median values of failure pressure and to provide standard deviations that would the be used with a normal distribution to calculated the failure probability for each pressure loading in the containment event tree. The values selected to represent the range of the medians are reproduced in Table B-13.

As indicated in the table, the reviewers had the most confidence in the most applicable and recent evaluation by Stone and Webster, and the weightings also reflect the potential for a higher pressure capability since the Stone and Webster calculation was based on yield strength, 
not ultimate. The weighting of Reviewer \#4 was based on comparison to a detailed evaluation of as similar containment design which was compared to the Stone and Webster calculation. The Seabrook containment is similar, and it was found during detailed study of that plant that the containment can accommodate strains well beyond yield without failure (due to the control of concrete cracking afforded by the close spacing of the rebar). Failure was found to be controlled by the strain limit of the liner or rebar, or by local interferences. For Seabrook, the median failure point was 3.6 times design [22]. Assuming the same ratio for Surry results in a failure pressure of $160 \mathrm{psi}$, which lends support to the two highest levels. Because a detailed study of Surry is not available, this reviewer weighted the lower pressure (Level 4) more heavily. There was also some discussion here as to the uncertainty associated with the different types of liners, and their roles in the failure pressure. It was concluded that there remained uncertainty in the liner performance, but detailed evaluation would be required to calculate more specific pressure capabilities than those provided in the references already outlined.

In this case, the composite was used directly in the LLH study. The Level 3 value is closest to the OCP central estimate, but the composite standard deviation is different. Thus the $\mathrm{LLH}$ results have a somewhat different central tendency than the OCP for this issue.

Table B-13

WEIGHTING FACTORS AND OUTCOMES FOR ISSUE 5: CONTAINMENT FAILURE PRESSURE

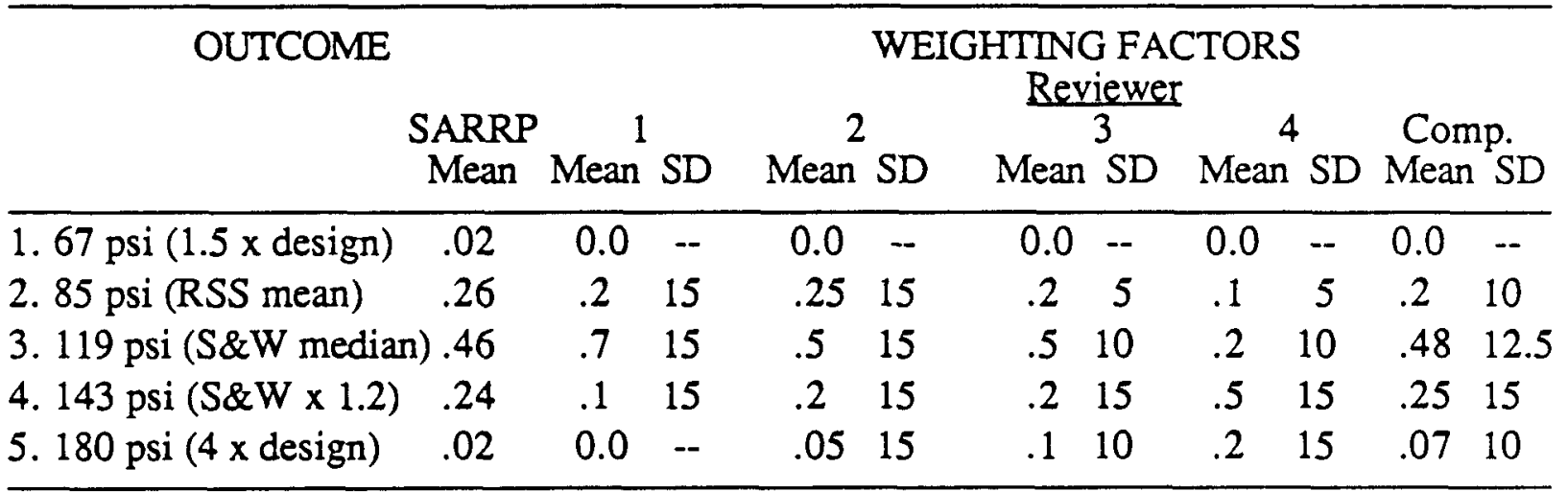

\section{B.7 ISSUE 6: CONTAINMENT FAILURE SIZE}

It was the conclusion of the review team that the size of the containment failure opening would have a significant impact on the progression of the accident and risk. The OCP study for Surry had considered two failure sizes: one small, but large enough to preclude further 
overpressurization and one large, resulting in gross rupture and depressurization in less than two hours. The Containment Performance Working Group (CPWG) studied this issue for several plants and provided upper bounds of leak area versus overpressurization for several plants. For Surry, these bounds indicated leaks at the personnel air lock of 0.004 square inches at 45 psig (technical specification leakage) to 0.36 square inches at 119 psi. This study did not provide central or lower bound estimates, but even the upper bound estimates are too small to preclude further overpressurization (a 25 square inch leak is required if heat sinks and wall condensation are not considered). If all heat sinks are considered, MARCH runs have indicated that leaks as small as 46 square inches could preclude overpressurization.

Other studies have estimated different failure sizes, for example IDCOR predicted a 0.1 square foot leak size for the Zion plant [3]. The most recent experimental evidence also indicated very little leakage up to the point of very gross containment failure; but the experiment does not correspond well to actual containments since it was performed on a steel vessel without consideration of concrete, while the Surry plant has a reinforced concrete containment with steel liner--not a steel containment. Overall the evidence is highly inconclusive, and even leakage size before failure still does not address the size of the final failure. A range of leak sizes were presented to the review group to represent the uncertainty in this issue, and as illustrated in Table B-14, the possibilities range from insignificant leaks to very large hole sizes. Recognizing that there could be a dependence on the type of pressurization, weighting factors were requested for slow overpressurizations and fast pressurizations associated with direct heating, steam spikes or hydrogen burns.

As illustrated in the table, the review group felt strongly that there should be a correlation to the containment failure pressure, Issue 4. There was a strong opinion among some of the reviewers that the lower containment failure pressures in Issue 4 reflected failure causes such as flaws or construction errors that would lead to local failure and a lower probability of a catastrophic failure. It was therefore decided that the weighting factors would be correlated to the failure pressure as illustrated in the table. There is a definite trend to larger failure openings with increasing ultimate failure pressure, since the higher failure pressures were thought to result from exceeding of the basic materials strengths and the potential for massive structural failure. In addition, there was a conclusion that the rate of pressurization was not as important as the total pressure relative to the failure pressure. It was proposed that the results in Table B-14 be used for slow pressurizations and rapid pressurizations to a total pressure below the median of the containment failure distribution. The results in Table B-15 are applicable for rapid pressurization to a value greater than the median of the failure 
distribution. There was little discussion of the actual assignment of each weighting factor. Reviewer 1 based his results on the Seabrook study which concluded a strong dependence of failure size on failure pressure. For that study, the probability of a leak exceeding 0.5 square feet was 0.4. He was also strongly convinced that the failure size was correlated to the level of pressurization versus the ultimate capacity.

Table B-14

WEIGHTING FACTORS FOR ISSUE 6: CONTAINMENT FAILURE SIZE GIVEN SLOW PRESSURIZATION OR RAPID PRESSURIZATION TO A PRESSURE LESS THAN THE MEDIAN OF THE CONTAINMENT CAPACITY DISTRIBUTION

\begin{tabular}{|c|c|c|c|c|c|}
\hline \multirow{3}{*}{$\begin{array}{c}\text { OUTCOME } \\
\text { Size of Opening }\end{array}$} & \multirow[b]{3}{*}{ SARRP } & \multicolumn{4}{|c|}{ WEIGHTING FACTORS } \\
\hline & & & & view & \\
\hline & & 1 & 2 & 3 & 4 \\
\hline \multicolumn{6}{|c|}{ For Containment Failure Pressure 119 psig } \\
\hline 1. 4 sq. in. & .15 & .35 & .1 & .2 & .25 \\
\hline 2. .1 sq. $\mathrm{ft}$. & .2 & .35 & .2 & .2 & .25 \\
\hline 3. .7 sq.ft. & .2 & .2 & .5 & .3 & .25 \\
\hline 4. 7 sq. ft. & .45 & .1 & .2 & .3 & .25 \\
\hline \multicolumn{6}{|c|}{ For Containment Failure Pressure 85 psig } \\
\hline 1. 4 sq. in. & .15 & .5 & .5 & .4 & .25 \\
\hline 2. .1 sq. ft. & .2 & .4 & .4 & .5 & .25 \\
\hline 3. .7 sq.ft. & .2 & .1 & .1 & .1 & .25 \\
\hline 4. 7 sq. ft. & .45 & 0.0 & 0.0 & 0.0 & .25 \\
\hline \multicolumn{6}{|c|}{ For Containment Failure Pressure 143 or 180 psig } \\
\hline 1. 4 sq. in. & .15 & .3 & 0.0 & 0.0 & .15 \\
\hline 2. . .1 sq. ft. & .2 & .3 & .1 & 0.0 & .15 \\
\hline 3. .7 sq.ft. & .2 & .2 & .6 & .4 & .35 \\
\hline 4. 7 sq. ft. & .45 & .2 & .3 & .6 & .35 \\
\hline
\end{tabular}


NUREG/CR-4551, VOL. 1: DRAFT REPORT FOR COMMENT (SEPTEMBER, 1986)

Table B-15

WEIGHTING FACTORS FOR ISSUE 6: CONTAINMENT FAILURE SIZE GIVEN RAPID PRESSURIZATION TO A TOTAL PRESSURE GREATER THAN THE MEDIAN OF THE CONTAINMENT CAPACITY DISTRIBUTION

\begin{tabular}{lcrrrr}
\hline OUTCOME & & \multicolumn{5}{c}{ WEIGHTING FACTORS } \\
Size of Opening & SARRP & 1 & 2 & 3 & 4 \\
\hline 1. 4 sq. in. & 0.0 & .1 & 0.0 & 0.0 & -- \\
2. .1 sq. ft. & 0.0 & .1 & 0.0 & .1 & - \\
3. .7 sq.ft. & 0.0 & .1 & .3 & .3 & - \\
4. 7 sq. ft. & 1.0 & .7 & .7 & .6 & - \\
\hline
\end{tabular}

The reviewer's inputs for this issue required several changes to allow timely inclusion in the LLH study. First, neither the containment nor source term analysis could distinguish all of the levels provided and the issue was instead divided into two levels, large and small. Large corresponds to greater than 0.7 square feet while small is any failure less than that. Figure B-3 illustrates the reviewers inputs in terms of failure size versus pressurization. It is clear that there is a correlation with containment failure pressure, and it is also clear that although the reviewers stated that the values should be the same for 143 psi as for 180 psi, a different function was actually intended. The graphs were therefore used to generate the values for the $180 \mathrm{psi}$ level. The second piece of input that could not be incorporated was the correlation to the total pressurization compared to the median capacity. As illustrated in the weighting factors for this issue, the reviewers provided different weights depending on the level of pressurization, with the median failure capacity selected as the crossover point. This could not be incorporated without major modeling changes and use of this input was delayed until future analysis is performed.

Due to the change in levels and the correlation of this issue to containment failure pressure, it was necessary to rework the input into a format compatible with the input as well as the correlations. Tables B-16 through 18 illustrate this process. The split fractions of large versus small failure openings were examined against the reviewer's inputs, with each reviewer weighted equally. Table B-16 delineates the fraction of reviewers who believed the split fraction was at least as great as the level indicated for each row, contingent on containment failure pressure. Table B-17 illustrates the same information except it is the reviewers' belief that the fraction is within the range (rather than at least as great as). Finally, the noncontingent 
voting fractions are listed in Table B-18: the results of Table B-17 were multiplied by the probability of mean containment failure pressure being at the level indicated by the column heading. The last row and column in Table B-18 indicate the values that were used in the LLH sampling along with the calculated correlation factor between Issues 4 and $5(r=-0.86)$.

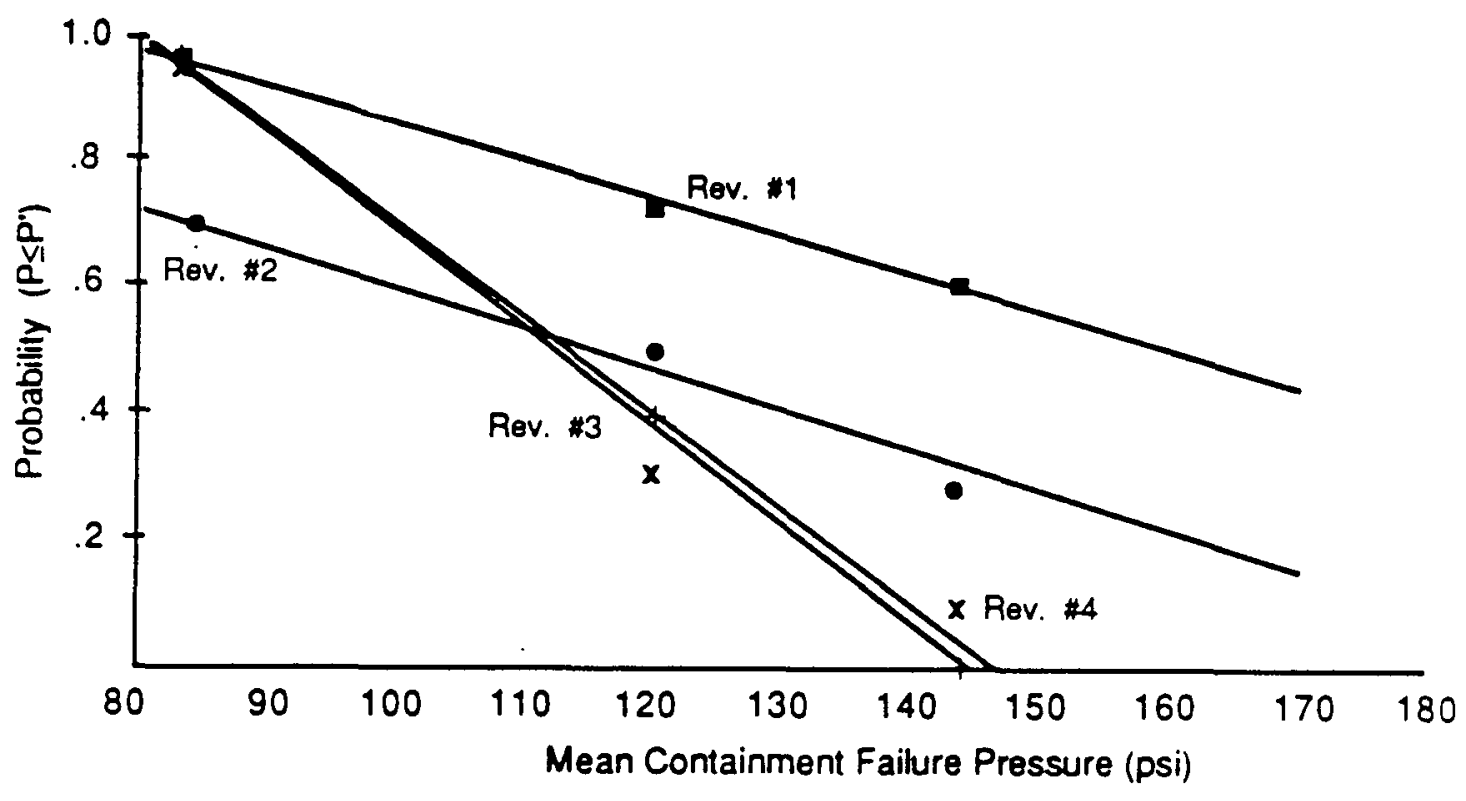

Figure B-3. Probability of a Small Opening as a Function of Mean Failure Pressure

Table B-16

REVIEWERS INPUT CONTINGENT ON CONTAINMENT PRESSURIZATION

FRACTION OF REVIEWERS BELIEVING THE PROBABILITY OF A SMALL FAILURE SIZE LIES AT

OR ABOVE THE SPECIFIED LEVEL CONTINGENT ON MEAN CONTAINMENT FAILURE PRESSURE

Mean Containment Failure Pressure Level

$\begin{array}{llll}1.85 & \text { psig } \quad 2.119 & \text { psig } & 3.143 \text { psig } \quad 4.180 \\ \text { psig }\end{array}$

Fraction of reviewers who believe $P \geq 0.9$

Fraction of reviewers who believe $P \geq 0.8$

Fraction of reviewers who believe $P \geq 0.6$

Fraction of reviewers who believe $P \geq 0.4$

Fraction of reviewers who believe $P \geq 0.2$

Fraction of reviewers who believe $P=0.0$

.75

.88

1.0

1.0

1.0

1.0
0.0

0.0

.38

.75

1.0

1.0
0.0

0.0

.25

.45

.67

1.0 
Table B-17

REVIEWERS' INPUT CONTINGENT ON CONTANMENT PRESSURIZATION

FRACTION OF REVIEWERS BELIEVING THE PROBABILITY OF A SMALL FAILURE SIZE LIES WITHIN THE SPECIFIED LEVEL CONTINGENT ON MEAN CONTAINMENT FAILURE PRESSURE Mean Containment Failure Pressure Level
1.85 psig
2. 119 psig 3. 143 psig
4. $180 \mathrm{psig}$

Fraction of reviewers who believe $.9 \leq \mathrm{P} \leq 1$ .75

0.0

0.0

0.0

Fraction of reviewers who believe $.8 \leq \mathrm{P} \leq .9$ $.13^{*}$

0.0

0.0

0.0

Fraction of reviewers who believe $.6 \leq \mathrm{P} \leq .8$

$.13^{*}$

$.38 *$

.25

0.0

Fraction of reviewers who believe $.4 \leq \mathrm{P} \leq .6$

0.0

.25

$.17^{*}$

.25

Fraction of reviewers who believe $.2 \leq \mathrm{P} \leq .4$

0.0

.25

.25

$.13^{*}$

Fraction of reviewers

who believe $0 \leq P \leq .2$

0.0

0.0

$.34^{*}$

$.63^{*}$

${ }^{*}$ Reviewer split between two levels.

Table B-18

NONCONTINGENT WEIGHTING FACTORS FOR SIZE OF CONTAINMENT FAILURE

\begin{tabular}{|c|c|c|c|c|c|}
\hline $\begin{array}{c}\text { Levels for Issue 5: } \\
\end{array}$ & $\begin{array}{r}\text { Mean } \\
\text { Mea }\end{array}$ & Containment & ailure Pressu & $\frac{\text { re Level }}{4}$ & $\begin{array}{l}\text { Issue } 5 \\
\text { Waiphtc }\end{array}$ \\
\hline & & & & & \\
\hline 1. $P=0$ & -- & - & .08 & .03 & .11 \\
\hline 2. $P=.2$ & -- & .12 & .06 & .008 & .19 \\
\hline 3. $P=.4$ & -- & .18 & .04 & .01 & .24 \\
\hline 4. $P=.6$ & .03 & .18 & .06 & .02 & .27 \\
\hline 5. $\mathrm{P}=.8$ & .03 & -- & .08 & .03 & .03 \\
\hline 6. $P=.9$ & .158 & - & .08 & .03 & .16 \\
\hline Issue 4 Weights & .2 & .48 & .25 & .27 & \\
\hline
\end{tabular}




\section{B.8 ISSUE 7: EFFECT OF CONTAINMENT FAILURE ON SPRAY PUMP OPERABILITY}

There is the potential for damage to operating equipment in containment due to the severe environment associated with post accident conditions. Hydrogen burns have been postulated as one potential failure mechanism of the containment sprays--the only system of interest in this regard for the Surry plant. Burning insulation and electrical failure or instrumentation damage have been the postulated failure modes. The hydrogen burn in the TMI containment did not cause such failure mechanisms and the sprays continued to operate normally. For the OCP analysis of Surry, a 0.001 probability of this type of failure mechanism was assigned for the central case.

Another potential mechanism of pump failure is the possibility of foreign material in the sump causing pump failure--either directly or by clogging the screens. The debris could be associated with energetic interactions such as direct heating. Even with out such interactions, debris would be expected. At TMI the sump water was laden with particulates; this did not cause failure, but the accident was not nearly as severe as those being postulated here.

Other mechanisms could also be postulated as a failure mode for the spray pumps--loss of NPSH or pipe integrity following containment depressurization (see Issue $9^{*}$ ). The same arguments apply, since the low pressure pumps and the spray pumps are very similar--the latter having a $8 \mathrm{ft} \mathrm{NPSH}$. References generally conclude that failure is unlikely [3,24], but the probability of failure is important to the results for Surry.

The results of the review discussion for this issue are listed below in Table B-19. (The results are nearly identical to the results for the Issue 9, and all the same justifications apply.) Two of the sets of weighting factors are allow for failure without containment failure or with small leaks, due to debris or hydrogen burn effects.

One reviewer provided considerable discussion of this issue. He pointed out that the spray system, once started, will run until the operator stops it or it fails mechanically. A gross structural failure of the spray piping could fail the system, but this would be very unlikely for the small failure sizes. However, since the piping is high in containment and spans the entire circumference, he assigned a probability of failure due to piping of $0.0,0.0,0.01,0.05$, and 0.25 for the small to large failure sizes respectively. He also pointed out that that the sump screens are very large ( 4 to 6 foot tall) and that it would be highly improbable for large

\footnotetext{
"Initially this issue was treated after Issue 9 , and the arguments concerning the failure mechanisms are discussed with that issue.
} 
NUREG/CR-4551, VOL. 1: DRAFT REPORT FOR COMMENT (SEPTEMBER, 1986)

blockage. Plastic sheets or other matter could block areas, but the central portion of the screens likely remain open. Large particulates would settle out and collect on the floor of the sump. The design include provision for limiting flow problems associated with debris, and he therefore applied a probability of 0.05 that sump blockage could occur.

Table B-19

WEIGHTING FACTORS FOR ISSUE 7: EFFECT OF CONTAINMENT ENVIRONMENT ON SPRAY PUMP OPERABILITY

\begin{tabular}{|c|c|c|c|c|c|}
\hline \multirow{2}{*}{$\begin{array}{c}\text { OUTCOME } \\
\text { CORRELATED } \\
\text { TO FAILURE SIZE }\end{array}$} & \multicolumn{5}{|c|}{ WEIGHTING FACTORS } \\
\hline & SARRP & 1 & $\begin{array}{l}\text { Sevieu } \\
\frac{2}{4}\end{array}$ & 3 & 4 \\
\hline 1. No Failure & .05 & .05 & 0.0 & 0.0 & 0.0 \\
\hline 2. 4 sq. in. & .1 & .05 & 0.0 & 0.0 & 0.0 \\
\hline 3. .1 sq. ft. & .2 & .05 & 0.0 & 0.0 & .05 \\
\hline 5. .7 sq.ft. & .2 & .15 & .2 & .2 & .15 \\
\hline 5. 7 sq. ft. & .4 & .35 & .45 & .3 & .25 \\
\hline \multicolumn{6}{|c|}{ Reviewers' Input Adjusted To Actual Failure Sizes Used in LLH } \\
\hline No Failure & .05 & .05 & 0.0 & 0.0 & 0.0 \\
\hline Small Failure & .15 & .05 & 0.0 & 0.0 & .02 \\
\hline Large Failure & .3 & .25 & .32 & .25 & .2 \\
\hline
\end{tabular}

\section{B.9 ISSUE 8: V SEQUENCE SUBMERGENCE}

This issue refers to the potential for the interfacing systems LOCA, or $\mathrm{V}$ sequence, to occur in a location that ensures that the bypass path from containment is covered by water, thus reducing the radiological release during and after core damage. This question is difficult to answer without a systematic, thermal-hydraulic and structural analysis of all the interface piping. In order to address this issue without initiating a full study of the problem, four experts were consulted concerning this issue:

James E. Metcalf, Stone and Webster

Walter A. von Riesemann, Sandia National Laboratories

Peter R. Davis, Intermountain Technology Corporation

Robert L. Ritzman, Electric Power Research Institute.

The Surry V sequence involves the failure of the check valves in one of the six inch cold leg ECCS injection lines. The RCS water at operating system temperature and pressure (2250 psi and $550 \mathrm{~F}$ ) would then rapidly flow through a locked-open motor operated valve at 
the outside of the containment boundary, whereupon it enters a 10-inch diameter, ASME class 2 pipe of lower pressure capacity ( $600 \mathrm{psi}$ design for temperatures less than $200 \mathrm{~F}$ ). The low pressure piping is postulated to break, causing a loss of coolant outside containment.

The first question raised by all four of the individuals listed above was uncertainty concerning whether or not the pipe would indeed rupture. If not, the coolant would be relieved through a one-inch relief valve to a waste collection system. This would be a less severe accident and might easily allow time for arresting the accident prior to core damage. On the other hand, a severe break of the pipe would cause the primary system and the contents of the refueling water storage tank to be discharged into the safeguards building, and eventually overflow into the auxiliary building. The location of the proposed pipe break would determine the depth of submergence. The experts on this subject reviewed the piping and estimated that $80 \%$ of the piping length is within two feet of the floor, and would therefore be covered by several feet of water in the auxiliary building. The other $20 \%$ would be covered by less than one-half foot of water.

Mr. Metcalf of Stone and Webster, the architecture engineering firm for the facility, indicated that the most likely point of rupture, if it occurred at all, would be at the first elbow following the transition to low pressure piping. His rationale was that 1) this elbow would be the first to see high stress levels, and 2) this elbow is restrained by an integral attachment which would increase the stress. Since this elbow is in a position that would be submerged, he assigned a $90 \%$ probability to the break site being submerged. Mr. von Riesemann corroborated Mr. Metcalfs observations qualitatively, but thought that a $90 \%$ chance was probably high given the uncertainty in the scenario. He offered no probabilistic judgment, however.

In a follow up letter, Mr. Metcalf added that Stone and Webster had earlier performed an analysis of operating stresses for the system in question. The situation analyzed is quite different from the interfacing systems LOCA since the direction, velocity and thermodynamic conditions of flow are different. However, the results can provide limited insights. In that study, the elbow was the point of second highest stress, the point of highest stress was another constrained elbow that would also be below two to three feet of water. Mr. Metcalf therefore concluded that the likelihood of submergence would be at least $90 \%$. Most of Mr Metcalf's views on the $\mathrm{V}$ sequence are summarized in Reference 25.

Mr. Davis of Intermountain Technology performed his own assessment of the Surry V sequence, based on a plant visit, review of drawings, and discussions with Virginia Electric 
Power Company and Stone and Wester. When asked for a subjective probability for submergence, he selected a 0.8 probability.

Mr. Ritzman analyzed the Surry V sequence while at Science Applications International Corporation under a contract with the Electric Power Research Institute. The results of this study are reported in Reference 26. In discussions with him, he indicated that he and the other authors could not justify any conclusions concerning the break location. Based on piping length considerations alone, he estimated an $80 \%$ probability of submergence. A key question among the authors and reviewers of the EPRI report was what he termed the morphology of the break. If a 10 inch double-ended guillotine break were to occur at a depth of two to three feet, there would be a real question as to whether bubble breakup could occur rapidly enough to assure any significant amount of fission product scrubbing. He noted that data from General Electric Company indicated that a depth of one to two pipe diameters is needed to ensure breakup of larger bubbles to smaller bubbles. Based on this, he and the other authors thought that it was unlikely that there would be any significant, classic pool scrubbing. He felt that it was more likely that the confined nature of the safeguards building made it more likely that there would be scrubbing as a result of two phase mixing and turbulence, a situation for which there are no models. He felt, based on personal judgment, that a large amount of fission products would end up in the water as a result of this turbulence. On further questioning he indicated that "fairly probable" meant "as likely as not" and that "significant scrubbing" meant a decontamination factor of about 10 . Considering other surfaces, he thought that it would be highly likely that the overall fraction of the release remaining in the safeguards building would be high.

Returning to the original question, there appears to be an $80-90 \%$ likelihood that the location would be submerged, given that the RCS and the RWST had been discharged in the building. One possibility not yet discussed is the potential for the operations staff to isolate the RWST and prevent its entering the building through the break. Further, the depth of water might not be enough to ensure scrubbing, the ultimate question of interest. For the OCP study, the SARRP analysts suggested that the probability of submergence would be $0.5,0.75$ and 0.9 for the pessimistic, central, and optimistic, respectively.

The reviewers did not have additional discussion on this issue, other than to state that their opinion would be less informed than the others who have studied the specifics of this issue. Based on the information provided, the reviewers did provide weighting factors. One reviewer did have more detailed comments. He developed weighting factors based on an event tree of the scenario. The key events in the tree included: 
- The potential for a more slowly developing sequence that could be arrested,

- The probability that he pipe would not rupture,

- The potential for leak before break,

- The possibility of the relief valve providing sufficient protection,

- The possibility of LPI seals to rupture and protect the piping, and

- The probability that the release would be submerged.

Obviously, some of the concerns listed above could be considered part of the front-end analysis of the frequency of the sequence, but the dividing line for this issue between front-end and back-end is not distinct. This reviewer evaluated the tree, using assumptions based on his best estimate of plant response. For example, he assumed:

- That the maximum equivalent static load is equal to the primary system pressure, not twice that values as assumed in the RSS;

- That if the pipe fails by a leak, that bubble collapse would be assured;

- That a gross rupture of the pipe would involve a $50 \%$ probability of bubble collapse and scrubbing; and

- That if the relief valve or pump seals failed, the release would be scrubbed $90 \%$ of the time, although this was an assumption that could be checked by plant-specific evaluation.

Through this process, this reviewer estimated a probability of 0.976 of a submerged failure. This did not include other adjustments to the sequence frequency that this reviewer thought would be appropriate to obtain a realistic portrayal of the V sequence threat at Surry.

The input for all reviewers is listed in Table B-20. Since the reviewers supplied probabilities, each input was weighted equally and used as a separate level in the LLH sampling.

Table B-20

WEIGHTING FACTORS FOR ISSUE 8: PROBABILITY OF SUBMERGENCE OF THE V SEQUENCE FAILURE SITE

\begin{tabular}{lllllllllllll}
\hline $\begin{array}{c}\text { OUTCOME } \\
\text { CORRELATED } \\
\text { TO FAILURE SIZES }\end{array}$ & SARRP & 2 & 3 & 4 & 5 & 6 & 7 & 8 & 9 & 10 \\
\hline $\begin{array}{l}\text { Probability Rupture Occurs } \\
\text { Several Pipe Diameters Below Water }\end{array}$ & .75 & .74 & .8 & .5 & .9 & .8 & .8 & .8 & .75 & .98 \\
\hline
\end{tabular}




\section{B.10 OTHER ISSUES}

There were other issues that were discussed during this portion of the LLH process but were not included in the containment issues that were sampled for one reason or another. These issues are discussed below

\section{B.10.1 Issue 9: Effect of Containment Failure on ECCS Operability}

Some of the most important accident sequences evaluated in the front-end analysis by ASEP involve failure of the containment service water system with resultant failure of containment heat removal. These sequences are in a somewhat unique state, termed core vulnerable, that involves successful injection and recirculation into the primary, but no ultimate heat removal from containment. The containment pressure therefore rises, and in a considerable time (two days), the pressure could reach the failure pressure. There are several phenomenological issues that are important to these scenarios which were discussed at the review meeting and are outlined below. The input derived here was actually implemented as part of the interface between the front-end sequence analysis and the containment analysis. These inputs were used to adjust sequence frequencies as described in Section A.4 of Appendix A, and also served as the last front-end uncertainty issue as described in Section 4 of the main report.

Following the containment failure in the core-vulnerable sequences, the continued success of core recirculation is uncertain, since there are several effects of containment failure that could lead to failure of the recirculation pumps. The first of these is the potential for cavitation and pump failure as a result of pumping a water steam mixture that could result from a rapid depressurization--i.e., the sump boils for some period of time. The pumps have a NPSH of 20 feet, and the loss of the suction head may be temporary enough that no damage results. (The Surry pumps are run dry for a short period of time as part of a test procedure once a year, so immediate damage on loss of head is unlikely.) The size of containment failure could impact the effect on the pumps, since the depressurization rate would determine the status of the sump water. The RSS assumed that pump failure was inevitable, whereas many more recent studies have indicated the potential for the pumps to continue operating under these conditions, and for the sump boiling effect to be very local (near the surface) such that the pumps are unaffected. References 3 and 25 have concluded that pump success can be likely under some conditions, depending on the specific design.

There is another potential problem that is more structural in nature. A gross failure of containment could lead to a physical displacement of the injection or suction piping, or could 
damage the check valves. Physical damage due to structural failure of other equipment is also a possibility.

The review team was asked to provide input on this question, correlated to the previous issue, the containment failure size. The results of their weighting factors are provided in Table B-21 and 22 for the two types of phenomenon. Weighting factors on a simple binary outcome of success or failure was requested.

Table B-21

WEIGHTING FACTORS FOR ISSUE 9.1: EFFECT OF CONTAINMENT FAILURE ON ECCS PUMP OPERABILITY

\begin{tabular}{|c|c|c|c|c|c|}
\hline \multirow{3}{*}{$\begin{array}{c}\text { OUTCOME } \\
\text { CORRELATED } \\
\text { TO FAILURE SIZE }\end{array}$} & \multirow[b]{3}{*}{ SARRP } & \multicolumn{4}{|c|}{ WEIGHTING FACTORS } \\
\hline & & & & evien & \\
\hline & & 1 & 2 & 3 & 4 \\
\hline 1. 4 sq. in. & 0.1 & 0.0 & 0.0 & 0.0 & -- \\
\hline 2. $.1 \mathrm{sq} . \mathrm{ft}$. & 0.2 & 0.0 & 0.0 & 0.0 & -- \\
\hline 3. .7 sq.ft. & 0.2 & .001 & .2 & .1 & -- \\
\hline 4. 7 sq. $\mathrm{ft}$. & 0.3 & .003 & .4 & .3 & - \\
\hline
\end{tabular}

Table B-22

WEIGHTING FACTORS FOR ISSUE 9.2: EFFECT OF CONTAINMENT FAILURE ON INJECTION AND SUCTION PIPING

\begin{tabular}{|c|c|c|c|c|c|}
\hline \multirow{2}{*}{$\begin{array}{c}\text { OUTCOME } \\
\text { CORRELATED } \\
\text { TO FAILURE SIZE }\end{array}$} & \multicolumn{5}{|c|}{ WEIGHTING FACTORS } \\
\hline & SARRP & 1 & 2 & $\frac{\text { Review }}{3}$ & 4 \\
\hline 1. 4 sq. in. & 0.0 & 0.0 & 0.0 & 0.0 & 0.0 \\
\hline 2. $.1 \mathrm{sq} . \mathrm{ft}$ & 0.0 & 0.0 & 0.0 & 0.0 & 0.0 \\
\hline 3. .7 sq.ft. & 0.0 & 0.0 & 0.0 & 0.0 & 0.0 \\
\hline 4. 7 sq. ft. & 0.001 & .1 & .01 & .1 & .1 \\
\hline
\end{tabular}

The results for the pump operability indicate a consensus that the failure would have to be of one of the two largest sizes proposed in order for the issue to be of any concern. Even for the gross failure of containment, the reviewers only weighted the failure with approximately a 0.3 factor--the general opinion being that the pumps had a good chance of surviving any 
temporary conditions associated with the sump saturation. Finally, there was some discussion of the potential for extensive operator action within a two day time frame. The sequence frequency coming into this issue already accounts for the nonrecovery of the cooling systems, but it was pointed out that even after pump failure, decay heat would be low enough that considerable repair opportunity would exist. One reviewer thought that the failure would be very unlikely since he found it nearly inconceivable that the operations staff would take no actions over two days to prevent gross overpressurization failure. He therefore conservatively assigned a 0.01 probability that the sequence would result in containment failure, and allowed an approximate one in ten chance of spray failure, given that containment failed grossly.

For the second case of structural damage to the piping, there was unanimous agreement that there would be no concern except in the gross rupture case. There was a wide variance on this issue, with the SARRP value being significantly lower than most of the others. Most of the reviewers felt that a one in ten chance of failure was more likely. It was pointed out that there was no data to apply, but the expert reviewers indicated that there was insignificant uncertainty in the possible modes of gross failure and that damage to lines that passed through or were supported by the liner could undergo serious damage. Reviewer \#1 applied the same probability $(0.01)$ that the failure of containment would actually occur, and a 0.1 probability of pipe failure for catastrophic containment failure.

\section{B.10.2 Size of V Sequence Break}

The size of the pipe rupture involved in the interfacing systems LOCA was also recognized as a potential uncertainty issue. The reviewers were polled on this issue, and the general conclusion was that it remained an uncertain area, but that the review group had no information to address the issue. It was generally stated that there are additional uncertainties in the entire $V$ sequence phenomenology that should be addressed by a plant specific, detailed study if the sequence is of great importance to the risk profile.

\section{B.10.3 Containment Failure by Detonation or Accelerated Burning}

The issue of containment failure caused by accelerated burning of hydrogen or detonation, either global or local, was raised by one or two of the reviewers at the first meeting. At a later review meeting the topic was discussed again, with the conclusion that the issue should be documented in this report, but that the current state of knowledge did not allow meaningful inclusion in the LLH study. Another conclusion was that the important issue relative to uncertainty is the local detonation issue, because the issue of global detonation could really be considered as having been treated within the outcomes for large hydrogen burns 
which could fail containment. In other words, since containment failure as a result of burning of hydrogen is included in the LLH, the results will show the sensitivity to hydrogen events which lead to containment failure and the inclusion of a global detonation in cases involving similar boundary conditions would not likely change the results. The issue of local detonation is not as clearly encompassed by the existing hydrogen treatment since it could apply when the overall hydrogen concentration would not be a containment threat; and it therefore could be more important.

At a review meeting, some research relative to the issue was discussed to collect the experts' opinions as to the treatment of this issue. The results of a recent study of local hydrogen detonations at another plant were presented to the group, along with some simple extrapolations to the Surry plant. In the study of the other plant, a fairly detailed model (42 compartments) of containment was established to track hydrogen concentrations for two accident scenarios. The results of that study showed that the hydrogen concentration was always greatest in the compartment where the hydrogen was leaving the primary system, and that all of the other compartments showed no buildup or stratification.[27] Thus the conclusion for the set of scenarios studied was that local detonation concentrations could only be reached at the source of the hydrogen. The peak concentrations seen in that study were about $15 \%$, and the hydrogen sources were based on core-damage accident scenarios that maximized hydrogen generation at about $75 \%$ metal-water reaction equivalent. In addition, the peak concentrations were very short in duration. Results of some simple calcualtions for Surry (15 compartments) were also shown to have similar conclusions to the more detailed study. Also presented were some results of detonation calculations for the local compartment with high concentration; these results pointed to minimal impact on containment. It was, however, pointed out that the determination of effects of any detonation is quite complex and would require more than a simple review.

A number of potential effects of local detonation were thought to be potentially important. One possibility is that of missile generation, which could possibly lead to penetration of containment or effects on other equipment. Another potential would be for stuctural failure; this would be highly dependent on geometry. Finally, there is the potential for equipment damage or structural failure which could lead to a failure of containment isolation, perhaps by pulling pipes away from penetrations, or due to missiles. There would be a great deal of uncertainty in most of these effects, and the threat could be very scenariodependent. 
Another uncertainty issue is that of trigger mechanisms for the local concentrations. These triggers would have to be present at the time period of detonable concentration, which is not that long, and would have to have the proper spatial relationship to the concentration of hydrogen. Thus the ignition source is another primary uncertainty.

There was a consensus that the uncertainties posed by this issue could not be addressed in the LLH without more calculations on which to base: 1) the probability of a detonable concentration occurring, 2) the probability of a detonation, and 3) the magnitude of the impulses and the potential for damage. It was urged that a set of calculations be performed, at least to a level of detail to assess the relative threat. It was also pointed out that the issue could not have an overwhelming effect on the current Surry result due to the importance of direct heating. This does not mean that the issue can be ignored, since further resolution of other uncertainties could cause them to become less important and local detonation could then be more important.

There was no consensus on the importance of the omission of this issue. Several reviewers stated their opinion that the issue would never be of great risk importance, while others thought that no such judgment could be made without further research. One reviewer wanted to emphasize that the conclusions of the study concerning hydrogen would be incomplete without treatment of this issue. 


\section{References for Appendix B}

1. Lyons, W. G, "Presentation at NRC/IDCOR Meeting on Containment Loads and Fission Product Behavior." U.S. Nuclear Regulatory Commission: May, 1984.

2. Estimates of Early Containment Loads from Core Melt Accidents, Report of the Containment Loads Working Group. U.S. Nuclear Regulatory Commission Report NUREG-1079 (Draft for Comment), Washington, DC: December, 1985.

3. IDCOR Task 23.1: Integrated Containment Analysis. Technology for Energy Corporation, Knoxville, TN: 1984.

4. Commonwealth Edison Company. Zion Probabilistic Safery Study Chicago, Illinois: 1980.

5. Power Authority of the State of New York and Consolidated Edison Company of New York, Inc. Indian Point Probabilistic Safety Study. New York, New york: 1980.

6. Reactor Safety Study--An Assessment of Accident Risks in U.S. Commercial Nuclear Power Plants. U.S. Nuclear Regulatory Commission Report WASH-1400 (NUREG75/014), Washington, DC: 1975.

7. Murfin, W. B., et al. Report of the Zion/Indian Point Study. U.S. Nuclear Regulatory Commission Report NUREG/CR-1410, Sandia National Laboratories, Albuquerque, NM: 1980.

8. Rivard, J. B., et al. Identification of Severe Accident Uncertainties. U.S. Nuclear Regulatory Commission Report NUREG/CR-3440, Sandia National Laboratories, Albuquerque, NM: 1984.

9. Berman, M., et al. An Uncertainty Study of PWR Steam Explosions. U.S. Nuclear Regulatory Commission Report NUREG/CR-3369, Sandia National Laboratories, Albuquerque, NM: 1984.

10. Steam Explosion Review Group. A Review of Current Understanding of the Potential for Containment Failure Arising from In-Vessel Steam Explosions. U.S. Nuclear Regulatory Commission Report NUREG-1116, Washington, DC: 1985.

11. Estimates of Early Containment Loads from Core Melt Accidents, Report of the Containment Loads Working Group. U.S. Nuclear Regulatory Commission Report NUREG-1079 (Draft for Comment), Washington, DC: December, 1985.

12. Containment Loads Working Group. "Consensus Summaries for Standard Problems 1 through 6." Letter Reports to J. Telford, U.S. Nuclear Regulatory Commission, MayJune, 1984.

13. Tarbell, W., et al. High-Pressure Melt Streaming (HIPS) Program Plan. U.S. Nuclear Regulatory Commisssion Report NUREG/CR-3025. Sandia National Laboratories, Albuquerque, NM: August, 1984.

14. Bergeron, K. D. , and D. C. Williams. "Calculations for PWR Standard Problem 1 and 2 with Direct Heating", Submittal to the Containment Loads Working Group, Sandia National Laboratories, Albuquerque, NM: April 1984.

15. Haskin, F. E. , et al. "Combustion-Induced Loads in Large Dry PWR Containments", Proceedings of the Second Containment Integrity Workshop: 1984.

16. Gieseke, J. A., et al. Radionuclide Release Under Specific Accident Conditions. Battelle Columbus Laboratories Report BMI-2104, Columbus, OH: 1984. 
17. Benjamin, A. S. , et al. "SARRP--Risk Rebaselining and Risk Reduction Analysis", Proceedings of the 11 th Water Reactor Safety Research Information Meeting. U. S. Nuclear Regulatory Commission, NUREG/CR-0048, Volume 3: 1984.

18. Camp, A. L., et al. Light Water Reactor Hydrogen Manual. U.S. Nuclear Regulatory Commission Report NUREG/CR-2726, Sandia National Laboratories, Albuquerque, NM: August, 1983.

19. Haskin, F. E. , et al. Analysis of Hypothetical Severe Core Damage Accidents for the Zion Pressurized Water Reactor, NUREG/CR-1410, Sandia National Laboratories, Albuquerque, MN: 1982.

20. Drozd, A., et al. "Parametric Study of Aerosol Behavior Following AB and TMLB Accidents," Proceedings of the American Nuclear Society 1984 Annual Meeting . TP8454, Stone and Webster Engineering Corp.: 1984.

21. Sizewell B Probabilistic Safety Study. Westinghouse Electric Co. Report WCAP-9991, Monroeville, PA: 1982.

22. Seabrook Plant Probabilistic Safety Study. Yankee Atomic Electric Co., Seabrook, NH: December, 1983.

23. GESSAR-II, BWR/6 Standard Plant Probabilistic Risk Assessment. General Electric Co., San Jose, CA: 1982.

24. Serkiz, A. W. Containment Emergency sump Performance, Technical Findings Related to Unresolved Safety Issue A-43. U.S. Nuclear Regulatory Commission Report NUREG-0897, Washington, DC: 1983.

25. Drozd, A. et al. " The V Sequence: An Engineering Viewpoint," ANS Topical Meeting on Fission Product Behavior and Source Term Research, Snowbird, Utah: July 15-19 1984.

26. Ritzman, R. L., et al. Surry Source term and Consequence Analysis, Electric Power Research Institute Report EPRI NP-4096, Palo Alto, CA: June 1985.

27. Peterson, A. C., "Gas Transport in Large, Dry Containments." Presentation to SARRP Expert Review Group, Sandia National Laboratories, Albuquerque, NM: September 2, 1986. 
NUREG/CR-4551, VOL. 1: DRAFT REPORT FOR COMMENT (SEPTEMBER, 1986)

\author{
Appendix C \\ SUPPORTING ANALYSIS FOR THE SURRY \\ SOURCE TERM UNCERTAINTY ASSESSMENT
}


-

-

-

-

- 


\section{CONTENTS}

Section Page

C.1 OCP REPRESENTATION OF SOURCE TERM UNCERTAINTIES............ C-1

C.1.1 Nature of the "Optimistic" and "Pessimistic" Estimates..................... C-1

C.1.2 Methodology and General Phenomenology ............................. 4

C.1.2.1 Combining Uncertainties....................................... -6

C.1.2.2 Consistency Requirements.................................. -8

C.1.2.3 Application of Salient QUEST Results......................... -9

C.1.2.4 Application of QUEST Results to Other Sequences..............C-22

C.1.2.5 Pessimistic "Worst Case" Source Terms.........................C-24

C.1.2.6 Other Phenomenological Uncertainty Factors................C-26

C.1.3 Pessimistic and Optimistic Source Terms for Surry ......................

C.2 SURRY SOURCE TERM ISSUE OUTCOMES AND WEIGHTING
FACTORS FOR THE LIMITED LATIN HYPERCUBE STUDY............... 46

C.2.1 Introduction............................................................. 46

C.2.2 Approạch....................................................... 47

C.2.3 Issues, Levels, and Weighting Factors............................... 49

C.2.4 Review Comments of Broader Applicability ......................... C-73

C.2.5 Implementation of Surry Source Term Calculations for the LLH.........C-75

C.3 SOURCE TERM CLUSTERING .....................................

C.3.1 Introduction...................................................... 93

C.3.2 Clustering Procedure.................................................

C.3.3 Surry Clustering Results.............................................

C.3.4 Limitations of Clustering Process....................................... -99

References for Appendix C ................................................... 107 
NUREG/CR-4551, VOL. 1: DRAFT REPORT FOR COMMENT (SEPTEMBER, 1986)

\section{LIST OF TABLES}

Table

C-1 Radionuclide Release Fractions for the Surry TMLB' Sequence With Early Containment Failure.

C-2 QUEST FCOR Values for "High" and "Low" Cases..........................

C-3 QUEST FCOR Values--WASH-1400 Groups............................... 14

C-4 "FVES" For RCS Aerosols in BMI-2104.....................................

C-5 FCCV(1-FCOR) Values From QUEST and BMI-2104....................... 21

C-6 Pessimistic Source Term Estimates........................................... 25

C-7 Surry Pessimistic Source Terms............................................ -43

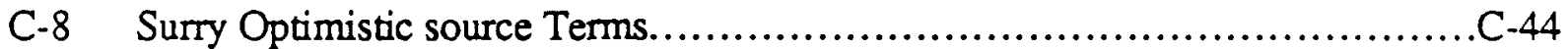

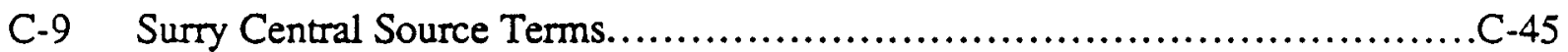

C-10 Summary of Source Term Bin Assignments For Surry........................ 51

C-11 Levels of FCOR for LLH Uncertainty Analysis............................ -52

C-12 Issue 1: In-Vessel Release From Fuel (FCOR)............................ 53

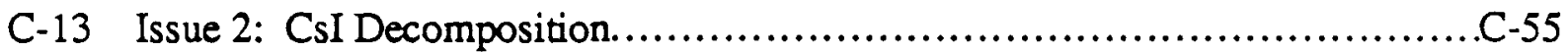

C-14 Surry Calculations for FVES: High Pressure Scenarios...................... C-56

C-15 Levels of FVES for LLH Uncertainty Analysis: High Pressure Core
Melt Sequences............................................................. 56

C-16 Levels of FVES for LLH Uncertainty Analysis: Intermediate Pressure Core Melt Sequences............................................................

C-17 Levels of FVES for LLH Uncertainty Analysis: Low Pressure Core Melt Sequences......................................................... 57

C-18 Levels of FVES for LLH Uncertainty Analysis: V Sequence.................... 58

C-19 Issue 3: In-Vessel Retention............................................. 59

C-20 Issue 4: Scrubbing Decontamination Factor for the $V$ Sequence With Overlying Water................................................................

C-21 Levels of Core Concrete Interaction Release Fraction For Each Fission Product Group ......................................................... 61

C-22 Issue 6: Core-Concrete Interaction Releases..............................6-61

C-23 Levels of Core-Concrete Interaction Release Fraction for Low Pressure Scenarios ....

C-24 Release of CCI Species From Containment for Late Containment Failure

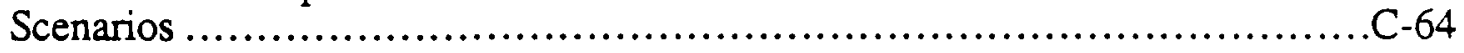

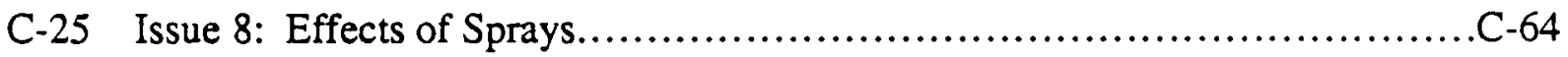

C-26 Aerosol Agglomeration Uncertainties.................................... 67

C-27 Issue 10: Late Releases of Iodine from the Containment.......................6-68 


\section{LIST OF TABLES (Continued)}

Table

C-28 Issue 11: Late Releases of Cesium and Iodine From the RCS.

C-29 Levels for the High Pressure Ejection Incremental Release. .C-71

C-30 Weighting Factors for the High Pressure Ejection Release. .C-72

C-31 Levels for the Direct Heating Incremental Release. C-72

C-32 Weighting Factors for the Direct Heating Release.. .C-72

C-33 Expression of Issues for Surry Source Term Bins. C-76

C-34 Listing of the SURSOR Code. C- -80

C-35 Early and Late Effect Weights of Source Term Groups. .C-98

C-36 Early and Late Effect Weights of Source Term Clusters. .C-104

C-37 Release Fractions for Surry Clusters. .C-105 


\section{LIST OF FIGURES}

Eigure

C-1 Airborne Radionuclides Calculated For Different Spray Drop Sizes and

$$
\text { Compared to Results for No Sprays }
$$

C-2 Sampled Surry Source Term Estimates From SURSOR for Group 2.............. 94

C-3 Sampled Surry Source Term Estimates From SURSOR for Group 3.............. 94

C-4 Sampled Surry Source Term Estimates From SURSOR for Group 4............. 95

C-5 Sampled Surry Source Term Estimates From SURSOR for Group 5............C-95

C-6 Sampled Surry Source Term Estimates From SURSOR for Group 6............C-96

C-7 Sampled Surry Source Term Estimates From SURSOR for Group 7..............-96

C-8 Spread in Early Effect Weight of Early Release By Cluster (Linear Scale)....... 101

C-9 Spread in Early Effect Weight of Early Release By Cluster (Log Scale)........ C-101

C-10 Spread in Early Effect Weight of Late Release By Cluster (Linear Scale)........ - 102

C-11 Spread in Early Effect Weight of Late Release By Cluster (Log Scale)..........C-102

C-12 Spread in Late Effect Weight By Cluster (Linear Scale)....................... 103

C-13 Spread in Late Effect Weight By Cluster (Log Scale)........................... 103

C-14 Comparison of CRAC2 OCP Early Fatality Results With and Without Clustering

C-15 Comparison of CRAC2 OCP Latent Fatality Results With and Without Clustering 


\title{
Appendix C \\ SUPPORTING ANALYSIS FOR THE SURRY SOURCE TERM UNCERTAINTY ASSESSMENT
}

\author{
David C. Williams \\ Sandia National Laboratories
}

The central estimates of source terms were based as closely as possible upon Source Term Code Package (STCP) runs. In some cases, it was necessary to infer source terms for scenarios not calculated in detail from those scenarios which had been analyzed. For the most part, applicable STCP calculations did exist for the dominant Surry scenarios, and the means by which they were used to derive source term bins for consequence analysis has been discussed in the main report. The STCP calculations themselves are described in the appropriate Battelle reports $[1,2,3]$. This information will not be repeated here.

This appendix is limited to discussion of the uncertainty analysis. Two methods will be described: the "optimistic, central, pessimistic" (OCP) approach and the limited Latin hypercube (LLH) approach. The OCP approach was completed before the LLH was initiated, and it will be described first, in Section C.1. The OCP analysis has not been revised or updated to reflect results of the LLH; hence, comparisons between the two results provides a representation of the degree to which these approaches might lead to different (or similar) conclusions. In Section C.2, the LLH approach is presented. It is not the principal purpose of this appendix to recapitulate results of the uncertainty analysis; most of these results have been presented in the main report. Here, the emphasis is on the methodology. Finally, the method used to calculate the source terms for the many different outcomes presented by the LLH analysis (clustering) is described in Section C.3

\section{C.1 OCP REPRESENTATION OF SOURCE TERM UNCERTAINTIES \\ C.1.1 Nature of the "Optimistic" and "Pessimistic" Estimates}

As in other aspects of the SARRP OCP uncertainty analysis, the source term analysis was intended to reflect source terms that might result from using assumptions and data considered, within the total spectrum of the reactor safety community, to be optimistic, central, or pessimistic. However, the capability of mechanistic source term analysis represented by codes such as those in the STCP is a new one, and time has not permitted their detailed application to explore the consequences of assumptions deemed "optimistic" or "pessimistic" in most instances. Hence, a considerable amount of subjective judgment was required, both in 
defining the optimistic and pessimistic assumptions and in estimating their likely consequences. It should also be acknowledged that the immediate implementation of this work was largely the task of a single individual, although the data base drawn upon, of course, reflects the work of many analysts. Indeed, one motivation for the LLH study was to reduce the dependence upon the judgment of one or a few individuals.

It should be acknowledged at the outset that it is necessary to accept considerably less detailed treatments of the optimistic and pessimistic source terms than in the case of the central values. For one thing, available relevant information is considerably less. More fundamentally, the concepts themselves are imprecise. Suppose, for example, that we somehow eventually obtain precise determinations as to the exact values of the source terms. It would then be meaningful to discuss the differences with respect to our current central estimates as representing the errors in the latter, but there would still be no objective way of determining whether the pessimistic and optimistic source terms had been "right" or "wrong". These estimates are not intended to be close approximations of actual source terms; they are only representions of the range of estimates within which we believe, based upon current understanding, the actual source term is likely (but not certain) to lie. The concepts defining these estimates are inherently subjective and fuzzy, with a fully objective definition of "correctness" not being possible even in principle.

Of course, one could say that the pessimistic (optimistic) source term is "right" if it is larger (smaller) than what the true source term actually is, and otherwise is "wrong". If that is our criterion, the task is simple: we set the release fractions equal to one and zero, respectively, and the job is done. This assures us that our results are "correct", though of little utility.

At this point, we are left with an attempt to derive source terms which are claimed to be pessimistic and optimistic, respectively, but which are also acknowledged to be other than truly bounding. The question inevitably arises, "How pessimistic (optimistic) are they?" The obvious response, and one commonly made, is to punt and not answer the question. Yet the question is a perfectly reasonable one, and an attempt will be made to address it here in terms of a subjective probability measure that will be referred to as "neutral betting odds".

The present discussion starts from the premise that any probabilities or confidence limits which might be associated with the optimistic and pessimistic source terms must inevitably be highly subjective. One reason is that there exists no model for which the input is existing knowledge (or some subset thereof) concerning source-term phenemenology and the output is the probability that a given set of release fractions will or will not be exceeded in a 
specified scenario. There are, of course, models that will link postulated input assumptions, including modeling assumptions, to calculated release fractions as output; however, to generate probability distributions for the output, probability measures for the phenomenological inputs would be needed. Any probabilities assigned to these inputs would be highly subjective, degree-of-believe representations, and output probability distributions resulting would be no more objective. Note the contrast between this situation and that existing in probabilistic treatments of system reliabilities in accident front-end analysis. In the latter case, input data include actual frequency data for initiating events, subsystem responses, etc, and fault tree/event tree models are used to generate corresponding probabilities for the various outputs. These probabilities are objective in the sense that they can be expressed in terms of a prediction of frequencies which could, in principle, be tested experimentally. Note also the contrast with quantum mechanics, in which the model itself explicitly predicts probabilities for output quantities (i.e., the experimental observables) from the purely deterministic input data, predictions which are again capable of being tested experimentally.

It should be emphasized that the term "subjective probabilities", as used here, is not intended to denote subjective estimates of probabilities that are objectively definable, e.g., in terms of frequencies of occurrence. An example of the latter might be a subjective "expert opinion" estimate of a pump failure frequency under conditions for which no data exist. Instead, the term is used here to refer to numerical representations of degrees of belief in specified hypotheses, e.g., that the release fractions will or will not exceed certain specified values under certain specified conditions. No frequency is even under consideration in the hypothesis about which a degree of belief is being expressed.

One way to discuss such probabilities is to view them as "neutral betting odds" (NBOs). These may be defined as constituting the answer to the question: given that the analyst is required to make a small wager as to whether the release fractions in a specified case will exceed a given value, at what betting odds would the analyst be indifferent as to which side of the bet he is taking? It is this approach that will be adopted here. The NBOs, thus defined, are not really about source terms; they are about the analyst's state of mind. The NBOs are "correct" so long as they are a reasonably accurate reflection of his state of mind, no matter how badly in error the source term estimates may turn out to be. To put it another way, an appropriate experimental test for determining whether NBOs are correct would be to give the analyst a polygraph test, not perform a series of fission product transport tests.

One very serious potential misinterpretation of the pessimistic, optimistic, and central source terms, and of the NBOs associated with the pessimistic and optimistic values, must be 
strongly warned against: in no sense do these correspond to a prediction as to the frequency distribution of source terms that would be obtained if one actually performed a large number of experimental tests designed to conform to a particular sequence of events for which the source term is estimated. Quite the contrary: it is likely that any such series of actual results would give a distribution considerably narrower than the range implied by the pessimistic and optimistic values taken together with their NBOs. The problem is that we do not know where in this wider range the narrower distribution would actually fall.

Given the above qualifications and explanations, the principal author of the present work offered the following NBOs for Surry risk profiles calculated with the source terms developed for this work. Assuming, in all cases, the probabilities assigned to the source term bins to be exact, NBOs in the range of 10:1 to 20:1 were offered that the actual risk profile will not exceed the profile calculated using the pessimistic source terms. NBOs in the range 5:1 to 10:1 were offered that the actual risk profile would not lie below the profile calculated using the optimistic source terms. NBOs that might be offered for individual source term bins, or for the release fractions of individual radionuclide species, could be quite different, but the NBOs for risk-dominant radionuclide species in the risk-dominant source terms obviously could not be very different.

The fact that the NBOs offered for the optimistic case are somewhat lower than for the pessimistic case reflected a belief that there is a small but persistent conservative bias in the source term analysis overall, even though a number of nonconservatisms can be identified in specific instances. Based upon the LLH results, it is now believed that the impact of these nonconservatisms may have been underestimated in developing the optimistic source terms, especially in the case of iodine releases. The phenomenologies considered in the LLH provide a number of parallel paths to increased iodine release, any one of which can easily exceed the present optimistic estimate unless quite optimistic assumptions are made concerning them.

To proceed further with this discussion is to risk getting involved in such subjects as "the uncertainty in the uncertainty". Experience has shown discussion of such subjects to be a leading cause of mental malfunction within the PRA community, and it shall therefore be avoided here.

\section{C.1.2 Methodology and General Phenomenology}

In the approach utilized here, the goal was to give some measure of the uncertainty in the source terms estimated in the central analyses. In a broad sense, the optimistic and pessimistic estimates remain within the framework of the BMI-2104 methodology, in that they are based upon estimates of what the methodology might yield given more optimistic or 
pessimistic sets of input assumptions and/or modeling approximations, subject to the constraints that these input and modeling variations be mutually consistent and within reasonably defensible uncertainty ranges. It must be emphasized that only a very limited subset of the innumerable uncertainties afflicting source term calculations have been examined quantitatively for their impact upon the final results. For this and other reasons, the optimistic and pessimistic source terms developed here can not be considered "bounding".

Limitations of time and resources available for this work here required that these estimates depend primarily upon previous analyses of uncertainties in the BMI-2104 methodology, and this in turn forced a heavy reliance upon the QUEST program, which is the most extensive assessment of these uncertainties presently available [4]. Other source term studies (WASH-1400, RSSMAP, NUREG-0772, DCOR) are referenced where their results are readily usable. However, time has not permitted any detailed evaluation of the implications of other source term studies for present purposes. One complicating factor is that other analyses typically differ significantly in the detailed assumptions concerning the progression of nominally similar accident sequences. Thus, the IDCOR analyses of a given sequence/pathway might differ substantially in its assumptions conceming system and operator response from what SARRP assumed, and it could prove difficult to estimate the source term that the IDCOR methodology would give were it to be applied to the SARRP scenario. (It is important to recognize that this appendix addresses only uncertainty in the source terms that arises due to uncertainty in the relevant phenomenology, given a well-defined sequence of events; in the SARRP methodology, uncertainty associated with uncertainty in the sequence of events is properly addressed through the containment event tree (CET) formalism.)

The estimation of optimistic and pessimistic source terms was carried out before calculations using the STCP itself were available, and thus the discussion that follows is based upon calculations reported in BMI-2104 as well as calculations with the revised versions of the BMI-2104 codes which were the immediate precursors of the STCP runs described in BMI2104. Only a few instances are known for which incorporating STCP results might have had significant impact upon the optimistic and pessimistic source terms; these instances are briefly noted in the relevant discussion.

The approach adopted in this work frequently involves making high and low estimates of the various quantities appearing in a simplified parametric representation of the source term, and combining these estimates, subject to consistency constraints, to obtain pessimistic and optimistic source terms. The source terms themselves were obtained either by applying the 
parametric representation directly or by applying correction factors to the central source terms. In its simplest form, this parametric representation may be written:

$$
F t(i)=F C O R(i) * F V E S(i) * F C O N V(i)+F C C I(i) * F C O N C(i) . \quad(C .1-1)
$$

The following notation and terminology are used:

$\mathrm{Ft}(\mathrm{i})=$ fraction of the initial core inventory of species $i$ that is ultimately released to the environment.

FCOR(i) = fraction of the initial core inventory of species $i$ released from the fuel prior to vessel failure.

FVES(i) = fraction of material released from the fuel which is released from the vessel.

FCONV(i) = fraction of material released from the vessel that is released from containment.

$\mathrm{FCCI}(\mathrm{i})=$ fraction of the initial inventory that is released from the melt during core-concrete interactions (CCI).

FCONC $(i)=$ fraction of the core-concrete interaction release that is released from containment.

Eq. C.1-1 should be thought of more as a conceptual framework for discussion than as an actual algorithm for calculation. Many complicating factors (sprays, aerosol transport phenomena, scrubbing in pools, etc.) must be allowed for in performing actual source term estimates. For present purposes, these may be conceptually represented through their effects upon the radionuclide transmission factors (i.e., FVES, FCONV, and FCONC) which appear in Eq. C. $1-1$.

\section{C.1.2.1 Combining Uncertainties}

Most of the uncertainties considered here are phenomenological in nature. There exists neither a data base nor an objective methodology for quantifying probability measures (frequencies, confidence limits, etc) associated with estimated ranges in parameter values representing such uncertainties. One consequence of this fact is that combining such uncertainties, to obtain an overall uncertainty in the final results, can pose important problems when many uncertainties affect the final result. One approach is to treat the estimated uncertainty ranges for the individual phenomenologies as bounds, and then combine these uncertainties in the ways that produce the most extreme results (allowing for consistency requirements); the range spanned by the final result is then viewed as bounding. Roughly speaking, the QUEST study followed this approach, with some important qualifications (e.g., no claim was made that the uncertainty ranges considered were truly bounding in any rigorous sense). 
The bounding approach just described is often criticized on the grounds that the final uncertainty range estimated reflects the compounding of many extremes in a way which is judged noncredible (i.e., of extremely low probability) even though it is acknowledged that no one of the inputs is excessively extreme and that the combination of the extremes is not logically inconsistent. For example, the QUEST maximum source term associated with early containment failure (CF) involved postulating the occurrence of maximum values of FCOR for all species, maximum values of FVES for all species, and a maximum value of FCONV, in Eq. C.1-1. This combination was assumed even though obtaining the maximum values of FCOR and FVES themselves required combining several limiting-case assumptions. It has been argued that the occurrence of all these "worst" values together is much less credible than would be judged from the plausibility of each of them considered individually.

To a greater degree than in QUEST, the above criticism has been acknowledged here. More specifically, the following "ground rules" were adopted as a guide to combining extreme results:

1) Two or even three extreme results were combined when they are phenomenologically independent, or when phenomenologically correlated in a positive sense; however, they were not combined if they are phenomenologically correlated in a negative sense, even though they may not be rigorously mutually inconsistent. In this context, two phenomenologies are considered independent if resolution of the uncertainties affecting one would do little to reduce the uncertainty band associated with the second. A positive correlation means that the demonstration that one of the phenomenologies does tend toward its extreme value would support the belief that the other tends toward its corresponding extreme, and conversely for a negative correlation.

2) In some cases, the results that might be combined are themselves composite, i.e., each requires several independent "worst (best) case" assumptions in itself. Two such results were generally not combined, unless it was judged that there would be a fairly significant positive phenomenological correlation between them.

Obviously, it is difficult to avoid considerable subjectivity in applying these guidelines, and it could be argued that it would be more objective to always combine uncertainties in such a way as to maximize the final uncertainty range. However, there is an unavoidable element of subjectivity in selecting which phenomenological uncertainties are to be included in the analysis of source term uncertainty, and there is usually considerable subjectivity in the estimates of the uncertainty range assigned for the parameters describing those phenomenologies which are included. A rigid insistence that all extremes be combined so as to maximize the width of the final uncertainty band probably does more to reduce the credibility and usefulness of the final result than it does to reduce its subjectivity. 


\section{C.1.2.2 Consistency Requirements}

In QUEST, it was found that sets of assumptions tending to maximize (minimize) the source term for early CF scenarios are, in some cases, more or less the inverse of those which maximize (minimize) releases given CF several hours or more after vessel breach (VB). This is not surprising, since early-CF scenarios are driven largely by release from the RCS while late-CF scenarios are driven largely by $\mathrm{CCI}$ releases, and clearly radionuclides postulated to be released from the fuel at or before VB are not available for late-time release during CCI.

A somewhat more subtle, but quite important, reason for the inverse relation concerns zirconium oxidation. For several reasons, scenarios involving maximum in-vessel release also tend to involve a high degree of in-vessel zirconium oxidation, while release of refractory species during $\mathrm{CCI}$ is favored by large amounts of metallic zirconium remaining unoxidized in the melt.

The requirements of internal consistency are reasonably clear for events which occur in series, i.e., along a single pathway through the event tree. It is less clear what consistency requirements should be imposed upon estimating the different source terms associated with parallel paths through the trees; e.g., consistency between early- and late-CF scenarios. Since so much of the uncertainty is phenomenological, and since the same laws of physics and chemistry must apply to all scenarios, it could be argued that all scenarios should be analyzed under the same set of assumptions concerning phenomenological uncertainty. Given this interpretation, for example, if pessimistic source terms were evaluated using assumptions maximizing early-CF releases, the same assumptions should be used for the late-CF cases, even though the resulting late-CF pessimistic source terms could easily be smaller than the central late-CF source terms.

For present purposes, it was judged inappropriate to impose the above strict interpretation of consistency: many difficulties could arise in constructing an over-all pessimistic or optimistic risk profile for a plant, and the nature of the profile and its sensitivities could depend upon whether the early-CF or late-CF source terms were chosen to be maximized (minimized), with there being no real basis for making this choice. Hence, both early-CF and late-CF source terms were calculated under the (different) sets of assumptions which tend to maximize (minimize) them individually. As a partial justification for this approach, it could be argued that there actually will be much variability from case to case among the possible accidents grouped into a single sequence in PRAs, and that it may happen that parameters favoring early CF do favor high (or low) radionuclide release at that time, while the different accident parameters favoring late $\mathrm{CF}$ also may favor high (low) release at late times. 
NUREG/CR-4551, VOL. 1: DRAFT REPORT FOR COMMENT (SEPTEMBER, 1986)

Time and resource limitations have precluded developing separate sets of source terms for each of the three containment event tree walk-throughs. Hence, with very few exceptions, the optimistic and pessimistic source terms assigned to a given pathway are independent of whether the pathway arises in the optimistic, central, or pessimistic CET walk-through. In some cases, certain inconsistencies do result. For example, the amount of zirconium oxidized in-vessel was typically specified for each CET walk-through and depended strongly upon the walk-through; in some cases, the values specified in SARRP differ greatly from the values associated with the QUEST analyses, in which the extent of $\mathrm{Zr}$ oxidation was a dependent variable. QUEST made no effort to estimate maximum/minimum source terms subject to the constraint that the extent of $\mathrm{Zr}$ oxidation be held fixed at some specified value, and attempting to do so would seriously complicate the present analyses.

More fundamentally, seeking this type of consistency is probably inappropriate. The phenomenology underlying the dependence of radionuclide release upon such parameters as zirconium oxidation is quite uncertain with only a very limited degree of experimental validation. It is probably more realistic to treat the uncertainty associated with the calculated dependence of the radionuclide release upon zirconium oxidation as representative of the kinds of uncertainties which will arise in any scenario, due to the complexities of fission product physical and chemical behavior. Insisting that the extent of zirconium oxidation be held fixed at the (somewhat arbitrary) values assumed in the various CETs would be misleading in its implication that the zirconium-related uncertainties are associated only with the amount of zirconium that would be present. In reality, the uncertainty associated with the physical and chemical effects of a given amount of zirconium, even assuming this amount were precisely known, are at least as important, to say nothing of many other chemical uncertainties which are not represented in existing modeling at all.

Some evidence is given below that the use of a single set of OCP source terms for a given scenario, independently of which CET walk-through leads to the scenario, does not lead to substantial error.

\section{C.1.2.3 Application of Salient QUEST Results}

The examination of uncertainties in the Surry TMLB' source term was by far the most thorough of the QUEST studies.[4] In addition, specific aspects of the Surry S2D [5] and Grand Gulf TC [6] sequences were treated. QUEST concentrated upon estimating what were called "high" and "low" source terms for each of two scenarios, one (called early) involving CF at VB and one (called late) involving CF many hours after VB (up to 15 hours after accident initiation). Thus four scenarios were treated in detail; these were dubbed the "early- 
NUREG/CR-4551, VOL. 1: DRAFT REPORT FOR COMMENT (SEPTEMBER, 1986)

high", "early-low", "late-high", and "late-low" cases. In addition, a base case which was quite similar to the BMI-2104 treatment of the Surry TMLB' sequence was analyzed. Many QUEST results were presented in terms of airborne radionuclides as a function of time within an intact containment, but for the five specific cases just noted, actual radionuclide releases were calculated for assumed containment failure modes. For the "low" cases, the containment failure mode was essentially the same as for SARRP level 2 leakage failure (pressure rise arrested but containment does not depressurize); for the "high" cases, large rupture failures were assumed (10 sq. m. or more failure area).

Obviously, the most severe risk profile results from combining pessimistic assumptions leading to early containment failure with pessimistic source term estimates, and it is therefore worth focusing considerable attention on the derivation of the source terms associated with early $\mathrm{CF}$, especially the pessimistic estimates. It is instructive to consider the range of environmental release fraction values obtained in QUEST for the Surry TMLB' sequence with early containment failure. These results are summarized in Table $\mathrm{C}-1$, along with the results of the two Battelle analyses of this sequence, i.e., the original BMI-2104 calculation and the more recent calculation utilized as the central source term estimate. (NOTE: The Battelle calculations cited in Table C-1 were substantially affected by the problem of excessive effects calculated for steam condensation on aerosols discussed below at the close of the present subsection; corrections have not been applied for these effects in Table $\mathrm{C}$-1.)

In Table C-1, Qe and Q1, respectively, are the summed MACCS early-effects and latent-cancer importance factors for all species. These are essentially the same importance factors as are used in defining the source term clusters for the LLH study discussed in Section C.3 of this appendix. Qe and Ql are listed in iodine-equivalents and cesium-equivalents, respectively. The row labeled "Qe-NG" gives Qe with the noble gas contribution, 0.0775, subtracted out (100\% noble gas release assumed in all cases). For small source terms, this may be a more meaningful measure than Qe because there are generally zero early health effects unless special environmental concentration factors apply (e.g., groundshine concentration due to rainout), and noble gases are less subject to these effects.

It is instructive to compare the difference between the two Battelle calculations with the difference between the two QUEST results. The Battelle calculations labeled "Central" were run with parameters chosen to maximize the pressure at or shortly after VB; among other things, these parameters tended to maximize the extent of in-vessel zirconium burnup, as in the SARRP pessimistic CET scenario. In the BMI-2104 calculation, there was no attempt to tune MARCH parameters to maximize containment loads; among other things, this lead to in-vessel 
Table C-1

RADIONUCLIDE RELEASE FRACTIONS FOR THE SURRY TMLB' SEQUENCE WITH EARLY CONTAINMENT FAILURE

\begin{tabular}{ccccc}
\hline $\begin{array}{c}\text { WASH-1400 } \\
\text { Group }\end{array}$ & Low & QUEST & \multicolumn{2}{c}{ Battelle Analyses } \\
& High & BMI-2104 & Central \\
\hline 2 & 0.006 & 0.8 & 0.046 & 0.075 \\
3 & 0.004 & 0.8 & 0.039 & 0.058 \\
4 & 0.002 & 0.8 & 0.10 & 0.055 \\
5 & $3 \mathrm{E}-6$ & 0.8 & 0.055 & 0.010 \\
6 & $3 \mathrm{E}-7$ & 0.2 & $5.0 \mathrm{E}-3$ & $1.3 \mathrm{E}-3$ \\
7 & $9 \mathrm{E}-9$ & 0.06 & $1.9 \mathrm{E}-4$ & $1.7 \mathrm{E}-4$ \\
& & & & \\
Qe & 0.086 & 4.1 & 0.29 & 0.22 \\
Qe-NG & 0.008 & 4.0 & 0.22 & 0.14 \\
Q1 & 0.0059 & 2.4 & 0.11 & 0.084 \\
\hline
\end{tabular}

zirconium burnup of only about $40 \%$, intermediate between the SARRP optimistic and central CET values for zirconium burnup. Resulting differences in the source term include less release of $\mathrm{CsI}$ and $\mathrm{CsOH}$ from the vessel in BMI-2104 but more release of Te and refractories during CCI. These differences are reasonably representative of the kinds of differences expected when the containment event tree phenomenologies are varied to obtain the OCP CET walkthroughs, but the phenomenologies unique to source term analysis are not varied. On the other hand, the difference between the QUEST results illustrate the range that arises when the uncertainties in the source term phenomenologies themselves are investigated.

The results summarized in Table $\mathrm{C}-1$ suggest that the source term implications associated with the CET phenomenologies are not trivial, but they are dwarfed by the implications of the uncertainties in the source term phenomenologies. Hence, it seems that, in general, the development of a single set of OCP source terms for the various sequence/pathway combinations (independently of whether the pathway arises in an optimistic, central, or pessimistic walk-through of the CET) seems to be reasonably justifiable. Where the differences among the CET walk-throughs does have implications for the source term which can be taken into account, the usual choice has been to associate optimistic CET phenomenologies with optimistic source term evaluation, and adopt the pessimistic CET phenomenologies for the pessimistic source term evaluation. 
It is also apparent from Table C-1 that the QUEST early-high source term is very large. It involves substantial releases of not only the volatile species but also Group 5, as well as quite significant releases of the refractories (Groups 6 and 7).

The QUEST early-high source term was heavily dominated by releases from the vessel (as opposed to $\mathrm{CCI}$ releases). As noted previously, it involved combining maximum estimates for FCOR, FVES, and FCONV. In what follows, the origins of these estimates are briefly reviewed and, in some cases, they are modified to bring their usage more in line with the ground rules for combining uncertainties discussed in Section C.1.2.1.

\section{In-Vessel Release From Fuel (FCOR)}

In QUEST, two classes of uncertainty affecting release from the fuel were considered. The first was uncertainty in the core thermal history, with conditions favoring maximum core temperatures and maximum time at temperature obviously favoring high values of FCOR. This uncertainty was addressed by varying MARCH parameters found to have an important effect upon core thermal histories. For example, the QUEST "high" thermal history was defined by setting the MARCH core-melt temperature parameters at the maxima of their credible ranges, postulating no core slump until most (75\%) of the entire core is melted, and maximizing the extent of zirconium oxidation, which maximizes heat generation (and also enhances release of tellurium from the fuel).

The second class of uncertainty is that associated with the rate of release of the various fission products at a given temperature, as calculated by CORSOR. CORSOR (i.e., the BMI2104 version, which was used in QUEST) assumes fission product release rates to vary as $\mathrm{Ae}^{\mathrm{BT}}$ ), where $\mathrm{T}$ is the temperature and $\mathrm{A}$ and $\mathrm{B}$ are constants over specified temperature ranges. The release rate uncertainty was addressed by varying the CORSOR release rate coefficients " $A$ " by a factor of ten in either direction with respect to the base case values, with the coefficients for all species being varied simultaneously (i.e., all were set to either their high or their low values simultaneously). The factor of ten variation was based upon information in NUREG-0772.

The effects of these two classes of uncertainties can be illustrated by examining the FCOR values for two representative species, iodine and barium, that result from the various combinations of assumed thermal histories and release rate coefficients. These results are summarized in Table C-2. 
Table C-2

QUEST FCOR VALUES FOR "HIGH" AND "LOW" CASES

\begin{tabular}{|c|c|c|c|c|c|c|}
\hline \multirow{2}{*}{$\begin{array}{l}\text { Thermal } \\
\text { History }\end{array}$} & \multicolumn{3}{|c|}{ Iodine Release } & \multicolumn{3}{|c|}{ Barium Release } \\
\hline & High & Base & Low & High & Base & Low \\
\hline High & 1.0 & 1.0 & 0.99 & 0.99 & 0.58 & 0.09 \\
\hline Base & 1.0 & 0.99 & 0.62 & 0.78 & 0.18 & 0.02 \\
\hline Low & 1.0 & 0.62 & 0.11 & 0.18 & 0.02 & 0.002 \\
\hline
\end{tabular}

Several observations are worth making concerning the results presented in the table:

1) The thermal-history and the release-rate uncertainties are each quite large and they produce roughly equal uncertainties in FCOR; when both uncertainties are varied in the same direction, the resulting effect is considerably greater than the effect of either by itself. In what follows, FCOR values obtained by maximizing (minimizing) both the thermal history and the release rate coefficients will be referred to as the "high-high" ("lowlow") cases, while results obtained by maximizing (minimizing) just one of these two factors, leaving the other at the base case values, will be referred to as the "high" ("low") cases. Among the latter, QUEST gives complete results only for the case where the thermal history is varied while the release coefficients are held constant, and these will be taken to represent the high and low cases. Based upon Table C-2 and some additional results in QUEST, it was assumed that these results are also representative of the values that could be obtained by varying the release coefficients while using the base case thermal history. (In general, variations associated with release rate coefficients tended to be somewhat greater than variations associated with thermal-hydraulics. The exception was $\mathrm{Te}$, for which the interrelation between thermal-hydraulics and $\mathrm{Zr}$ oxidation resulted in sensitivity to the thermal-hydraulic parameters being the greater.)

2) When the thermal-hydraulic uncertainties and the release rate uncertainties are varied in opposite directions, the effects roughly cancel, yielding FCOR values not far from the base case. The latter means that base case values of FCOR are consistent with either extreme in either the thermal history or the release coefficients, provided only that the other factor is varied in the opposite direction.

3) Since volatile species (iodine) are almost totally released in the base case, more severe conditions have little effect upon their release fractions. However, less severe conditions can result in substantial quantities of these species remaining in the melt at vessel failure time.

4) Semi-refractory species (barium) are quite sensitive to both uncertainties; both very large and very small values of FCOR are conceivable.

5) Although the variations used in QUEST were such as to drive all releases toward either high or low values, this is by no means necessary. Thermal- 
hydraulic variations would tend to act in this way, but the release rate coefficients for species with very different chemical and physical properties may be regarded as being largely independent. One could easily have high (or low) FCOR values for some species and central values for others; one could even have high values for some and low values for others.

In Table C-3, QUEST high-high, high, base case, low, and low-low values of FCOR are summarized for the seven WASH-1400 fission product groups.

Table C-3

\section{QUEST FCOR VALUES--WASH-1400 GROUPS}

\begin{tabular}{cccccc}
\hline Group & High-High & High & Base & Low & Low-Low \\
\hline 1 & 1.0 & 1.0 & 0.986 & 0.63 & 0.13 \\
2 & 1.0 & 1.0 & 0.986 & 0.62 & 0.11 \\
3 & 1.0 & 1.0 & 0.986 & 0.63 & 0.13 \\
4 & 1.0 & 0.90 & 0.33 & 0.026 & 0.022 \\
5 & 0.98 & 0.49 & 0.13 & 0.014 & $9.5 \mathrm{E}-4$ \\
6 & 0.53 & 0.11 & 0.041 & $8.0 \mathrm{E}-3$ & $8.1 \mathrm{E}-4$ \\
7 & 0.074 & $7.7 \mathrm{E}-3$ & $2.5 \mathrm{E}-4$ & $1.4 \mathrm{E}-5$ & $1.6 \mathrm{E}-6$ \\
\hline
\end{tabular}

In evaluating the assumptions involved in obtaining these results, it was judged that both the uncertainties associated with the thermal history and those associated with the release rate coefficients are composite in the sense intended in the second of the guidelines for combining uncertainties (Section C.1.2.1). Thus, several MARCH phenomenological parameters affecting thermal history must be adjusted to obtain the corresponding high and low cases, while obtaining the high and low release rate cases requires postulating that the release rate coefficients are near their upper (lower) limits for each of many different fission products characterized by very different chemical and physical behaviors. Postulating that either of these sets of assumptions might be valid by itself was judged sufficiently pessimistic (optimistic) for present purposes. Hence, in the present work, the high and low values of FCOR were employed for estimating pessimistic and optimistic source terms, and the more extreme highhigh and low-low cases were not used.

It should be acknowledged that even the QUEST high-high and low-low values are not truly bounding, in that they are based upon examining only the two classes of uncertainty discussed above. There are many other uncertainties which could affect FCOR values, some of which are discussed in Reference 4. Thus, restricting the uncertainty range for FCOR to 
that spanned by the high and low values in Table C-3 is judged to be rather restrained. One motivation for this restraint was not to exhaust the credit for combining uncertainties within the spirit of the guidelines discussed in Section C.1.2.1. Except when inconsistencies would result, the high and low FCOR estimates were freely combined with pessimistic or optimistic estimates of other parameters in deriving pessimistic and optimistic source terms.

In calculating optimistic source terms, postulating the low values of FCOR for Cs and I would imply significant quantities of these species remain in the melt at VB and would thus be available for release during CCI. The net result could be to increase their release to the environment. Hence, central FCOR values were usually postulated for these species in calculating optimistic source terms. As is implied by the discussion of Table C-2, this is by no means inconsistent with postulating low FCOR values for other species.

Estimation of FCOR values for pessimistic source terms provides the closest approach currently known to an example of an instance in which the STCP might have lead to conclusions different from those based upon the BMI-2104 code suite. The STCP uses a revised version of CORSOR, called CORSOR-M, in which the release rates are assumed to be governed by an expression of the Arrhenius form, $A E^{-B / T}$, and the constants $A$ and $B$ are such that the calculated releases of refractory species (WASH-1400 Groups 5-7) are generally one to several orders of magnitude smaller than in the older code; releases of the more volatile species are little changed. Had the procedure employed in QUEST been applied using CORSOR-M, refractory releases approaching the high-high values of Table C-3 would not have been obtained, and the refractory releases in the early high source term of Table C-1 would have been significantly reduced. Since the decision had already been made not to apply the QUEST high-high FCOR releases to the present work, it is still not clear that the present work would have been significantly affected by the differences between CORSOR and CORSOR-M.

\section{Belease from the Vessel (FVES)}

In QUEST, it was noted that MERGE and TRAP-MELT do not take into account heat and mass transport due to natural convection between control volumes, though the correlations used do implicitly take into account natural convection within a control volume. Simple scoping calculations indicated that, in high-pressure sequences such as TMLB', flow velocities due to natural convection are likely to be one to three orders of magnitude greater than the net flow velocity due to steam generation. MERGE and TRAP-MELT only treat the latter in calculating transport between control volumes. The implications of this fact are quite different for the volatile species and for the more refractory species, and the two are therefore discussed separately below. 
Yolatile Species: $\mathrm{C}, \mathrm{Cs}$, and Te Simple scoping calculations in QUEST indicated that natural convective transport could lead to efficient deposition of radionuclide vapors, with FVES values of less than 0.01 being possible, provided sufficiently cool (or chemically reactive) deposition surfaces were available. If these species were present as aerosol, MAEROS calculations again indicated that over $99 \%$ retention was possible, also assuming revolatilization does not occur. On the other hand, simple scoping calculations also indicated that natural convective heat transport, thermal radiation, and decay heating effects could be sufficient to heat deposition surfaces to the point where deposition of volatiles would not occur or else deposited materials would be revolatilized.

Quantitative calculation of the net retention was thus found to require a reasonably accurate evaluation of strongly opposing effects. Such an evaluation requires a detailed modeling of natural convection, radiative heat transport, decay heating, and fission product chemistry; it is far beyond the capability of simple scoping calculations of the type available to QUEST and must await the development and validation of detailed codes such as MELPROG [7]. In the mean time, it was judged in QUEST that FVES for these species must be considered highly uncertain, with high and low values of 0.9 and 0.01 , respectively, being assigned.

BMI-2104 results published since the QUEST analyses were performed lend indirect support to the existence of a wide uncertainty range. The dominance of natural circulation transport over net flow transport is expected to exist for all high-pressure sequences, independent of reactor type. BMI-2104 FVES values for CsI and CsOH in high-pressure sequences range from a high of 0.8-0.9 for the Peach Bottom TC sequence to a low of 0.02 for the Zion TMLB' sequence. These variations represent the response of the fission product transport model to thermal-hydraulic variations which, though substantial, are probably less than the thermal-hydraulic uncertainties introduced by the incomplete treatment of natural convection. Since the variations in FVES introduced by those thermal-hydraulic variations that are treated in BMI-2104 essentially span the QUEST uncertainty range, it is highly credible that the (probably larger) thermal-hydraulic uncertainties associated with natural convection introduce a comparable uncertainty range in the results of any given analysis.

One possible cause of high iodine release from the primary system was not considered in QUEST, namely, the reported decomposition of CsI when in a high-temperature steam environment subjected to high radiation fields. Based partly upon this phenomenon, one reviewer suggested that, for pessimistic source term estimates, the WASH-1400 value of 
FVES, 1.0, might still be appropriate. This suggestion was adopted, and FVES $=1.0$ is assumed for the pessimistic estimates of iodine release.

The arguments for a large "high" value of FVES are weakest in the case of tellurium: evidence favoring chemical retention of $\mathrm{Te}$ is stronger than in the case of $\mathrm{CsOH}$ and $\mathrm{CsI}$, and no BMI-2104 analysis gave high values of FCOR*FVES for Te. Nonetheless, in the absence better thermal-hydraulic analyses of high-pressure sequences, and better understanding of the thermal stability limits of the tellurium compounds formed upon deposition within the RCS, the QUEST uncertainty range for FVES(Te), 0.01 to 0.9 , was also accepted for the present work.

Arguments for high FVES values in high-pressure sequences depend, in part, upon the assumption of a large amount of heating of deposition surfaces by natural convection and radiative transfer, and such heating would no doubt be favored by the thermal histories leading to high FCOR values; similarly, the availability of cool deposition surfaces would be favored by thermal histories yielding low FCOR values. Thus, FVES and FCOR are positively correlated in a phenomenological sense, and it was judged reasonable to combine high (low) FCOR values with high (low) FVES values for these species.

Refractory Species (Groups 5, 6,7) Once these species leave the hottest part of the core, they are expected to transport only as aerosols, not vapors. Hence, their retention is unlikely to be significantly affected by even severe natural convective heating of deposition surfaces. Natural convection will alter aerosol concentrations, and hence agglomeration and deposition rates, in the various control volumes. However, it will not greatly affect the mean aerosol residence time within the RCS, which is probably the most important single parameter governing aerosol retention factors. Hence, it was judged that natural convection introduces significantly less uncertainty in the FVES values of low-volatility aerosol species than it does for the more volatile species.

In general, phenomenological assumptions favoring high FCOR values also favor high aerosol generation, which in turn favors high retention factors for aerosols, with the reverse being true for assumptions favoring low FCOR values. Hence, for the more refractory species, it was judged that FCOR and FVES have a significant negative phenomenological correlation, and extreme values of these parameters should not be combined. In effect, the base case value of FVES is already mildly pessimistic when combined with the pessimistic (i.e., high) values of FCOR, and mildly optimistic when combined with the optimistic (i.e., low) FCOR values. 
In the present work, therefore, the central values of FVES were assigned for use with both the low and the high FCOR values, in the case of the less volatile species. Values of FVES for RCS aerosols in BMI-2104 are summarized in Table C-4.

Table C-4

"FVES" FOR RCS AEROSOLS IN BMI-2104

\begin{tabular}{|c|c|c|c|c|c|c|}
\hline \multicolumn{2}{|c|}{ Surry } & \multicolumn{2}{|c|}{ Zion } & & \multicolumn{2}{|c|}{ Sequoyah } \\
\hline TMLB' & 0.07 & TMLB' & 0.057 & & TMLB' & 0.081 \\
\hline S2D- $\gamma$ & 0.34 & S2D & 0.35 & & S2D & 0.15 \\
\hline$S 2 D-\varepsilon$ & 0.27 & & & & & \\
\hline $\mathrm{V}$ & 0.33 & & & & & \\
\hline \multirow[t]{6}{*}{$\mathrm{AB}$} & 0.52 & & & & & \\
\hline & & attom & & Grand & ulf & \\
\hline & $\mathrm{AE}$ & 0.30 & & TC & 0.29 & \\
\hline & TC & 0.42 & & TPI & 0.20 & \\
\hline & TW & 0.27 & & TQUV & 0.59 & \\
\hline & & & & S2E & 0.80 & \\
\hline
\end{tabular}

The PWR results show a fairly regular trend toward increasing FVES with decreasing system pressurization, as expected; trends among the BWR data are less clear and the results are more difficult to interpret. Based upon these results, FVES values of 0.07 were judged reasonably appropriate for PWR high-pressure sequences, and 0.33 was adopted for the V sequence and PWR sequences with S2 thermal-hydraulics (see, however, discussion of Surry Bins 11 and 12 for recent changes in the Battelle $V$-sequence analysis).

The present treatment differs substantially from that used in QUEST, which assigned an FVES value of 0.9 for all species in estimating the early-high source terms. The rationale given was the possibility of massive aerosol resuspension upon vessel failure and blowdown. Significant resuspension cannot be ruled out and more investigation is certainly warranted. However, the QUEST evaluation of resuspension was oriented primarily toward resuspension within the containment, not the RCS; the data base reviewed there included conditions very favorable to resuspension; and some factors opposing high resuspension in the RCS (such as the likely presence of molten constituents in the total aerosol deposited in the RCS) were not taken into account. Furthermore, if massive resuspension did occur, it is likely that much of the material would be in the form of larger agglomerates, which would not contribute as effectively to the source term as does the micron-sized aerosol generally assumed in 
consequence calculations. Hence, the QUEST value of 0.9 was not used for aerosol species in the present work.

\section{Release of RCS Species to the Environment (FCONV)}

Within the containment, most radionuclide species are expected to transport as aerosols, and it might be argued that values of FCONV derived from BMI-2104 would be appropriate for the optimistic and pessimistic cases, as was argued in the case of FVES for aerosol species. However, the Battelle analyses of the Surry TMLB'- $\delta$ (early) sequence (both in BMI-2104 and in the SARRP central analysis) imply FCONV $=0.3$; in particular, only $30 \%$ of the CSI released to the containment is also released to the environment. The reason for this rather low value was not immediately apparent: given the rather large ( $7 \mathrm{sq} . \mathrm{ft}$.) rupture failure assumed, a rapid blowdown expelling most of the airborne radionuclides is expected, and it is not at all clear that the time interval between radionuclide release from the RCS and the release from the containment is sufficient to permit significant aerosol deposition, in the absence of sprays or other ESFs.

Hence the value of 0.3 was judged insufficiently conservative for the pessimistic estimates, and a value of 0.8 was assumed for FCONV in the case of early CF pessimistic scenarios. This value is the same as that assumed for the oxidation release accompanying direct heating (Bins 16-19 discussed in Section C.1.3), and was based upon an isentropic expansion of hot gases from an assumed Surry failure pressure of 9 bars down to 1 bar.

For the optimistic assessments, the value of 0.3 implied by the Battelle calculations was judged sufficiently small for scenarios such as TMLB'- $\delta$ (early), considering the small amounts of aerosol to be expected in the optimistic scenarios.

After this work had been largely completed, a likely reason for the low value of FCONV implied by the Battelle calculations was identified. Based upon prior experience with the behavior of the MARCH-NAUA link, it was suggested that the Battelle calculation was likely to include an exaggerated effect of condensation upon aerosols during containment blowdown. Subsequent checking of the detailed output confirmed this supposition: in effect, the radionuclides "rained out" within the containment during the blowdown. This behavior is judged unrealistic for two reasons:

1) Calculations using the CONTAIN code, which gives a coupled treatment of thermal-hydraulic phenomena (including condensation) and aerosol phenomena, indicate that little decontamination due to the "rainout" effect could be expected for the rapid blowdown implied by the $7 \mathrm{sq} \mathrm{ft}(0.65 \mathrm{sq}$ $\mathrm{m}$ ) failure size assumed, although the condensation effect is itself real and can reduce source terms somewhat when the blowdown is much slower 
(e.g., following a 0.02 sq $\mathrm{m}$ containment failure). These calculations are described further in Reference 8.

2) In the Battelle calculations, a failure due to steam overpressurization was postulated, with the containment atmosphere being near saturation at failure time. In the SARRP scenarios of actual interest, however, the failures involve either hydrogen burns or direct heating (or both). In these cases, the containment atmosphere at the time of failure would be highly superheated, and little or no condensation during blowdown would be expected.

It appears, therefore, that the value of FCONV derived from the Battelle calculations is nonconservative; the original central source term calculations (which are those cited in Table C1) underestimate the Cs and I release by factors of 2.5 to 3 for the TMLB' sequence with early CF, as well as for the other scenarios binned with this case. The central source term used in SARRP for these scenarios was recalculated with steam condensation upon aerosols in NAUA switched off. Results imply an FCONV value of about 0.8 , in line with present expectations. This recalculated source term is the one actually used for the central source term estimates discussed in the main report.

\section{Releases}

Assuming a dry cavity and the absence of ESFs such as sprays, the source term associated with late containment failures is generally dominated by radionuclides released during core-concrete interactions, and these species can be significant contributors even when containment failure is early. QUEST presented some rather extensive sensitivity studies of CCI releases as calculated by CORCON-VANESA (Chapter 6 and Appendices $\mathrm{K}$ and L, Reference 4). However, reliance upon these results was minimized in the present work for two reasons:

(1) These studies were carried out primarily to identify those input parameters which most affected the results, not make realistic evaluations of the uncertainties. Thus, the parameter ranges used did not necessarily reflect realistic estimates of the actual uncertainty ranges for these parameters, and most of the studies were restricted to one-at-a-time parameter variations about the base case.

(2) During the course of these sensitivity studies, it was discovered that a modeling defect in the CORCON code could, under some conditions, result in excessive and unrealistic excursions in the calculated melt temperature, which in turn led to excessive release rates for refractory species in the VANESA calculations.

Hence, the present evaluation relies primarily upon the results of Chapter 7 of Ref. 4 which summarizes the VANESA releases for several important species for the four "bounding" QUEST cases (i.e., early-high, etc) and the base case. The CORCON modeling problem had 
been fixed when these results were obtained, and the range of releases spanned by these results was used in the present work. QUEST reported the results for $\mathrm{CCI}$ release normalized to the amount of the various species in the melt at the time of onset of CCI, i.e., in terms of the quantity FCCI/(1-FCOR), in the notation of Eq. C.1-1. The high and low values, along with the base case and the (very similar) values derived from BMI-2104, are summarized in Table C-5.

Table C-5

FCCV/(1-FCOR) VALUES FROM QUEST AND BMI-2104:

(SURRY TMLB' SEQUENCE)

\begin{tabular}{ccccc}
\hline $\begin{array}{c}\text { WASH-1400 } \\
\text { Group }\end{array}$ & High & $\begin{array}{c}\text { QUEST } \\
\text { Base }\end{array}$ & Low & BMI-2104 \\
\hline 4 & 0.63 & 0.40 & 0.23 & 0.33 \\
5 & 0.34 & 0.11 & 0.09 & 0.11 \\
6 & negl. & negl. & negl. & negl. \\
7 & 0.02 & $7.7 \mathrm{E}-4$ & $2.1 \mathrm{E}-4$ & $5.4 \mathrm{E}-4$ \\
\hline
\end{tabular}

In selecting values from Table $\mathrm{C}-5$ for a given application, consistency requirements with respect to choices made for release from the RCS must be considered. Interpreted rigorously (as was done in the QUEST approach), consistency would require the use of low values from Table $C-5$ whenever high values of FCOR and FVES had been assumed, and vice versa, because FCOR and FCCI are negatively correlated phenomenologically. However, a rigorous interpretation was judged overly restrictive for present purposes, because this phenomenological relationship between FCOR and FCCI itself involves considerable uncertainty. Hence, use of the BMI-2104 (i.e., central) values from Table C-5 was also judged consistent with use of either the low or the high values of FCOR and FVES. Imposing strict consistency would have trivial impact upon the pessimistic source terms, but would affect the optimistic values because the relatively large releases of refractories implied by the high values in Table C-5 would be used whenever the optimistic estimates of RCS releases were assumed. The crucial phenomenology involved is the enhanced refractory release predicted when large amounts of unoxidized zirconium are present in the melt. The range of relevant parameters for which VANESA predicts this enhancement has not been determined. More importantly, the VANESA experimental validation program has not yet addressed scenarios involving enhanced refractory release associated with zirconium. Hence, imposing these 
enhanced releases upon the optimistic source term calculations was considered to be quite unjustified. In deriving FCCI values from the Table C-5 results, the value of FCOR assumed was, of course, required to be fully consistent with the value assumed in the RCS analysis.

\section{Release from the Containment (FCONC)}

BMI-2104 releases for the Surry TMLB'- $\delta$ (early) sequence implied values of FCONC of about $0.43,0.54$, and 0.36 for Groups 4,5 , and 7 , respectively. No reasons for substantially increasing these values for the pessimistic case were identified, and the Group 4 value, 0.43 , was adopted as representative for the release of $C C I$ species given an early failure. For scenarios with late CF, central values of FCONC taken from the most appropriate BMI2104 results were used. The optimistic values of FCONC were reduced substantially in accordance with the QUEST aerosol phenomenological uncertainty studies as discussed below (Section C.1.2.5).

\section{C.1.2.4 Application of QUEST Results to Other Sequences}

There is very little information to go on in applying the Surry TMLB' uncertainty estimates to other sequences. Time has not permitted a detailed examination of the many ways other sequence/pathway combinations differ from the Surry TMLB' sequence and, even if it had, there would usually be no way to make quantitative allowance for the effect of these differences upon the uncertainties in the source term. All that can be done is to devise a rather broad-brush approach, with some attempt at consistency of treatment to the extent needed to preserve any qualitative differences between sequences and pathways that are judged to reflect reality, but not introduce the appearance of such differences in the absence of reasonable belief that they do reflect reality.

The following discussion is restricted to a limited number of rather general phenomenologies. Discussion of many scenario-specific issues has been deferred to the discussion of individual bin assignments.

\section{In-Vessel Release from the Fuel (ECOR)}

Though the FCOR values obtained in BMI-2104 did vary significantly among the various plant/sequences treated, the variations were generally smaller than the range spanned by the high and low values of Table C-3. Hence, these ranges were taken to be applicable to other sequences. Admittedly, the justification for this assumption is weak, but the limitations in the available data permit little else; QUEST-type studies of FCOR for other sequences have not been performed. 


\section{Release from the RCS (FVES)}

The BMI-2104 results for PWRs indicated rather similar behavior for a given RCS thermal-hydraulic state, and thus the results discussed above for in-vessel releases are applied to all sequences which were binned with TMLB' in terms of RCS thermal-hydraulics in deriving the central source term estimates (e.g., TML, S3D).

In sequences characterized by $S 2$ thermal-hydraulics (intermediate RCS pressures), natural convective transport probably still dominates net flow transport, and the arguments for wide uncertainty ranges in the retention of volatile species raised in QUEST still apply. However, the degree of dominance by natural convection is certainly less than in the TMLB'type sequence, and residence times are considerably shorter, as is reflected in the substantially larger values of FVES obtained in the Battelle calculations for the central source terms. The pessimistic values of FVES for Cs, I, and Te were therefore left at the TMLB' value, 0.9. For the optimistic case, it was assumed that the ratio (optimistic/central) would be as in the TMLB' sequence (i.e., $0.01 / .15=.067$ ), and this factor was then applied to the central RCS release estimates from the Battelle calculations. Quantitatively, this procedure obviously has little to recommend it; qualitatively, it does preserve the expectation that the optimistic release should be larger for $S 2$ thermal-hydraulics than for TMLB' while also preserving the judgment that the uncertainty range in RCS release is still large.

The $\mathrm{V}$-sequence is singularly vexatious in that it is important to the risk profile for Surry, yet RCS transport is dominated by net flow and the QUEST arguments based upon natural circulation are largely irrelevant. Further discussion of the $\mathrm{V}$ sequence is deferred to the specific bins developed for it, except to note that the pessimistic value FVES $=0.9$ for volatile species was retained here also.

For aerosol species in all sequences, the central values of FVES were judged sufficiently optimistic when aerosol sources are small (optimistic case) and pessimistic when the sources are large (pessimistic case) so that central values were generally used without change.

FCCI values were generally taken from the values summarized in Table C-5, since the range in possible melt compositions was judged to be similar. As noted above, the modified consistency arguments sometimes dictated use of a Battelle "central" value rather than a "high" or "low" value; when this was done, the value derived from the Battelle calculation for the specific sequence of interest was used instead of the Table C-5 value, unless it made little difference. 
NUREG/CR-4551, VOL. 1: DRAFT REPORT FOR COMMENT (SEPTEMBER, 1986)

The TMLB' values of FCONV and FCONC were sometimes judged applicable, but in many cases sequence-specific modifications were required. These are discussed in connection with the bins for which they apply.

\section{C.1.2.5 Pessimistic "Worst Case" Source Terms}

In this subsection, pessimistic source terms are derived for "worst case" scenarios involving early $C F$ or bypass without mitigation features. The modified QUEST results discussed above are used and the results compared with the widely-known Siting Source Term 1 (SST1).

In Table C-6, pessimistic source terms are summarized for TMLB', S2D without mitigation by sprays, the Surry $V$ sequence, and the $A B / T M L B$ ' hybrid sequence used to represent "S2C" (Bin 5) in some of the earlier Surry central source term estimates. In all cases, the values were derived using the approach summarized above: "high" values of FCOR were assumed, FVES was assumed to be 1.0 for iodine and 0.9 for Groups 3 and 4 while FVES was based upon Table C-4 for aerosol species, FCONV was taken to be 0.8 , and central values of the $\mathrm{CCI}$ releases were assumed. The latter were derived from the BMI-2104 analyses, with FCCI adjusted for the different value of FCOR assumed in calculating the RCS releases. Results are compared with SST1 in the last column of Table C- 6 . The summed MACCS importance factors for early effects $(\mathrm{Qe})$ and latent cancers $(\mathrm{Ql})$ are also given.

In a relative sense, the release fractions of Groups 5 - 7 vary considerably, but not enough to have a dominant impact upon the risk, since consequences will remain dominated by the release of Groups 2 - 4 in all cases. Furthermore, SST-1 values provide a reasonable representation of Groups 5-7, albeit somewhat on the low side for Group 5 and somewhat on the high side for Groups 6 and 7 (however, the present estimates for Group 6 would increase if an oxidation release were included). Probably the most risk-significant difference between the present result and SST-1 is the lower iodine release in the latter. Nothing in QUEST or the BMI-2104 methodology supports a lower upper bound for iodine release than for Cs and Te; on the contrary, some of the factors favoring retention of the latter species in the RCS seem the most questionable for iodine. It should be recalled that SST1 was derived from a BWR sequence, and the lower iodine release may reflect suppression pool effects not relevant to Surry. In any case, it is evident from these comparisons that SST1 remains a quite conservative estimate for the "worst case" scenarios, though some release fractions are not quite as large as in the present pessimistic source terms. 
NUREG/CR-4551, VOL. 1: DRAFT REPORT FOR COMMENT (SEPTEMBER, 1986)

Table C-6

PESSIMISTIC SOURCE TERM ESTIMATES

\begin{tabular}{cccccc}
\hline $\begin{array}{c}\text { WASH-1400 } \\
\text { Group }\end{array}$ & TMLB' & $\begin{array}{c}\mathrm{S}_{2} \mathrm{D} \\
\text { (No Spray) }\end{array}$ & $\mathrm{V}$ & $\begin{array}{c}\text { AB/TMLB' } \\
\left(" \mathrm{~S}_{2} \mathrm{C}^{\prime \prime}\right)\end{array}$ & SST1 \\
\hline 1 & 1.0 & 1.0 & 1.0 & 1.0 & 1.0 \\
2 & 0.8 & 0.8 & 0.8 & 0.8 & 0.45 \\
3 & 0.72 & 0.72 & 0.72 & 0.72 & 0.67 \\
4 & 0.65 & 0.65 & 0.65 & 0.65 & 0.64 \\
5 & 0.052 & 0.19 & 0.18 & 0.28 & 0.07 \\
6 & 0.0062 & 0.036 & 0.036 & 0.057 & 0.05 \\
7 & $6.6 \mathrm{E}-4$ & 0.0028 & 0.0058 & 0.0042 & 0.009 \\
$\mathrm{Qe}$ & 1.6 & 1.8 & 1.9 & 2.0 & 1.5 \\
$\mathrm{Q} 1$ & 0.93 & 1.1 & 1.1 & 1.2 & 0.97 \\
\hline
\end{tabular}

Comparing the results of Table C- 6 with those of Table $\mathrm{C}-1$, it is apparent that all these pessimistic source terms are substantially larger than the TMLB' source terms calculated by Battelle. On the other hand, they are still significantly smaller than the QUEST "early high" TMLB' source term.

It is instructive to examine the robustness of these pessimistic source terms to variations in the assumptions used to derive them, especially greater extremes of conservatism. If the QUEST high-high values of FCOR are used, the TMLB' release fractions of Groups 5, 6, and 7 become $0.079,0.030$, and 0.0037 , respectively, which are not enough higher to significantly augment overall consequences. Likewise, use of the FVES values of 0.9 with the high FCOR values yields release fractions of $0.37,0.073$, and 0.0057 for these species, again not a big enough change to impact overall risk greatly, although the higher $\mathrm{Sr}$ releases certainly would not be negligible in their implications. Finally, if consistency requirements are dropped and the high values in Table C-5 are used to derive FCCI, TMLB' releases of Groups 5 and 7 would increase to 0.10 and 0.0087 , respectively, which is still within the range already considered.

In the context of risk assessment, therefore, the pessimistic source terms appear to be quite robust toward increases in the degree of conservatism invoked in their derivation. Only the severest combinations of the QUEST extremes would result in substantial increases. It is, of course, much less robust against reductions in some of the conservatisms concerning release of the more volatile species. It is quite possible that reduction in just a few phenomenological 
uncertainties (natural convective transport, thermal radiative heating, decay heating and revolatilization, possibly aerosol resuspension) would permit substantial reductions for many scenarios, even in the context of pessimistic estimates.

It should be remembered that the preceding estimates include no allowance for releases associated with high pressure melt ejection and direct containment heating. These will be discussed in connection with the bins derived specifically to cover these scenarios.

\section{C.1.2.6 Other Phenomenological Uncertainty Factors}

In this subsection, a number of phenomenologies are discussed in terms of their uncertainties and how they might affect source term estimates. Some, but not all, of the discussion is based upon QUEST results. Discussion of some phenomenologies which arise in only a limited number of scenarios is deferred to the discussion of the particular source term $\operatorname{bin}(s)$ they affect.

\section{Aerosol Agglomeration Uncertainties}

NAUA calculations reported by Battelle were performed assuming unit shape factors for all aerosol particles and neglected turbulent agglomeration. In QUEST, it was concluded that these assumptions would yield airborne radionuclide concentrations near the upper limit of the uncertainty band associated with aerosol agglomeration processes, and no modifications were judged needed for the pessimistic estimates in the present work. However, QUEST indicated that there could be a substantial downward uncertainty in the source term due to aerosol shape factors and turbulent effects. With these parameters chosen from within their uncertainty bands so as to maximize agglomeration and settling rates, aerosols and radionuclides airborne could be reduced by an order of magnitude or even more.

The lower bound of the uncertainty band for aerosol agglomeration varied substantially, depending upon the scenario. During CCI releases, there is typically an approximate steady state in airborne concentrations, with deposition rates roughly equal to the source rates. Under these conditions, QUEST results implied a reduction in airborne radionuclides by a factor of about 0.072 , given the optimistic parameter choices. Hence, in the present work, FCONC was reduced by this factor in deriving optimistic source term estimates for those scenarios for which the QUEST results were judged to apply. This reduction factor was applied to FCONV only when there was sufficient time between release from the vessel and containment failure to permit the agglomeration and settling processes to be effective.

Physically, the conditions corresponding to the enhanced deposition rates in QUEST correspond to assuming aerosol shape factors derived from experiments with dry UO2 
aerosols, combined with assuming convective turbulence driven by a substantial heat source. The enhanced deposition rates were not credited in deriving optimistic source terms for scenarios clearly at variance with these assumptions. In particular, the dry aerosol shape factors were considered inconsistent with high relative humidities (about $80 \%$ or greater) and thus were not assumed for scenarios also involving sprays.

In QUEST it was pointed out that the CONTAIN and NAUA kernels for gravitational agglomeration of aerosols differed by a factor of three, but the effect of this difference upon the source term was not explored. Since that time, some limited calculations with the CONTAIN code, modified to have the same gravitational kernel as NAUA, indicate that an uncertainty factor of 1.4 to 1.8 in aerosol concentrations may arise from this source, with NAUA yielding the higher aerosol concentrations. At present, there are no experimental data that would permit a choice to be made between the two treatments. Since the NAUA treatment would usually give the higher source terms, no allowance for this uncertainty is needed in the pessimistic source term estimates, but it does increase the total uncertainty associated with aerosol agglomeration processes. No additional aerosol agglomeration uncertainty beyond that evaluated in QUEST was included in the present work, however.

\section{Scrubbing in Pools}

In many accident scenarios, aerosols and associated radionuclides can be released to the environment only after passing through water. Decontamination factors associated with the resulting scrubbing effect are often substantial, but are sensitive to many parameters and can be quite uncertain. The only case QUEST examined in any detail involved scrubbing in the suppression pool for the Grand Gulf TC sequence [6]. In this case, it was concluded that there was an order of magnitude uncertainty in either direction in the effective DF, which was about 50 in the base case for the instance considered (scrubbing of Te). Thus, it was concluded that the DF for this scenario could span the range 5 to 500 .

In SARRP, many scenarios involving scrubbing must be considered, and some simplified means is needed for allowing for the uncertainty in scrubbing when estimating optimistic and pessimistic source terms. Obviously, no simple prescription of the type required can be more than a very crude approximation. The best that can be hoped for is something that can be applied in a reasonably consistent fashion, that acknowledges the substantial uncertainties in the effective DFs for pool scrubbing scenarios, and that will not unnecessarily distort expected trends in going from one scenario to another. 
In the present work, it was assumed that the DF depends exponentially upon a scrubbing parameter, $S c P$, where the decontamination factor, $D F=\exp (S c P)$, where $S c P$ depends upon pool depth plus the many parameters which affect the removal rate of aerosol particles of given characteristics as the bubble rises through the pool. (For realistic problems, with a wide range of particle sizes, this is a very crude approximation at best.) The QUEST results then translate into a central value of 3.9 for $\mathrm{ScP}$, with an uncertainty of 2.3 , or $59 \%$, in either direction. To obtain pessimistic and optimistic values of the scrubbing DF in other scenarios, central values of $\mathrm{ScP}$ were estimated from the Battelle results and it was assumed that the same percentage uncertainty in ScP, 59\%, would apply in these cases also.

The specific case of interest here is the Surry $V$ sequence with water present in the release pathway. For this scenario, Battelle calculations imply a DF of about 5 . The present approach then gives $\mathrm{ScP}=1.58$ with a range of 0.65 to 2.52 , implying DFs of 1.9 (rounded to 2 ) and 12.4 for the pessimistic and optimistic estimates, respectively.

It is freely acknowledged that this procedure is very crude. It does have the desired characteristics noted above, however, and available information did not permit a more refined approach within the time available for this study.

\section{Effects of Containment Spravs.}

Uncertainty in the effectiveness of sprays was studied in QUEST in connection with the Surry S2D sequence [5]. Some results are reproduced in Figure C-1, expressed as total radionuclides airborne as a function of time. The range of results spanned by varying the drop size from 400 to 2000 microns was judged to give a reasonable representation of the uncertainty; although the actual uncertainty in effective drop size is probably less than this, drop size was used to some extent as a surrogate for other uncertainties in the spray collection efficiency for aerosols. Comparison with the results obtained for the same aerosol sources but with no sprays suggest a DF in the range of 10 to 100 , at least during the $\mathrm{CCI}$ phase of the accident. These calculations were performed with a value of the spray header height which was too great, especially for the recirculation phase, and a DF of 5 may be a better estimate of the lower limit; 100 remains reasonable for the upper limit. It should be noted that these DFs are based upon airborne radionuclide concentrations in the containment with the same radionuclide sources assumed present in all cases. Thus, they make no allowance for either scrubbing in a flooded cavity or altered thermal-hydraulic conditions, both of which are likely to affect the source terms for sequences involving spray operation. 


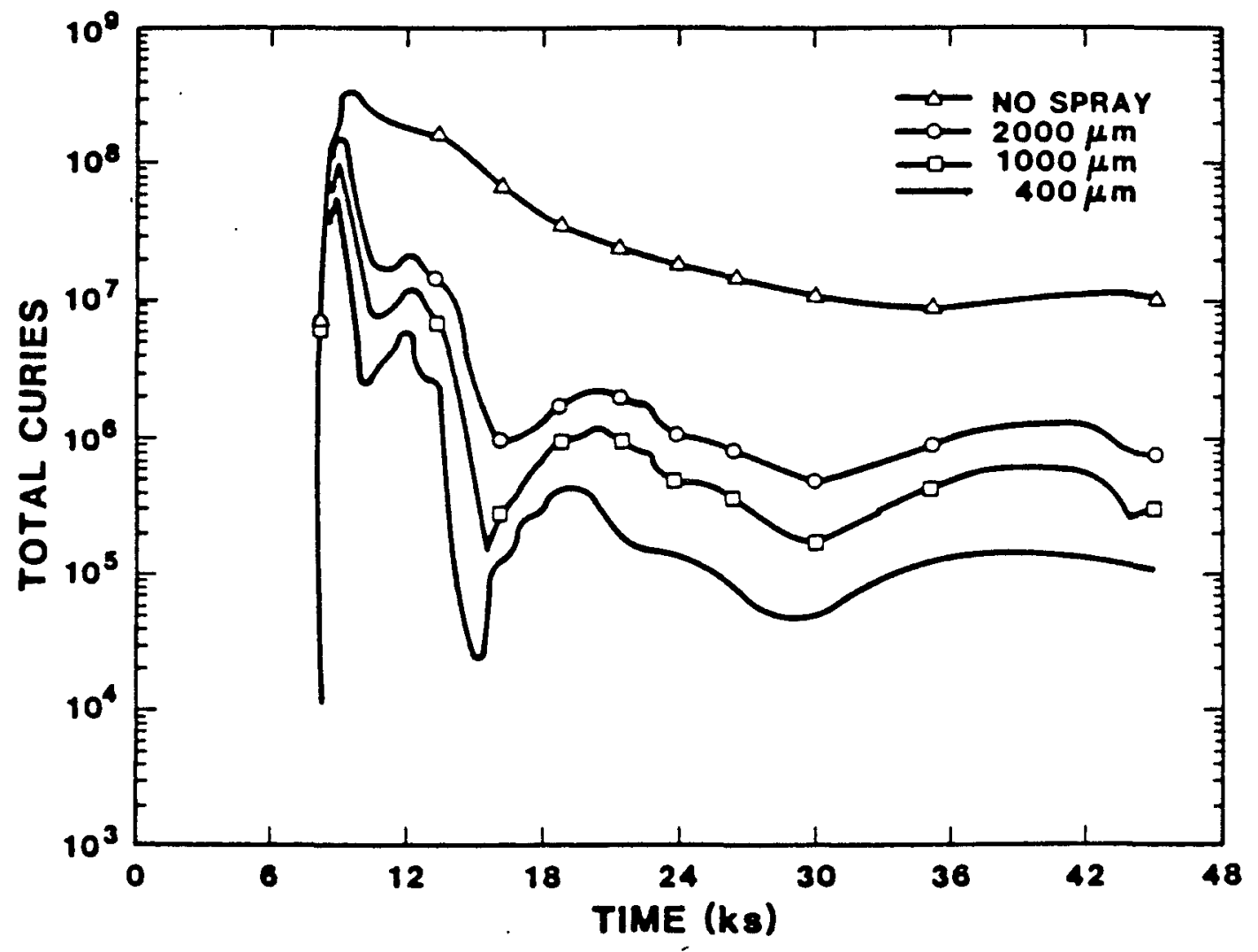

Figure C-1. Airborne Radionuclides Calculated For Different Spray Drop Sizes and Compared to Results for No Sprays [4]

The actual impact of the sprays depends upon many parameters and can vary widely. Sprays may not have time for significant decontamination during periods of very rapid release of radionuclides to the containment; to a limited degree, results at early times in Figure C-1 illustrate this effect. On the other hand, if the source of a given species to containment ceases, the residual quantities airborne are depleted fairly rapidly by the sprays, and the effective DFs at late times can be very large (several orders of magnitude).

Battelle calculations for Surry reported in BMI-2104 assumed a spray drop diameter of 400 microns in NAUA, and results generally implied a spray effectiveness at least as high as indicated by the lower bound of the QUEST uncertainty range for airborne radionuclides ( 400 micron curve in Figure C-1), with effective "DFs" exceeding 100 and even 1000 sometimes being obtained. More recent Battelle calculations (i.e., those adopted for the SARRP central estimates) assuming a 1000 micron drop, and also involving some changes to the NAUA spray model, gave substantially less decontamination, with results more in line with the upper portion of the QUEST uncertainty range in Figure $\mathrm{C}-1$, although exact comparisons can not be made. 
In the present work, a pessimistic DF of 5 was taken for the effect of sprays upon releases from the RCS, except that no DF was credited when there might be insufficient time for the sprays to take effect before release from the containment would occur. For CCI releases in the pessimistic case, a DF of 10 was taken relative to the release that would occur with no sprays and no cavity scrubbing.

In the optimistic analysis, a DF of 100 was assumed for the sprays, relative to the optimistic estimate without sprays. Note that, for the latter, the $\mathrm{CCI}$ releases were generally reduced by an order of magnitude by the allowance for the aerosol agglomeration uncertainties, so that there is a net reduction of three orders of magnitude in the optimistic estimate with sprays operating, relative to the central estimates without sprays. Although the agglomeration uncertainties may not apply when the sprays are operating, the large reduction in releases can still be justified by noting that the optimistic estimate should take some credit for scrubbing in the flooded cavity, and an additional factor of ten is far from excessive. Furthermore, some Battelle calculations (with a 400 micron drop) imply reductions due to sprays that are this large, even though full allowance for the flooded cavity was not always included in those calculations.

Several potentially-important sequences involve failure of a pressurized RCS with CF following immediately thereafter. In the pessimistic and central cases, it was assumed that most of the RCS releases would be released as a puff at VB, and that there would be insufficient time for the sprays to have an effect before this material was driven out of the containment. However, some CONTAIN calculations for the Surry TMLB' sequence with postulated spray recovery have shown that very substantial decontamination could $\propto c c u r$ within a few tens of seconds, provided sufficient time had elapsed before spray recovery for aerosol aging to enhance the particle size. Calculations for these conditions indicated characteristic residence times for $\mathrm{CsI}$ and $\mathrm{CsOH}$ were about 10 seconds, and about 20 seconds for other species. (The effect was largest for $\mathrm{CsI}$ and $\mathrm{CsOH}$ because they were released at earlier times and were therefore concentrated in the larger particles.) Since containment blowdown times were of the order of $100-200$ seconds (assuming a 7 sq. ft. rupture failure of containment as in BMI-2104), DFs of at least 10 are implied for CsI and $\mathrm{CsOH}$, and at least 5 for other species. In evaluating the optimistic source terms, it was assumed that aerosol aging would occur within the RCS prior to VB, and that these DFs would therefore apply.

Recent detailed review of the Surry TMLB' calculations indicates that the primary system release is not restricted to a puff at VB, as had been assumed previously; much of the volatile release occurs a few minutes before VB. This fact strengthens the arguments offered 
above for giving some credit to the sprays in the optimistic case. It also raises questions as to whether the central treatment, which gives no credit to the sprays in these scenarios, may be unduly conservative. Giving no credit to the sprays continues to be appropriate for the pessimistic representation of these scenarios.

The various guidelines discussed above for generating DFs for sequences involving sprays could not be imposed rigidly in this work. Some variations were required on a case-bycase basis.

\section{Qxidation_Release}

An oxidation release is assumed to arise only in scenarios involving direct containment heating $(D C H)$. It is not treated in the STCP, but crude allowances were made for it in the pessimistic source terms estimated for Bins $16-19$ as described below in Section C.1.3.

\section{Organic Jedine}

Neither the BMI-2104 methodolgy nor QUEST have provided significant basis for reappraisal of release fractions for organic iodine. NUREG-0772 derived an organic iodine release fraction of $0.03 \%$ of the total iodine inventory. A recent NRC assessment concluded that the NUREG-0772 evaluation was on the optimistic side, and its results are therefore used in the optimistic source terms. Pessimistic estimates were based upon the WASH-1233 conclusion that $3.2 \%$ would be a reasonable upper bound for conversion of iodine within containment to organic form. For present purposes, it was assumed this would imply about $2 \%$ of the core inventory being converted to organic iodine, corresponding to about $60 \%$ of the core inventory being within containment (i.e., released from the RCS but not being released to the environment). For those scenarios to which the $80 \%$ iodine release was assigned (Table C6), any additional release associated with organic iodine was neglected.

For sequences in which there was no $\mathrm{CF}$, or there was a basemat melt-through $\mathrm{CF}$ only, it was assumed that $0.03 \%$ (optimistic) or $2 \%$ (pessimistic) of the core inventory would be airborne in organic form within containment at all times, and the actual release was calculated from the leak rate.

\section{Late Releases of $\mathrm{Cs}$ and Jodine.}

A major limitation of the BMI-2104 methodology was that it did not address sources of volatile species (primarily $\mathrm{Cs}$ and $\mathrm{I}$ ) to the containment that might exist at late times. QUEST also addressed this question to only a limited degree. Since the BMI-2104 scenarios generally included features (ESFs or interaction with $\mathrm{CCI}$ aerosols) that more or less rapidly removed 
RCS aerosols from the containment atmosphere, it is hardly surprising that the BMI-2104 calculations, and most of the QUEST studies, indicated release of these species would be very small in any scenario dominated by late containment failures.

In reality, there are several phenomenologies that could lead to significant release of Cs and I at late times, and any source term estimates that ignored them would be difficult to justify as being pessimistic. Some of these phenomenologies include:

1) Revolatilization from the RCS. If substantial amounts of I, Cs, and Te are retained in the RCS, decay heating could ultimately result in revolatilization of at least some of these species. The amounts and timing of the release of this material from the RCS are very uncertain. If it is released gradually over many hours, its subsequent behavior would likely be similar to that of Te released from the melt, which also occurs gradually over many hours. The BMI-2104 analysis for the Surry TMLB' sequence with late CF (TMLB'-epsilon) implies FCONC $=0.36$ for Te. If it is assumed that $50 \%$ of the core inventories of Cs and I are released from the RCS at late times, and that a similar value of FCONC applies, the environmental release fractions for these species would be about 0.18 .

It is possible that the volatile species in the RCS might vaporize, but remain within the RCS for lack of a driving force, as postulated in some IDCOR analyses. Then, given a containment rupture failure, depressurization could transport most of this material into the containment, from which substantial fractions could be released to the environment. Such a scenario might give higher release fractions than the one treated here.

2) Slow settling of RCS releases. If debris beds are coolable, as has been argued by IDCOR and other analysts, or if scrubbing in a flooded cavity is very effective, CCI aerosols will be absent and removal of RCS aerosol from the containment atmosphere will be much slower. In QUEST, calculations with a stand-alone version of MAEROS with aerosol parameters chosen to simulate the BMI-2104 calculations (unit shape factors, no turbulence) indicated as much as $47 \mathrm{~kg}$ of RCS aerosols could remain airborne at seven hours after VB, with concentrations falling off only as the reciprocal of the time. This amount corresponds to about $30 \%$ of the total aerosol calculated to be released from the RCS in the BMI-2104 Surry TMLB' analysis, and would contain about $5 \%$ of the core inventories of iodine and cesium. Substantially higher amounts of these species could remain airborne at late times if it were postulated that the RCS aerosol were richer in Cs and I than calculated in BMI-2104: $47 \mathrm{~kg}$ corresponds to about $30 \%$ of the total core inventories of these species, and amounts remaining airborne could approach these values if RCS aerosol releases were largely limited to these two species. Furthermore, these results were obtained with MAEROS using the standard CONTAIN gravitational agglomeration kernel; using the NAUA kernel could increase them somewhat. (On the other hand, these calculations included no phoretic deposition, although this would not have had a large effect upon the results.) Although this scenario can lead to significant late-time releases of Cs and I, it does so at the expense of there being any significant CCI releases. 
3) Radioactive Decay Chains. In QUEST, it was noted that Te-132 in the melt during CCI could result in a strong and continuing source of I-132 to the containment atmosphere. Although the latter species has a 2.3-hour half life, it could be continually replenished at a rate limited in principle only by the 78-hour half life of Te-132. (In practice, depletion of Te in the melt and cooling of the melt would probably reduce the I-132 source somewhat more rapidly than this.) QUEST calculations with the CONTAIN code, which includes treatment of radioactive decay chains, indicated that as much as $30 \mathrm{MCi}$ of I-132 activity could be airborne at any one time from this source, in the Surry TMLB' sequence. This corresponds to somewhat over $10 \%$ of the total curies of iodine activity in the Surry core inventory at these times. Since I-132 is a potent gamma source, release of $30 \mathrm{MCi}$ could yield short-term gamma doses equivalent to release of as much as $20 \%$ of the total iodine inventory. The short half life would mitigate some of the effects of the I-132 release, relative to a release of all iodine isotopes, but even this mitigation is limited by the presence of the continuing I-132 source within the failed containment. There is no comparable late source of $\mathrm{Cs}$ isotopes due to radioactive decay chains.

4) Resuspension at CE. In QUEST, it was concluded that rapid depressurization of containment following a catastrophic failure could lead to flashing of superheated pools within containment, notably the refueling canal pool and the basement pool. Radionuclides contained therein could be resuspended and released from containment by this mechanism. Results indicted that as much as $20 \%$ of the core inventories of Cs and I might be resuspended and released.

Very large containment failure sizes (100 sq. m. or more) are required for this mechanism to operate effectively. It is also likely that consequences would be mitigated somewhat by a large effective particle size for the resuspended material, although the particle size to be expected has not been quantitatively evaluated.

It also has been argued, with some experimental support, that energetic events such as hydrogen burns could lead to substantial resuspension of previously-deposited aerosols [2]. If such an event were to be the cause of containment failure, a significant contribution to the associated source term could result. No explicit allowance for such effects has been made in the present work, however.

5) Retention in the Melt Until After VB. QUEST calculations indicated that substantial fractions of the core inventories of $\mathrm{Cs}$ and I could remain in the melt at VB, and thus be available for later release during CCI. This behavior would increase the amounts airborne at later times. However, the QUEST results that included this effect still indicated rather rapid release from the melt and subsequent sweepout by $\mathrm{CCI}$ aerosols, so that the period during which Cs and I were available for release was prolonged only 1-2 hours. The scenarios treated were not such as to maximize this effect, but it remains true that the VANESA model appears to predict rather rapid release of $\mathrm{Cs}$ and I for any credible conditions, and greatly prolonged releases of $\mathrm{Cs}$ and I due to this mechanism may be possible only for rather specialized scenarios. An example might be scenarios involving initiallycoolable debris beds, which eventually dry out and heat up after the 
overlying water is boiled off. No calculations are available for any such scenarios.

It is apparent that there are several mechanisms which could conceivably lead to release fractions of $\mathrm{Cs}$ and I in the range $0.05-0.3$, even given late containment failure. It is true that some of these mechanisms place additional restrictions upon the scenario, or apply principally to only a single isotope (i.e., I-132), but the RCS revolatilization scenario is not limited in this way. Hence, pessimistic source terms derived here for sequences with a late rupture $C F$ include release fractions of 0.15 for both Cs and I, except when additional mitigation factors (e.g., sprays) were present.

Since the BMI-2104 methodology used to derive central source terms does not include any of these mechanisms for late releases of Cs and I, they were also neglected in the optimistic source term estimates. For at least two of these mechanisms, RCS revolatilization and decay chains, this neglect should be reconsidered in future work for the central and even the optimistic source terms. These effects could easily equal or exceed the releases associated with organic iodine, which is routinely considered in all source term evaluations.

\section{Pressure Suppression of CCI Vaporization (PSOCV)}

It has been hypothesized that pressurization of the containment can yield a substantial reduction in the release rate of aerosols and radionuclides during $\mathrm{CCI}$, a possible effect which is not treated in the BMI-2104 code suite or the STCP. The principle reason for the effect is that high pressure reduces the volume of gases sparging the melt, since gas volume is proportional to the reciprocal of the pressure. Pressure can also have significant effect upon some of the chemical equilibria involved in vaporization as calculated by VANESA. The magnitude of the effect is very scenario-dependent: it is of no great importance for scenarios involving early rupture CF, but an approximate assessment of the potential effect of PSOCV for some Peach Bottom scenarios involving leakage from the drywell without depressurization showed that order-of-magnitude effects could arise in certain cases. The effect is generally to reduce the rate of release of CCI species but it prolongs the period during which RCS species remain airborne within the containment, and can prolong the time during which volatile constituents remain available for release from the melt. It can therefore change the character of the source term by increasing releases of some species while decreasing releases of others, and no generalizations can be made as to whether it is "optimistic" or "pessimistic" to neglect it.

Since CCI releases do not generally dominate the more important Surry source terms, and since the effect of PSOCV is difficult to characterize in terms of being optimistic or 
pessimistic, it was neglected here. This neglect should not be taken to imply a judgment that it need not be included in future modeling efforts.

\section{C.1.3 Pessimistic and Optimistic Source Terms for Surry}

In the consequence assessment portion of the SARRP analyses, considerable inconvenience would result if the binning structure used for the pessimistic and/or optimistic source terms were redefined to be different from that developed for the central estimate source terms. It was found that this constraint did not unduly interfere with the development of the pessimistic and optimistic source terms and, hence, the binning structure and assignment of sequence/pathway combinations to bins is the same as for the central estimates. Most of the rationale for the derivation of release fractions for these bins has been described in Section 2 of the main report and details are given here only when this is not the case. The discussion of iodine release given here applies only to the particulate release; releases of organic iodine were added in as discussed in Section C.1.2.

\section{Bin 1 (TMLB' with early CE)}

Pessimistic. The TMLB' source term of Table C-6 is assigned for the pessimistic case. It does not include an oxidation release and thus does not apply to scenarios involving direct containment heating.

Optimistic: This source term is derived by assuming the low values of FCOR in Table C.2-3 (except that central Cs and I values were assumed), FVES $=0.01$ for Groups $2-4$ and 0.07 for Groups 5-7, and central values of FCCU/(1-FCOR) derived from the BMI-2104 analysis of the Surry TMLB' sequence. The reduction factor of 0.072 for optimistic aerosol agglomeration assumptions was applied to FCONC but not to FCONV. The rationale for these choices is given in Section C.1.2.

\section{Bin 2 (TML, S3D, etc, with early CF)}

This bin is applied to scenarios similar to those represented by Bin 1 except that the sprays are operating.

Pessimistic: The Bin 1 source term is overwhelmingly dominated by RCS releases; these appear as a puff at $C F$ and sprays are therefore ineffective in the pessimistic analysis, and the continued operation of sprays after CF could make little difference. This bin is therefore the same as Bin 1.

Optimistic: Derived from Bin 1 (optimistic) by crediting spray DFs of 10 for Cs and I, 5 for other RCS releases, and 100 for all CCI releases as discussed in connection with sprays in Section C.1.2. 


\section{Bin 3 (S2D and similar, $C F$ at VB, sprays faill}

Pessimistic: Releases from the RCS are similar to Bin 1, but they do not appear as a puff occurring simultaneously with $\mathrm{CF}$ and sprays have time to act. The pessimistic spray DF for $\mathrm{RCS}$ releases, $\mathrm{DF}=5$, was credited for all species.

Optimistic: For Cs and I, the releases calculated by Battelle assuming a 400 micron drop (BMI-2104) were judged to be quite optimistic enough and were used as given. The Group 6 release in those calculations was also used after multiplying by the low/central FCOR ratio (0.2; see Table C-3). CCI releases were derived by applying the optimistic aerosol agglomeration factor, 0.072 , to central values of $\mathrm{FCCI} /(1-\mathrm{FCOR})$, with the low FCOR values (central for Cs and I) being assumed.

\section{Bin 4 (Similar to Bin 3 except sprays survive after CF)}

Pessimistic: RCS releases strongly dominate Bin 3 (pessimistic) and operation of sprays after CF makes little difference; Bin 4 is the same as Bin 3.

Optimistic: Apply DF of 100 to CCI releases of Bin 3 (optimistic) to credit sprays and cavity scrubbing.

\section{Bin 5. (AG and other scenarios with CF preceding core melt)}

Pessimistic: "High" values of FCOR were taken from Table C-3, and the usual "high" values of FVES were assumed for volatile species; for Groups 5,6 , and 7 , the FVES value $(0.77)$ derived from the STCP calculation for the Surry AG sequence was used. The standard pessimistic value of FCONV, 0.8, was assumed. Since the cavity is flooded in this scenario, $\mathrm{CCI}$ releases contribute little. The resulting source term, given in Table C-7, differs only slightly from the $A B / T M L B$ ' source term in Table $C-6$.

Optimistic: Derived from the central Bin 5 by applying the reduction factor for optimistic agglomeration parameters to both FONC and FCONV (RCS releases are at low pressure and there is little basis for postulating large reductions in FVES, but the RCS species are not released as a puff and the agglomeration reduction factor is therefore credited.) Group 6 release was also reduced by 0.2 (low FCOR). (This calculation was based upon an earlier approximate version of Bin 5 derived by assuming RCS releases were as in the Surry AB sequence and CCI releases as in TMLB'; a derivation based upon the STCP AG calculation would not differ greatly.)

\section{Bin 6 (Isolation failure without spravs)}

Pessimistic: Derived from the central estimate by scaling up in proportion to the (FCOR*FVES+FCCI) values corresponding to the pessimistic scenario used for early releases. For aerosol species, FVES = 0.33 was assumed. (A different source term, with more Group 5 and 7 releases but less Group 2-4 and 6 releases, could be derived from the late-CF pessimistic scenario values for FCOR and FCCI). 
Optimistic: Derived from the central estimate by scaling Groups 2, 3, and 6 in proportion to optimistic values of FCOR*FVES*FCONV and Groups 4, 5 and 7 by corresponding values of (1FCOR)*FCONC (the latter groups are dominated by CCI releases, in the optimistic scenario). Optimistic agglomeration parameters were credited for both FCONC and FCONV (the 6" diameter isolation failure assumed allows time for agglomeration to be effective even if releases from the RCS are as a puff).

\section{Bin 7 (Isolation failure with sprays on)}

Pessimistic: Derived from Bin 6 (pessimistic) by applying the pessimistic spray $\mathrm{DF}$ for RCS releases $(\mathrm{DF}=5$, all species dominated by $\mathrm{RCS}$ releases).

Optimistic: Groups 2, 3, and 6 (released from the RCS) were derived from Bin 6 (optimistic) by crediting a spray DF of 100 but rescinding the credit for optimistic agglomeration parameters, which was judged inapplicable to sprays-on conditions. Groups 4, 5, and 7 (dominated by CCI releases) were derived from Bin 6 (optimistic) by applying the DF of 100 for sprays and cavity scrubbing. As an exercise, an alternate version was derived from the Battelle S2Dbeta calculation with a 400 micron drop [1] by scaling Group 2, 3, and 6 releases to the optimistic FCOR and FVES values, and making no adjustments to Groups 4,5 , and 7 . Results agreed with the present optimistic source term to about a factor of two for all species, indicating once again the present belief that the Battelle spray calculations with a 400 micron drop size are actually quite optimistic.

\section{Bin 8 (Late rupture CF with sprays on: see Bin 9)}

Pessimistic: Derived from Bin 9 (pessimistic) by crediting a spray DF of 5 for RCS species (Groups 2, 3, and 6) and DF of 10 for sprays plus cavity scrubbing for CCI species (Groups 4, 5, and 7).

Optimistic: For Groups 2, 3, and 6 releases were assumed to be effectively zero; "<1E-7" was specified here, but some Battelle calculations for this scenario with a 400 micron drop $[1,3]$ actually gave still smaller release fractions. Groups 4, 5, and 7 were derived from Bin 9 (optimistic) by crediting the DF of 100 for sprays plus cavity scrubbing.

\section{Bin 9 (Late rupture CF with no sprays)}

Pessimistic: Groups 2 and 3 release fractions were estimated to be 0.15 . The base case scenario is gradual revolatilization of about half the CsI and $\mathrm{CsOH}$ that BMI-2104 calculates to be initially deposited in the RCS; however, there are other scenarios yielding comparable results, as discussed in Section C.1.2. Groups 4, 5, and 7 (dominated by CCI releases) were scaled up from the central estimate of Bin 9 in proportion to $\mathrm{FCCI}$ values calculated assuming low values of F COR (Table $C-3$ ) and high values of FCCI/(1-FCOR) (Table C-5). Group 6 was scaled up from the central value by assuming the $S 2$ thermal-hydraulics value of 
FVES, 0.33, and the high value of FCOR; the latter only marginally satisfies consistency requirements. Even so, the resulting release fraction, $4.2 \mathrm{E}-5$, still seems low for a pessimistic estimate.

Optimistic: QUEST results indicated that, with optimistic agglomeration parameters, RCS aerosols are swept out rapidly by CCI aerosols, almost as if sprays were operating, and release fractions of essentially zero ( $<1 \times 10^{-7}$ specified) were therefore assumed for Groups 2, 3, and 6 (there are no late sources of these species in the optimistic scenario). Release of Groups 4, 5, and 7 was derived from the central estimate for Bin 9 by adjusting for high values of FCOR, low values of FCCU/(1-FCOR), and crediting the reduction factor for optimistic aerosol agglomeration parameters.

\section{Bin 10 (Late leakage $C F$ )}

The Battelle analysis used for the central estimate for Bin 10 actually assumed a pressure-dependent leak rate which allowed significant leakage during a period of high pressure following VB (a TMLB' sequence was analyzed). If the leakage failure actually began only at late times, releases of Groups 2,3 , and 6 might be greatly reduced; however,.for Groups 2 and 3, this could easily be compensated for by a delayed release from the RCS.

Pessimistic: Groups 4, 5, and 7 were derived from the central estimate of Bin 10 by scaling to the pessimistic estimate of FCCI. Release of Cs and I was estimated by assuming a gradual $50 \%$ revolatilization from the RCS and a value of FCONC equal to 0.060 (the value implied by the Battelle results for Te). Group 6 was estimated by scaling up the central value by allowing for FVES $=0.33$ (S2 thermal-hydraulics) and a high value of FCOR.

Optimistic: Here it was assumed that the scenario would be as in the Battelle central estimate calculation, i.e., with some early leakage. Groups 2,3 , and 6 were scaled down from the central values in proportion to the low values of FCOR and FVES, while Groups 4, 5, and 7 were reduced by allowing for optimistic agglomeration parameters. Central values of FCCI/(1-FCOR) and low values of FCOR were assumed, since the latter had been assumed in evaluating RCS releases. If leakage actually did not begin until late times, a smaller source term could be derived because there would be no need to minimize RCS release. Releases of Groups 2,3 , and 6 could be virtually eliminated and release of Te reduced by almost a factor of ten by postulating the high value of FCOR instead of the low value.

\section{Bins 11 and 12 (V sequence, with and without water)}

Pessimistic: BMI-2104 implies FVES values of about 0.5 for $\mathrm{CsI}$ and $\mathrm{CsOH}$ in the Surry $V$ sequence, with most of this being deposited by aerosol processes. However, the report also points out that the calculations imply a deposition of about 50 grams of aerosol per centimeter in the 6" pipe forming the release path, and raises the 
question of whether this aerosol might form an obstruction, increasing the pressure and flow rates to the point where the aerosol might be resuspended. Furthermore, the BMI-2104 analysis neglected decay heating and revolatilization. Hence, the pessimistic value of FVES $=0.9$ was retained for this case also, even though many of the QUEST-based arguments of Section C.1.2 are not applicable because the $\mathrm{V}$ sequence is dominated by net flow transport rather than by natural circulation as in the cases considered in QUEST. Given this value of FVES, the V-sequence source term in Table C- 6 is applicable to the $\mathrm{V}$ sequence without water and this source term was therefore assigned to Bin 12. Bin 11 (water in the pathway) was then derived from this result by applying the pessimistic DF of 2 for scrubbing derived as discussed in Section C.1.2.

Optimistic: The usual optimistic FCOR values were assumed. It was judged that uncertainties in FVES for the volatile species should be somewhat smaller than for the higher-pressure sequences, since the transport process treated by the BMI-2104 methodology (net flow) is the dominant process for this sequence. On the other hand, it was judged that uncertainties remain substantial. A value of 0.1 was assigned, with very little justification being possible.

After most of this work had been performed, an error in the TRAP-MELT treatment of turbulent deposition of aerosols was identified and corrected, and the V-sequence TRAPMELT calculations were redone [3]. As a result, FVES was reduced from 0.5 to 0.2 for CsI and $\mathrm{CsOH}$, with corresponding reductions being made in the $\mathrm{V}$-sequence central source term estimates (NAUA calculations were not repeated). Still more recent calculations at BNL using the STCP [7]), which includes effects of decay heating upon revolatilization prior to vessel breach, yielded FVES values for Cs and I close to the original values (i.e, about 0.5 ). Releases of refractories (Groups 5, 6, and 7) were significantly reduced by other effects in the STCP treatment, however.

Since there was so little relation between the optimistic and pessimistic values and any calculated result to begin with, the change in the calculated results still provides little basis for changing the optimistic and pessimistic values. Hence, no changes were made in FVES for the volatile species. Since central values of FVES for aerosol species were used for all cases, the value assumed for the optimistic source term was reduced by a factor of 2.5 from the BMI2104 value in order to bring it into better agreement with the more recent calculations.

In the $\mathrm{V}$ sequence, "FCONV" represents the fractional release from the safeguards building and was about 0.85 in the BMI-2104 calculations, for the case without water in the pathway. For this case, it was judged reasonable to take some credit for the aerosol agglomeration uncertainties for the optimistic source term. The factor of 0.072 used elsewhere 
is not directly applicable in this case because, in the central case, most aerosol is being released rather than deposited and because the aerosol source strength is smaller by a factor of five in the optimistic case. An approximate model was used to define a base case for which the approximate model predicted FCONV $=0.85$ as in BMI-2104. The input to the approximate model was then changed to correspond to the aerosol agglomeration parameters and source strength characterizing the optimistic case. The resulting value of FCONV, 0.24 , was then used to estimate the optimistic source term.

For the CCI release, the full agglomeration uncertainty factor of 0.072 was credited for enhanced deposition within the containment in the optimistic case. No additional credit was taken for enhanced deposition in the safeguards building.

In the $\mathrm{V}$ sequence with water (Bin 11), an optimistic DF of 12.4 for scrubbing, derived as in Section C.1.2.6, was applied to all releases in Bin 12 (optimistic). However, the credit for enhanced aerosol deposition in the safeguards building was rescinded for the $\mathrm{V}$ sequence with water in the pathway, because this scenario is inconsistent with the physical assumptions underlying the enhanced deposition rates in QUEST, namely, dry aerosol shape factors combined with convective turbulence due to a substantial heat source.

Bins 13 and 14 (Basemat melt-through)

Battelle has defined two bins for basemat melt-through scenarios. In one it was assumed that the containment is highly pressurized due to the absence of containment heat removal systems, and that this pressurization forces a complete release of noble gas (but not organic iodine). In the other scenario, pressurization was limited and only a leakage source term applies, which was calculated by integrating the design basis leakage over 30 days. For the present work, two separate scenarios were not treated. Instead, the first scenario (complete noble gas release) was assumed for the pessimistic case and the second (leakage only) was assumed for the optimistic case. Releases of aerosol species were as in Bin 15.

Bin 15 (No CF--design basis leakage only)

Pessimistic: Calculated as for the central estimate except using the high values of FCOR and FVES, central values of FCCV/(1-FCOR), and the pessimistic estimate $(2 \%)$ of the iodine fraction present as organic iodine in the containment atmosphere.

Optimistic: Calculated as for the central estimate except using the low values of FCOR and FVES, central values of FCCI/(1-FCOR), the reduction factor for optimistic aerosol agglomeration parameters was applied to the aerosol residence time, and the optimistic estimate $(0.03 \%)$ of the iodine fraction present as organic iodine was used. 
In both cases, the leakage was integrated over five days in estimating the release of organic iodine and noble gas. In neither case was credit for sprays taken; substantial reductions in the aerosol species leaked would apply when sprays are available.

\section{Bins 16 - 20 (Direct Heating Scenarios)}

Bins $16-19$ are analogous to Bins $1-4$, respectively, except that direct containment heating $(\mathrm{DCH})$ is assumed to be a factor in causing the early containment failure, and an oxidation release is therefore added in to the releases otherwise calculated for these bins. DCH does not exist in the optimist's world; hence, in the optimistic analysis, Bins $16-19$ are identical to Bins $1-4$, respectively.

Bin 20 represents a recent addition made as the result of recent ASEP analyses and the LLH results which, taken together, imply that the risk-dominant scenario at Surry, in terms of contributing to the mean LLH risk, involves plant damage state SNNN. This state arises as a result of a station blackout with large pump seal LOCAs (S2 total effective size) or PORV failure to close, leading to core melt at intermediate pressure with no ESFs available. Containment failure in this scenario is at vessel breach and involves direct heating. The central and pessimistic estimates are derived from Bin 1 by adding in an oxidation release equivalent to half the Bin 16 oxidation release, in keeping with the reduced DCH associated with intermediate primary system pressure postulated in the CET.

The oxidation release is assumed to be proportional to the amount of fuel participating in DCH. For the Bin 16 pessimistic analysis, it is assumed that $50 \%$ of the fuel participates in $\mathrm{DCH}$, corresponding to the pessimistic CET estimate; for Bins 17 - 20 the amounts are reduced by factors of $0.5,0.2,0.2$, and 0.5 , respectively. These reduction factors correspond approximately to the assumptions used in the CET for analyzing containment loads in the scenarios to which these bins are applied.

The direct heating release itself is calculated by assuming that, for the fuel which actually participates in direct heating, $100 \%$ of the $\mathrm{Te}$ and $90 \%$ of the $\mathrm{Ru}$ (as in the WASH1400 oxidation release) are released. It was also assumed that $1 \%$ of all the ejected corium was aerosolized and therefore contributes to the source term. Since the core ejection fraction was $100 \%$ in the pessimistic CET walk-through, this implies a minimum environmental release of $0.8 \times 0.01=8 \times 10^{-3}$ for all the direct heating cases. The latter contribution is not important except for Group 7 and has therefore been calculated only for the latter. All the oxidation release fractions given above apply, of course, only to those radionuclides remaining in the fuel at the time of vessel breach. 
Finally, $80 \%$ of all radionuclides released to the containment during direct heating are assumed to be released to the environment, provided the containment fails at the time of vessel breach (or earlier). This $80 \%$ release factor was based upon an isentropic blowdown calculation performed by hand.

The pessimistic source terms derived here are summarized in Table C-7, while the optimistic source terms are summarized in Table C-8. For the sake of completeness, the central source terms are also given in Table C-9. The MACCS importance measures, Qe and Ql, are also given in the last two columns of the table. In most of the optimistic source terms, and some of the central, the early consequence measure $\mathrm{Qe}$ is dominated by noble gas $(\mathrm{Qe}=0.0775$ for $100 \%$ noble gas release). In these cases, the importance measure is defined in a way that overestimates the significance of this noble gas release; it might be more meaningful to subtract out the noble gas contribution in such cases. When the source term is larger, the importance measures provide a more realistic accounting of the (relatively small) noble gas contribution to the total. Of course, the timing of the release also has very important implications for early health effects.

The release fractions given in the tables are those actually used in the Surry pessimistic and optimistic consequence calculations. For the $\mathrm{V}$ sequence, the pessimistic releases differ slightly from those in Table C- 6 because the consequence calculations were actually performed using an earlier estimate, and the changes to the source term reflected by the Table C- 6 values were not judged sufficiently important to demand recalculation at this time, considering other demands upon the available resources. Future recalculations of the Surry risk profile will employ the Table C-6 source term for Bin 12 (pessimistic) and an analogous source term for Bin 11. 
NUREG/CR-4551, VOL. 1: DRAFT REPORT FOR COMMENT (SEPTEMBER, 1986)

Table C-7

SURRY PESSIMISTIC SOURCE TERMS

\begin{tabular}{|c|c|c|c|c|c|c|c|c|c|}
\hline \multirow[b]{2}{*}{ Bin } & \multirow[b]{2}{*}{1} & \multicolumn{6}{|c|}{ WASH-1400 Release Groups } & \multicolumn{2}{|c|}{$\begin{array}{l}\text { MACCS } \\
\text { 'Importance' }\end{array}$} \\
\hline & & 2 & 3 & 4 & 5 & 6 & 7 & $\mathrm{Qe}$ & Q1 \\
\hline 1 & $1.0 \mathrm{E}+0$ & $8.0 \mathrm{E}-1$ & $7.2 \mathrm{E}-1$ & $6.5 \mathrm{E}-1$ & $5.2 \mathrm{E}-2$ & $6.2 \mathrm{E}-3$ & $6.6 \mathrm{E}-4$ & $1.56 \mathrm{E}+0$ & $9.28 \mathrm{E}-1$ \\
\hline 2 & $1.0 \mathrm{E}+0$ & $8.0 \mathrm{E}-1$ & $7.2 \mathrm{E}-1$ & $6.5 \mathrm{E}-1$ & $5.2 \mathrm{E}-2$ & $6.2 \mathrm{E}-3$ & $6.6 \mathrm{E}-4$ & $1.56 \mathrm{E}+0$ & $9.28 \mathrm{E}-1$ \\
\hline 3 & $1.0 \mathrm{E}+0$ & $1.8 \mathrm{E}-1$ & $1.4 \mathrm{E}-1$ & $1.4 \mathrm{E}-1$ & $5.0 \mathrm{E}-2$ & $5.8 \mathrm{E}-3$ & $6.4 \mathrm{E}-4$ & $4.73 \mathrm{E}-1$ & $2.30 \mathrm{E}-1$ \\
\hline 4 & $1.0 \mathrm{E}+0$ & $1.8 \mathrm{E}-1$ & $1.4 \mathrm{E}-1$ & $1.4 \mathrm{E}-1$ & $5.0 \mathrm{E}-2$ & $5.8 \mathrm{E}-3$ & $6.4 \mathrm{E}-4$ & $4.73 \mathrm{E}-1$ & $2.30 \mathrm{E}-1$ \\
\hline 5 & $1.0 \mathrm{E}+0$ & $8.0 \mathrm{E}-1$ & $7.2 \mathrm{E}-1$ & $6.5 \mathrm{E}-1$ & $3.0 \mathrm{E}-1$ & $6.8 \mathrm{E}-2$ & 4.7E-3 & $2.09 \mathrm{E}+0$ & $1.25 \mathrm{E}+0$ \\
\hline 6 & $1.0 \mathrm{E}+0$ & $1.8 \mathrm{E}-1$ & $8.4 \mathrm{E}-2$ & $3.6 \mathrm{E}-1$ & $1.3 \mathrm{E}-1$ & $2.0 \mathrm{E}-2$ & $1.8 \mathrm{E}-3$ & $7.95 \mathrm{E}-1$ & $2.94 \mathrm{E}-1$ \\
\hline 7 & $1.0 \mathrm{E}+0$ & $5.2 \mathrm{E}-2$ & $1.7 \mathrm{E}-2$ & 7.2E-2 & $2.6 \mathrm{E}-2$ & 4.1E-3 & $3.6 E-4$ & $2.37 \mathrm{E}-$ & $6.17 \mathrm{E}-2$ \\
\hline 8 & $1.0 \mathrm{E}+0$ & $5.0 \mathrm{E}-2$ & $3.0 \mathrm{E}-2$ & $2.4 \mathrm{E}-2$ & $5.3 \mathrm{E}-3$ & $8.0 \mathrm{E}-6$ & $3.4 E-4$ & $1.62 \mathrm{E}-1$ & $4.61 \mathrm{E}-2$ \\
\hline 9 & $1.0 \mathrm{E}+0$ & $1.7 \mathrm{E}-1$ & $1.5 \mathrm{E}-1$ & $2.4 \mathrm{E}-1$ & $5.3 \mathrm{E}-2$ & 4.2E-5 & $3.4 \mathrm{E}-3$ & $5.79 \mathrm{E}-1$ & $2.68 \mathrm{E}-1$ \\
\hline 10 & $1.0 \mathrm{E}+0$ & $5.0 \mathrm{E}-2$ & $3.0 \mathrm{E}-2$ & $3.7 \mathrm{E}-2$ & 2.1E-2 & $2.2 \mathrm{E}-3$ & $8.9 E-4$ & $2.07 \mathrm{E}-1$ & $6.85 \mathrm{E}-2$ \\
\hline 11 & $1.0 \mathrm{E}+0$ & 3.5E-1 & $3.3 \mathrm{E}-1$ & $3.2 \mathrm{E}-1$ & 3.5E-2 & 2.5E-2 & 4.5E-3 & $8.9 \mathrm{E}-1$ & $4.95 \mathrm{E}-1$ \\
\hline 12 & $1.0 \mathrm{E}+0$ & $6.7 \mathrm{E}-1$ & $6.7 \mathrm{E}-1$ & $6.4 \mathrm{E}-1$ & $7.0 \mathrm{E}-2$ & $5.0 \mathrm{E}-2$ & $9.0 \mathrm{E}-3$ & $1.67 \mathrm{E}+0$ & $9.96 \mathrm{E}-1$ \\
\hline 13 & $1.0 \mathrm{E}+0$ & $1.7 \mathrm{E}-4$ & $1.0 \mathrm{E}-4$ & $9.7 \mathrm{E}-5$ & $1.1 \mathrm{E}-5$ & $8.9 \mathrm{E}-7$ & $1.3 \mathrm{E}-7$ & $7.78 \mathrm{E}-2$ & $1.24 \mathrm{E}-3$ \\
\hline 14 & $1.0 \mathrm{E}+0$ & $1.7 \mathrm{E}-4$ & $1.0 \mathrm{E}-4$ & $9.7 \mathrm{E}-5$ & $1.1 \mathrm{E}-5$ & $8.9 \mathrm{E}-7$ & $1.3 \mathrm{E}-7$ & $7.78 \mathrm{E}-2$ & $1.24 \mathrm{E}-3$ \\
\hline 15 & $5.0 \mathrm{E}-3$ & $1.2 \mathrm{E}-4$ & $1.0 \mathrm{E}-4$ & $9.7 \mathrm{E}-5$ & $1.1 \mathrm{E}-5$ & $8.9 \mathrm{E}-7$ & $1.3 \mathrm{E}-7$ & $6.14 \mathrm{E}-4$ & $1.40 \mathrm{E}-4$ \\
\hline 16 & $1.0 \mathrm{E}+0$ & $8.0 \mathrm{E}-1$ & $7.2 \mathrm{E}-1$ & $6.9 \mathrm{E}-1$ & $5.6 \mathrm{E}-2$ & 3.3E-1 & $8.7 \mathrm{E}-3$ & $2.42 E+0$ & $1.36 \mathrm{E}+0$ \\
\hline 17 & $1.0 \mathrm{E}+0$ & 8.0E-1 & $7.2 \mathrm{E}-1$ & $6.7 \mathrm{E}-1$ & $5.6 \mathrm{E}-2$ & $1.7 \mathrm{E}-1$ & 8.7E-3 & $2.06 \mathrm{E}+0$ & $1.18 \mathrm{E}+0$ \\
\hline 18 & $1.0 \mathrm{E}+0$ & $1.8 \mathrm{E}-1$ & $1.4 \mathrm{E}-1$ & $1.5 \mathrm{E}-1$ & $5.4 \mathrm{E}-2$ & $7.6 \mathrm{E}-2$ & $8.6 \mathrm{E}-3$ & $7.65 \mathrm{E}-1$ & $3.78 \mathrm{E}-1$ \\
\hline 19 & $1.0 \mathrm{E}+0$ & $1.8 \mathrm{E}-1$ & $1.4 \mathrm{E}-1$ & $1.5 \mathrm{E}-1$ & $5.4 \mathrm{E}-2$ & $7.6 \mathrm{E}-2$ & $8.6 \mathrm{E}-3$ & $7.65 \mathrm{E}-1$ & $3.78 \mathrm{E}-1$ \\
\hline 20 & $1.0 \mathrm{E}+0$ & $8.0 \mathrm{E}-1$ & $7.2 \mathrm{E}-1$ & $6.7 \mathrm{E}-1$ & $5.6 \mathrm{E}-2$ & $1.7 \mathrm{E}-1$ & $8.7 \mathrm{E}-3$ & $2.06 \mathrm{E}+0$ & $1.18 \mathrm{E}+0$ \\
\hline
\end{tabular}


NUREG/CR-4551, VOL. 1: DRAFT REPORT FOR COMMENT (SEPTEMBER, 1986)

Table C-8

SURRY OPTIMISTIC SOURCE TERMS

\begin{tabular}{|c|c|c|c|c|c|c|c|c|c|}
\hline \multirow[b]{2}{*}{ Bin } & \multirow[b]{2}{*}{1} & \multicolumn{6}{|c|}{ WASH-1400 Release Groups } & \multicolumn{2}{|c|}{$\begin{array}{l}\text { MACCS } \\
\text { 'Importance' }\end{array}$} \\
\hline & & 2 & 3 & 4 & 5 & 6 & 7 & $\mathrm{Qe}$ & $\frac{\text { Tance }}{\text { QI }}$ \\
\hline 1 & $1.0 \mathrm{E}+0$ & $3.3 \mathrm{E}-3$ & $3.0 \mathrm{E}-3$ & $1.0 \mathrm{E}-2$ & $3.7 \mathrm{E}-3$ & $1.7 \mathrm{E}-4$ & $1.7 \mathrm{E}-5$ & $9.46 \mathrm{E}-2$ & $9.13 \mathrm{E}-$ \\
\hline 2 & $1.0 \mathrm{E}+0$ & $6.0 \mathrm{E}-4$ & $3.0 \mathrm{E}-4$ & $1.2 \mathrm{E}-4$ & $9.2 \mathrm{E}-5$ & $3.4 \mathrm{E}-5$ & $2.3 E-7$ & $7.84 \mathrm{E}-2$ & $1.60 \mathrm{E}-3$ \\
\hline 3 & $1.0 \mathrm{E}+0$ & $4.2 \mathrm{E}-4$ & $1.1 \mathrm{E}-4$ & $1.2 \mathrm{E}-3$ & 2.6E-3 & $2.2 E-4$ & $8.0 \mathrm{E}-6$ & $8.30 \mathrm{E}-2$ & $4.03 \mathrm{E}-3$ \\
\hline 4 & $1.0 \mathrm{E}+0$ & $4.2 E-4$ & $1.1 \mathrm{E}-4$ & $1.2 \mathrm{E}-5$ & $2.6 \mathrm{E}-5$ & $4.4 \mathrm{E}-5$ & $6.8 \mathrm{E}-7$ & $7.81 \mathrm{E}-2$ & $1.33 \mathrm{E}-3$ \\
\hline 5 & $1.0 \mathrm{E}+0$ & $2.8 \mathrm{E}-2$ & $2.7 \mathrm{E}-2$ & $1.4 \mathrm{E}-2$ & $6.2 \mathrm{E}-3$ & $1.7 \mathrm{E}-$ & $41.2 \mathrm{E}-5$ & $1.28 \mathrm{E}-1$ & $3.82 \mathrm{E}-2$ \\
\hline 6 & $1.0 \mathrm{E}+0$ & $4.1 \mathrm{E}-4$ & $6.2 E-5$ & $1.3 \mathrm{E}-2$ & $4.5 \mathrm{E}-3$ & $2.3 \mathrm{E}-5$ & $1.9 \mathrm{E}-5$ & $9.45 \mathrm{E}-2$ & $6.78 \mathrm{E}-3$ \\
\hline 7 & $1.0 \mathrm{E}+0$ & $3.2 \mathrm{E}-4$ & 8.6E-6 & $1.3 \mathrm{E}-4$ & $4.5 \mathrm{E}-5$ & $3.2 \mathrm{E}-6$ & $1.9 \mathrm{E}-7$ & $7.80 \mathrm{E}-2$ & $1.20 \mathrm{E}-3$ \\
\hline 8 & $1.0 \mathrm{E}+0$ & $3.0 \mathrm{E}-4$ & $1.0 \mathrm{E}-7$ & $6.7 \mathrm{E}-6$ & $6.9 \mathrm{E}-6$ & $1.0 \mathrm{E}-7$ & $2.5 \mathrm{E}-8$ & $7.78 \mathrm{E}-2$ & $1.14 \mathrm{E}-3$ \\
\hline 9 & $1.0 \mathrm{E}+0$ & $3.0 \mathrm{E}-4$ & $1.0 \mathrm{E}-7$ & $6.7 \mathrm{E}-4$ & $6.9 \mathrm{E}-4$ & $1.0 \mathrm{E}-7$ & $2.5 \mathrm{E}-6$ & $7.93 \mathrm{E}-2$ & $1.84 \mathrm{E}-3$ \\
\hline 10 & $1.0 \mathrm{E}+0$ & $3.1 \mathrm{E}-4$ & $5.3 \mathrm{E}-6$ & $1.3 \mathrm{E}-3$ & 4.7E-4 & $2.4 \mathrm{E}-6$ & $1.7 \mathrm{E}-6$ & $7.95 \mathrm{E}-2$ & $1.71 \mathrm{E}-3$ \\
\hline 11 & $1.0 \mathrm{E}+0$ & $6.6 \mathrm{E}-3$ & $6.5 \mathrm{E}-3$ & $7.2 \mathrm{E}-4$ & $5.3 \mathrm{E}-4$ & $2.0 \mathrm{E}-4$ & $1.9 \mathrm{E}-5$ & $8.69 \mathrm{E}-2$ & $9.19 \mathrm{E}-$ \\
\hline 12 & $1.0 \mathrm{E}+0$ & $2.3 \mathrm{E}-2$ & 2.3E-2 & $8.2 \mathrm{E}-3$ & $3.6 \mathrm{E}-3$ & $7.1 \mathrm{E}-3$ & $2.4 \mathrm{E}-4$ & $1.33 \mathrm{E}-1$ & $4.04 \mathrm{E}-$ \\
\hline 13 & $3.0 \mathrm{E}-2$ & $9.1 \mathrm{E}-6$ & $7.7 \mathrm{E}-8$ & $2.2 \mathrm{E}-6$ & $8.6 \mathrm{E}-7$ & $2.7 \mathrm{E}-8$ & $4.7 \mathrm{E}-9$ & $2.34 \mathrm{E}-3$ & $3.51 \mathrm{E}-$ \\
\hline 14 & $3.0 \mathrm{E}-2$ & $9.1 \mathrm{E}-6$ & $7.7 \mathrm{E}-8$ & $2.2 \mathrm{E}-6$ & $8.6 \mathrm{E}-7$ & $2.7 \mathrm{E}-8$ & $4.7 \mathrm{E}-9$ & $2.34 \mathrm{E}-3$ & $3.51 \mathrm{E}$ \\
\hline 15 & $5.0 \mathrm{E}-3$ & $1.6 \mathrm{E}-6$ & 7.7E-8 & $2.2 \mathrm{E}-6$ & $8.6 \mathrm{E}-7$ & $2.7 \mathrm{E}-8$ & 4.7E-9 & $3.92 \mathrm{E}-4$ & $6.81 \mathrm{E}-6$ \\
\hline 16 & $1.0 \mathrm{E}+0$ & 3.3E-3 & 3.0E-3 & $1.0 \mathrm{E}-2$ & $3.7 \mathrm{E}-3$ & $1.7 \mathrm{E}-4$ & $1.7 \mathrm{E}-5$ & $9.46 \mathrm{E}-2$ & $9.13 \mathrm{E}-3$ \\
\hline 17 & $1.0 \mathrm{E}+0$ & $6.0 \mathrm{E}-4$ & $3.0 \mathrm{E}-4$ & $1.2 \mathrm{E}-4$ & $9.2 \mathrm{E}-5$ & $3.4 \mathrm{E}-5$ & $2.3 \mathrm{E}-7$ & $7.84 \mathrm{E}-2$ & $1.60 \mathrm{E}-3$ \\
\hline 18 & $81.0 \mathrm{E}+0$ & 4.2E-4 & $1.1 \mathrm{E}-4$ & $1.2 \mathrm{E}-3$ & $2.6 \mathrm{E}-3$ & $2.2 E-4$ & $8.0 \mathrm{E}-6$ & $8.30 \mathrm{E}-2$ & $4.03 \mathrm{E}-3$ \\
\hline 19 & $1.0 \mathrm{E}+0$ & $4.2 \mathrm{E}-4$ & $1.1 \mathrm{E}-4$ & $1.2 \mathrm{E}-5$ & $2.6 \mathrm{E}-5$ & $4.4 \mathrm{E}-5$ & $6.8 \mathrm{E}-7$ & $7.81 \mathrm{E}-2$ & $1.33 \mathrm{E}-3$ \\
\hline 20 & $1.0 \mathrm{E}+0$ & 3.3E-3 & 3.0E-3 & $1.0 \mathrm{E}-2$ & $3.7 \mathrm{E}-3$ & $1.7 \mathrm{E}-4$ & $1.7 \mathrm{E}-5$ & $9.46 \mathrm{E}-2$ & $9.13 \mathrm{E}-3$ \\
\hline
\end{tabular}


NUREG/CR-4551, VOL. 1: DRAFT REPORT FOR COMMENT (SEPTEMBER, 1986)

Table C-9

SURRY CENTRAL SOURCE TERMS

\begin{tabular}{|c|c|c|c|c|c|c|c|c|c|}
\hline \multirow[b]{2}{*}{$\mathrm{Bin}$} & \multirow[b]{2}{*}{1} & \multicolumn{6}{|c|}{ WASH-1400 Release Groups } & \multicolumn{2}{|c|}{$\begin{array}{l}\text { MACCS } \\
\text { 'Importance' }\end{array}$} \\
\hline & & 2 & 3 & 4 & 5 & 6 & 7 & Qe & $\mathrm{Ql}$ \\
\hline 1 & $1.0 \mathrm{E}+0$ & $1.9 \mathrm{E}-1$ & $1.6 \mathrm{E}-1$ & $1.0 \mathrm{E}-1$ & $1.7 \mathrm{E}-2$ & $3.0 \mathrm{E}-3$ & $1.9 \mathrm{E}-4$ & $3.96 \mathrm{E}-1$ & $2.11 \mathrm{E}-1$ \\
\hline 2 & $1.0 \mathrm{E}+0$ & $1.9 \mathrm{E}-1$ & $1.6 \mathrm{E}-1$ & 8.7E-2 & $1.2 \mathrm{E}-3$ & $3.0 \mathrm{E}-3$ & $3.4 \mathrm{E}-5$ & $3.62 \mathrm{E}-1$ & $1.94 \mathrm{E}-1$ \\
\hline 3 & $1.0 \mathrm{E}+0$ & $1.5 \mathrm{E}-2$ & $1.8 \mathrm{E}-2$ & $1.2 \mathrm{E}-2$ & $3.4 \mathrm{E}-2$ & $3.0 \mathrm{E}-3$ & $9.9 \mathrm{E}-4$ & $1.72 \mathrm{E}-1$ & $6.38 \mathrm{E}-2$ \\
\hline 4 & $1.0 \mathrm{E}+0$ & $1.0 \mathrm{E}-2$ & $4.4 E-3$ & $5.4 \mathrm{E}-4$ & $1.8 E-3$ & $5.8 E-4$ & $3.9 \mathrm{E}-6$ & $9.22 E-2$ & $8.86 \mathrm{E}-3$ \\
\hline 5 & $1.0 \mathrm{E}+0$ & $5.8 \mathrm{E}-1$ & 5.7E-1 & 4.7E-1 & $1.0 \mathrm{E}$ & 8.0E-7 & $1.0 \mathrm{E}-4$ & $1.10 \mathrm{E}+0$ & $6.88 \mathrm{E}-1$ \\
\hline 6 & $1.0 \mathrm{E}+0$ & 2.7E-2 & $1.3 \mathrm{E}-2$ & $1.2 \mathrm{E}-1$ & $6.2 \mathrm{E}-2$ & $1.6 \mathrm{E}-3$ & $2.6 \mathrm{E}-4$ & $2.91 \mathrm{E}-1$ & $8.90 \mathrm{E}-2$ \\
\hline 7 & $1.0 \mathrm{E}+0$ & 1.7E-2 & $1.0 \mathrm{E}-2$ & $3.2 \mathrm{E}-3$ & $4.2 \mathrm{E}-3$ & $1.0 \mathrm{E}-3$ & $1.2 \mathrm{E}-5$ & $1.06 \mathrm{E}-1$ & $1.81 \mathrm{E}-2$ \\
\hline 8 & $1.0 \mathrm{E}+0$ & $5.0 \mathrm{E}-3$ & $2.5 \mathrm{E}-9$ & $6.4 \mathrm{E}-3$ & $5.5 \mathrm{E}-5$ & $2.0 \mathrm{E}-7$ & $1.2 \mathrm{E}-7$ & $8.76 \mathrm{E}-2$ & $2.32 \mathrm{E}-3$ \\
\hline 9 & $1.0 \mathrm{E}+0$ & $7.8 \mathrm{E}-3$ & $3.9 \mathrm{E}-4$ & 8.5E-2 & $1.8 \mathrm{E}-2$ & $3.3 \mathrm{E}-6$ & $8.1 \mathrm{E}-5$ & $1.77 \mathrm{E}-1$ & $2.80 \mathrm{E}-2$ \\
\hline 10 & $1.0 \mathrm{E}+0$ & $6.9 E-3$ & $1.1 \mathrm{E}-3$ & $1.3 \mathrm{E}-2$ & $5.8 \mathrm{E}-3$ & $1.7 \mathrm{E}-4$ & $2.4 \mathrm{E}-5$ & $1.03 \mathrm{E}-1$ & $9.85 \mathrm{E}-3$ \\
\hline 11 & $1.0 \mathrm{E}+0$ & 4.1E-2 & 3.5E- & $2.0 \mathrm{E}-2$ & $1.1 \mathrm{E}-2$ & $1.5 \mathrm{E}-3$ & $9.3 E-4$ & $1.71 \mathrm{E}-1$ & $6.13 \mathrm{E}-2$ \\
\hline 12 & $1.0 \mathrm{E}+0$ & $1.7 \mathrm{E}-1$ & $1.5 \mathrm{E}-1$ & $1.1 \mathrm{E}-1$ & $6.2 \mathrm{E}-2$ & $9.0 \mathrm{E}-3$ & $5.5 \mathrm{E}-3$ & 5.43E-1 & $2.90 \mathrm{E}-1$ \\
\hline 13 & $1.0 \mathrm{E}+0$ & $1.5 \mathrm{E}-4$ & $1.6 \mathrm{E}-5$ & $3.1 E-5$ & $1.2 \mathrm{E}-5$ & $3.7 \mathrm{E}-7$ & $6.5 \mathrm{E}-8$ & $7.77 \mathrm{E}-2$ & $1.15 \mathrm{E}-3$ \\
\hline 14 & $3.0 \mathrm{E}-2$ & $1.5 \mathrm{E}-4$ & $1.6 \mathrm{E}-5$ & $3.1 \mathrm{E}-5$ & $1.2 \mathrm{E}-5$ & $3.7 E-7$ & $6.5 \mathrm{E}-8$ & $2.52 \mathrm{E}-3$ & $7.90 \mathrm{E}-5$ \\
\hline 15 & $2.5 \mathrm{E}-3^{*}$ & $4.2 \mathrm{E}-5$ & $1.6 \mathrm{E}-5$ & $3.1 \mathrm{E}-5$ & $1.2 \mathrm{E}-5$ & $3.7 \mathrm{E}-7$ & $6.5 \mathrm{E}-8$ & $2.80 \mathrm{E}-4$ & $3.79 \mathrm{E}-5$ \\
\hline 16 & $1.0 \mathrm{E}+0$ & $1.9 \mathrm{E}-1$ & $1.6 \mathrm{E}-1$ & $1.3 \mathrm{E}-1$ & $1.7 \mathrm{E}-2$ & 7.5E-2 & $1.7 \mathrm{E}-3$ & 5.97E-1 & $3.07 \mathrm{E}-1$ \\
\hline 17 & $1.0 \mathrm{E}+0$ & $1.9 \mathrm{E}-1$ & $1.6 \mathrm{E}-1$ & $1.0 \mathrm{E}-1$ & $1.2 \mathrm{E}-2$ & $3.9 \mathrm{E}-2$ & $7.8 \mathrm{E}-4$ & $4.76 \mathrm{E}-1$ & $2.51 \mathrm{E}-1$ \\
\hline 18 & $1.0 \mathrm{E}+0$ & $1.5 \mathrm{E}-2$ & $1.8 \mathrm{E}-2$ & $1.9 \mathrm{E}-2$ & $3.4 \mathrm{E}-2$ & $1.7 \mathrm{E}-2$ & $1.3 \mathrm{E}-3$ & 2.13E-1 & 8.27E-2 \\
\hline 19 & $1.0 \mathrm{E}+0$ & $1.0 \mathrm{E}-2$ & $4.4 \mathrm{E}-3$ & $7.1 \mathrm{E}-3$ & $1.8 \mathrm{E}-3$ & $1.5 \mathrm{E}-2$ & $3.0 \mathrm{E}-4$ & $1.33 \mathrm{E}-1$ & $2.81 \mathrm{E}-2$ \\
\hline 20 & $1.0 \mathrm{E}+0$ & $1.9 \mathrm{E}-1$ & $1.6 \mathrm{E}-1$ & $1.1 \mathrm{E}-1$ & $1.7 \mathrm{E}-2$ & $3.9 \mathrm{E}-2$ & $9.4 \mathrm{E}-4$ & 4.93E-1 & $2.58 \mathrm{E}-1$ \\
\hline
\end{tabular}

*Later verification indicated that this should have been 5.0E-3, an error with no risk implication. 


\section{C.2. SURRY SOURCE TERM ISSUE OUTCOMES AND WEIGHTING FACTORS FOR THE LIMITED LATIN HYPERCUBE STUDY}

\section{C.2.1 Introduction}

This section describes the representation of Surry source term issues as finally implemented in the Limited Latin Hypercube (LLH) study of uncertainties in the Surry risk profile. It is based upon the earlier "strawman" proposal, but with substantial revisions in order to reflect the comments and revisions made by the source term review group, and to document the representation of issues and weighting factors as actually carried out in the LLH study. In order to complete the documentation, the listing of the SURSOR code that carries out the source term evaluations used in the LLH, given the required level specifications, has been included in Section C.2.5. The present report also gives, in Section C.2.3, the weighting factor "votes" of the individual reviewers, as well as the composite weighting factors which are the values actually employed by the LLH; with the consent of the review group, these composite values were simply taken to be the arithmetic means of the votes of the individual reviewers on each issue. The section on each issue also includes a summary of the comments provided by the review team at or following the meeting.

The input required for the study employing a limited application of Latin hypercube (LLH) sampling is of two types: first, a representative set of two to four values of the possible outcomes associated with each of several specific source term issues (referred to as the "issue levels" in this discussion); second, a weighting factor appropriate for each outcome or issue level. The latter may be conditional upon what level is assigned to other issues. The "issue levels" were specified primarily by the SARRP team, while the weighting factors were based primarily upon values provided by the review group; when necessary, issue outcomes were also revised to reflect points raised by the review group.

The next section gives a few more details on what is done and the approach used. Section C.2.3 presents the issues considered, the specific values characterizing the issue outcome levels, and the weighting factors chosen for these outcome levels. It should be noted that the discussion of the uncertainty associated with most issues was presented in Section C.1, and that discussion is not repeated here. The information in Section C. 1 served as the basis for the strawman proposal on issues and weighting factors that was initially sent to each reviewer. Section C.2.5 describes, briefly, the SURSOR routine which implements the actual calculation of the needed source term information, given the assignment of levels for the various issues; it also gives the SURSOR code listing. 


\section{C.2.2 Approach}

For the issue outcomes, the approach used here is closely related to that which was used to derive estimates of optimistic and pessimistic source terms in the earlier study based upon the OCP representation of source terms and their uncertainties. The arguments for the effects which the various issues might have upon the source term were given in Section C.1 which discusses the OCP study, and these arguments will not be repeated here. The actual numbers given here sometimes differ from any that appear in the OCP work because the intended usage of the results is different.

In defining issues and issue levels, the results of actual calculations were usually relied upon whenever possible. In all cases, three to four levels have been specified for each issue. For most issues, one level is based upon the results of a Battelle STCP calculation. (Which level this is varies from issue to issue, but it is specified in Section C.2.3 below.) Exceptions arise because, in a few cases, the review group made changes to the level definitions in such a way that no level corresponds exactly to the result of a STCP or BMI-2104 calculation, or else because the issue addressed is not even modeled in the STCP. The highest and lowest levels are based upon QUEST [4] results insofar as is possible. In many of these cases, substantial extrapolations have been required, with outright guesses being necessary in some instances. Brief mention of the source of these values is given in Section C.2.3, with more details being given in Section C.1 in some instances.

Since the possible values of the issue outcomes form a continuum, it might seem more appropriate to define the outcomes in terms of a continuous range of values, rather than a set of discrete values. However, the present LLH study employed sets of discrete values; changing this feature would have presented difficulties not justifiable in terms of any possible improvement in the results. Since it is the discrete values which actually are used in the calculation, it is these same values that were presented to the reviewers for the assignments of weighting factors.

The results presented here are discussed in the context of the following simple equation for the release fractions of the various radionuclide species:

$$
\mathrm{Ft}(\mathrm{i})=\mathrm{FCOR}(\mathrm{i}) * \mathrm{FVES}(\mathrm{i}) * \mathrm{FCONV}(\mathrm{i})+\mathrm{FCCI}(\mathrm{i}) * \mathrm{FCONC}(\mathrm{i}) .(\mathrm{C} .2-1)
$$

The following notation and terminology are used:

$\mathrm{Ft}(\mathrm{i})=$ fraction of the initial core inventory of species $\mathrm{i}$ that is ultimately released to the environment.

FCOR(i) $=$ fraction of initial core inventory of species $i$ released from the fuel prior to vessel failure. 
NUREG/CR-4551, VOL. 1: DRAFT REPORT FOR COMMENT (SEPTEMBER, 1986)

FVES(i) $=$ fraction of material released from the fuel which is released from the vessel.

FCONV(i) $=$ fraction of material released from vessel that is released from containment.

$\mathrm{FCCI}(\mathrm{i})=$ fraction of initial inventory that is released from the melt during coreconcrete interactions (CCI).

FCONC(i) $=$ fraction of the core-concrete interaction release that is released from containment.

The Source Term Code Package (STCP) assumes that iodine will be present as CsI. Recent experimental data indicate that, in the presence of steam and radiation (as in the primary system), CsI tends to decompose to elemental iodine (or some other highly volatile form) which may be released from the primary system with zero retention, although the cesium derived from the CsI may still be retained. In order to allow for this possibility, Eq. C.2-1 is modified for iodine as follows:

$\mathrm{Ft}(\mathrm{I})=\mathrm{FCOR}(\mathrm{I}) *[\mathrm{FI} 2+(1-\mathrm{FI} 2) * \mathrm{FVES}(\mathrm{I})] * \mathrm{FCONV}(\mathrm{I})+\mathrm{FCCI}(\mathrm{I}) * \mathrm{FCONC}(\mathrm{I})$

Here, FI2 is the fraction of the iodine released from the fuel which is converted to the more volatile form (elemental 12 , hydrogen iodide, etc.); no in-vessel retention is credited for this more volatile form.

It must be emphasized that the actual construction of a set of source terms from a set of issue level specifications requires consideration of many features not represented explicitly in Eq. C.2-1 which should be thought of more as providing a conceptual framework for discussion than as an actual algorithm for calculation, except when suitably modified and/or restricted. It also must be emphasized that the approach being used to construct source terms from issue specifications is a purely parametric one. No mechanistic or phenomenological modeling is used; the possible effects one issue may have upon another, or possible restrictions as to when a given result is applicable, are taken into account only if explicit provision is made for them.

In general, it is not possible to meaningfully express the effect of a source term "issue" upon the source term directly, but the possible effects of an issue can be expressed in terms of its effect upon one or more of the quantities appearing in Eq. C.2-1, at least as a first approximation. The possible outcomes and associated sample weights are therefore discussed in terms of these quantities. To keep the number of issues manageable and yet also provide reasonable coverage of the myriads of phenomena that are actually involved, the definition of "issues" often must be very broad. 


\section{C.2.3 Issues. Levels, and Weighting Factors}

Thirteen issues are offered for consideration below. Although this number is clearly inadequate (many facets of the problem discussed in the OCP analyses are not reflected here), it was originally feared that practical limitations associated with the LLH study would force pruning of the list still further. To deal with this problem, issue priorities were suggested, and reviewers were asked to vote on the priorities to be assigned. However, it turned out that all the issues discussed below could be treated in the LLH, and these priorities therefore will not be discussed further.

In choosing the issues to be treated, an attempt was made to take into account the potential risk significance of the issue in a global sense with respect to the relevant parameter space, with some emphasis on major regions of parameter space that might generate risk estimates significantly larger than the central values. This pessimistic emphasis applies only to issue selection, not to level specification and weighting factors; no conscious conservative loading of the latter has been employed.

To illustrate this point, it is instructive to compare the perspectives on dominant issues given by the central versus the pessimistic walk-throughs of the containment event tree, source term uncertainties, and consequence uncertainties. Based upon a run of the risk code made $12 / 19 / 85$ (shortly before the strawman proposal and the January review meeting), the $\mathrm{V}$ sequence was dominant for the central case, contributing $70-80 \%$ of the total risk, for both early and late consequence measures; high pressure sequences made up most of the rest. For the pessimistic case, the high pressure sequences (some with induced LOCAs) with early containment failure strongly dominated, with the V-sequence being much less significant, contributing under $10 \%$ of the risk. (The same sequence frequencies were assigned in both the central and the pessimistic cases.) The LLH judgments on issue selection have been guided by the belief that it is also important to treat those issues which likely drive the more pessimistic results, rather than just issues unique to the $V$-sequence, which dominates the central results.

\section{Correlations Between Issues}

The LLH technique allows for the specification of correlations between the various issues; that is, the weighting factors specified for one issue may depend upon the level specified for one or more other issues. However, significant complications can be introduced when this is done, and it is not clear that the available data base is adequate to justify specification of such correlations among the source term issues considered here. In general, correlations were not offered in the original strawman proposal, except for possible 
correlations between Issue 1 (in-vessel release from fuel) and Issue 3 (fractional release from the primary system). The review group agreed that the justification was not adequate for defining correlations among the weighting factors in this case or in any of the others. Hence, no correlations of this kind were defined.

It is important that this statement not be misinterpreted. There are a great many instances in which the assumptions made about one or more issues will affect what is reasonable to assume about some other issue. However, these effects have been built into the model, rather than represented through correlations imposed upon the weighting factors applied to the relevant issue levels. For example, specification that an induced LOCA does/does not occur in a station blackout sequence affects both the direct heating level definitions and the release from the primary system; the amount of direct heating specified in the containment event tree affects not only the possible radionuclide release due to direct heating but also the amount of fuel assumed available for participation in core-concrete interactions after vessel failure, and the radionuclide content of this fuel. Representations of all these relationships have been built into the model; thus, the weighting factors chosen for the issues describing these phenomena need not (or at least do not) depend upon the level chosen for the other issues involved.

\section{Source Term Bins}

In the original OCP approach, a total of 17 source term "bins" were defined for representing the consequences of the innumerable sequence/pathway outcome combinations generated by the containment event tree (CET) analysis. The assignment of representative cases to these bins is indicated in Table $\mathrm{C}-10$. For more details on these bins, the descriptions of the OCP source term analyses in Section C.1 and in the main report may be consulted.

In response to review recommendations, the representation of direct heating was upgraded from that initially used in the OCP analysis. The result was to define four bins (16 19) which are analogs of Bins 1 - 4, respectively, but with the effects of direct heating included in Bins $16-19$ while they are omitted in Bins $1-4$. 
Table C-10

SUMMARY OF SOURCE TERM BIN ASSIGNMENTS FOR SURRY

\begin{tabular}{|c|c|c|c|c|c|c|c|c|c|c|c|}
\hline Sequence & $\begin{array}{l}\text { Cont. Fails } \\
\text { Before } \\
\text { Core Melt }\end{array}$ & \multicolumn{2}{|c|}{ Early Overpressure } & \multicolumn{2}{|c|}{$\begin{array}{l}\text { Late Hydrogen } \\
\text { Bum Failure }\end{array}$} & \multicolumn{2}{|c|}{ Late Overpressure } & $\begin{array}{c}\text { Basemat } \\
\text { Meltthrough }\end{array}$ & $\begin{array}{l}\text { Isolation } \\
\text { Failure }\end{array}$ & Bypass & $\begin{array}{l}\text { No } \\
\text { Failure }\end{array}$ \\
\hline TMLB' & - & $1,16^{b}$ & -- & 9 & 8 & 9 & 10 & $13,14^{\mathrm{c}}$ & $6^{d}$ & -- & 15 \\
\hline$S_{3} D$ & -- & 1,16 & $2,17^{b}$ & 9 & 8 & 9 & 10 & 13,14 & $7^{d}$ & -- & 15 \\
\hline V & -- & -- & -- & -- & -- & - & -- & -- & -- & $11,12^{\mathrm{e}}$ & -- \\
\hline TMQD & -- & $3,18^{b}$ & $4,19^{b}$ & 9 & 8 & 9 & 10 & 13,14 & 7 & -- & 15 \\
\hline TKMU & -- & 1,16 & 2,17 & 9 & 8 & 9 & 10 & 13,14 & 7 & -- & 15 \\
\hline$A G$ & 5 & -- & -- & -- & -- & -- & -- & - & 7 & -- & -- \\
\hline$S_{2} D$ & -- & 3,18 & 4,19 & 9 & 8 & 9 & 10 & 13,14 & 7 & -- & 15 \\
\hline $\mathrm{AD}$ & - & -- & -- & -- & -- & 9 & 10 & 13,14 & 7 & -- & 15 \\
\hline $\mathrm{AH}$ & -- & -- & -- & -- & -- & 9 & 10 & 13,14 & 7 & -- & 15 \\
\hline
\end{tabular}

a Dashes (--) mean that the scenario does not arrise in the central walk-through.

b Bins 1-4 and 16-19, respectively, are equivalent except that Bins 16-19 include effects due to direct heating and Bins 1-4 do not.

c Bin 13 is used for a pressurized containment (no sprays); Bin 14 for unpressurized (sprays on).

d Bins 6 and 7 are for isolation failure sequencces with and without containment sprays, respectively.

e Bins 11 and 12 represent the $V$ sequence with and without scrubbing by water in the pathway, respectively. 


\section{Issue 1: In-Vessel_Release from Fuel}

As indicated in Table C-11, the QUEST values of FCOR are the basis of the present estimates (see Section C.1). Level 2 is essentially the same as the BMI-2104 TMLB' calculation, but it is not the same as the later Battelle STCP calculation, reported on about $4 / 1 / 85$, which was used previously as the SARRP central case when there was no direct heating: the latter involved setting MARCH parameters to values that gave high in-vessel zirconium oxidation, which in turn can have significant implications for the FCOR values of some species, notably Group 4. Values for this calculation are given below as "Level 2a". The differences are significant only for tellurium. It is felt that the BMI-2104 calculations may actually provide a more representative value. In conjunction with the other levels, it also provides a better sampling of the full range of possible FCOR values for tellurium. Hence, it was recommended that the values labeled "Level 2", rather than the "Level $2 \mathrm{a}$ " values, be used as the second level in the LLH study.

The other levels specified here are taken from the QUEST results, except that the "lowlow" levels (see Section C.1) have been dropped in order to limit the number of outcomes to four, and some of the "low" values modified (reduced) slightly to reflect this.

Table C-11

LEVELS OF FCOR FOR LLH UNCERTAINTY ANALYSIS

\begin{tabular}{clllcc}
\hline $\begin{array}{c}\text { WASH-1400 } \\
\text { GROUP }\end{array}$ & $\begin{array}{c}\text { LEVEL 1 } \\
\text { LOW }\end{array}$ & $\begin{array}{c}\text { LEVEL 2 } \\
\text { BASE }\end{array}$ & LEVEL 2a ${ }^{1}$ & $\begin{array}{c}\text { LEVEL 3 } \\
\text { HIGH }\end{array}$ & $\begin{array}{c}\text { LEVEL 4 } \\
\text { HIGH-HIGH }\end{array}$ \\
\hline 1. & 0.5 & 0.986 & 1.0 & 1.0 & 1.0 \\
2. & 0.5 & 0.986 & 1.0 & 1.0 & 1.0 \\
3. & 0.5 & 0.986 & 1.0 & 1.0 & 1.0 \\
4. & 0.026 & 0.33 & 0.78 & 0.90 & 1.0 \\
5. & 0.014 & 0.13 & 0.14 & 0.49 & 0.98 \\
6. & 0.008 & 0.041 & 0.037 & 0.11 & 0.53 \\
7. & $1.4 \times 10^{-5}$ & $2.5 \times 10^{-4}$ & $4.2 \times 10^{-4}$ & 0.0077 & 0.074 \\
\hline
\end{tabular}

1 Based on Battelle calculations of $4 / 1 / 85$

There are no dependencies upon sequence imposed upon the above values; although STCP calculations do sometimes show significant dependence upon the sequence, these dependencies are considerably smaller than the ranges spanned by the above values. The latter ranges are intended to represent phenomenological uncertainties applicable in substantial degree 
to all sequences. Hence, defining sequence-dependent values of FCOR does not seem justified at this time.

As is discussed in Section C.1, there is no intrinsic need to assign a given level to all species; that is, the releases of the different species are partially independent and could be Level 4 for one species and Level 2 or 3 for another. This complication is also being ignored here, though it was considered in the OCP analysis. Thus, in the present work, the same level will be assigned to all species, in any given LLH sample member. (Doing otherwise would require treating different species as different issues in the LLH study.)

These results and a summary of their origin were provided to the review team described above. The SARRP analyst's weighting factors for each of the levels was also provided and is repeated in Table C-12 in the column labeled "SARRP". At the source term review meeting the four members of the review team provided their weighting factors and justifications thereof. The weighting factors of the reviewers for each level are provided in Table C-12. The key points of discussion are highlighted below to allow an understanding of the primary considerations that the reviewers included in the development of their weighting factors, as well as limitations that might affect the interpretation of the results.

Table C-12

ISSUE 1: IN-VESSEL RELEASE FROM FUEL (FCOR)

\begin{tabular}{llcccccc}
\hline \multirow{2}{*}{ FCOR LEVEL } & \multicolumn{5}{c}{ WEIGHTING FACTOR } \\
& & SARRP & Rev.1 & Rev.2 & Rev.3 & Rev.4 Composite \\
\hline 1. & Low & .30 & .25 & .10 & .30 & .25 & .24 \\
2. & Level 2 & .35 & .45 & .40 & .40 & .70 & .46 \\
3. & High & .25 & .25 & .40 & .20 & .05 & .23 \\
4. & High-High & .10 & .05 & .10 & .10 & 0.0 & .07 \\
\hline
\end{tabular}

This issue was selected unanimously as a first priority issue because of the large uncertainties involved and their potential for significant impact on the risk results. All reviewers felt comfortable with Level 2 as indicated by their weighting factors. As for the lower values associated with Level 1, all reviewers agreed that the release fractions were certainly a possible outcome. One reviewer noted that limited experimental evidence nearly always pointed to releases less than the base case and that the experiments that come out with values higher than the base case nearly always include special conditions that may not be 
representative. It was pointed out that other uncertainties could also result in lower releases such as saturation in the gas phase associated with in-vessel recirculation were not modeled.

Another reviewer would have weighted Levels 1 and 2 more heavily if it were not for his uncertainty concerning the applicability of the evidence to core-melt accidents occurring with high primary system pressure. Several others also pointed out the importance of high primary-system pressure relative to the potential to increase the release due to revaporization and higher fuel releases upon vessel failure. There was some disagreement on the impact of invessel recirculation, one outcome being that the phenomenon could result in prolonged heating of the core and a convective component to the heatup that would lead to higher releases rather than low releases due to gas saturation as described in the previous paragraph. Several of the reviewers stated that based on their experience and limited experimental evidence that the highhigh case for this issue was unreasonable--the values for release fractions being unrealistically high. Nevertheless weighting factors were provided for this case and these were used directly in the analysis.

Other experts at the meeting discussed recent results with other computer codes, notably MELPROG, that indicated different temperature distributions in the core than predicted with other methods. These results were disputed by others but the discussion clearly pointed out the realism of the thermodynamic component of the uncertainty because state-of-the art models can be in rather significant disagreement. Finally, the other topic discussed at length relative to this issue is discussed in Section C.2.4 below, because it was a more general concern: the loss of information and insight due to the broadness of the defined issues and the lumping together of all fission product groups within a level, whereas the uncertainties within the groups might be independent or correlated negatively.

\section{Issue 2: CsI Decomposition}

This issue is reflected by the values of FI2 used in Eq. C.2-1a. The STCP assumes a value of zero; recent experimental results can be interpreted as implying values close to unity can not be ruled out. Under these circumstances, a wide distribution spanning the full range of possibilities was offered in the original straw man proposal.

The review group unanimously selected this issue as a priority one. The results of the weighting factors provided for the fraction of CsI that is decomposed are listed below in Table C-13. Three of the reviewers expressed an opinion that if the reaction did take place that it would not result in complete conversion of the CsI as indicated by Level 4; and this level was weighted less heavily than the others. Particular evidence was cited that CsI would not be 
stable because of radiation and reaction with other gases. Because of the experimental evidence however, one reviewer also gave a zero weighting factor on Level 1 since it would imply that the phenomenon did not apply. In general, it was concluded that the current state knowledge concerning this issue is inconclusive, although the individual interpretation of how to express this in weighting factors varied somewhat. No dependencies upon sequence were specified.

Table C-13

ISSUE 2: CSI DECOMPOSITION

\begin{tabular}{clcccccc}
\hline \multirow{2}{*}{$\begin{array}{c}\text { DECOMPOSITION } \\
\text { LEVEL }\end{array}$} & SARRP & Rev.1 & Rev.2 & Rev.3 & Rev.4 Composite \\
\hline 1. & 0.0 & .25 & .25 & .40 & .10 & 0.0 & .2 \\
2. & 0.33 & .25 & .25 & .40 & .50 & .50 & .38 \\
3. & 0.67 & .25 & .25 & .20 & .30 & .50 & .3 \\
4. & 1.0 & .25 & .25 & 0.0 & .10 & 0.0 & .12 \\
\hline
\end{tabular}

Issue 3: In-Yessel Retention (FVES)

Representation of this issue is complex because it is desirable to acknowledge dependence of FVES upon the accident sequence in addition to the radionuclide species of interest. It is to be remembered that, for Group 2 (iodine), the FVES value given here applies only to that portion of the iodine which is not converted to the more volatile form (Issue 2): for the more volatile form, FVES $=1.0$ is postulated in the present treatment.

In Section C.1, arguments are offered that the following trends are to be expected: the likelihood of large FVES for volatile species varies in the order $\mathrm{CsI}>\mathrm{CsOH}>\mathrm{Te}$; conditions favoring large FCOR also favor large FVES for these species; while conditions favoring large FCOR for Groups 5, 6, and 7 favor small FVES for those species; and that the STCP may have overestimated aerosol production from control rod materials which could lead to underestimating FVES for Groups 5, 6, and 7 under some conditions. However, preserving all these trends in the present study would present difficulties and some simplifications have been made; in particular, the justification for assuming the suggested correlations between FCOR and FVES was deemed weak by the review group and such correlations were not included. 


\section{Issue Levels (FVES Values)}

FVES values from the Battelle calculations correspond to Level 2 below, while most Level 1 and Level 4 outcomes are based upon QUEST results. Unfortunately, this connection is loose at best because QUEST treated in-vessel release only for TMLB' and, for this sequence, it was apparent in QUEST that the dominant uncertainties could not be addressed with the BMI-2104 code suite (or the STCP) and, hence, simple bounding calculations were resorted to.

High-Pressure Sequences. For the high pressure sequences, the two Surry TMLB' calculations provide two "Level 2" candidates, as discussed above in connection with FCOR. The values of FVES yielded by these analyses are listed in Table $\mathrm{C}-14$ below.

Table C-14

SURRY CALCULATIONS FOR FVES: HIGH PRESSURE SCENARIOS

\begin{tabular}{ccc}
\hline $\begin{array}{c}\text { WASH-1400 } \\
\text { GROUP }\end{array}$ & BMI-2104 & $\begin{array}{c}\text { BATTELLE } \\
4 / 1 / 85\end{array}$ \\
\hline 2 & 0.14 & 0.24 \\
3 & 0.14 & 0.20 \\
4 & 0.15 & 0.15 \\
5 & 0.11 & 0.11 \\
6 & 0.19 & 0.10 \\
7 & 0.10 & 0.10 \\
\hline
\end{tabular}

As in the case of FCOR, the BMI-2104 values were recommended for Level 2. The actual level structure adopted is simplified somewhat by combining some groups as follows in Table C-15.

Table C-15

LEVELS OF FVES FOR LLH UNCERTAINTY ANALYSIS: HIGH PRESSURE CORE-MELT SEQUENCES

\begin{tabular}{ccllc}
\hline $\begin{array}{c}\text { WASH-1400 } \\
\text { GROUPS }\end{array}$ & LEVEL 1 & $\begin{array}{c}\text { LEVEL 2 } \\
\text { BASE }\end{array}$ & LEVEL 3 & LEVEL 4 \\
\hline $1 \& 2$ & 0.01 & 0.14 & 0.4 & 0.9 \\
4 & 0.01 & 0.15 & 0.3 & 0.9 \\
$5,6, \& 7$ & 0.03 & 0.11 & 0.2 & 0.4 \\
\hline
\end{tabular}


Intermediate Pressure Except for the case of Bin 2, the intermediate pressure values of FVES are also used whenever an S2 induced LOCA is specified by the CET for high pressure sequences. This treatment is believed to be conservative, perhaps significantly so. The reason for exempting Bin 2 is noted in connection with Issue 8, below. The levels are listed in Table C-16.

Table C-16

LEVELS OF FVES FOR LLH UNCERTAINTY ANALYSIS:

INTERMEDIATE PRESSURE CORE-MELT SEQUENCES

\begin{tabular}{cllll}
\hline $\begin{array}{c}\text { WASH-1400 } \\
\text { GROUPS }\end{array}$ & LEVEL 1 & $\begin{array}{c}\text { LEVEL 2 } \\
\text { BASE }\end{array}$ & LEVEL 3 & LEVEL 4 \\
\hline $1 \& 2$ & 0.04 & 0.5 & 0.7 & 0.9 \\
4 & 0.01 & 0.02 & 0.2 & 0.9 \\
$5,6, \& 7$ & 0.1 & 0.34 & 0.5 & 0.7 \\
\hline
\end{tabular}

Low Pressure--Bin 5 The Level 2 FVES values are based upon the recent STCP calculation for the AG sequence, which shows very little retention in the primary system. The issue levels are listed in Table C-17.

Table C-17

LEVELS OF FVES FOR LLH UNCERTAINTY ANALYSIS:

LOW PRESSURE CORE-MELT SEQUENCES

\begin{tabular}{ccccc}
\hline $\begin{array}{c}\text { WASH-1400 } \\
\text { GROUPS }\end{array}$ & LEVEL 1 & $\begin{array}{c}\text { LEVEL 2 } \\
\text { BASE }\end{array}$ & LEVEL 3 & LEVEL 4 \\
\hline $1 \& 2$ & 0.2 & 0.87 & 0.95 & 1.0 \\
4 & 0.1 & 0.83 & 0.90 & 0.95 \\
$5,6, \& 7$ & 0.1 & 0.77 & 0.85 & 0.95 \\
\hline
\end{tabular}

These FVES values are also used for the high pressure sequences when the CET specifies a large induced LOCA, except in the case of Bin 2; again, significant conservatism may result.

V Sequence In the Battelle V-sequence calculations, aerosol processes dominate the transport and deposition processes for all species except Te; hence, the FVES values for the various species are more nearly similar than in the other sequences. Most of the tellurium that 
is released from the RCS is released to the containment (not the safeguards building) when the vessel fails; this behavior is not well represented in the current treatment and the FVES value cited here is an approximate "effective" value, crudely taking into account the fraction that is transported back through the primary system. The behavior of the $\mathrm{Te}$ in the Battelle calculations is a result of stagnant conditions in the vessel for a considerable period prior to vessel failure and may not be entirely realistic.

Table C-18

LEVELS OF FVES FOR LIH UNCERTAINTY ANALYSIS: V SEQUENCE

\begin{tabular}{ccccc}
\hline $\begin{array}{c}\text { WASH-1400 } \\
\text { GROUPS }\end{array}$ & LEVEL 1 & $\begin{array}{c}\text { LEVEL 2 } \\
\text { BASE }\end{array}$ & LEVEL 3 & LEVEL 4 \\
\hline $1 \& 2$ & 0.05 & 0.2 & 0.4 & 0.9 \\
4 & 0.05 & 0.1 & 0.4 & 0.9 \\
$5,6, \& 7$ & 0.05 & 0.1 & 0.4 & 0.9 \\
\hline
\end{tabular}

In all cases, the constraint that one of the levels correspond directly to the results of Battelle calculations means that the full range of outcomes is not always sampled as effectively as might otherwise be the case. The choice of associating Level 2 with the Battelle calculations was made because it was judged to yield the best level distribution for the high-pressure sequences and the $V$ sequence, which are expected to be the most risk-significant.

\section{Weighting Factors}

It is argued that weighting factors should be be correlated with FCOR in Section C.1. However, it is also argued that the correlations should be different for different species, with positive correlations for the volatile species and negative correlations for the less volatile species. Specification of different weights for different species would require definition of a new issue for each species. The review group, however, judged that available information was not adequate to justify specifying a correlation between FVES and FCOR. Hence, a single set of weighting factors was adopted as listed in Table C-19.

The focus of discussion on this issue centered on the methodology used in the proposed LLH analysis. These comments all had more general implications and they have been collected in Section C.2.4, but the discussions are particularly germane to this issue and should be reviewed prior to drawing conclusions on this issue. It should be noted that several 
Table C-19

ISSUE 3: IN-VESSEL RETENTION (FVES)

\begin{tabular}{ccccccc}
\hline FVES LEVEL & \multicolumn{5}{c}{ WEIGHTING FACTORS } \\
& SARRP & Rev.1 & Rev.2 & Rev.3 & Rev.4 Composite \\
\hline 1. & .15 & .15 & .15 & .10 & 0.0 & .11 \\
2. & .35 & .35 & .35 & .40 & .5 & .39 \\
3. & .35 & .35 & .35 & .40 & .5 & .39 \\
4. & .15 & .15 & .15 & .10 & 0.0 & .11 \\
\hline
\end{tabular}

of the reviewers felt that the compromises required to make this issue manageable tended to eliminate significant information.

There was considerable discussion at the review meeting concerning the phenomenology of this issue. It was generally recognized that the sequence dependence of this issue is very strong and that the uncertainty in this issue was a priority one with relatively large ranges of uncertainty and significant impact on risk. Some concerns about the issue included:

- The treatment of recirculation--which could either increase the retention due to the larger volumes involved, or decrease retention due to increased temperatures;

- Impaction is neglected, which could bias the results too high; and

- The coefficients of retention are only correlated to flow while other physical parameters are obviously important.

One reviewer also noted that the low pressure sequences would show less uncertainty than the SARRP range, but this could not be reflected in the assignment of a single set of weighting factors. Finally, it was generally concluded that the sequence variations would lead to wider uncertainty than could be modeled by the limited number of sequences being considered here, but that practical limitations would prevent fuller incorporation in the LLH study.

\section{Issue 4: Scrubbing DF for V-Sequence With Water}

For this issue, Level 3 corresponds to the Battelle calculations; Levels 2 and 4 reflect extrapolations of some QUEST results on suppression pool DFs discussed in the OCP writeup (the extrapolations involved are very large and quite uncertain); Level 1 is arbitrary and is introduced in order to reflect a review comment that the OCP range offered was too narrow. The DF levels and weighting factors are provided in Table C-20. 
The reviewers concurred with the large uncertainty associated with the issue. Reviewer \#1 agreed with the SARRP assessment, while the others weighted the highest decontamination factor less heavily because it was their opinion that the very high values of DF would not be expected for the conditions of this scenario. It was generally agreed that information on this issue was lacking, however.

Table C-20

ISSUE 4: SCRUBBING DECONTAMINATION (DF) FACTOR FOR THE $\checkmark$ SEQUENCE WITH OVERLYING WATER

\begin{tabular}{ccccccc}
\hline DF & \multicolumn{5}{c}{ WEIGHTING FACTOR } \\
LEVEL & SARRP & Rev.1 & Rev.2 & Rev.3 & Rev.4 & Composite \\
\hline 1. $\mathrm{DF}=100$ & .25 & .25 & .05 & .10 & 0.0 & .13 \\
2. $\mathrm{DF}=12$ & .25 & .25 & .15 & .30 & .33 & .26 \\
3. $\mathrm{DF}=5$ & .25 & .25 & .70 & .40 & .34 & .38 \\
4. $\mathrm{DF}=2$ & .25 & .25 & .10 & .20 & .33 & .23 \\
\hline
\end{tabular}

\section{Issue 5: Release of RCS Species from the Containment (FCONV)}

This issue was originally introduced primarily because of an apparent anomaly in the BMI-2104 calculations for Surry TMLB', which is believed to result in substantial underprediction of the containment release fraction due to excessive steam condensation upon aerosols calculated by the MARCH-NAUA link (see Section C.1 for more details). A recent Battelle calculation with NAUA condensation processes switched off yielded an FCONV value of 0.8 , essentially the same as the results of simple hand calculations for failures induced by direct heating. The review group agreed that it is acceptable to simply postulate this value of FCONV for all high-pressure sequences with early containment failure, and this issue has been dropped from consideration. For other sequences, fixed values of FCONV are used, with the values being chosen to agree as best possible with the values implied by STCP calculations.

\section{Issue 6: Core-Concrete Interaction (CCI) Releases}

Other things being equal, the fractional releases of a given radionuclide species from the melt, as calculated by VANESA, are almost independent of the amount present in the melt at the time of the start of CCI. Hence, outcomes are tabulated in terms of the value of FCCI/(1FCOR), in terms of the quantities appearing in Eq. C.2-1. The levels listed in Table C-21 are 
the result of deliberations of the review group, and no one level exactly corresponds to the results of any one STCP calculation for all species.

Table C-21

LEVELS OF CORE-CONCRETE INTERACTION

RELEASE FRACTIONS FOR EACH FISSION PRODUCT GROUP

\begin{tabular}{cccc}
\hline $\begin{array}{c}\text { WASH-1400 } \\
\text { GROUP }\end{array}$ & LEVEL 1 & $\begin{array}{c}\text { LEVELS--FCCV/1-FCOR) } \\
\text { LEVEL 2 }\end{array}$ & LEVEL 3 \\
\hline $2 \& 3$ & 0.02 & 1.0 & 1.0 \\
4 & 0.008 & 0.4 & 0.8 \\
5 & 0.002 & 0.05 & 0.2 \\
6 & $1.0 \times 10^{-6}$ & $3.0 \times 10^{-5}$ & 0.07 \\
7 & $2.0 \times 10^{-5}$ & $7.7 \times 10^{-4}$ & $2.0 \times 10^{-2}$ \\
\hline
\end{tabular}

After discussion of the basis for the levels summarized above each of the reviewers weighted the levels as illustrated in Table C-22. Overall, the reviewers were fairly satisfied that the current code results, as modified to reflect more recent thinking on Group 5 releases, reflected the best estimate. Thus Level 2 is rather heavily weighted. It should be noted that the reviewer most familiar (\#4) with core-concrete interactions who had the largest part in developing the levels weighted each level equally, but did stress that the distribution would be cut off around the limits represented by the Levels 1 and 3. In other words, his weighting factors for values more extreme than those levels would be very low. There was little more discussion of the issues during the weighting process, except that one reviewer did indicate his view that the result here would be somehow correlated with the next issue, release of the CCI species from containment. For example, the amount of aerosol generation of non-radioactive species would affect the phenomenology within the containment and therefore the actual release if containment failed. Since correlations would be difficult to define, it was decided that the issue would not be correlated with any others.

Table C-22

ISSUE 6: CORE-CONCRETE INTERACTION (CCI) RELEASES

\begin{tabular}{ccccccc}
\hline FCCI/(1-FCOR) & \multicolumn{5}{c}{ WEIGHTING FACTOR } \\
LEVEL & SARRP & Rev.1 & Rev.2 & Rev.3 & Rev.4 Composite \\
\hline 1. Low & .25 & .15 & .15 & .20 & .33 & .215 \\
2. Base & .45 & .70 & .70 & .65 & .33 & .57 \\
3. High & .30 & .15 & .15 & .15 & .33 & .215 \\
\hline
\end{tabular}




\section{Issue 7: Release of CCL Species from the Containment (FCONC)}

Again, effects of sprays and aerosol agglomeration uncertainties are treated as separate issues. Early containment failure and late failures must also be distinguished. For the early containment failure cases, the review group judged that there was not sufficient uncertainty to justify defining levels for FCONC; hence, for the early failure cases, fixed FCONC values chosen to give a reasonable match to the STCP calculations were used.

For late failures, Level 3 values are based upon TMLB'- $\varepsilon$ results in BMI-2104 (Group 2-3 values are crude estimates only); values depend substantially on the species because of the differing time histories of their release to the containment. Obviously, they would also depend upon the time of containment failure. The levels specified here (in Table C-23) are intended to serve as a crude surrogate for sensitivity to containment failure time, something which has not been investigated explicitly with the STCP. Level 1 was introduced as a result of the review group's observation that the time delay between the start of $\mathrm{CCI}$ and containment failure could be very long, even days, and that this should result in very small release fractions.

Table C-23

LEVELS OF CORE CONCRETE INTERACTION RELEASE FRACTION FOR LOW PRESSURE SCENARIOS

\begin{tabular}{ccccc}
\hline $\begin{array}{c}\text { WASH-1400 } \\
\text { GROUP }\end{array}$ & $\begin{array}{c}\text { LEVEL 1 } \\
\text { LOW-Low }\end{array}$ & $\begin{array}{c}\text { LEVEL } \\
\text { Low }\end{array}$ & $\begin{array}{c}\text { ECONC } \\
\text { LEVEL 3 }\end{array}$ & $\begin{array}{c}\text { LEVEL 4 } \\
\text { High }\end{array}$ \\
\hline $2 \& 3$ & 0.001 & 0.01 & $0.1^{*}$ & 0.3 \\
4 & 0.015 & 0.15 & 0.37 & 0.4 \\
5 & 0.003 & 0.03 & 0.16 & 0.3 \\
6 & 0.015 & 0.15 & 0.37 & 0.4 \\
7 & 0.003 & 0.03 & 0.16 & 0.3 \\
\hline
\end{tabular}

*Although this is the base case, this value could be only roughly estimated from code results.

The review group selected weighting factors for these levels based upon their judgment of the effect of the timing on these late containment failures. It was acknowledged that the timing of the accident could vary substantially, particularly when considering the amount of water that could be present in the cavity that could delay the onset of core-concrete interactions and containment failures. There are two timing uncertainties: 1) the timing until containment failure and 2) the timing between start of the core-concrete interaction and the failure of the containment. It was also a concern of several reviewers that the timing was directly tied to the 
individual accidents being quantified, but the uncertainty being represented here did not include that correlation and therefore the level of uncertainty might be misrepresented. In effect, these weighting factors apply to a group of levels that will then be applied across a group of sequences, but if the weighting factors had been assigned on a sequence specific basis, the reviewers would have likely selected different weighting factors. The weighting factors require an integration of all of the uncertainties including timing of the sequence, strength of containment, and other phenomenology in the containment. It was concluded that this should be recognized as a deficiency, but that the current method was all that was possible for the LLH study. Once again, it was stressed that this issue was a lower priority than others which would likely affect risk.

The weighting factors are listed in Table C-24 below. As illustrated, the majority opinion was that the release fractions would be at or below the base case predicted by the code, principally because there are a number of different scenarios that can be postulated which would lead to longer failure times. It was also pointed out that there was a correlation with the containment uncertainty issue associated with failure pressure. For example, a case with a low containment failure pressure would have an earlier release than one with a very high value for containment pressure capacity. This too should be recognized when reviewing the results associated with this uncertainty issue.

Finally, there was some discussion concerning operator action associated with this issue. Although this is also a generic issue discussed in Section C.2.4, it is important here because of the time scale involved. Although operator action at this point would not stop the core-melt process, there are certainly actions that are possible within a two day period which could limit the release. These options could include assurance of containment cooling functions, flooding the containment, or even establishing a provision for spraying the external surface of the entire containment to limit releases at the time of containment failure. This issue is not reflected in the current weighting factors, but it is a potentially important uncertainty for these late scenarios.

\section{Issue 8: Effects of Spravs}

Although it is very crude, the effects of sprays are expressed here in terms of a DF, designated DFSPR below, to be applied to FCONV and FCONC. Values for FCONC are sometimes higher than for FCONV to allow for scrubbing in the cavity, which will contain water if the sprays are on. Values for FCONV apply only if there is early containment failure. 
Table C-24

ISSUE 7: RELEASE OF CCI SPECIES FROM CONTAINMENT (FCONC) FOR LATE CONTAINMENT FAILURE SCENARIOS

FCONC LEVEL

WEIGHTING FACTOR

SARRP Rev.1 Rev.2 Rev.3 Rev.4 Composite

\begin{tabular}{lllllll}
\hline 1a. Low-Low $(.1 \times$ Low $)$ & .2 & .28 & .20 & .20 & .10 & .20 \\
1. Low & .3 & .33 & .40 & .30 & .50 & .37 \\
2. Base & .3 & .33 & .30 & .40 & .20 & .30 \\
3. High & .2 & .05 & .10 & .10 & .20 & .13 \\
\hline
\end{tabular}

For late containment failure, $\mathrm{FCONV}=0$ if the sprays are operating prior to containment failure (except for cases in which they have been operating only briefly). Three cases are distinguished:

1) FCONV, high pressure sequences (puff release from the RCS at the time of containment failure); scrubbing by sprays assumed to be ineffective (DFSPR = 1).

2) FCONV, low and intermediate pressure sequences.

3) FCONC, all.

If sprays fail at containment failure time, only the DF for Case 2 applies. No DF for sprays is applied for the $\mathrm{V}$-sequence. Except for Case 1, Level 2 values correspond approximately to the results of the Battelle calculations with a 1000 micron spray drop. The value of 45 chosen for Level 2, (Case 2) may seem large, but it is required in order to give a reasonable fit to the STCP results for Bins 3 and 4; it no doubt reflects the fact that there is a considerable time interval between the release of the radionuclides from the RCS and the time of containment failure (at vessel breach) in the scenarios analyzed with the STCP. The weighting factors are listed below in Table C-25.

Table C-25

ISSUE 8: EFFECTS OF SPRAYS

\begin{tabular}{|c|c|c|c|c|c|c|c|c|}
\hline & & & & & IGHTI & $\mathrm{GFACl}$ & DRS & \\
\hline & & & SARRP & Rev.1 & Rev.2 & Rev.3 & Rev.4 & Composite \\
\hline & Decontan & ctor & & & & & & \\
\hline 1. & $\begin{array}{l}\text { Case } 2 \\
100\end{array}$ & $\begin{array}{l}\text { Case } 3 \\
1000\end{array}$ & .30 & .25 & .10 & .40 & .20 & .25 \\
\hline 2. & 30 & 30 & .40 & .50 & .60 & .30 & .60 & .48 \\
\hline 3. & 5 & 10 & .30 & .25 & .30 & .30 & .20 & .28 \\
\hline
\end{tabular}


The weighting factors indicate that the Level 2 decontamination factor was the most heavily weighted by a majority of the reviewers but that there remains significant uncertainty because the other two levels were also weighted with rather significant values. Only one reviewer (\#3) indicated that the highest level of decontamination was most appropriate.

There is one potentially serious defect in this representation. Some recent executions of the risk code suggest high-pressure sequences in which sprays operate at least to the time of containment failure (which occurs at vessel failure) can be major risk contributors. At present, no credit is given for the action of the sprays upon the RCS releases in these scenarios, on the grounds that most of the release from the RCS was assumed to occur as a puff at vessel failure time and the sprays would have no time to act to mitigate the release from the containment. However, it now appears that this treatment is overly conservative for at least one, and possibly two, reasons:

1) Recent re-examination of the details of the STCP calculations indicates that the assumption of the puff release from the RCS is not valid; over $80 \%$ of the $\mathrm{CsOH}$ and CsI release comes before vessel breach (VB). The delay between release from the RCS and VB in the calculations is not long, of the order of 2 to 5 minutes, but this can be sufficient to permit the sprays to have a significant effect. Furthermore, the magnitude of this delay is itself highly uncertain and could easily be longer than calculated. Giving no credit for the sprays may be excessively conservative.

2) Depending upon the size of the containment rupture, sprays may have a significant effect upon even a puff release, given that the sprays do survive containment failure.

These questions affect all scenarios assigned to source term Bin 2 and the first also affects a significant fraction of the Bin 1 assignments, since this bin is also assigned to high pressure scenarios in which the sprays operate up to the time of containment failure but then fail.

The review group agreed that these questions were of considerable concern, but expressed no consensus as to how they should be treated except to endorse the suggestion that, if possible, their impact should be bounded by re-evaluating the LLH risk profile after reassigning all the affected Bin 1 and Bin 2 scenarios to Bin 3 and Bin 4 , respectively. The latter is probably bounding because these bins give a representation of the effect of the sprays that would likely be nonconservative for these scenarios, although these bins also include (higher) FVES values that may be inappropriate for these high pressure scenarios. 
The impact of these questions is aggravated in the event of an induced LOCA, since the assumptions of a puff release and no credit for the sprays then become totally indefensible. If containment failure occurs at the time of VB, as is generally the case for the $\mathrm{S} 2$ induced LOCA, the problem is resolved in a straight forward manner by reassignment to Bins 3 or 4 (depending upon whether the sprays survive containment failure). If containment failure is earlier than VB (e.g., at the time of the LOCA), Bins 3 and 4 are expected to be nonconservative. Bin 2 remains as a possible compromise assignment, although it may be conservative; it would certainly be overly conservative if it were evaluated with the large LOCA value of FVES. It is for this reason that induced LOCAs are ignored in evaluating FVES for Bin 2. Finally, if both spray failure and containment failure occur at the time of the induced LOCA, Bin 1 may not be excessively conservative, since most of the RCS release is expected to follow the induced LOCA and thus escape the action of the sprays.

\section{Issue 2: Aerosol Agglomeration Uncertainties}

QUEST results implied that aerosol agglomeration uncertainties introduce significant downward uncertainty, but little upward uncertainty, relative to the STCP calculations. For present purposes, the effects of this uncertainty may be crudely represented as a DF applied to FCONV and FCONC. This DF, designated "DFAER" below, is to be applied only when sprays are not operating and is not to be applied to FCONV for the V sequence with water (Bin 11 ) or to FCONV for any high pressure sequence with early CF (Bin 1 without induced LOCA), or to any direct heating releases; values are different (smaller) for the RCS releases in the V-sequence without water (Bin 12) than for the other cases. These values are based upon QUEST results; see Section C.1 for more discussion. Level 3 corresponds to the Battelle calculations. The reviewer input is provided in Table C-26.

There was nearly uniform agreement at the review meeting that both the proposed levels and the suggested weighting factors would suffice in characterizing the issue. Essentially, the QUEST results provided the sole basis for this issue and there was little additional discussion by the review group.

For the AG sequence, the STCP calculations implied a value of FCONV of about 0.66 , which is somewhat less than the value of 0.8 implied for the safeguard building in the $\mathrm{V}$ sequence without water but greater than the value of about 0.4 applicable to the QUEST scenario (TMLB' after vessel breach). Hence, the V-sequence values of DFAER were also used for this case. By analogy, these values were also applied to the Bin 1 scenarios in which induced LOCAs occur. 
Table C-26

AEROSOL AGGLOMERATION UNCERTAINTIES

\begin{tabular}{ccccccc}
\hline $\begin{array}{c}\text { DECONTAMINATION } \\
\text { LEVEL }\end{array}$ & SARRP & Rev.1 & Rev.2 & Rev.3 & Rev.4 Composite \\
\hline SEQUENCE WITHQUT SPRAYS & & & & & & \\
\hline 1. DF $=10$ & .20 & .20 & .20 & .20 & .33 & .23 \\
2. DF $=3$ & .40 & .40 & .40 & .40 & .34 & .39 \\
3. DF $=1$ & .40 & .40 & .40 & .40 & .33 & .38 \\
V SEQUENCE WITHOUT WATER & & & & & & \\
\hline 1. DF $=3$ & .20 & .20 & .20 & .20 & .33 & .23 \\
2. DF $=1.5$ & .40 & .40 & .40 & .40 & .34 & .39 \\
3. DF $=1$ & .40 & .40 & .40 & .40 & .33 & .38 \\
\hline
\end{tabular}

\section{Issue 10: Late Iodine Releases from Containment Sources}

Originally, this issue was intended to be restricted to organic iodine; however, the review group decided that it should include all of the various sources of volatile iodine that may arise within containment at times later than vessel breach, except that iodine revolatilized from the primary system is treated under Issue 11 and excluded here. As a result, the levels specified were redefined to values considerably higher than might be judged reasonable for organic iodine alone. Additional sources of iodine considered potentially significant include I132 generated by decay of Te-132 in the melt, and then released from the melt; and iodine released from pools and/or other reservoirs within containment, perhaps as a result of radiation chemistry effects. The levels and weighting factors used are as follows:

The review group developed levels of the iodine source term that could result from any of the phenomena described above, none of which are included in the current code calculations. These levels are listed in Table C-27--once again the levels listed include any organic iodine contribution. The weighting factors of the review group are also presented. Once again, the extreme values associated with Levels 1 and 4 were weighted significantly less than the two central values.

It is important to recognize that these values represent fractions of the core inventory released to the environment for all scenarios except those without any containment failure or with basemat melt-through failure only. Thus, it is assumed that none of the mitigating phenomena represented by FCONC, DFSPR, or DFAER apply. In effect, all these sources of 
Table C-27

ISSUE 10: LATE RELEASES OF IODINE FROM THE CONTAINMENT

LATE RELEASE

LEVEL
WEIGHTING FACTOR

SARRP Rev.1 Rev.2 Rev.3 Rev.4 Composite

1. 0.0

.1

3

0.0

.45

.3

.45

.3

.1

$\begin{array}{cccc}.10 & 0.0 & 0.0 & .04 \\ .40 & .45 & .2 & .32 \\ .30 & .45 & .4 & .38 \\ .20 & .10 & .4 & .26\end{array}$

late iodine are being treated as if they were organic iodine. As a result of the levels and weights chosen, all source terms have an average of about 0.05 "late iodine" release, which occurs virtually independently of anything else. (Here, "average" means the result of averaging over this particular issue.) This feature must be kept in mind when interpreting the results of the LLH study.

At the Sequoyah LLH issues meeting (April 2-3, 1986), the review group clarified its preferences on the treatment of this issue. The reviewers continued to hold the belief that no DFs for mitigation affects should be applied, but they also expressed the belief that the levels defined should be applied only to that iodine which was released to the containment but not otherwise released to the environment. It was also agreed that this approach should be applied to Surry. However, this meeting was held after the Surry LLH source term implementation had to be frozen in order to meet the SARRP schedule. Adopting the review group's final recommendation on this issue would reduce the mean contribution of the late iodine release somewhat, but it could still be a significant contributor to those source terms which would otherwise be small.

\section{Issue 11: Releases of $\mathrm{Cs}$ and Iodine from the $\mathrm{RCS}$}

It was originally suggested that Issue 11 include a variety of possible sources that might yield significant Cs and I within containment at late times (i.e., well after vessel breach). However, the review group redefined it to include only $\mathrm{Cs}$ and I revolatilized from the primary system. This issue is characterized in terms of a parameter 'FLATE' which represents the fraction of whatever Cs and I initially deposited in the primary system that is revolatilized at later times, i.e., after VB. In calculating the actual contribution to the source term, 'FLATE' is multiplied by FCOR*(1-FVES) for cesium and FCOR*(1-FVES)*(1-FI2) for iodine. In all cases except the $\mathrm{V}$ sequence, the release is multiplied by FCONC for Te, after applying the 
applicable value of DFSPR or DFAER. In the V sequence, the revolatilized Cs and I release is treated as is the initial release from the primary system, since the release pathway is expected to be essentially the same. This treatment tends to enhance the importance of the revolatilization release for the $\mathrm{V}$ sequence, in comparison with other sequences.

Table C-28

ISSUE 11: LATE RELEASES OF CESIUM AND IODINE FROM THE RCS

\begin{tabular}{ccccccc}
\hline LATE RELEASE & \multicolumn{6}{c}{ WEIGHTING FACTOR } \\
LEVEL-FLATE & SARRP & Rev.1 & Rev.2 & Rev.3 & Rev.4 Composite \\
\hline 1. 0.00 & .20 & .20 & .10 & .10 & 0.0 & .12 \\
2. 0.07 & .30 & .40 & .30 & .20 & .10 & .26 \\
3. 0.23 & .30 & .30 & .40 & .40 & .60 & .40 \\
4. 0.70 & .20 & .10 & .20 & .30 & .30 & .22 \\
\hline
\end{tabular}

Issues 12 and 13: Release Associated with High Pressure Ejection (HPE) and Direct Containment_Heating_(DCH)

When the primary system is incompletely depressurized at the time of vessel breach, fuel is expected to be ejected under pressure in a process which can result in significant aerosol generation, as has been demonstrated experimentally. This process is expected to occur more or less independently of whether dispersed debris results in significant containment pressurization due to DCH. If DCH does occur, it implies additional exposure of highly heated and fragmented debris to a possibly oxidizing atmospheric environment, and this exposure is expected to lead to additional aerosol and radionuclide release from the fuel. In the original LLH straw man proposal, some allowance was made for these effects, but they were not offered as source term issues; that is, postulated HPE/DCH effects were "hard wired" into the source term evaluations. The source term review group, however, reached a consensus that these releases should be treated as source term issues in there own right, and this treatment is described here.

Dana Powers prepared a review of the phenomenology involved in these processes, and this review was distributed to the source term review group [10]. Hence, only a brief summary of some of the salient features is offered here. Powers' review included a definition of levels for representing the HPE and DCH contributions to the source term, and his representation has been adopted for the LLH study, as has Powers' recommendation that the HPE and DCH releases be treated as separate issues in the LLH. 
In calculating radionuclide release fractions for HPE, the level values given below are to be multiplied by the fraction of the core ejected, FREJ; the level values for DCH releases are to be multiplied by the quantity FREJ*FRDH, which may be taken as a measure of the amount of direct heating (Powers' discussion should be consulted for details). These parameters obviously should be functions of the amount of direct heating postulated in the event tree analysis, but they were not voted upon directly by the event tree analysts--only the total pressure rise (steam spike plus direct heating) was voted upon.

Although there is no unique relation between the pressure rise and the values of these parameters in the direct heating model, the variation in the latter values which is consistent with a specified pressure rise is limited. Some parametric calculations of pressure rise as a function of direct heating have been used to define sets of these parameters which are judged reasonable for each of the pressure rise levels in the event tree analysis. For the case of a high pressure failure with a dry cavity, these values are as follows:

\begin{tabular}{llc} 
Bressure rise & EREJ & EREJ*FRDH \\
\hline $11 \mathrm{psi}$ & 0.38 & 0.0 \\
45 & 0.38 & 0.095 \\
75 & 0.38 & 0.285 \\
104 & 0.54 & 0.405 \\
130 & 0.73 & 0.548
\end{tabular}

The above apply as given only to high pressure sequences with a dry cavity (the worst case, in the CET). For cases with intermediate pressure or a wet cavity, the CET prescribed somewhat reduced pressure rises, with further reductions prescribed for the case with both a wet cavity and intermediate pressure. Analogous downward adjustments were made in the corresponding values of FREJ and FRDH in evaluating the impact upon the source term. No HPE or DCH release was assumed for the $V$ sequence or for any case involving a large LOCA, induced or otherwise. The HPE and DCH release fractions calculated apply only to that fraction of the inventory not released prior to vessel breach. For the radionuclides released from fuel due to either HPE or DCH, FCONV $=0.8$; i.e., it was assumed that $80 \%$ of this material is released to the environment, given containment rupture at or before vessel breach. No mitigation factors apply.

If containment failure is by leakage only, or occurs significantly later than at vessel breach, HPE and DCH releases have two opposing effects upon the source term: on the one hand, they increase the amount of radionuclides airborne within containment immediately after VB; on the other hand, the increase in total aerosol concentrations can substantially shorten aerosol and radionuclide residence times in the containment atmosphere. The net effect could be 
either to increase or to reduce the radionuclide release to the environment. Since the net effect is expected to be very scenario-dependent, and since no mechanistic calculations of the effect are available, it is neglected in the LLH. It is not known whether the net impact of this neglect is "conservative" or "nonconservative", but it is believed to be small.

It was assumed that a fraction of the core equal to (FREJ*FRDH) will not be available for core concrete interactions, on the grounds that it will be too widely dispersed to reheat and attack the concrete. This reduction of CCI release is also applied to the other bins derived from high-pressure and intermediate-pressure scenarios, in which the containment does not rupture due to the direct heating (Bins 6-10 and 13). The quantity FREJ*FRDH is approximately the minimum core fraction participating in direct heating which could yield the indicated pressure rises. The fraction dispersed could be larger if the dispersed material does not contribute to direct heating with $100 \%$ efficiency. On the other hand, it is possible that not all the dispersed material should be "disqualified" from core-concrete interactions. Thus, this quantity is judged to be a reasonable, though probably conservative, measure of the degree to which occurrence of a direct heating event reduces the availability of fuel for core-concrete interactions. Fuel which participates in HPE but does not participate in DCH is not removed from the CCI inventory, although any radionuclides released due to HPE are removed from the CCI inventory. For the exact prescriptions used in these calculations, the SURSOR code listing and its comments may be consulted (Section C.2.5).

The radionuclide release fractions and weighting factors associated with HPE (Issue 12) are expressed through the parameter 'RADEJL'. Levels and weighting factors for RADEJL are listed below in Tables C-29 and C- 30 .

Table C-29

LEVELS FOR THE HIGH PRESSURE EJECTION INCREMENTAL RELEASE

\begin{tabular}{cllll}
\hline $\begin{array}{c}\text { WASH-1400 } \\
\text { GROUP }\end{array}$ & LEVEL 1 & LEVEL 2 & LEVEL 3 & LEVEL 4 \\
\hline 1 & 1.0 & 1.0 & 1.0 & 1.0 \\
$2 \& 3$ & 0.1 & 0.5 & 1.0 & 1.0 \\
4 & 0.01 & 0.1 & 0.1 & 1.0 \\
5 & $1.0 \times 10^{-4}$ & 0.01 & 0.01 & 0.1 \\
6 & $1.0 \times 10^{-4}$ & $1.0 \times 10^{-3}$ & 0.01 & 0.1 \\
7 & $1.0 \times 10^{-4}$ & $1.0 \times 10^{-3}$ & 0.01 & 0.1 \\
\hline
\end{tabular}


NUREG/CR-4551, VOL. 1: DRAFT REPORT FOR COMMENT (SEPTEMBER, 1986)

Table C-30

WEIGHTING FACTORS FOR THE HIGH PRESSURE EJECTION RELEASE

\begin{tabular}{cccccccc}
\hline & & \multicolumn{6}{c}{ WEIGHTING FACTOR } \\
LEVEI & SARRP & Rev.1 & Rev.2 & Rev.3 & Rev.4 Composite \\
\hline 1. & .20 & 0.0 & .15 & 0.0 & 0.0 & .07 \\
2. & .40 & .1 & .75 & .3 & .05 & .32 \\
3. & .40 & .85 & .1 & .65 & .9 & .58 \\
4. & 0.0 & .05 & 0.0 & .05 & .05 & .03 \\
\hline
\end{tabular}

For the $\mathrm{DCH}$ release (Issue 13), the releases are expressed through the parameter 'RADDHL'. Level specifications and weighting factors for RADDHL are listed in Tables C-31 and $\mathrm{C}-32$. The actual contributions to the source term from these processes are given by the following equations:

HPE: $0.8 *(1-\mathrm{FCOR}) *$ FREJ*RADEJL

DCH: $\quad 0.8 *(1-F C O R) * F R E J * F R D H *(1-R A D E J) * R A D D H L$

Table C-31

LEVEIS FOR THE DIRECT HEATING INCREMENTAL RELEASE

\begin{tabular}{cllll}
\hline $\begin{array}{c}\text { WASH-1400 } \\
\text { GROUP }\end{array}$ & LEVEL 1 & LEVEL 2 & LEVEL 3 & LEVEL 4 \\
\hline 1 & $1.0^{*}$ & $1.0^{*}$ & $1.0^{*}$ & $1.0^{*}$ \\
$2,3 \& 4$ & 0.5 & 1.0 & 1.0 & 1.0 \\
5 & $1.0 \times 10^{-4}$ & 0.01 & 0.2 & 0.2 \\
6 & $1.0 \times 10^{-6}$ & .05 & 0.2 & 0.9 \\
7 & $1.0 \times 10^{-6}$ & .01 & 0.05 & 0.1 \\
\hline
\end{tabular}

*Irrelevant, since $100 \%$ NG release in HPE already has been postulated to occur.

Table C-32

WEIGHTING FACTORS FOR THE DIRECT HEATING RELEASES

\begin{tabular}{cccccccc}
\hline & \multicolumn{6}{c}{ WEIGHTING FACTOR } \\
LEVEL & SARRP & Rev.1 & Rev.2 & Rev.3 & Rev.4 Composite \\
\hline 1. & .20 & 0.0 & .5 & 0.0 & 0.0 & .14 \\
2. & .35 & .4 & .4 & .1 & .1 & .27 \\
3. & .35 & .5 & .1 & .6 & .8 & .47 \\
4. & .1 & .1 & 0.0 & .3 & .1 & .12 \\
\hline
\end{tabular}




\section{Qther Issues}

In addition to the issues discussed above, other issues could certainly be defined. Some of these may well be significant; for example, it has been argued that source terms associated with late containment failures could be substantially augmented by resuspension if the late failure involves an energetic process (e.g., hydrogen burns or detonations, or even slow overpressure failures if the failure itself is a catastrophic rupture). In addition, the QUEST study showed that I-132 produced by decay of Te-132 is a potentially important contributor to the source term associated with late containment failures. Although this decay chain was noted in connection with Issue 10 , it is not explicitly represented by any of the issues as they were finally defined for the LLH study.

It also may be noted that none of the issues addressed scenarios involving isolation failure, basemat melt-through, or leakage at or somewhat above the design basis. However, the risk-significance of such issues is expected to be small, due to low probability of occurrence or low consequences.

The details of the actual implementation of the source term calculations for the Surry LLH study are given in Section C.2.5.

\section{C.2.4 Review Comments of Broader Applicability}

During the review session on the LLH inputs for the source term analysis there were several discussions that arose during the discussion of a specific issue, but that had a more general applicability to the methods and interpretation of the LLH study. The comments concerning the approach itself and its interpretation are discussed in Appendix $F$. These comments are important to the understanding of the LLH method and the differing interpretations of the weighting factors and the ranges. This section describes some other comments, somewhat more specific and less philosophical in nature. These comments too are important to a fuller understanding of the inputs of the review group and the limitations of the results or the specific source term review input.

One issue that was raised several times during the source term meeting was that the limitations associated with the scope of the uncertainty project could result in a potentially significant loss of information concerning the state of the art in source term research. This problem is particularly germane to the source term analysis because the size of the problem forces simplifying steps, such as the creation of source term bins and radionuclide groups, and the LLH analysis requires yet further simplifications. There are too many degrees of freedom for a reasonable approach to handle the more complete level of detail. For example, the 
NUREG/CR-4551, VOL. 1: DRAFT REPORT FOR COMMENT (SEPTEMBER, 1986)

selection of a level of values for a issue such as \# 3, requires that all of the values for each radionuclide groups be accepted, whereas an individual reviewer may have very strong opinions concerning the effect of the issue on a single fission product group. In addition, the research to resolve these uncertainties is continuing, but at a level of detail that would not appear in this analysis. This resolution is therefore sacrificed to the method, aside from the documentation of such opinion in this report. The result of this is that special care will be required in the development and presentation of the results and insights of the LLH study. For several of the source term issues, some reviewers felt that there was a potential for this type of problem, but recognizing limitations to approaching the problem more thoroughly, all agreed to provide their weighting factors as requested.

One other issue that was discussed at length concerned the selection of the ranges for the issues. One reviewer was particularly alerted to this issue by the relatively uniform range associated with the voting on weighting factors for several of the issues. This raised the question of whether the selected ranges really represent the reasonable limits on the issue. For example, if the ranges were doubled for an issue currently weighted somewhat uniformly, the weightings might not change, indicating that the selected ranges were too small. Two features of the analysis tended to limit this potential problem. The first was that the reviewers were asked to approve the ranges and, if the method and schedule allowed, any changes in levels were incorporated, as was actually the case for several issues. The second feature is that the issues weighted most uniformly involved levels with ranges from 0.0 to 1.0 and the limits could not actually be changed. After discussion of this issue, the reviewers were particularly aware of the potential problem and on several occasions re-examined the range to be assured that it was large enough. It should also be noted that if the levels were defined too near the extreme, low weighting factors could prevent their being sampled in the limited number of sample members (100) used in the LLH.

Finally one other concern relative to the levels was that the reviewers could have differing interpretations of what their weighting factors meant relative to the levels. For example, it could be possible that one reviewer would treat the levels at the extreme as limits, and provide weighting factors accordingly, while another could treat the same level as being representative of a range of outcomes both greater and lesser than the actual level specification. The discussion during the specification of levels helped to limit this problem.

A second area of concern by a participant outside the source term review group dealt with the potential for operator action that could affect the source term release from the site. Even after an accident had proceeded to core melt and/or vessel breach, there are activities that 
could and probably would be implemented that would have an impact on the source term. This is particularly possible for accident sequences with long times before containment failure. Any major accident would quickly involve many experts who could act to mitigate the effects of an accident. The actions could include repair of equipment, unique solutions to problems, and innovative ways to reduce the threat of release. While it is difficult to quantify these effects, it was the opinion of the reviewer that the uncertainty would be misrepresented without some sort of consideration of these effects, and that the difficulty in quantifying these may not really be different than some of the other issues that were quantified with little or no evidence.

\section{C.2.5 Implementation of Surry Source Term Calculations for the LLH}

As noted previously, Surry source terms in the LLH were described in terms of 19 bins, each corresponding to a set of sequence/pathway combinations which were judged to give comparable source terms. The impact of all issues must be considered for all bins, although not every bin is affected by every issue. Thus, each of the 19 bins must be evaluated for each specification of the source term issues, i.e., for each member of the LLH sample. This subsection documents the means by which the source terms are evaluated, and includes the listing of the SURSOR routine which performs the required calculations. The source term derivation is based upon Eq. C.2-1 of Section C.2.3, with the restrictions and conditions specified in Section C.2.3 included. The evaluation of the individual bins is summarized in a somewhat opaque form in Table C-33.

In Table C-33, a cross (X) means that the value assigned to the indicated quantity is assigned in a straight-forward manner as discussed in Section C.2.3. Absence of any entry means the particular issue does not arise in connection with the bin. Where specific numerical values are entered, these are always used, independently of the level assignments; such cases arise for reasons to be discussed shortly.

The following points require further explanation:

FVES: The values of FVES chosen are for whichever of the four RCS thermal-hydraulic states (discussed in Section C.2.3) is appropriate for the bin. These states are designated high pressure (HP), intermediate pressure (IP), low pressure (LP), and V-sequence (V).

FCONV: Fixed values are now assigned for all scenarios.

FCONC: Fixed values are assigned for early failure, bypass, and leakage scenarios; " $L$ " means the value discussed in Section C.2.3 for late containment failures is assigned (Issue 7).

DFAER: An entry "RCS" means that the DF used to represent aerosol agglomeration uncertainties is applied to FCONV, while a "CCI" entry means it is applied to FCONC. 
Table C-33

Expression of Issues for Surry Source Term Bins

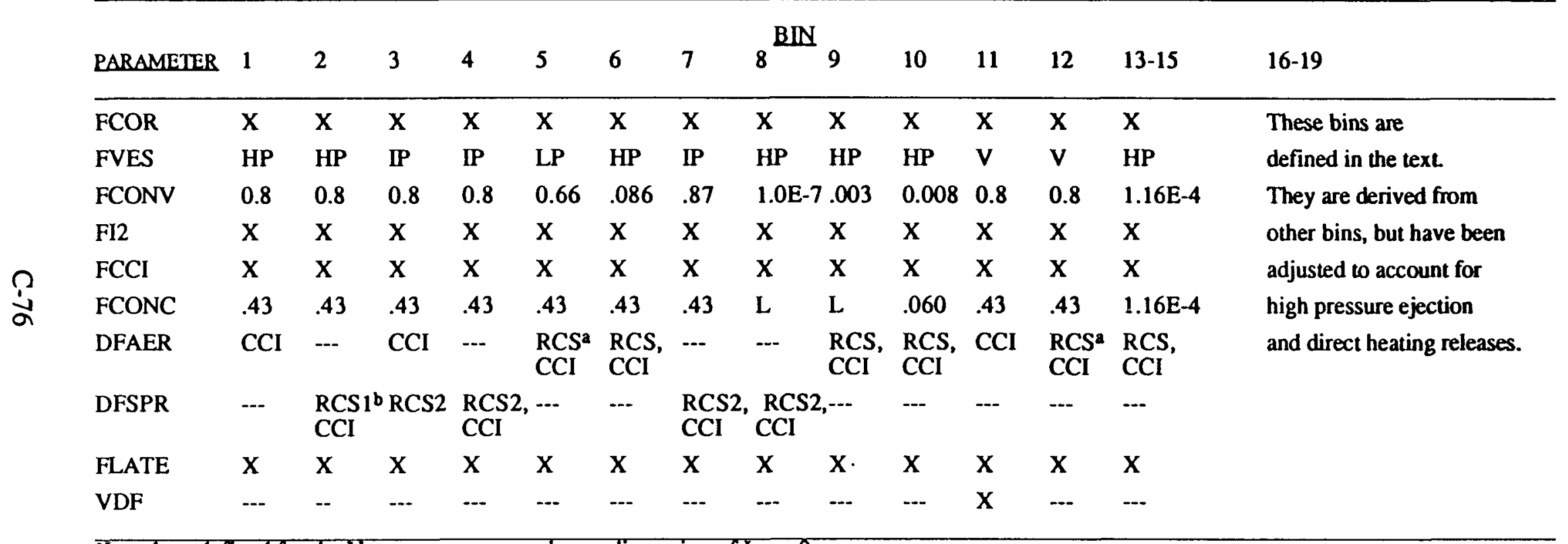

a Level set defined for the $V$ sequence was used: see discussion of Issue 9 ..

${ }^{b}$ Currently set equal to unity, i.e., no decontamination. 
NUREG/CR-4551, VOL. 1: DRAFT REPORT FOR COMMENT (SEPTEMBER, 1986)

DFSPR: As for DFAER, with the added specification that "RCS1" means that the value appropriate for a puff release from the RCS occurring at containment failure time is assigned; "RCS2" designates the value appropriate for all other cases, as discussed in Section C.2.3. Currently, the RCS1 value is always unity; as discussed in Section C.2.3 this choice stemmed from a misconception as to the nature of the RCS release and is probably too conservative.

Bins 13, 14, 15. Except for late sources of volatile iodine (Issue 10) and noble gases, these bins are the same. Noble gas releases are fixed at the central values $(1.0,0.03,0.005$, respectively) while volatile iodine releases are $(0.03,0.03,0.005$, respectively) times the volatile iodine fraction specified under Issue 10 in Section C.2.3.

Bins $16-19$. Derived from Bins $1-4$, respectively, by increasing the environmental release by an amount equal to the extra release from fuel that is specified in Issues 12 and 13 due to high pressure ejection and direct containment heating.

It was previously noted that scenarios involving containment leakage failure modes, isolation failures, basemat failures, and design leakage with no CF were not discussed in deriving the issue levels. In general, these scenarios will not be risk-dominant and defining issues specifically for them is not justified. The rationale underlying the values of FCONV and FCONC (Section C.2.3) is not applicable to these scenarios; hence, the values of these parameters are held fixed at values chosen so that the resulting source terms will be reasonably close to the central source term estimates when the other parameters are fixed at values corresponding to the central source term calculations. This rationale applies to all the specific numerical values entered into Table C-33.

The actual evaluation of source terms for the LLH study is carried out by the SURSOR routine. This routine has undergone substantial testing and debugging, although, as in any code, the absence of bugs can not be guaranteed--though the "mathematics" involved is trivial, the logic is somewhat complex and it is hard to be certain that something unintended can't occur for some of the innumerable possible combinations of input. Hence, if anyone using this routine sees results that they question, the author should be consulted immediately.

Most of the input level assignments and the source term bins generated are straightforward in terms of the discussion in Section C.2.3 and will not be discussed in detail here. The code listing includes a brief specification of the inputs required. The listing is provided as Table C-34 at the end of this section.

There are some points concerning application to scenarios involving induced LOCAs and direct heating that are not intuitively obvious. First, induced LOCAs are assumed to apply 
only to the high pressure sequences; hence, they have no effect upon the intermediate pressure cases (Bins 3, 4, 18, and 19). For the case of a high pressure sequence without sprays and with an induced LOCA, Bin 1 (and hence Bin 16) is calculated assuming FVES values appropriate for the size of the LOCA. The effect is to significantly increase releases from the RCS in most cases.

As has been discussed on many occasions, Bin 2 contains no allowance for the effect of the sprays upon the releases from the RCS. This is now believed to be overly conservative even for the case without an induced LOCA, since the RCS releases are not truly a puff and the sprays would have some limited time in which to decontaminate the containment atmosphere. It is even more conservative for the induced LOCA cases, since the RCS releases are expected to be more protracted when there is an induced LOCA, thereby giving the sprays more time to have an effect. Hence, a source term analogous to Bin 1 with induced LOCA is not calculated for Bin 2; instead, the standard Bin 2 (i.e., ignoring induced LOCAs) is returned. This is crudely equivalent to giving the sprays a modest amount of credit, since the high-pressure sequence releases of RCS species are typically a factor of 2 to 10 lower than the low- or intermediate-pressure cases. However, exceptions arise--Bin 2 can give a higher RCS release than Bin 1 for some combinations of level specifications. In all cases, Bin 17 is based upon the RCS releases of Bin 2 (i.e., without the induced LOCA), but the induced LOCA is included in estimating the direct heating effects (the sprays do not introduce any special ambiguities in interpreting the effects of the LOCA upon the direct heating releases).

The suggested binning for high pressure sequences with sprays and with induced LOCAs is as follows. If containment failure is thought to occur at about the time of maximum radionuclide release from the vessel (e.g., during the time interval between start of core melt and start of core slump), the time available for decontamination by the sprays is likely limited and $\mathrm{Bin} 2$ (Bin 17 with $\mathrm{DCH}$ ) may be a reasonable assignment. (However, it can be rather conservative in some cases, notably when there is substantial CsI decomposition within the RCS specified.) If containment failure is expected to occur significantly later (e.g., at vessel breach), Bin 3 or Bin 4 (18 or 19 with $\mathrm{DCH}$ ) is the appropriate assignment, depending upon whether sprays fail or survive at containment failure. For the small induced LOCAs, containment failure at vessel breach is the likely alternative and, hence, Bins 3 and 4 (18 and 19) are appropriate, just as if the LOCA were the accident initiator. For large induced LOCAs, the choice is less clear.

It must be remembered that Bins 1 and 16 are currently used for high pressure sequences in which sprays operate up to containment failure and then fail, in addition to those 
cases for which the sprays were never operating. As in the discussion of Bins 2 and 17, this is conservative when there is no induced LOCA and still more so when there is an induced LOCA. For small induced LOCAs, the obvious solution is to reassign these scenarios to Bins 3 and 18. For large induced LOCAs, the choice is less clear. Indeed, the (lack of) treatment of the effects of sprays upon the RCS releases in high pressure sequences continues to be an embarrassment in a number of contexts. The effects can probably be bounded by running the complete analysis assigning these scenarios to Bins $1,2,16$, and 17 and then repeating with assignment to Bins $3,4,18$, and 19 . This bounding approach has been tentatively accepted by the review group, although with some misgivings and with a recommendation that the treatment be refined, if possible, should the bounding approach show the question to be important.

Another orphan scenario in the present treatment is the case with a LOCA as a core melt initiator but with no sprays. At present, Bin 1 is probably the best assignment but it has serious limitations: the contents of Bin 1 will depend upon how the induced LOCA flag has been set for the high pressure sequences, and direct heating will also be calculated in $\mathrm{Bin} 16$ for the current induced LOCA flag setting; obviously, the induced LOCA status for the highpressure sequences is irrelevant for the initiating LOCAs that are actually of interest here. The LLH results showed that station blackout scenarios dominate the mean risk by rather wide margins. Among the station blackouts, plant damage state SNNN (S2 pump seal LOCA) exceeds TNNN (fully pressurized primary system) by a factor of three to four. This means that the absence of a bin for LOCA-induced scenarios without sprays does, in fact, impact the risk-dominant scenario in the LLH. Time has not permitted changes to address this difficulty, hence, SNNN continues to be treated as if it were TNNN in terms of source term issues. A new bin (Bin 20) was defined for these scenarios as noted in the main report, but the results depicted throughout the report have not yet been updated to reflect this new bin.

The dominance of station blackout in the LLH does mean that the issues discussed above in connection with sprays in high- pressure sequences are less important. These issues do affect plant damage states TYYBW and TYYBD, which together contribute about 10-12\% to the mean risk.

Many assumptions were required in order to implement the SURSOR representation of the Surry source terms for the LLH study. Most of those not discussed previously in this report are flagged by comments in the code listing in Table C-34. The FORTRAN source code listing follows and is sufficiently commented to permit a reasonably motivated (or masochistic) reviewer to determine any specific details of the calculation that may be of interest. 
Table C-34

LISTING OF THE SURSOR CODE

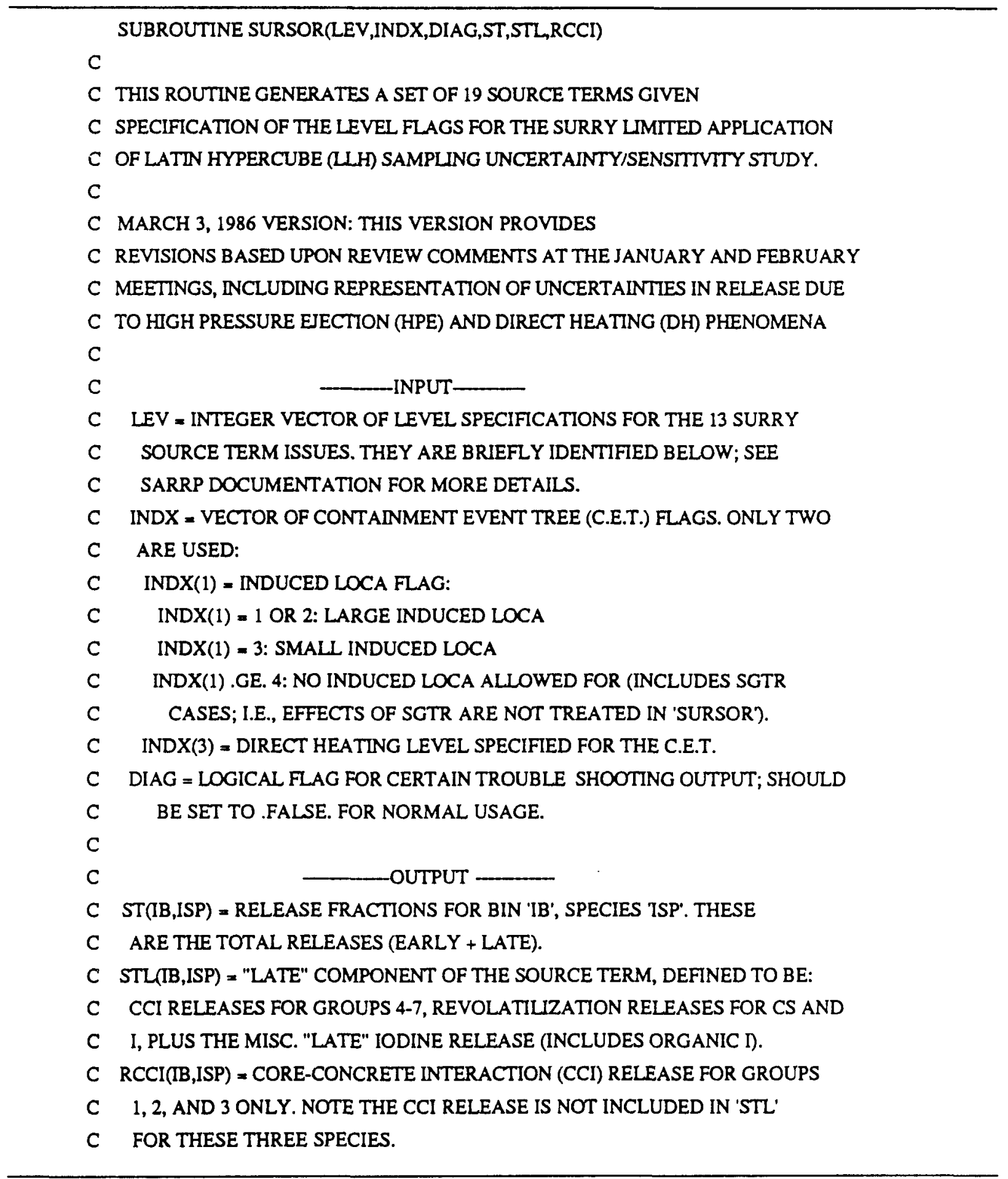


NUREG/CR-4551, VOL. 1: DRAFT REPORT FOR COMMENT (SEPTEMBER, 1986)

Table C-34 (Continued)

DIMENSION INDX(10),LEV(20),ST(20,7),STL(20,7),RCCI(20,3)

DIMENSION FVHP $(4,7), F V I P(4,7), F V L P(4,7), F V V(4,7)$

DIMENSION FCORL(4,7),FI2(4),FCONVE(4),VDF(4),CCI(4,7),FCONCE(4)

1,FCONCL(4,7),DFSPR1(4),DFSPR2(4),DFSPRC(4),DFAERL(4),DFAERV(4),

2LATEIL(4),FLATE(4)

DIMENSION FCOR(10),FVES(10),FCCI(10),FCONC(10),FCVFIX(20),

1FCONTE(20),MAXL(20),ICASE(4)

DIMENSION FREJ(4,5),FRDH(4,5),RADEJL(4,7),RADDHL(4,7)

REAL LATEIL

C 'MAXL(几)' IS THE NO. OF LEVELS ASSOCIATED WTTH THE JLTH ISSUE; IF

C A HIGHER LEVEL IS SPECIFIED, 'MAXL(IL)' IS THE LEVEL ACTUALLY USED

DATA (MAXL(JL),JL=1,13)/4,4,4,4,3, 3,4,3,3,4, 4,4,4/

C LEVEL SPECIFICATIONS FOR 'FCOR'

DATA ((FCORL(IL,IS),IS $=1,7), \mathrm{IL}=1,4) / 3 * 0.5,0.026,0.014,0.008$,

$11.4 \mathrm{E}-5,3 * 0.986,0.33,0.13,0.041,0.00025,3 * 1.0,0.90,0.49,0.11$,

$20.0077,4 * 1.0,0.98,0.53,0.074 /$

C FRACTION OF CSI THAT DECOMPOSES TO ELEMENTAL IODINE

DATA (FI2(1),I=1,4)/0.0,0.33,0.67,1.0/

C RELEASE FRACTIONS FROM THE PRIMARY SYSTEM - HIGH PRESSURE SEQUENCES

DATA ((FVHP(IL,IS),IS=1,7),IL=1,4)/1.,3*.01,3*.03,

$11 ., 2 * 14, .15,3 * .11,1 ., 2 * 0.4,0.3,3 * 0.2,1 ., 3 * 0.9,3 * 0.41$

C DITTO, MEDIUM - PRESSURE SEQUENCES

DATA ((FVIP(IL,IS),IS=1,7),IL=1,4)/1.0,2*.04,.01,3*.1,

$1 \quad 1 ., 2 * .5, .02,3 * .34,1 ., 2 * .7, .2,3 * .5,1 ., 3 * .9,3 * .71$

C DITTO, LOW PRESSURE SEQUENCES

DATA ((FVLP(IL,IS),IS=1,7),IL=1,4)/1.,2*.2,4*.1,

$11 ., 2 * .87, .83,3 * .77,1 ., 2 * .95, .9,3 * .85,3 * 1 ., 4 * .95 /$

C DITTO, V-SEQUENCE

DATA ((FVV(IL,IS),IS=1,7),IL=1,4)/1.,6*.05, 1.,2*.2,.1,3*.11,

$11 ., 6^{*} .4,1 ., 6^{*} .91$

C DF FOR V-SEQUENCE WTTH WATER

DATA (VDF(I), $\mathrm{I}=1,4) / 100.0,12.0,5.0,2.0 /$

C CONTAINMENT RELEASE FRACTIONS FOR RCS SPECIES

C -THESE NOW A DUMMY: ALL LEVELS $=0.8-$ DATA $(\operatorname{FCONVE}(1), I=1,3) / 0.8,0.8,0.81$

C FRACTIONAL RELEASES FROM THE MELT DURING CCI

DATA ((CCI(IL,IS),IS=1,7),IL=1,3)/1.,2*.02,.008,.002,1.E-6,2.E-5,

$13 * 1 ., 0.40,0.05,3 . \mathrm{E}-5,7.7 \mathrm{E}-4,3 * 1 ., 0.8,0.2,0.07,0.02 J$

C CONTAINMENT RELEASE FRACTIONS FOR CCI SPECIES, EARLY CONT. FAIL 
Table C-34 (Continued)

C - THESE NOW A DUMMY: ALL LEVELS $=0.43$

DATA (FCONCE(I), $1=1,4) / 0.43,0.43,0.43,0.43 /$

C SAME, FOR LATE CONTAINMENT FAILURES

DATA ((FCONCL(IL,IS),IS=1,7),IL=1,4)/1.,2*.001,.015,.003,.015

$1, .003,1 ., 2 * .01, .15, .03, .15, .03,1 ., 2 * .1, .37, .16, .37, .16$,

$11 ., 2 * 3, .4,3, .4, .31$

C "DF" FOR SPRAYS: PUFF RELEASE FROM RCS, SLOW RELEASE FROM RCS,

C AND CCI RELEASES

C -DFSPR 1 (PUFF RELEASE) NOW DUMMY: $=1$, ALL LEVELS

DATA (DFSPR1(IL),DFSPR2(IL),DFSPRC(IL),IL=1,3)/1.,100.,1000.,

1 1.,45.,30., 1.,5.,10.1

C "DF" TO REPRESENT AEROSOL AGGLOMERATION UNCERTAINTIES

DATA (DFAERL(IL),DFAERV(IL),IL=1,3)/10.,3., 3.,1.5, 1.0,1.0/

C LATE VOLATILE IODINE FRACTION (ORGANIC, FROM POOLS, DECAY CHAINS,

C ETC.

DATA (LATEIL(IL),IL=1,4)/0.0,0.01,0.05,0.1/

C FRACTION OF CS AND I THAT IS RELEASED TO THE CONTAINMENT AT

C LATE TIMES

DATA (FLATE(IL),IL $=1,4) / 0.0,0.07,0.23,0.70 /$

C CONTAINMENT REIEASE FRACTIONS FOR BINS $6-13$ (NOT TREATED AS A

C SOURCE TERM ISSUE)

DATA (FCVFIX(IB),IB=1,9)/0.66,0.0855,0.87,1.E-7,0.003, 0.008,

$10.8,0.8,1.16 \mathrm{E}-4 /$

C FRACTIONS OF CORE EJECTED IN HPE, DIRECT HEATING FRACTIONS

DATA ((FREJ(IC,IL),IL $=1,5), \mathrm{IC}=1,4) / 3 * .38, .54, .73$,

$1.15,2 * .38, .54, .73, .075,2 * .38, .54, .73,5 * 0.0 /$

DATA ((FRDH(IC,IL),IL $=1,5), \mathrm{IC}=1,4) / 0 ., 0.25,3 * .75,0 ., 0.2,0.38$

$1,0.55,0.50,0 ., 0.07,0.18,0.33,0.25,5 * 0.01$

C RADIONUCLIDE REIEASE LEVELS FOR HPE AND DH

DATA ((RADEJL(IL,IS),IS=1,7),IL $=1,4) / 1.0,2 * 0.1,0.01,3 * 1 . E-4$,

$11.0,2 * 0.5,0.1,0.01,2 * 1 . E-3,3 * 1 ., 0.1,3 * 0.01,4 * 1.0,3 * 11$

DATA ((RADDHL(IL,IS),IS=1,7),IL $=1,4) / 1.0,3 * 0.5,1 . \mathrm{E}-4,2 * 1 . \mathrm{E}-6$,

$14 * 1.0,0.01,0.05,0.01,4 * 1.0,2 * 0.2,0.05,4 * 1.0,0.2,0.9,0.1$,

LOGICAL DIAG

C SET 'DIAG' .TRUE. TO CHECK ON DATA INITIALIZATION (ALSO CHECKS

C ON ASSIGNING DFS' FOR ISSUES 8 AND 9)

IF(.NOT.DIAG) GO TO 1

PRINT 1020

1020 FORMAT( $/ /$ FCOR LEVEL VALUES_-_') 
NUREG/CR-4551, VOL 1: DRAFT REPORT FOR COMMENT (SEPTEMBER, 1986)

Table C-34 (Continued)

DO $1001 \mathrm{IL}=1,4$

PRINT 1010,IL,(FCORL(IL,IS),IS=1,7)

1010 FORMAT $(14,4 X, 1$ P7E10.2)

1001 CONTINUE

PRINT 1030

1030 FORMAT( $/ /$ FVES VALUES; HP, IP, LP, V SEQUENCES - ' $)$

DO 1002 IL $=1,4$

1002 PRINT 1010,IL,(FVHP(IL,IS),IS=1,7)

DO 1003 IL $-1,4$

1003 PRINT 1010,IL,(FVIP(IL,IS),IS=1,7)

DO $1004 \mathrm{IL}=1,4$

1004 PRINT 1010,IL,(FVLP(IL,IS),IS=1,7)

DO 1005 IL $=1,4$

1005 PRINT 1010,IL,(FVV(IL,IS),IS=1,7)

PRINT 1040,(FI2(IL), VDF(IL),FCONVE(IL),IL $=1,4)$

1040 FORMAT(/ FI2 VDF FCONVE'//1P(3E10.2))

PRINT 1080,(FCVFIX(I),I=1,9)

1080 FORMAT( $/$ FCVFIX VALUES, BINS 5 THRU $13-\%(5 X, 1$ P5E10.2))

PRINT 1060

1060 FORMAT $\left({ }^{\prime}\right.$ CCI VALUES - ' $)$

DO 1007 IL $=1,4$

1007 PRINT 1010,IL,(CCI(IL,IS),IS=1,7)

PRINT 1050

1050 FORMAT( $($ FCONCL VALUES - $'$ )

DO 1006 IL $=1,4$

1006 PRINT 1010,IL,(FCONCL(IL,IS),IS=1,7)

PRINT 1070,(FCONCE(IL),DFSPR1(IL),DFSPR2(IL),DFSPRC(IL),

1DFAERL(IL),DFAERV(IL),LATEIL(IL),FLATE(IL),IL $>1,4)$

1070 FORMAT( $/{ }^{\circ}$ FCONCE DFSPR1 DFSPR2 DFSPRC DFAERL

1 DFAERV LATEIL FLATE'//1P(8E10.2))

PRINT 1090,(IC,(FREJ(IC,IL),IL=1,J),IC=1,4),(IC,(FRDH(IC,IL),IL

$1=1,5, I C=1,4)$

1090 FORMAT(/' FREJ VALUES - $/ 4(/ 15,1$ P5E10.2)/' FRDH VALUES --

14(/15,5E10.2))

PRINT 1100,(IL,(RADEJL(IL,IS),IS=1,7),IL=1,4),(IL,(RADDHL

IIL,IS),IS=1,7),IL=1,4)

1100 FORMAT( $/$ RADEJL VALUES - $4(/ 15,1$ P4E10.2,2X,3E10.2)/' RADDHL

IVALUES -'4(/15,1P4E10.2,2X,3E10.2))

1 CONTINUE 
NUREG/CR-4551, VOL. 1: DRAFT REPORT FOR COMMENT (SEPTEMBER, 1986)

Table C-34 (Continued)

C SET INDUCED LOCA FLAG

ILOCA $=$ MINO(3,5-INDX(1))

ILOCA $=$ MAXO(ILOCA,1)

$\mathrm{NL}=11$

NSPEC $=7$

C START CONCOCTING THE SOURCE TERMS

C 13 BINS CALCULATED IN DETAIL — OTHERS DERIVED FROM THESE

NBIN $=13$

C ISSUE 1. IN-VESSEL RELEASE FROM FUEL (FCOR)

ISS $=1$

IL = MINO(LEV(ISS),MAXL(ISS))

DO 2 ISP $=1$,NSPEC

2 FCOR(ISP) - FCORL(IL,ISP)

DO 101 IB $=1, N B I N$

C ISSUE 2: CSI DECOMPOSITION WTTHIN RCS (TREATED BELOW)

$C$ ISSUE 3. RELEASE FROM THE RCS (FVES)

ISS $=3$

$\mathrm{IL}=$ MINO(LEV(ISS),MAXL(ISS))

GO TO $(11,211,12,12,13,11,12,11,11,11,14,14,11)$,IB

C HIGH RCS PRESSURE

11 CONTINUE

GO TO $(211,12,13)$, ILOCA

211 CONTINUE

DO 15 ISP $=1$,NSPEC

15 FVES(ISP) = FVHP(IL,ISP)

GO TO 19

C INTERMEDIATE RCS PRESSURE

12 CONTINUE

DO 16 ISP $=1$,NSPEC

16 FVES(ISP) = FVIP(IL,ISP)

GO TO 19

C LOW RCS PRESSURE

13 CONTINUE

DO 17 ISP $=1$,NSPEC

17 FVES(ISP) = FVLP(IL,ISP)

GO TO 19

C V-SEQUENCE

14 CONTINUE 
NUREG/CR-4551, VOL 1: DRAFT REPORT FOR COMMENT (SEPTEMBER, 1986)

Table C-34 (Continued)

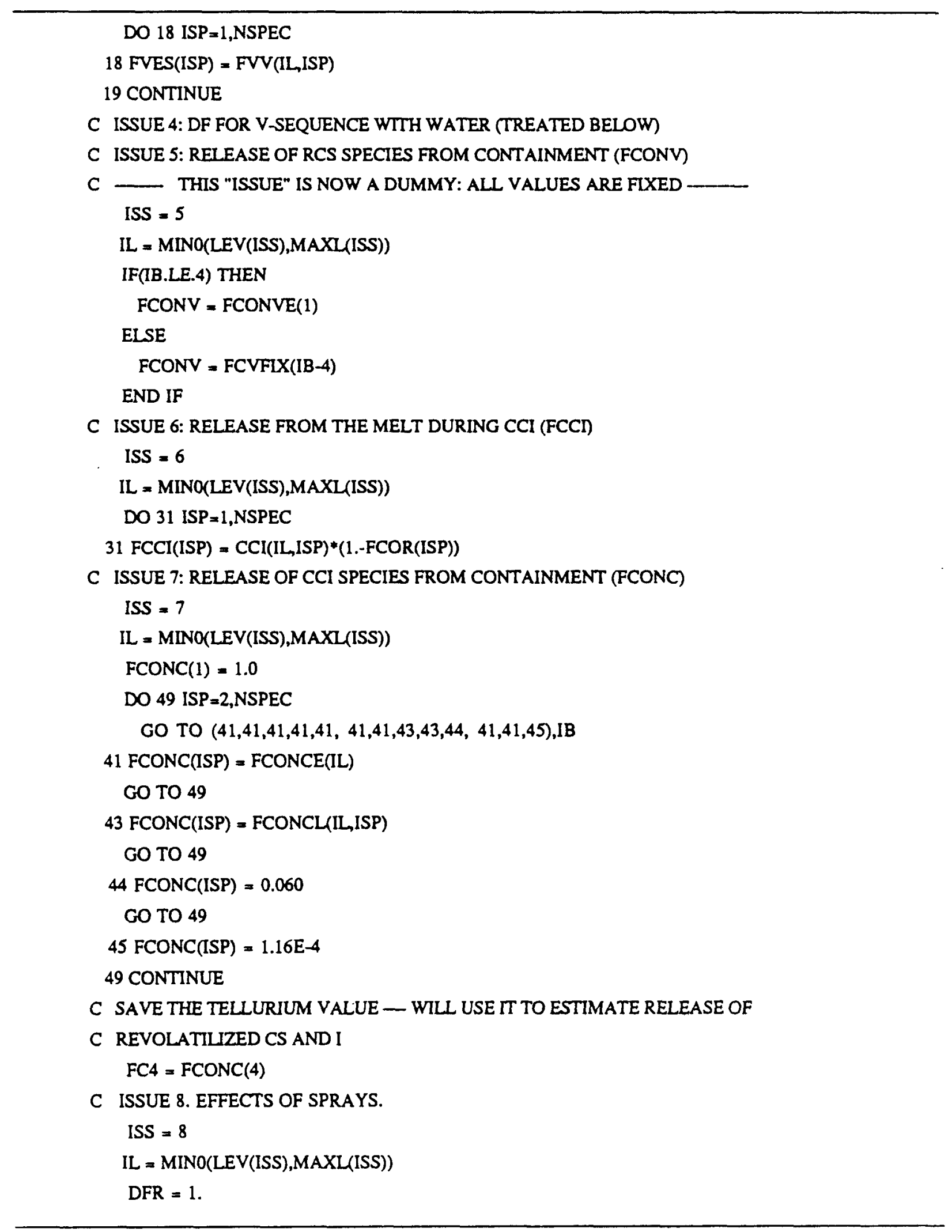




\section{Table C-34 (Continued)}

$\mathrm{DFC}=1$.

DFRS $=$ DFSPR2(IL)

$\mathrm{DFCMX}=1$.

GO TO $(51,52,53,54,51,51,54,55,51,51,51,51,51)$, IB

C SPRAYS SURVIVE, BUT PUFF RCS RELEASE (MINIMAL SPRAY EFFECTS

C FOR RCS SPECIES: CURRENTLY A DUMMY, ALL LEVELS $=1.0$ )

52 DFR - DFSPR1(IL)

DFC $=$ DFSPRC(IL)

GO TO 51

C REGULAR EFFECT ON RCS RELEASE, SPRAYS FAIL AT CF

53 DFR = DFSPR2(IL)

GO TO 51

C SPRAYS SURVIVE

54 DFR = DFSPR2(IL)

DFC $=$ DFSPRC(IL)

GO TO 51

C PROVISION TO FUDGE BIN 8 TO AGREE BETTER WITH BATTELLE CALC. FOR

C 'CENTRAL' TE REIEASE (FUDGE = APPROX. 2). NOT CURRENTLY USED.

55 FUDGE $=1$.

DFR $=$ DFSPR2(IL)/FUDGE

DFC $=$ DFSPRC(IL)/FUDGE

$51 \mathrm{FCONV}=\mathrm{FCONV} / \mathrm{DFR}$

DO 56 ISP $=2$,NSPEC

$56 \mathrm{FCONC}(\mathrm{ISP})=\mathrm{FCONC}(\mathrm{ISP}) / \mathrm{DFC}$

C SAVE 'DFCMX' TO ESTIMATE 'FCONC' FOR LATE CS AND I RELEASES;

C CREDIT FOR SPRAYS NOT TO EXCEED MAX. VALUE FOR RCS RELEASES.

DFCMX = AMAX1(DFC,DFCMX)

DFCMX = AMIN1(DFCMX,DFRS)

IF(DIAG) PRINT 510,IB,DFR,DFC

510 FORMAT $\left({ }^{\prime} \quad\right.$ SPRAY DF FOR BIN',13,' DFR, DFC ='1P2E10.3)

C ISSUE 9. AEROSOL AGGLOMERATION AND DEPOSITION UNCERTAINTIES

ISS $=9$

$\mathrm{IL}=\mathrm{MINO}(\mathrm{LEV}(\mathrm{ISS}), \mathrm{MAXL}(\mathrm{ISS}))$

$\mathrm{DFC}=1$.

$\mathrm{DFR}=1$.

GO TO $(61,69,61,69,63,62,69,69,62,62,61,63,62)$,IB

C APPLIES TO BOTH RCS AND CCI RELEASES

62 DFR = DFAERL(IL)

C APPLIES TO CCI RELEASES ONLY 
Table C-34 (Continued)

61 DFC = DFAERL(IL)

IF((IB.NE.1).OR.(ILOCA.EQ.1))GO TO 69

C V-SEQUENCE WITHOUT WATER, AG SEQUENCE, AND HIGH PRESSURE SEQUENCE W/O

C SPRAYS BUT WITH INDUCED LOCA: ONLY LIMITED EFFECT ON RCS SPECIES 63 DFR = DFAERV(IL)

DFC $=$ DFAERL(IL)

69 FCONV $=$ FCONV/DFR

C USE 'DFCMX' TO ESTIMATE 'FCONC' FOR REVOLATILIZATION RELEASES OF CS AND I.

C BASE IT ON THE VALUE FOR TELLURIUM. EXCEPTION: TREAT REVOLATILIZATION

C IN THE V-SEQUENCE AS BEING SIMILAR TO PRIMARY SYSTEM RELEASES.

DFCMX $\approx$ AMAX1(DFC,DFCMX)

FCONTE(IB) $=$ FC4/DFCMX

IF(IB.EQ.12) FCONTE(IB) $=$ FCONV

DO 68 ISP $=2$,NSPEC

$68 \mathrm{FCONC}($ ISP $)=\mathrm{FCONC}(\mathrm{ISP}) / \mathrm{DFC}$

IF(DIAG) PRINT 610,IB,DFR,DFC

610 FORMAT(' AEROSOL DF FOR BIN',13,'. DFR, DFC ='1P2E10.3)

C ASSEMBLE INITIAL SOURCE TERM VALUES. KEEP CCI RELEASES SEPARATE

C FROM RCS RELEASE.

C NOBLE GAS

$S T(I B, 1)=F C O R(1)$

$\operatorname{STL}(\mathrm{IB}, 1)=\mathrm{FCCI}(1) * \mathrm{FCONC}(1)$

C ISSUE 2: ALLOW FOR VOLATTLE IODINE RELEASE FROM RCS

ISS $=2$

IL = MINO(LEV(ISS),MAXL(ISS))

$\mathrm{ST}(\mathrm{IB}, 2)=\mathrm{FCOR}(2) *\left(\mathrm{FI}(\mathrm{IL})+(1 .-\mathrm{FI} 2(\mathrm{IL}))^{*} \mathrm{FVES}(2)\right)^{*} \mathrm{FCONV}$

$\operatorname{STL}(\mathrm{IB}, 2)=\mathrm{FCCl}(2) * \mathrm{FCONC}(2)$

C CALCULATE OTHER SPECIES

DO 71 ISP $=3$,NSPEC

STL(IB,ISP) $=$ FCCI(ISP)*FCONC(ISP)

71 ST $($ IB,ISP) $=$ FCOR(ISP)*FVES(ISP)*FCONV

C FOR THE LATE RELEASES OF CS AND I, ADJUST FCONTE(IB) FOR FCOR

$C$ AND FVES VALUES. NOTE THIS WILL HAVE TO BE DONE SEPARATELY FOR CS

C AND I IF THE VALUES EVER DIFFER FOR THESE SPECIES - CURRENTLY ARE

C ALWAYS THE SAME. CORRECT FOR VOLATTLE IODINE IN RCS LATER.

FCONTE $(\mathrm{IB})=\mathrm{FCOR}(3) *(1-\mathrm{FVES}(3)) * \mathrm{FCONTE}(\mathrm{IB})$

101 CONTINUE

C ISSUE 4: CORRECT BIN 11 (V-SEQUENCE WITH WATER) FOR SCRUBBING

ISS $=4$ 
Table C-34 (Continued)

$$
\begin{gathered}
\text { IL }=\text { MINO(LEV(ISS),MAXL(ISS)) } \\
\text { IB }=11 \\
\text { DO } 102 \text { ISP }=2, \text { NSPEC } \\
\text { STL(IB,ISP) }=\text { STL(IB,ISP)/VDF(IL) } \\
102 \text { ST(IB,ISP) }=\text { ST(IB,ISP)/VDF(IL) }
\end{gathered}
$$

C FOR THE REVOLATILIZATION OF CS AND I, USE THE BIN 12 RELEASE

C FACTOR, DIVIDED BY THE SCRUBBING 'DF

FCONTE(IB) $=$ FCONTE (12)/VDF(IL)

$\mathrm{C}$

C - ISSUES 12 AND 13

C ADD THE HIGH PRESSURE EJECTION (HPE) AND DIRECT HEATING (DH)

C CONTRIBUTIONS TO BINS 1 THRU 4 TO GET BINS 16 THRU 19, RESPECTIVELY.

$\mathrm{C}$

C SET FLAGS FOR THE CASE: CASE 1 = HP SEQUENCE, DRY CAVITY; CASE 2

C CASE 2 = HP SEQ. WTTH WET CAVTTY OR INTERMED. P WITH DRY CAVITY;

C CASE 3 = INTERMED. P WITH WET CAVITY; CASE 4 = LARGE INDUCED LOCA

C (NO HPE OR DH RADIONUCLIDE RELEASE FOR BINS 16 AND 17).

ICASE $(1)=$ IL.OCA

IF(ILOCA.EQ.3) ICASE(1) $=4$

C BIN 17 WILL HAVE THE HPE AND DH CONTRIBUTIONS APPROPRIATE TO A

C WET CAVITY AND A HIGH PRESSURE RCS AS MODIFIED BY ILOCA'; OTHER

C RELEASES ARE AS IN BIN 2, I.E., WITHOUT INDUCED LOCA.

$$
\begin{aligned}
& \operatorname{ICASE}(2)=\operatorname{ILOCA}+1 \\
& \operatorname{ICASE}(3)=3 \\
& \operatorname{ICASE}(4)=3
\end{aligned}
$$

C SET FLAG FOR AMOUNT OF DH IN THE CONTAINMENT EVENT TREE

$\mathrm{LDH}=\operatorname{INDX}(3)$

C SET FLAGS FOR THE LEVELS OF RADIONUCLIDE RELEASE SPECIFIED

$$
\begin{aligned}
& \text { ISS }=12 \\
& \text { LREJ }=\text { MINO(LEV(ISS),MAXL(ISS)) } \\
& \text { ISS }=13 \\
& \text { LRDH }=\text { MINO(LEV(ISS),MAXL(ISS)) } \\
& \text { DO } 103 \text { IB }=16,19 \\
& \text { IC }=\text { ICASE(IB-15) } \\
& \text { DHM }=\text { FREJ(IC,LDH)*FRDH(IC,LDH) } \\
& \text { C DELM }=\text { MASS PARTICIPATING IN HPE BUT NOT DH } \\
& \text { DELM }=\text { FREJ(IC,LDH)-DHM } \\
& \text { DO } 103 \text { ISP=1,NSPEC }
\end{aligned}
$$




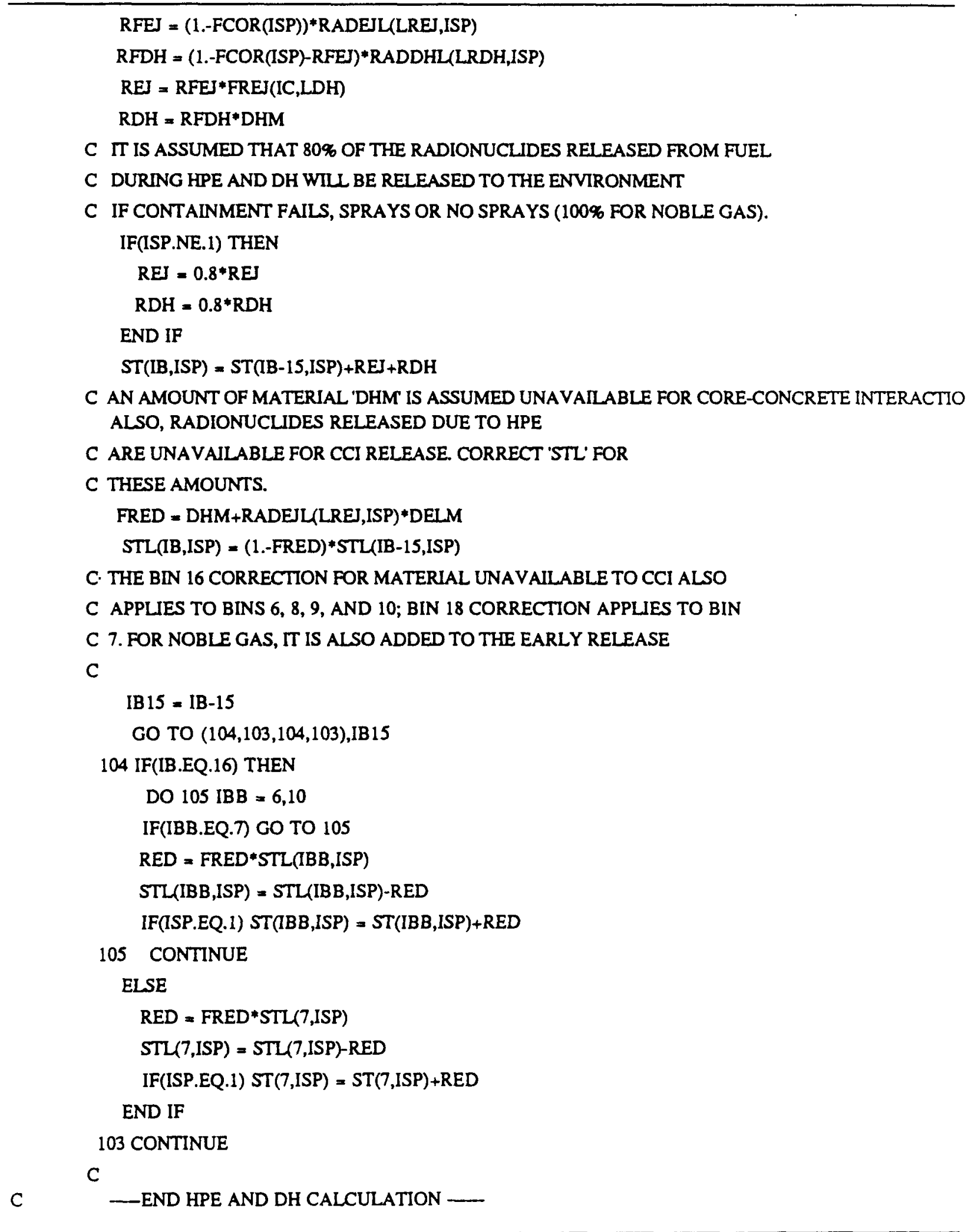


NUREG/CR-4551, VOL 1: DRAFT REPORT FOR COMMENT (SEPTEMBER, 1986)

Table C-34 (Continued)

C AT THIS POINT, WE ASSUME THAT THE CCI RELEASES OF GROUPS $1-3$

C COME TOO EARLY TO COUNT AS A "LATE" RELEASE, BUT WE STILL SAVE THEM

C SEPARATELY AS "RCCI".

C

DO $141 \mathrm{IB}=1,19$

DO 141 ISP $=1,3$

RCCI(IB,ISP) $=$ STL(IB,ISP)

$S T(I B, I S P)=S T(I B, I S P)+S T L(I B, I S P)$

141 STL(IB,ISP) $=0.0$

C

C ISSUE 11: REVOLATILIZATION OF CS AND I FROM THE RCS.

C ASSUME RELEASE FRACTIONS FROM

C CONTAINMENT, FCONC, EQUAL TO THE TELLURIUM VALUES FOR CCI RELEASES.

C 'FCONTE' WAS SET EQUAL TO FCONC(TE)*FCOR(CS)*(1.-FVES(CS)).

ISS $=11$

IL = MINO(LEV(ISS),MAXL(ISS))

IL2 = MINO(LEV(2),MAXL(2))

FCSI $=1$. FI2(IL2)

DO $111 \mathrm{IB}=1,13$

STL(IB 2 2) = FLATE(IL)*FCSI*FCONTE(IB)

111 STL(IB,3) = FLATE(IL)*FCONTE(IB)

DO 112 IB $=16,19$

DO 112 ISP $=2,3$

112 STL(IB,ISP) $=$ STL(IB-15,ISP)

C

C. ISSUE 10: MISC. "LATE" SOURCE OF VOLATTLE IODINE TO THE CONTAINMENT.

C E.G., INCLUDES DECAY CHAIN EFFECTS, ORGANIC IODINE, RELEASE FROM

C POOLS, ETC. NO "DFS" APPLY.

C

ISS $=10$

$\mathrm{IL}=\mathrm{MINO}(\mathrm{LEV}(\mathrm{ISS}), \mathrm{MAXL}(\mathrm{ISS}))$

DO 121 IB $=1,12$

C REDUCE VOLATILE I RELEASE IF I RELEASE ALREADY EXCEEDS $50 \%$

$\mathrm{RELI}=\mathrm{ST}(\mathrm{IB}, 2)+\mathrm{STL}(\mathrm{B}, 2)$

121 STL(IB,2) $=$ STL(IB,2)+2.*(1.-AMAX1 $(0.5$, RELI) $) *$ LATEIL(IL)

DO $122 \mathrm{lB}=16,19$

RELI $=S T(I B, 2)+S T L(I B, 2)$

122 STL(IB,2) $=$ STL(IB,2)+2.*(1.-AMAXI $(0.5$, RELI) $) *$ LATEIL(IL)

$\mathrm{IB}=13$ 
Table C-34 (Continued)

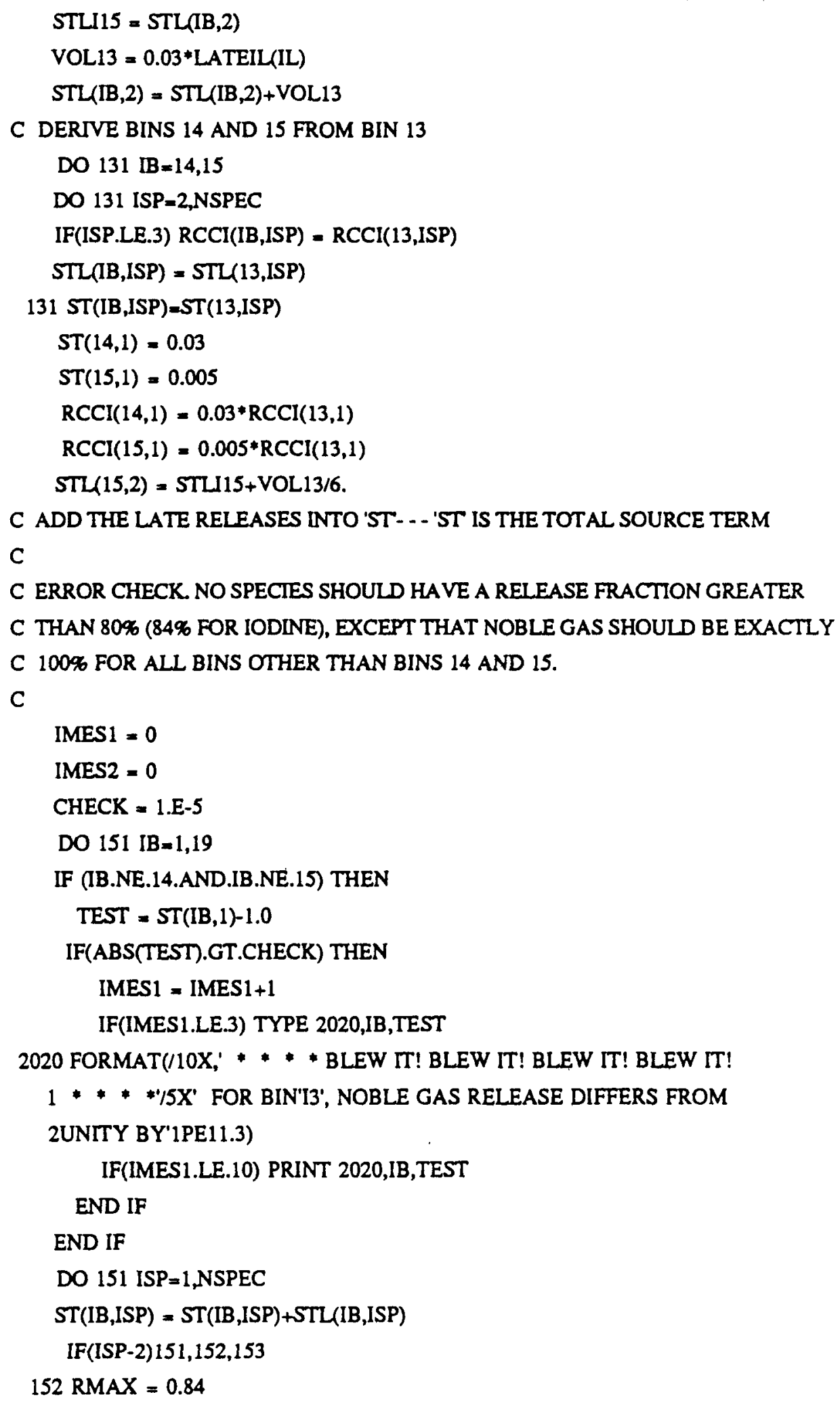


NUREG/CR-4551, VOL. 1: DRAFT REPORT FOR COMMENT (SEPTEMBER, 1986)

\section{Table C-34 (Continued)}

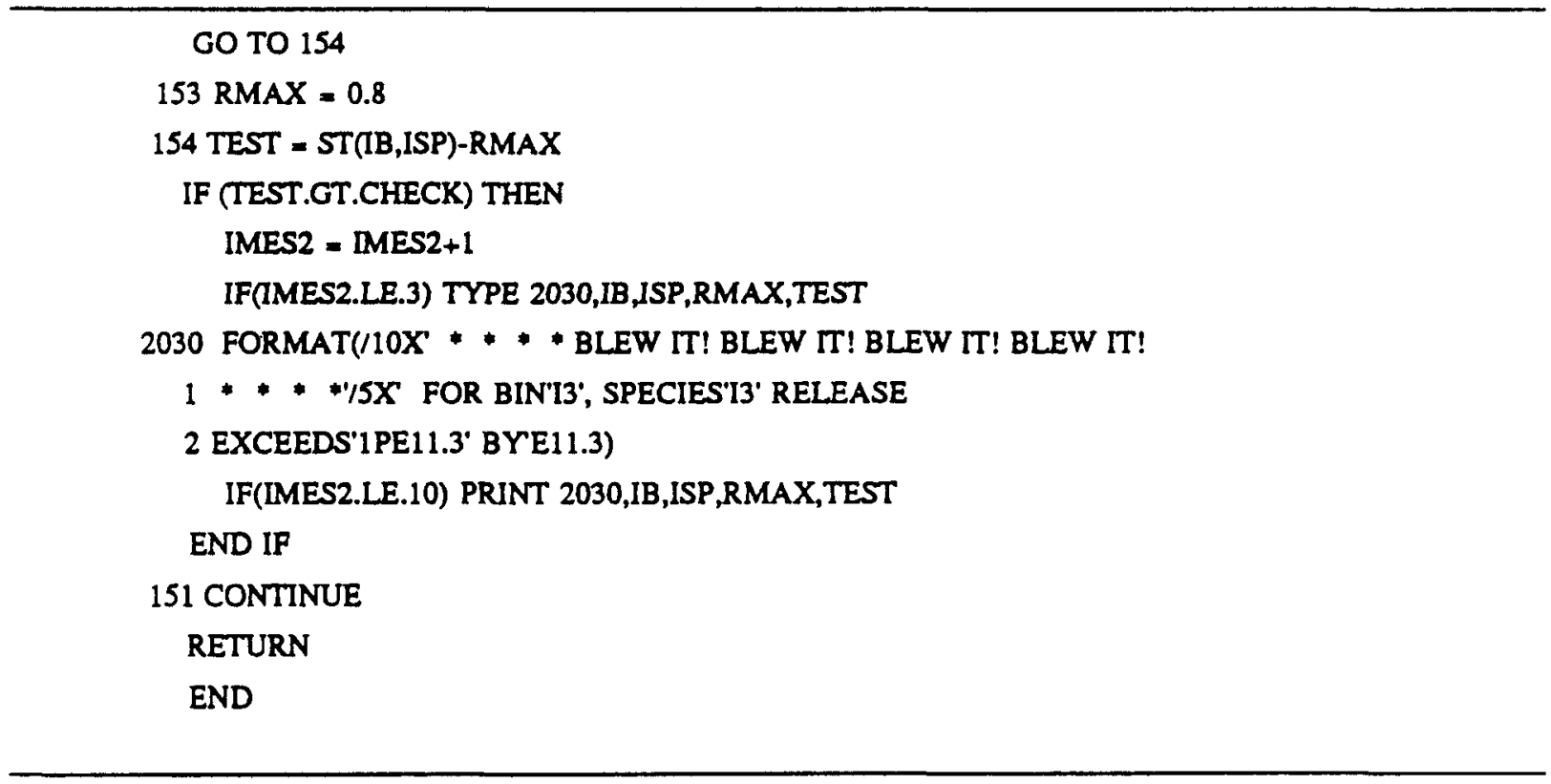




\title{
C.3 CLUSTERING OF SURRY SOURCE TERM ESTIMATES
}

\author{
J. Michael Griesmeyer \\ Sandia National Laboratories
}

\section{C.3.1 Introduction}

This section describes the clustering of the Surry LLH source terms for consequence calculations. The limited Latin hypercube sampling described in Section 2.2.2 of the main report involved thirteen source term issues. The values for these issues, which are described in Section 4.3 , were varied in the one hundred observations of the sample.

For each observation, or sample member, A source term corresponding to each of the nineteen original source term bins was estimating resulting in a total of 1900 source term estimates. Figures C-2 through C-7 show the range of release fractions for release Groups 2 through 6 , plotted by release bin. The central estimates for each source term are also plotted. The large variation in estimated source terms among and within bins and the overlap of the source term ranges for different categories suggest that the source term estimates should be grouped for the CRAC2 and MACCS consequence calculations, and that the grouping should attempt to discretize the source term space rather than find the "best" estimate for the original individual bins.

The discretization of the source term space assigned each source term estimate to a cluster. Consequence calculations were performed using the average characteristics of the source term estimates assigned to the cluster. The general criteria for acceptable clustering were that the clustering (1) not introduce errors that were significant compared to the errors already present in the analysis, and (2) preserve the relationships between important inputs to the analysis and consequences of interest. The clustering process is based upon consideration of the source term characteristics that most strongly influence the consequences. Previous studies [11,12] indicate that the most important source term characteristics are in order:

- Magnitude of the release,

- Warning time before the release,

- Duration of the release,

- Time of release,

- Energy of the release, and

- Height of the release,

The magnitude of the release is by far the most important. Warning time impacts the early effects though, by influencing whether emergency measures can be effective. Duration 


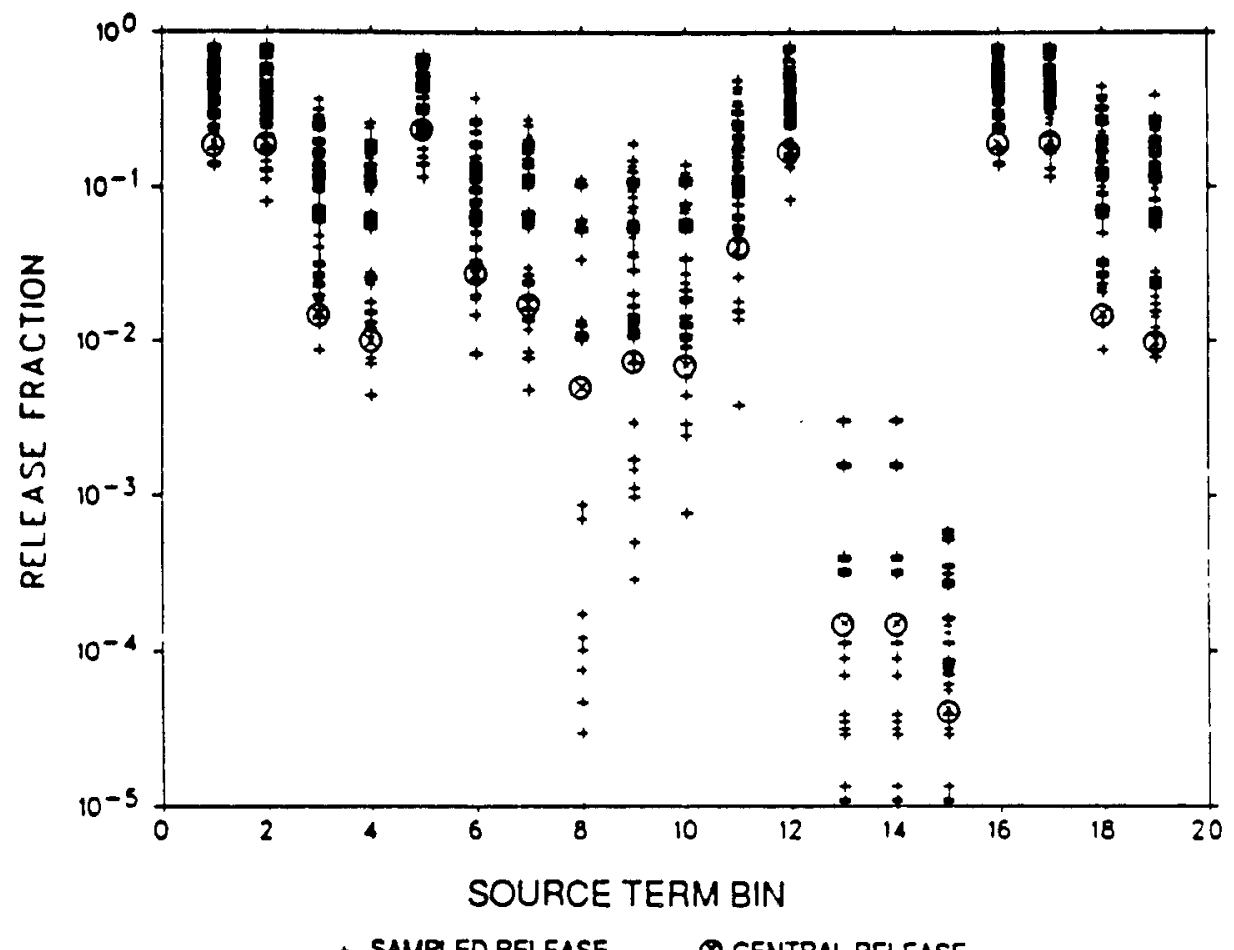

Figure C-2. Sampled Surry Source Term Estimates From ȘURSOR for Group 2

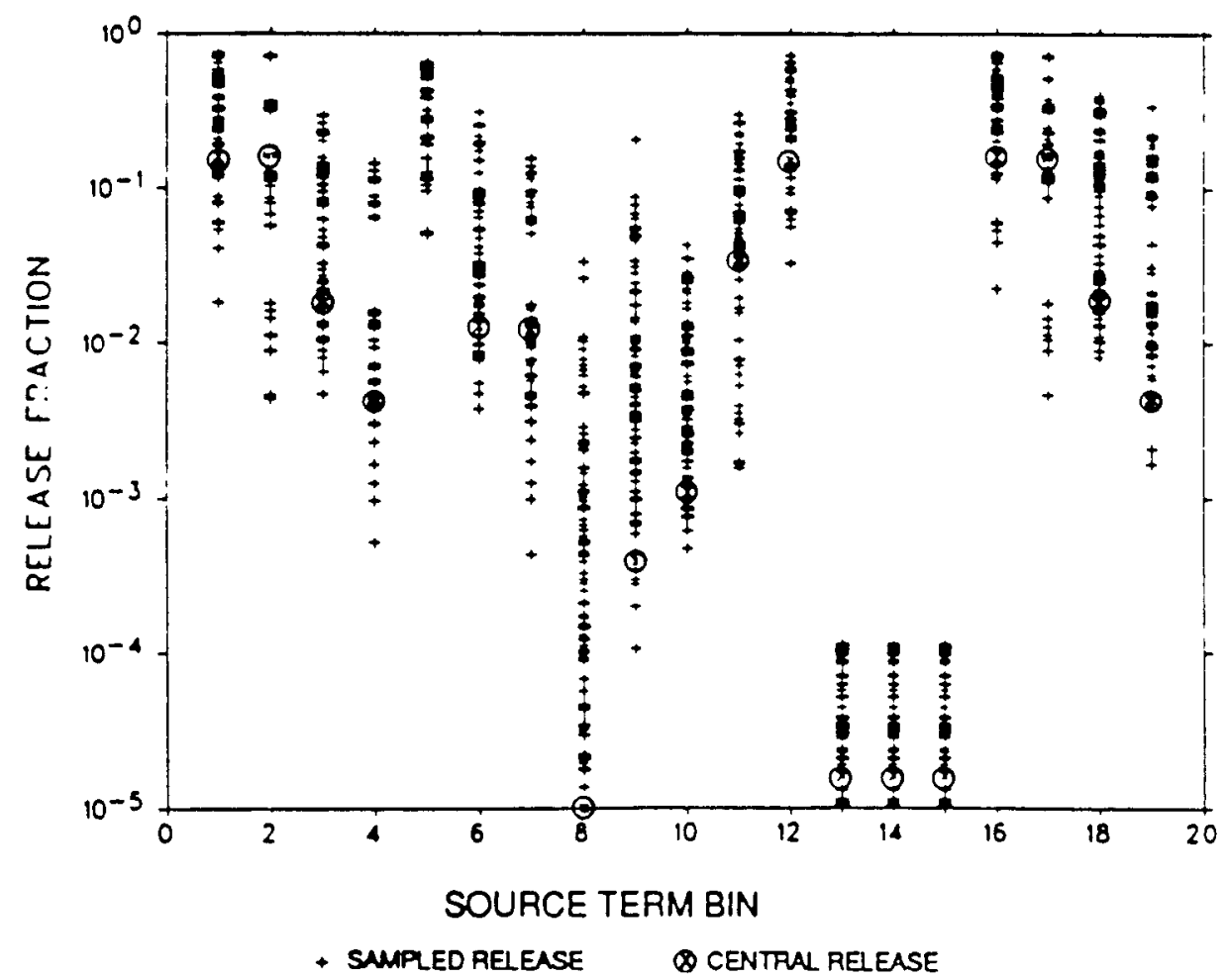

Figure C-3. Sampled Surry Source Term Estimates From SURSOR for Group 3 
NUREG/CR-4551, VOL. 1: DRAFT REPORT FOR COMMENT (SEPTEMBER, 1986)

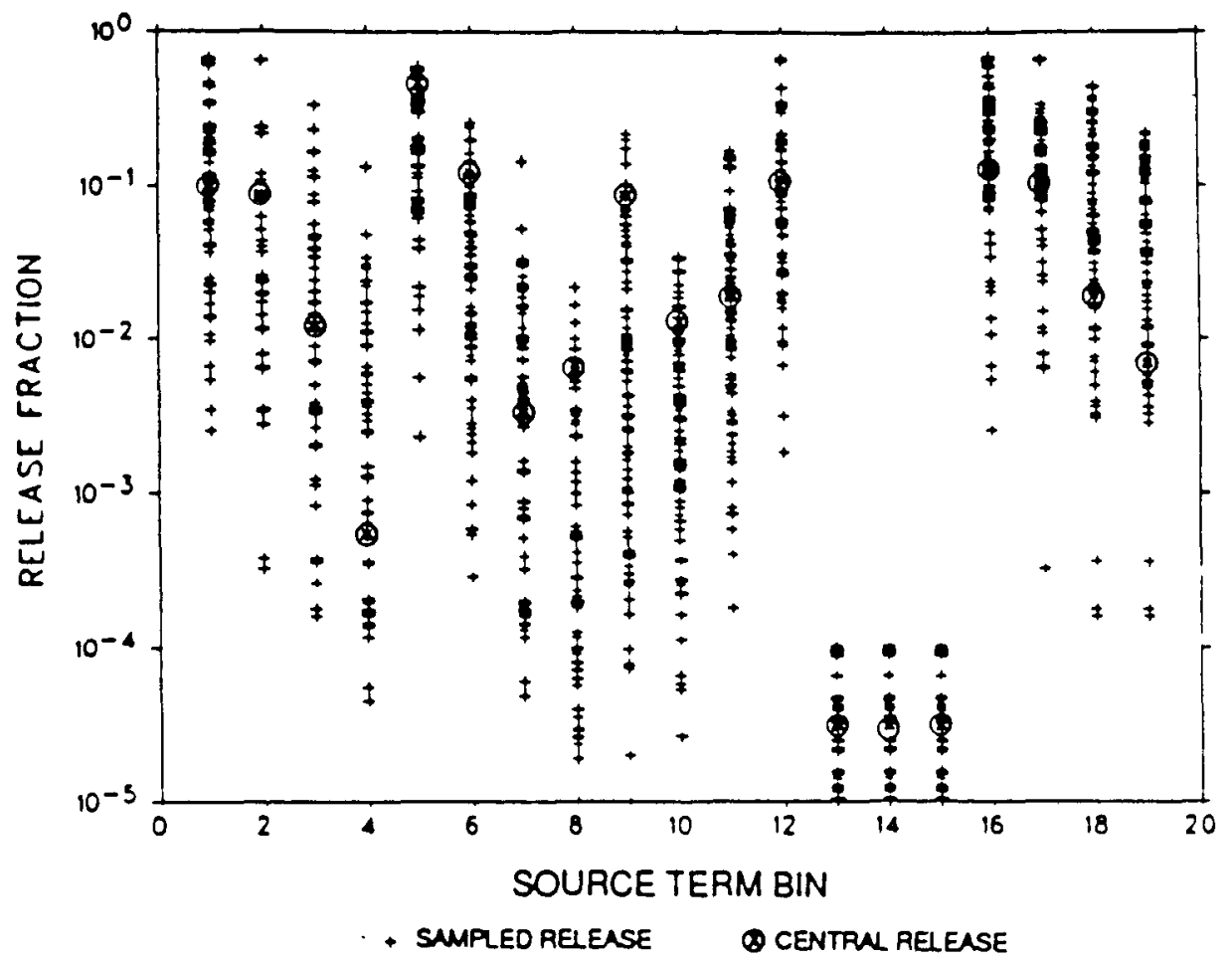

Figure C-4. Sampled Surry Source Term Estimates From SURSOR for Group 4

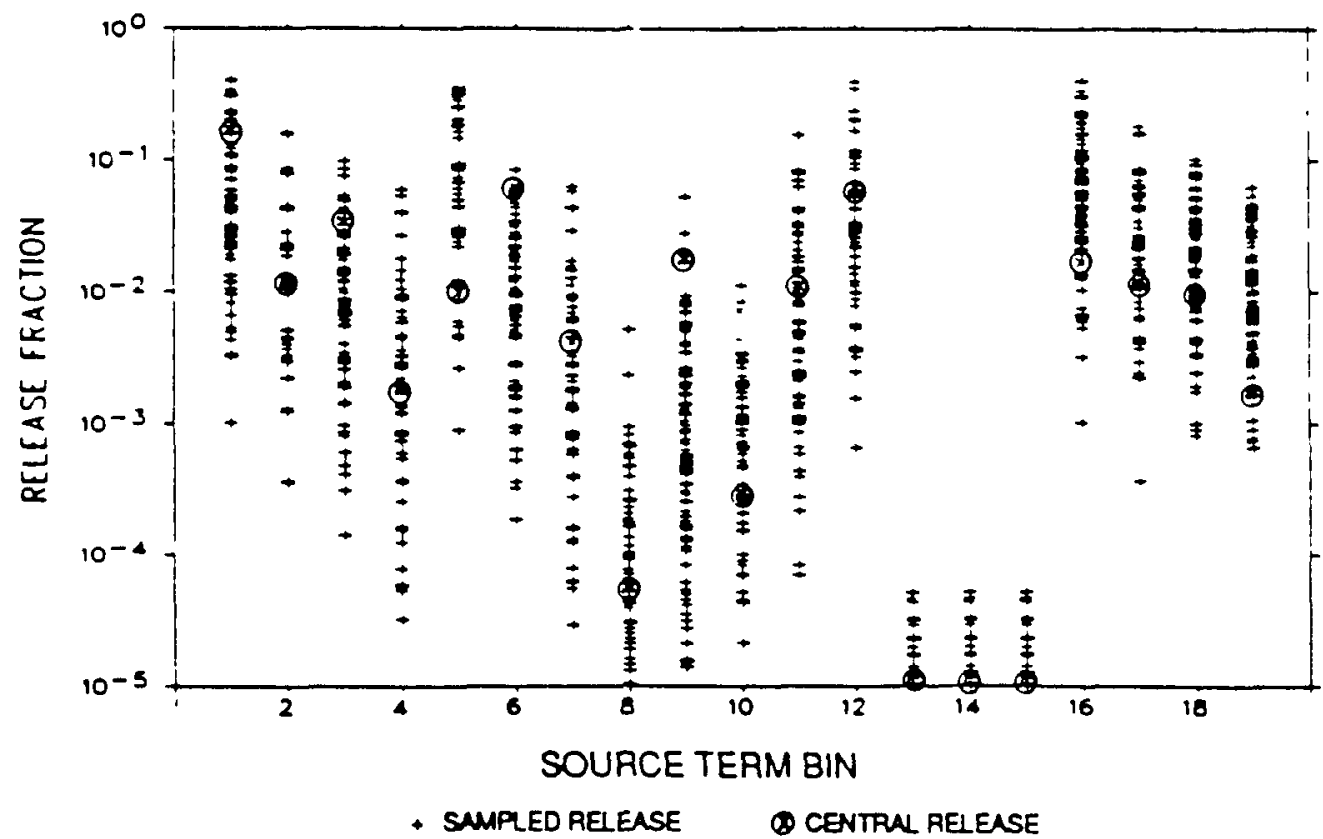

Figure C-5. Sampled Surry Source Term Estimates From SURSOR for Group 5 
NUREG/CR-4551, VOL 1: DRAFT REPORT FOR COMMENT (SEPTEMBER, 1986)

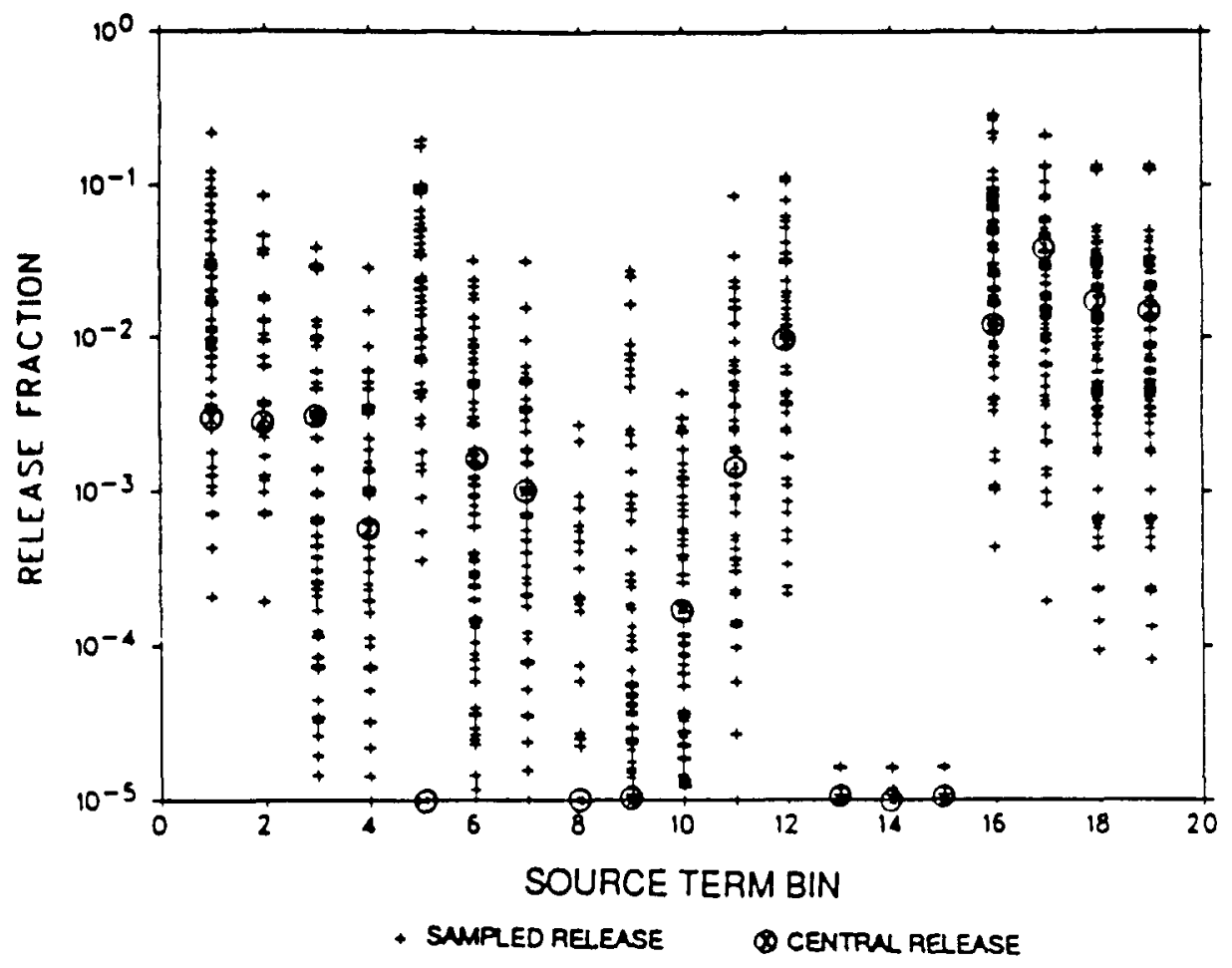

Figure C-6. Sampled Surry Source Term Estimates From SURSOR for Group 6

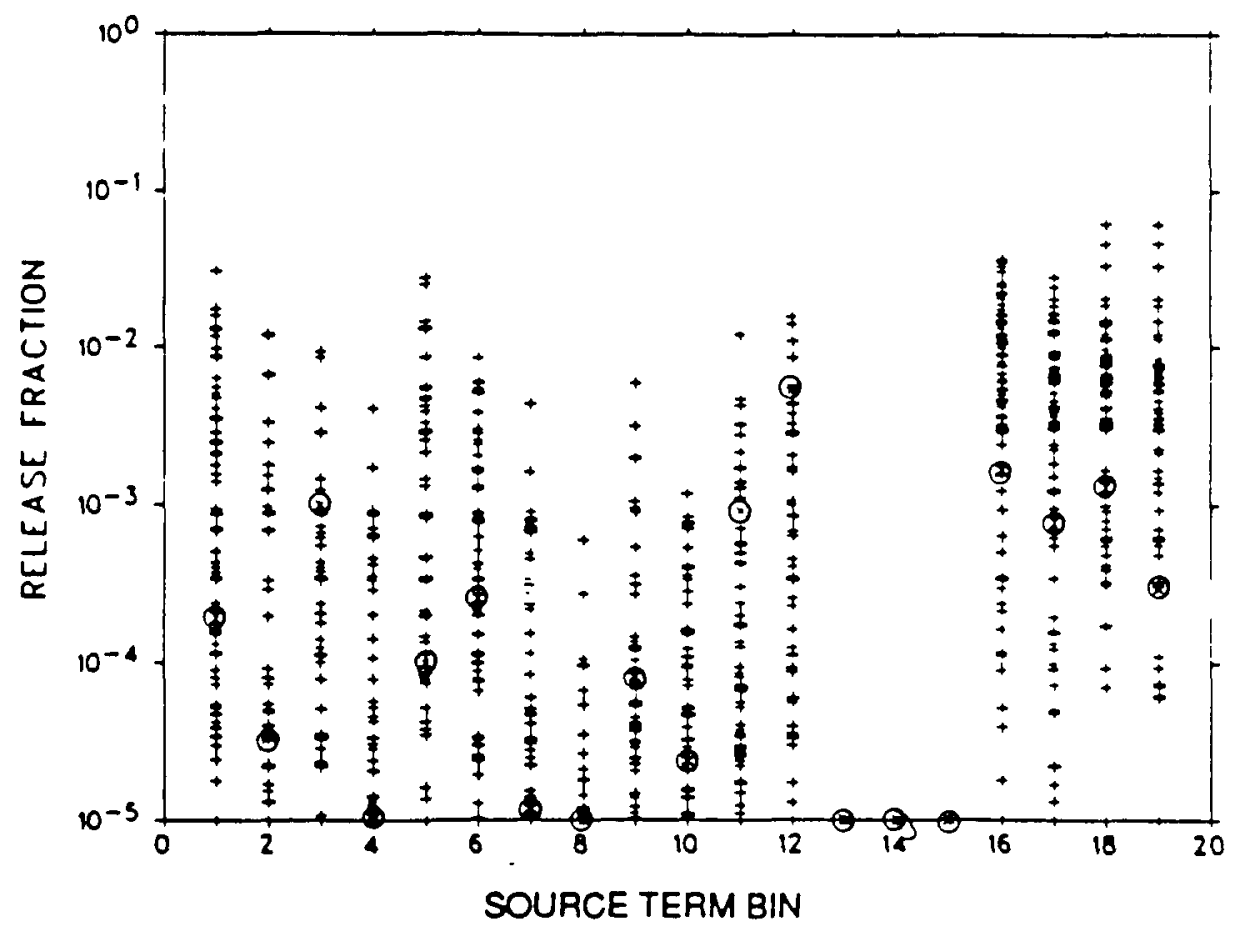

- sumpled release ocentrul reiease

Figure C-7. Sampled Surry Source Term Estimates From SURSOR for Group 7 
determines whether the later part of the release is late enough to allow evacuation. The other characteristics are of secondary importance. Timing, aside from duration and warning time, may allow for decay of some short-lived radionuclides before release and thus reduce some early effects. Energy and height of release influence close-in doses by determining the location and time of plume touchdown.

The magnitude of the release for each observation and release category was estimated using SURSOR (described in Section C.2). The other characteristics of each source term estimates were assumed to be those of the corresponding release bin. These characteristics are listed in Section 5 of the main report, along with the central estimates of the release fraction for each category.

In order to facilitate the clustering process, the dimensionality of the source term characteristic space was reduced. After the cluster centers in the reduced space were found, they were transformed back to the original space. A main approximation used in the reduction of the space was that all consequences behave either as early health effects or as late health effects with respect to release magnitudes.

Thus, the magnitude of the release for each of the seven chemical groupings was combined into an early effect weighting (iodine equivalent) and a late effect weight (cesium equivalent). The weights for the chemical groups were assembled from Table 3 of Reference 13. The iodine and cesium equivalents used in the Surry source term clustering are shown in Table C-35.

Warning time was reflected by the early effect weight of early releases, EEER, and by the early effect weight of the late release, EELR. Early release was defined as the release that occurs before there is adequate time for emergency response (i.e., within 3-4 hours of warning). These early effect weights (EEER and EELR), together with the late effect weight, $L R$, result in a three-dimensional reduced space for the purpose of clustering. The high power density in PWRs causes the duration of significant release to be short. Thus, no Surry release bin has both an early release and a late release. The following bins were classified by early release: 1 through 7,10 through 12 , and 16 through 19 . Bins 8,9 , and 13 through 15 were classified as late releases. 
Table C-35

EARLY AND LATE EFFECT WEIGHTS OF SOURCE TERM GROUPS

\begin{tabular}{|c|c|c|c|c|}
\hline \multirow[t]{2}{*}{ GROUP } & \multicolumn{3}{|c|}{ EARLY EEEECT WEIGHTS } & \multirow{2}{*}{ LATE EFFECT WEIGHTS 2} \\
\hline & Bone & Lung & Average & \\
\hline 1 & 0.1 & 0.1 & 0.1 & 0.001 \\
\hline 2 & 1.0 & 1.0 & 1.0 & 0.1 \\
\hline 3 & 0.1 & 0.09 & 0.1 & 1.0 \\
\hline 4 & 0.9 & 0.65 & 0.8 & 0.14 \\
\hline 5 & 2.2 & 1.1 & 1.65 & 0.9 \\
\hline 6 & 0.5 & 3.8 & 2.15 & 1.3 \\
\hline 7 & 4.5 & 27 & 16 & 7.8 \\
\hline
\end{tabular}

24 Hour bone dose

2 Total cancers

\section{C.3.2 Clustering Procedure}

The clustering process was carried out using an interactive cluster analysis code, CLUSTER, that was developed in the Probability and Risk Uncertainty Evaluation Program (PRUEP). The clustering is based on a nearest neighbor approach using a modified Eudidian distance:

$$
\left.\mathrm{d}_{\mathrm{ij}}=\underset{\mathrm{k}}{\sum \sum_{k}}\left(\mathrm{x}_{\mathrm{kj}}-\mathrm{x}_{\mathrm{kj}}\right)^{2}\right\}^{1 / 2}
$$

where

$$
\begin{aligned}
& d_{i j}=\text { the distance between points } i \text { and } j \\
& x_{k l}=\text { the } k^{\text {th }} \text { coordinate of the } l^{\text {th }} \text { point } \\
& W_{k}=\text { the weight of the } k^{\text {th }} \text { coordinate. }
\end{aligned}
$$

In this analysis, the three coordinates for each point are EEER, EELR, and LE defined above. The weights, $W_{k}$, are chosen to reduce dispersion within the dimensions for the resulting clusters, but are otherwise arbitrary.

The clustering of the source terms in the reduced dimensions was accomplished using the following iterative procedure:

1) Obtain an Initial Set of Clusters. Several procedures are available for obtaining initial cluster centers: random selection of points from observation, 
discretizing the distance from the origin $\left(X_{1}=0, X_{2}=0, X_{3}=0\right)$, or a maximum distance algorithm [14].

2) Use the $\mathrm{K}$ means algorithm [15] to optimize the center positions and assignments. The $\mathrm{K}$ means algorithm is:

a. Obtain starting cluster centers,

b. Map all data points on to the centers based upon shortest distance to a center,

c. Find the new center positions by averaging the position of the points assigned to the individual clusters, and

d. If any center position has changed, go back to step a, otherwise stop.

3) For the reduced dimension, plot the value versus the assigned cluster.

4) If the dispersion in a dimension for a cluster is unacceptable, split the cluster and return to step 2, otherwise go to step 5.

5) Back out the average cluster centers in the original space using the cluster assignments found in step 2 .

The stopping criterion is not rigorous. In practice, the clustering was considered acceptable if the dispersion within a dimension was less than a factor of two for clusters with large releases, and less than a factor of 10 for small releases.

\section{C.3.3 Surry Clustering Results}

The clustering of the sampled Surry source terms resulted in 24 clusters. Figures C-8 through $\mathrm{C}-13$ show the within cluster dispersion in the reduced dimensions. The centers in the reduced space are given in Table C-36. A zero in the table for EEER or EELR indicates that the center is associated with an early release or a late release respectively. Table C-37 gives the release fraction space based on the cluster assignments in the reduced space. The other characteristics of the clusters (warning time, duration, height, time of release, energy, and height) are obtained by averaging values from the source term bins associated with the points in the clusters. These are given in Table 5-8 of the main report.

\section{C.3.4 Limitations of Clustering Process}

One of the concerns of the analysts and reviewers of the present work was the potential for error introduced by the clustering process. While this has not been fully investigated at this time due to schedule constraints, one test of the clustering process was performed, with results that suggest the process is not a major source of error in the risk calculation. The test was performed using the OCP methodology because this provided a limited size problem yet covered ranges similar to the LLH problem. The OCP risk calculation including the source 
terms for the optimistic, central, and pessimistic cases was performed as described in Section 2 of the main report, except that for this test, the OCP source terms were processed by the same clustering code used in the LLH. The results were then recalculated in terms of the CRAC2 consequences assigned to the clusters, rather than the consequences calculated specifically for the OCP source terms. These results were then compared to the base case OCP study, as illustrated in Figures C-14 and C-15. As illustrated, the results compare quite well, and it would appear from this test that the clustering process itself added little uncertainty to the risk calculation. This is not a verification of clustering since it was performed on a different problem than the one of interest (the OCP versus the $L L H$ ), but the ranges of source terms covered in the OCP are similar to the LLH and the test does help to provide confidence in the clustering process. The fact that the clusters used were not even optimized for the OCP study could be interpreted as further support for the adequacy of the clustering process, since even using the LLH clusters, the OCP result was nearly duplicated. Based on a review of these results and a review of the specific risk results, the analysts believe that clustering does not impose large uncertainties on the risk results for Surry. This belief is subject to further verification however, particularly for risk profiles which may have higher influence of radionuclide groups that might not be considered as fully in the clustering process. 
NUREG/CR-4551, VOL. 1: DRAFT REPORT FOR COMMENT (SEPTEMBER, 1986)

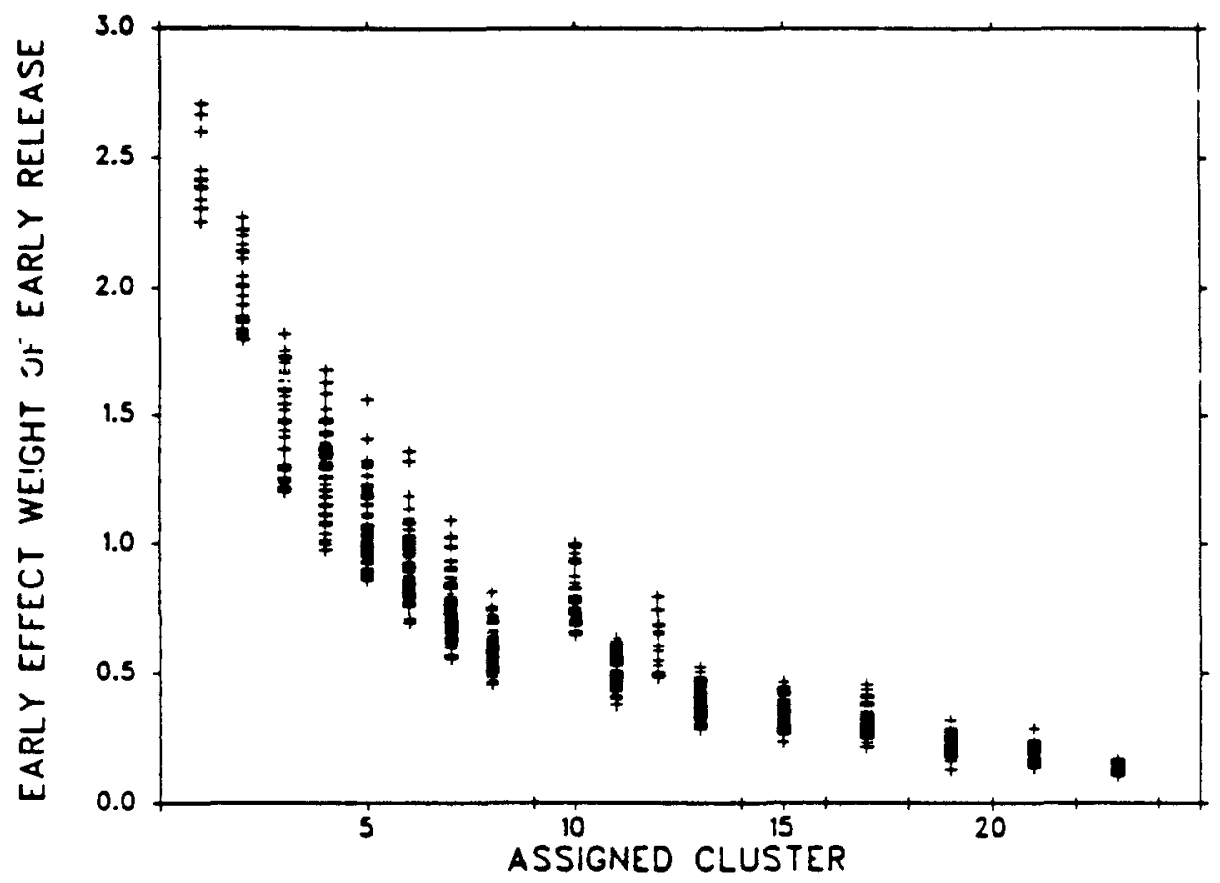

Figure C-8. Spread in Early Effect Weight of Eariy Release By Cluster (Linear Scale)

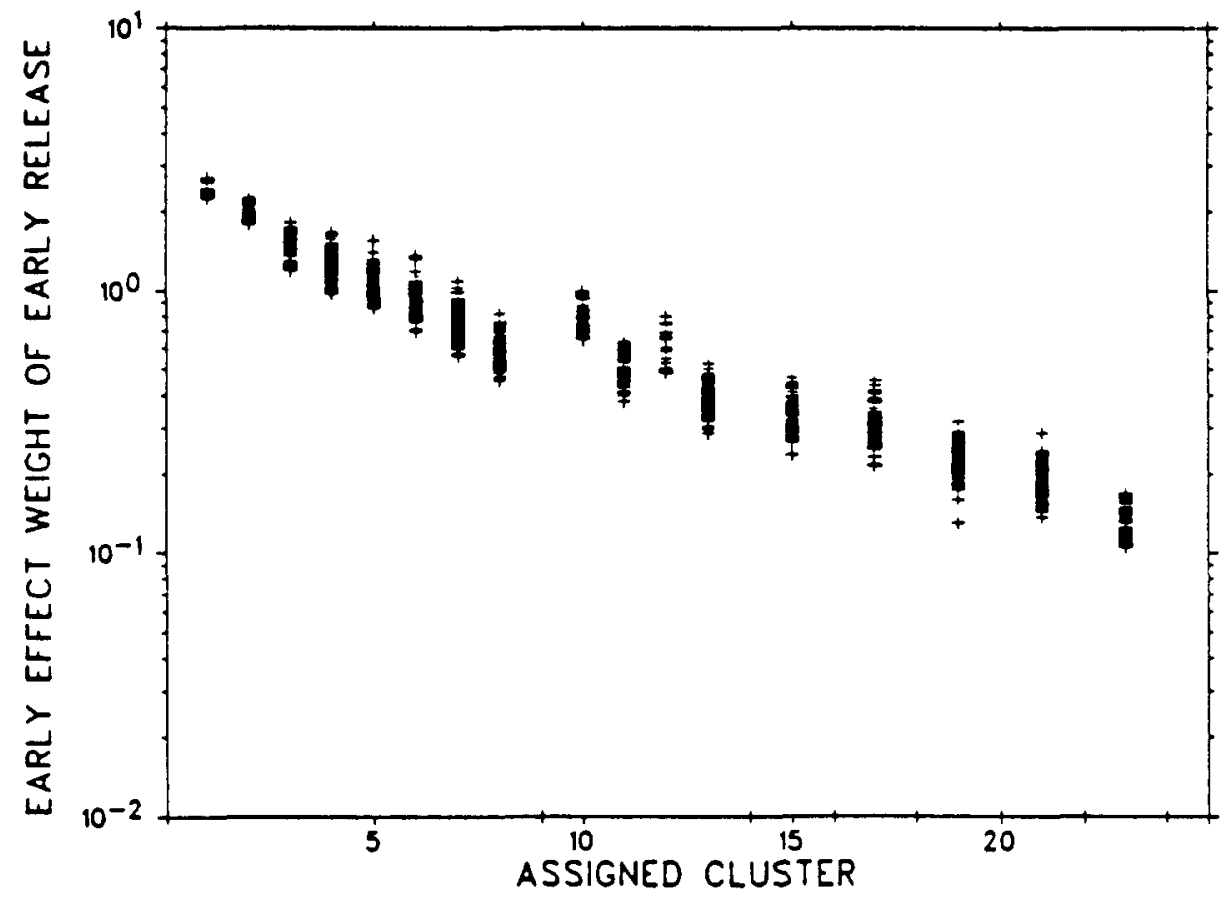

Figure C-9. Spread in Early Effect Weight of Early Release By Cluster (Log Scale) 
NUREG/CR 4551, VOL 1: DRAFT REPORT FOR COMMENT (SEPTEMBER, 1986)

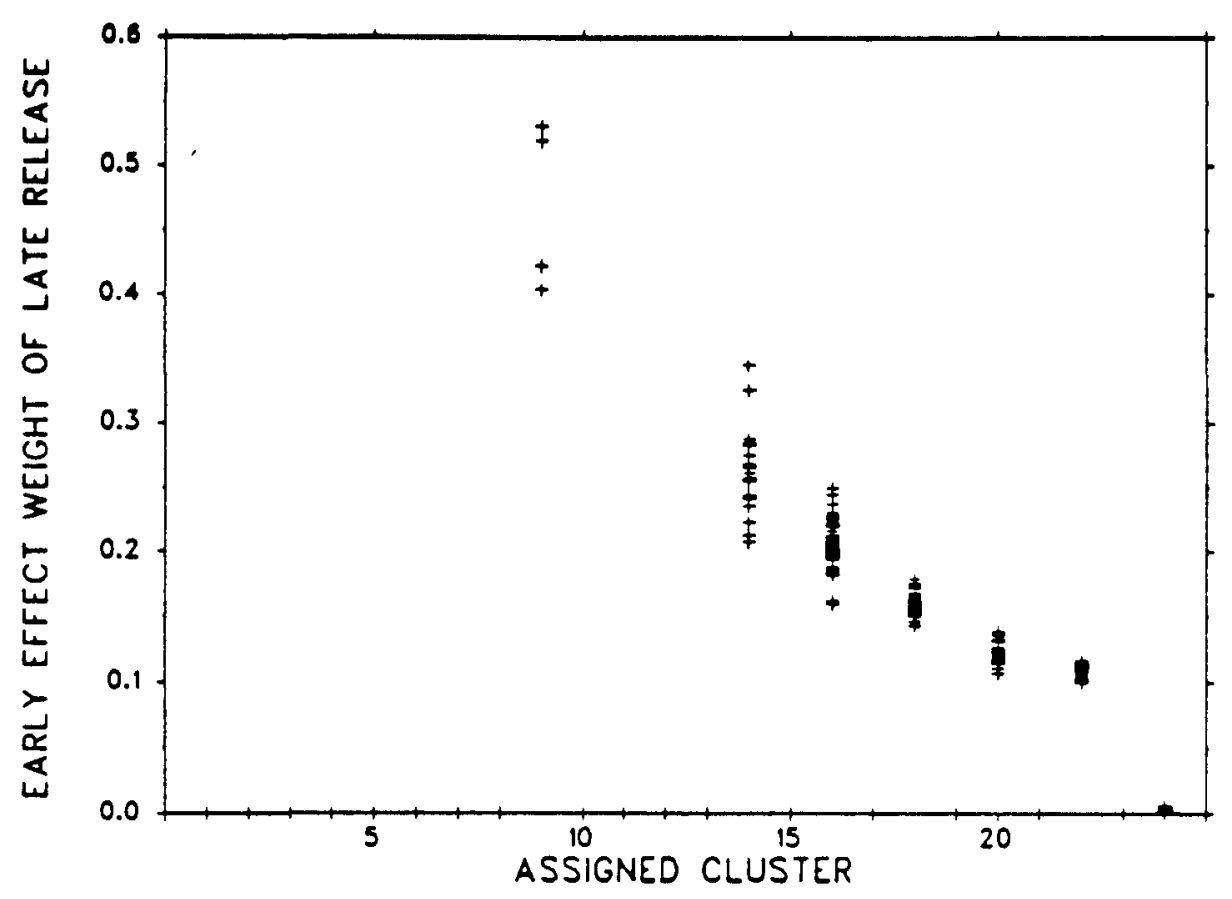

Figure C-10. Spread in Early Effect Weight of Late Release By Cluster (Linear Scale)

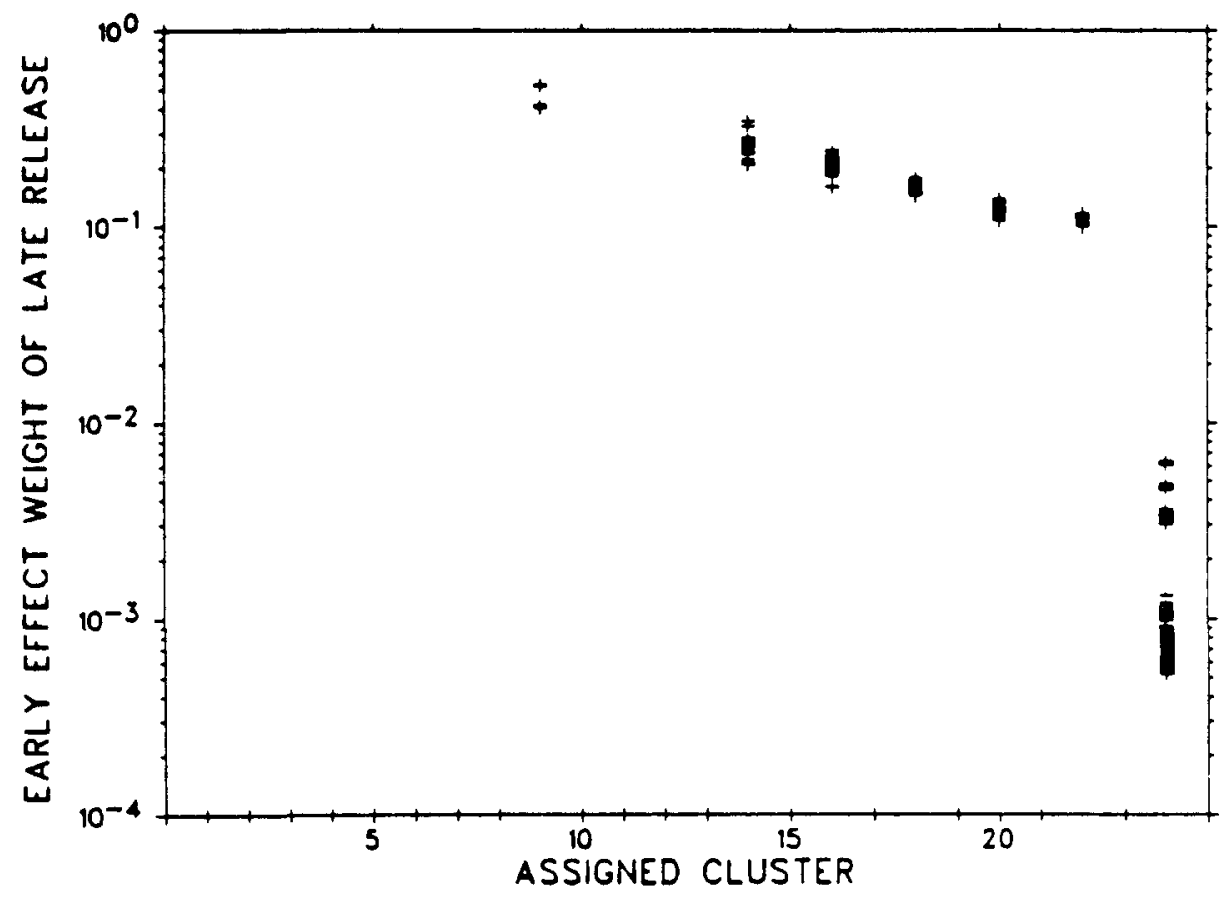

Figure C-11. Spread in Early Effect Weight of Late Release By Cluster (Log Scale) 
NUREG/CR-4551, VOL 1: DRAFT REPORT FOR COMMENT (SEPTEMBER, 1986)

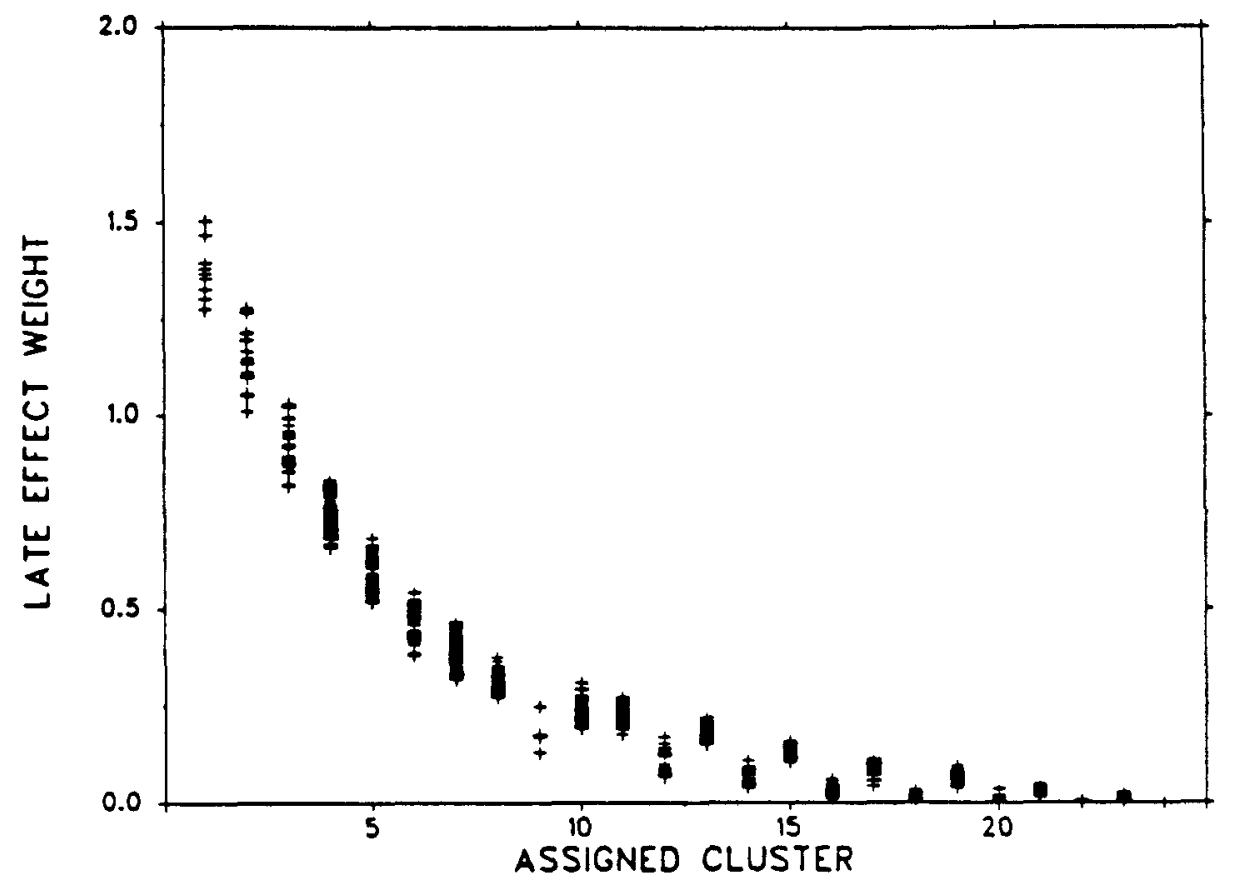

Figure C-12. Spread in Late Effect Weight By Cluster (Linear Scale)

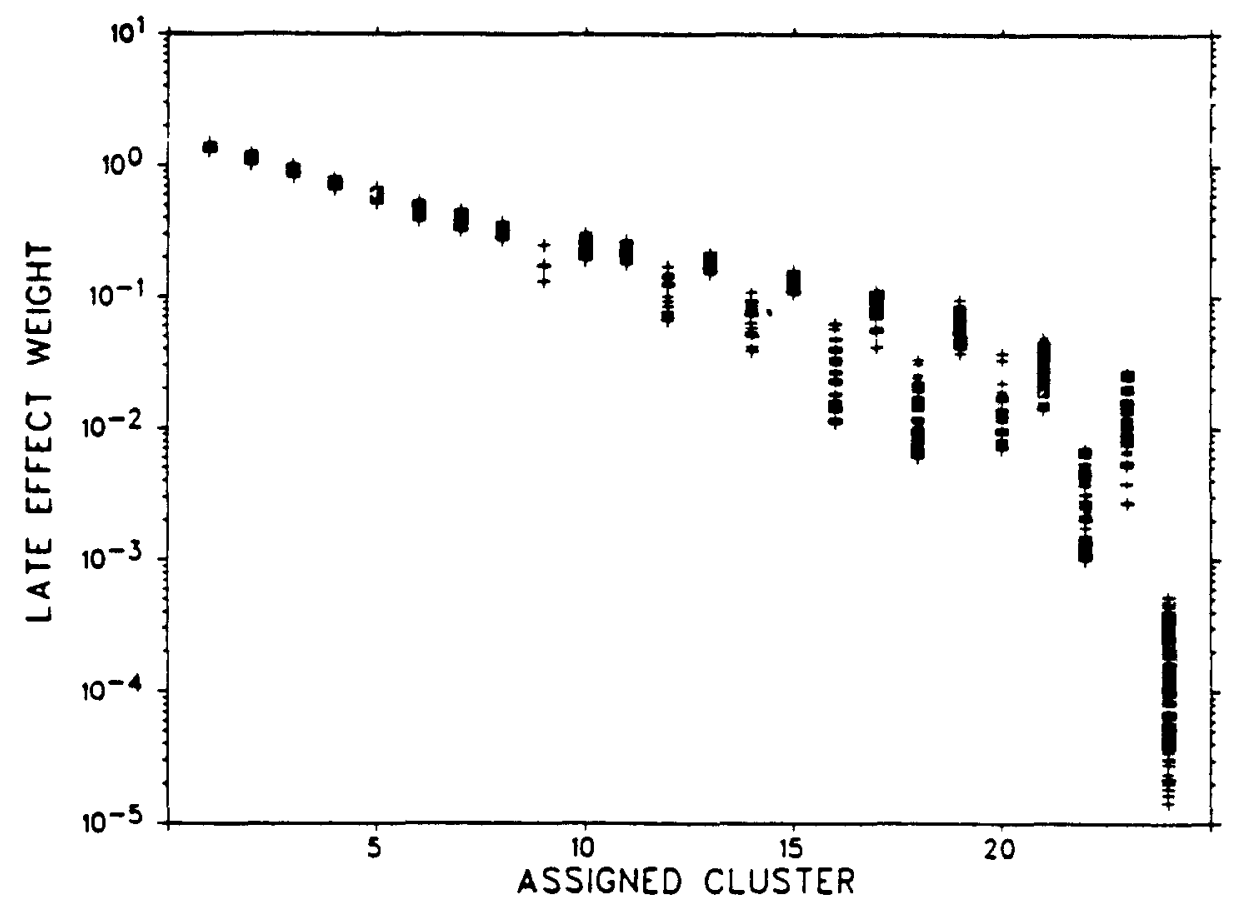

Figure C-13. Spread in Late Effect Weight By Cluster (Log Scale) 
NUREG/CR-4551, VOL. 1: DRAFT REPORT FOR COMMENT (SEPTEMBER, 1986)

Table C-36

EARLY AND LATE EFFECT WEIGHTS OF SOURCE TERM CLUSTERS

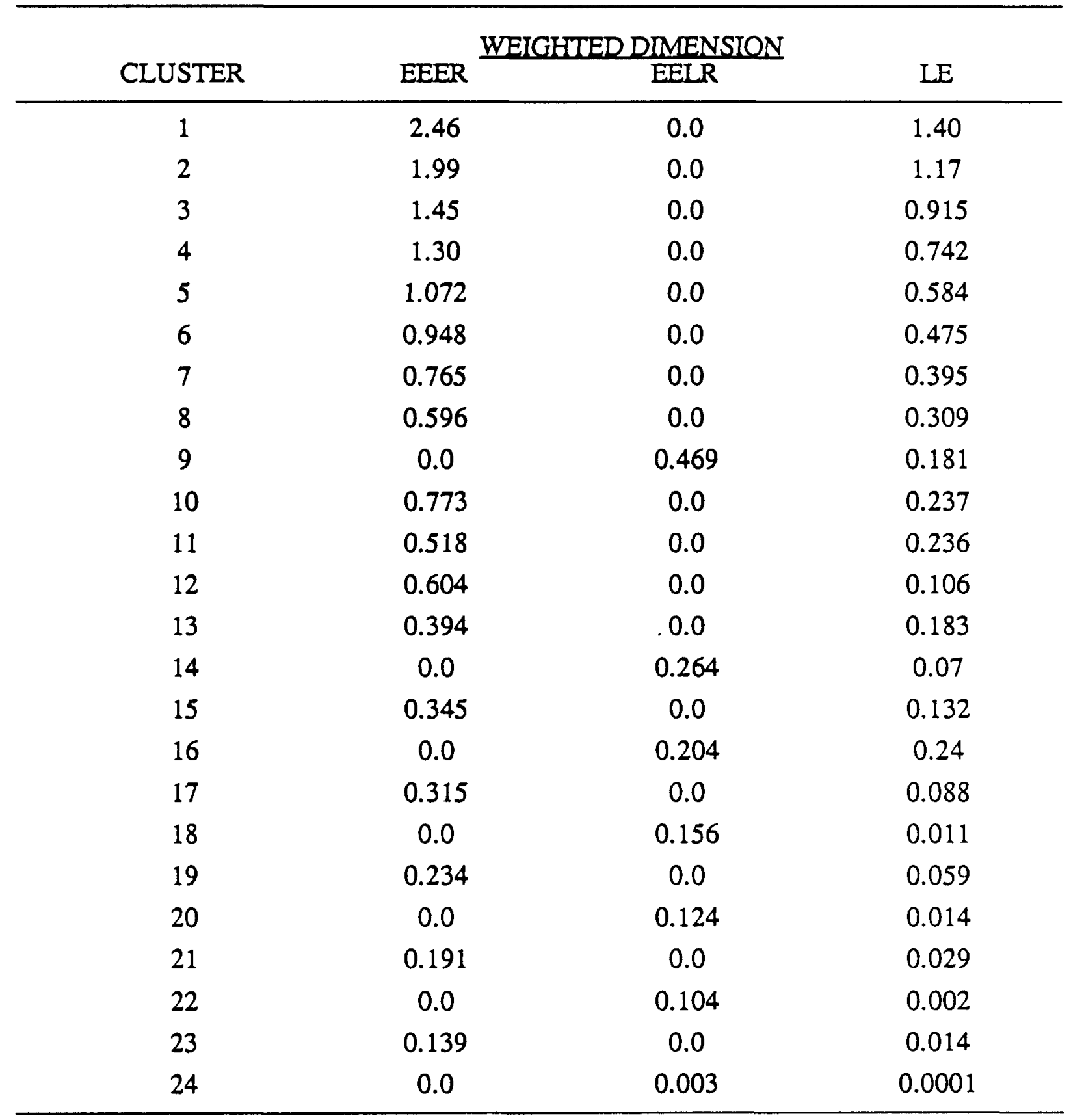


NUREG/CR-4551, VOL. 1: DRAFT REPORT FOR COMMENT (SEPTEMBER, 1986)

Table C-37

RELEASE FRACTIONS FOR SURRY LLH CLUSTERS

\begin{tabular}{|c|c|c|c|c|c|c|c|}
\hline Cluster & $\underset{\mathrm{Kr}-\mathrm{Xe}}{1}$ & 2 & $\begin{array}{c}3 \\
C s-R b\end{array}$ & $\begin{array}{l}4 \\
\mathrm{Te}\end{array}$ & $\begin{array}{c}5 \\
\text { Sr-Ba }\end{array}$ & $\begin{array}{c}6 \\
\mathrm{Ru}\end{array}$ & $\begin{array}{r}7 \\
\mathrm{La}\end{array}$ \\
\hline 1 & 1.0 & $6.93-1$ & $6.18-1$ & $5.64-1$ & $3.25-1$ & $1.42-1$ & $1.97-2$ \\
\hline 2 & 1.0 & $6.99-1$ & $6.42-1$ & $5.30-1$ & $2.29-1$ & $8.09-2$ & $9.31-3$ \\
\hline 3 & 1.0 & $6.91-1$ & $6.14-1$ & $3.15-1$ & $1.02-1$ & $4.21-2$ & $5.28-3$ \\
\hline 4 & 1.0 & $5.31-1$ & $4.25-1$ & $2.63-1$ & $1.08-1$ & $5.00-2$ & $8.15-3$ \\
\hline 5 & 1.0 & $4.90-1$ & $3.55-1$ & $2.08-1$ & $6.21-2$ & $2.89-2$ & $7.20-3$ \\
\hline 6 & 1.0 & $5.08-1$ & $3.00-1$ & $1.51-1$ & $4.76-2$ & $2.17-2$ & $4.03-3$ \\
\hline 7 & 1.0 & $3.75-1$ & $2.47-1$ & $1.17-1$ & $3.83-2$ & $2.20-2$ & $3.82-3$ \\
\hline 8 & 1.0 & $2.88-1$ & $2.07-1$ & $1.02-1$ & $3.13-2$ & $1.07-2$ & $1.97-3$ \\
\hline 9 & 1.0 & $9.73-2$ & $7.77-2$ & $1.72-1$ & $2.69-2$ & $1.71-2$ & $2.84-3$ \\
\hline 10 & 1.0 & $4.86-1$ & $1.18-1$ & $8.63-2$ & $3.11-2$ & $1.07-2$ & $1.97-3$ \\
\hline 11 & 1.0 & $2.41-1$ & $1.45-1$ & $6.87-2$ & $2.42-2$ & $1.06-2$ & $2.76-3$ \\
\hline 12 & 1.0 & $4.37-1$ & $3.41-2$ & $2.39-2$ & $1.03-2$ & $4.25-3$ & $1.19-3$ \\
\hline 13 & 1.0 & $1.68-1$ & $1.18-1$ & $5.06-2$ & $2.02-2$ & $7.32-3$ & $1.58-3$ \\
\hline 14 & 1.0 & $9.73-2$ & $4.05-2$ & $4.99-2$ & $6.26-3$ & $2.29-3$ & $4.54-4$ \\
\hline 15 & 1.0 & $1.52-1$ & $8.14-2$ & $3.59-2$ & $1.31-2$ & $5.54-3$ & $1.35-3$ \\
\hline 16 & 1.0 & $8.74-2$ & $9.10-3$ & $1.20-2$ & $1.78-3$ & $6.55-4$ & $1.29-4$ \\
\hline 17 & 1.0 & $1.47-1$ & $4.71-2$ & $2.88-2$ & $7.75-3$ & $3.57-3$ & $1.20-3$ \\
\hline 18 & 1.0 & $5.14-2$ & $3.64-3$ & $3.63-3$ & $6.24-4$ & $1.33-4$ & $2.45-5$ \\
\hline 19 & 1.0 & $8.48-2$ & $3.02-2$ & $1.97-2$ & $6.43-3$ & $2.64-3$ & $8.87-4$ \\
\hline 20 & 1.0 & $1.37-2$ & $8.22-3$ & $6.84-3$ & $1.41-3$ & $3.98-4$ & $6.54-5$ \\
\hline 21 & 1.0 & $7.38-2$ & $1.42-2$ & $7.74-3$ & $3.00-3$ & $7.97-4$ & $1.83-4$ \\
\hline 22 & 1.0 & $3.56-3$ & $4.57-4$ & $6.13-4$ & $8.19-5$ & $3.39-5$ & $5.77-6$ \\
\hline 23 & 1.0 & $3.22-2$ & $7.21-3$ & $2.98-3$ & $1.29-3$ & $3.39-4$ & $6.41-5$ \\
\hline 24 & $1.75-2$ & $8.30-4$ & $4.39-5$ & $2.76-5$ & $1.01-5$ & $2.83-6$ & $4.44-7$ \\
\hline $25^{*}$ & $2.5-3$ & $4.2-5$ & $1.6-5$ & $3.1-5$ & $1.2-5$ & $3.7-7$ & $6.5-8$ \\
\hline
\end{tabular}

*A 25th cluster was added to more properly characterize the lower range of the sample results. This cluster was defined by inspection. 
NUREG/CR-4551, VOL 1: DRAFT REPORT FOR COMMENT (SEPTEMBER, 1986)

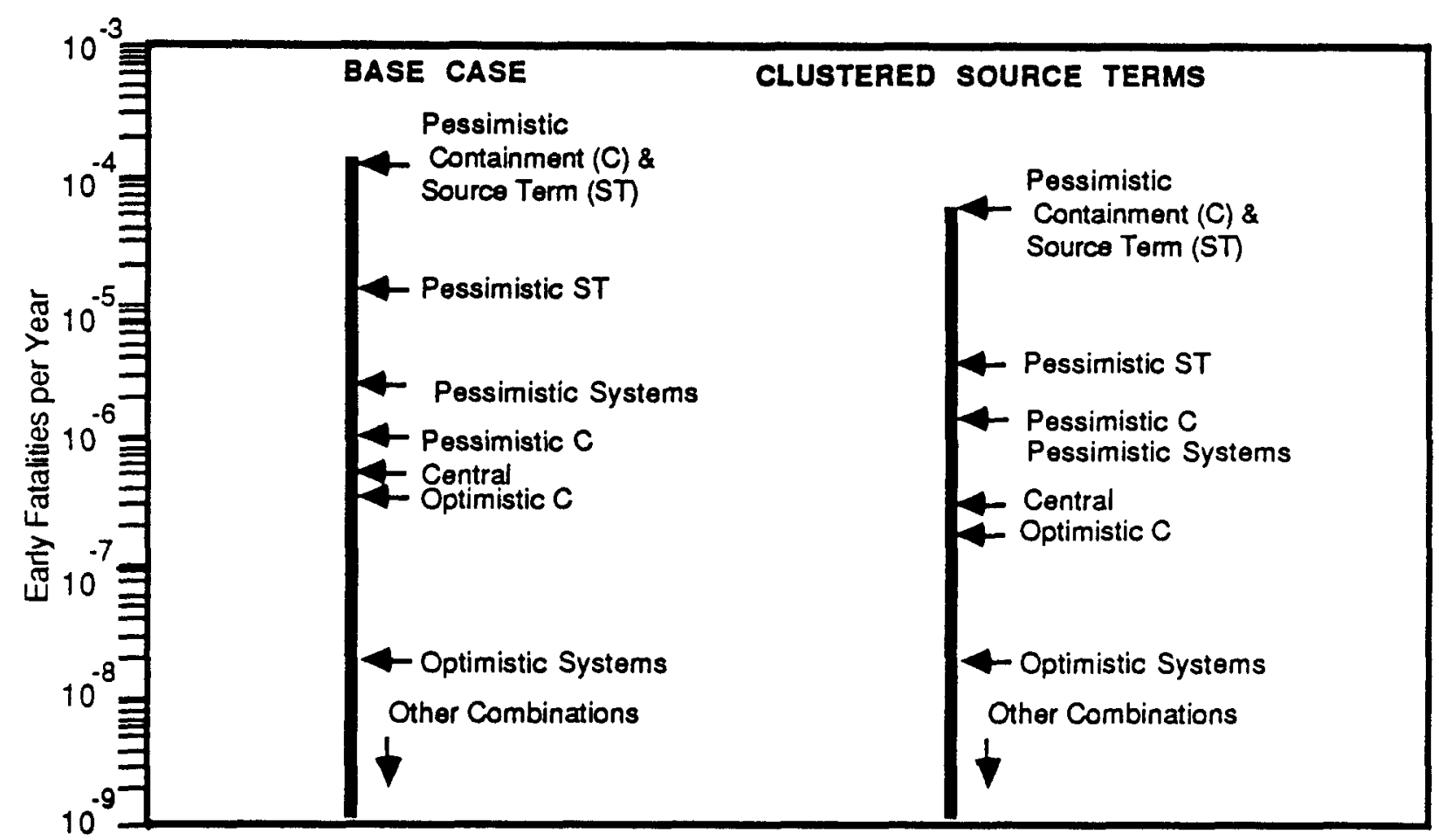

Figure C-14. Comparison of CRAC2 OCP Early Fatality Results With and Without Clustering

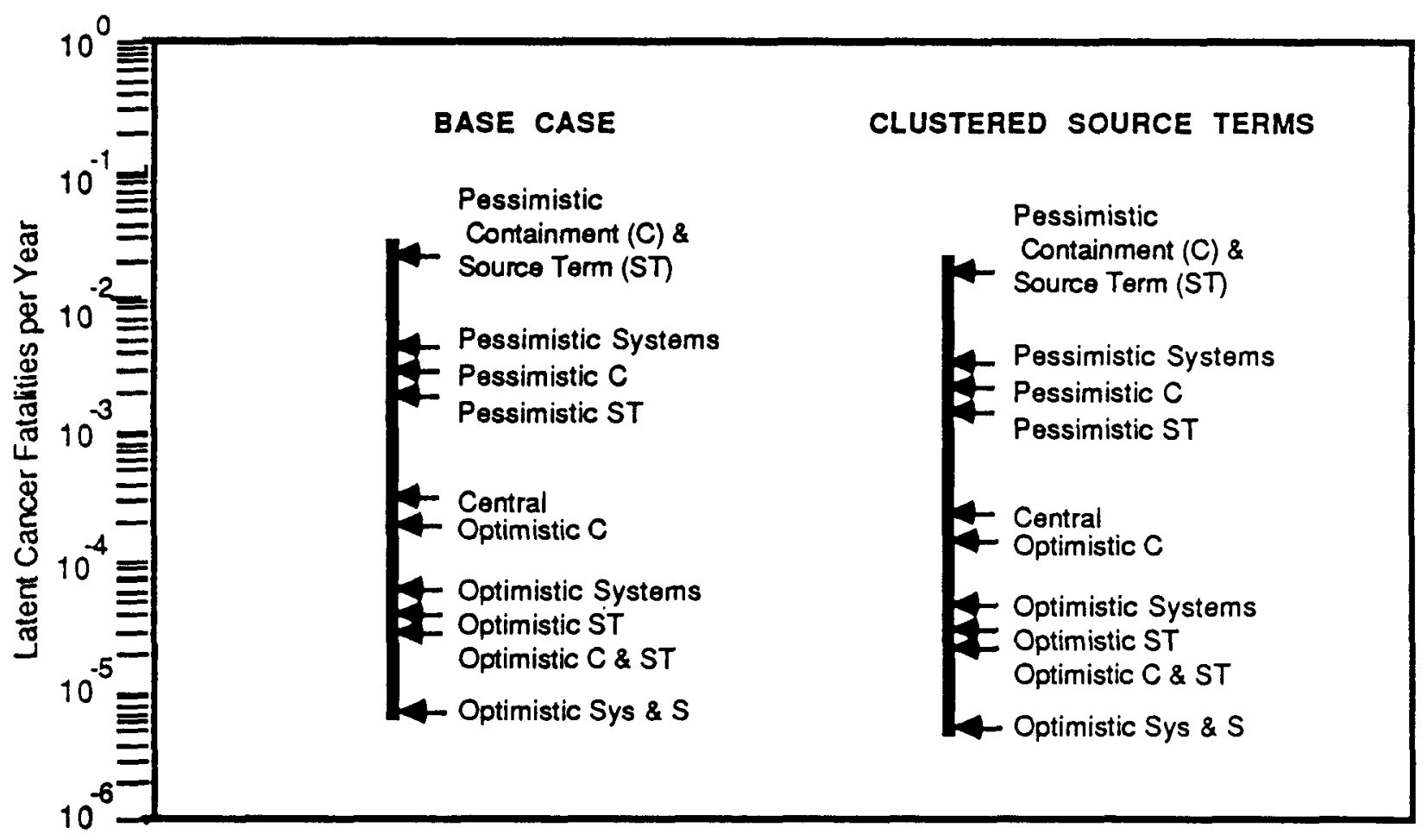

Figure C-15. Comparison of CRAC2 OCP Latent Fatality Results With and Without Clustering 


\section{References for Appendix C}

1. Gieseke, J. A., el al. Radionuclide Release Under Specific Accident Conditions. Battelle Columbus Laboratories Report BMI-2104, Vol. 5, Columbus, OH: 1984.

2. Gieseke, J. A., et al. Informal Report on Source Term Predictions for Various Containment Failure Assumptions. Battelle Columbus Laboratories, Columbus, $\mathrm{OH}$ : August, 1984.

3. Denning, R. S., et al. Report on Radionuclide Release Calculations for Selected Severe Accident Scenarios: Volume III, PWR Subatmospheric Containment Design (Draft Report). Battelle Columbus Laboratories, Columbus, OH: January, 1986.

4. Lipinski, R. J., et al. Uncertainty in Radionuclide Release Under Specific LWR Accident Conditions. SAND84-0410, Vol. II, Sandia National Laboratories, Albuquerque, NM: 1985.

5. Lipinski, R. J., et al. Uncertainty in Radionuclide Release Under Specific LWR Accident Conditions. SAND84-0410, Vol. III, Sandia National Laboratories, Albuquerque, NM: 1985.

6. Lipinski, R. J., et al. Uncertainty in Radionuclide Release Under Specific LWR Accident Conditions. SAND84-0410, Vol. IV, Sandia National Laboratories, Albuquerque, NM: 1985.

7. Reassessment of the Technical Bases for Estimating Source Terms. U.S. Nuclear Regulatory Commission Report NUREG-0956, Washington, DC: August, 1985.

8 Williams, D. C., K. D. Bergeron. P. E. Rexroth and J. L. Tills, "Integrated Phenomenological Analysis of Containment Response to Severe Core Damage Accidents", Progress Nuclear. Engineering., to be published.

9. Bergeron, K. D. , A. L. Camp and D. A. Powers, "Some Unresolved Issues in the Analysis of Severe Accident Phenomena," Proceedings of the International ANS/ENS Topical Mtg. on Thermal Reactor Safety, San Diego, CA: Feb. 2-6, 1986.

10. Powers, D., Memo to SARRP Surry Source Term LLH Group on High Pressure Ejection and Direct Heating Scenarios: February, 1985.

11. Aldrich, D. C, et al. Technical Guidance for Siting Criteria Development . SAND811549, NUREG/CR-2239, Sandia National Laboratories, Albuquerque, NM: 1981.

12. Alpert, D. J, et al. A Demonstration Uncertainty/Sensitivity Analysis Using the Health and Economic Consequence Model CRAC2. SAND81-1824, NUREG/CR-4199, Sandia National Laboratories, Albuquerque, NM: 1985.

13. Alpert, D. J, et al. Relative Importance of Individual Elements to Reactor Accident Consequences Assuming Equal Release Fractions. SAND81-2575, NUREG/CR-446, Sandia National Laboratories, Albuquerque, NM: March 1986.

14. Batchelor, B. G. and P. R. Wilkens, "Methods for Location of Clusters of Patterns to Initialize a Learning Machine," Electronic Letters, Vol. 5, No. 20, pp 481-483 : 1969.

15. MacQueen, J., "Some Methods for Classification and Analysis of Multivariate Data," Proceedings of the Fifth Berkeley Symposium on Probability and Statistics. University of California Press, Berkeley, CA : 1967. 
NUREG/CR-4551, VOL. 1: DRAFT REPORT FOR COMMENT (SEPTEMBER, 1986)

Appendix D

DETAILED LISTINGS OF RISK RESULTS

$D-i / i$ i 
-

-

-

-

- 


\section{CONTENTS}

Section

D.1 RISQUE CODE OUTPUT FOR THE LLH ANALYSIS USING CRAC2 ....... D-1

D.2 RISQUE CODE OUTPUT FOR THE LLH ANALYSIS USING MACCS....... D-72

D.3 OUTPUT FOR THE OCP ANALYSIS.....................................

D.4 OUTPUT FOR THE SENSITIVITY CASE WITHOUT DIRECT HEATING..D-157

D.5 REVIEW OF HIGH AND LOW RISK SAMPLE MEMBERS OF THE LLH RESULT ................................................................ 180

\section{LIST OF TABLES}

Section

Page

D.1 Review of LLH Uncertainty Issues Relative to Early Fatality Risk..............

D.1 Review of LLH Uncertainty Issues Relative to Latent Cancer Fatality Risk...... D-184 
-

•

-

-

- 
NUREG/CR-4551, VOL. 1: DRAFT REPORT FOR COMMENT (SEPTEMBER, 1986)

\section{Appendix D \\ DETAILED LISTINGS OF RISK RESULTS}

The primary risk results are provided in Section 5 of the main report. Additional information that is provided in the output of the RISQUE code is included in this appendix. The complete LLH results calculated using the CRAC2 code are contained in Section D.1. Section D.2 provides the same output for the results calculated using MACCS. The detailed results for the OCP methodology (using CRAC2) are similarly provided in Section D.3. The CRAC2 results for early and latent cancer fatalities for the sensitivity case involving no direct heating are listed in Section D.4. Finally, a review of some of the individual sample members for the base case risk results that was used to study the risk results is included in Section D.5.

\section{D.1 RISQUE CODE OUTPUT FOR THE LLH ANALYSIS USING CRAC2}

The complete output of the RISKEE code is reproduced in this section. All of the results described in the main report may be found in this listing. In addition, the results included in the listing allow an understanding of the detailed contributors to each of the consequence measures. The LLH results are also amenable to detailed review, since the issue outcomes and risk results for each consequence measure are provided for each of the 100 sample members. The first part of the output is a listing of each uncertainty issue and the level of the issue in each of the 100 sample members. The issues and levels are defined as in Section 4 of the main report. [In the output, the issues are either numbered consecutively by groups (group 1 for sequence issues, group 2 for containment issues and group 3 for source term issues) or consecutively for all of the issues starting with group 1.] This first part of the code listing provides the short title of each issue as a cross reference. The issue level definitions are then followed in the listing by the core-melt frequencies for each of the 100 sample members. Finally, for each of the risk measures, the following results are presented:

- The risk results for each of the individual sample members in order of increasing risk;

- The risk values in the same order, along with the individual issue levels ;

- The fractional contribution of each plant damage state to the mean risk,

- The fractional contribution of each source term bin to mean risk;

- The fractional contribution of each cluster to mean risk;

- The distribution of risk at each level and the rank regression analysis of the results; and

- The chi-squared study of each issue, by individual level and by total issue. 
NUREG/CR-4551, VOL. 1: DRAFT REPORT FOR COMMENT (SEPTEMBER, 1986)

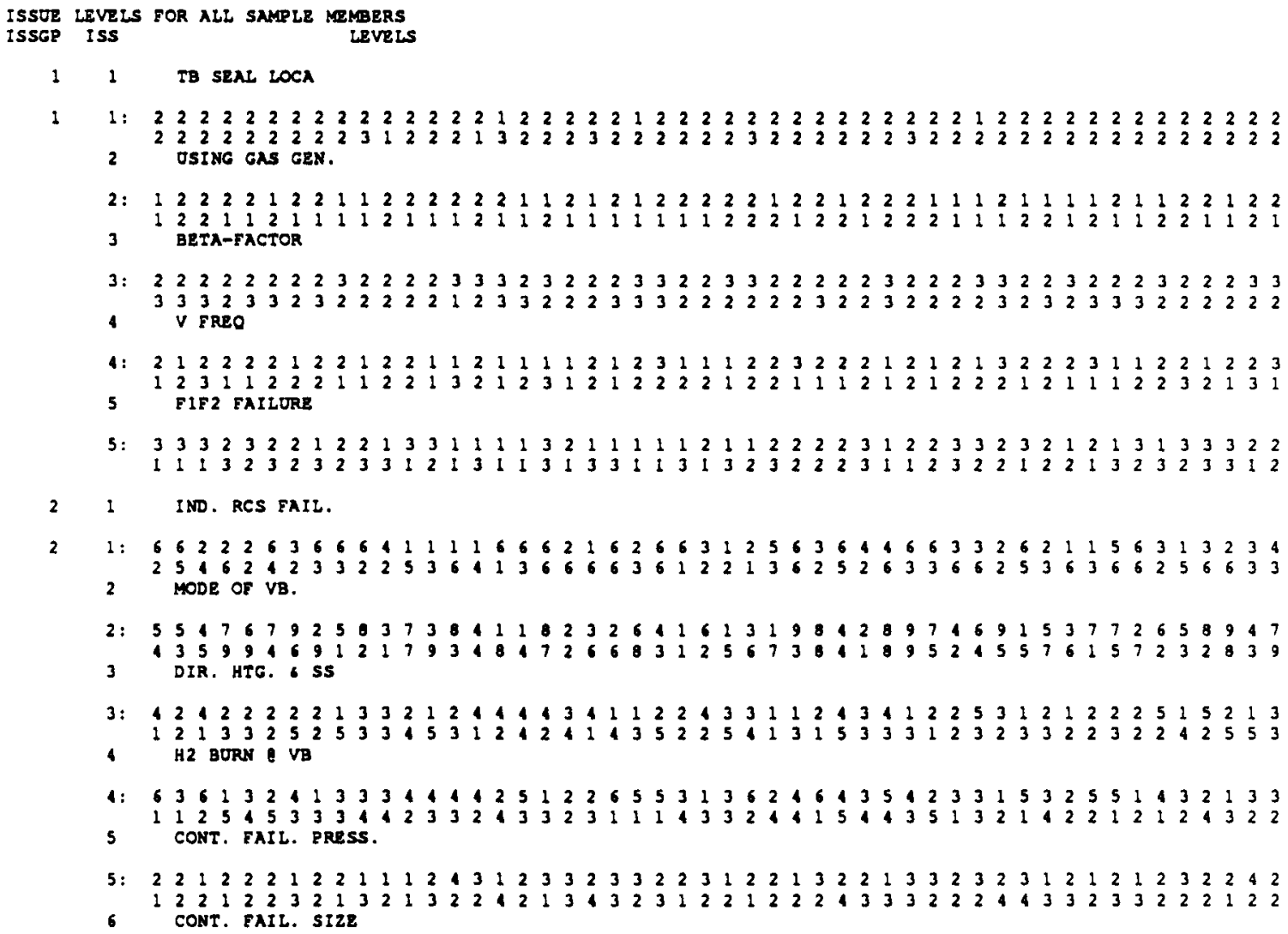

5: $\begin{array}{llllllllllllllllllllllllllllllllllllllllllllllll}3 & 3 & 2 & 3 & 2 & 1 & 2 & 2 & 1 & 3 & 3 & 1 & 1 & 1 & 1 & 3 & 2 & 1 & 1 & 1 & 1 & 1 & 2 & 1 & 1 & 2 & 2 & 2 & 2 & 3 & 1 & 2 & 2 & 3 & 3 & 2 & 3 & 2 & 1 & 2 & 1 & 3 & 1 & 3 & 3 & 3 & 2 & 2\end{array}$ $\begin{array}{llllllllllllllllllllllllllllllllllllllllllllllllll}1 & 1 & 3 & 2 & 3 & 2 & 3 & 2 & 3 & 3 & 1 & 2 & 1 & 3 & 1 & 1 & 3 & 1 & 3 & 3 & 1 & 1 & 3 & 1 & 3 & 2 & 3 & 2 & 2 & 2 & 3 & 1 & 1 & 2 & 3 & 2 & 2 & 1 & 2 & 2 & 2 & 3 & 2 & 3 & 2 & 3 & 3 & 1 & 2\end{array}$

21 IND. RCS FAIL.

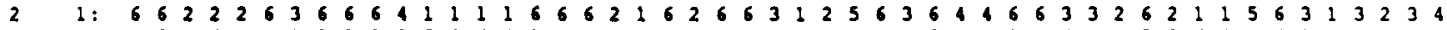
2546242332253641366666361225136625526336662533636662566633 MODE OF VB. 2: $\left.\begin{array}{lllllllllllllllllllllllllllllllllllllllllllllllll}5 & 5 & 4 & 7 & 6 & 7 & 9 & 2 & 5 & 0 & 3 & 7 & 3 & 8 & 1 & 1 & 1 & 8 & 2 & 3 & 2 & 6 & 4 & 1 & 6 & 1 & 3 & 1 & 9 & 8 & 1 & 2 & 0 & 9 & 7 & 4 & 6 & 9 & 1 & 5 & 3 & 7 & 7 & 2 & 6 & 5 & 8 & 9 & 4\end{array}\right]$ DIR. HTG. 6 SS

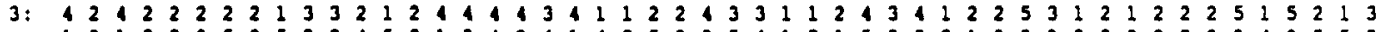

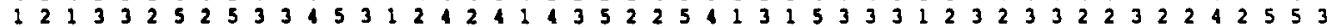
H2 BURN VB

4: 636132413334414251226553136624643542331532551432133

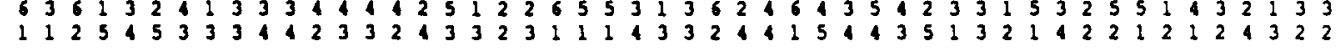
CONT. FAIL. RRESS.

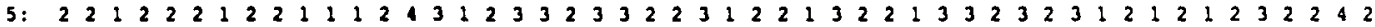

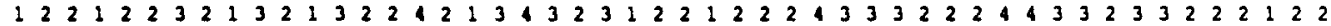
CONT. EAIL. SIZZ

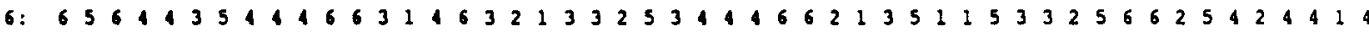
55453334413424414631144644442513333451133231345635 SPRAY FAILURE

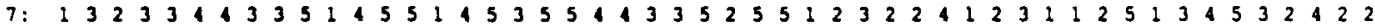

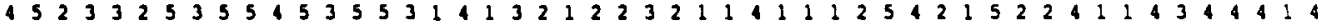
$\checkmark$ seo. SURM.

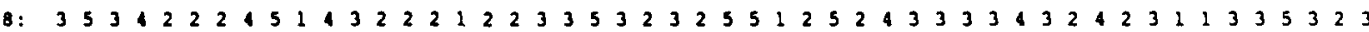
$\begin{array}{llllllllllllllllllllllllllllllllllllllllllllllll}4 & 2 & 1 & 4 & 3 & 4 & 2 & 3 & 2 & 3 & 5 & 3 & 3 & 3 & 3 & 3 & 3 & 5 & 2 & 2 & 3 & 3 & 2 & 2 & 3 & 3 & 2 & 3 & 3 & 1 & 3 & 1 & 3 & 3 & 1 & 2 & 2 & 1 & 3 & 3 & 2 & 2 & 3 & 3 & 4 & 2 & 3 & 2\end{array}$ 31 IN VESS RELEASE

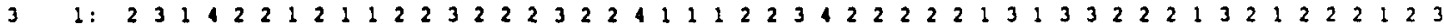

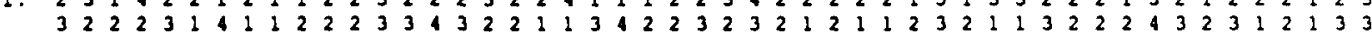
2 CSI DRCOMP

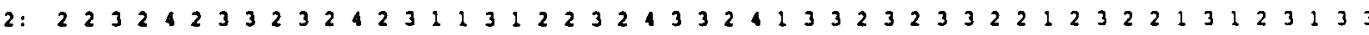

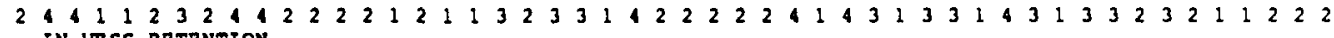
IN VESS RETENTION

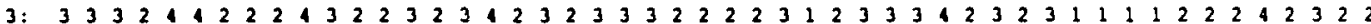
$\begin{array}{lllllllllllllllllllllllllllllllllllllllllllllllll}3 & 3 & 4 & 2 & 3 & 2 & 3 & 2 & 2 & 2 & 1 & 3 & 3 & 2 & 3 & 3 & 3 & 1 & 2 & 2 & 3 & 2 & 3 & 3 & 2 & 1 & 2 & 3 & 4 & 3 & 2 & 3 & 3 & 3 & 4 & 1 & 2 & 3 & 3 & 2 & 2 & 1 & 2 & 2 & 1 & 3 & 3 & 1 & 4\end{array}$

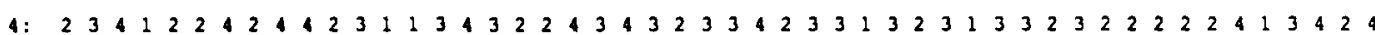

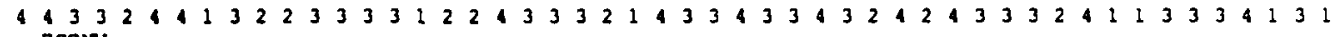
FCON

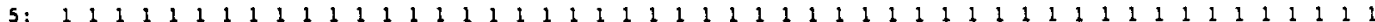

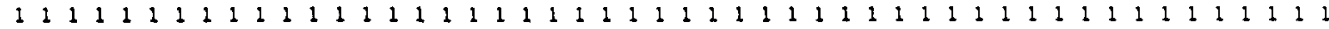
CEI REL

6: $\begin{array}{lllllllllllllllllllllllllllllllllllllllllllllllll} & 2 & 1 & 3 & 2 & 2 & 2 & 2 & 2 & 2 & 2 & 1 & 1 & 2 & 1 & 2 & 1 & 1 & 2 & 1 & 2 & 2 & 3 & 2 & 2 & 3 & 2 & 1 & 1 & 3 & 3 & 2 & 2 & 2 & 2 & 2 & 1 & 3 & 2 & 2 & 1 & 2 & 2 & 2 & 2 & 2 & 2 & 1 & 2\end{array}$ $\begin{array}{llllllllllllllllllllllllllllllllllllllllllllllllll}2 & 1 & 1 & 2 & 3 & 3 & 3 & 2 & 3 & 2 & 3 & 3 & 1 & 1 & 1 & 2 & 2 & 2 & 3 & 2 & 3 & 2 & 2 & 3 & 2 & 2 & 2 & 2 & 3 & 2 & 3 & 1 & 1 & 2 & 1 & 3 & 3 & 2 & 2 & 3 & 1 & 2 & 2 & 2 & 2 & 2 & 1 & 2 & 3 & 2\end{array}$ FCONC

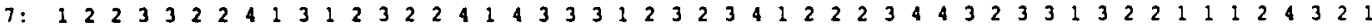

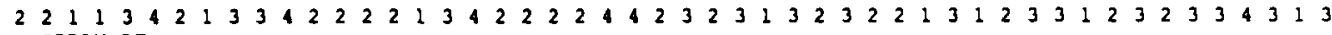
SPRAY DF

8: $\quad \begin{array}{lllllllllllllllllllllllllllllllllllllllllllllllll} & 2 & 2 & 2 & 2 & 1 & 3 & 2 & 3 & 2 & 3 & 3 & 3 & 2 & 2 & 1 & 1 & 3 & 3 & 1 & 2 & 2 & 2 & 2 & 3 & 2 & 3 & 2 & 2 & 3 & 2 & 2 & 1 & 2 & 3 & 3 & 1 & 1 & 1 & 2 & 2 & 2 & 2 & 3 & 2 & 2 & 3 & 1 & 1\end{array}$ $\begin{array}{lllllllllllllllllllllllllllllllllllllllllllllllll}3 & 2 & 1 & 1 & 2 & 1 & 2 & 1 & 2 & 1 & 3 & 3 & 3 & 1 & 2 & 1 & 2 & 2 & 2 & 1 & 2 & 2 & 3 & 1 & 2 & 2 & 1 & 1 & 1 & 2 & 3 & 2 & 2 & 3 & 3 & 2 & 3 & 2 & 1 & 3 & 1 & 2 & 2 & 2 & 2 & 3 & 3 & 1 & 3\end{array}$ ARR AGGLOM

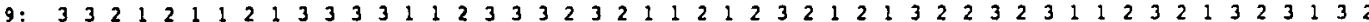

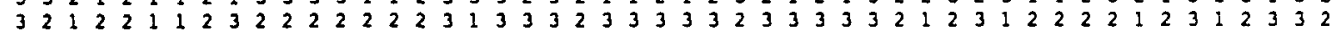


NUREG/CR-4551, VOL. 1: DRAFT REPORT FOR COMMENT (SEPTEMBER, 1986)

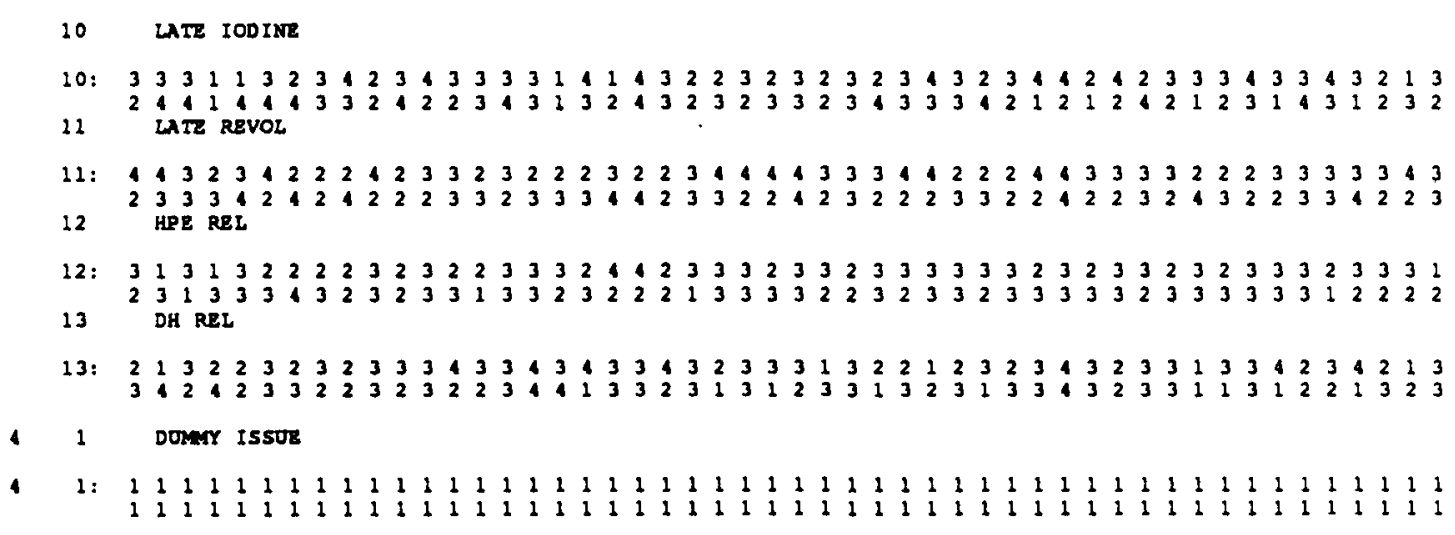
HPE REL

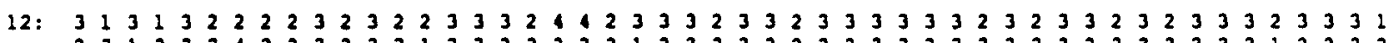

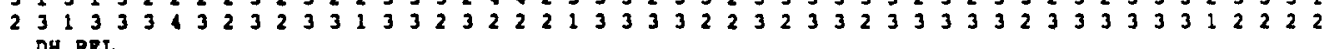
DH REL

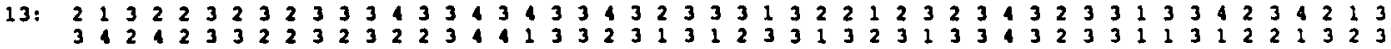
11 DONAY I5SOE

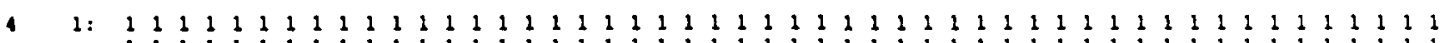

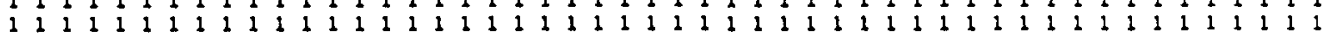


BASE CASE: SURRY LALHC

Specifle inforration concerning input date follows:

HEAOER : ALL ISSOES VARY IN SAMPLE

SEOUENCES : SEQUENCES FROM ASEP 2/20/86

CONTAINRENT FAILURE NODES : CFM ISSCES

RELEASES : RELEASES BY ISSUES $(1-13)$

CONSEqUENCES : CONSEgOENCES $4 / 23 / 86$ CRAC2

SORTED MELTDOWN FREOCENCIRS

PROBABILITY

COM. PROB.

$1.0008-02$

1. $000 \mathrm{E}-02$

1. $000 \mathrm{E}-02$

2. $000 \mathrm{~B}-02$

3. $0008-02$

4. $000 \mathrm{E}-02$

$1.000 \mathrm{E}-02$

$5.0008-02$

$6.000 \mathrm{~B}-02$
$7.000 \mathrm{E}-02$

$1.000 \mathrm{E}-02$

$1.0008-02$

8. 0008-02

$9.0008-02$

$1.0008-02$

$1.0008-02$

$1.0008-02$

$1.000 \mathrm{E}-02$
$1.000 \mathrm{E}-02$

$1.000 \mathrm{E}-02$

$1.000 \mathrm{E}-02$

$1.000 \mathrm{~B}-02$

$1.0008-02$

$1.000 \mathrm{E}-02$

$1.0008-02$

1. 000 E-02

$1.000 \mathrm{E}-02$

$1.0008-02$

$1.0008-02$

1. $000 \mathrm{R}-02$

1. $000 \mathrm{E}-02$

1. $0008-02$

1. $000 \mathrm{E}-02$

$1.000 \mathrm{E}-02$

$1.000 \mathrm{E}-02$

$1.000 \mathrm{e}-02$

1. $000 \mathrm{E}-02$

$1.000 \mathrm{E}-02$

$1.0008-02$

$1.000 \mathrm{E}-02$

$1.000 \mathrm{E}-02$
$1.000 \mathrm{E}-02$

$1.000 \mathrm{E}-02$
$1.000 \mathrm{E}-02$

$1.000 \mathrm{E}-02$

$1.000 \mathrm{E}-02$

$1.000 \mathrm{R}-02$

$1.000 \mathrm{E}-02$

$1.0008-02$

$1.0008-02$

$.0008-02$

$1.000 \mathrm{E}-02$
$1.000 \mathrm{E}-02$

$1.000 \mathrm{E}-02$

$1.000 \mathrm{E}-02$

$1.000 \mathrm{~B}-02$

$1.000 \mathrm{E}-02$

$1.000 \mathrm{E}-02$

$1.000 \mathrm{E}-02$

$.000 \mathrm{E}-02$

$1.000 E-02$
$1.0008-02$

1. $1.000 \mathrm{E}=02$

$1.0008-02$

$1.0008-02$

1. $000 \mathrm{E}-02$

$1.0008-02$

1. $000 \mathrm{~B}-02$

$1.000 \mathrm{E}-02$

$1.000 \mathrm{E}-02$

$1.000 \mathrm{E}-02$

$1.000 \mathrm{E}-02$

1. $000 \mathrm{E}-02$

1. $000 \mathrm{OE}-02$

$1.0008-02$

$1.0008-02$

1. $000 \mathrm{E}-02$

$1.0008-02$

$1.000 \mathrm{E}-02$

$1.000 \mathrm{E}-02$

1. $000 \mathrm{E}-02$

$1.000 \mathrm{E}=02$

$1.000 \mathrm{E}-02$

$1.000 \mathrm{E}-02$

1. $000 \mathrm{E}-02$

$1.000 \mathrm{E}-02$

$1.000 \mathrm{E}-02$

$1.000 \mathrm{E}-02$

1. $000 \mathrm{z}-01$

1. $1008-01$

1. 2008-01

1. $300 \mathrm{E}-01$

1. $4008-01$

1. $500 \mathrm{~B}-01$

1. $600 \mathrm{E}-01$

$1.700 \mathrm{~B}-01$

1. $800 \mathrm{E}-01$
$1.900 \mathrm{~B}-01$

2. $0008-01$

2. $1008-01$

2. $200 \mathrm{~B}-01$

2. 3008-01

2. $1008-01$

2. 500E-01

2. $600 \mathrm{z}-01$

2. $700 \mathrm{E}-01$

2. $8008-01$

2. $900 \mathrm{E}-01$

3. $000 \mathrm{E} \rightarrow 01$

3. 1008-01

3. $200 \mathrm{~B}-01$

3. $3008-01$

3. $1008-01$

3. $500 \mathrm{E}-01$

3. $600 \mathrm{E}-01$

3. $700 \mathrm{E}-01$

3. $800 \mathrm{E}-01$

3. $900 \mathrm{O}-01$

1. $0008-01$

1. $100 \mathrm{E}-01$

1. 200z-01

1. $300 \mathrm{OB}-01$

1. 1008-01

4. $500 \mathrm{E}-01$

4. $6008-01$

4. 700E-01

1.8008-01

4. $9008-01$

5. $000 \mathrm{E}-01$

5. $100 \mathrm{O}-01$

5. $200 \mathrm{E}-01$

5. $300 \mathrm{z}-01$

5. $1008-01$

5. 5008-01

5. 600B-01

5. $700 \mathrm{~B}-01$

5. $800 \mathrm{E}-01$

5. $900 \mathrm{E}-01$

6. $000 \mathrm{E}-01$

6. $1008-01$

6. $200 \mathrm{E}-01$

6. $3008-01$

6. $400 \mathrm{E}-01$

6. $500 \mathrm{E}-01$

6. $600 \mathrm{~B}-01$

6. $7008-01$

6. $800 \mathrm{~B}-01$

6. $900 \mathrm{E}-01$

7. $000 \mathrm{E}-01$

7. 1008-01

7. $200 \mathrm{OB}-01$

7. 3008-01

7. $400 \mathrm{E}-01$

7. $500 \mathrm{E}-01$

7. $600 \mathrm{~B}-01$

$7.7008-01$

T. $800 \mathrm{E}-01$

FREOUENCY

1. 4738-05

$1.473 \mathrm{E}-05$

$1.4788-05$

1.4788-05

$1.4788-05$

$1.4788-05$

$1.4780-05$

$1.4788-05$

$1.4788-05$

$.486 \mathrm{E}-05$

$1.486 \mathrm{E}-05$

1. 486R-05

1.5548-05

1. $554 \mathrm{E}-0.5$

$1.5588-05$

$1.558 \mathrm{E}-05$

$1.558 \mathrm{E}-0 \mathrm{~S}$

1. $558 \mathrm{E}-05$

$1.558 \mathrm{E}-05$

1. 5588-05

1.5678-05

1. $567 \mathrm{E}-05$

$1.5678-05$

$1.567 \mathrm{E}-05$

$1.567 \mathrm{E}-05$

1. $567 \mathrm{E}-05$

1. $567 \mathrm{E}-05$

$1.567 \mathrm{E}-05$

1. $601 \mathrm{E}-05$

$1.6018-05$

$1.018 \mathrm{E}-05$

1. 872 $\mathrm{B}-05$

$1.073 E-05$

1. $73 \mathrm{~B}-05$

1. $873 \mathrm{E}-05$

$1.873 \mathrm{E}-05$

1. $378 \mathrm{E}-05$

1. $878 \mathrm{E}-05$

1. 8788-05

$1.8788-05$

1. $8788-05$

$1.886 \mathrm{E}-05$

1. 886E-05

1.8868-05

$1.8268-05$

$1.8998-05$

1. $9548-05$

.

$1.954 \mathrm{E}-05$

1. $954 \mathrm{E}-05$

1. $954 \mathrm{E}-05$

1. 9548-05

1. $958 \mathrm{E}-0.5$

1. $958 \mathrm{E}-05$

$1.958 \mathrm{E}-05$

1. $587-05$

1. $967 \mathrm{E}-05$

1. $967 \mathrm{E}-05$

1. $967 \mathrm{E}-05$

1. $967 \mathrm{E}-05$

$1.9678-05$

1.9678-05

$1.9678-05$

$1.350-05$

$2.1308-05$

$2.138 \mathrm{E}-05$

2.138E-0S

2.138E-05

2. 206E-05

$2.2068-05$

$2.2068-05$

.2068-05

2.206E-05

2. $2108-05$

2.2108-05

2.2108-05

$2,219 \mathrm{E}-05$

2.268E-05 
NUREG/CR-4551, VOL. 1: DRAFT REPORT FOR COMMENT (SEPTEMBER, 1986)

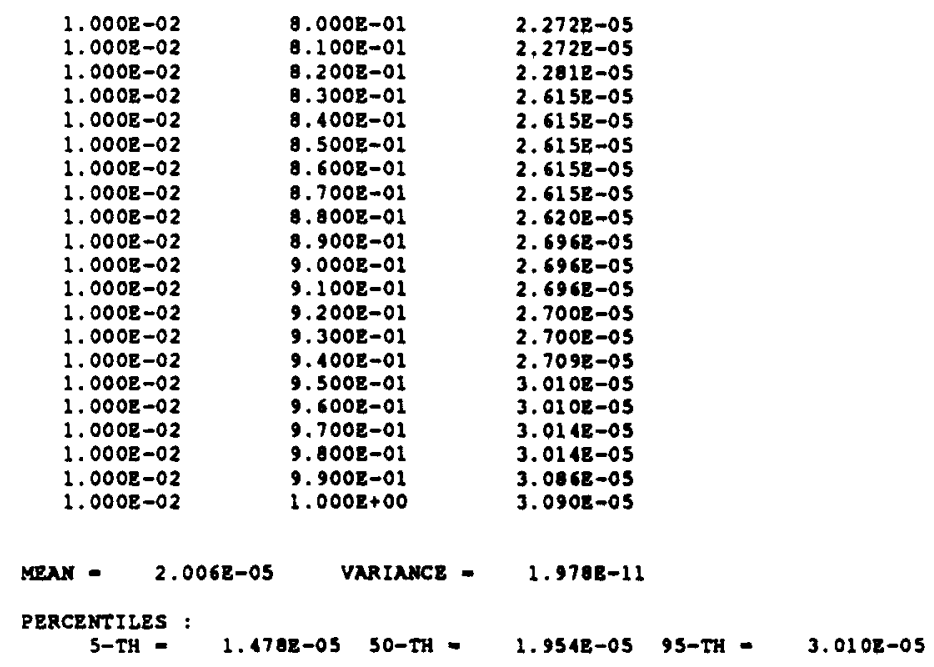


NUREG/CR-4551, VOL. 1: DRAFT REPORT FOR COMMENT (SEPTEMBER, 1986)

CONSEQUENCE MEASURE:

EARLY DEATHS RER YEAR

BASE CASE

SORRY UAHC (CRAC)

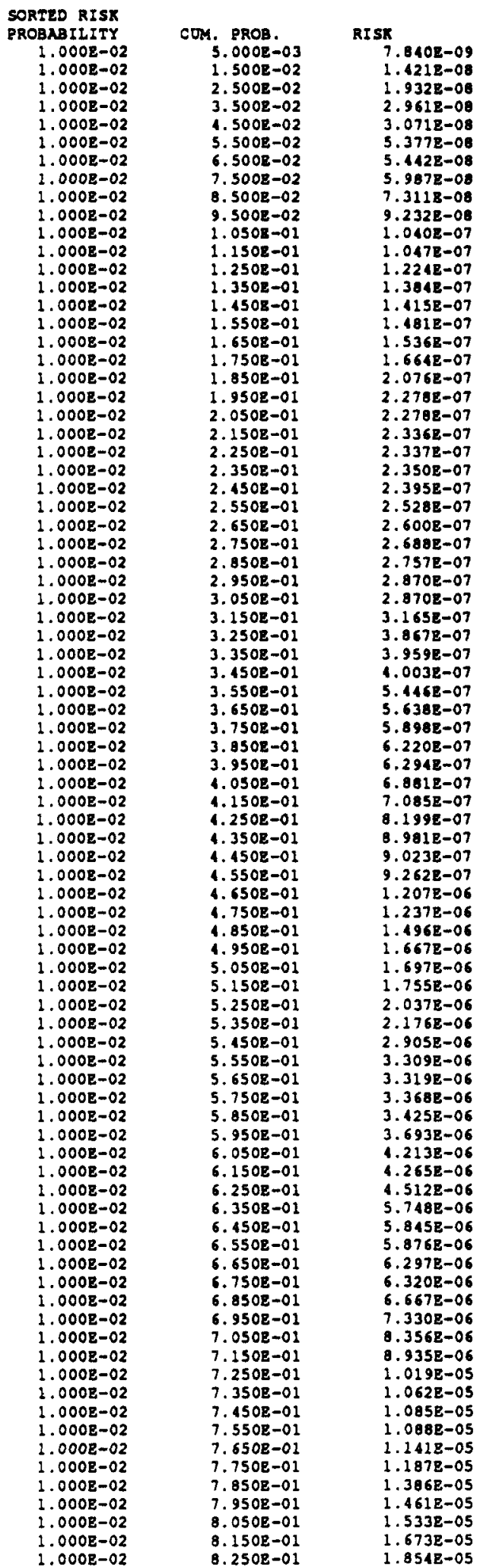


NUREG/CR-4551, VOL. 1: DRAFT REPORT FOR COMMENT (SEPTEMBER, 1986)

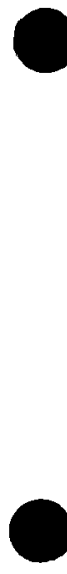

$$
\begin{aligned}
& 1.000 \mathrm{E}-02 \\
& 1.000 \mathrm{z}-02 \\
& 1.000 \mathrm{E}-02 \\
& 1.000 \mathrm{E}-02 \\
& 1.000 \mathrm{E}-02 \\
& 1.000 \mathrm{E}-02 \\
& 1.000 \mathrm{z}-02 \\
& 1.000 \mathrm{E}-02 \\
& 1.000 \mathrm{E}-02 \\
& 1.000 \mathrm{E}-02 \\
& 1.000 \mathrm{E}-02 \\
& 1.000 \mathrm{E}-02 \\
& 1.000 \mathrm{z}-02 \\
& 1.000 \mathrm{z}-02 \\
& 1.000 \mathrm{E}-02 \\
& 1.000 \mathrm{E}-02 \\
& 1.000 \mathrm{~B}-02
\end{aligned}
$$

B. 350E-01

0. 4508-01

8. 550E-01

6. 650z-01

8.7508-01

8. 850E-01

0.950E-01

9.050E-01

9.15OE-01

9.250E-01

9.350E-01

9. $450 \mathrm{~B}-01$

$9.5508-02$

$9.650 \mathrm{z}-01$

9.750E-01

$9.050 \mathrm{E}-01$

9.950z-01

1. 9402-05

2. 05 4 $2-03$

2. $0802-05$

$2.137 \mathrm{E}-05$

$2.2668-05$

$2.3678-05$

$2.4028-05$

$2.4028-05$

2. 637E-05

3. 545E-05

5. 51 OE-OS

1. $2192-04$

$1.2722-04$

1. $4408-04$

$1.9752-04$

2. $507 \mathrm{E}-04$

2. $5072=04$

$2.3452-04$
$2.8982-04$

MENN = $1.922 \mathrm{~B}-05$

VARTANCE $=\quad 2.792 \mathrm{E}-09$

PERCENTILES :

5-TH - 4.0648-08 50-TH

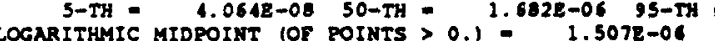

NUMBE ABOVE MIDPOINT - 51

NTMER ABOVE MTDPOIN

CHI-SODARED (1) - $4.0008-02$

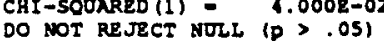


NUREG/CR-4551, VOL. 1: DRAFT REPORT FOR COMMENT (SEPTEMBER, 1986)

\begin{abstract}
CONseguence Mensone:
\end{abstract}
EARLY DEATHS PER YENR

Buse case.

SURRY LALHC (CRAC)

ISSUE LEVELS FOR EACH SANQPE MEARER. IN ORDER OF INCREASING RISK RISK ISSTE LEVELS, FOR ALL ISSURS

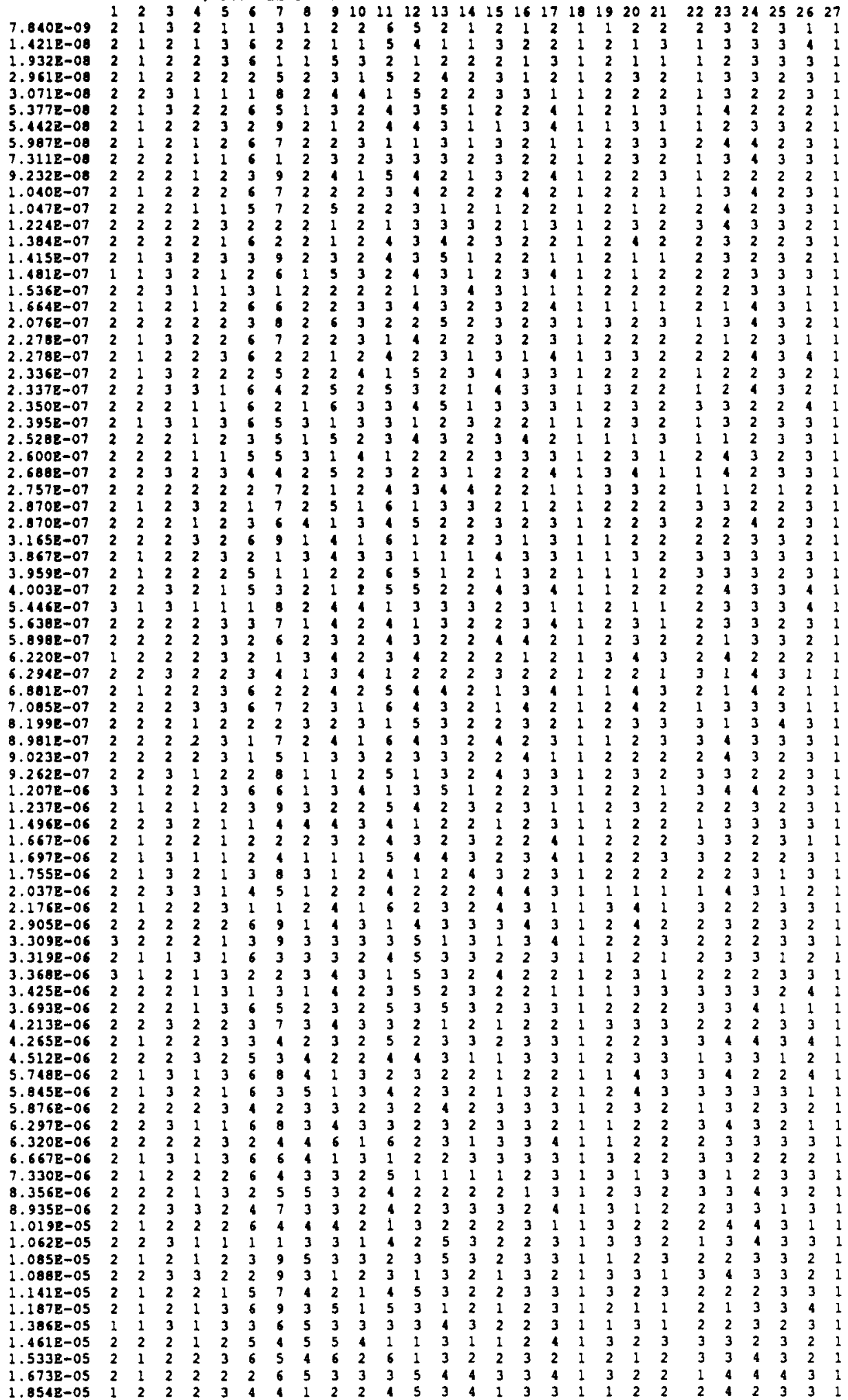


NUREG/CR-4551, VOL. 1: DRAFT REPORT FOR COMMENT (SEPTEMBER, 1986)

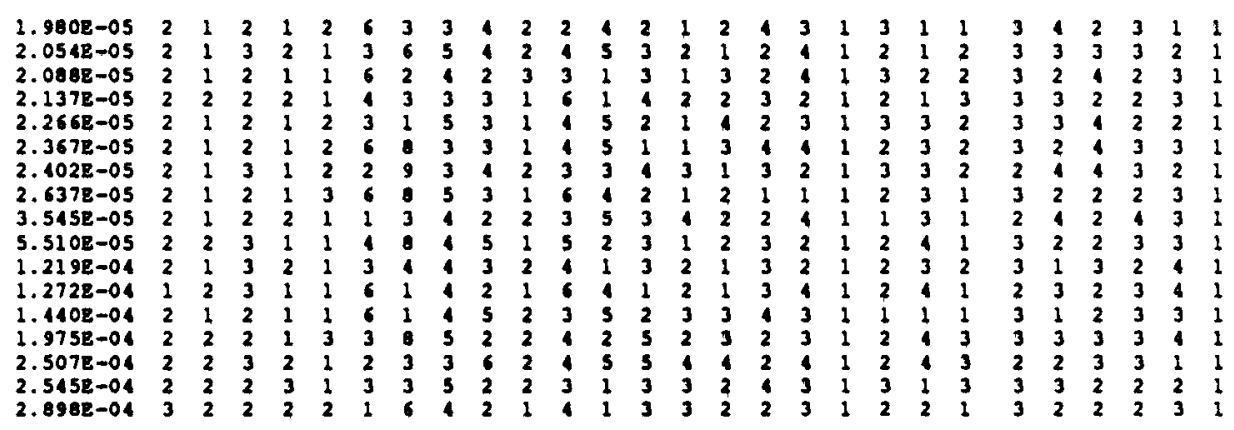


CONSEQUENCE MEASORE :

EARLY DEATHS PER YRAR

BASE CASE :

SURRY LALHC (CRAC)

FRACTIONAL CONTRIBUTION OF EACH SEOJENCE TO MEAN RISR

$\begin{array}{lr}\text { SEQUENCE } & \text { CONTRIBUTION } \\ \text { SYYB } & 0.0171 \\ \text { SYNI } & 0.0001 \\ \text { SNRN } & 0.6734 \\ \text { SYNN } & 0.0006 \\ \text { ANNN } & 0.0000 \\ \text { AYNN } & 0.0075 \\ \text { AYNI } & 0.0180 \\ \text { AYYB } & 0.0004 \\ \text { TYNN } & 0.1525 \\ \text { TYYBN } & 0.0532 \\ \text { TYYBD } & 0.0562 \\ \text { TYNI } & 0.0002 \\ \text { Y } & 0.0208\end{array}$

CONSEOUENCE NASTRE:

EARLY DEATHS PER YEAR

BASE CASE :

SURRY LALHC (CRAC)

FRACTIONAL CONTRIBUTION OP EACH BIN TO MRAN RIST

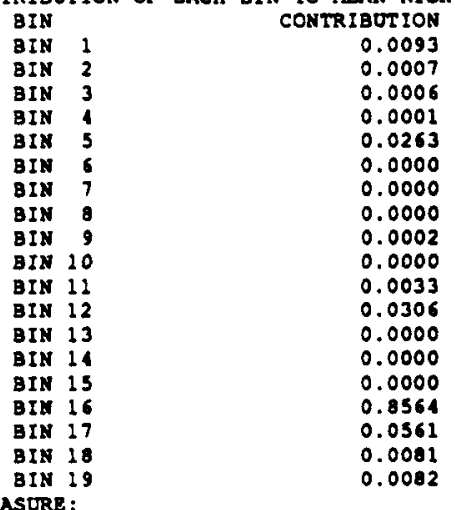

CONSEOUENCE MRASURE:

EARLY DEATHS PER YEAR

BASE CASE :

SURRY LALHC (CRAC)

FRACTIONAL CONTRIBOTIONS OF RACH RELEASE TO MENN RISK

\begin{tabular}{|c|c|c|}
\hline \multicolumn{3}{|r|}{ CONTRIBOTION } \\
\hline SUR & $1 G P$ & 0.1424 \\
\hline SUR & $2 G P$ & 0.3126 \\
\hline SOR & 3GP & 0.0874 \\
\hline SUR & $4 G P$ & 0.0709 \\
\hline SUR & 562 & 0.0339 \\
\hline son & $6 C P$ & 0.0311 \\
\hline SUR & $7 \mathrm{GP}$ & 0.0085 \\
\hline SUR & 8GP & 0.0042 \\
\hline SUR & 96 & 0.0001 \\
\hline SOR 1 & $10 G 2$ & 0.0023 \\
\hline SUR 1 & $11 G 2$ & 0.0026 \\
\hline SOR & $12 \mathrm{GP}$ & 0.0002 \\
\hline SUR : & 1368 & 0.0021 \\
\hline SUR I & 146 & 0.0001 \\
\hline SOR 1 & $15 G R$ & 0.0006 \\
\hline SOR & $16 G$ & 0.0000 \\
\hline SOR 1 & $17 \mathrm{GP}$ & 0.0006 \\
\hline SUR 1 & $18 G$ & 0.0000 \\
\hline SUR 1 & 1962 & 0.0005 \\
\hline SUR 2 & 206 & 0.0000 \\
\hline SUR 2 & 2162 & 0.0000 \\
\hline SUR 2 & $22 G$ & 0.0000 \\
\hline SUR & $23 G P$ & 0.0000 \\
\hline SUR 2 & 245 & 0.0000 \\
\hline
\end{tabular}


CONSEQUENCE MRASURE:

EARLY DEATHS PER YEAR

SENSITIVITY OF RIST TO ISSUES

DISTRIBUTION OF RISK AT EACH LEVEL OF RACH ISSOE

BASE CASE :

SORRY LALHC (CRAC)

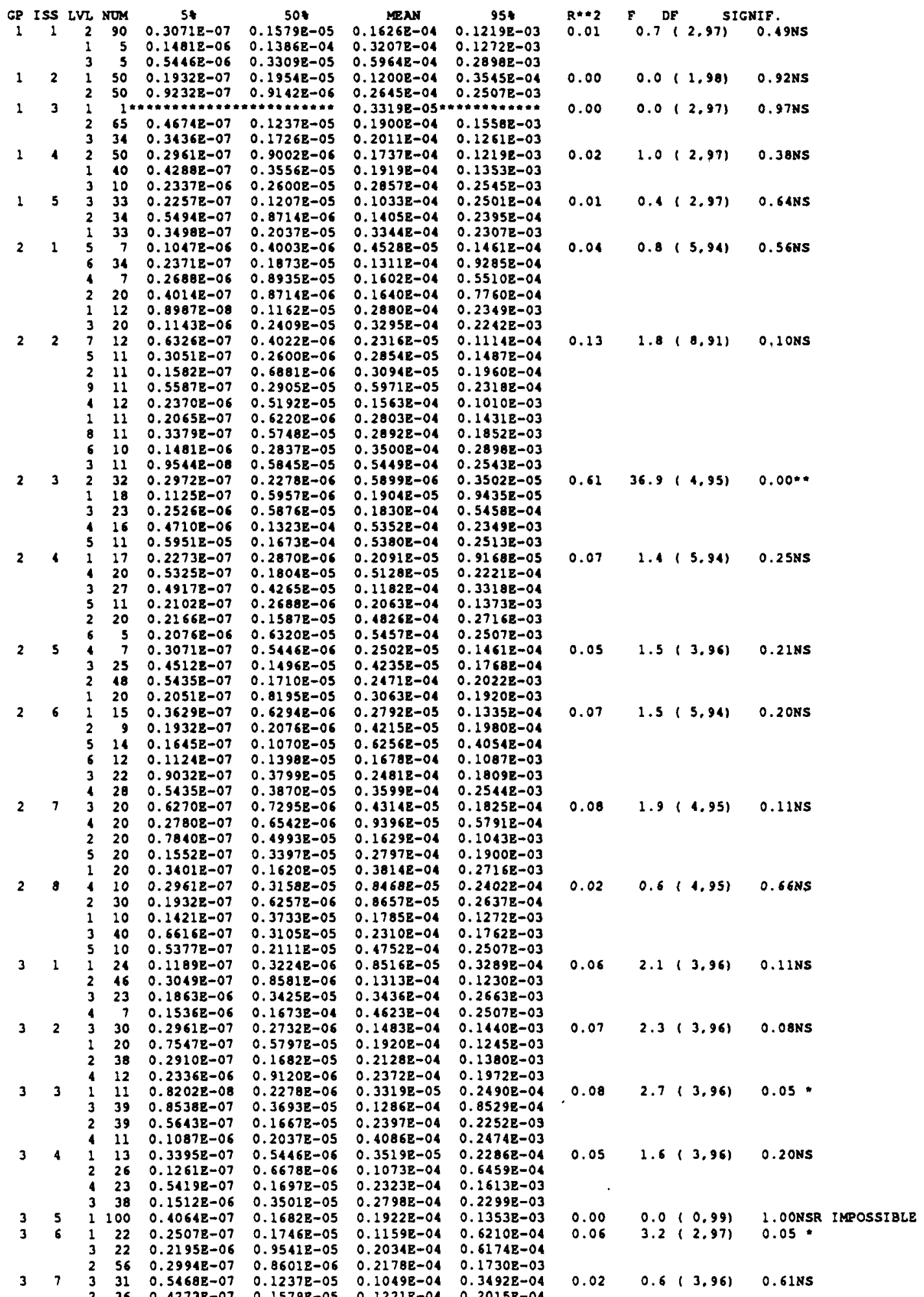


NUREG/CR-4551, VOL. 1: DRAFT REPORT FOR COMMENT (SEPTEMBER, 1986)

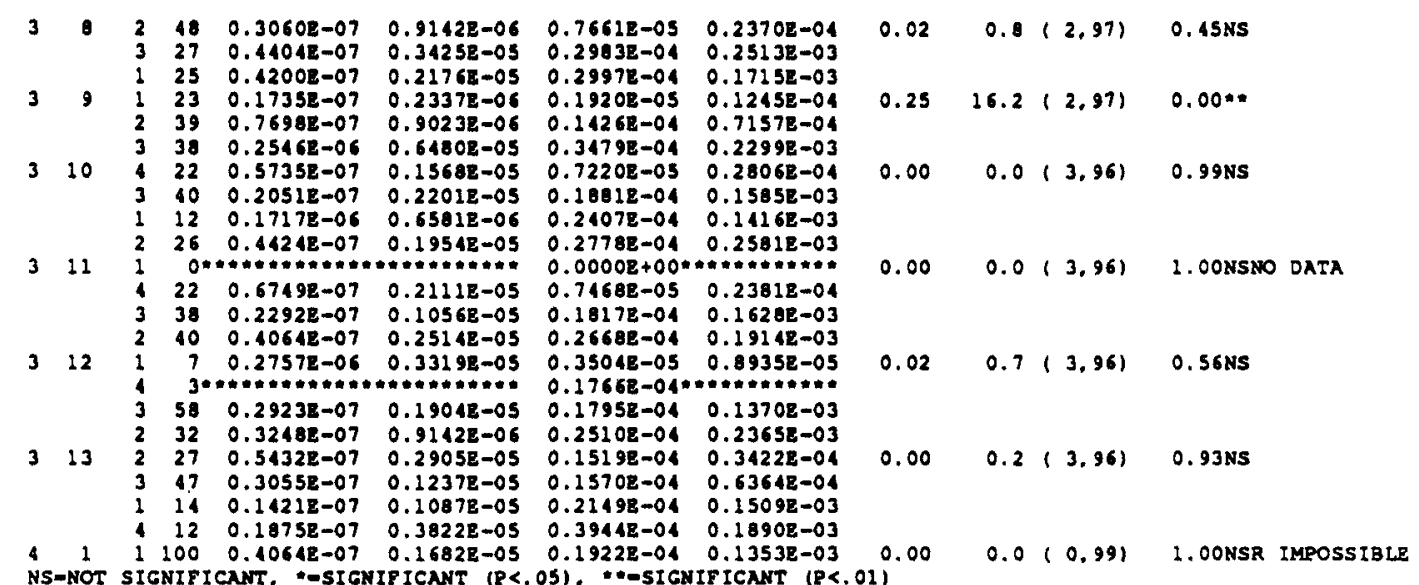




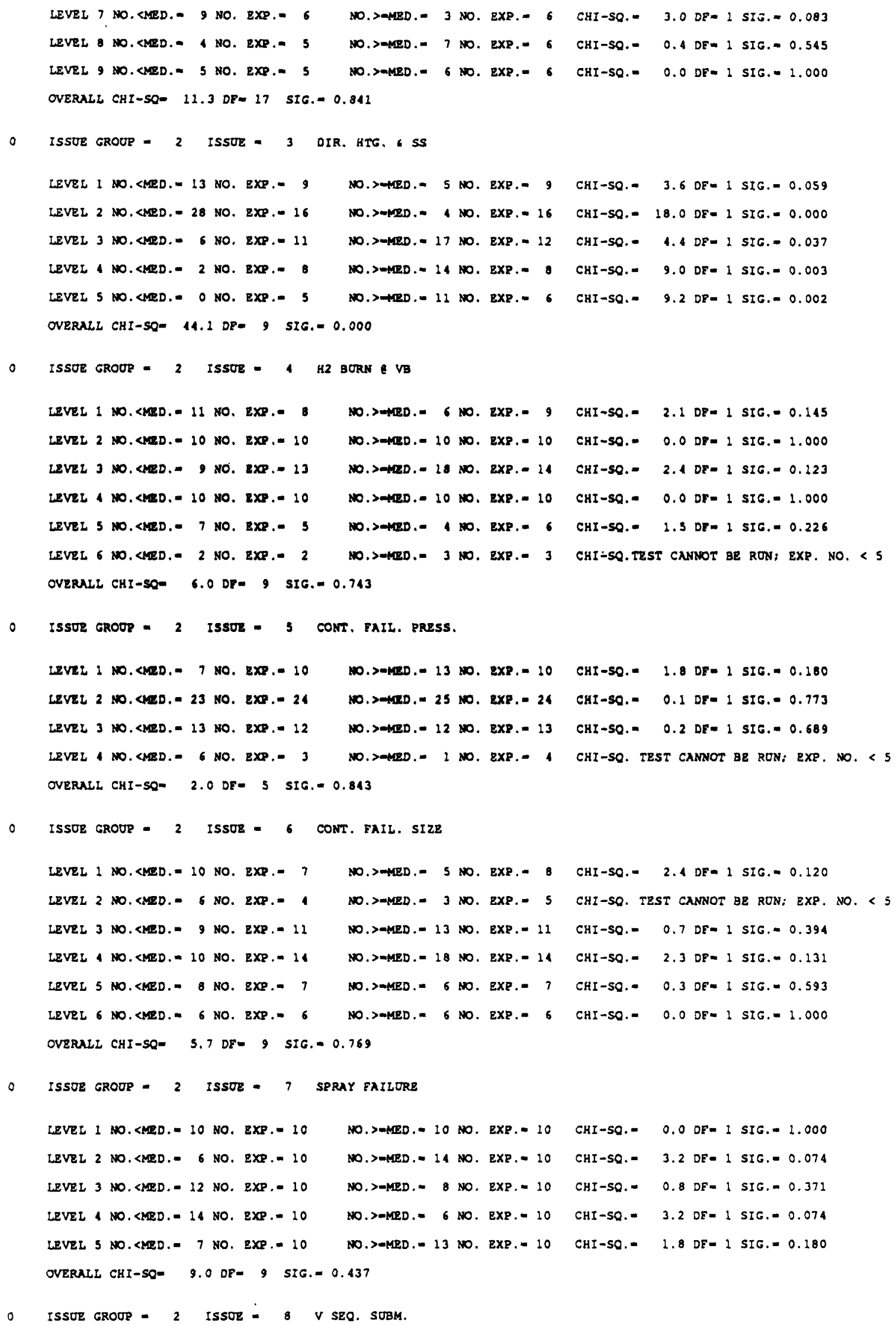

CHI-SO. 2.4 DF- 1 SIG. 0.120 CHI-SO. TEST CANNOT BE RON; EXP. NO. < 5 CHI-SO. $=0.7$ DF- 1 SIG. $=0.394$ CHI-SQ. $2.3 \mathrm{DF}=1$ SIG. $=0.131$ CHI-SO. $=0.3$ DFE I SIG. $=0.593$ CHI-SO. 0.0 DEW I SIG. $=1.000$

CHI-SQ.- 0.0 OFW 1 SIG.- 1.000

CHI-SO. - 3.2 DF- 1 SIG.= 0.074

$\mathrm{CHI}-\mathrm{SO}=0.8 \mathrm{DF}=1 \mathrm{SIG}=0.371$

CHI-SQ. $\quad 3.2$ DF= 1 SIG. $=0.074$

CHI-SO. $\quad 1.8 \mathrm{DF}=1$ SIG. $=0.180$ 


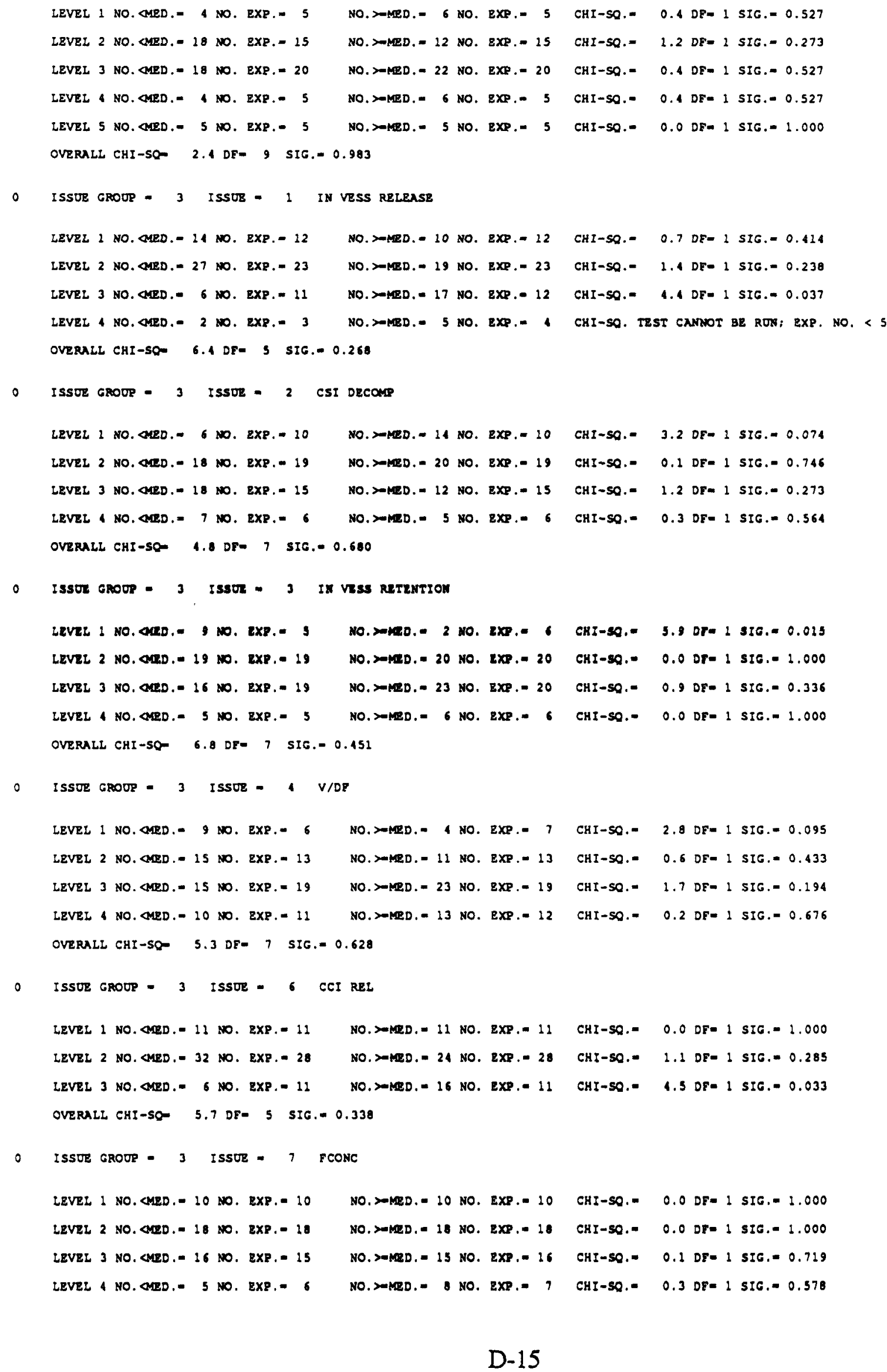

CHI-SO.= 0.4 DF 1 SIG. $=0.527$

$C H I-S Q=1.2 \mathrm{DF}=1$ SIG. $=0.273$

CHI-SQ.= 0.4 DF= 1 SIG.- 0.527

CHI-SO.- 0.4 DFE I SIG.- 0.527

CHI-SO.- 0.0 DF- 1 SIG.- 1.000

CHI-SQ. $\quad 0.7$ DF= 1 SIG. $=0.414$

CHI-SO.- 1.4 DF- 1 SIG. $=0.238$

CHI-SO.= 4.4 DF- 1 SIG.- 0.037

CHI-SO. TEST CANNOT BE RUN; RXP. NO. < 5

CHI-SO.= 3.2 OF- 1 SIG.=0.074

CHI $50 .=0.1$ DF= 1 SIG. $=0.746$

CHI-SO. = 1.2 DF $=1$ SIG. -0.273

CHI-SO.= 0.3 DF= 1 SIG. $=0.564$

CKI-50.= 5.9 Ora 1 sI6.=0.025 CHI-8Q.= 0.0 Dr- 1 SIG.- 1.000 CHI-SO.= 0.9 DF- I SIC. 0.336 CHI-SO.- 0.0 DF- 1 SIG.- 1.000

CHI-SO.= 2.8 DF= 1 SIG $=0.095$ CHI-SO.= 0.6 DF $=1$ SIG. $=0.433$ CHI-SO.= 1.7 DF- 1 SIG.=0.194 CHI-SO.= 0.2 DFE 1 SIG.= 0.676

CHI-SO.= 0.0 DF= 1 SIG.= 1.000 CH:-SO.= 1.1 OF- 1 SIG. $=0.285$ CHI-SO.= 4.5 DF= 1 SIG. $=0.033$

CHI-SO.= 0.0 DFE I SIG.= 1.000 CHI-SO.- 0.0 DF= 1 SIG.- 1.000 CHI-SO.= 0.1 DF- 1 SIG. $=0.719$ CHI-SO.= 0.3 DF= 1 SIG.=0.578 


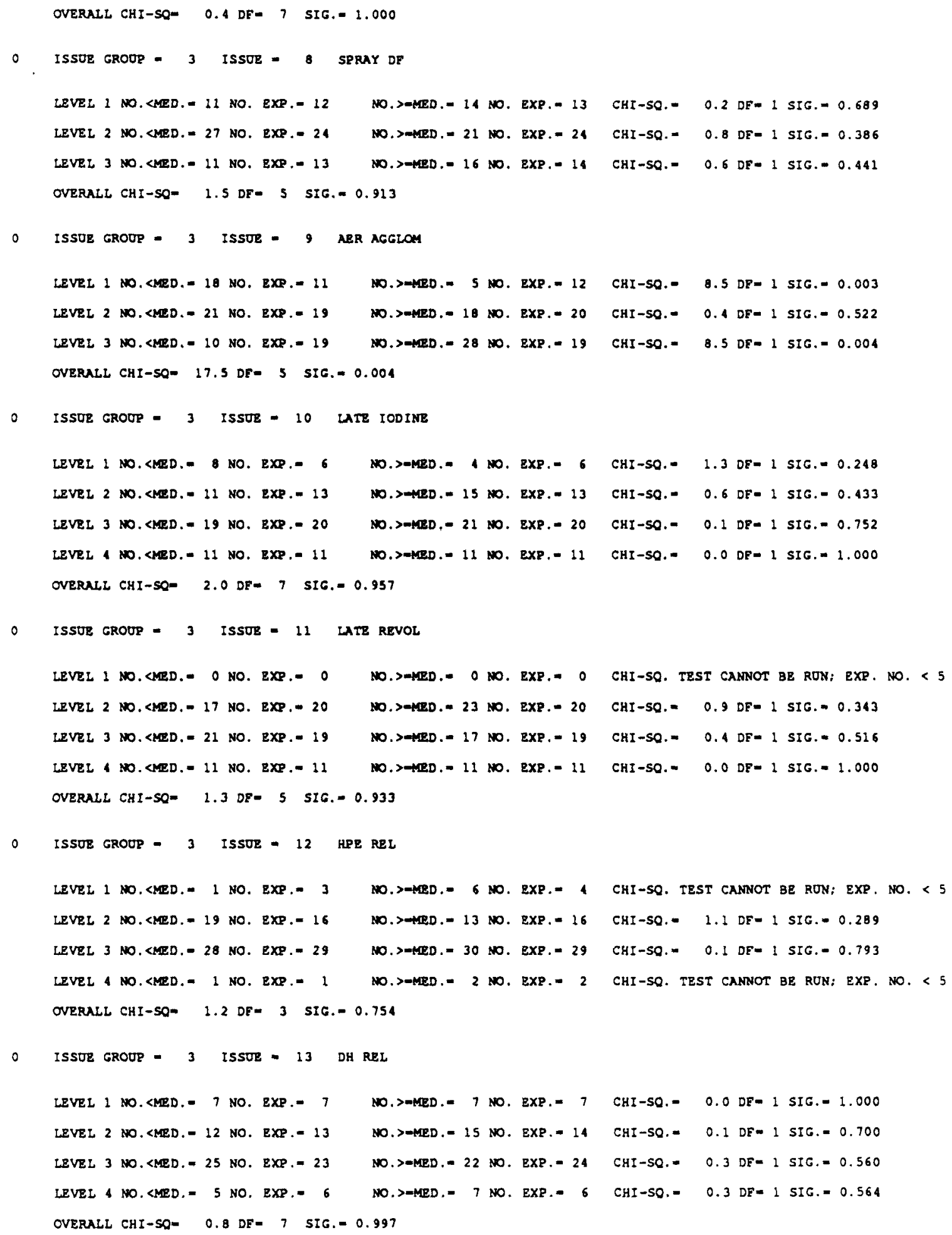

CHI-SQ. TEST CANNOT BE RUN; EXP. NO. < 5 CHI-SQ $=0.9$ DF $=1$ SIG. $=0.343$

CHI-SO. $=0.4$ DF $=1$ SIG. $=0.516$

CHI-50.- 0.0 DF $=1$ SIG. $=1.000$

CHI-SO. TEST CANNOT BE RUN; EXP, NO. < 5

CHI-SQ. = 1.1 DF= 1 SIG.- 0.289

CHI-SQ. 0.1 DF $=1$ SIG. -0.793

CHI-SO. TEST CANNOT BE RUN; EXP. NO. < 5 
NUREG/CR-4551, VOL. 1: DRAFT REPORT FOR COMMENT (SEPTEMBER, 1986)

CONSEOUENCE MEASORE;

BARLY ILINESS PER YEAR

BASE CASz

SORRY WALHC (CRAC)

SORTED RISK

ROBABILITY

$1.000 \mathrm{E}-02$

$1.000 \mathrm{~B}-02$

1. $000 \mathrm{~B}-02$

1. $000 \mathrm{E}-02$

1. $0008-02$

1. $000 \mathrm{E}-02$

$1.000 \mathrm{E}-02$

$1.000 \mathrm{z}-02$

$1.0008-02$

$1.000 \mathrm{E}-02$

1. $000 \mathrm{E}-02$

$1.000 \mathrm{z}-02$

$1.0008-02$

$1.000 \mathrm{z}-02$

$.0008-02$

$1.000 \mathrm{E}-02$

$.0008-02$

$1.0008-02$
$1.0008-02$

$1.0008-02$

$1.000 \mathrm{E}-02$

1. $0008-02$

$1.000 \mathrm{z}-02$

$1.000 \mathrm{z}-02$

$1.0008-02$

$1.0008-02$

$1.000 \mathrm{E}-02$

$1.000 \mathrm{E}-02$

$1.000 \mathrm{~B}-02$

$1.000 \mathrm{E}-02$

1. $000 \mathrm{E}-02$

1. 0008-02

1. $000 \mathrm{E}-02$

1. $0008-02$

1. $000 \mathrm{E}-02$

$1.0008-02$

$1.000 \mathrm{E}-02$

$1.000 \mathrm{E}-02$

1. $000 \mathrm{E}-02$

$1.000 \mathrm{~B}-02$

1. $000 \mathrm{E}-02$

$1.000 \mathrm{E}-02$

$1.000 \mathrm{E}-02$

$1.000 \mathrm{E}-02$

$1.000 \mathrm{E}-02$

$1.000 \mathrm{~B}-02$

1. $000 \mathrm{~B}-02$

1. $000 \mathrm{E}-02$

1. $000 \mathrm{E}-02$

$1.0008-02$

1. $0008-02$

$1.000 \mathrm{~B}-02$

$1.000 \mathrm{E}-02$

$1.000 \mathrm{E}-02$

1. $0008-02$

$1.0008-02$

$1.000 \mathrm{E}-02$

$1.0008-02$

$1.000 \mathrm{E}-02$

1. $000 \mathrm{E}-02$

$1.000 \mathrm{~B}-02$

1. $000 \mathrm{E}-02$

1. $000 \mathrm{~B}-02$

1. $0008-02$

$1.000 \mathrm{E}-02$

1. $000 \mathrm{E}-02$

$1.000 \mathrm{~B}-02$

1. $000 \mathrm{E}-02$

1. $000 \mathrm{E}-02$

$1.000 \mathrm{E}-02$

$1.0008-02$

$1.000 \mathrm{z}-02$

1. $000 \mathrm{E}-02$

$1.000 \mathrm{E}-02$

1. $000 \mathrm{E}-02$

1. $000 \mathrm{E}-02$

$1.000 \mathrm{E}-02$

$1.000 \mathrm{E}-02$

$1.000 \mathrm{E}-02$

$1.000 \mathrm{E}-02$

$1.000 \mathrm{E}-02$

$1.000 \mathrm{E}-02$

$1.0008-02$

CUM. PROB.

5. $000 \mathrm{E}-03$

$1.500 \mathrm{E}-02$

2.5008-02

3. $500 \mathrm{E}-02$

4. 500E-02

5. 50OE-02

$6.500 \mathrm{E}-02$

$7.5008-02$

8. 500E-02

$9.5008-02$

$1.0508-01$

1. 1508-01

1. $250 \mathrm{E}-01$

$1.350 \mathrm{E}-01$

$1.450 \mathrm{R}-01$

$1.550 \mathrm{E}-01$

1. $650 \mathrm{E}-01$

$1.7508-01$

$1.850 \mathrm{E}-01$

1. 9508-01

2. 0508-01

2.1508-01

$2.250 \mathrm{~B}-01$

2.3508-01

2. 4508-01

2. 5508-01

2. $650 \mathrm{E}-01$

2.730E-01

2. .50R-01

2. $950 \mathrm{E}-01$

3. $050 \mathrm{E}-01$

3.1508-01

3.2508-01

3. $350 \mathrm{~B}-01$

$3.450 \mathrm{~B}-01$

$3.550 \mathrm{~B}-01$

3. 650R-01

3. $7508=01$

$3.8508-01$

3. $950 \mathrm{~B}-01$

1.0508-01

$4.150 \mathrm{E}-01$

1.250E-01

1. 3508-01

4. $450 \mathrm{E}-01$

1.5508-01

1. 6508-01

1.750z-01

4.8508-01

$4.950 \mathrm{E}-01$

5. 050B-01

5. $150 \mathrm{E}-01$

5. $2508-01$

5. $350 \mathrm{E}-01$

$5.450 \mathrm{E}-01$

5. 550E-01

$5.650 \mathrm{E}-01$

$5.750 \mathrm{E}-01$

$5.850 \mathrm{E}-01$

$5.950 \mathrm{E}-01$

6.0508-01

6.150R-01

6. $250 \mathrm{E}-01$

$6.350 \mathrm{z}-0$.

$6.450 \mathrm{~B}-01$

6. $550 \mathrm{E}-01$

6. $650 \mathrm{~B}-01$

6. $750 \mathrm{~B}-01$

$6.950 z-01$

6.9508-01

$7,050 \mathrm{~B}-01$

$7.150 \mathrm{~B}-01$

7. 250E-01

$7.3308-01$

$7.4508-01$

$7.550 \mathrm{~B}-01$

$7.650 \mathrm{~B}-01$

7. $750 \mathrm{~B}-01$

$7.850 \mathrm{E}-01$

$7.950 \mathrm{E}-01$

8. 0508-01

8. $1508-01$

ISK

1. $799 \mathrm{E}-07$

$7.657 \mathrm{e}-07$

9. $302 \mathrm{E}-07$

2. 243E-06

$2.523 \mathrm{E}-06$

2. $9718-06$

3. $288 \mathrm{Be}-06$

3. $512 \mathrm{E}-06$

3. $7628-06$

5. $6688-06$

6. 1518-06

6. $9818-06$

$7.862 \mathrm{E}-06$

-. 043E-06

8. $3308-06$

$1.0068-05$

1.088E-05

1. $301 \mathrm{E}-0 \mathrm{~S}$

1. $313 \mathrm{~B}-05$

1. $3208-05$

. $5958-05$

1. 5998-05

$1.650 \mathrm{~B}-05$

$1.6778-05$
$1.7228-05$

1. $751 \mathrm{E}-05$

1. $8008-05$

1. $917 \mathrm{E}-05$

1. 8338-05

$1.807 \mathrm{E}-05$

$2.0408-05$

$2.1388-05$

$3.123 \mathrm{E}-0.5$

3. $2108-05$

3. $942 \mathrm{E}-0 \mathrm{~S}$

1. 238E-05

4.2408-05

1. $946 \mathrm{E}-05$

5. 345E-0S

5. $410 \mathrm{OB}-05$

. $4108-05$

5. $7568-05$

6. 458z-05

6. $536 \mathrm{E}-05$

6. 940E-05

6. $967 \mathrm{E}-05$

7.1288-05

8. $022 \mathrm{E}-05$

0. $3458-05$

$9.823 \mathrm{E}-05$

1. $242 \mathrm{E}-04$

1. $3638-04$

$1.3818-04$

$1.120 \mathrm{E}-04$

$1.642 \mathrm{E}-04$

$1.671 \mathrm{E}-04$

$2.060 \mathrm{E}-04$

$2.369 \mathrm{E}-04$

$2.635 z-04$

$2.808 \mathrm{E}-04$

3. $2768-04$

$3.466 \mathrm{E}-04$

3. $908 \mathrm{E}-04$

$3.977 \mathrm{~B}-04$

$4.038 \mathrm{E}-04$

4. $1212-04$

$4.936 \mathrm{z}-04$

5. $3318-04$

5. $659 \mathrm{E}-04$

5. $996 \mathrm{~B}-04$

$6.5258-04$

$7.167 \mathrm{E}-04$

7. $332 \mathrm{E}-04$

8. $3028-04$

9. $608 \mathrm{~B}-04$

8. 8308-04

$9.257 \mathrm{~B}-04$

$9.387 \mathrm{~B}-04$

$9.7618-04$

$1.1678-03$

$1.181 \mathrm{E}-03$ 
NUREG/CR-4551, VOL. 1: DRAFT REPORT FOR COMMENT (SEPTEMBER, 1986)

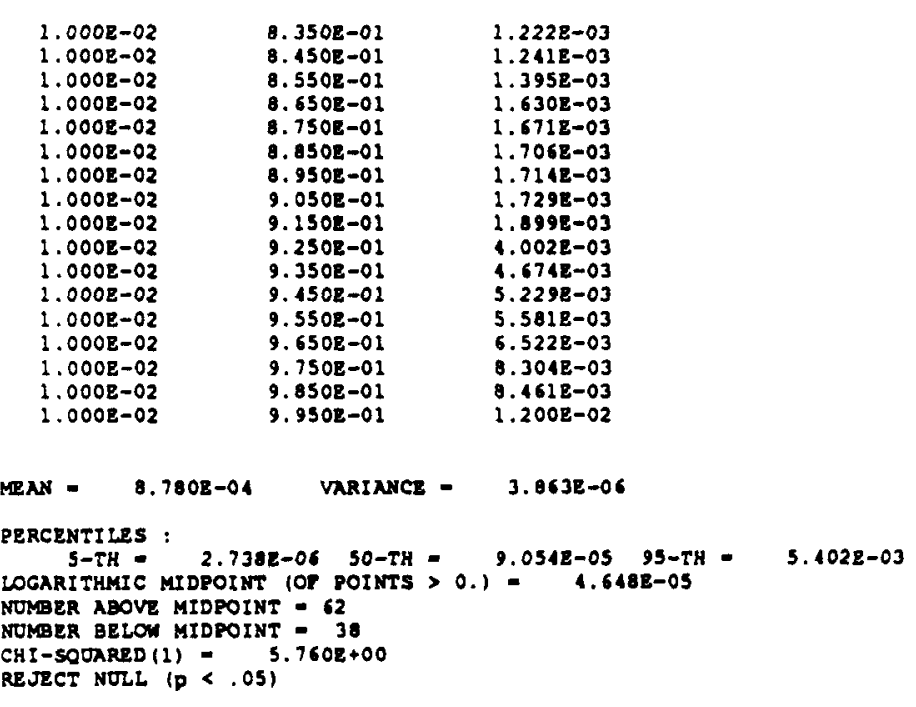


CONSEQJENCE MEASURE

EARLY ILLNESS PER YEAR

BASE CASE

SURRY LALHC (CRAC)

ISSUE LEVELS FOR EACH SAMPLE MEMBR, IN ORDER OF INCREASING RISR

RIST ISSOE LEVELS, FOR ALL ISSUES

\begin{tabular}{|c|c|c|c|c|c|c|c|c|c|c|c|c|c|c|c|c|c|}
\hline & & & & & & & & & & & & & & & & & \\
\hline $1.799 \mathrm{~g}-07$ & 2 & 1 & 3 & 2 & 1 & i & 3 & 1 & 2 & 2 & 6 & 5 & 2 & 1 & 2 & 1 & 2 \\
\hline $.657 \mathrm{E}-07$ & 2 & 1 & 2 & 2 & 3 & 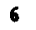 & 1 & 1 & 5 & 3 & 2 & 1 & 2 & 2 & 2 & 1 & 3 \\
\hline $.3028-07$ & 2 & 1 & 2 & 1 & 3 & 6 & 2 & 2 & 1 & 1 & 5 & 4 & 1 & 1 & 3 & 2 & 2 \\
\hline .06 & 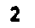 & 1 & 2 & 2 & 2 & 2 & & 2 & 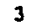 & $i$ & 5 & 2 & 4 & 2 & 3 & 1 & 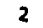 \\
\hline $.523 \mathrm{z}-06$ & 2 & 2 & 3 & 1 & 1 & 1 & a & 2 & 4 & 4 & 1 & $\mathbf{5}$ & 2 & 2 & 3 & 3 & $i$ \\
\hline$E-06$ & 2 & 1 & 2 & 1 & 2 & 0 & 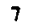 & 2 & 2 & 3 & 1 & 1 & 3 & 1 & 3 & 2 & 1 \\
\hline 06 & 2 & 1 & 2 & 2 & 3 & 2 & 9 & 2 & 1 & 2 & 4 & 4 & 3 & 1 & 1 & 3 & 4 \\
\hline $.5128-06$ & 2 & 2 & 2 & 1 & 2 & 3 & 9 & & 4 & 1 & 5 & 4 & 2 & 1 & 3 & 2 & 4 \\
\hline-06 & 2 & 1. & 3 & 2 & 2 & 6 & 5 & & 3 & 2 & 1 & 3 & 5 & 2 & 2 & 2 & 4 \\
\hline $.4688-06$ & 2 & 2 & 3 & 3 & 1 & 6 & 1 & & 5 & 2 & 5 & 3 & 2 & 1 & 4 & 3 & 3 \\
\hline .1528 & 2 & 2 & 2 & 1 & 1 & 6 & 1 & 2 & 3 & $?$ & 3 & 3 & 3 & 2 & 3 & 2 & \\
\hline . 981z-06 & 1 & 1 & 3 & 2 & 1 & 2 & 6 & 1 & 3 & 3 & 2 & 1 & 3 & 1 & 2 & 3 & 4 \\
\hline $\begin{array}{l}.8628-06 \\
.043 E-06\end{array}$ & $\begin{array}{l}2 \\
2\end{array}$ & $\begin{array}{l}2 \\
1\end{array}$ & 2 & & $\frac{1}{2}$ & & 7 & & $\begin{array}{l}5 \\
2\end{array}$ & ? & $\begin{array}{l}2 \\
3\end{array}$ & 3 & $\frac{1}{2}$ & 2 & & 2 & \\
\hline $.3308-06$ & 2 & 1 & 3 & 2 & 3 & 3 & 9 & 2 & 3 & $i$ & 4 & 3 & 3 & 3 & 2 & 2 & 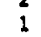 \\
\hline 05 & 2 & 2 & 2 & 2 & 3 & 2 & 2 & 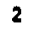 & 1 & 2 & $i$ & 3 & 3 & 3 & 2 & 1 & 3 \\
\hline-05 & 2 & 2 & 2 & 2 & 1 & 6 & 2 & 2 & 1 & . & 4 & 3 & 4 & 2 & 3 & 2 & \\
\hline 05 & 2 & & 3 & 1 & 1 & 3 & 1 & & 2 & 2 & 2 & 1 & 3 & 4 & 3 & 1 & \\
\hline 9 & 2 & 1 & 2 & 1 & 2 & 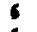 & 6 & 2 & 2 & 3 & 3 & 4 & 3 & 2 & 3 & 2 & 4 \\
\hline 3 & 2 & 1 & $\frac{2}{3}$ & & $\begin{array}{l}3 \\
2\end{array}$ & 6 & 2 & 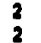 & ! & 3 & 4 & 2 & 3 & 1 & 3 & $!$ & 4 \\
\hline 59 & 2 & 2 & 2 & 2 & 2 & 3 & 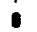 & 2 & 6 & & ? & 2 & 3 & 2 & 3 & 2 & \\
\hline .05 & 2 & 2 & 2 & 3 & 3 & 6 & 9 & $j$ & 4 & & 6 & 1 & 2 & 2 & 3 & $\overrightarrow{1}$ & 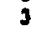 \\
\hline 09 & 2 & 2 & 3 & 2 & 3 & 4 & 4 & 2 & 5 & 2 & 3 & 2 & 3 & 1 & 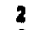 & 2 & \\
\hline .7 & 2 & & $\begin{array}{l}2 \\
2\end{array}$ & $\frac{1}{1}$ & $\frac{1}{2}$ & 3 & $\begin{array}{l}2 \\
5\end{array}$ & 1 & 6 & & 3 & 4 & 3 & 1 & & 1 & \\
\hline $0 S$ & 2 & 3 & $i$ & 3 & 2 & $i$ & 7 & 2 & 5 & 4 & 3 & 4 & 3 & 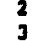 & 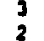 & 4 & \\
\hline 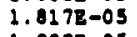 & 2 & 2 & 2 & 2 & 2 & 2 & 7 & 2 & 1 & 2 & 4 & 3 & 4 & 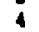 & 2 & 2 & \\
\hline 5 & 2 & 1 & 3 & 2 & 2 & 2 & 5 & 2 & 2 & 4 & 1 & 5 & 2 & 3 & 4 & 3 & \\
\hline .8 & 2 & 2 & 2 & 1 & 1 & 5 & 5 & 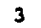 & 1 & 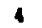 & 1 & 2 & 2 & 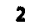 & 3 & 3 & \\
\hline .0 & 2 & 1 & 3 & 1 & 3 & 6 & 5 & 3 & 1 & 3 & 3 & 1 & 2 & & 2 & 2 & \\
\hline 5 & 2 & 2 & 2 & 1 & 2 & 3 & 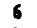 & 4 & 1 & 3 & 4 & 5 & 2 & & 3 & 2 & \\
\hline-05 & 2 & 1 & 2 & 2 & 2 & 5 & 1 & 1 & 2 & 2 & 6 & 5 & 1 & & 1 & 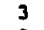 & \\
\hline .1 & 2 & 2 & 3 & 2 & 1 & 5 & 3 & 2 & 1 & 2 & 5 & 5 & 2 & & 4 & 3 & \\
\hline 2 & 2 & 1 & ? & 2 & 3 & 2 & 1 & 3 & 4 & 1 & 3 & 1 & & & & 3 & \\
\hline-05 & 2 & 2 & 2 & 2 & 3 & 2 & & 2 & 3 & & 4 & 3 & & & 4 & 4 & \\
\hline 4.23 & 2 & 2 & 2 & 2 & 3 & 3 & 7 & 1 & 1 & & 4 & 1 & 3 & & 0 & 3 & \\
\hline 4.2 & 3 & 1 & 3 & 1 & 1 & 1 & 8 & 2 & 4 & & 1 & 3 & 3 & & & 3 & \\
\hline-05 & 2 & 2 & 3 & 2 & 2 & 3 & 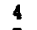 & 1 & 3 & 4 & 1 & 2 & 2 & & 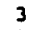 & 2 & \\
\hline .3 & 2 & 2 & 2 & 3 & & 6 & 7 & 2 & 3 & 1 & 6 & 4 & 3 & & 1 & 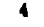 & \\
\hline .4 & 1 & 2 & 2 & 2 & 3 & 2 & 1 & 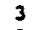 & 1 & , & 3 & 4 & 2 & & , & 1 & \\
\hline 05 & 2 & 1 & 2 & 2 & 3 & 6 & 2 & 2 & 4 & 2 & 5 & 4 & 4 & & 1 & 3 & \\
\hline .75 & 2 & 2 & 2 & 1 & 2 & 2 & 2 & 3 & 2 & 3 & 1 & 5 & 3 & & 2 & 3 & \\
\hline 6.45 & 2 & 2 & 2 & 2 & 3 & 1 & 5 & 1 & 3 & & 2 & 3 & & & $d$ & 4 & \\
\hline-05 & & 2 & 3 & 1 & & 2 & & 1 & 1 & 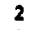 & 5 & 1 & 3 & & 4 & 3 & 3 \\
\hline 6.94 & 2 & 2 & 2 & 2 & 3 & 1 & 7 & 2 & 4 & 1 & 6 & 4 & 3 & & 4 & 2 & 3 \\
\hline 1.9 & 2 & 1 & 2 & 2 & 2 & 2 & 2 & $\mathbf{2}$ & 3 & 2 & 4 & 3 & 2 & & 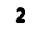 & 2 & 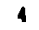 \\
\hline 3 & $z$ & 1 & 3 & 1 & 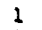 & 3 & & 1 & 1 & & 5 & 4 & 4 & 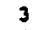 & 2 & 3 & 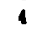 \\
\hline-05 & 2 & 1 & 3 & 2 & 1 & 3 & 8 & 3 & 1 & 2 & 4 & 1 & 2 & 4 & 3 & 2 & 3 \\
\hline .3 & 3 & 1 & 2 & 2 & 3 & 6 & 6 & 1 & 3 & 4 & 1 & 3 & 5 & 2 & 2 & 2 & $s$ \\
\hline .8 & 2 & 1 & 2 & 1 & 2 & 3 & 9 & 7 & 2 & 2 & 5 & 4 & 2 & 3 & 2 & 3 & $i$ \\
\hline 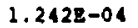 & & 2 & 3 & 2 & & 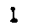 & & 4 & 4 & 7 & 1 & 1 & 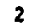 & 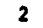 & 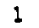 & 2 & 3 \\
\hline-04 & 2 & 2 & 3 & 3 & 1 & 1 & 5 & 1 & 2 & 2 & 4 & 2 & 2 & & 4 & 4 & 3 \\
\hline 1.38 & & 1 & 2 & 2 & 3 & 1 & 2 & 2 & 4 & , & 6 & 2 & 3 & & 4 & 3 & \\
\hline-01 & 2 & 2 & 2 & 1 & 3 & 1 & & 1 & 4 & 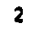 & 3 & 5 & 2 & & 2 & 2 & \\
\hline 1.6 & 2 & 2 & 2 & 2 & 2 & 6 & 9 & 1 & 4 & 3 & 1 & 4 & 3 & 3 & 3 & 4 & 3 \\
\hline 1.6 & 2 & 2 & 2 & 1 & 3 & 6 & 5 & 2 & 3 & 2 & 5 & 3 & 5 & & 2 & 3 & \\
\hline 2.06 & & 1 & 2 & 2 & 3 & 3 & 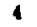 & 2 & 3 & ? & 5 & 2 & 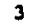 & 3 & 2 & 3 & \\
\hline .3691 & & 1 & & 1 & & 2 & 2 & 3 & 4 & 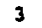 & 1 & 5 & 3 & 2 & 4 & 2 & 2 \\
\hline 04 & 3 & 2 & 2 & 2 & 1 & 3 & 9 & 3 & 3 & & 3 & 5 & 1 & & 1 & 3 & \\
\hline 04 & 2 & 1 & 1 & 3 & 1 & 6 & 3 & 3 & 3 & 2 & 4 & J & 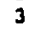 & 3 & 2 & 2 & 3 \\
\hline .27 & 9 & 2 & $?$ & 2 & , & 3 & 7 & 3 & 4 & 3 & 3 & 2 & 1 & 2 & 1 & , & 2 \\
\hline 3. & 2 & 2 & 2 & 3 & 2 & 5 & 3 & 4 & 2 & 2 & 4 & 4 & 3 & 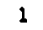 & 1 & 3 & 3 \\
\hline & 2 & 2 & 2 & 2 & 3 & 4 & 2 & 3 & 3 & 2 & 3 & 2 & 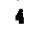 & & 3 & 3 & \\
\hline 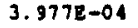 & 2 & 1 & 3 & 1 & 3 & 6 & 6 & 4 & 1 & & 1 & & & & 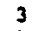 & 3 & 3 \\
\hline-04 & 2 & 1 & 3 & 2 & & 6 & & 5 & 1 & 3 & 4 & 2 & 3 & 2 & 1 & 3 & 2 \\
\hline 1.1 & 2 & 1 & 3 & 1 & 3 & 6 & 8 & 4 & 1 & 3 & 2 & 3 & 2 & & 1 & 2 & \\
\hline 4.93 & 2 & 2 & 3 & 1 & 1 & 6 & 8 & 3 & 4 & 3 & 3 & 2 & 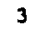 & & 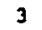 & 3 & \\
\hline 5. & 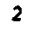 & 2 & 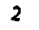 & 2 & 3 & , & 4 & & 6 & 1 & 6 & 2 & & & 2 & 2 & \\
\hline .65 & 2 & 2 & 3 & 3 & 2 & 2 & 9 & 3 & 1 & 2 & 3 & 1 & 3 & 2 & 1 & 3 & 2 \\
\hline & 2 & 1 & 2 & 2 & 2 & 6 & 4 & 3 & 3 & 2 & 5 & 1 & , & & 1 & 2 & \\
\hline & 2 & 2 & 2 & 1 & 3 & 2 & 5 & " & 3 & 2 & 4 & 2 & 2 & & 2 & 1 & \\
\hline 7.1 & , & 1 & 2 & 2 & & 6 & 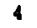 & 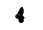 & 4 & , & 1 & 3 & , & & 2 & 3 & 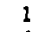 \\
\hline 7. & 2 & 2 & 3 & 3 & 2 & 1 & 7 & 3 & 3 & 2 & 4 & 2 & 3 & & & 2 & 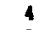 \\
\hline .04 & 2 & 2 & 3 & 1 & 1 & 1 & 1 & & 3 & 1 & 4 & 2 & 5 & & 2 & 2 & 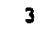 \\
\hline 8.60 & 2 & 1 & 2 & 1 & 2 & 3 & 9 & & 3 & & 2 & & 5 & 3 & 2 & 3 & 3 \\
\hline $8.830 \mathrm{E}-04$ & 2 & 1 & 2 & 1 & 1 & 6 & 2 & 4 & 2 & 3 & 3 & 1 & 3 & 1 & 3 & 2 & A \\
\hline 9.2 & & 1 & & 1 & 3 & 6 & 9 & 3 & 5 & 1 & 5 & 3 & 1 & & 2 & 2 & 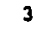 \\
\hline $72-04$ & 2 & 1 & 2 & 2 & 1 & 5 & 7 & & 2 & 1 & 4 & 5 & 3 & & 2 & 3 & 3 \\
\hline $61 \mathrm{z}-04$ & 1 & 1 & 3 & 1 & 3 & 3 & 0 & 5 & 3 & 3 & 3 & 3 & 4 & 3 & 2 & 2 & 3 \\
\hline-0.3 & 2 & 1 & 2 & 2 & 1 & 1 & 3 & 4 & 2 & 2 & 3 & 5 & 3 & 4 & 2 & 2 & 4 \\
\hline-03 & 2 & 2 & 2 & 1 & 2 & 5 & 1 & 5 & 5 & 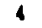 & 1 & 1 & 3 & & 2 & 2 & 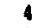 \\
\hline-03 & & 1 & 2 & 2 & & & & & 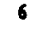 & & 6 & & 3 & & & & \\
\hline
\end{tabular}


NUREG/CR-4551, VOL. 1: DRAFT REPORT FOR COMMENT (SEPTEMBER, 1986)

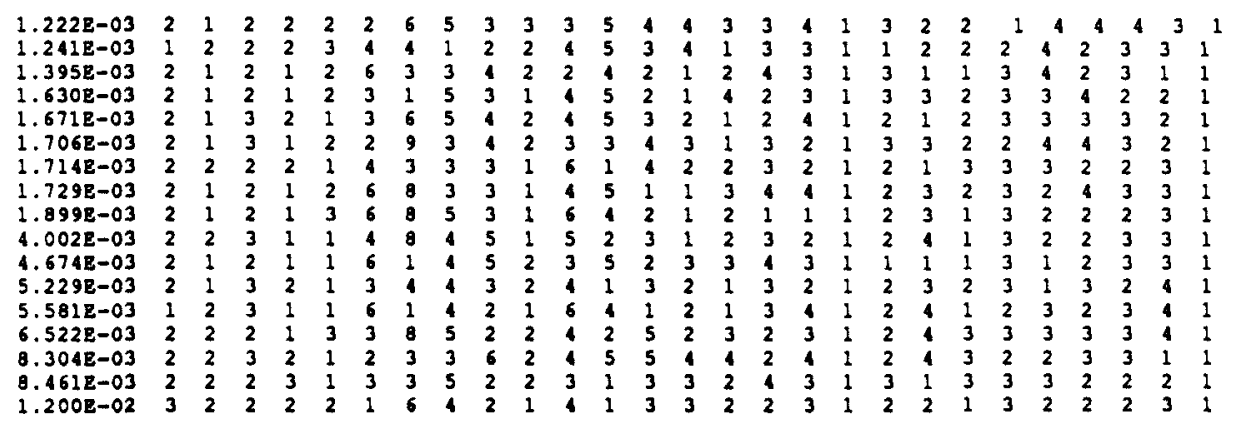




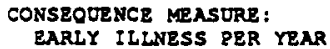

0.0000
0.0000

0.0000
0.0000

0.0000 
NUREG/CR-4551, VOL. 1: DRAFT REPORT FOR COMMENT (SEPTEMBER, 1986)

CONSEQUENCE MEASURE:

EARLY ILLNESS PER YEAR

SENSITIVITY OF RISK TO ISSUES

DISTRIBUTION OF RISK AT RACH LEVEL OF EACH ISSOE

BASE CASE

SORRY LALHC (CRAC)

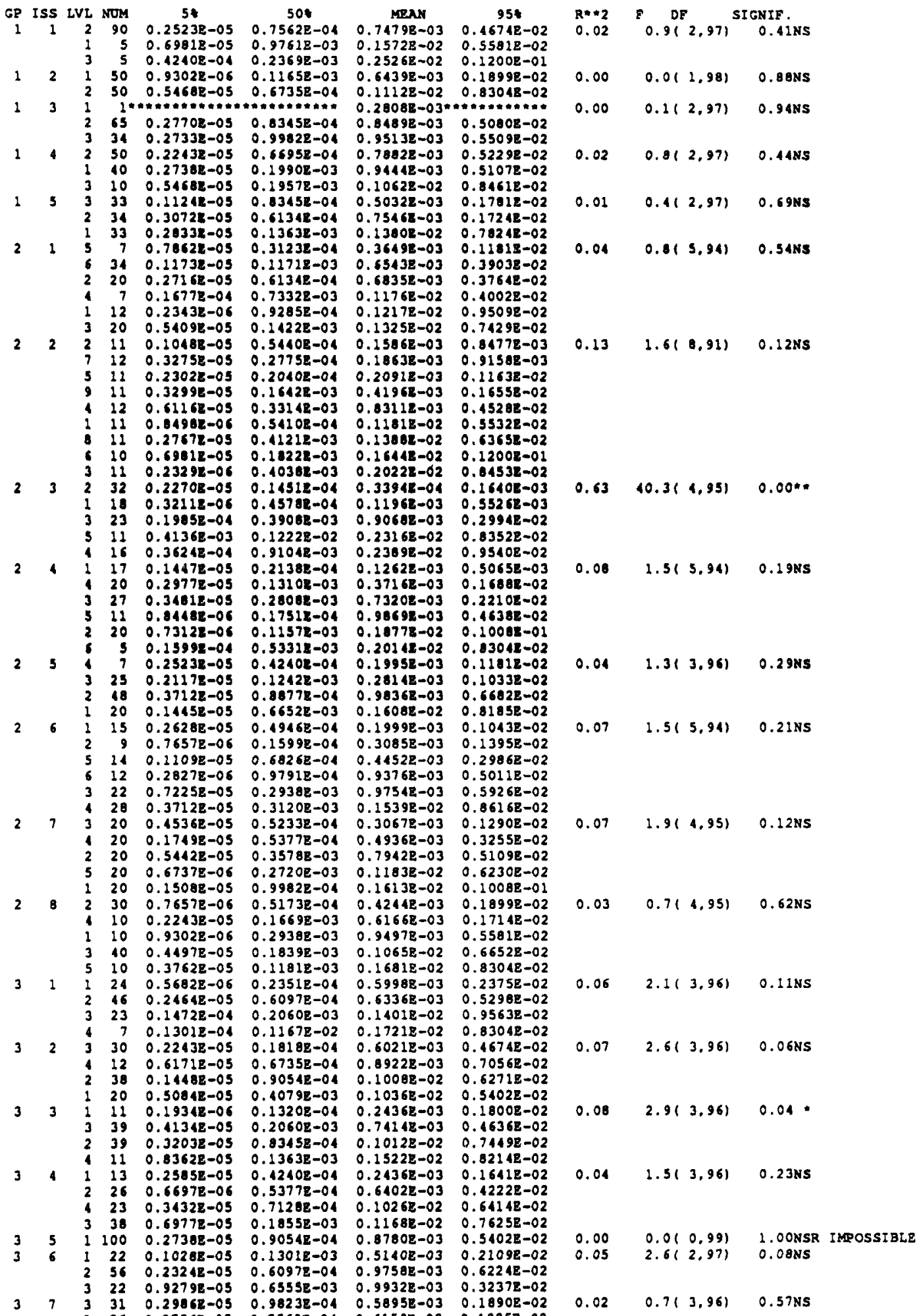


NUREG/CR-4551, VOL. 1: DRAFT REPORT FOR COMMENT (SEPTEMBER, 1986)

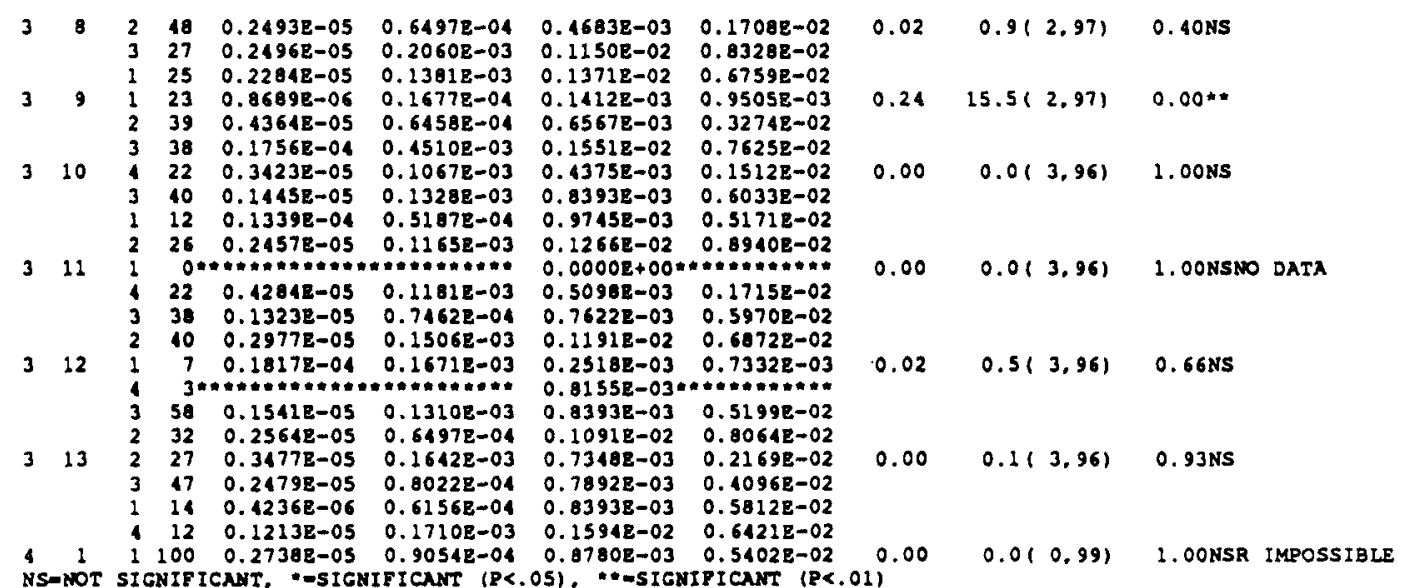


NUREG/CR-4551, VOL. 1: DRAFT REPORT FOR COMMENT (SEPTEMBER, 1986)

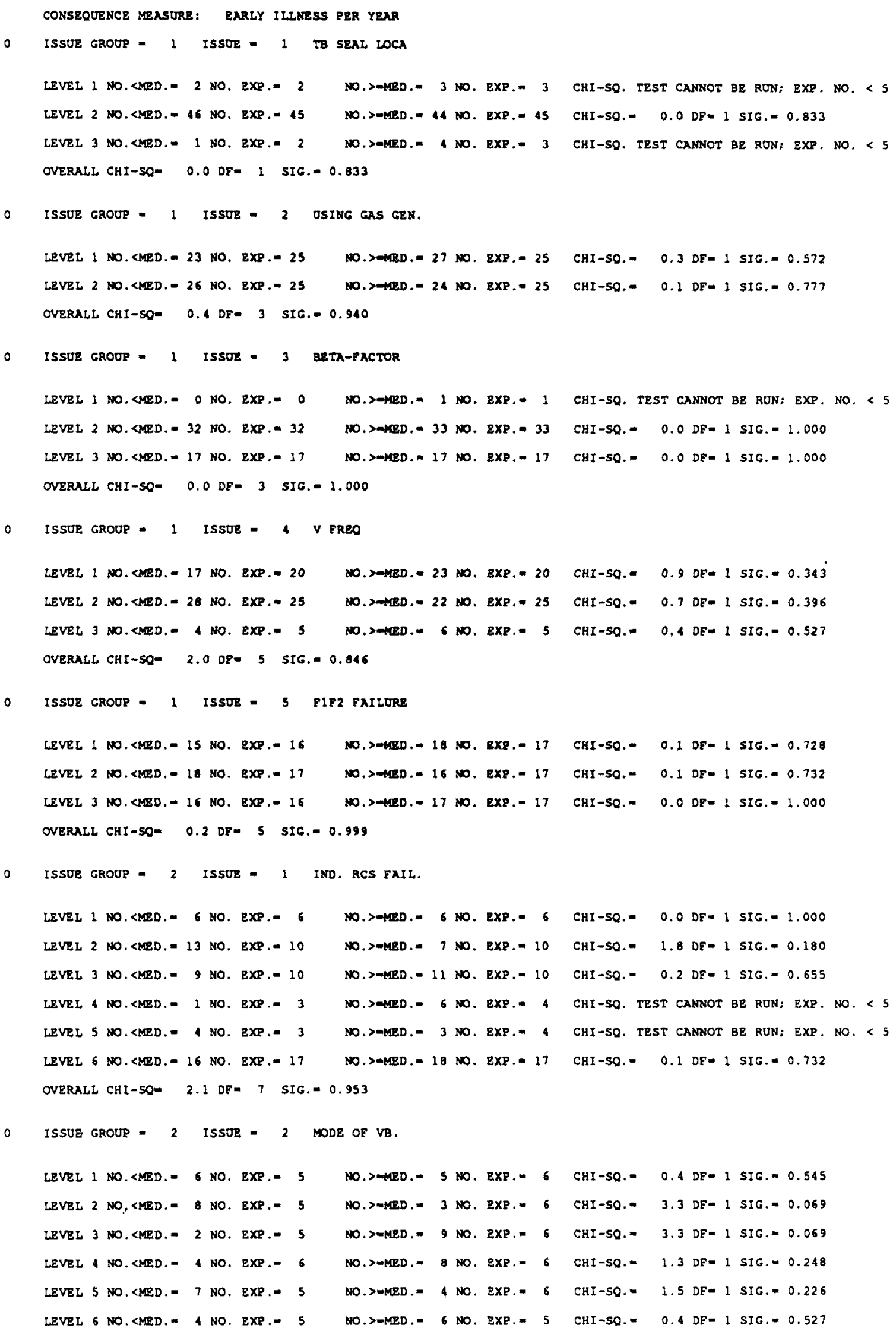




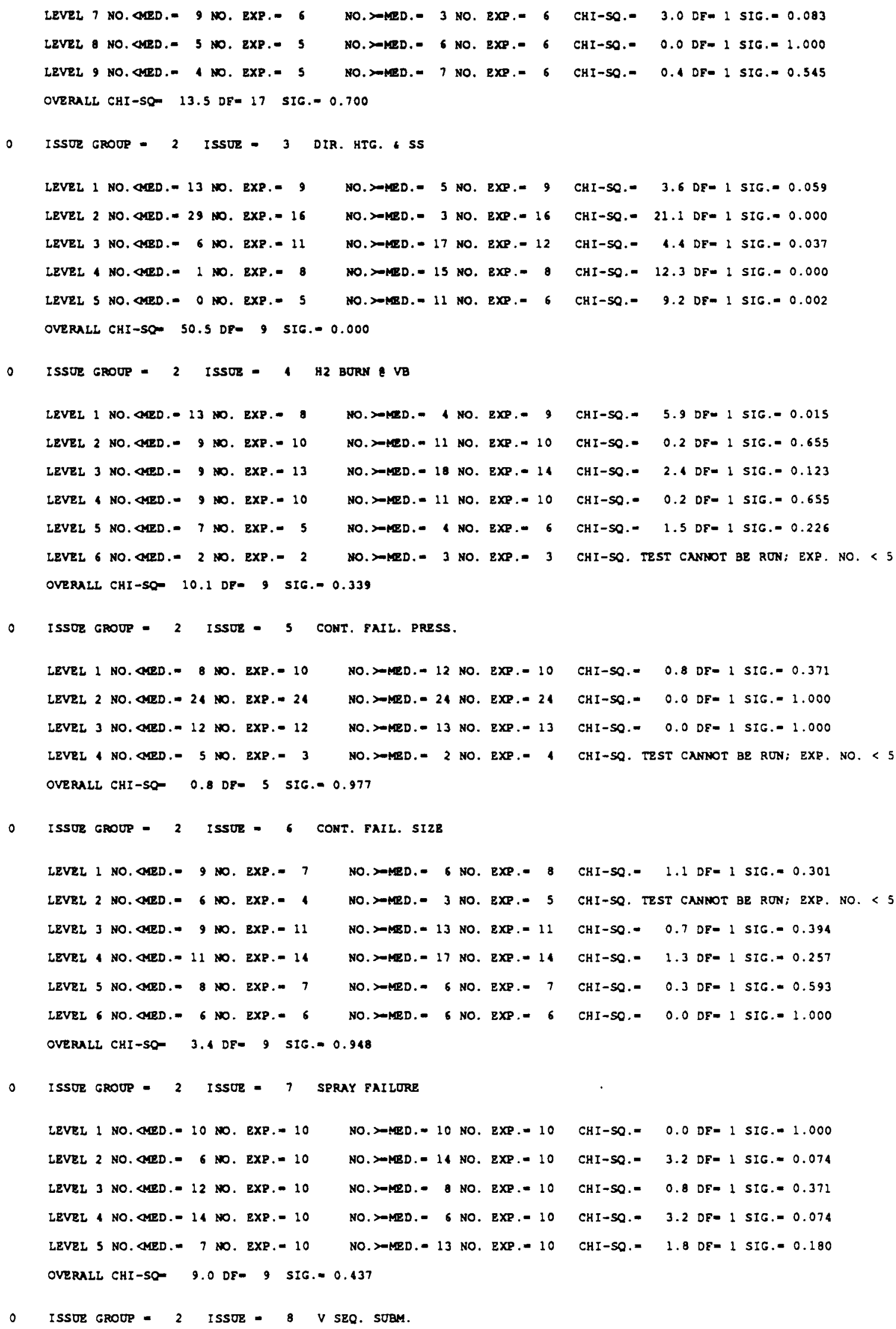

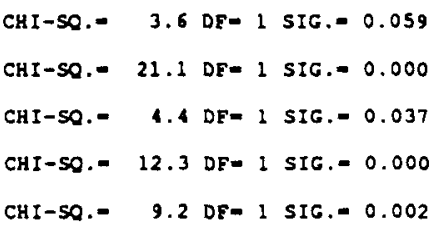

CHI-SO.- 5.9 DF 1 5IG. $=0.015$ CHI-SO.= 0.2 DF- 1 SIG.- 0.655 CHI-SO.- 2.1 DF- 1 SIG.- 0.123

CHI-SO.- 0.2 DF- 1 SIG. $=0.655$

CHI-SO. 1.5 DF= 1 SIC. -0.226 CHI-SO. TEST CANHOT BE RON; EXP. NO. < 5

CHI-SO.= 0.8 OF- 1 SIG. $=0.371$

CHI-SQ.- 0.0 DF= 1 SIG.= 1.000

CHI-SO.- 0.0 DF= 1 SIG. 1.000

CHI-SQ. TEST CNNNOT BE RUN; EXP. NO. < 5

CHI-SQ.- 1.1 DF= 1 SIG. $=0.301$ CHI-SQ. TEST CANHOT BE RON; EXP. NO. < 5

CHI-SO.- 0.7 DF- I SIG.- 0.394

CHI-\$Q. 1.3 DF 1 SIG. $=0.257$

CHI-SQ.- 0.3 DF= 1 SIG. $=0.593$

CHI-SO.= 0.0 DF 1 SIG. $=1.000$

CHI-SO.= 0.0 DF= 1 SIG. 1.000

CHI-SQ.- $\quad 3.2$ DF= 1 SIG. $=0.074$

CHI-SO.= 0.8 DFF 1 SIG. $=0.371$

CHI-SO.= 3.2 DF= I SIG.= 0.074

CHI-SO. 1.8 DE $=1$ SIG $=0.180$ 


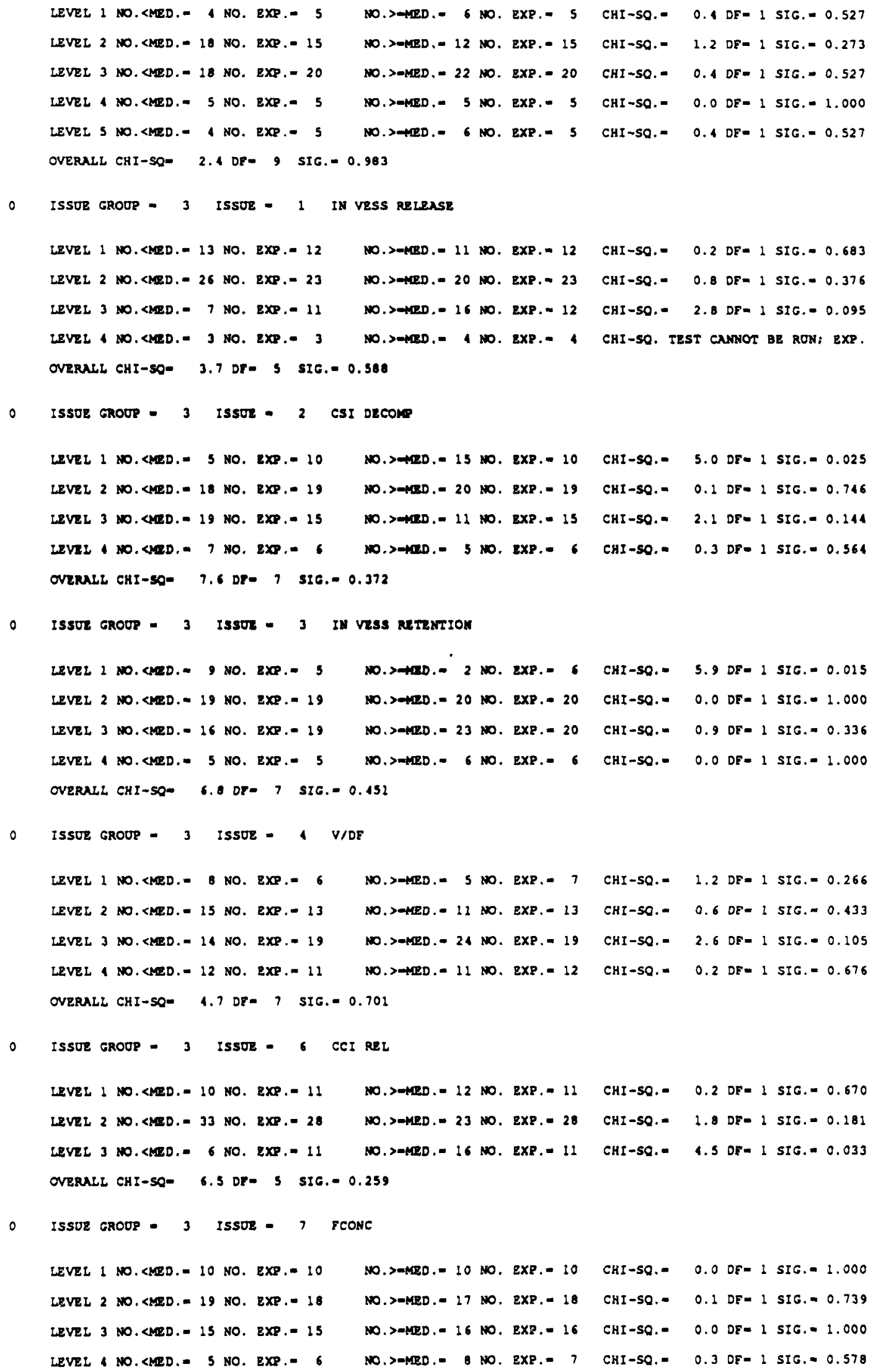




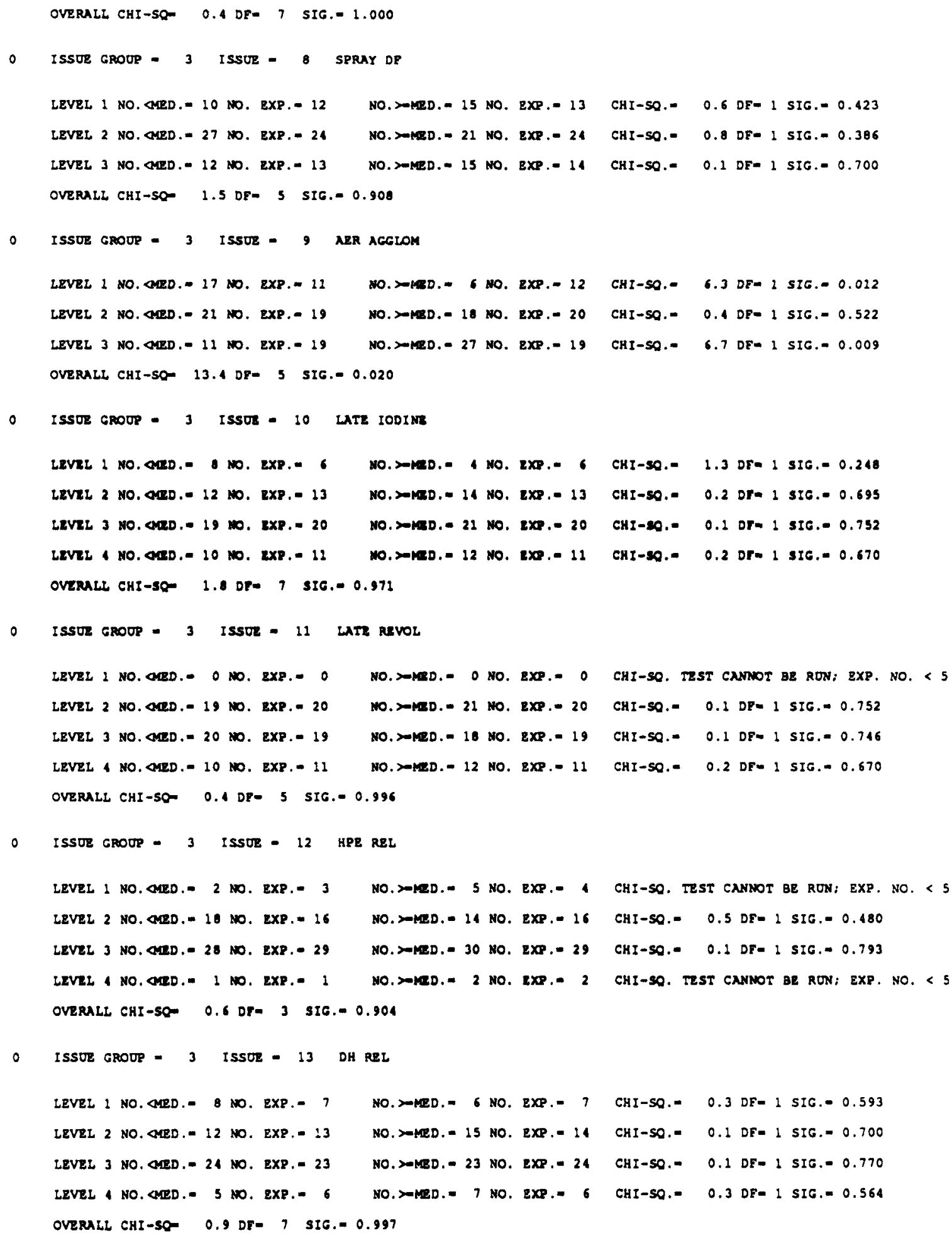


NUREG/CR-4551, VOL. 1: DRAFT REPORT FOR COMMENT (SEPTEMBER, 1986)

CONSEOUENCE MEASORE:

IND. RISK OF DEATH

EAsE CASE :

SORRY LALHC (CRAC)

SORTED RISK

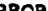

$1.000 \mathrm{~B}-0.25$

$1.0008-02$

$1.0008-02$

$1.000 \mathrm{E}-02$

1. $000 \mathrm{E}-02$

$1.000 \mathrm{e}-02$

$1.000 \mathrm{E}-02$

$1.0008-02$

$1.0008-02$

$1.000 \mathrm{E}-02$

$1.000 \mathrm{~B}-02$

$1.000 \mathrm{E}-02$

$.000 \mathrm{E}-02$

$1.000 \mathrm{~B}-02$

$1.000 E-02$

$1.000 \mathrm{E}-02$

$1.000 \mathrm{E}-02$

$1.0008-02$

1. $000 \mathrm{E}-02$

1. $000 \mathrm{E}-02$

$.000 \mathrm{R}-02$

$1.000 \mathrm{E}-02$

1. $000 \mathrm{E}-02$

$1.000 \mathrm{E}-02$

$1.000 \mathrm{E}-02$

$1.000 \mathrm{E}-02$

$1.000 \mathrm{E}-02$

$1.0008-02$

$.0008-02$

$1.0002-02$

$1.000 \mathrm{E}-02$

1. $0008-02$

$1.000 \mathrm{z}-02$

$1.000 \mathrm{E}-02$

$1.0008-02$

$.000 \mathrm{E}-02$

$1.000 \mathrm{E}-02$

1. $000 \mathrm{~B}-02$

1. $000 \mathrm{E}-02$

$1.0008-02$

$1.000 \mathrm{E}-02$

$1.0008-02$

$1.000 \mathrm{E}-02$

$1.000 \mathrm{E}-02$

$1.000 \mathrm{~B}-02$

1. $000 \mathrm{E}-02$

$1.000 \mathrm{~B}-02$

$1.000 \mathrm{E}-02$

$1.0008-02$

1. $000 \mathrm{~B}-02$

$1.0008-02$

$1.000 \mathrm{E}-02$

1. $000 \mathrm{E}-02$

1. $000 \mathrm{~B}-02$

$1.000 \mathrm{E}-02$

1. $0008-02$

$1.000 \mathrm{E}-02$

$1.000 \mathrm{E}-02$

$1.000 \mathrm{E}-02$

$1.000 \mathrm{~B}-02$

$1.000 \mathrm{z}-02$

$1.000 \mathrm{E}-02$

$1.000 \mathrm{E}-02$

$1.000 \mathrm{E}-02$

1. $000 \mathrm{E}-02$

$1.000 \mathrm{E}-02$

$1.000 \mathrm{E}-02$

$1.000 \mathrm{E}-02$

1. $000 \mathrm{E}-02$

$1.000 \mathrm{E}-02$

$1.0008-02$

$1.000 \mathrm{E}-02$

$1.000 \mathrm{E}-02$

$1.000 \mathrm{E}-02$

$1.000 \mathrm{~B}-02$

$1.000 \mathrm{E}-02$

1. $000 \mathrm{z}-02$

$1.000 \mathrm{E}-02$

$1.000 \mathrm{~B}-02$

$1.000 \mathrm{E}-02$

$1.000 \mathrm{E}-02$

$1.000 \mathrm{E}-02$

$1.000 \mathrm{E}-02$

COM. PROB. RIST

5. $0008-03$

. $500 \mathrm{OB}-02$

2. $500 \mathrm{E}-02$

3. 5008-02

1. $500 \mathrm{R}=02$

5, $5008-02$

$6,5008-02$

7, $500 \mathrm{~B}-02$

7, $500 \mathrm{~B}-02$

5008-02

9. $50 \mathrm{OE}-02$
1. $050 \mathrm{OE}-01$

$.150 \mathrm{~B}-01$

$1.250 \mathrm{OB}-01$

1. $350 \mathrm{O}-01$

1. $450 \mathrm{E}-01$

. $550 \mathrm{~B}-01$

$1.650 \mathrm{z}-01$

1. 75OE-0

$1.850 \mathrm{E}-01$

. $9508-01$

2. $050 \mathrm{E}-01$

2. 150z-01

$2.250 \mathrm{~B}-01$

2. 3508-01

2. $4508-01$

2. 5508-01

2. $650 \mathrm{O}-01$

.7508-01

2. $8508-01$

2. $9508-01$

3. $050 \mathrm{0}-01$

$3.1502-01$

$3.250 \mathrm{~B}-01$

$3.2508-01$

. 3508-01

3. $450 \mathrm{~B}-01$

3. $550 z-01$

$3.7508-02$

3. 8508-01

3. $950 \mathrm{E}-01$

$4.0508-01$

$1.0508-01$

1.1508-01

1. 2508-01

1. 3508-01

1. $150 \mathrm{OB}-01$

4. 550R-01

1. $650 \mathrm{~B}-01$

1. 750E-01

4.850E-01

4. $950 \mathrm{E}-01$

. 050E-01

$5.150 \mathrm{E}-01$

5. 25OR-O

5. $350 \mathrm{O}-01$

5. 4508-01

5. 5508-01

5. 6502-01

$5.7508-01$

5. $850 \mathrm{OE}-01$

$5.950 \mathrm{Q}-01$

$6.050 \mathrm{E}-0$

$6.150 \mathrm{z}-0$.

$6.250 \mathrm{E}-0$

$6.250 \mathrm{E}-0$

$6.350 \mathrm{~B}-0$

6. $450 \mathrm{OB}-01$
6. $550 \mathrm{O}-01$

6. $550 z-01$

6. $6508-01$

$6.850 \mathrm{E}-01$

6. $950 \mathrm{~B}-01$

7. $500 \mathrm{~B}-01$

1. $215 \mathrm{E}-10$

$1.767 \mathrm{e}-10$

$1.873 \mathrm{E}-10$

$1.906 \mathrm{E}-10$

$2.031 \mathrm{E}-10$

$2.170 \mathrm{E}-10$

$3.140 \mathrm{E}-10$

$3.459 \mathrm{~B}-10$

$3.5848-10$
$3.608 \mathrm{e}-10$

. $668 \mathrm{z}-10$

$4.0978-10$

$4.170 \mathrm{E}-10$

4. $676 \mathrm{E}-10$

$5.096 \mathrm{E}-10$

$5.123 \mathrm{E}-10$

5. $240 \mathrm{z}-10$

$5.611 \mathrm{E}-10$

6. $018 \mathrm{~B}-10$

$6.997 \mathrm{E}-10$

$7.272 \mathrm{E}-10$

$7.279 \mathrm{E}-10$

7. $298-10$

$7.553 \mathrm{E}-10$

$8.442 \mathrm{~B}-10$

-.5758-10

. $9198-10$

$9.177 \mathrm{E}-10$

$9.962 \mathrm{E}-10$

$1.0018-09$

$1.0068-09$

$1.0098-09$

1.1048-09

$1.130 \mathrm{~B}-09$

$1.1438-09$

$1.1698-09$

$1.214 z-09$

$1.2168-09$

$1.217 \mathrm{E}-09$

$1.317 \mathrm{E}-09$

1. $4048-09$

$1.120 \mathrm{E}-09$

$1.4368-09$

1. $483 \mathrm{E}-09$

1. 5298-09

$1.885 \mathrm{E}-09$

1.956z-09

2.0148-09

2.0518-09

2.0538-09

$2.562 \mathrm{E}-09$

2. $969 \mathrm{~B}-09$

3.2178-09

3. 2418-09

3. $2408-09$

3. $3008-09$

3. $504 \mathrm{E}-09$

3. $671 \mathrm{E}-09$

$4.069 \mathrm{E}-09$

$1.480 \mathrm{~B}-09$

5. 650R-09

5.6518-09

6. $066 \mathrm{~B}-09$

6. $428 \mathrm{~B}-09$

$6.953 \mathrm{~B}-09$

$7.291 \mathrm{E}-09$

$7.676 \mathrm{E}-09$

8.092 E-09

8.857 E-09

$9.768 \mathrm{E}-09$

$9.788-09$

$1.0518-08$

$1.066 E-08$

$1.084 \mathrm{E}-08$

$1.144 \mathrm{E}-08$

$1.180 \mathrm{E}-08$

1. $253 \mathrm{E}-08$

$1.261 \mathrm{E}-08$

$\begin{array}{ll}7.950 \mathrm{E}-01 & 1.344 \mathrm{E}-08 \\ 8.050 \mathrm{E}-01 & 1.364 \mathrm{E}-08 \\ 8.150 \mathrm{E}-01 & 1.427 \mathrm{E}-08 \\ 8.250 \mathrm{E}-01 & 1.438 \mathrm{E}-08\end{array}$

$\begin{array}{ll}7.950 \mathrm{E}-01 & 1.344 \mathrm{E}-08 \\ 8.050 \mathrm{E}-01 & 1.364 \mathrm{E}-08 \\ 8.15 \mathrm{OB}-01 & 1.427 \mathrm{E}-08 \\ 8.250 \mathrm{E}-01 & 1.438 \mathrm{E}-08\end{array}$

$\begin{array}{ll}7.950 \mathrm{E}-01 & 1.344 \mathrm{E}-08 \\ 8.050 \mathrm{E}-01 & 1.364 \mathrm{E}-08 \\ 8.15 \mathrm{OB}-01 & 1.427 \mathrm{E}-08 \\ 8.250 \mathrm{E}-01 & 1.438 \mathrm{E}-08\end{array}$ 
NUREG/CR-4551, VOL. 1: DRAFT REPORT FOR COMMENT (SEPTEMBER, 1986)

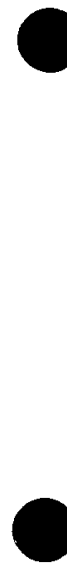

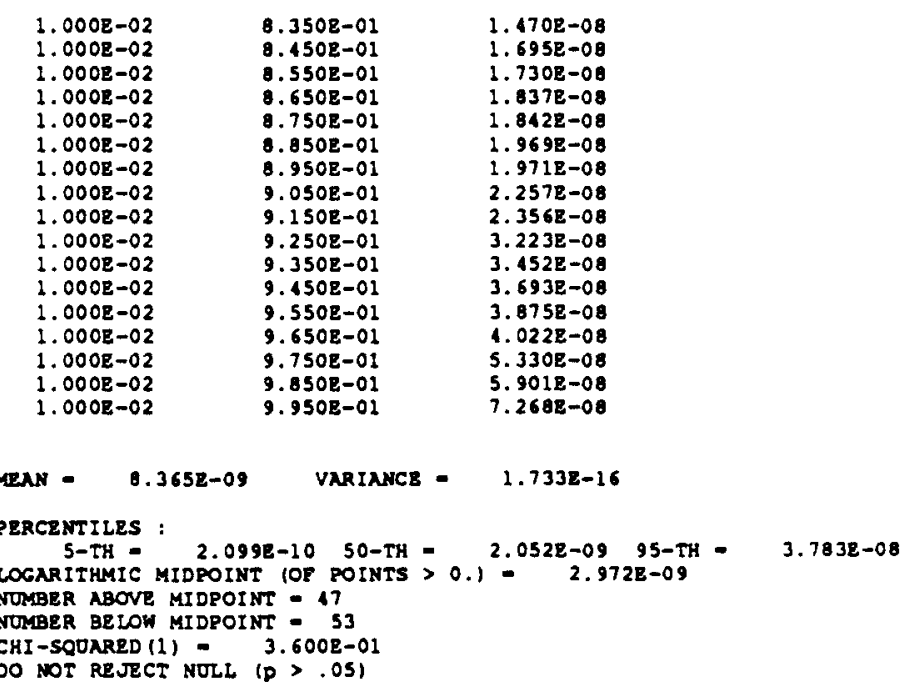


NUREG/CR-4551, VOL. 1: DRAFT REPORT FOR COMMENT (SEPTEMBER, 1986)

CONSEQTENCE MEASORE:

BASE CASE

SURRY LALHC (CRAC)

ISSOE LEVELS FOR EACH SAPLE MEMER. IH ORDER OF INCREASING RISK

RISX ISSUE LEVELS, FOR ALL ISSOES

\begin{tabular}{|c|c|c|c|c|c|c|c|c|c|c|c|c|c|c|c|}
\hline $1.215 \mathrm{E}-10$ & 2 & 1 & 3 & 2 & 1 & 1 & 3 & 1 & $\begin{array}{l}9 \\
2\end{array}$ & $\begin{array}{r}10 \\
2\end{array}$ & 11 & 12 & $\begin{array}{r}13 \\
2\end{array}$ & 14 & $\begin{array}{r}15 \\
2\end{array}$ \\
\hline $1.7678-10$ & 2 & 1 & 2 & 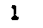 & 3 & 6 & 2 & 2 & 1 & 1 & 5 & 4 & 1 & 1 & 3 \\
\hline $1.8738-10$ & 2 & 2 & 3 & 1 & 1 & 1 & 8 & 2 & 4 & 4 & 1 & 5 & 2 & 2 & 3 \\
\hline $68-10$ & 2 & 2 & 2 & 1 & 1 & 5 & 7 & 2 & 5 & 2 & 2 & 3 & 1 & 2 & 1 \\
\hline $.031 \mathrm{E}-10$ & 2 & 2 & 2 & 1 & 1 & 6 & 1 & 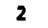 & 3 & 2 & 3 & 3 & 3 & 2 & 3 \\
\hline $1708-10$ & 2 & 1 & 2 & 1 & 2 & 6 & & 2 & 2 & 3 & 3 & 4 & 3 & 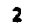 & 3 \\
\hline $3 \mathrm{E}-10$ & 2 & 2 & 3 & 1 & 1 & 3 & 1 & 2 & 2 & 2 & 2 & 1 & 3 & 4 & 3 \\
\hline $08-10$ & 2 & 1 & 2 & 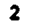 & 3 & 6 & & 1 & s & 3 & 2 & 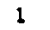 & 2 & 2 & 2 \\
\hline $.4598-10$ & 2 & 2 & 2 & 1 & 2 & 3 & & 4 & 1 & 3 & 4 & 5 & 2 & & 3 \\
\hline $42-10$ & 2 & 2 & 2 & 1 & 1 & 6 & 2 & 1 & 6 & 3 & 3 & 4 & 3 & 1 & 3 \\
\hline $.6088-10$ & 2 & 2 & 2 & 1 & 1 & 5 & 3 & 3 & 1 & 4 & 1 & 2 & 2 & 2 & 3 \\
\hline 3. $6608-10$ & 2 & 1 & 2 & 2 & 2 & 2 & 5 & , & 3 & 1 & 5 & 2 & 4 & 2 & 3 \\
\hline 1.0 & 2 & 1 & 3 & 1 & 3 & 6 & 5 & 3 & 1 & 3 & 3 & 1 & 2 & & 2 \\
\hline $08-10$ & 2 & 2 & 2 & 2 & 1 & 6 & & 2 & 1 & 2 & 4 & 3 & 4 & & 3 \\
\hline $1.6768-10$ & 3 & 1 & 3 & 1 & 1 & 1 & & 2 & 4 & e & 1 & . & 3 & & 2 \\
\hline $.096 \mathrm{~B}$ & 2 & 2 & 2 & 2 & & 2 & 7 & 2 & 1 & 2 & 4 & 3 & 4 & 4 & 2 \\
\hline $5.123 z-10$ & 2 & 2 & 2 & 1 & 2 & 2 & 2 & 3 & 2 & 3 & 1 & 5 & 3 & 2 & 2 \\
\hline $0 z-10$ & 2 & 1 & 3 & 1 & 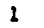 & 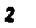 & & 1 & 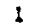 & 1 & 5 & 4 & 4 & & 2 \\
\hline $1.6112-10$ & 2 & 2 & 3 & 1 & 2 & 2 & B & 1 & 1 & 2 & 5 & 1 & 3 & 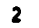 & 1 \\
\hline $6.018 z-10$ & 2 & 2 & 2 & 1 & 2 & 3 & 3 & 1 & 3 & 2 & 3 & 4 & 3 & & 3 \\
\hline 6.9 & 2 & 1 & 2 & 2 & 2 & 6 & 7 & 2 & & 2 & 3 & 4 & 2 & & 2 \\
\hline $7.272 z-10$ & 2 & 1 & 2 & 1 & 2 & 6 & 7 & 2 & 2 & 3 & 1 & 1 & 3 & & 3 \\
\hline 7.2 & 2 & 1 & 3 & 2 & 2 & 6 & 9 & 1 & 3 & 2 & 4 & 3 & 3 & 1 & 2 \\
\hline $7.429 \mathrm{E}-10$ & 2 & 1 & 2 & 2 & 3 & 2 & & 2 & & 2 & & & 3 & & , \\
\hline $7.553 \mathrm{E}-10$ & 2 & 1 & 3 & 2 & 2 & 6 & 7 & 2 & 2 & 3 & 1 & 4 & 2 & & 3 \\
\hline o.1428-10 & 2 & 1 & 3 & 2 & 2 & 2 & s & 2 & 2 & 1 & 1 & 5 & 2 & & 1 \\
\hline $8.5758-10$ & 2 & 2 & 2 & 1 & 3 & 1 & & 1 & & 2 & 3 & A & 2 & & 2 \\
\hline $98-10$ & 2 & 2 & 2 & 2 & 3 & 2 & 2 & 2 & 1 & 2 & 1 & 3 & 3 & & 2 \\
\hline$e-10$ & 2 & 1 & 2 & 2 & 2 & 5 & 1 & 1 & 2 & 2 & 6 & 5 & 1 & 2 & 1 \\
\hline $9.9628-10$ & 2 & 2 & 2 & 2 & 3 & 1 & 3 & 1 & 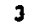 & 3 & 2 & 3 & 3 & 2 & 2 \\
\hline 8-09 & 2 & 1 & 3 & 2 & 1 & 3 & & 3 & 1 & 2 & 4 & 1 & 2 & & 3 \\
\hline$z-09$ & 2 & 2 & 3 & 2 & 2 & 3 & & 1 & 3 & 1 & 1 & 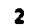 & 2 & & 3 \\
\hline $1.0098-09$ & 2 & 2 & 2 & 2 & 3 & 2 & & 2 & 3 & 2 & 1 & 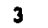 & 2 & & 4 \\
\hline $1.1048-09$ & 2 & 1 & 2 & 1 & 2 & 3 & & 3 & 2 & 2 & s & 4 & 2 & & 2 \\
\hline$e-09$ & 2 & 1 & 3 & 2 & 3 & 3 & & 2 & 3 & 2 & 4 & 3 & 5 & & 2 \\
\hline $8-09$ & 1 & 1 & 3 & 2 & 1 & 2 & 6 & 1 & 5 & 3 & 2 & 4 & 3 & & 2 \\
\hline $2-09$ & 2 & 2 & 2 & 1 & 3 & 6 & s & 2 & 3 & 2 & 3 & 3 & 3 & 3 & 2 \\
\hline-09 & 2 & 2 & 3 & 2 & 1 & 5 & 3 & 2 & & 2 & 5 & 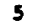 & 2 & & 4 \\
\hline $58-09$ & 2 & 2 & 2 & 2 & 3 & 3 & & 1 & 4 & 2 & 1 & 1 & 3 & & 2 \\
\hline$E-09$ & 2 & 2 & 2 & 2 & 2 & 3 & & 2 & 6 & 3 & 2 & 2 & 5 & & 3 \\
\hline$z-09$ & 2 & 2 & 2 & 1 & 2 & 3 & 9 & 2 & 4 & 1 & 5 & 4 & 2 & & 3 \\
\hline 1.4 & 2 & 1 & 2 & 2 & 3 & 6 & 2 & 2 & 4 & 2 & 3 & 4 & 4 & & 1 \\
\hline $2-09$ & 3 & 1 & 2 & 2 & 3 & 6 & 6 & 1 & 3 & 4 & 1 & 3 & 5 & & 2 \\
\hline $1.1368-09$ & 2 & 1 & 2 & 2 & 1 & 2 & 2 & 2 & 3 & 2 & 4 & 3 & 2 & & 2 \\
\hline 1.4 & 2 & 1 & & 2 & & 6 & & , & & 2 & & 2 & 3 & & 3 \\
\hline $1.529 \mathrm{E}-09$ & 2 & 1 & 2 & 2 & 3 & 2 & & 3 & 4 & 3 & 3 & 1 & 1 & & 4 \\
\hline 1.885E-09 & 2 & 2 & 2 & 2 & 3 & 1 & 7 & 2 & 4 & 1 & 6 & 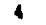 & 3 & & 4 \\
\hline $6 z-09$ & $?$ & 2 & 2 & 2 & 2 & 6 & 9 & 1 & & 3 & 1 & & 3 & & 3 \\
\hline 2.0 & 1 & 2 & 2 & 2 & 3 & 2 & 1 & 3 & 4 & 2 & 3 & 4 & 2 & & 2 \\
\hline $2-09$ & 2 & 1 & 2 & 2 & 3 & 1 & 1 & 2 & 4 & 1 & 6 & 2 & 3 & & 4 \\
\hline $2.0538-09$ & 2 & 1 & 2 & 2 & 3 & 3 & & 2 & 3 & 2 & 5 & 2 & 3 & & 2 \\
\hline 2.5 & , & 1 & 2 & 3 & & 1 & & , & & 1 & & & 3 & & 2 \\
\hline $\mathrm{E}-09$ & 2 & 2 & 3 & 3 & 1 & 6 & 4 & 2 & 5 & 2 & 5 & 3 & 2 & & 4 \\
\hline$e-09$ & 2 & 2 & 2 & 3 & 3 & 6 & 7 & 2 & 3 & 1 & 6 & 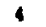 & ר & & 1 \\
\hline $8-09$ & 2 & 1 & 3 & 1 & 3 & 6 & & 4 & 1 & 3 & 2 & 3 & 2 & 2 & 1 \\
\hline 3.2 & , & 2 & 2 & 3 & 3 & 6 & 9 & 1 & 4 & 1 & 6 & 1 & 2 & 2 & 3 \\
\hline B- -09 & 3 & 1 & 2 & $i$ & 3 & 2 & 2 & 3 & 4 & 3 & i & 5 & 3 & 2 & 4 \\
\hline $8-09$ & 2 & 1 & 3 & 1 & 3 & 6 & 6 & 4 & 1 & 3 & 1 & 2 & 2 & 3 & 3 \\
\hline 1.6 & , & 2 & 3 & 2 & , & 4 & & 2 & 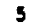 & 2 & 3 & 2 & 3 & 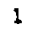 & 2 \\
\hline $\mathrm{E}-09$ & 3 & 2 & 2 & 2 & 1 & 3 & 9 & 3 & 3 & 3 & 3 & 5 & 1 & 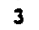 & 1 \\
\hline 1.4 & 2 & 2 & 3 & 2 & 2 & 3 & 7 & 3 & 4 & 3 & 3 & 2 & 1 & & 1 \\
\hline 5. & $?$ & 1 & 2 & 1 & 1 & 6 & 0 & 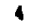 & & 3 & & & 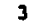 & & 3 \\
\hline 5.6 & , & 1 & 1 & 3 & 1 & 6 & 3 & 3 & 3 & 2 & & 5 & 3 & 3 & 2 \\
\hline 6.0 & 2 & 1 & 3 & 2 & 1 & 6 & 3 & 3 & 1 & 3 & 1 & 2 & 3 & 2 & 1 \\
\hline-09 & 2 & 2 & 3 & 1 & 1 & 6 & 8 & 3 & 4 & 3 & & 2 & 3 & & 3 \\
\hline 6.9 & 2 & 1 & 2 & 2 & 2 & 6 & & & & 2 & & & & & 1 \\
\hline E-09 & ? & 2 & , & 3 & 2 & 5 & 3 & 4 & 7 & 2 & 4 & 4 & 3 & 1 & 1 \\
\hline 7.6 & 2 & 1 & 2 & 2 & 1 & 1 & 3 & 4 & 2 & 2 & 3 & 5 & 3 & 4 & 2 \\
\hline 8.0 & 2 & 2 & 3 & 3 & 2 & 2 & 9 & 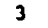 & 1 & 2 & & & 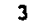 & 2 & 1 \\
\hline 8.8 & 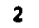 & 2 & 3 & 2 & , & 1 & 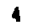 & 4 & 4 & 3 & 4 & 1 & 2 & 2 & 1 \\
\hline 9.768 & 2 & 2 & 2 & 2 & 3 & 4 & & 3 & 3 & 2 & 3 & 2 & 4 & 2 & 3 \\
\hline $9.812 \mathrm{E}-09$ & 2 & 1 & 2 & 1 & 2 & 3 & 9 & 5 & 3 & 3 & 2 & 3 & 5 & 3 & 2 \\
\hline 1.0 & 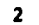 & 2 & 3 & 3 & & 4 & & 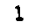 & & 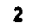 & & 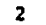 & 2 & 2 & 4 \\
\hline 1.06 & 2 & 1 & 2 & 2 & 2 & 2 & 6 & 5 & 3 & 3 & 3 & 5 & 4 & 4 & 3 \\
\hline 1.0 & 2 & 2 & 2 & 1 & 3 & 2 & 5 & 5 & 3 & 2 & 4 & 2 & 2 & 2 & 2 \\
\hline 1.1 & 2 & 1 & 2 & 1 & 2 & 6 & 3 & 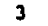 & 4 & & 2 & & 6 & 4 & 2 \\
\hline $1.180 \mathrm{E}-08$ & 2 & 1 & 2 & 2 & 2 & 6 & 4 & 4 & 4 & 2 & 9 & 3 & 2 & 2 & 2 \\
\hline $\mathbf{E}-08$ & 2 & 2 & 2 & 1 & 2 & 5 & 4 & 5 & 5 & 4 & 1 & 1 & 3 & 1 & 1 \\
\hline $.261 \mathrm{z}-08$ & 1 & 2 & 2 & 2 & 3 & 4 & 4 & 1 & 2 & 2 & 4 & 5 & 3 & 4 & 1 \\
\hline $344 \mathrm{~B}-0$ & 2 & 2 & 3 & 1 & 1 & 1 & & 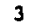 & & & 4 & & 5 & & 2 \\
\hline $.364 \mathrm{~B}-08$ & 2 & 1 & 3 & 1 & 2 & 2 & 9 & 3 & a & 2 & 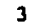 & 3 & 4 & 3 & 1 \\
\hline 08 & 2 & 2 & 3 & 3 & 2 & 4 & 7 & 3 & 3 & 2 & 4 & 2 & 3 & 3 & 3 \\
\hline $438 \mathrm{E}-\mathrm{C}$ & & 1 & & & & & & & & & & & & & \\
\hline
\end{tabular}


NUREG/CR-4551, VOL. 1: DRAFT REPORT FOR COMMENT (SEPTEMBER, 1986)

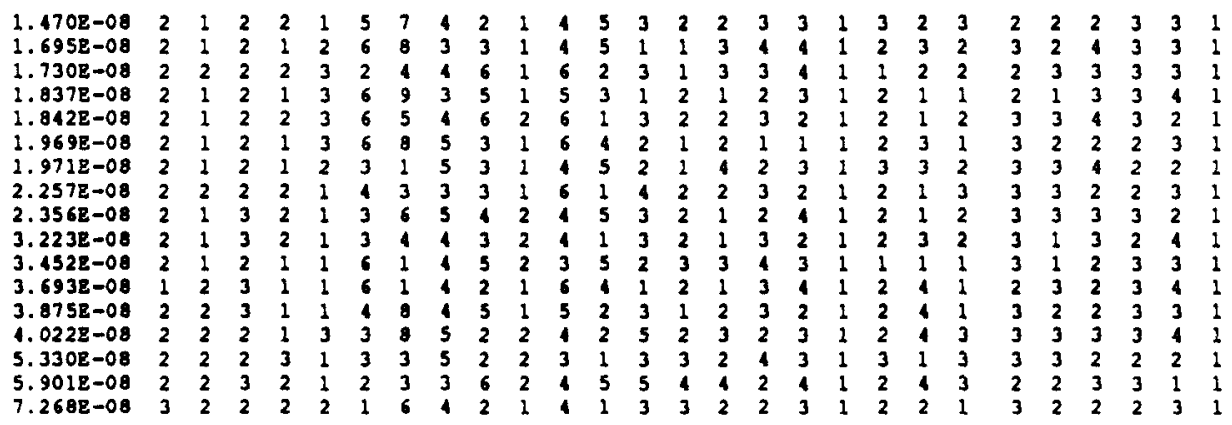


CONSEQUENCE MEASORR:

IND. RISK OF DEATH

BASE CASE :

SURRY LALHC (CRAC)

FRACTIONAL CONTRIBUTION OF EACH SEOUENCE TO MEAN RISK

SEQUENCE CONTRIBUTION

SYYB 0.1230

SYNI 0.0002

$\begin{array}{ll}\text { SNRN } & 0.4915 \\ \text { SYNN } & 0.0007\end{array}$

ANNN 0.0000

AYNN

AYN I

AYYB

INNS

0.0089

0.0214

0.0064

0.1623

TYYBW

0.0588

TYYBD 0.0644

1 CONSEQTENCE MEASURE:

IND. RISK OF DEATH

0.0002
0.0823

BASE CASE :

SORRY LALHC (CRAC)

ERACTIONAL CONTRIBUTION OF RACH BIN TO MEAN RISK

BIN

CONTRIBUTION

BIN 120.0101

BIN 3

BIN 4

0.0029

BIN $5 \quad 0.0312$

$\begin{array}{lll}\text { BIN } 5 & 0.0001 \\ \operatorname{BIN} 7 & 0.0011\end{array}$

$\begin{array}{ll}\text { BIN } 7 & 0.0011 \\ \text { BIN } & 0.0000\end{array}$

BIN 9

8 IN 10

BIN 11

BIN $12 \quad 0.0685$

BIN 13

BIN $14 \quad 0.0000$

BIN 15

0.6298

0.0693

BIY 18

BIN $19 \quad 0.0805$

1 CONSEOUENCE MEASURE:

IND. RISK OF DEATH

BASE CASE

SORRY LALHC ICRAC

FRACTIONAL CONTRIBUTIONS OP EACH RELEASE TO MEAM RISK RELEASE CONTRIBOTION SUR 1GP 0.1915 SUR 2GP 0.1710

SUR 3GP 0.1039

SUR 4GP 0.1376

SUR SGP 0.0739

SUR 6CP $\quad 0.0727$

SUR 7GR 0.0406

SUR BGP 0.0547

SUR $9 G \quad 0.0032$

SUR IOGP 0.0085

SUR 11GP $\quad 0.0405$

SOR 12GR 0.0016

SUR $13 G 2 \quad 0.0401$

SUR $14 \mathrm{G} \quad 0.0029$

SUR $15 \mathrm{GP}$

SUR $16 \mathrm{G}$

SUR $17 \mathrm{GP} \quad 0.0187$

SOR $18 \mathrm{G} \quad 0.0000$

SUR 19GP 0.0171

0.0000

SUR 21GP 0.0022

SUR $22 \mathrm{G} \quad 0.0000$

SUR $2362 \quad 0.0001$

SUR $24 \mathrm{G} \quad 0.0000$

SUR $25 \mathrm{C}$

0.0000 
CONSBOUENCE MASURE:

IND. RISK OE DEATH

SENSITIVITY OF RIST TO ISSOES

DISTRIBUTION OF RISK AT EACH LEVEL OF EACH ISSOE aASE CASE :

SURRY LALHC (CRAC)

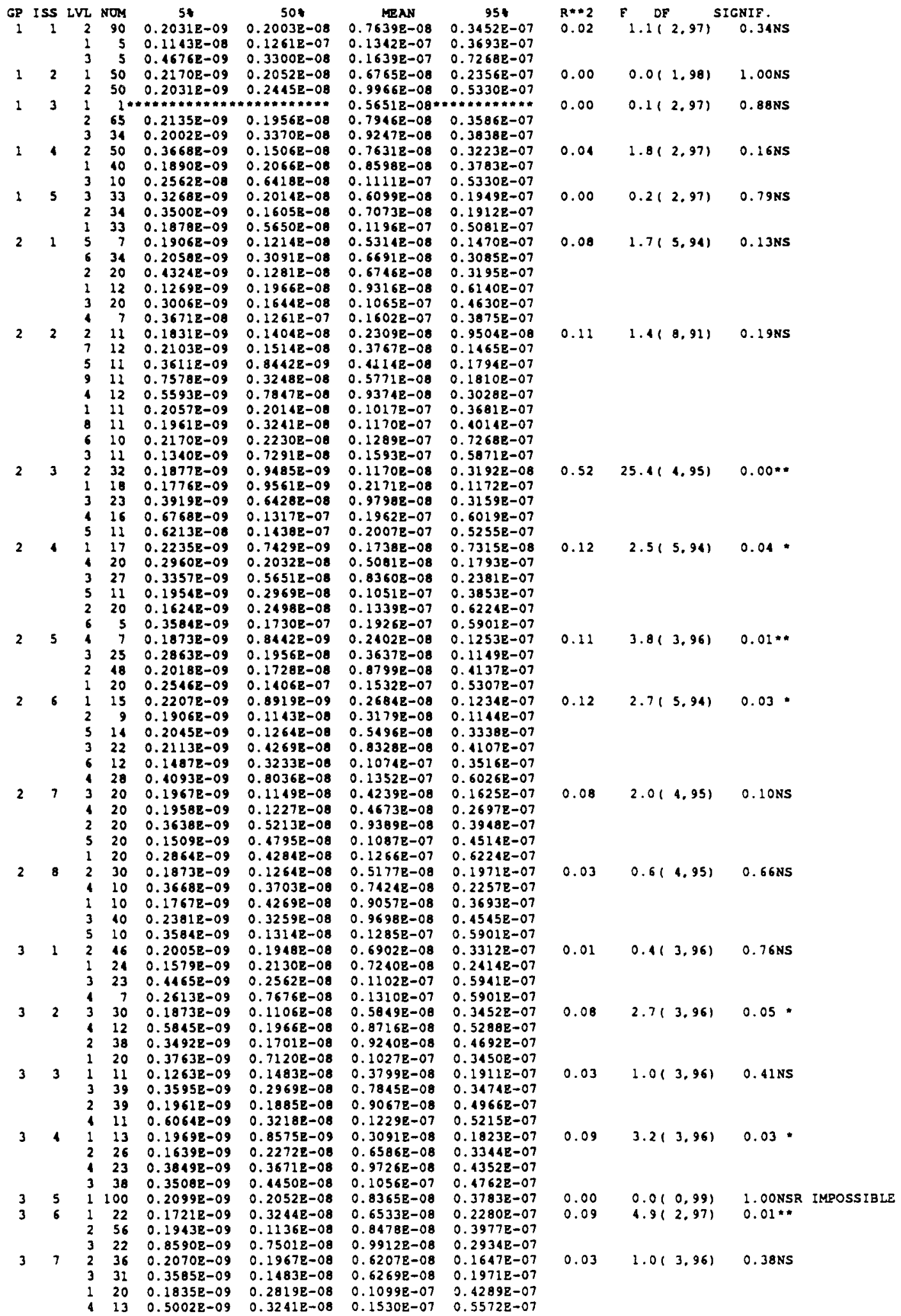


NUREG/CR-4551, VOL. 1: DRAFT REPORT FOR COMMENT (SEPTEMBER, 1986)

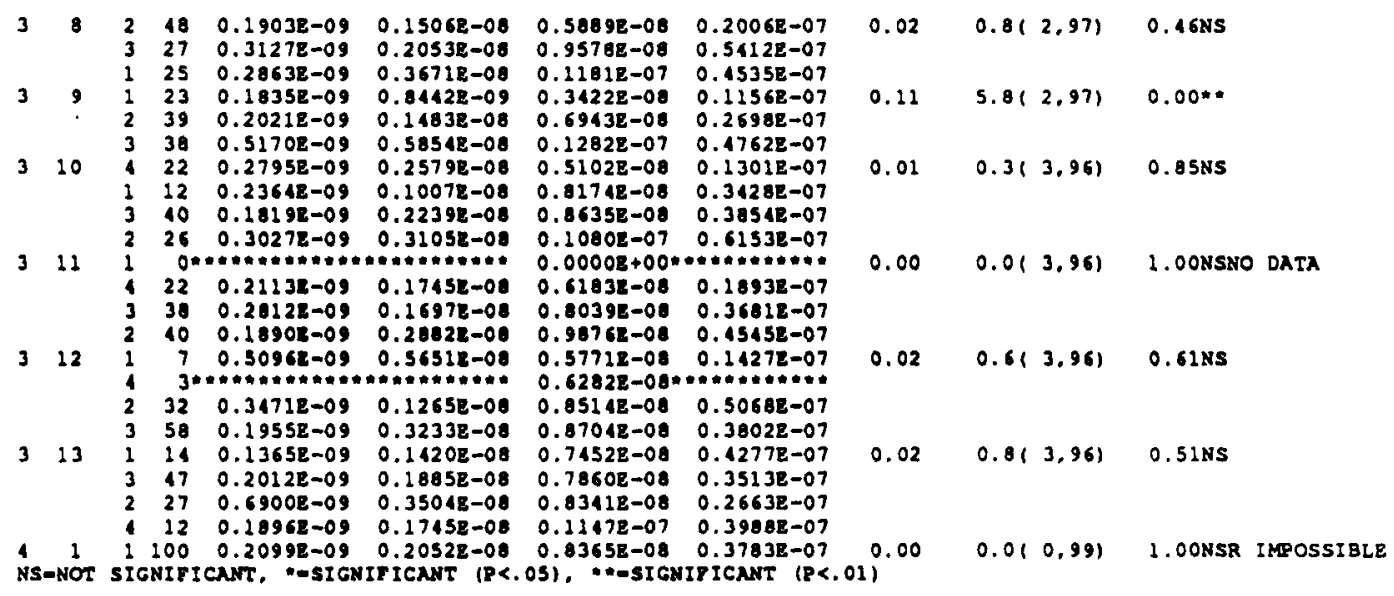


CONSEOUENCE MEASURE: IND. RISK OF DEATH

- ISSUE GROUP - 1 ISSUE - 1 TB SEAL LOCA

LEVEL 1 NO. QRED.- 2 No. EXP.= 2

LEVEL 2 NO. QRED. = 45 NO. EXP. $=45$

LEVEL 3 NO. QRED.- 2 No. EXP. $=2$

OVERALL CHI-SO 0.0 DE= 1 SIG. $=1.000$

0

LEVEL 1 NO. GMD. $=24$ NO, EXP. - 25

LEVEL 2 No. QRed. 25 No. ExP. -25

NO. > MED. - 26 NO. EXP.- 25

NO. $\rightarrow$ Red. -25 No. EXP. $=25$

OVERALE CHI-SO 0.1 DFE 3 SIG.=0.994

O ISSOE GROOR = 1 ISSUZ - 3 BETA-EACTOR

LEVEL I NO. SED.= 0 No. EXP.= 0

LEVEL 2 NO. QED. $=34$ NO. EXP. $=32$

LEVEL J NO. CED. - 15 NO. EXP. - 17

NO.>MED.- 1 No. EXP.= 1

NO. >MED. - 31 NO. EXP. -33

MO. DKED. - 19 MO. EXP. $=17$

OVERALL CHI-SO= 0.7 DF- 3 5TG.- 0.869

0

ISSTE GROOP - 1 ISSOZ - I V FREO

LEVEL I NO. QED. = 20 NO. EXP. $=20$

LEVEL 2 NO. QRE. - 29 NO. EXP. = 25

MO. $>$ KLD. $=20$ MO. EXP. -20

MO.>MED. - 21 No. EXP.- 25

LEVEL 3 No. QMed.- 0 No. Exp.- 5

NO. $\rightarrow$ MED. - 10 NO. EXP.= 5

OVERALL CHI-SO 11.3 DF- 5 SIE.= 0.046

ISSTE GROUR - 1 ISSTE - S P1F2 FAILORE

LEVEL I NO. QRD.- 14 NO. EXP.- 16

LEVEL 2 NO. QUED. - 18 No. EXP. - 17

NO. >MED. - 19 NO. ExP. $=17$

NO. > MED. = 16 พO. $2 X P=17$

LEVEL 3 NO. ARD. - 17 NO. EXP. -16

NO. XIED. -16 No. EXP. $=17$

OVERALL CHI-SO- 0.7 DFE 5 SIG.- 0.982

ISSUE GROOP - 2 ISSUE - 1 IND. RCS FAIL.

LEVEL I NO. QRED.- 6 NO. EXP.- 6

LEVEL 2 NO. QRED. - 13 NO. EXP. = 10

LEVEL 3 NO. QYED. - 10 No. EXP. = 10

LEVEL \& NO. QRED = 0 NO. EXP.= 3

LEVEL 5 NO. KMED. = 4 No. EXP.- 3

LEVEL 6 NO. QRED. - 16 NO. EXP. - 17

OVERALL CHI-SO 1.9 DFE 7 SIG.= 0.964

ISSUE GROUP - 2 ISSUE - 2 MODE OF VB.

LEVEL 1 NO. QMED. = 6 NO. EXP.- 5

LEVEL 2 NO. KMED.- o NO. EXP.- 5

LEVEL 3 NO. LMED. = 3 NO. EXP. $=5$

LEVEL 4 NO. KMED = 2 NO. EXP.= 6

LEVEL 5 NO. LMED.- 8 NO. EXP.- 5

LEVEL 6 NO. KMED. = 5 NO. BXP. = 5
NO. $>$ MED. $=5$ NO. EXP. $=6$ NO.>MED. = NO. EXP.- 6 NO.>MED. - 10 NO. EXP. $=6$ No.>MED. = 3 No. EXP. $=6$ NO. PAMED. - 5 NO. EXP.=5 NO.>MED. - 3 NO. EXP. - 6
CHI-SO. TEST CANNOT BE RUN; EXP. NO. < 5 CHI-SO.= 0.0 DF- 1 SIG.= 1.000

CHI-SO. IEST CANNOT BE RUN; EXP. NO. $<5$
CHI-SO.= 0.1 DFE 1 SIG. $=0.777$

CHI-SO.- 0.0 DF= 1 SIG. 1.000
CHI-SO. TRST CANNOT BE RUN; EXP. NO. < 5

CHI-SQ. $=0.2$ DF= 1 SIC $=0.620$

CHI-SO. $=0.5$ DF 1 SIC. $=0.493$
CHI-SO.= 0.0 DF= I SIG. $=1.000$

CHI-SO. 1.3 DF- 1 SIG. $=0.250$

CHI-SO. $=10.0 \mathrm{DF}=1 \mathrm{SIG}=0.002$

CHI-SO.= 0.5 DF= 1 SIG. $=0.486$ CHI-5O.- 0.1 DF- 1 SIG. $=0.732$ CHI-SO.- 0.1 DF 1 SIG. $=0.728$
CHI-SO= 0.0 DF= 1 SIG. $=1.000$

CHI-SO. 1.8 DE- 1 SIG. $=0.180$ CHI-SO.- 0.0 DF $=1$ SIG. $=1.000$ CHI-SO. TEST CANNOT BE RUN; EXP. NO. < 5 CHI-SO. TEST CANNOT BE RUN; EXP. NO. < 5 CHI-SO. $=0.1$ DE 1 SIG. $=0.732$

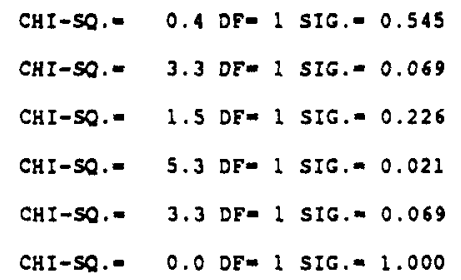




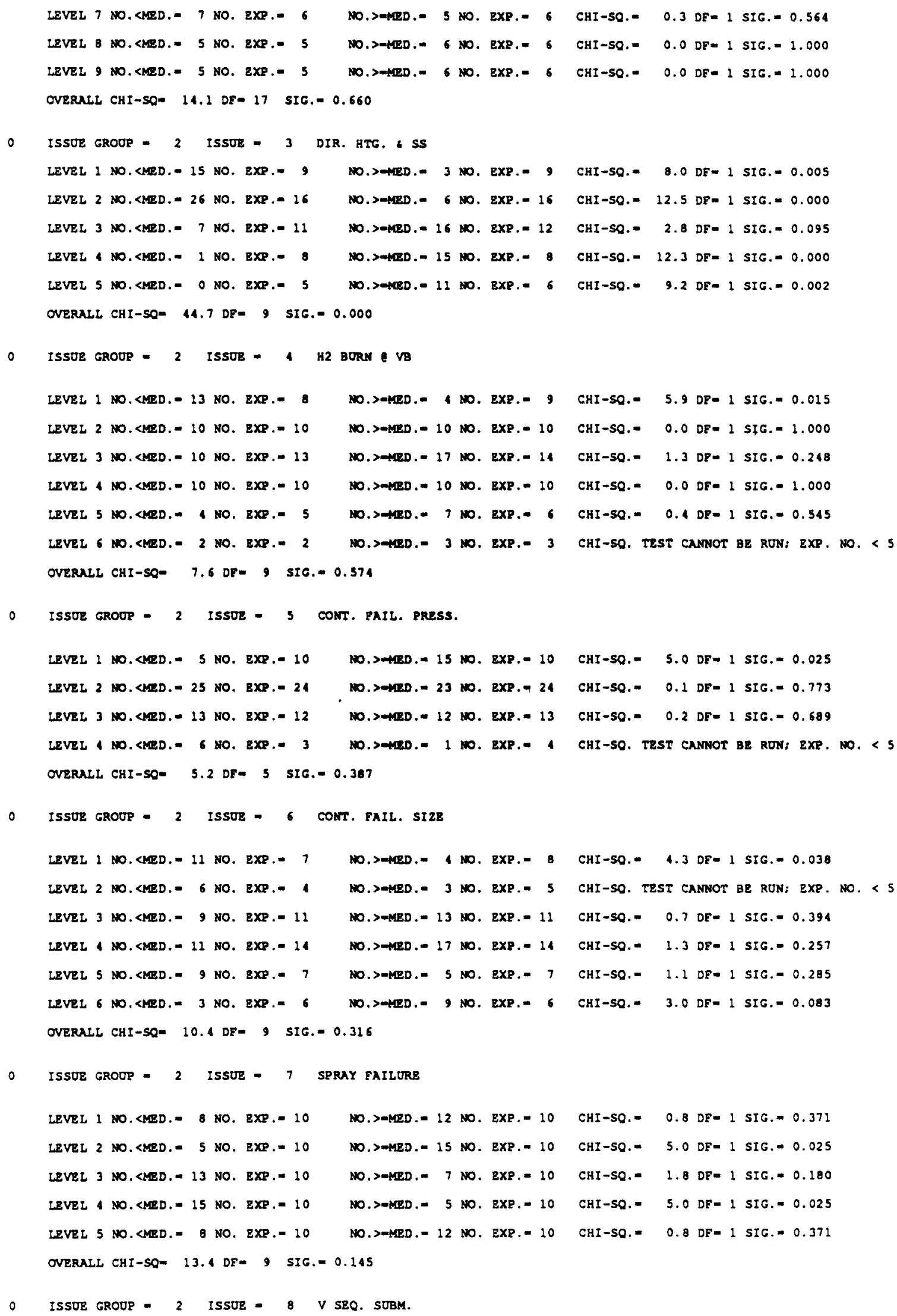




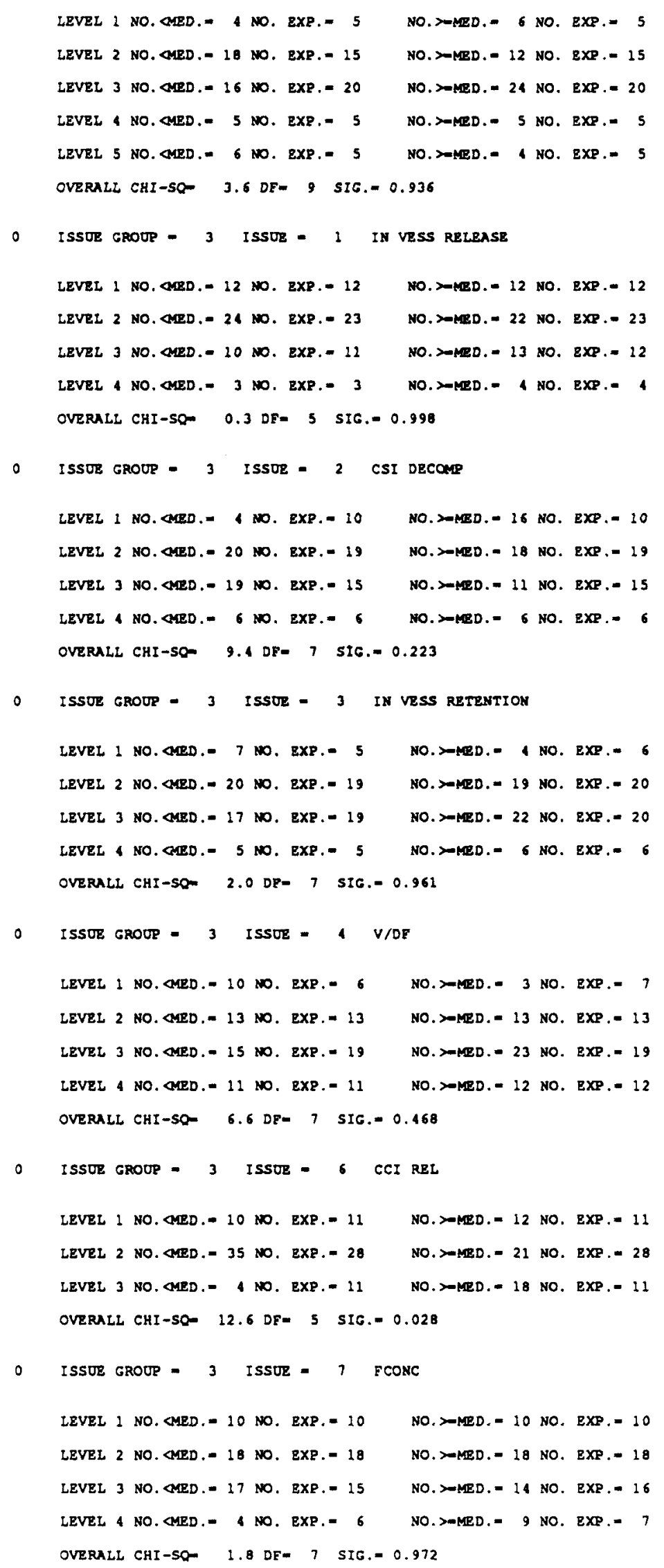


ISSOE GROUP - 3 ISSUE - 8 SPRAY DP

LEVEL 1 NO. CMED. = 10 NO. EXP. $=12$

No. >AKED $=15$ NO, EXP. $=13$

CHI-SO. $=0.6$ DF= I SIG. $=0.423$

LEVEL 2 NO. S\&RD. - 26 NO. EXP. - 24

NO. $>M E D .=22$ NO. EXP. - 24

CHI-5O. 0.3 DF= 1 SIG. 0.564

LEVEL 3 NO. SMED. = 13 NO. EXP. - 13

No.>-4R. - 14 No. EXP. - 14

CHI-SQ. $=0.0$ DF $=1$ SIG. -1.000

OVRRALL CHI-SQ- 1.0 DF- 5 SIG.- 0.965

ISSUR GROUP - 3 ISSUE - 9 ARR AGCLOM

LEVEL 1 NO. <MED. - 14 NO. BXP.- 11

No. >AME.- 9 No. EXP.= 12

CHI-SO.- $1.6 \mathrm{DF}=1 \mathrm{SIG}=0.210$

LEVEL 2 No. <LED. - 22 No. EXP.- 19

No.>mede. 17 wo. Exp. $=20$

CHI-SO.- 0.9 DF- 1 SIG. $=0.336$

LeVEL 3 NO.<RED. - 13 NO. BXP. - 19

No.>Mede. 25 No. ExP. $=19$

CHI-SO. $\quad 3 . \&$ DE $=1$ SIG. $=0.052$

OVERALL CHI-sO- 6.3 DFe 5 sIG. $=0.280$

ISSUE GROUR - 3 ISSUE - 10 LATR IOOIM

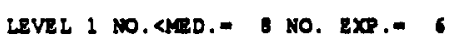

No.>mede. 4 so. Exp.- 6

CHI-SO.= 1.3 DF $=1$ SIG. $=0.248$

LEVEL 2 wo. LARD. - 11 No. EXP. $=13$

No.>-7ad = 15 No. EXP. = 13

CHI-so. $=0.6$ DF $=1$ SIG. $=0.433$

LEVEL 3 NO. SLED. - 20 NO. EXP. -20

10.>ARE.- 20 No. EXP.- 20

CHI-SO.- 0.0 DF- 1 SIG.- 1.000

LEVEL \& 10. KMED. - 10 MO. EXP.- 11

No. >mied. - 12 No. Exp. - 11

CHI-SO.- 0.2 DF= 1 SIG. 0.670

OVERALL CHI-5O= 2.1 DF= 7 sIG. 0.952

ISSOE GROOP - 3 IsSUE - 11 LATE RIVOL

LEVEL 1 NO. SRED. - O NO. EXP.- 0

LEVEL 2 No. <MED. - 18 NO. EXP. - 20

LEVEL 3 No. SED. - 20 NO. EXP. - 19

LEVEL 4 No. KMED. = 11 No. EXP. - 11

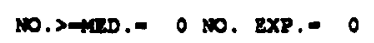

NO. SANED. - 22 No. EXP. $=20$

W. >MRD. - 10 No. EXP.= 19

No.>AED. - 11 No. EXP. $=11$
CHI-50. TEST CXNNOT BE RON: 8XP. NO. < 5

CHI-SO. $=0.4 \mathrm{DP}=1 \mathrm{SIG}=0.527$

CHI-SO.= 0.1 DF $=1$ SIG. $=0.746$

CHI-SQ. $\quad 0.0$ DF= 1 SIG. $=1.000$

OVERALL CHI-SO- 0.5 DF- 5 SIG.= 0.992

ISSTE GROUP - 3 ISSUE - 12 HPE REL

LEVEL I NO. <MED.- 3 No. EXP.- 3

No.>-MED. = 4 No. EXP.- 4

LEVEL 2 NO. LMED. - 20 NO. BXP, - 16

LEVEL 3 No. CMED. = 25 NO. EXP. = 29

LEVEL 4 No. KMED.- 1 No. EXP.- 1

NO. > MED. - 12 No. EXP. -16

No.>MAD. -33 No. EXP. $=29$

SO.>-MED. - 2 NO. EXP.= 2
CHI-SO. TEST CANNOT BE RUN; EXP. NO. < 5

CHI-SO. $=2.0 \mathrm{DF}=1$ SIG.=0.157

CHI-SO.= 1.1 DF= 1 SIG.=0.293

CHI-SO. TEST CANNOT BE RUN; EXP. NO. < 5

OVERALL CHI-SO- 3.1 DF- 3 SIG.- 0.376

ISSUE GROUP - 3 ISSOE - 13 PH REL

LEVEL \& NO. CMED.- NO. EXP.- 7

LEVEL 2 No. SMED.- 11 No. EXP. - 13

LEVEL 3 No. LMED. - 24 No. EXP. - 23

LEVEL 4 NO. LMED.- 6 No. EXP.- 6

OVERALL CHI-SOE 1.0 DFE 7 SIG.= 0.995

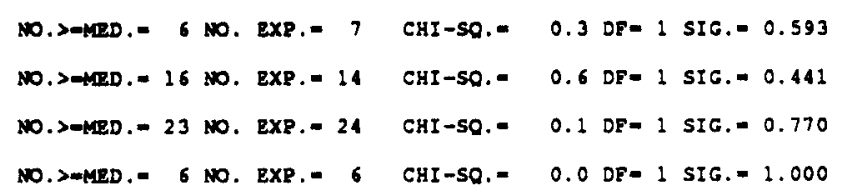

No. $>M E R$. 6 NO. EXP. $=6$

$C H I-S Q=0.0$ DF $=1$ SIG. $=1.000$ 
CONSEOUENCE MRASURE:

CANCER DEATHS PER YEAR

BASE CASE

SURRY LALHC (CRAC)

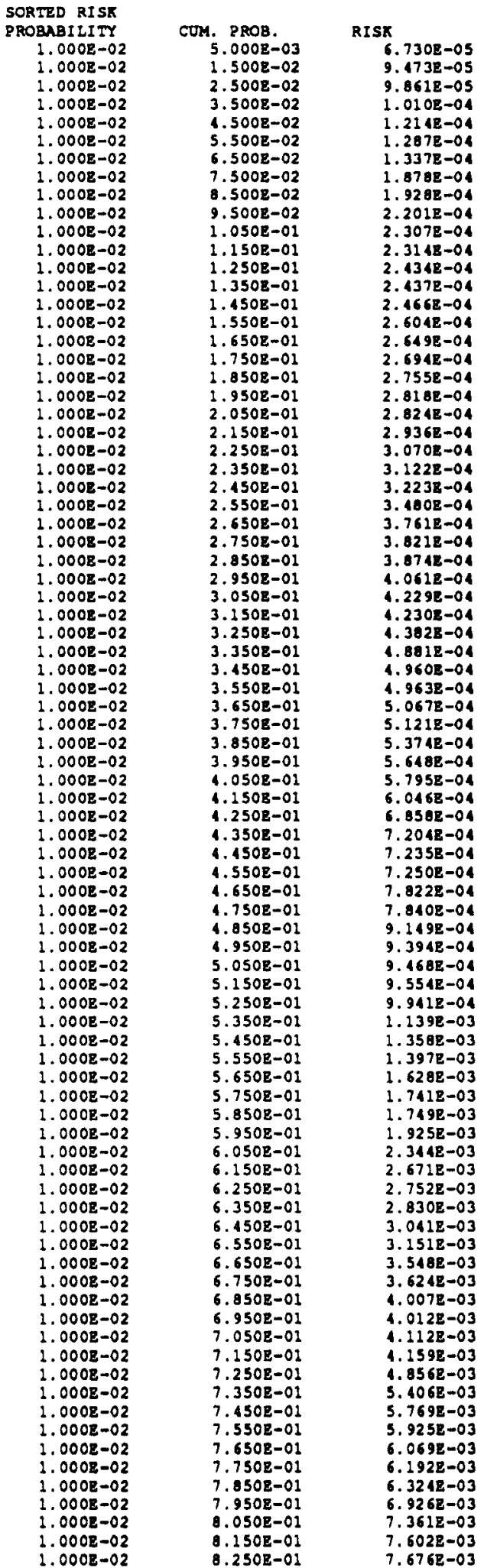


NUREG/CR-4551, VOL. 1: DRAFT REPORT FOR COMMENT (SEPTEMBER, 1986)

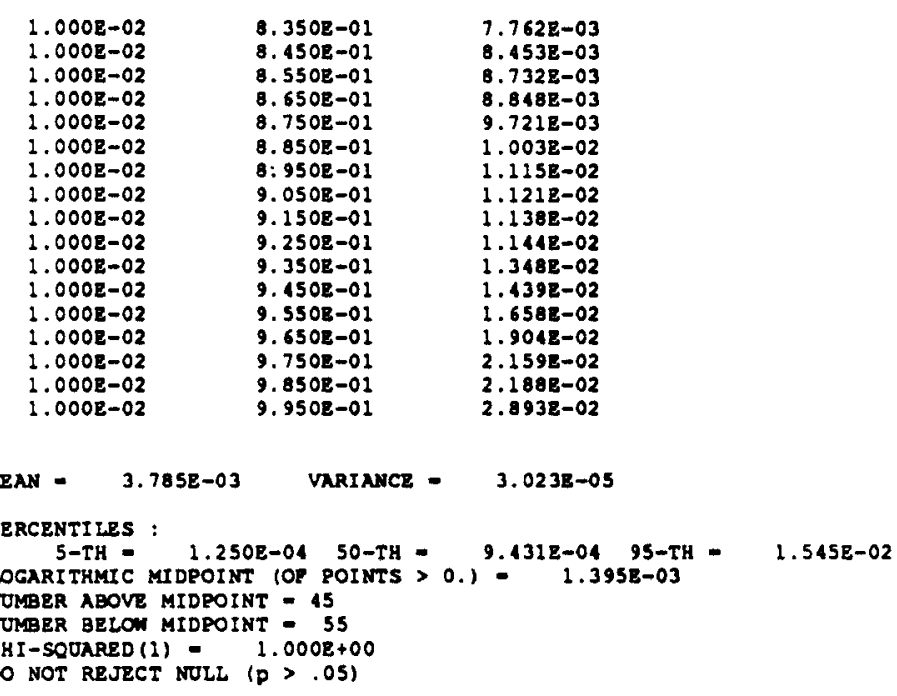


NUREG/CR-4551, VOL. 1: DRAFT REPORT FOR COMMENT (SEPTEMBER, 1986)

CONSEOUENCE MEASURE:

CANCER DEATHS PER YEAR

BASE CASE :

SORRY WALHC (CRAC)

ISSUE LEVELS FOR EACH SAMLE MEMER. IN ORDER OR INCREASING RISK RISK ISSUE LEVELS, FOR ALL ISSUES

\begin{tabular}{|c|c|c|c|c|c|c|c|c|c|c|c|c|c|c|}
\hline & & & & & & 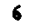 & 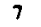 & 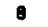 & 91 & 10 & 111 & 121 & 131 & 14 \\
\hline $730 z-05$ & 2 & $i$ & 3 & 2 & 1 & 1 & 3 & 1 & 2 & 2 & 6 & 3 & 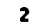 & 1 \\
\hline $.473 E-05$ & $?$ & 2 & 3 & 1 & 1 & 1 & 8 & 2 & 4 & 4 & 1 & 5 & 2 & 2 \\
\hline $.861 E-05$ & 2 & 2 & 2 & 1 & 1 & 5 & 7 & 2 & 5 & 2 & 2 & 3 & 1 & 2 \\
\hline $.0108-04$ & $?$ & 2 & 2 & 1 & 1 & 6 & 1 & & 3 & 2 & 3 & 3 & 3 & 2 \\
\hline $.214 E-04$ & $2+2$ & 1 & 2 & 1 & 3 & 6 & 2 & 2 & 1 & 1 & 5 & 4 & 1 & 1 \\
\hline $.287 E-04$ & 2 & 1 & 2 & 1 & 7 & 6 & 6 & 2 & 2 & 3 & 3 & 6 & 3 & 2 \\
\hline $.337 \mathrm{E}-04$ & 2 & 1 & 2 & 2 & 3 & 6 & 1 & 1 & 5 & 3 & 2 & 1 & $\boldsymbol{2}$ & 2 \\
\hline $.8788-04$ & 2 & 2 & 2 & 1 & 1 & & & 1 & 6 & 3 & 3 & 4 & 5 & 1 \\
\hline . 928z-04 & 2 & 2 & 2 & 1 & 2 & $\mathbf{3}$ & 6 & 4 & 1 & 3 & 4 & 5 & 2 & 2 \\
\hline $.2018-04$ & 2 & 2 & 2 & 2 & 1 & 6 & 2 & 2 & 1 & 2 & 4 & 3 & 4 & 2 \\
\hline 04 & 2 & 1 & 2 & 2 & 2 & 2 & 5 & 2 & 3 & 1 & 5 & 2 & 4 & 2 \\
\hline $.3148-04$ & 2 & 2 & 2 & 2 & 2 & ? & 7 & 2 & 1 & 2 & 4 & 3 & 1 & 4 \\
\hline $.434 \mathrm{E}-04$ & 2 & 2 & 3 & 1 & 1 & 3 & 1 & 2 & 2 & 2 & 2 & 1 & 3 & 4 \\
\hline $.437 z-04$ & 2 & 2 & 2 & 1 & 2 & 3 & 5 & 1 & 5 & 2 & 3 & 4 & 3 & 2 \\
\hline $.466 \mathrm{~B}-04$ & 2 & 2 & 2 & 1 & 2 & 2 & 2 & 3 & 2 & 3 & 1 & 5 & 3 & 2 \\
\hline .604 & 2 & 1 & 3 & 1 & 1 & 2 & & 1 & 2 & 1 & 5 & 4 & 4 & 3 \\
\hline $.6498-04$ & 2 & 1 & 3 & 2 & 2 & 6 & 7 & 2 & 2 & 3 & 1 & 4 & 2 & 2 \\
\hline .694 & 2 & 2 & 2 & 1 & 1 & 5 & 5 & 3 & 1 & 4 & 1 & 2 & 2 & 2 \\
\hline 04 & 2 & 1 & 3 & 2 & 2 & 6 & 5 & 1 & 3 & 2 & 4 & 3 & 5 & 1 \\
\hline $.818 \mathrm{E}-04$ & 2 & 1 & 2 & 2 & 2 & 6 & 7 & 2 & 2 & 2 & 3 & 4 & 2 & 2 \\
\hline .82 & 2 & 1 & 2 & 2 & 3 & 2 & 9 & 2 & 1 & 2 & 4 & 4 & 3 & 1 \\
\hline .938 & 3 & 1 & 3 & 1 & 1 & 1 & 8 & ? & 4 & 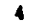 & 1 & 3 & 3 & 3 \\
\hline .07 & 2 & 1 & 2 & 1 & 2 & 6 & 7 & 2 & 2 & 3 & 1 & 1 & 3 & 1 \\
\hline-04 & 2 & 1 & 2 & 2 & 2 & 5 & 1 & 1 & 2 & 2 & 6 & 5 & 1 & 2 \\
\hline $.28>-1$ & 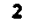 & 1 & 3 & 1 & 3 & 6 & 5 & $\mathbf{3}$ & 1 & 3 & 3 & 1 & 2 & 3 \\
\hline-04 & 2 & 2 & 2 & $i$ & 3 & 2 & 3 & 1 & 4 & 2 & 3 & 5 & 2 & 3 \\
\hline .76 & 1 & 1. & 3 & 2 & 1 & 2 & 6 & 1 & 5 & 3 & 2 & 4 & 3 & 1 \\
\hline 3.82 & 2 & 2 & 2 & 2 & 3 & 2 & 2 & 2 & 1 & 2 & 2 & 3 & 3 & 3 \\
\hline $3.8748-04$ & 2 & 2 & 2 & 2 & 3 & 2 & & 2 & 3 & 2 & 4 & 3 & 2 & 2 \\
\hline-04 & 2 & 2 & 3 & 1 & 2 & 2 & & 1 & 1 & 2 & $\mathbf{5}$ & 1 & 3 & 2 \\
\hline $1.229 E-04$ & 2 & 1 & 3 & 2 & 3 & 3 & & 2 & 3 & 2 & 4 & 3 & 5 & 1 \\
\hline .23 & 2 & 2 & 2 & $=$ & & 3 & & 2 & & 1 & $\mathbf{s}$ & 4 & 2 & 1 \\
\hline .38 & 2 & 1 & 3 & 2 & 2 & 2 & 5 & 2 & 2 & 4 & 1 & 5 & 2 & 3 \\
\hline 1.88 & 2 & 1 & 2 & 2 & 1 & 2 & 2 & 2 & 3 & 2 & 4 & 3 & 2 & 3 \\
\hline 4.96 & 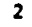 & 2 & 3 & 2 & 2 & 3 & & 1 & 7 & 4 & 2 & 2 & 2 & 2 \\
\hline $4.963 \mathrm{E}-04$ & 2 & 2 & 2 & 1 & 3 & 6 & 5 & 2 & 3 & 2 & 5 & 3 & 5 & 3 \\
\hline 5.06 & 2 & 2 & 2 & 2 & 3 & 1 & & 1 & 3 & 3 & 2 & 3 & 3 & 2 \\
\hline .12 & 4 & 1 & 3 & 2 & 1 & 3 & & 3 & 1 & 2 & 4 & 1 & 2 & 4 \\
\hline $5.3742-04$ & 2 & 2 & 2 & 2 & 2 & 3 & & 2 & 6 & 3 & 2 & 2 & 5 & 2 \\
\hline 5.64 & 2 & 1 & 2 & 1 & 2 & 3 & 9 & 3 & 2 & 2 & 5 & 4 & 2 & 3 \\
\hline 5.79 & 2 & 1 & 2 & 2 & 3 & 6 & 2 & 2 & 1 & 2 & 1 & 2 & 3 & 1 \\
\hline 6.0 & 2 & 2 & 3 & 2 & 1 & 5 & & 9 & & $=$ & 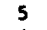 & 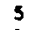 & 2 & 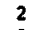 \\
\hline .85 & 2 & 2 & 2 & 2 & 3 & 3 & 7 & 1 & 4 & 2 & 4 & 1 & 3 & 2 \\
\hline $7.204 E-04$ & 2 & 2 & 3 & 3 & 1 & 6 & 4 & 2 & 5 & 2 & 5 & 3 & 2 & 1 \\
\hline $7.235 \mathrm{E}-04$ & 3 & 1 & 2 & 2 & 3 & 6 & & 1 & & 4 & 1 & - & 5 & 1 \\
\hline $7.250 \mathrm{e}-04$ & 2 & 1 & 2 & 2 & & 6 & & 2 & & $?$ & 5 & 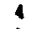 & 4 & 7 \\
\hline 7.82 & 2 & 1 & 2 & 2 & 3 & 2 & 1 & 3 & 4 & 3 & 3 & 1 & 1 & 1 \\
\hline $7.840 \mathrm{E}+04$ & 1 & 2 & 2 & 2 & 3 & 2 & 1 & 3 & A & 2 & 3 & 4 & 2 & 2 \\
\hline $9.149 \mathrm{~B}-04$ & 2 & 2 & 2 & 2 & & 6 & 9 & 1 & 4 & 3 & 1 & 4 & 3 & 3 \\
\hline $.394 z-04$ & 2 & 1 & & 2 & & 1 & & 2 & & & 6 & 7 & 3 & \\
\hline $9.468 \mathrm{~B}$ & 2 & 2 & 2 & 3 & 2 & 6 & 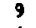 & 1 & 4 & 1 & 6 & 1 & 2 & 2 \\
\hline $9.5548-04$ & 2 & 1 & 2 & 2 & 3 & 3 & t & 2 & 3 & 2 & 5 & 2 & 3 & 3 \\
\hline $9.9418-04$ & 2 & 2 & 2 & 2 & & 1 & & 2 & 4 & 1 & 6 & 4 & 3 & 2 \\
\hline 1.13 & 2 & 1 & 2 & 3 & & 1 & & 2 & & 1 & & , & & \\
\hline $1.35 \mathrm{gE}-03$ & 2 & 2 & 3 & 2 & & 4 & 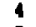 & 2 & 5 & 2 & 3 & 2 & 3 & 1 \\
\hline 1.39 & 2 & 2 & 2 & 3 & & 6 & & 2 & 3 & 1 & 6 & 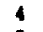 & 3 & 2 \\
\hline 1. $628 \mathrm{~g}-$ & 2 & 1 & 3 & 1 & 3 & 6 & & 4 & 1 & 3 & 2 & 3 & 2 & 2 \\
\hline $1.7418-$ & 2 & 1 & 3 & 9 & & 6 & & 4 & 1 & 3 & & & 2 & $3+3+2$ \\
\hline 1.7 & 3 & 1 & 2 & 1 & 3 & 2 & & 3 & 4 & 3 & 1 & 5 & 3 & 2 \\
\hline 1. 92 & 3 & 2 & 2 & 2 & 1 & 3 & & 3 & 3 & 3 & 3 & 5 & 1 & 3 \\
\hline 2.34 & 2 & 1 & 2 & 1 & 1 & 6 & 2 & 4 & 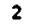 & 3 & 3 & 1 & 3 & 1 \\
\hline 2.673 & 2 & 1 & & 3 & & 6 & & 3 & & & & & 3 & 3 \\
\hline $2.752 \mathrm{E}-03$ & 2 & 1 & 2 & 2 & & 1 & & 4 & 2 & 2 & 3 & 5 & 3 & 4 \\
\hline 2.83 & 2 & 2 & 3 & 2 & 2 & 3 & & 3 & 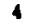 & 3 & 3 & $-1+2$ & 1 & 2 \\
\hline 3.04 & 2 & 1 & 3 & 2 & 1 & 6 & 3 & 5 & 1 & 3 & & 2 & 3 & 2 \\
\hline 3.1 & $\lambda$ & 2 & 3 & 3 & 2 & 2 & S & 3 & & & 3 & 1 & 3 & 2 \\
\hline $3.5488-03$ & 2 & 1 & 2 & 2 & 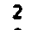 & 6 & 4 & 3 & 3 & 2 & 5 & 1 & 1 & 1 \\
\hline 3.62 & 2 & 2 & 2 & 3 & 2 & 5 & 3 & 4 & 2 & 2 & 1 & 4 & 3 & , \\
\hline 1.0 & 2 & 2 & 3 & - 12 & 1 & 6 & $\varepsilon$ & 3 & & & & 2 & & . \\
\hline $4.0228-0$ & 2 & 2 & 3 & 2 & & 1 & & 4 & & 3 & 4 & 1 & 2 & 2 \\
\hline 4.11 & 2 & 2 & 2 & 2 & 3 & 4 & & 3 & 3 & 2 & 3 & 2 & 4 & 2 \\
\hline $1.1598-$ & 2 & 2 & 3 & 3 & 1 & 1 & 5 & 1 & 2 & 2 & 4 & $?$ & 2 & 2 \\
\hline $4.8568-$ & 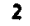 & 1 & 2 & 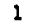 & 2 & 3 & 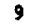 & - $+2+$ & 3 & 3 & 2 & 3 & 5 & 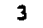 \\
\hline 5.40 & 2 & 1 & 2 & 2 & & 2 & & 5 & $=$ & 3 & 3 & 5 & 4 & 4 \\
\hline 5.7 & 2 & 1 & 2 & 1 & 2 & 6 & 3 & 3 & 4 & 2 & 2 & 4 & 2 & 1 \\
\hline 50 & 2 & 1 & 2 & 2 & & 6 & & 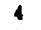 & 4 & & 1 & 3 & 2 & 2 \\
\hline 6.06 & 1 & 2 & 2 & 2 & 3 & 4 & 1 & $*$ & 2 & 2 & 4 & 5 & 3 & 4 \\
\hline 6.19 & 3 & 2 & 2 & 1 & 3 & 2 & & 5 & 3 & 2 & 4 & 2 & 2 & 2 \\
\hline $6.324 E-03$ & 2 & 2 & 2 & 1 & 2 & 5 & 4 & 5 & 5 & 4 & 1 & 1 & 3 & 1 \\
\hline $69 ? 0$ & 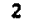 & 1 & 3 & 1 & 2 & 2 & 9 & 3 & 4 & 2 & 3 & 3 & 4 & 3 \\
\hline 7.3618 & 2 & 1 & 2 & 2 & & 5 & & 4 & 2 & & 4 & $\mathbf{5}$ & 3 & 2 \\
\hline 7.60 & 1 & 1 & 3 & 1 & 3 & 3 & 6 & 5 & 3 & 3 & 3 & 3 & 4 & 3 \\
\hline $6 \mathrm{E}$ & 2 & 2 & 3 & 1 & & 1 & & 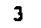 & 3 & 1 & 4 & 2 & 5 & 3 \\
\hline
\end{tabular}


NUREG/CR-4551, VOL. 1: DRAFT REPORT FOR COMMENT (SEPTEMBER, 1986)

\begin{tabular}{|c|c|c|c|c|c|c|c|c|c|c|c|c|c|c|c|c|c|c|c|c|c|c|c|c|c|c|c|}
\hline $62 z-03$ & 2 & 1 & 2 & 1 & 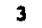 & 6 & 9 & 3 & 5 & 1 & 5 & 3 & 1 & 2 & 1 & 2 & 3 & 1 & 2 & 1 & 1 & 2 & 1 & 3 & & & 4 \\
\hline$B-03$ & 2 & 1 & 2 & 1 & 2 & 6 & 8 & 3 & 3 & 1 & 4 & 5 & 1 & 1 & 3 & 4 & & 1 & 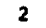 & 3 & 2 & 3 & 2 & & & & \\
\hline $32 \mathrm{E}-03$ & 2 & 1 & 2 & 2 & 3 & 6 & 5 & 4 & 6 & 2 & 6 & 1 & 3 & 2 & 2 & 3 & 2 & 1 & 2 & 1 & 2 & 3 & 3 & & & & 2 \\
\hline 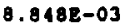 & 2 & 2 & 3 & 3 & 2 & 4 & 7 & 3 & 3 & 2 & 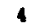 & 2 & 3 & 3 & 3 & 2 & & 1 & 3 & 1 & 2 & 2 & 3 & 3 & & & \\
\hline-03 & 2 & 2 & 2 & 2 & 3 & 2 & 4 & 4 & 6 & 1 & 6 & 2 & 3 & 1 & 3 & 3 & & 1 & & 2 & 2 & & 3 & & & & \\
\hline 2 & 2 & 1 & 2 & 1 & 2 & 3 & 1 & 5 & 3 & 1 & 4 & 5 & 2 & 1 & 4 & 2 & 3 & 1 & 3 & 3 & 3 & 3 & 3 & & & & \\
\hline 12 & 2 & 1 & 2 & 1 & 3 & 6 & a & 5 & $\mathbf{3}$ & 1 & 6 & 4 & 2 & 1 & 2 & 1 & & 1 & & 3 & & 3 & 2 & & & & \\
\hline$z-02$ & 2 & 1 & 3 & 2 & 1 & 3 & 6 & 5 & 4 & 2 & 4 & 5 & 3 & 2 & 1 & 2 & & 1 & 2 & 1 & 2 & 3 & 3 & & & & \\
\hline 22 & 2 & 1 & 2 & 1 & 1 & 6 & 1 & 4 & 5 & 2 & 3 & 5 & 2 & 3 & 3 & 4 & & 1 & & 1 & 1 & 3 & 1 & & & & \\
\hline$E-02$ & 2 & 2 & 2 & 2 & 1 & 4 & 3 & 3 & 3 & 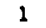 & 6 & 1 & 4 & 2 & 2 & 3 & & 1 & & 1 & 3 & 3 & 3 & & & & \\
\hline-02 & 2 & 1 & 3 & 2 & 1 & 3 & 1 & 4 & 3 & 2 & 4 & 1 & 3 & 2 & 1 & 3 & & 1 & & 3 & 2 & 3 & 1 & & & & \\
\hline-02 & 2 & 2 & 2 & 1 & 3 & 3 & 8 & 5 & 2 & 2 & 4 & 2 & 5 & 2 & 3 & 2 & 3 & 1 & & 4 & 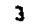 & & 3 & & & & \\
\hline$E-02$ & 1 & 2 & 3 & 1 & 1 & 6 & 1 & 4 & 2 & 1 & 6 & 4 & 1 & 2 & 1 & 3 & 4 & 2 & & 4 & 1 & 2 & 3 & & & & \\
\hline & 2 & 2 & 2 & 3 & 1 & 3 & 3 & 5 & 2 & 2 & 3 & 1 & 3 & 3 & 2 & 4 & 3 & 1 & & 1 & 3 & & 3 & & & & \\
\hline 02 & 2 & 2 & 3 & 1 & 1 & 4 & 8 & 1 & 5 & 1 & 5 & 2 & 3 & 1 & 2 & 3 & 2 & 1 & 2 & 4 & l & 3 & 2 & & & & \\
\hline 868 & 2 & 2 & 3 & & 1 & ? & 3 & ? & 6 & & 4 & $=$ & 5 & 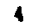 & 4 & 2 & & 1 & & 4 & 3 & & 2 & & & & \\
\hline $.8938-02$ & 3 & 2 & 2 & 2 & 2 & 1 & 6 & & 2 & $\downarrow$ & & 1 & 3 & & 2 & 2 & & 1 & & 2 & 1 & & 2 & & & & \\
\hline
\end{tabular}




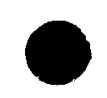

CONSEOUENCE HEASURE:

CANCER DEATHS RER YEAR

BASE CASE :

SURRY LALHC (CRAC)

FRACIIONAL CONTRIBUTION OF EACH SEQUENCE TO MEAN RISK

$\begin{array}{lr}\text { SEQUENCE } & \text { CONTRIBUTION } \\ \text { SYYB } & 0.1347 \\ \text { SYNI } & 0.0001 \\ \text { SNNN } & 0.4777 \\ \text { SYNN } & 0.0007 \\ \text { ANNN } & 0.0000 \\ \text { AYNN } & 0.0092 \\ \text { AYNI } & 0.0224 \\ \text { AYYB } & 0.0070 \\ \text { TNMN } & 0.1457 \\ \text { TYYBH } & 0.0627 \\ \text { IYYBD } & 0.0682 \\ \text { IYNI } & 0.0002 \\ \text { Y } & 0.0713\end{array}$

1 CONSEOUENCE MEASURE:

$$
0.0713
$$

CANCER DEATHS PER YEAR

BASE CASE :

SORRY LALHC (CRAC)

FRACTIONAL CONTRIBOTION OF EACH BIN TO MAN RISK

BIA COMTRIBOTIOA

BIN $1 \quad 0.0104$

$\begin{array}{ll}\operatorname{BIN} 1 & 0.0104 \\ \operatorname{BIN} 2 & 0.0020\end{array}$

$\begin{array}{ll}\text { BIN } 2 & 0.0020 \\ \text { SIN } 3 & 0.0071\end{array}$

BIN 0.0037

BIX $5 \quad 0.0325$

BIN $6 \quad 0.0000$

BIN $7 \quad 0.0010$

BIN 80.0010

BIN $9 \quad 0.007$

BIN $10 \quad 0.0029$

BIN 11

BIN $12 \quad 0.0755$

BIN 13 0.0000

BII $14 \quad 0.0005$

BIN $15 \quad 0.0003$

8IX $16 \quad 0.6114$

BIN $17 \quad 0.0760$

aIN $10 \quad 0.0360$

1 CONSEOUENCE MEASURE:

0.0805

CANCER DEATHS PRR YEAR

BASE CASE

SURRY LALHC (CRAC)

FRACTIONAL CONTRIBOTIONS OF EACH RELEASE TO MEAH RISK RELEASE

SOR 1 GP

SOR 2 GP

SUR $3 G E$

SUR 408

SUR TGP

$\begin{array}{ll}\text { SUR } & 5 G P \\ \text { SUR } & 6 G P \\ \text { SUR } & 1 G P\end{array}$

SOR TGP

SOR $A G P$

SUR 96

SUR 10G?

SUR $11 \mathrm{GP}$

SOR $12 \mathrm{GP}$

SUR $13 G ?$

SUR $14 G$

SOR $15 G R$

SUR $16 \mathrm{G}$

SOR $17 \mathrm{GP}$

SUR 180

SOR $196 P$

STR 200

SUR 2168

SUR 220

SUR 23GP

SUR 240

CONTRIBUTION

0.1442

0.1500

0.1157

0.1514

0.0854

0.0988

0.0665

0.0556

0.0017

0.0091

0.0395

0.0010

0.0250

0.0026

0.0181

0.0036

0.0147

0.0029

0.0083

0.0011

0.0029

0.0002

0.0002

0.0019

SUR $25 \mathrm{C}$

0.0003 
CONSEQUENCE MEASURE:

CANCER DEATHS PRR YEAR

SENSITIVITY OF RISK TO ISSTES

DISTRIBOTION OF RISK AT EACH LEVEL OF BACH ISSOB BASE CASE :

SURRY LALHC (CRAC)

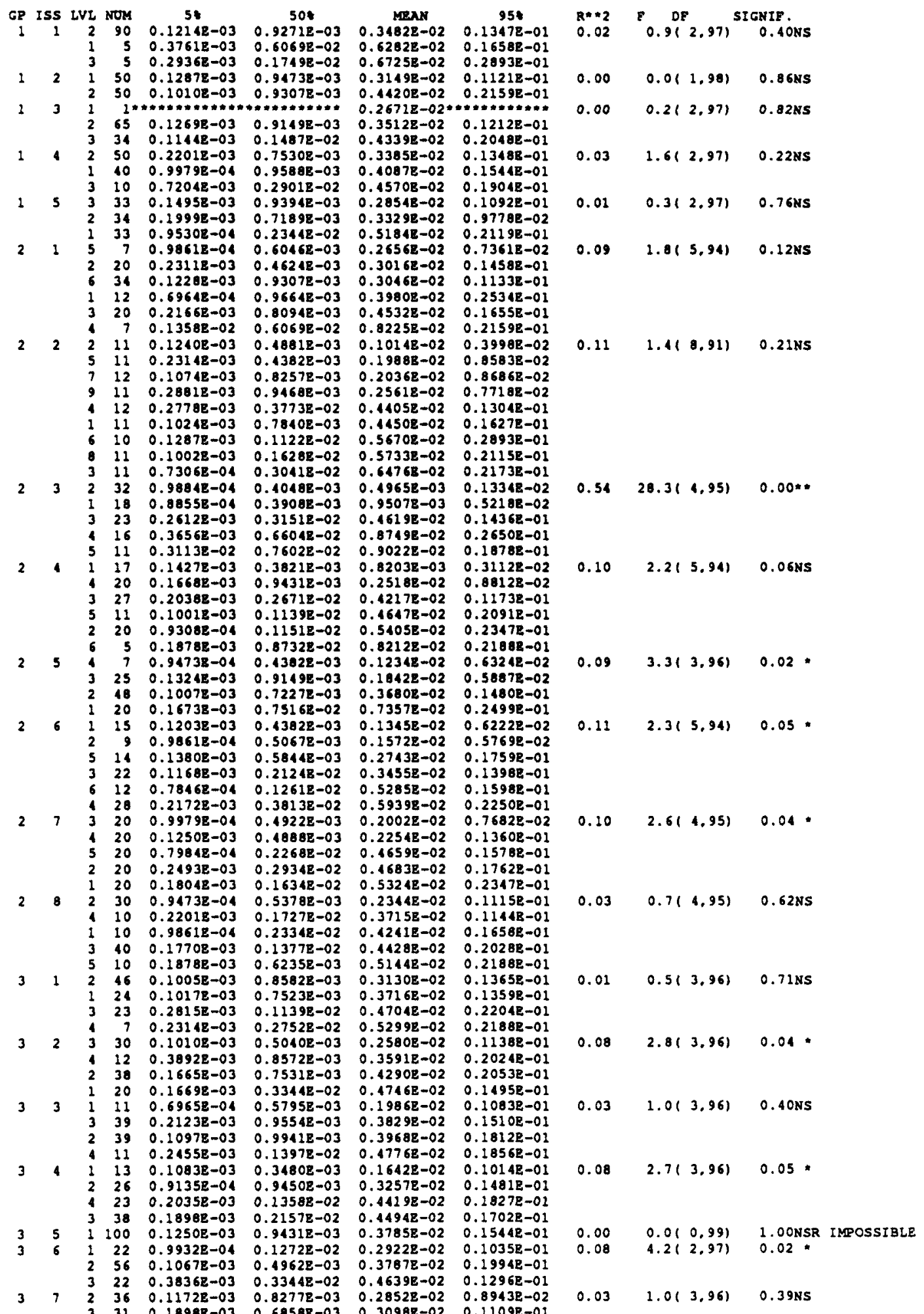

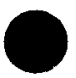


NUREG/CR-4551, VOL. 1: DRAFT REPORT FOR COMMENT (SEPTEMBER, 1986)

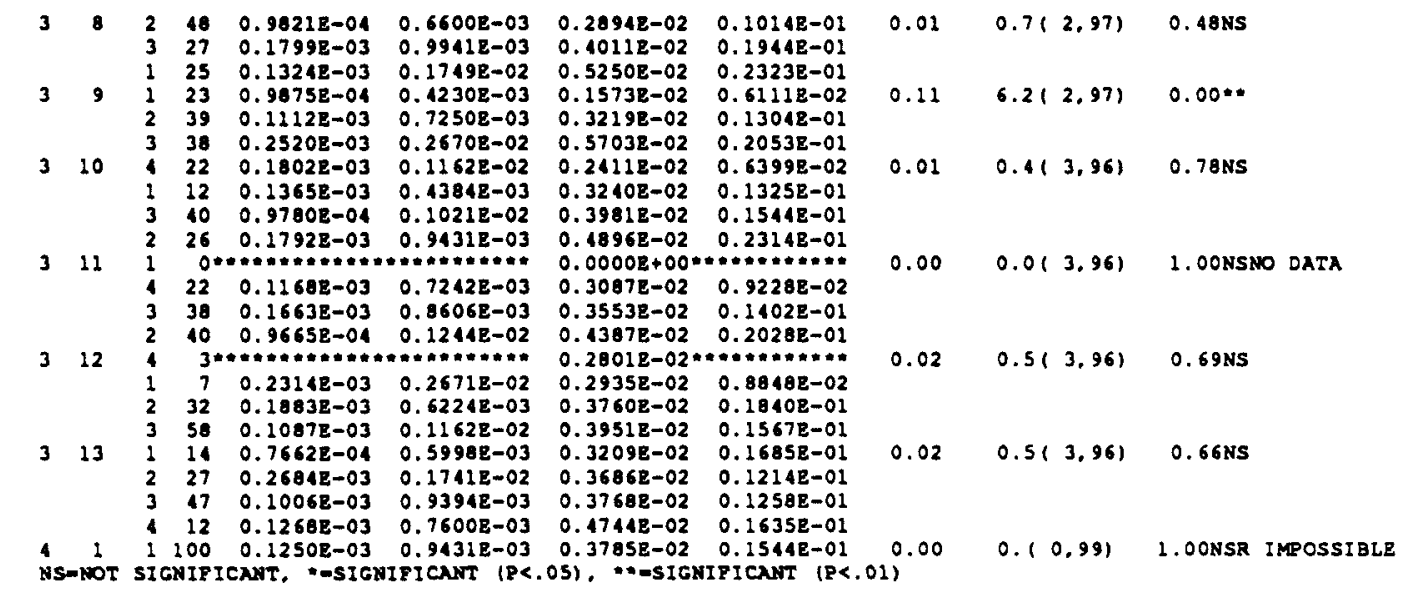


CONSROUENCE MEASORE: CANCER DEATHS PER YEAR

LEVEL 1 No. <MED.- 2 NO. EXP.- 2 LEVEL 2 NO. LAED. - 15 พO. EXP. - 45

No.>AED.- 3 No. $\exp .=3$ NO.>MRD. = 45 No. EXP. $=45$

NO.> LEVEL 3 NO. LMED.- 2 NO. $\exp ,-2$

OVERALL CHI-SO- 0.0 OF- 1 SIG. 1.000
LLVEL 1 No. <MED.- 6 NO. EXP.- 5

LEVEL 2 No. LYED. - o NO. EXP.- $s$

LEVEL 3 NO. <MEO. = 3 NO. EXP. = 5

LEVEL 4 M. <LED. = 3 NO. EXP.- 6

LEVEL 5 MO. $\angle M E D .=8$ NO. $\operatorname{eXP}=5$

LEVEL 6 NO. SMED.- 5 NO. EXP." 5
CHI-SO. TEST CANNOT BE RUN; EXP. NO. < 5 CHI-SQ.- 0.0 DF= 1 SIG $=1.000$ CHI-SO. TEST CANNOT BE RON; EXP. NO. < 5

CHI-5O.= 0.1 DF- 1 SIC. $=0.777$

CHI-SO.- 0.0 DF- 1 SIG. $=1.000$

CHI-5Q. TEST CANNOT BE RON; EXP. NO. < 5 CHI-SO. = 0.1 DF- 1 SIG. $=0.804$ CHI-SO.- 0.1 DF $=1$ SIG. $=0.732$

CHI-SQ. $\quad 0.0$ DF- 1 SIG. $=1.000$ CHI-SO. $=0.7 \mathrm{DF}=1$ SIG. $=0.396$ CHI-SO.= 6.4 DF= 1 SIG.- 0.011

CHI-SQ. $=0.1$ DF- 1 SIG. $=0.728$ CHI-SO.- 0.1 DF $=1$ SIG. $=0.732$ CHI-SO.= 0.0 DF 1 SIG. 1.000

CHI-SO.= 0.3 DF 1 SIG. $=0.564$ CHI-SQ.- 1.8 DF- 1 SIG. 0.180 CHI-SO.= 0.0 DF 1 SIG. $=1.000$ CHI-SQ. TEST CANNOT BE RON; EXP. NO. < 5 CHI-SO. TEST CANNOT BE RUN; EXP. NO. < 5 CHI-SQ.- 0.0 DF= I SIG. $=1.000$

CHI-SO. $=0.4 \mathrm{DF}=1$ SIG. $=0.545$

CHI-SO. $\quad 3.3$ DE- 1 SIG. $=0.069$

CHI-SO. $=1.5$ DF 1 SIG. $=0.226$

CHI-SQ. 3.0 DF= 1 SIG. $=0.083$

CHI-5Q $=3.3$ DF 1 SIG $=0.069$

CHI-SQ. $=0.0$ DP= 1 SIG. $=1.000$ 


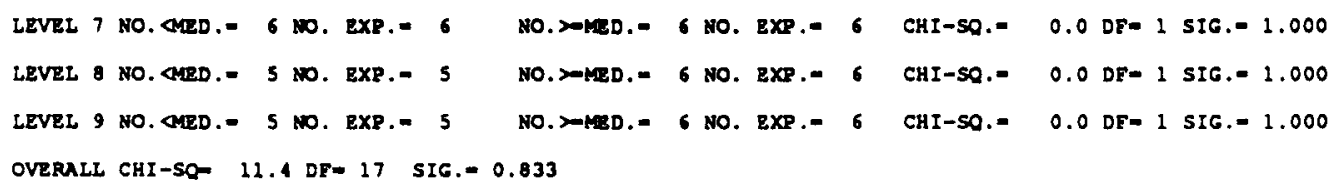




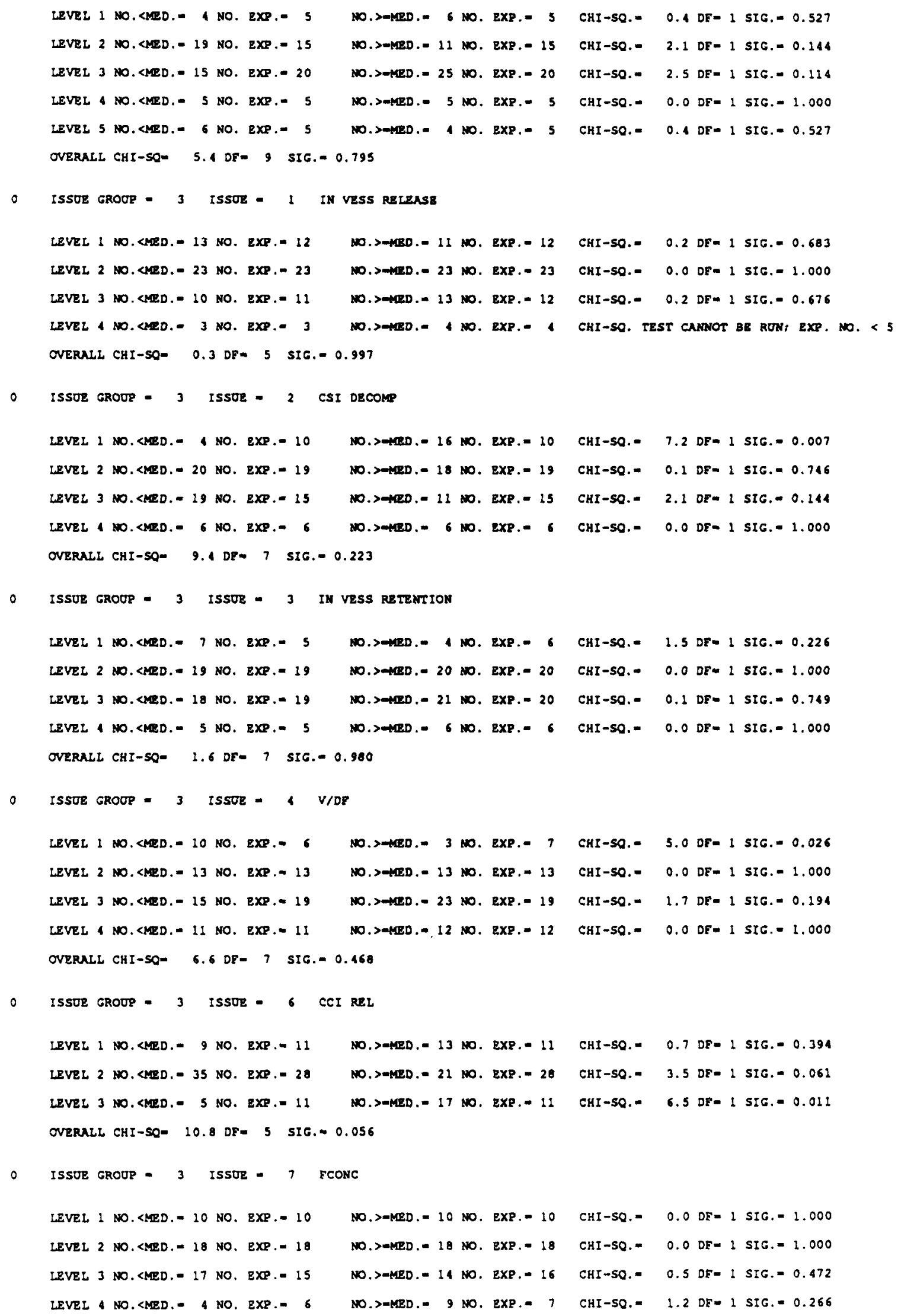

CHI-SQ. $=0.7$ DF= I SIG. $=0.394$ CHI-SO.= 3.5 DE= 1 SIG. $=0.061$ CHI-SQ.= 6.5 DF- 1 SIG. $=0.011$

CHI-SO $=0.0 \mathrm{DE}=1$ SIG $=1.000$

CHI-SO. 0.0 DF= 1 SIG. $=1.000$

CHI-SO. $=0.5$ DF 1 SIG. $=0.472$

CHI-SQ. $=1.2$ DE $=1$ SIG. $=0.266$ 


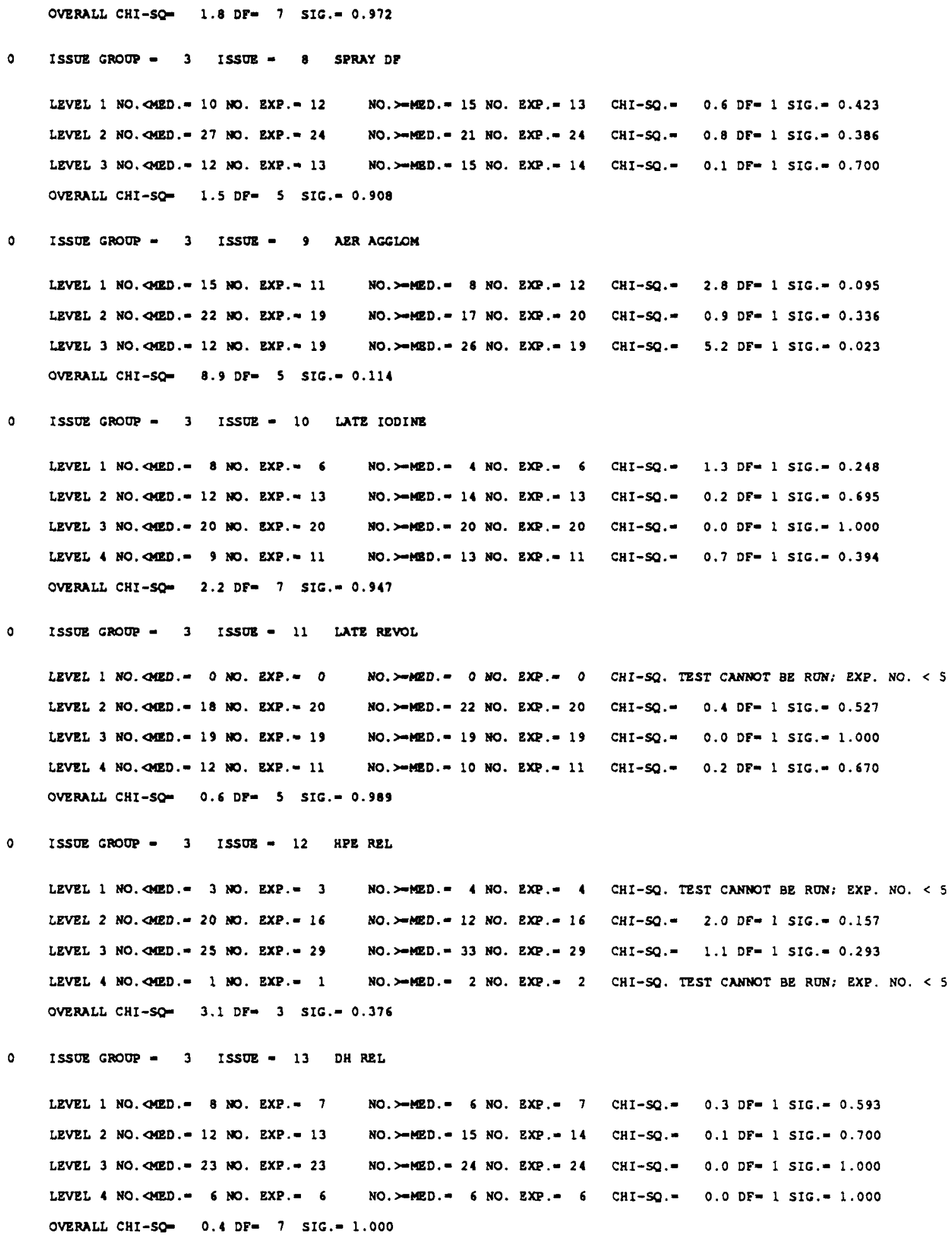

ISSOE GROOP - 3 ISSUE - 13 DH REI

LEVEL 1 NO. GED.- 8 No. EXP.= 7

NO. X-KD. $=6$ NO. BXP.- 7 LEVEL 2 NO. QRD. - 12 NO. EXP.= 13 No. >-KED. - 15 No. EXP. - 14 LEVEL 3 NO. AED. = 23 NO. EXP. $=23$ NO. $>$ KED. $=24$ No. EXP. -24 LEVEL \&O. ARED. 6 NO. EXP.= 6 NO. $>M E D .=6$ NO. EXP. $=6$ CHI-SQ.- $1.3 \mathrm{DF}=1$ SIG.= 0.248 CHI-SO.- 0.2 DF= 1 SIG. $=0.695$ CHI-SO.= 0.0 DF- 2 SIG.= 1.000 CHI-SO.= 0.7 DF- 1 SIG.= 0.394 CHI-SQ. TEST CANNOT BE RUN; EXP. NO. < 5 CHI-SO.- 0.4 DF- 1 SIG.= 0.527 CHI-SO.= 0.0 DF + SIG. $=1.000$ CHI-SO. $=0.2$ DF- 1 SIC. $=0.670$

CHI-SO. TEST CANNOT BE RUN: EXP, NO. < 5 CHI-SO.= 2.0 DF- 1 SIG. $=0.157$ CHI-SQ.= 1.1 DF= 1 SIG.= 0.293 CHI-SO, IRST CNNNOT BE RUN; EXP. NO. < 5 OVERALL CHI-SO- 0.4 DFE 7 SIG.= 1.000 
CONSEQUENCE MEASURE:

POP. DOSE 50 MI PER YR

BASE CASE

SURRY LALHC (CRAC)

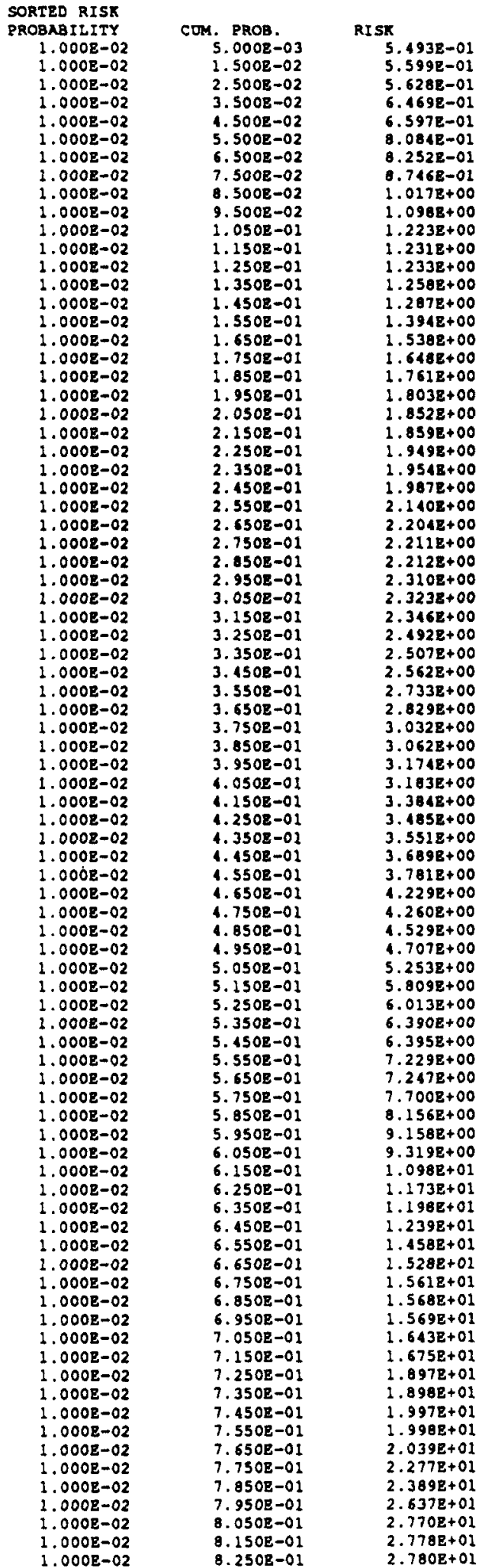


NUREG/CR-4551, VOL. 1: DRAFT REPORT FOR COMMENT (SEPTEMBER, 1986)

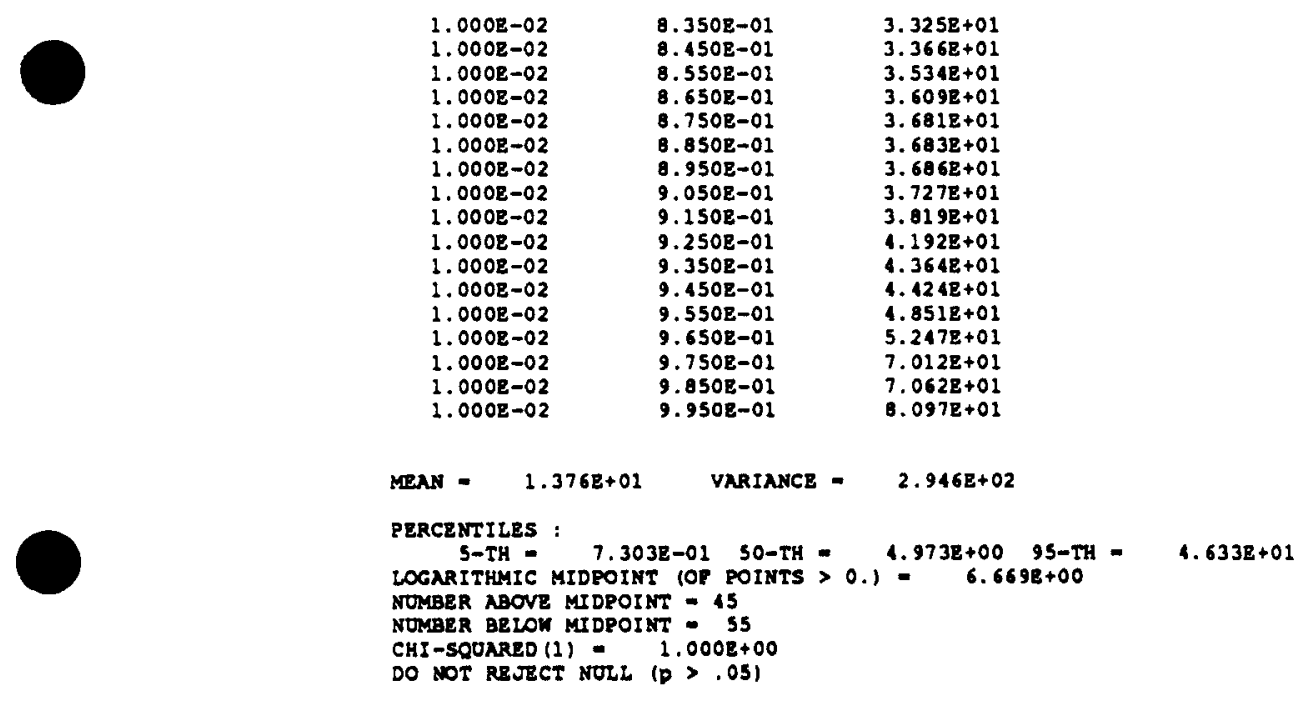


NUREG/CR-4551, VOL. 1: DRAFT REPORT FOR COMMENT (SEPTEMBER, 1986)

CONSEOUENCE MEASURE

POP. DOSE $50 \mathrm{MI}$ PER YR

BASE CASE :

SURRY TALHC (CRAC)

ISSOE LEVELS FOR EACH SARLE MEMER, IN ORDER OF INCREASING RISK

RISR ISSUE LEVEIS, FOR ALL ISSUES

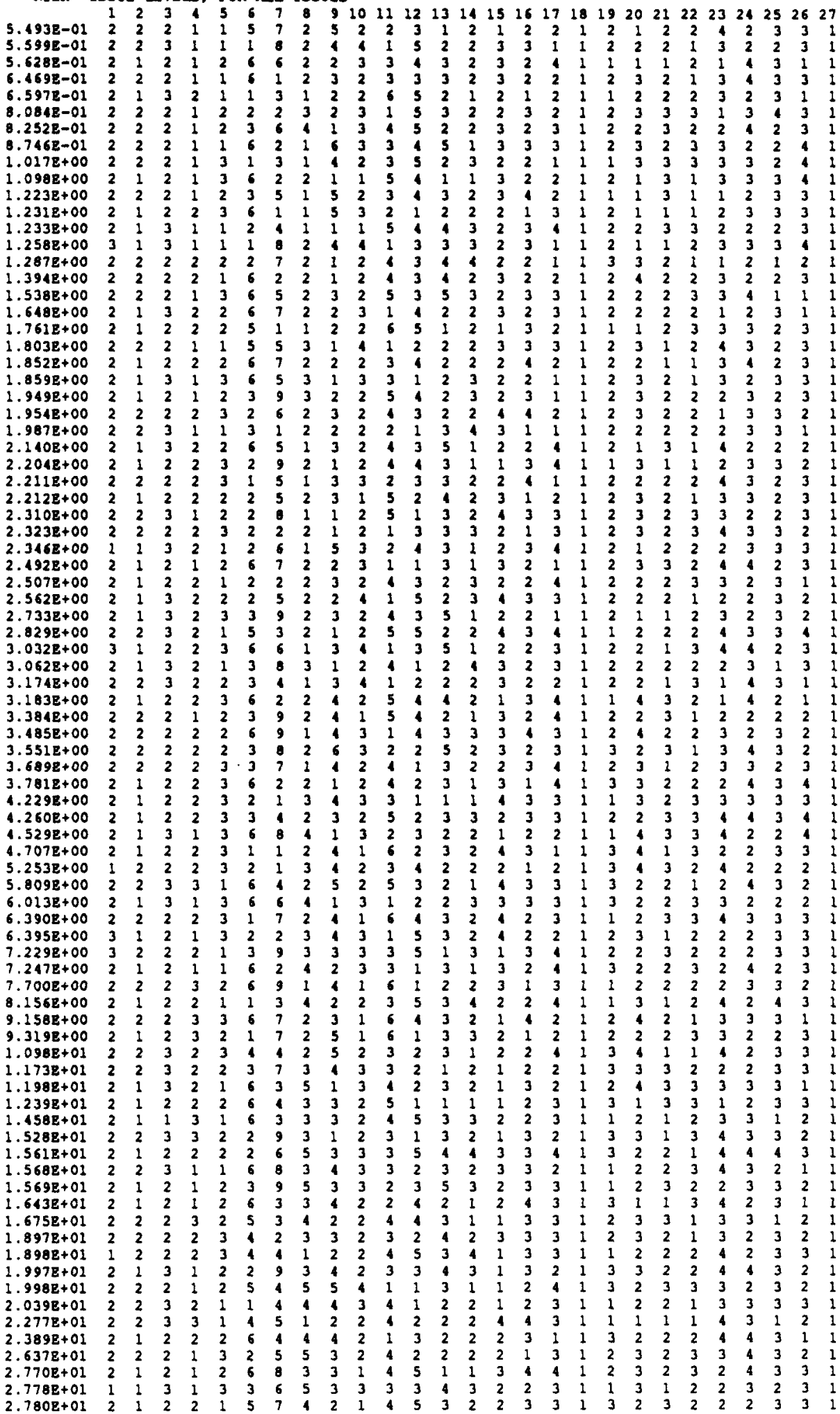


NUREG/CR-4551, VOL. 1: DRAFT REPORT FOR COMMENT (SEPIEMBER, 1986)

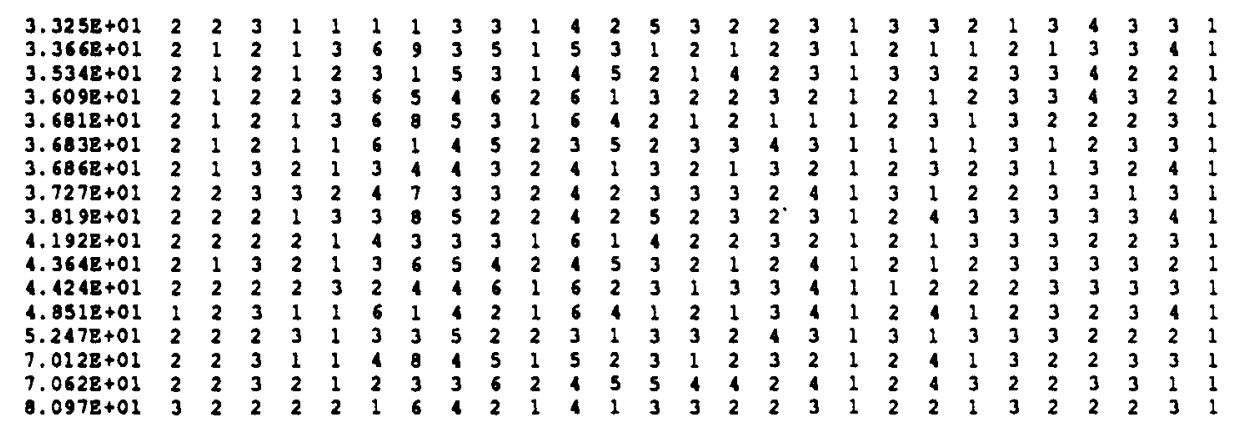


CONSEQUENCE MRASURE:

POR. DOSE 50 MI RER YR

BASE CASE :

SURRY LALHC (CRAC)

FRACTIONAL CONTRIBOTION OF EACH SEOOENCE TO MEAN RISK

$\begin{array}{lr}\text { SEQUENCE } & \text { CONTRIBOTION } \\ \text { SYYB } & 0.1959 \\ \text { SYNI } & 0.0001 \\ \text { SNNA } & 0.3922 \\ \text { SYNN } & 0.0007 \\ \text { ANRA } & 0.0000 \\ \text { AYNI } & 0.0085 \\ \text { AYNI } & 0.0211 \\ \text { AYYB } & 0.0142 \\ \text { INNA } & 0.1301 \\ \text { TYYBN } & 0.0586 \\ \text { TYYBD } & 0.0660 \\ \text { IYYI } & 0.0002 \\ \text { Y } & 0.1112\end{array}$

1 CONSEOUENCE MEASTRE:

POP. DOSE 50 MI PER YR

BASE CASE :

SORRY LALHC (CRAC)

FRACTYONAL CONTRIBUTION OR BACH BIN TO MRAN RISR

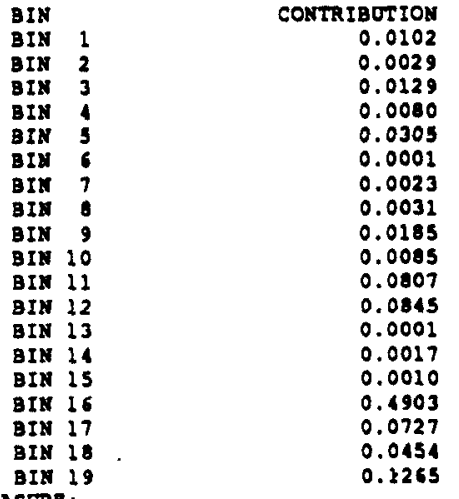

1

CONSEOUENCE MEASORE:

POR, DOSE 50 MI PER YR

BASE CASE :

SORRY LALHC (CRAC)

FRACTIONAL CONTRIBUTIONS OF EACH RELEASE TO MEAN RISK RELEASE

SOR IGP

SUR $2 G P$

SUR 3GP

SOR IGP

SUR SGE

SOR GGP

SUR 7GP

SUR OGP

SUR $10 G P$

SOR $11 G P$

SUR $12 G 2$

SOR I3GR

SOR $14 G$

SOR 15GP

SUR $16 G$

SUR $17 G P$

SOR $10 \mathrm{G}$

SOR $19 \mathrm{GP}$

SUR 206

SUR 21GP

SOR $22 G$

SUR $236 P$

SUR 246

CONTRIBUTION

0.0983

0.1050

0.0828

0.1257

0.0824

0.1009

0.0743

0.0742

0.0034

0.0135

0.0625

0.0021

0.0450

0.0450

.0066

0.0354

0.0106

0.0317

0.0059

0.0199

0.0031

0.0031

0.0006

0.0055

0.0017 


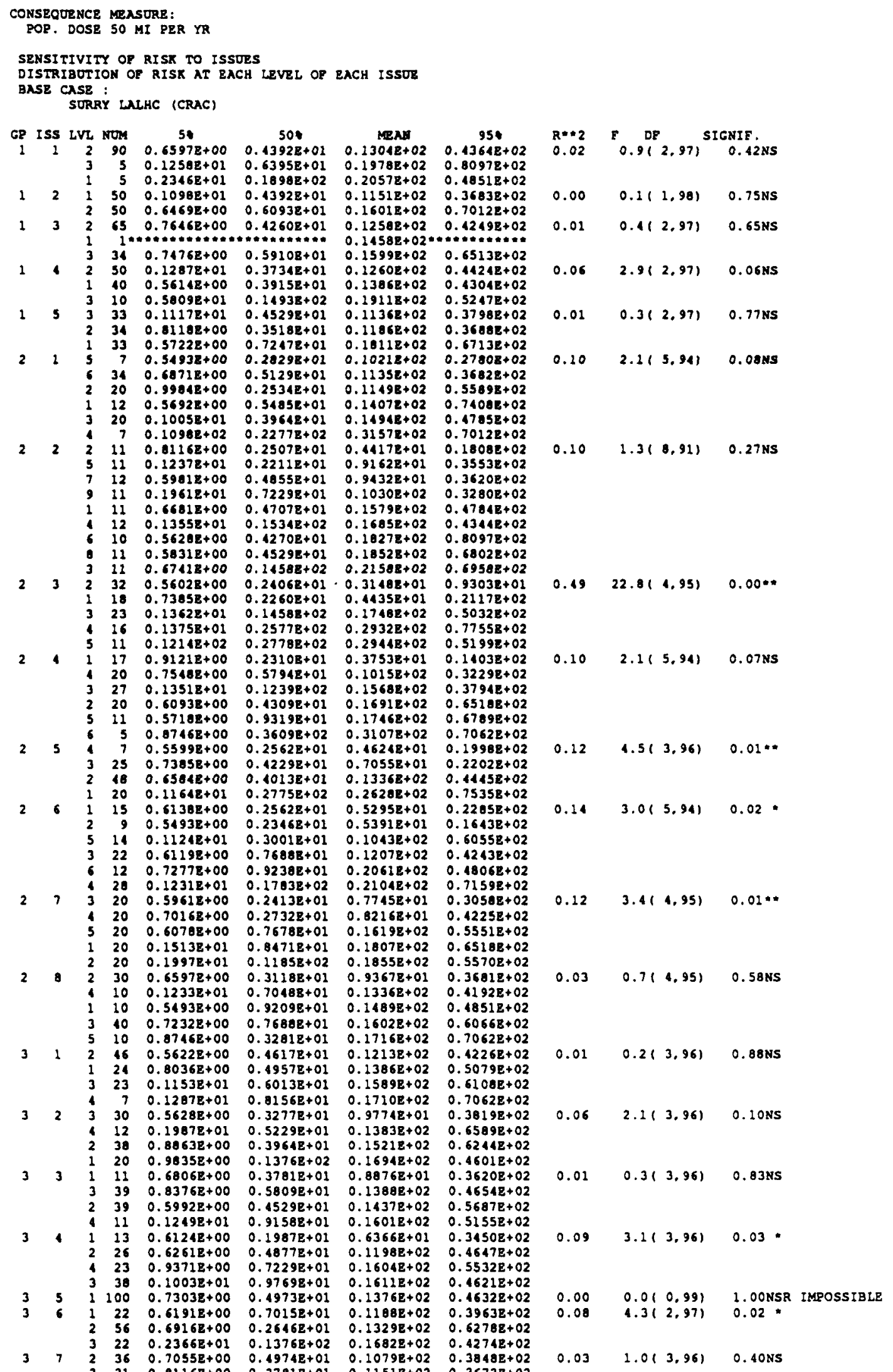


NUREG/CR-4551, VOL. 1: DRAFT REPORT FOR COMMENT (SEPTEMBER, 1986)

\begin{tabular}{|c|c|}
\hline 3 & 8 \\
\hline \multirow{2}{*}{3} & 9 \\
\hline & \\
\hline \multirow[t]{3}{*}{3} & 10 \\
\hline & \\
\hline & \\
\hline \multirow[t]{3}{*}{3} & 11 \\
\hline & \\
\hline & \\
\hline \multirow[t]{2}{*}{3} & 12 \\
\hline & \\
\hline 3 & 13 \\
\hline & \\
\hline & \\
\hline
\end{tabular}

$0.11508+02 \quad 0.37878+02$

$0.13638+02$

$0.1022 \mathrm{E}+02$

$20.7269 E+02$

$0.78048+010.72598+02$

$0.12158+02 \quad 0.46548+02$

$0.19018+02$ 0.62448+02

$0.93438+01 \quad 0.23218+02$

$0.11138+02 \quad 0.3685 z+02$

$0.1509 \mathrm{E}+02 \quad 0.1632 \mathrm{E}+02$

$\begin{array}{ccccc}10 & 0.6533 \mathrm{E}+00 & 0.5063 \mathrm{E}+01 & 0.1509 \mathrm{E}+02 & 0.1632 \mathrm{E}+02 \\ 26 & 0.1137 \mathrm{E}+01 & 0.6095 \mathrm{E}+01 & 0.1665 \mathrm{E}+02 & 0.7258 \mathrm{E}+02\end{array}$

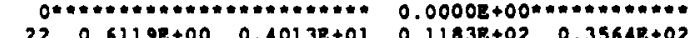

$\begin{array}{lllll}38 & 0.1049 \mathrm{E}+01 & 0.5199 \mathrm{E}+01 & 0.1391 \mathrm{E}+02 & 0.4400 \mathrm{E}+02\end{array}$

3e.

$\begin{array}{lllll}32 & 0.8300 \mathrm{E}+00 & 0.3106 \mathrm{E}+01 & 0.1244 \mathrm{E}+02 & 0.5131 \mathrm{z}+02\end{array}$

$\begin{array}{lllll}7 & 0.12878+01 & 0.14588+02 & 0.13898+02 & 0.37278+02\end{array}$

$\begin{array}{lllll}58 & 0.65208+00 & 0.67998+01 & 0.1475 E+02 & 0.4675 E+02\end{array}$

$1140.5810 \mathrm{E}+00 \quad 0.3178 \mathrm{E}+01 \quad 0.1164 \mathrm{E}+02 \quad 0.5686 \mathrm{E}+02$

$\begin{array}{llllll}17 & 0.63308+00 & 0.47078+01 & 0.13788+02 & 0.47408+02\end{array}$

$\begin{array}{lllll}27 & 0.1836 \mathrm{E}+01 & 0.7700 \mathrm{E}+01 & 0.1438 \mathrm{~B}+02 & 0.14868+02\end{array}$

$\begin{array}{lllll}12 & 0.8879 \mathrm{E}+00 & 0.1013 \mathrm{E}+01 & 0.14748+02 & 0.47368+02\end{array}$

NS-NOT SIGNIFICANT. "OSIGNIFICANT (P<. 05)." "-SIGAIPICANT (P<.01)
0.02
$0.7(2.97$
$0.48 \mathrm{NS}$
0.07
$3.5(2.97$
$0.04 *$
0.02
$0.6(3.96)$
$0.64 \mathrm{NS}$
0.00
0.013 .961
1. OONSNO DATX
0.02
$0.8(3.96)$
$0.52 \mathrm{NS}$
0.03
$0.813 .96) \quad 0.48$ NS

0.010 .991 1.00KSR IKTOSSIBLE 
CONSEQUENCE GEASURE: POP. DOSE 50 MI PER YR

LEVEL 1 NO. QRED . 26 NO. EXP. $=25$

LEVEL 2 NO. QMRD. - 23 10. EXP. - 25

OVERALL CHI-SO 0.4 DP- 3 SIG.- 0.940

LEVEL 1 No. ARD.- 0 No. EXP.- 0

LEVEL 2 NO. QRE. - 33 แ0. EXP. - 32

LEVEL 3 NO. ARD. - 16 NO. EXP. - 17

OVERALL CHI-SO- 0.2 DF- 3 SIG.- 0.981

ISSUE GROUP - 1 ISSUE - I V FREO

LEVEL 1 NO. ARD. - 21 NO. ExP. - 20

LEVEL 2 NO. QED. $=28$ NO. EXP.- 25

LEVEL 3 NO. QRBD.- 0 No. EXP.- 5

OVERALL CHI-SO 10.8 DFW 5 SIG.- 0.055

LEVEL 1 NO. SMO . - is NO. EXP. - 16

LEVEL 2 NO. QRD. = 18 NO. EXP. - 17

LEVEL 3 NO. QRE. - 17 NO. EXP, - 16

OVERALL CHI-SO 0.7 DPO S SIG.= 0.982

ISSUE GROUP = 2 ISSUE - 1 IND. RCS FAIL.

ISSUE GROUP $=2$ ISSUE -2 MODE OF VB.
LEVEL 1 NO. QED.- 1 No. BXP.- 2

LEVEL 2 NO. QNED. - 46 NO. EXP. - 43

LEVEL 3 NO. QRE.- 2 No. EXP.- 2

OVERALI CHI-SO 0.0 DF- I SIC. $=0.833$

พO. -MBD.- 24 nO. EXP. -23

NO.>MED. - 27 NO. BXP.- 25

NO. >MED. - 19 NO. EXP. - 20

NO. TIED. - 22 NO. EXP. - 25

NO. >ABD. - 10 No. EXP.- 5

No. $>$ MED. - 19 พ0. EXP. -17

NO. YAED. - 16 NO. EXP. = 17

NO. -WED. = 16 NO. EXP. $=17$

CHI-SO. $=0.5$ OF- ISIG. $=0.486$

CHI-SQ. 0.1 DF= 1 SIG. $=0.732$

CHI-SO.= $\quad 0.1$ DP- 1 SIG. $=0.728$
CHI-SO. TEST CANNOT BE RON: EXP. NO. < 5

CHI-SO. TEST CANNOT BE RUN; EXP. NO. < 5 CHI-SQ. $=0.0$ DF 1 SIG.- 0.833
CHI-SO.= 0.1 DF- I SIG.- 0.777

CHI-SO.= 0.3 DE- 1 SIG. $=0.572$
CHI-SO.= 0.1 DFE 1 SIG.- 0.752

CHI-SO. 0.7 DF $=1$ SIG. $=0.396$

CHI-SO= 10.0 DF- I SIG. $=0.002$
NO. - YRD. = 7 NO. ExP.- 6

NO. >-MED. = NO. $\exp =10$

No. >ARD.- 9 NO, EXP.- 10

No. >MED. 7 NO. EXP. $=4$

NO. > WRD. - 3 NO. EXP.= 4

NO. >MED. 17 NO. EXP, - 17
CHI-SO.= 0.3 DF= 1 SIC. $=0.564$

CHI-SO.= 0.8 DF= 1 SIG. $=0.371$

CHI-SO. $\quad 0.2$ DF $=1$ SIG. $=0.655$

CHI-SO. TEST CANNOT BE RUN: EXP. NO. < 5 CHI-SQ. TEST CANNOT BE RON; EXP. NO. < 5 CHI-SO.= 0.0 DF= 1 SIG. $=1.000$
LEVEL 1 NO. QMED.- 5 NO. EXP. - 5

LEVEL 2 NO. QRD.- A NO. EXP.= 5

LEVEL 3 NO. QRE.= 3 NO. EXP.= 5

LEVEL 4 NO. GRD.- 3 NO. EXP. 6

LEVEL 5 NO. QRED.- 8 NO. EXP. $=5$

LEVEL 6 NO. ARE. = 5 NO. EXP.- 5

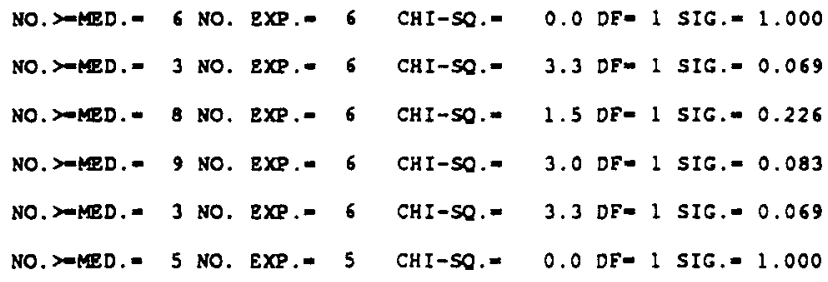




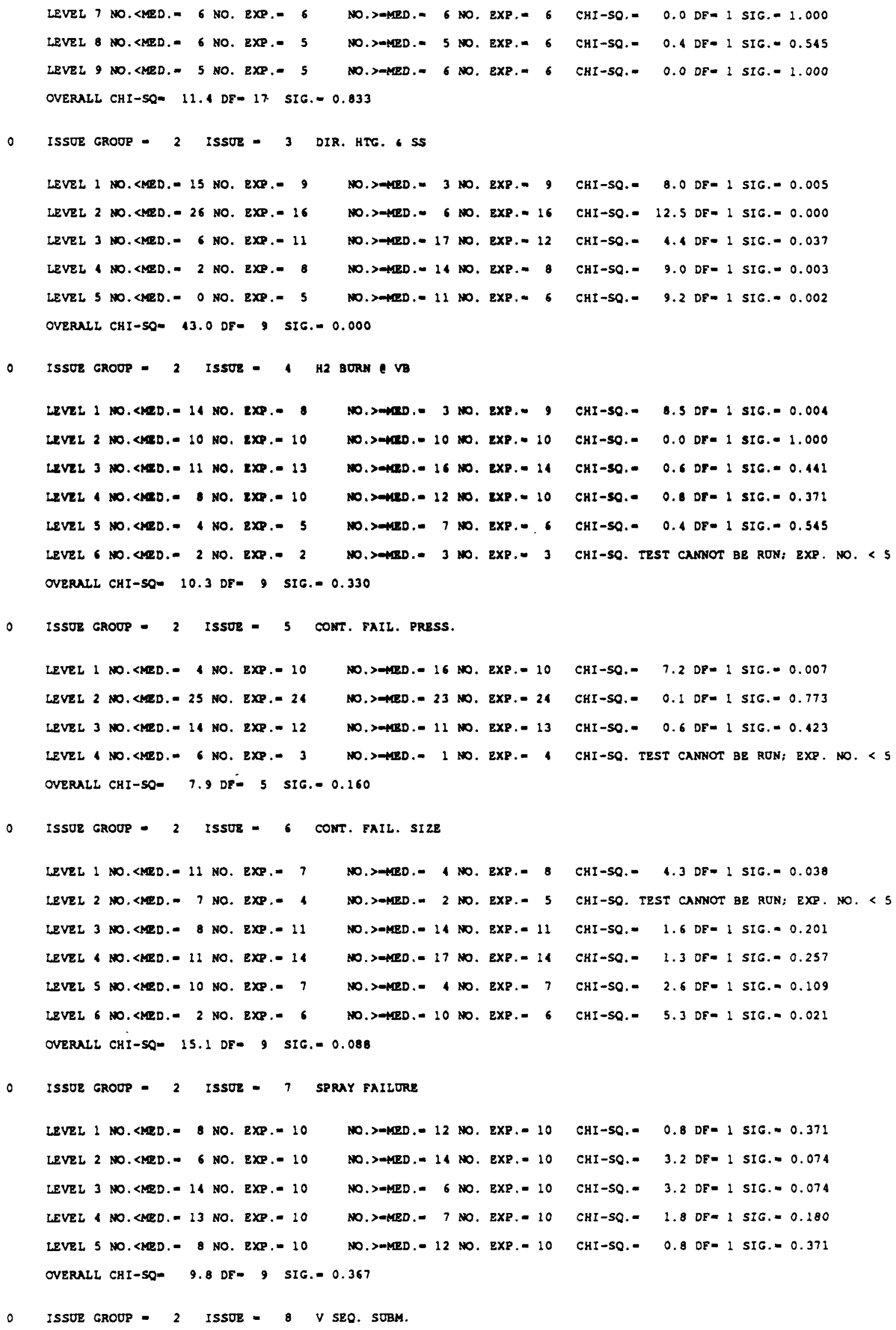

CHI-SO. $=0.0 \mathrm{DF}=1$ SIG. -1.000 CHI-SQ. $\quad 0.4$ DF= 1 SIG. $=0.545$ CHI-SQ.- 0.0 DF- 1 SIG.- 1.000

CHI-SO.- 12.5 DF $=1$ SIG. 0.000

CHI-SO.= 4.4 DF= 1 SIG.-0.037

CHI-SQ.- 9.0 DF= 1 SIG. $=0.003$ CHI-SO.= 9.2 DF -1 SIG. $=0.002$

CHI-5O.= B.5 DF- 1 SIG.=0.004 CHI-SO.- 0.0 DFE 1 SIG. 1.000 CHI-SO.= 0.6 DF- 1 SIC. $=0.441$ CHI-50.- 0.8 DT= 1 SIG.- 0.371 CHI-SO. $=0.4$ DF $=1$ SIG. $=0.545$ CHI-SQ. TEST CANNOT BE RON: EXP. NO. < S

CHI-5O, $=7.2$ DF= 1 SIG. $=0.007$

CHI-SO.- 0.1 DF= 1 SIG. $=0.773$

CHI-50.- 0.6 DF= 1 SIG. $=0.123$ CHI-SO. TEST CANHOT BE RON; EXP. NO. < 5

CHI-SO. - 4.3 DF- I SIG. 0.038 CHI-SO. TEST CANNOT BE RUN; EXP. NO. < 5

CHI-SQ.= 1.6 DF- 1 SIG. $=0.201$

CHI-SO. = 1.3 OF- 1 SIG. $=0.257$

CHI-SO. $\quad 2.6$ DF= 1 SIG.= 0.109 CHI-SO.= 5.3 DF- I SIG. 0.021

CHI-SO.- 0.8 DF- 1 SIG. 0.371

CHI-SQ.= 3.2 DF= 1 SIG. $=0.074$

$\mathrm{CHI}-\mathrm{SQ}=3.2 \mathrm{DF}=1 \mathrm{SIC}=0.074$

$C H I-5 Q .=1.8 \mathrm{DF}=15 \mathrm{SIG}=0.180$

CHI-SO.- 0.8 DF= 1 SIG.=0.371 


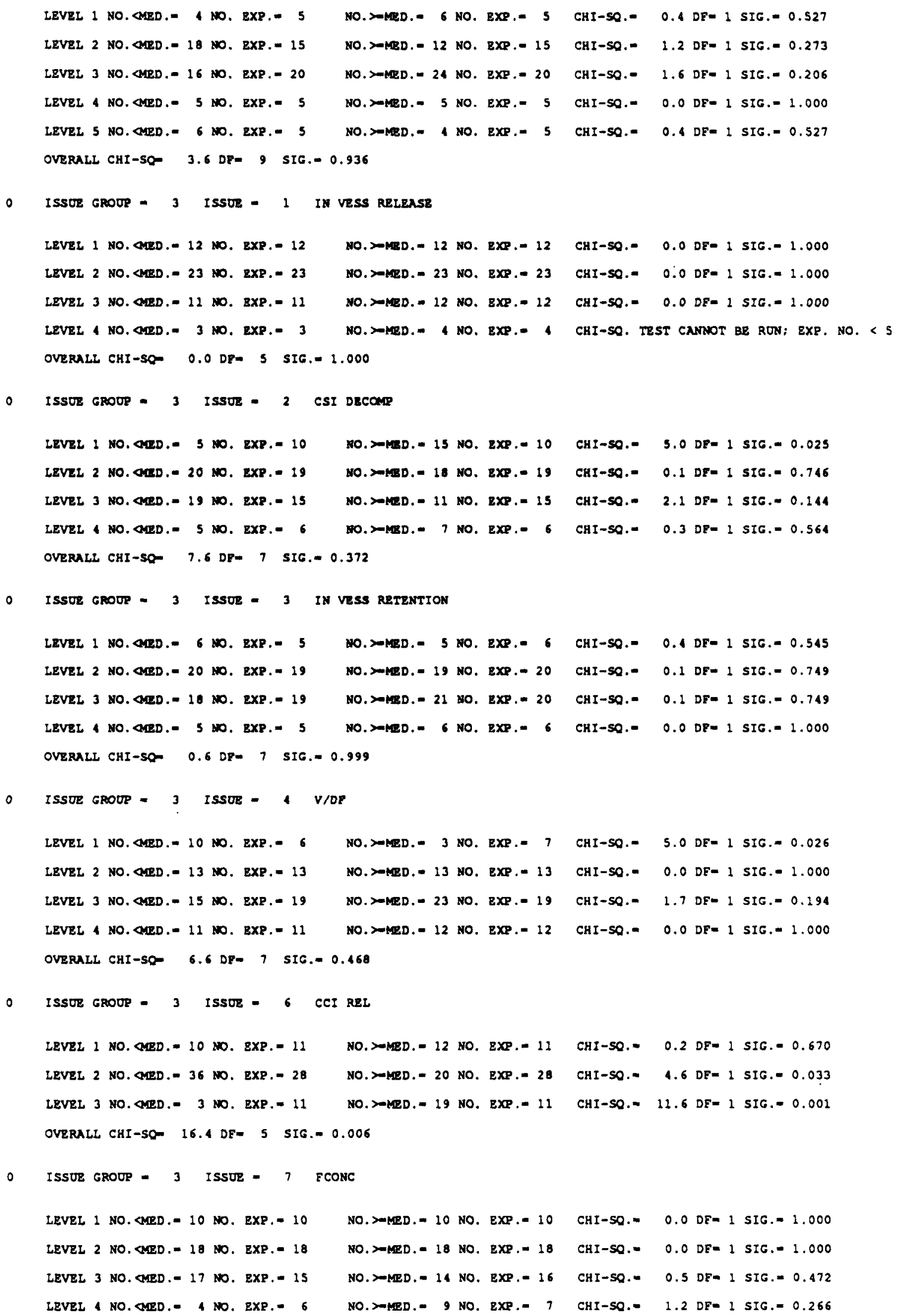

CHI-SO.- $\quad 0.4$ DF= I SIG. $=0.527$

CHI-SO.- $1.2 \mathrm{DF}=1$ SIG. $=0.273$

CHI-SO.= 1.6 DFE 1 SIG. $=0.206$

CHI-SO. $=0.0$ DF 1 SIG. $=1.000$

$\mathrm{CHI}-\mathrm{SO}=0.4 \mathrm{DF}=1 \mathrm{SIG}=0.527$

CHI-SO.= 0.0 DE- I SIG.- 1.000 CHI-SO.= 0.0 DF- 1 SIG $=1.000$ CHI-50.= 0.0 DF= 1 SIG. $=1.000$ CHI-SO. TEST CANNOT BE RON; EXP. NO. < 5

CHI-SO.- S.O DF= 1 SIG.=0.025 CHI-SQ.= 0.1 DF- 1 SIG.= 0.746 CHI-SO.= 2.1 DFm I SIG. $=0.144$ CHI-SO.= 0.3 DF= 1 SIG. $=0.564$

CHI-SO.- 0.1 DF- 1 SIG. $=0.545$ CHI-SO.- 0.1 DFE 1 SIG. $=0.749$ CHI-5O.= 0.1 DF= 1 SIG.=0.749 CHI-SO.- 0.0 DF- 1 SIG.= 1.000

CHI-SO.- S.O DF= 1 SIG.=0.026 CHI-SO.- 0.0 DF= 1 SIC. $=1.000$ CHI-SO.= 1.7 DF- I SIG. $=0.294$ CHI-SO.= 0.0 DF= 1 SIG. $=1.000$

CHI-SO. $=0.2 D F=1 S I G=0.670$ CHI-SO. 4.6 DF 1 SIG. $=0.033$ CHI-SO.= 11.6 DF= 1 SIG. $=0.001$

CHI-SO.= 0.0 DE= 1 SIG. $=1.000$ CHI-SO.- 0.0 DF= 1 SIG. -1.000 CHI-SO. $=0.5 \mathrm{DF}=1$ SIG $=0.472$ CHI-SO.= 1.2 DF= I SIG. $=0.266$ 


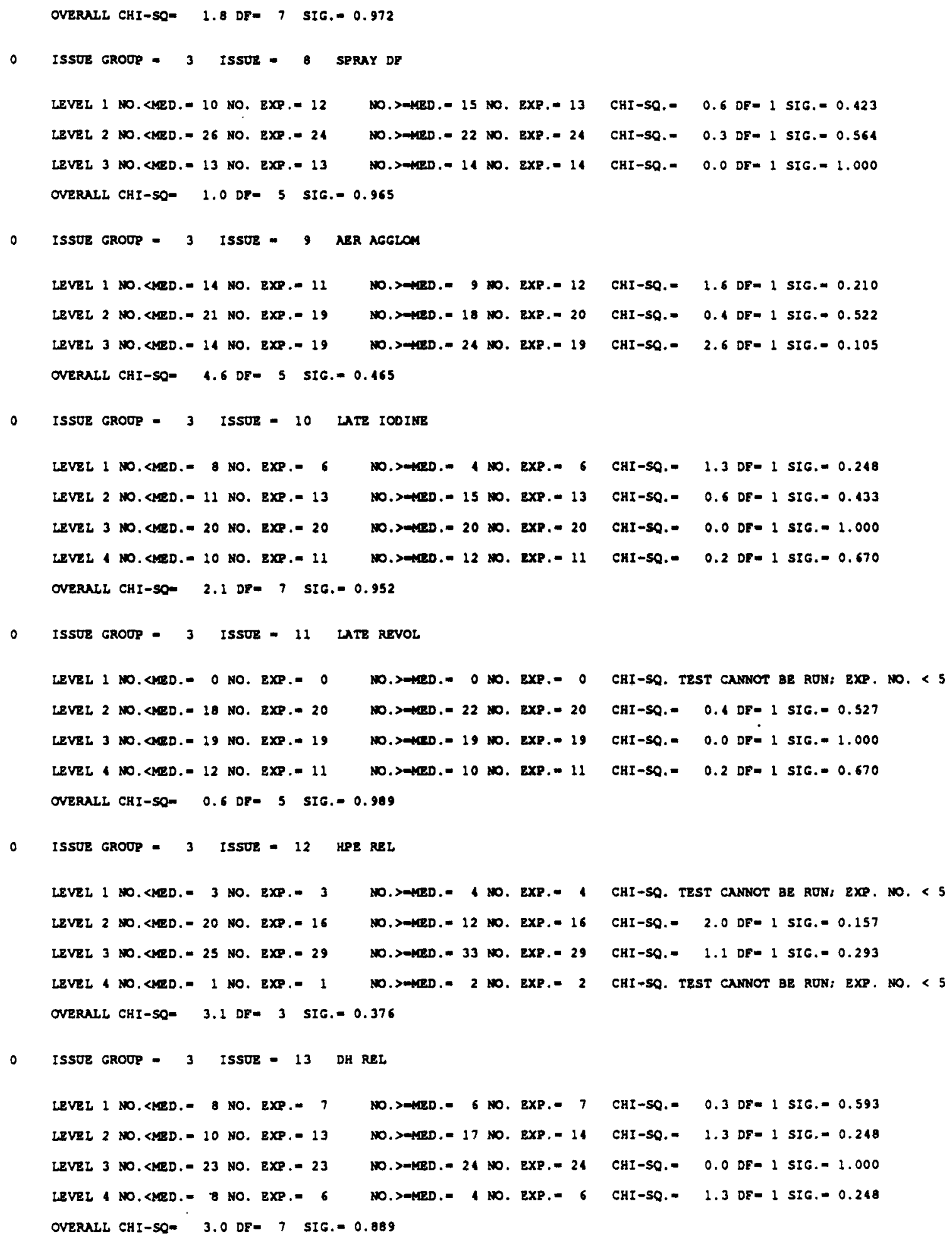


NUREG/CR-4551, VOL. 1: DRAFT REPORT FOR COMMENT (SEPTEMBER, 1986)

CONSEOUENCE GRASORE:

OFFSITE cOSTS PER YRAR

BASE CASE :

SURRY LALHC (CRAC)

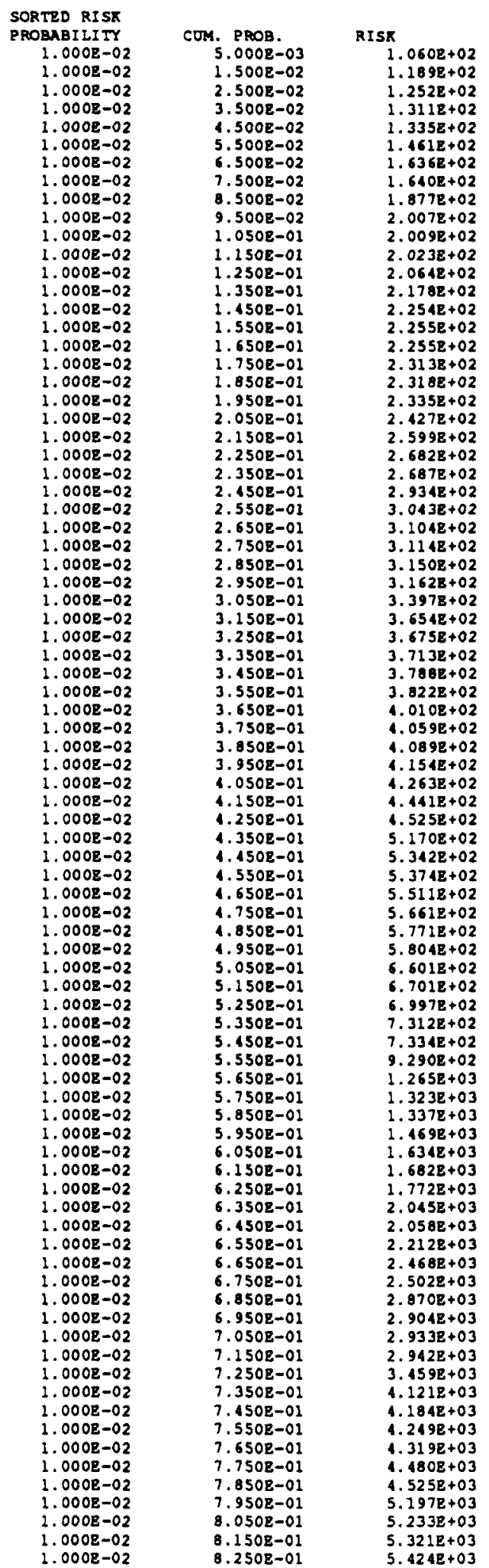


NUREG/CR-4551, VOL. 1: DRAFT REPORT FOR COMMENT (SEPTEMBER, 1986)

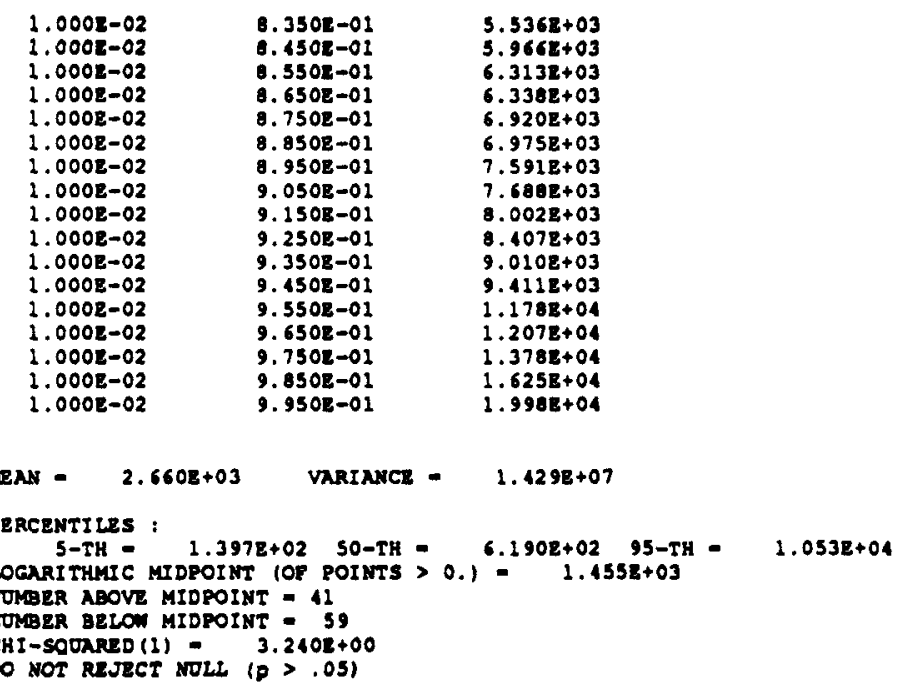


CONSEQUENCE MEASURE

OFESITE COSTS PER YEAR

BASE CASE

SURRY LALHC (CRAC)

ISSOE LEVELS FOR EACH SAYRE MEHBER, IN OROER OF INCREASING RISK RISK ISSUE LEVELS, FOR ALL ISSUES

\begin{tabular}{|c|c|c|c|c|c|c|c|c|c|c|c|c|c|c|c|c|c|c|c|c|c|c|c|c|c|c|c|}
\hline \\
\hline & 1 & & 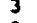 & & & & & & & & 11 & & & 64 & & 10 & & 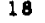 & 2. & & & 22 & 23 & 24 & 23 & 60 & 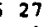 \\
\hline $\mathrm{OOZ}+02$ & 2 & 1 & 3 & 2 & 1 & 1 & 3 & 1 & 2 & 2 & 6 & 5 & 2 & 1 & 2 & 1 & 2 & 1 & 1 & 2 & 2 & 2 & 3 & 2 & 3 & 1 & 1 \\
\hline . $189 \mathrm{~g}+02$ & 2 & 1 & 2 & 2 & 3 & 6 & 1 & 1 & 5 & 3 & 2 & 1 & 2 & 2 & 2 & 1 & 3 & 1 & 2 & 1 & 1 & 1 & 2 & 3 & 3 & 3 & \\
\hline $.252 E+02$ & 2 & 1 & 2 & 1 & 3 & 6 & 2 & 2 & 1 & 1 & 5 & 4 & 1 & 1 & 3 & 2 & 2 & 1 & 2 & 1 & 3 & 1 & 3 & 3 & 3 & & \\
\hline $.3118+02$ & 2 & 2 & 2 & 1 & 1 & 6 & 1 & 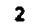 & 3 & 2 & 3 & 3 & 3 & 2 & 3 & ; & 2 & 1 & 2 & 3 & 2 & 1 & 3 & 4 & 3 & & \\
\hline $.335 \mathrm{E}+02$ & 2 & 2 & 2 & 1 & 1 & 5 & 7 & 2 & 5 & 2 & 2 & 3 & 1 & 2 & 1 & 2 & 2 & 1 & 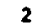 & 1 & & 2 & 4 & 2 & 3 & & \\
\hline $8+02$ & 2 & 1 & 2 & 1 & 2 & 6 & 6 & 2 & 2 & 3 & 3 & 1 & 3 & 2 & 3 & 2 & 4 & 1 & $i$ & 1 & 1 & 2 & 1 & 1 & 3 & 1 & \\
\hline+02 & 2 & 1 & 2 & 2 & 2 & 2 & 5 & 2 & 3 & 1 & 5 & 2 & 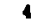 & & 3 & & 2 & 1 & 7 & & 9 & & 3 & 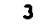 & & & \\
\hline $.640 \mathrm{OE}+02$ & 2 & 2 & 3 & 1 & 1 & 1 & 8 & 2 & 4 & 4 & 1 & 5 & 2 & 2 & 3 & 3 & 1 & 1 & 2 & 2 & & L & 3 & 2 & & 3 & \\
\hline+02 & 2 & 1 & 2 & 1 & 2 & 6 & 7 & 2 & 2 & 3 & 1 & 1 & 3 & 1 & 3 & 2 & 1 & 1 & 2 & 3 & & 2 & 4 & 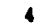 & & 3 & \\
\hline+02 & ? & 2 & 2 & 1 & 1 & 6 & & & & 9 & 3 & & & & 3 & & & & & 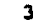 & & 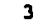 & 3 & 2 & & & \\
\hline$B+02$ & 2 & 2 & 2 & 2 & 1 & 6 & 2 & 2 & 1 & 2 & 1 & 3 & 1 & 2 & 3 & 2 & 2 & 1 & & 4 & & 2 & 3 & 2 & & 3 & \\
\hline $2.023 E+02$ & 2 & 1 & 2 & 2 & 3 & 2 & 9 & 2 & 1 & 2 & 4 & 4 & 3 & 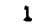 & 1 & 3 & & 1 & & 3 & & 1 & 2 & 3 & 3 & 2 & \\
\hline $.0648+02$ & 2 & 2 & 2 & 1 & 2 & 3 & 6 & 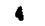 & 1 & 3 & 4 & 5 & , & ? & 7 & 2 & 3 & & 2 & 2 & & 2 & 2 & 4 & & 3 & \\
\hline $18+02$ & 2 & & 2 & 2 & 9 & 2 & 7 & 2 & 1 & 2 & 4 & 3 & 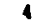 & 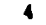 & 2 & 2 & & 1 & & 3 & & & 1 & 2 & & 2 & \\
\hline $2.254 E+02$ & 2 & 1 & 2 & 2 & 2 & 6 & 7 & 2 & 2 & 2 & 3 & 1 & 2 & 2 & 2 & 4 & 2 & 1 & 2 & 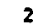 & & 1 & 3 & 4 & 2 & 3 & \\
\hline $.2558+02$ & 2 & 2 & 3 & 1 & 1 & 3 & 1 & 2 & 2 & 2 & 2 & 1 & 3 & 4 & 3 & 1 & 1 & & & 2 & & 2 & 2 & 3 & & & \\
\hline $.2558+02$ & 2 & & 3 & 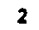 & 2 & 6 & 5 & & 3 & 2 & 4 & 3 & s & 1 & 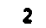 & 2 & 4 & & & & 3 & & 4 & 2 & & & \\
\hline $3132+02$ & 2 & & 2 & 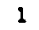 & 1 & 5 & , & , & 1 & 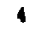 & 1 & 2 & 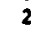 & 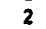 & 3 & 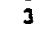 & 3 & & & & & 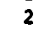 & 4 & 3 & & 3 & \\
\hline $17+02$ & 2 & & 3 & 2 & 2 & 6 & 7 & 2 & 2 & 3 & $\mathfrak{l}$ & 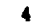 & 2 & ? & ? & 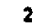 & & & & & & & 1 & 2 & & 1 & \\
\hline $5 E+02$ & $2+2$ & & 2 & 2 & & & & & & & & & & & & & & & & & & & 3 & 3 & 2 & & \\
\hline
\end{tabular}
$\begin{array}{lllllllllllllllllllllllllllll}2.353 E+02 & 2 & 1 & 3 & 2 & 2 & 6 & 7 & 2 & 2 & 3 & 1 & 4 & 2 & 2 & 3 & 2 & 3 & 1 & 2 & 2 & 2 & 2 & 1 & 2 & 3 & 1 & 1\end{array}$

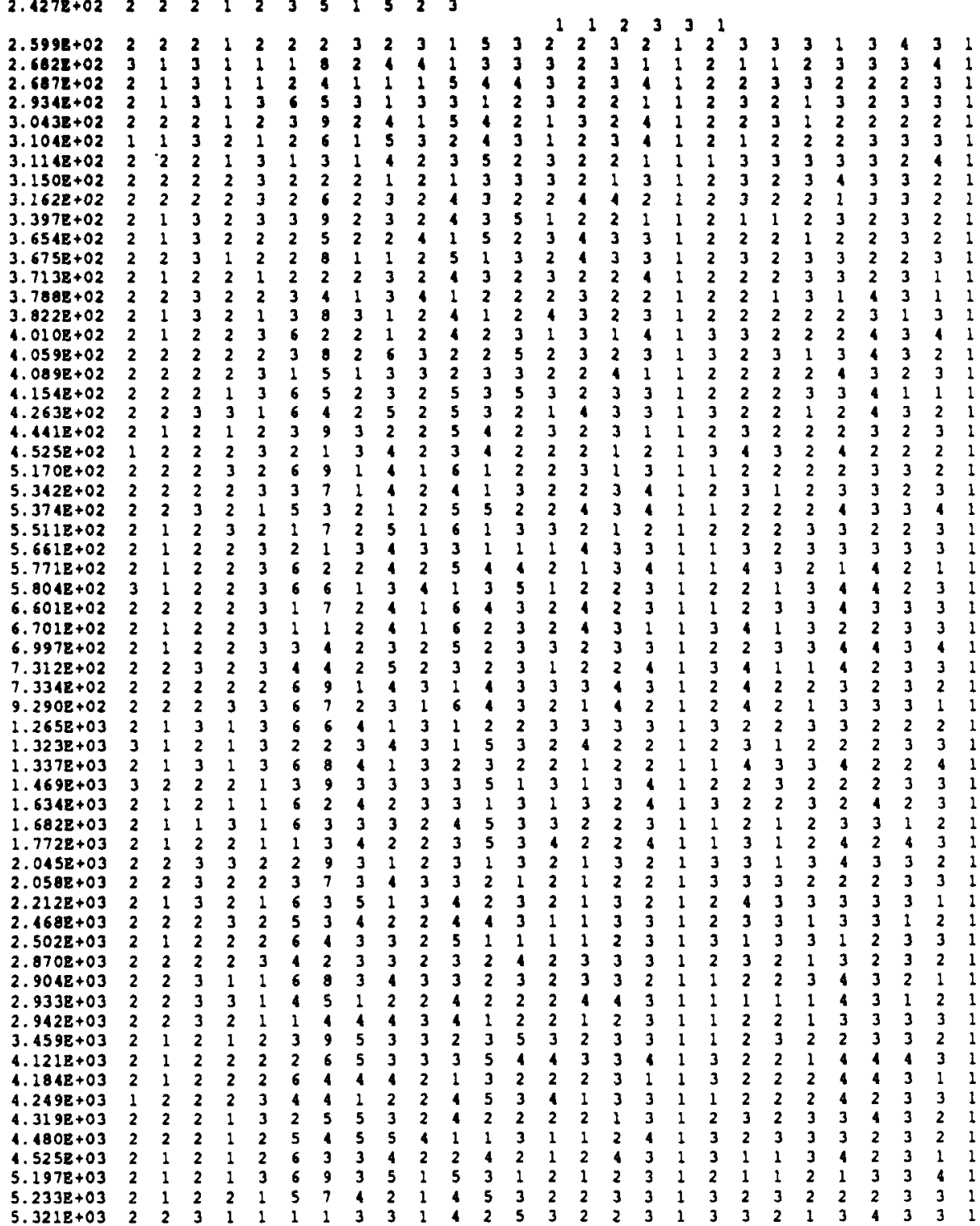


NUREG/CR-4551, VOL. 1: DRAFT REPORT FOR COMMENT (SEPTEMBER, 1986)
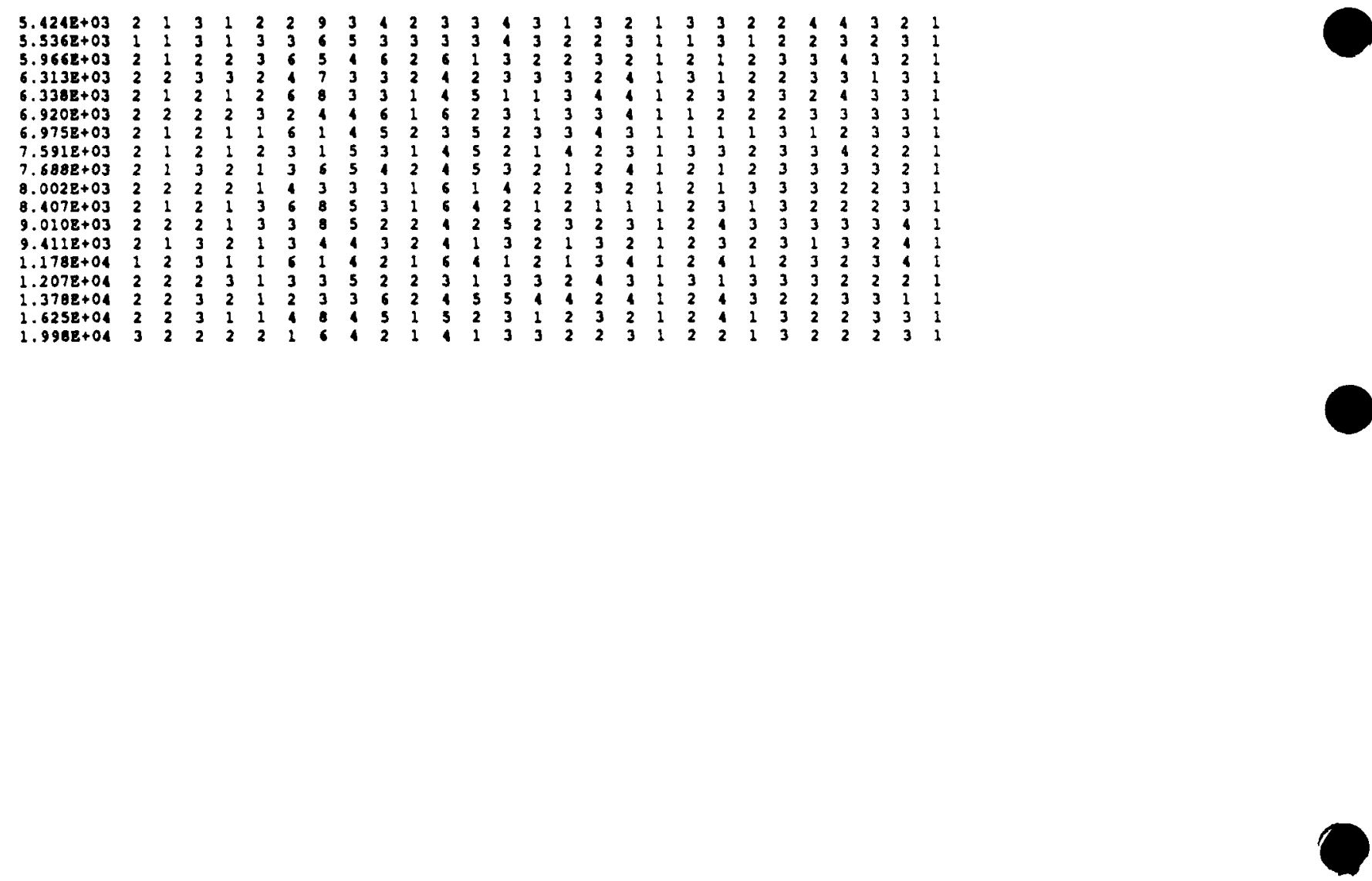


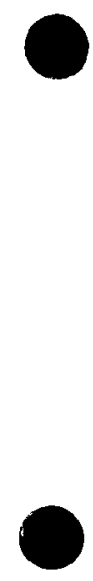

CONSEOTENCE MEASURE:

OFFSITE COSTS PER YEAR

BASE CASE :

SURRY LALHC (CRAC)

FRACTIONAL CONTRIBOTION OF EACH SEOUENCR TO MEAN RIST SROURNCE

SYYB

SYNI

SNNX

SYNA

ANMN

AYN

AYNI

AYYB

TNNA

TYYBN

IYS

$Y$

1 CONSEOUENCE MEASURE:

OFFSITE COSTS PER YEAR

CONTRIBUTION

0.1376

0.0001
0.4790

0.4790

0.0000

0.0093

0.0225

0.0085

0.0634

0.0687

0.0002

0.0630

BASE CASE :

SURRY LALHC (CRAC)

PRACTIONaL COMTRIBOTIOA OF EACH BIN TO MEAN RISK

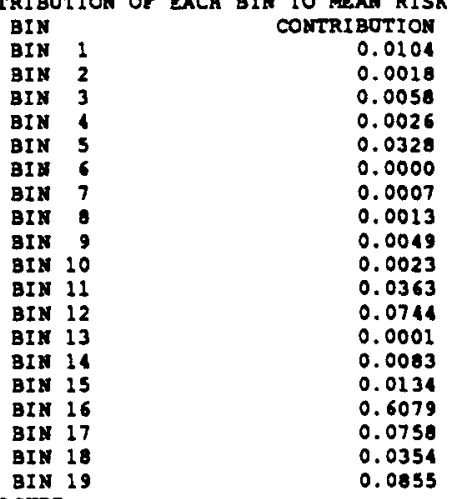

1

CONSEOOENCE MEASURE:

OFFSITE COSTS PER YEAR

BAsz case :

SURRY LALHC (CRAC)

FRACTIONAL CONTRIBOTIONS OP EACH RELEASE TO MEAN RIST ReIRAs?

SUR 1 GP

SUR $2 G$

SUR $3 G P$

SUR $19 P$

SOR $5 G P$

SUR 6GP

SOR 7GP

SUR OGP

SUR 96

SUR $10 G P$

SUR $11 G P$

SUR $12 G E$

SUR 136

SUR 146

SUR $15 G R$

SOR $16 \mathrm{G}$

SUR $17 \mathrm{GP}$

SUR $18 \mathrm{C}$

SUR 19GP

SUR $20 \mathrm{G}$

SUR 21GP

SUR 22

SUR 236

$\begin{array}{lll}\text { SUR } & 24 & G \\ \text { SUR } & 25 & C\end{array}$

CONTRIBUTION

0.1251

0.1483

0.1305

0.1510

0.0887

0.1003

0.10679

0.0679

0.057

0.0013

0.0079

0.0373

0.0006

0.0217

0.001

0.0013

0.015

0.002

0.0104

0.0019

0.0044

0.0008

0.0013

0.0013

0.0083

0.0134 


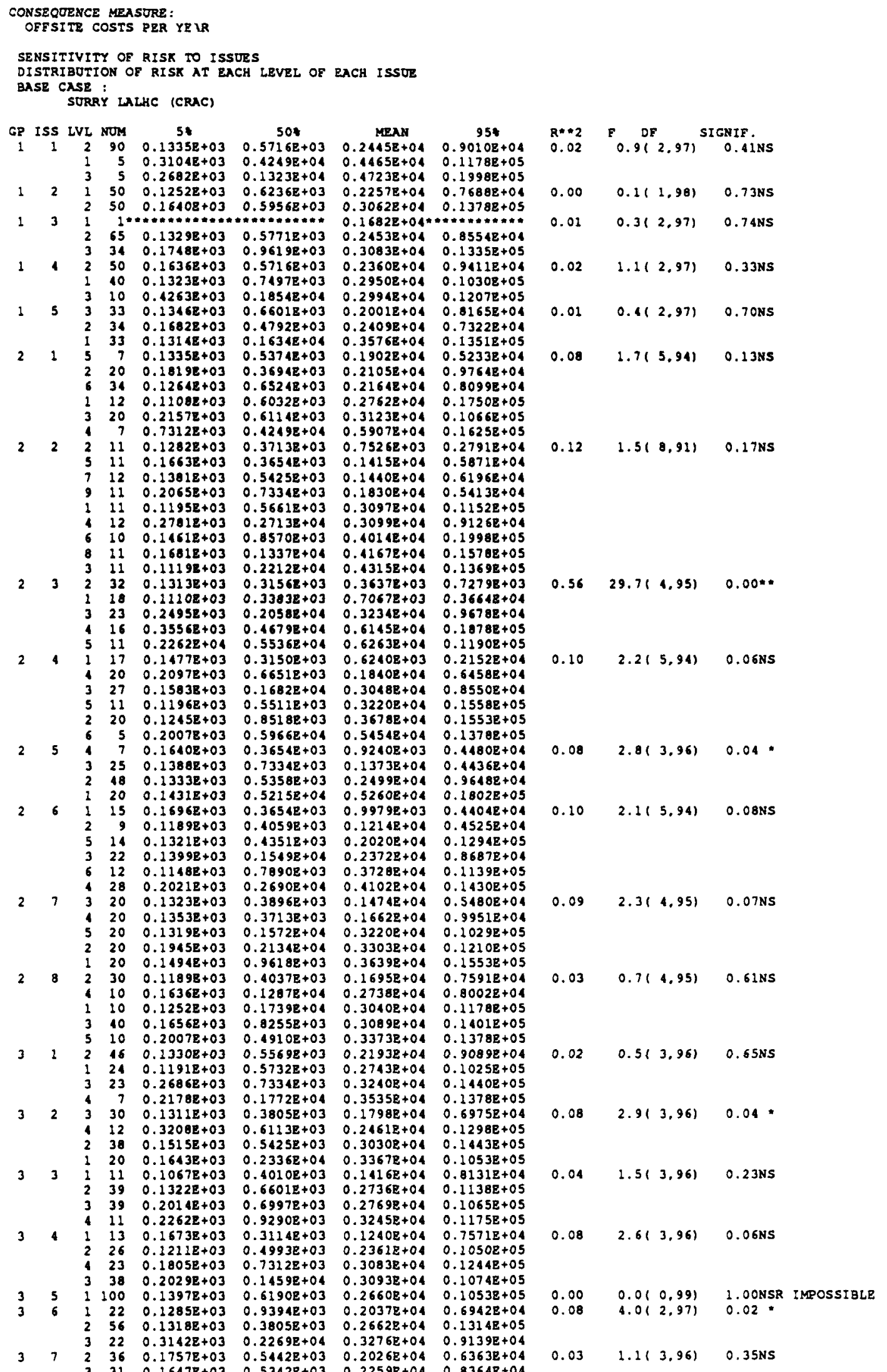


NUREG/CR-4551, VOL. 1: DRAFT REPORT FOR COMMENT (SEPTEMBER, 1986)

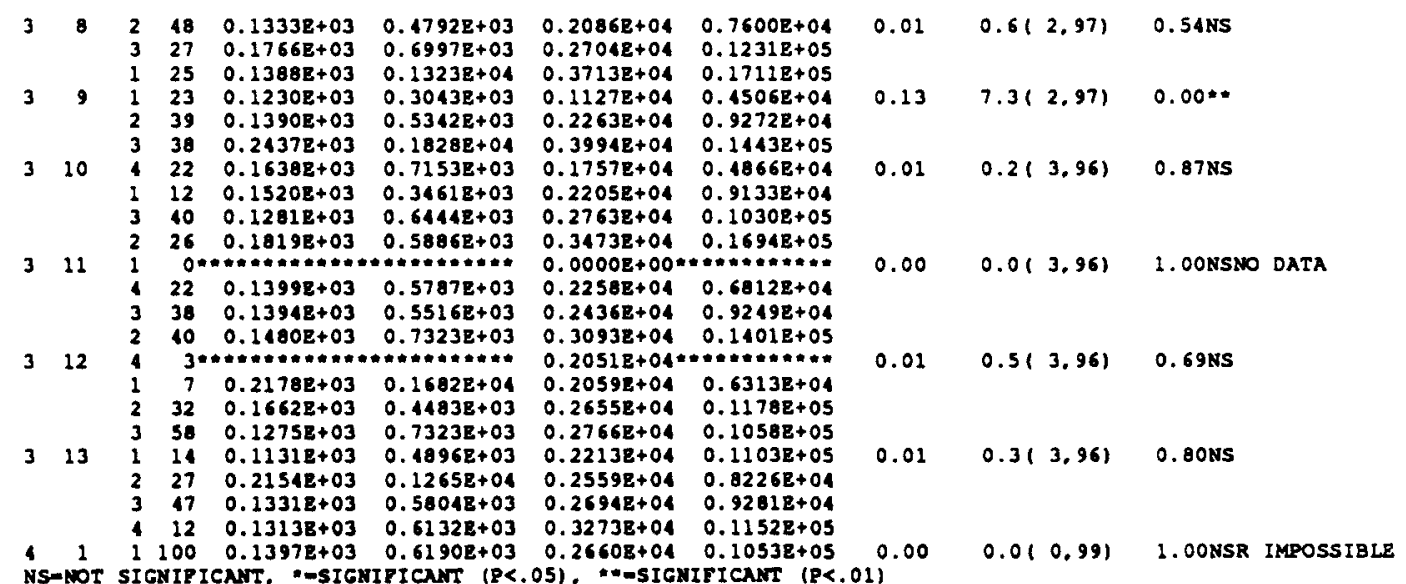


CONSEOUENCE MRASURE: OFFSITE COSTS PER YEAR 


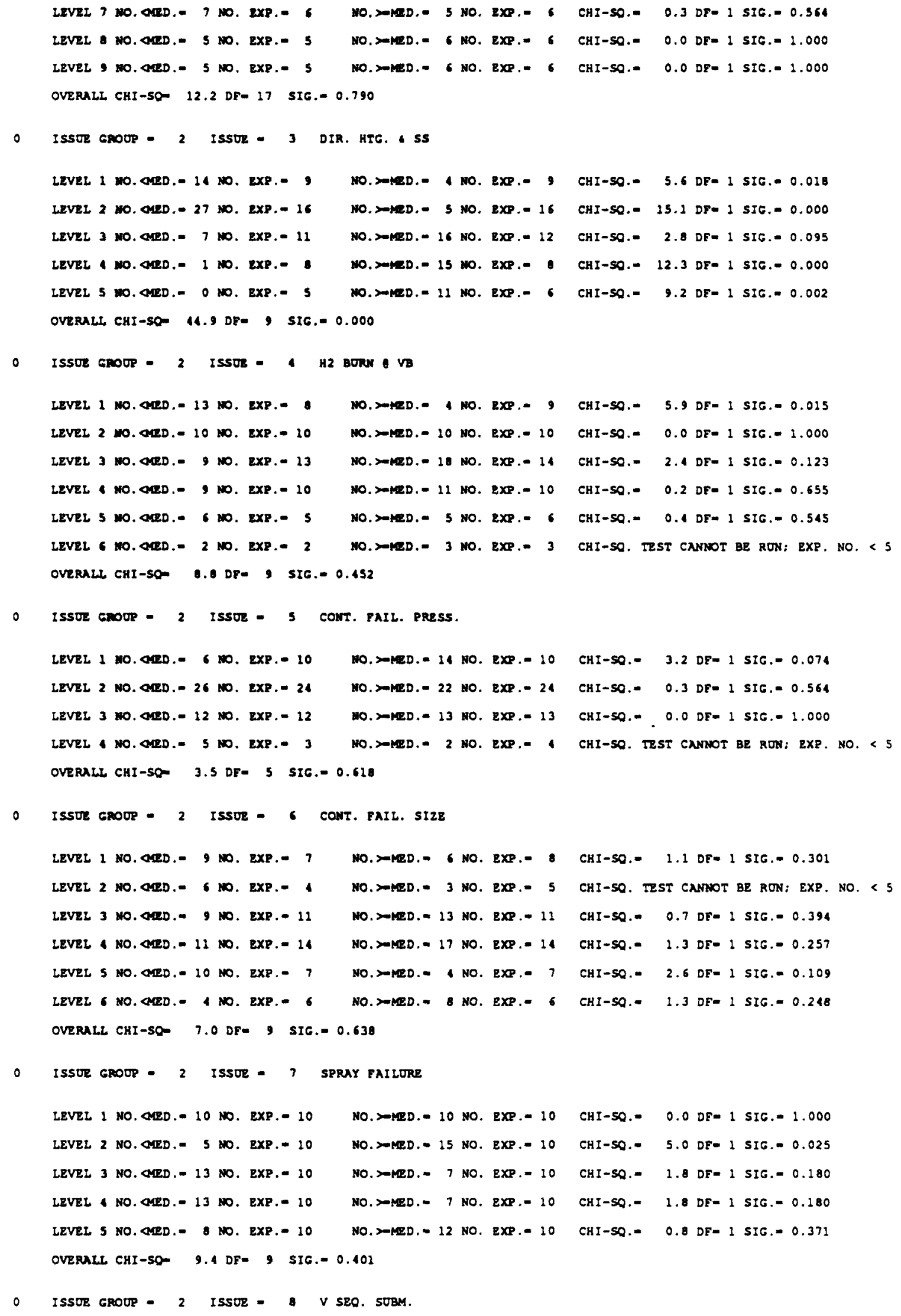

CHI-SO.= 5.9 DF- I SIE.- 0.015 CHI-SO.= 0.0 DE 1 SIG. $=1.000$ CHI-SO.- 2.4 DF- 1 SIG.- 0.123 CHI-SO.- 0.2 DF- 1 SIG.- 0.655 CHI-50.= 0.0 DF= 1 SIG. $=1.000$ CHI-SO. TEST CANNOT BE RUN: EXP. NO. < 5

CHI-SO.= 1.1 OE= 1 SIG. $=0.301$ CHI-SO. IEST CANNOT BE RON; EXP. NO. < 5 CHI-SO.= 0.7 DF= 1 SIG. $=0.394$ CHI-SO.= 1.3 DF- I SIG. $=0.257$ CHI-SQ.- 2.6 DF= 1 SIG.- 0.109 


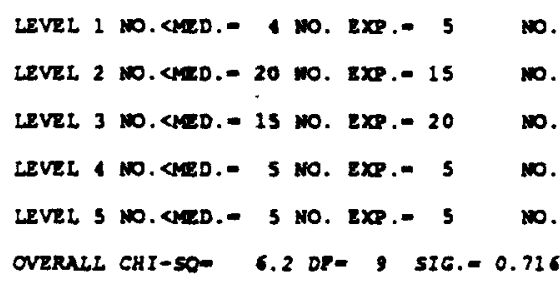

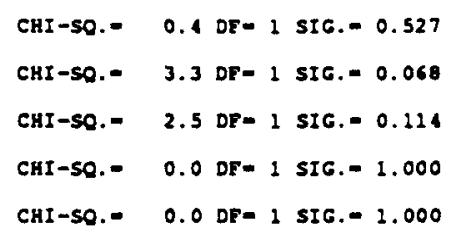

CHI-SQ.- 0.2 DF- I SIG. $=0.670$ CHI-SO.- 2.6 DF- 1 SIG. $=0.109$ CHI-SO.= 6.5 DE= 1 SIG. $=0.011$

CHI $50 .=0.0$ DF= 1 SIC. $=1.000$ CHI-5O. 0.1 DE- 1 SIG. $=0.739$ CHI-5O.= 0.5 DF- 1 SIG. $=0.472$ CHI-5O.- 2.8 DF- 1 SIG. $=0.095$ 

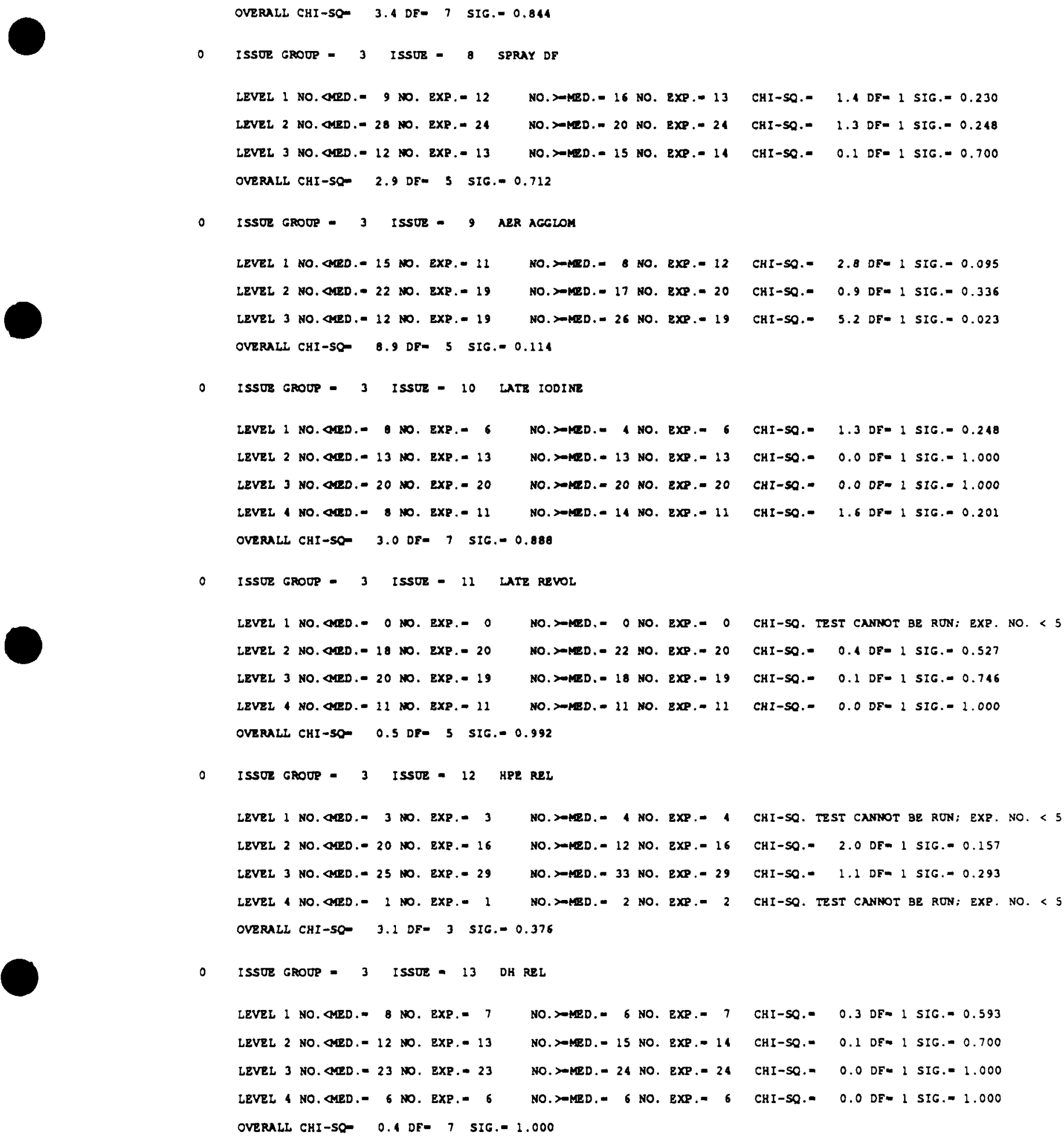


\section{D.2 RISQUE CODE OUTPUT FOR THE LLH ANALYSIS USING MACCS}

The computer output for risk as calculated with MACCS is provided in this section. The printout follows the same format as the CRAC2 output presented in the previous section. The first part of the output is a listing of each uncertainty issue and the level of the issue in each of the 100 sample members. The issues and levels are defined as in Section 4 of the main report. [In the output, the issues are either numbered consecutively by groups (group 1 for sequence issues, group 2 for containment issues and group 3 for source term issues) or consecutively for all of the issues starting with group 1.] This first part of the code listing provides the short title of each issue as a cross reference. The issue level definitions are then followed in the listing by the core-melt frequencies for each of the 100 sample members. Finally, for each of the risk measures, the following results are presented:

- The risk results for each of the individual sample members in order of increasing risk;

- The risk values in the same order, along with the individual issue outcomes (levels);

- The fractional contribution of each plant damage state to the mean risk,

- The fractional contribution of each source term bin to mean risk;

- The fractional contribution of each cluster to mean risk;

- The distribution of risk at each level and the rank regression analysis of the results; and

- The chi-squared analysis of each issue, first by individual level and then by total issue. 
NUREG/CR-4551, VOL. 1: DRAFT REPORT FOR COMMENT (SEPTEMBER, 1986)

1 ISSUE LEVELS FOR ALI SAMPLZ MERKBRS

ISSGP ISS

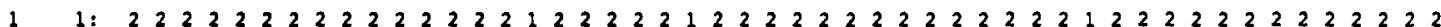

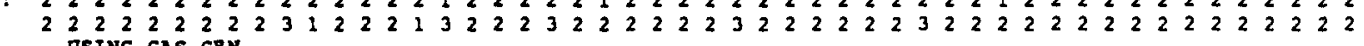
DSING GAS GEN.

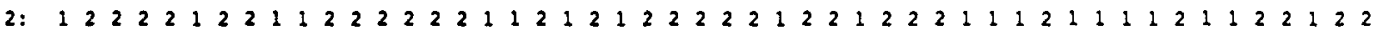

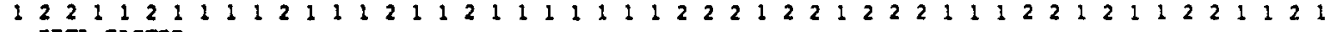
BE TA-PACTOR

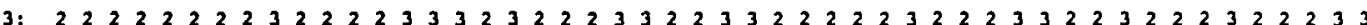
$\begin{array}{lllllllllllllllllllllllllllllllllllllllllllllllllll}3 & 3 & 3 & 2 & 3 & 3 & 2 & 3 & 2 & 2 & 2 & 2 & 2 & 1 & 2 & 3 & 3 & 2 & 2 & 2 & 3 & 3 & 3 & 2 & 2 & 2 & 2 & 2 & 2 & 3 & 2 & 2 & 3 & 2 & 2 & 2 & 2 & 3 & 2 & 3 & 2 & 3 & 3 & 3 & 2 & 2 & 2 & 2 & 2 & 2\end{array}$ $\checkmark$ Freo

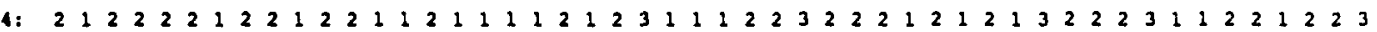

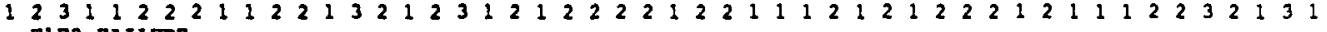
F1F2 FXILORE

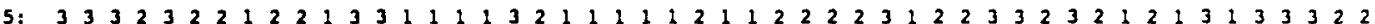
$\begin{array}{llllllllllllllllllllllllllllllllllllllllllllllllll}1 & 1 & 1 & 3 & 2 & 3 & 2 & 3 & 2 & 3 & 3 & 1 & 2 & 1 & 3 & 1 & 1 & 3 & 1 & 3 & 3 & 1 & 1 & 3 & 1 & 3 & 2 & 3 & 2 & 2 & 2 & 3 & 1 & 1 & 2 & 3 & 2 & 2 & 1 & 2 & 2 & 1 & 3 & 2 & 3 & 2 & 3 & 3 & 1 & 2\end{array}$

21 IND. RCS EAIL.

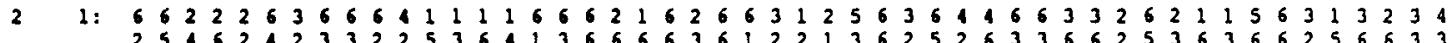

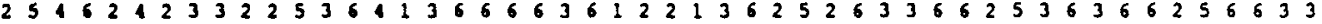
HODE OF $\mathrm{VB}$.

2. 554767925837384148232641643998428974691553772658947 13599.69121793484726683125673841895245575157232839 DIR. HTE. IS

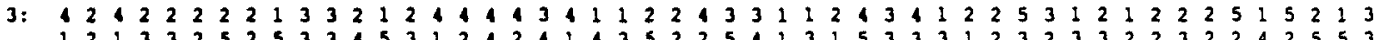

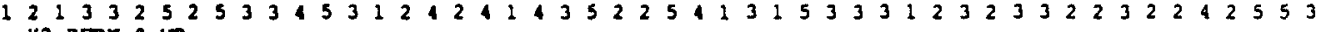
H2 BURM $\mathrm{E}$ VB

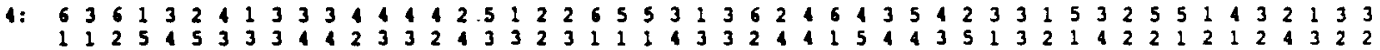
CONT. FAIL. PRESS.

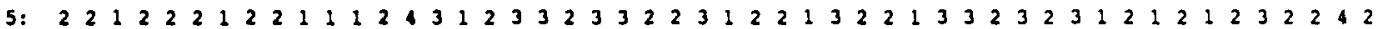

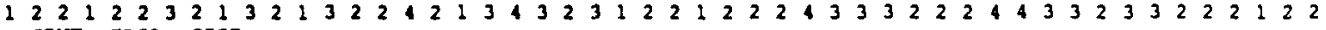
CONT. PAIL. SIZE

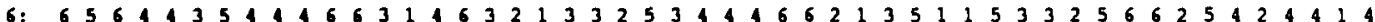
55153334413424114631344644442513333451133231445635 SPRAY PAILURB

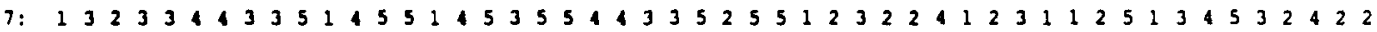

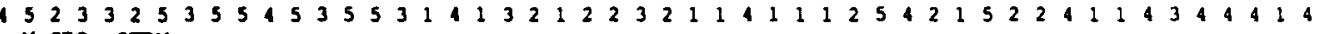
$\checkmark$ SEO. SURM.

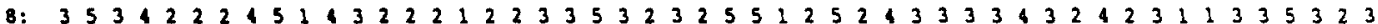

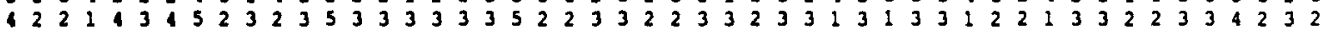

31 IN VESS ReLEASE

$\begin{array}{llllllllllllllllllllllllllllllllllllllllllllllllllllll}3 & 1 & 0 & 2 & 3 & 1 & 1 & 2 & 2 & 1 & 2 & 1 & 1 & 2 & 2 & 3 & 2 & 2 & 2 & 3 & 2 & 2 & 4 & 1 & 1 & 1 & 2 & 2 & 3 & 4 & 2 & 2 & 2 & 2 & 2 & 1 & 3 & 1 & 3 & 3 & 2 & 2 & 2 & 1 & 3 & 2 & 1 & 2 & 2 & 2 & 1 & 2 & 3\end{array}$ $\begin{array}{lllllllllllllllllllllllllllllllllllllllllllllllllll} & 2 & 2 & 2 & 3 & 1 & 4 & 1 & 1 & 2 & 2 & 2 & 3 & 3 & 4 & 3 & 2 & 2 & 1 & 1 & 3 & 4 & 2 & 2 & 3 & 2 & 3 & 2 & 1 & 2 & 1 & 1 & 2 & 3 & 2 & 1 & 1 & 3 & 2 & 2 & 2 & 4 & 3 & 2 & 3 & 1 & 2 & 1 & 3 & 3\end{array}$ CSI DECOMP

2: $\quad 2 \begin{array}{llllllllllllllllllllllllllllllllllllllllllllllllll} & 2 & 2 & 4 & 3 & 3 & 2 & 3 & 2 & 2 & 3 & 1 & 1 & 3 & 1 & 2 & 2 & 3 & 2 & 4 & 3 & 3 & 2 & 4 & 1 & 3 & 3 & 2 & 3 & 2 & 3 & 3 & 2 & 2 & 1 & 2 & 3 & 2 & 2 & 1 & 3 & 1 & 2 & 3 & 1 & 3 & 3\end{array}$

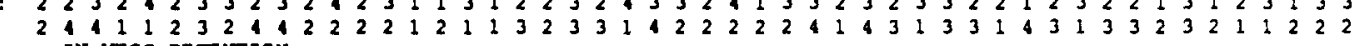
IN VESS RETENTION

3: $\quad \begin{array}{lllllllllllllllllllllllllllllllllllllllllllllllllll}3 & 3 & 2 & 1 & 2 & 2 & 2 & 4 & 3 & 2 & 2 & 3 & 2 & 3 & 4 & 2 & 3 & 2 & 3 & 3 & 3 & 2 & 2 & 2 & 2 & 3 & 1 & 2 & 3 & 3 & 3 & 4 & 2 & 3 & 2 & 3 & 1 & 1 & 1 & 1 & 2 & 2 & 2 & 4 & 2 & 3 & 2 & 2\end{array}$ $\begin{array}{llllllllllllllllllllllllllllllllllllllllllllllllllll}3 & 3 & 2 & 3 & 2 & 3 & 2 & 2 & 2 & 1 & 3 & 3 & 2 & 3 & 3 & 3 & 1 & 2 & 2 & 3 & 2 & 3 & 3 & 2 & 1 & 2 & 3 & 4 & 3 & 2 & 3 & 3 & 3 & 4 & 1 & 2 & 3 & 3 & 2 & 2 & 1 & 2 & 2 & 1 & 3 & 3 & 1 & 4 & 3\end{array}$

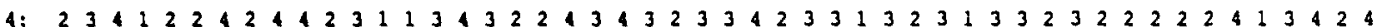

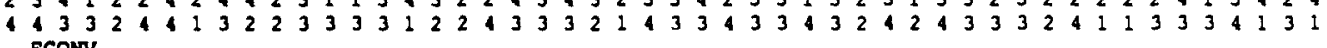
FCONV

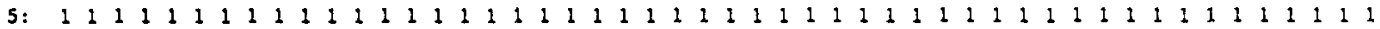

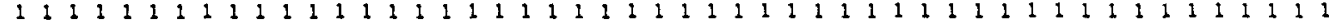
$\operatorname{ccI} \operatorname{Rez}$

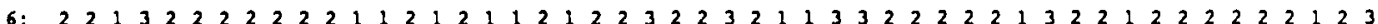

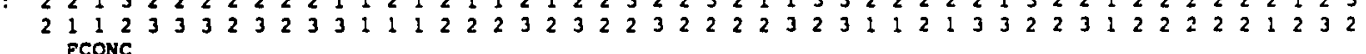

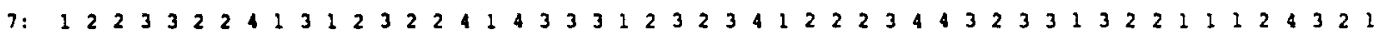

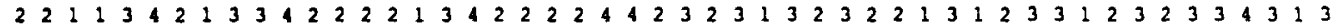
SPRAY DF

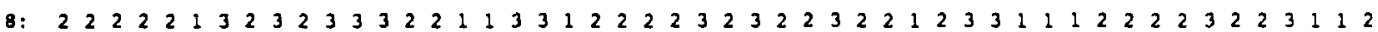

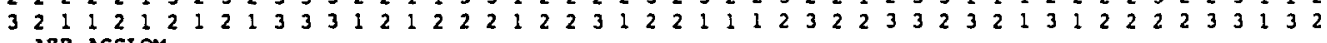
AER AGGLOH

9: $\quad \begin{array}{lllllllllllllllllllllllllllllllllllllllllllllllll}3 & 2 & 1 & 2 & 1 & 1 & 2 & 1 & 3 & 3 & 3 & 3 & 1 & 1 & 2 & 3 & 3 & 3 & 2 & 3 & 2 & 1 & 1 & 2 & 1 & 2 & 3 & 2 & 1 & 2 & 1 & 3 & 2 & 2 & 3 & 2 & 3 & 1 & 1 & 2 & 3 & 2 & 1 & 3 & 2 & 3 & 1 & 3 & 2\end{array}$

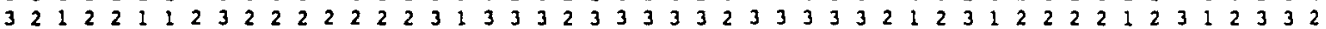


NUREG/CR-4551, VOL. 1: DRAFT REPORT FOR COMMENT (SEPTEMBER, 1986)

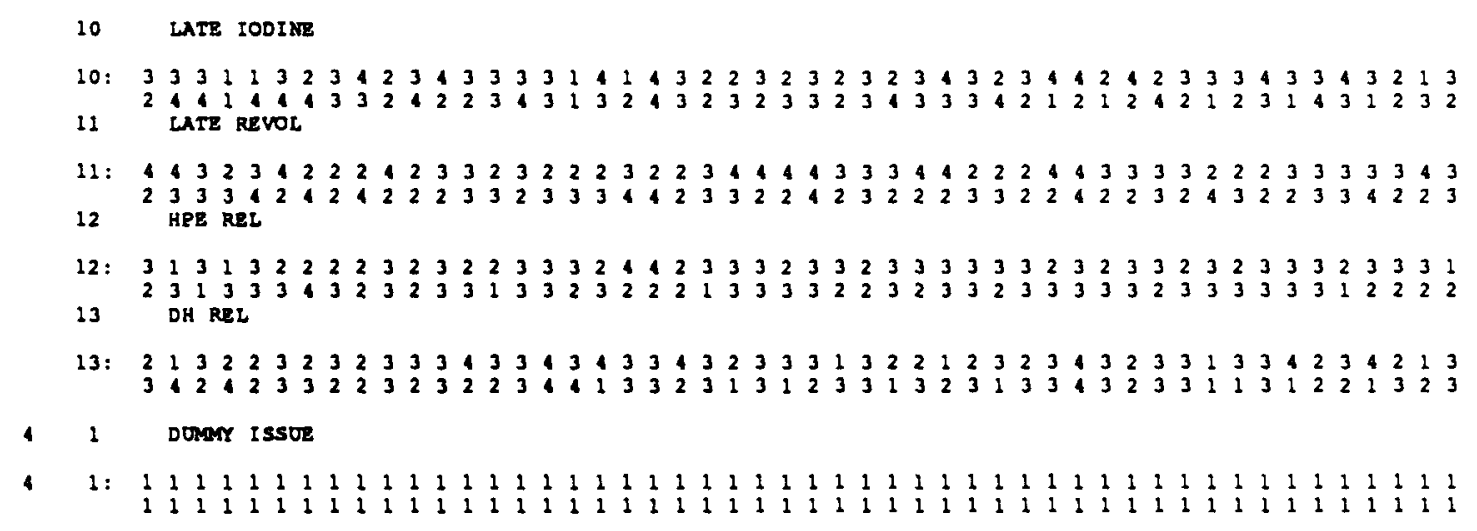


BASE CASE: SURY LALHC (M

Specifie information concerning input deta followe. HEADER : ALL ISSOZS VARY IN SAMPL

SEQCENCES : SEOUENCES FROY ASEP 2/20/86

CONTAINMENT FAIIURE MODES : CFM ISSUES

RELEASES : RELEASES BY ISSUES $(1-13)$

CONSEOUENCES : CONSEOUENCES $4 / 23 / 86$ MACCS

SORTED MELTDOWN FREOUENCIES

$3.000 \mathrm{x}-02$

$1.0008-02$

$1.0008-02$

$1.000 \mathrm{E}-02$

2.0008-02

3. $000 \mathrm{~B}-02$

$1.0008-02$

$1.0008-02$

$5.000 \mathrm{z}-02$

$1.0008-02$

$6.000 \mathrm{~B}-02$

7.0008-02

7. $000 \mathrm{z}-02$
8. $000 \mathrm{~g}-02$

$1.000 \mathrm{E}-02$

$1.0008-02$

8.0008-02

$9.000 \mathrm{E}-02$
$1.000 \mathrm{E}-01$

$1.1008-01$

1. 2008-01

$1.0008-02$

$1.000 \mathrm{E}-02$

$1.3008-01$

$1.0008-02$

$1.4008-01$

1. $500 \mathrm{z}-01$

$1.000 \mathrm{E}-02$

$1.500 \mathrm{E}-0$

$1.600 \mathrm{~B}-01$
$1.700 \mathrm{~B}-01$

$1.0008-1000$

$1.700 \mathrm{E}-01$
$1.800 \mathrm{E}-01$

1. $900 \mathrm{E}-01$

2. $000 \mathrm{~B}-01$

2. 1008-01

FReouexcr

$1.0008-02$

$1.000 \mathrm{E}-02$

$1.0008-02$

$1.0008-02$

$1.0008-02$

2. 3008-01

2. 4008-01

2.5008-01

2. 6008-01

2. 7005-01

2. $0008-01$

2. $9008-01$

3.0008-01

3. 100E-01

$3.2008-01$

3. 3008-01

3. 4008-01

1. 4738-05

1. $473 \mathrm{E}-05$

1. 47 8B-05

1. $4788-05$

1. $47 \mathrm{az}-05$

1. $178 \mathrm{E}-05$

$1.478 \mathrm{~B}-05$

1. $478 \mathrm{E}-05$

1. $478 \mathrm{E}-05$

1. $486 \mathrm{~B}-0.5$

1. $486 \mathrm{~B}-05$

1. $4868-05$

$1.5548-05$

$1.5548-05$

1.5548-05

$5508-05$

1. 558z-0.5

$1.5588-05$

$1.558 \mathrm{z}-05$

$1.5588-05$

$1.558 \mathrm{z}-05$

$1.5678-05$

.567E-0.5

1.5678-05

1. 5672-05

$1.3678-05$

$1.567 \mathrm{E}-05$

. $.5678-05$

$1.5678-05$

1. $6012-05$

$1.6012-05$

$1.8182-05$

$1.8728-05$

$1.8738-0.5$

$1.8738-05$

$1.8738-05$

3. $6008-01$

1. $7782-05$

3. $900 \mathrm{E}-01$

$3.9000-01$

$4.000 \mathrm{e}-01$

$4.100 \mathrm{E}-01$

4.2008-01 $1.878 \mathrm{E}-05$

4. 3008-01

$4.100 \mathrm{E}-01 \quad 1.866 \mathrm{~B}-0 \mathrm{~S}$

4.500R-01 1.886z-05

4. $600 \mathrm{E}-01 \quad 1.806 \mathrm{E}-05$

$4.700 \mathrm{z}-01$

$\begin{array}{ll}4.700 \mathrm{E}-01 & 1.899 \mathrm{E}-05 \\ 4.800 \mathrm{E}-01 & 1.954 \mathrm{E}-0.5\end{array}$

$\begin{array}{ll}4.800 \mathrm{E}-01 & 1.954 \mathrm{E}-05 \\ 4.900 \mathrm{z}-01 & 1.954 \mathrm{~B}-05\end{array}$

$5.000 \mathrm{E}-01 \quad 1.954 \mathrm{E}-05$

5.1008-01 $\quad 1.954 \mathrm{~B}-05$

5. $2008-01 \quad 1.954 E-05$

5. $3008-01$

5. $1008-01 \quad 1.9588-05$

5.5008-01 1.958z-05

5. 6008-01 1.9588-05

$5.7008-01 \quad 1.9678-05$

$5.800 \mathrm{E}-01 \quad 1.967 \mathrm{E}-05$

5.9008-01 $1.9678-05$

6.0008-01 1.9678-05

$6.1008-01 \quad 1.9678-05$

$6.200 \mathrm{E}-01 \quad 1.967 \mathrm{E}-05$

$6.300 \mathrm{E}-01 \quad 1.967 \mathrm{E}-05$

$6.400 \mathrm{E}-01 \quad 1.967 \mathrm{E}-05$

$6.500 \mathrm{E}-01 \quad 2.125 \mathrm{~B}-05$

6. 600 E-01 2.1308-05

$6.700 \mathrm{E}-01 \quad 2.138 \mathrm{E}-05$

6. $300 \mathrm{~g}-01 \quad 2.138 \mathrm{E}-05$

$6.900 \mathrm{E}-01 \quad 2.138 \mathrm{E}-05$

$7.000 \mathrm{E}-01 \quad 2.206 \mathrm{E}-05$

$7.1008-01 \quad 2.206 \mathrm{E}-05$

7.2008-01 2.2068-05

$7.300 \mathrm{e}-01 \quad 2.206 \mathrm{~B}-05$

$7.100 \mathrm{E}-01 \quad 2.206 \mathrm{E}-05$

$7.5008-01 \quad 2.2108-05$

7. $6008-01 \quad 2.210 \mathrm{E}-05$

$7.700 \mathrm{~B}-01 \quad 2.210 \mathrm{~B}-05$

$7.800 \mathrm{E}-01 \quad 2.219 \mathrm{E}-05$

$7.900 \mathrm{E}-01 \quad 2.268 \mathrm{E}-05$

8. $000 \mathrm{E}-01 \quad 2.2728-05$

8. $1008-01$

$8.2008-01$

$\begin{array}{ll}8.300 \mathrm{E}-01 & 2.615 \mathrm{E}-05 \\ 8.400 \mathrm{E}-01 & 2.615 \mathrm{E}-05\end{array}$ 
NUREG/CR-4551, VOL. 1: DRAFT REPORT FOR COMMENT (SEPTEMBER, 1986)

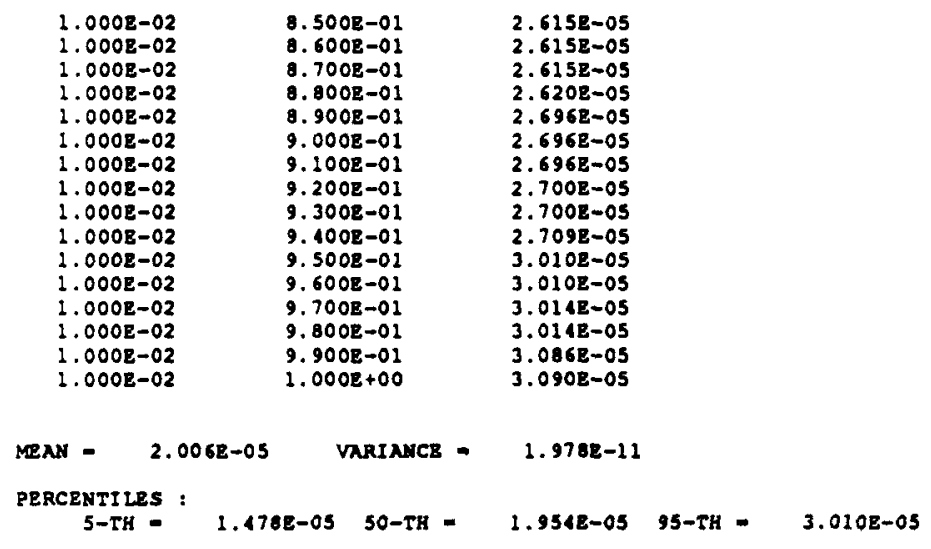


NUREG/CR-4551, VOL. 1: DRAFT REPORT FOR COMMENT (SEPTEMBER, 1986)

CONSEOUENCE MRASURE:

EARLY DEATHS PER YEAR

BASE CASE :

SURRY LALHC (MACCS)

SORTED RISK PBOBABTL

1.0008-02

1.000e-02

$1.000 \mathrm{E}-02$

1. $000 \mathrm{E}-02$

1. $000 \mathrm{E}-02$

$1.000 \mathrm{E}-02$

1. $000 \mathrm{R}-02$

$1.000 \mathrm{~B}-02$

$1.000 \mathrm{E}-02$

$1.000 \mathrm{E}-02$

$1.000 \mathrm{E}-02$

$1.0008-02$

1. $000 \mathrm{E}-02$

$1.000 \mathrm{E}-02$

$1.0008-02$

$1.000 \mathrm{E}-0$

$1.000 \mathrm{E}-0$

1. $000 \mathrm{E}-02$

1. $000 \mathrm{E}-02$
$1.00 \mathrm{E}-02$

$1.000 \mathrm{E}-02$

$1.0008-02$

$1.0008-02$

$1.000 \mathrm{E}-02$

$1.0000-02$

$1.000 \mathrm{~B}-02$

$3.000 \mathrm{E}-02$

$1.0008-02$

$1.0008-02$

$1.0008-02$

$1.0008-02$

$1.000 \mathrm{~B}-02$

$1.000 \mathrm{E}-02$

$.000 \mathrm{E}=02$

$1.000 \mathrm{~B}-02$
$1.00 \mathrm{OB}-02$

$.000 \mathrm{E}-02$

$1.000 \mathrm{R}-02$

1. $000 \mathrm{E}-02$

1. $000 \mathrm{OE}-02$

1. $000 \mathrm{~B}-02$

1. $000 \mathrm{E}-02$

$1.000 \mathrm{E}-02$

1. $0008-02$

$1.0008-02$

$1.000 \mathrm{E}-02$

1. $000 \mathrm{OE}-02$

$1.000 \mathrm{E}-02$

$1.000 \mathrm{E}-02$

$1.0008-02$

1. $0008-02$

$1.000 \mathrm{~B}-02$

$1.000 \mathrm{E}-02$

1. $000 \mathrm{E}-02$

1. $0008-02$

$1.000 \mathrm{E}-02$

$1.000 \mathrm{z}-02$

$1.0008-02$

$1.000 \mathrm{E}-02$

$1.000 \mathrm{E}-02$

1. $000 \mathrm{E}-02$

1. $0008-02$

1. $000 \mathrm{E}-02$

$1.000 \mathrm{E}-02$

$1.000 \mathrm{~B}-02$

$1.000 \mathrm{E}-02$

$1.000 \mathrm{E}-02$

$1.000 \mathrm{E}-02$

$1.0008-02$

$1.000 \mathrm{E}-02$

1. $000 \mathrm{E}-02$

1. $000 \mathrm{E}-02$

$1.000 \mathrm{E}-02$

1. $000 \mathrm{E}-02$

1. $000 \mathrm{E}-02$

1. $000 \mathrm{E}-02$

$1.000 \mathrm{E}-02$

1. $000 \mathrm{E}-02$

$1.000 \mathrm{E}-02$

$1.0008-02$

$1.0008-02$

$1.0000-02$

$1.0008-02$

$1.0008-02$
$1.0008-02$

COM. PROB.

5. 000B-03

1. $500 \mathrm{~B}-02$

2. $500 \mathrm{E}-02$

3. 500E-02

4. 5008-02

5. 5008-02

6. $500 \mathrm{E}-02$

7. 5002-02

8. 500 z-02

$9.500 \mathrm{z}-02$

1. $050 \mathrm{~B}-01$

$1.150 \mathrm{E}-01$

1. $250 \mathrm{~B}-01$

$1.350 \mathrm{E}-01$

1. $450 \mathrm{E}-01$

1. 5508-01

1. $650 \mathrm{~B}-01$

$1.750 \mathrm{E}-01$

1. $850 \mathrm{~B}-01$

1. $950 \mathrm{~B}-01$

2.0508-01

$2.150 \mathrm{E}-01$

2. 250E-01

$2.350 \mathrm{~B}-01$

2. $450 \mathrm{~B}-01$

2. 5508-01

2. $650 \mathrm{E}-01$

$2.750 \mathrm{E}-01$

2.8508-01

$2.9508-01$

$3.050 \mathrm{~B}-01$

$3.1508-01$

3. 2508-01

3. $3508-01$

3. 4508-01

3. $550 \mathrm{E}-01$

3. $650 \mathrm{~B}-01$

3.7508-01

3. $850 \mathrm{E}-01$

3. $950 \mathrm{~B}-01$

4.050E-01

4.15OE-01

1. 2508-01

1.3508-01

4. 4508-01

4.5508-01

4. 650B-01

1. $750 \mathrm{E}-01$

4. 8508-01

$4.950 \mathrm{E}-01$

$5.050 \mathrm{E}-01$

$5.150 \mathrm{E}-01$

$5.250 \mathrm{E}-01$

5.350E-01

5. 450E-01

$5.550 \mathrm{E}-01$

$5.650 \mathrm{z}-01$

$5.750 \mathrm{E}-01$

5. $850 \mathrm{~B}-01$

5. $950 \mathrm{E}-01$

6. $050 \mathrm{R}-01$

6. $150 \mathrm{E}-01$

6. 250E-01

$6.350 \mathrm{E}-01$

$6.450 \mathrm{E}-01$

6. $550 \mathrm{E}-01$

6. 6508-01

6. 7508-01

$6.850 \mathrm{z}-01$

6. $950 \mathrm{~B}-01$

$7.050 \mathrm{E}-01$

$7.1508-01$

7. 250E-01

$7.3508-01$

7. 450E-01

$7.550 \mathrm{E}-01$

$7.650 \mathrm{E}-01$

$7.750 \mathrm{~B}-01$

$7.850 \mathrm{~B}-01$

7. $950 \mathrm{~B}-01$

8. 0508-01

8. 150Z-01

8. $250 \mathrm{E}-01$ 8.5x

. $3778-09$

. $4398-08$

$2.646 \mathrm{E}-08$

4. $1768-08$

5. $789 E-08$

$6.349 \mathrm{E}-08$

$6.4218-08$

9. $355 \mathrm{z}-08$

$1.031 z-07$
$1.095 z-07$

1. $1248-07$

1. 2508-07

1. $9668-07$

2.1518-07

2. 242E-07

2. 3018-07

$2.7298-07$

$2.9758-07$

3. 105E-07

3. $354 \mathrm{E}-07$

3. $173 \mathrm{E}-07$

3. $718 \mathrm{E}-07$

3. $7748-07$

3. $854 E-07$

1.0188-07

4. 236E-07

4. 505E-07

4. 653E-07

1. $9478-07$

4607

$5.4168-07$

5. $489 \mathrm{E}-07$

$.827 \mathrm{e}-07$

6. $9778-07$

7.836z-07

9. $652 z-07$

. $713 \mathrm{E}-07$

. $9908-07$

. $7828-07$

$9608-07$

$9.9608-07$

$.0008-06$

$1.051 \mathrm{E}-06$

$.083 \mathrm{E}-06$

$1.104 \mathrm{E}-06$

$1.135 \mathrm{E}-06$

$1.2528-06$

$1.7308-06$

$1.731 \mathrm{E}-06$

$1.822 \mathrm{E}-06$

$2.037 \mathrm{E}-06$

$2.261 \mathrm{E}-06$

2. $5848-06$

. $7908-06$

$2.9238-06$

$2.923 \mathrm{~B}-06$

3. $300 \mathrm{E}-06$

4. 364E-06

4. $5028-06$

$5.067 \mathrm{E}-06$

5. $2608-06$

$5.2878-06$

6. $652 \mathrm{E}-06$

6. $676 \mathrm{E}-06$

6. $8918-06$

7. 336E-06

$7.505 E-06$

7. $953 \mathrm{~B}-06$

$9.231 \mathrm{E}-06$

$9.4228-06$

9. $635 \mathrm{E}-06$

1.001E-05

$1.011 \mathrm{E}-05$

$1.013 \mathrm{E}-05$

1. $048 \mathrm{BE}-05$

$1.0908-05$

1. $096 \mathrm{E}-05$

1. 2318-05

1. 332E-05

1. $341 \mathrm{E}-05$

1. $385 \mathrm{E}-05$

1. $406 \mathrm{E}-05$

$1.427 \mathrm{E}-0.5$

1. $578 \mathrm{E}-05$

1. $343 \mathrm{E}-05$ 
NUREG/CR-4551, VOL. 1: DRAFT REPORT FOR COMMENT (SEPTEMBER, 1986)

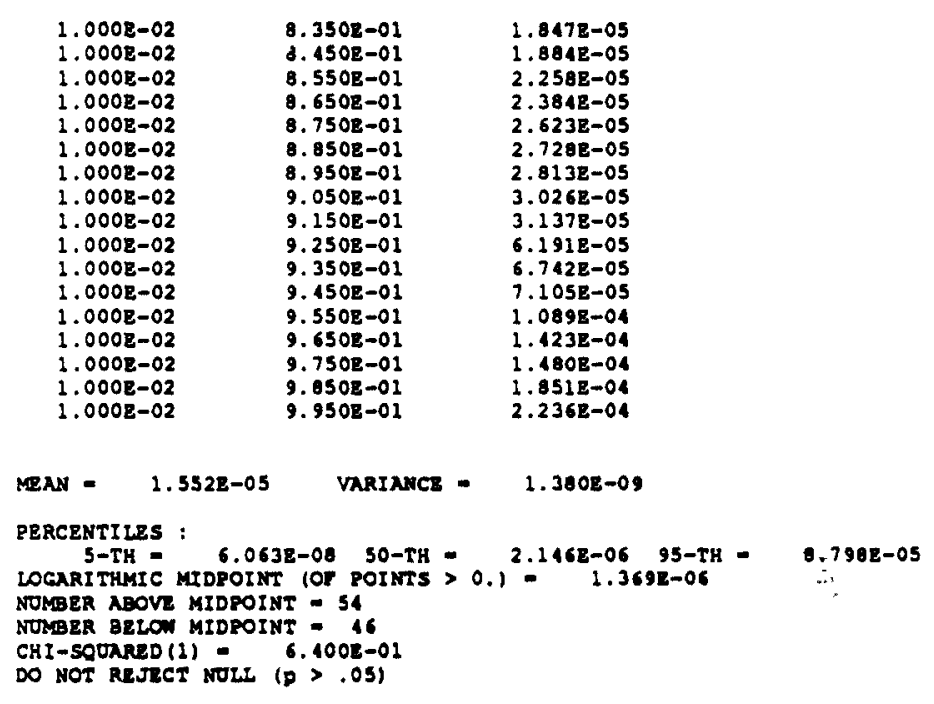




\begin{abstract}
CONSEQUENCE MEASURE:
\end{abstract}
EARLY DEATHS PER YEAR

BASE CASE :

SURRY LALHC (MACCS)

ISSUR LEVELS FOR EACH SAMPLE MEMPER. IN ORDER OF INCREASING RISK

RISK ISSUE LEVELS, FOR ALL ISSOES

\begin{tabular}{|c|c|c|c|c|c|c|c|c|c|c|c|c|c|c|c|}
\hline & & & & & & & & & & & 1 & 12 & 13 & 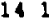 & 6 \\
\hline E-09 & & & 3 & 2 & 1 & 1 & 3 & 1 & 2 & 2 & 6 & 5 & 2 & 1 & 2 \\
\hline $139 E-08$ & 2 & 1 & 2 & 1 & 3 & 6 & 2 & 2 & 1 & $\overline{1}$ & 5 & 4 & 1 & $i$ & 3 \\
\hline $.646 z-08$ & 2 & 2 & 3 & 1 & 1 & 1 & 8 & 2 & 4 & 4 & 1 & 5 & 2 & 2 & 3 \\
\hline $.176 E-08$ & 2 & 1 & 2 & 1 & ? & 6 & 7 & 2 & 2 & 3 & 1 & 1 & 3 & 1 & 3 \\
\hline $5.789 \mathrm{e}-08$ & 2 & 1 & 2 & 2 & 3 & 2 & 9 & 2 & 1 & 2 & 4 & 4 & 3 & 1 & 1 \\
\hline $.3498-08$ & 2 & 1 & 2 & 2 & 3 & 6 & 2 & 1 & 5 & 3 & 2 & 1 & 2 & 2 & 2 \\
\hline $5.121 E-08$ & 2 & 1 & 3 & 2 & 2 & 6 & 5 & 1 & 3 & 2 & 4 & 3 & 5 & 1 & 2 \\
\hline $9.355 \mathrm{E}-0 \mathrm{~B}$ & 2 & 1 & 2 & 2 & 2 & 2 & 5 & 2 & 3 & 1 & 5 & 2 & 4 & 2 & 3 \\
\hline & & & & & & & & & & & & & & & \\
\hline $.0958-07$ & 2 & 1 & 3 & 2 & 3 & 3 & 9 & 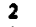 & 3 & 2 & 4 & 3 & 5 & 1 & 2 \\
\hline $.1242-07$ & 2 & 1 & 2 & 2 & 2 & 6 & 7 & 2 & 2 & 2 & 3 & 4 & 2 & 2 & 2 \\
\hline $.2508-07$ & & 2 & 2 & 2 & 3 & 2 & 2 & 2 & 1 & 2 & 1 & 3 & 3 & 3 & 2 \\
\hline $2-07$ & & 2 & 2 & 1 & & 6 & 1 & & & & & & & & \\
\hline $2-07$ & 2 & 2 & 2 & & 1 & 6 & 2 & , & 1 & 2 & 4 & 3 & 4 & 2 & 3 \\
\hline $.2428-07$ & 2 & 1 & 2 & 2 & 3 & 6 & 2 & 2 & 1 & 2 & 4 & 2 & 3 & 1 & 3 \\
\hline $.3012-07$ & & 2 & 2 & 2 & & 2 & 7 & & 1 & 2 & 4 & & 4 & 4 & 2 \\
\hline $92-07$ & 2 & 1 & 2 & 1 & 2 & 6 & 6 & 2 & 2 & 3 & 3 & 4 & 3 & 2 & 3 \\
\hline $2-07$ & 2 & 2 & 2 & 1 & 1 & 5 & 7 & 2 & 5 & 2 & 2 & 3 & 1 & 2 & 1 \\
\hline $.1058-07$ & & 2 & 2 & 1 & 1 & 5 & 5 & 3 & 1 & 4 & 1 & 2 & 2 & 2 & 3 \\
\hline $.3548-07$ & 2 & 1 & 3 & 2 & 2 & 6 & 7 & 2 & 2 & 3 & 1 & 4 & 2 & 2 & 3 \\
\hline-07 & 2 & 1 & 3 & 2 & 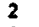 & 2 & 5 & 4 & ? & 4 & 1 & 5 & 2 & 3 & 4 \\
\hline $82-07$ & 2 & 1 & 2 & 2 & 2 & 5 & 1 & 1 & 2 & 2 & 6 & 5 & 1 & 2 & 1 \\
\hline $2-07$ & 2 & 2 & 2 & 1 & 2 & 3 & 6 & 1 & 1 & 3 & 4 & 3 & 2 & 2 & 3 \\
\hline $.8542-07$ & 2 & 2 & 3 & 2 & $\downarrow$ & 5 & 3 & 2 & $\downarrow$ & 2 & $\mathbf{5}$ & 5 & 2 & & 4 \\
\hline $.0188-07$ & 2 & 2 & 2 & 1 & 2 & 3 & 5 & 1 & 5 & 2 & 3 & 4 & 3 & & 3 \\
\hline $.2362-07$ & & & 2 & & 2 & & & 2 & & $\mathbf{3}$ & 2 & 2 & & & 3 \\
\hline 1. $505 E-07$ & 2 & 2 & 3 & 1 & 1 & 3 & 1 & 2 & 2 & 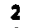 & 2 & 1 & 3 & & 3 \\
\hline $1.6532-07$ & 2 & 1 & 3 & 1 & 3 & 6 & 5 & 3 & 1 & 3 & 3 & 1 & 2 & 3 & 2 \\
\hline $8-07$ & & 2 & 2 & 2 & 3 & 2 & 6 & 2 & 3 & 2 & 1 & 3 & 2 & 2 & 4 \\
\hline $\begin{array}{l}07 \\
.07\end{array}$ & $\begin{array}{l}2 \\
2\end{array}$ & 2 & 2 & 1 & 2 & 3 & 9 & 2 & 4 & 1 & 5 & 4 & 2 & 1 & 3 \\
\hline $5.4898-07$ & 2 & 2 & 3 & 2 & 2 & 3 & 1 & 1 & 3 & 4 & 1 & 2 & 2 & & \\
\hline $8-07$ & 2 & 2 & 2 & 2 & 3 & 3 & 7 & 1 & 4 & 2 & 4 & 2 & 3 & 2 & 2 \\
\hline $6.977 \mathrm{E}-07$ & 3 & 1 & 3 & 1 & 1 & 1 & B & 2 & 4 & 4 & 1 & 3 & 3 & & 2 \\
\hline $.8368-07$ & 2 & 2 & 2 & 2 & 3 & 1 & 5 & 1 & 3 & 3 & 2 & 3 & 3 & 2 & 2 \\
\hline $8.652 \mathrm{E}-07$ & 2 & 1 & 2 & 2 & 1 & 2 & 2 & 2 & 3 & 2 & 4 & 3 & 2 & 3 & 2 \\
\hline $0.713 \mathrm{E}-07$ & 2 & 1 & 3 & 1 & 1 & 2 & 4 & 2 & 1 & 1 & 5 & 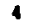 & 4 & 3 & 2 \\
\hline 8. 9 & 2 & 2 & 3 & 2 & 3 & 4 & 4 & 2 & 5 & 2 & 3 & 2 & 3 & & 2 \\
\hline $9.782 \mathrm{E}-07$ & 2 & 1 & 3 & 2 & 1 & 3 & 8 & 3 & 1 & 2 & 4 & 1 & 2 & 4 & 3 \\
\hline $9.9608-07$ & 2 & 1 & 2 & 3 & 2 & 1 & 7 & 2 & 5 & 1 & 6 & 1 & 3 & 3 & 2 \\
\hline $1.0008-06$ & 2 & 2 & 2 & 1 & 2 & 2 & 2 & 3 & 2 & 3 & 1 & 5 & 3 & & $?$ \\
\hline $1.051 \mathrm{E}-06$ & 3 & 1 & 2 & 2 & 3 & 6 & 6 & 1 & 3 & 4 & 1 & 3 & 5 & 1 & 2 \\
\hline $1.0832-06$ & 2 & 1 & 2 & 2 & 3 & 6 & 2 & 2 & 1 & 2 & 5 & 4 & 4 & 2 & 1 \\
\hline $1.1048-06$ & 2 & 1 & 2 & 1 & 2 & 3 & 9 & 3 & 2 & 2 & $\mathbf{5}$ & 4 & 2 & 3 & 2 \\
\hline $1.1358-06$ & 2 & 2 & 3 & 1 & 2 & 2 & 8 & 1 & 1 & 2 & 5 & 1 & 3 & 2 & 4 \\
\hline $1.252 \mathrm{E}-06$ & 2 & 2 & 3 & 2 & 1 & 1 & 4 & 4 & 4 & 3 & 4 & 1 & 2 & 2 & 1 \\
\hline $1.730 \mathrm{~B}-06$ & 2 & 2 & 2 & 1 & 3 & 1 & 3 & 1 & 1 & 2 & 3 & 5 & 2 & 3 & 2 \\
\hline $1.7312-06$ & 2 & 2 & 3 & 3 & 1 & 4 & 5 & 1 & 2 & 2 & 4 & 2 & 2 & 2 & 4 \\
\hline e-06 & 2 & 2 & 2 & 1 & 1 & 6 & 2 & 1 & 6 & 3 & 3 & 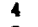 & 5 & 1 & 3 \\
\hline $1-06$ & 2 & 2 & 2 & 1 & 3 & 6 & 5 & 2 & 3 & 2 & 5 & 3 & 5 & 3 & 2 \\
\hline $2.2618-06$ & 2 & 2 & 2 & 2 & 2 & 6 & 9 & 1 & 4 & 3 & 1 & 1 & 3 & 3 & 3 \\
\hline $.504 \mathrm{E}-06$ & 1 & 2 & 2 & 2 & 3 & 2 & 1 & 3 & 4 & 2 & 3 & 4 & 2 & 2 & 2 \\
\hline $.7908-06$ & 2 & 1 & 2 & 2 & 3 & 3 & 4 & 2 & 3 & 2 & 5 & 2 & 3 & 3 & 2 \\
\hline$z-06$ & 3 & 2 & 2 & 2 & 1 & 3 & 9 & 3 & 3 & 3 & 3 & 5 & 1 & & 1 \\
\hline 3. $300 \mathrm{~B}-06$ & 3 & 1. & 2 & 1 & 3 & 2 & 2 & 3 & 4 & 3 & 1 & 5 & 3 & 2 & 4 \\
\hline $4.364 z-06$ & 2 & 2 & 3 & 3 & 1 & 6 & 4 & 2 & 5 & 2 & 5 & 3 & 2 & 1 & 1 \\
\hline 4.50 & 2 & 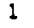 & 3 & 1 & 3 & 6 & 6 & 4 & 1 & 3 & 1 & 2 & 2 & & 3 \\
\hline $0.067 z-06$ & 2 & 2 & 2 & 2 & 3 & 4 & 2 & 3 & 3 & 2 & 3 & 2 & 4 & 2 & 3 \\
\hline $5.2608-06$ & 2 & 2 & 2 & 3 & 2 & 6 & 9 & 1 & 4 & 1 & 6 & 1 & 2 & 2 & 3 \\
\hline$z-06$ & 2 & 1 & 3 & 2 & 1 & 6 & 3 & 5 & 1 & 3 & 4 & 2 & 3 & 2 & 1 \\
\hline 6.65 & 2 & 2 & 2 & 2 & 2 & 6 & 4 & 3 & 3 & 2 & E & 1 & 1 & 1 & 1 \\
\hline 6.67 & 2 & 2 & 3 & 2 & 2 & 3 & 7 & 3 & 4 & 3 & 3 & 2 & 1 & 2 & 1 \\
\hline $6.891 z-06$ & 2 & 1 & 3 & 1 & 3 & 6 & 8 & 4 & 1 & 3 & 2 & 3 & 2 & 2 & 1 \\
\hline$z-06$ & 2 & 2 & 2 & 3 & 2 & 5 & 3 & 4 & 2 & 2 & 4 & 4 & 3 & 1 & 1 \\
\hline $7.505 B-06$ & 2 & 2 & 3 & 3 & 2 & 2 & 9 & 3 & 1 & 2 & 7 & 1 & 3 & 2 & 1 \\
\hline 7.95 & 2 & 2 & 2 & 3 & 3 & 6 & 7 & 2 & 7 & 1 & 6 & 4 & 3 & 2 & 1 \\
\hline $9.231 \mathrm{E}-06$ & 2 & 1 & 1 & 3 & 1 & 6 & 3 & 3 & 3 & 2 & 4 & 5 & 3 & 3 & 2 \\
\hline 9.422 & 2 & 2 & 2 & 2 & 3 & 2 & 4 & 4 & 6 & 1 & 6 & 2 & 3 & 1 & 3 \\
\hline-06 & 2 & 1 & 2 & 1 & 2 & 3 & 9 & 5 & 3 & 3 & ? & 3 & 5 & 3 & 2 \\
\hline $1.0018-05$ & 2 & 1 & 2 & 2 & 3 & 2 & 1 & 2 & 4 & 1 & 6 & 2 & 3 & 2 & 4 \\
\hline $1.011 \mathrm{E}-05$ & 2 & 2 & 3 & 1 & 1 & 6 & 8 & 3 & 4 & 3 & 3 & 2 & 3 & 2 & 3 \\
\hline 1.012 & 2 & 1 & 2 & 2 & 2 & 6 & 4 & 4 & 4 & 2 & 1 & 3 & 2 & 2 & 2 \\
\hline 1.0 & 2 & 1 & 2 & 1 & 1 & 6 & 2 & 4 & 2 & 3 & 3 & 1 & 3 & 1 & 3 \\
\hline 1.09 & 2 & 1 & 2 & 1 & 3 & 6 & 9 & 3 & 5 & 1 & 5 & 3 & 1 & 2 & 1 \\
\hline $1.096 \mathrm{E}-05$ & 2 & 1 & 2 & 2 & 1 & 5 & 7 & 4 & 2 & 1 & 4 & 5 & 3 & 2 & 2 \\
\hline 1.2 & 1 & 1 & 3 & 1 & 3 & 3 & 6 & 5 & 3 & 3 & 3 & 3 & 4 & 3 & 2 \\
\hline $1.332 \mathrm{E}-05$ & 2 & 2 & 2 & 2 & 3 & 1 & 7 & 2 & 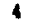 & 1 & 6 & 4 & 3 & 2 & \\
\hline 1.3418 & 2 & 2 & 2 & 1 & 3 & 2 & 5 & 5 & 3 & 2 & 4 & 2 & 2 & 2 & 2 \\
\hline 1. $3852-05$ & 2 & 2 & 2 & 1 & 2 & 5 & 4 & 5 & 5 & 4 & 1 & 1 & 3 & 1 & 1 \\
\hline .4 & 2 & 2 & 3 & 3 & 2 & 4 & 7 & 3 & 3 & 2 & 4 & 2 & 3 & 3 & \\
\hline $1.427 \mathrm{E}-05$ & 2 & 1 & 2 & 2 & 3 & 6 & 5 & 4 & 6 & 2 & 6 & 1 & 3 & 2 & 2 \\
\hline 5 & 1 & 2 & 2 & 2 & 3 & 4 & 4 & 1 & 2 & 2 & 4 & 5 & 3 & 4 & 1 \\
\hline $1.843 z-05$ & 2 & 1 & 3 & 2 & 1 & 3 & 6 & 5 & 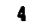 & 2 & 4 & 5 & 3 & 2 & 1 \\
\hline
\end{tabular}


NUREG/CR-4551, VOL. 1: DRAFT REPORT FOR COMMENT (SEPTEMBER, 1986)

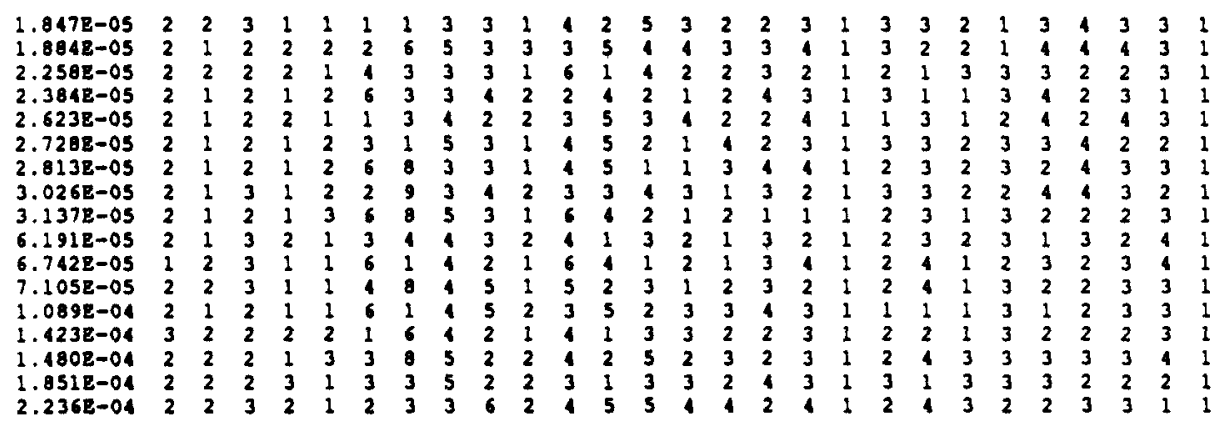




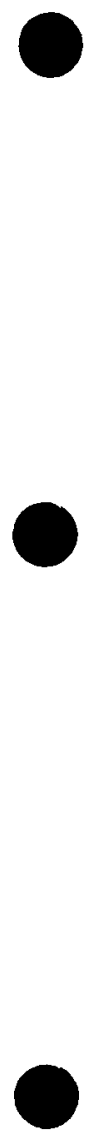

CONSEQUENCE MEASURE:

EARLY DEATHS PER YEAR

BASE CASE :

SURRY WALHC (MACCS)

FRACTIONAL CONTRIBUTION OF RACH SEQUENCE TO MEAN RISK SEOUENCE

SYYB

CONTRIBUTION

SYYB

0.0218

SYNI

0.0001

SYM:

ANon

AYMN

AYNI

0.0005

0.0000

0.0000
0.0070

AYYB

TYYBN

0.0168

TYYBD

$\mathrm{VYNI}$

0.0008

0.0551

0.0586

0.0588

0.0227

1

CONSEOUENCE MRASURE:

EARLY DEATHS PER YEAR

BASE CASE :

SURRY WALHC (MACCS)

FRACTIONAL CONTRIBUTION OF EACH BIN TO REN RISK

BIN

CONTRIBUTION

BIN

BIN 2

BIN 3

BIA 4

BIN 5

BIN 6

BIM

BIN

BII 9

Bra 10

BIN 11

BIN 13

BIA 14

BIN 15
BIN 16

BIX 17

BIX 1

BIx 19

1 CONSEOUENCE MEASURE:

$$
0.0369
$$

0.0004

0.0009

0.0002

0.0245

0.0246

0.0000

0.0000

0.0000

0.0002

0.0000

0.0037

0.0387

0.0000

0.0000

0.0000

0.0000

0.8138

0.0594

0.0098

0.0114

EARLY DEATHS RER YEAR

BAsE CAse

SORRY WALHC (MACCS)

FRACTIONAL CONTRIBOTIONS OP EACH RELEASE TO MEAN RISK RELEASE

SoR 16

SOR $2 G$

SOR 30

SOR 16

SOR $5 \mathrm{G}$

SUR $6 G$

SOR 76

SOR

SUR 9

SUR 11 G

SUR $12 G$

SUR $13 G$

SUR $14 G$

SUR $15 \mathrm{G}$

SUR 160

SUR 170

SUR 186

SUR 196

SUR 206

SOR $21 G$

SUR $22 G$

SOR $23 G$

SUR $24 G$

SUR 256

CONTRIBUTION

0.4321

0.1964

0.1466

0.0807

0.0369

0.0675

0.0149

0.0047

0.0002

0.0087

0.0037

0.0043

0.0014

0.0000

0.0000

0.0005

0.0009

0.0000

0.0003

0.0000

0.0001

0.0000

0.0001

0.0000

0.0000 


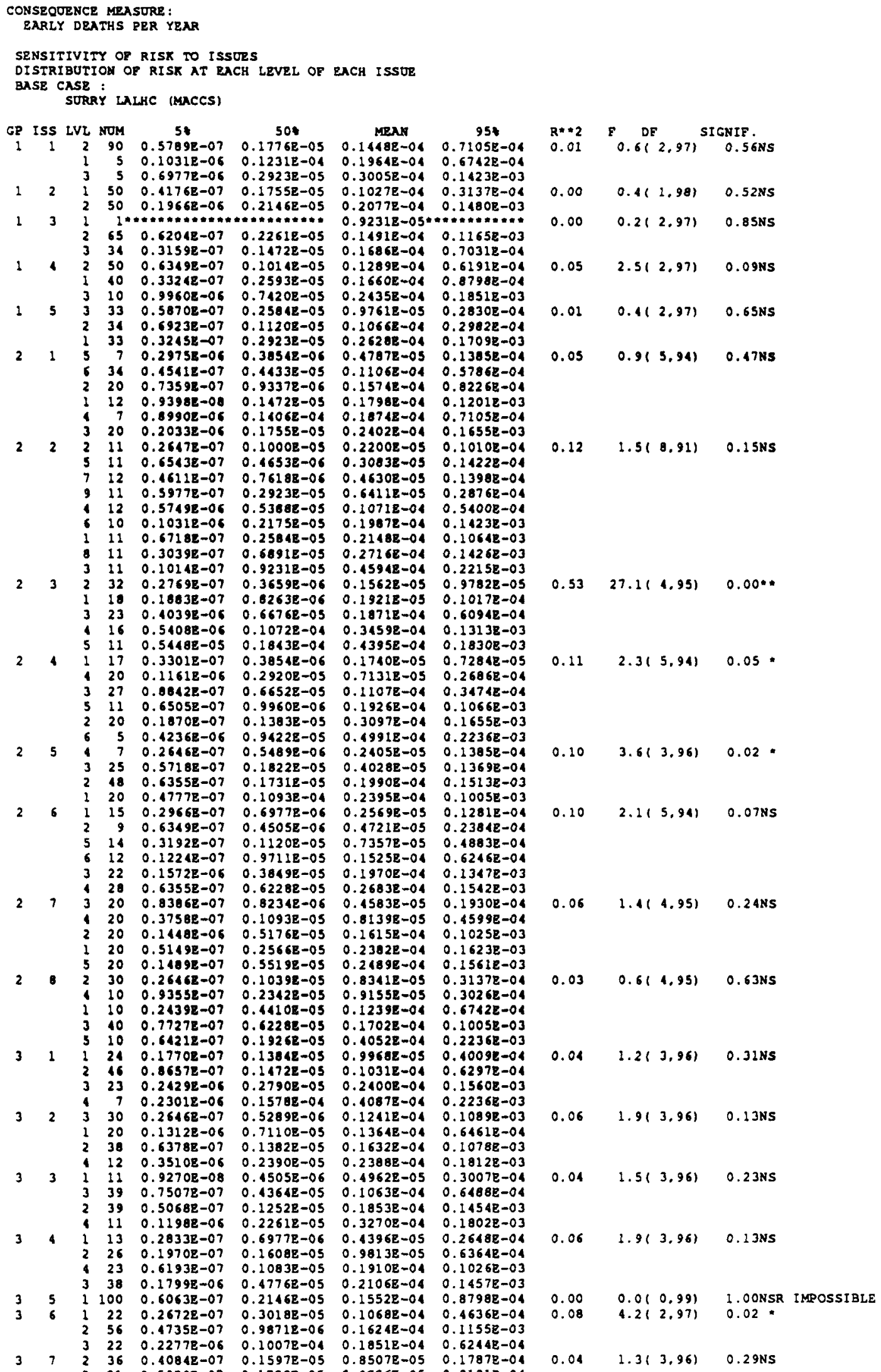


NUREG/CR-4551, VOL. 1: DRAFT REPORT FOR COMMENT (SEPTEMBER, 1986)
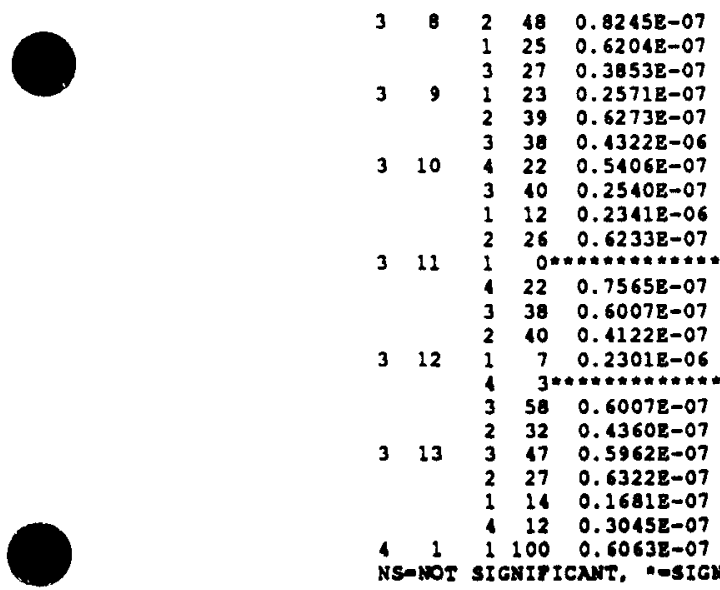

$250.62048-07$

$\begin{array}{lll}3 & 27 & 0.3853 \mathrm{E}-07 \\ 1 & 23 & 0.2571 \mathrm{E}-07\end{array}$

$239 \quad 0.6273 \mathrm{E}-07$

$3380.1322 \mathrm{E}-0$

$340 \quad 0.2540 \mathrm{z}-07$

$1 \quad 12 \quad 0.2341 \mathrm{E}-06$

$260.62338-07$

$0.1192 \mathrm{z}-05 \quad 0.7214 \mathrm{E}-05 \quad 0.2834 \mathrm{~g}-04$

$0.33008-05 \quad 0.21238-04$

$0.2923 \mathrm{E}-05 \quad 0.2499 \mathrm{E}-04 \quad 0.1904 \mathrm{E}-03$

$0.1236 \mathrm{E}-06 \quad 0.2997 \mathrm{E}-05 \quad 0.1860 \mathrm{~B}-04$

$0.1104 \mathrm{E}-05$

$0.1235 \mathrm{E}-0.04$

$0.1860 \mathrm{~B}-04$
$0.4702 \mathrm{E}-04$

$0.8667 \mathrm{E}-05 \quad 0.2634 \mathrm{E}-04 \quad 0.1457 \mathrm{E}-03$

$0.74118-06$

$0.1606 \mathrm{E}-04 \quad 0.1030 \mathrm{~B}-03$

311

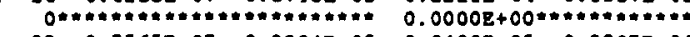

$\begin{array}{lllll}22 & 0.7565 \mathrm{E}-07 & 0.2384 \mathrm{E}-05 & 0.8400 \mathrm{E}-05 & 0.2897 \mathrm{E}-04\end{array}$

$338 \quad 0.6007 \mathrm{E}-07 \quad 0.1472 \mathrm{E}-05 \quad 0.1545 \mathrm{E}-04 \quad 0.1044 \mathrm{E}-03$

$240 \quad 0.4122 \mathrm{E}-07 \quad 0.2748 \mathrm{E}-05 \quad 0.1950 \mathrm{E}-04 \quad 0.1245 \mathrm{E}-03$

$312170.2301 \mathrm{E}-06 \quad 0.2037 \mathrm{E}-05 \quad 0.5086 \mathrm{E}-05 \quad 0.1406 \mathrm{E}-04$

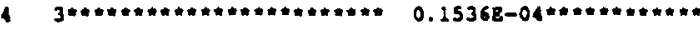

$358 \quad 0.6007 \mathrm{E}-07 \quad 0.3795 \mathrm{E}-05 \quad 0.1620 \mathrm{E}-04 \quad 0.9182 \mathrm{E}-04$

$232 \quad 0.4360 \mathrm{E}-07 \quad 0.1093 \mathrm{E}-05 \quad 0.1658 \mathrm{~g}-04 \quad 0.1309 \mathrm{E}-03$

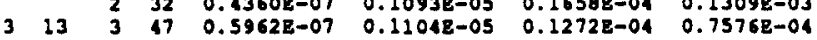

$2 \quad 27 \quad 0.63228-07 \quad 0.43028-05 \quad 0.1350 \mathrm{E}-04 \quad 0.39718-04$

$12140.1681 \mathrm{E}-07 \quad 0.1485 \mathrm{E}-05$ 0.20478-04 $0.1429 \mathrm{E}-03$

$1120.3045 \mathrm{z}-07 \quad 0.2255 \mathrm{E}-05 \quad 0.2523 \mathrm{z}-04 \quad 0.1368 \mathrm{E}-03$

$\begin{array}{llllllll}1 & 1 & 100 & 0.6063 \mathrm{E}-07 & 0.2146 \mathrm{E}-05 & 0.1552 \mathrm{z}-04 & 0.3790 \mathrm{E}-04 & 0.00\end{array}$

$0.01 \quad 0.6(2.97) \quad 0.54 \mathrm{NS}$

0.19

$11.7(2.97)$

$0.00 \%$

0.01

$0.2(3.96)$

0.90 Ns

0.00

$0.0(3.96)$

1. OONSNO DATA

0.02

$0.7(3.96)$

$0.57 \mathrm{NS}$

0.00

$0.1(3.961$

$0.95 N S$

$0.0(0.99) \quad 1.00$ NSR IMPOSSIBLE 


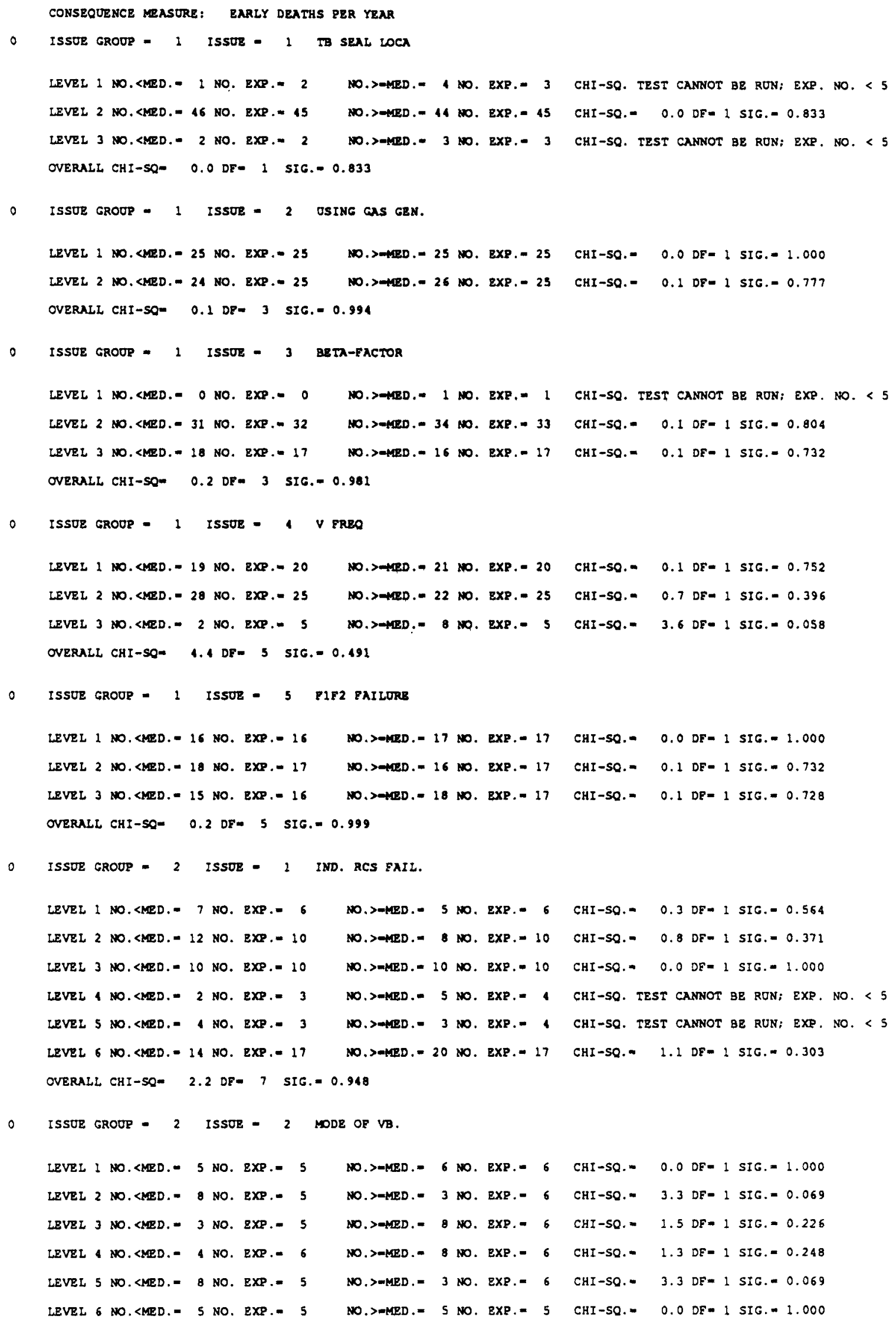




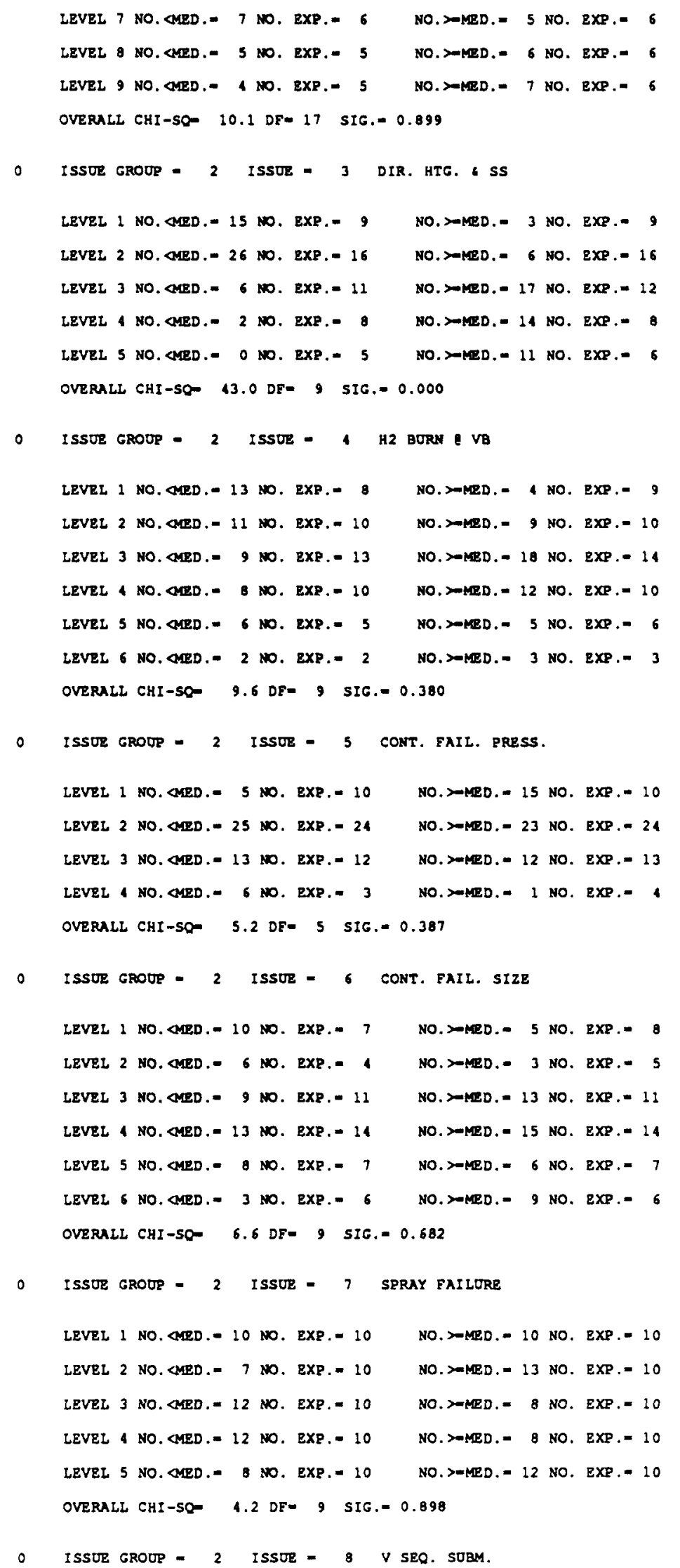




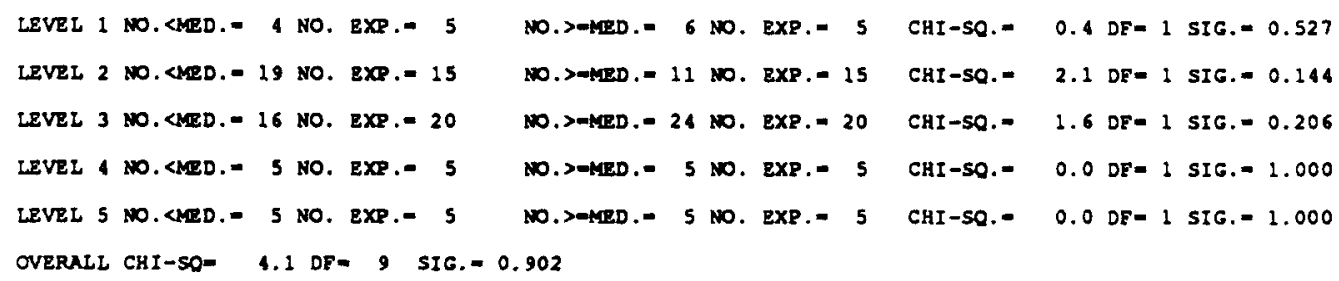




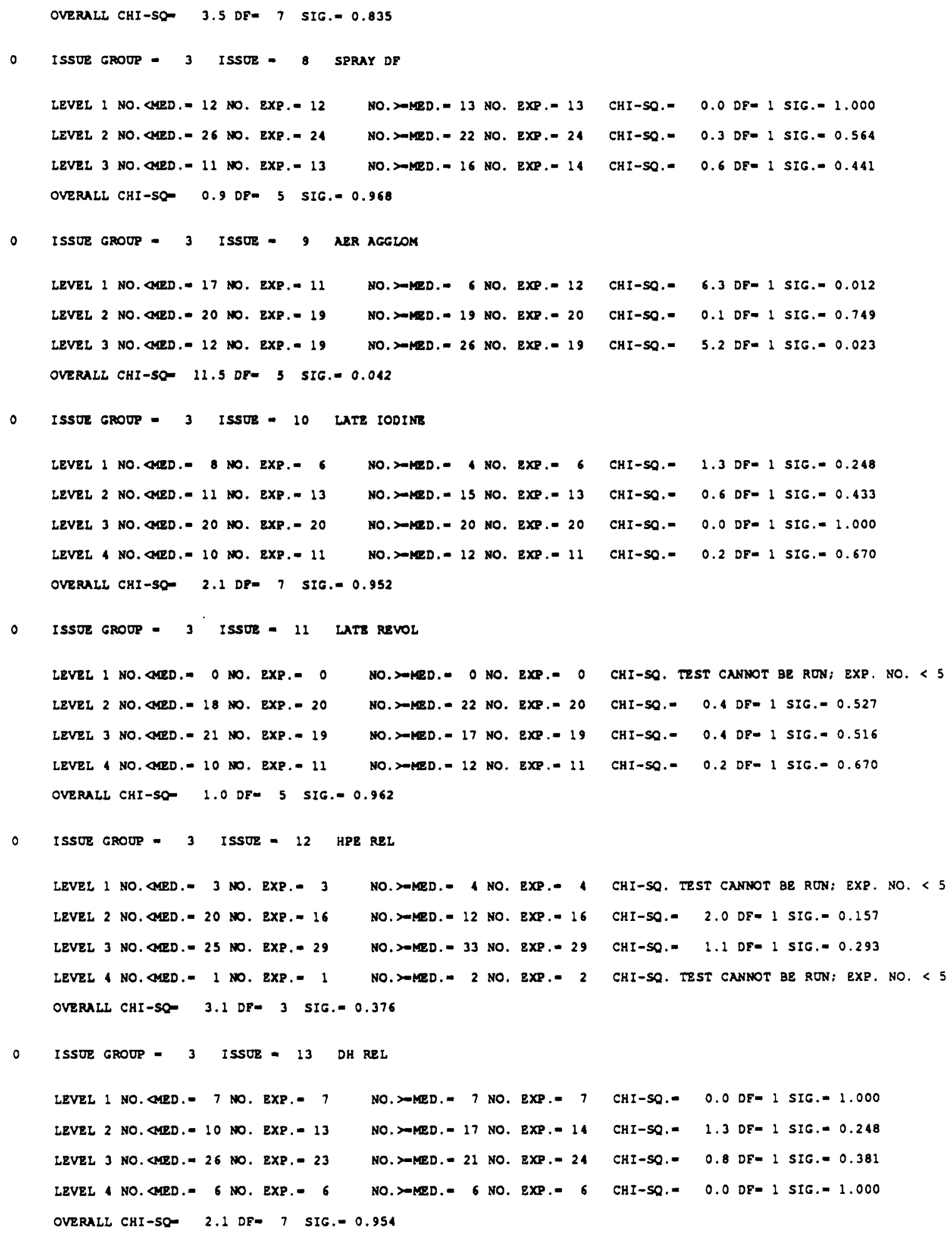


NUREG/CR-4551, VOL. 1: DRAFT REPORT FOR COMMENT (SEPTEMBER, 1986)

CONSEOUENCE MEASURE:

EARLY ILLNESS PER YEAR

BASE CASE :

SORRY LALHC (MACCS)

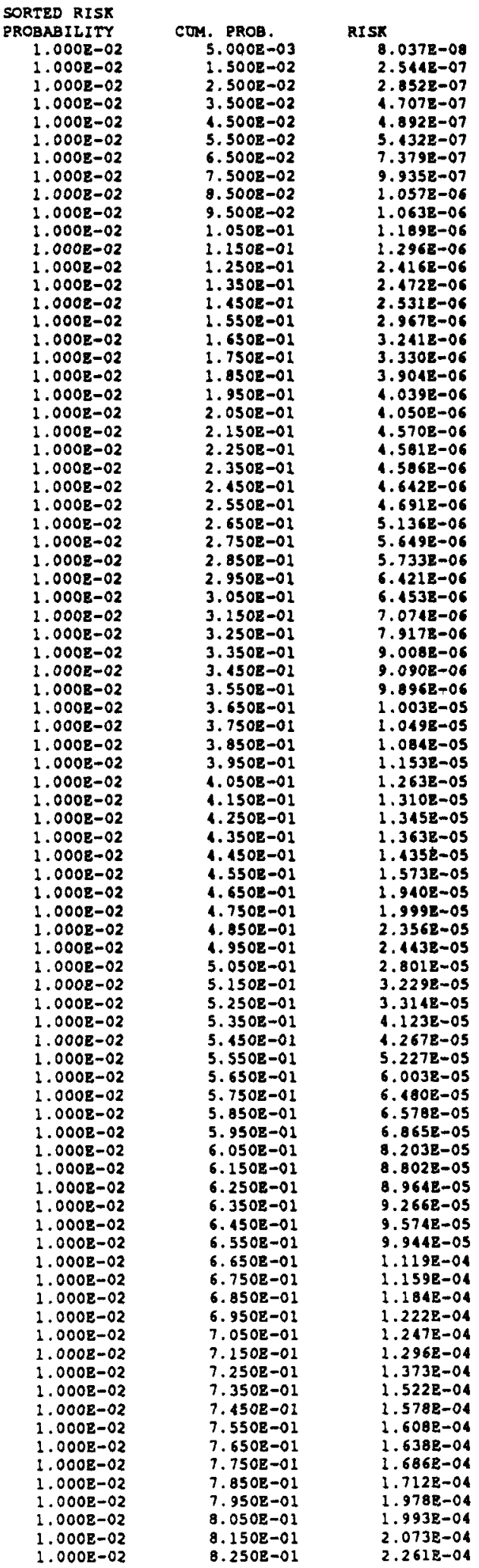


NUREG/CR-4551, VOL. 1: DRAFT REPORT FOR COMMENT (SEPTEMBER, 1986)

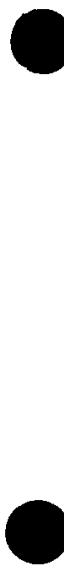

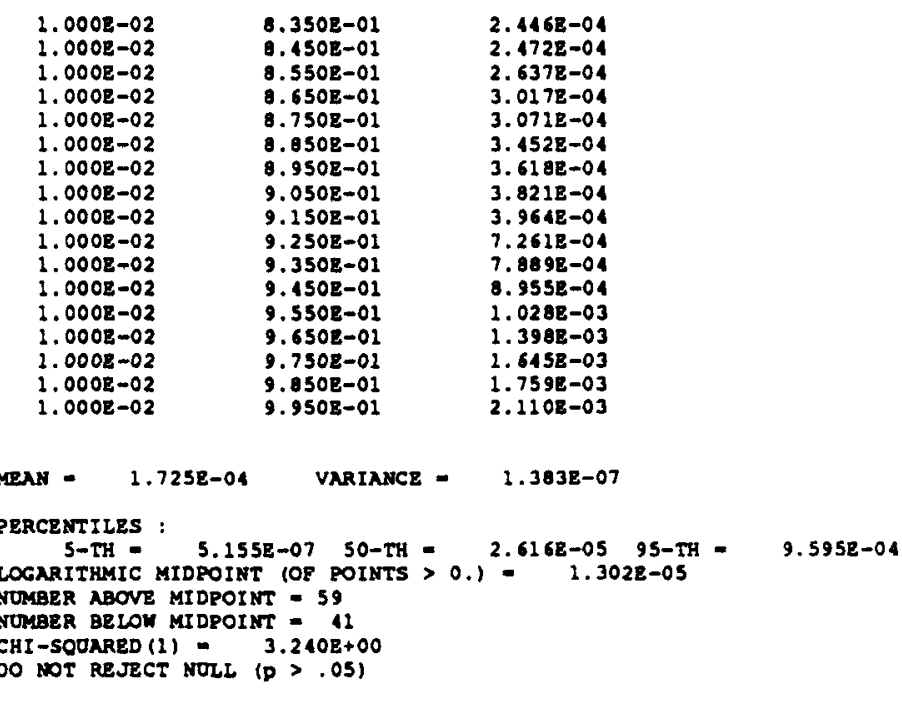


NUREG/CR-4551, VOL. 1: DRAFT REPORT FOR COMMENT (SEPTEMBER, 1986)

\begin{tabular}{|c|c|c|c|c|c|c|c|c|c|c|c|c|c|c|c|c|c|c|c|c|c|c|c|c|c|c|}
\hline & $\begin{array}{l}2 \\
2\end{array}$ & $\begin{array}{l}1 \\
1\end{array}$ & $\begin{array}{l}2 \\
2\end{array}$ & $\begin{array}{l}2 \\
2\end{array}$ & $\begin{array}{l}2 \\
1\end{array}$ & 2 & 6 & $\begin{array}{l}5 \\
4\end{array}$ & $\begin{array}{l}3 \\
2\end{array}$ & 3 & 3 & $\begin{array}{l}5 \\
5\end{array}$ & 4 & 4 & 3 & $\begin{array}{l}3 \\
2\end{array}$ & 4 & $\begin{array}{l}1 \\
1\end{array}$ & 3 & $\begin{array}{l}2 \\
3\end{array}$ & 2 & $\begin{array}{l}1 \\
2\end{array}$ & 4 & $\begin{array}{l}4 \\
2\end{array}$ & 4 & 3 \\
\hline & 2 & 1 & 3 & 2 & 1 & 3 & 6 & 5 & 4 & 2 & 8 & 5 & 3 & 2 & 1 & 2 & 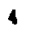 & 1 & 2 & 1 & 2 & 3 & 3 & 3 & 3 & 2 \\
\hline & 2 & 1 & 2 & 1 & 2 & 6 & 3 & 3 & 4 & 2 & 2 & 4 & 2 & 1 & 2 & 4 & 3 & 1 & 3 & 1 & 1 & 3 & 4 & 2 & 3 & \\
\hline $3.071 E-01$ & 2 & 2 & 2 & 2 & 1 & 4 & 3 & 3 & 3 & 1 & 6 & 1 & 4 & 2 & 2 & 3 & 2 & 1 & 2 & 1 & 3 & 3 & 3 & 2 & 2 & 3 \\
\hline $3.4528-04$ & 2 & 1 & 2 & 1 & 2 & 3 & 1 & 5 & 3 & 1 & 4 & 5 & 2 & 1 & 4 & 2 & 3 & 1 & 3 & 3 & 2 & 3 & 3 & 4 & 2 & 2 \\
\hline $.618 \mathrm{~B}-04$ & 2 & 1 & 2 & 1 & 2 & 6 & 8 & 3 & 3 & 1 & 4 & 5 & 1 & 1 & 3 & 4 & 4 & 1 & 2 & 3 & & 3 & 2 & 4 & 3 & 3 \\
\hline $3.8218-04$ & 2 & 1 & 3 & 1 & 2 & 2 & 9 & 3 & 4 & 2 & 3 & 3 & 4 & 3 & 1 & 3 & 2 & 1 & & 3 & & 2 & 4 & 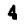 & 3 & \\
\hline $3.964 z-04$ & 2 & 1 & 2 & 1 & 3 & 6 & 8 & 5 & 3 & 1 & 6 & 4 & 2 & 1 & 2 & 1 & 1 & 1 & 2 & 3 & 1 & 3 & 2 & 2 & 2 & 3 \\
\hline $1.262 \mathrm{E}-04$ & 2 & 1 & 3 & 2 & 1 & 3 & 4 & 1 & 3 & 2 & 4 & 1 & 3 & 2 & 1 & 3 & 2 & 1 & 2 & 3 & 2 & 3 & 1 & 3 & 2 & 4 \\
\hline $7.8898-04$ & 1 & 2 & 3 & 1 & 1 & 6 & 1 & 1 & 2 & 1 & 6 & 4 & 1 & 2 & 1 & 3 & 4 & 1 & 2 & 4 & 1 & 2 & 3 & 2 & 3 & 4 \\
\hline $8.955 z-04$ & 2 & 2 & 3 & 1 & 1 & 1 & 8 & 1 & 5 & 1 & 5 & 2 & 3 & 1 & 2 & 3 & 2 & 1 & 2 & 4 & 1 & 3 & 2 & 2 & 3 & 3 \\
\hline $1.028 z=03$ & 2 & 1 & 2 & 1 & 1 & 6 & 1 & 4 & 5 & 2 & 3 & 5 & 2 & 3 & 3 & 4 & 3 & 1 & 1 & 1 & 1 & 3 & 1 & 2 & 3 & 3 \\
\hline 1. $3988-03$ & 2 & 2 & 2 & 1 & 3 & 3 & 8 & 5 & 2 & 2 & 4 & 2 & 5 & 2 & 3 & 2 & 3 & 1 & 2 & 4 & 3 & 3 & 3 & 3 & 3 & 4 \\
\hline $1.645 E-03$ & 3 & 2 & 2 & 2 & 2 & 1 & 6 & 4 & 2 & 1 & 4 & 1 & 3 & 3 & 2 & 2 & 3 & 1 & 2 & 2 & 1 & 3 & 2 & 2 & 2 & 3 \\
\hline $7598-03$ & 2 & 2 & 2 & 3 & 1 & 3 & 3 & 5 & 2 & 2 & 3 & 1 & 3 & 3 & 2 & 4 & 3 & 2 & 3 & 1 & 3 & 3 & 3 & 2 & 2 & 2 \\
\hline $10 \mathrm{~g}-03$ & 2 & 2 & 3 & 2 & 1 & 2 & 3 & 3 & 6 & 2 & 4 & $\mathbf{5}$ & 5 & 4 & 4 & 2 & 4 & 1 & 2 & 4 & 3 & 2 & 2 & 3 & 3 & 1 \\
\hline
\end{tabular}


CONSEQOENCE YEASURE:

EARLY ILLNESS PER YEAR

BAsE CASE :

SURRY LALHC (MACCS)

FRACTIONAL, CONTRIBUTION OF RACH SEQUENCE TO MAN RISK

SEOUENCE CONTRIBUTION

SYYB $\quad 0.0232$

syni

0.0001

STrin

Sniv

STNN

ANN

AYNN

0.6296

AYNI

TNNN

Tryan

TrYas

0.0006

0.0000

0.0077

0.0193

0.0008

0.1800

0.0571

TYNI

0.0603

0.0002

1 CONSEOUENCE TENORE:

BARLY ILLNESS PER YEAR

BASE CASE :

SURRY LALHC (MACCS)

FRACTIONAL CONTRIBUTION OE EACH BIN TO MEAN RISK

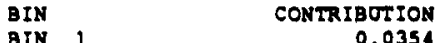

BIN $1 \quad 0.0354$

BIN 20.0005

$\begin{array}{ll}\operatorname{BIN} 3 & 0.0010 \\ \operatorname{BIN} 1 & 0.0001\end{array}$

BIN 5

BIN $6 \quad 0.0000$

BIN 7

BIN $8 \quad 0.0000$

BIN $9 \quad 0.0001$

BIR $10 \quad 0.0000$

BIN $11 \quad 0.0033$

BIN $12 \quad 0.0416$

BIN $13 \quad 0.0000$

BIN $14 \quad 0.0000$

BIN $15 \quad 0.0000$

BIN $16 \quad 0.8061$

BIN $17 \quad 0.0623$

BIN $18 \quad 0.0110$

CONSEOUENCE MRASURE:

EARLY ILLNESS PER YEAR

0.0116

BASE CASE :

SURRY LALHC (MACCS)

FRACTIONAL CONTRIBOTIONS OE BACH RELEASE TO RRAN RISK RELRASE

SUR 16

SUR 26

SUR $3 G$

SUR 16

SUR 5 G

$\operatorname{SUR} 6 \frac{6}{10}$

SUR $T$ SUR

SUR 96

SOR $10 \mathrm{G}$

SUR $11 G$

SUR $12 G$

SUR 136

SUR 146

SUR $15 \mathrm{G}$

SOR $16 \mathrm{G}$

SUR $17 G$

SUR $18 \mathrm{G}$

SOR 196

SUR 200

SUR $21 G$

SUR 226

SUR 236

SOR $24 G$

CONTRIBOTION

0.3644

0.2043

0.1666

0.1055

0.0434

0.0745

0.0163

0.0046

0.0001

0.0098

0.0030

0.0049

0.0049

0.0000

0.0004

0.0000

0.0007

0.0000

0.0002

0.0002

0.0001

0.0000

0.0001

0.0000

SUR 256

0.0000 


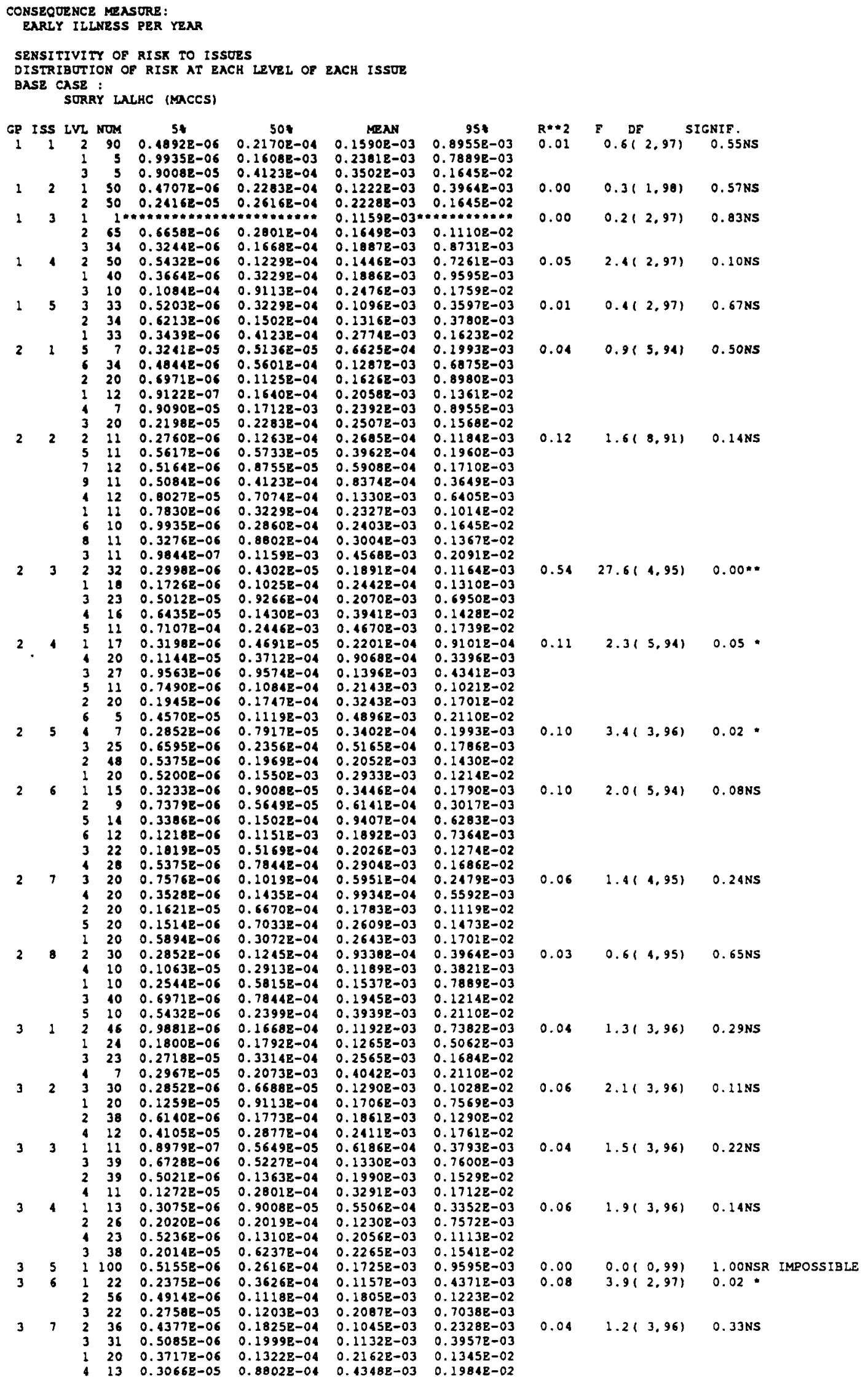


NUREG/CR-4551, VOL. 1: DRAFT REPORT FOR COMMENT (SEPTEMBER, 1986)

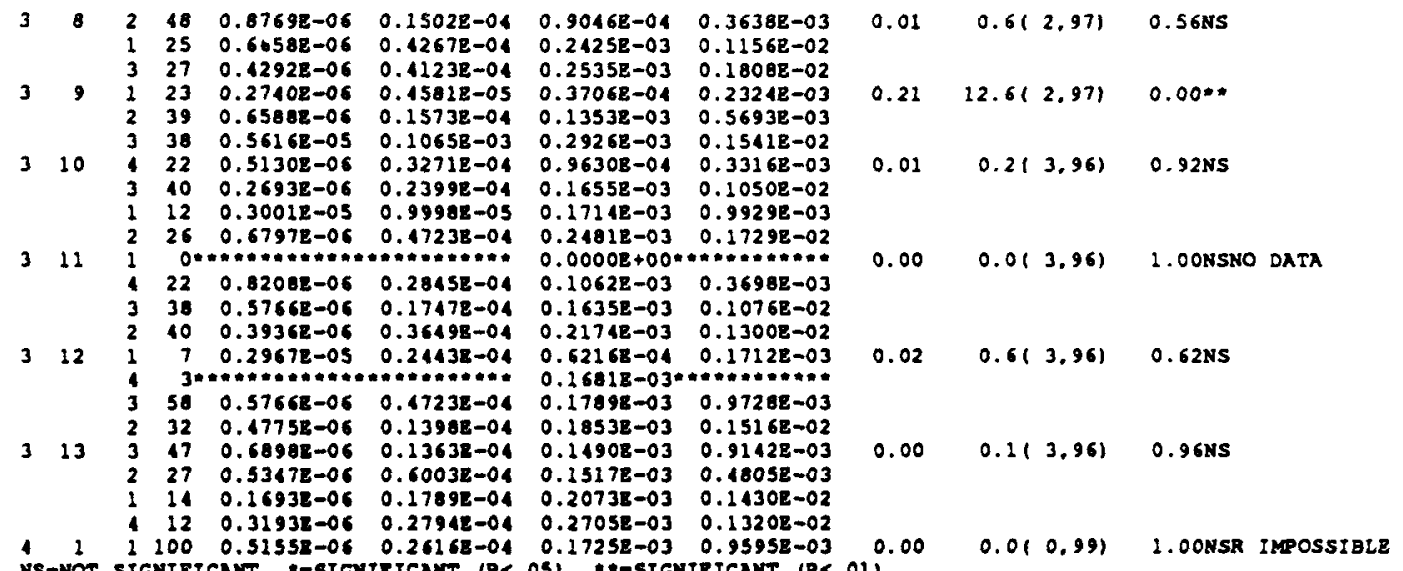
NS-NOT SIGNIFICNAT. "-SIGAITICNIT (P<. OSI. "-SIGNITICNNT (P<.01) 
CONSEQUENCE MEASURE: EARLY ILLNESS PER YEAR 


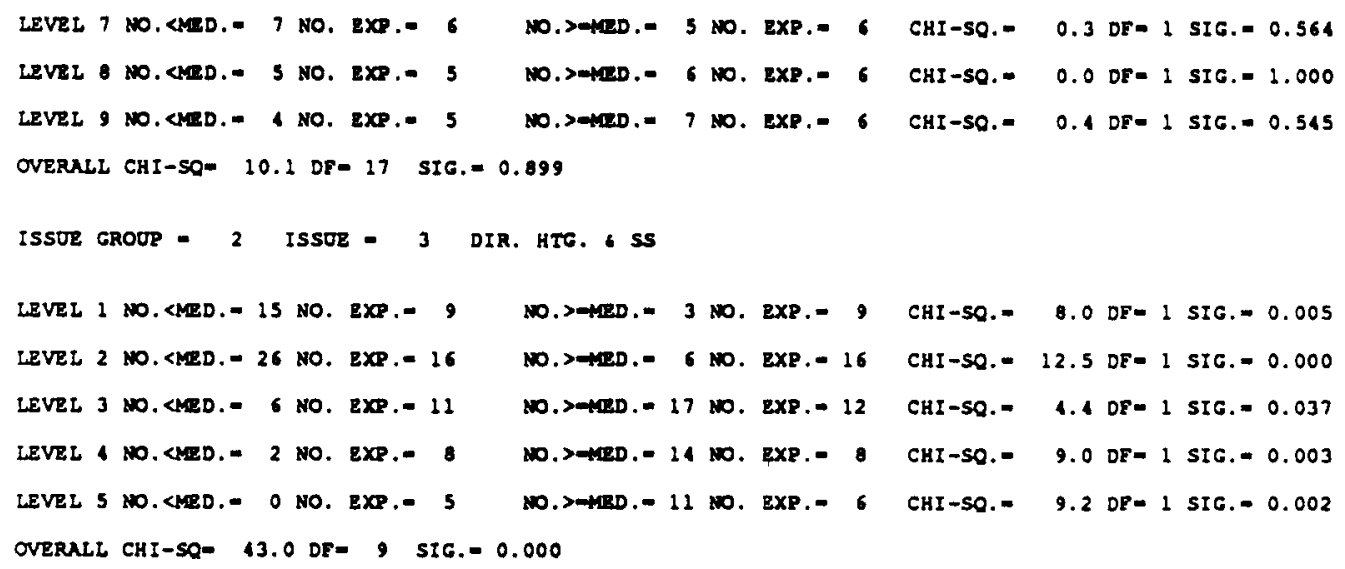




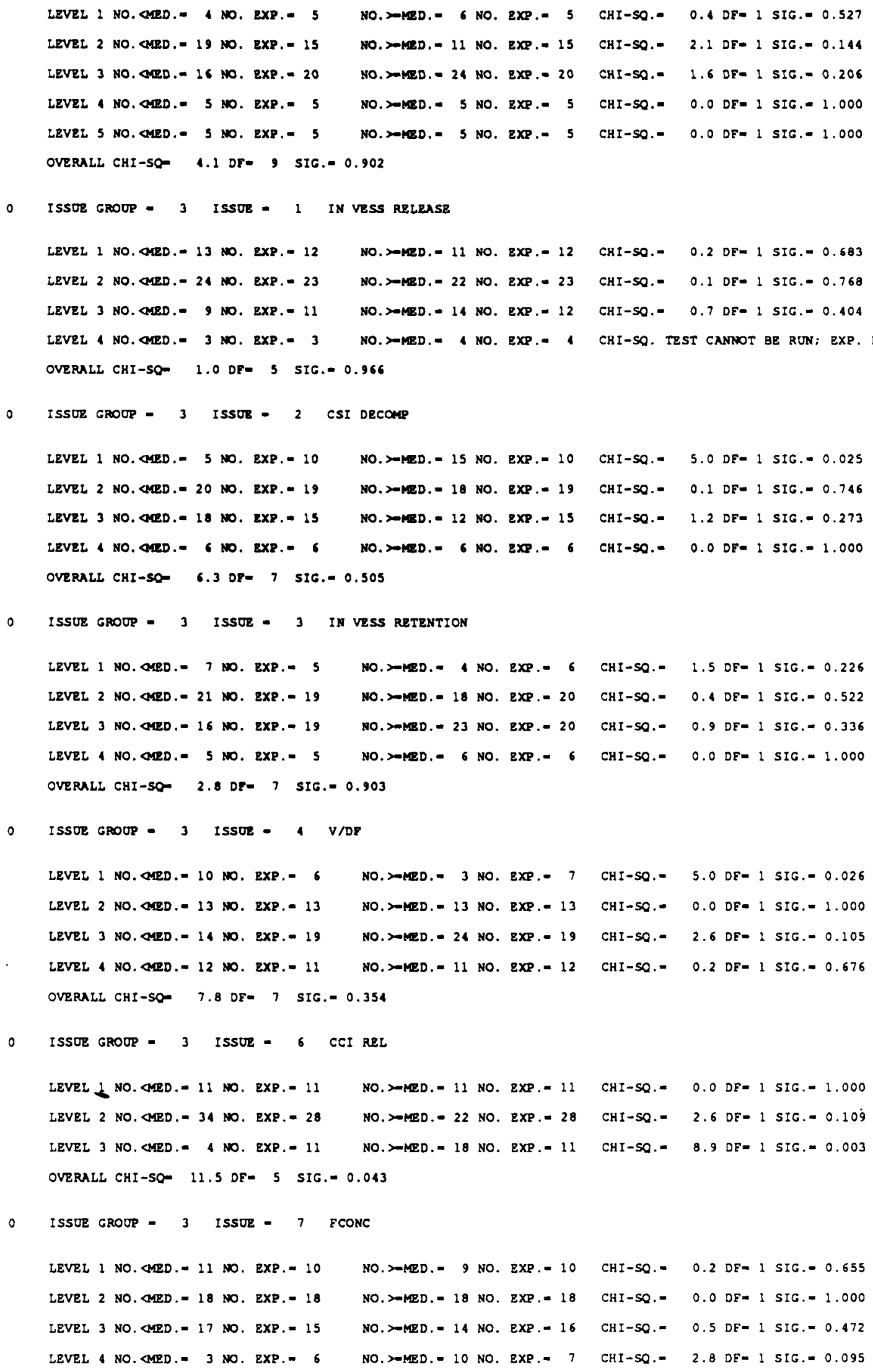




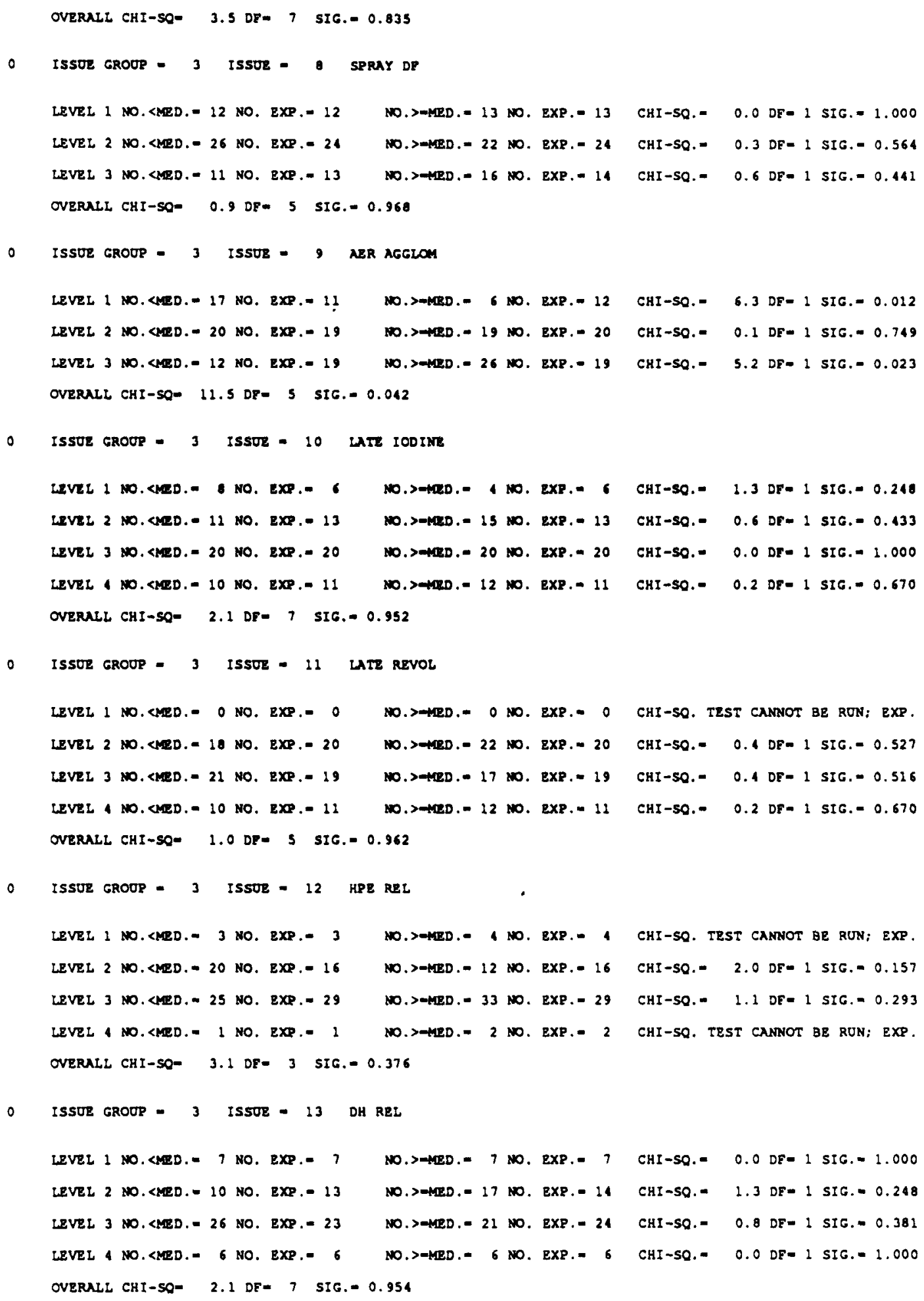


CONSEQUENCE MEASURE

IND. RISK OF FATALITY

BASE CASE :

SORRY LALHC (MACCS)

SORTED RISK

PROBABILITY

$1.000 \mathrm{~B}-02$

1. $000 \mathrm{E}-02$

1. $000 \mathrm{E}-02$

$1.0008-02$

1. $000 \mathrm{~B}-02$

$1.000 \mathrm{E}-02$

1. $000 \mathrm{E}-02$

1. $000 \mathrm{OE}-02$

1. $000 \mathrm{E}-02$

$1.000 \mathrm{E}-02$

$1.000 \mathrm{~B}-02$

1. $000 \mathrm{~B}-02$

1. $000 \mathrm{~B}-02$

$1.000 \mathrm{E}-02$

1. $000 \mathrm{E}-02$

1. $000 \mathrm{~B}-02$

1. $000 \mathrm{~B}-02$

$1.0002-02$

$1.0002-02$

1. $0008-02$

1. $000 \mathrm{z}-02$

$1.0008-02$

1. $000 \mathrm{z}-02$

$.0008-02$

$1.0008-02$

$1.000 \mathrm{z}-02$

$1.000 \mathrm{z}-02$

1. $000 \mathrm{z}-02$

$1.0008-02$

$1.0008-02$

$1.0008-02$

$.000 \mathrm{z}-02$

$1.000 \mathrm{z}-02$

1. $000 \mathrm{~B}-02$

$1.0008-02$

$1.0008-02$

$1.0008-02$

$1.000 \mathrm{~B}-02$

$1.000 \mathrm{~B}-02$

$1.0008-02$

$1.000 \mathrm{E}-02$

1. $000 \mathrm{E}-02$

. $000 \mathrm{E}-02$

$1.000 \mathrm{e}-02$

1. $000 \mathrm{O}-02$

$1.0008-02$

$1.000 \mathrm{z}-02$

$1.000 \mathrm{~B}-02$

1. $000 \mathrm{E}-02$

1. $000 \mathrm{E}-02$

$1.0008-02$

1. $000 \mathrm{CO}-02$

1. $000 \mathrm{~B}-02$

$1.000 \mathrm{~B}-02$

$1.000 \mathrm{E}-02$

$1.000 \mathrm{~B}-02$

1. $0008-02$

$1.000 \mathrm{z}-02$

$1.000 \mathrm{E}-02$

$1.000 \mathrm{E}-02$

$1.000 \mathrm{E}-02$

1. $000 \mathrm{~B}-02$

$1.0008-02$

.0008-02

$1.000 \mathrm{E}-02$

1. $000 \mathrm{O}-02$

$1.000 \mathrm{~B}=02$

$1.000 \mathrm{E}-02$

$1.000 \mathrm{E}-02$

$1.000 \mathrm{E}-02$

$1.0008-02$

$1.000 \mathrm{z}-02$

$1.000 \mathrm{E}-02$

$1.000 \mathrm{~B}-02$

$1.000 \mathrm{E}-02$

$1.000 \mathrm{E}-02$

1. $000 \mathrm{E}-02$
$1.000 \mathrm{E}-02$

1.000e-02

$1.000 \mathrm{e}-02$

$1.000 \mathrm{E}-02$

$1.000 \mathrm{E}-02$

$1.000 \mathrm{E}-02$

1. $000 \mathrm{E}-02$

$1.000 \mathrm{E}-02$
$1.00 \mathrm{OE}-02$
COM. PROB.

5.0008-03

$1.500 \mathrm{E}-02$

2. 50OE-02

$3.500 \mathrm{E}-02$

$4.5008-02$

5. 500B-02

$6.500 \mathrm{z}-02$

$7.500 \mathrm{E}-02$

$8.5008-02$

$9.500 \mathrm{E}-02$

$1.050 \mathrm{E}-01$

1. $150 \mathrm{~B}-01$

1. 250E-01

1. 350E-01

1. 450 E-01

$1.5508-01$

$1.6508-01$

$1.750 \mathrm{E}-01$

$1.050 \mathrm{z}-01$

$1.9508-01$

$2.0508-01$

1.0501

$2.150 \mathrm{E}-01$

$2.2508-01$

$2.350 \mathrm{z}-01$

$2.450 \mathrm{~B}-01$

$2.5508-01$

$2.650 \mathrm{~B}-01$

2.7508-01

2. 850B-01

$2.0502-01$

$2.9308-01$

3. $050 \mathrm{z}-01$

$3.150 z-01$

3.2508-01

3.350z-01

3. 450z-01

3.5508-01

3. 650E-01

3.750E-01

3.850E-01

3.950E-01

. $050 \mathrm{E}-01$

1.1508-01

4. $250 \mathrm{E}-01$

4.350E-01

$4.4508-01$

$4.550 \mathrm{E}-01$

$4.650 \mathrm{E}-01$

$4.750 \mathrm{E}-01$

$4.850 \mathrm{e}-01$

4.9508-01

5. 0508-01

$5.150 \mathrm{~B}-01$

5.250E-01

5. $250 \mathrm{E}-01$

5. $350 \mathrm{~B}-01$

5.5508-01

5.6508-01

5.7502-01

5.850E-01

5. 9508-01

6. $050 \mathrm{~B}-01$

$6.150 \mathrm{~B}-01$

$6.250 \mathrm{E}-01$

6.350E-01

$6.4508-01$

$6.550 \mathrm{~B}-01$

$6.650 \mathrm{~B}-01$

$6.750 \mathrm{E}-01$

6.850z-01

$6.9508-01$

$7.050 \mathrm{E}-01$

$7.150 \mathrm{~B}-01$

$7.250 \mathrm{E}-01$

7. $3508-01$

7. 450B-01

7. 5508-01

$7.650 \mathrm{E}-01$

$7.750 \mathrm{E}-01$

$7.850 \mathrm{~B}-01$

$7.950 \mathrm{z}-01$

. $50 \mathrm{O}-01$

$8.050 \mathrm{E}-01$

$8.150 z-01$
$8.250 \mathrm{E}-01$
RISK

1. $572 \mathrm{E}-10$

2. $334 \mathrm{~B}-10$

$2.701 \mathrm{E}-10$

4. 1178-10

$6.754 \mathrm{~B}-10$

$7.802 \mathrm{E}-10$

$8.455 \mathrm{E}-10$

8. $915 \mathrm{SE}-10$

$9.060 \mathrm{t}-10$

$9.649 \mathrm{E}-10$

$9.738 \mathrm{~B}-10$

$1.065 \mathrm{~B}-09$

$1.1332-09$

1. $1498-09$

$1.180 \mathrm{E}-09$

1.1961-09

1. 2921-09

1. 32 6R-09

$1.306 \mathrm{t}-09$

$1.3992-09$

1. $1138-09$

1. $3568-09$

. $6522-09$

1. 6918-09

$1.7008-09$

1.741E-09

1. $9048-09$

1. $936 \mathrm{~B}-09$

$1.9948-09$

$2.0572-09$

$2.1408-09$

2.3401-09

2. 4592-09

. $1888-09$

2. 5508-09

2. $5718-09$

$2.665 \mathrm{~B}-09$

2.778B-09

2. $9158-09$

3.1338-09

3. $137 \mathrm{E}-09$

3. $173 \mathrm{e}-09$

3. $5948-09$

5. $108 \mathrm{e}-09$

$5.2008-09$

$5.2598-09$

5. $3268-09$

5. $713 \mathrm{~B}-09$

6. 106E-09

$6.3828-09$

8. $1018-09$

9. 1068-09

$9.8518-09$

$1.0458-08$

1. $046 \mathrm{~B}-08$

1. $1978-08$

$1.234 \mathrm{E}-08$

1. $385 \mathrm{~B}-08$

$1.4028-08$

1. $639 \mathrm{E}-08$

1. $749 \mathrm{~B}-08$

1. $765 \mathrm{E}-08$

1. $828 \mathrm{~g}-08$

1. $935 \mathrm{E}-08$

2. $0148-08$

$2.3798-08$

2. $4308-08$

2. $457 \mathrm{~B}-0 \mathrm{~B}$

2. $479 \mathrm{E}-08$

$2.82 \mathrm{BB}-0 \mathrm{~B}$

3. $229 \mathrm{E}-0 \mathrm{~B}$

3. $240 \mathrm{O}-0 \mathrm{~B}$

3. $260 \mathrm{E}-08$

3. $279 \mathrm{E}-08$

3. $3718-08$

3. $6908-08$

3. $702 \mathrm{E}-08$

$3.768 \mathrm{E}-0 \mathrm{~B}$

4. $173 \mathrm{E}-08$

$4.8438-08$

$4.8430-08$

$4.864 \mathrm{E}-08$

$5.320 \mathrm{O}-0 \mathrm{~B}$
$5.904 \mathrm{E}-0 \mathrm{~B}$ 
NUREG/CR-4551, VOL. 1: DRAFT REPORT FOR COMMENT (SEPTEMBER, 1986)

\begin{tabular}{|c|c|c|}
\hline $\begin{array}{l}.000 \mathrm{E}-02 \\
.000 \mathrm{E}-02 \\
.000 \mathrm{E}-02 \\
.000 \mathrm{E}-02 \\
.000 \mathrm{E}-02 \\
.000 \mathrm{E}-02 \\
.000 \mathrm{E}-02 \\
.000 \mathrm{E}-02 \\
.000 \mathrm{E}-02 \\
.000 \mathrm{E}-02 \\
.000 \mathrm{E}-02 \\
.000 \mathrm{E}-02 \\
.000 \mathrm{E}-02 \\
.000 \mathrm{E}-02 \\
.000 \mathrm{E}-02 \\
.000 \mathrm{E}-02 \\
.000 \mathrm{E}-02\end{array}$ & 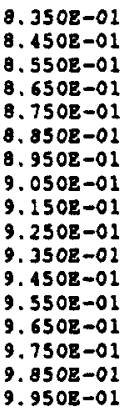 & 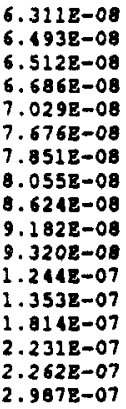 \\
\hline
\end{tabular}

MEAN $=3.0008-08$ VARIANCE $=2.634 \mathrm{E}-15$

5-TH $=7.260 \mathrm{E}-10 \quad 50-\mathrm{TH}=7.322 \mathrm{E}-09$ 95-TH $=1.297 \mathrm{E}-07$

LOGARITHMIC MIDPOINT (OR POINTS > 0.1-6.853E-09

NOMBER ABOVE MIDPOINT - 50

NUMER BELON MTDPOINT - 50

CHI-SOTARED (1) - $0.000 E+00$

DO NOT REJECT NOLL (p $>.05$ ) 
CONSEQUENCE MEASORE:

IND. RISK OF FATALITY

BASE CASE :

SURRY WALHC (MACCS)

ISSOR LEVELS FOR EACH SAYPLE MEMBER, IN OROER OP INCREASING RIST

RISR ISSOE LEVELS. FOR ALL ISSUES

$\begin{array}{cccccccccccccccccccccccccccc}5728 & 10 & 2 & 3 & 1 & 5 & 6 & 7 & 8 & 9 & 10 & 11 & 12 & 13 & 14 & 15 & 16 & 17 & 18 & 19 & 20 & 21 & 22 & 23 & 24 & 25 & 26 & 27\end{array}$ \begin{tabular}{lllllllllllllllllllllllllllll}
$2.3348-10$ & 2 & 2 & 3 & 1 & 1 & 1 & 3 & 1 & 2 & 2 & 6 & 5 & 2 & 1 & 2 & 1 & 2 & 1 & 1 & 2 & 2 & 2 & 3 & 2 & 3 & 1 & 1 \\
\hline
\end{tabular}

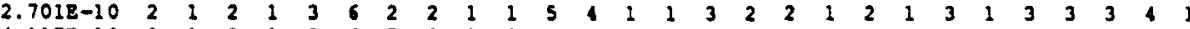

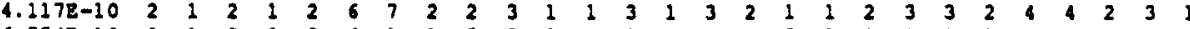

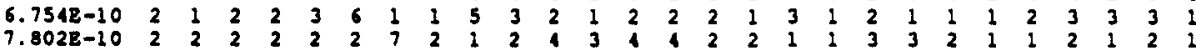

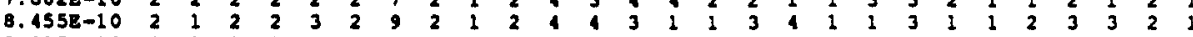

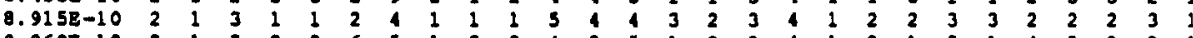

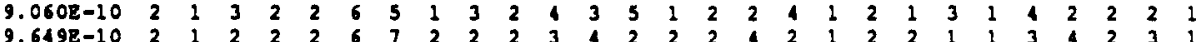
$\begin{array}{llllllllllllllllllllllllllll}9.649 \mathrm{E}-10 & 2 & 1 & 2 & 2 & 2 & 6 & 7 & 2 & 2 & 2 & 3 & 1 & 2 & 2 & 2 & 1 & 2 & 1 & 2 & 2 & 1 & 1 & 3 & 4 & 2 & 3 & 1 \\ 9.738 \mathrm{E}-10 & 2 & 1 & 2 & 2 & 2 & 2 & 5 & 2 & 3 & 1 & 5 & 2 & 1 & 2 & 3 & 1 & 2 & 1 & 2 & 3 & 2 & 1 & 3 & 3 & 2 & 3 & 1\end{array}$ $\begin{array}{lllllllllllllllllllllllllllll}0.0658-09 & 1 & 1 & 3 & 2 & 1 & 2 & 6 & 1 & 5 & 3 & 2 & 4 & 3 & 1 & 2 & 3 & 4 & 1 & 2 & 1 & 2 & 2 & 2 & 3 & 3 & 3 & 1\end{array}$

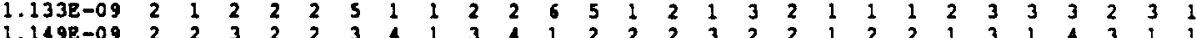

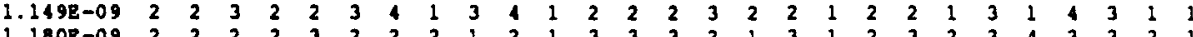

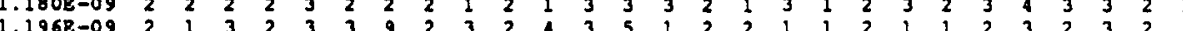

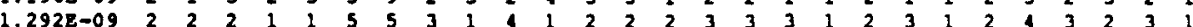

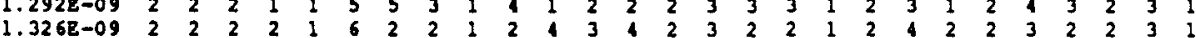
$\begin{array}{lllllllllllllllllllllllllllllll}1.386 \mathrm{E}-09 & 2 & 1 & 2 & 1 & 2 & 6 & 6 & 2 & 2 & 3 & 3 & 4 & 3 & 2 & 3 & 2 & 1 & 1 & 1 & 1 & 1 & 2 & 1 & 4 & 3 & 1 & 1\end{array}$ $1.399 \mathrm{E}-09 \begin{array}{lllllllllllllllllllllllllllllllll}1 & 2 & 2 & 2 & 1 & 1 & 6 & 1 & 2 & 3 & 2 & 3 & 3 & 3 & 2 & 3 & 2 & 2 & 1 & 2 & 3 & 2 & 1 & 3 & 4 & 3 & 3 & 1\end{array}$ $\begin{array}{lllllllllllllllllllllllllllllll}1.413 \mathrm{~B}-09 & 2 & 2 & 2 & 2 & 3 & 2 & 6 & 2 & 3 & 2 & 4 & 3 & 2 & 2 & 4 & 4 & 2 & 1 & 2 & 3 & 2 & 2 & 1 & 3 & 3 & 2 & 1\end{array}$ $\begin{array}{llllllllllllllllllllllllllllll}1.556 \mathrm{~B}-09 & 2 & 2 & 2 & 1 & 3 & 1 & 3 & 1 & 4 & 2 & 3 & 5 & 2 & 3 & 2 & 2 & 1 & 1 & 1 & 3 & 3 & 3 & 3 & 3 & 2 & 1 & 1\end{array}$ $\begin{array}{lllllllllllllllllllllllllllllll}1.6918 & -09 & 2 & 1 & 2 & 2 & 1 & 2 & 2 & 2 & 3 & 2 & 4 & 3 & 2 & 3 & 2 & 2 & 4 & 1 & 2 & 2 & 2 & 3 & 3 & 2 & 3 & 1 & 1\end{array}$

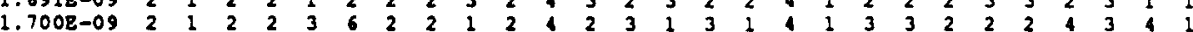
$\begin{array}{llllllllllllllllllllllllllll}1.7008-09 & 2 & 1 & 2 & 2 & 3 & 6 & 2 & 2 & 1 & 2 & 1 & 2 & 3 & 1 & 3 & 1 & 1 & 1 & 3 & 3 & 2 & 2 & 2 & 4 & 3 & 4 & 1\end{array}$ $\begin{array}{llllllllllllllllllllllllllll}1.741 \mathrm{E}-09 & 2 & 1 & 3 & 2 & 1 & 3 & 8 & 3 & 1 & 2 & 4 & 1 & 2 & 1 & 3 & 2 & 3 & 1 & 2 & 2 & 2 & 2 & 2 & 3 & 1 & 3 & 1 \\ 1.904 \mathrm{E}-09 & 2 & 1 & 3 & 2 & 2 & 6 & 7 & 2 & 2 & 3 & 1 & 1 & 2 & 2 & 3 & 2 & 3 & 1 & 2 & 2 & 2 & 2 & 1 & 2 & 3 & 1 & 1\end{array}$ $\begin{array}{cccccccccccccccccccccccccccc}1.904 E-09 & 2 & 1 & 3 & 2 & 2 & 6 & 7 & 2 & 2 & 3 & 1 & 4 & 2 & 2 & 3 & 2 & 3 & 1 & 2 & 2 & 2 & 2 & 1 & 2 & 3 & 1 & 1 \\ 1.936 E-09 & 2 & 2 & 2 & 2 & 3 & 1 & 5 & 1 & 3 & 3 & 2 & 3 & 3 & 2 & 2 & 1 & 1 & 1 & 2 & 2 & 2 & 2 & 1 & 3 & 2 & 3 & 1\end{array}$

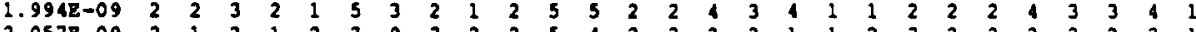
$\begin{array}{lllllllllllllllllllllllllllll}2.057 \mathrm{E}-09 & 2 & 1 & 2 & 1 & 2 & 3 & 9 & 3 & 2 & 2 & 5 & 4 & 2 & 3 & 2 & 3 & 1 & 1 & 2 & 3 & 2 & 2 & 2 & 3 & 2 & 3 & 1\end{array}$ $\begin{array}{lllllllllllllll}2 & 3 & 1 & 3 & 3 & 1 & 2 & 2 & 2 & 1 & 2 & 2 & 3 & 2\end{array}$ $\begin{array}{llllllllllllllllllllllllllllll}2.348 E-09 & 2 & 2 & 2 & 1 & 3 & 6 & 5 & 2 & 3 & 2 & 5 & 3 & 5 & 3 & 2 & 3 & 3 & 1 & 2 & 2 & 2 & 3 & 3 & 4 & 1 & 1 & 1 \\ 2.4598-09 & 2 & 2 & 2 & 1 & 1 & 5 & 7 & 2 & 5 & 2 & 2 & 3 & 1 & 2 & 1 & 2 & 2 & 1 & 2 & 1 & 2 & 2 & 4 & 2 & 3 & 3 & 1\end{array}$

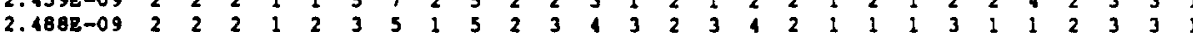
$\begin{array}{llllllllllllllllllllllllllll}2.488 \mathrm{E}-09 & 2 & 2 & 2 & 1 & 2 & 3 & 5 & 1 & 5 & 2 & 3 & 1 & 3 & 2 & 3 & 4 & 2 & 1 & 1 & 1 & 3 & 1 & 1 & 2 & 3 & 3 & 1 \\ 2.550 \mathrm{E}-09 & 2 & 2 & 2 & 2 & 3 & 3 & 7 & 1 & 4 & 2 & 4 & 1 & 3 & 2 & 2 & 3 & 4 & 1 & 2 & 3 & 1 & 2 & 3 & 3 & 2 & 3 & 1\end{array}$ $\begin{array}{lllllllllllllllllllllllllllllll}2.571 \mathrm{E}-09 & 3 & 1 & 3 & 1 & 1 & 1 & 8 & 2 & 4 & 4 & 1 & 3 & 3 & 3 & 2 & 3 & 1 & 1 & 2 & 1 & 1 & 2 & 3 & 3 & 3 & 4 & 1\end{array}$

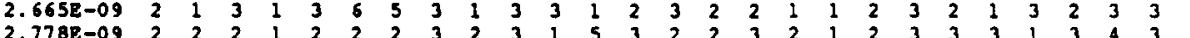

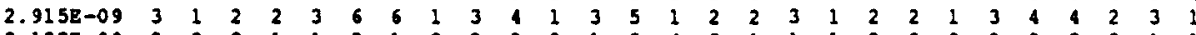
$\begin{array}{llllllllllllllllllllllllllllllllll}3.133 \mathrm{E}-09 & 2 & 2 & 3 & 1 & 1 & 3 & 1 & 2 & 2 & 2 & 2 & 1 & 3 & 4 & 3 & 1 & 1 & 1 & 2 & 2 & 2 & 2 & 2 & 3 & 3 & 1 & 1\end{array}$ $\begin{array}{lllllllllllllllllllllllllllll}3.1378-09 & 2 & 1 & 2 & 2 & 3 & 2 & 1 & 3 & 1 & 3 & 3 & 1 & 1 & 1 & 4 & 3 & 3 & 1 & 1 & 3 & 2 & 3 & 3 & 3 & 3 & 3 & 1\end{array}$

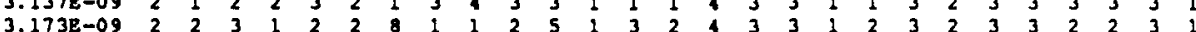
$\begin{array}{llllllllllllllllllllllllllll}3.1738-09 & 2 & 2 & 3 & 1 & 2 & 2 & 8 & 1 & 1 & 2 & 5 & 1 & 3 & 2 & 4 & 3 & 3 & 1 & 2 & 3 & 2 & 3 & 3 & 2 & 2 & 3 & 1 \\ 3.5948-09 & 2 & 2 & 2 & 2 & 2 & 3 & 8 & 2 & 6 & 3 & 2 & 2 & 5 & 2 & 3 & 2 & 3 & 1 & 3 & 2 & 3 & 1 & 3 & 4 & 3 & 2 & 1\end{array}$ $\begin{array}{ccccccccccccccccccccccccccccc}5.108 \mathrm{E}-09 & 2 & 2 & 2 & 2 & 2 & 6 & 9 & 1 & 1 & 3 & 1 & 4 & 3 & 3 & 3 & 4 & 3 & 1 & 2 & 4 & 2 & 2 & 3 & 2 & 3 & 2 & 1\end{array}$ $\begin{array}{lllllllllllllllllllllllllllll}5.200 \mathrm{OB}-09 & 2 & 2 & 2 & 1 & 2 & 3 & 9 & 2 & 1 & 1 & 5 & 1 & 2 & 1 & 3 & 2 & 4 & 1 & 2 & 2 & 3 & 1 & 2 & 2 & 2 & 2 & 1\end{array}$

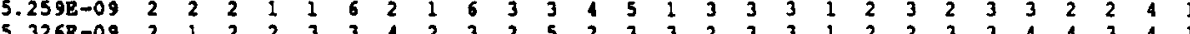

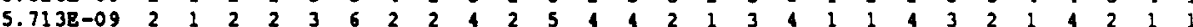

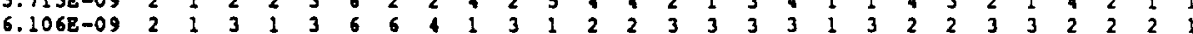
$\begin{array}{lllllllllllllllllllllllllllll}6.106 \mathrm{E}-09 & 2 & 1 & 3 & 1 & 3 & 6 & 6 & 4 & 1 & 3 & 1 & 2 & 2 & 3 & 3 & 3 & 3 & 1 & 3 & 2 & 2 & 3 & 3 & 2 & 2 & 2 & 1 \\ 6.382 \mathrm{E}-09 & 3 & 2 & 2 & 2 & 1 & 3 & 9 & 3 & 3 & 3 & 3 & 5 & 1 & 3 & 1 & 3 & 4 & 1 & 2 & 2 & 3 & 2 & 2 & 2 & 3 & 3 & 1\end{array}$

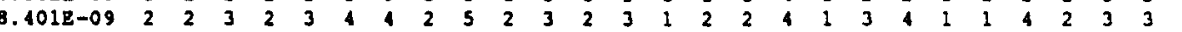
$\begin{array}{lllllllllllllllllllllllllllll}9.4068-09 & 2 & 1 & 2 & 3 & 2 & 1 & 7 & 2 & 5 & 1 & 6 & 1 & 3 & 3 & 2 & 1 & 2 & 1 & 2 & 2 & 2 & 3 & 3 & 2 & 2 & 3 & 1\end{array}$

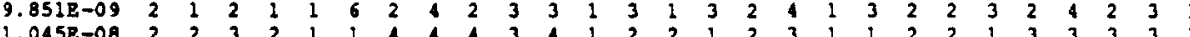

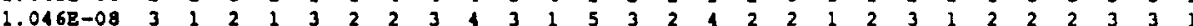
$\begin{array}{lllllllllllllllllllllllllllll}1.046 \mathrm{E}-08 & 3 & 1 & 2 & 1 & 3 & 2 & 2 & 3 & 1 & 3 & 1 & 5 & 3 & 2 & 4 & 2 & 2 & 1 & 2 & 3 & 1 & 2 & 2 & 2 & 3 & 3 & 1 \\ 1.197 \mathrm{E}-08 & 2 & 2 & 3 & 3 & 1 & 1 & 5 & 1 & 2 & 2 & 1 & 2 & 2 & 2 & 1 & 4 & 3 & 1 & 1 & 1 & 1 & 1 & 4 & 3 & 1 & 2 & 1\end{array}$ $\begin{array}{llllllllllllllllllllllllllllllllllll}1.197 \mathrm{E}-08 & 2 & 2 & 3 & 3 & 1 & 1 & 5 & 1 & 2 & 2 & 1 & 2 & 2 & 2 & 1 & 1 & 3 & 1 & 1 & 1 & 1 & 1 & 4 & 3 & 1 & 2 & 1 \\ 1.234 \mathrm{E}-08 & 2 & 1 & 2 & 2 & 2 & 6 & 1 & 3 & 3 & 2 & 5 & 1 & 1 & 1 & 1 & 2 & 3 & 1 & 3 & 1 & 3 & 3 & 1 & 2 & 3 & 3 & 1\end{array}$ $\begin{array}{llllllllllllllllllllllllllllllllll}1.385 E-08 & 2 & 1 & 2 & 2 & 3 & 1 & 1 & 2 & 4 & 1 & 6 & 2 & 3 & 2 & 4 & 3 & 1 & 1 & 3 & 4 & 1 & 3 & 2 & 2 & 3 & 3 & 1\end{array}$

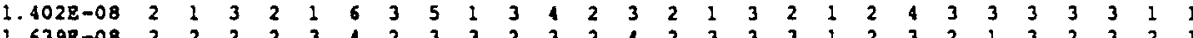

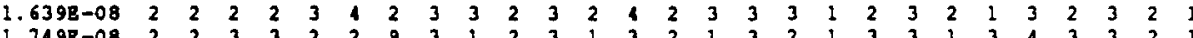

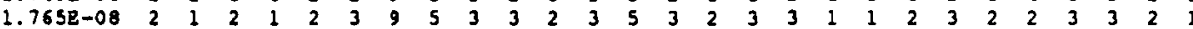
$\begin{array}{lllllllllllllllllllllllllllllllllll}1.8288-08 & 2 & 1 & 3 & 1 & 3 & 6 & 8 & 4 & 1 & 3 & 2 & 3 & 2 & 2 & 1 & 2 & 2 & 1 & 1 & 4 & 3 & 3 & 4 & 2 & 2 & 4 & 1\end{array}$ $\begin{array}{llllllllllllllllllllllllllllll}1.935 E-08 & 1 & 2 & 2 & 2 & 3 & 2 & 1 & 3 & 4 & 2 & 3 & 1 & 2 & 2 & 2 & 1 & 2 & 1 & 3 & 4 & 3 & 2 & 4 & 2 & 2 & 2 & 1\end{array}$ $\begin{array}{llllllllllllllllllllllllllllllllllll}2.0148-08 & 2 & 1 & 2 & 2 & 1 & 5 & 7 & 1 & 2 & 1 & 4 & 5 & 3 & 2 & 2 & 3 & 3 & 1 & 3 & 2 & 3 & 2 & 2 & 2 & 3 & 3 & 1\end{array}$ $\begin{array}{llllllllllllllllllllllllllllllll}2.379 \mathrm{P}-08 & 2 & 2 & 3 & 3 & 1 & 6 & 4 & 2 & 5 & 2 & 5 & 3 & 2 & 1 & 4 & 3 & 3 & 1 & 3 & 2 & 2 & 1 & 2 & 4 & 3 & 2 & 1\end{array}$ $\begin{array}{llllllllllllllllllllllllllllll}2.4308-08 & 2 & 2 & 2 & 1 & 2 & 5 & 4 & 5 & 5 & 4 & 1 & 1 & 3 & 1 & 1 & 2 & 4 & 1 & 3 & 2 & 3 & 3 & 3 & 2 & 3 & 2 & 1\end{array}$ $\begin{array}{lllllllllllllllllllllllllllll}2.4578-08 & 2 & 2 & 2 & 3 & 3 & 6 & 7 & 2 & 3 & 1 & 6 & 4 & 3 & 2 & 1 & 4 & 2 & 1 & 2 & 4 & 2 & 1 & 3 & 3 & 3 & 1 & 1\end{array}$

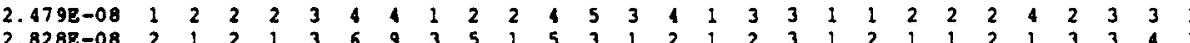
$\begin{array}{llllllllllllllllllllllllllllllllll}2.828 \mathrm{E}-08 & 2 & 1 & 2 & 1 & 3 & 6 & 9 & 3 & 5 & 1 & 5 & 3 & 1 & 2 & 1 & 2 & 3 & 1 & 2 & 1 & 1 & 2 & 1 & 3 & 3 & 4 & 1 \\ 3.229 \mathrm{E}-08 & 2 & 2 & 3 & 2 & 2 & 3 & 7 & 3 & 4 & 3 & 3 & 2 & 1 & 2 & 1 & 2 & 2 & 1 & 3 & 3 & 3 & 2 & 2 & 2 & 3 & 3 & 1\end{array}$ $\begin{array}{lllllllllllllllllllllllllllll}3.229 \mathrm{E}-08 & 2 & 2 & 3 & 2 & 2 & 3 & 7 & 3 & 4 & 3 & 3 & 2 & 1 & 2 & 1 & 2 & 2 & 1 & 3 & 3 & 3 & 2 & 2 & 2 & 3 & 3 & 1 \\ 3.240 \mathrm{~B}-08 & 2 & 1 & 2 & 2 & 1 & 1 & 3 & 1 & 2 & 2 & 3 & 5 & 3 & 4 & 2 & 2 & 4 & 1 & 1 & 3 & 1 & 2 & 4 & 2 & 4 & 3 & 1\end{array}$ $\begin{array}{llllllllllllllllllllllllllll}3.260 \mathrm{~B}-08 & 1 & 1 & 3 & 1 & 3 & 3 & 6 & 5 & 3 & 3 & 3 & 3 & 4 & 3 & 2 & 2 & 3 & 1 & 1 & 3 & 1 & 2 & 2 & 3 & 2 & 3 & 1\end{array}$ $\begin{array}{lllllllllllllllllllllllllllll}3.2798-08 & 2 & 1 & 2 & 2 & 3 & 6 & 5 & 4 & 6 & 2 & 6 & 1 & 3 & 2 & 2 & 3 & 2 & 1 & 2 & 1 & 2 & 3 & 3 & 4 & 3 & 2 & 1\end{array}$ $\begin{array}{lllllllllllllllllllllllllllllllll}3.371 \mathrm{R}-08 & 2 & 1 & 2 & 2 & 2 & 6 & 4 & 4 & 4 & 2 & 1 & 3 & 2 & 2 & 2 & 3 & 1 & 1 & 3 & 2 & 2 & 2 & 4 & 4 & 3 & 1 & 1\end{array}$ $\begin{array}{lllllllllllllllllllllllllllllllll}3.7028-08 & 2 & 2 & 2 & 3 & 2 & 5 & 3 & 4 & 2 & 2 & 4 & 4 & 3 & 1 & 1 & 3 & 3 & 1 & 2 & 3 & 3 & 1 & 3 & 3 & 1 & 2 & 1\end{array}$ $\begin{array}{llllllllllllllllllllllllllll}3.7028-08 & 2 & 2 & 2 & 3 & 2 & 5 & 3 & 4 & 2 & 2 & 4 & 4 & 3 & 1 & 1 & 3 & 3 & 1 & 2 & 3 & 3 & 1 & 3 & 3 & 1 & 2 & 1 \\ 3.7688-08 & 2 & 2 & 2 & 2 & 3 & 1 & 7 & 2 & 4 & 1 & 6 & 4 & 3 & 2 & 4 & 2 & 3 & 1 & 1 & 2 & 3 & 3 & 4 & 3 & 3 & 3 & 1\end{array}$ $\begin{array}{lllllllllllllllllllllllllllll}3.7688-08 & 2 & 2 & 2 & 2 & 3 & 1 & 7 & 2 & 4 & 1 & 6 & 4 & 3 & 2 & 4 & 2 & 3 & 1 & 1 & 2 & 3 & 3 & 4 & 3 & 3 & 3 & 1 \\ 4.1738-08 & 2 & 2 & 2 & 3 & 2 & 6 & 9 & 1 & 4 & 1 & 6 & 1 & 2 & 2 & 3 & 1 & 3 & 1 & 1 & 2 & 2 & 2 & 2 & 3 & 3 & 2 & 1\end{array}$

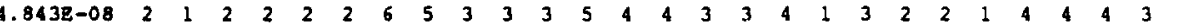
$\begin{array}{cccccccccccccccccccccccccccc}4.864 Z-08 & 2 & 2 & 3 & 1 & 1 & 6 & 8 & 3 & 4 & 3 & 3 & 2 & 3 & 2 & 3 & 3 & 2 & 1 & 1 & 2 & 2 & 3 & 4 & 3 & 2 & 1 & 1\end{array}$

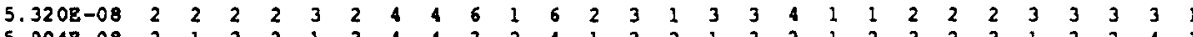


NUREG/CR-4551, VOL. 1: DRAFT REPORT FOR COMMENT (SEPTEMBER, 1986)

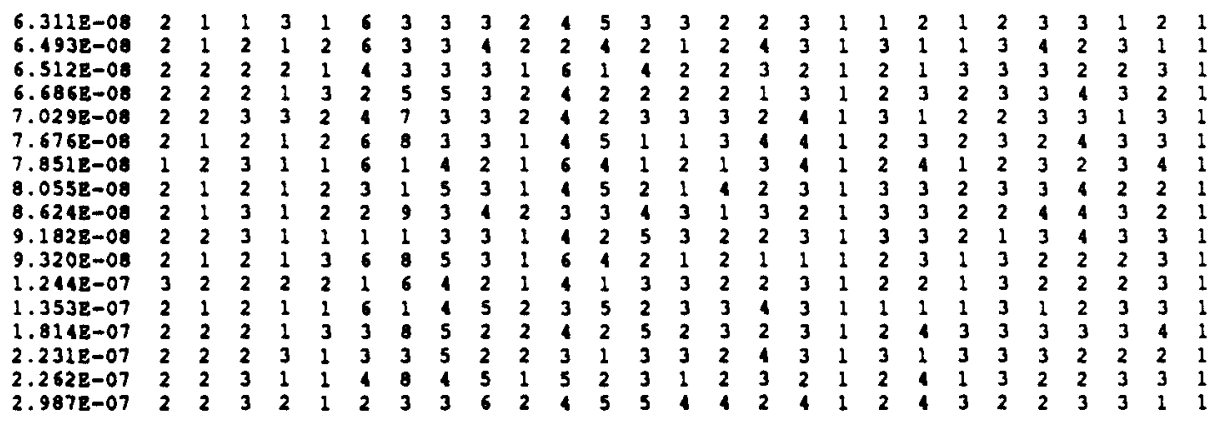

\section{D-102}


CONSEQUENCE MEASURE:

IND. RISK OF FATALITY

BASE CASE :

SURRY LALHC (MACCS)

FRACTIONAL CONTRIBOTION OF EACH SEOUENCE TO MEAN RISK

\begin{tabular}{|c|c|}
\hline SYYB & $\begin{array}{r}\text { ONTRIBUTIOA } \\
0.0619\end{array}$ \\
\hline SYNI & 0.0001 \\
\hline SNMN & 0.5595 \\
\hline SYNA & 0.0006 \\
\hline ANNS & 0.0000 \\
\hline AYNN & 0.0074 \\
\hline AYNI & 0.0179 \\
\hline AYYB & 0.0036 \\
\hline man: & 0.1817 \\
\hline IYYBN & 0.0591 \\
\hline TYYBD & 0.0679 \\
\hline$q^{\ln }$ & 0.0398 \\
\hline
\end{tabular}

CONSEOTERCE MasARE:

0.0398

IND. RISR OF FATALITY

BAse case :

SURRY LALHC (MACCS)

FRACTIONAL CONTRIBOTION OF RACH BIN TO MRAN RISK

BIN CONTRIBOTION

BIN

CONTRI BOTION

0.0369

0.0038

0.0010

0.0260

0.0000

0.0003

0.0000

0.0000

0.0001

0.0001

0.0176

0.0623

0.0000

0.0000

0.0000

0.7198

0.0706

0.0706

0.0215
0.0383

CONSEOTENCR BIN 1

IND. RISK OF FATALITY

BASE CASE :

SURRY LALHC (Maces)

FRACTIONAL CONTRIBOTIONS OP EACH RELEASE TO MEAN RIST

REIEASE CONTRIBUTION

SUR 16

SUR 20

SUR $3 G$

SUR 50

$\operatorname{son} 60$

SUR 70

SUR 86

SUR 96

SOR $10 \mathrm{G}$

SUR $11 G$

SUR 126

SUR $13 \mathrm{G}$

SUR $14 G$

SOR $15 \mathrm{G}$

SOR $16 G$

SOR 176

SUR $18 G$

SUR 196

SOR $20 \mathrm{G}$

SUR $21 G$

SUR $22 G$

SUR $23 G$

SUR $24 G$

0.2689

0.0882

0.2069

0.0683

0.0471

0.1704

0.0389

0.0200

0.0007

0.0311

0.0178

0.0173

0.0090

0.0002

0.0041

0.0001

0.0069

0.0000

0.0024

0.0000

0.0000

0.0000

0.0006

0.0000

0.0000 
NUREG/CR-4551, VOL. 1: DRAFT REPORT FOR COMMENT (SEPTEMBER, 1986)

CONSEOUENCE MEASURE:

IND. RIST OF FATALITY

SENSITIVITY OF RISK TO ISSUES

DISTRIBUTION OF RISK AT EACH LEVEL OP RACH ISSUE

BASE CASE :

SURRY LALHC (MACCS)

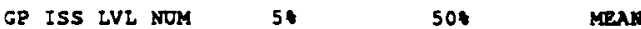

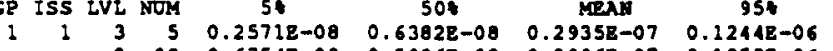

$290 \quad 0.6754 \mathrm{E}-09 \quad 0.5906 \mathrm{E}-08 \quad 0.2996 \mathrm{E}-07 \quad 0.2353 \mathrm{E}-06$

$155 \quad 0.1065 \mathrm{E}-08 \quad 0.2479 \mathrm{E}-07 \quad 0.3126 \mathrm{E}-07 \quad 0.7851 \mathrm{z}-07$

$121 \quad 50 \quad 0.1117 \mathrm{E}-09 \quad 0.5906 \mathrm{~B}-08 \quad 0.2162 \mathrm{E}-07 \quad 0.8624 \mathrm{E}-07$

$\begin{array}{lllllll}2 & 50 & 0.11498-08 & 0.93688-08 & 0.38378-07 & 0.22318-06\end{array}$

$\begin{array}{llllll}3 & 34 & 0.30528-09 & 0.9368 \mathrm{E}-08 & 0.3519 \mathrm{~B}-07 & 0.1888 \mathrm{E}-06\end{array}$

$14250 \quad 0.78028-090.1285 \mathrm{E}-08$ 0.21128-07 $0.6512 \mathrm{E}-07$

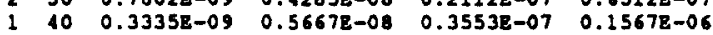

$3 \quad 10 \quad 0.9406 \mathrm{E}-08 \quad 0.3016 \mathrm{E}-07 \quad 0.5225 \mathrm{E}-07 \quad 0.2231 \mathrm{E}-06$

$153 \quad 33 \quad 0.6986 \mathrm{E}-09 \quad 0.6106 \mathrm{~B}-08 \quad 0.21328-07 \quad 0.8867 \mathrm{~B}-07$

$\begin{array}{lllllll}2 & 34 & 0.80398-09 & 0.5154 \mathrm{E}-08 & 0.2398 \mathrm{E}-07 & 0.8507 \mathrm{z}-07\end{array}$

$133 \quad 0.2854 \mathrm{E}-090.1045 \mathrm{E}-07 \quad 0.4487 \mathrm{E}-07 \quad 0.2257 \mathrm{E}-0.07$

$2157 \quad 0.1133 \mathrm{E}-08 \quad 0.2459 \mathrm{E}-08 \quad 0.1262 \mathrm{E}-07 \quad 0.3702 \mathrm{E}-07$

$6 \quad 340.4545 \mathrm{E}-09 \quad 0.7756 \mathrm{E}-0 \mathrm{~B} \quad 0.2473 \mathrm{~B}-07 \quad 0.9006 \mathrm{~B}-07$

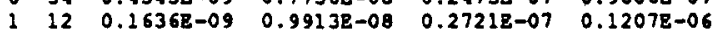

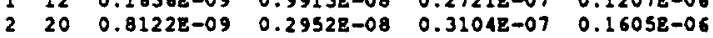

$320 \quad 0.1172 \mathrm{E}-08 \quad 0.5262 \mathrm{E}-08 \quad 0.3500 \mathrm{E}-07 \quad 0.2012 \mathrm{E}-06$

$\begin{array}{llllll}1 & 0.8401 \mathrm{E}-08 & 0.2479 \mathrm{E}-07 & 0.6045 \mathrm{E}-07 & 0.2262 \mathrm{E}-06 \\ 2 & 13 & 0.2908 \mathrm{E}-09 & 0.2778 \mathrm{E}-08 & 0.5149 \mathrm{E}-0 \mathrm{~g} & 0.1603 \mathrm{E}-07\end{array}$

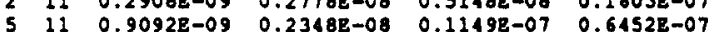

$0.6452 \mathrm{E}-07$

$\begin{array}{lllllll}7 & 12 & 0.4388 \mathrm{E}-09 & 0.4898 \mathrm{E}-08 & 0.1695 \mathrm{E}-07 & 0.6604 \mathrm{E}-07 \\ 9 & 11 & 0.8602 \mathrm{E}-09 & 0.6382 \mathrm{E}-08 & 0.1929 \mathrm{E}-07 & 0.8316 \mathrm{E}-07\end{array}$

$412 \quad 0.9144 \mathrm{E}-09 \quad 0.1714 \mathrm{E}-07 \quad 0.2145 \mathrm{~B}-07 \quad 0.5843 \mathrm{~B}-07$

$6 \quad 10 \quad 0.1065 \mathrm{E}-08 \quad 0.4219 \mathrm{E}-08 \quad 0.2569 \mathrm{E}-07 \quad 0.1244 \mathrm{E}-06$

$111 \quad 0.69318-090.13858-07 \quad 0.38998-07 \quad 0.1327 \mathrm{E}-06$

B $11 \quad 0.2581 \mathrm{~B}-09 \quad 0.1828 \mathrm{E}-07 \quad 0.5961 \mathrm{~B}-07 \quad 0.2237 \mathrm{E}-06$

$311 \quad 0.17638-090.37028-07 \quad 0.72928-07 \quad 0.29438-06$

$32320.28108-090.19498-000.53088-080.24498-07$

$1 \quad 18 \quad 0.2817 \mathrm{z}-090.2195 \mathrm{~B}-08 \quad 0.6080 \mathrm{z}-08 \quad 0.3388 \mathrm{z}-07$

$\begin{array}{llllll}1 & 23 & 0.1568 \mathrm{E}-08 & 0.1935 \mathrm{E}-07 & 0.4444 \mathrm{~B}-07 & 0.2387 \mathrm{E}-06\end{array}$

$126 \quad 0.2445 E-08 \quad 0.3325 E-07 \quad 0.54948-07 \quad 0.19398-06$

5 11 $0.1418 \mathrm{E}-07 \quad 0.4843 \mathrm{~B}-07 \quad 0.7446 \mathrm{E}-07 \quad 0.22088-06$

$24117 \quad 0.3916 \mathrm{~B}-09 \quad 0.17008-08 \quad 0.4435 \mathrm{E}-08 \quad 0.1800 \mathrm{~B}-07$

$1200.60278-0$

$3270.96338-090.1639 \mathrm{E}-07 \quad 0.3135 \mathrm{~B}-07 \quad 0.9203 \mathrm{E}-07$

$220 \quad 0.2544 \mathrm{E}-09 \quad 0.5555 \mathrm{E}-08 \quad 0.3798 \mathrm{E}-07 \quad 0.2012 \mathrm{E}-06$

5 11 $0.6910 \mathrm{~B}-09 \quad 0.9406 \mathrm{~B}-08$ 0.12038-07 $0.2204 \mathrm{~B}-06$

$65 \quad 0.3594 \mathrm{E}-08 \quad 0.3279 \mathrm{E}-07 \quad 0.7870 \mathrm{E}-07 \quad 0.2987 \mathrm{E}-06$

$\begin{array}{llllll}7 & 0.2334 \mathrm{E}-09 & 0.2140 \mathrm{~B}-08 & 0.4943 \mathrm{E}-08 & 0.2430 \mathrm{~B}-07\end{array}$

$2 \quad 18 \quad 0.83878-09 \quad 0.55168-08 \quad 0.33458-07 \quad 0.18528-05$

$\begin{array}{llllllll} & & 1 & 20 & 0.49078-09 & 0.3965 \mathrm{E}-07 & 0.5364 \mathrm{E}-07 & 0.1677 \mathrm{E}-06 \\ 6 & 1 & 15 & 0.2690 \mathrm{E}-09 & 0.2571 \mathrm{E}-08 & 0.6418 \mathrm{E}-08 & 0.3106 \mathrm{E}-07\end{array}$

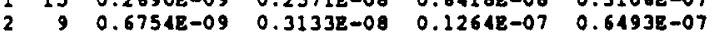

$514 \quad 0.3430 \mathrm{~B}-09 \quad 0.1062 \mathrm{E}-08 \quad 0.2275 \mathrm{~B}-07 \quad 0.1492 \mathrm{E}-06$

$322 \quad 0.1199 \mathrm{E}-08 \quad 0.1271 \mathrm{~B}-07 \quad 0.3344 \mathrm{E}-07 \quad 0.1653 \mathrm{E}-06$

$6 \quad 12 \quad 0.1916 \mathrm{E}-090.35158-07 \quad 0.3761 \mathrm{E}-07 \quad 0.9161 \mathrm{E}-07$

$428 \quad 0.8387 \mathrm{E}-09 \quad 0.1681 \mathrm{E}-07 \quad 0.4586 \mathrm{E}-07 \quad 0.1907 \mathrm{E}-06$

$7320 \quad 0.04088-090.24038-080.13138-07 \quad 0.53928-07$

$4200.47798-090.51548-080.19428-07 \quad 0.85548-07$

$120 \quad 0.52738-09 \quad 0.10148-07 \quad 0.3238 \mathrm{~B}-07 \quad 0.1666 \mathrm{z}-06$

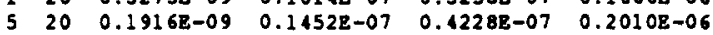

$2 \quad 20 \quad 0.1058 \mathrm{E}-08 \quad 0.13948-07 \quad 0.42778-07 \quad 0.2025 \mathrm{E}-08$

$\begin{array}{lllllll}2 & 2 & 30 & 0.2334 \mathrm{E}-09 & 0.2389 \mathrm{E}-08 & 0.2116 \mathrm{E}-07 & 0.9320 \mathrm{E}-07\end{array}$

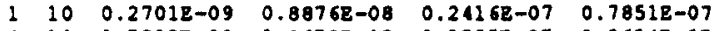

$\begin{array}{llllll}10 & 0.7802 \mathrm{E}-09 & 0.9678 \mathrm{E}-08 & 0.2585 \mathrm{E}-07 & 0.8624 \mathrm{E}-07\end{array}$

$\begin{array}{lllllll}3 & 40 & 0.9488 \mathrm{E}-09 & 0.1204 \mathrm{E}-07 & 0.3148 \mathrm{E}-07 & 0.1666 \mathrm{E}-06 \\ 5 & 10 & 0.9060 \mathrm{E}-09 & 0.4348 \mathrm{E}-08 & 0.6057 \mathrm{E}-07 & 0.2987 \mathrm{E}-06\end{array}$

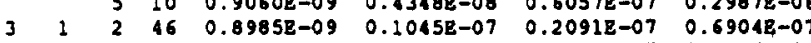

$124 \quad 0.2297 \mathrm{E}-09 \quad 0.6647 \mathrm{E}-08 \quad 0.3057 \mathrm{E}-07 \quad 0.1216 \mathrm{E}-06$

$\begin{array}{llllll}3 & 23 & 0.1070 \mathrm{E}-08 & 0.6106 \mathrm{E}-08 & 0.3887 \mathrm{~g}-07 & 0.1612 \mathrm{E}-06\end{array}$

$47 \begin{array}{llllll}7 & 0.7802 \mathrm{E}-09 & 0.2479 \mathrm{~B}-07 & 0.5856 \mathrm{Z}-07 & 0.2987 \mathrm{E}-06\end{array}$

$120 \quad 0.97858-09 \quad 0.2108 \mathrm{~B}-07 \quad 0.2605 \mathrm{~B}-07 \quad 0.8228 \mathrm{~B}-07$

$238 \quad 0.7156 \mathrm{E}-09 \quad 0.3940 \mathrm{~B}-08080.3319 \mathrm{~B}-07 \quad 0.1766 \mathrm{z}-06$

$\begin{array}{llllllll} & & 4 & 12 & 0.1462 \mathrm{E}-08 & 0.1119 \mathrm{E}-07 & 0.4073 \mathrm{E}-07 & 0.2620 \mathrm{E}-06 \\ 3 & 1 & 11 & 0.1691 \mathrm{E}-09 & 0.3133 \mathrm{E}-08 & 0.2167 \mathrm{~B}-07 & 0.9166 \mathrm{E}-07\end{array}$

$\begin{array}{llllll}3 & 39 & 0.8659 \mathrm{E}-09 & 0.6382 \mathrm{E}-08 & 0.2497 \mathrm{E}-07 & 0.8267 \mathrm{E}-07\end{array}$

$\begin{array}{lllllll}2 & 39 & 0.5489 \mathrm{~B}-09 & 0.8401 \mathrm{~B}-08 & 0.3176 \mathrm{~B}-07 & 0.1531 \mathrm{~B}-06\end{array}$

$411 \quad 0.9835 \mathrm{~B}-09 \quad 0.1197 \mathrm{~B}-07 \quad 0.49878-07 \quad 0.2176 \mathrm{~B}-06$

$41130.25428-090.2057 \mathrm{E}-080.1210 \mathrm{~B}-07 \quad 0.8001 \mathrm{~B}-07$

$2 \quad 260.24248-090.29208-080.26178-07 \quad 0.10468-06$

$423 \quad 0.8751 \mathrm{E}-09 \quad 0.6382 \mathrm{E}-08 \quad 0.3339 \mathrm{E}-07 \quad 0.1253 \mathrm{E}-06$

$\begin{array}{llllll}38 & 0.1224 \mathrm{E}-08 & 0.1423 \mathrm{E}-07 & 0.3668 \mathrm{~g}-07 & 0.1613 \mathrm{E}-06\end{array}$

$5 \quad 1100 \quad 0.7260 \mathrm{E}-09 \quad 0.73228-08 \quad 0.3000 \mathrm{E}-07 \quad 0.1297 \mathrm{E}-06$

$\begin{array}{lllllll}1 & 22 & 0.4314 \mathrm{E}-09 & 0.1454 \mathrm{E}-07 & 0.2483 \mathrm{~B}-07 & 0.8562 \mathrm{~B}-07\end{array}$

$256 \quad 0.4776 \mathrm{E}-09 \quad 0.2721 \mathrm{E}-08 \quad 0.2786 \mathrm{~B}-07 \quad 0.1620 \mathrm{~B}-06$

$\begin{array}{llllll}3 & 22 & 0.12458-08 & 0.2189 \mathrm{~B}-07 & 0.40598\end{array}$

$\begin{array}{lllllll}2 & 36 & 0.3489 \mathrm{E}-09 & 0.5702 \mathrm{E}-08 & 0.1785 \mathrm{E}-07 & 0.5996 \mathrm{E}-07 \\ 3 & 31 & 0.7834 \mathrm{E}-09 & 0.3173 \mathrm{E}-08 & 0.2472 \mathrm{E}-07 & 0.9153 \mathrm{E}-07\end{array}$

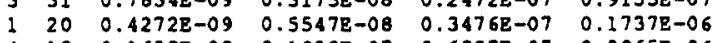

R**2 F DE SIGNIF

$0.02 \quad 1.7(1,98) \quad 0.19 \mathrm{NS}$

$0.01 \quad 0.7(2.97) \quad 0.50 \mathrm{Ns}$

$0.09 \quad 4.7(2.97) \quad 0.01 *$

$0.01 \quad 0.3(2.97) \quad 0.74 \mathrm{NS}$

$0.06 \quad 1.2(5.94) \quad 0.33 \mathrm{NS}$

$0.11 \quad 1.4(8.91) \quad 0.22 \mathrm{NS}$

$0.4922 .7(4.95) \quad 0.00 *$

$0.14 \quad 3.0(5.94) \quad 0.01 *$

$0.12 \quad 4.4(3.96) \quad 0.01 *$

$0.12 \quad 2.5(5.94) \quad 0.04$

$0.06 \quad 1.6(4.95) \quad 0.19 \mathrm{NS}$

$0.03 \quad 0.6(4.95) \quad 0.65 \mathrm{Ns}$

$0.01 \quad 0.3(3.96) \quad 0.79 \mathrm{NS}$

$0.04 \quad 1.5(3.96) \quad 0.22 \mathrm{NS}$

$0.02 \quad 0.5(3.96) \quad 0.68 \mathrm{NS}$

$0.07 \quad 2.5(3.96) \quad 0.06 \mathrm{NS}$

$0.00 \quad 0.0(0,99) \quad 1.00 N S R$ IMPOSSIBLE

$0.09 \quad 4.8(2.97) \quad 0.01 *$

$0.04 \quad 1.5(3.96) \quad 0.23 \mathrm{NS}$ 
NUREG/CR-4551, VOL. 1: DRAFT REPORT FOR COMMENT (SEPTEMBER, 1986)

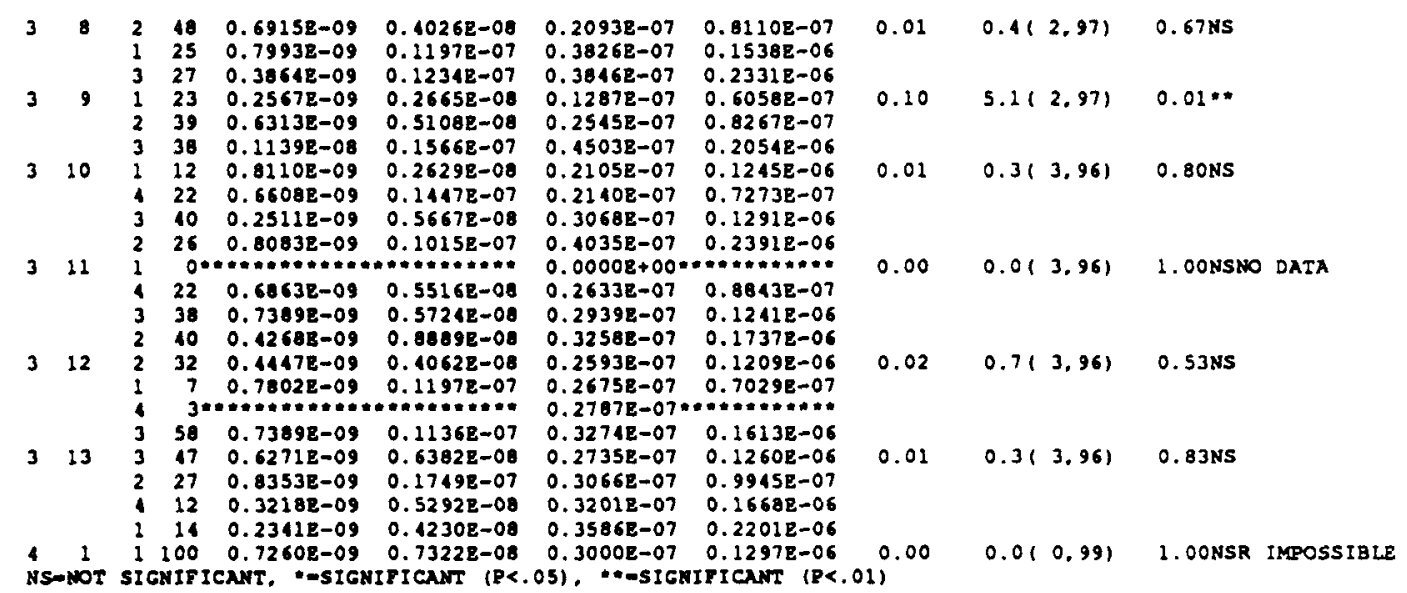


CONSEOUENCE MEASORE: IND. RISR OF FATALITY

LEVEL 1 NO. CYRD.- 1 NO. EXP.= 2

LEVEL 2 No. LMeD. - 16 No. EXe. $=45$

10.>-XRD. - 40. ExP.- 3

NO.>ARD. - 44 No. BXP. = 45

LEVEL 3 no. CMED.- 2 No. EXP.- 2

No. >AED. = 3 NOE. EXP. $=3$

OVERALI CHI-SO- 0.0 DT- 1 SIG.- 0.833

- ISSUE GROUP - 1 ISSUR - 2 USING GAS GI.

LEVEL 1 10. CHED. - 26 NO0, EXP. 25

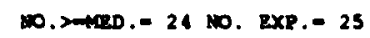

LEVEL 2 NO. CAED. - 23 NO. EXP. -25

10. >-AED. -27 No. exp. -25

OVERALL CHI-SO- 0.4 DT- 3 SIG. 0.940

ISSOE GROOR - 1 ISSOL = 3 BETA-PACTOR

Lever 1 to. Lede. 0 No. exp.= 0

10.>-100.- 1 10. Exp.e 1

LEVEL 2 No. LRE. - 33 No. ExP. 32

No.>-Nite. 32 10. EXP. -33

LEVEL 3 No. <Hed.- 16 No. ExP.- 17

No.> Da. = 18 No. Exp. - 17

OVERALL CHI-SO- 0.2 DTE 3 SIG.- 0.901

ISSUE GROUP - I ISSUE - 1 V FREO

LEVEL \& No. CABD. - 21 No. ExP. - 20

LEVEL 2 No. CNED. - 20 NO. EXP. - 25

Bo.>-Mad. 19 100. BXP. $=20$

No.>-NED. 22 so. Exp. 25

LEVEL 3 No. <HRD.- O NO. EXP.- 5

10. >

OVERALL CHI-SO- 10.8 DF- 5 SIG.=0.05S

ISSUE GROUP -

Issue =

PIF2 PATLURS

LEVEL 1 No. CQED.- 14 No. ExP.- 16

LEVEL 2 No. CMED. = 10 NO. ExP.= 17

LEVEL 3 No. SMED. - 17 Mo. ExP.- 16

OVERALL CHI-SO- 0.7 DF- S SIG.- 0.982

ISSUE GROOP - 2 ISSUE - 1 IND. RCS PAIL.

LEVEL 1 No. CUED. = 5 NO. EXP. - 6

LEVEL 1 No. SMED.- 5 NO. EXP.= 5

LEVEL 2 NO. LED. - 8 NO. EXP.- 5

LEVEL 3 NO. CMED." 3 NO. EXP.- 5

LEVEL A No. CMED.- 3 NO. EXP." 6

LEVEL 5 NO. SAED. = 8 NO. EXP. = 5

LEVEL 6 NO. LMED." 6 NO. EXP.- 5
10. >atad. - 19 so. Exp. $=17$

No. > NED. - 16 No. EXP. - 17

No.>-tad - 16 No. Exp. - 17

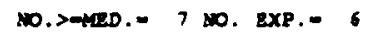

MODz or vB.

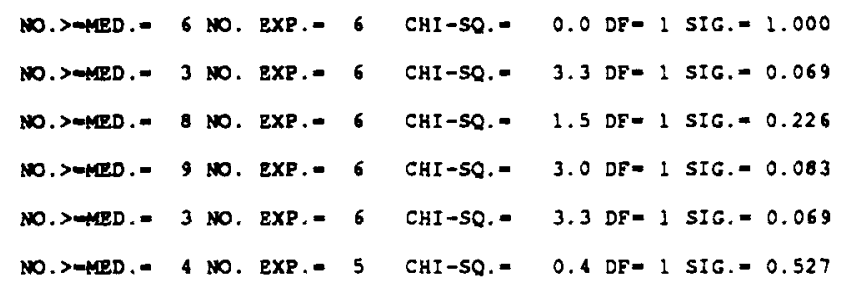

CHI-SO.- 0.3 DF- 1 SIG. $=0.564$ CHI-SO.- 0.8 DF= I SIG.=0.371 CHI-SO. 0.2 DF- 1 SIG. $=0.655$ CHI-SO. IEST CANNOT BE RON: EXP. NO. < 5 CHI-SO. TEST CANNOT BE RON; EXP. NO. < 5 CHI-SQ. $\quad 0.0$ DE= 1 SIC. $=1.000$

\author{
\begin{tabular}{l} 
№. $<5$ \\
№. 5 \\
\hline
\end{tabular}
}

CHI-SO. $=0.7$ DF- 1 SIG. $=0.396$

CHI-SO. $=10.0$ DF 1 SIC. $=0.002$

CHI-SQ. TEST CANWOT BE RON; EXP. No. < 5

CHI-SO.- 0.1 DF= 1 SIG. $=0.777$

CHI-SO. TEST CNNNOT BE RON; EXP. NO. < 5

CHI-SO.- 0.1 DF- 1 SIG. 0.804

2

CHI-SO.- 0.0 DF= 1 SIG. $=0.833$
CHI-SO. IEST CANNOT BE RON; EXP. NO. < 5

\author{
,
}




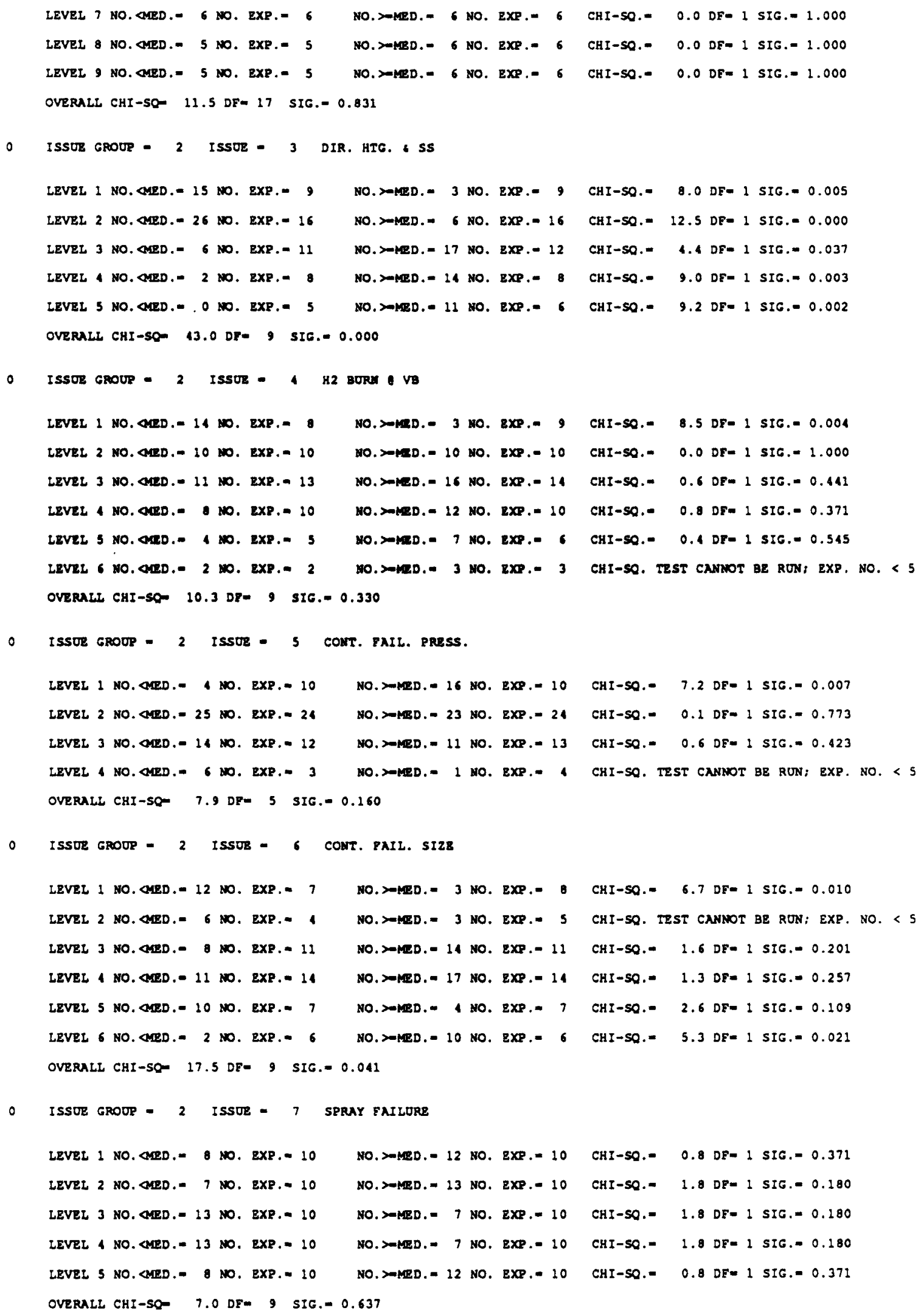

CHI-SO.- $\quad 8.0$ DF- 1 SIG.=0.005 CHI-SQ.= 12.5 DF= 1 SIG. $=0.000$ CHI-SO.= 4.4 DF- 1 SIG.=0.037 CHI-SQ.- 9.0 DE- 1 SIG.= 0.003 CHI-SO.- 9.2 DF- 2 SIG.- 0.002

CHI-SO.- 8.5 DF= I SIG.- 0.004 CHI-SO.= 0.0 OF $=1$ SIG. $=1.000$ CHI-SO. = 0.6 OF= 1 SIG.=0.441 CHI-5Q.- 0.8 DF- I SIG.- 0.371 CHI-SO.- 0.4 DF= 1 SIG. $=0.545$ CAI-SO. TEST CANROT BE RON; EXP, NO. < 5 CHI-SO.- 7.2 DF- 1 SIG.= 0.007 CHI-SO.- 0.1 DF- 1 SIG. $=0.773$ CHI-SO.= 0.6 DF= 1 SIG. $=0.423$ CHI-SQ. TEST CANNOT BE RUN; EXP. NO. < 5

CHI-SO.= 6.7 DF= 1 SIC. $=0.010$ CHI-SO. IEST CANNOT BE RON; EXP. NO. < S CHI-SO.- 1.6 DF= 1 SIG.- 0.201 CHI-SQ.= 1.3 DF= 1 SIG.= 0.257 CHI-SO.= 2.6 DFE ISIG. 0.109 CHI-SQ. $=5.3$ DF $=1$ SIG. $=0.021$

CHI-SO.= $0.8 \mathrm{DF}=1$ SIG.=0.371 CHI-SO.= 1.8 DF= 1 SIG. 0.180 CHI-SQ.= $1.8 \mathrm{DF}=1 \mathrm{SIG}=0.180$ CHI-SQ.= 1.8 DF= 1 SIG.= 0.180 CHI-SO.= 0.8 DF= 1 SIG.= 0.371 


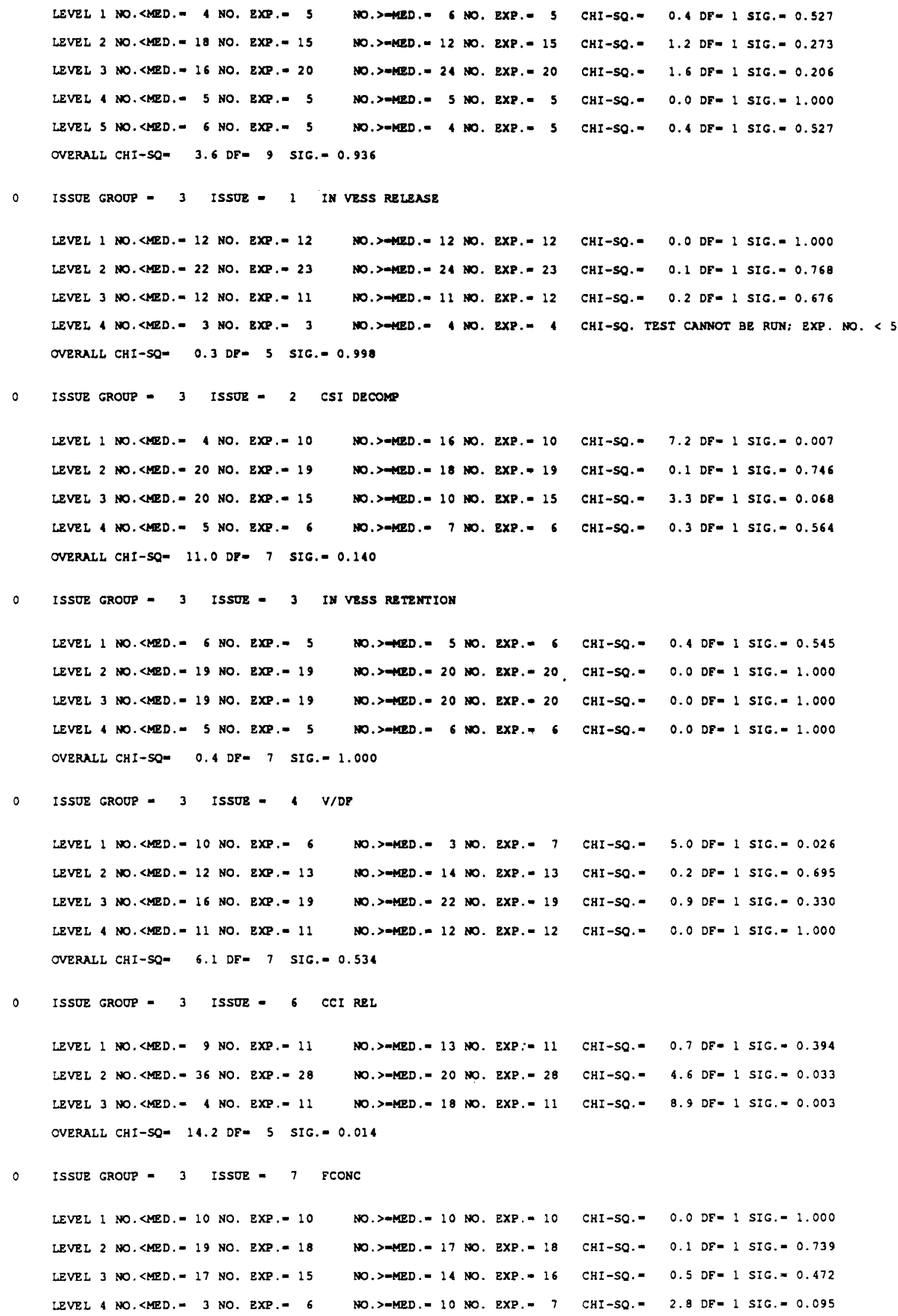

ISSOR GROUP - 3 ISSUR - 3 IN VESS RETERTION

LEVEL 1 NO. CMED. = 6 NO. EXP. $=5$ LEVEL 2 No. CMED. = 19 NO. $\operatorname{EXP}=19$ LEVEL 3 NO. CMED. = 19 NO. EXP. $=19$ LEVEL 4 NO. KMED. - 5 NO. EXP. - 5 No.>-MED. = 5 No. EXP. = 6 No. SARD. = 20 so. EXP. $=20$. NO. >AED. - 20 NO. EXP. $=20$ No.>-MED. - 6 No. EXP. 6 OVERALI CHI-SO= 0.4 DF- 7 SIG. -1.000

ISSUE GROUP $=3$ ISSUE - 1 V/DF

LEVRL 1 No. CMED. = 10 NO. EXP = 6 NO.>ARD.- 3 No. EXP.= 7 LEVEL 2 NO. $\angle M E D=12$ NO. EXP. = 13 No. >MED. = 14 NO. $\operatorname{exP}=13$ LEVEL 3 No. <MED. - 16 NO. EXP. $=19$ No. >MED. $=22$ No. EXP.- 19 LEVEL 4 NO. <LED. = 11 NO. EXP. = 11 NO.>-MED. = 12 NO. EXP. $=12$ OVERALL CHI-SO- 6.1 DF= 7 SIG. $=0.534$

ISSUE GROUP = 3 ISSUE $=6$ CCI REL

LEVEL 1 NO. $\angle M E D .=9$ NO. EXP. - 11 NO. >MED $=13$ NO. EXP; $=11$ LEVEL 2 NO. KMED. $=36$ NO. EXP. $=28$ NO. >-MED. $=20$ NO. EXP. $=28$ LEVEL 3 NO. LMED. = 4 NO. EXP, = 11 No. >-MED. = 18 No. EXP. $=11$ OVERALL CHI-SO- 14.2 DE- S SIG.=0.014

ISSUE GROUP = 3 ISSUE $=7$ FCONC

LEVEL I NO. LMED. - 10 NO. EXP. - 10 LEVEL 2 NO. LMED. = 19 NO. EXP . $=18$ LEVEL 3 NO. <MED. = 17 NO. EXP. $=15$ LEVEL 4 NO. $\angle$ MED. $=3$ NO. EXP. $=6$

NO. $>-M E D .=10$ NO. EXP. $=10$ No. > MED. -17 NO. $\operatorname{EXP}=18$ NO. >MMED. $=14$ NO. EXP. $=16$ NO. >-MED. = 10 NO. EXP. $=7$

CHI-SQ. $\quad 0.4$ DF $=1$ SIC. $=0.545$ CHI-SO. 0.0 DF $=1$ SIG. $=1.000$ CHI-SO. 0.0 DF= I SIG. $=1.000$ CHI-SO. 0.0 DF= 1 SIG. $=1.000$ CHI-SO. = 5.0 DF- 1 SIG. $=0.026$ CHI-SO.- 0.2 DF- 1 SIG.= 0.695 CHI-SO. 0.9 DF- I SIG. 0.330 CHI-SO. 0.0 DF- 1 SIG. $=1.000$

CHI-SO.= 0.7 DE= 1 SIG.=0.394 CHI-SO.= 4.6 DF= 1 SIG. $=0.033$ CHI-SQ. $\quad 8.9 \mathrm{DF}=1 \mathrm{SIG}=0.003$ CHI-SO. $=0.0$ DE 1 SIG. $=1.000$ CHI-SQ. 0.1 DF= 1 SIG. $=0.739$ CHI-SO. $\quad 0.5$ DF 1 SIG. $=0.472$ CHI-SQ. $2.8 \mathrm{DF}=1 \mathrm{SIG}=0.095$ 


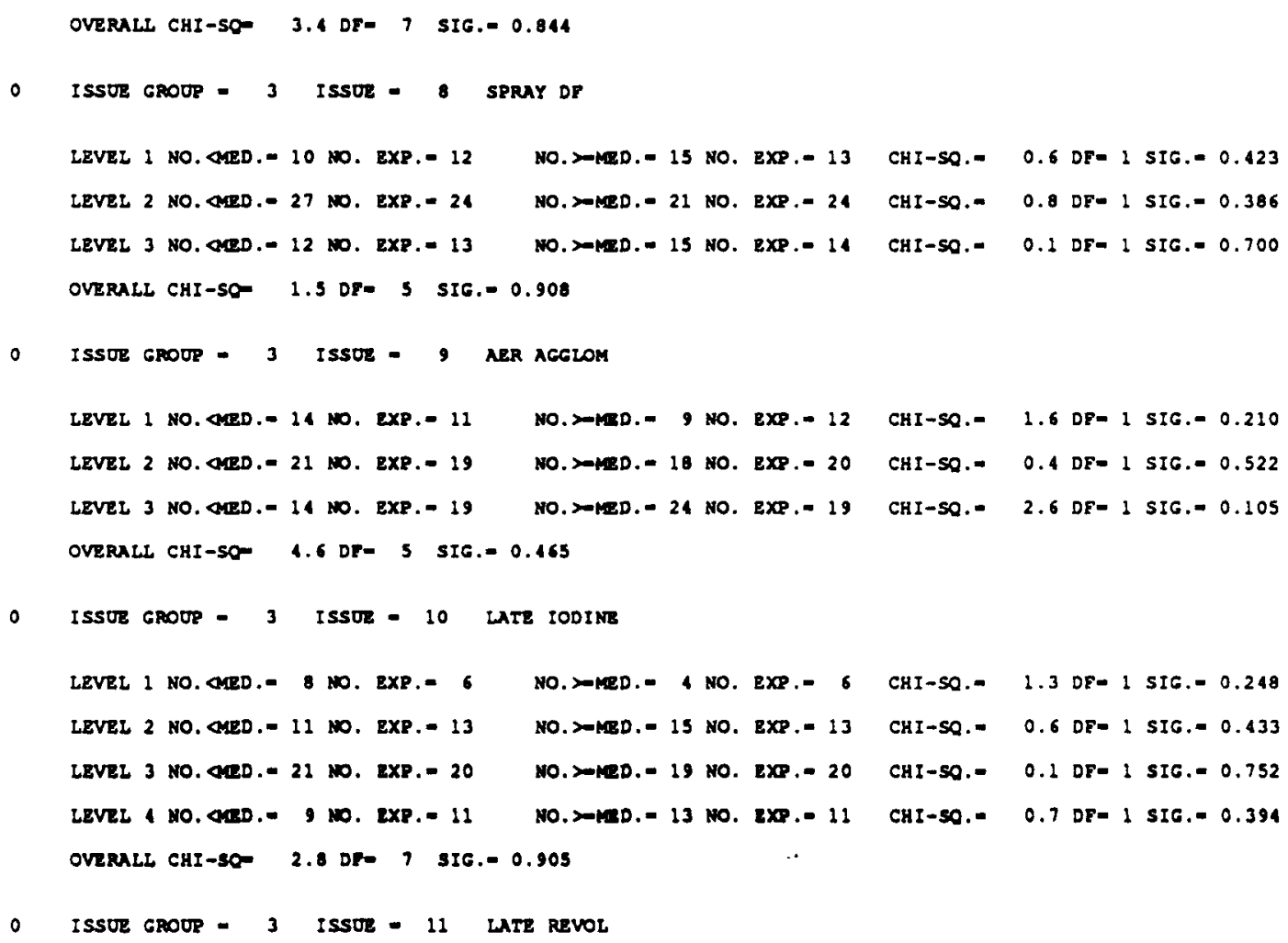
NO. SAED. 5 NO. EXP.= 6 CHI-SQ.= 0.3 DF- 1 SIG.= 0.564

CHI-SO. TEST CANNOT BE RUN; EXP. NO. < 5 CHI-SO.- 2.0 DF- 1 SIG.- 0.157 CHI-SO.- 1.1 DE- 1 SIG.= 0.293 CHI-SO. TEST CANNOT BE RON; EXP. NO. < 5 LEVEL \& NO. ARE. = 7 NO. EXP. = 6

CHI-SO.= 0.3 DFE 1 SIC. $=0.593$

CHI-SQ.= 0.6 DF- 1 SIG. $=0.441$

CHI-SQ.- 0.0 DF- 1 SIG.= 1.000

CHI-SQ.= 0.3 DF- 1 SIG.= 0.564 
CONSEOUENCE MEASORE:

CANCER DEATHS PER YEAR

BASE CASE :

SURRY LALHC (MACCS)

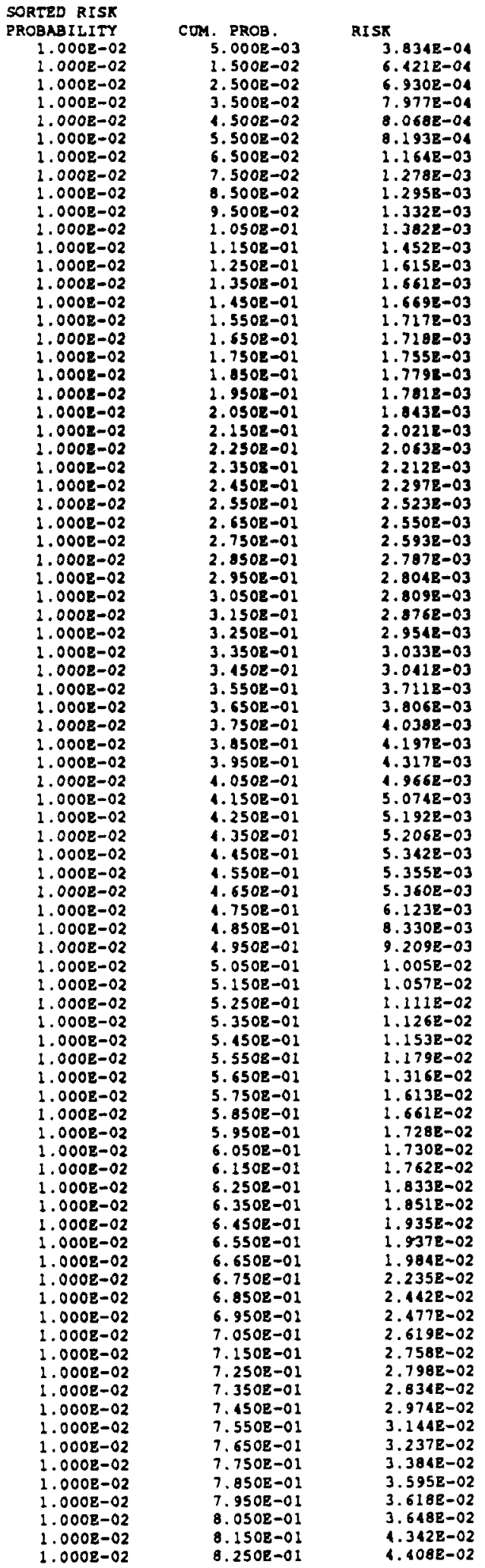


NUREG/CR-4551, VOL. 1: DRAFT REPORT FOR COMMENT (SEPTEMBER, 1986)

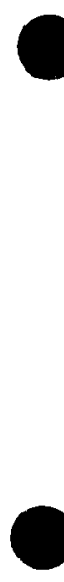

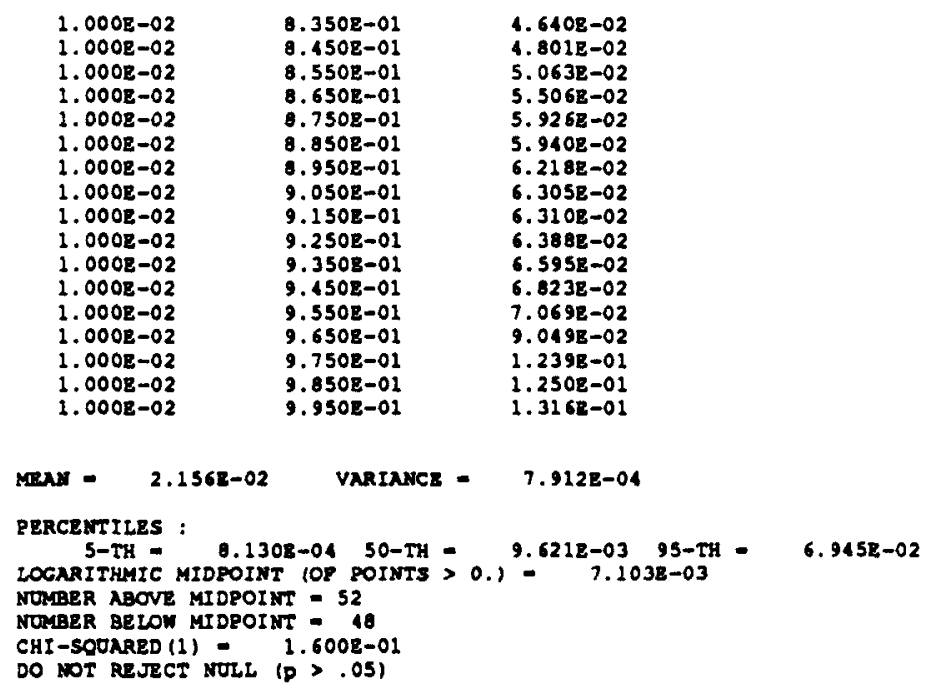


NUREG/CR-4551, VOL. 1: DRAFT REPORT FOR COMMENT (SEPTEMBER, 1986)

CONSEOUENCE MEASURE

CANCER DEATHS PER YEAR

BASE CASE

SURRY LALHC (MACCS)

ISSUE LEVELS FOR RACH SAMPLE MEMBER, IN ORDER OF INCREASING RISK RISK ISSUE LEVELS. POR ALL ISSUES

\begin{tabular}{|c|c|c|c|c|c|c|c|c|c|c|c|c|c|c|c|c|}
\hline $\begin{array}{l}3.834 \mathrm{E}-04 \\
6.121 \mathrm{E}-04\end{array}$ & $\begin{array}{l}2 \\
2\end{array}$ & $\begin{array}{l}1 \\
2\end{array}$ & 3 & 2 & $\begin{array}{l}5 \\
1 \\
1\end{array}$ & $\begin{array}{l}6 \\
1 \\
1\end{array}$ & $\begin{array}{l}7 \\
3 \\
8\end{array}$ & $\begin{array}{l}8 \\
1 \\
2\end{array}$ & $\begin{array}{l}91 \\
2 \\
4\end{array}$ & $\begin{array}{r}101 \\
2\end{array}$ & $\begin{array}{rl}11 & 1 \\
6 & \end{array}$ & $\begin{array}{r}121 \\
5 \\
5\end{array}$ & $\begin{array}{rl}13 & 1 \\
2 & \\
2 & \end{array}$ & $\begin{array}{cc}14 & 1 \\
1 & \end{array}$ & $\begin{array}{r}151 \\
2 \\
3\end{array}$ & $\begin{array}{r}16 \\
1 \\
3\end{array}$ \\
\hline $5.9308-04$ & 2 & 2 & 2 & 1 & 1 & 6 & $i$ & $\begin{array}{l}2 \\
2\end{array}$ & 3 & 2 & 1 & 3 & $\begin{array}{l}2 \\
3\end{array}$ & 2 & 3 & $\begin{array}{l}3 \\
2\end{array}$ \\
\hline $7.9778-04$ & 2 & 1 & 2 & 1 & 2 & 6 & 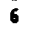 & 2 & 2 & 3 & 3 & 1 & 3 & 2 & 3 & 2 \\
\hline $8.068 \mathrm{~B}-04$ & 2 & 1 & 2 & 1 & 3 & 6 & 2 & 2 & 1 & 1 & 5 & 4 & 1 & 1 & 3 & 2 \\
\hline $3.1938-04$ & 2 & 1 & 2 & 2 & 3 & 6 & 1 & 1 & 5 & 3 & 2 & 1 & 2 & 2 & 2 & 1 \\
\hline $1.164 E-0.3$ & 2 & 2 & 2 & 1 & 2 & 3 & 6 & 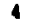 & 1 & 3 & 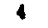 & 5 & $?$ & 2 & & 2 \\
\hline $1.2788-03$ & 2 & 1 & 2 & 2 & 2 & 2 & 5 & 2 & 3 & 1 & $\mathbf{s}$ & 2 & 4 & 2 & 3 & 1 \\
\hline $.2958-03$ & 2 & 2 & 2 & 1 & 2 & 2 & 2 & 3 & 2 & 3 & 1 & 5 & 3 & 2 & 2 & 3 \\
\hline $1.332 \mathrm{z}-03$ & 2 & 2 & 2 & 2 & 1 & 6 & & 2 & & 2 & 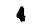 & 3 & a & 2 & 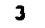 & 2 \\
\hline $1.3828-03$ & 2 & 2 & 2 & 2 & 2 & 2 & 7 & 2 & 1 & 2 & 4 & 3 & 4 & 4 & 2 & 2 \\
\hline $1.452 \mathrm{E}-03$ & 2 & 1 & 3 & 1 & 1 & 2 & 4 & 1 & 1 & 1 & 5 & 4 & & 3 & ? & 3 \\
\hline 1.6 & 2 & 1 & 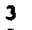 & 4 & 2 & 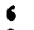 & & 2 & & 3 & 1 & 4 & 2 & 2 & 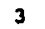 & 2 \\
\hline $1.661 \mathrm{e}-0.3$ & 2 & 2 & 2 & 1 & 1 & 5 & 5 & 3 & 1 & 4 & 1 & 2 & 7 & 2 & 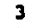 & 3 \\
\hline $2-03$ & 3 & 1 & 3 & 1 & 1 & 1 & - & 2 & 4 & 4 & 1 & 3 & 3 & 3 & & 3 \\
\hline. .7 & 2 & 2 & 3 & 1 & 1 & 3 & 1 & 2 & & 2 & 9 & 1 & 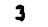 & 4 & ? & 1 \\
\hline $1.718 E-03$ & 2 & 2 & 2 & 1 & 3 & 1 & 3 & 1 & 1 & 2 & 3 & 5 & 7 & 3 & 2 & 2 \\
\hline-03 & 2 & 1 & 3 & 2 & 2 & 6 & 5 & 1 & 3 & 2 & 4 & 3 & & 1 & 2 & 2 \\
\hline 1.77 & 2 & 1 & 2 & 2 & 2 & 5 & 1 & 1 & 2 & 2 & 6 & 5 & 1 & 2 & 1 & 3 \\
\hline 1.78 & 2 & 1 & 2 & 2 & 3 & 2 & & 2 & 4 & 2 & & 4 & 3 & 1 & & 3 \\
\hline 1.843 & 2 & 1 & 2 & 2 & 2 & 6 & 7 & 2 & 2 & 2 & 3 & 1 & 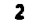 & 2 & 2 & 4 \\
\hline $2.0218-03$ & 2 & 1 & 2 & 1 & 2 & 6 & 7 & 2 & 2 & 3 & 1 & 1 & 3 & 1 & 3 & 2 \\
\hline $2.063 \mathrm{E}-$ & 2 & 1 & 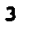 & 1 & 3 & 6 & & 3 & 1 & 3 & 3 & 1 & 2 & 3 & 2 & 2 \\
\hline $2.212 \mathrm{~B}-03$ & 2 & 2 & 2 & 2 & 3 & 2 & & 2 & 3 & 2 & 4 & 3 & 2 & 2 & 4 & 4 \\
\hline .2 & 2 & 2 & 3 & 1 & 2 & 2 & 8 & 1 & 1 & 2 & 5 & 1 & 3 & 2 & 4 & 3 \\
\hline .523 & 2 & 2 & ? & 1 & 3 & 6 & 5 & 2 & 3 & 2 & 5 & 3 & 5 & 3 & 2 & 3 \\
\hline $2.550 \mathrm{E}-03$ & 2 & 2 & 2 & 2 & 3 & 2 & & 2 & & 2 & 1 & 3 & 3 & 3 & 2 & 1 \\
\hline .55 & 1 & 1 & 3 & 2 & 1 & 2 & 6 & $i$ & 5 & 3 & 2 & 4 & & 1 & 2 & 3 \\
\hline $2.787 \mathrm{E}-03$ & 2 & 1 & 3 & 2 & 2 & 2 & 5 & 2 & 2 & 4 & 1 & 5 & 2 & 3 & 4 & 3 \\
\hline $2.804 \mathrm{~B}-03$ & 2 & 1 & 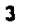 & 2 & 3 & 3 & 9 & 2 & & 2 & 4 & 3 & 5 & 1 & 2 & 2 \\
\hline 2.8 & 2 & 2 & 2 & 2 & 3 & 1 & 5 & 1 & 3 & 3 & 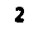 & 3 & 3 & 2 & & 4 \\
\hline $2.876 \mathrm{E}-03$ & 2 & 2 & 3 & 2 & 2 & 3 & 4 & 1 & 3 & 1 & 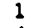 & 2 & 2 & 2 & 3 & 2 \\
\hline $2.954 \mathrm{E}-03$ & 2 & 1 & 2 & 2 & 1 & 2 & 2 & 2 & 3 & 2 & 4 & 3 & 2 & 3 & 2 & 2 \\
\hline $3.033 \mathrm{E}-03$ & 2 & 1 & 3 & 2 & 1 & 3 & 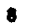 & 3 & 1 & 2 & 4 & 1 & 2 & 4 & 3 & 2 \\
\hline 3.041 & 2 & 1 & 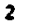 & 1 & 2 & 3 & 9 & 3 & & 2 & 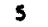 & 4 & 2 & 3 & 2 & 3 \\
\hline $3.711 \mathrm{E}-03$ & 2 & 2 & 2 & 1 & 1 & 5 & 7 & 2 & 5 & 2 & 2 & 3 & 1 & 2 & 1 & 2 \\
\hline $3.806 \mathrm{E}-$ & 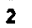 & 1 & 2 & 2 & 3 & 6 & 2 & 2 & 1 & 2 & 4 & 2 & 3 & $\bar{l}$ & 3 & 1 \\
\hline 4.038 & 2 & 2 & 3 & 2 & 1 & 5 & & 2 & & 2 & 5 & 5 & & 2 & & 3 \\
\hline 4.197 & 3 & 1 & 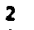 & 2 & 3 & 6 & 6 & 9 & & 4 & & 3 & & 1 & & 2 \\
\hline $4.317 \mathrm{E}-03$ & 2 & 2 & 2 & 2 & 3 & 3 & 7 & 1 & 1 & 2 & 1 & 1 & 3 & 2 & 2 & 3 \\
\hline $4.966 \mathrm{E}-03$ & 2 & 1 & 2 & 2 & 3 & 2 & 1 & 3 & 1 & 3 & 3 & 1 & 1 & 1 & 4 & 3 \\
\hline 5.07 & 1 & 2 & 4 & 2 & 3 & 2 & 1 & 7 & & 2 & 3 & 4 & & 2 & & 1 \\
\hline 5.19 & 2 & 1 & 2 & 2 & 3 & 6 & 2 & 2 & 1 & 2 & & 4 & 4 & 2 & & 3 \\
\hline 5.206 & 2 & 2 & 2 & 1 & 2 & 3 & 5 & 1 & 5 & 2 & 3 & 4 & 3 & 2 & 3 & 4 \\
\hline $.342 \mathrm{E}-$ & 2 & 1 & 2 & 2 & 3 & 3 & 1 & 2 & 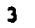 & 2 & 5 & 2 & 3 & 3 & & 3 \\
\hline $5.355 z-03$ & 2 & 2 & 2 & 2 & 2 & 3 & $B$ & 2 & 6 & 3 & & 2 & & 2 & & 2 \\
\hline 5.3 & 2 & 2 & 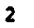 & 2 & 2 & 6 & 9 & 1 & 1 & 3 & 1 & 4 & 3 & 3 & 3 & 4 \\
\hline $6.123 \mathrm{E}-03$ & 2 & 2 & 2 & 1 & 1 & 6 & 2 & 1 & 6 & 3 & 3 & 4 & 5 & 1 & 3 & 3 \\
\hline $8.330 \mathrm{E}-$ & 2 & 1 & 3 & 1 & 3 & 6 & 8 & 1 & 1 & 3 & 2 & 3 & 2 & 2 & $i$ & 2 \\
\hline $9.209 \mathrm{E}-$ & 2 & 1 & 3 & 1 & 3 & 6 & 6 & 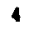 & 1 & 3 & & 2 & & 3 & & 3 \\
\hline $1.005 E-$ & 3 & 1 & 2 & 1 & 3 & 2 & 2 & 3 & 4 & 3 & 1 & 5 & 3 & 2 & 4 & 2 \\
\hline $1.057 \mathrm{E}$ & 2 & 2 & 2 & 3 & 2 & 6 & 9 & 1 & 1 & 1 & 6 & 1 & 2 & 2 & 3 & 1 \\
\hline $1.111 \mathrm{E}-02$ & 2 & 2 & 2 & 1 & 2 & 3 & 9 & 2 & 4 & 1 & 5 & 4 & 2 & 1 & 3 & 2 \\
\hline 1.2 & 3 & 2 & 3 & 2 & 1 & 3 & 0 & 7 & 7 & 3 & & 5 & & 3 & & 3 \\
\hline 1.153 & 2 & 1 & 2 & 1 & 1 & 6 & 2 & 1 & 2 & 3 & 3 & 1 & 3 & 1 & 3 & 2 \\
\hline $1.179 \mathrm{E}-$ & 2 & 1 & 2 & 2 & 3 & 1 & 1 & 2 & 4 & 1 & 6 & 2 & 3 & 2 & 1 & 3 \\
\hline $1.316 \mathrm{E}-$ & 2 & 1 & 2 & 2 & 1 & 1 & 3 & 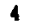 & 2 & 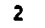 & 3 & 5 & & 1 & & 2 \\
\hline 1.613 & 2 & 2 & 2 & 3 & 3 & 6 & 7 & 2 & 3 & 1 & 6 & 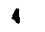 & & 2 & & 4 \\
\hline $1.661 \mathrm{R}-0$ & , & 2 & 3 & 2 & 3 & 4 & 4 & 2 & 5 & 2 & 3 & 2 & 3 & 1 & 2 & 2 \\
\hline $1.728 \mathrm{~g}-02$ & 2 & 2 & 3 & 2 & 2 & 3 & 7 & 3 & 4 & 3 & 3 & 2 & 1 & 2 & 1 & 2 \\
\hline $1.730 \mathrm{E}-$ & 2 & 1 & 3 & 2 & 1 & 6 & 3 & 5 & 1 & 3 & 4 & 2 & & 2 & & 3 \\
\hline $1.762 \mathrm{E}-$ & 2 & 2 & 3 & 3 & 2 & 2 & 9 & 3 & 1 & 2 & 3 & 1 & 3 & 2 & 1 & 3 \\
\hline $1.8338-02$ & 2 & 2 & 2 & 2 & 3 & 1 & 7 & 2 & 1 & 1 & 6 & 4 & 3 & 2 & 4 & 2 \\
\hline 1.85 & 2 & 1 & 1 & 3 & 1 & 6 & 3 & 3 & 3 & 2 & 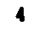 & 5 & 3 & 3 & 2 & 2 \\
\hline 1.935 & 2 & 2 & 3 & 3 & 1 & 6 & 4 & 2 & 5 & 2 & 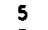 & 3 & & 1 & & 3 \\
\hline 1.9 & 2 & 1 & 2 & 2 & 2 & 6 & 4 & 3 & 3 & 2 & 5 & 1 & 1 & 1 & 1 & 2 \\
\hline 1.9 & 2 & 1 & 2 & 3 & 2 & 1 & 7 & 2 & 5 & 1 & 6 & 1 & 3 & 3 & 2 & 1 \\
\hline 2.2 & 2 & 2 & 2 & 3 & 2 & 5 & 3 & 4 & 2 & 2 & 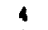 & 4 & 3 & 1 & + & 3 \\
\hline $2.442 \mathrm{E}-02$ & 2 & 2 & 3 & 1 & 1 & 6 & 8 & 3 & 4 & 3 & & 2 & 3 & 2 & 3 & 3 \\
\hline $2.4778-02$ & 2 & 2 & 2 & 2 & 3 & 4 & 2 & 3 & 3 & 2 & 3 & 2 & 4 & 2 & 3 & 3 \\
\hline 2.619 & 2 & 1 & 2 & 1 & 2 & 3 & 9 & 5 & 3 & 3 & 2 & 3 & 5 & 3 & 2 & 3 \\
\hline $2.758 \mathrm{E}-$ & 2 & 2 & 3 & 2 & 1 & 1 & 4 & 4 & 4 & 3 & 8 & 1 & 2 & 2 & & 2 \\
\hline $2.798 \mathrm{E}-$ & 2 & 1 & 2 & 2 & 2 & 2 & 6 & 5 & 3 & 3 & 3 & 5 & 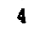 & 4 & 3 & 3 \\
\hline $2.8348-0$ & 2 & 2 & 3 & 3 & 1 & 4 & 5 & 1 & 2 & 2 & 4 & 2 & 2 & 2 & 4 & 4 \\
\hline $2.974 \mathrm{E}-0$ & 2 & 1 & 2 & 1 & 2 & 6 & 3 & 3 & 4 & 2 & 2 & 4 & 2 & 1 & 2 & 4 \\
\hline $3.1448-$ & 2 & 1 & 2 & 2 & 1 & 5 & 7 & 4 & 2 & 1 & & 5 & & 2 & 2 & 3 \\
\hline $3.237 \mathrm{E}-02$ & 1 & 2 & 2 & 2 & 3 & 4 & 4 & 1 & 2 & 2 & 4 & 5 & 3 & 4 & 1 & 3 \\
\hline 3.38 & 2 & 1 & 2 & 2 & 2 & 6 & 4 & 4 & 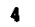 & 2 & 1 & 3 & 2 & 2 & 2 & 3 \\
\hline $3.595 \mathrm{E}-0$ & 2 & 2 & 2 & 1 & 2 & 5 & 4 & 5 & 5 & 4 & 1 & 1 & 3 & 1 & 1 & 2 \\
\hline 3. & 2 & 1 & 3 & 1 & 2 & 2 & 9 & 3 & 4 & 2 & 3 & 3 & 4 & 3 & 1 & 3 \\
\hline $3.648 \mathrm{E}-02$ & 2 & 2 & 2 & 1 & 3 & 2 & 5 & 5 & 3 & 2 & 4 & 2 & 2 & 2 & 2 & 1 \\
\hline .3 & 1 & 1 & 3 & 1 & 3 & 3 & 6 & 5 & 3 & 3 & 3 & 3 & 4 & 3 & 2 & 2 \\
\hline & 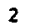 & 1 & & 1 & 2 & 6 & 8 & $\mathrm{~J}$ & 3 & 1 & 4 & 5 & 1 & 1 & 3 & 4 \\
\hline
\end{tabular}


NUREG/CR-4551, VOL. 1: DRAFT REPORT FOR COMMENT (SEPTEMBER, 1986)

\begin{tabular}{|c|c|c|c|c|c|c|c|c|c|c|c|c|c|c|c|c|c|c|c|c|c|c|c|c|c|c|c|c|c|}
\hline 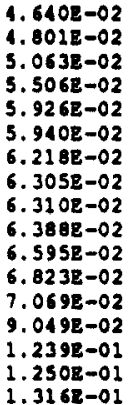 & $\begin{array}{l}2 \\
2 \\
2 \\
2 \\
2 \\
2 \\
2 \\
2 \\
2 \\
2 \\
2 \\
1 \\
2 \\
2 \\
2 \\
2\end{array}$ & $\begin{array}{l}1 \\
2 \\
1 \\
2 \\
1 \\
1 \\
1 \\
1 \\
2 \\
1 \\
2 \\
2 \\
2 \\
2 \\
2 \\
2 \\
2\end{array}$ & $\begin{array}{l}2 \\
3 \\
2 \\
3 \\
2 \\
2 \\
3 \\
2 \\
2 \\
3 \\
2 \\
3 \\
2 \\
2 \\
3 \\
3 \\
2\end{array}$ & $\begin{array}{l}1 \\
1 \\
2 \\
3 \\
1 \\
1 \\
2 \\
1 \\
2 \\
2 \\
1 \\
1 \\
2 \\
3 \\
2 \\
1 \\
2\end{array}$ & $\begin{array}{l}3 \\
1 \\
3 \\
2 \\
2 \\
1 \\
1 \\
3 \\
1 \\
1 \\
3 \\
1 \\
3 \\
1 \\
1 \\
1 \\
2\end{array}$ & $\begin{array}{l}6 \\
1 \\
6 \\
4 \\
3 \\
6 \\
3 \\
6 \\
4 \\
3 \\
3 \\
6 \\
2 \\
3 \\
2 \\
4 \\
1\end{array}$ & $\begin{array}{l}9 \\
1 \\
5 \\
7 \\
1 \\
1 \\
6 \\
8 \\
3 \\
4 \\
8 \\
1 \\
4 \\
3 \\
3 \\
6 \\
6\end{array}$ & $\begin{array}{l}4 \\
3 \\
5 \\
4 \\
5 \\
5 \\
3 \\
4 \\
5 \\
4 \\
4 \\
5 \\
3 \\
4\end{array}$ & $\begin{array}{l}6 \\
3 \\
3 \\
5 \\
4 \\
3 \\
3 \\
3 \\
2 \\
2 \\
6 \\
2 \\
6 \\
5 \\
2\end{array}$ & $\begin{array}{l}1 \\
1 \\
2 \\
2 \\
1 \\
2 \\
2 \\
1 \\
1 \\
2 \\
2 \\
1 \\
1 \\
2 \\
2 \\
1 \\
1\end{array}$ & $\begin{array}{l}5 \\
1 \\
6 \\
1 \\
4 \\
3 \\
4 \\
6 \\
6 \\
4 \\
4 \\
6 \\
6 \\
3 \\
4 \\
5 \\
4\end{array}$ & $\begin{array}{l}3 \\
2 \\
1 \\
2 \\
5 \\
5 \\
5 \\
4 \\
1 \\
1 \\
2 \\
1 \\
2 \\
1 \\
3 \\
2 \\
1\end{array}$ & $\begin{array}{l}1 \\
5 \\
3 \\
3 \\
2 \\
2 \\
3 \\
2 \\
1 \\
3 \\
5 \\
1 \\
3 \\
3 \\
5 \\
3 \\
3\end{array}$ & $\begin{array}{l}2 \\
3 \\
1 \\
3 \\
2 \\
1 \\
2 \\
2 \\
2 \\
2 \\
1 \\
3 \\
1\end{array}$ & $\begin{array}{l}3 \\
1 \\
3 \\
2 \\
4\end{array}$ & $\begin{array}{l}3 \\
3 \\
2 \\
3 \\
3 \\
4 \\
2\end{array}$ & $\begin{array}{l}4 \\
1 \\
2 \\
2\end{array}$ & 1 & & & $\begin{array}{l}1 \\
3 \\
1 \\
1 \\
3 \\
1 \\
1 \\
3\end{array}$ & $\begin{array}{l}2 \\
2\end{array}$ & $\begin{array}{l}3 \\
2 \\
3 \\
3\end{array}$ & & $\begin{array}{l}3 \\
3 \\
3 \\
3 \\
1 \\
3 \\
2 \\
3 \\
1 \\
3\end{array}$ & $\begin{array}{l}3 \\
4\end{array}$ & 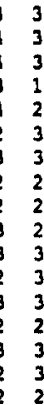 & 4 & 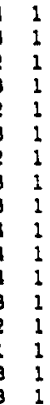 \\
\hline
\end{tabular}




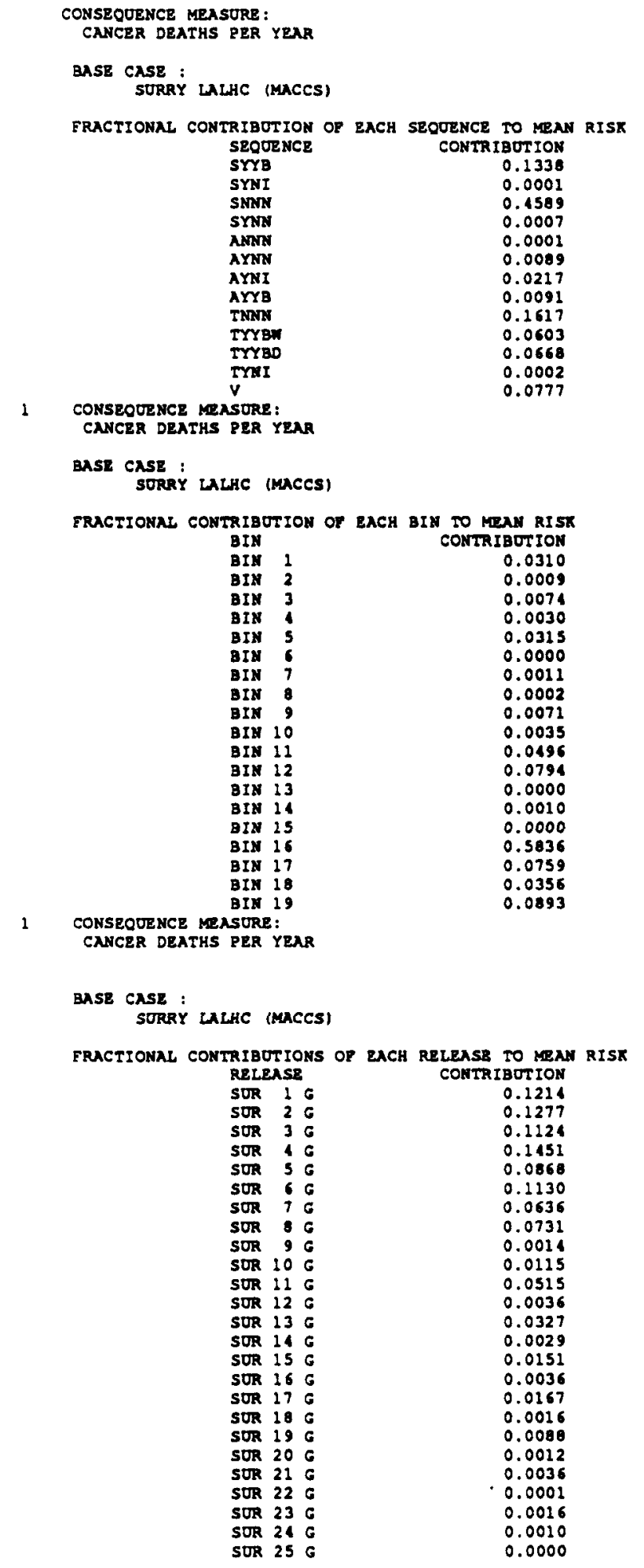


CONSEQUENCE MEASURE:

CANCER DEATHS PER YEAR

SENSITIVITY OF RISK TO ISSUES

DISTRIBUTION OF RISK AT EACH LEVEL OR EACH ISSUE BASE CASE :

SURRY LALKC (MACCS)

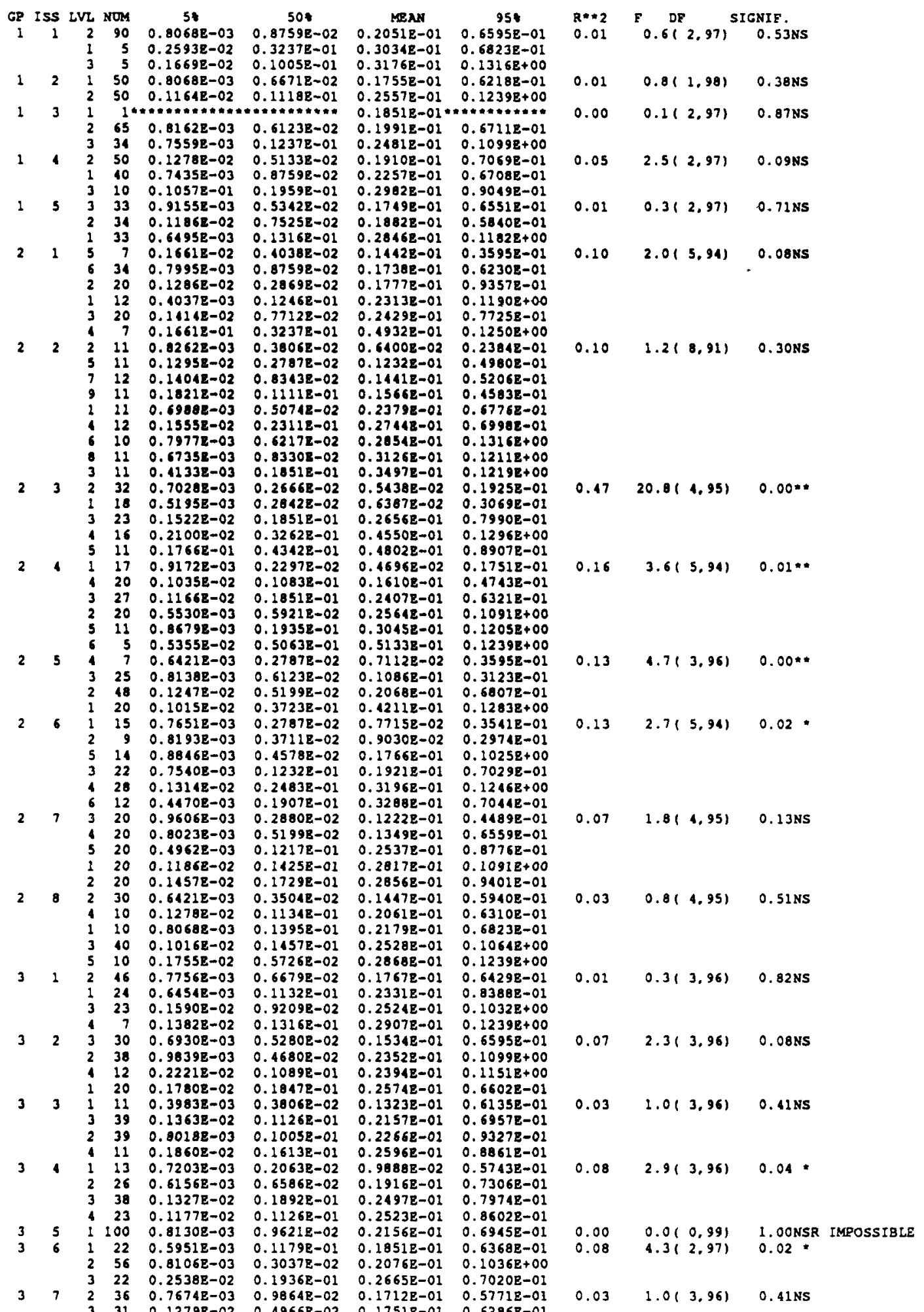


NUREG/CR-4551, VOL. 1: DRAFT REPORT FOR COMMENT (SEPTEMBER, 1986)

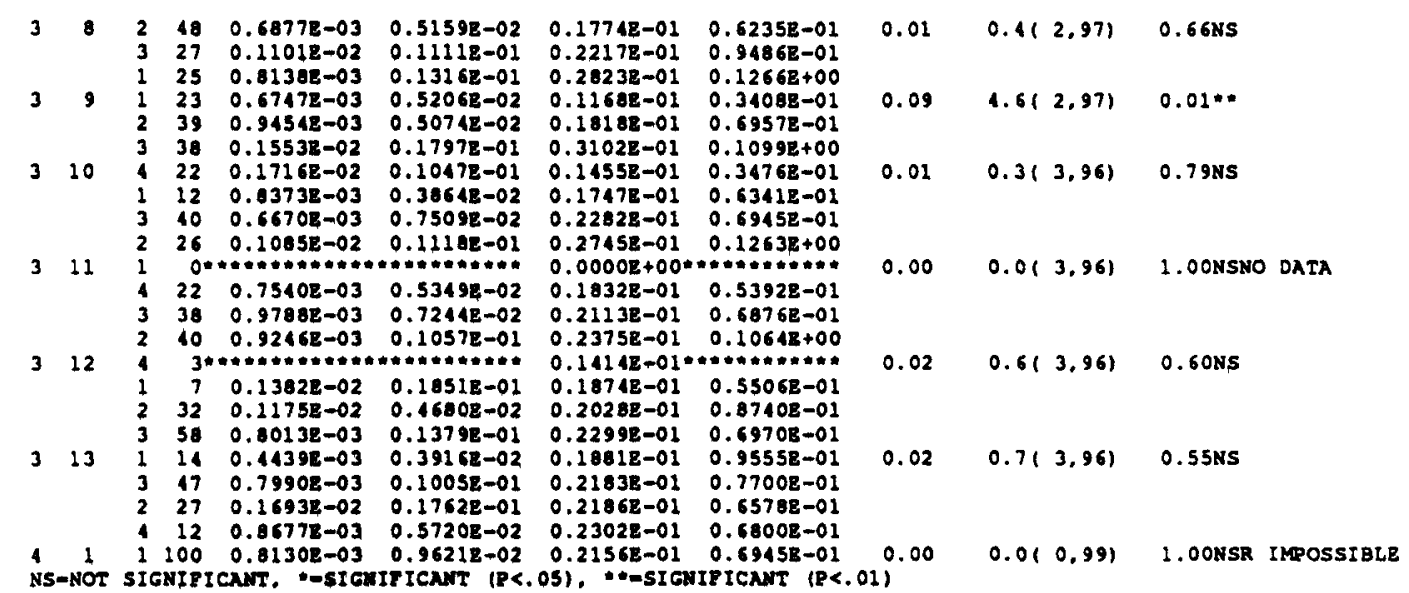


CONSEOUENCE MEASURE: CANCER DEATHS RER YEAR

ISSUE GROP - 1 ISSUE - 1 TB SEAL LOCA

LEVEL I NO. GED. = 2 WO. EXP. = 2

พO.>ME.= 3 No. EXP.= 3

LEVEL 2 NO. QGED. = 45 WO. EXP. 45

MO. >-MED. - 45 No. EXP. - 45

LEVEL 3 NO. ARD.- 2 No. EXP.- 2

NO.>MED.= 3 No. EXP.= 3

OVERALL CHI-SO= 0.0 OF= 1 SIG. 1.000

0

ISSTE GROUP - 1 ISSUE - 2 USING GAS GBN.

LEVEL 1 NO. GRD. $=26$ NO. EXP. $=25$

NO. 2 MeD. - 24 NO. BXP. $=25$

LEVEL 2 NO. QRE. - 23 so. EXP. - 23

MO. JMED. 27 NO. EXP. -25

OVRRALL CHI-SO 0.4 DF- 3 SIG. $=0.940$

0

ISSOE GROOR - 1 ISSUE - 3 BETA-FACTOR

LEVEL 1 No. QRE. = 0 No. Exp.= 0

No.s-mo.- 1 No. exp.- 1

LEVEL 2 NO. QAED. - 33 No. EXP. - 32

NO.>ted. 32 NO. EXP.- 33

LEVEL 3 NO. QRED. - 16 No. EXP. - 17

NO. >MED. - 18 NO. EXP.= 17

OVERALL CHI-SO 0.2 DF- 3 SIG.- 0.901

0

ISSUE GROUP - 1 ISSUE - 4 V PREQ

LEVEL I NO. QED.- 20 No. EXP. $=20$

พ0.>MED. - 20 พ0. EXP. -20

LEVEL 2 NO. GXED. 29 NO. EXP, 25

MO. >MED. - 21 NO. EXP.- 25

LEVEL 3 NO. QRED.- 0 No. EXP.= 5

MO. $>$ MED. - 10 พо. EXP.= 5

OVERALL CHI-SO 11.3 DPE 3 SIG.- 0.046

ISSUE GROUR - 1 ISSOE - 5 FIF2 FAILURE

LEVEL I NO. AMED.- 14 NO. EXP. - 16

IEVEL 2 No. QED.- 17 No. EXP.- 17

HO. JaED. - 19 No. EXP. - 17

No.>MED. $=17$ No. EXP.= 17

LEVEL 3 NO. QED. - 18 No. EXP. - 16

NO. XMED. - 15 NO. EXP. = 17

OVERALL CHI-SQ 1.0 DF- 5 SIG.- 0.965

$\circ$

ISSUE GROUP - 2 ISSOE - 1 IND. RCS FAIL.

LEVEL 1 NO. QTED.- 5 NO. EXP. - 6

LEVEL 2 MO. QRE.- 13 NO. exP. - 10

LEVEL 3 No. QRD.- 10 No. EXP. - 10

LEVEL 4 NO. GRD.- 0 NO. EXP.= 3

LEVEL 5 NO. QMED.- 4 No. EXP.= 3

LEVEL 6 NO. QRED.- 17 NO. EXP.- 17

NO. S-MRD. - 7 NO. EXP.- 6

NO. $>=\mathrm{MED}=7$ NO. $\mathrm{EXP}=10$

No. $>$ KED. = 10 NO. EXP. -10

NO.>KED. $=7$ NO. EXP.= 4

NO. >MED.- 3 NO. EXP.-

NO. 工MED. - 17 NO. $\operatorname{EXP.-17}$

OVERALL CHI-SO 2.1 DFE 7 SIG. $=0.952$

0
ISSUE GROUP - 2 ISSUE - 2 MODE OP VB.

LEVEL I NO. QED.- 6 NO. EXP.- 5

LEVEL 2 NO. QMED.- 8 NO. EXP.= 5

LEVEL 3 NO. CMED.- 3 NO. EXP. $=5$

LEVEL \& NO. QRD. = 3 NO. EXP. = 6

LEVEL 5 NO. QMED.= NO. EXP.= 5

LEVEL 6 NO. QED.= 5 NO. EXP. $=5$
CHI-5Q. TEST CANNOT BE RON: EXP, NO. < 5

$\mathrm{CH}$ I-SO. $=0.0$ DE- 1 SIG. $=1.000$

CHI-SO. TEST CANNOT BE RUN; EXP. NO. < 5

CHI-SO.= 0.1 DF= 1 SIG. $=0.777$

CHI-SO.- 0.3 DF- 1 SIC. $=0.572$

CHI-SO. TEST CANNOT BE RUN; EXP. NO. < 5

CHI-SO.= 0.1 DF- I SIC. $=0.804$

CHI-SO.- 0.1 DF- 1 SIG.- 0.732

CHI-SO.= 0.0 DF- 1 SIG.- 1.000

CHI-SQ. $\quad 1.3$ DE= 1 SIG. $=0.258$

CHI-SO. 10.0 DFE 1 SIG.=0.002

CHI-SO.= 0.5 DF- I SIG. $=0.486$

CHI-SO.- 0.0 DF- 1 SIG. $=1.000$

CHI-SO.= 0.5 DF= I SIG. 0.186

CHI-SQ. $\quad 0.3$ DF= 1 SIG $=0.564$

CHI-SO.- 1.8 DF= 1 SIG. 0.180

CHI-SO.= 0.0 DE- 1 SIG. 1.000

CHI-SO. TEST CANNOT BE RUN: EXP. NO. < 5 CHI-SO. TEST CANNOT BE RUN; EXP. NO. < 5

CHI-SO.- 0.0 DF= 1 SIG. 1.000
NO. XKED. = 5 NO. EXP. $=6$

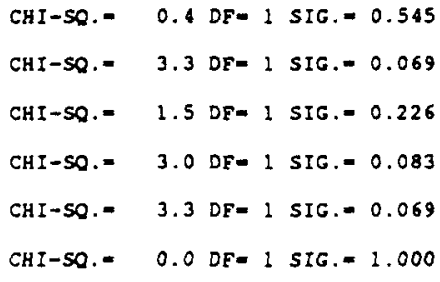

NO. X-MED. - 3 NO. EXP.= 6

NO. $>$ MD. $=8$ NO. $\operatorname{ExP} .=6$

NO. $\triangle$ MED. $=9$ NO. EXP.= 6

NO, $>M E D .=3$ No. EXP.= 6

NO. $>M E D .=5$ NO. EXP.= 5 


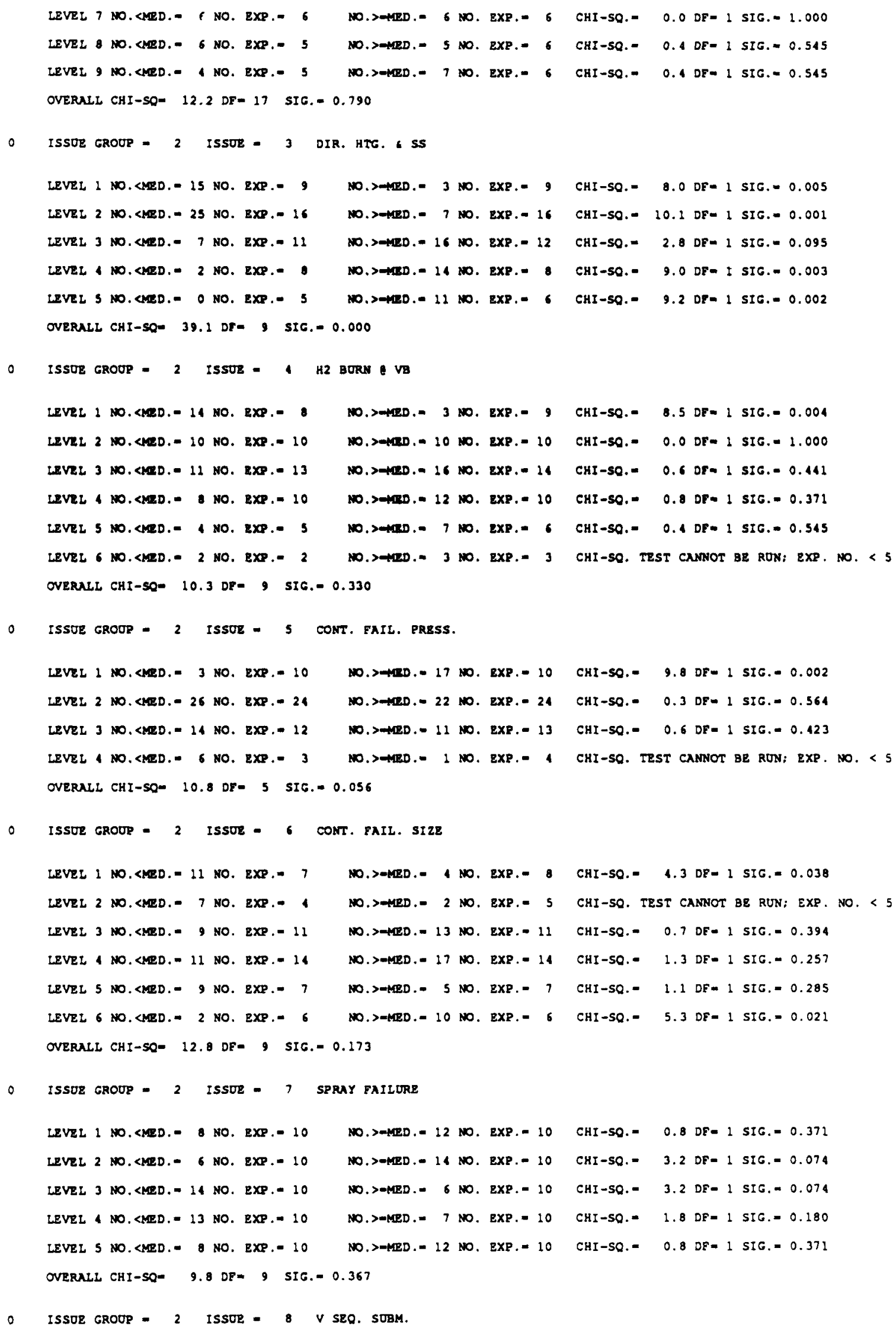

CHI-SO.- 4.3 DF- I SIG. $=0.038$ CHI-SQ. IEST CANNOT BE RUN: EXP. NO. < 5 CHI-SO.- 0.7 OF- 1 SIG. $=0.394$ CHI-SO.- 1.3 DF $=1$ SIG. -0.257 CHI-SQ.- 1.1 DF- 1 SIG.= 0.285 CHI-SQ.- 5.3 DE $=1$ SIG. $=0.021$

CHI-SO.= 0.8 DF= 1 SIG. 0.371 CHI-SO.= 3.2 DF- 1 SIG. $=0.074$ CHI-SO.- $\quad 3.2$ DF- 1 SIG. 0.074 CHI-SO.= 1.8 DF 1 SIG. 0.180 CHI-SQ. $\quad 0.8 \mathrm{DF}=1$ SIG. $=0.371$ 


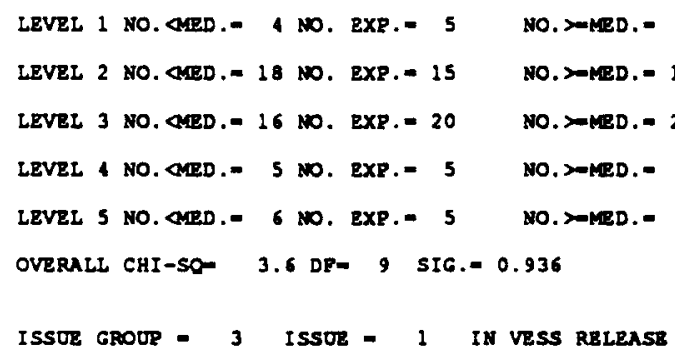

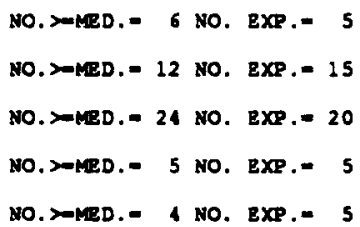
REL

NO. >-MED. - 12 NO. EXP. = 11 NO.>MED. -21 NO. $2 \times P .=28$ NO. SMED. = 18 NO. ExP.- 11

$\mathrm{CHI}-\mathrm{SO}=0.2 \mathrm{DF}=1 \mathrm{SIG}=0.670$ $\mathrm{CHI}-\mathrm{SO} .=3.5 \mathrm{DF}=1 \mathrm{SIG}=0.061$ CHI-SO.= 0.9 DF- 1 SIC. $=0.003$

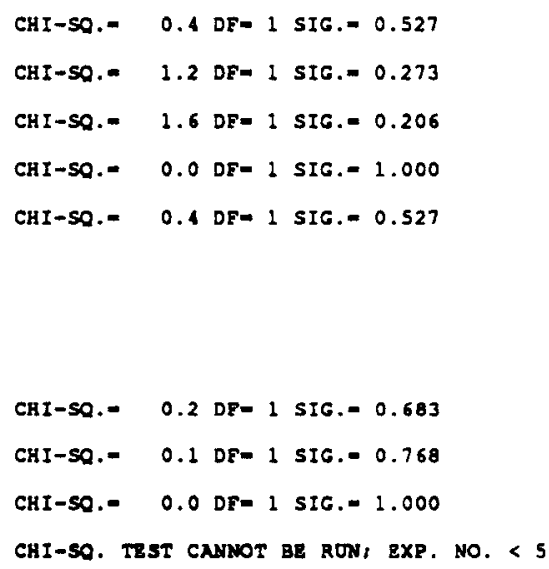


OVERALL CHI-SO- 0.9 DFE 1 SIG.= 0.996

ISSUE GROUR - 3 ISSUE - 8 SPRAY DF

LEVEL 1 NO. <LED. = 10 NO. EXP. $=12$

10. $>-4 B D .=15$ 10. EXP. $=13$

LEVEL 2 NO. CMED. - 26 NO. EXP. -24

No.>-NGD. = 22 No, ExP. -24

Wo. > ALD. - 14 10. EXE.- 14

LEVEL 3 NO. CAED. - 13 NO. EXP. - 13

OVERALL CHI-SO- 1.0 DF- S SIG.= 0.965

ISSUE GROUR - 3 ISSUE - , AER AGCLOY

LEVEL 1 No. <Med. - 13 No. exp. - 11

No.>-1ED, - 10 W0. EXP. - 12

LEVEL 2 NO. <MED. $=22$ No. EXP. $=29$

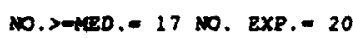

LEVEL 3 No. <MED. - 14 No. EXP. - 19

No.>-1E0. -24 No. EXP. -19

OVERALL CHI-SOE 4.3 DTE 5 SIG.- 0.314

0

ISSUE GROUP - 3 ISSUE - 10 LATE IODIKE

LEVEL I No. <HED. - NO. EXP.-

No.>-4RD. - \& No. ExR.- 6

LEVEL 2 No. CMED. = 10 No. EXP, -13

No.>AED. - 16 No. Exp. - 13

so.>-ALD. $=20$ No. Exp. $=20$

LEVEL 3 NO. KMED.- 20 NO, EXP.- 20

10.>-10. - 11 เo. Exp. - 11

Level 4 No. 4leo. - 11 No. exp. - 11

.910

ISSUE GROOP - 3 ISSUE - 11 LATR REVOR

LEVEL 1 NO. KRD. - 0 No. EXP.- 0

No.>ARE.- 0 No. EXP.: 0

LEVEL 2 No. LRD. 18 NO. EXP. 20

\$o. >med, = 22 No, ExP, -20

LEVEL 3 No. <MeD. - 19 No. EXe. - 19

No.>-ALD. - 19 ko. exp.- 19

LEVEL + No. 4ao. - 12 No. $\exp .911$

No. $2 M E D .=10$ mo. axp. - 21

OVERALL CHI-SQ- 0.6 DF= 5 SIG. 0.989

ISSUE GROUP - 3 ISSUE - 12 HPE REL

LEVEL \& No. CHED.- 3 No. EXP.- 3

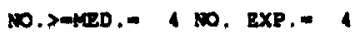

LEVEL 2 NO. CMED. - 20 NO. EXP, = 16

Wo. >MED. $=12$ MO. EXP.- 16

LEVEL 3 No. CMED.- 25 NO. EXP.- 29

No. $>+180 .=33$ No. EXP. - 29

LEVEL \& NO. SEED.= I NO. EXP.- 1

B.>ARD.- 2 NO. EXP.- 2

OVERALL CHI-SO- 3.1 DFP 3 SIG.=0.376

ISSUE GROUP - 3 ISSUE - 13 DH REL

LEVEL 1 NO. CAED.- 8 NO. EXP.- 7

No.>MED.- 6 No. ExP. $=7$ No.>AED. $=17$ NO. EXP. $=14$

NO.>MED. = 24 No. EXP, $=24$

NO.>-MED. $=1$ NO. EXP. $=6$

CHI-SQ. $=0.3$ DF 1 SIG. $=0.593$

CHI-5O. $=1.3$ DF- 1 SIG. $=0.248$

CHI-SO. $=0.0$ DF= 1 SIG. $=1.000$

$\mathrm{CHI}-\mathrm{SQ}=1.3 \mathrm{DF}=1 \mathrm{SIG}=0.248$

OVERALL CHI-SQ- 3.0 DFE 7 SIG. $=0.889$ 
CONSEQUENCE MEASURE:

POP. DOSE 50 MI PER YEAR

BASE CASE

SURRY LALHC (MACCS)

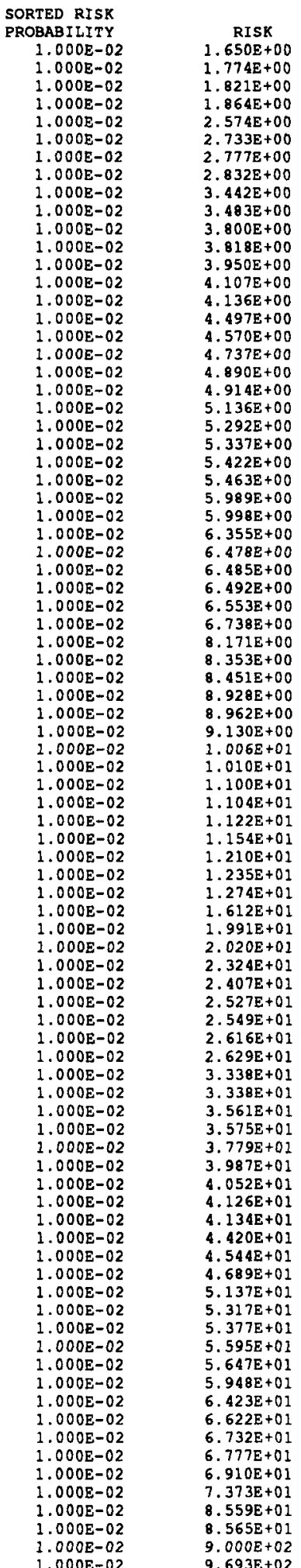


NUREG/CR-4551, VOL. 1: DRAFT REPORT FOR COMMENT (SEPTEMBER, 1986)

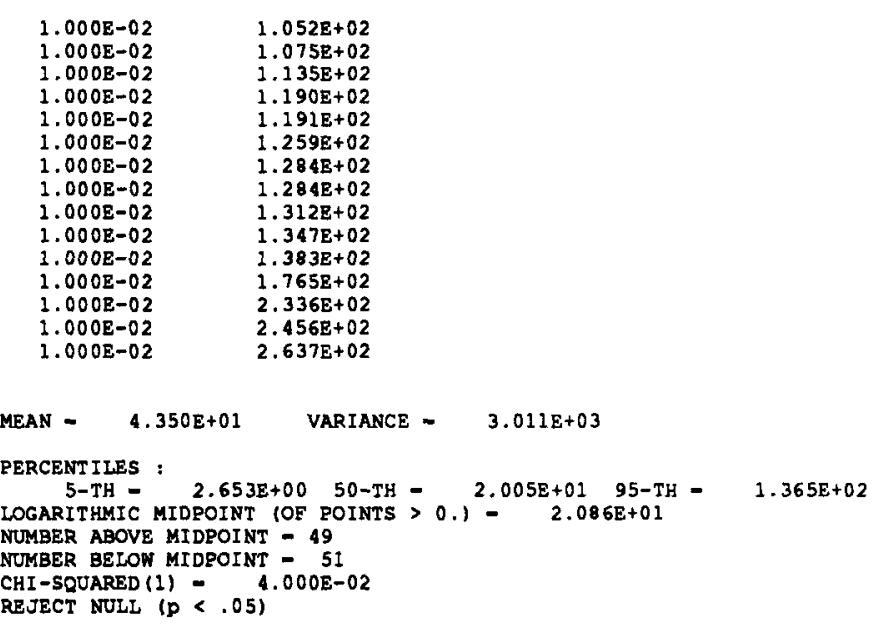

D-122 
NUREG/CR-4551, VOL. 1: DRAFT REPORT FOR COMMENT (SEPTEMBER, 1986)

CONSEQUENCE MEASURE:

POP. DOSE 50 MI PER YEAR

BASE CASE

SURRY LALHC (MACCS)

ISSUE LEVELS FOR EACH SAMPLE MEMBER, IN ORDER OF INCREASING RISK

RISK ISSUE LEVELS, FOR ALL ISSUES

$\begin{array}{llllllllllllllllllllllllllll}1.650 \mathrm{E}+00 & 2 & 2 & 3 & 4 & 5 & 6 & 7 & 8 & 9 & 10 & 11 & 12 & 13 & 14 & 15 & 16 & 17 & 18 & 19 & 20 & 21 & 22 & 23 & 24 & 25 & 26 & 27\end{array}$ $\begin{array}{lllllllllllllllllllllllllllll}774 \mathrm{~B}+00 & 2 & 1 & 2 & 1 & 2 & 6 & 6 & 2 & 2 & 3 & 3 & 4 & 3 & 2 & 3 & 2 & 4 & 1 & 1 & 1 & 1 & 2 & 1 & 4 & 3 & 1 & 1\end{array}$ $\begin{array}{lllllllllllllllllllllllllllllllll}1.821 E+00 & 2 & 2 & 3 & 1 & 1 & 1 & 8 & 2 & 4 & 4 & 1 & 5 & 2 & 2 & 3 & 3 & 1 & 1 & 2 & 2 & 2 & 1 & 3 & 2 & 2 & 3 & 1\end{array}$ $\begin{array}{lllllllllllllllllllllllllllllllll}1.864 E+00 & 2 & 2 & 2 & 1 & 1 & 6 & 1 & 2 & 3 & 2 & 3 & 3 & 3 & 2 & 3 & 2 & 2 & 1 & 2 & 3 & 2 & 1 & 3 & 4 & 3 & 3 & 1\end{array}$ $\begin{array}{llllllllllllllllllllllllllll}2.574 \mathrm{E}+00 & 2 & 2 & 2 & 1 & 2 & 3 & 6 & 4 & 1 & 3 & 4 & 5 & 2 & 2 & 3 & 2 & 3 & 1 & 2 & 2 & 3 & 2 & 2 & 4 & 2 & 3 & 1 \\ 2.733 \mathrm{E}+00 & 2 & 1 & 2 & 1 & 3 & 6 & 2 & 2 & 1 & 1 & 5 & 4 & 1 & 1 & 3 & 2 & 2 & 1 & 2 & 1 & 3 & 1 & 3 & 3 & 3 & 4 & 1\end{array}$

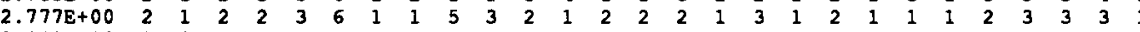
$\begin{array}{llllllllllllllllllllllllllllll}2.832 \mathrm{E}+00 & 2 & 2 & 2 & 1 & 2 & 2 & 2 & 3 & 2 & 3 & 1 & 5 & 3 & 2 & 2 & 3 & 2 & 1 & 2 & 3 & 3 & 3 & 1 & 3 & 4 & 3 & 1 \\ 3.4442 \mathrm{E}+00 & 2 & 2 & 2 & 2 & 1 & 6 & 2 & 2 & 1 & 2 & 4 & 3 & 4 & 2 & 3 & 2 & 2 & 1 & 2 & 4 & 2 & 2 & 3 & 2 & 2 & 3 & 1\end{array}$ $\begin{array}{llllllllllllllllllllllllllllll}3.442 E+00 & 2 & 2 & 2 & 2 & 1 & 6 & 2 & 2 & 1 & 2 & 4 & 3 & 4 & 2 & 3 & 2 & 2 & 1 & 2 & 4 & 2 & 2 & 3 & 2 & 2 & 3 & 1\end{array}$

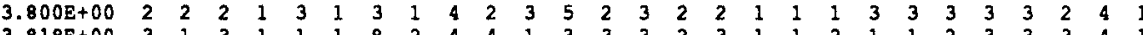
$\begin{array}{rllllllllllllllllllllllllllllll}3.818 E+00 & 3 & 1 & 3 & 1 & 1 & 1 & 8 & 2 & 4 & 4 & 1 & 3 & 3 & 3 & 2 & 3 & 1 & 1 & 2 & 1 & 1 & 2 & 3 & 3 & 3 & 4 & 1 \\ 3.950 E+00 & 2 & 1 & 3 & 1 & 1 & 2 & 4 & 1 & 1 & 1 & 5 & 4 & 4 & 3 & 2 & 3 & 4 & 1 & 2 & 2 & 3 & 3 & 2 & 2 & 2 & 3 & 1\end{array}$

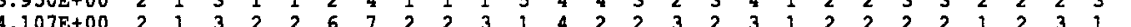

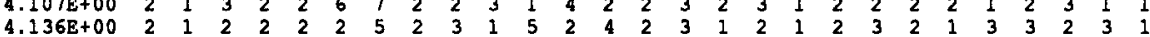
$\begin{array}{llllllllllllllllllllllllllllll}4.496 \mathrm{E}+00 & 2 & 1 & 2 & 2 & 2 & 5 & 1 & 1 & 2 & 2 & 6 & 5 & 1 & 2 & 1 & 3 & 2 & 1 & 1 & 1 & 2 & 3 & 3 & 3 & 2 & 3 & 1\end{array}$ $\begin{array}{lllllllllllllllllllllllllllllll}4.496 \mathrm{E}+00 & 2 & 1 & 2 & 2 & 2 & 5 & 1 & 1 & 2 & 2 & 6 & 5 & 1 & 2 & 1 & 3 & 2 & 1 & 1 & 1 & 2 & 3 & 3 & 3 & 2 & 3 & 1 \\ 4.570 \mathrm{E}+00 & 2 & 1 & 2 & 2 & 2 & 6 & 7 & 2 & 2 & 2 & 3 & 4 & 2 & 2 & 2 & 4 & 2 & 1 & 2 & 2 & 1 & 1 & 3 & 4 & 2 & 3 & 1\end{array}$ $\begin{array}{lllllllllllllllllllllllllllll}4.570 \mathrm{E}+00 & 2 & 1 & 2 & 2 & 2 & 6 & 7 & 2 & 2 & 2 & 3 & 4 & 2 & 2 & 2 & 4 & 2 & 1 & 2 & 2 & 1 & 1 & 3 & 4 & 2 & 3 & 1 \\ 4.737 \mathrm{E}+00 & 2 & 2 & 2 & 1 & 1 & 5 & 5 & 3 & 1 & 4 & 1 & 2 & 2 & 2 & 3 & 3 & 3 & 1 & 2 & 3 & 1 & 2 & 4 & 3 & 2 & 3 & 1\end{array}$ $\begin{array}{lllllllllllllllllllllllllllllllllll}4.890 E+00 & 2 & 1 & 3 & 2 & 2 & 6 & 5 & 1 & 3 & 2 & 4 & 3 & 5 & 1 & 2 & 2 & 4 & 1 & 2 & 1 & 3 & 1 & 4 & 2 & 2 & 2 & 1 \\ 4.914 E+00 & 2 & 1 & 2 & 2 & 3 & 2 & 9 & 2 & 1 & 2 & 4 & 4 & 3 & 1 & 1 & 3 & 4 & 1 & 1 & 3 & 1 & 1 & 2 & 3 & 3 & 2 & 1\end{array}$

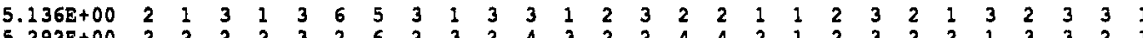

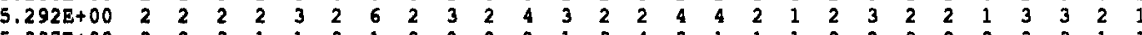

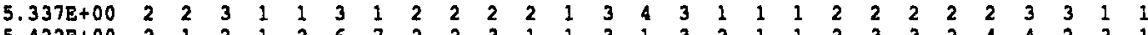
$\begin{array}{lllllllllllllllllllllllllllll}5.422 \mathrm{E}+00 & 2 & 1 & 2 & 1 & 2 & 6 & 7 & 2 & 2 & 3 & 1 & 1 & 3 & 1 & 3 & 2 & 1 & 1 & 2 & 3 & 3 & 2 & 4 & 4 & 2 & 3 & 1 \\ 5.463 \mathrm{E}+00 & 2 & 2 & 2 & 1 & 3 & 6 & 5 & 2 & 3 & 2 & 5 & 3 & 5 & 3 & 2 & 3 & 3 & 1 & 2 & 2 & 2 & 3 & 3 & 4 & 1 & 1 & 1\end{array}$

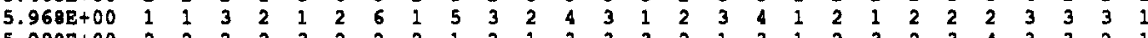

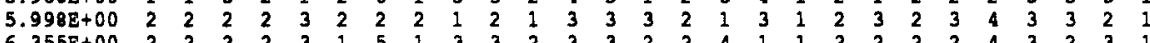

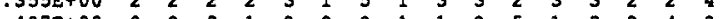

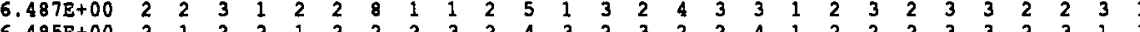
$\begin{array}{lllllllllllllllllllllllllllll}6.485 \mathrm{E}+00 & 2 & 1 & 2 & 2 & 1 & 2 & 2 & 2 & 3 & 2 & 4 & 3 & 2 & 3 & 2 & 2 & 4 & 1 & 2 & 2 & 2 & 3 & 3 & 2 & 3 & 1 & 1 \\ 6.492 \mathrm{E}+00 & 2 & 1 & 3 & 2 & 2 & 2 & 5 & 2 & 2 & 4 & 1 & 5 & 2 & 3 & 4 & 3 & 3 & 1 & 2 & 2 & 2 & 1 & 2 & 2 & 3 & 2 & 1\end{array}$ $\begin{array}{llllllllllllllllllllllllllllll}6.492 \mathrm{E}+00 & 2 & 1 & 3 & 2 & 2 & 2 & 5 & 2 & 2 & 4 & 1 & 5 & 2 & 3 & 4 & 3 & 3 & 1 & 2 & 2 & 2 & 1 & 2 & 2 & 3 & 2 & 1 \\ 6.553 \mathrm{E}+00 & 2 & 2 & 2 & 1 & 3 & 6 & 5 & 2 & 3 & 2 & 5 & 3 & 5 & 3 & 2 & 3 & 3 & 1 & 2 & 2 & 2 & 3 & 3 & 4 & 1 & 1 & 1\end{array}$

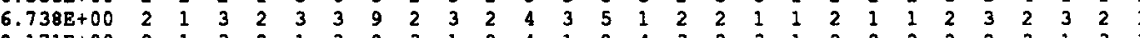
$\begin{array}{llllllllllllllllllllllllllllllll}8.171 E+00 & 2 & 1 & 3 & 2 & 1 & 3 & 8 & 3 & 1 & 2 & 4 & 1 & 2 & 4 & 3 & 2 & 3 & 1 & 2 & 2 & 2 & 2 & 2 & 3 & 1 & 3 & 1 \\ 8.353 E+00 & 2 & 2 & 3 & 2 & 2 & 3 & 4 & 1 & 3 & 4 & 1 & 2 & 2 & 2 & 3 & 2 & 2 & 1 & 2 & 2 & 1 & 3 & 1 & 4 & 3 & 1 & 1\end{array}$ $\begin{array}{lllllllllllllllllllllllllllll}8.353 \mathrm{E}+00 & 2 & 2 & 3 & 2 & 2 & 3 & 4 & 1 & 3 & 4 & 1 & 2 & 2 & 2 & 3 & 2 & 2 & 1 & 2 & 2 & 1 & 3 & 1 & 4 & 3 & 1 & 1 \\ 8.451 E+00 & 2 & 2 & 3 & 2 & 1 & 5 & 3 & 2 & 1 & 2 & 5 & 5 & 2 & 2 & 4 & 3 & 4 & 1 & 1 & 2 & 2 & 2 & 4 & 3 & 3 & 4 & 1\end{array}$

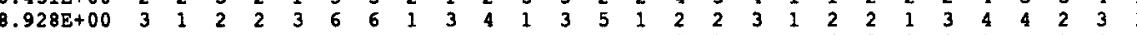

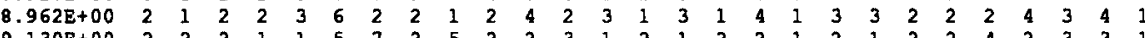

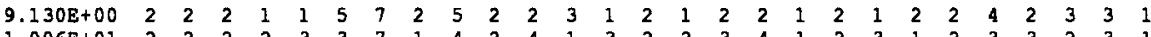

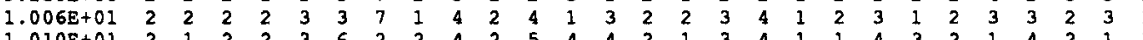

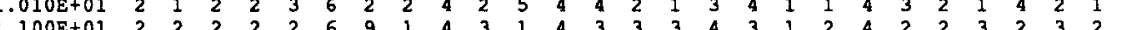
$\begin{array}{llllllllllllllllllllllllllllll}1.100 \mathrm{E}+01 & 2 & 2 & 2 & 2 & 2 & 6 & 9 & 1 & 4 & 3 & 1 & 4 & 3 & 3 & 3 & 4 & 3 & 1 & 2 & 4 & 2 & 2 & 3 & 2 & 3 & 2 & 1 \\ 1.104 \mathrm{E}+01 & 2 & 1 & 2 & 2 & 3 & 2 & 1 & 3 & 4 & 3 & 3 & 1 & 1 & 1 & 4 & 3 & 3 & 1 & 1 & 3 & 2 & 3 & 3 & 3 & 3 & 3 & 1\end{array}$

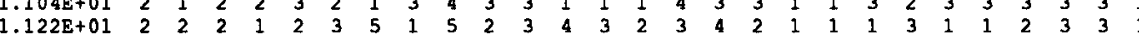
$\begin{array}{lllllllllllllllllllllllllllllll}1.122 \mathrm{E}+01 & 2 & 2 & 2 & 1 & 2 & 3 & 5 & 1 & 5 & 2 & 3 & 4 & 3 & 2 & 3 & 4 & 2 & 1 & 1 & 1 & 3 & 1 & 1 & 2 & 3 & 3 & 1 \\ 1.154 \mathrm{E}+01 & 1 & 2 & 2 & 2 & 3 & 2 & 1 & 3 & 4 & 2 & 3 & 4 & 2 & 2 & 2 & 1 & 2 & 1 & 3 & 4 & 3 & 2 & 4 & 2 & 2 & 2 & 1\end{array}$ $\begin{array}{llllllllllllllllllllllllllllllllll}1.210 E+01 & 2 & 1 & 2 & 2 & 3 & 3 & 4 & 2 & 3 & 2 & 5 & 2 & 3 & 3 & 2 & 3 & 3 & 1 & 2 & 2 & 3 & 3 & 4 & 4 & 3 & 4 & 1\end{array}$ $\begin{array}{lllllllllllllllllllllllllllll}1.235 E+01 & 2 & 2 & 2 & 1 & 1 & 6 & 1 & 2 & 3 & 2 & 3 & 3 & 3 & 2 & 3 & 2 & 2 & 1 & 2 & 3 & 2 & 1 & 3 & 4 & 3 & 3 & 1\end{array}$ $\begin{array}{llllllllllllllllllllllllllllllll}1.274 \mathrm{E}+01 & 2 & 2 & 2 & 2 & 2 & 3 & 8 & 2 & 6 & 3 & 2 & 2 & 5 & 2 & 3 & 2 & 3 & 1 & 3 & 2 & 3 & 1 & 3 & 4 & 3 & 2 & 1 \\ 1.612 \mathrm{E}+01 & 2 & 1 & 3 & 1 & 3 & 6 & 8 & 4 & 1 & 3 & 2 & 3 & 2 & 2 & 1 & 2 & 2 & 1 & 1 & 4 & 3 & 3 & 4 & 2 & 2 & 4 & 1\end{array}$

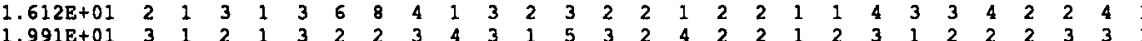
$\begin{array}{lllllllllllllllllllllllllllll}2.020 \mathrm{E}+01 & 2 & 1 & 3 & 1 & 3 & 6 & 6 & 4 & 1 & 3 & 1 & 2 & 2 & 2 & 4 & 2 & 2 & 1 & 2 & 3 & 1 & 2 & 2 & 2 & 3 & 3 & 1 \\ 3 & 3 & 3 & 1 & 3 & 2 & 2 & 3 & 3 & 2 & 2 & 2 & 1\end{array}$

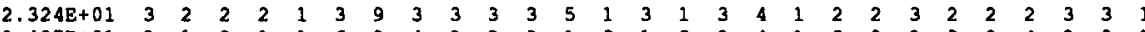

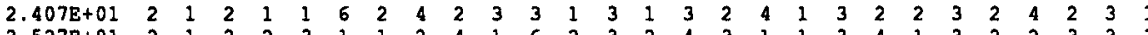
$\begin{array}{llllllllllllllllllllllllllllllllll}2 & 1 & 2 & 2 & 3 & 1 & 1 & 2 & 4 & 1 & 6 & 2 & 3 & 2 & 4 & 3 & 1 & 1 & 3 & 4 & 1 & 3 & 2 & 2 & 3 & 3 & 1\end{array}$ $\begin{array}{lllllllllllllllllllllllllllll}2.549 \mathrm{E}+01 & 2 & 2 & 2 & 1 & 2 & 3 & 9 & 2 & 4 & 1 & 5 & 4 & 2 & 1 & 3 & 2 & 4 & 1 & 2 & 2 & 3 & 1 & 2 & 2 & 2 & 2 & 1 \\ 2.616 \mathrm{E}+01 & 2 & 2 & 2 & 3 & 2 & 6 & 9 & 1 & 4 & 1 & 6 & 1 & 2 & 2 & 3 & 1 & 3 & 1 & 1 & 2 & 2 & 2 & 2 & 3 & 3 & 2 & 1\end{array}$ $\begin{array}{llllllllllllllllllllllllllll}2.616 \mathrm{E}+01 & 2 & 2 & 2 & 3 & 2 & 6 & 9 & 1 & 4 & 1 & 6 & 1 & 2 & 2 & 3 & 1 & 3 & 1 & 1 & 2 & 2 & 2 & 2 & 3 & 3 & 2 & 1 \\ 2.629 \mathrm{E}+01 & 2 & 1 & 2 & 2 & 1 & 1 & 3 & 4 & 2 & 2 & 3 & 5 & 3 & 4 & 2 & 2 & 4 & 1 & 1 & 3 & 1 & 2 & 4 & 2 & 4 & 3 & 3\end{array}$ $\begin{array}{llllllllllllllllllllllllllll}2.629 \mathrm{E}+01 & 2 & 1 & 2 & 2 & 1 & 1 & 3 & 4 & 2 & 2 & 3 & 5 & 3 & 4 & 2 & 2 & 4 & 1 & 1 & 3 & 1 & 2 & 4 & 2 & 4 & 3 & 1 \\ 3.338 \mathrm{E}+01 & 2 & 2 & 3 & 2 & 2 & 3 & 7 & 3 & 4 & 3 & 3 & 2 & 1 & 2 & 1 & 2 & 2 & 1 & 3 & 3 & 3 & 2 & 2 & 2 & 3 & 3 & 1\end{array}$ $\begin{array}{llllllllllllllllllllllllllll}3.383 \mathrm{E}+01 & 2 & 2 & 2 & 3 & 3 & 6 & 7 & 2 & 3 & 1 & 6 & 4 & 3 & 2 & 1 & 4 & 2 & 1 & 2 & 4 & 2 & 1 & 3 & 3 & 3 & 1 & 1\end{array}$ $\begin{array}{llllllllllllllllllllllllllllll}3.561 E+01 & 2 & 1 & 3 & 2 & 1 & 6 & 3 & 5 & 1 & 3 & 4 & 2 & 3 & 2 & 1 & 3 & 2 & 1 & 2 & 4 & 3 & 3 & 3 & 3 & 3 & 1 & 1 \\ 3.575 E+01 & 2 & 2 & 2 & 2 & 3 & 1 & 7 & 2 & 4 & 1 & 6 & 4 & 3 & 2 & 4 & 2 & 3 & 1 & 1 & 2 & 3 & 3 & 4 & 3 & 3 & 3 & 1\end{array}$ $\begin{array}{lllllllllllllllllllllllllllllllll}3.575 \mathrm{E}+01 & 2 & 2 & 2 & 2 & 3 & 1 & 7 & 2 & 4 & 1 & 6 & 4 & 3 & 2 & 4 & 2 & 3 & 1 & 1 & 2 & 3 & 3 & 4 & 3 & 3 & 3 & 1 \\ 3.779 E+01 & 2 & 1 & 1 & 3 & 1 & 6 & 3 & 3 & 3 & 2 & 4 & 5 & 3 & 3 & 2 & 2 & 3 & 1 & 1 & 2 & 1 & 2 & 3 & 3 & 1 & 2 & 1\end{array}$ $\begin{array}{llllllllllllllllllllllllllllll}3.987 \mathrm{E}+01 & 2 & 2 & 3 & 2 & 3 & 4 & 4 & 2 & 5 & 2 & 3 & 2 & 3 & 1 & 2 & 2 & 4 & 1 & 3 & 4 & 1 & 1 & 4 & 2 & 3 & 3 & 1\end{array}$ $\begin{array}{lllllllllllllllllllllllllllll}4.052 \mathrm{E}+01 & 2 & 1 & 2 & 2 & 2 & 6 & 4 & 3 & 3 & 2 & 5 & 1 & 1 & 1 & 1 & 2 & 3 & 1 & 3 & 1 & 3 & 3 & 1 & 2 & 3 & 3 & 1\end{array}$ $\begin{array}{llllllllllllllllllllllllllll}4.126 \mathrm{E}+01 & 2 & 2 & 3 & 3 & 2 & 2 & 9 & 3 & 1 & 2 & 3 & 1 & 3 & 2 & 1 & 3 & 2 & 1 & 3 & 3 & 1 & 3 & 4 & 3 & 3 & 2 & 1\end{array}$ $\begin{array}{lllllllllllllllllllllllllllllll}4.134 E+01 & 2 & 2 & 3 & 3 & 1 & 6 & 4 & 2 & 5 & 2 & 5 & 3 & 2 & 1 & 4 & 3 & 3 & 1 & 3 & 2 & 2 & 1 & 2 & 4 & 3 & 2 & 1\end{array}$ $\begin{array}{llllllllllllllllllllllllllllll}4.420 \mathrm{E}+01 & 2 & 2 & 2 & 3 & 2 & 5 & 3 & 4 & 2 & 2 & 4 & 4 & 3 & 1 & 1 & 3 & 3 & 1 & 2 & 3 & 3 & 1 & 3 & 3 & 1 & 2 & 1 \\ 4.554 \varepsilon+01 & 2 & 2 & 3 & 1 & 1 & 6 & 8 & 3 & 4 & 3 & 3 & 2 & 3 & 2 & 3 & 3 & 2 & 1 & 1 & 2 & 2 & 3 & 4 & 3 & 2 & 1 & 1\end{array}$ $\begin{array}{llllllllllllllllllllllllllll}4.554 E+01 & 2 & 2 & 3 & 1 & 1 & 6 & 8 & 3 & 4 & 3 & 3 & 2 & 3 & 2 & 3 & 3 & 2 & 1 & 1 & 2 & 2 & 3 & 4 & 3 & 2 & 1 & 1 \\ 4.689 \mathrm{E}+01 & 2 & 1 & 2 & 3 & 2 & 1 & 7 & 2 & 5 & 1 & 6 & 1 & 3 & 3 & 2 & 1 & 2 & 1 & 2 & 2 & 2 & 3 & 3 & 2 & 2 & 3 & 1\end{array}$ $\begin{array}{lllllllllllllllllllllllllllll}5.137 E+01 & 2 & 2 & 2 & 2 & 3 & 4 & 2 & 3 & 3 & 2 & 3 & 2 & 4 & 2 & 3 & 3 & 3 & 1 & 2 & 3 & 2 & 1 & 3 & 2 & 3 & 2 & 1\end{array}$

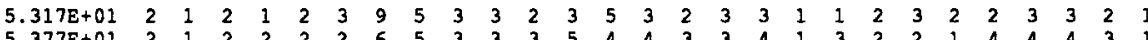

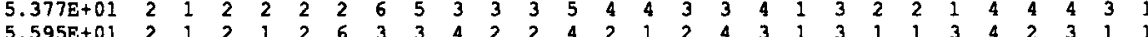

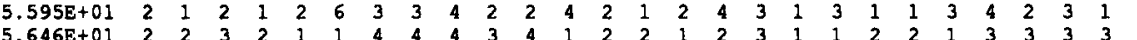

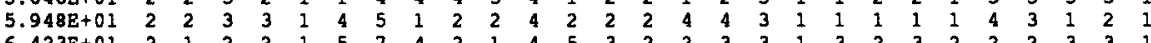

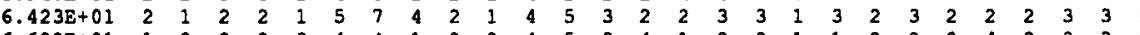

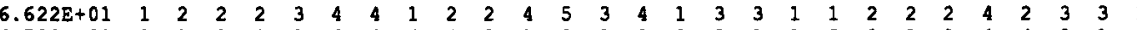

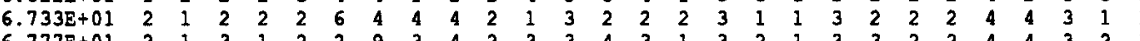
$\begin{array}{llllllllllllllllllllllllllll}6.777 \mathrm{E}+01 & 2 & 1 & 3 & 1 & 2 & 2 & 9 & 3 & 4 & 2 & 3 & 3 & 4 & 3 & 1 & 3 & 2 & 1 & 3 & 3 & 2 & 2 & 4 & 4 & 3 & 2 & 1 \\ 6.910 \mathrm{E}+01 & 2 & 2 & 2 & 1 & 3 & 2 & 5 & 5 & 3 & 2 & 4 & 2 & 2 & 2 & 2 & 1 & 3 & 1 & 2 & 3 & 2 & 3 & 3 & 4 & 3 & 2 & 1\end{array}$ $\begin{array}{llllllllllllllllllllllllllll}6.910 \mathrm{E}+01 & 2 & 2 & 2 & 1 & 3 & 2 & 5 & 5 & 3 & 2 & 4 & 2 & 2 & 2 & 2 & 1 & 3 & 1 & 2 & 3 & 2 & 3 & 3 & 4 & 3 & 2 & 1 \\ 7.373 \mathrm{E}+01 & 2 & 2 & 2 & 1 & 2 & 5 & 4 & 5 & 5 & 4 & 1 & 1 & 3 & 1 & 1 & 2 & 4 & 1 & 3 & 2 & 3 & 3 & 3 & 2 & 3 & 2 & 1\end{array}$ $\begin{array}{lllllllllllllllllllllllllllllll}8.559 \mathrm{~B}+01 & 1 & 1 & 3 & 1 & 3 & 3 & 6 & 5 & 3 & 3 & 3 & 3 & 4 & 3 & 2 & 2 & 3 & 1 & 1 & 3 & 1 & 2 & 2 & 3 & 2 & 3 & 1\end{array}$

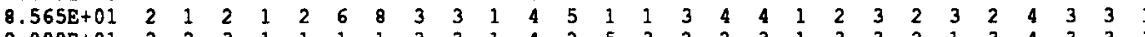
$\begin{array}{lllllllllllllllllllllllllllll}9.000 \mathrm{E}+01 & 2 & 2 & 3 & 1 & 1 & 1 & 1 & 3 & 3 & 1 & 4 & 2 & 5 & 3 & 2 & 2 & 3 & 1 & 3 & 3 & 2 & 1 & 3 & 4 & 3 & 3 & 1 \\ 9.693 \mathrm{E}+01 & 2 & 1 & 2 & 1 & 3 & 6 & 9 & 3 & 5 & 1 & 5 & 3 & 1 & 2 & 1 & 2 & 3 & 1 & 2 & 1 & 1 & 2 & 1 & 3 & 3 & 4 & 1\end{array}$ 
NUREG/CR-4551, VOL. 1: DRAFT REPORT FOR COMMENT (SEPTEMBER, 1986)

$\begin{array}{llllllllllllllllllllllllllll}1.052 \mathrm{E}+02 & 2 & 2 & 3 & 3 & 2 & 4 & 7 & 3 & 3 & 2 & 4 & 2 & 3 & 3 & 3 & 2 & 4 & 1 & 3 & 1 & 2 & 2 & 3 & 3 & 1 & 3 & 1 \\ 1.075 \mathrm{E}+02 & 2 & 1 & 2 & 2 & 3 & 6 & 5 & 4 & 6 & 2 & 6 & 1 & 3 & 2 & 2 & 3 & 2 & 1 & 2 & 1 & 2 & 3 & 3 & 4 & 3 & 2 & 1 \\ 1.135 \mathrm{E}+02 & 2 & 1 & 2 & 1 & 2 & 3 & 1 & 5 & 3 & 1 & 4 & 5 & 2 & 1 & 4 & 2 & 3 & 1 & 3 & 3 & 2 & 3 & 3 & 4 & 2 & 2 & 1 \\ 1.190 \mathrm{E}+02 & 2 & 1 & 2 & 1 & 3 & 6 & 8 & 5 & 3 & 1 & 6 & 4 & 2 & 1 & 2 & 1 & 1 & 1 & 2 & 3 & 1 & 3 & 2 & 2 & 2 & 3 & 1 \\ 1.191 \mathrm{E}+02 & 2 & 1 & 2 & 1 & 1 & 6 & 1 & 4 & 5 & 2 & 3 & 5 & 2 & 3 & 3 & 4 & 3 & 1 & 1 & 1 & 1 & 3 & 1 & 2 & 3 & 3 & 1 \\ 1.259 \mathrm{E}+02 & 2 & 2 & 2 & 2 & 1 & 4 & 3 & 3 & 3 & 1 & 6 & 1 & 4 & 2 & 2 & 3 & 2 & 1 & 2 & 1 & 3 & 3 & 3 & 2 & 2 & 3 & 1 \\ 1.284 \mathrm{~B}+02 & 2 & 2 & 2 & 1 & 3 & 3 & 8 & 5 & 2 & 2 & 4 & 2 & 5 & 2 & 3 & 2 & 3 & 1 & 2 & 4 & 3 & 3 & 3 & 3 & 3 & 4 & 1 \\ 1.284 E+02 & 2 & 1 & 3 & 2 & 1 & 3 & 4 & 4 & 3 & 2 & 4 & 1 & 3 & 2 & 1 & 3 & 2 & 1 & 2 & 3 & 2 & 3 & 1 & 3 & 2 & 4 & 1 \\ 1.312 \mathrm{E}+02 & 2 & 1 & 3 & 2 & 1 & 3 & 6 & 5 & 4 & 2 & 4 & 5 & 3 & 2 & 1 & 2 & 4 & 1 & 2 & 1 & 2 & 3 & 3 & 3 & 3 & 2 & 1 \\ 1.347 \mathrm{E}+02 & 1 & 2 & 3 & 1 & 1 & 6 & 1 & 4 & 2 & 1 & 6 & 4 & 1 & 2 & 1 & 3 & 4 & 1 & 2 & 4 & 1 & 2 & 3 & 2 & 3 & 4 & 1 \\ 1.383 E+02 & 2 & 2 & 2 & 2 & 3 & 2 & 4 & 4 & 6 & 1 & 6 & 2 & 3 & 1 & 3 & 3 & 4 & 1 & 1 & 2 & 2 & 2 & 3 & 3 & 3 & 3 & 1 \\ 1.765 E+02 & 2 & 2 & 2 & 3 & 1 & 3 & 3 & 5 & 2 & 2 & 3 & 1 & 3 & 3 & 2 & 4 & 3 & 1 & 3 & 1 & 3 & 3 & 3 & 2 & 2 & 2 & 1 \\ 2.336 \mathrm{E}+02 & 2 & 2 & 3 & 1 & 1 & 4 & 8 & 4 & 5 & 1 & 5 & 2 & 3 & 1 & 2 & 3 & 2 & 1 & 2 & 4 & 1 & 3 & 2 & 2 & 3 & 3 & 1 \\ 2.456 \mathrm{E}+02 & 2 & 2 & 3 & 2 & 1 & 2 & 3 & 3 & 6 & 2 & 4 & 5 & 5 & 4 & 4 & 2 & 4 & 1 & 2 & 4 & 3 & 2 & 2 & 3 & 3 & 1 & 1 \\ 2.537 \mathrm{E}+02 & 3 & 2 & 2 & 2 & 2 & 1 & 6 & 4 & 2 & 1 & 4 & 1 & 3 & 3 & 2 & 2 & 3 & 1 & 2 & 2 & 1 & 3 & 2 & 2 & 2 & 3 & 1\end{array}$


NUREG/CR-4551, VOL. 1: DRAFT REPORT FOR COMMENT (SEPTEMBER, 1986)

CONSEQUENCE MEASURE:

ROP. DOSE 50 MI PER YEAR

BASE CASE :

SURRY LALHC (MACCS)

FRACTIONAL CONTRIBUTION OF BACH SEQUENCE TO MEAN RISK

SEQUENCE

0.1449

SNNN

SYNN

ANNN

AYNN

AYNI

AYYB

TNNN

TYYBW

TYYBD

0.0001

0.4431
0.0007

0.0001

0.0087

0.0212

0.02126

0.1573

0.0593

0.0655

0.0002

1 CONSEOUENCE MEASURE:

POP. DOSE 50 MI PER YEAR

BASE CASE :

SURRY LALHC (MACCS)

FRACTIONAL CONTRIBUTION OF EACH BIN TO MEAN RISK BIN

BIN 1
BIN

BIN 2

BIN

BIN

BIN 5

$\begin{array}{ll}\text { BIN } & 6 \\ \text { BIN } 7\end{array}$

BIN 8

BIN 9

BIN 10

BIN 11

BIN 12

BIN 13

BIN 14

BIN 15

BIN 16

BIN 17

BIN 18

1 CONSEOUENCE MEASURE

CONTRIBUTION

0.0300

0.0009

0.0084

0.0041

0.0308

0.000

0.0015

0.0003

0.0117

0.0064

0.0583

0.0776

0.0001

0.0027

0.0043

0.5597

0.0738

0.0364

0.0929

POP DOSE 50 MI PER YEAR

BASE CASE :

SURRY LALHC (MACCS)

FRACTIONAL CONTRIBUTIONS OF EACH RELEASE TO MEAN RISK

RELEASE

SUR 1 G

SUR 2

SUR 3 G

SUR $4 \mathrm{G}$

SUR 5 G

SUR 6

SUR 8

SUR $9 \mathrm{G}$

SUR $10 \mathrm{G}$

SUR 12 G

SUR $14 \mathrm{G}$

SUR $15 \mathrm{G}$

SUR $16 G$

SUR 18

SUR $19 \mathrm{G}$

SUR $20 \mathrm{G}$

SUR $21 \mathrm{G}$

SUR 22

SUR $23 \mathrm{G}$

SUR 24

SUR $25 \mathrm{G}$

CONTRIBUTION

0.1162

0.1259

0.1027

0.1463

0.0844

0.0995

0.0590

0.0717

0.0021

0.0114

0.0565

0.0040

0.0353

0.0039

0.0181

0.0067

0.0218

0.0032

0.0127

0.0023

0.0058

0.0003

0.0032

0.0027

0.0043 


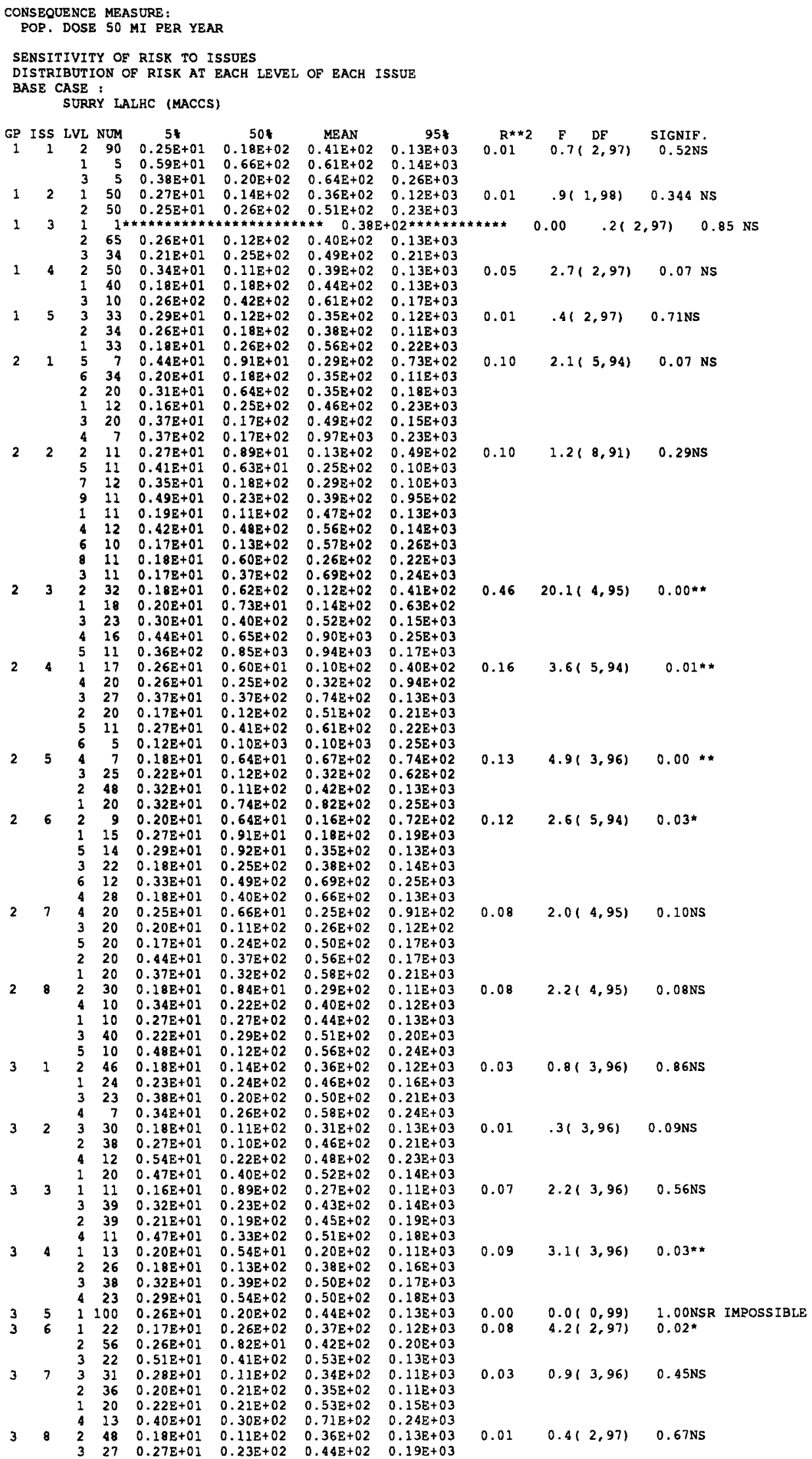


NUREG/CR-4551, VOL. 1: DRAFT REPORT FOR COMMENT (SEPTEMBER, 1986)

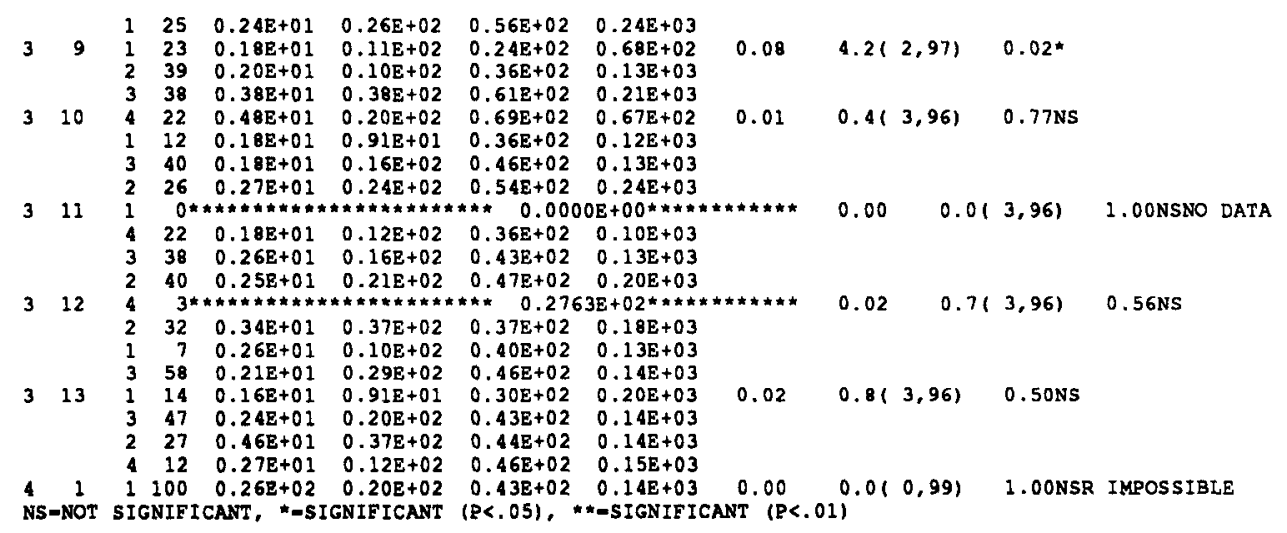


CONSEQUENCE MEASURE: POP, DOSE 50 MI PER YEAR

- ISSUE GROUR - 1 ISSUE - 1 TB SEAL LOCA

LEVEL 1 NO. $\angle M E D .-2$ NO. EXP.- 2

LEVEL 2 NO. LMED. - 45 so. EXP. - 45

NO. >-MED. - 3 NO. EXP.- 3

NO.>-MED. -45 NO. EXP. $=45$

NO.>-MED. -3 NO. EXP. 3

LEVEL 3 NO. $\angle M E D .-2$ NO. EXP.- 2

OVERALL CHI-SQ- $0.0 \mathrm{DF}=1$ SIG.- 1.000

ISSUE GROUP - 1 ISSUE - 2 USING GAS GEN.

LEVEL 1 NO. $\triangle M E D .-26$ NO. EXP. -25

NO.>-MED. -24 NO. EXP. $=25$

NO.>-MED. -27 NO. EXP. $=25$

LEVEL 2 NO. $\angle M E D .-23$ NO. EXP. -25

OVERALL CHI-SQ- 1.0 DF- 4 SIG.= 0.94

ISSUE GROUP - 1 ISSUE = 3 BETA-FACTOR

LEVEL 1 NO. $\angle$ MED.- 0 NO. EXP.- 0

NO. >-MED.- 1 NO. EXP.- 1

NO. >-MED.- 32 NO. EXP. -33

LEVEL 2 NO. <MED. -35 NO. EXP. -33

NO. >-MED. 18 NO. EXP. $=17$

LEVEL 3 NO. CMED. - 14 NO. EXP. = 16

918

ISSUE GROUP - 1 ISSUE - 4 V FREQ

LEVEL 1 NO. <MED. - 20 NO. EXP. - 20

NO. >-MED. -20 NO. EXP. -20

LEVEL 2 NO. $\angle M E D .-29$ NO. EXP. 25

NO.>-MED, $=21$ No. EXP. -25

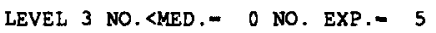

NO. >-MED. -10 NO. EXP. $=5$

OVERALI CHI-SO- 12.3 DF- 5 SIG.- 0.046

ISSUE GROUP = 1 ISSUE $=5$ F1F2 FAILURE

LEVEL 1 NO. $\angle M E D .=14$ NO. EXP.- 16

NO.>-MED. - 19 No. EXP.- 17

LEVEL 2 NO. $\angle M E D .-17$ NO. EXP.- 17

NO.>-MED. - 17 NO. EXP.- 17

LEVEL 3 NO. <MED. - 18 NO, EXP.- 16

NO. >-MED. = 15 NO. EXP.- 17

OVERALL CHI-5Q- 1.0 DF- 5 SIG.- 0.965

0

ISSUE GROUP - 2 ISSUE - 1 IND. RCS FAIL.

LEVEL 1 NO. $\angle M E D .-5$ NO. EXP.- 6

NO. >-MED. - 7 NO. EXP. - 6

LEVEL 2 NO. MED. - 13 NO. EXP.- 10

NO. >-MED. - 7 NO. EXP. -10

NO. >-MED. - 10 NO. EXP. -10

NO.>-MED. -7 NO. EXP. $=4$

NO. >-MED.- 3 NO. EXP.- 4

NO. >-MED. - 17 NO. EXP. 17

LEVEL 6 NO. $M$ MED - 17 NO. EXP. - 17

gag

OVERALL CHI-SO- 2.1 DF- 7 SIG. 0.998

0

ISSUE GROUP - 2 ISSUE - 2 MODE OF VB.

LEVEL 1 NO. MMED.- 6 NO. EXP.- 5

LEVEL 2 NO. $\angle M E D .-8$ NO. EXP.- 5

LEVEL 3 NO. $\angle M E D .-3$ NO. EXP.- 5

LEVEL 4 NO. $\angle$ MED.- 3 NO. EXP.- 6

LEVEL 5 NO. CMED.- 8 NO. EXP.- 5

LEVEL 6 NO. $\angle M E D .-5$ NO. EXP. -5

LEVEL 7 NO. \MED. - 6 NO. EXP. - 6
NO. >-MED. = 5 NO. EXP.- 6 NO.>-MED. 3 NO. EXP. $=6$ NO. >-MED. -8 NO. EXP. -6 NO.>-MED. - 9 NO. EXP.= 6 NO.>-MED. -3 NO. EXP. $=6$ NO. >-MED. - 5 NO. EXP. - 5 NO.>-MED. - 6 NO. EXF.- 6
CHI-SO. TEST CANNOT BE RUN: EXP. NO. < 5 CHI-SO.- 0.0 DF- 1 SIG. 1.000 CHI-SQ. TEST CANNOT BE RUN; EXP. NO. $<5$

CHI-SQ. 0.2 DF- 1 SIG. $=0.777$

CHI-SQ. $\quad 0.3$ DF- 1 SIG. $=0.572$

CHI-SQ. TEST CANNOT BE RUN: EXP. NO. $<5$ CHI-SQ. 0.1 DF- $\{$ SIG. $=0.804$ CHI-SQ.- 0.1 DE- 1 SIG. $=0.732$

CHI-SQ. $\quad 0.0$ DF- 1 SIG. -1.000 CHI-SQ. $\quad 1.3$ DF- $\downarrow S I G=0.258$ CHI-SQ. 10.0 DF- 1 SIG. 0.002

CHI-SQ.- 0.5 DF= 1 SIG.- 0.486 CHI-SQ.- 0.0 DF- 1 SIG. 1.000 CHI-SQ.- 0.5 DF- 1 SIG. $=0.486$

CHI-SQ. 0.3 DF- 1 SIG.- .564 CHI-SQ.- 1.8 DF- 1 SIG. 0.180 CHI-SQ. 0.0 DF- 1 SIG.- 1.000 CHI-SQ. 0.0 DE- 1 SIG. $=1.000$ CHI-SQ. $\quad 0.0$ DF- 1 SIG. 1.000 CHI-SO.- 0.0 DF- 1 SIG.- 1.000

$\mathrm{CHI}-\mathrm{SQ}=.4 \mathrm{DF}-1 \mathrm{SIG} .=0.545$ CHI-SQ. $\quad 3.3$ DF- 1 SIG.= 0.069 CHI-SQ.- 1.5 DF- 1 SIG.= 0.226 CHI-SQ. $\quad 3.0$ DF- 1 SIG. $=0.083$ CHI-SQ. $\quad 3.3$ DF- I SIG. 0.069 CHI-SQ. - 0.0 DF- 1 SIG.- 1.000 CHI-SQ. $=0.0$ DE- 1 SIG. 1.000 


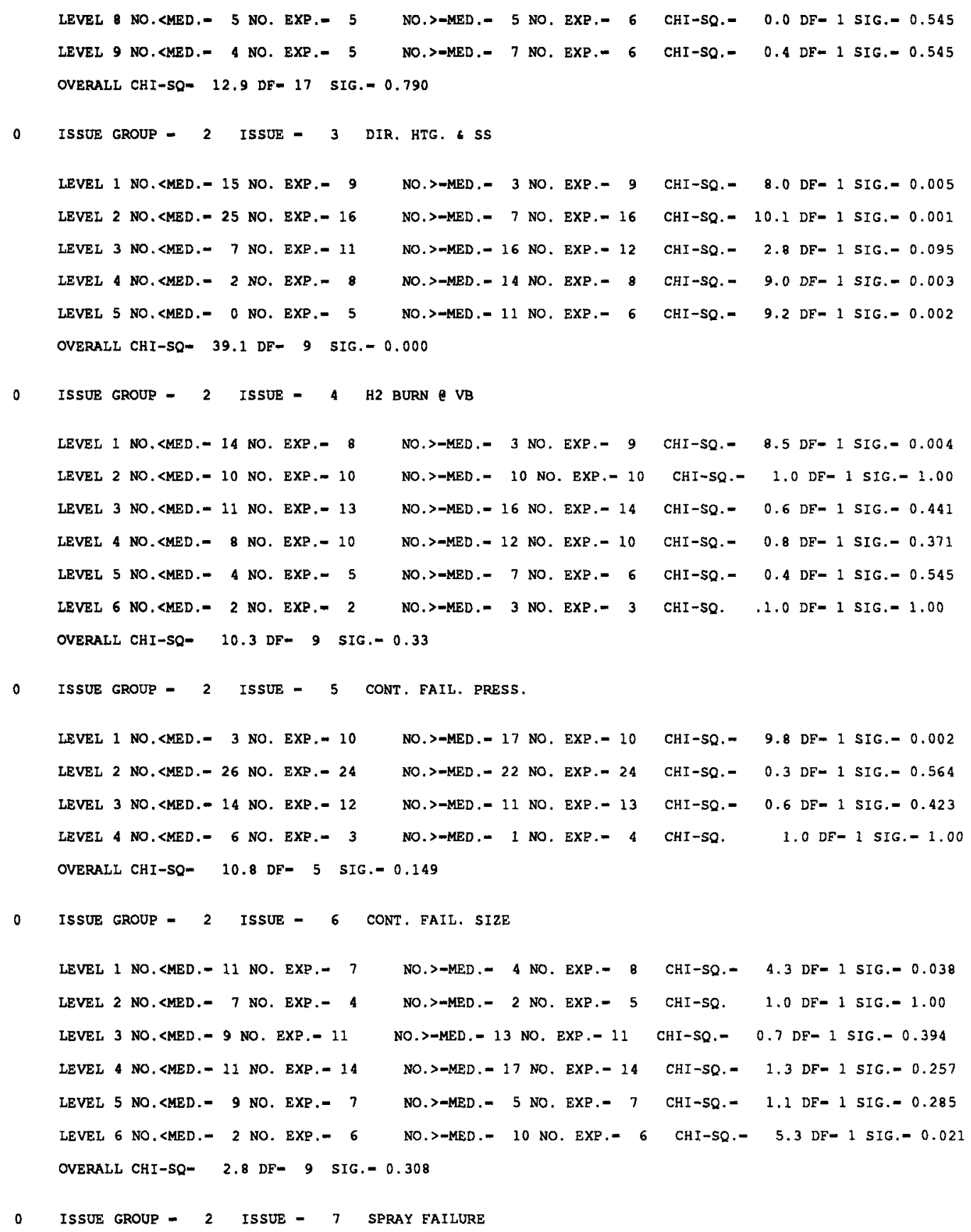

CHI-SQ. 0.8 DF- 1 SIG. -0.371 CHI-SQ. 3.3 DF- I SIG. 0.074 CHI-SQ.- 3.2 DF- 1 SIG.- 0.074 CHI-SQ. 1.8 DF- 1 SIG.- 0.180 CHI-SQ. $\quad 0.8$ DF= 1 SIG. 0.371

$\mathrm{CHI}-\mathrm{SQ}=0.4 \mathrm{DF}-1 \mathrm{SIG}=0.527$ CHI-SQ. $\quad 1.2$ DF= 1 SIG. 0.273 
LEVEL 3 NO. \MED. = 16 NO. EXP. - 20 LEVEL 4 NO. <MED. - 5 NO. EXP. - 5 LEVEL 5 NO. $\angle M E D .-6$ NO. EXP.- 5 OVERALL CHI-SO- 3.6 DF- 9 SIG.- 0.936

LEVEL 1 NO. $\angle M E D$ - 10 NO. EXP.- 10 LEVEL 2 NO. $\angle M E D=17$ NO. EXP.- 18 LEVEL 3 NO. <MED, - 17 NO. EXP. - 15 OVERALL CHI-SQ- 0.9 DF- 7 SIG.- 0.996

LEVEL 1 NO. <MED. - 11 NO, EXP - 12 LEVEL 2 NO. $\angle$ MED. -24 NO. EXP. $=23$ LEVEL 3 NO. <MED. - 11 No. EXP.- 11 LEVEL 4 NO. $\angle$ MED.- 3 NO. EXP. = 3 OVERALL CHI-SQ- $\quad 0.3$ DF- 5 SIG.- 1.000

LEVEL 1 NO. $\angle$ MED.- 5 NO. EXP.- 10

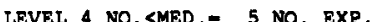
OVERALI CHI-SQ- 7.0 DF- 7 SIG. $=0.434$

LEVEL 1 NO. CMED.- 7 NO. EXP.- 5

LEVEL 2 NO. CMED . - 19 NO. EXP. - 19 LEVEL 3 NO. $\angle M E D .-18$ NO. EXP. - 19 OVERALL CHI-SQ- $\quad 1.6 \mathrm{DF}-7$ SIG. 0.980

ISSUE GROUR - 3 ISSUE - $4 \mathrm{~V} / \mathrm{DF}$

LEVEL 1 NO. <MED.- 10 NO. EXP.- 6 LEVEL 2 NO. $\angle M E D .-14$ NO. EXP.- 13 LEVEL 3 No. ८MED. - 15 No. EXP.- 19 LEVEL 4 NO. <MED. - 10 NO. EXP. - 11 OVERALL CHI-SQ- $7.0 \mathrm{DF}-7$ SIG. $=0.433$

ISSUE GROUP - 3 ISSUE - $\begin{array}{llll}6 & \text { CCI REL }\end{array}$

LEVEL 1 NO. <MED.- 10 NO. EXP. - 11 LEVEL 2 NO. $M$ MED $=35$ NO. EXP. -28 LEVEL 3 NO. $\angle M E D .=4$ NO. EXP.- 11 OVERALL CHI-SQ- $12.6 \mathrm{DF}=5$ SIG. $=0.028$

ISSUE GROUP - 3 ISSUE - $\begin{array}{llll} & 7 & \text { FCONC }\end{array}$
NO. >-MED.- 22 NO. EXP.- 20 NO.>-MED. -5 No. $\operatorname{EXP} .-5$ NO.>-MED. -4 NO. EXP.- 5 CHI-SQ.- 1.6 DF- 1 SIG.- 0.206 CHI-SQ.- 0.0 DF- 1 SIG.- 1.000 CHI-SQ.- 0.4 DF- 1 SIG. -0.527 LEVEL 4 NO. ২MED.- 5 NO. EXP.- 6

ISSUE GROUP - 3 ISSUE - 2 CSI DECOMP

NO. >-MED. - 15 NO. EXP.- 10 NO. >-MED. - 17 NO. EXP.- 19 NO. >-MED. - 12 NO. EXP.- 15 NO.>-MED. 7 NO. EXP.- 6

ISSUE GROUP - 3 ISSUE - 3 IN VESS RETENTION

NO. >-MED. -4 NO. EXP.- 6

NO. >-MED. -20 NO. EXP. $=20$ NO. >-MED. $=21$ NO. EXP. $=20$ LEVEL 4 NO. CMED.- 5 NO. EXP.- 5 NO.>-MED.- 6 NO. EXP.- 6 NO. >-MED.- 3 NO. EXP.- 7 NO. >-MED. = 12 NO. EXP. -13 NO. >-MED. -23 NO. EXP. -19 NO. >-MED. -13 NO. EXP. -12

NO.>-MED.- 12 NO. EXP.- 11 NO.>-MED. - 21 NO. EXP. -28 NO. >-MED. -18 NO. EXP. $=11$ ISSUE GROUP - 3 ISSUE - 8 SPRAY DF
NO. >-MED. - 10 NO. EXP. = 10 NO.>-MED.- 19 NO. EXP.- 18 NO.>-MED. -14 NO. EXP. -16 NO.>-MED. - 8 NO. EXP.- 7
NO. >-MED. $=13$ NO. EXP. -12

CHI-SQ.- $\quad 0.2$ DF- 1 SIG. $=683$

CHI-SQ.- 0.1 DF- 1 SIG.- 768

CHI-SQ.- 0.0 DF- 1 SIG. $=1.000$

CHI-SQ. $\quad 1.0$ DF- I SIG.- 1.00
CHI-SQ. $\quad 7.2$ DF- 1 SIG.- 0.007

CHI-SQ.- 0.4 DF= 1 SIG.- 0.516

CHI-SQ.- 2.1 DF- I SIG.= 0.144

CHI-SQ.- 0.3 DF- 1 SIG. -0.564
CHI-SQ. -1.5 DF- 1 SIG. $=0.226$ CHI-SQ.- 0.0 DF- 1 SIG.- 1.000 CHI-SQ.- $\quad 0.1$ DF- 1 SIG.= 0.749 CHI-SQ. $\quad 0.0$ DF- 1 SIG. -1.000

CHI-SO. $\quad 5.0$ DF- 1 SIG.- 0.026 CHI-SO.- .2 DF- 1 SIG. .695 CHI-SQ.- 1.7 DF- 1 SIG.- 0.194 CHI-SQ. 0.2 DF- 1 SIG. -0.676

CHI-5Q. .2 DF- 1 SIG $=0.67$ CHI-SQ.- $\quad 3.5$ DF- 1 SIG. $=0.061$ CHI-SQ.- 8.4 DE- 1 SIG.- 0.003

CHI-SQ.= 0.0 DF- 1 SIG.- 1.000 CHI-SO.- 0.1 DF= 1 SIG. $=0.739$ CHI-SQ.- 0.5 DF- I SIG. 0.472 CHI-SQ. 0.3 DEW 1 SIG. 0.578 


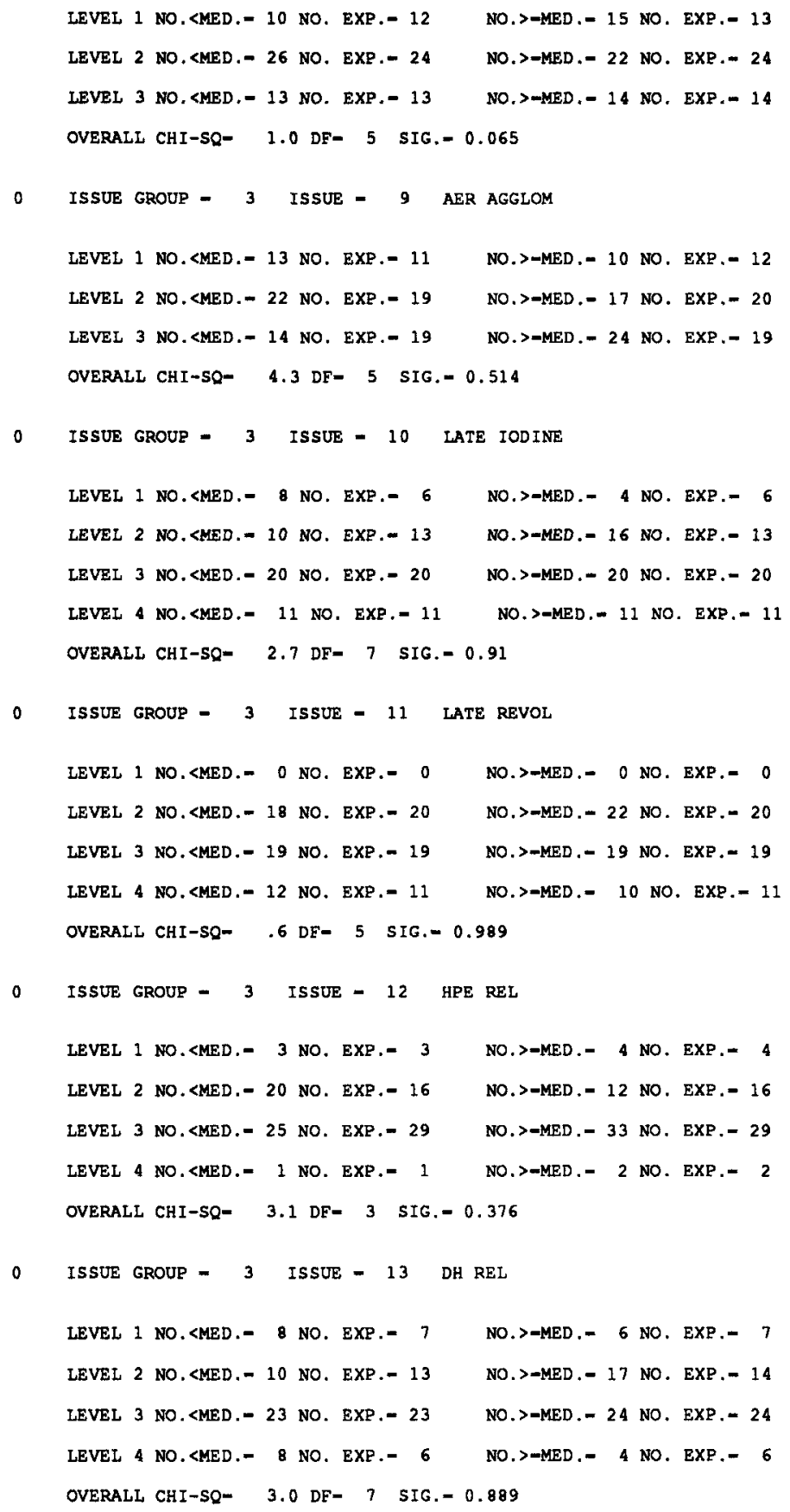




\begin{tabular}{|c|c|c|}
\hline 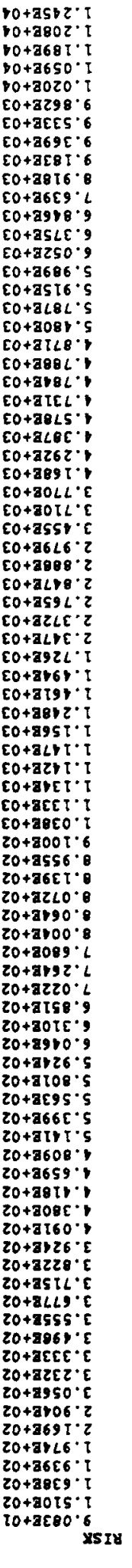 & 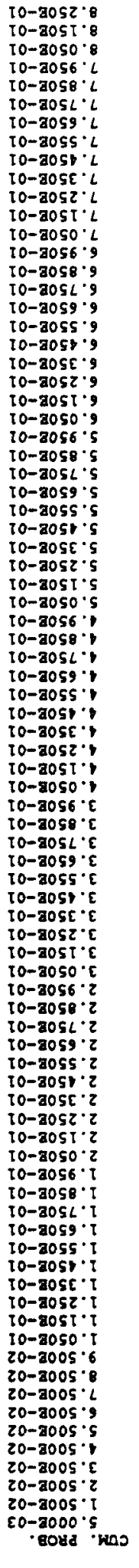 & 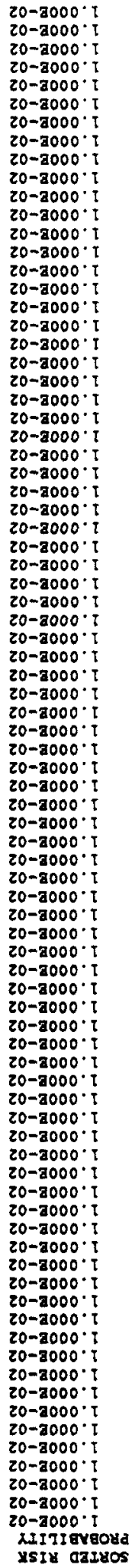 \\
\hline
\end{tabular}

(SoON) SHTVI dxens

: asYo asve

yrax yza SASOS arisaso ; gans Yras aonaodasnos 
NUREG/CR-4551, VOL. 1: DRAFT REPORT FOR COMMENT (SEPTEMBER, 1986)

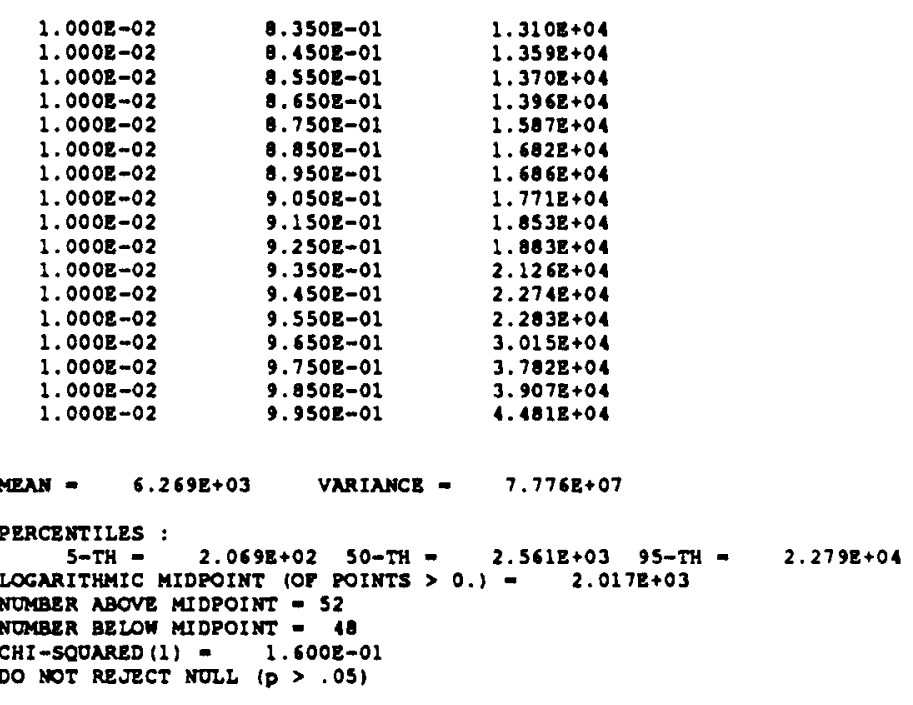


NUREG/CR-4551, VOL. 1: DRAFT REPORT FOR COMMENT (SEPTEMBER, 1986)

CONSEQOENCE MEASURE:

OFFSITE COSTS RER YEAR

BASE CASE

SORRY LALHC (MACCS)

ISSOE LEVELS FOR EACH SAMLIS MEPER, IN ORDER OP INCREASING RISR RISK ISSUE LEVELS. FOR ALL ISSUES

\begin{tabular}{|c|c|c|c|c|c|c|c|c|c|c|c|c|c|c|c|c|}
\hline & & & & & & & & & & & & & & & & \\
\hline $\begin{array}{l}.083 \mathrm{E}+01 \\
510 \mathrm{E}+02\end{array}$ & 2 & 2 & 3 & $\begin{array}{l}2 \\
1\end{array}$ & : & $\frac{1}{1}$ & 8 & $\frac{1}{2}$ & 2 & 2 & 6 & $\begin{array}{l}5 \\
5\end{array}$ & 2 & $\frac{1}{2}$ & 2 & 1 \\
\hline $.638 \mathrm{E}+\mathrm{C2}$ & 2 & 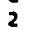 & 2 & 1 & 1 & & 1 & 2 & 3 & 2 & 3 & 3 & 3 & 2 & 3 & 2 \\
\hline $.939 \mathrm{E}+02$ & 2 & 1 & 2 & $i$ & 3 & & 2 & 2 & $i$ & $i$ & 5 & 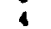 & $i$ & 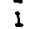 & 3 & 2 \\
\hline $.974 E+02$ & 2 & 1 & 2 & 2 & 3 & 6 & 1 & 1 & 5 & 3 & 2 & 1 & 2 & 2 & 2 & 1 \\
\hline $.1698+02$ & 2 & 1 & $?$ & & 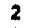 & & & 2 & & 了 & 7 & 4 & 3 & & 3 & 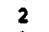 \\
\hline $.9048+02$ & 2 & 2 & 3 & 1 & 2 & 3 & 1 & 2 & 2 & 2 & 2 & 1 & 3 & 4 & 3 & 1 \\
\hline $.056 E+02$ & 2 & 1 & 2 & 2 & 3 & 2 & s & 2 & 3 & 1 & 5 & 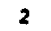 & 4 & 2 & 3 & 2 \\
\hline 02 & & 2 & , & & ? & & 6 & & I & 3 & 4 & 5 & 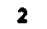 & 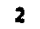 & 3 & 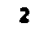 \\
\hline $3+02$ & 2 & 2 & 2 & 2 & 1 & 6 & 2 & 2 & $i$ & 2 & 4 & 3 & 4 & 2 & 3 & 2 \\
\hline 02 & & 2 & , & 2 & 2 & 2 & 7 & 2 & 1 & 2 & 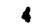 & 3 & 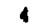 & 6 & 2 & 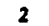 \\
\hline $.5552+02$ & 2 & 1 & 2 & 1 & 2 & 6 & 7 & 2 & 2 & 3 & 1 & 1 & 3 & 1 & 3 & 2 \\
\hline $.6778+02$ & 2 & 1 & 3 & 2 & 2 & 6 & 5 & 1 & 3 & 2 & 4 & j & 5 & $i$ & 2 & 2 \\
\hline $.715 z+02$ & & 1 & 2 & 2 & 3 & 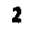 & 9 & 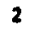 & 1 & 2 & 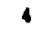 & 4 & 3 & 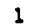 & 1 & 3 \\
\hline $.022 z+02$ & 2 & 1 & 3 & 1 & 1 & 2 & 1 & 1 & 1 & 1 & 5 & 4 & 4 & 3 & 2 & 3 \\
\hline $.924 z+02$ & 2 & 1 & 2 & 2 & 2 & 6 & 7 & 2 & 2 & 2 & 3 & 4 & 2 & 2 & 2 & 4 \\
\hline $.091 E+02$ & 2 & 2 & 3 & 2 & 2 & 6 & 7 & 2 & 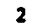 & 3 & 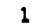 & 4 & 2 & 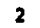 & 3 & 2 \\
\hline $.380 E+02$ & 2 & 2 & 2 & I & $\overline{1}$ & 5 & 5 & 3 & 1 & 4 & $i$ & 2 & 2 & 2 & 3 & 3 \\
\hline $1.418 B+02$ & 2 & 2 & 2 & 1 & 2 & 2 & 2 & 3 & 2 & 3 & 1 & $\mathbf{s}$ & 3 & 2 & 2 & 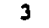 \\
\hline $4.6592+02$ & 2 & 1 & 2 & 2 & 2 & 5 & 1 & 1 & 2 & 2 & 6 & 5 & 1 & 2 & 1 & 3 \\
\hline $.809 \mathrm{E}+02$ & & & & 1 & & 1 & & 2 & & 4 & 1 & 3 & 3 & 3 & 2 & 3 \\
\hline $.1438+02$ & 2 & 1 & 3 & 1 & 3 & 6 & 5 & 3 & 1 & 3 & 3 & 1 & 2 & 3 & 2 & 2 \\
\hline $2+02$ & 2 & 2 & 2 & 2 & 3 & 2 & 2 & , & 2 & 2 & 1 & 3 & 7 & 3 & 2 & 1 \\
\hline $.563 z+02$ & 2 & 1 & $\mathbf{3}$ & 2 & 1 & 2 & 6 & 1 & 5 & 3 & 2 & 4 & 3 & 1 & 2 & 3 \\
\hline+02 & 2 & 2 & 2 & 1 & 3 & 1 & 3 & 1 & 1 & 2 & 3 & 5 & 2 & 3 & 2 & 2 \\
\hline .9 & 2 & $\frac{1}{2}$ & 3 & 2 & 3 & 3 & 3 & 2 & 3 & 2 & 4 & J & 5 & 1 & 2 & 2 \\
\hline $\begin{array}{l}+02 \\
+02\end{array}$ & 2 & $\begin{array}{l}2 \\
1\end{array}$ & & 2 & 2 & 2 & s & 2 & 2 & 4 & 1 & 3 & $\begin{array}{l}2 \\
2\end{array}$ & $\begin{array}{l}2 \\
3\end{array}$ & 4 & 3 \\
\hline $.8518+02$ & 2 & $i$ & 3 & 2 & 2 & 3 & 8 & 3 & 1 & 2 & 4 & $i$ & 2 & 4 & 3 & 2 \\
\hline 02 & 2 & 2 & 3 & 1 & 2 & 2 & 8 & 1 & 1 & 2 & 5 & 1 & 3 & 2 & 4 & 3 \\
\hline 1.26 & 2 & 1 & 2 & 2 & 1 & 2 & 2 & 2 & 3 & 2 & 4 & 3 & 2 & 3 & 2 & 2 \\
\hline$E+02$ & 2 & 2 & 3 & 2 & 2 & 3 & 1 & 1 & 3 & 4 & 1 & 2 & 2 & 2 & 3 & 2 \\
\hline $3.004 E+02$ & 2 & 1 & 2 & 2 & 3 & 6 & 2 & 2 & 1 & 2 & 1 & 2 & 3 & 1 & 3 & 1 \\
\hline$E+02$ & 2 & 2 & 2 & 1 & 1 & 5 & 7 & 2 & 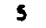 & 2 & 2 & 3 & 1 & 2 & 1 & 2 \\
\hline+02 & 2 & 2 & 2 & 2 & 3 & 1 & 5 & 1 & 3 & 3 & 2 & 3 & 3 & 2 & 2 & \\
\hline $1.1392+02$ & 2 & 2 & 2 & 2 & 3 & 6 & 5 & 2 & 3 & 2 & 5 & 3 & 5 & 3 & 2 & 3 \\
\hline $3.9 !$ & 2 & 1 & 7 & 1 & 2 & 3 & 9 & 3 & 2 & 2 & 5 & 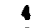 & 2 & 3 & 2 & 3 \\
\hline+02 & 2 & 2 & 3 & 2 & 1 & 3 & 3 & 2 & 1 & 2 & 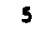 & $\mathbf{s}$ & 4 & 2 & 4 & \\
\hline $8+03$ & 2 & 2 & 2 & 2 & 3 & 3 & 7 & 1 & 4 & 2 & 4 & 1 & 3 & 2 & 2 & 3 \\
\hline 1.1 & 2 & 1 & , & 2 & 3 & 2 & 1 & 3 & 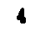 & 3 & 3 & 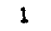 & I & 1 & 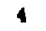 & \\
\hline $1.1348+03$ & 2 & 2 & 2 & 1 & 2 & 3 & 5 & 1 & 5 & 2 & 3 & 4 & 3 & 2 & 3 & 4 \\
\hline+03 & 3 & 1 & 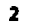 & 2 & 3 & 6 & 6 & 1 & 3 & 1 & 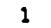 & 3 & 5 & 1 & 2 & 2 \\
\hline 1.1 & 1 & 2 & 2 & 2 & 3 & 2 & 1 & 3 & 4 & 3 & 3 & 4 & & 2 & 7 & \\
\hline $\mathrm{E}+03$ & 2 & 2 & 2 & 2 & 2 & 3 & 8 & 2 & 6 & 3 & 2 & 2 & $\mathbf{s}$ & 2 & 3 & 2 \\
\hline 1.24 & 2 & 1 & 2 & 2 & 3 & 6 & 2 & & 4 & 2 & 5 & 4 & 4 & 2 & 1 & 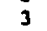 \\
\hline 1.46 & 2 & 2 & 2 & 2 & 2 & 6 & 9 & 1 & 4 & 3 & 1 & 4 & 3 & 3 & 3 & 4 \\
\hline+03 & 2 & 1 & 2 & 2 & 3 & 3 & 4 & & 3 & 2 & & 2 & 3 & 3 & 2 & 3 \\
\hline $1.726 \mathrm{E}+03$ & 2 & 2 & 2 & 1 & 1 & 6 & 2 & 1 & 6 & 3 & 3 & 4 & 5 & 1 & 3 & 3 \\
\hline .34 & 2 & 2 & 2 & $i$ & 2 & 3 & 9 & & 4 & 1 & 5 & 4 & 2 & 1 & 3 & 2 \\
\hline+03 & 2 & 2 & 2 & 3 & 2 & 6 & 9 & 1 & 4 & 1 & 6 & 1 & 2 & 2 & 3 & 1 \\
\hline .76 & 2 & 1 & 3 & 1 & 3 & 6 & 6 & 4 & 1 & 3 & 1 & 2 & 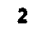 & 3 & 3 & 3 \\
\hline 2.8 & 3 & 1 & 2 & $i$ & 3 & 2 & 2 & & 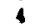 & 3 & 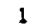 & 5 & 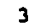 & 2 & 4 & 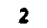 \\
\hline $2.888 \mathrm{E}+03$ & 2 & 1 & 3 & 1 & 3 & 6 & 8 & & 1 & 3 & 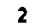 & 3 & 2 & 2 & 1 & 2 \\
\hline .979 & 3 & 2 & 2 & 2 & 1 & 3 & 9 & & 3 & 3 & & 3 & & 3 & & 3 \\
\hline $3.4358+03$ & 2 & 2 & 3 & , & 3 & 4 & 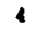 & & 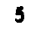 & 2 & 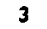 & 2 & 2 & , & & 2 \\
\hline 1.710 & 2 & 1 & 2 & 1 & 1 & 6 & 2 & 4 & 2 & 3 & 3 & 1 & 3 & 1 & 3 & 2 \\
\hline $3.770 \mathrm{E}+0$ & 2 & 1 & 2 & 2 & 3 & 1 & 1 & & 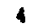 & 1 & 6 & 2 & 3 & 2 & 4 & 3 \\
\hline 4.16 & 2 & 1 & 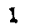 & 3 & 1 & 6 & 3 & & 3 & 2 & & 5 & 3 & 3 & 2 & 2 \\
\hline $.292 \mathrm{E}+03$ & 2 & 1 & 2 & 2 & 1 & 1 & 3 & 4 & 2 & 2 & 7 & 5 & 3 & 4 & 2 & 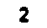 \\
\hline $1.3878+03$ & 2 & 1 & 2 & 3 & 2 & 1 & 7 & 2 & 5 & 1 & 6 & 1 & 3 & 3 & 2 & 1 \\
\hline 4.5 & 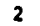 & 2 & 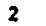 & 3 & 3 & 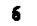 & 7 & & 3 & , & & 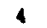 & 3 & 2 & 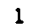 & 4 \\
\hline $1.731 \bar{E}+03$ & 2 & 2 & 3 & 2 & 2 & 3 & 7 & 3 & 4 & 3 & & 2 & 1 & 2 & 1 & 2 \\
\hline 1.78 & 2 & 2 & 3 & 3 & 1 & 6 & 4 & 2 & 5 & 2 & 5 & 3 & 2 & 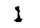 & 4 & 3 \\
\hline 1.78 & & 2 & 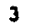 & 3 & 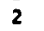 & & 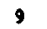 & & 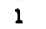 & 2 & & 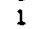 & 3 & 2 & i & 3 \\
\hline 4.87 & 2 & 1 & 3 & 2 & 1 & 6 & 3 & 5 & 1 & 3 & & 2 & 3 & 2 & 1 & 3 \\
\hline 5.48 & 2 & 1 & 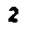 & 2 & 2 & 6 & 4 & & $\pi$ & 2 & & 1 & & 2 & & 2 \\
\hline 5.78 & 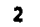 & 2 & 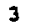 & , & , & & 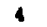 & & 4 & 3 & & $i$ & 2 & 2 & 1 & 2 \\
\hline 3.91 & 2 & 2 & 2 & 3 & 2 & 5 & 3 & & 2 & 2 & & 4 & 3 & 1 & 1 & 3 \\
\hline 5.98 & 2 & 2 & 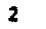 & 2 & 3 & 1 & 7 & & 4 & 1 & & 4 & & 2 & & 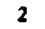 \\
\hline 6.05 & 2 & 2 & 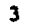 & 7 & , & & $\mathbf{s}$ & & 7 & , & & 2 & 2 & & 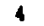 & 4 \\
\hline 6.37 & 2 & 2 & 2 & 2 & 3 & & 2 & 3 & 3 & 2 & 3 & 2 & 4 & 2 & 3 & 3 \\
\hline 6.84 & 2 & 2 & 3 & 1 & 1 & 6 & 8 & 3 & 4 & 3 & & 2 & 3 & 2 & 3 & 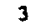 \\
\hline & 2 & 1 & 2 & & 2 & & A & & - & 3 & & 3 & $\mathbf{5}$ & & 2 & 3 \\
\hline 8.91 & 2 & 1 & 2 & 2 & 1 & 5 & 7 & 4 & 2 & 1 & 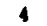 & 5 & 3 & 2 & 2 & 3 \\
\hline 9.183 & 2 & 1 & 2 & 2 & 2 & 2 & 6 & 5 & 3 & 3 & 3 & 5 & 4 & 4 & 3 & 2 \\
\hline 9.36 & 2 & 1 & 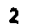 & & $=$ & & 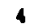 & & 4 & 2 & & 3 & ? & 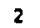 & 2 & 3 \\
\hline $9.533 \mathrm{E}+03$ & 1 & 2 & 2 & 2 & 3 & 4 & 4 & 1 & 2 & 2 & & 5 & 3 & & 1 & 7 \\
\hline 9.8 & 2 & 2 & 2 & & 3 & 2 & 5 & 5 & 3 & 2 & & 2 & 2 & 2 & 2 & 1 \\
\hline $1.0208+04$ & 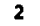 & 1 & 2 & 1 & 2 & & 3 & 3 & 4 & 2 & 2 & 4 & $\rightarrow$ & & 2 & 4 \\
\hline .05 & 2 & 2 & 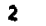 & 1 & 2 & 5 & 4 & 5 & 5 & 4 & 1 & 1 & 3 & 1 & 1 & 2 \\
\hline .1698 & 2 & 1 & , & 1 & 3 & 6 & 9 & 3 & 5 & 1 & 5 & 3 & 1 & 2 & 1 & 2 \\
\hline $.208 \mathrm{E}+0$ & 1 & 1 & 3 & 1 & 3 & & 6 & 5 & 3 & 3 & 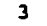 & 3 & 4 & 3 & 2 & 2 \\
\hline $1.245 \mathrm{E}+04$ & & & & & 2 & & 9 & & & 2 & & 3 & & & 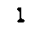 & 3 \\
\hline
\end{tabular}


NUREG/CR-4551, VOL. 1: DRAFT REPORT FOR COMMENT (SEPTEMBER, 1986)

\begin{tabular}{|c|c|c|c|c|c|c|c|c|c|c|c|c|c|c|c|c|c|c|c|c|c|c|c|c|c|c|}
\hline $3108+04$ & 2 & 2 & 3 & 1 & 1 & 1 & 1 & 3 & 3 & 1 & 4 & 2 & 5 & 3 & 2 & 2 & 3 & 1 & 3 & 3 & 2 & 2 & 3 & & 3 & 3 \\
\hline $.359 \mathrm{E}+04$ & 2 & 1 & 2 & 2 & 3 & 6 & 5 & 4 & 6 & 2 & 6 & 1 & 3 & 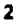 & 2 & 3 & 2 & 1 & 2 & 1 & 2 & 3 & 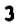 & 4 & 3 & \\
\hline $.3708+04$ & 2 & 2 & 3 & 3 & 2 & 4 & 7 & 3 & 3 & 2 & 4 & 2 & 3 & 3 & 3 & 2 & 4 & 1 & 3 & 1 & 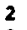 & 2 & 3 & 3 & 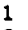 & \\
\hline $.3968+04$ & 2 & 1 & 2 & 1 & 2 & 6 & 8 & 3 & 3 & 1 & 4 & 5 & 1 & 1 & 3 & 4 & 4 & 1 & 2 & & 2 & 3 & 2 & & 3 & \\
\hline $.587 z+04$ & 2 & 2 & 2 & 2 & 3 & 2 & 4 & 4 & 6 & 1 & 6 & 2 & 3 & 1 & 3 & 3 & 4 & 1 & 1 & 2 & 2 & 2 & 3 & 3 & 3 & \\
\hline $1.682 \mathrm{E}+04$ & 2 & 1 & 2 & 1 & 2 & 3 & 1 & 5 & 3 & 1 & 4 & 5 & 2 & 1 & 1 & 2 & 3 & 1 & 3 & 3 & 2 & 3 & 3 & 4 & 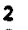 & \\
\hline $.686 E+04$ & 2 & 1 & 3 & 2 & 1 & 3 & 6 & 5 & 4 & 2 & 4 & 5 & 3 & 2 & 1 & 2 & 1 & 1 & 2 & 1 & 2 & 3 & 3 & 3 & 3 & \\
\hline $.7718+04$ & 2 & 2 & 2 & 2 & 1 & 4 & 3 & 3 & 3 & 1 & 6 & 1 & 4 & 2 & 2 & 3 & 2 & 1 & 2 & 1 & 3 & 3 & 3 & 2 & 2 & \\
\hline $.853 \mathrm{E}+04$ & 2 & 1 & 2 & 1 & 3 & 6 & 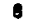 & 5 & 3 & 1 & 6 & 4 & 2 & 1 & 2 & 1 & 1 & 1 & 2 & 3 & 1 & 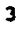 & 2 & 2 & 6 & \\
\hline $.083 E+04$ & 2 & 1 & 2 & 1 & 1 & 6 & 1 & 4 & 5 & 2 & 3 & 5 & 2 & 3 & 3 & 4 & 3 & 1 & 1 & 1 & 1 & 3 & 1 & 2 & 3 & \\
\hline$E+04$ & 2 & 1 & 3 & 2 & 1 & 3 & 4 & 4 & 3 & 2 & 4 & 1 & 3 & 2 & 1 & 3 & 2 & 1 & 2 & 3 & 2 & 3 & 1 & 3 & 2 & \\
\hline $8+04$ & 2 & 2 & 2 & 1 & 3 & 3 & 8 & 5 & 2 & 2 & 4 & 2 & 5 & 2 & 3 & 2 & 3 & 1 & 2 & 4 & 3 & 3 & 3 & 3 & 3 & 4 \\
\hline $.283 E+04$ & 1 & 2 & 3 & 1 & 1 & 6 & 2 & 4 & 2 & 1 & 6 & 4 & 1 & 2 & 1 & 3 & 4 & 1 & 2 & 4 & 1 & & 3 & 2 & 3 & 4 \\
\hline $025 E+04$ & 2 & 2 & 2 & 3 & 1 & 3 & 3 & 5 & 2 & 2 & 3 & 1 & 3 & 3 & 2 & 4 & 3 & 1 & 3 & 1 & 3 & 3 & 3 & 2 & 2 & 2 \\
\hline $.782 E+04$ & 2 & 2 & 3 & 1 & 1 & 4 & 8 & 4 & 5 & 1 & 5 & 2 & 3 & 2 & 2 & 3 & 2 & 1 & 2 & 1 & 1 & 3 & 2 & 2 & 3 & 3 \\
\hline $3078+04$ & 2 & 2 & 3 & 2 & 1 & 2 & 3 & 3 & 6 & 2 & 4 & 5 & 5 & 4 & 4 & 2 & 4 & 1 & 2 & & 3 & & 2 & 3 & 3 & \\
\hline $1812+04$ & 3 & ? & 2 & 2 & 2 & 1 & 6 & 4 & 2 & 1 & 4 & 1 & 3 & 3 & 2 & 2 & 3 & 1 & 2 & 2 & 1 & & 2 & 2 & 2 & \\
\hline
\end{tabular}


CONSEQUENCE MEASURE:

OFFSITE COSTS PER YEAR

BASE CASE

SURRY LALHC (MACCS)

FRACTIONAL CONTRIBOTION OF EACH SEOUENCE TO MEAN RISK

$\begin{array}{lr}\text { SEQUENCE } & \text { CONTRIBOTION } \\ \text { SYYB } & 0.1045 \\ \text { SYNI } & 0.0001 \\ \text { SNNN } & 0.5004 \\ \text { SYNN } & 0.0007 \\ \text { MYNN } & 0.0000 \\ \text { AYNN } & 0.0090 \\ \text { AYNI } & 0.0216 \\ \text { IYYB } & 0.0068 \\ \text { IYNN } & 0.1673 \\ \text { TYYBM } & 0.0610 \\ \text { TYYBD } & 0.0661 \\ \text { IYNI } & 0.0002 \\ \text { V } & 0.0620\end{array}$

1 CONSEOUENCE MEASORE:

OFFSITE COSTS PER YEAR

BASE CASE :

SURRY LALHC (MACCS)

FRACTIONAL CONTRIBUTION OP RACH BIN TO MEAN RISK

$$
\text { BIN }
$$

BIN 1

BIN 2

BIN 3

BIN 4

BIN 5

BIN

BIN 7

BIN 8

BIN 9

BIN 10

BIN 11

BIN 12

BIN 13

BIN 14

BIN 15

BIN 16

BIS 17

BIN 19

1 CONSEOUENCE MANSURE:

OFFSITE COSTS PER YEAR

CONTRIBUTION

0.0309

0.0055

0.0021

0.0315

0.0000

0.0008

0.0001

0.0050

0.0025

0.0367

0.0367

0.0000

0.0019

0.0000

0.6394

0.0746

0.0303

0.0670

BASE CASE

SORRY LALHC (MACCS)

FRACTIONAL CONTRIBUTIONS OF RACH RELEASE TO MRN RISK

RELEASE

SUR $1 G$

sण 2

SOR $3 G$

CR 46

SOR 56

SUR $6 \mathrm{G}$

SUR $7 \mathrm{G}$

SUR $8 G$

SOR 96

SOR $10 G$

SUR $11 G$

SUR $12 G$

SUR $13 G$

SUR I4G

SOR $15 \mathrm{G}$

SUR I6G

SUR 17

SUR $19 \mathrm{G}$

SUR $20 \mathrm{G}$

SUR $21 G$

SUR $22 G$

SUR $23 G$

SUR 246

SUR 256

0.1483

0.1390

0.1390

0.0855

0.1092

0.0505

0.0524

0.0013

0.008

0.0089

0.0028

0.0236

0.0015

0.0105

0.0029

0.0117

0.0015

0.0058

0.0033

0.0001

0.0011

0.0019

0.0000 


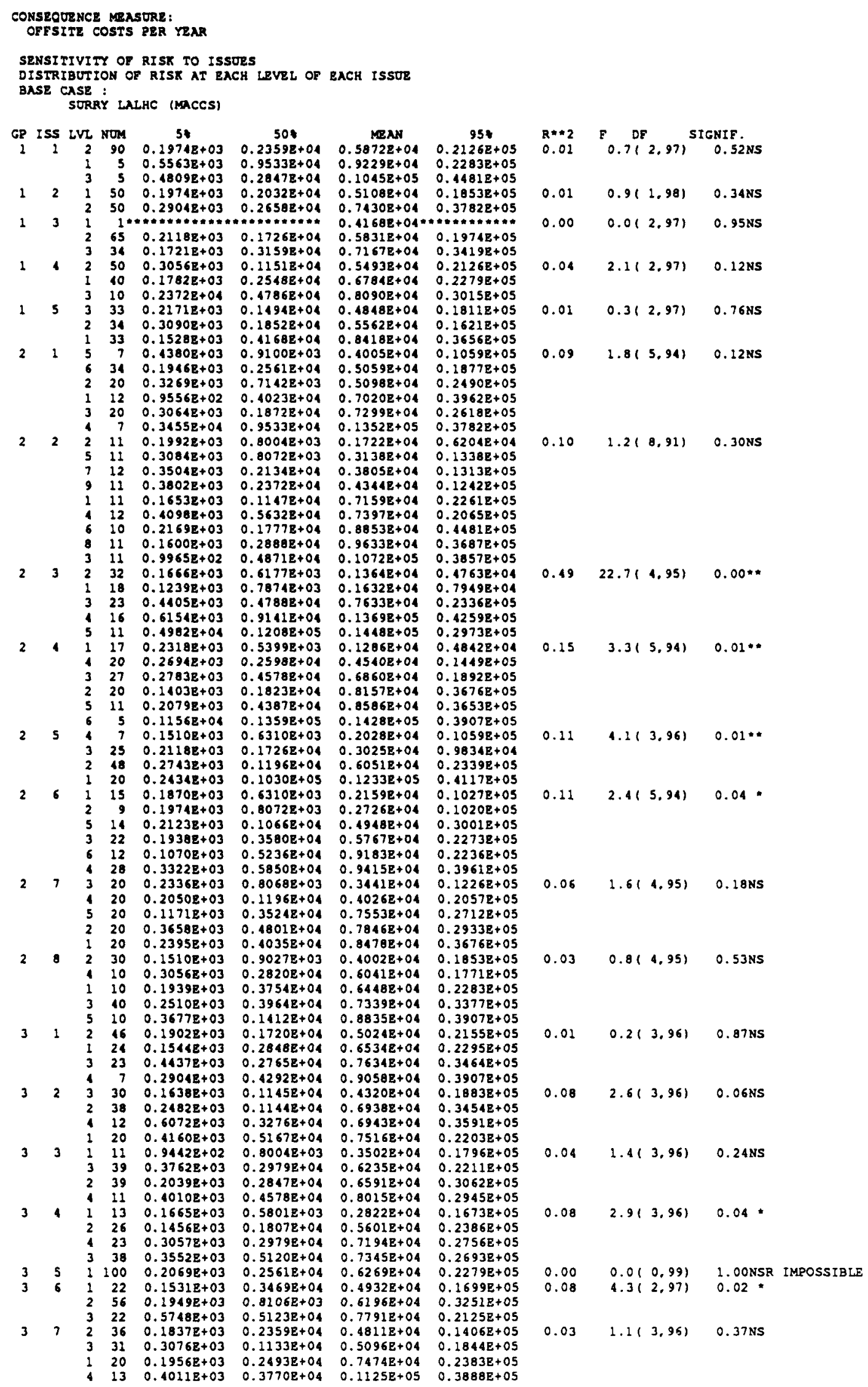


NUREG/CR-4551, VOL. 1: DRAFT REPORT FOR COMMENT (SEPTEMBER, 1986)

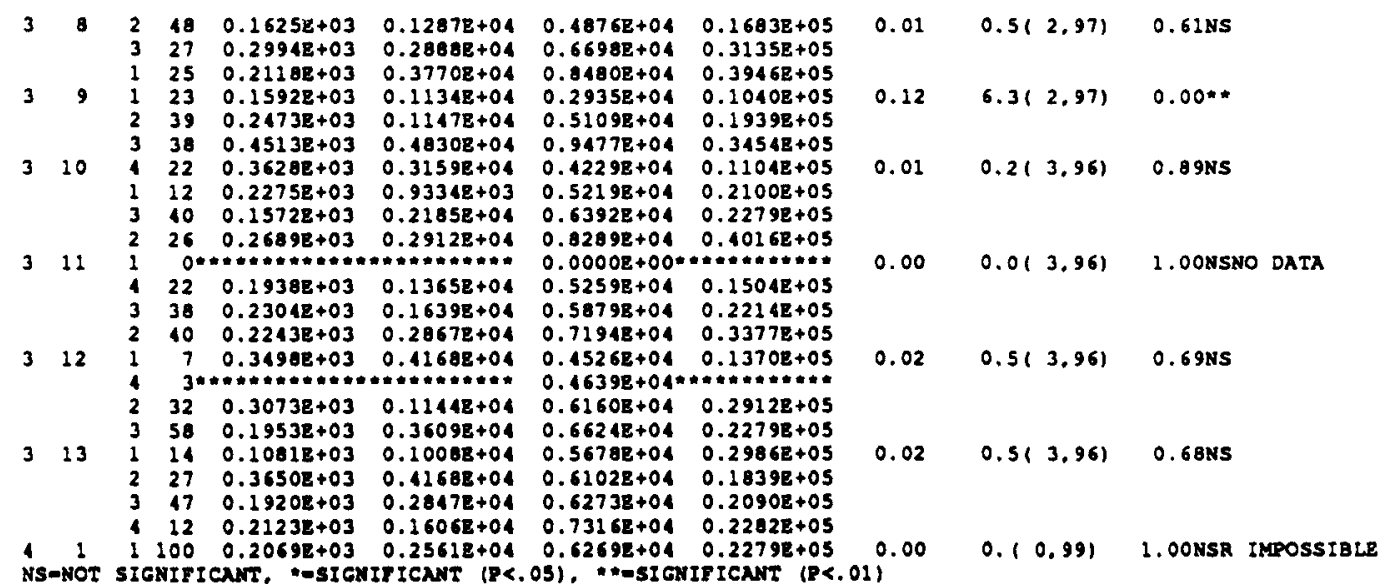


CONSEOUENCE MEASURE: OFFSITE COSTS PER YEAR

- ISSUE GROUP $=1$ ISSTB $=1$ TB SEAL LOCA

LEVEL 1 NO. QEED.- 2 NO. EXP.- 2

LEVEL 2 MO. QMRD.- 45 NO. EXP. $=45$

LEVEI 3 NO. ARD.- 2 NO. EXP.= 2

OVERALL CHI-SO 0.0 DFE I SIG." 1.000 ISSUE GROOP - 1 ISSTE - 2 USING GAS GEM.

LEVEL 1 NO. QRE. - 25 NO. EXP. $=25$

NO. >MED. 25 พO. BXP.- 25

LEVEL 2 NO. ARD.- 24 NO. EXP. - 25

NO. $>$ NED. -26 NO. EXP. -25

OVERALI CHI-SO- 0.1 DF- 3 SIG.- 0.994

ISSUR GROUP - 1 ISSUR - 3 BETA-PACTOR

LEVEL 1 NO. QED.- 0 No. EXP.= 0

No. >MED.- 1 No. Exe.- 1

LEVEL 2 No. QRD. - 34 100. Exp. - 32

No. >med. - 31 Mo. $2 \times P .-33$

LEVEL 3 No. QED. = 15 10. EXP. - 17

NO. >IED. - 19 NO. BXP.= 17

OVERALL CHI-SO= 0.7 DF= 3 SIG.- 0.869

- ISSUE GROUP - 1 ISSUE - 4 V EREO

LEVEL 1 MO. QXED. - 20 10. EXP.- 20

HO. TKED. $=20$ Ho. EXP. $=20$

LEVEL 2 No. QRED. = 29 NO. EXP. - 25

MO. LAED. - 21 NO. EXP. -25

LEVEL 3 NO. QRED.- 0 NO. EXP.= 5

No. >MED. - 10 No. EXP. $=5$

OVERALL CHI-SO- 11.3 DF= 5 SIG. $=0.046$

ISSUE GROUP - 1 ISSUE - 5 PIP2 FAILURB

LEVEL I NO. QRD.- 14 NO. EXP. = 16

MO. $>\operatorname{MgD}=19$ NO. EXP. $=17$

LEVEL 2 NO. ARE. = 18 No. EXP. - 17

NO. $>$ MD. -16 NO, $E X P .=17$

LEVEL 3 NO. QED. = 17 No. EXP. - 16

No. TED. - 16 NO. EXP. -17

OVERALL CHI-SO- 0.7 DF- 3 SIG.= 0.962

ISSUE GROOP - 2 ISSUE $=1$ IND. RCS FAIL.

LEVEL 1 NO. ORED.- 5 NO. EXP.= 6

No. >MED. - 7 No. EXP.= 6

LEVEL 2 NO. QRE. = 13 NO. EXP.= 10

NO. LMBD. = 7 NO, EXP. $=10$

NO. S-MRD. = NO. EXP.= 10

NO.>LED.- 7 NO. EXP.- 4

LEVEL 4 No. ARE.- 0 No. EXP.= 3

LEVEL 5 NO. QRE. = 4 NO. EXP.= 3

NO. XMED.= 3 NO. EXP.= 4

LEVEL 6 NO. SAED. - 16 NO. EXP. = 17

NO. >-MED. = 18 NO. EXP. $=17$

OVERALL CHI-SQ- 2.5 DF= 7 SIG.= 0.931

0

ISSUE GROUP $=2$ ISSUE $=2$ MODE OF VB.

LEVEL I NO. QRED.- 6 No. EXP.- 5

NO. >MRD. - 5 NO. $\mathrm{EXP}=6$

LEVEL 2 NO. QRED.= 8 NO. EXP.- 5

LEVEL 3 NO. QRE. = 3 NO. EXP.= 5

LEVEL 4 HO. QRED. = 3 NO. EXP.- 6

LEVEL 5 NO. QRD. = 0 NO. EXP.- 5

LEVEL 6 NO. AED.- 5 NO. EXP.- 5
No. XKED. = 3 No. EXP.- 6

NO, XMED. - \& NO. EXP.= 6

NO. $>$ MBD. $=$ NO. $\mathrm{EXP} .=6$

MO. XMED. - 3 NO. EXP.- 6

NO. $>$ MED. $=5$ NO. EXP.- 5
CHI-SO. TEST CANWOT aE RON; EXP. NO, < 5

CHI-SO.- $0.0 \mathrm{DF}=1$ SIG. -1.000

CHI-SO. IEST CNNOT BE RON; EXP. NO. < 5

CHI-SO.= 0.0 DE- I SIG. $=1.000$

CHI-SO.= 0.1 DF= 1 SIG.- 0.777

CHI-SO. TEST CANHOT BE RON, EXP. NO. < 5

CHI-SO.- 0.2 DF- 1 SIC. $=0.620$

CHI-SO.- 0.5 DF= 1 SIG. 0.493

CHI-SO.= 0.0 DF- 1 SIG. $=1.000$ CHI-SO.= $1.3 \mathrm{DF}=1 \mathrm{SIG}=0.258$

CHI-SO.- 10.0 DF= 1 SIG.=0.002

CHI-SO.- $\quad 0.5$ DF- 1 SIG. $=0.486$

CHI-SO.= 0.1 DF= 1 SIG. $=0.732$

CHI-SO.- 0.1 DF= I SIG. $=0.728$

CHI-SO.= 0.3 DF= I SIG. 0.564

CHI-SO.- 1.8 DE $=1$ SIG. $=0.180$

CHI-SQ.- 0.2 DF= 1 SIG.= 0.655

CHI-SO. TEST CANNOT BE RON; EXP. NO. $<5$

CHI-SQ. TEST CANNOT BE RUN: EXP. NO. < 5

CHI-SO. $=0.1$ DF= 1 SIG $=0.732$

$C H I-S Q=0.4 D F=1$ SIG. $=0.545$

$\mathrm{CHI}-\mathrm{SO}=3.3 \mathrm{DF}=1 \mathrm{SIG}=0.069$

CHI-SQ.- 1.5 DE= 1 SIG.=0.226

CHI-SQ.- 3.0 DF= 1 SIC. $=0.083$

CHI-SO.= 3.3 DF= I SIG.=0.069

CHI-SO.- $0.0 \mathrm{DF}=1$ SIG. $=1.000$ 


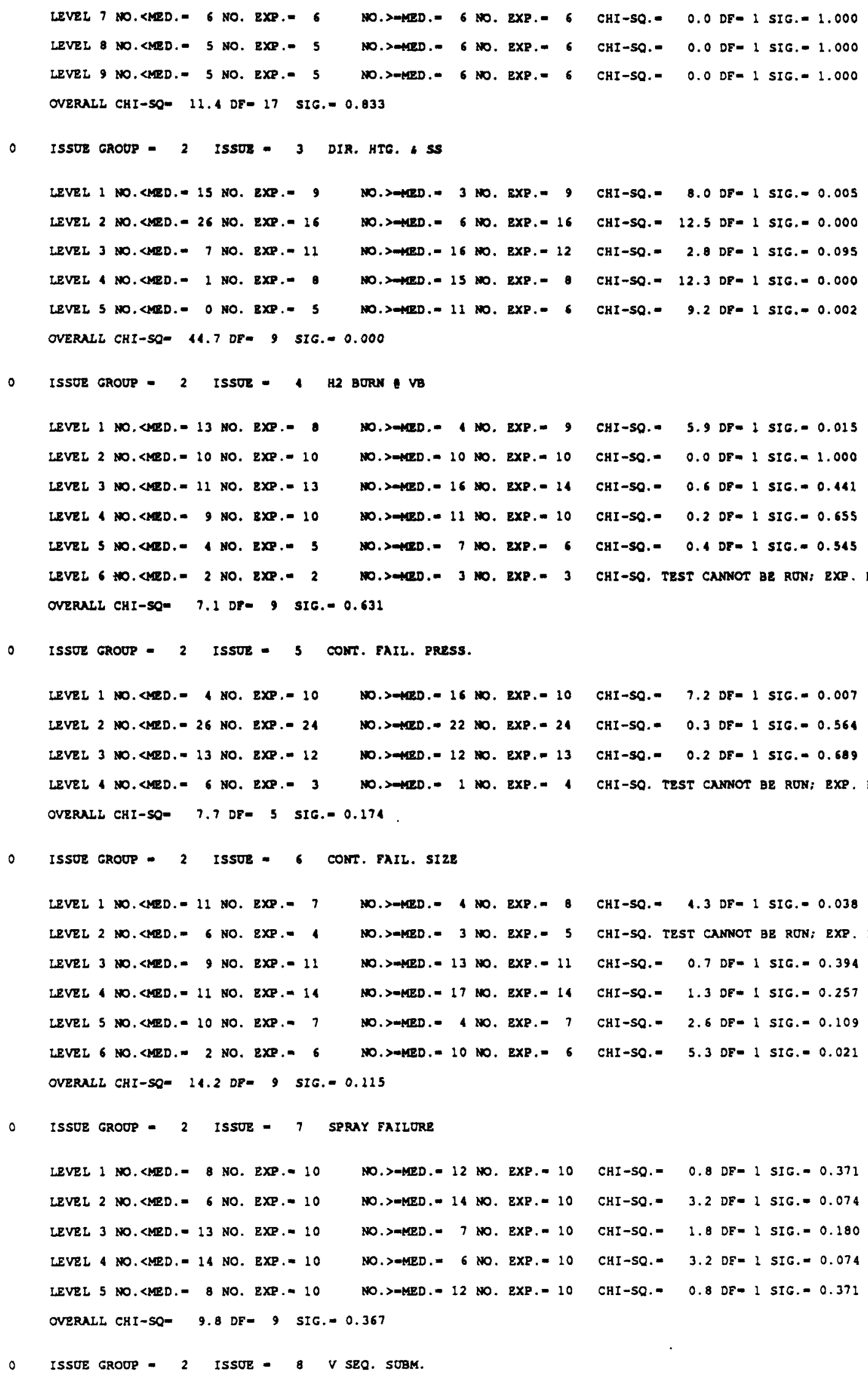




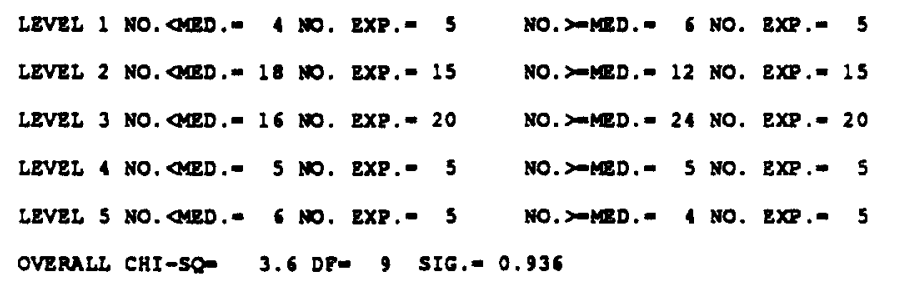

No. XMED.- 12 No. EXP. $=15$

No. >MED. -24 No. $\exp .=20$

No. $>$ Med.- 5 No. EXP.- 5

No.>-NeD.- 4 No. exp.- 5 168

No. 2-MED.- 12 NO. $\operatorname{EXP}=12$ No. $>$ ARD. -23 พo. EXP. -23 YO. $>$ Med.- I NO. exp.= 4

CHI-SQ.- 0.0 DFM 1 SIG.- 1.000

CHI-SO.- 0.0 DF- 1 SIG.- 1.000 CHI-SO.= 0.0 DF- 1 SIG.- 1.000 CHI-SO. TEST CANMOT BE RUN: EXP. NO. < 5
CHI-SO.- 1.5 DF- 1 SIG.- 0.226 CHI-SQ.- 0.0 DF- 1 SIG.= 1.000 CHI-SO.- 0.1 DF- 1 SIG. 0.749 CHI-SO.- 0.0 DP= 1 SIG. $=1.000$
CHI-SO.- $\quad 5.0$ DF- 1 SIG.= 0.026 CHI-SO.- 0.0 DF- 1 SIG.- 1.000 CHI-SO. $=1.7$ DF= 1 SIE. $=0.194$ CHI-SO.- 0.0 DF- I SIG. $=1.000$
CHI-SO.- 0.7 DF= 1 SIG. $=0.394$ CRI-SQ $=4.6 \mathrm{DF}=1$ SIG. $=0.033$ CHI-SO.- 8.9 DF= 1 SIC. $=0.003$
NO. T-MED. - 10 NO. EXP. $=10$ NO. - MED. $=18$ NO. EXP.- 18 No. $>$ Med.- 14 No. EXP. $=16$ NO. >MED. = 9 NO. EXP.- 7
CHI-SO. $=0.0$ DF- 1 SIG $=1.000$ CHI-SO. 0.0 DF- 1 SIG. $=1.000$ CHI-SO. $=0.5$ DF- 1 SIG. $=0.472$ CHI-SQ. $=1.2$ DF= 1 SIG. $=0.266$ 


\section{OVERALL CHI-SO= 1.8 DF= 7 SIG. 0.972 \\ 0 ISSUE GROOP - 3 ISSUE - 8 SPRAY DF}

LEVEL 1 NO. LEED. - 10 NO. EXP. = 22

No. > $\times \mathbb{R D} .-15$ 10. ExP. $=13$

LEVEL 2 No. LMED. - 26 No. EXP.- 24

LEVEL 3 NO. SMED. - 13 NO. EXP. $=13$

No.>-tED. -22 No. EXP. -24

NO. >MED. - 14 No. EXP. - 14

OVERALL CHI-SO- $1.0 \mathrm{DF}=5$ SIG.- 0.965

ISSUE GROOP - 3 ISSUE -, AER ACCLOM

LEVEL 1 No. CARD. - 14 NO. EXP. - 11

No. > ARD.. 9 No. EXP. $=12$

EEVEL 2 No. LMED.- 22 No. ExP.- 19

No.>-NED. = 17 No. ExP. -20

LEVEL 3 No. KNED. - 13 NO. EXP. - 19

No.>-tied. 25 No. exp. -19

OVERALL CHI-SO- 6.3 DF= 5 SIG. $=0.280$

ISSUE GROUP - 3 ISSUE - 10 LATE TODINB

LEVEL I NO. LLED.- B NO. EXP.- 6

NO. >-MED. = 4 NO. EXP.- 6

LEVEL 2 No. CMED.- 11 No. EXP. $=13$

No.>AED. - 15 NO. EXP. $=13$

LEVEL 3 NO. CAED. - 20 NO. EXP.- 20

No.>AED. 20 No. Exp. $=20$

LEVEL \& No. CMED. - 10 NO. EXP. - 11

No. >-WED. = 12 Ho. EXP.- 11

OVRRALL CHI-SO- 2.1 DTE 7 SIG.= 0.952

0

ISSUE GROOP $=3$ ISSOR - 11 LATE REVOL

LEVEL I NO. <HED. = O NO. EXP.= 0

SO.>ARD. = 0 NO. EXP. $=0$

LEVEL 2 NO. KMED. - 18 NO. exp. $=20$

No.>MED. $=22$ No. EXP. 20

LEVEL 3 NO. LMD. = 19 NO. EXP. = 19

No.>-Weo.- 19 No. exp. = 19

LEVEL 4 No. <HED.- 12 No. EXP. - 11

\$o. >-ARD. - 10 so. EXP. = 11

OVERALL CHI-5O- 0.6 OF" 5 SIG.=0.949

0

ISSOE GROOP - 3 ISSUE - 12 HPE REL

LEVEL 1 NO. LMED.- 3 NO. EXP.= 3

LEVEL 2 NO. SMED. - 20 NO. EXP. - 16

LEVEL 3 NO. LEED. - 25 NO. EXP. $=29$

No.>ARD. - 1 No. EXP.= 4

No.>AED, -12 NO. EXP. $=16$

NO.>-NED. $=33$ NO. EXP. $=29$

LEVEL 4 NO. LWED.- 1 NO. EXP.- 1

NO. >AED, = 2 NO. EXP.- 2

OVERALL CHI-SO= $3.1 \mathrm{DF}=3$ SIG. $=0.376$

0

ISSOE GROUP - 3 ISSUE $=13$ DH REL
LEVEL I NO. KMED.- 8 NO. EXP. $=7$

LEVEL 2 NO. <MED. - 11 NO. EXP. - 13

LEVEL 3 NO. <HED. - 23 NO. EXP. = 23

LEVEL + NO. <KED. - 7 NO. EXP.- 6

OVERALI CHI-SO= 1.2 OF= 7 SIG. $=0.991$
CHI-SO.= 0.6 DE- 1 SIG. $=0.423$

CHI-SO. = 0.3 DF $=1$ SIG. $=0.564$

CHI-SO. $=0.0$ DF $=1$ SIG. $=1.000$

CHI-SO.= 1.6 DF= 1 SIG. $=0.210$

CHI-SO.- 0.9 DF= 1 SIG. $=0.336$

CHI-SO.= 3.8 DF- 1 SIG. $=0.052$

CHI-SO.= 1.3 DF- 1 SIG.= 0.248

CHI-SO. $=0.6$ DE $=1$ SIG. $=0.433$

CHI-SQ.= 0.0 DF- 1 SIG. $=1.000$

CHI-SO.= 0.2 DF= I SIG. $=0.670$

CHI-SO. TEST CANNOT BE RON; EXP. NO. < 5

CHI-SO.= 0.4 DF= 1 SIG. $=0.527$

CHI-SO.= 0.0 DF- 1 SIG. $=1.000$

CHI-SQ.- 0.2 DF= 1 SIG. $=0.670$

CHI-5O. TEST CANNOT BE RON; EXP. NO. < 5

CHI-SO.= 2.0 DF- 1 SIG. $=0.157$

CHI-SQ.- $\quad 1.1$ DF- 1 SIG. $=0.293$

CHI-SO. TEST CANNOT gE RON; EXP. NO. < 5 


\section{D.3 OUTPUT FOR THE OCP ANALYSIS}

The output describing the $\mathrm{OCP}$ results for each consequence measure as calculated by CRAC2 is provided in this section. The results are intended to supplement the information provided in Section 5 of the main report. Since the MACCS results provide little additional insight, they are not included here but are summarized in the main report. The main report focused on the central results, which were intended as the best estimate of the SARRP program. The listing provided here also includes the optimistic and pessimistic results, as well as the uncertainty as measured by examining the uncertainties from each of the analysis areas, taken two or three at a time. These results allow further comparison of the OCP uncertainty methodology with the LLH uncertainty approach. The results focus on the uncertainty as calculated by the OCP method, considering single sources of uncertainty first, and then considering multiple sources up to combinations of four sources of uncertainty. The areas of uncertainty considered in this output include: sequence data, sequence phenomenology, containment failure modes (or containment event tree results), source term uncertainty, and consequence uncertainty (not studied in detail, but included in the calculations). The risk results are followed by tables describing the fractional contribution of each plant-damage state to each risk measure. 


\section{OCP RESULTS FOR THE SURRY PLANT}

BASE CASE: SURRY WITH DIRECT HEATING SEQUENCES: PLANT DAMAGE STATES 4/25/86 CONTAINMENT FAILURE MODES: CET, 4/22/86 CRAC2

CONSEQUENCES: 95\% EVACUATION, PUFF RELEASES FOR PUFF SCENARIOS

BASE CASE: SURRY WITH DIRECT HEATING

CONSEQUENCE MEASURE: EARLY FATALITIES PER YEAR

RISK DATA:

OPTIMISTIC $=0.0 \mathrm{E}+00 \quad$ CENTRAL $=2.9 \mathrm{E}-07 \quad$ PESSIMISTIC $=1.1 \mathrm{E}-02$ UNCERTAINTY ANALYSIS

SINGLE SOURCES OF UNCERTAINTY

$\begin{array}{lllllll} & \text { SEQ.PHEN } & \text { SEODATA } & \text { CEM } & \text { BEL. } & \text { CONSO } & \text { ENVELOPE } \\ \text { MAX. } & 2.9 \mathrm{E}-06 & 1.1 \mathrm{E}-06 & 7.0 \mathrm{E}-07 & 8.7 \mathrm{E}-06 & 4.5 \mathrm{E}-06 & 8.7 \mathrm{E}-06 \\ \mathrm{MIN} & 7.1 \mathrm{E}-08 & 1.0 \mathrm{E}-08 & 2.1 \mathrm{E}-07 & 2.8 \mathrm{E}-10 & 0.0 \mathrm{E}+00 & 0.0 \mathrm{E}+00\end{array}$

DOUBLE SOURCES OF UNCERTAINTY

SEQ PHEN SEOPHEN SEQ PHEN CEM CEM REL ENV \&CEM \&DATA \&CONS \&REL \&CONS \&CONS

$\begin{array}{llllllll}\text { MAX. } & 6.7 \mathrm{E}-06 & 1.4 \mathrm{E}-04 & 4.3 \mathrm{E}-05 & 9.9 \mathrm{E}-05 & 1.1 \mathrm{E}-05 & 1.3 \mathrm{E}-04 & 1.4 \mathrm{E}-04 \\ \mathrm{MIN} & 7.0 \mathrm{E}-08 & 1.3 \mathrm{E}-12 & 0.0 \mathrm{E}+00 & 3.3 \mathrm{E}-10 & 0.0 \mathrm{E}+00 & 0.0 \mathrm{E}+00 & 0.0 \mathrm{E}+00\end{array}$

\section{TRIPLE SOURCES OE UNCERTAINTY}

SEO PHEN SEO PHEN SEO PHEN REL\&CEM ENV CEM\&REL CEM\&CONS REL\&CONS \& CONS

$\begin{array}{llllll}\mathrm{MAX} & 7.2 \mathrm{E}-04 & 9.9 \mathrm{E}-05 & 2.0 \mathrm{E}-03 & 1.5 \mathrm{E}-03 & 2.0 \mathrm{E}-03 \\ \mathrm{MIN} & 1.3 \mathrm{E}-12 & 0.0 \mathrm{E}+00 & 0.0 \mathrm{E}+00 & 0.0 \mathrm{E}+00 & 0.0 \mathrm{E}+00\end{array}$

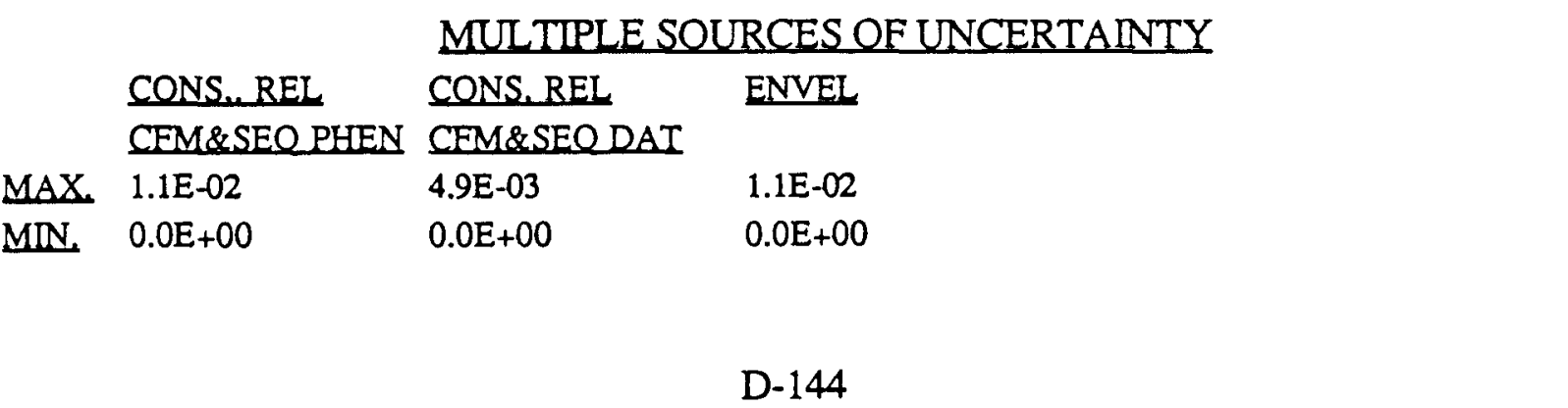


BASE CASE: SURRY WITH DIRECT HEATING

CONSEQUENCE MEASURE: EARLY INJURIES PER YEAR

RISK DATA:

\begin{tabular}{|c|c|c|c|c|c|c|c|}
\hline \multicolumn{3}{|c|}{ OPTIMISTIC $=0.0 \mathrm{E}+\infty$} & \multicolumn{2}{|c|}{ CENTRAL $=6.2 \mathrm{E}-06$} & \multicolumn{2}{|c|}{ PESSIMISTIC $=$} & $1.7 \mathrm{E}-01$ \\
\hline & \multicolumn{7}{|c|}{ UNCERTAINTY ANALYSIS } \\
\hline & \multicolumn{7}{|c|}{ SINGLE SOURCES OF UNCERTAINTY } \\
\hline & SEO RHEN & SEODATA & CEM & BEL. & conse & ENVELOPE & \\
\hline$\Delta x$. & $6.4 \mathrm{E}-05$ & $2.2 \mathrm{E}-05$ & $3.2 \mathrm{E}-05$ & $1.1 \mathrm{E}-0$ & $6.2 \mathrm{E}-05$ & $1.1 \mathrm{E}-04$ & \\
\hline \multirow[t]{3}{*}{ MIN } & $1.4 \mathrm{E}-06$ & 2.2E-07 & 4.1E-06 & $1.2 \mathrm{E}-07$ & $0.0 \mathrm{E}+00$ & $0.0 \mathrm{E}+00$ & \\
\hline & \multicolumn{7}{|c|}{ DOUBLE SOURCES OF UNCERTAINTY } \\
\hline & $\begin{array}{l}\text { SEQ PHEN } \\
\text { CEM }\end{array}$ & $\begin{array}{l}\text { SEO. PHEN } \\
\text { \&DATA }\end{array}$ & $\begin{array}{l}\text { SEO. PHEN } \\
\text { \& CONS. }\end{array}$ & $\frac{\text { CEM }}{\& \text { BEL }}$ & $\begin{array}{l}\text { CFM } \\
\text { CONS }\end{array}$ & $\begin{array}{l}\text { BEL } \\
\& \text { CONS }\end{array}$ & ENV \\
\hline$\Delta x$ & $2.0 \mathrm{~L}^{\circ}$ & SE-03 & $5.4 \mathrm{E}-04$ & $3.0 \mathrm{E}-03$ & $3.4 \mathrm{E}-04$ & & $3.0 \mathrm{E}-03$ \\
\hline $\mathrm{N}$ & $1.4 \mathrm{E}-06$ & $1.7 \mathrm{E}-09$ & $0.0 \mathrm{E}+00$ & $1.2 \mathrm{E}-07$ & $0.0 \mathrm{E}+\infty 0$ & $0.0 \mathrm{E}+\infty$ & $0.0 \mathrm{E}+00$ \\
\hline
\end{tabular}

TRIPLE SOURCES OF UNCERTAINTY

SEO PHEN SEO PHEN SEO PHEN REL\&CEM ENV

CEM\&REL CEM\&CONS REL\&CONS \& CONS

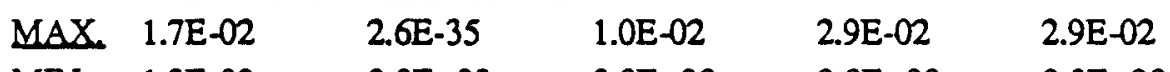

MIN 1.3E-09 $\quad 0.0 \mathrm{E}+00 \quad 0.0 \mathrm{E}+00 \quad 0.0 \mathrm{E}+00 \quad 0.0 \mathrm{E}+00$

\begin{tabular}{llll} 
& & \multicolumn{2}{c}{ MULTHPLE SOURCES OF UNCERTAINTY } \\
\cline { 3 - 3 } CONS.REL & CONS. REL & ENVEL \\
\cline { 2 - 3 } CEM\&SEQ PHEN CEM\&SEQDAT & & \\
MAX. & $1.7 \mathrm{E}-02$ & $9.6 \mathrm{E}-02$ & $1.7 \mathrm{E}-01$ \\
MIN & $0.0 \mathrm{E}+00$ & $0.0 \mathrm{E}+00$ & $0.0 \mathrm{E}+00$
\end{tabular}


BASE CASE: SURRY WITH DIRECT HEATING

CONSEQUENCE MEASURE: LATENT CANCER FATALITIES PER YEAR

BISKDATA:

OPTIMISTIC $=1.2 \mathrm{E}-06 \quad$ CENTRAL $=3.2 \mathrm{E}-04 \quad$ PESSIMISTIC $=1.9 \mathrm{E}-01$

UNCERTAINTY ANALYSIS

SINGLE SOURCES OE UNCERTAINTY

$\begin{array}{lllllll} & \text { SEO PHEN } & \text { SEODATA } & \text { CEM } & \text { REL. } & \text { CONSO } & \text { ENVELOPE } \\ \text { MAX. } & 3.7 \mathrm{E}-03 & 1.1 \mathrm{E}-03 & 3.7 \mathrm{E}-03 & 1.7 \mathrm{E}-03 & 6.4 \mathrm{E}-04 & 3.7 \mathrm{E}-03 \\ \text { MIN } & 4.4 \mathrm{E}-05 & 1.7 \mathrm{E}-05 & 2.3 \mathrm{E}-04 & 6.4 \mathrm{E}-05 & 1.6 \mathrm{E}-04 & 1.7 \mathrm{E}-05\end{array}$

DOUBLE SOURCES OE UNCERTAINTY

$\begin{array}{llllll}\text { SEQ PHEN SEQ PHEN } & \text { SEQ PHEN CEM } & \text { CEM } & \text { BEL } & \text { ENV } \\ \text { \& CEM } & \text { EATA } & \text { \& CONS } & \text { \&BEL } & \text { CONS } & \text { \&ONS }\end{array}$

$\begin{array}{llllllll}\text { MAX. } & 2.5 \mathrm{E}-02 & 1.7 \mathrm{E}-02 & 7.4 \mathrm{E}-03 & 1.4 \mathrm{E}-02 & 7.4 \mathrm{E}-03 & 3.4 \mathrm{E}-03 & 2.5 \mathrm{E}-02 \\ \mathrm{MIN} & 3.9 \mathrm{E}-05 & 5.6 \mathrm{E}-06 & 2.1 \mathrm{E}-05 & 4.9 \mathrm{E}-05 & 1.1 \mathrm{E}-04 & 3.2 \mathrm{E}-05 & 5.7 \mathrm{E}-06\end{array}$

TRIPLE SOURCES OF UNCERTAINTY

SEQ PHEN SEQ PHEN SEQ PHEN BEL\&CEM ENV

CEM\&REL CEM\&CONS REL\&CONS \& CONS

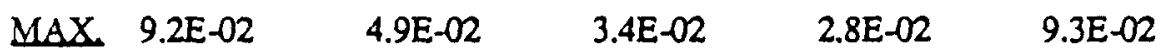

MIN $5.4 \mathrm{E}-06 \quad 1.9 \mathrm{E}-05 \quad 2.8 \mathrm{E}-06 \quad 2.4 \mathrm{E}-05 \quad 2.8 \mathrm{E}-06$

\begin{tabular}{|c|c|c|c|}
\hline \multirow{2}{*}{\multicolumn{2}{|c|}{$\frac{\text { CONS REL }}{\text { CEM\&SEO PHEN }}$}} & \multicolumn{2}{|c|}{ MULTIPLE SOURCES OF UNCERTAINTY } \\
\hline & & $\begin{array}{l}\text { CONS.REL } \\
\text { CEM\&SEODAT }\end{array}$ & ENVEL \\
\hline$\Delta x$ & $1.7 \mathrm{E}-01$ & $9.6 \mathrm{E}-02$ & $1.7 \mathrm{E}-01$ \\
\hline & -06 & $1.2 E-06$ & $1.1 \mathrm{E}-06$ \\
\hline
\end{tabular}


BASE CASE: SURRY WITH DIRECT HEATING

CONSEQUENCE MEASURE: INDIVIDUAL RISK OF EARLY FATALITY

RISKDATA:

OPTIMISTIC $=0.0 \mathrm{E}+00 \quad$ CENTRAL $=1.3 \mathrm{E}-09 \quad$ PESSIMISTIC $=4.4 \mathrm{E}-06$

UNCERTAINTY ANALYSIS

SINGLE SOURCES OE UNCERTAINTY

$\begin{array}{lllllll} & \text { SEQ PHEN } & \text { SEQDATA } & \text { CEM } & \text { REL } & \text { CONSQ } & \text { ENVELOPE } \\ \text { MAX } & 1.6 \mathrm{E}-08 & 4.7 \mathrm{E}-09 & 4.7 \mathrm{E}-09 & 7.4 \mathrm{E}-09 & 2.1 \mathrm{E}-08 & 2.1 \mathrm{E}-08 \\ \text { MTN } & 1.2 \mathrm{E}-10 & 8.4 \mathrm{E}-11 & 7.3 \mathrm{E}-10 & 5.7 \mathrm{E}-12 & 0.0 \mathrm{E}+00 & 0.0 \mathrm{E}+00\end{array}$

DOUBLE SOURCES OF UNCERTAINTY

SEO PHEN SEQ PHEN SEO PHEN CEM CEM REL ENV

\&CEM \&DATA \&CONS \&REL \&CONS \& CONS

$\begin{array}{llllllll}\text { MAX. } & 3.7 \mathrm{E}-08 & 9.2 \mathrm{E}-08 & 2.4 \mathrm{E}-07 & 3.8 \mathrm{E}-08 & 5.9 \mathrm{E}-08 & 1.1 \mathrm{E}-07 & 2.4 \mathrm{E}-07 \\ \text { MIN. } & 9.3 \mathrm{E}-11 & 2.7 \mathrm{E}-14 & 0.0 \mathrm{E}+00 & 6.7 \mathrm{E}-12 & 0.0 \mathrm{E}+00 & 0.0 \mathrm{E}+00 & 0.0 \mathrm{E}+00\end{array}$

TRIPLE SOURCES OF UNCERTAINTY

SEO PHEN SEQ PHEN SEO PHEN REL\&CEM. ENV

CEM\&REL CEM\&CONS BEL\&CONS \& CONS

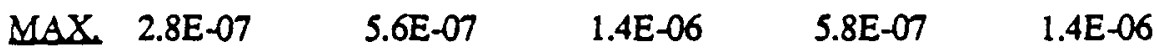

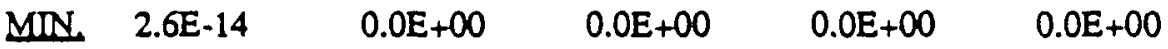

\begin{tabular}{llll} 
& & \multicolumn{2}{c}{ MULTIPLE SOURCES OF UNCERTAINTY } \\
CONS. REL & CONS. REL & ENVEL \\
CEM\&SEQ PHEN CEM\&SEQDAT & \\
MAX. & $4.4 E-06$ & $1.9 E-06$ & $4.4 \mathrm{E}-06$ \\
MIN $0.0 \mathrm{E}+00$ & $0.0 \mathrm{E}+00$ & $0.0 \mathrm{E}+00$
\end{tabular}


BASE CASE: SURRY WITH DIRECT HEATING

CONSEQUENCE MEASURE: OFFSITE COSTS PER YEAR

RISK DATA:

OPTIMISTIC $=3.9 \mathrm{E}+00 \quad$ CENTRAL $=2.3+02 \quad$ PESSIMISTIC $=1.2 \mathrm{E}+05$

UNCERTAINTY ANALYSIS

SINGLE SOURCES OE UNCERTAINTY

$\begin{array}{lllllll} & \text { SEQ PHEN } & \text { SEODATA } & \text { CEM } & \text { BEL. } & \text { CONSO } & \text { ENVELOPE } \\ \text { MAX. } & 2.2 \mathrm{E}+03 & 8.1 \mathrm{E}+02 & 1.9 \mathrm{E}+03 & 1.1 \mathrm{E}+03 & 4.6 \mathrm{E}+02 & 2.1 \mathrm{E}+03 \\ \text { MNN } & 3.7 \mathrm{E}+01 & 8.4 \mathrm{E}+01 & 2.1 \mathrm{E}+02 & 9.8 \mathrm{E}+01 & 1.1 \mathrm{E}+02 & 1.4 \mathrm{E}+01\end{array}$

DOUBLE SOURCES OE UNCERTAINTY $\begin{array}{lllll}\text { SEQ PHEN SEQ PHEN } & \text { SEQ PHEN CEM. } & \text { CFM } & \text { BEL } & \text { ENV } \\ \text { \& CEM } & \text { \&DATA } & \begin{array}{l}\text { \& CONS. } \\ \text { \& REL }\end{array} \text { \&CNS } & \text { \&CONS } & \end{array}$

$\begin{array}{llllllll}\mathrm{MAX} & 1.3 \mathrm{E}+04 & 1.1 \mathrm{E}+04 & 4.4 \mathrm{E}+03 & 8.7 \mathrm{E}+03 & 3.8 \mathrm{E}+03 & 2.1 \mathrm{E}+03 & 1.3 \mathrm{E}+04 \\ \mathrm{MIN} & 3.6 \mathrm{E}+01 & 7.8 \mathrm{E}+00 & 1.8 \mathrm{E}+01 & 1.1 \mathrm{E}+02 & 1.0 \mathrm{E}+02 & 4.9 \mathrm{E}+01 & 7.8 \mathrm{E}+00\end{array}$

TRIPLE SOURCES OE UNCERTAINTY

SEO PHEN SEO PHEN SEO PHEN REL\&CEM ENV CEM\&REL CEM\&CONS REL\&CONS \& CONS

$\begin{array}{llllll}\mathrm{MAX} & 5.9 \mathrm{E}+04 & 2.6 \mathrm{E}+04 & 2.2 \mathrm{E}+04 & 1.7 \mathrm{E}+04 & 5.9 \mathrm{E}+04 \\ \mathrm{MIN} & 8.6 \mathrm{E}+00 & 1.8 \mathrm{E}+01 & 3.8 \mathrm{E}+00 & 5.2 \mathrm{E}+01 & 3.8 \mathrm{E}+00\end{array}$

\begin{tabular}{|c|c|c|c|}
\hline \multirow{2}{*}{\multicolumn{2}{|c|}{ CONS. REL }} & \multicolumn{2}{|c|}{ MUITIPLE SOURCES OF UNCERTAINTY } \\
\hline & & CONS.REL & ENVEL \\
\hline$\Delta x$ & $1.2 \mathrm{E}+05$ & $5.7 \mathrm{E}+04$ & $1.1 \mathrm{E}+05$ \\
\hline & $4.3 \mathrm{E}+00$ & $4.9 \mathrm{E}+00$ & $4.3 \mathrm{E}+00$ \\
\hline
\end{tabular}


BASE CASE: SURRY WITH DIRECT HEATING

CONSEQUENCE MEASURE: POPULATION DOSE PER YEAR (WITHIN 50 MULES)

RISKDATA:

OPTIMISTIC $=1.1 \mathrm{E}-02 \quad$ CENTRAL $=2.3+00 \quad$ PESSIMISTIC $=7.9 \mathrm{E}+02$

UNCERTAINTY ANALYSIS

SINGLE SOURCES OF UNCERTAINTY

$\begin{array}{lllllll} & \text { SEO PHEN } & \text { SEODATA } & \text { CEM } & \text { BEL } & \text { CONSO } & \text { ENVELOPE } \\ \text { MAX } & 2.8 E+01 & 8.1 \mathrm{E}+00 & 2.2 \mathrm{E}+01 & 9.5 \mathrm{E}+00 & 4.6 \mathrm{E}+00 & 2.8 \mathrm{E}+01 \\ \mathrm{MIN} & 2.0 \mathrm{E}-01 & 1.5 \mathrm{E}-01 & 1.6 \mathrm{E}+00 & 5.6 \mathrm{E}-01 & 1.2 \mathrm{E}+00 & 1.5 \mathrm{E}-01\end{array}$

DOUBLE SOURCES OF UNCERTAINTY

SEQ. PHEN SEQ PHEN SEQ PHEN CEM CEM REL ENV

\&CEM \&DATA \&CONS \& REL \& CONS \& CONS

\begin{tabular}{|c|c|c|c|}
\hline $\mathrm{MAX} \quad 1.5 \mathrm{E}+02$ & $8.5 E+01$ & $5.5 \mathrm{E}+01$ & $5.9 E+01$ \\
\hline
\end{tabular}

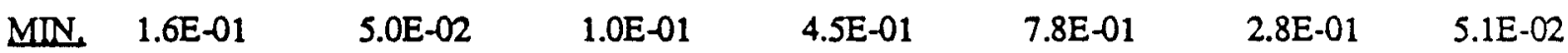

TRIPLE SOURCES OF UNCERTAINTY

SEO PHEN SEO PHEN SEO PHEN REL\&CEM ENV

CEM\&REL CEM\&CONS REL\&CONS \& CONS

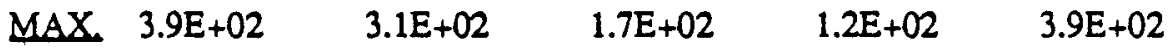

$\begin{array}{lllll}M I N & 4.8 \mathrm{E}-02 & 8.1 \mathrm{E}-02 & 2.5 \mathrm{E}-02 & 2.3 \mathrm{E}-01\end{array}$

\begin{tabular}{llll} 
& \multicolumn{3}{c}{ MULTIPLE SOURCES OF UNCERTAINTY } \\
CONS.REL & CONS. REL & ENVEL \\
\cline { 3 - 3 } CEM\&SEQ PHEN CEM\&SEQDAT & \\
MAX & $7.9 \mathrm{E}+02$ & $3.9 \mathrm{E}+02$ & $7.9 \mathrm{E}+02$ \\
MIN & $2.4 \mathrm{E}-02$ & $1.1 \mathrm{E}-02$ & $1.1 \mathrm{E}-02$
\end{tabular}


FREQUENCIES OF CORE MELT

OPTIMISTIC $=\$ 2.0 \mathrm{E}-06 \quad$ CENTRAL $=\$ 2.0-05 \quad$ PESSIMIISTIC $=\$ 6.4 \mathrm{E}-05$

ONSITE COSTS (DISCOUNTED TO PRESENT YEAR):

(AVERAGE COST $\times$ FREQUENCY OF CORE MELT)

MINIMUM $=\$ 2.1 \mathrm{E}+03 \quad$ CENTRAL $=\$ 3.4+04 \quad$ MAXIMUM $=\$ 1.6 \mathrm{E}+05$

MAXIMUM COST IN ANY ONE YEAR $=\$ 4.7 \mathrm{E}+09$

ONSITE COSTS UNCERTAINTY:

\begin{tabular}{|c|c|c|c|c|}
\hline & \multicolumn{3}{|c|}{ EREQUENCIES } \\
\hline & & $21 F_{+03}$ & CENTRAL & FESSINIS II \\
\hline COSTS & $\begin{array}{l}\text { OPT } \\
\text { CEN } \\
\text { PESS }\end{array}$ & $\begin{array}{l}2.1 \mathrm{E}+03 \\
3.5 \mathrm{E}+03 \\
4.9 \mathrm{E}+03\end{array}$ & $\begin{array}{l}2.1 \mathrm{E}+04 \\
3.4 \mathrm{E}+04 \\
4.8 \mathrm{E}+04\end{array}$ & $\begin{array}{l}0.7 \mathrm{E}+04 \\
1.1 \mathrm{E}+05 \\
1.6 \mathrm{E}+05\end{array}$ \\
\hline
\end{tabular}


CONSEQUENCE: EARLY FATALITIES PER YEAR

FRACTIONAL CONTRIBUTIONS OF DAMAGE STATES TO RISK, FOR OPTIMISTIC, CENTRAL AND PESSIMISTIC VALUES OF SEQUENCE DATA WITH ALL OTHER INPUTS AT CENTRAL VALUES, AND FOR SEQUENCE DATA AT CENTRAL VALUES WITH CFM'S RELEASES AND CONSEQUENCES ALL AT EITHER OPTIMISTIC OR PESSIMISTIC VALUES

DAMAGE STATE

$\begin{array}{ll} & \text { OPT } \\ \text { SYYB } & .13 \\ \text { SYNI } & 0.0 \\ \text { SNNN } & .04 \\ \text { SYNN } & .01 \\ \text { ANNN } & 0.0 \\ \text { AYNN } & \varepsilon \\ \text { AYNI } & .08 \\ \text { AYYB } & .01 \\ \text { TNNN } & .02 \\ \text { TYYBW } & .01 \\ \text { TYYBD } & .01 \\ \text { TYNI } & \varepsilon \\ \text { V } & .67\end{array}$

SEOUENCEDATA

CEN

.03

$\varepsilon$

.02

.01

$\varepsilon$

$\varepsilon$

.44

.01

.01

$\varepsilon$

$\varepsilon$

0.01

.45
CEM. RELEASES \&

CONSEQUENCES

PESS

.02

$\varepsilon$

.02

.01

$\varepsilon$

$\varepsilon$

.47

.01

.01

e

$\varepsilon$

.01

.44
QPT

PESS

0.0

0.0

0.0

0.0

0.0

0.0

0.0

0.0

0.0

0.0

0.0

0.0

0.0 $\varepsilon$

0.0

.01

$\varepsilon$

$\varepsilon$

$\varepsilon$

.01

$\varepsilon$

.01

$\varepsilon$

$\varepsilon$

$\varepsilon$

.94 
CONSEQUENCE: EARLY INJURIES PER YEAR

FRACTIONAL CONTRIBUTIONS OF DAMAGE STATES TO RISK, FOR OPTIMISTIC, CENTRAL AND PESSIMISTIC VALUES OF SEQUENCE DATA WITH ALL OTHER INPUTS AT CENTRAL VALUES, AND FOR SEQUENCE DATA AT CENTRAL VALUES WITH CFM'S RELEASES AND CONSEQUENCES ALL AT EITHER OPTIMISTIC OR PESSIMISTIC VALUES

\begin{tabular}{llllll} 
DAMAGE & \multicolumn{2}{c}{ SEQUENCEDATA } & & \multicolumn{2}{c}{ CEM. RELEASES \& } \\
STATE & & & & \multicolumn{2}{c}{ CONSEQUENCES } \\
& OPT & CEN & PESS & OPT & PESS \\
& & & & & \\
SYYB & .09 & .03 & .02 & 0.0 & $\varepsilon$ \\
SYNI & 0.0 & $\varepsilon$ & $\varepsilon$ & 0.0 & 0.0 \\
SNNN & .03 & .02 & .02 & 0.0 & .01 \\
SYNN & .01 & .01 & .01 & 0.0 & .10 \\
ANNN & 0.0 & $\varepsilon$ & $\varepsilon$ & 0.0 & 0.0 \\
AYNN & $\varepsilon$ & $\varepsilon$ & $\varepsilon$ & 0.0 & $\varepsilon$ \\
AYNI & .08 & .41 & .44 & 0.0 & $\varepsilon$ \\
AYYB & .01 & .01 & .01 & 0.0 & $\varepsilon$ \\
TNNN & .02 & .01 & .01 & 0.0 & .32 \\
TYYBW & .01 & $\varepsilon$ & $\varepsilon$ & 0.0 & .24 \\
TYYBD & .01 & $\varepsilon$ & $\varepsilon$ & 0.0 & .27 \\
TYNI & $\varepsilon$ & 0.01 & .01 & 0.0 & $\varepsilon$ \\
V & .71 & .49 & .47 & 0.0 & .04
\end{tabular}


CONSEQUENCE: LATENT CANCER FATALITY PER YEAR

FRACTIONAL CONTRIBUTIONS OF DAMAGE STATES TO RISK, FOR OPTIMISTIC, CENTRAL AND PESSIMISTIC VALUES OF SEQUENCE DATA WITH ALL OTHER INPUTS AT CENTRAL VALUES, AND FOR SEQUENCE DATA AT CENTRAL VALUES WITH CFM'S RELEASES AND CONSEQUENCES ALL AT EITHER OPTIMISTIC OR PESSIMISTIC VALUES

\begin{tabular}{llllll} 
DAMAGE & \multicolumn{2}{c}{ SEOUENCEDATA } & & \multicolumn{2}{c}{ CFM, RELEASES \& } \\
STATE & & & & \multicolumn{2}{c}{ CONSEOUENCES } \\
& OPT & CEN & PESS & OPT & PESS \\
& & & & & \\
SYYB & .24 & .09 & .08 & .07 & .12 \\
SYNI & 0.0 & $\varepsilon$ & $\varepsilon$ & $\varepsilon$ & $\varepsilon$ \\
SNNN & .08 & .07 & .06 & $\varepsilon$ & .36 \\
SYNN & $\varepsilon$ & .01 & .01 & .03 & $\varepsilon$ \\
ANNN & $\varepsilon$ & $\varepsilon$ & $\varepsilon$ & $\varepsilon$ & $\varepsilon$ \\
AYNN & $\varepsilon$ & $\varepsilon$ & $\varepsilon$ & $\varepsilon$ & $\varepsilon$ \\
AYNI & .03 & .22 & .24 & .81 & .01 \\
AYYB & .03 & .02 & .03 & .01 & .03 \\
TNNN & .04 & .01 & .01 & .01 & .17 \\
TYYBW & .02 & .01 & .01 & .01 & .06 \\
TYYBD & .03 & .01 & .01 & .01 & .07 \\
TYNI & $\varepsilon$ & $\varepsilon$ & $\varepsilon$ & .01 & $\varepsilon$ \\
V & .50 & .53 & .53 & .03 & .18
\end{tabular}


CONSEQUENCE: INDIVIDUAL RISK OF EARLY FATALITY

FRACTIONAL CONTRIBUTIONS OF DAMAGE STATES TO RISK, FOR OPTIMISTIC, CENTRAL AND PESSIMISTIC VALUES OF SEQUENCE DATA WITH ALL OTHER INPUTS AT CENTRAL VALUES, AND FOR SEQUENCE DATA AT CENTRAL VALUES WITH CFM'S RELEASES AND CONSEQUENCES ALL AT EITHER OPTIMISTIC OR PESSIMISTIC VALUES

\begin{tabular}{|c|c|c|c|c|c|}
\hline \multirow[t]{2}{*}{$\begin{array}{l}\text { DAMAGE } \\
\text { STATE }\end{array}$} & \multicolumn{3}{|c|}{ SEQUENCEDATA } & \multicolumn{2}{|c|}{$\begin{array}{l}\text { CEM.RELEASES \& } \\
\text { CONSEQUENCES }\end{array}$} \\
\hline & OPT & CEN & PESS & OPT & PESS \\
\hline SYYB & .31 & .13 & .12 & 0.0 & .04 \\
\hline SYNI & 0.0 & $\varepsilon$ & $\varepsilon$ & 0.0 & 0.0 \\
\hline SNNN & .10 & .09 & .09 & 0.0 & .08 \\
\hline SYNN & $\varepsilon$ & .01 & .01 & 0.0 & $\varepsilon$ \\
\hline ANNN & $\varepsilon$ & $\varepsilon$ & $\varepsilon$ & 0.0 & $\varepsilon$ \\
\hline AYNN & $\varepsilon$ & $\varepsilon$ & $\varepsilon$ & 0.0 & $\varepsilon$ \\
\hline AYNI & .01 & .13 & .14 & 0.0 & .02 \\
\hline AYYB & .02 & .04 & .03 & 0.0 & $\varepsilon$ \\
\hline TNNN & .02 & .02 & .02 & 0.0 & .01 \\
\hline TYYBW & .02 & .01 & .01 & 0.0 & $\varepsilon$ \\
\hline TYYBD & .02 & .01 & .01 & 0.0 & $\varepsilon$ \\
\hline TYNI & $\varepsilon$ & .01 & $\varepsilon$ & 0.0 & $\varepsilon$ \\
\hline V & .45 & .54 & .55 & 0.0 & .81 \\
\hline
\end{tabular}


CONSEQUENCE: OFFSITE COSTS PER YEAR

FRACTIONAL CONTRIBUTIONS OF DAMAGE STATES TO RISK, FOR OPTIMISTIC, CENTRAL AND PESSIMISTIC VALUES OF SEQUENCE DATA WITH ALL OTHER INPUTS AT CENTRAL VALUES, AND FOR SEQUENCE DATA AT CENTRAL VALUES WITH CFM'S RELEASES AND CONSEQUENCES ALL AT EITHER OPTIMISTIC OR PESSIMISTIC VALUES

DAMAGE SEQUENCEDATA

STATE

$\begin{array}{llll} & \text { OET } & \text { CEN } & \text { PES } \\ \text { SYYB } & .31 & .13 & .12 \\ \text { SYNI } & 0.0 & \varepsilon & \varepsilon \\ \text { SNNN } & .12 & .09 & .11 \\ \text { SYNN } & \varepsilon & .01 & .01 \\ \text { ANNN } & \varepsilon & \varepsilon & \varepsilon \\ \text { AYNN } & \varepsilon & \varepsilon & \varepsilon \\ \text { AYNI } & .02 & .24 & .27 \\ \text { AYYB } & .04 & .04 & .03 \\ \text { TNNN } & .06 & .05 & .05 \\ \text { TYYBW } & .04 & .02 & .02 \\ \text { TYYBD } & .05 & .02 & .02 \\ \text { TYNI } & \varepsilon & \varepsilon & \varepsilon \\ \text { V } & .31 & .37 & .37\end{array}$

CEM.RELEASES \&

CONSEQUENCES

QPT $\quad$ PESS

$\begin{array}{ll}.52 & .06 \\ .02 & \varepsilon \\ .02 & .38 \\ .01 & \varepsilon \\ \varepsilon & \varepsilon \\ \varepsilon & \varepsilon \\ .21 & .01 \\ .05 & .01 \\ .02 & .19 \\ .06 & .07 \\ .0 & .08 \\ \varepsilon & \varepsilon \\ \varepsilon & .19\end{array}$


CONSEQUENCE: POPULATION DOSE

FRACTIONAL CONTRIBUTIONS OF DAMAGE STATES TO RISK, FOR OPTIMISTIC, CENTRAL AND PESSIMISTIC VALUES OF SEQUENCE DATA WITH ALL OTHER INPUTS AT CENTRAL VALUES, AND FOR SEQUENCE DATA AT CENTRAL VALUES WITH CFM'S RELEASES AND CONSEQUENCES ALL AT EITHER OPTIMISTIC OR PESSIMISTIC VALUES

\begin{tabular}{|c|c|c|c|c|c|}
\hline \multirow[t]{2}{*}{$\begin{array}{l}\text { DAMAGE } \\
\text { STATE }\end{array}$} & \multicolumn{3}{|c|}{ SEQUENCEDATA } & \multicolumn{2}{|c|}{$\begin{array}{l}\text { CEM. RELEASES \& } \\
\text { CONSEQUENCES }\end{array}$} \\
\hline & OPT & CEN & PESS & OPT & PESS \\
\hline SYYB & .32 & .15 & .15 & .07 & .17 \\
\hline SYNI & 0.0 & $\varepsilon$ & $\varepsilon$ & $\varepsilon$ & $\varepsilon$ \\
\hline SNNN & .11 & .10 & .10 & $\varepsilon$ & .39 \\
\hline SYNN & $\varepsilon$ & $\varepsilon$ & $\varepsilon$ & .03 & $\varepsilon$ \\
\hline ANNN & $\varepsilon$ & $\varepsilon$ & $\varepsilon$ & $\varepsilon$ & $\varepsilon$ \\
\hline AYNN & $\varepsilon$ & $\varepsilon$ & $\varepsilon$ & $\varepsilon$ & $\varepsilon$ \\
\hline AYNI & .04 & .11 & .12 & .82 & $\varepsilon$ \\
\hline AYYB & .04 & .04 & .04 & .01 & .04 \\
\hline TNNN & .04 & .03 & .03 & $\varepsilon$ & .12 \\
\hline TYYBW & .03 & .02 & .01 & .01 & .04 \\
\hline TYYBD & .03 & .02 & .01 & .01 & .08 \\
\hline TYNI & $\varepsilon$ & $\varepsilon$ & $\varepsilon$ & .01 & $\varepsilon$ \\
\hline V & .41 & .52 & .54 & .03 & .18 \\
\hline
\end{tabular}


NUREG/CR-4551, VOL. 1: DRAFT REPORT FOR COMMENT (SEPTEMBER, 1986)

\section{D.4 OUTPUT FOR THE SENSITIVITY CASE WITHOUT DIRECT HEATING}

As described in Section 5.5 of the main report, a sensitivity analysis was performed for a case with all inputs the same as the base case except that direct heating was always precluded. This led to a number of insights concerning the importance of uncertainty issues. The detailed output for the early fatality and the latent cancer fatality risk measures as calculated with CRAC2 is provided here. This output enables more detailed comparison to the base case. 
CONSEQUENCE MEASURE:

EARLY DEATHS PER YEAR

SENSITIVITY CASE : NO DIRECT HEATING SURRY WALHC (CRAC)

\begin{tabular}{|c|c|c|}
\hline 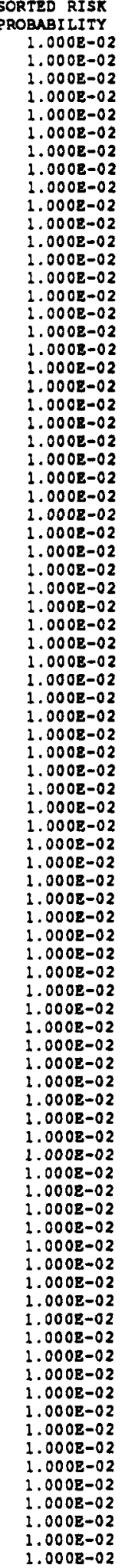 & 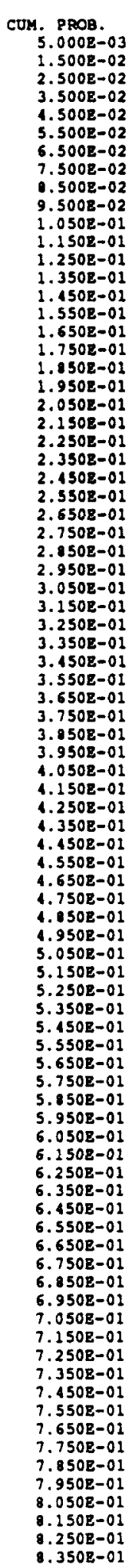 & 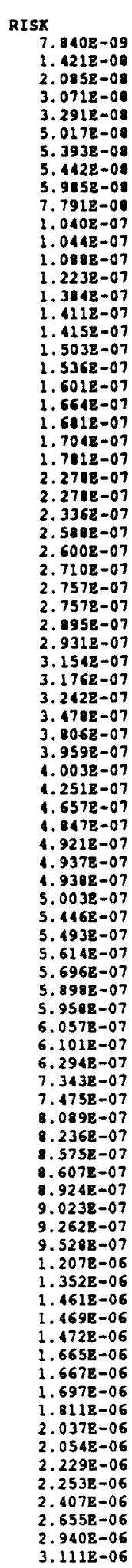 \\
\hline
\end{tabular}


NUREG/CR-4551, VOL. 1: DRAFT REPORT FOR COMMENT (SEPTEMBER, 1986)

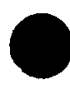

\begin{tabular}{|c|c|c|}
\hline $\begin{array}{l}1.000 \mathrm{E}-02 \\
1.000 \mathrm{E}-02 \\
1.000 \mathrm{E}-02 \\
1.000 \mathrm{E}-02 \\
1.000 \mathrm{E}-02 \\
1.000 \mathrm{E}-02 \\
1.000 \mathrm{E}-02 \\
1.000 \mathrm{E}-02 \\
1.000 \mathrm{E}-02 \\
1.000 \mathrm{E}-02 \\
1.000 \mathrm{E}-02 \\
1.000 \mathrm{E}-02 \\
1.000 \mathrm{E}-02 \\
1.000 \mathrm{E}-02 \\
1.000 \mathrm{E}-02 \\
1.000 \mathrm{E}-02\end{array}$ & $\begin{array}{l}9.450 \mathrm{E}-01 \\
8.550 \mathrm{z}-01 \\
8.650 \mathrm{z}-01 \\
8.750 \mathrm{z}-01 \\
9.850 \mathrm{z}-01 \\
8.950 \mathrm{z}-01 \\
9.050 \mathrm{z}-01 \\
9.150 \mathrm{z}-01 \\
9.250 \mathrm{z}-01 \\
9.350 \mathrm{z}-01 \\
9.450 \mathrm{z}-01 \\
9.550 \mathrm{z}-01 \\
9.650 \mathrm{z}-01 \\
9.750 \mathrm{z}-01 \\
9.950 \mathrm{z}-01 \\
9.930 \mathrm{z}-01\end{array}$ & $\begin{array}{l}3.512 \mathrm{E}-06 \\
3.699 \mathrm{E}-06 \\
3.713 \mathrm{E}-06 \\
4.264 \mathrm{E}-06 \\
4.271 \mathrm{E}-06 \\
5.205 \mathrm{E}-06 \\
6.507 \mathrm{E}-06 \\
.399 \mathrm{E}-06 \\
1.021 \mathrm{E}-05 \\
1.021 \mathrm{E}-05 \\
1.713 \mathrm{E}-05 \\
1.773 \mathrm{E}-05 \\
1.854 \mathrm{E}-05 \\
2.512 \mathrm{E}-05 \\
3.240 \mathrm{E}-05 \\
1.968 \mathrm{E}-04\end{array}$ \\
\hline
\end{tabular}

MEAN - $4.2648-06$ VARIANCE $=4.047 \mathrm{E}-10$

PERCENTILES : 4.0648-08 50-TH 5 -5.5538-07 95-TH -

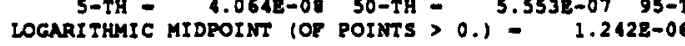

NOMBER ABOVE MIDPOINT - 32

NOMBER BELOM MIDPOINT - 68

CHI-SOOARED (1) - $1.296 \mathrm{E}+01$

REJECT NOLL (P $<.01$ ) 
SENSITIVITY CASE : NO DIRECT HEATING SURRY LALHC (CRAC) ISSUE LEVELS FOR EACH SAMPLE MEMBER, IN ORDER OF INCREASING RISK
RISK ISSOE LEVEL, FOR ALL ISSUES

\begin{tabular}{|c|c|c|c|c|c|c|c|c|c|c|c|c|c|}
\hline & & & & & & & & 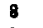 & & 10 & 111 & 12 & 13 \\
\hline $\begin{array}{l}7.840 \mathrm{E}-09 \\
1.421 \mathrm{E}-0 \mathrm{~g}\end{array}$ & $\begin{array}{l}2 \\
2\end{array}$ & $\frac{1}{1}$ & $\begin{array}{l}3 \\
2\end{array}$ & 2 & $\frac{1}{3}$ & $\frac{1}{6}$ & $\begin{array}{l}3 \\
2\end{array}$ & $\begin{array}{l}1 \\
2\end{array}$ & $\begin{array}{l}2 \\
1\end{array}$ & $\begin{array}{l}2 \\
1\end{array}$ & $\begin{array}{l}6 \\
5\end{array}$ & $\begin{array}{l}5 \\
4\end{array}$ & $\begin{array}{l}2 \\
1\end{array}$ \\
\hline $.085 E-08$ & 2 & 1 & 2 & & 3 & 6 & 1 & 1 & 5 & 3 & 2 & $i$ & 2 \\
\hline & 2 & 2 & 3 & & 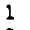 & & 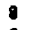 & 2 & & 4 & 1 & 5 & 2 \\
\hline 291 & 2 & 1 & 2 & 2 & 2 & 2 & 5 & 2 & 3 & 1 & 5 & 2 & 4 \\
\hline & 2 & 2 & 7 & & & & & & & 3 & & & \\
\hline & 2 & 1 & 7 & & 2 & & & 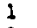 & 3 & 2 & 4 & 3 & 5 \\
\hline 08 & 2 & 1 & 2 & & 3 & & & 2 & 1 & 2 & 4 & 1 & 3 \\
\hline & & 1 & 2 & & 2 & & 7 & & 2 & 3 & 1 & & 3 \\
\hline 08 & 2 & 2 & 2 & 1 & 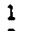 & & 1 & 2 & 3 & 2 & 3 & 3 & 3 \\
\hline .040 & & 1 & 2 & 2 & 2 & & 7 & 2 & & 2 & 3 & 4 & 2 \\
\hline & & & & & 3 & & & & & 2 & 3 & 4 & 2 \\
\hline 1.088 & 2 & 1 & 3 & 2 & 3 & & 1 & 3 & 4 & 3 & 3 & 1 & 1 \\
\hline & & 2 & & & & 2 & & & & 2 & 1 & & \\
\hline 3 & & 2 & 7 & & 1 & & & & & 2 & 4 & 3 & 4 \\
\hline 9 & & 2 & 2 & 1 & 1 & 5 & 7 & 2 & 5 & 2 & 2 & 3 & 1 \\
\hline & & 1 & & & 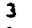 & & & 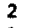 & & 2 & 4 & 3 & 5 \\
\hline .5 & 1 & 1 & 3 & & 1 & 2 & 6 & 1 & 5 & 3 & 2 & 4 & 3 \\
\hline 7 & & 2 & 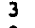 & 1 & 1 & 3 & 1 & & & 2 & 2 & 1 & 3 \\
\hline 27 & $-5+2+$ & 1 & 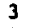 & 1 & 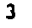 & 6 & - & 3 & 1 & 3 & 3 & 1 & 2 \\
\hline 07 & 2 & 1 & 2 & 1 & 2 & 6 & 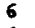 & 2 & 2 & 3 & 3 & 4 & 3 \\
\hline 1.6 & & 2 & 2 & 1 & 2 & 3 & 6 & 4 & 1 & 3 & 4 & 5 & 2 \\
\hline & 2 & 2 & - & 1 & 2 & & 3 & 2 & 4 & 2 & 5 & 4 & 2 \\
\hline 07 & 2 & 2 & 2 & 3 & 2 & 5 & 3 & 4 & $?$ & 2 & 4 & 4 & 3 \\
\hline .07 & 2 & 1 & 3 & 2 & 2 & 6 & 7 & 2 & 2 & 3 & 1 & 1 & 2 \\
\hline 07 & 2 & 1 & 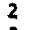 & 2 & 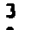 & 6 & 4 & 2 & & 2 & 4 & 2 & 3 \\
\hline 07 & 2 & 1 & 3 & 2 & 2 & 2 & & 2 & 2 & 4 & 1 & 5 & 9 \\
\hline 07 & & 1 & 2 & 2 & 1 & 5 & $?$ & 1 & 2 & 1 & 4 & 5 & 3 \\
\hline 07 & 2 & 2 & 2 & 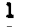 & 1 & 5 & & 3 & & 1 & 1 & 2 & 2 \\
\hline-07 & 2 & 2 & 3 & 2 & 2 & 3 & & & & 3 & 3 & 2 & \\
\hline 7 & 2 & & 2 & 2 & 2 & 2 & 7 & 2 & 1 & 2 & 4 & 3 & 4 \\
\hline & 2 & & & & 2 & & & & & 3 & 2 & 2 & 5 \\
\hline 17 & 2 & 2 & 3 & & 3 & s & 4 & 2 & & 2 & 3 & 2 & 3 \\
\hline 07 & $\mathrm{~J}$ & 1 & 2 & 1 & 3 & 2 & 2 & 3 & & 3 & 1 & 5 & 3 \\
\hline & 2 & 2 & 4 & 1 & 2 & & 5 & 1 & & 2 & 3 & 4 & 3 \\
\hline 07 & 2 & 1 & 1 & 3 & 1 & 6 & 3 & 3 & 3 & 2 & 4 & 5 & 3 \\
\hline 07 & 2 & 1 & 2 & 1 & I & 6 & 2 & 1 & 2 & 3 & 3 & 1 & 3 \\
\hline & $2+2>$ & & 2 & 1 & 2 & & & 5 & & 4 & 1 & 1 & \\
\hline 7 & 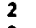 & 2 & 3 & 1 & 1 & 6 & 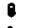 & 3 & 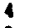 & 3 & 3 & 2 & 3 \\
\hline 9 & 2 & 1 & 2 & 2 & 2 & 5 & I & , & 2 & 2 & 6 & 5 & 1 \\
\hline $003 E-07$ & 2 & 2 & 3 & 2 & 1 & 5 & 3 & 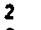 & 1 & 2 & 5 & 5 & 2 \\
\hline 7 & 2 & 1 & 2 & 1 & 2 & 3 & 9 & 3 & & 2 & 5 & 4 & 2 \\
\hline & 2 & 1 & 2 & 1 & 3 & 6 & & 5 & & 1 & 6 & 4 & 2 \\
\hline 7 & 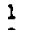 & 2 & 3 & 1 & 1 & 6 & 1 & 4 & & 1 & 6 & 4 & 1 \\
\hline $.9218-07$ & & 1 & 2 & 2 & 2 & 6 & 4 & 3 & 3 & 2 & 5 & 1 & 1 \\
\hline & 2 & 2 & 3 & 3 & 1 & 6 & & 2 & & 2 & 5 & 3 & 2 \\
\hline 7 & 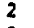 & 1 & 2 & 1 & 2 & 3 & 9 & 5 & 3 & 3 & 2 & 3 & 5 \\
\hline 7 & $7+2>$ & 2 & 2 & 1 & 3 & 2 & 5 & 5 & & 2 & 4 & 2 & 2 \\
\hline & 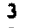 & 1 & 3 & 1 & 1 & 1 & e & 2 & & 4 & 1 & 3 & 3 \\
\hline 4 & 2 & 1 & 3 & 2 & 1 & 3 & 6 & 5 & & 2 & 4 & 5 & 3 \\
\hline 7 & & 2 & 2 & 2 & 3 & 4 & 2 & 3 & 3 & 2 & 3 & 2 & 4 \\
\hline 7 & 2 & 2 & 2 & 2 & 3 & 3 & & 1 & & . & 4 & e & 3 \\
\hline $898 \mathrm{E}-07$ & 2 & 2 & 2 & 2 & 3 & 2 & 6 & 2 & 3 & 2 & 4 & 3 & 2 \\
\hline $958 \mathrm{E}-07$ & 2 & 1 & 3 & 1 & 3 & 6 & 8 & 4 & 1 & 3 & 2 & 3 & 2 \\
\hline & 2 & 2 & 2 & 1 & 3 & 3 & & s & & 2 & 4 & 2 & 5 \\
\hline $1018-07$ & 1 & 1 & 3 & $1+2+3$ & 3 & 3 & & 5 & & 3 & 3 & & \\
\hline 7 & 2 & 2 & 3 & 2 & 2 & 3 & d & 1 & 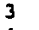 & 4 & 1 & 2 & 2 \\
\hline 7 & 2 & 1 & 2 & 2 & 3 & 6 & & $-2+3$ & & 2 & 5 & 4 & 4 \\
\hline $4758-07$ & 2 & 2 & 2 & 1 & 2 & 2 & & 7 & & 3 & 1 & 5 & 3 \\
\hline$=-07$ & 2 & 1 & 3 & 2 & 1 & 3 & 4 & 4 & 3 & 2 & 4 & 1 & 3 \\
\hline 7 & 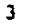 & 2 & 2 & 2 & 1 & 3 & & 3 & & 3 & 3 & & 1 \\
\hline 5 & 2 & 1 & 2 & 3 & 2 & 1 & & 2 & & 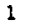 & 6 & 1 & 3 \\
\hline-07 & 2 & 1 & 3 & 2 & 1 & 6 & & 5 & 1 & 3 & 4 & 2 & 3 \\
\hline 9 & $2+2>$ & 2 & 2 & & 2 & 6 & & 1 & & & 6 & & 2 \\
\hline 0 & 2 & 2 & 2 & 2 & 3 & 1 & & 1 & 3 & 3 & 2 & 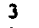 & 3 \\
\hline 2 & 2 & 2 & 3 & 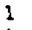 & 2 & 2 & & 1 & & 2 & 5 & 1 & 3 \\
\hline 9.5 & 2 & 1 & 2 & & 3 & $6+2>$ & & $a$ & & 1 & 5 & & \\
\hline 3 & 3 & 1 & 2 & $\lambda$ & 3 & 6 & 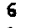 & 1 & 3 & 4 & 1 & 3 & 5 \\
\hline 3 & 2 & 2 & 3 & 1 & 1 & 1 & & 3 & & 1 & 4 & 2 & 5 \\
\hline & 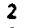 & 1 & 2 & 2 & 1 & 1 & & 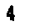 & & 2 & 3 & 5 & \\
\hline 06 & 2 & 2 & 2 & & 1 & 6 & & 1 & 6 & 3 & 3 & 4 & 5 \\
\hline & 2 & 1 & 2 & 2 & 2 & 6 & & 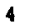 & & 2 & $\because$ & 3 & 2 \\
\hline & 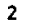 & 1 & 3 & & 1 & 3 & & 3 & & 2 & 4 & 1 & 2 \\
\hline 1.6 & 2 & 1 & 2 & 2 & 1 & 2 & 2 & 2 & 3 & 2 & 4 & 3 & 2 \\
\hline 6 & 2 & 1 & 3 & 1 & I & 2 & & 1 & & 1 & 5 & $a$ & 4 \\
\hline & 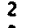 & 1 & 2 & & 2 & 2 & & 5 & & 3 & 3 & 5 & 4 \\
\hline-06 & 2 & 2 & 3 & 3 & 1 & 4 & 5 & 1 & & 2 & 4 & 2 & 2 \\
\hline & 2 & 2 & 2 & 2 & 3 & 2 & & 4 & & 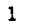 & 0 & 2 & 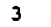 \\
\hline 2 & 2 & 1 & 3 & 1 & 2 & 2 & 9 & 3 & & 2 & 3 & 3 & 4 \\
\hline & 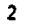 & 1 & 2 & I & 2 & 3 & 1 & 5 & 7 & 1 & 4 & 5 & 2 \\
\hline & 3 & 2 & 2 & 2 & 2 & 1 & 6 & 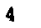 & & 1 & 4 & 1 & 3 \\
\hline E- & $2+>$ & 1 & 2 & 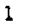 & 2 & 6 & & $a$ & & 2 & 2 & 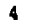 & 2 \\
\hline $20100-06$ & ? & 2 & 2 & 7 & 2 & 6 & 9 & ; & & 3 & 1 & 4 & 3 \\
\hline & 2 & 2 & 3 & 3 & 2 & 4 & 7 & 3 & 3 & 2 & 4 & 2 & 3 \\
\hline & & 2 & 2 & & 3 & & 3 & & & 2 & 3 & 5 & 2 \\
\hline
\end{tabular}


NUREG/CR-4551, VOL. 1: DRAFT REPORT FOR COMMENT (SEPTEMBER, 1986)

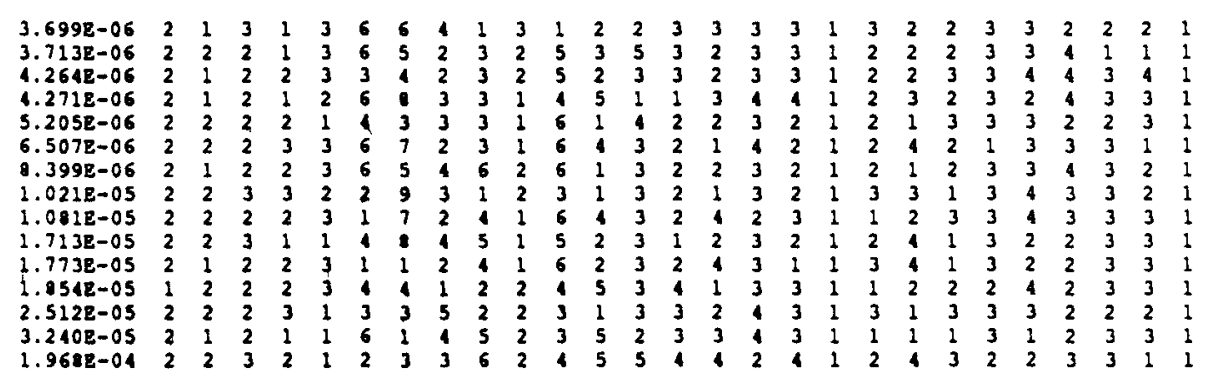


NUREG/CR-4551, VOL. 1: DRAFT REPORT FOR COMMENT (SEPTEMBER, 1986)

CONSEOURNCE MEASURE.

EARLY DEATHS PER YEAR

SENSITIVITY CASE : NO DIRECT HEATING

SURRY LALHC (CRAC)

FRACTIONAL CONTRIBUTION OF EACH SEQUENCE TO MEAN RISK

SEQUENCE CONTRIBDTION

SYYB $\quad 0.0014$

sYr?

SMin

SYNN

ANMN

0.0001

0.4808

0.0026

AYMN

0.0339

AYNI

0.0339

AYrI

0.0012

TANN

0.2284

TYYBN

TYYBD

0.0280

0.0457

0.0009

TYNI

0.0939

CONSZOUENCE MEASURE

EARLY DEATHS PER YEAR

ERACTIONAL CONTRIBOTIOH OF EACH BIY TO MEN RIST

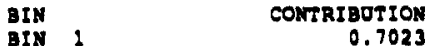

BIN $1 \quad 0.7023$

BIN 2

0.0025

0.0004

BIA 5

BIN 6

BIN $7 \quad 0.0002$

BIM 0.0000

BIN 90.0009

BIX 10

Btw $11 \quad 0.0148$

BIN $12 \quad 0.1380$

BIS $13 \quad 0.0000$

BIN 14

0.0000

BIN $17 \quad 0.0000$

CONSEOUENCE MEASURE

EARLY DEATHS PER YEAR

0.0000

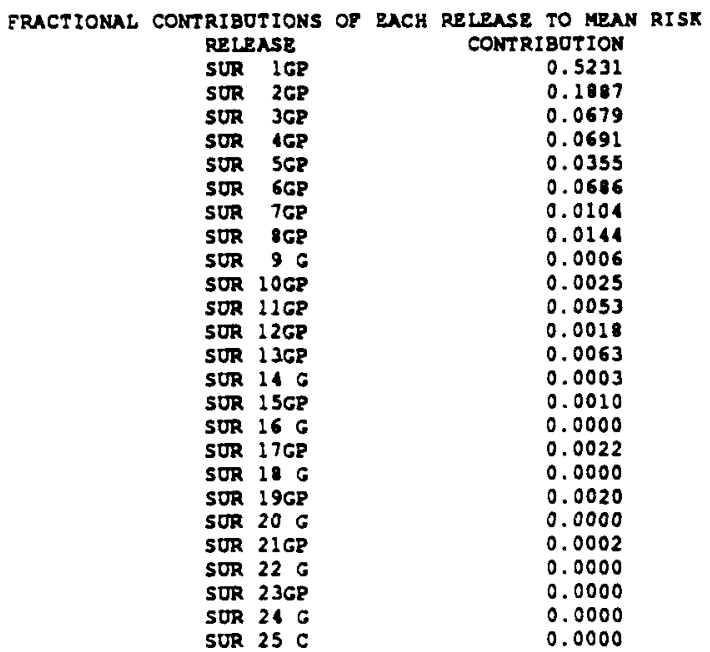




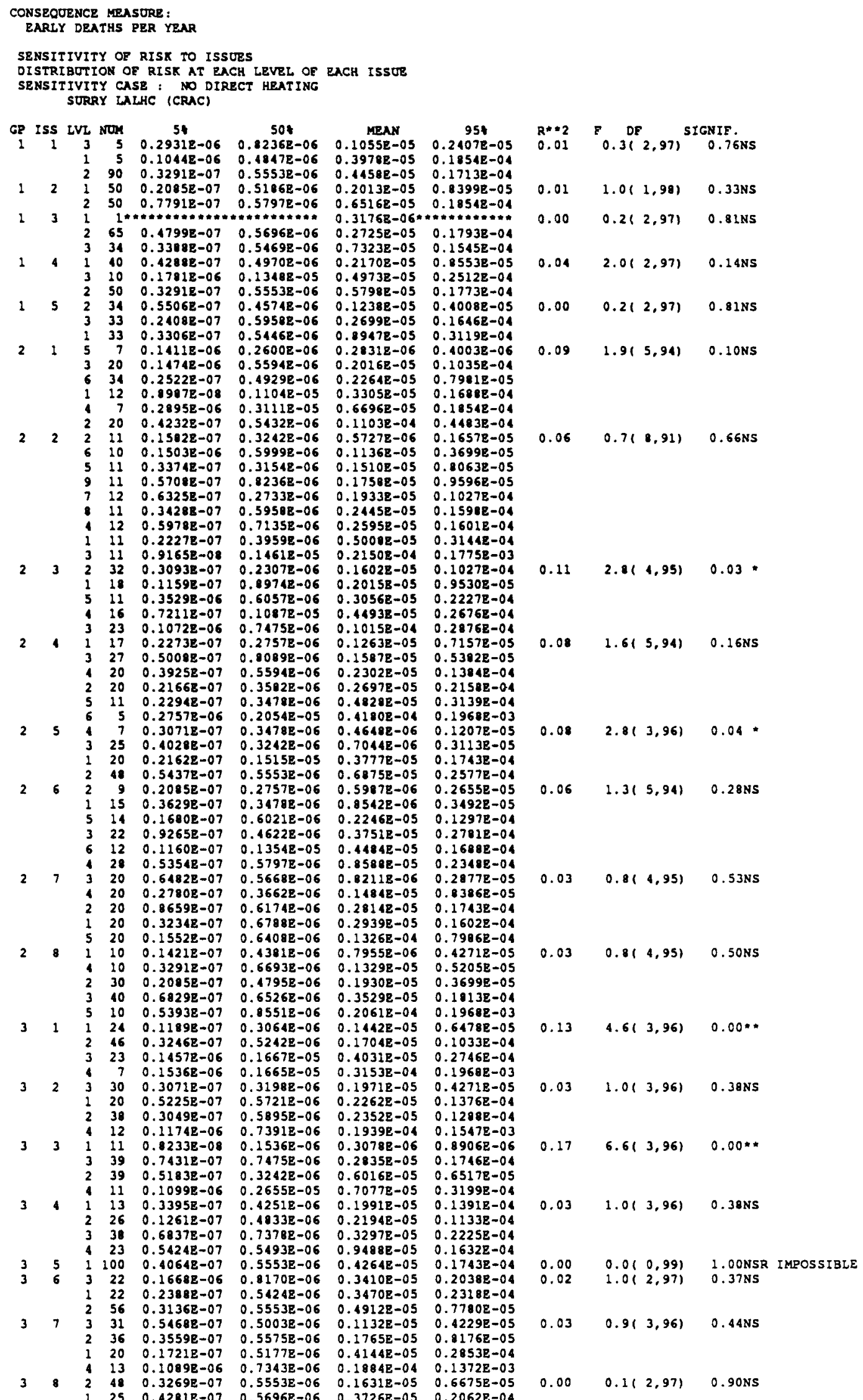


NUREG/CR-4551, VOL. 1: DRAFT REPORT FOR COMMENT (SEPTEMBER, 1986)

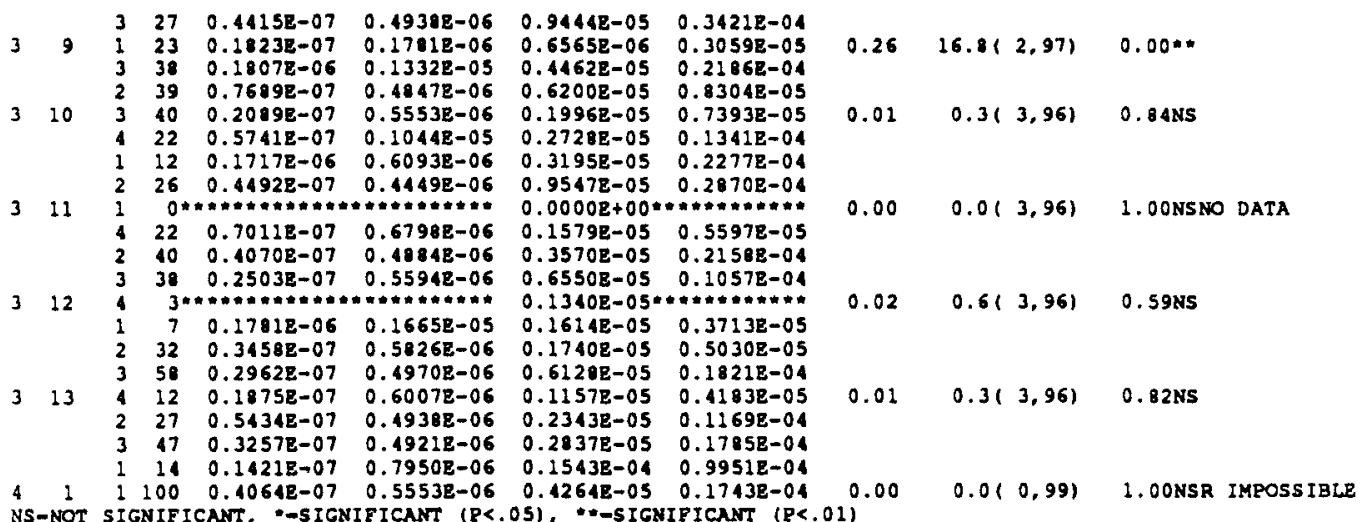


CONSEQUENCE MRASURE; EARYY DEATHS PER YRAR

ISSUE GROUP - 1 ISSUE - 5 FIF2 FAILURE

LEVEL 1 NO. SNED, - 17 NO. EXP. - 16

NO. >-NED. - 16 NO. EXP. - 17

LEVEL 2 No. LHED. - 19 No. gxe. - 17

No.>-HED. - is No. EXP.- 17

LEVEL 3 NO. KMED. - 13 HO. EXP. - 16

No.>-MED. -20 No. EXP. -17

OVERALL CHI-SO- 1.7 DF- 5 5IG.- 0.891

IS\$UE GROOP - 2 IS\$OE - 1 INO. RCS EAIL.

LEVEL \& NO. <MED. - 4 NO. EXP.- 6

NO.> TED. - DO. EXP. - 6

LEVEL 2 NO. MED. - 10 NO. EXP. - 10

NO.> MED. - 10 NO. EXP. -10

LEVEL 3 NO. SMED. - 9 NO. EXP. - 10

No.> WED. - 11 No. EXP.- 10

No.>HRD.- 6 No. EXP.-

No.>ARO.- 0 No. EXP.- 4

NO.> HED. - 16 NO. EXP. -17

LEVEL 6 NO. LMRD. - 18 NO. EXP. - 17

OVERALE CHI-SO- 1.7 DF- 7 SIG. 0.977
ISSUR GROUP - 2 ISSUE - 2 WODE OF VB.

LEVEL I NO. SHED.- 7 NO. EXP.- 5 LEVEL 2 NO. CMED.- 6 NO. EXP.- 5 LEVEL 3 NO. <MED.- 4 NO. EXP.- 5 LEVEL 4 NO. <HED.- 5 NO. EXP.- 6 LEVEL 5 NO. <MED.- 7 NO. EXP.- 5 LEVEL 6 NO. CMED. - 3 NO. EXP.- 5 LEVEL 7 NO. CMED. - 7 NO. EXP.- 6

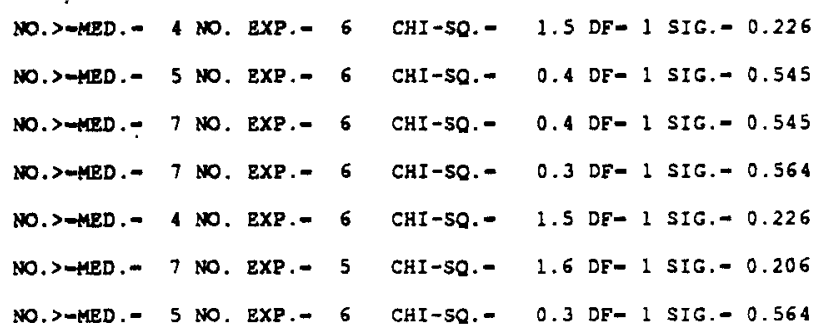

CHI-50. - 1.5 DF- 1 SIG. $=0.226$ CHI-5O.- 0.4 DE- 1 SIG. $=0.545$ CHI-SO.- 0.4 DF- 1 SIG. 0.545 CHI-SQ.- 0.3 DF- 1 SIG.- 0.564 CHI-5O.- 1.5 DF- 1 SIG. $=0.226$ CHI-SO.- 1.6 DF- 1 SIG. $=0.206$ CHI-5O.- 0.3 DF- I SIG. -0.564

CHI-SO. TEST CANHOT BE RUN: EXP. NO. < 5 CHI-SQ.- 0.0 DF 1 SIG. 0.833 CHI-SO. TEST CANNOT BE RON: EXP. NO. < 5

CHI-SO. $=0.1$ DF $=1$ SIG. $=0.777$

CHI-5Q. $=0.3$ DF- I SIG. 0.572

CHI-SQ. TEST CANNOT BE RUN: EXP. NO. <5 CHI-50.- 0.1 DF= 1 SIG.- 0.804 CHI-5Q.- 0.0 DF- 1 SIG. -1.000

CHI-SO.- 0.4 DF- 1 SIG.- 0.527 CHI-SO.- 0.1 DF- 1 SIG. 0.777 CHI-sQ.- 1.6 DF- 1 sIG.- 0.206

CHI-5O. $=0.1$ DF- 1 SIG. -0.728 CHI-SQ.- 0.5 DF- 1 SIG.- 0.493 CHI-SQ.- 1.1 DE- 1 SIG.- 0.296

CHI-SO.- 1.3 DF- 1 SIG. 0.248 CHI-50.- 0.0 DF- 1 SIG.- 1.000 CHI-SQ. - 0.2 DF- I SIG. 0.655 CHI-SO. TEST CANNOT BE RUN; EXP. NO. < 5 CHI-SO. TEST CANNOT BE RUN; EXP. NO. < 5 CHI-SO. - 0.1 DF- 1 SIG. -0.732 


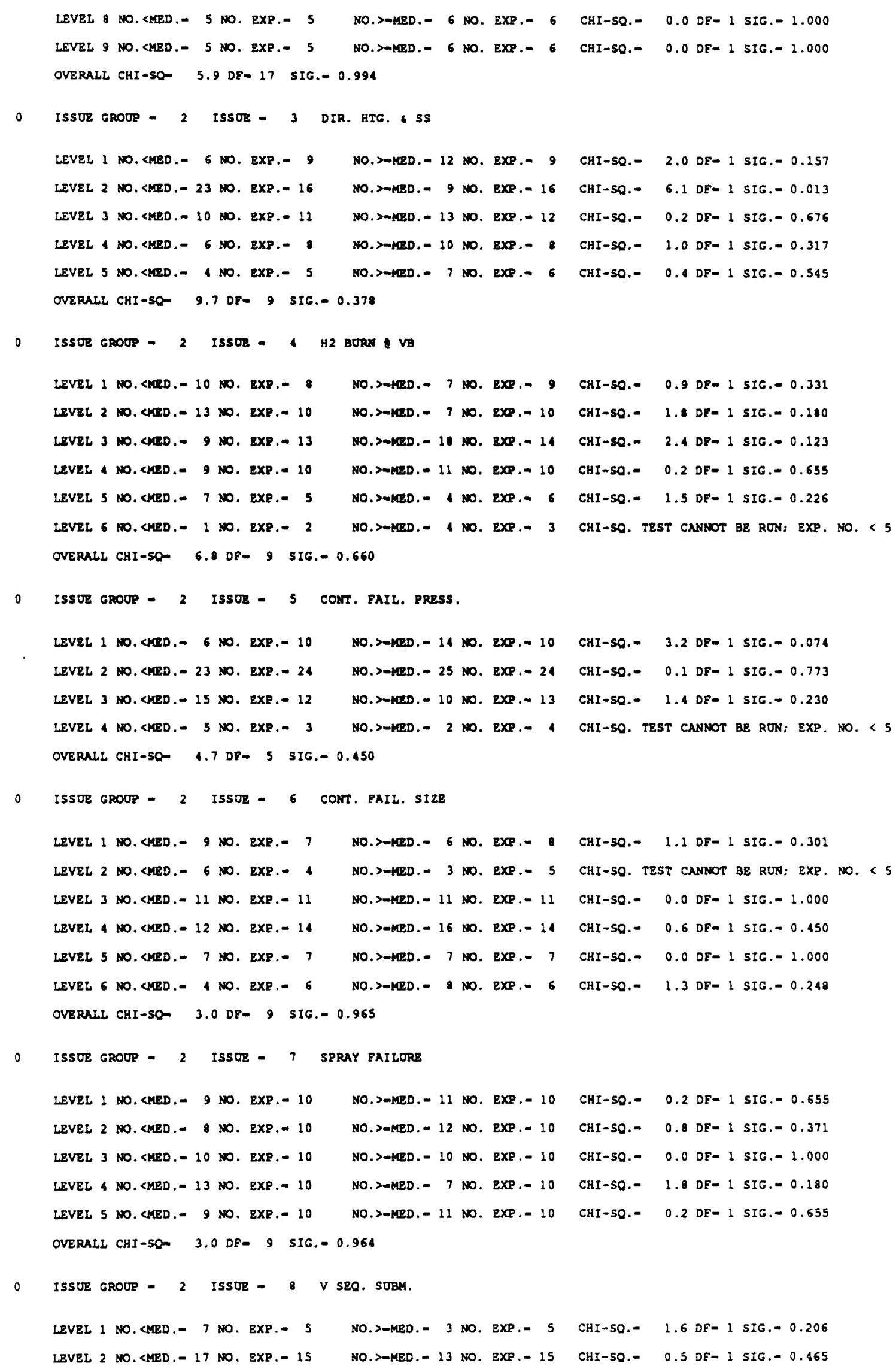

CHI-50.- 0.9 DF- 1 SIC. -0.331

CHI-so.- 1.8 DF- 1 SIG.- 0.180

CHI-5O.- 2.4 DF- 1 SIG. -0.123

CHI-SO.- 0.2 DF- 1 SIG. -0.655

CHI-50.- 1.5 DF- 1 SIG. 0.226

CHI-SO. TEST CANNOT BE RON: EXP. NO. < 5

CHI-SO.- 3.2 DF- 1 SIG.- 0.074

CHI-50.- 0.1 DF- I SIG. 0.773

CHI-SO.- 1.4 DF- 1 SIG. -0.230

CHI-SO. TEST CANNOT BE RUN; EXP. NO. $<5$

CHI-50.- 1.1 DF- 1 SIG.- 0.301 CHI-SO. TEST CANNOT BE RUN: EXP. NO. < 5 CHI-SO.- 0.0 DF- 1 SIG. 1.000 CHI-SO.- 0.6 DF- 1 SIG. -0.450 CHI-SO.- 0.0 DF- 1 SIG.- 1.000 CHI-SO.- 1.3 DF- 1 SIG. 0.248

CHI-SO.- 0.2 DF- 1 SIG.- 0.655 CHI-SO.- 0.8 DF- 1 SIG.- 0.371 CHI-SQ.- 0.0 DF- 1 SIG.- 1.000 CHI-SQ.- 1.8 DF- 1 SIG. -0.180 CHI-SQ.- 0.2 DF- 1 SIG. -0.655 
LEVEL 3 NO. LMED. - 18 NO. EXP. - 20 LEVEL 4 No. CHeD.- 3 NO. Exe.- 5 LEVEL 5 NO. CHED.- 4 NO. EXP.- 5 OVERALL CHI-SO- $1.5 \mathrm{DF}-9$ STG.- 0.873

LEVEL 1 NO. LMED.- 17 NO. EXP. - 12 LEVEL 2 NO. SHED.- 23 NO. EXP.- 23 LEVEL 3 NO. <MED.- 7 No. EXP.- 11 LZVEL 4 No. 〈MED.- 2 No. EXP.- 3 OVERALL CHI-5Q- 7.0 DP- 5 SIG. -0.22

ISSUE GROOP - 3 ISSUE - 6 CCI REL

LEVEL 1 NO. <LRD. - 11 NO. EXP . - 11

NO. $>-N D 0 .-11$ NO. EXP. - 11 LEVEL 2 No. SMED. - 27 NO. EXP. - 28 NO.> KIDD. - 29 NO. EXP. - 28 No.>ABD. - 11 No. EXE.- 11 LEVEL 3 No. <MED. - 11 No. EXP. - 11 OVERALL CHI-SQ- 0.1 DF- 5 SIG.- 1.000 ISSUE GROUP - 3 ISSUE - 7 FCONC

LEVEL 1 NO. SMED. - 11 NO. EXP. - 10 LEVEL 2 NO. LMED. - 18 NO. EXP. - 18 NO. > HED. - 9 NO. EXP.- 10 NO.>-MED. - 18 NO. EXP. - 18 LEVEL 3 NO. SMED.- 16 NO. EXP. - 15 No.>-100. - 15 NO. EXP. - 16 LEVEL 4 NO. QYED.- 4 NO. EXP.- 6 NO.>MED.- 9 NO. EXR.- 7 OVERALL CHI-SO- $1.6 \mathrm{DF}-7$ SIG.- 0.980

ISSUE GROUP - 3 ISSUE - 8 SPRAY DF
CHI-5Q. $=0.4$ DF- 1 SIG. $=0.527$ CHI-SO.- 1.6 DF- 1 SIG.- 0.206 CHI-50. $=0.4$ DF- 1 SIG. -0.527

CHI-50.- 4.2 DF- I SIG.- 0.041 CHI-50.- 0.0 DF- 1 SIG.- 1.000 CHI-5Q.- 2.9 DF- 1 SIG.- 0.095 CHI-5O. TEST CANLOT BE RCN; EXP. NO. < 5

CHI-SQ. $=0.2$ DF- 1 SIG. -0.655 CHI-SO.- 0.1 DF- 1 SIG. 0.746 CHI-50.- 0.5 DF- I SIG.- 0.465 CHI-50.- 0.3 DF $=1$ SIG. -0.564

CHI-50.- 5.9 DF- 1 SIG.- 0.015 CHI-50.- 0.9 DF- 1 SIG. -0.336 CHI-SO. - 0.9 DF- 2 SIG.- 0.336 CHI-SO. - 3.3 DF= 1 SIG. 0.069

CHI-SO.- 2.8 DF- 1 SIG.- 0.095 CHI-SQ.- 0.0 DF- 1 SIG.- 1.000 CHI-SO. $\quad 0.9$ DF- 1 SIG.- 0.330 CHI-SO.- 0.0 DF- 1 SIG.- 1.000

CHI-SQ. $=0.0$ DF- 1 SIG.- 1.000 CHI-50.- 0.1 DF- 1 SIG.- 0.789 CHI-SO.- 0.0 DF- 1 SIG. -1.000

CHI-SO.- 0.2 DF- 1 SIG.- 0.655 CHI-SQ.- 0.0 DF- 1 SIG.- 1.000 CHI-50.- 0.1 DF- 1 SIG. 0.719 CHI-SO.- 1.2 DF- 1 SIG. 0.266 


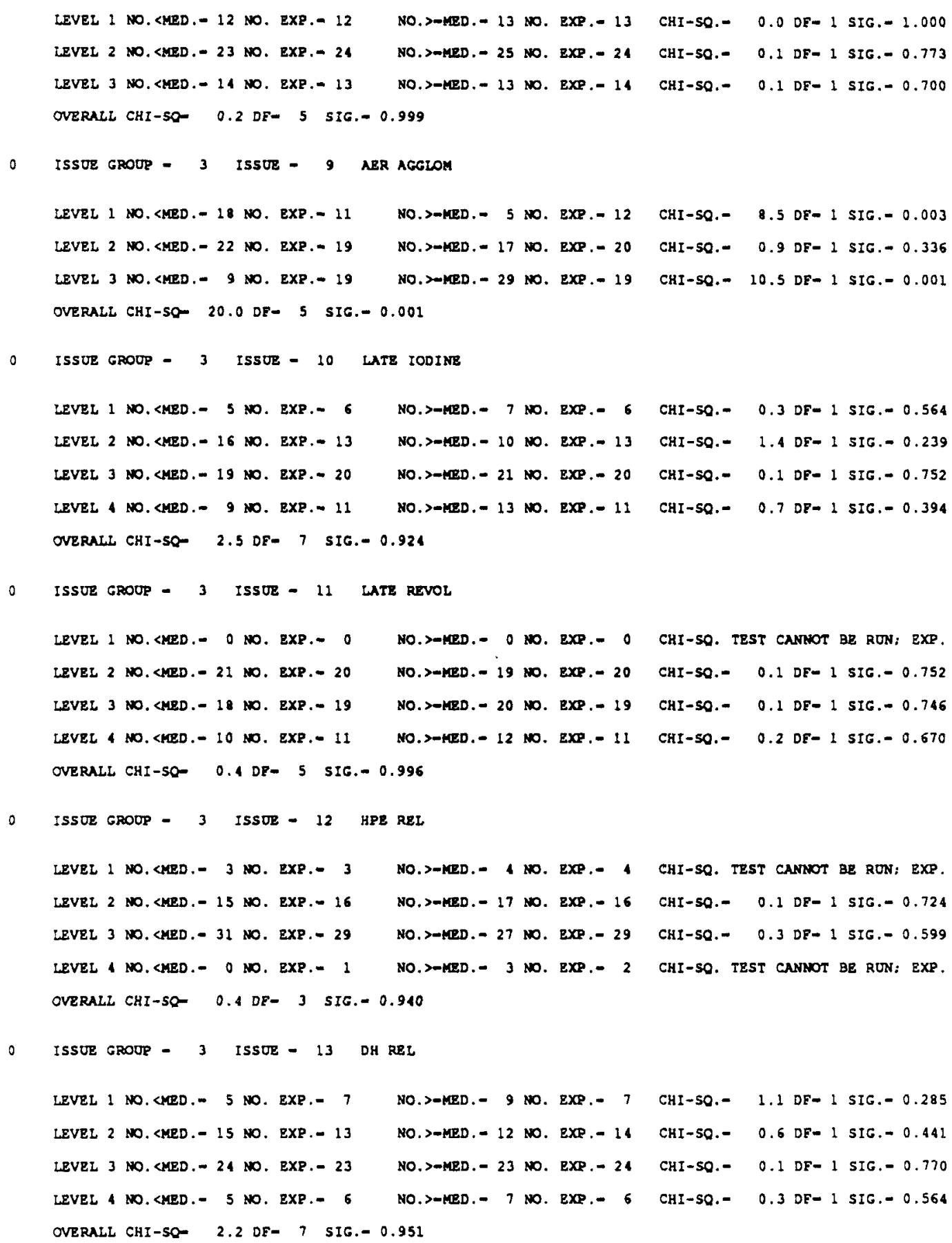


NUREG/C.2-4551, VOL. 1: DRAFT REPORT FOR COMMENT (SEPTEMBER, 1986)

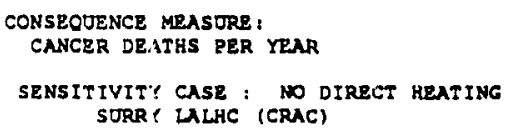

7. 350z-41

7. $350 z-01$

$7.550 \mathrm{e}-(1) 1$

$7.650 \mathrm{E}-01$

7. $750 \mathrm{E}-02$

$7.850 \mathrm{E}-91$

$7.950 \mathrm{E}-01$

8. $050 \mathrm{E}-01$

. 15OE- 11

b. $250 \mathrm{E}-121$

9. $350 E-(1)$

RISK

i. $675 \mathrm{E}-05$

1. $+728-05$

$\ldots .0418-04$

$.2878-04$

. $3318-04$

$+.3318-04$

.. $128-04$

1. $9038-04$

$1.916 E-04$

2.0208-04

2. $1428-04$

$2.201 \mathrm{E}-04$

2.265B-04

2. 3148-04

$3.127 \mathrm{E}-04$

2. $429 \mathrm{E}-04$

$2.4298-04$

$2.4418-04$

$2.6048-04$

$2.649 \mathrm{E}-04$

2. $693 \mathrm{E}-04$

$2.728 \mathrm{~g}-04$

2.7288-04

2. $7528-04$

2.015E-04

$2.824 \mathrm{E}-04$

2. $8448-04$

$2.936 \mathrm{z}-04$

$2.936 \mathrm{E}-04$

3. $0698-04$

$3.1198-04$

$3.1478-04$

3. $200 \mathrm{z}-04$

1. 2628-04

1, 2078-04

3. $3148-04$

3, $521 \mathrm{~B}-04$

3. $556 \mathrm{E}-04$

3. $6512-04$

$3.7978-04$

3. $819 e-04$

$3.0708-04$

1.0508-04

$4,060 \mathrm{z}-04$

$4.1858-04$

1. 224R-04

1. 3928-0

1. $4438-04$

1. $4540-04$

$4542-04$
$1.7462-04$

1. $7462-04$

I. $878 \mathrm{E}-04$

$.960 \mathrm{E}-04$

1. $9778-04$

$5.0338-04$

5. $0412-04$

5. $0662-04$

j. $227 z-04$

5. $4298-04$

$5.476 \mathrm{E}-04$

5. $603 \mathrm{E}-04$

'. $631 \mathrm{E}-04$

j. $1948-04$

6. $046 \mathrm{z}-04$

$6.886 \mathrm{E}-04$

$1.0648-04$

$7.233 \mathrm{E}-04$

1. $3058-04$

7. $5078-04$

$7.5078-04$

. $9578-04$

$7.9978-04$

$3.090 \mathrm{E}-04$

a. 3638-04

3. $7728-04$

9. 135E-04

$130-04$

$4558-04$

.5438-04

$9.8748-04$

$1.0398-0.3$

$1.1448-0$

1. 440E-0.3

1. $472 \mathrm{~B}-0.3$

1. $5198-03$

1. $6218-0$

$1.621 \mathrm{E}-03$

$2.140 \mathrm{~B}-03$ 
NUREG/CR-4551, VOL. 1: DRAFT REPORT FOR COMMENT (SEPTEMBER, 1986)

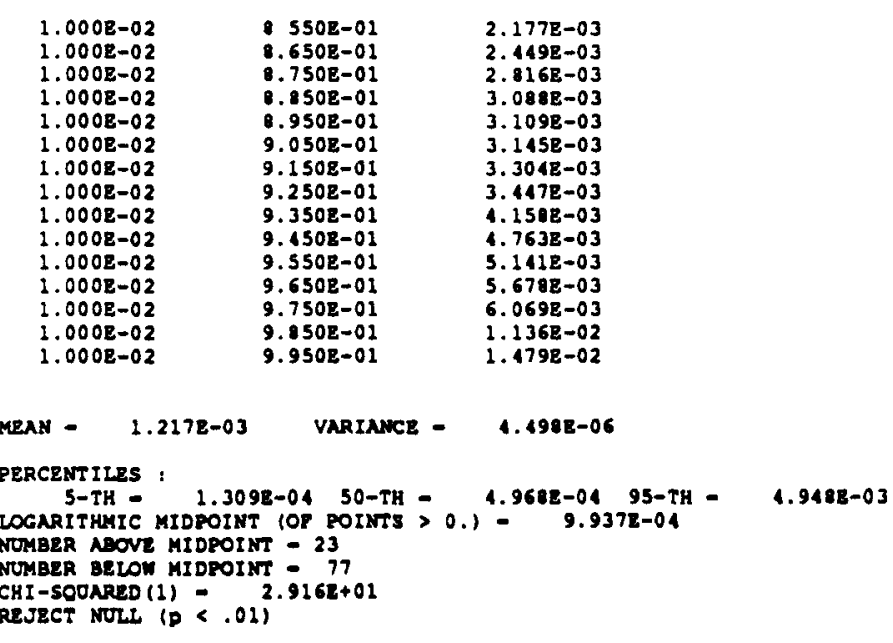


NUREG/CR-4551, VOL. 1: DRAFT REPORT FOR COMMENT (SEPTEMBER, 1986)

CONSEOUENCE KEASURE:

CANCER DEATHS PER YEAR

SENSITIVITY CASE : NO DIRECT HEATING SORRY LALHC (CRAC)

ISSUE LEVELS FOR EACH SAYPLE MEMBER, IN ORDER OP INCREASING RISK RIST ISSUE LEVEIS, FOR ALL ISSUES

\begin{tabular}{|c|c|c|c|c|c|c|c|c|c|c|c|c|}
\hline & & 2 & 3 & 4 & 5 & 6 & 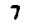 & 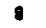 & & 10 & 111 & 12 \\
\hline $6.675 \mathrm{~B}-0.05$ & 2 & 1 & 3 & 2 & 1 & 1 & 3 & 1 & 2 & 2 & 6 & 5 \\
\hline $.472 \mathrm{E}-05$ & 2 & 2 & 3 & 1 & 1 & 1 & 8 & 2 & 4 & 4 & 1 & 5 \\
\hline $1.0418-04$ & 2 & 2 & 2 & 1 & 1 & 6 & 1 & 2 & 3 & 2 & 3 & 3 \\
\hline $1.2148-04$ & 2 & 1 & 2 & 1 & 3 & 6 & 2 & 2 & 1 & 1 & 5 & 4 \\
\hline $1.267 \mathrm{E}-04$ & 2 & 1 & 2 & 1 & 2 & 6 & 6 & 2 & 2 & 3 & 3 & 4 \\
\hline $1.331 \mathrm{E}-04$ & 2 & 2 & 2 & 1 & 2 & 3 & 6 & 4 & 1 & 3 & 4 & 5 \\
\hline $1.412 \mathrm{E}-04$ & 2 & 1 & 2 & 2 & 3 & 6 & 1 & 1 & 5 & 3 & 2 & 1 \\
\hline $1.903 \mathrm{E}-04$ & 2 & 2 & 2 & 1 & 1 & 5 & 7 & 2 & 3 & 2 & 2 & 3 \\
\hline $1.9148-04$ & 3 & 1 & 2 & 1 & 3 & 2 & 2 & 3 & 4 & 3 & 1 & 5 \\
\hline $2.0808-04$ & 2 & 1 & 2 & 2 & 2 & 2 & 5 & 2 & 3 & 1 & 5 & 2 \\
\hline $2.1428-04$ & 2 & 1 & 2 & 1 & 2 & 3 & 9 & 3 & 2 & 2 & 5 & 4 \\
\hline $2.2018-04$ & 2 & 2 & 2 & 2 & 1 & 6 & 2 & 2 & 1 & 2 & 4 & 3 \\
\hline $2.265 \mathrm{E}-04$ & 2 & 2 & 2 & 1 & 2 & 2 & 2 & 3 & 2 & 3 & 1 & 5 \\
\hline $2.3148-04$ & 2 & 2 & 2 & 2 & 2 & 2 & 7 & 2 & 1 & 2 & 4 & 3 \\
\hline $2.427 \mathrm{E}-04$ & 1 & 2 & 2 & 2 & 3 & 2 & 1 & 3 & 4 & 2 & 3 & 4 \\
\hline $2.4298-04$ & 2 & 2 & 3 & 1 & 1 & 3 & 1 & 2 & 2 & 2 & 2 & 1 \\
\hline $2.1418-04$ & 2 & 2 & 3 & 2 & 1 & 1 & 1 & 1 & 4 & 3 & 4 & 1 \\
\hline $2.604 \mathrm{E}-04$ & 2 & 1 & 3 & 1 & 1 & 2 & 1 & 1 & 1 & 1 & 5 & 4 \\
\hline $2.6498-04$ & 2 & 1 & 3 & 2 & 2 & 6 & 7 & 2 & 2 & 3 & 1 & 4 \\
\hline $2.6938-04$ & 2 & 2 & 2 & 1 & 1 & 5 & 5 & 3 & 1 & 4 & 1 & 2 \\
\hline $2.720 \mathrm{E}-04$ & 2 & 1 & 3 & 1 & 3 & 6 & 5 & 3 & 1 & 3 & 3 & 1 \\
\hline $2.728 \mathrm{R}-04$ & 2 & 2 & 2 & 1 & 2 & 5 & 4 & 5 & 5 & 1 & 1 & 1 \\
\hline $2.752 \mathrm{E}-04$ & 2 & 1 & 3 & 2 & 2 & 6 & 5 & 1 & 3 & 2 & 4 & 3 \\
\hline $2.8152-04$ & 2 & 1 & 2 & 2 & 2 & 6 & 7 & 2 & 2 & 2 & 3 & 4 \\
\hline $2.9248-04$ & 2 & 1 & 2 & 2 & 3 & 2 & 9 & 2 & 1 & 2 & 4 & 4 \\
\hline $2.8448-04$ & 2 & 1 & 3 & 1 & 3 & 6 & 1 & 4 & 1 & 3 & 2 & 3 \\
\hline $2.936 \mathrm{E}-04$ & 3 & 1 & 3 & 1 & 1 & 1 & - & 2 & 4 & 4 & 1 & 3 \\
\hline $3.069 \mathrm{E}-04$ & 2 & 1 & 2 & 1 & 2 & 6 & 7 & 2 & 2 & 3 & 1 & 1 \\
\hline $3.1192-04$ & 2 & 1 & 2 & 2 & 2 & 5 & 1 & 1 & 2 & 2 & 6 & $\mathbf{s}$ \\
\hline $3.1472-04$ & 2 & 1 & 2 & 1 & 2 & 3 & 9 & 5 & 3 & 3 & 2 & 3 \\
\hline $3.200 \mathrm{z}-04$ & 2 & 1 & 2 & 1 & 1 & 6 & 2 & 4 & 2 & 3 & 3 & 1 \\
\hline $3.2628-04$ & 2 & 1 & 2 & 2 & 3 & 2 & 1 & 3 & 4 & 3 & 3 & 1 \\
\hline $3.2878-04$ & 2 & 2 & 3 & 2 & 2 & 3 & 7 & 3 & 4 & 3 & 3 & 2 \\
\hline $3.3148-04$ & 2 & 2 & 3 & 2 & 1 & 6 & • & 3 & 4 & 3 & 3 & 2 \\
\hline $3.5218-04$ & 2 & 1 & 2 & 2 & 1 & 5 & 7 & 4 & 2 & 1 & 4 & 5 \\
\hline $3.556 \mathrm{E}-04$ & 2 & 2 & 2 & 1 & 3 & 1 & 3 & 1 & 4 & 2 & 3 & $\mathbf{s}$ \\
\hline $3.651 \mathrm{E}-04$ & 2 & 1 & 1 & 3 & 1 & 6 & 3 & $\mathbf{3}$ & 3 & 2 & 4 & 5 \\
\hline $3.797 \mathrm{E}-04$ & 1 & 1 & 3 & 2 & 1 & 2 & 6 & 1 & 5 & 3 & 2 & 1 \\
\hline $3.8198-04$ & 2 & 2 & 2 & 2 & 3 & 2 & 2 & 2 & 1 & 2 & 1 & 3 \\
\hline $3.8708-04$ & 2 & 2 & 2 & 2 & 3 & 2 & 6 & 2 & 3 & 2 & 4 & 3 \\
\hline $4.0502-04$ & 2 & 2 & 2 & 1 & 2 & 3 & 5 & 1 & 5 & 2 & 3 & 4 \\
\hline $1.0608-04$ & 2 & 2 & 3 & 1 & 2 & 2 & 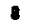 & 1 & 1 & 2 & 5 & 1 \\
\hline 1.2858-04 & 2 & 2 & 2 & 2 & 3 & 3 & 8 & 5 & 2 & 2 & 1 & 2 \\
\hline $4.2248-04$ & 2 & 1 & 3 & 2 & 3 & 3 & 9 & 2 & 3 & 2 & 4 & 3 \\
\hline $1.3828-04$ & 2 & 1 & 3 & 2 & 2 & 2 & 5 & 2 & 2 & 4 & 1 & 3 \\
\hline $4.4438-04$ & 1 & 1 & 3 & 1 & 3 & 3 & 6 & 5 & 3 & 3 & 3 & 3 \\
\hline $4.454 \mathrm{E}-04$ & 2 & 1 & 3 & 2 & 1 & 3 & 4 & 1 & 3 & 2 & 4 & 1 \\
\hline $4.7468-04$ & 2 & 1 & 3 & 2 & 1 & 3 & 8 & 3 & 1 & 2 & 4 & 1 \\
\hline $4.878 \mathrm{E}-04$ & 2 & 1 & 2 & 2 & 1 & 2 & 2 & 2 & 3 & 2 & 4 & 3 \\
\hline $4.960 \mathrm{~B}-04$ & 2 & 2 & 3 & 2 & 2 & 3 & 4 & 1 & 3 & 1 & 1 & 2 \\
\hline $4.9778-04$ & 2 & 1 & 3 & 1 & 3 & 6 & 6 & 4 & 1 & 3 & I & 2 \\
\hline $5.0338-04$ & 2 & 2 & 2 & 1 & 3 & 6 & 5 & 2 & 3 & 2 & 5 & 3 \\
\hline $5.0418-04$ & 2 & 1 & 2 & 2 & 2 & 6 & 1 & 3 & 3 & 2 & 5 & 1 \\
\hline $5.0668-04$ & 2 & 2 & 2 & 2 & 3 & 1 & 5 & 1 & 3 & 3 & 2 & 3 \\
\hline $5.2278-04$ & 2 & 1 & 2 & 2 & 1 & 1 & 3 & 4 & 2 & 2 & 3 & 5 \\
\hline $5.429 \mathrm{~B}-04$ & 3 & 2 & 2 & 2 & 2 & 1 & 6 & 4 & 2 & 1 & 4 & 1 \\
\hline $5.4768-04$ & 2 & 1 & 3 & 2 & 1 & 3 & 6 & 5 & 1 & 2 & 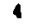 & 5 \\
\hline $5.6038-04$ & 2 & 2 & 2 & 1 & 3 & 2 & 5 & 5 & 3 & 2 & 4 & 2 \\
\hline $5.6318-04$ & 2 & 1 & 3 & 2 & 1 & 6 & 3 & 5 & 1 & 3 & 4 & 2 \\
\hline $5.7948-04$ & 2 & 1 & 2 & 2 & 3 & 6 & 2 & 2 & 1 & 2 & 4 & 2 \\
\hline $6.0468-04$ & 2 & 2 & 3 & 2 & 1 & 5 & 3 & 2 & 1 & 2 & 5 & 5 \\
\hline $6.086 z-04$ & 2 & 2 & 2 & 2 & 3 & 3 & 7 & 1 & 4 & 2 & 4 & 1 \\
\hline $7.064 E-04$ & 2 & 2 & 2 & 3 & 2 & 5 & 3 & 4 & 2 & 2 & & 4 \\
\hline $7.233 \mathrm{E}-04$ & 3 & 1 & 2 & 2 & 3 & 6 & 6 & 1 & 3 & 4 & 1 & 3 \\
\hline $7.3058-04$ & 2 & 1 & 2 & 1 & 2 & 6 & 3 & 3 & 4 & 2 & 2 & 4 \\
\hline $7.5078-04$ & 2 & 2 & 2 & 2 & 2 & 3 & 8 & 2 & 6 & 3 & 2 & 2 \\
\hline $7.7868-04$ & 2 & 1 & 3 & 1 & 2 & 2 & 9 & 3 & 4 & 2 & 3 & 3 \\
\hline $7.9578-04$ & 2 & 1 & 2 & 2 & 3 & 6 & 2 & 2 & 4 & 2 & 5 & 4 \\
\hline $7.9978-04$ & 1 & , & 3 & 1 & 1 & 6 & 1 & 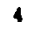 & 2 & 1 & 6 & 4 \\
\hline $0.090 \mathrm{E}-04$ & 2 & 1 & 2 & 2 & 2 & 6 & 4 & 4 & 4 & 2 & 1 & 3 \\
\hline $0.363 \mathrm{E}-$ & 2 & 2 & 2 & 1 & 2 & 3 & 9 & 2 & 4 & 1 & 5 & 4 \\
\hline $0.7728-04$ & 3 & , & 2 & 2 & 1 & 3 & 9 & 3 & 3 & 3 & 3 & 5 \\
\hline $9.135 \mathrm{E}-04$ & 2 & 1 & 2 & 2 & 2 & 2 & 6 & 5 & 3 & 3 & 3 & 5 \\
\hline $9.1: 18-04$ & 2 & 2 & 2 & 2 & 2 & 6 & 9 & 1 & 4 & 3 & 1 & 4 \\
\hline $9.455 \mathrm{E}-04$ & 2 & 2 & 2 & 1 & 1 & 6 & 2 & 1 & 6 & 3 & 3 & 4 \\
\hline $9.543 \mathrm{E}-04$ & 2 & 1 & 2 & 2 & 3 & 3 & 4 & 2 & 3 & 2 & 5 & 2 \\
\hline $9.74 E-0$ & 2 & 2 & 3 & 1 & 1 & 1 & 1 & & 3 & 1 & 1 & 2 \\
\hline $.0398-03$ & 2 & 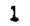 & 2 & 1 & , & 3 & 1 & 5 & 3 & 1 & 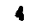 & 5 \\
\hline $.1448-03$ & 2 & 1 & 2 & 1 & 3 & 6 & 9 & 5 & 3 & 1 & 6 & 4 \\
\hline .14 & 2 & 2 & 2 & 2 & 3 & 4 & 2 & & & 2 & & 2 \\
\hline $1.472 \mathrm{E}-0$ & 2 & 2 & 3 & 2 & 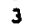 & 4 & 4 & 7 & 5 & 2 & 3 & 2 \\
\hline $.519 \mathrm{E}-03$ & 2 & 2 & 3 & 3 & 1 & 6 & 4 & 2 & 5 & 2 & $\mathbf{5}$ & 3 \\
\hline $.621 \mathrm{E}-03$ & 2 & & 2 & 3 & 2 & 6 & 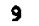 & 1 & 4 & 1 & 6 & 1 \\
\hline $.0398-03$ & 2 & & 2 & 1 & 2 & 6 & 8 & & 3 & 1 & & 5 \\
\hline $1408-03$ & & & & & & & & & & & & \\
\hline
\end{tabular}


NUREG/CR-4551, VOL. 1: DRAFT REPORT FOR COMMENT (SEPTEMBER, 1986)

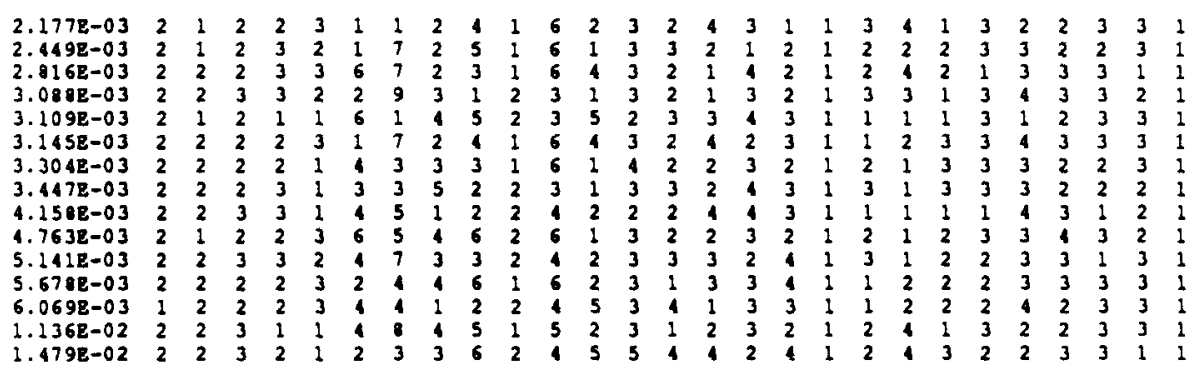


NUREG/CR-4551, VOL. 1: DRAFT REPORT FOR COMMENT (SEPTEMBER, 1986)

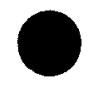

CONSEOUENCE MEASTIRE

CANCER DEATHS FER YEAR

SENSITIVITY CASE : NO DIRECT HEATING

SURRY LALHC (CRAC

FRACTIONAL CONTRIBUTION OF EACH SEOUENCE TO MEAN RISK

SEQURNCE CONTRIBTTION

sYYB $\quad 0.0228$

SYYB

.022

SMNN

0.0001

InN

0.277

ANNN

0.0022

AYNN

0.0002

AYN I

0.0287

AYYs

0.0695

TMNes

0.0252

0.2411

TYYBD

TrYBD 0.0743

$\begin{array}{ll}\text { TYNI } & 0.0007 \\ Y & 0.2216\end{array}$

CONSEOUENCE MUSTRE:

CANCER DEATHS PER YEAR

0.2216

FRACTIONLL CONTRIBOTION OF EACH BIN TO MEAN RISK

CONTRIBOTION

BIN

BIN 1

BIN

BIN

BIN

aIN 6

BIN 7

3IX

BIN 9

BIN 10

aIx 11

3is 12

atr 14

arin 15

BIM 16

BIN 17

BIN 18

BIN 19

CONSEQUENCE MEASORE

CANCER DEATHS PER YEAR

0.1199

0.0402

0.0184

0.0074

0.1012

0.0002

0.0038

0.0005

0.0224

0.0114

0.1368

0.2347

0.0002

0.0019

0.0019

0.0011

0.0000

0.0000

0.0000

0.0000

FRACTIONAL CONTRIBUTIONS OF EACH RELEASE TO MEAN RISK

\begin{tabular}{|c|c|c|}
\hline \multicolumn{2}{|c|}{ RRLEASE } & CONTRIBOTION \\
\hline SOR & $2 \mathrm{GP}$ & 0.0625 \\
\hline SOR & $3 G P$ & 0.0620 \\
\hline SOR & 4GP & 0.1018 \\
\hline SOR & $5 G P$ & 0.0620 \\
\hline SOR & 6GP & 0.1502 \\
\hline SOR & $7 G P$ & 0.0563 \\
\hline SOR & BGP & 0.1315 \\
\hline SUR & 96 & 0.0050 \\
\hline SOR & $10 G P$ & 0.0066 \\
\hline SOR & $11 \mathrm{GP}$ & 0.0564 \\
\hline SOR & $12 \mathrm{GP}$ & 0.0002 \\
\hline SOR & 1369 & 0.0532 \\
\hline SOR & 146 & 0.0088 \\
\hline SOR & $15 \mathrm{GP}$ & 0.0190 \\
\hline SOR & $16 G$ & 0.0111 \\
\hline SOR & 2768 & 0.0381 \\
\hline SOR & 186 & 0.0054 \\
\hline SUR & $196 P$ & 0.0233 \\
\hline SOR & $20 G$ & 0.0038 \\
\hline 5OR & $21 G R$ & 0.0090 \\
\hline SOR & 226 & 0.0004 \\
\hline SOR & $23 \mathrm{GP}$ & 0.0049 \\
\hline SOR & 246 & 0.0019 \\
\hline SUR & $25 \mathrm{C}$ & 0.0011 \\
\hline
\end{tabular}




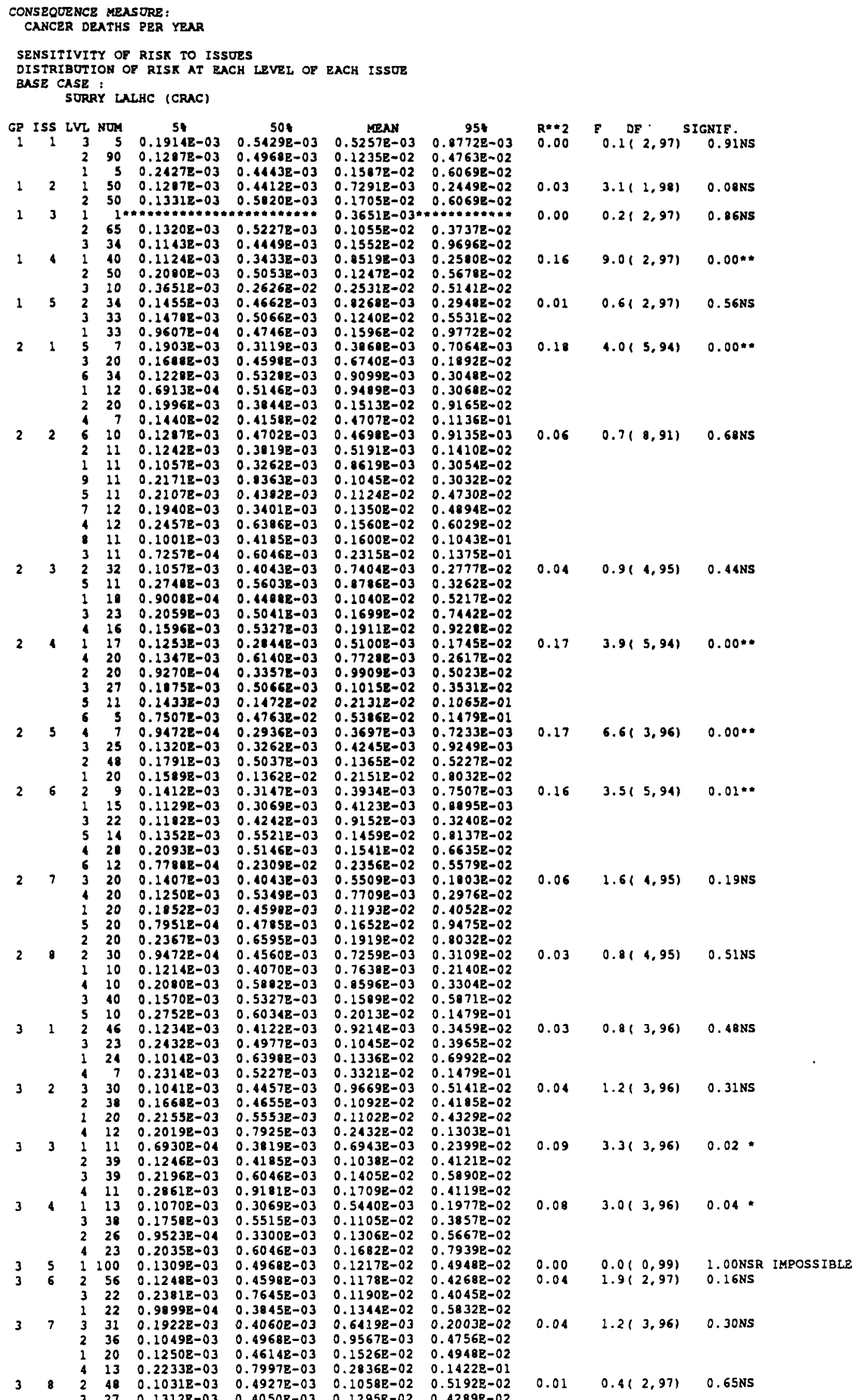


NUREG/CR-4551, VOL 1: DRAFT REPORT FOR COMMENT (SEPTEMBER, 1986)

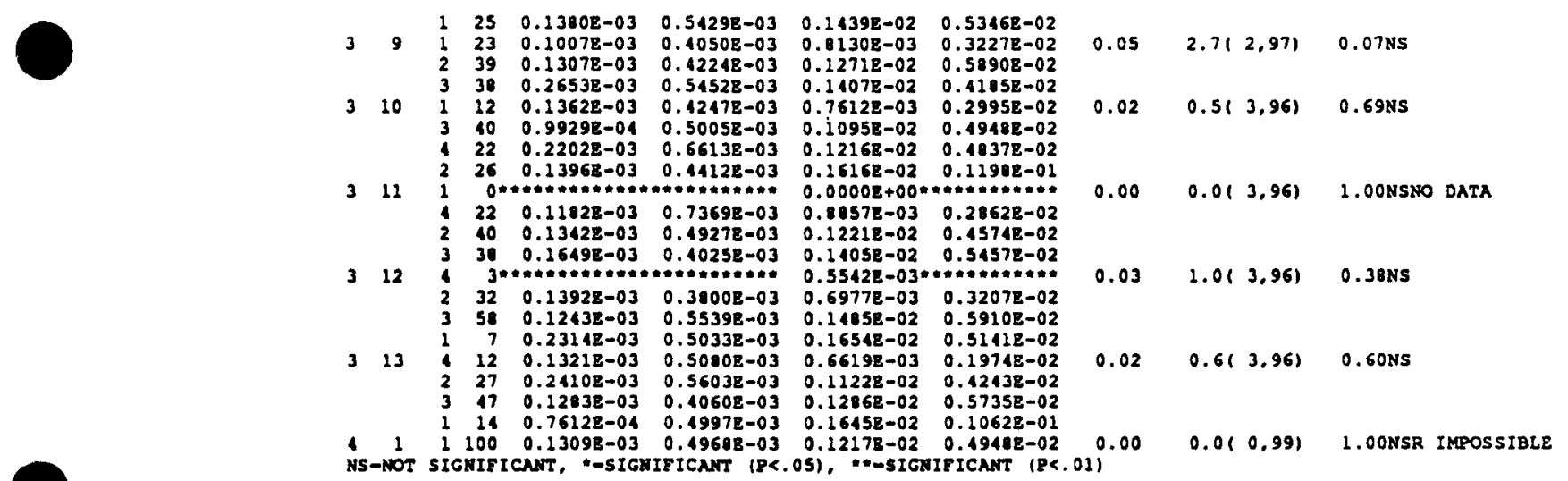


CONSEOCENCE MEASTRE: CANCER DEATHS PER YRAR

0 ISSUE GROUP - 1 ISSUE - 1 TB SEAL LOCA

LEVEL 1 NO. \&MED.- 3 NO. EXP.- 2

LEVEL 2 No. <MED. - 44 NO. EXP. - 45

LEVEL 3 NO. ८MED.- 2 No. EXP.- 2

OVERALL CHI-SO 0.0 DF- 1 SIG.- 0.833

0
NO.>-MED.- 2 NO. EXP.- 3 NO.>-MED. - 46 NO. $8 X P .=45$ NO.>-MED. - 3 NO. EXP. $=3$
CHI-SO. TEST CANNOT BE RUN; EXP. NO. < 5 CHI-SO.- 0.0 DF- 1 SIG.- 0.833 CHI-SO. TEST CANNOT BE RUN; EXP. NO. < 5
LEVEL 1 SO. SARD. - 29 NO. EXP.- 25

LEVEL 2 NO. CMED. - 20 MO. EXP.- 25

OVERALI CHI-SO 3.3 DF- 3 SIG.- 0.350

ISSUE GROUR - 1 ISSUE - 3 BETA-FACTOR

LEVEI 1 No. ২MED.- 1 NO. EXR.- 0

LEVEL 2 NO. <MRD. - 29 NO. EXP.- 32

LEVEL 3 No. <MED.- 19 No. EXP.- 17

NO.>-ARD.- 0 No. EXP.- 1 No.>-AED. - 36 No. EXP.- 33 NO. >-MED. - 15 No. EXP. - 17

OVERALL CHI-SO- 1.0 DF- 3 SIG.- 0.795

ISSUE GROUP - 1 ISSUE - I V FREO

LEVEL 1 NO. $\angle$ KRD. - 25 N0. EXP. - 20

LEVEL 2 NO. SMED. 23 NO. EXR.- 25

NO.>-AED. - 15 No. EXP. - 20 No. $2-\mathrm{AED} .-27$ No. $2 \times 5 .-25$

LEVEL 3 NO. SMRD.- 1 NO. RXP.- 5 NO.>-MED. - 9 No. EXP.- 5

OVERALL CHI-SO 9.2 DF- 5 SIG.- 0.101

\section{LEVRL 1 NO. SMRD. - 19 NO. EXP. - 16}

LEVEL 2 No. CARD. - 17 No. Exp. - 17

LEVEL 3 No. <MED. - 14 No. EXP. - 16

OVERALL CHI-5O- 1.0 DF- 5 SIG.- 0.965

\begin{abstract}
ISSUR GROUP - 2 ISSUR - 1 IND. RCS FAIL.
\end{abstract}
LEVEL 1 No. <MED.- 5 NO. EXP. - 6

LEVEL 2 NO. <MED.- 14 No. EXP.- 10

LEVEL 3 NO. SMED. - 11 NO. EXP. -10

LEVEL 4 NO. SMED.- 0 No. EXP.- 3

LEVEL 5 NO. <MED.- 5 NO. EXP. - 3

LEVEL 6 No. <MED. - 11 No. EXP. - 17

OVERALL CHI-SO- 4.8 DF- 7 SIG.- 0.685 O ISSUE GROUP - 2 ISSUR - 2 MODE OP VB.

IEVEL 1 No. SMED.- 6 NO. EXP.- 5

LEVEL 2 NO. <MED.- 7 NO. EXP.- 5

LEVEL 3 No. CMED. - 3 No. EXP.- 5

LEVEL 4 NO. LMED.- 4 SO. EXP.- 6

LEVEL 5 No. <MED.- 6 No. EXP.- 5

LEVEL 6 No. <MED.- 5 NO. EXP.- 5

LEVEL 7 NO. <MED. - 7 NO. EXP.- 6
No. >-MED. - 7 No. EXP.- 6 No.>-MED. - 6 NO. EXP. -10 No.2 MED. - 7 MO. EXP.- 4 No. >-MED.- 2 NO. EXP. - 4 NO. >-MED. - 20 NO. EXP. -27 NO. > MED. - 9 NO. $\operatorname{EXP} .-10$
CHI-SO.- 1.3 DF- 1 SIG.- 0.258

CHI-5O.- 2.0 DF- 1 SIG.- 0.157

CHI-SO. TEST CANHOT BE RON: EXP. NO. < 5 CHI-SO.- 0.6 DF- 1 SIG. 0.457 CHI-50.- 0.5 DF- 1 SIG. -0.493

CHI-SQ.- 2.5 DF- 1 SIG.- 0.114 CHI-50.- 0.3 DF- 1 SIG.- 0.572 CHI-50.- 6.4 DF- 1 SIG.- 0.011

CHI-SO.- 0.5 DF- 1 SIG.- 0.486 CHI-SO.- 0.0 DF- 1 SIG.- 1.000 CHI-SQ.- 0.5 DF- 1 SIG.- 0.486

CHI-50.- 0.3 DF- 1 SIG. 0.564 CHI-5Q.- 3.2 OF- 1 SIG.- 0.074 CHI-50.- 0.2 DF- 1 SIG.- 0.655 CHI-SQ. TEST CANHOT BE RON: EXP. NO. < 5 CHI-SO. TEST CANNOT BE RUN: EXP. NO. $<5$ CHI-SO.- 1.1 DF- 1 SIG.- 0.303

CHI-50.- 0.4 DF- I SIG.- 0.545 CHI-SQ.- 1.5 DF- 1 SIG. 0.226 CHI-SQ.- 1.5 DF- 1 SIG. 0.226 CHI-SQ.- 1.3 DF- 1 SIG.- 0.248 CHI-50.- 0.4 DF- 1 SIG. -0.545 CHI-SQ.- 0.0 DF- 1 SIG.- 1.000 CHI-SQ.- 0.3 OF- 1 SIG. 0.564 


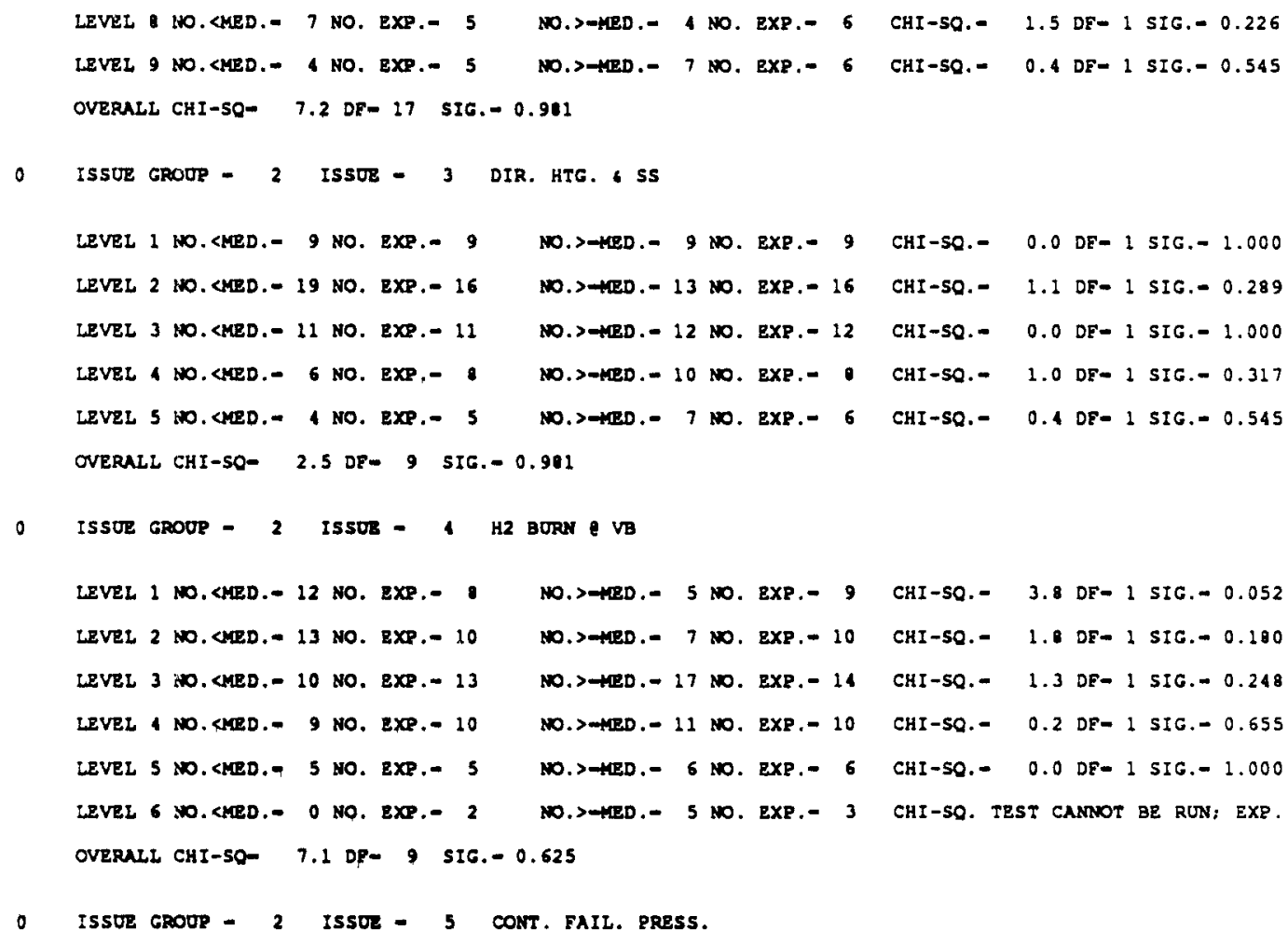




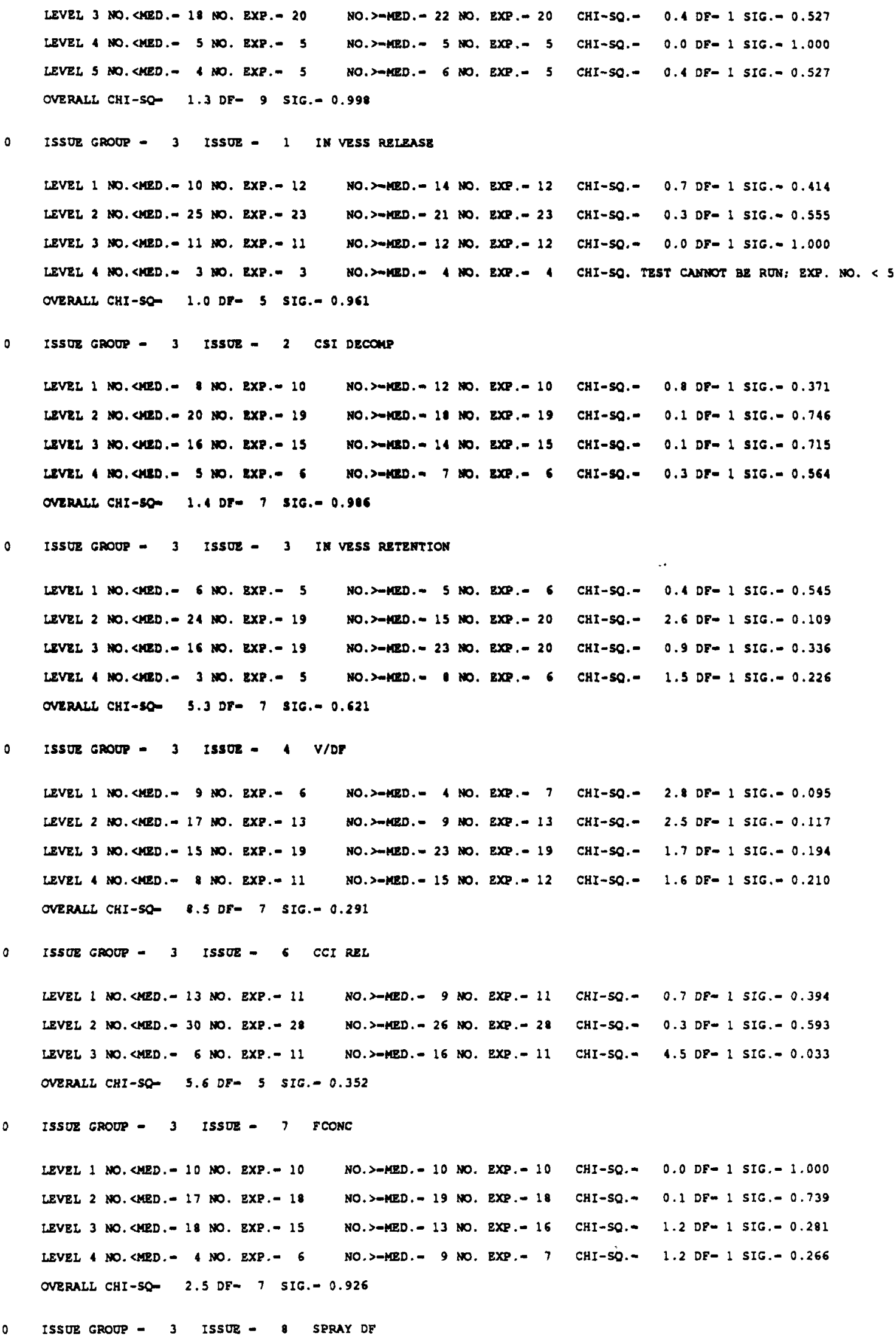

CHI-SO.- 0.7 DE- 1 SIG. $=0.414$ CHI-SQ.- 0.3 DF- I SIG. -0.555 CHI-SO.- 0.0 DF- 1 STC.- 1.000 CHI-SO. TEST CANNOT aE RON; EXP. NO. < 5

CHI-SQ.- 0.8 OF- 1 SIG.- 0.371 CHI-SO.- 0.1 DF- 1 SIG.= 0.746 CHI-s0.- $0.1 \mathrm{DF}-1$ SIG.- 0.715 CHI-SO.- 0.3 DF- 1 5IG. $=0.364$

CHI-SO.- 0.4 DE- 1 SIG.- 0.545 CHI-SO.- 2.6 DF- 1 SIG.- 0.109 CHI-50.- 0.9 DF- 1 SIG. -0.336 CHI-SO.- 1.5 DF- I SIG.- 0.226

CHI-SO.- 2.2 DF- 1 SIG.- 0.095 CHI-SQ.- 2.5 OF- 1 SIG.- 0.117 CHI-SO.- 1.7 DF- 1 SIG.=0.194 CHI-SO.- 1.6 DF- 1 SIG. -0.210

CHI-5O.- 0.7 DF- 1 SIG. -0.394 CHI-50.= 0.3 DF- I SIG. 0.593 CHI-SQ. 4.5 DF- 1 SIC. $=0.033$

CHI-SQ.- 0.0 DF- 1 SIG.- 1.000 CHI-SQ.- 0.1 DFW 1 SIG. $=0.739$ CHI-SO.- 1.2 DF- 1 SIG.- 0.281 CHI-SO.- 1.2 DF- 1 SIG. 0.266 


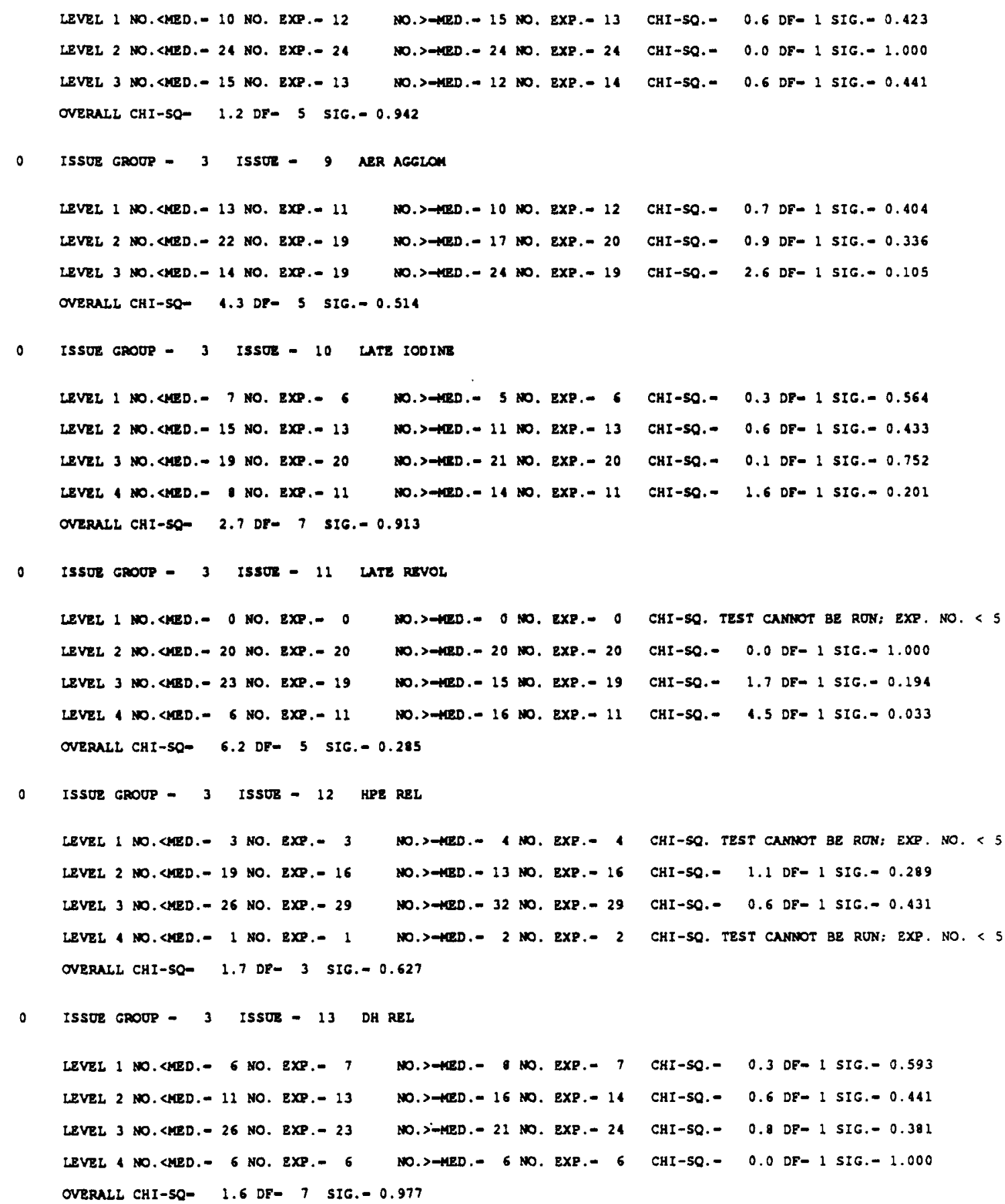

OVERALL CHI-SO- 1.6 DF- 7 SIG. 0.977

CHI-SO. TEST CNMNOT BE RON; EXP. NO. < 5 CHI-SO. $\quad 0.0$ OF- 1 SIG.- 1.000 CHI-50.- 1.7 DF- 1 SIG. $=0.194$ CHI-SO.- 4.5 DF- 1 SIG.- 0.033

CHI-SO. TEST CAMNOT BE RON: EXP. NO. < 5 CHI-50.- 1.1 DF- 1 SIG. 0.289 CHI-SO.- 0.6 DF- 1 SIG.- 0.431 CHI-SO. TEST CANNOT BE RUN; EXP. NO. \& 5 
NUREG/CR-4551, VOL. 1: DRAFT REPORT FOR COMMENT (SEPTEMBER, 1986)

\section{D.5 REVIEW OF HIGH AND LOW RISK SAMPLE MEMBERS OF THE LLH RESULT}

In an attempt to help identify important uncertainties, the sample members at either end of the range for two risk measures were reviewed in detail. Of the 100 LLH sample members, the top 20 and bottom 20 contributors to early and latent fatalities were examined individually and compared to each other. Those issues found to have the same proportion of outcomes in the top 20 risk samples as the bottom 20 risk samples probably are not significant to the overall uncertainty, although insights concerning these observations are also limited by the sample size. However, those issues with substantially different outcome distributions in the top 20 versus bottom 20 may be important with respect to the overall uncertainty. This method of review is limited in statistical meaning, but was performed to help provide qualitative insights. Table D-1 summarizes the results of this process for the early fatality risk measure as calculated with CRAC2. Only the issues with different distributions of a given level in the top and bottom regimes are included (variation less than $15 \%$ was assumed to be negligible). Some issues involve many levels, and the comparison had to be more qualitative as discussed in the table.

The first insight of this review confirms the result of the other analyses that the direct heating issue is clearly the most important uncertainty: $90 \%$ of the low risk samples involve little or no direct heating, while $90 \%$ of the high risk samples involve the three highest levels of direct heating. The other issues are of less significance, but the review generated some insights in addition to those provided by the regression method. A majority of low risk sample members had high values of aerosol agglomeration decontamination, and a majority of high risk sample members had low values. Part of this may be a spurious correlation resulting from the limited sample size and from the chance correlation of inputs, but as seen in Section 5.5, the sensitivity study that excluded direct heating still pointed to this as an important issue.

The next most important issue determined by this process was the containment failure pressure. Note that the high risk samples have the two lowest levels of pressure capacity $90 \%$ of the time, while the low risk samples have these two levels in only $65 \%$ of the samples. Containment failure size is coupled with the failure pressure and therefore shows some effect.

The issue of hydrogen burn at vessel breach may also be important, but the reason is more subtle. The table indicates that the highest risk samples never have the lowest level of hydrogen burning. Therefore, all of the risk-dominant scenarios in which direct heating occurs have some level of hydrogen burn pressure increment. Prior to the input of the LLH review 
Table D-1

REVIEW OF LLH UNCERTANTY ISSUES RELATIVE TO EARLY FATALITY RISK ${ }^{a, b}$

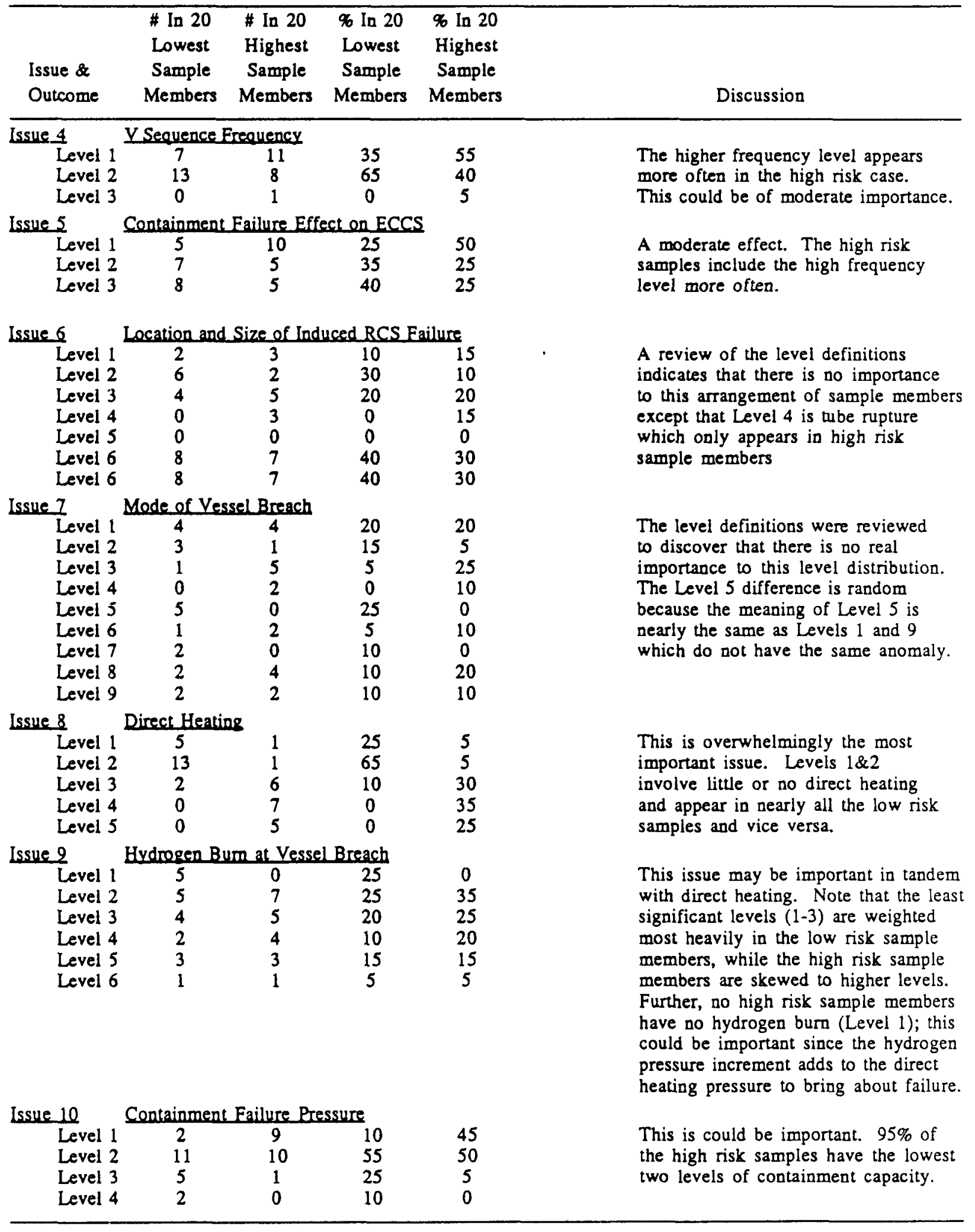


Table D-1 (Continued)

REVIEW OF LLH UNCERTAINTY ISSUES RELATTVE TO EARLY FATALITY RISK

\begin{tabular}{|c|c|c|c|c|c|}
\hline $\begin{array}{l}\text { Issue \& } \\
\text { Outcome }\end{array}$ & $\begin{array}{l}\text { \# In } 20 \\
\text { Lowest } \\
\text { Sample } \\
\text { Members }\end{array}$ & $\begin{array}{l}\text { \# In } 20 \\
\text { Highest } \\
\text { Sample } \\
\text { Members }\end{array}$ & $\begin{array}{l}\text { \% In } 20 \\
\text { Lowest } \\
\text { Sample } \\
\text { Members }\end{array}$ & $\begin{array}{l}\text { \% In } 20 \\
\text { Highest } \\
\text { Sample } \\
\text { Members }\end{array}$ & Discussion \\
\hline Issue 11 & \multicolumn{5}{|c|}{ Centainment Failure Size } \\
\hline $\begin{array}{l}\text { Level } 1 \\
\text { Level } 2 \\
\text { Level } 3 \\
\text { Level } 4 \\
\text { Level } 5 \\
\text { Level } 6\end{array}$ & $\begin{array}{l}4 \\
4 \\
5 \\
4 \\
2 \\
1\end{array}$ & $\begin{array}{l}0 \\
1 \\
5 \\
8 \\
2 \\
4\end{array}$ & $\begin{array}{l}20 \\
20 \\
25 \\
20 \\
10 \\
5\end{array}$ & $\begin{array}{l}0 \\
5 \\
25 \\
40 \\
10 \\
20\end{array}$ & $\begin{array}{l}\text { The levels increase in the frequency } \\
\text { of large hole sizes. Note that the } \\
\text { high risk samples are skewed to } \\
\text { the higher levels. This is primarily } \\
\text { because large hole sizes are correlated } \\
\text { with high containment failure } \\
\text { pressures. }\end{array}$ \\
\hline Issue 15 & \multicolumn{5}{|c|}{ CsIDecomposition } \\
\hline $\begin{array}{l}\text { Level } 1 \\
\text { Level } 2 \\
\text { Level } 3 \\
\text { Level } 4\end{array}$ & $\begin{array}{l}1 \\
9 \\
9 \\
1\end{array}$ & $\begin{array}{l}6 \\
7 \\
4 \\
3\end{array}$ & $\begin{array}{l}5 \\
45 \\
45 \\
5\end{array}$ & $\begin{array}{l}30 \\
35 \\
20 \\
15\end{array}$ & $\begin{array}{l}\text { The reason for this difference is not } \\
\text { known. It would be expected that the } \\
\text { low risk samples would have little } \\
\text { decomposition, but that is not the } \\
\text { case; this could be an anomaly. }\end{array}$ \\
\hline Issue 16 & \multicolumn{5}{|c|}{ In-Vessel Retention } \\
\hline $\begin{array}{l}\text { Level } 1 \\
\text { Level } 2 \\
\text { Level } 3 \\
\text { Level } 4\end{array}$ & $\begin{array}{l}7 \\
7 \\
4 \\
2\end{array}$ & $\begin{array}{l}1 \\
8 \\
7 \\
4\end{array}$ & $\begin{array}{l}35 \\
35 \\
20 \\
10\end{array}$ & $\begin{array}{l}5 \\
40 \\
35 \\
20\end{array}$ & $\begin{array}{l}70 \% \text { of the low risk samples have } \\
\text { high retention, compared to } 45 \% \\
\text { of the high risk. This may be of } \\
\text { moderate importance. }\end{array}$ \\
\hline Issue 17 & \multicolumn{5}{|c|}{ Y Sequence Decontamination } \\
\hline $\begin{array}{l}\text { Level } 1 \\
\text { Level } 2 \\
\text { Level } 3 \\
\text { Level } 4\end{array}$ & $\begin{array}{l}5 \\
7 \\
4 \\
4\end{array}$ & $\begin{array}{l}2 \\
4 \\
8 \\
6\end{array}$ & $\begin{array}{l}25 \\
35 \\
20 \\
20\end{array}$ & $\begin{array}{l}10 \\
20 \\
40 \\
30\end{array}$ & $\begin{array}{l}60 \% \text { of the low risk samples have } \\
\text { high decontamination, compared to } \\
30 \% \text { of the high risk samples. This } \\
\text { could be a moderate effect. }\end{array}$ \\
\hline Issue 20 & \multicolumn{5}{|c|}{ CCL Releases from Containment } \\
\hline $\begin{array}{l}\text { Level } 1 \\
\text { Level } 2 \\
\text { Level } 3 \\
\text { Level } 4\end{array}$ & $\begin{array}{l}6 \\
6 \\
7 \\
1\end{array}$ & $\begin{array}{l}6 \\
3 \\
6 \\
5\end{array}$ & $\begin{array}{l}30 \\
30 \\
35 \\
5\end{array}$ & $\begin{array}{l}30 \\
15 \\
30 \\
25\end{array}$ & $\begin{array}{l}\text { A relatively minor effect, although } \\
\text { the high releases of Level } 4 \text { appear } \\
\text { nearly always with high risk cases. }\end{array}$ \\
\hline sue 21 & \multicolumn{5}{|c|}{ Spray Decontamination } \\
\hline $\begin{array}{l}\text { Level } 1 \\
\text { Level } 2 \\
\text { Level } 3\end{array}$ & $\begin{array}{l}4 \\
10 \\
6\end{array}$ & $\begin{array}{l}9 \\
7 \\
4\end{array}$ & $\begin{array}{l}20 \\
50 \\
30\end{array}$ & $\begin{array}{l}45 \\
35 \\
20\end{array}$ & $\begin{array}{l}\text { The differences do not seem to have a } \\
\text { physical meaning, since the low } \\
\text { decontamination appears more with } \\
\text { low risk samples. }\end{array}$ \\
\hline Issue 22 & \multicolumn{5}{|c|}{ Aerosol Agglomeration } \\
\hline $\begin{array}{l}\text { Level } 1 \\
\text { Level } 2 \\
\text { Level } 3\end{array}$ & $\begin{array}{l}12 \\
7 \\
1\end{array}$ & $\begin{array}{l}0 \\
6 \\
14\end{array}$ & $\begin{array}{l}60 \\
35 \\
5\end{array}$ & $\begin{array}{l}0 \\
30 \\
70\end{array}$ & $\begin{array}{l}\text { Input levels for this issue were chance } \\
\text { correlated with direct heating }(\mathrm{r}=.27) \text {. } \\
\text { There is thus a possibility that the } \\
\text { apparent importance of this issue is } \\
\text { due partly or wholly to sampling } \\
\text { variation. It is not possible to state } \\
\text { whether or not this issue is important } \\
\text { in its own right. }\end{array}$ \\
\hline
\end{tabular}

aAll results calculated with CRAC2.

bOnly issues with different level distributions are shown. 
group, hydrogen burns were not allowed for most of these cases, since nearly all of the important scenarios are station blackout accidents with no containment heat removal. These scenarios were thought to produce a steam-inerted containment, precluding the possibility of hydrogen burning. The review group assigned fairly high likelihood of significant pressure increments due to hydrogen burns in direct heating scenarios, regardless of the steam concentration. The phenomenon cited was the possibility of local burning or recombination of hydrogen in the proximity of the hot fuel particles (or a change in inerting criteria at very high pressures) with a pressure increment similar to the case of global hydrogen deflagration. For many of the accidents, it appears that this additional pressure increment may be one of the actual causes for exceeding the containment pressure capacity. Direct heating alone does not account fully for the early containment failure probabilities seen in the LLH result. Of course, both hydrogen burning and direct heating pressure increments are intimately coupled with the containment failure pressure issue.

Several other issues of potentially moderate importance are also identified in Table D-1. For example, in-vessel retention of the radioactive releases appears to have an effect, as do both issues affecting the risk of the $\mathrm{V}$ sequence. The results for the issue of CsI decomposition are very difficult to understand and may be anomalous; further investigation is underway. The low risk samples have a distribution that primarily includes the two central levels to the exclusion of either extreme, while the high risk samples have a nearly uniform distribution of outcomes for this issue. The reasons for this effect are unclear at this time, and are difficult to establish because of the complex coupling among nearly all of the source term issues.

Table D-2 delineates the results for the top and bottom twenty risk sample members for the latent cancer fatality risk measure as calculated with CRAC2. The conclusions are nearly the same as the review of early fatality results. 
TABLE D-2

REVIEW OF LLH UNCERTAINTY ISSUES RELATIVE TO LATENT CANCER FATALITY RISKa,b

\begin{tabular}{|c|c|c|c|c|c|}
\hline $\begin{array}{l}\text { Issue \& } \\
\text { Outcome }\end{array}$ & $\begin{array}{c}\# \\
\text { In } 20 \\
\text { Lowest } \\
\text { Risk } \\
\text { Samples }\end{array}$ & $\begin{array}{c}\# \\
\text { In } 20 \\
\text { Highest } \\
\text { Risk } \\
\text { Samples }\end{array}$ & $\begin{array}{c}\% \\
\text { In } 20 \\
\text { Lowest } \\
\text { Risk } \\
\text { Samples }\end{array}$ & $\begin{array}{c}\% \\
\text { In } 20 \\
\text { Highest } \\
\text { Risk } \\
\text { Samples }\end{array}$ & Discussion \\
\hline
\end{tabular}

Issue 6 Location and Size of Induced Eailure

$\begin{array}{lllll}\text { Level 1 } & 2 & 2 & 10 & 10 \\ \text { Level 2 } & 5 & 3 & 25 & 15 \\ \text { Level 3 } & 3 & 6 & 15 & 30 \\ \text { Level 4 } & 0 & 2 & 0 & 10 \\ \text { Level 5 } & 2 & 0 & 10 & 0 \\ \text { Level 6 } & 8 & 7 & 40 & 35\end{array}$

\begin{abstract}
A review of the level definitions indicates that there is no significance to this arrangement of samples except that Level 4 is tube rupture which only appears in high risk samples.
\end{abstract}

The level definitions were reviewed to discover that there is no real significance to this level distribution.

$\begin{array}{llccc}\text { Level 1 } & 4 & 2 & 20 & 10 \\ \text { Level 2 } & 3 & 0 & 15 & 0 \\ \text { Level 3 } & 1 & 4 & 5 & 20 \\ \text { Level 4 } & 0 & 2 & 0 & 10 \\ \text { Level 5 } & 5 & 2 & 25 & 10 \\ \text { Level 6 } & 1 & 3 & 5 & 15 \\ \text { Level 7 } & 4 & 0 & 20 & 0 \\ \text { Level 8 } & 1 & 4 & 5 & 20 \\ \text { Level 9 } & 1 & 3 & 5 & 15\end{array}$

\section{Issue 8 Direct Heating}

$\begin{array}{lcccc}\text { Level 1 } & 4 & 0 & 20 & 0 \\ \text { Level 2 } & 11 & 0 & 55 & 0 \\ \text { Level 3 } & 4 & 7 & 20 & 35 \\ \text { Level 4 } & 1 & 7 & 5 & 35 \\ \text { Level 5 } & 0 & 6 & 0 & 30\end{array}$

This is overwhelmingly the most significant issue. Levels $1 \& 2$ involve little or no direct heating and appear in nearly all the low risk samples and vice versa.

\section{Issue 9 Hydrogen Bum at Vessel Breach}

$\begin{array}{lllll}\text { Level 1 } & 7 & 1 & 35 & 5 \\ \text { Level 2 } & 4 & 4 & 20 & 20 \\ \text { Level 3 } & 3 & 6 & 15 & 30 \\ \text { Level 4 } & 2 & 3 & 10 & 15 \\ \text { Level 5 } & 3 & 3 & 15 & 15 \\ \text { Level 6 } & 1 & 3 & 5 & 15\end{array}$

The issue may be of importance. Note that the least severe levels (1-3) are weighted most heavily in the low risk samples while the high risk samples are skewed to higher levels. This may be important in tandem with direct heating. Further, no high risk sample members have no hydrogen burn (Level 1). This could be important because the hydrogen pressure increment is adding to the direct heating pressure to bring about failure. See the discussion in the main report. 
NUREG/CR-4551, VOL. 1: DRAFT REPORT FOR COMMENT (SEPTEMBER, 1986)

Appendix E ASSESSMENT OF RISK-REDUCTION MEASURES 
-

-

-

-

• 


\section{CONTENTS}

Section Page

E.1 SUMMARY OF SAFETY OPTIONS EVALUATED.......................... E-1

E.1.1 Description of Accident-Prevention Options...........................E-3

E.1.2 Description of Options Intended to Mitigate Accidents...................E-9

E.2 EVALUATION OF EFFECTS ON RISK MEASURES......................E-12

E.2.1 Effect of Accident-Prevention Options on Core-Melt Sequences...........E-12

E.2.2 Effect of Mitigation Options on Containment Response..................E-13

E.2.3 Effect of All Options on Risk Measures..............................E-16

E.3 ASSESSMENT OF IMPACT FOR RISK-REDUCTION MEASURES...........E-24

E.3.1 Estimated Costs of Risk-Reduction Measures...........................E-24

E.3.2 Estimated Personnel Doses Due to the Risk-Reduction Measures..........E-30

E.3.3 Insights Concerning Replacement Power.............................E-31

E.4 COSTS VERSUS BENEFITS........................................... 32

E.5 OBSERVATIONS CONCERNING RISK-REDUCTION ANALYSIS..........E-52 


\section{LIST OF TABLES}

Table

E-1 Important Sequences Affected by Potential Accident-Prevention Options...........E-3

E-2 Reduction Factors for Option P4 for Affected Damage States.................... E-7

E-3 Reduction in Core-Damage Frequency For Prevention Modifications............E-13

E-4 Relative Contribution of Source Term Bins to Latent Cancer Fatality Risk........E-15

E-5 Latent Cancer Fatality Risk Results for the Safety Options: LLH Method..........E-18

E-6 Early Fatality Risk Results for the Safety Options: LLH Method.................E-19

E-7 Latent Cancer Fatality Risk Results for the Safety Options: OCP Method..........E-20

E-8 Early Fatality Risk Results for the Safety Options: OCP Method.................E-21

E-9 LLH Latent Cancer Fatality Risk Reduction for the Safety Options...............E-22

E-10 LLH Early Fatality Risk Reduction for the Safety Options......................E-22

E-11 OCP Latent Cancer Fatality Risk Reduction for the Safety Options................ E-23

E-12 OCP Early Fatality Risk Reduction for the Safety Options......................E-23

E-13 Estimated One-Time Cost for Risk-Reduction Measures......................E-25

E-14 Annual Maintenance and Operations Costs for Proposed Options.................E-27

E-15 Estimated Present Value Total Cost for the Risk-Reduction Measures.............E-29

E-16 Dose in Person-Rems for Risk-Reduction Measures.........................E-30

E-17 Replacement Power Insights for Risk-Reduction Measures....................E-31

E-18 Comparison of Costs and Benefits for Safety Options: LLH Method.............E-37

E-19 Comparison of Costs and Benefits for Safety Options: OCP Method.............E-46

E-20 Value of Risk Reduction to Zero for Surry ................................E-54 
NUREG/CR-4551, VOL. 1: DRAFT REPORT FOR COMMENT (SEPTEMBER, 1986)

\section{LIST OF FIGURES}

Eigure

Page

E-1 Configuration of LPIS Interfacing Check Valves............................

E-2 Cost-Benefit Comparison for Prevention Options (CRAC2)......................

E-3 Cost-benefit Comparison for Mitigation Options (CRAC2).....................E-34

E-2 Cost-Benefit Comparison for Prevention Options (MACCS)....................E-35

E-3 Cost-benefit Comparison for Mitigation Options (MACCS).....................E-36 
-

-

-

-

- 


\section{Appendix E \\ ASSESSMENT OF RISK-REDUCTION MEASURES}

In order to gain additional perspective on the significance of the risk associated with the operation of the Surry plant, it is useful to examine the value and effectiveness of potential improvements in plant systems or operating practices. This section outlines the selection of possible modifications aimed at reducing the various risk measures for Surry, describes the value and impact associated with the implementation of each of the measures and summarizes the cost/benefit comparison for each option.

\section{E.1 SUMMARY OF SAFETY OPTIONS EVALUATED}

The assessment of modifications for Surry included a review of both the generic options identified in Section 3 of the main report and measures aimed specifically at the aspects of Surry design and operation that were assessed to be important to risk. The proposed options were developed in an attempt to address the risk associated with two types of consequence measures: the health and safety of the public and the potential for financial losses, both offsite and onsite. Offsite risks, including both health and cost measures, were assessed to be dominated in the LLH study by accident sequences involving station blackout (i.e., the sustained loss of offsite and onsite ac power), with smaller contributions from several other types of accidents. In the central estimate of the OCP study, the accidents initiated by the corevulnerable sequences (in which containment fails before core damage) and the interfacing systems LOCA (or $\mathrm{V}$ sequence) dominate the risk measures.

Onsite financial risks are directly related to the frequency of the core-melt sequences; since the onsite costs are dominated by the costs of replacement power and loss of capital investment, the type of core-melt sequence is not particularly important. No single type of sequence was assessed to dominate the frequency of core melt, although station-blackout sequences contribute more than one-third. It is therefore difficult to identify a single, simple modification that offers a promise of having a significant overall effect on core-melt frequency. One other type of sequence deserves mention, although it was not explicitly considered in the value/impact assessment. Accidents in which containment failure would be predicted but which would not necessarily be expected to result in core melt were calculated to have significant frequencies. The costs associated with such an accident could be very large, although the offsite effects would be negligible compared to core-melt events. 


\section{E.1.1 Descrintion of Accident-Prevention Options}

Table E-1 summarizes the options intended to reduce the frequency of core damage, and the sequences each has the potential to affect. Note that some of the generic options were not included in the analysis for Surry. For example, one involved the improvement of procedures for effecting recirculation of emergency core cooling after depletion of the refueling water storage tank (RWST). Because an automatic system for accomplishing the switchover task has already been implemented at Surry, it was judged that such an option would have no potential to be cost-effective. The following sections describe each of the options as they have been defined to address the important aspects of the Surry sequences.

\section{Option P1: Improved Testing of Interfacing Check Valves}

This purpose of the first option would be to reduce the likelihood of a LOCA that occurred outside containment due to failure of isolation between the high-pressure reactorcoolant system (RCS) and systems at lower design pressures (event $\mathrm{V}$ ). Of primary concern are the cold-leg injection lines for the low-pressure injection system (LPIS). The configuration of the check valves in the interfacing systems is indicated in Figure E-1. There is a similar set of injection lines for each of the three cold legs at Surry.

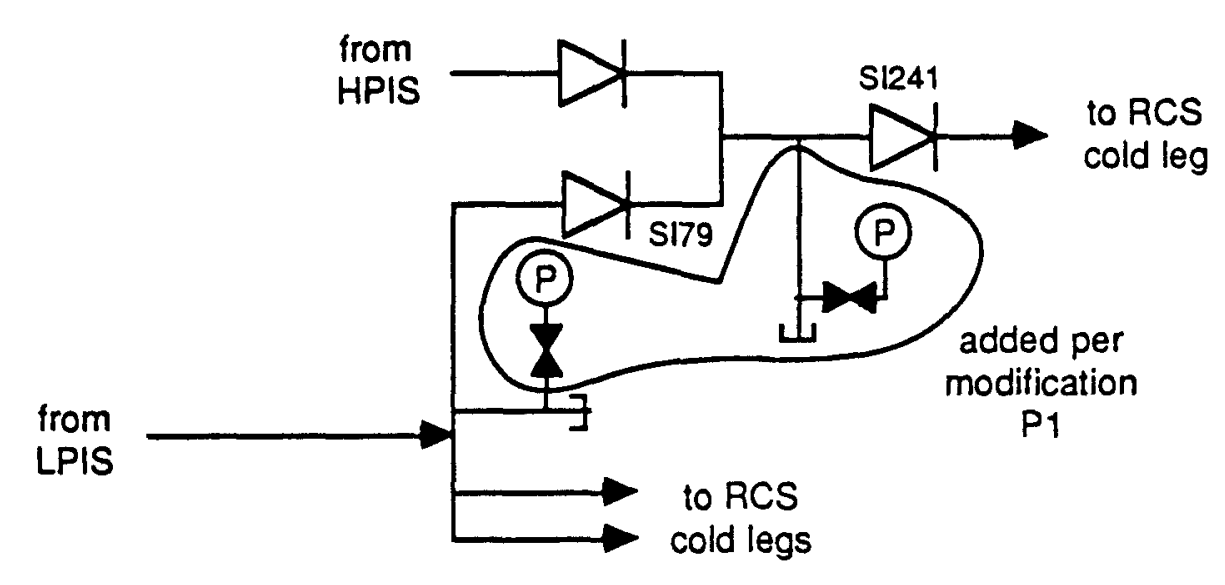

Figure E-1. Configuration of LPIS Interfacing Check Valves

Currently, the status of the check valves is verified on an annual basis. In the existing ASEP assessment, the sequences of events leading to an interfacing-systems LOCA are the following: 
Table E-1

IMPORTANT SEQUENCES AFFECTED BY POTENTIAL ACCIDENT-PREVENTION OPTIONS

\begin{tabular}{|c|c|c|c|c|c|c|c|c|c|c|c|c|c|}
\hline \multirow[b]{2}{*}{ Option } & \multirow[b]{2}{*}{$\begin{array}{c}T_{1} \mathrm{~L}(\mathrm{SL}) \mathrm{DCF} \mathrm{F}_{1} \\
5.6 \times 10^{-6}\end{array}$} & \multirow[b]{2}{*}{$\begin{array}{l}\mathrm{T}_{4} \mathrm{QH}_{1} \\
1.5 \times 10^{-6}\end{array}$} & \multirow[b]{2}{*}{$\begin{array}{l}\mathrm{T}_{4 \mathrm{H}} \mathrm{QH}_{1} \\
1.3 \times 10^{-6}\end{array}$} & \multicolumn{5}{|c|}{ Sequences Affecled and Their Base Case Froquencies* } & \multirow[b]{2}{*}{$\begin{array}{c}\mathrm{T}_{45} \mathrm{QH}_{2} \\
89.2 \times 10^{-7}\end{array}$} & \multirow[b]{2}{*}{$\begin{array}{c}V \\
9.0 \times 10^{-7}\end{array}$} & \multirow[b]{2}{*}{$\begin{array}{c}S_{2} D_{1} \\
7.7 \times 10^{-7}\end{array}$} & \multirow[b]{2}{*}{$\begin{array}{l}S_{1} D_{1} \\
7.7 \times 10^{-7}\end{array}$} & \multirow[b]{2}{*}{$\begin{array}{l}\text { Others } \\
-3 \times 10^{-}\end{array}$} \\
\hline & & & & $\begin{array}{l}T_{1} \mathrm{LP} \\
1.4 \times 10^{-6}\end{array}$ & $\begin{array}{l}\mathrm{T}_{1}(\mathrm{LT}) \mathrm{DCF}_{1} \\
3.8 \times 10^{-6}\end{array}$ & $\begin{array}{l}1 \mathrm{TH}(\mathrm{ST}) \mathrm{DCF}_{1} \\
1.1 \times 10^{-6}\end{array}$ & $\begin{array}{r}1 . \mathrm{T}_{4} \mathrm{QH}_{2} \\
1.110^{-6}\end{array}$ & $\begin{array}{l}\mathrm{TKRD}_{4} \\
9.8 \times 10^{-7}\end{array}$ & & & & & \\
\hline $\begin{array}{l}\text { PI. Improved testing of interfacing } \\
\text { systems check valves }\end{array}$ & - & - & - & - & - & - & -- & - & - & $\mathbf{x}$ & - & - & - \\
\hline $\begin{array}{l}\text { P2 Improved emergency ac power } \\
\text { for loss of offsite powa }\end{array}$ & $\mathbf{x}$ & - & - & - & $\mathbf{X}$ & $\mathbf{X}$ & - & - & - & - & - & - & $\mathbf{x}$ \\
\hline $\begin{array}{l}\text { P3. Improved de power system } \\
\text { through extension of batuery life }\end{array}$ & - & - & - & - & $\mathbf{X}$ & - & - & - & - & - & - & - & - \\
\hline $\begin{array}{l}\text { P4. Additional, independent train } \\
\text { of high-pressure injection }\end{array}$ & $\mathbf{x}$ & - & - & $\mathbf{X}$ & $\mathbf{x}$ & $\mathbf{x}$ & $\mathbf{X}$ & $X$ & $\mathbf{X}$ & - & $\mathbf{X}$ & $\mathbf{X}$ & $\mathbf{X}$ \\
\hline $\begin{array}{l}\text { P5. Additional, independent train } \\
\text { of auxiliary feedwaler }\end{array}$ & - & - & - & $\mathbf{x}$ & $\mathbf{x}$ & $\mathbf{x}$ & - & - & - & - & - & - & $\mathbf{x}$ \\
\hline $\begin{array}{l}\text { P6. Long-term residual beat removal/ } \\
\text { low-pressure rocirculation }\end{array}$ & - & $\mathbf{X}$ & $x$ & - & - & - & - & - & - & - & - & - & $\mathbf{X}$ \\
\hline $\begin{array}{l}\text { P7. New service water } \\
\text { system for containment }\end{array}$ & - & - & - & - & - & - & - & - & - & - & - & - & $\mathbf{X}$ \\
\hline $\begin{array}{l}\text { P8 Rapid primary system } \\
\text { depressurization }\end{array}$ & - & -- & - & $\mathbf{X}$ & - & - & $\mathbf{X}$ & - & $\mathbf{x}$ & $\cdots$ & $\mathbf{X}$ & $\mathbf{x}$ & $\mathbf{x}$ \\
\hline
\end{tabular}


1. Transfer open (leakage) through the valve closest to the LPIS (e.g., valve SI241) and rupture of the valve closest to the RCS (e.g., valve SI79);

2. Failure of either valve to close after use of the low-pressure injection system and rupture of the other, and

3. Rupture of the valve closest to the RCS, followed by rupture of the other.

One option that could significantly reduce the frequency of these failure combinations would be to provide for testing that would allow for positive determination of the status of the valves at more frequent intervals. This was accomplished at another PWR by the installation of a test apparatus similar to the configuration illustrated by Figure E-1. For Surry, it would be necessary to add a tee off the line between the check valves for a pressure gage, and to add a pressure gage to a drain tap (presumably already in existence) upstream from the check valve closest to the LPIS. Testing could be accomplished by first pressurizing through the highpressure injection line and monitoring the upstream pressure, verifying the status of the upstream check valve, then draining and monitoring the pressure between the valves to verify the status of the downstream valve.

If this testing were to be accomplished following each use of the LPIS and on a quarterly basis, it would have the following impact on the base-case analysis:

- The frequencies for the first and third types of sequences noted above would be reduced by a factor of 16 ; and

- The second type would be eliminated altogether, since failures to reclose would be detected prior to plant startup.

The base case frequency would therefore be reduced from $9.0 \times 10^{-7} / \mathrm{yr}$ to $5.0 \times 10^{-8} / \mathrm{yr}$.

The modification would also have an impact on the sensitivity cases. The high value was estimated by applying a higher failure rate for the rupture mode. The effect of the modification would be to reduce the annual frequency of this sequence from $4.0 \times 10^{-6}$ to $2.0 \times 10^{-7}$. The low value was originally calculated by reducing the failure rates for both the leak and rupture modes. In this case, the effect of the modification would be to reduce the frequency of the $\mathrm{V}$ sequence from $5.3 \times 10^{-8}$ to $1.6 \times 10^{-9}$.

\section{Option P2: Improved Onsite Ac Power System}

Because of the importance of sequences involving the total loss of ac power, the second option considered was the improvement of the emergency ac power system. Although there are several options for accomplishing this improvement, a specific proposal is required for value/impact analysis. For this study, the addition of a diesel generator with other changes to allow crossties between Surry units in the event of multiple diesel failures was used as a 
representative improvement. The analysis assumes that the new diesel generator would of the same size as those currently in service, but that it would be of different manufacturer and subject to different maintenance practices. Thus it was assumed that the new diesel would not be subject to the common-cause failure mode that has been evaluated for the existing diesels.

The new frequency for failure of the onsite emergency power system was calculated based on the following design assumptions:

- The new diesel (DG-4) is nominally aligned to Unit 1 , but can be crossconnected to either unit.

- The existing swing diesel (DG-3) is nominally aligned to Unit 2, but can be used for Unit 1 if and only if DG-2 is available to supply power to Unit 2.

- Simultaneous maintenance on DG-1 and DG-4 is precluded, as is maintenance on DG-2 and DG-3.

- DG-4 is not subject to the common-cause failure modes affecting the other diesels.

- The cross-connect is performed manually from the control room, and the actions are proceduralized such that the probability of failure to crossconnect is dominated by hardware failures of the diesels.

The effect of this modification is to reduce the frequencies of plant-damage states TNNN and ANNN by a factor of 0.051 (both are totally dominated by station-blackout sequences). For the sensitivity case when the $\beta$-factors are eliminated, this factor rises slightly to 0.059 ; as the $\beta$-factors are increased in the sensitivity study, the factor changes to 0.042 .

\section{Option P3: Extension of Battery Life}

The evaluation of the Surry plant assumed that the station batteries have a seven-hour life following the loss of all ac power. This is one of the limiting features for station blackout sequences; because although the turbine-driven auxiliary feedwater (AFW) pump could continue to operate after depletion of the batteries, it was assumed that it would be difficult to ensure continued long-term cooling with no instrumentation to maintain control. Thus, the third option considered was the improvement of the dc power system. A significant improvement would be estimated if the batteries could supply power reliably for greater than about $12 \mathrm{hr}$, since additional time available for restoring ac power provides a lower value for non-recovery. The improvement could be achieved by the addition of new batteries; by procedures to reduce loads and extend the availability of power when all ac power is lost; or even possibly through a testing program to demonstrate the viability of the current batteries in the longer term. For purposes of this analysis, the addition of new batteries was selected as representative of this option. 
This option would result in the reduction of the frequency of one of the dominant coremelt sequences, sequence $T_{1} L(L T) D_{1} C F_{1}$ (a station blackout followed by successful operation of the turbine-driven AFW pump until the batteries are depleted). It was assumed that the unreliability of the batteries during the mission time was negligible compared to the probability of failure to restore ac power. The extension from the current assumption of depletion of the batteries in $7 \mathrm{hr}$ to $12 \mathrm{hr}$ reduces the frequency for plant-damage state TNNN, to which this sequence is assigned, by 0.65 . None of the sensitivity issues impact this factor.

\section{Qption P4: Addition of an Independent High-Pressure Injection System}

A number of the important sequences for Surry included the failure of high-pressure injection and/or seal injection for the reactor-coolant pumps (RCPs). The fourth option considered consists of an independent means of providing high-pressure primary inventory makeup and RCP seal injection. In developing this option, it was assumed that a single-train of high-pressure injection (HPI) with dedicated power and other support systems would be added to the current design. The system was assumed to have sufficiently high shut-off head to provide feed-and-bleed cooling using the pressurizer safety relief valves. Manual initiation from the control room was also assumed.

The hardware unavailability of the system was estimated through comparison to the existing charging system. The following values were used:

Failure of the system with station ac power available: $\quad 0.019$

Failure when ac power not available from station: $\quad 0.049$

These values were applied to the individual cut sets of the sequences in the affected plantdamage states. The factor to be applied to the damage-state frequencies for the base case and the two sensitivity cases (with $\beta$-factors eliminated and with them increased) are summarized in Table E-2 below.

\section{Option P5: Addition of an Independent Train of Auxiliary Feedwater}

This option involves the addition of a train of auxiliary feedwater, assumed to be independent of all current plant support systems, with the possible exception that it would share a suction source with the current AFW system. The system is assumed to be selfpowered, either through the use of a diesel generator or a steam-driven pump. The system would also require special design consideration to limit the potential for common-cause failure with the existing system, particularly relative to steam binding which has been experienced at the Surry plant. It was assumed that the system would be capable of delivering feedwater for a 
NUREG/CR-4551, VOL. 1: DRAFT REPORT FOR COMMENT (SEPTEMBER, 1986)

Table E-2

REDUCTION FACTORS FOR OPTION P4 FOR AFFECTED DAMAGE STATES

\begin{tabular}{cccccc}
\hline $\begin{array}{c}\text { Plant-Damage } \\
\text { State }\end{array}$ & $\begin{array}{c}\text { Power } \\
\text { Available }\end{array}$ & $\begin{array}{c}\text { Power } \\
\text { Not Available }\end{array}$ & \multicolumn{3}{c}{ Beduction Factor } \\
SYYB & $\mathbf{X}$ & & 0.89 & 0.95 & 0.85 \\
SNNN & & $\mathbf{X}$ & 0.049 & 0.049 & 0.049 \\
TNNN & & $\mathbf{X}$ & 0.049 & 0.049 & 0.049 \\
TYYBW & $\mathbf{X}$ & & 0.019 & 0.019 & 0.019 \\
TYYBD & $\mathbf{X}$ & & 0.019 & 0.019 & 0.019 \\
AYYB & $\mathbf{X}$ & & 0.58 & 0.76 & 0.59 \\
\hline
\end{tabular}

relatively long time period (at least 12 hours). This addition would reduce the frequency of damage states TNNN, TYYBD, and TYNI by a factor comparable to its unavailability. The unavailability was assumed to be similar to that of the existing turbine-driven train, 0.031 .

\section{Option P6: New Low-Pressure Cooling Capability}

Because of the risk significance of sequences involving the loss of core cooling as a consequence of failure of containment heat removal, the provision for an independent train of low-pressure cooling was considered. This option would encompass the capability to circulate either primary coolant, in the residual heat removal (RHR) mode, or containment sump water, in the low-pressure recirculation mode, through a heat exchanger and back to the RCS. The system was assumed to require manual initiation, and to have an independent source of cooling water and electric power. No detailed analysis was performed; however, because this would be a single-train system, and unavailability of 0.05 was assumed to be representative.

This modification would directly impact sequences involving failure of low-pressure recirculation or those involving failure of long-term containment heat removal. The affected plant-damage states are the following:

\begin{tabular}{cccc} 
Blant-Damage State & Base Case & \multicolumn{2}{c}{ Reduction Factor } \\
No B's & Higher B's \\
SYYB & 0.52 & 0.57 & 0.46 \\
SYNN & 0.05 & 0.05 & 0.05 \\
AYYB & 0.60 & 0.91 & 0.37 \\
AYNN & 0.05 & 0.05 & 0.05 \\
AYNI & 0.05 & 0.05 & 0.05
\end{tabular}




\section{Qption P7: New Service Water System for Containment Cooling}

The Surry ASEP analysis indicated that the service water system designed to remove heat from containment systems is potentially subject to common-cause failures that could defeat the containment heat removal function. The sequences resulting from this scenario could result in particularly severe consequences, since the containment would already be in a failed state at the time of core melt.

This option would require careful design consideration to ensure that it was not subject to the same common-cause problem associated with biofouling of the valves or heat exchangers that affects the assessed reliability of the existing system. Although the same effect could be obtained by preventing future occurrences of the biofouling failure mode, it is not clear what changes, if any, would be adequate, and a new, independent system was therefore selected to represent this option for the value/impact analysis. No detailed design was developed, and the option was assumed to have an unavailability of 0.05 . The impact of this modification would be to reduce the frequencies of damage states AYNI and TYNI by the factor of 0.05 .

\section{Qption P8--RCS Depressurization System}

Some of the sequences contributing to the risk for Surry involved failure of the highpressure injection system, or of the high-pressure recirculation system after successful injection, in the event of a small LOCA or total loss of feedwater. If the RCS pressure could be reduced sufficiently, core-heat removal could be sustained by low-pressure injection or recirculation, as appropriate. Therefore, an option was considered that would facilitate the operators' ability to blow down the RCS to a lower pressure. This option was evaluated in a functional manner by applying the equivalent of a recovery event to appropriate sequences. A detailed design was not considered, but the system was assumed to have the following characteristics:

1. It would have sufficient capacity for pressure relief to allow depressurization to the operating pressure for the low-pressure injection system;

2. It would be manually initiated; and

3. Its unavailability would be no greater than 0.01 per demand (assumed to be dominated by failure of the operators to initiate the depressurization).

In order to make best use of the available resources, no detailed analysis of competing risks was performed, since it was anticipated that this modification would prove to be of limited benefit. However, it is very likely that the provision for an additional means to depressurize the RCS would carry with it the potential for an increase in the frequency of LOCAs. The system might also be employed inadvertently during some upset events, possibly 
precluding other, preferred options. The overall impact on the response of containment as a consequence of altering the nature of some sequences would also require evaluation prior to. concluding that the implementation of this option might be warranted.

The unavailability of the depressurization system was assumed to be 0.01 . Its impact on affected plant-damage states is as follows:

Plant-Damage State

SYYB

TYYBW

TYYBD

\section{Base Case}

0.58

0.01

0.01
Reduction Factor

No $\beta$ 's Higher $\beta$ 's

0.62

0.45

0.01

0.01

0.01

0.01

\section{E.1.2 Description of Options Intended to Mitigate Accidents}

A number of safety options intended to reduce the likelihood of containment failure and/or the severity of releases following a core melt have been identified. Several of the options which were initially proposed for their mitigative effects also had an impact on the frequency of the core-vulnerable sequences $-S_{1} F_{1} F_{2}$ and $A F_{1} F_{2}$. All of the mitigative options are described below and their impact in terms of both prevention and mitigation are discussed.

It should be noted that all of the systems that perform the mitigative functions described below are assumed to be independent of existing support systems. The costs assessed for the options reflect the new support systems required for each option, including electric power and heat rejection. As with the prevention options, there are critical assumptions concerning the applicability of the common-cause events to the new system that introduce uncertainty in the results. Generally, the method used here for initial calculation of safety option value would tend to overstate the positive impact of the proposed modifications.

\section{Option M1--Missile Shield}

The provision of a shield to preclude the possibility of containment failure as a consequence of a missile generated due to an in-vessel steam explosion (the failure mode designated $\alpha$ ) was considered. The design capability of the structure would be difficult to establish because there is widely varying scientific opinion on the phenomenon of steam explosions. In addition, it would be very difficult to design a structure that provided protection against all postulated missiles, but that did not interfere with normal plant operations, particularly refueling. Because the risk reduction associated with this option was expected to 
be small, details of the design were not considered. Instead, the risk was recalculated by eliminating all $\alpha$-mode failures.

\section{Option_M2--Hydrogen_Ignition_ System}

The containment event tree for Surry provided the opportunity for hydrogen combustion whenever the containment atmosphere reached potentially flammable conditions. An ignition system would be intended to burn off hydrogen at lower concentrations to prevent buildup and combustion on a scale large enough to lead to containment failure. The system design was assumed to be an ignition system similar to that discussed in Section 3 of the main report. A study would be required to verify that the placement of igniters would ensure complete buming without a local concentration of hydrogen. The impact of this option on risk was assessed by forcing ignition to occur at each node in the containment event tree at which hydrogen buming would be possible. A $1.0 \times 10^{-3}$ unavailability was estimated to be appropriate for the igniter system, and the system was assumed to have its own, independent power supply.

\section{Option M3--Prevention of Hydrogen Combustion}

As an alternative to option $\mathrm{M} 2$, the provision for preventing the combustion of hydrogen (e.g., through inerting of the containment atmosphere) was considered. The system was assumed to be similar to the containment inerting currently in use at some BWRs. The effectiveness of such an option was investigated by precluding the possibility of combustion at each of the associated nodes in the containment event tree. ${ }^{*}$ The inerting option could present some problems in terms of long-term stabilization of the inerted, but hydrogen-rich, containment environment. Because these potential problems were not fully considered in the evaluation of the this option, its associated risk reduction would tend to be somewhat overestimated. As with the igniters, a $1.0 \times 10^{-3}$ unavailability was used in the calculations.

\section{Option M4--Early Containment Venting}

Venting of the containment atmosphere through filters to prevent overpressurization and a more serious release was investigated. It was assumed that the system design would be similar to that described in Section 3, and that it would have an independent power supply. The effects of venting early in the accident were simulated by setting the probability of a moderate-sized (less than one square foot) failure of containment isolation to 1.0, and assigning the resulting release to the bin corresponding to meltthrough of the containment

\footnotetext{
${ }^{*}$ The inerting option would also have an impact on the severity of direct heating, but this was not considered in this study.
} 
basemat at high pressure. A more detailed assessment would entail creating a new release bin that more accurately reflects the filter effectiveness, and performing an additional set of consequence calculations. It should also be noted that a venting strategy would also preclude accident the accident sequences resulting from a loss of containment cooling and these effects were also included as part of the value of the option. In the calculations of the effect of this modification, a $1.0 \times 10^{-2}$ probability of failure was estimated.

\section{Qption M5--Late Containment Venting}

This system would be the same as option M4, but the venting strategy would be different. The value of venting the containment later in the accident progression, following breach of the reactor vessel, was evaluated in a manner similar to option M4. The resulting release was again assessed as a high-pressure meltthrough of the basemat, corresponding to a filter of moderate effectiveness.

\section{Option M6-Additional Containment Cooling-.-Containment Spray}

Because of the significance to risk of sequences involving overpressurization due to failure of containment cooling, followed by the consequential failure of long-term core cooling, the addition of an alternative containment spray system was considered. The containment event tree was reevaluated considering the impact of increased spray availability for each branch point involving spray operation or containment cooling. The system evaluated was assumed to be independent of the current support systems, and includes the capability for recirculation and heat rejection through a new service water system. A $5.0 \times 10^{-2}$ unavailability was used in the estimates of risk reduction. This modification was also assumed to reduce the frequency of the core-vulnerable sequences in which the loss of containment cooling was the principal system fault (e.g., the sequences $S_{1} F_{1} F_{2}$ and $A F_{1} F_{2}$ ).

\section{Option M7--Additional Containment Cooling--Ean Coolers}

This option is similar in effect to the option described above, and the containment event tree questions regarding fan coolers which were included in the tree development so that the logic would have wider applicability were changed to reflect the addition of fan coolers for Surry. As with the sprays, a $5.0 \times 10^{-2}$ unavailability was used in the calculations. However, for this option, the containment cooling capability was assumed to be smaller than that required to prevent the containment failure for the core-vulnerable sequences. 


\section{Eption_M8--Containment_Flooding_System}

This option was proposed because of the importance of the direct heating phenomenon to the results of the LLH study. The flooding system was assumed to have the capability of delivering the contents of another RWST-size tank directly onto the reactor cavity. This would have two effects: 1) it would reduce the pressure increments associated with direct heating and 2) it would increase the probability of debris coolability in the cavity for some sequences. ${ }^{*}$ A system unavailability of $1.0 \times 10^{-3}$ was considered in the calculation.

\section{Qption P8/M8--RCS Depressurization_System}

Although this option was already listed as a preventative option, it has a substantial mitigative effect in terms of reducing the potential for containment failure due to direct heating. The potential for reducing system pressure and thus precluding direct heating at vessel breach was included in the calculation of risk-reduction for this option.

\section{E.2 EVALUATION OF EFFECTS ON RISK MEASURES}

The expected benefit associated with each of the safety options was estimated by recalculating the risk measures accounting for the effects described in the previous sections. Section E.2.1 describes the resultant impact on the frequencies of the core-melt sequences and the plant damage states, and Section E.2.2 summarizes the effects on containment response that would be expected as a consequence of implementing the mitigation options. Section E.2.3 details the integrated effects of each option in terms of its associated reduction of risk.

\section{E.2.1 Effect of Accident-Prevention Options on Core-Melt Sequences}

For each of the options identified in Section E.1.1, the changes that would result to the dominant core-meit sequences were evaluated on a cut-set by cut-set basis. The sequence results are summarized in Table E-3. As can be seen from the table, only a few of the options have significant effects on the total frequency of core melt. Due to the dominance of stationblackout sequences, options that lead to enhanced reliability of the power systems do have some effect, as do the options involving methods of heat removal independent of current means and with their own power source. The most effective option relative to reduction of overall core-melt frequency is the addition of a new high pressure injection system along with changes to the PORV power supplies to ensure their operability in the event of loss of all ac power. As

\footnotetext{
"This option was initially proposed as a limited-volume flooding system. It was later proposed that a deep flooding system with a great deal of water inventory could essentially eliminate direct heating. Thus this option, as defined, may not be optimal in terms of benefit.
} 
illustrated in Table E-3, this option, P4, results in a frequency reduction for eight of the twelve most frequent sequences. However, there are a sufficient number of additional sequences that contribute to the total that the reduction in core-melt frequency is limited for any particular type of preventive measure--for option P4 the reduction is less than a factor of four which is small compared to the uncertainties involved.

Table E-3

REDUCTION IN CORE-DAMAGE FREQUENCY ASSOCIATED WITH PREVENTION MODIFICATIONS

\begin{tabular}{|c|c|c|c|c|c|c|c|c|c|}
\hline \multirow[b]{2}{*}{$\begin{array}{l}\text { Damage } \\
\text { State }\end{array}$} & \multirow[b]{2}{*}{ Frequency } & \multicolumn{8}{|c|}{ Erequency With Option Divided by Erequency Withour 1} \\
\hline & & P1 & $\mathrm{P} 2$ & P3 & P4 & P5 & P6 & $\mathrm{P} 7$ & P8 \\
\hline SYYB & $7.1 \times 10^{-6}$ & - & - & - & .9 & - & .5 & - & .6 \\
\hline SNNN & $5.7 \times 10^{-6}$ & - & .05 & - & .05 & - & - & - & - \\
\hline TNNN & $1.9 \times 10^{-6}$ & - & .05 & .65 & .05 & .03 & - & - & - \\
\hline AYYB & $1.8 \times 10^{-6}$ & - & - & - & .58 & - & .6 & - & - \\
\hline TYYBD & $1.1 \times 10^{-6}$ & - & - & - & .02 & .03 & - & - & .01 \\
\hline TYYBW & $1.0 \times 10^{-6}$ & - & - & - & .02 & - & - & - & .01 \\
\hline V & $9.0 \times 10^{-7}$ & .06 & - & - & - & - & - & - & - \\
\hline AYNI & $8.2 \times 10^{-8}$ & - & - & - & - & - & .05 & .05 & - \\
\hline TYNI & $8.0 \times 10^{-8}$ & - & - & - & - & .03 & - & .05 & - \\
\hline SYNN & $\varepsilon$ & - & - & - & - & - & .05 & - & - \\
\hline AYNN & $\varepsilon$ & - & - & - & - & - & .05 & - & - \\
\hline $\begin{array}{l}\text { Total } \\
\text { Frequency }\end{array}$ & $y^{2.1} \times 10^{-5}$ & $2.1 \times 10$ & $\times 10$ & 10 & $\times 10$ & $\times 10^{-}$ & $\times 10^{-}$ & $\times 10$ & $6 \times 10^{-5}$ \\
\hline
\end{tabular}

The ratio is only shown for damage states that changed.

\section{E.2.2 Effect of Mitigation Options on Containment Response}

The mitigation option effects described in the previous section were implemented by rerunning the containment event tree with adjustments to simulate each option. This task involves a great deal of calculation and output, since the tree had to be rerun for each plant damage state for each option. In addition, the LLH output require calculation of response for 
every sample member. Because the documentation of each output is impractical, the CET effects of the options reported here are in terms of the final risk results, broken down to illustrate the impact of the options on the containment response.

One good measure of effectiveness can, however, be extrapolated from an examination of the relative contribution of source term bins to the risk as displayed in Table E-4. The source term bins correspond to the major containment phenomena and the impact of the issues can be seen more fully than simply through the descriptions above. For example, the first option is intended to eliminate the steam explosion-induced failure of containment, and the relative contribution of $B$ in 1 decreased; however, the effect was limited because the failure mode was not very important to the base case. Option M2, the hydrogen igniters, had little impact of the mean risk of latent cancer fatalities. Option M3 did cause a reduction in hydrogen burn failure modes, but more importantly, it reduced the contribution of direct heating. This resulted from a feature seen in Section 5: the direct heating failure mode is affected by the hydrogen burn pressure increment that the LLH reviewers assigned for this case, including a hydrogen increment in a steam-inert environment.

The early containment venting option (\#4) decreased the probability of early containment failure, including that associated with direct heating since the base containment pressure is reduced. The late filtered venting option had almost no impact since early failures dominate the risk. The most effective option is M6, the new train of containment spray. This option increases the availability of containment cooling for nearly all scenarios, while at the same time providing a high assurance of a flooded cavity which has a significant impact on the direct heating pressure increments. Therefore, the relative contributions of most early failures is less, with the result that many sequences contribute approximately equally to the risk. The fan coolers, option M7, are not as effective as sprays since they do not impact direct heating-the flooding of the cavity associated with option M6 does not happen for option M7. The final option was intended to reduce the direct heating contribution, but the effect was somewhat limited because the review group assigned fairly high pressure even with a full cavity, and the hydrogen burn pressure increments are still possible. One option that has preventative and mitigative effects is also included in table, option P8, RCS depressurization. As indicated the option had a large impact on the direct heating scenarios, essentially eliminating them. The risk reduction was greatest for this option.

It should be noted that the results above apply to the LLH sample. The OCP results were somewhat different, due principally to the reduced importance of the direct heating phenomenon. The mitigation options had less impact for the OCP central estimate since no 
single phenomenon dominated the containment response--the preventative options generally had more impact in this case. The addition of containment sprays had the most significant impact on containment response. The risk results presented in the next section allow more insight into the results.

Table E-4

\section{RELATIVE CONTRIBUTION OF SOURCE TERM BINS TO LATENT CANCER FATALITY RISK FOR MITIGATION OPTIONS ${ }^{1,2,3}$}

\begin{tabular}{|c|c|c|c|c|c|c|c|c|c|c|}
\hline \multirow[b]{2}{*}{$\begin{array}{l}\text { Source } \\
\text { Term } \\
\text { Bin }\end{array}$} & \multicolumn{10}{|c|}{ Eraction of Base Case Bisk Contributed by Each Bin for Each Option } \\
\hline & Base & M1 & M2 & M3 & M4 & M5 & M6 & M7 & M8 & P8 \\
\hline 1 Early rupture including $\alpha$ & $3.6 \%$ & $2.5 \%$ & $3.5 \%$ & $1 \%$ & $2.5 \%$ & $3.5 \%$ & $.2 \%$ & $2 \%$ & $2.7 \%$ & $9.5 \%$ \\
\hline 2 Same as 1 with sprays & - & - & - & - & - & - & - & - & - & - \\
\hline 3 As 1 , with lower RCS pres & $.2 \%$ & $.2 \%$ & $.5 \%$ & $.3 \%$ & $.2 \%$ & $.5 \%$ & $.6 \%$ & $.5 \%$ & $.3 \%$ & $.8 \%$ \\
\hline 4 As 2 with lower RCS pres & $.2 \%$ & $.2 \%$ & $.2 \%$ & - & $.1 \%$ & $.2 \%$ & $.2 \%$ & $.2 \%$ & $.1 \%$ & $.2 \%$ \\
\hline 5 Failure before core melt & $6 \%$ & $6 \%$ & $6 \%$ & $6 \%$ & $4 \%$ & $6 \%$ & $6 \%$ & $6 \%$ & $3 \%$ & $6 \%$ \\
\hline 6 Isolation, no sprays & - & - & - & - & - & - & - & - & - & - \\
\hline 7 Isolation, sprays & $.1 \%$ & $.1 \%$ & $.1 \%$ & $.1 \%$ & - & $.1 \%$ & $.1 \%$ & $.1 \%$ & $.1 \%$ & $.1 \%$ \\
\hline 8 Late rupture, with spray & - & - & - & - & - & - & - & - & - & - \\
\hline 9 Late rupture, no spray & $.6 \%$ & $.6 \%$ & $.6 \%$ & $.6 \%$ & - & - & $.7 \%$ & $.6 \%$ & $.4 \%$ & $.5 \%$ \\
\hline 10 Late overpressure & - & - & - & - & - & - & - & - & - & - \\
\hline $11 \mathrm{~V}$ sequence, wet & $4.7 \%$ & $4.7 \%$ & $4.7 \%$ & $4,7 \%$ & $4.7 \%$ & $4.7 \%$ & $4.7 \%$ & $4.7 \%$ & $2.5 \%$ & $4.7 \%$ \\
\hline $12 \mathrm{~V}$ sequence, dry & $7.3 \%$ & $7.3 \%$ & $7.3 \%$ & $7.3 \%$ & $7.3 \%$ & $7.3 \%$ & $4.6 \%$ & $7.3 \%$ & $4 \%$ & $3 \%$ \\
\hline \multicolumn{2}{|c|}{13 Basemat meitthrough, high pres } & - & - & - & - & - & - & - & - & - \\
\hline \multicolumn{2}{|c|}{14 Basemat meltthrough, low pres } & - & - & - & - & - & - & - & - & - \\
\hline 15 Design Leakage & - & - & - & - & - & - & - & - & - & - \\
\hline 16 Direct Heating, high pres & $72 \%$ & $71 \%$ & $71 \%$ & $38 \%$ & $51 \%$ & $71 \%$ & $4.2 \%$ & $56 \%$ & $29 \%$ & $1 \%$ \\
\hline 17 As 16 , with sprays & $3.3 \%$ & $3.3 \%$ & $3.3 \%$ & $2.7 \%$ & $8.8 \%$ & $3.3 \%$ & $3.3 \%$ & $9.5 \%$ & $2 \%$ & - \\
\hline 18 Direct Heating, lower pres & $.7 \%$ & $.6 \%$ & $.7 \%$ & $.2 \%$ & - & $.6 \%$ & $1 \%$ & $.6 \%$ & $.4 \%$ & - \\
\hline 19 As 18 with sprays & $17 \%$ & $1.7 \%$ & $1.7 \%$ & $5 \%$ & $10 \%$ & $1.7 \%$ & $2.8 \%$ & $2.8 \%$ & $1 \%$ & $=$ \\
\hline Total & $100 \%$ & $99 \%$ & $100 \%$ & $61 \%$ & $89 \%$ & $99 \%$ & $29 \%$ & $91 \%$ & $46 \%$ & $26 \%$ \\
\hline
\end{tabular}

1Preventive option P8, RCS depressurization, has a mitigative effect which is listed in this table.

2 These values were obtained from the $\mathrm{LLH}$ mean.

${ }^{3}$ Risk as calculated by CRAC2. 


\section{E.2.3 Effect of All Options_on_Risk_Measures}

The risk was calculated for each of the prevention and mitigation options described above. The results for early and latent cancer fatality risk measures are reproduced in Tables E5 through E-8 for both the LLH and the OCP methodology." The other risk measures are not reproduced here since the early fatality, latent cancer fatality, and reduction in core-damage frequency tell most of the story concerning the risk reduction. The other risk measures behave similarly to one of these three measures. (The comparison of costs and benefits at the end of this appendix includes all the risk measures.) Tables E-9 through E-12 list the same results in terms of the risk-reduction compared to the base case. These tables allow a quick review of the most effective options. ${ }^{* *}$

For the latent cancer fatality risk measure as calculated in the $\mathrm{LLH}$, the most effective options are:

\section{P4 Independent HPI \\ M6 Additional, independent containment sprays \\ P8 RCS depressurization}

P6 \& P8 RCS depressurization and a new, independent HPI

These results reflect the importance of the direct heating failure mode since all of the effective options have a significant impact in preventing direct heating or reducing the resultant pressure rise. It is also interesting to note that a few of the option options lead to increased risk in the .5th or 95 th percentile, although never in the mean or median. For example, the early venting option can increase risk since it allows a release for some accidents in which the release might have been contained.

These same options are effective for the early fatality risk measure, as calculated in the .LLH. However, in this case other options appear to have a significant effect:

\section{P2 Additional diesel generator}

M3 Containment inerting

P2 \& P4 Additional diesel generator and early filtered venting

The reduction of direct heating was important and was the reason why the same options as the latent cancer fatality were effective here. The improved ac power both reduced core-damage frequency and the probability of having sequences involving dry a dry cavity, which results in

\footnotetext{
${ }^{*}$ All risk information presented in this section was calculated using CRAC2. MACCS results were somewhat different, but no new conclusions or insights were derived, and in the interest of space, only one set of results is provided. Section E.4 does include the cost/benefit comparisons for both consequence codes.

** Much of this information is also presented graphically in Section 6 of the main report.
} 
the largest direct heating pressure increment. The containment inerting option was effective because it is preventing hydrogen combustion concurrent with direct heating, thus reducing the probability of early containment failure.

The OCP results for latent cancer fatalities are listed in Table E-11. As indicated, none of the options appeared particularly effective for the central estimate, although P1, improved V testing, and M6, containment sprays, reduced the risk about 50\%. In the OCP study the V sequence was of much greater significance to risk and a reduction in its frequency or consequences has a significant impact. The containment sprays are effective by reducing the pressure increments for most sequences. The optimistic and pessimistic results illustrated the fact that the reasons for high are low risk are different, since the options did generally not have the same effect across the entire OCP range. For example, options M4 through M7 had significant impact in the optimistic case because they all reduced the probability of late overpressure and two reduced the frequency of the core-vulnerable sequences. Another good example is the RCS depressurization option. It had no impact on the central, increased risk in the optimistic (by decreasing the probability of a coolable debris bed), and decreased risk in the pessimistic (by reducing the frequency of direct heating).

The early fatality results for the OCP method indicate similar trends, although more of the options had an impact on the central estimate. The optimistic case is not illustrated since the base case evaluation predicts no fatalities.

All of the risk-reduction results are included in Section E.4 of this appendix in terms of costs and comparison to the costs of the safety-improvement options. 
NUREG/CR-4551, VOL. 1: DRAFT REPORT FOR COMMENT (SEPTEMBER, 1986)

Table E-5

LATENT CANCER FATALITY RISK RESULTS FOR THE SAFETY OPTIONS:

LLH METHOD

\begin{tabular}{lccccc}
\hline \multicolumn{1}{c}{ OPTION } & MEAN & MEDIAN & 5TH & 95TH & VARIANCE \\
\hline BASE CASE & $3.68 \mathrm{E}-03$ & $1.35 \mathrm{E}-03$ & $2.41 \mathrm{E}-04$ & $1.34 \mathrm{E}-02$ & $2.46 \mathrm{E}-05$ \\
P1: Improved V & $3.40 \mathrm{E}-03$ & $1.23 \mathrm{E}-03$ & $1.46 \mathrm{E}-04$ & $1.32 \mathrm{E}-02$ & $2.37 \mathrm{E}-05$ \\
P2: Improved ac Power & $1.00 \mathrm{E}-03$ & $6.63 \mathrm{E}-04$ & $1.84 \mathrm{E}-04$ & $3.64 \mathrm{E}-03$ & $1.24 \mathrm{E}-06$ \\
P3: Improved dc Power & $3.46 \mathrm{E}-03$ & $1.16 \mathrm{E}-03$ & $2.36 \mathrm{E}-04$ & $1.24 \mathrm{E}-02$ & $2.25 \mathrm{E}-05$ \\
P4: Independent HPI & $7.59 \mathrm{E}-04$ & $5.94 \mathrm{E}-04$ & $1.69 \mathrm{E}-04$ & $1.97 \mathrm{E}-03$ & $4.84 \mathrm{E}-07$ \\
P5: New Aux Feedwater & $2.92 \mathrm{E}-03$ & $9.40 \mathrm{E}-04$ & $2.22 \mathrm{E}-03$ & $9.92 \mathrm{E}-03$ & $1.81 \mathrm{E}-05$ \\
P6: Independent LPI & $3.41 \mathrm{E}-03$ & $1.12 \mathrm{E}-03$ & $1.02 \mathrm{E}-04$ & $1.31 \mathrm{E}-02$ & $2.36 \mathrm{E}-05$ \\
P7: New Service Water & $3.61 \mathrm{E}-03$ & $1.31 \mathrm{E}-03$ & $2.18 \mathrm{E}-04$ & $1.32 \mathrm{E}-02$ & $2.46 \mathrm{E}-05$ \\
P8: RCS Depressurization & $9.69 \mathrm{E}-04$ & $6.06 \mathrm{E}-04$ & $2.28 \mathrm{E}-04$ & $2.99 \mathrm{E}-03$ & $1.61 \mathrm{E}-06$ \\
M1: Missile Shield & $3.65 \mathrm{E}-03$ & $1.12 \mathrm{E}-03$ & $2.23 \mathrm{E}-04$ & $1.34 \mathrm{E}-02$ & $2.47 \mathrm{E}-05$ \\
M2: Hydrogen Igniters & $3.68 \mathrm{E}-03$ & $1.35 \mathrm{E}-03$ & $2.41 \mathrm{E}-04$ & $1.34 \mathrm{E}-02$ & $2.47 \mathrm{E}-05$ \\
M3: Containment Inerting & $2.67 \mathrm{E}-03$ & $7.40 \mathrm{E}-04$ & $1.76 \mathrm{E}-04$ & $1.12 \mathrm{E}-02$ & $1.59 \mathrm{E}-05$ \\
M4: Early Venting & $3.28 \mathrm{E}-03$ & $7.16 \mathrm{E}-04$ & $1.58 \mathrm{E}-04$ & $1.60 \mathrm{E}-02$ & $2.69 \mathrm{E}-05$ \\
M5: Late Venting & $3.65 \mathrm{E}-03$ & $1.20 \mathrm{E}-03$ & $1.95 \mathrm{E}-04$ & $1.33 \mathrm{E}-02$ & $2.46 \mathrm{E}-05$ \\
M6: Containment Sprays & $1.08 \mathrm{E}-03$ & $6.62 \mathrm{E}-04$ & $1.91 \mathrm{E}-04$ & $4.01 \mathrm{E}-03$ & $1.87 \mathrm{E}-06$ \\
M7: Fan Coolers & $3.34 \mathrm{E}-03$ & $9.70 \mathrm{E}-04$ & $2.22 \mathrm{E}-04$ & $1.33 \mathrm{E}-02$ & $2.39 \mathrm{E}-05$ \\
M8: Containment Flooding & $3.13 \mathrm{E}-03$ & $1.00 \mathrm{E}-03$ & $2.52 \mathrm{E}-04$ & $1.25 \mathrm{E}-02$ & $2.14 \mathrm{E}-05$ \\
P6 \& P8: Depress. and LPI & $3.37 \mathrm{E}-04$ & $2.50 \mathrm{E}-04$ & $4.25 \mathrm{E}-05$ & $7.16 \mathrm{E}-04$ & $1.42 \mathrm{E}-07$ \\
P2 \& M4: DG and Venting & $1.79 \mathrm{E}-03$ & $6.67 \mathrm{E}-04$ & $1.37 \mathrm{E}-04$ & $7.45 \mathrm{E}-03$ & $5.57 \mathrm{E}-06$ \\
\hline
\end{tabular}


NUREG/CR-4551, VOL. 1: DRAFT REPORT FOR COMMENT (SEPTEMBER, 1986)

Table E-6

EARLY FATALITY RISK RESULTS FOR THE SAFETY OPTIONS:

LLH METHOD

\begin{tabular}{lccccc}
\hline \multicolumn{1}{c}{ OPTION } & MEAN & MEDIAN & TTH & $95 T H$ & VARIANCE \\
\hline BASE CASE & $2.10 \mathrm{E}-05$ & $3.32 \mathrm{E}-06$ & $5.84 \mathrm{E}-08$ & $1.53 \mathrm{E}-04$ & $3.08 \mathrm{E}-09$ \\
P1: Improved V & $2.05 \mathrm{E}-05$ & $3.12 \mathrm{E}-06$ & $2.51 \mathrm{E}-08$ & $1.54 \mathrm{E}-04$ & $2.98 \mathrm{E}-09$ \\
P2: Improved ac Power & $3.30 \mathrm{E}-06$ & $8.69 \mathrm{E}-07$ & $4.58 \mathrm{E}-08$ & $1.86 \mathrm{E}-05$ & $5.55 \mathrm{E}-11$ \\
P3: Improved dc Power & $1.97 \mathrm{E}-05$ & $3.10 \mathrm{E}-06$ & $5.70 \mathrm{E}-08$ & $1.42 \mathrm{E}-04$ & $2.79 \mathrm{E}-09$ \\
P4: Independent HPI & $2.37 \mathrm{E}-06$ & $8.10 \mathrm{E}-07$ & $3.64 \mathrm{E}-08$ & $9.36 \mathrm{E}-06$ & $2.40 \mathrm{E}-11$ \\
P5: New Aux Feedwater & $1.70 \mathrm{E}-05$ & $2.10 \mathrm{E}-06$ & $5.38 \mathrm{E}-08$ & $1.13 \mathrm{E}-04$ & $2.26 \mathrm{E}-09$ \\
P6: Independent LPI & $2.00 \mathrm{E}-05$ & $1.42 \mathrm{E}-06$ & $3.07 \mathrm{E}-08$ & $1.53 \mathrm{E}-04$ & $3.01 \mathrm{E}-09$ \\
P7: New Service Water & $2.07 \mathrm{E}-05$ & $2.92 \mathrm{E}-06$ & $5.33 \mathrm{E}-08$ & $1.54 \mathrm{E}-04$ & $3.07 \mathrm{E}-09$ \\
P8: RCS Depressurization & $3.52 \mathrm{E}-06$ & $7.69 \mathrm{E}-07$ & $6.10 \mathrm{E}-08$ & $1.04 \mathrm{E}-05$ & $1.79 \mathrm{E}-10$ \\
M1: Missile Shield & $2.09 \mathrm{E}-05$ & $2.62 \mathrm{E}-06$ & $4.17 \mathrm{E}-08$ & $1.54 \mathrm{E}-04$ & $3.09 \mathrm{E}-09$ \\
M2: Hydrogen Igniters & $2.10 \mathrm{E}-05$ & $3.32 \mathrm{E}-06$ & $5.84 \mathrm{E}-08$ & $1.53 \mathrm{E}-04$ & $3.08 \mathrm{E}-09$ \\
M3: Containment Inerting & $1.24 \mathrm{E}-05$ & $1.04 \mathrm{E}-06$ & $4.20 \mathrm{E}-08$ & $5.68 \mathrm{E}-05$ & $1.59 \mathrm{E}-09$ \\
M4: Early Venting & $1.59 \mathrm{E}-05$ & $1.08 \mathrm{E}-06$ & $3.99 \mathrm{E}-08$ & $7.85 \mathrm{E}-05$ & $2.30 \mathrm{E}-09$ \\
M5: Late Venting & $2.10 \mathrm{E}-05$ & $3.32 \mathrm{E}-06$ & $5.84 \mathrm{E}-08$ & $1.59 \mathrm{E}-04$ & $3.08 \mathrm{E}-09$ \\
M6: Containment Sprays & $3.18 \mathrm{E}-06$ & $8.30 \mathrm{E}-07$ & $4.51 \mathrm{E}-08$ & $1.72 \mathrm{E}-05$ & $5.05 \mathrm{E}-11$ \\
M7: Fan Coolers & $1.82 \mathrm{E}-05$ & $1.65 \mathrm{E}-06$ & $5.84 \mathrm{E}-08$ & $1.26 \mathrm{E}-04$ & $2.62 \mathrm{E}-09$ \\
M8: Containment Flooding & $1.69 \mathrm{E}-05$ & $1.54 \mathrm{E}-06$ & $5.84 \mathrm{E}-08$ & $8.02 \mathrm{E}-05$ & $2.47 \mathrm{E}-09$ \\
P6 \& P8: Depress. and LPI & $6.28 \mathrm{E}-07$ & $1.80 \mathrm{E}-07$ & $1.35 \mathrm{E}-08$ & $1.40 \mathrm{E}-06$ & $6.02 \mathrm{E}-12$ \\
P2 \& M4: DG and Venting & $6.08 \mathrm{E}-07$ & $8.64 \mathrm{E}-07$ & $3.4 \mathrm{E}-08$ & $2.80 \mathrm{E}-05$ & $2.44 \mathrm{E}-10$ \\
& & & & &
\end{tabular}


NUREG/CR-4551, VOL. 1: DRAFT REPORT FOR COMMENT (SEPTEMBER, 1986)

Table E-7

LATENT CANCER FATALITY RISK RESULTS FOR THE SAFETY OPTIONS: OCP METHOD

\begin{tabular}{|c|c|c|c|c|c|}
\hline \multirow{3}{*}{$\frac{\text { OPTION }}{\text { BASE CASE }}$} & \multirow{3}{*}{$\begin{array}{l}\text { CENTRAL } \\
3.22 \mathrm{E}-04\end{array}$} & \multirow{2}{*}{\multicolumn{2}{|c|}{ OPTIMISTIC PESSIMISTIC }} & \multicolumn{2}{|c|}{ UNCERTAINTY DOUBLES } \\
\hline & & & & LOW & $\mathrm{HIGH}$ \\
\hline & & $1.17 \mathrm{E}-06$ & $1.85 \mathrm{E}-01$ & 5.67E-06 & $2.47 \mathrm{E}-02$ \\
\hline P1: Improved V & $1.61 \mathrm{E}-04$ & 3.35E-07 & $1.67 \mathrm{E}-01$ & $5.49 \mathrm{E}-06$ & $2.05 \mathrm{E}-02$ \\
\hline P2: Improved ac Power & $2.93 \mathrm{E}-04$ & $1.11 \mathrm{E}-06$ & 8.77E-02 & $5.64 \mathrm{E}-06$ & $1.35 \mathrm{E}-02$ \\
\hline P3: Improved dc Power & $3.19 \mathrm{E}-04$ & $1.16 \mathrm{E}-06$ & $1.75 \mathrm{E}-01$ & $5.66 \mathrm{E}-06$ & $2.32 \mathrm{E}-02$ \\
\hline P4: Independent HPI & $2.75 \mathrm{E}-04$ & $1.05 \mathrm{E}-06$ & $5.74 \mathrm{E}-02$ & $5.44 \mathrm{E}-06$ & $1.25 \mathrm{E}-02$ \\
\hline P5: New Aux Feedwater & $3.08 \mathrm{E}-04$ & $1.14 \mathrm{E}-06$ & $1.47 \mathrm{E}-01$ & $5.54 \mathrm{E}-06$ & $1.89 \mathrm{E}-02$ \\
\hline P6: Independent LPI & $2.32 \mathrm{E}-04$ & 3.37E-07 & $1.64 \mathrm{E}-01$ & 8.98E-07 & $2.22 \mathrm{E}-02$ \\
\hline P7: New Service Water & $2.54 \mathrm{E}-04$ & 4.59E-07 & $1.85 \mathrm{E}-01$ & $1.23 \mathrm{E}-06$ & 2.42E-02 \\
\hline P8: RCS Depressurization & $3.04 \mathrm{E}-04$ & $1.39 \mathrm{E}-06$ & $9.00 \mathrm{E}-02$ & $5.66 \mathrm{E}-06$ & $3.39 \mathrm{E}-02$ \\
\hline M1: Missile Shield & $3.22 \mathrm{E}-04$ & $1.17 \mathrm{E}-06$ & $1.65 \mathrm{E}-01$ & $5.67 \mathrm{E}-06$ & $2.06 \mathrm{E}-02$ \\
\hline M2: Hydrogen Igniters & $3.22 \mathrm{E}-04$ & $1.17 \mathrm{E}-06$ & $1.85 \mathrm{E}-01$ & 5.67E-06 & $2.50 \mathrm{E}-02$ \\
\hline M3: Containment Inerting & $3.20 \mathrm{E}-04$ & $1.17 \mathrm{E}-06$ & $1.53 \mathrm{E}-01$ & $5.66 \mathrm{E}-06$ & $1.98 \mathrm{E}-06$ \\
\hline M4: Early Venting & $2.00 \mathrm{E}-04$ & $1.25 \mathrm{E}-07$ & $9.56 \mathrm{E}-02$ & $2.98 \mathrm{E}-07$ & $1.55 \mathrm{E}-02$ \\
\hline MS: Late Venting & 2.09E-04 & $1.25 \mathrm{E}-07$ & $1.54 \mathrm{E}-01$ & $3.15 \mathrm{E}-07$ & $2.37 \mathrm{E}-02$ \\
\hline M6: Containment Sprays & $1.89 \mathrm{E}-04$ & $2.05 E-07$ & $1.29 \mathrm{E}-01$ & $4.97 \mathrm{E}-07$ & $1.58 \mathrm{E}-02$ \\
\hline M7: Fan Coolers & $2.62 \mathrm{E}-04$ & $2.22 \mathrm{E}-07$ & $1.79 \mathrm{E}-01$ & $8.56 \mathrm{E}-07$ & $2.31 \mathrm{E}-02$ \\
\hline M8: Containment Flooding & & & TEVALUAT & ED & \\
\hline $\mathrm{P} 1$ \& $\mathrm{P} 2: \mathrm{V}$ and $\mathrm{AC}$ Power & $1.31 \mathrm{E}-04$ & 2.70E-07 & $6.26 \mathrm{E}-02$ & $5.46 \mathrm{E}-06$ & $7.98 \mathrm{E}-03$ \\
\hline
\end{tabular}


NUREG/CR-4551, VOL. 1: DRAFT REPORT FOR COMMENT (SEPTEMBER, 1986)

Table E-8

EARLY FATALITY RISK RESULTS FOR THE SAFETY OPTIONS: OCP METHOD

\begin{tabular}{|c|c|c|c|c|c|}
\hline \multirow[b]{2}{*}{ OPTION } & \multirow[b]{2}{*}{ CENTRAL } & \multirow{2}{*}{\multicolumn{2}{|c|}{ OPTIMISTIC PESSIMISTIC }} & \multicolumn{2}{|c|}{ UNCERTAINTY DOUBLES } \\
\hline & & & & LOW & HIGH \\
\hline BASE CASE & $2.96 \mathrm{E}-07$ & $0.00 \mathrm{E}+00$ & $1.08 \mathrm{E}-02$ & $0.00 \mathrm{E}+00$ & $1.40 \mathrm{E}-04$ \\
\hline P1: Improved V & $1.69 \mathrm{E}-07$ & $0.00 \mathrm{E}+00$ & $7.40 \mathrm{E}-03$ & $0.00 \mathrm{E}+00$ & $8.60 \mathrm{E}-05$ \\
\hline P2: Improved ac Power & $2.88 \mathrm{E}-07$ & $0.00 \mathrm{E}+\infty 0$ & $5.90 \mathrm{E}-03$ & $0.00 \mathrm{E}+00$ & $1.30 \mathrm{E}-04$ \\
\hline P3: Improved dc Power & $2.96 \mathrm{E}-07$ & $0.00 \mathrm{E}+00$ & $9.80 \mathrm{E}-03$ & $0.00 \mathrm{E}+00$ & $1.40 \mathrm{E}-04$ \\
\hline P4: Independent HPI & $1.39 \mathrm{E}-08$ & $0.00 \mathrm{E}+00$ & $6.80 \mathrm{E}-03$ & $0.00 \mathrm{E}+00$ & $8.00 \mathrm{E}-05$ \\
\hline P5: New Aux Feedwater & $2.92 \mathrm{E}-07$ & $0.00 \mathrm{E}+00$ & 7.06E-03 & $0.00 \mathrm{E}+00$ & $1.36 \mathrm{E}-04$ \\
\hline P6: Independent LPI & $1.59 \mathrm{E}-07$ & $0.00 \mathrm{E}+00$ & $1.07 \mathrm{E}-02$ & $0.00 \mathrm{E}+00$ & $1.33 \mathrm{E}-04$ \\
\hline P7: New Service Water & $1.64 \mathrm{E}-07$ & $0.00 \mathrm{E}+00$ & $1.08 \mathrm{E}-02$ & $0.00 \mathrm{E}+00$ & $1.34 \mathrm{E}-04$ \\
\hline P8: RCS Depressurization & 2.92E-07 & $0.00 \mathrm{E}+00$ & $3.90 \mathrm{E}-03$ & $0.00 \mathrm{E}+00$ & $1.00 \mathrm{E}-04$ \\
\hline M1: Missile Shield & $3.00 \mathrm{E}-07$ & $0.00 \mathrm{E}+00$ & $9.74 \mathrm{E}-03$ & $0.00 \mathrm{E}+00$ & $1.40 \mathrm{E}-04$ \\
\hline M2: Hydrogen Igniters & $2.97 \mathrm{E}-07$ & $0.00 \mathrm{E}+00$ & $1.10 \mathrm{E}-02$ & $0.00 \mathrm{E}+00$ & $1.40 \mathrm{E}-04$ \\
\hline M3: Containment Inerting & $2.96 \mathrm{E}-07$ & $0.00 \mathrm{E}+00$ & $9.40 \mathrm{E}-03$ & $0.00 \mathrm{E}+00$ & $1.35 \mathrm{E}-04$ \\
\hline M4: Early Venting & $1.37 \mathrm{E}-07$ & $0.00 \mathrm{E}+00$ & $8.90 \mathrm{E}-03$ & $0.00 \mathrm{E}+00$ & $1.28 \mathrm{E}-04$ \\
\hline M5: Late Venting & $1.38 \mathrm{E}-07$ & $0.00 \mathrm{E}+00$ & $1.07 \mathrm{E}-02$ & $0.00 \mathrm{E}+00$ & $1.30 \mathrm{E}-04$ \\
\hline M6: Containment Sprays & $1.44 \mathrm{E}-07$ & $0.00 \mathrm{E}+00$ & $8.58 \mathrm{E}-03$ & $0.00 \mathrm{E}+00$ & $1.30 \mathrm{E}-04$ \\
\hline M7: Fan Coolers & $1.60 \mathrm{E}-07$ & $0.00 \mathrm{E}+00$ & $1.05 \mathrm{E}-02$ & $0.00 E+00$ & $1.40 \mathrm{E}-04$ \\
\hline M8: Containment Flooding & & NC & TEVALUAT & $\mathrm{ED}$ & \\
\hline $\mathrm{P} 1$ \& $\mathrm{P} 2: \mathrm{V}$ and $\mathrm{AC}$ Power & $1.60 \mathrm{E}-07$ & $0.00 \mathrm{E}+00$ & $2.49 \mathrm{E}-03$ & $0.00 \mathrm{E}+00$ & $4.60 \mathrm{E}-05$ \\
\hline
\end{tabular}


NUREG/CR-4551, VOL. 1: DRAFT REPORT FOR COMMENT (SEPTEMBER, 1986)

Table E-9

\section{LLH LATENT CANCER FATALITY RISK REDUCTION FOR THE SAFETY OPTIONS}

\begin{tabular}{lcccc}
\hline \multicolumn{1}{c}{ OPTION } & MEAN & MEDIAN & 5 TH & $95 T H$ \\
\hline P1: Improved V & $92 \%$ & $91 \%$ & $60 \%$ & $98 \%$ \\
P2: Improved ac Power & $27 \%$ & $49 \%$ & $76 \%$ & $27 \%$ \\
P3: Improved dc Power & $94 \%$ & $85 \%$ & $97 \%$ & $92 \%$ \\
P4: Independent HPI & $20 \%$ & $44 \%$ & $70 \%$ & $14 \%$ \\
P5: New Aux Feedwater & $79 \%$ & $69 \%$ & $92 \%$ & $74 \%$ \\
P6: Independent LPI & $92 \%$ & $82 \%$ & $42 \%$ & $97 \%$ \\
P7: New Service Water & $98 \%$ & $97 \%$ & $90 \%$ & $98 \%$ \\
P8: RCS Depressurization & $26 \%$ & $44 \%$ & $94 \%$ & $22 \%$ \\
M1: Missile Shield & $99 \%$ & $82 \%$ & $92 \%$ & $100 \%$ \\
M2: Hydrogen Igniters & $100 \%$ & $100 \%$ & $100 \%$ & $100 \%$ \\
M3: Containment Inerting & $72 \%$ & $54 \%$ & $73 \%$ & $83 \%$ \\
M4: Early Venting & $89 \%$ & $53 \%$ & $65 \%$ & $119 \%$ \\
M5: Late Venting & $99 \%$ & $88 \%$ & $80 \%$ & $99 \%$ \\
M6: Containment Sprays & $29 \%$ & $49 \%$ & $79 \%$ & $29 \%$ \\
M7: Fan Coolers & $90 \%$ & $71 \%$ & $92 \%$ & $99 \%$ \\
M8: Containment Flooding & $85 \%$ & $74 \%$ & $104 \%$ & $93 \%$ \\
P6 \& P8: Depress. and LPI & $9 \%$ & $18 \%$ & $17 \%$ & $63 \%$ \\
P2 \& M4: DG and Venting & $48 \%$ & $49 \%$ & $56 \%$ & $55 \%$ \\
\hline
\end{tabular}

Table E-10

LLH EARLY FATALITY RISK REDUCTION FOR THE SAFETY OPTIONS

\begin{tabular}{lcccc}
\hline \multicolumn{1}{c}{ OPTION } & MEAN & MEDIAN & 5TH & $95 T H$ \\
\hline P1: Improved V & $97 \%$ & $93 \%$ & $42 \%$ & $100 \%$ \\
P2: Improved ac Power & $15 \%$ & $26 \%$ & $78 \%$ & $12 \%$ \\
P3: Improved dc Power & $93 \%$ & $93 \%$ & $97 \%$ & $92 \%$ \\
P4: Independent HPI & $11 \%$ & $24 \%$ & $62 \%$ & $6 \%$ \\
P5: New Aux Feedwater & $80 \%$ & $63 \%$ & $92 \%$ & $73 \%$ \\
P6: Independent LPI & $95 \%$ & $42 \%$ & $52 \%$ & $100 \%$ \\
P7: New Service Water & $98 \%$ & $87 \%$ & $91 \%$ & $100 \%$ \\
P8: RCS Depressurization & $16 \%$ & $23 \%$ & $104 \%$ & $6 \%$ \\
M1: Missile Shield & $99 \%$ & $78 \%$ & $71 \%$ & $100 \%$ \\
M2: Hydrogen Igniters & $100 \%$ & $100 \%$ & $100 \%$ & $100 \%$ \\
M3: Containment Inerting & $59 \%$ & $31 \%$ & $71 \%$ & $37 \%$ \\
M4: Early Venting & $75 \%$ & $32 \%$ & $68 \%$ & $51 \%$ \\
M5: Late Venting & $100 \%$ & $100 \%$ & $100 \%$ & $103 \%$ \\
M6: Containment Sprays & $15 \%$ & $25 \%$ & $77 \%$ & $11 \%$ \\
M7: Fan Coolers & $86 \%$ & $49 \%$ & $100 \%$ & $82 \%$ \\
M8: Containment Flooding & $80 \%$ & $46 \%$ & $100 \%$ & $52 \%$ \\
P6 \& P8: Depress. and LPI & $2 \%$ & $5 \%$ & $23 \%$ & $1 \%$ \\
P2 \& M4: DG and Venting & $28 \%$ & $26 \%$ & $58 \%$ & $18 \%$ \\
\hline
\end{tabular}


Table E-11

OCP LATENT CANCER FATALITY RISK REDUCTION FOR THE SAFETY OPTIONS

\begin{tabular}{lccc}
\hline \multicolumn{1}{c}{ OPTION } & CENTRAL & OPTIMISTIC & PESSIMISTIC \\
\hline P1: Improved V & $50 \%$ & $28 \%$ & $90 \%$ \\
P2: Improved ac Power & $90 \%$ & $94 \%$ & $47 \%$ \\
P3: Improved dc Power & $99 \%$ & $99 \%$ & $94 \%$ \\
P4: Independent HPI & $85 \%$ & $89 \%$ & $31 \%$ \\
P5: New Aux Feedwater & $95 \%$ & $97 \%$ & $79 \%$ \\
P6: Independent LPI & $72 \%$ & $28 \%$ & $88 \%$ \\
P7: New Service Water & $78 \%$ & $39 \%$ & $100 \%$ \\
P8: RCS Depressurization & $94 \%$ & $118 \%$ & $48 \%$ \\
M1: Missile Shield & $100 \%$ & $100 \%$ & $89 \%$ \\
M2: Hydrogen Igniters & $100 \%$ & $100 \%$ & $100 \%$ \\
M3: Containment Inerting & $99 \%$ & $100 \%$ & $82 \%$ \\
M4: Early Venting & $62 \%$ & $10 \%$ & $51 \%$ \\
M5: Late Venting & $64 \%$ & $10 \%$ & $83 \%$ \\
M6: Containment Sprays & $58 \%$ & $17 \%$ & $69 \%$ \\
M7: Fan Coolers & $81 \%$ & $18 \%$ & $96 \%$ \\
M8: Containment Flooding & & NOT EVALUATED & \\
P1 \& P2: V and AC Power & $40 \%$ & $23 \%$ & $34 \%$ \\
\hline
\end{tabular}

Table E-12

OCP EARLY FATALITY RISK REDUCTION FOR THE SAFETY OPTIONS

\begin{tabular}{lccc}
\hline \multicolumn{1}{c}{ OPTION } & CENTRAL & OPTIMISTIC & PESSIMISTIC \\
\hline P1: Improved V & $57 \%$ & - & $68 \%$ \\
P2: Improved ac Power & $97 \%$ & - & $54 \%$ \\
P3: Improved dc Power & $100 \%$ & - & $90 \%$ \\
P4: Independent HPI & $4 \%$ & - & $62 \%$ \\
P5: New Aux Feedwater & $98 \%$ & - & $65 \%$ \\
P6: Independent LPI & $53 \%$ & - & $99 \%$ \\
P7: New Service Water & $55 \%$ & - & $100 \%$ \\
P8: RCS Depressurization & $98 \%$ & - & $36 \%$ \\
M1: Missile Shield & $101 \%$ & - & $90 \%$ \\
M2: Hydrogen Igniters & $100 \%$ & - & $101 \%$ \\
M3: Containment Inerting & $100 \%$ & - & $87 \%$ \\
M4: Early Venting & $46 \%$ & - & $82 \%$ \\
M5: Late Venting & $46 \%$ & - & $99 \%$ \\
M6: Containment Sprays & $48 \%$ & - & $79 \%$ \\
M7: Fan Coolers & $54 \%$ & NOT EVALUATED & $97 \%$ \\
M8: Containment Flooding & & - & $23 \%$ \\
P1 \& P2: V and AC Power & $54 \%$ & & \\
\hline
\end{tabular}




\section{E.3 ASSESSMENT OF IMPACT FOR RISK-REDUCTION MEASURES}

The investigation of the merit of the proposed risk-reduction measures requires a thorough evaluation of impact that would result from their implementation. As discussed in Section 3 of the main report, there are three principal types of impact considered in the first-cut evaluation of each option:

1. The total cost associated with installation of the proposed modification.

2. The ongoing costs to the utility (maintenance, increased plant downtime, etc.) that arise from the modification.

3. The personnel dose associated with installation and maintenance of the option.

While there may be other types of impacts that are also significant, a value/impact assessment that is limited to consideration of the costs noted above serves as an effective screening evaluation. Because the baseline risk estimated for Surry is already relatively low, few potential modifications will be cost effective. The costs and other impacts of any modifications that appear as a result of the screening analysis to be beneficial can be evaluated more thoroughly.

The costs for the modifications considered for Surry are delineated in Section E.3.1, and estimated personnel doses associated with the options are included in Section E.3.2. All of these estimates are based on the impact information included in Appendix G. Although the cost of replacement power is not included in the basic cost estimates, Section E.3.3 includes a brief discussion of this potential impact. That discussion provides additional perspective for consideration of the risk-reduction measures and insights useful to a more complete assessment of replacement-power costs.

\section{E.3.1 Estimated Costs of Risk-Reduction Measures}

As stated above, the information compiled in Appendix $G$ was used to estimate costs for each of the proposed risk-reduction measures. Table E-13 provides the installation cost for each option, including an estimate of the uncertainty (presented in terms of an upper and a lower estimate). The basis for each estimate is included in the notes that follow the tables. The recurring costs associated with increased maintenance and operation costs for the plant after installation of the option are presented in Table E-14. The present value of the total cost of each option, considering the year in which an accident could occur, are listed in Table E-15. 
NUREG/CR-4551, VOL. 1: DRAFT REPORT FOR COMMENT (SEPTEMBER, 1986)

Table E-13

ESTIMATED ONE-TIME COST FOR RISK-REDUCTION MEASURES

Option

Total Installation Cost

Central Upper Lower Notes

P1. Improved V Testing

Procedures

$\$ 100,000 \quad \$ 180,000 \quad \$ 30,000 \quad 1$

P2. Improved ac power through the addition of a diesel generator

$\$ 16,000,000 \$ \$ 32,000,000 \quad \$ 11,000,000 \quad 2$

P3. Improved de power system through extension of battery life

$\$ 1,200,000$

$\$ 2,400,000$

$\$ 600,000 \quad 3$

P4. Additional, independent train of high-pressure injection

$\$ 26,800,000$

$\$ 53,600,000$

$\$ 18,800,000$

4

P5. Additional, independent train of auxiliary feedwater

$\$ 7,000,000$

$\$ 14,000,000$

$\$ 5,000,000 \quad 5$

P6. Long-term residual heat removal/ low-pressure recirculation

$\$ 25,000,000 \quad \$ 50,000,000 \quad \$ 17,000,000 \quad 6$

P7. New service water system for containment

$\$ 8,000,000$

$\$ 16,000,000$

$\$ 6,000,000$

7

P8. Rapid primary system depressurization

$\$ 3,0000,00$

$\$ 6,0000,00$

$\$ 2,0000,00 \quad 8$

M1. Missile shield

$\$ 10,000,000$

$\$ 30,000,000$

$\$ 7,000,000 \quad 9$

M2. Hydrogen ignition system

$\$ 7,600,000$

$\$ 15,200,000$

$\$ 2,000,000 \quad 10$

M3. Prevention of hydrogen combustion

$\$ 3,000,000$

$\$ 7,000,000$

$\$ 1,500,000$

M4. Early containment venting

$\$ 13,500,000$

$\$ 27,000,000$

$\$ 9,500,000$

12

M5. Late containment venting

$\$ 13,500,000$

$\$ 27,000,000$

$\$ 9,500,000 \quad 12$

M6. Additional contairment cooling--sprays

$\$ 8,000,000$

$\$ 16,000,000$

$\$ 5,600,000$

13

M7. Additional containment cooling--fans

$\$ 3,700,000$

$\$ 7,400,000$

$\$ 2,600,000 \quad 14$

M8. Containment flooding system

$\$ 200,000$

$\$ 1,000,000$

$\$ 60,000$ 


\section{Notes to Table E-13:}

1. There are no records in Appendix $G$ that are directly applicable to this option, but several of the records did included line item costs for valves (records 6,38 and 47). The total cost associated with this change would appear to be less than that of record 16 but more than record 12 .

2. Record 55 in Appendix $\mathrm{G}$ is directly applicable and it was derived from the reference that included the most detailed cost investigation; it was therefore used directly.

3. The basic assumption was that the batteries would need to be replaced. Record 31 is directly applicable, and record 27 is also relevant. Because detailed information was not available and the possibility exists that the batteries would not have to be replaced, the lower cost reflects upgrading of the batteries and improved testing to ensure eight-hour lifetime. The lower cost was estimated to be a factor of two less than the central for this case.

4. Record 58 applies directly.

5. This is a subset of the modification in record 59 which also includes a primary makeup pump. However, record 59 includes a new diesel and all new buildings which would not seem to be needed in this case. It would appear that the cost associated with the addition of a diesel-or turbine-driven AFW pump would be most similar to record 49 but the pump would be more expensive, as would the tie-ins to the steam generators.

6. Record 56 applies directly.

7. Records 49 and 50 bound this modification.

8. The addition of a dedicated depressurization system (for a BWR) is included in the modifications in record 56 along with other system additions. Since this option would require extensive study for application to a PWR it seems likely that the costs could be very high. For scoping purposes $\$ 6,000,000$ was selected as a central estimate but this would require additional research if this option should prove to be of net benefit in the first-cut analysis.

9. There is no data record that would apply. In addition, no detailed design was considered. Engineering judgment was used to estimate a range of costs.

10. Data from record 51 was used directly for the central and high estimate. The low estimate was reduced because of the potential for installation of a much simpler system than defined in record 51.

11. Although this has been accomplished for many BWRs, time did not permit detailed investigation of installed costs. It would appear that this would be more than the costs for the option in record 38, but probably less than that in record 50 . This cost estimate is quite uncertain.

12. Containment venting through filters is reflected in records 53 and 54 . The system in record 60 includes-high efficiency filters. This option was simulated in estimating the source term as a basemat meltthrough, and it will be assumed here that the former vent systems are more applicable than the high-efficiency version. There is no difference in the system cost for the early versus late vent strategy.

13. Records 39,50 and 52 (somewhat different since it is BWR-specific) apply. The value selected lies between that of the systems in records 50 and 52 .

14. No fans are represented in the data, but the major cost would be the independent heat rejection system. Record 39 applies most directly.

15. This system is essentially a tank and a pump and the costs were estimated through comparison to several of the records, for example, records 28 and 29 would be projects of similar scope. 
NUREG/CR-4551, VOL. 1: DRAFT REPORT FOR COMMENT (SEPTEMBER, 1986)

Table E-14

ANNUAL MAINTENANCE AND OPERATIONS COSTS FOR PROPOSED OPTIONS

Option
Central
Total Annual Cost

Upper Lower Notes

P1. Addition of new interfacing check valves

$\$ 50,000$

$\$ 100,000$

$\$ 20,000 \quad 1$

P2. Improved ac power through the addition of a diesel generator

$\$ 200,000$

$\$ 400,000$

$\$ 140,000 \quad 2$

P3. Improved dc power system through extension of battery life

$$
\$ 7,000
$$

$\$ 14,000$

$\$ 5,000$

P4. Additional, independent train of high-pressure injection

$\$ 150,000$

$\$ 300,000$

$\$ 110,000 \quad 4$

P5. Additional, independent train of auxiliary feedwater

$\$ 75,000$

$\$ 150,000$

$\$ 50,000 \quad 5$

P6. Long-term residual heat removal/ low-pressure recirculation

$\$ 150,000 \quad \$ 300,000 \quad \$ 110,000 \quad 6$

P7. New service water system for containment

$\$ 20,000$

$\$ 40,000$

$\$ 14,000$ 7

P8. Rapid primary system depressurization

$\$ 40,000$

$\$ 100,000$

$\$ 5,000$

$\$ 10,000$

$\$ 25,000 \quad 8$

M1. Missile shield

$\$ 50,000$

$\$ 100,000$

$\$ 2,500 \quad 9$

M2. Hydrogen ignition system

M3. Prevention of hydrogen combustion

$\$ 300,000$

$\$ 600,000$

$\$ 35,000 \quad 10$

M4. Early containment venting

M5. Late containment venting

$\$ 100,000$

$\$ 100,000$

$\$ 200,000$

$\$ 210,000$

11

$\$ 70,000 \quad 12$

$\$ 200,000$

$\$ 70,000 \quad 12$

M6. Additional containment cooling--sprays

$\$ 90,000$

$\$ 180,000$

$\$ 63,000$

13

M7. Additional containment cooling--fans

$\$ 50,000$

$\$ 100,000$

$\$ 70,000 \quad 14$

M8. Containment flooding system

$\$ 15,000$

$\$ 10,000$ 


\section{Notes to Table E-14:}

1. Based on recurring costs in records $17,26,27$ and 30 , a $\$ 5000$ cost was estimated for this option.

2. Record 55 was used directly.

3. The basic assumption was that the batteries would need to be replaced. Record 31 is directly applicable and record 27 is also relevant.

4. No recurring costs were given in record 56. The recurring costs would probably be most similar to those associated with the options in records 52 and 55 .

5. This would be expected to require less maintenance than risk-reduction measure P4.

6. This would require more maintenance than that associated with the change in record 52 , but less than record 61 . It is also similar to option P4, and that value was used for this option as well.

7. The maintenance cost would lie between the costs described in records 49 and 50 .

8. For scoping purposes $\$ 100,000$ was selected as a central estimate, but additional research would be required if this option should prove to be of net benefit in the firstcut analysis. This would require more maintenance than that associated with the change in record 44, but less than record 52. It is also similar to option P2, and that value was used for this option as well.

9. There is no data record that would apply. In addition, no detailed design was considered. Engineering judgment was used to estimate a range of costs assuming that only minimal inspection would be required.

10. Data from record 51 was used directly.

11. Although this has been accomplished for many BWRs, time did not permit detailed investigation of installed costs. It would appear that this could have significant annual costs, and $\$ 300,000$ was selected as a scoping estimate.

12. It was assumed that the vent systems would require maintenance of similar magnitude to that associated with record 52 .

13. This option includes additional sprays and service water for containment cooling. Records 50 and 52 (somewhat different since it is BWR-specific) apply.

14. The maintenance cost would be similar those associated with the changes in records 44,49 and 50.

15. The maintenance cost would be similar those associated with the changes in records $33,40,20$. 
Table E-15

ESTIMATED PRESENT VALUE TOTAL COST FOR RISK-REDUCTION MEASURES

\begin{tabular}{|c|c|c|c|c|}
\hline & Option & Central & $\begin{array}{l}\text { Total Cost } \\
\text { Upper }\end{array}$ & Lower \\
\hline P1. & $\begin{array}{l}\text { New test procedure for } \\
\text { interfacing check valves }\end{array}$ & $\$ 930,000$ & $\$ 1,800,000$ & $\$ 360,000$ \\
\hline P2. & $\begin{array}{l}\text { Improved ac power through the } \\
\text { addition of a diesel generator }\end{array}$ & $\$ 19,000,000$ & $\$ 38,600,000$ & $\$ 13,000,000$ \\
\hline P3. & $\begin{array}{l}\text { Improved dc power system } \\
\text { through extension of battery life }\end{array}$ & $\$ 1,300,000$ & $\$ 2,600,000$ & $\$ 680,000$ \\
\hline P4. & $\begin{array}{l}\text { Additional, independent train } \\
\text { of high-pressure injection }\end{array}$ & $\$ 29,000,000$ & $\$ 58,600,000$ & $\$ 20,000,000$ \\
\hline P5. & $\begin{array}{l}\text { Additional, independent train } \\
\text { of auxiliary feedwater }\end{array}$ & $\$ 8,200,000$ & $\$ 16,400,000$ & $\$ 5,800,000$ \\
\hline P6. & $\begin{array}{l}\text { Long-term residual heat removal/ } \\
\text { low-pressure recirculation }\end{array}$ & $\$ 27,000,000$ & $\$ 55,000,000$ & $\$ 18,800,000$ \\
\hline P7. & $\begin{array}{l}\text { New service water system } \\
\text { for containment }\end{array}$ & $\$ 6,200,000$ & $\$ 8,300,000$ & $\$ 16,000,000$ \\
\hline P8. & $\begin{array}{l}\text { Rapid primary system } \\
\text { depressurization }\end{array}$ & $\$ 3,700,000$ & $\$ 6,000,000$ & $\$ 2,400,000$ \\
\hline M1. & Missile shield & $\$ 10,000,000$ & $\$ 30,000,000$ & $\$ 7,000,000$ \\
\hline M2. & Hydrogen ignition system & $\$ 8,400,000$ & $\$ 16,800,000$ & $\$ 2,600,000$ \\
\hline M3. & $\begin{array}{l}\text { Prevention of hydrogen } \\
\text { combustion }\end{array}$ & $\$ 8,000,000$ & $\$ 17,000,000$ & $\$ 4,900,000$ \\
\hline M4. & Early containment venting & $\$ 15,100,000$ & $\$ 30,300,000$ & $\$ 10,500,000$ \\
\hline M5. & Late containment venting & $\$ 15,200,000$ & $\$ 30,300,000$ & $\$ 10,100,000$ \\
\hline M6. & $\begin{array}{l}\text { Additional containment } \\
\text { cooling-sprays }\end{array}$ & $\$ 9,500,000$ & $\$ 19,000,000$ & $\$ 6,600,000$ \\
\hline M7. & $\begin{array}{l}\text { Additional containment } \\
\text { cooling--fans }\end{array}$ & $\$ 4,900,000$ & $\$ 9,100,000$ & $\$ 3,400,000$ \\
\hline M8. & $\begin{array}{l}\text { Containment flooding } \\
\text { system }\end{array}$ & $\$ 1,200,000$ & $\$ 2,500,000$ & $\$ 750,000$ \\
\hline
\end{tabular}




\section{E.3.2 Estimated Personnel_Doses Due to the Risk-Reduction Measures}

The radiation exposure associated with the installation of a modification is strongly dependent on the layout and practices at a specific plant, the schedule for implementation, and other factors that could only be determined through detailed planning of a specific option. Since this type of information was unavailable, the estimates of personnel dose are largely judgmental, and are intended only to be representative. If a proposed modification appeared to be beneficial, more detailed assessment of this factor would be required. The estimates of personnel dose used in the value/impact analysis are presented in Table E-16.

Table E-16

DOSE IN PERSON-REMS FOR RISK-REDUCTION MEASURES

\begin{tabular}{|c|c|c|c|c|c|c|}
\hline & & Total Ins & ion Dose & son-rem) & Annual & \\
\hline & Option & Central & Upper & Lower & Dose & Notes \\
\hline P1. & $\begin{array}{l}\text { Improved testing of } \\
\text { interfacing check valves }\end{array}$ & 10 & 20 & 1 & 0.4 & 1 \\
\hline P2. & $\begin{array}{l}\text { Improved ac power through } \\
\text { the addition of a diesel generator }\end{array}$ & 0.0 & 0.0 & 0.0 & 0.0 & 2 \\
\hline P3. & $\begin{array}{l}\text { Improved de power system } \\
\text { through extension of battery life }\end{array}$ & 0.0 & 0.0 & 0.0 & 0.0 & 2 \\
\hline P4. & $\begin{array}{l}\text { Additional, independent train } \\
\text { of high-pressure injection }\end{array}$ & 150 & 400 & 50 & 1.0 & 3 \\
\hline P5. & $\begin{array}{l}\text { Additional, independent train } \\
\text { of auxiliary feedwater }\end{array}$ & 0.0 & 0.0 & 0.0 & 0.0 & 2 \\
\hline P6. & $\begin{array}{l}\text { Long-term residual heat removal/ } \\
\text { low-pressure recirculation }\end{array}$ & 150 & 300 & 50 & 1.0 & 3 \\
\hline P7. & $\begin{array}{l}\text { New service water system } \\
\text { for containment }\end{array}$ & 50 & 200 & 20 & 1.0 & 3 \\
\hline P8. & $\begin{array}{l}\text { Rapid primary system } \\
\text { depressurization }\end{array}$ & 100 & 1000 & 20 & 1.0 & 3 \\
\hline M1. & Missile shield & 800 & 1500 & 300 & 0.0 & 3 \\
\hline M2. & Hydrogen ignition system & 500 & 800 & 200 & 1.0 & 3 \\
\hline M3. & $\begin{array}{l}\text { Prevention of hydrogen } \\
\text { combustion }\end{array}$ & 50 & 200 & 20 & 1.0 & 3 \\
\hline M4. & Early containment venting & 50 & 200 & 20 & 0.0 & 3 \\
\hline M5. & Late containment venting & 50 & 200 & 20 & 0.0 & 3 \\
\hline M6. & $\begin{array}{l}\text { Additional containment } \\
\text { cooling-sprays }\end{array}$ & 300 & 600 & 50 & 1.0 & 3 \\
\hline M7. & $\begin{array}{l}\text { Additional containment } \\
\text { cooling--fans }\end{array}$ & 300 & 600 & 50 & 1.0 & 3 \\
\hline M8. & Containment flooding & 0 & 0 & 0 & 0 & 2 \\
\hline
\end{tabular}

Notes: 1. This is done regularly and would involve minimal dose.

2. All work would be remote from radiation areas.

3. Based on comparison to entries in Table G-1, Appendix G. 


\section{E.3.3 Insights Concerning_Replacement_Power}

Although replacement power costs are not included for reasons cited in Appendix G, Table E-17 presents insights concerning this issue in order to more fully assess the impact of the proposed options. All of the proposed modifications could involve downtime due to the difficulties in scheduling a major modification during a normal shutdown period. This would be a very specific consideration which could not be judged with the amount of detail considered in this study. The table only includes entries for those items that could be expected to have significant downtime in addition to that which could result from problems in scheduling a major modification activity. Costs for downtime also vary widely, but $\$ 500,000 /$ day is a fairly representative value.

Table E-17

REPLACEMENT POWER INSIGHTS FOR RISK-REDUCTION MEASURES

\begin{tabular}{|c|c|c|c|}
\hline & Option & $\begin{array}{c}\text { Possible } \\
\text { Downtime (days) }\end{array}$ & Insights \\
\hline P1. & $\begin{array}{l}\text { Addition of new } \\
\text { interfacing check valves }\end{array}$ & $0.5-1.0$ & $\begin{array}{l}\text { This could involve either a longer } \\
\text { startup or possibly the need to } \\
\text { shut down for installation. }\end{array}$ \\
\hline P4. & $\begin{array}{l}\text { Additional, independent train } \\
\text { of high-pressure injection }\end{array}$ & $1-30$ & $\begin{array}{l}\text { This option would require tie-ins } \\
\text { to the primary. See data record } \\
49^{2} \text {. }\end{array}$ \\
\hline P6. & $\begin{array}{l}\text { Long-term residual heat } \\
\text { removal/low-pressure } \\
\text { recirculation }\end{array}$ & $1-30$ & $\begin{array}{l}\text { This would require containment } \\
\text { activities and a primary system } \\
\text { interface. }\end{array}$ \\
\hline P8. & $\begin{array}{l}\text { Rapid primary system } \\
\text { depressurization }\end{array}$ & $5-30$ & $\begin{array}{l}\text { This would require extensive } \\
\text { work on the vessel or pressurizer } \\
\text { and would probably involve } \\
\text { significant downtime. }\end{array}$ \\
\hline M1. & Missile shield & $20-200$ & $\begin{array}{l}\text { This would probably require } \\
\text { extensive construction work } \\
\text { in the containment. }\end{array}$ \\
\hline M2. & Hydrogen ignition system & $10-30$ & $\begin{array}{l}\text { Major activities in containment } \\
\text { would be required. }\end{array}$ \\
\hline M3. & $\begin{array}{l}\text { Prevention of hydrogen } \\
\text { combustion }\end{array}$ & $2-10$ & $\begin{array}{l}\text { This would involve work both } \\
\text { inside and outside containment. }\end{array}$ \\
\hline M4. & Early containment venting & $5-15$ & See Appendix G, Section G.2.2. \\
\hline M5. & Late containment venting & $5-15$ & See Appendix G, Section G.2.2. \\
\hline M6. & $\begin{array}{l}\text { Additional containment } \\
\text { cooling--sprays }\end{array}$ & $5 \cdot 20$ & $\begin{array}{l}\text { This would require extra activities } \\
\text { in containment. }\end{array}$ \\
\hline M7. & $\begin{array}{l}\text { Additional containment } \\
\text { cooling-fans }\end{array}$ & $5-20$ & $\begin{array}{l}\text { Requires construction in the } \\
\text { containment. }\end{array}$ \\
\hline
\end{tabular}

Notes: 1. This table only includes items for which there could be particular cause for delay in addition to the scheduling delays that could result for any option.

2. All data records referred to are in Appendix G. 


\section{E.4 COSTS VERSUS BENEFITS}

The benefits were calculated in terms of averted accident costs to allow comparison to the cost of implementing the options. Due to the differing opinions within the community on the appropriate way to calculate the averted accident costs, several measures were used here. Four calculations were performed:

- Averted actual offsite costs, consisting of property damage costs (as calculated by CRAC2 and MACCS) and health effects costs (see Section 3);

- Averted offsite costs calculated as reduction in person rems (as estimated by CRAC2 and MACCS) times $\$ 1000$ dollars per person-rem averted;

- Averted actual offsite costs (as in the first method) plus averted onsite costs (calculated as described in Section 3);

- Averted offsite costs at $\$ 1000$ per rem plus averted onsite costs.

The results are illustrated in Figures E-2 Through E-5. It is fairly straightforward to see that none of the options appears cost effective. The bars represent the 5th to 95th percentiles of the LLH sample result for the value of averted risk and none really approaches the cost of the applicable option. The MACCS code results did predict higher costs and some of the options are closer to being effective, but the overall conclusions are the same between the CRAC2 and MACCS results. It should be noted that there are some sample members outside the LLH range illustrated which could show cost effectiveness, but the conclusions should be drawn from the reasonable ranges presented. The figures illustrate the OCP central as well and a similar conclusion holds. Only option $\mathrm{P} 1$, the improved $\mathrm{V}$ testing, approaches cost effectiveness and only for lowest costs compared to highest averted costs.

For the interested reader, Tables E-18 and E-19 list the results for the LLH and OCP methods, respectively, as calculated with CRAC2." These tables include the breakdown of option costs and averted costs as calculated by each method. The low, central and high columns in Table E-18 refer to the 5th, 50th and 95th of the distribution of the 100 sample members for the risk reduction for each option. In Table E-19, these labels refer to the averted risk for the optimistic, central and pessimistic walkthroughs, and low, central and high estimates of cost (since the costs could not be defined in terms of optimistic or pessimistic).

\footnotetext{
"Since the results were not dramatically different, the detailed results are not repeated for
} MACCS calculations. 


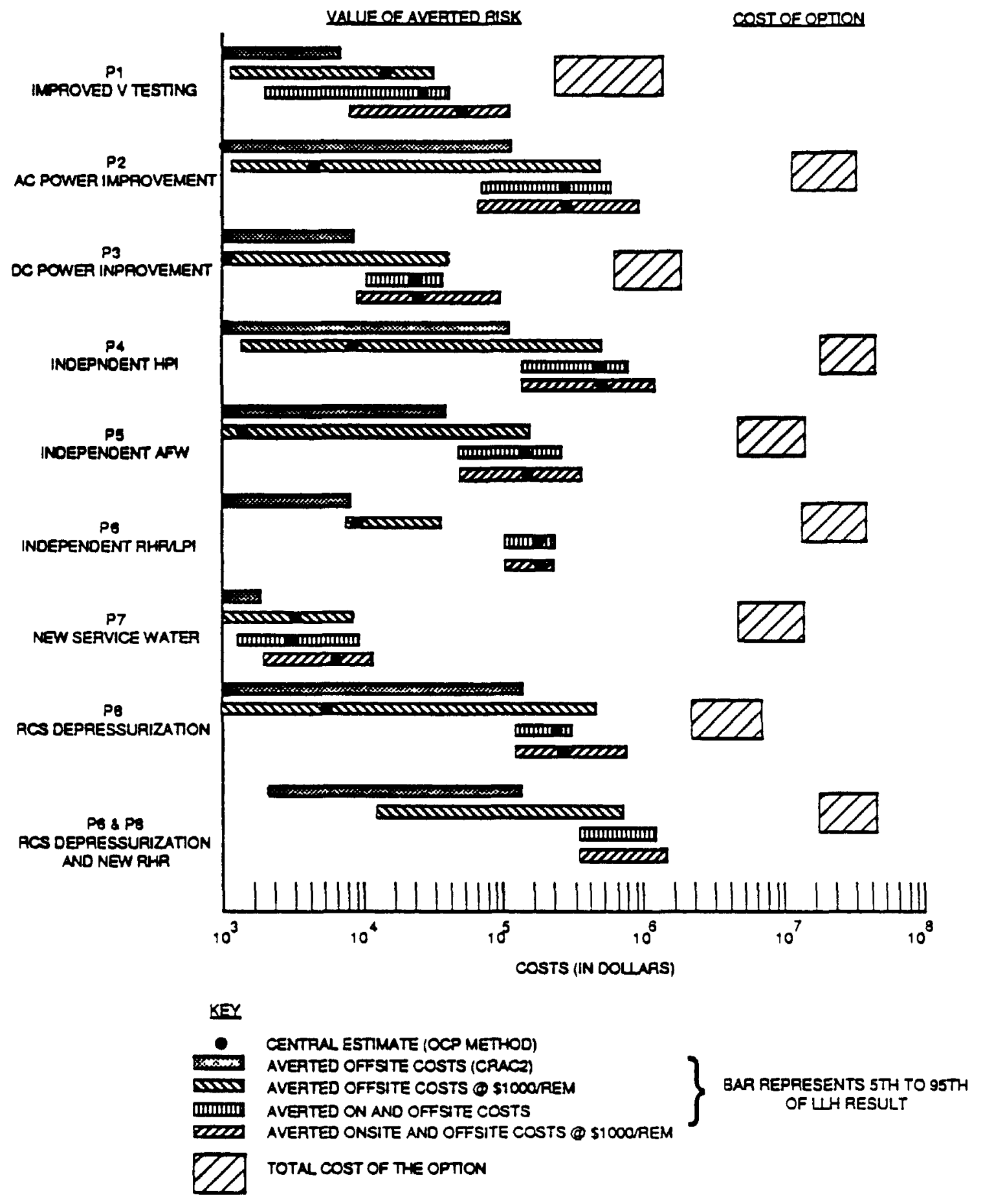

Figure E-2. Cost/Benefit Comparison for Prevention Options (CRAC2) 


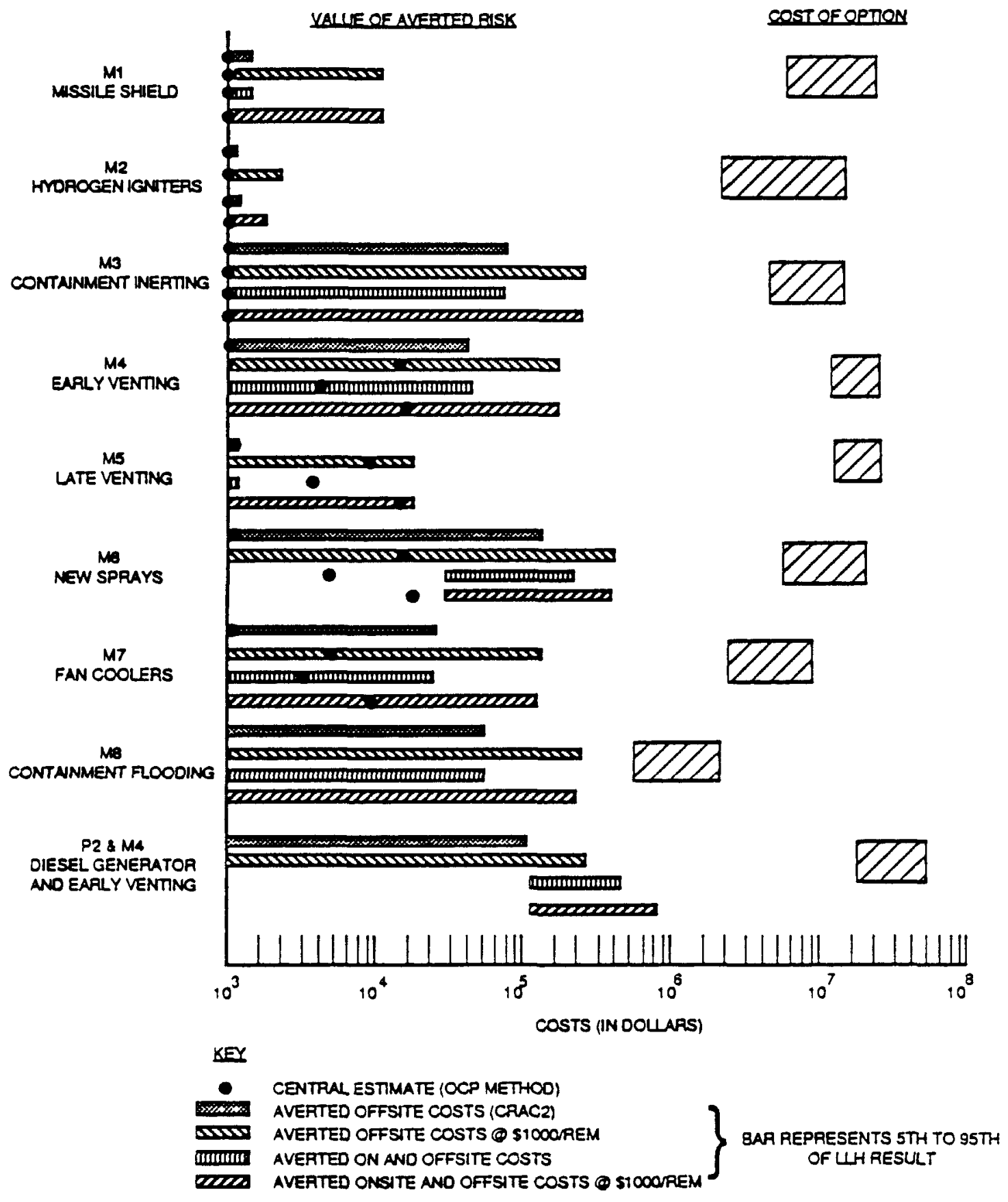

Figure E-3. Cost/Benefit Comparison for Mitigation Options (CRAC2) 


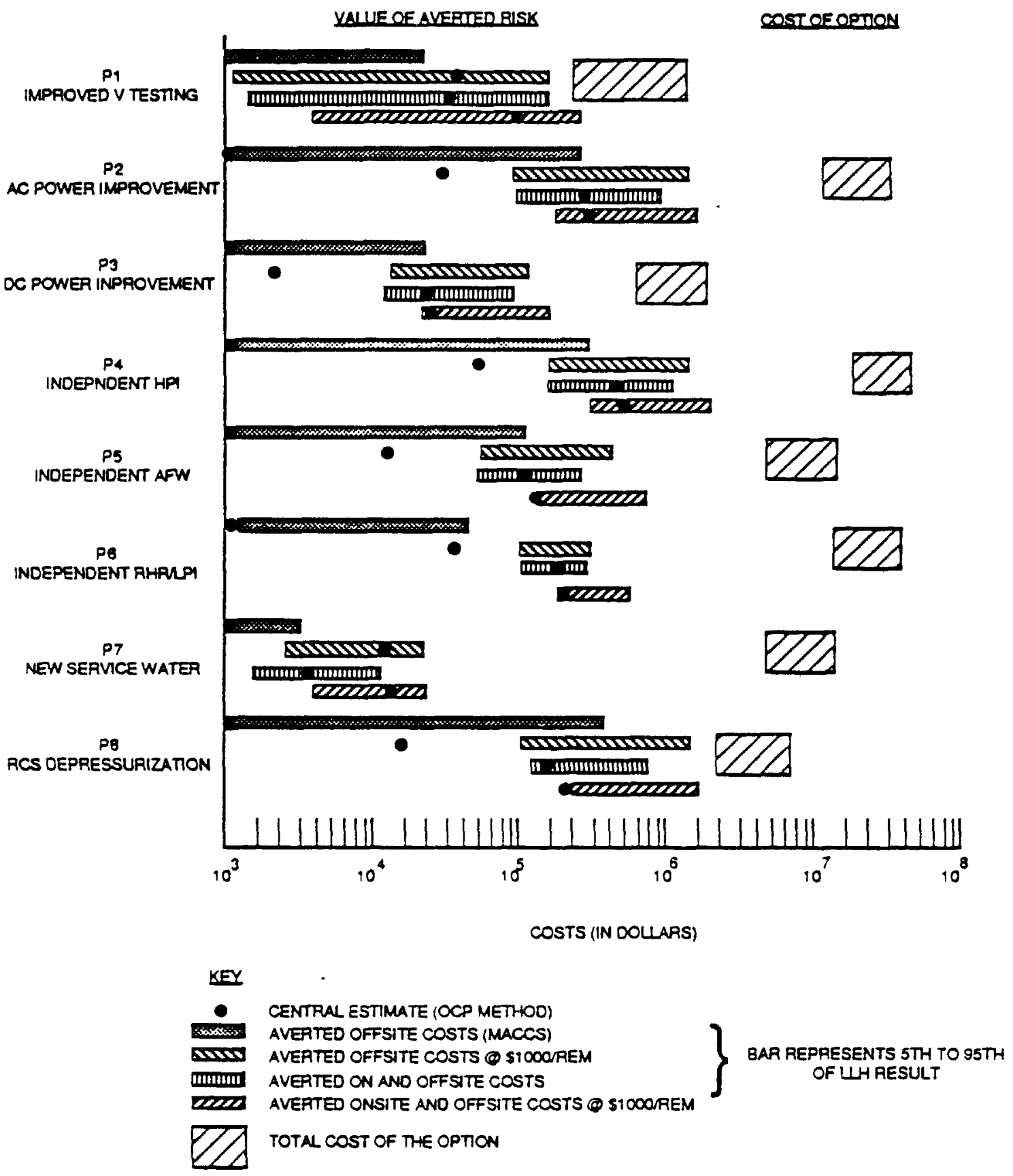

Figure E-4. Cost/Benefit Comparison for Prevention Options (MACCS) 
NUREG/CR-4551, VOL. 1: DRAFT REPORT FOR COMMENT (SEPTEMBER, 1986)

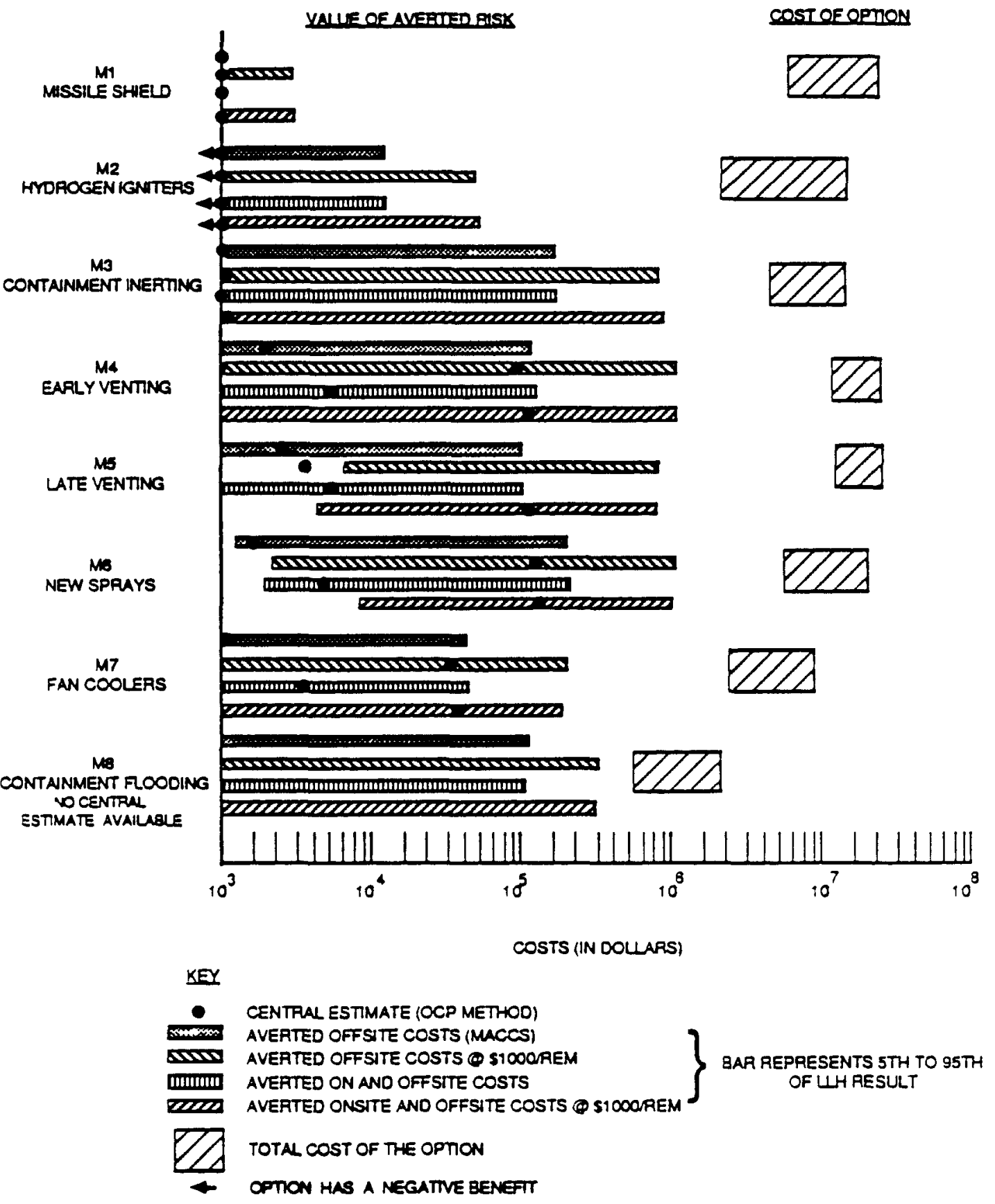

Figure E-5. Cost/Benefit Comparison for Mitigation Options (MACCS) 
Table E-18

COMPARISON OF COSTS AND BENEFITS FOR SAFETY OPTIONS: LLH METHOD (COSTS IN DOLLARS)

\begin{tabular}{|c|c|c|c|}
\hline \multicolumn{4}{|c|}{ SAFETY OPTION :P1: Improved $V$ procedures } \\
\hline \multicolumn{4}{|c|}{ VALUE-IMPACT ANALYSIS } \\
\hline & LOW & CENTRAL & HIGH \\
\hline CHANGE IN CORE MELT FREQUENCY & $4.998 \mathrm{E}-08$ & 8.110E-07 & $8.110 \mathrm{E}-07$ \\
\hline \multicolumn{4}{|l|}{ costs: } \\
\hline INSTALLATION \& ENG. COSTS & $3.000 \mathrm{E}+04$ & $1.000 \mathrm{E}+05$ & $1.800 \mathrm{E}+05$ \\
\hline OPERATING \& MAINT. COSTS & $3.302 E+05$ & $8.255 \mathrm{E}+05$ & $1.651 E+06$ \\
\hline REPLACEMENT POWER DURING INST. & $0.000 \mathrm{E}+00$ & $0.000 \mathrm{E}+00$ & $0.000 \mathrm{E}+00$ \\
\hline REPLACEMENT POWER IN OPERATION & $0.000 \mathrm{E}_{+}+00$ & $0.000 E_{+}+0$ & $2.000 E_{+00}$ \\
\hline TOTAL COSTS & $3.602 E+05$ & $9.255 \mathrm{E}+05$ & $1.831 E+06$ \\
\hline \multicolumn{4}{|l|}{ BENEFITS } \\
\hline AVERTED ON-SITE COST & $2.351 E+03$ & $3.814 E+04$ & $5.399 E+04$ \\
\hline \multicolumn{4}{|l|}{ AVERTED OFFSTTE COSTS } \\
\hline AVERTED PROPERTY DAMAGE & $1.108 E+02$ & $1.978 \mathrm{E}+03$ & $6.901 E+03$ \\
\hline VALUE OF AVERTED PROMPT FATALTY & $5.313 \mathrm{E}-02$ & $1.693 E+00$ & $1.376 \mathrm{E}+01$ \\
\hline VALUE OF AVERTED EARLY ILLNESS & $3.415 E-01$ & $1.006 \mathrm{E}+01$ & $1.047 \mathrm{E}+02$ \\
\hline VALUE OF AVERTED LAT. CANCER & $1.977 E+01$ & $3.333 \mathrm{E}+02$ & $1.024 \mathrm{E}_{+}+03$ \\
\hline TOTAL AVERTED OFFSITE COSTS & $1.313 \mathrm{E}+02$ & $2.338 \mathrm{E}+03$ & $7.955 E+03$ \\
\hline OFFSITE COSTS AT \$1000/P-REM & $1.402 \mathrm{E}+03$ & $2.319 \mathrm{E}+04$ & $4.913 E+04$ \\
\hline \multicolumn{4}{|l|}{ TOTAL AVERTED ON \& OFFSITE COSTS } \\
\hline (BASED ON COMPUTED COSTS) & $2.786 \mathrm{E}+03$ & $4.014 \mathrm{E}+04$ & $5.983 E+04$ \\
\hline (BASED ON \$1000/P-REM) & $9.221 E+03$ & $6.178 E+04$ & $1.265 \mathrm{E}+05$ \\
\hline \multicolumn{4}{|c|}{$\begin{array}{l}\text { SAFETY OPTION :P2: New dlesel generator } \\
\text { VALUE-IMPACT ANALYSIS }\end{array}$} \\
\hline & LOW & CENTRAL & $\mathrm{HIGH}$ \\
\hline CHANGE IN CORE MELT FREQUENCY & $3.416 \mathrm{E}-06$ & $7.212 \mathrm{E}-06$ & $1.139 E-05$ \\
\hline \multicolumn{4}{|l|}{ COSTS: } \\
\hline INSTALLATION \& ENG. COSTS & $1.100 \mathrm{E}+07$ & $1.600 \mathrm{E}+07$ & $3.200 E+07$ \\
\hline OPERATING \& MAINT. COSTS & $2.311 \mathrm{E}+06$ & $3.302 \mathrm{E}+06$ & $6.604 \mathrm{E}+06$ \\
\hline REPLACEMENT POWER DURING INST. & $0.000 \mathrm{E}+00$ & $0.000 \mathrm{E}+00$ & $0.000 \mathrm{E}+00$ \\
\hline REPLACEMENT POWER IN OPERATION & $0.000 \mathrm{E}+00$ & $0.000 \mathrm{E}+00$ & $2.000 \mathrm{E}+00$ \\
\hline TOTAL COSTS & $1.331 \mathrm{E}+07$ & $1.930 \mathrm{E}+07$ & $3.860 \mathrm{E}+07$ \\
\hline \multicolumn{4}{|l|}{ BENEFITS } \\
\hline AVERTED ON-SITE COST & $9.657 \mathrm{E}+04$ & $3.392 E+05$ & $5.356 \mathrm{E}+05$ \\
\hline \multicolumn{4}{|l|}{ AVERTED OFFSITE COSTS } \\
\hline AVERTED PROPERTY DAMAGE & $4.816 \mathrm{E}+02$ & $9.499 E+03$ & $1.171 E+05$ \\
\hline VALUE OF AVERTED PROMPT FATALTYY & $7.342 \mathrm{E}-02$ & $2.062 \mathrm{E}+01$ & $2.267 \mathrm{E}+03$ \\
\hline VALUE OF AVERTED EARLY ILLNESS & $5.540 \mathrm{E}-01$ & $1.237 \mathrm{E}+02$ & $8.289 E+03$ \\
\hline VALUE OF AVERTED LAT. CANCER & $1246 E_{+}+01$ & $1.396 \mathrm{E}+03$ & $1.766 \mathrm{E}+04$ \\
\hline TOTAL AVERTED OFFSITE COSTS & $5.108 \mathrm{E}+02$ & $1.108 \mathrm{E}+04$ & $1.434 \mathrm{E}+05$ \\
\hline OFFSITE COSTS AT \$1000/P-REM & $1.404 \mathrm{E}+03$ & $6.481 E+04$ & $5.597 \mathrm{E}+05$ \\
\hline \multicolumn{4}{|l|}{ TOTAL A VERTED ON \& OFFSITE COSTS } \\
\hline (BASED ON COMPUTED COSTS) & $9.716 \mathrm{E}+04$ & $3.445 \mathrm{E}+05$ & $6.244 E+05$ \\
\hline (BASED ON \$1000/P-REM) & $9.915 \mathrm{E}+04$ & $4.819 E+05$ & $1.006 \mathrm{E}+06$ \\
\hline
\end{tabular}


NUREG/CR-4551, VOL. 1: DRAFT REPORT FOR COMMENT (SEPTEMBER, 1986)

Table E-18 (Continued)

COMPARISON OF COSTS AND BENEFITS FOR SAFETY OPTIONS:

LLH METHOD (COSTS IN DOLLARS)

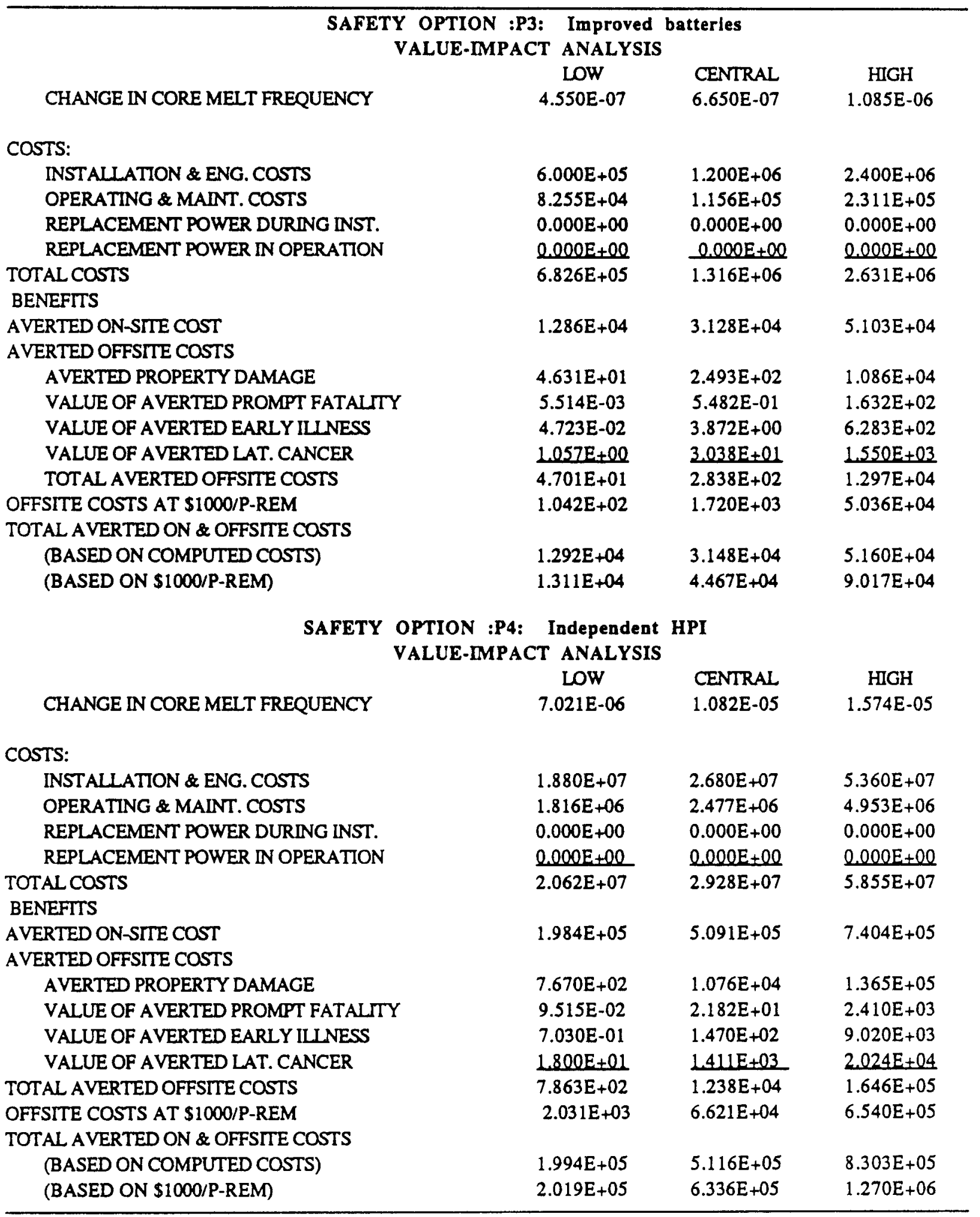


Table E-18 (Continued)

COMPARISON OF COSTS AND BENEFITS FOR SAFETY OPTIONS: LLH METHOD (COSTS IN DOLLARS)

\begin{tabular}{|c|c|c|c|}
\hline \multicolumn{4}{|c|}{$\begin{array}{l}\text { SAFETY OPTION :P5: Independent AFW } \\
\text { VALUE-IMPACT ANALYSIS }\end{array}$} \\
\hline & LOW & CENTRAL & HIGH \\
\hline CHANGE IN CORE MELT FREQUENCY & $2.327 \mathrm{E}-06$ & $2.908 \mathrm{E}-06$ & $4.071 \mathrm{E}-06$ \\
\hline \multicolumn{4}{|l|}{ COSTS: } \\
\hline INSTALLATION \& ENG. COSTS & $5.000 \mathrm{E}+06$ & $7.000 \mathrm{E}+06$ & $1.400 \mathrm{E}+07$ \\
\hline OPERATING \& MAINT. COSTS & $8.255 E+05$ & $1.238 \mathrm{E}+06$ & $2.477 \mathrm{E}+06$ \\
\hline REPLACEMENT POWER DURING INST. & $0.000 E+00$ & $0.000 \mathrm{E}+00$ & $0.000 \mathrm{E}+00$ \\
\hline REPLACEMENT POWER IN OPERATION & $0.000 E+00$ & $0.000 E_{+}+00$ & $0.000 \mathrm{E}+00$ \\
\hline TOTAL COSTS & $5.826 E+06$ & $8.238 E+06$ & $1.648 E+07$ \\
\hline \multicolumn{4}{|l|}{ BENEFITS } \\
\hline AVERTED ON-STTE COST & $6.576 \mathrm{E}+04$ & $1.368 \mathrm{E}+05$ & $1.936 \mathrm{E}+05$ \\
\hline \multicolumn{4}{|l|}{ AVERTED OFFSITE COSTS } \\
\hline AVERTED PROPERTY DAMAGE & $2.169 \mathrm{E}+02$ & $9.344 \mathrm{E}+02$ & $3.785 \mathrm{E}+04$ \\
\hline VALUE OF AVERTED PROMPT FATALTY & $3.360 \mathrm{E}-02$ & $1.766 \mathrm{E}+00$ & $5.004 \mathrm{E}+02$ \\
\hline VALUE OF AVERTED EARLY ILLNESS & 2.322E-01 & $1.350 \mathrm{E}+01$ & $1.841 \mathrm{E}+03$ \\
\hline VALUE OF AVERTED LAT. CANCER & $6.240 \mathrm{E}+00$ & $108 E+02$ & $5.460 \mathrm{E}+03$ \\
\hline TOTAL AVERTED OFFSITE COSTS & $2.223 E+02$ & $1.064 E+03$ & $4.595 \mathrm{E}+04$ \\
\hline OFFSITE COSTS AT \$1000/P-REM & $5.501 \mathrm{E}+02$ & $5.513 \mathrm{E}+03$ & $1.842 E+05$ \\
\hline \multicolumn{4}{|l|}{ TOTAL A VERTED ON \& OFFSITE COSTS } \\
\hline (BASED ON COMPUTED COSTS) & $6.604 E+04$ & $1.392 \mathrm{E}+05$ & $2.236 \mathrm{E}+05$ \\
\hline (BASED ON \$1000/P-REM) & $6.706 \mathrm{E}+04$ & $1.779 \mathrm{E}+05$ & $3.428 \mathrm{E}+05$ \\
\hline \multicolumn{4}{|c|}{$\begin{array}{l}\text { SAFETY OPTION :P6: Indep. RHR/LPR } \\
\text { VALUE-IMPACT ANALYSIS }\end{array}$} \\
\hline & Low & CENTRAL & $\mathrm{HIGH}$ \\
\hline CHANGE IN CORE MELT FREQUENCY & $4.321 E-06$ & $4.360 E-06$ & $5.677 \mathrm{E}-06$ \\
\hline \multicolumn{4}{|l|}{ COSTS: } \\
\hline INSTALLATION \& ENG. COSTS & $1.700 \mathrm{E}+07$ & $2.500 \mathrm{E}+07$ & $5.000 \mathrm{E}+07$ \\
\hline OPERATING \& MAINT. COSTS & $1.816 \mathrm{E}+06$ & $2.477 \mathrm{E}+06$ & $4.953 E+06$ \\
\hline REPLACEMENT POWER DURING INST. & $0.000 \mathrm{E}+00$ & $0.000 \mathrm{E}+00$ & $0.000 \mathrm{E}+00$ \\
\hline REPLACEMENT POWER IN OPERATION & $2000 E+00$ & $0.000 E_{+} 00$ & $0.000 \mathrm{E}+00$ \\
\hline TOTAL COSTS & $1.882 E+07$ & $2.748 E+07$ & $5.495 \mathrm{E}+07$ \\
\hline \multicolumn{4}{|l|}{ BENEFITS } \\
\hline AVERTED ON-SITE COST & $1.221 E+05$ & $2.050 \mathrm{E}+05$ & $2.927 E+05$ \\
\hline \multicolumn{4}{|l|}{ AVERTED OFFSITE COSTS } \\
\hline AVERTED PROPERTY DAMAGE & $9.964 \mathrm{E}+02$ & $3.142 \mathrm{E}+03$ & $6.492 \mathrm{E}+03$ \\
\hline VALUE OF AVERTED PROMPT FATALTY & $2.263 \mathrm{E}-01$ & $7.945 \mathrm{E}+00$ & $7.211 \mathrm{E}+01$ \\
\hline VALUE OF AVERTED EARLY ILLNESS & $1.259 \mathrm{E}+00$ & $5.495 \mathrm{E}+01$ & $2.962 E+02$ \\
\hline VALUE OF AVERTED LAT. CANCER & $1.245 E_{+} 02$ & $4.124 \mathrm{E}+02$ & $8.868 E+02$ \\
\hline TOTAL AVERTED OFFSITE COSTS & $1.123 \mathrm{E}+03$ & $3.657 \mathrm{E}+03$ & $7.622 \mathrm{E}+03$ \\
\hline OFFSITE COSTS AT $\$ 1000 / P$-REM & $9.811 \mathrm{E}+03$ & $1.775 \mathrm{E}+04$ & $4.187 E+04$ \\
\hline \multicolumn{4}{|l|}{ TOTAL AVERTED ON \& OFFSTIE COSTS } \\
\hline (BASED ON COMPUTED COSTS) & $1.239 \mathrm{E}+05$ & $2.084 \mathrm{E}+05$ & $2.976 \mathrm{E}+05$ \\
\hline (BASED ON \$1000/P-REM) & $1.346 \mathrm{E}+05$ & $2.228 \mathrm{E}+05$ & $3.193 E+05$ \\
\hline
\end{tabular}


Table E-18 (Continued)

COMPARISON OF COSTS AND BENEFITS FOR SAFETY OPTIONS: LLH METHOD (COSTS IN DOLLARS)

\begin{tabular}{|c|c|c|c|}
\hline \multicolumn{4}{|c|}{ SAFETY OPTION :P7: New containment service water } \\
\hline & LOW & CENTRAL & HIGH \\
\hline CHANGE IN CORE MELT FREQUENCY & $3.987 E-08$ & $7.882 \mathrm{E}-08$ & $1.149 \mathrm{E}-07$ \\
\hline \multicolumn{4}{|l|}{ costs: } \\
\hline INSTALLATION \& ENG. COSTS & $6.000 E+06$ & $8.000 E+06$ & $1.600 \mathrm{E}+07$ \\
\hline OPERATING \& MAINT. COSTS & $2.311 E+05$ & $3.302 E+05$ & $6.604 \mathrm{E}+05$ \\
\hline REPLACEMENT POWER DURING INST. & $0.000 \mathrm{E}+00$ & $0.000 E+00$ & $0.000 E+00$ \\
\hline REPLACEMENT POWER IN OPERATION & $0.000 E_{+}+00$ & $0.000 E_{+} 00$ & $0.000 \mathrm{E}+00$ \\
\hline TOTAL COSTS & $6.231 E+06$ & $8.330 \mathrm{E}+06$ & $1.666 \mathrm{E}+07$ \\
\hline \multicolumn{4}{|l|}{ BENEFITS } \\
\hline AVERTED ON-SITE COST & $1.127 E+03$ & $3.248 E+03$ & $7.651 E+03$ \\
\hline \multicolumn{4}{|l|}{ AVERTED OFFSITE COSTS } \\
\hline AVERTED PROPERTY DAMAGE & $1.889 \mathrm{E}+02$ & $7.076 E+02$ & $1.992 E+03$ \\
\hline VALUE OF AVERTED PROMPT FATALTTY & 7.085E-02 & $1.738 \mathrm{E}+00$ & $2.561 E+01$ \\
\hline VALUE OF AVERTED EARLY ILLNESS & $3.761 \mathrm{E}-01$ & $1.422 \mathrm{E}+01$ & $1.061 E+02$ \\
\hline VALUE OF AVERTED LAT. CANCER & $2850 \mathrm{E}+01$ & $1031 E+02$ & $2.685 \mathrm{E}+02$ \\
\hline TOTAL AVERTED OFFSITE COSTS & $2.183 E+02$ & $8.644 E+02$ & $2.377 E+03$ \\
\hline OFFSITE COSTS AT $\$ 1000 /$ P-REM & $1.958 E+03$ & $4.735 \mathrm{E}+03$ & $8.901 E+03$ \\
\hline \multicolumn{4}{|l|}{ TOTAL AVERTED ON \& OFFSITE COSTS } \\
\hline (BASED ON COMPUTED COSTS) & $1.456 \mathrm{E}+03$ & $4.130 \mathrm{E}+03$ & $9.129 \mathrm{E}+03$ \\
\hline (BASED ON S1000/P-REM) & $3.474 E+03$ & $8.072 E+03$ & $1.442 \mathrm{E}+04$ \\
\hline \multicolumn{4}{|c|}{$\begin{array}{c}\text { SAFETY OPTION :P8/M9: RCS depressurization } \\
\text { VALUE-IMPACT ANALYSIS }\end{array}$} \\
\hline \multirow[b]{2}{*}{ CHANGE IN CORE MELT FREQUENCY } & Low & CENTRAL & HIGH \\
\hline & $5.061 \mathrm{E}-06$ & $5.061 \mathrm{E}-06$ & $5.775 E-06$ \\
\hline \multicolumn{4}{|l|}{ costs: } \\
\hline INSTALLATION \& ENG. COSTS & $2.000 \mathrm{E}+06$ & $3.000 E+06$ & $6.000 \mathrm{E}+06$ \\
\hline OPERATING \& MAINT. COSTS & $4.128 \mathrm{E}+05$ & $6.604 \mathrm{E}+05$ & $1.651 E+06$ \\
\hline REPLACEMENT POWER DURING INST. & $0.000 \mathrm{E}+00$ & $0.000 \mathrm{E}+00$ & $0.000 \mathrm{E}+00$ \\
\hline REPLACEMENT POWER IN OPERATION & $2.000 \mathrm{E}+00$ & $0.000 \mathrm{E}+00$ & $2.000 E_{+}+00$ \\
\hline TOTAL COSTS & $2.413 E+06$ & $3.660 E+06$ & $7.651 E+06$ \\
\hline BENEFITS & & & \\
\hline AVERTED ON-SITE COST & $1.431 E+05$ & $2.380 \mathrm{E}+05$ & $3.370 \mathrm{E}+05$ \\
\hline A VERTED OFFSITE COSTS & & & \\
\hline AVERTED PROPERTY DAMAGE & $-5.742 E+02$ & $6.136 \mathrm{E}+03$ & $1.368 \mathrm{E}+05$ \\
\hline VALUE OF AVERTED PROMPT FATALITY & $-4.103 E+00$ & $4.038 \mathrm{E}+00$ & $2.272 E+03$ \\
\hline VALUE OF AVERTED EARLY ILLNESS & $-2.444 E+01$ & $3.817 \mathrm{E}+01$ & $8.480 E+03$ \\
\hline VALUE OF AVERTED LAT. CANCER & $-1.151 E_{+02}$ & $8.675 \mathrm{E}+02$ & $2.018 \mathrm{E}+04$ \\
\hline TOTAL AVERTED OFFSITE COSTS & $6.853 E+02$ & $6.989 E+03$ & $1.703 \mathrm{E}+05$ \\
\hline OFFSITE COSTS AT \$1000/P-REM & $-1.020 \mathrm{E}+04$ & $5.098 \mathrm{E}+0.4$ & $6.843 E+05$ \\
\hline TOTAL AVERTED ON \& OFFSTTE COSTS & & & \\
\hline (BASED ON COMPUTED COSTS) & $1.431 \mathrm{E}+05$ & $2.607 E+05$ & $4.501 E+05$ \\
\hline (BASED ON \$1000/P-REM) & $1.435 E+05$ & $3.377 \mathrm{E}+05$ & $8.935 E+05$ \\
\hline
\end{tabular}


NUREG/CR-4551, VOL. 1: DRAFT REPORT FOR COMMENT (SEPTEMBER, 1986)

Table E-18 (Continued)

COMPARISON OF COSTS AND BENEFITS FOR SAFETY OPTIONS:

LLH METHOD (COSTS IN DOLLARS)

\begin{tabular}{|c|c|c|c|}
\hline \multicolumn{4}{|c|}{$\begin{array}{l}\text { SAFETY OPTION :M1: MISSILE SHIELD } \\
\text { VALUE-IMPACT ANALYSIS }\end{array}$} \\
\hline & LOW & CENTRAL & HIGH \\
\hline CHANGE IN CORE MELT FREQUENCY & $0.000 \mathrm{E}+00$ & $0.000 \mathrm{E}+00$ & $0.000 \mathrm{E}+00$ \\
\hline \multicolumn{4}{|l|}{ COSTS: } \\
\hline INSTALLATION \& ENG. COSTS & $7.000 \mathrm{E}+06$ & $1.000 \mathrm{E}+07$ & $3.000 \mathrm{E}+07$ \\
\hline OPERATING \& MAINT. COSTS & $4.128 \mathrm{E}+04$ & $8.255 \mathrm{E}+04$ & $1.651 E+05$ \\
\hline REPLACEMENT POWER DURING INST. & $0.000 \mathrm{E}+00$ & $0.000 \mathrm{E}+00$ & $0.000 \mathrm{E}+00$ \\
\hline REPLACEMENT POWER IN OPERATION & $0.000 E+00$ & $2000 E_{+}+00$ & $2.000 E_{+}+00$ \\
\hline TOTAL COSTS & $7.041 E+06$ & $1.008 \mathrm{E}+07$ & $3.017 E+07$ \\
\hline \multicolumn{4}{|l|}{ BENEFITS } \\
\hline AVERTED ON-SITE COST & $0.000 \mathrm{E}+00$ & $0.000 \mathrm{E}+00$ & $0.000 E+00$ \\
\hline \multicolumn{4}{|l|}{ AVERTED OFFSITE COSTS } \\
\hline AVERTED PROPERTY DAMAGE & $4.491 E+00$ & $1.270 E+02$ & $1.313 E+03$ \\
\hline VALUE OF AVERTED PROMPT FATALTY & $-4.800 \mathrm{E}-01$ & $1.624 \mathrm{E}-01$ & $6.058 E+00$ \\
\hline VALUE OF AVERTED EARLY ILLNESS & $-4.470 \mathrm{E}+00$ & $1.057 \mathrm{E}+00$ & $3.697 \mathrm{E}+01$ \\
\hline VALUE OF AVERTED LAT. CANCER & $2.961 \mathrm{E}-01$ & $1.873 E_{+}+01$ & $1.951 \mathrm{E}+02$ \\
\hline TOTAL AVERTED OFFSITE COSTS & $5.528 E+00$ & $1.485 \mathrm{E}+02$ & $1.540 \mathrm{E}+03$ \\
\hline OFFSITE COSTS AT \$1000/P-REM & $8.155 E+01$ & $9.651 E+02$ & $1.134 E+04$ \\
\hline \multicolumn{4}{|l|}{ TOTAL A VERTED ON \& OFFSTTE COSTS } \\
\hline (BASED ON COMPUTED COSTS) & $4.579 E+00$ & $1.482 \mathrm{E}+02$ & $1.361 E+03$ \\
\hline (BASED ON \$1000/P-REM) & $7.152 \mathrm{E}+01$ & $9.647 \mathrm{E}+02$ & $1.133 \mathrm{E}+04$ \\
\hline \multicolumn{4}{|c|}{$\begin{array}{c}\text { SAFETY OPTION :M2: H2 IGNITERS } \\
\text { VALUE-IMPACT ANALYSIS }\end{array}$} \\
\hline & Low & CENTRAL & HIGH \\
\hline CHANGE IN CORE MELT FREQUENCY & $0.000 \mathrm{E}+00$ & $0.000 \mathrm{E}+00$ & $0.000 \mathrm{E}+00$ \\
\hline \multicolumn{4}{|l|}{ COSTS: } \\
\hline INSTALLATION \& ENG. COSTS & $2.000 \mathrm{E}+06$ & $7.600 \mathrm{E}+06$ & $1.520 \mathrm{E}+07$ \\
\hline OPERATING \& MAINT. COSTS & $5.779 E+05$ & $8.255 E+05$ & $1.651 E+06$ \\
\hline REPLACEMENT POWER DURING INST. & $0.000 \mathrm{E}+00$ & $0.000 \mathrm{E}+00$ & $0.000 \mathrm{E}+00$ \\
\hline REPLACEMENT POWER N OPERATION & $2.000 \mathrm{E}_{+} 00$ & $0.000 \mathrm{E}_{+}+00$ & $0.000 \mathrm{E}+00$ \\
\hline TOTAL COSTS & $2.578 \mathrm{E}+06$ & $8.426 E+06$ & $1.685 \mathrm{E}+07$ \\
\hline \multicolumn{4}{|l|}{ BENEFITS } \\
\hline AVERTED ON-SITE COST & $0.000 \mathrm{E}+00$ & $0.000 \mathrm{E}+00$ & $0.000 \mathrm{E}+00$ \\
\hline \multicolumn{4}{|l|}{ AVERTED OFFSITE COSTS } \\
\hline AVERTED PROPERTY DAMAGE & $-5.894 \mathrm{E}+00$ & $0.000 \mathrm{E}+00$ & $8.446 \mathrm{E}+01$ \\
\hline VALUE OF AVERTED PROMPT FATALITY & $-3.013 E-03$ & $0.000 \mathrm{E}+00$ & $4.842 E-02$ \\
\hline VALUE OF AVERTED EARLY ILLNESS & $-2.318 \mathrm{E}-02$ & $0.000 \mathrm{E}+00$ & $1.677 \mathrm{E}-01$ \\
\hline VALUE OF AVERTED LAT. CANCER & $=1005 E_{+}+00$ & $0.000 \mathrm{E}+00$ & $2.265 E+01$ \\
\hline TOTAL AVERTED OFFSITE COSTS & $-6.925 E+00$ & $\overline{0.000 E+00}$ & $\overline{1.161 \mathrm{E}+02}$ \\
\hline OFFSITE COSTS AT \$1000/P-REM & $-6.927 \mathrm{E}+01$ & $0.000 \mathrm{E}+00$ & $2.147 E+03$ \\
\hline \multicolumn{4}{|l|}{ TOTAL AVERTED ON \& OFFSTTE COSTS } \\
\hline (BASED ON COMPUTED COSTS) & $-1.311 E+01$ & $0.000 \mathrm{E}+00$ & $1.111 \mathrm{E}+02$ \\
\hline (BASED ON $\$ 1000 / \mathrm{P}-\mathrm{REM}$ ) & $-1.310 \mathrm{E}+02$ & $0.000 E+00$ & $1.784 E+03$ \\
\hline
\end{tabular}


Table E-18 (Continued)

COMPARISON OF COSTS AND BENEFITS FOR SAFETY OPTIONS:

LLH METHOD (COSTS IN DOLLARS)

SAFETY OPTION
VAL
CHANGE IN CORE MELT FREQUENCY
COSTS:
INSTALLATION \& ENG. COSTS
OPERATING \& MAINT. COSTS
REPLACEMENT POWER DUR)
REPLACEMENT POWER IN OPERATION
TOTAL COSTS
BENEFITS
AVERTED ON-SITE COST
AVERTED OFFSITE COSTS
AVERTED PROPERTY DAMAGE
VALUE OF AVERTED PROMPT FATALTY
VALUE OF AVERTED EARLY ILLNESS
VALUE OF AVERTED LAT. CANCER
TOTALAVERTED OFFSTE COSTS
OFFSITE COSTS AT S1OOO/P-REM
TOTAL AVERTED ON \& OFFSITE COSTS
(BASED ON COMPUTED COSTS)
(BASED ON \$1OOO/P-REM)

TION :M3: CONTAINMENT INERTING

VALUE-IMPACT ANALYSIS

$\begin{array}{ccc}\text { LOW } & \text { CENTRAL } & \text { HIGH } \\ 0.000 \mathrm{E}+00 & 0.000 \mathrm{E}+00 & 0.000 \mathrm{E}+00\end{array}$

SAFETY OPTION :M4: Early filtered venting

VALUE-IMPACT ANALYSIS

\begin{tabular}{lll}
$1.500 \mathrm{E}+06$ & $3.000 \mathrm{E}+06$ & $7.000 \mathrm{E}+06$ \\
$3.467 \mathrm{E}+06$ & $4.953 \mathrm{E}+06$ & $9.906 \mathrm{E}+06$ \\
$0.000 \mathrm{E}+00$ & $0.000 \mathrm{E}+00$ & $0.000 \mathrm{E}+00$ \\
$0.000 \mathrm{E}+00$ & $\frac{0.000 \mathrm{E}+00}{2.000 \mathrm{E}_{+}+00}$ \\
\hline $4.967 \mathrm{E}+06$ & $7.953 \mathrm{E}+06$ & $1.691 \mathrm{E}+07$ \\
$0.000 \mathrm{E}+00$ & $0.000 \mathrm{E}+00$ & $0.000 \mathrm{E}+00$ \\
$-1.260 \mathrm{E}-03$ & $1.797 \mathrm{E}+03$ & $7.124 \mathrm{E}+04$ \\
$-6.100 \mathrm{E}-06$ & $1.664 \mathrm{E}+00$ & $5.760 \mathrm{E}+02$ \\
$-5.706 \mathrm{E}-05$ & $1.400 \mathrm{E}+01$ & $2.460 \mathrm{E}+03$ \\
$-2.403 \mathrm{E}-04$ & $2.624 \mathrm{E}+02$ & $2.533 \mathrm{E}+03$ \\
$-1.583 \mathrm{E}-03$ & $2.068 \mathrm{E}+03$ & $8.256 \mathrm{E}+04$ \\
$-7.416 \mathrm{E}-03$ & $1.748 \mathrm{E}+04$ & $3.811 \mathrm{E}+05$ \\
$-1.994 \mathrm{E}-03$ & $1.879 \mathrm{E}+03$ & $7.778 \mathrm{E}+04$ \\
$-7.873 \mathrm{E}-03$ & $1.657 \mathrm{E}+04$ & $3.417 \mathrm{E}+05$
\end{tabular}

CHANGE IN CORE MELT FREQUENCY

COSTS:

INSTALLATION \& ENG. COSTS

OPERATING \& MAINT. COSTS

REPLACEMENT POWER DURING INST.

REPLACEMENT POWER IN OPERATION

TOTAL COSTS

BENEFITS

AVERTED ON-SITE COST

AVERTED OFFSITE COSTS

AVERTED PROPERTY DAMAGE

VALUE OF AVERTED PROMPT FATALTY

VALUE OF AVERTED EARLY ILLNESS

VALUE OF AVERTED LAT. CANCER

TOTAL AVERTED OFFSITE COSTS

OFFSITE COSTS AT \$1000/P-REM

TOTAL AVERTED ON \& OFFSTTE COSTS

(BASED ON COMPUTED COSTS)

(BASED ON \$1000/P-REM)
LOW

4.155E-08

CENTRAL

8.214E-08

HIGH

1.198E-07

$\begin{array}{lll}9.500 \mathrm{E}+06 & 1.350 \mathrm{E}+07 & 2.700 \mathrm{E}+07 \\ 1.156 \mathrm{E}+06 & 1.651 \mathrm{E}+06 & 3.302 \mathrm{E}+06 \\ 0.000 \mathrm{E}+00 & 0.000 \mathrm{E}+00 & 0.000 \mathrm{E}+00 \\ \frac{0.000 \mathrm{E}+00}{1.066 \mathrm{E}+07} & \frac{0.000 \mathrm{E}+00}{1.515 \mathrm{E}+07} & \frac{0.000 \mathrm{E}+00}{3.030 \mathrm{E}+07} \\ & & \\ 1.174 \mathrm{E}+03 & 3.385 \mathrm{E}+03 & 7.973 \mathrm{E}+03 \\ & & \\ -4.273 \mathrm{E}+04 & 1.594 \mathrm{E}+03 & 4.496 \mathrm{E}+04 \\ -1.017 \mathrm{E}+02 & 3.290 \mathrm{E}+00 & 4.923 \mathrm{E}+02 \\ -8.054 \mathrm{E}+02 & 2.396 \mathrm{E}+01 & 1.875 \mathrm{E}+03 \\ -6.096 \mathrm{E}+03 & 2.200 \mathrm{E}+02 & 5.496 \mathrm{E}+03 \\ -5.000 \mathrm{E}+04 & 1.877 \mathrm{E}+03 & 5.247 \mathrm{E}+04 \\ -2.779 \mathrm{E}+05 & 1.383 \mathrm{E}+04 & 1.999 \mathrm{E}+05 \\ & & \\ -5.185 \mathrm{E}+04 & 5.793 \mathrm{E}+03 & 5.277 \mathrm{E}+04 \\ -2.772 \mathrm{E}+05 & 1.749 \mathrm{E}+04 & 1.985 \mathrm{E}+05\end{array}$


NUREG/CR-4551, VOL 1: DRAFT REPORT FOR COMMENT (SEPTEMBER, 1986)

Table E-18 (Continued)

COMPARISON OF COSTS AND BENEFTTS FOR SAFETY OPTIONS: LLH METHOD (COSTS IN DOLLARS)

\begin{tabular}{|c|c|c|c|}
\hline \multicolumn{4}{|c|}{$\begin{array}{c}\text { SAFETY OPTION :M5: LATE FILTERED VENTING } \\
\text { VALUE-IMPACT ANALYSIS }\end{array}$} \\
\hline & LOW & CENTRAL & HIGH \\
\hline CHANGE IN CORE MELT FREQUENCY & $0.000 \mathrm{E}+00$ & $0.00 \mathrm{E}+00$ & $0.000 \mathrm{E}+00$ \\
\hline \multicolumn{4}{|l|}{ costs: } \\
\hline INSTALLATION \& ENG. COSTS & $9.500 \mathrm{E}+06$ & $1.350 \mathrm{E}+07$ & $2.700 \mathrm{E}+07$ \\
\hline OPERATING \& MAINT. COSTS & $1.156 \mathrm{E}+06$ & $1.651 E+06$ & $3.302 \mathrm{E}+06$ \\
\hline REPLACEMENT POWER DURING INST. & $0.000 \mathrm{E}+00$ & $0.000 \mathrm{E}+00$ & $0.000 \mathrm{E}+00$ \\
\hline REPLACEMENT POWER IN OPERATION & $0,000 \mathrm{E}+00$ & $0.000 E+00$ & $0.000 \mathrm{E}+00$ \\
\hline TOTAL COSTS & $1.066 \mathrm{E}+07$ & $1.515 \mathrm{E}+07$ & $3.030 \mathrm{E}+07$ \\
\hline \multicolumn{4}{|l|}{ BENEFITS } \\
\hline AVERTED ON-SITE COST & $0.000 \mathrm{E}+00$ & $0.000 \mathrm{E}+00$ & $0.000 \mathrm{E}+00$ \\
\hline \multicolumn{4}{|l|}{ AVERTED OFFSITE COSTS } \\
\hline AVERTED PROPERTY DAMAGE & $-7.021 E+00$ & $3.697 \mathrm{E}+00$ & $9.644 E+02$ \\
\hline VALUE OF AVERTED PROMPT FATALTY & $-1.748 E-02$ & $6.371 E-02$ & $4.332 \mathrm{E}-01$ \\
\hline VALUE OF AVERTED LAT. CANCER & $-4.542 \mathrm{E}+00$ & $-1702 \mathrm{E}+00$ & $2.334 E+02$ \\
\hline TOTAL AVERTED OFFSITE COSTS & $-1.162 E+01$ & $1.915 E+00$ & $1.213 E+03$ \\
\hline OFFSITE COSTS AT \$1000/P-REM & $-5.406 \mathrm{E}+02$ & $-2.157 \mathrm{E}+02$ & $2.273 E+04$ \\
\hline \multicolumn{4}{|l|}{ TOTAL A VERTED ON \& OFFSTIE COSTS } \\
\hline (BASED ON COMPUTED COSTS) & $-1.168 \mathrm{E}+01$ & $1.656 \mathrm{E}+00$ & $1.131 \mathrm{E}+03$ \\
\hline (BASED ON \$1000/P-REM) & $-5.484 \mathrm{E}+02$ & $-2.367 \mathrm{E}+02$ & $2.243 E+04$ \\
\hline
\end{tabular}

SAFETY OPTION :M6: New sprays

VALUE-IMPACT ANALYSIS

LOW

$1.235 \mathrm{E}-06$

CENTRAL

1.805E-06

$\mathrm{HIGH}$

2.945E-06

COSTS:

INSTALLATTON \& ENG. COSTS

OPERATING \& MAINT. COSTS

REPLACEMENT POWER DURING INST.

REPLACEMENT POWER IN OPERATION

TOTAL COSTS

BENEFTTS

AVERTED ON-SITE COST

AVERTED OFFSITE COSTS

AVERTED PROPERTY DAMAGE

VALUE OF AVERTED PROMPT FATALITY

VALUE OF AVERTED EARLY ILLNESS

VALUE OF AVERTED LAT. CANCER

TOTAL AVERTED OFFSITE COSTS

OFFSITE COSTS AT \$1000/P-REM

TOTAL AVERTED ON \& OFFSTTE COSTS

(BASED ON COMPUTED COSTS)

(BASED ON \$1000/P-REM)

$\begin{array}{lll}5.600 \mathrm{E}+06 & 8.000 \mathrm{E}+06 & 1.600 \mathrm{E}+07 \\ 1.040 \mathrm{E}+06 & 1.486 \mathrm{E}+06 & 2.972 \mathrm{E}+06 \\ 0.000 \mathrm{E}+00 & 0.000 \mathrm{E}+00 & 0.000 \mathrm{E}+00 \\ \frac{0.000 \mathrm{E}+00}{6.640 \mathrm{E}+06} & \frac{0.000 \mathrm{E}+00}{9.486 \mathrm{E}+06} & \frac{0.000 \mathrm{E}+00}{1.897 \mathrm{E}+07} \\ & & \\ 3.491 \mathrm{E}+04 & 8.489 \mathrm{E}+04 & 1.385 \mathrm{E}+05 \\ & & \\ 1.515 \mathrm{E}+02 & 7.351 \mathrm{E}+03 & 1.180 \mathrm{E}+05 \\ 4.703 \mathrm{E}-02 & 1.475 \mathrm{E}+01 & 2.298 \mathrm{E}+03 \\ 3.687 \mathrm{E}-01 & 8.770 \mathrm{E}+01 & 8.402 \mathrm{E}+03 \\ 7.651 \mathrm{E}+00 & 1.278 \mathrm{E}+03 & 1.784 \mathrm{E}+04 \\ 1.580 \mathrm{E}+02 & 8.647 \mathrm{E}+03 & 1.447 \mathrm{E}+05 \\ 5.660 \mathrm{E}+02 & 6.470 \mathrm{E}+04 & 5.605 \mathrm{E}+05 \\ & & \\ 3.517 \mathrm{E}+04 & 1.035 \mathrm{E}+05 & 2.463 \mathrm{E}+05 \\ 3.636 \mathrm{E}+04 & 1.423 \mathrm{E}+05 & 6.490 \mathrm{E}+05\end{array}$


Table E-18 (Continued)

COMPARISON OF COSTS AND BENEFITS FOR SAFETY OPTIONS: LLH METHOD (COSTS IN DOLLARS)

\begin{tabular}{|c|c|c|c|}
\hline \multicolumn{4}{|c|}{$\begin{array}{c}\text { SAFETY OPTION :M7: Fan coolers } \\
\text { VALUE-IMPACT ANALYSIS }\end{array}$} \\
\hline & LOW & CENTRAL & HIGH \\
\hline CHANGE IN CORE MELT FREQUENCY & $0.000 \mathrm{E}+00$ & $0.000 \mathrm{E}+00$ & $0.000 \mathrm{E}+00$ \\
\hline \multicolumn{4}{|l|}{ COSTS: } \\
\hline INSTALLATION \& ENG. COSTS & $2.600 \mathrm{E}+06$ & $3.700 \mathrm{E}+06$ & $7.400 E+06$ \\
\hline OPERATING \& MAINT. COSTS & $8.255 E+05$ & $1.156 \mathrm{E}+06$ & $1.651 E+06$ \\
\hline REPLACEMENT POWER DURING INST. & $0.000 \mathrm{E}+00$ & $0.000 E+00$ & $0.000 \mathrm{E}+00$ \\
\hline REPLACEMENT POWER IN OPERATION & $0.000 E_{+}+00$ & $0.000 \mathrm{E}_{ \pm}+00$ & $0.000 E+00$ \\
\hline TOTAL COSTS & $3.426 \mathrm{E}+06$ & $4.856 \mathrm{E}+06$ & $9.051 \mathrm{E}+06$ \\
\hline \multicolumn{4}{|l|}{ BENEFITS } \\
\hline AVERTED ON-SITE COST & $0.000 \mathrm{E}+00$ & $0.000 \mathrm{E}+\infty 0$ & $0.000 \mathrm{E}+00$ \\
\hline \multicolumn{4}{|l|}{ AVERTED OFFSITE COSTS } \\
\hline AVERTED PROPERTY DAMAGE & $-1.044 E+04$ & $1.472 E+01$ & $2.766 E+04$ \\
\hline VALUE OF AVERTED PROMPT FATALTY & $-2.051 E+01$ & $5.888 \mathrm{E}-02$ & $2.854 \mathrm{E}+02$ \\
\hline VALUE OF AVERTED EARL Y ILLNESS & $-1.786 E+02$ & $4.537 \mathrm{E}-01$ & $1.107 \mathrm{E}+03$ \\
\hline VALUE OF AVERTED LAT. CANCER & $-1.556 \mathrm{E}_{+} 03$ & $3.949 E_{+}+0$ & $3.882 E+03$ \\
\hline TOTAL AVERTED OFFSTTE COSTS & $-1.219 \mathrm{E}+04$ & $2.885 E+01$ & $3.410 \mathrm{E}+04$ \\
\hline OFFSITE COSTS AT \$1000/P-REM & $-7.086 E+04$ & $2.813 E+02$ & $1.408 E+05$ \\
\hline \multicolumn{4}{|l|}{ TOTAL A VERTED ON \& OFFSTTE COSTS } \\
\hline (BASED ON COMPUTED COSTS) & $-1.320 \mathrm{E}+04$ & $2.828 \mathrm{E}+01$ & $3.274 \mathrm{E}+04$ \\
\hline (BASED ON \$1000/P-REM) & $-7.092 E+04$ & $2.745 E+02$ & $1.350 \mathrm{E}+05$ \\
\hline \multirow[t]{2}{*}{$\begin{aligned} \text { SAFETY OPTION :M8: } \\
\text { VALUE-IMPACT }\end{aligned}$} & $\begin{array}{l}\text { Containment } \\
\text { ANALYSIS }\end{array}$ & flooding & \\
\hline & Low & CENTRAL & $\mathrm{HIGH}$ \\
\hline CHANGE IN CORE MELT FREQUENCY & $0.000 E+00$ & $0.000 \mathrm{E}+00$ & $0.000 \mathrm{E}+00$ \\
\hline \multicolumn{4}{|l|}{ costs: } \\
\hline INSTALLATION \& ENG. COSTS & $6.000 E+05$ & $1.000 E+06$ & $2.000 \mathrm{E}+06$ \\
\hline OPERATING \& MAINT. COSTS & $1.651 E+05$ & $2.477 E+05$ & $4.953 E+05$ \\
\hline REPLACEMENT POWER DURING INST. & $0.000 \mathrm{E}+00$ & $0.000 E+00$ & $0.000 \mathrm{E}+00$ \\
\hline REPLACEMENT POWER IN OPERATION & $0.000 \mathrm{E}+00$ & $0.000 \mathrm{E}+00$ & $2.000 \mathrm{E}+00$ \\
\hline TOTAL COSTS & $7.651 E+05$ & $1.248 E+06$ & $2.495 E+06$ \\
\hline \multicolumn{4}{|l|}{ BENEFTTS } \\
\hline AVERTED ON-STTE COST & $0.000 \mathrm{E}+00$ & $0.000 \mathrm{E}+00$ & $0.000 \mathrm{E}+00$ \\
\hline \multicolumn{4}{|l|}{ AVERTED OFFSITE COSTS } \\
\hline AVERTED PROPERTY DAMAGE & $-1.469 E+04$ & $0.000 \mathrm{E}+00$ & $5.735 E+04$ \\
\hline VALUE OF AVERTED PROMPT FATALTY & $-2.231 E+01$ & $0.000 \mathrm{E}+00$ & $3.145 \mathrm{E}+02$ \\
\hline VALUE OF AVERTED EARLY IILNESS & $-1.651 E+02$ & $0.000 \mathrm{E}+00$ & $1.604 \mathrm{E}+03$ \\
\hline VALUE OF AVERTED LAT. CANCER & $-2.150 \mathrm{E}_{+} 03$ & $0.000 E_{+} 00$ & $8.062 \mathrm{E}+03$ \\
\hline TOTAL AVERTED OFFSITE COSTS & $-1.694 E+04$ & $\overline{0.000 E+00}$ & $6.694 \mathrm{E}+04$ \\
\hline OFFSITE COSTS AT \$1000/P-REM & $-9.151 E+04$ & $0.000 \mathrm{E}+00$ & $2.924 \mathrm{E}+05$ \\
\hline \multicolumn{4}{|l|}{ TOTAL AVERTED ON \& OFFSITE COSTS } \\
\hline (BASED ON COMPUTED COSTS) & $-1.752 \mathrm{E}+04$ & $0.000 \mathrm{E}+00$ & $6.482 \mathrm{E}+04$ \\
\hline (BASED ON \$1000/P-REM) & $-9.336 E+04$ & $0.000 E+00$ & $2.729 E+05$ \\
\hline
\end{tabular}


Table E-18 (Continued)

COMPARISON OF COSTS AND BENEFITS FOR SAFETY OPTIONS: LLH METHOD (COSTS IN DOLLARS)

\begin{tabular}{|c|c|c|c|}
\hline $\begin{array}{r}\text { SAFETY OPTION :P2 \& M4: } \\
\text { VALUE-IMPACT }\end{array}$ & $\begin{array}{l}\text { New DG and } \\
\text { ANALYSIS }\end{array}$ & Early Vent & \\
\hline & Low & CENTRAL & $\mathrm{HIGH}$ \\
\hline CHANGE IN CORE MELT FREQUENCY & $3.499 \mathrm{E}-06$ & $7.295 \mathrm{E}-06$ & $1.145 \mathrm{E}-05$ \\
\hline \multicolumn{4}{|l|}{ costs: } \\
\hline INSTALLATION \& ENG. COSTS & $2.100 \mathrm{E}+07$ & $3.000 \mathrm{E}+07$ & $5.900 \mathrm{E}+07$ \\
\hline OPERATING \& MAINT. COSTS & $3.467 \mathrm{E}+06$ & $4.953 E+06$ & $9.906 E+06$ \\
\hline REPLACEMENT POWER DURING INST. & $0.000 \mathrm{E}+00$ & $0.000 \mathrm{E}+00$ & $0.000 \mathrm{E}+00$ \\
\hline REPLACEMENT POWER IN OPERATION & $0.000 E+00$ & $0.000 \mathrm{E}+00$ & $0.000 E_{+00}$ \\
\hline TOTAL COSTS & $\overline{2.447 E+07}$ & $3.495 \mathrm{E}+07$ & $6.891 \mathrm{E}+07$ \\
\hline \multicolumn{4}{|l|}{ BENEFITS } \\
\hline AVERTED ON-SITE COST & $9.995 E+04$ & $3.412 E+05$ & $5.375 E+05$ \\
\hline \multicolumn{4}{|l|}{ AVERTED OFFSTTE COSTS } \\
\hline AVERTED PROPERTY DAMAGE & $5.451 E+02$ & $6.019 E+03$ & $8.194 \mathrm{E}+04$ \\
\hline VALUE OF AVERTED PROMPT FATALTY & $1.191 \mathrm{E}-01$ & $2.077 E+01$ & $1.683 E+03$ \\
\hline VALUE OF A VERTED EARLY ILLNESS & $7.869 \mathrm{E}-01$ & $1.356 E+02$ & $6.086 \mathrm{E}+03$ \\
\hline VALUE OF AVERTED LAT. CANCER & $2.401 E_{+01}$ & $2.988 \mathrm{E}_{+}+02$ & $1.137 E_{+} 04$ \\
\hline TOTAL AVERTED OFFSITE COSTS & $\overline{5.736 \mathrm{E}+02}$ & $7.617 E+03$ & $\overline{9.855 \mathrm{E}+04}$ \\
\hline OFFSITE COSTS AT \$1000/P-REM & $-1.174 E+04$ & $4.467 \mathrm{E}+04$ & $3.522 \mathrm{E}+05$ \\
\hline \multicolumn{4}{|l|}{ TOTAL A VERTED ON \& OFFSITE COSTS } \\
\hline (BASED ON COMPUTED COSTS) & $1.009 \mathrm{E}+05$ & $3.449 \mathrm{E}+05$ & $5.925 \mathrm{E}+05$ \\
\hline (BASED ON \$1000/P-REM) & $1.047 \mathrm{E}+05$ & $4.203 E+05$ & $7.833 E+05$ \\
\hline \multicolumn{4}{|c|}{ SAFETY OPTION :P6 \& P8/M9: Inde;. LPR \& RCS depr. } \\
\hline & Low & CENTRAL & $\mathrm{HIGH}$ \\
\hline CHANGE IN CORE MELT FREQUENCY & $1.330 \mathrm{E}-05$ & $1.706 \mathrm{E}-05$ & $2.337 \mathrm{E}-05$ \\
\hline \multicolumn{4}{|l|}{ COSTS: } \\
\hline INSTALLATION \& ENG. COSTS & $1.900 \mathrm{E}+07$ & $2.800 \mathrm{E}+07$ & $5.600 E+07$ \\
\hline OPERATING \& MAINT. COSTS & $2.311 E+06$ & $3.137 E+06$ & $6.604 E+06$ \\
\hline REPLACEMENT POWER DURING INST. & $0.000 \mathrm{E}+00$ & $0.000 \mathrm{E}+00$ & $0.000 \mathrm{E}+00$ \\
\hline REPLACEMENT POWER IN OPERATION & $0.000 \mathrm{E}+00$ & $0.000 \mathrm{E}+00$ & $0.000 \mathrm{E}+00$ \\
\hline TOTAL COSTS & $\overline{2.131 E+07}$ & $3.114 \mathrm{E}+07$ & $6.260 \mathrm{E}+07$ \\
\hline \multicolumn{4}{|l|}{ BENEFITS } \\
\hline AVERTED ON-SITE COST & $3.771 E+05$ & $8.025 E+05$ & $1.138 \mathrm{E}+06$ \\
\hline \multicolumn{4}{|l|}{ AVERTED OFFSITE COSTS } \\
\hline AVERTED PROPERTY DAMAGE & $2.364 E+03$ & $1.267 \mathrm{E}+04$ & $1.509 \mathrm{E}+05$ \\
\hline VALUE OF AVERTED PROMPT FATALITY & $3.532 \mathrm{E}-01$ & $5.000 \mathrm{E}+01$ & $2.537 \mathrm{E}+03$ \\
\hline VALUE OF AVERTED EARLY ILLNESS & $2.175 E+00$ & $2.365 E+02$ & $9.527 \varepsilon+03$ \\
\hline VALUE OF AVERTED LAT. CANCER & $2.081 E_{ \pm} 02$ & $1786 \mathrm{E}_{+}+03$ & $2.174 \mathrm{E}+04$ \\
\hline TOTAL AVERTED OFFSITE COSTS & $2.579 \mathrm{E}+03$ & $1.478 \mathrm{E}+04$ & $1.827 \mathrm{E}+05$ \\
\hline OFFSITE COSTS AT \$1000/P-REM & $1.413 E+04$ & $9.158 \mathrm{E}+04$ & $7.394 \mathrm{E}+05$ \\
\hline \multicolumn{4}{|l|}{ TOTAL AVERTED ON \& OFFSITE COSTS } \\
\hline (BASED ON COMPUTED COSTS) & $3.805 E+05$ & $8.176 E+05$ & $1.257 \mathrm{E}+06$ \\
\hline (BASED ON \$1000/P-REM) & $3.952 \mathrm{E}+05$ & $9.214 E+05$ & $1.717 \mathrm{E}+06$ \\
\hline
\end{tabular}


Table E-19

COMPARISON OF COSTS AND BENEFITS FOR SAFETY OPTIONS: OCP METHOD (COSTS IN DOLLARS)

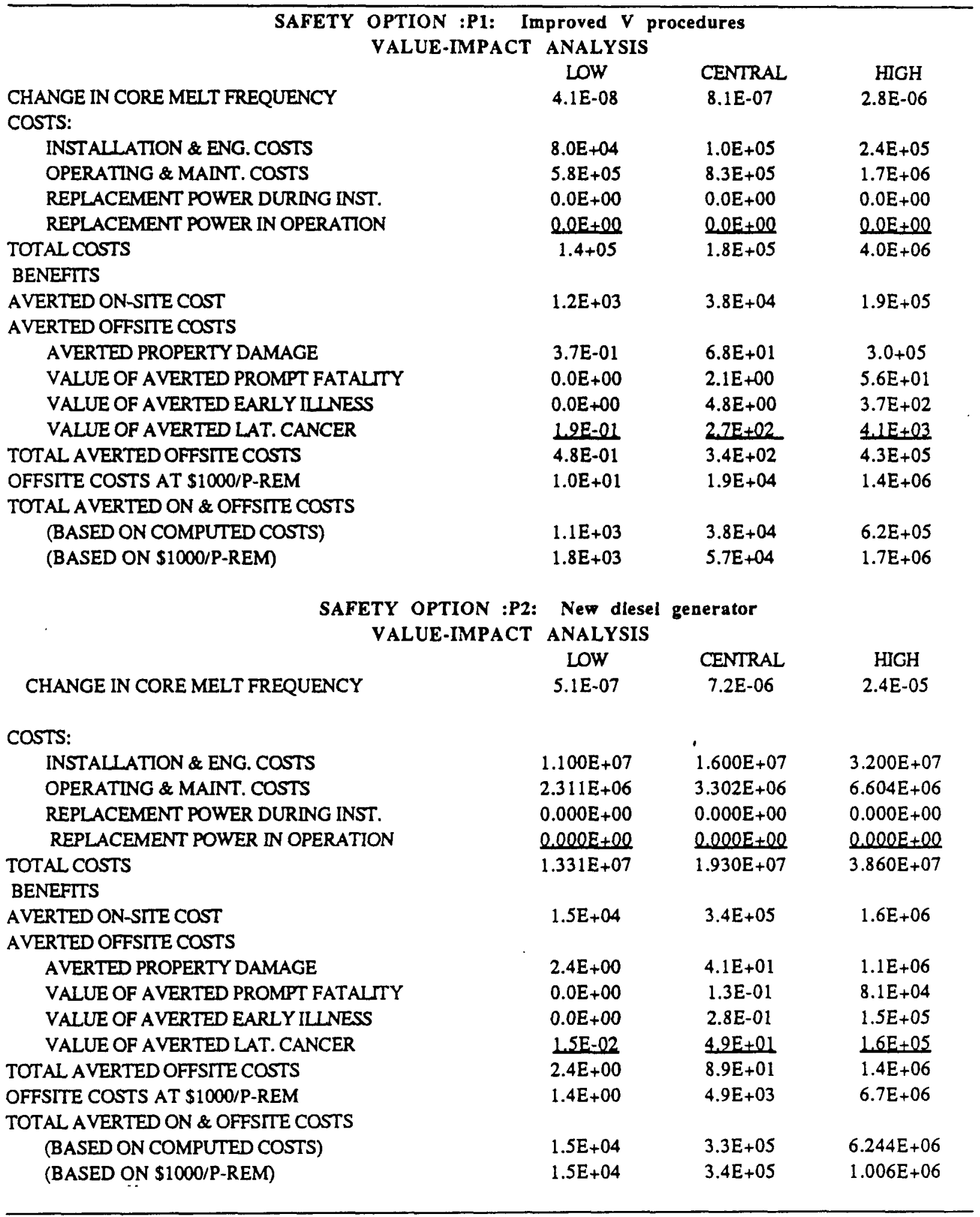


Table E-19 (Continued)

COMPARISON OF COSTS AND BENEFITS FOR SAFETY OPTIONS: OCP METHOD (COSTS IN DOLLARS)

\begin{tabular}{|c|c|c|c|}
\hline $\begin{array}{r}\text { SAFETY } \\
V\end{array}$ & $\begin{array}{l}Y \text { OPTION :P3: Improve } \\
\text { VALUE-IMPACT ANALY }\end{array}$ & batterles & \\
\hline & LOW & CENTRAL & HIGH \\
\hline CHANGE IN CORE MELT FREQUENCY & $5.2 \mathrm{E}-08$ & $6.6 \mathrm{E}-07$ & 2.2E-06 \\
\hline COSTS: & & & \\
\hline INSTALLATION \& ENG. COSTS & $6.000 E+05$ & $1.200 E+06$ & $2.400 \mathrm{E}+06$ \\
\hline OPERATING \& MAINT. COSTS & $8.255 E+04$ & $1.156 E+05$ & $2.311 E+05$ \\
\hline REPLACEMENT POWER DURING INST. & $0.000 \mathrm{E}+00$ & $0.000 \mathrm{E}+00$ & $0.000 \mathrm{E}+00$ \\
\hline REPLACEMENT POWER IN OPERATION & $0.000 \mathrm{E}+00$ & $0.000 \mathrm{E}+00$ & $2.000 E+00$ \\
\hline TOTAL COSTS & $6.826 E+05$ & $1.316 E+06$ & $2.631 E+06$ \\
\hline BENEFITS & & & \\
\hline AVERTED ON-SITE COST & $1.4 \mathrm{E}+03$ & $3.1 E+04$ & $1.5 \mathrm{E}+05$ \\
\hline AVERTED OFFSITE COSTS & & & \\
\hline AVERTED PROPERTY DAMAGE & $5.6 \mathrm{E}-01$ & $5.0 \mathrm{E}+00$ & $1.1 E+05$ \\
\hline VALUE OF AVERTED PROMPT FATALTY & $0.0 \mathrm{E}+00$ & $1.2 E-02$ & $1.6 E+04$ \\
\hline VALUE OF AVERTED EARLY ILLNESS & $0.0 \mathrm{E}+00$ & 4.3E-02 & $3.8 E+04$ \\
\hline VALUE OF AVERTED LAT. CANCER & $3,3 E_{-}-03$ & $5.4 E_{+}+00$ & $1.7 \varepsilon_{+}+04$ \\
\hline TOTAL AVERTED OFFSITE COSTS & $\overline{5.6 \mathrm{E}-01}$ & $1.0 \mathrm{E}+01$ & $1.8 \mathrm{E}+05$ \\
\hline OFFSITE COSTS AT \$1000/P-REM & $3.6 \mathrm{E}-01$ & $4.3 E+02$ & $4.4 E+05$ \\
\hline TOTAL A VERTED ON \& OFFSTIE COSTS & & & \\
\hline (BASED ON COMPUTED COSTS) & $1.5 \mathrm{E}+03$ & $3.1 \mathrm{E}+04$ & $3.3 \mathrm{E}+05$ \\
\hline (BASED ON \$1000/P-REM) & $1.5 \mathrm{E}+03$ & $3.1 E+04$ & $5.9 E+05$ \\
\hline SAFETY 0 & OPTION :P4: Independ & HPI & \\
\hline & VALUE-IMPACT ANALYS & & \\
\hline & LOW & CENTRAL & HIGH \\
\hline CHANGE IN CORE MELT FREQUENCY & $1.0 \mathrm{E}-06$ & $1.1 \mathrm{E}-05$ & $3.8 \mathrm{E}-05$ \\
\hline costs: & & & \\
\hline INSTALLATION \& ENG. COSTS & $1.880 \mathrm{E}+07$ & $2.680 \mathrm{E}+07$ & $5.360 E+07$ \\
\hline OPERATING \& MAINT. COSTS & $1.816 \mathrm{E}+06$ & $2.477 E+06$ & $4.953 E+06$ \\
\hline REPLACEMENT POWER DURING INST. & $0.000 E+00$ & $0.000 E+00$ & $0.000 \mathrm{E}+00$ \\
\hline REPLACEMENT POWER IN OPERATION & $0.000 \mathrm{E}+00$ & $0.000 \mathrm{E}+00$ & $2,000 E_{+}+0$ \\
\hline TOTAL COSTS & $2.062 E+07$ & $2.928 \mathrm{E}+07$ & $5.855 E+07$ \\
\hline BENEFITS & & & \\
\hline AVERTED ON-SITE COST & $1.8 E+04$ & $5.5 E+05$ & $2.6 \mathrm{E}+06$ \\
\hline AVERTED OFFSITE COSTS & & & \\
\hline AVERTED PROPERTY DAMAGE & $1.8 \mathrm{E}+01$ & $7.8 \mathrm{E}+01$ & $1.3 \mathrm{E}+06$ \\
\hline VALUE OF AVERTED PROMPT FATALTY & $0.0 \mathrm{E}+00$ & $2.1 \mathrm{E}-01$ & $1.1 \mathrm{E}+05$ \\
\hline VALUE OF AVERTED EARLY ILLNESS & $0.0 \mathrm{E}+00$ & 4.4E-01 & $2.3 \mathrm{E}+05$ \\
\hline VALUE OF AVERTED LAT. CANCER & $1.1 \mathrm{E}-01$ & $2.9 E_{+}+01$ & $2.1 E+05$ \\
\hline TOTAL AVERTED OFFSITE COSTS & $1.8 \mathrm{E}-01$ & $1.5 \mathrm{E}+02$ & $1.9 \mathrm{E}+06$ \\
\hline OFFSITE COSTS AT \$1000/P-REM & 1.1E-01 & $8.1 E+03$ & $8.6 \mathrm{E}+06$ \\
\hline TOTAL A VERTED ON \& OFFSTTE COSTS & & & \\
\hline (BASED ON COMPUTED COSTS) & $2.8 E+04$ & $5.5 \mathrm{E}+05$ & $4.5 E+06$ \\
\hline (BASED ON \$1000/P-REM) & $2.8 E+04$ & $5.6 \mathrm{E}+05$ & $1.1 E+07$ \\
\hline
\end{tabular}


Table E-19 (Continued)

COMPARISON OF COSTS AND BENEFITS FOR SAFETY OPTIONS: OCP METHOD (COSTS IN DOLLARS)

\begin{tabular}{|c|c|c|c|}
\hline $\begin{array}{r}\text { SAFETY } \\
\mathrm{V}\end{array}$ & $\begin{array}{l}\text { P5: Indeper } \\
\text { CT ANALY }\end{array}$ & AFW & \\
\hline & Low & CENTRAL & HIGH \\
\hline CHANGE IN CORE MELT FREQUENCY & 2.8E-07 & $2.9 \mathrm{E}-06$ & $9.5 \mathrm{E}-06$ \\
\hline costs: & & & \\
\hline INSTALLATION \& ENG. COSTS & $5.000 \mathrm{E}+06$ & $7.000 E+06$ & $1.400 E+07$ \\
\hline OPERATING \& MAINT. COSTS & $8.255 E+05$ & $1.238 E+06$ & $2.477 \mathrm{E}+06$ \\
\hline REPLACEMENT POWER DURING INST. & $0.000 E+\infty 0$ & $0.000 E+00$ & $0.000 E+00$ \\
\hline REPLACEMENT POWER IN OPERATION & $0.000 \mathrm{E}+00$ & $0.000 \mathrm{E}+00$ & $0.000 \mathrm{E}+00$ \\
\hline TOTAL COSTS & $5.826 E+06$ & $8.238 E+06$ & $1.648 E+07$ \\
\hline BENEFTIS & & & \\
\hline AVERTED ON-STTE COST & $7.9 E+03$ & $1.3 \mathrm{E}+05$ & $6.4 E+05$ \\
\hline AVERTED OFFSITE COSTS & & & \\
\hline AVERTED PROPERTY DAMAGE & $6.4 \mathrm{E}+00$ & $2.5 E+01$ & $4.2 E+05$ \\
\hline VALUE OF AVERTED PROMPT FATALTY & $0.0 \mathrm{E}+\infty 0$ & 7.2E-02 & $6.2 E+04$ \\
\hline VALUE OF AVERTED EARLY ILLNESS & $0.0 \mathrm{E}+00$ & 2.1E-01 & $1.5 \mathrm{E}+05$ \\
\hline VALUE OF AVERTED LAT. CANCER & $5.9 E_{-}-02$ & $2.2 E+02$ & $6.2 E+04$ \\
\hline TOTAL A VERTED OFFSITE COSTS & $6.5 \mathrm{E}+00$ & $4.8 E+01$ & $6.9 E+05$ \\
\hline OFFSITE COSTS AT \$1000/P-REM & $5.7 E+00$ & $1.8 \mathrm{E}+03$ & $1.6 \mathrm{E}+06$ \\
\hline TOTAL A VERTED ON \& OFFSTTE COSTS & & & \\
\hline (BASED ON COMPUTED COSTS) & $7.9 \mathrm{E}+03$ & $1.3 \mathrm{E}+05$ & $1.3 E+06$ \\
\hline (BASED ON \$1000/P-REM) & $7.9 \mathrm{E}+03$ & $1.7 \mathrm{E}+05$ & $2.3 E+06$ \\
\hline $\begin{array}{r}\text { SAFETY } \\
\text { VAL }\end{array}$ & $\begin{array}{l}\text { P6: Indep. } \\
\text { ANALYSI }\end{array}$ & RPR & \\
\hline & LOW & CENTRAL & HIGH \\
\hline CHANGE IN CORE MELT FREQUENCY & $6.1 E-7$ & $5.2 \mathrm{E}-06$ & $1.7 \mathrm{E}-05$ \\
\hline COSTS: & & & \\
\hline INSTALLATION \& ENG. COSTS & $1.700 E+07$ & $2.500 E+07$ & $5.000 \mathrm{E}+07$ \\
\hline OPERATING \& MAINT. COSTS & $1.816 E+06$ & $2.477 \mathrm{E}+06$ & $4.953 \mathrm{E}+06$ \\
\hline REPLACEMENT POWER DURING INST. & $0.000 \mathrm{E}+00$ & $0.000 \mathrm{E}+00$ & $0.000 \mathrm{E}+00$ \\
\hline REPLACEMENT POWER IN OPERATION & $0.000 \mathrm{E}+00$ & $2.000 \mathrm{E}+00$ & $2000= \pm 00$ \\
\hline TOTAL COSTS & $1.882 E+07$ & $2.748 \mathrm{E}+07$ & $5.495 E+07$ \\
\hline BENEFITS & & & \\
\hline AVERTED ON-STTE COST & $1.7 \mathrm{E}+04$ & $2.4 E+05$ & $1.1 \mathrm{E}+06$ \\
\hline AVERTED OFFSITE COSTS & & & \\
\hline AVERTED PROPERTY DAMAGE & $2.1 \mathrm{E}+01$ & $5.1 E+01$ & $2.0 \mathrm{E}+05$ \\
\hline VALUE OF AVERTED PROMPT FATALTY & $0.0 \mathrm{E}+00$ & $2.2 \mathrm{E}+00$ & $1.6 \mathrm{E}+03$ \\
\hline VALUE OF AVERTED EARLY ILLNESS & $0.0 \mathrm{E}+00$ & $4.3 E+00$ & $3.1 E+03$ \\
\hline VALUE OF AVERTED LAT. CANCER & 1.8E-01' & $1.5 E_{+}+02$ & $3,8 \mathrm{E}+04$ \\
\hline TOTAL AVERTED OFFSITE COSTS & $2.1 \mathrm{E}+01$ & $2.0 \mathrm{E}+2$ & $2.5 E+05$ \\
\hline OFFSITE COSTS AT \$1000/P-REM & $1.8 \mathrm{E}+01$ & $8.2 \mathrm{E}+03$ & $2.4 \mathrm{E}+06$ \\
\hline TOTAL AVERTED ON \& OFFSITE COSTS & & & \\
\hline (BASED ON COMPUTED COSTS) & $1.7 \mathrm{E}+04$ & 2.5E+05 & $1.4 E+06$ \\
\hline (BASED ON \$1000/P-REM) & $1.7 \mathrm{E}+04$ & $2.5 \mathrm{E}+05$ & $3.5 \mathrm{E}+06$ \\
\hline
\end{tabular}


Table E-19 (Continued)

COMPARISON OF COSTS AND BENEFITS FOR SAFETY OPTIONS: OCP METHOD (COSTS IN DOLLARS)

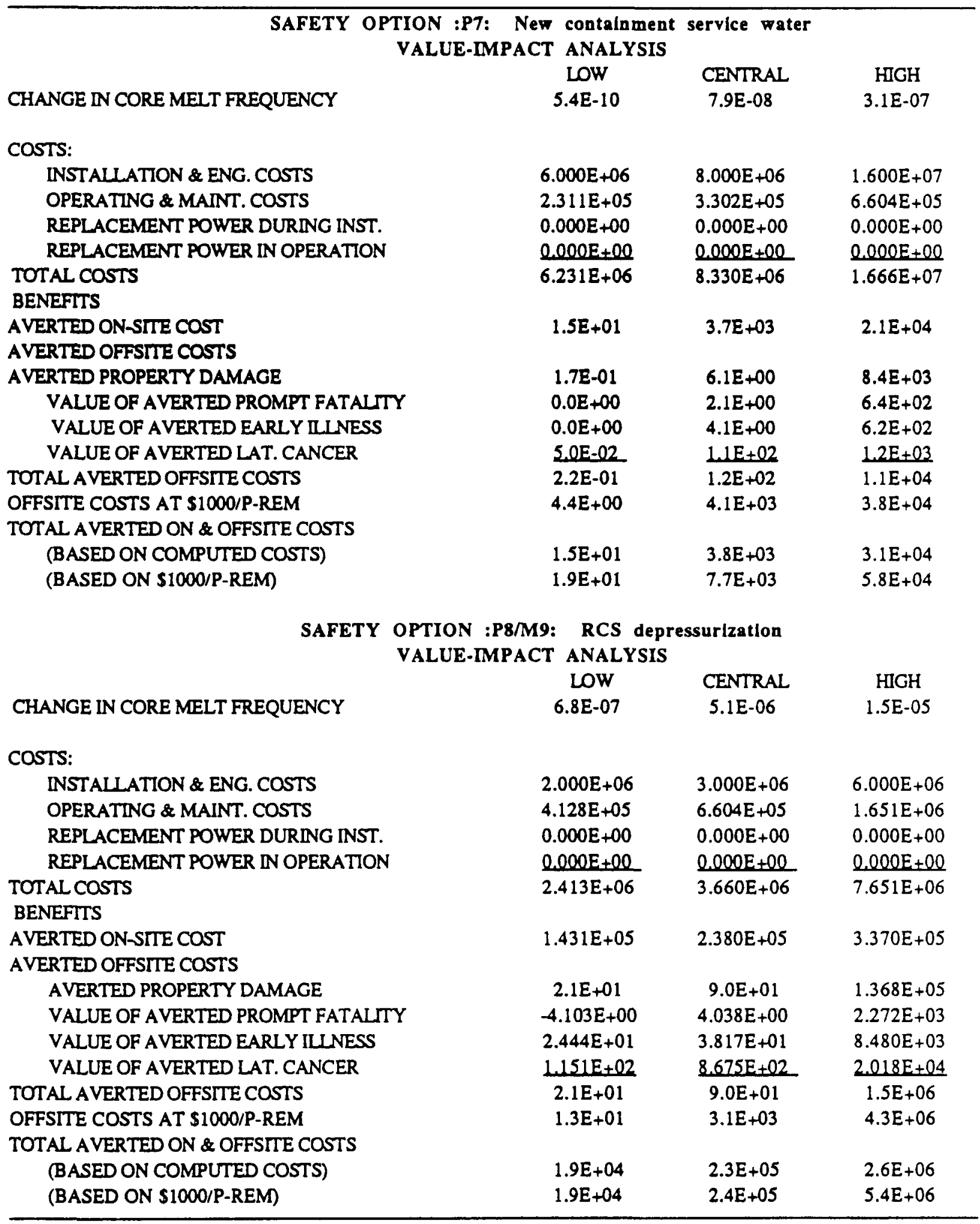


Table E-19 (Continued)

COMPARISON OF COSTS AND BENEFITS FOR SAFETY OPTIONS: OCP METHOD (COSTS IN DOLLARS)

\begin{tabular}{|c|c|c|c|}
\hline \multicolumn{4}{|c|}{$\begin{array}{l}\text { SAFETY OPTION :M1: MISSILE SHIELD } \\
\text { VALUE-IMPACT ANALYSIS }\end{array}$} \\
\hline \multirow[b]{2}{*}{ CHANGE IN CORE MELT FREQUENCY } & Low & CENTRAL & HIGH \\
\hline & $0.000 \mathrm{E}+00$ & $0.000 \mathrm{E}+00$ & $0.000 E+00$ \\
\hline \multicolumn{4}{|l|}{ COSTS: } \\
\hline INSTALLATION \& ENG. COSTS & $7.000 \mathrm{E}+06$ & $1.000 \mathrm{E}+07$ & $3.000 \mathrm{E}+07$ \\
\hline OPERATING \& MAINT. COSTS & $4.128 \mathrm{E}+04$ & $8.255 \mathrm{E}+04$ & $1.651 \mathrm{E}+05$ \\
\hline REPLACEMENT POWER DURING INST. & $0.000 \mathrm{E}+00$ & $0.000 \mathrm{E}+00$ & $0.000 \mathrm{E}+00$ \\
\hline REPLACEMENT POWER IN OPERATION & $0.000 \mathrm{E}+00$ & $0.000 E+00$ & $2.000 \mathrm{E}+00$ \\
\hline TOTAL COSTS & $7.041 \mathrm{E}+06$ & $1.008 \mathrm{E}+07$ & $\overline{3.017 E+07}$ \\
\hline \multicolumn{4}{|l|}{ BENEFITS } \\
\hline AVERTED ON-STTE COST & $0.000 \mathrm{E}+00$ & $0.000 E+00$ & $0.000 E+00$ \\
\hline \multicolumn{4}{|l|}{ AVERTED OFFSITE COSTS } \\
\hline AVERTED PROPERTY DAMAGE & $-1.0 \mathrm{E}-03$ & $8.0 \mathrm{E}-02$ & $2.4 \mathrm{E}+05$ \\
\hline VALUE OF AVERTED PROMPT FATALTY & $0.0 \mathrm{E}+00$ & $1.3 \mathrm{E}-04$ & $1.8 \mathrm{E}+04$ \\
\hline VALUE OF AVERTED EARLY ILLNESS & $0.0 \mathrm{E}+00$ & $3.8 \mathrm{E}-04$ & $3.1 E+04$ \\
\hline VALUE OF AVERTED LAT. CANCER & $2.6 \mathrm{E}-06$ & $2.5 \mathrm{E}-01$ & $3,4 E+04$ \\
\hline TOTAL A VERTED OFFSITE COSTS & $-1.0 \mathrm{E}-03$ & $3.5 \mathrm{E}-01$ & $3.2 \mathrm{E}+05$ \\
\hline OFFSITE COSTS AT \$1000/P-REM & $2.5 \mathrm{E}-04$ & $2.4 \mathrm{E}+01$ & $9.6 E+05$ \\
\hline \multicolumn{4}{|l|}{ TOTAL AVERTED ON \& OFFSITE COSTS } \\
\hline (BASED ON COMPUTED COSTS) & $-1.0 \mathrm{E}-03$ & $3.3 \mathrm{E}-01$ & $3.2 E+05$ \\
\hline \multirow[t]{3}{*}{ (BASED ON \$1000/P-REM) } & 2.4E-04 & $2.3 E+01$ & $9.6 \mathrm{E}+05$ \\
\hline & $\begin{array}{l}\text { 12: H2 IGNI } \\
\text { CT ANALYS }\end{array}$ & & \\
\hline & Low & CENTRAL & $\mathrm{HIGH}$ \\
\hline CHANGE IN CORE MELT FREQUENCY & $0.000 \mathrm{E}+\infty$ & $0.000 E+00$ & $0.000 \mathrm{E}+00$ \\
\hline \multicolumn{4}{|l|}{ costs: } \\
\hline INSTALLATION \& ENG. COSTS & $2.000 E+06$ & $7.600 \mathrm{E}+06$ & $1.520 \mathrm{E}+07$ \\
\hline OPERATING \& MAINT. COSTS & $5.779 E+05$ & $8.255 E+05$ & $1.651 \mathrm{E}+06$ \\
\hline REPLACEMENT POWER DURNG INST. & $0.000 \mathrm{E}+00$ & $0.000 \mathrm{E}+00$ & $0.000 \mathrm{E}+00$ \\
\hline REPLACEMENT POWER IN OPERATION & $0.000 E+00$ & $0.000 E_{+00}$ & $2.000 \mathrm{E}+00$ \\
\hline TOTAL COSTS & $2.578 E+06$ & $8.426 E+06$ & $1.685 \mathrm{E}+07$ \\
\hline \multicolumn{4}{|l|}{ BENEFITS } \\
\hline AVERTED ON_STTE COST & $0.000 \mathrm{E}+00$ & $0.000 \mathrm{E}+00$ & $0.000 E+00$ \\
\hline \multicolumn{4}{|l|}{ AVERTED OFFSITE COSTS } \\
\hline AVERTED PROPERTY DAMAGE & $-1.4 E+04$ & $-2.9 \mathrm{E}-01$ & $1.1 E+01$ \\
\hline VALUE OF AVERTED PROMPT FATALTY & $0.0 \mathrm{E}+00$ & $-4.1 \mathrm{E}-0.4$ & $-2.7 \mathrm{E}-03$ \\
\hline VALUE OF AVERTED EARLY ILLNESS & $0.0 \mathrm{E}+00$ & $-3.8 E-03$ & $-6.4 \mathrm{E}-03$ \\
\hline VALUE OF AVERTED LAT. CANCER & $-3,3 \mathrm{E}-06$ & $-3.2 \mathrm{E} \cdot 01$ & $=11 \mathrm{E}+03$ \\
\hline TOTAL AVERTED OFFSITE COSTS & $-1.3 E+04$ & $-6.1 E-01$ & $-1.0 \mathrm{E}+04$ \\
\hline OFFSITE COSTS AT \$1000/P-REM & $-2.6 \mathrm{E}-04$ & $-1.2 \mathrm{E}+01$ & $-1.0 \mathrm{E}+05$ \\
\hline \multicolumn{4}{|l|}{ TOTAL AVERTED ON \& OFFSTTE COSTS } \\
\hline (BASED ON COMPUTED COSTS) & $-1.4 \mathrm{E}+04$ & $-6.1 E-01$ & $-1.0 \mathrm{E}+04$ \\
\hline (BASED ON \$1000/P-REM) & $-2.6 \mathrm{E}-04$ & $-1.2 E+01$ & $1.1 E+05$ \\
\hline
\end{tabular}


Table E-19 (Continued)

COMPARISON OF COSTS AND BENEFITS FOR SAFETY OPTIONS: OCP METHOD (COSTS IN DOLLARS)

\begin{tabular}{|c|c|c|c|}
\hline SAFETY OPTIO & NTAINMENT & VERTING & \\
\hline & ANALYSIS & & \\
\hline & LOW & CENTRAL & $\mathrm{HIGH}$ \\
\hline CHANGE IN CORE MELT FREQUENCY & $0.000 \mathrm{E}+00$ & $0.000 \mathrm{E}+00$ & $0.000 \mathrm{E}+00$ \\
\hline cosTs: & & & \\
\hline INSTALLATION \& ENG. COSTS & $1.500 \mathrm{E}+06$ & $3.000 E+06$ & $7.000 \mathrm{E}+06$ \\
\hline OPERATING \& MAINT. COSTS & $3.467 E+06$ & $4.953 E+06$ & $9.906 \mathrm{E}+06$ \\
\hline REPLACEMENT POWER DUR) & $0.000 \mathrm{E}+00$ & $0.000 \mathrm{E}+00$ & $0.000 E+00$ \\
\hline REPLACEMENT POWER IN OPERATION & $0.000 \mathrm{E}+00$ & $0.000 \mathrm{E}+00$ & $0.000 E+00$ \\
\hline TOTAL COSTS & $4.967 \mathrm{E}+06$ & $7.953 E+06$ & $1.691 E+07$ \\
\hline BENEFITS & & & \\
\hline AVERTED ON-STTE COST & $0.000 \mathrm{E}+00$ & $0.000 \mathrm{E}+00$ & $0.000 \mathrm{E}+00$ \\
\hline AVERTED OFFSTTE COSTS & & & \\
\hline AVERTED PROPERTY DAMAGE & $-3.3 E-00$ & $2.8 \mathrm{E}+00$ & $3.1 E+05$ \\
\hline VALUE OF AVERTED PROMPT FATALTY & $0.0 \mathrm{E}+00$ & $7.8 \mathrm{E}-03$ & $2.3 E+04$ \\
\hline VALUE OF AVERTED EARLY ILLNESS & $0.0 \mathrm{E}+00$ & 4.4E-02 & $5.9 E+04$ \\
\hline VALUE OF AVERTED LAT. CANCER & $1.2 E_{-}-04$ & $4.6 \mathrm{E}+00$ & $5.2 \mathrm{E}+04$ \\
\hline TOTAL AVERTED OFFSITE COSTS & $-3.4 E+00$ & $7.5 \mathrm{E}+00$ & $4.4 E+05$ \\
\hline OFFSITE COSTS AT \$1000/P-REM & $1.1 \mathrm{E}-02$ & $2.7 E+02$ & $2.3 E+06$ \\
\hline TOTAL A VERTED ON \& OFFSITE COSTS & & & \\
\hline (BASED ON COMPUTED COSTS) & $-3.3 E+00$ & $7.5 \mathrm{E}+00$ & $4.4 \mathrm{E}+05$ \\
\hline (BASED ON \$1000/P-REM) & $1.1 \mathrm{E}-02$ & $2.7 \mathrm{E}+02$ & $2.5 \mathrm{E}+06$ \\
\hline $\begin{array}{r}\text { SAFETY } \\
\text { VAL }\end{array}$ & $\begin{array}{l}\text { 4: Early nit } \\
\Gamma \text { ANALYSIS }\end{array}$ & venting & \\
\hline & LoW & CENTRAL & HIGH \\
\hline CHANGE IN CORE MELT FREQUENCY & $6.7 \mathrm{E}-10$ & $8.6 \mathrm{E}-08$ & $3.3 \mathrm{E}-07$ \\
\hline COSTS: & & & \\
\hline INSTALLATION \& ENG. COSTS & $9.500 \mathrm{E}+06$ & $1.350 \mathrm{E}+07$ & $2.700 \mathrm{E}+07$ \\
\hline OPERATING \& MAINT. COSTS & $1.156 \mathrm{E}+06$ & $1.651 \mathrm{E}+06$ & $3.302 E+06$ \\
\hline REPLACEMENT POWER DURING INST. & $0.000 \mathrm{E}+00$ & $0.000 \mathrm{E}+00$ & $0.000 \mathrm{E}+00$ \\
\hline REPLACEMENT POWER IN OPERATION & $0.000 E_{+}+00$ & $0.000 E_{+}+0$ & $0.000 \mathrm{E}+00$ \\
\hline TOTAL COSTS & $1.066 \mathrm{E}+07$ & $1.515 \mathrm{E}+07$ & $3.030 \mathrm{E}+07$ \\
\hline BENEFITS & & & \\
\hline AVERTED ON-SITE COST & $1.8 \mathrm{E}+01$ & $4.0 \mathrm{E}+03$ & $2.2 E+04$ \\
\hline AVERTED OFFSITE COSTS & & & \\
\hline AVERTED PROPERTY DAMAGE & $3.6 \mathrm{E}+00$ & $2.7 \mathrm{E}+01$ & $8.9 E+05$ \\
\hline VALUE OF AVERTED PROMPT FATALTY & $0.0 \mathrm{E}+00$ & $2.6 E+00$ & $3.2 E+04$ \\
\hline VALUE OF AVERTED EARLY ILLNESS & $0.0 \mathrm{E}+00$ & $5.1 E+00$ & $5.7 E+04$ \\
\hline VALUE OF AVERTED LAT. CANCER & $3.9 \mathrm{E}-00$ & $2.0 \mathrm{E}+02$ & $1.5 \mathrm{E}+0.5$ \\
\hline TOTAL AVERTED OFFSITE COSTS & $4.0 \mathrm{E}+\infty 0$ & $2.3 \mathrm{E}+02$ & $1.1 \mathrm{E}+06$ \\
\hline OFFSITE COSTS AT \$1000/P-REM & $4.0 \mathrm{E}+01$ & $1.5 \mathrm{E}+04$ & $8.0 E+06$ \\
\hline TOTAL AVERTED ON \& OFFSITE COSTS & & & \\
\hline (BASED ON COMPUTED COSTS) & $2.3 E+01$ & $4.6 E+03$ & $1.2 E+06$ \\
\hline (BASED ON \$1000/P-REM) & $5.8 \mathrm{E}+01$ & $1.9 \mathrm{E}+04$ & $8.0 E+06$ \\
\hline
\end{tabular}


Table E-19 (Continued)

COMPARISON OF COSTS AND BENEFITS FOR SAFETY OPTIONS: OCP METHOD (COSTS IN DOLLARS)

\begin{tabular}{|c|c|c|c|}
\hline SAFETY OPT & LATE FILTERE & VENTING & \\
\hline & Low & CENTRAL & $\mathrm{HIGH}$ \\
\hline CHANGE IN CORE MELT FREQUENCY & $6.6 \mathrm{E}-10$ & 8.5E-08 & $3.3 \mathrm{E}-07$ \\
\hline COSTS: & & & \\
\hline INSTALLATION \& ENG. COSTS & $9.500 E+06$ & $1.350 \mathrm{E}+07$ & $2.700 E+07$ \\
\hline OPERATING \& MAINT. COSTS & $1.156 \mathrm{E}+06$ & $1.651 E+06$ & $3.302 \mathrm{E}+06$ \\
\hline REPLACEMENT POWER DURING INST. & $0.000 \mathrm{E}+00$ & $0.000 \mathrm{E}+00$ & $0.000 \mathrm{E}+00$ \\
\hline REPLACEMENT POWER IN OPERATION & $0.000 \mathrm{E}+00$ & $0.000 E_{+}+00$ & $2000 \mathrm{E}+00$ \\
\hline TOTAL COSTS & $1.066 \mathrm{E}+07$ & $1.515 E+07$ & $3.030 \mathrm{E}+07$ \\
\hline BENEFITS & & & \\
\hline AVERTED ON-SITE COST & $1.8 \mathrm{E}+01$ & $4.0 E+03$ & $2.2 \mathrm{E}+04$ \\
\hline AVERTED OFFSITE COSTS & & & \\
\hline AVERTED PROPERTY DAMAGE & $-1.9 \mathrm{E}+01$ & $1.9 \mathrm{E}+01$ & $2.8 E+05$ \\
\hline VALUE OF AVERTED PROMPT FATALTY & $-0.0 \mathrm{E}+00$ & $2.6 \mathrm{E}+00$ & $1.8 \mathrm{E}+03$ \\
\hline VALUE OP AVERTED EARLY ILLNESS & $0.0 \mathrm{E}+\infty 0$ & $5.1 E+00$ & $2.0 E+03$ \\
\hline VALUE OF AVERTED LAT. CANCER & $3,9 \mathrm{E}-01$ & $1.8 E+02$ & $5.2 E+04$ \\
\hline TOTAL AVERTED OFFSTTE COSTS & $-1.9 E+01$ & $2.1 E+02$ & $3.4 E+05$ \\
\hline OFFSITE COSTS AT \$1000/P-REM & $4.0 E+01$ & $1.4 \mathrm{E}+04$ & $3.6 \mathrm{E}+06$ \\
\hline TOTAL A VERTED ON \& OFFSTTE COSTS & & & \\
\hline (BASED ON COMPUTED COSTS) & $-5.9 \mathrm{E}-01$ & $4.2 \mathrm{E}+03$ & $3.6 E+05$ \\
\hline (BASED ON \$1000/P-REM) & $5.9 E+01$ & $1.8 \mathrm{E}+04$ & $3.6 \mathrm{E}+06$ \\
\hline $\begin{array}{r}\text { SAFETY } \\
\text { VAL }\end{array}$ & $\begin{array}{l}\text { :M6: New spr } \\
\text { CT ANALYSIS }\end{array}$ & & \\
\hline & Low & CENTRAL & HIGH \\
\hline CHANGE IN CORE MELT FREQUENCY & $6.4 \mathrm{E}-10$ & $8.2 \mathrm{E}-8$ & $3.1 E-07$ \\
\hline COSTS: & & & \\
\hline INSTALLATION \& ENG. COSTS & $5.600 \mathrm{E}+06$ & $8.000 \mathrm{E}+06$ & $1.600 \mathrm{E}+07$ \\
\hline OPERATING \& MAINT. COSTS & $1.040 \mathrm{E}+06$ & $1.486 \mathrm{E}+06$ & $2.972 \mathrm{E}+06$ \\
\hline REPLACEMENT POWER DURING INST. & $0.000 \mathrm{E}+00$ & $0.000 \mathrm{E}+00$ & $0.000 \mathrm{E}+00$ \\
\hline REPLACEMENT POWER IN OPERATION & $0.000 \mathrm{E}_{ \pm}+00$ & $0.000 E_{+00}$ & $0.000 E_{+00}$ \\
\hline TOTAL COSTS & $6.640 \mathrm{E}+06$ & $9.486 E+06$ & $1.897 \mathrm{E}+07$ \\
\hline BENEFITS & & & \\
\hline AVERTED ON-STTE COST & $1.8 \mathrm{E}+01$ & $3.89 \mathrm{E}+03$ & $2.2 \mathrm{E}+04$ \\
\hline AVERTED OFFSITE COSTS & & & \\
\hline AVERTED PROPERTY DAMAGE & $3.1 E+00$ & $1.9 E+01$ & $6.5 \mathrm{E}+05$ \\
\hline VALUE OF AVERTED PROMPT FATALTY & $0.0 \mathrm{E}+00$ & $2.5 \mathrm{E}+00$ & $3.7 \mathrm{E}+04$ \\
\hline VALUE OF AVERTED EARLY ILLNESS & $0.0 \mathrm{E}+00$ & $4.8 \mathrm{E}+00$ & $5.1 E+04$ \\
\hline VALUE OF AVERTED LAT. CANCER & $4.1 E-01$ & $2.2 E+02$ & $2.3 E+04$ \\
\hline TOTAL AVERTED OFFSITE COSTS & $3.6 \mathrm{E}+00$ & $2.5 E+02$ & $8.2 \mathrm{E}+05$ \\
\hline OFFSITE COSTS AT $\$ 1000 / P-R E M$ & $4.1 E+01$ & $1.6 E+04$ & $3.4 E+06$ \\
\hline TOTAL AVERTED ON \& OFFSITE COSTS & & & \\
\hline (BASED ON COMPUTED COSTS) & 2.1E-01 & $4.1 E+03$ & $8.5 E+05$ \\
\hline (BASED ON $\$ 1000 /$ P-REM) & $5.9 \mathrm{E}-01$ & $1.9 \mathrm{E}+04$ & $3.5 \mathrm{E}+06$ \\
\hline
\end{tabular}


Table E-19 (Continued)

COMPARISON OF COSTS AND BENEFITS FOR SAFETY OPTIONS: OCP METHOD (COSTS IN DOLLARS)

\begin{tabular}{|c|c|c|c|}
\hline \multicolumn{4}{|c|}{$\begin{array}{c}\text { SAFETY OPTION :M7: Fan coolers } \\
\text { VALUE-IMPACT ANALYSIS }\end{array}$} \\
\hline & Low & CENTRAL & $\mathrm{HIGH}$ \\
\hline CHANGE IN CORE MELT FREQUENCY & $6.3 E-10$ & $8.2 \mathrm{E}-08$ & $3.2 \mathrm{E}-07$ \\
\hline \multicolumn{4}{|l|}{ COSTS: } \\
\hline INSTALLATION \& ENG. COSTS & $2.600 \mathrm{E}+06$ & $3.700 E+06$ & $7.400 E+06$ \\
\hline OPERATING \& MAINT. COSTS & $8.255 \mathrm{E}+05$ & $1.156 E+06$ & $1.651 E+06$ \\
\hline REPLACEMENT POWER DURING INST. & $0.000 \mathrm{E}+00$ & $0.000 \mathrm{E}+00$ & $0.000 \mathrm{E}+00$ \\
\hline REPLACEMENT POWER IN OPERATION & $0.000 \mathrm{E}+00$ & $0.000 \mathrm{E}+00$ & $0.000 E+00$ \\
\hline TOTAL COSTS & $3.426 E+06$ & $4.856 \mathrm{E}+06$ & $9.051 E+06$ \\
\hline \multicolumn{4}{|l|}{ BENEFITS } \\
\hline AVERTED ON-SITE COST & $1.8 \mathrm{E}+01$ & $3.8 \mathrm{E}+03$ & $2.1 E+04$ \\
\hline \multicolumn{4}{|l|}{ AVERTED OFFSITE COSTS } \\
\hline AVERTED PROPERTY DAMAGE & $-7.1 E+01$ & $-3.5 E+01$ & $7.5 E+04$ \\
\hline VALUE OF AVERTED PROMPT FATALTY & $0.0 \mathrm{E}+00$ & $2.2 \mathrm{E}+00$ & $5.0 E+03$ \\
\hline VALUE OF AVERTED EARLY ILLNESS & $0.0 \mathrm{E}+\infty$ & $3.8 E+00$ & $5.4 E+03$ \\
\hline VALUE OF AVERTED LAT. CANCER & $3.9 \mathrm{E}-01$ & $1.0 E_{+}+02$ & $1.0 \mathrm{E}+04$ \\
\hline TOTAL AVERTED OFFSITE COSTS & $-6.9 \mathrm{E}+01$ & $7.1 \mathrm{E}+01$ & $9.7 E+05$ \\
\hline OFFSITE COSTS AT $\$ 1000 / P$-REM & $3.8 \mathrm{E}+01$ & $6.4 \mathrm{E}+03$ & $2.9 E+05$ \\
\hline \multicolumn{4}{|l|}{ TOTAL A VERTED ON \& OFFSTTE COSTS } \\
\hline (BASED ON COMPUTED COSTS) & $-5.2 \mathrm{E}+01$ & $3.9 E+03$ & $1.2 \mathrm{E}+05$ \\
\hline (BASED ON \$1000/P-REM) & $5.6 \mathrm{E}+01$ & $1.0 \mathrm{E}+04$ & $3.1 \mathrm{E}+05$ \\
\hline \multicolumn{4}{|c|}{$\begin{array}{r}\text { SAFETY OPTION :P1 \& P2: V Procedures } \\
\text { VALUE.IMPACT ANALYSIS }\end{array}$} \\
\hline & LOW & CENTRAL & $\mathrm{HIGH}$ \\
\hline CHANGE IN CORE MEIT FREQUENCY & $5.5 \mathrm{E}-07$ & 8.1E-06 & $2.7 \mathrm{E}-05$ \\
\hline \multicolumn{4}{|l|}{ COSTS: } \\
\hline INSTALLATION \& ENG. COSTS & $1.100 \mathrm{E}+07$ & $1.600 \mathrm{E}+07$ & $3.200 \mathrm{E}+07$ \\
\hline OPERATING \& MAINT. COSTS & $2.367 E+06$ & $3.353 E+06$ & $6.606 \mathrm{E}+06$ \\
\hline REPLACEMENT POWER DURING INST. & $0.000 \mathrm{E}+00$ & $0.000 \mathrm{E}+00$ & $0.000 \mathrm{E}+00$ \\
\hline REPLACEMENT POWER IN OPERATION & $0.000 \mathrm{E}_{+}+00$ & $0.000 E+00$ & $0.000 \mathrm{E}+00$ \\
\hline TOTAL COSTS & $1.333 \mathrm{E}+07$ & $\overline{1.995 \mathrm{E}+07}$ & $3.891 E+07$ \\
\hline \multicolumn{4}{|l|}{ BENEFITS } \\
\hline AVERTED ON-SITE COST & $1.5 \mathrm{E}+04$ & $3.7 \mathrm{E}+05$ & $1.8 E+06$ \\
\hline \multicolumn{4}{|l|}{ AVERTED OFFSITE COSTS } \\
\hline AVERTED PROPERTY DAMAGE & $2.9 \mathrm{E}+00$ & $1.1 \mathrm{E}+02$ & $1.3 \mathrm{E}+06$ \\
\hline VALUE OF AVERTED PROMPT FATALTY & $0.0 \mathrm{E}+00$ & $2.2 \mathrm{E}+00$ & $1.3 \mathrm{E}+05$ \\
\hline VALUE OF AVERTED EARLY ILLNESS & $0.0 \mathrm{E}+00$ & $5.1 E+00$ & $1.8 \mathrm{E}+05$ \\
\hline VALUE OF AVERTED LAT. CANCER & $12 \mathrm{E}-01$ & $3.2 E_{+} 02$ & $20 \mathrm{E}+05$ \\
\hline TOTAL AVERTED OFFSITE COSTS & $3.1 \mathrm{E}+00$ & $4.3 E+02$ & $1.9 \mathrm{E}+06$ \\
\hline OFFSITE COSTS AT $\$ 1000 / P-R E M$ & $1.1 E+01$ & $2.3 \mathrm{E}+04$ & $8.0 E+06$ \\
\hline \multicolumn{4}{|l|}{ TOTAL AVERTED ON \& OFFSITE COSTS } \\
\hline (BASED ON COMPUTED COSTS) & $1.6 \mathrm{E}+04$ & $3.7 E+05$ & $3.7 \mathrm{E}+06$ \\
\hline (BASED ON $\$ 1000 /$ P-REM) & $1.6 \mathrm{E}+04$ & $4.0 \mathrm{E}+05$ & $9.9 \mathrm{E}+06$ \\
\hline
\end{tabular}




\section{E.5 OBSERVATIONS CONCERNING RISK-REDUCTION ANALYSIS}

As illustrated in the previous section, none of the options appeared to be cost effective. One reason for this is that Surry was not assessed to have one or two risk outliers that could be reduced resulting in a large effect, rather there are many risk contributors. In addition, there were no subtle, simple fixes discovered and all of the options involve significant cost.

Another way to view this result is to consider the amount of money that could be allocated to reduce the Surry public risk to zero. This was calculated the same way as the other options discussed previously by suggesting a hypothetical option that eliminated all the coredamage accidents. The result is listed in Table E-20 below. As illustrated, the highest value of complete removal of the risk is two million dollars, based on the use of CRAC2. Obviously, this does not allow for any significant hardware changes and only other options to improve safety through better operations have any chance of being cost effective. The MACCS code does predict higher costs, but only by about a factor of two.

Table E-20

VALUE OF REDUCTION OF RISK TO ZERO FOR SURRY

\begin{tabular}{lccc}
\hline \multicolumn{1}{c}{ Option } & \multicolumn{3}{c}{ TOTAL VALUE OF AVERTED RISK } \\
& LLH Median & LLH High & LLH Low \\
\hline TOTAL AVERTED OFFSITE COSTS & $\$ 16,000$ & $\$ 186,000$ & $\$ 3,700$ \\
TOTAL AVERTED OFFSITE COSTS & $\$ 110,000$ & $\$ 787,000$ & $\$ 25,000$ \\
COMPUTED AT \$1000/PERSON-REM & & & \\
TOTAL AVERTED OFFSITE COSTS & $\$ 940,000$ & $\$ 1,400,000$ & $\$ 450,000$ \\
PLUS ONSITE COSTS & & & $\$ 480,000$ \\
TOTAL AVERTED OFFSITE COSTS & $\$ 1,000,000$ & $\$ 2,000,000$ & \\
COMPUTED AT \$1000/PERSON-REM & & & \\
PLUS ONSITE COSTS & & & \\
\hline
\end{tabular}

As already stated, the risk reduction measures were calculated using both the $\mathrm{OCP}$ and LLH methods. Several of the options had quite different effects between the two, due to the different contributors to risk (presented in Section 5.5 of the main report). For example, option P1 appeared attractive for some combinations of costs versus estimated risk reduction in the OCP due to the fact that the $\mathrm{V}$ sequence was more important, whereas RCS depressurization was most important to the $L L H$ due to the importance of direct heating. The list of dominant contributors, either to sequence frequency or phenomenology, is critical to the identification of effective options. The comparison of the methods illustrates considerable uncertainty in the dominant contributors, and establishing options with clear effectiveness is difficult. 
NUREG/CR-4551,VOL.1: DRAFT REPORT FOR COMMENT (SEPTEMBER, 1986)

\section{Appendix F}

SUMMARY OF REVIEW TEAM COMMENTS CONCERNING THE LIMITED LATIN HYPERCUBE ANALYSIS AS IMPLEMENTED IN SARRP 
•

-

-

-

- 


\section{Appendix F}

\section{SUMMARY OF REVIEW TEAM COMMENTS CONCERNING THE LIMITED LATIN HYPERCUBE ANALYSIS AS IMPLEMENTED IN SARRP}

The members of the expert review group assembled for SARRP were asked to provide comments concerning their views on the limited Latin hypercube (LLH) methodology being utilized for uncertainty and sensitivity analysis. This appendix summarizes the reviewers' comments which were provided at their meeting of April 3,1986. The attendees at this meeting each had direct familiarity with the LLH process, through their participation in the process of providing expert opinion regarding the formulation and characterization of the uncertainty issues. The comments that follow were taken directly from a tape recording of the meeting. It should be noted that time for discussion was limited at the meeting, and several participants hoped to follow their comments with more specific, written comments. Some of the discussion related specifically to the interim risk results for Surry that were presented to illustrate the process and to stimulate the discussion, and those comments are discussed as appropriate in other parts of this report. The summary that follows assumes fairly intimate knowledge of the SARRP methodology, as well as the history involved in its development.

In general, the reviewers felt that the uncertainty approach being pursued by SARRP was about the best that could be done within time and budget constraints. Most then focused their discussion on areas of concern regarding possible biases or misinterpretation of the results.

\section{Introduction by SARRP Management}

Dr. Allan Benjamin of Sandia initiated the session with a brief review of the uncertainty approaches employed in SARRP. He also provided some initial results for the Surry plant that were obtained using both the LLH and optimistic/central/pessimistic (OCP) approaches. $\mathrm{He}$ explained that through interactions of SARRP personnel, review groups, and NRC staff, the LLH approach was developed in order to address the significant drawback of the OCP approach, which was the initial method selected. That drawback concerns the lack of ability to characterize the relative likelihoods of the outcomes, i.e., the degree of optimism and pessimism in the results. The LLH method allowed the development of a distribution of possible outcomes (in terms of the risk results) generated by variation of a specific number of uncertain inputs, which were termed issues in this study. The method was generally developed to allow study of phenomenological uncertainties associated with core, containment, and 
source term processes. The distributions are developed from weighting factors provided by a group of individuals knowledgeable about the issues and current research and opinions on the subjects. The results therefore provide insights concerning the relative importance of particular sources of uncertainty selected for study. The method does not produce a statistical definition of uncertainty due to the inherent limitations on scope. While the LLH approach did not provide a perfect solution, it was agreed that it was the best available under the circumstances. The uncertainty approaches are described in Sections 2 and 4 of the main report.

The presentation of the interim results served to stimulate the discussion of the appropriateness of the LLH methods proposed for use in the program. Some discussion of details ensued, but the participants in the meeting generally offered higher-level comments concerning the methodology itself.

The attendees at the LLH meeting who participated in this discussion are listed below. The specific comments discussed in this report are attributed to individuals by number only, and the listing below does not correspond to the numerical listing in the text.

\section{Participants in April 3, 1986 Discussion of LLH Methodology}
M. Emst, U.S. Nuclear Regulatory Commission
A. Benjamin, Sandia National Laboratories
R. Denning, Battelle Columbus Laboratories
G. Greene, Brookhaven National Laboratory
S. Hodge, Oak Ridge National Laboratory
M. Corradini, University of Wisconsin
J. Gieseke, Battelle Columbus Laboratories
C. Hofmayer, Brookhaven National Laboratory
J. Mitchell, U.S. Nuclear Regulatory Commission
H. Ashar, U.S. Nuclear Regulatory Commission
T. Kress, Oak Ridge National Laboratory
G. Boyd, SAROS, Inc.
M. Berman, Sandia National Laboratories*

\section{Objectives of the Discussion}

The SARRP and NRC managers provided guidance concerning their objectives in soliciting comments from those involved in the LLH process. There are obviously limitations in the process used in SARRP to assess uncertainty, and these need to be clearly understood before conclusions are drawn. There are several intended uses of the products of the SARRP analyses relative to NUREG- 1150 that also require consideration. The SARRP results will be examined to determine if the estimated risks are acceptable, including the risk represented by reasonable bounds about the central estimates. The key drivers of risk need to be identified in order to understand the system features and phenomena that contribute to the risk estimates.

\footnotetext{
This reviewer was not able to attend the April 3 meeting, but provided written comments subsequent to the meeting.
} 
Reasonable uncertainty estimates are also needed to draw conclusions regarding these contributors. The goal of the LLH process was to establish "reasonable" ranges of uncertainty. The definition of a reasonable range is clearly a matter of judgment, and it is very important that all terminology used in the study be carefully defined. After months of consideration, a statement of the scope and objectives of the uncertainty analysis has been developed by Sandia and NRC. Additional input concerning the LLH methodology was sought from the attendees at this meeting to ensure that the analysis is as fully understood as possible, including both its benefits and limitations.

Concerning the relationship of the SARRP analyses to the NRC position papers being developed for specific issues, it was stated that the position papers will definitely be developed by the NRC staff, based on all available inputs. If the SARRP study suggests different conclusions from those in a NRC position paper, it will be necessary to attain some sort of resolution. Currently the position papers are generally lagging the treatment of the same issues in SARRP, and interaction has been somewhat limited.

One of the concerns is the need to translate the discussion and assignment of weighting factors provided in the meetings of the experts into inputs for the LLH analysis in a clear and appropriate manner. The meetings have been dynamic in nature, and it is not clear that all of the input provided in the meetings can be properly characterized within the framework of the study. The process of developing inputs based on expert opinion will be subject to criticism due to the degree of interaction that took place, and the views of those participating in the program are needed in order for SARRP and the NRC to have a full appreciation of the results.

There is also reason for concern about limitations that would cause an important contributor to be missed. Time and budget constraints alone have limited the efforts in the program. The questions of whether or not all of the actual contributors to risk and to uncertainty have been identified and to what degree small variations in the input could change the results are also important in this regard. It is also important to assess the degree to which the plant-specific analyses can be used to form the basis for drawing more generic conclusions. Some of the results appear to be due to relatively subtle plant features that will limit the ability to draw conclusions for other plants.

In summary, the meeting attendees were asked to provide high-level comments concerning the following aspects:

- Their overall opinions regarding the usefulness of the process;

- Their greatest concems about the LLH approach and the manner in which it was implemented; 
- Their opinions concerning how to characterize the results; and

- Their views regarding the ability to use this information to draw more generic conclusions.

\section{Beviewer Comments}

The meeting attendees provided comments in response to the objectives stated above. Due to time constraints at the meeting, the discussion was limited to general areas rather than specific items, except as examples. Several participants stated their intent to provide written comments at a later date. The comments are provided for individuals below. Group discussion is also included where appropriate.

Reviewer \#1. Reviewer \#1 offered a number of observations concerning the collection of the input information and the meaning and presentation of the results. Specific comments are summarized below.

- The results presentation should include a breakdown of the output to allow examination of the relative sensitivities of the uncertainty issues not only to risk, but also to the interim products of the study. For example, the uncertainty in containment loading and response should be examined relative to key measures, such as the frequency of early containment failure. The source terms should be similarly characterized in terms of release fractions of key species. After publication of the results, most of the users will want to carry the results further to assess their broader implicationș. This will involve the need to decompose the results as described above, a task best performed by the SARRP analysts. The reporting will need to include discussion of the subtleties and the limitations of the methods used. The most desirable situation would be for the SARRP analysts to have thought through this process before the same questions are asked by others.

- The process of soliciting expert opinion used in this program will be the subject of considerable criticism, and it will be argued that this is a fatal flaw. The key area of concern is the reviewer interaction. It is essentially a no-win situation; if there were no interactions, some would say that the reviewers might not have been supplying input coherently, while others will say that the interaction led to an inappropriate narrowing of the uncertainty range. There is no good solution to this dilemma, although there may be ways to partially assess the impact of the interactions. The input for the Surry plant was somewhat unique since it was the first plant, and the reviewers had generally prepared their responses in advance of the meeting. The Surry input therefore offers the potential for some examination of the sensitivity of the methodology. One test would be to compare the original inputs of one or two reviewers with their final input. Another test would involve the use of the reviewers' inputs as the sensitivity cases, rather than use of the composite input of all of the reviewers. These tests may provide insight to help respond to future criticism.

- The results should also address their applicability to other plants. It would appear that the results indicate the importance of plant-specific features as 
subtle as relative geometries of reactor cavities. These insights should be included in the report.

- The results will be difficult to characterize, since they cannot be represented in statistical terms. If the LLH output is referred to as a representation of uncertainty rather than the result of a sensitivity study, the limitations will have to be clearly stated, and all terms will have to be carefully defined.

Reviewer \#2. Reviewer \#2 discussed both the methodology and its implementation in SARRP. He planned to supply written comments at a later date.

- All supporting analyses for NUREG-1150 must include uncertainty measures in order to have any viable use. The method developed for SARRP has very serious flaws, but overall this was an appropriate method given all the constraints and the difficulty of the problem. In fact, this appears to be the best method available.

- The LLH methodology allows for correction of known deficiencies in the Source Term Code Package (STCP). He would have more reservations about the program if there were no method for correcting for these deficiencies.

- There are many problems involving the subjectivity of the process. "First, the participants were forced to provide unsubstantiated guesses as input for many issues that could at least have been based on calculations had schedule and budget allowed. In some cases, nothing could have been done to narrow the uncertainty, short of years of research. In many other cases, the input could have been based on evidence and calculation, but the participants had to provide input without benefit of additional calculation. It would have cost millions of dollars to do that for each issue, but very important issues should be based on the best knowledge available. It should be one of the promises of NUREG-1150 that these issues will be addressed fully in the future.

- There is a significant risk that the LLH process will narrow the uncertainty bounds associated with accident phenomenology, since not all issues have been included and the range of input in terms of participants was somewhat limited. The users of the output must be careful to eliminate a concern based on the LLH output alone, and the quantitative results must be used very carefully.

- The results should be characterized as a qualified uncertainty analysis. The project should not be wishy-washy in its statement of what was done by calling it a sensitivity study. The uses of NUREG-1150 will require an uncertainty characterization, and this LLH process is the uncertainty analysis for the program. The report will need to define the terminology and limitations very carefully.

- When asked to compare the SARRP analyses to the Reactor Safety Study, the reviewer stated that there were very clear advances in the technology, and the uncertainty approach was obviously an improvement, since the RSS did not treat phenomenological uncertainties. However, the RSS fell on its face because even though it contained a significant amount of good work, weaknesses in presentation tended to hide the advances made. SARRP will 
run the same risk unless the presentation of the entire process allows others to understand the full scope of the activities and their implications.

Reviewer \#3. Reviewer \#3 was most concerned about two issues: (1) the guessing during the expert input process, and (2) the deficiencies in the STCP.

- There was a significant amount of unmitigated guessing during the LLH input collection that could have been prevented if participants abstained on issues about which their qualifications were limited. In some cases, it was not clear that the right people were selected to provide the input. In all cases, code calculations should have been given more weight than expert input. Reviewer \#3 was questioned as to whether the results were useful given the amount of guessing that was involved. It was his opinion that the results were useful for the objectives of SARRP, but that extensions of the results for other purposes would have many pitfalls.

- The STCP does not include models for a number of phenomena that could be important. This situation is not necessary since there are other models available, although in many cases the models are simple, parametric ones. Good examples include steam explosion, direct heating, and more realistic models for core-concrete interactions. It would be better if the STCP included the effects of direct heating through a code such as DHEAT rather than the addition of the pressures in the LLH process.

- The community is still on the learning curve relative to more unique phenomena that may be very important to risk. For example, SARRP should be praised for including alternative containment failure modes such as liner meltthrough and suppression pool bypass. Phenomena such as these are very plant-specific, but could easily be more important than traditional failure modes such as overpressurization.

- The analyses of some plants benefited from the presence at the LLH input meeting of experts who had actually visited the facilities. There is no substitute for onsite inspection, particularly relative to plant-specific subtleties. Additional input of this type would be desirable.

- The consideration of a full range of radionuclides is important for complete characterization of the risk. The SARRP program included a good breakdown of the types of releases and their severity.

- From this reviewer's perspective, the front-end analysis appears to have serious flaws. The treatment of the operator action to turn on the igniters and the lack of information on the critical SRV tailpipe vacuum breakers were problem areas. These types of issues could drive the risk.

Reviewer\#4. Reviewer \#4 echoed the opinions of most of the previous reviewers and added one further point:

- The plant-to-plant variations include more than physical differences; the procedural differences could lead to dramatic differences in response for very similar plants. A good example is containment venting at BWRs, which has very different guidance at different plants. This problem also precludes generalization based on these plant-specific studies. 
Reviewer \#5. Reviewer \#5 had some additional comments on the lack of analysis associated with some issues.

- The LLH process requires too much guessing. There is a lack of analysis upon which to base the weighting factors, particularly considering the fact that most participants are using the same basic sources.

- The guessing comes about as a result of an imbalance in the project activities. Some very important issues have had almost no effort devoted to them, whereas a great deal of effort is spent on less important issues. A good example is the request to vote on a pressure increment due to hydrogen burns with limited or no analysis.

Beviewer \#6. Reviewer \#6 offered some views on similar processes in other programs.

- The Seismic Safety Margins Research Program (SSMRP) included a similar expert-opinion process, although many more experts were involved. The project analysis had difficulty resolving all of the input, because it was not always clear what the participants had intended. In addition, there is considerable interpretation of the request for information which can lead to disconnects among those providing the input. The process used here led to a great advantage in that the participants had the benefit of discussion of the issue to ensure that they were all responding to the same question, the discussion allowed a sharing of insights which did lead to more informed assignment of weighting factors, and the project analysis had access to the reviewers to fully understand how the input was to be used.

- There could be additional benefits to a concise re-review of all of the input provided to date. There has been considerable learning associated with the analysis of the later plants which could be fed back to the first plants studies with the LLH process.

Reviewer \#7. Reviewer \#7 expanded on some of the concerns that had been raised by other reviewers.

- The approach used in the study will tend to miss the interactions among the issues. The number of correlations included in the LLH study was limited, and although the SARRP analysts have included extensive considerations of correlations and consistency, the LLH study does not allow for full modeling of these correlations. Many of the synergisms are perhaps lost in this process, leading to an oversimplified view of the final output, and perhaps a distorted view of the importance of individual issues. The latest LLH study of the Grand Gulf plant did include one good example of the importance of these synergisms, the scrubbing DFs associated with the various processes in containment. In many cases, the synergisms are predicted, but there is not enough understanding to effectively model the interactions.

- It would have been better if there had been less interaction and subsequent influence within the voting process. The first round, the input for the Surry 
plant, involved less interaction and more independent thinking. There were some changes as a result of conversation, but nothing like the later plants where the reviewers assessed the situation and developed their inputs within the meeting with limited independent thinking. The discussions in the meeting tended to narrow the uncertainty ranges to the point at which, if discussion lasted long enough, everyone tended to agree. Even when two or more individuals had polarized viewpoints, the output eventually tended to be the same.

Several others noted that while the reviewers did tend to agree on the weighting factors, the ranges still reflected the wide variation in opinion. Reviewer \#7 still believed that the weighting factors and ranges would have been quite different without the interactions within the meetings.

- Again, there were too many guesses; in some cases the guesses resulted from entirely uninformed opinions. One way to test the process is to select a few cases in which reviewer input was used in lieu of code calculations. A code calculation could be performed for some issues such as pressure increments, and the results could be compared to the weighting factors assigned without benefit of the calculations. This could provide some level of confidence that the process works.

- Another potential problem with the process involved the selection of the rainges for the possible outcomes associated with each issue. The SARRP analysts provided ranges which very heavily influenced the reviewer input. If it were possible, the discussion of the issue should have been used by a third party to develop a range based on what people had said. The discussion at the meeting appears to be a better source of information than the voting outcomes which indicated less uncertainty. Once again, the process appeared to work toward agreement rather than a full display of uncertainty. Another good test would be to conduct a second vote for some issues after changing the ranges. There is a psychological factor involved in the process, since people tend to have preconceived notions of how their input should fit into a range that is supplied, and removal of levels at either end of the range would likely lead to different input, perhaps producing different interpretations and conclusions.

Reviewer \#8. Reviewer \#8 offered considerable praise to the SARRP analysts for implementing a difficult process, although he was concerned about the acceptance of the results by others.

- The results of this study must include uncertainty measures. The central estimates are misleading without the additional insight provided by the uncertainty analysis.

- The process used in the study is a good one, and the best available under the circumstances. This is an efficient way of including expert opinion. The SARRP analysts should be commended for their responsiveness in implementing the input of the group.

- There were, however, too many guesses on the part of the expert reviewers that were not based on any calculations. 
- The display of the results should include both a breakdown of the importance of the issues to measures of risk as well as to intermediate products, such as source terms and containment failure modes.

- This reviewer, along with others at the meeting, had some concerns about the methods used to link the consequence analysis to the rest of the analysis, particularly with respect to the treatment of waming times. It will require care to avoid losing information and perhaps overlooking some subtleties.

- Others have been promised that NUREG-1150 will provide a risk perspective to the new source term technology. However, it is a no-win situation for the following reasons:

- If the results, and particularly the uncertainty ranges, tell us that the new source term technology does not allow for any relaxation of regulatory requirements, there will be an outcry from those who believe that there has been considerable progress which is not demonstrated in the SARRP results.

- If the results and the uncertainty ranges both indicate that the risk is very low and some regulatory changes might be called for, they will be impossible to implement. None of the analysis performed would stand up under scrutiny. The input cannot be defended as having a sound basis, particularly when considering the health and safety of the public. Anyone who wants to make a case against the results or the process used to obtain them will be able to.

The results, therefore, will be criticized, and many of the criticisms will be valid. Reviewers will easily be able to see that the study was hurried, the important issues involved guesswork rather than analysis, and there was a somewhat incestuous relationship among reviewers, particularly since most of those providing input were basing it on the same sources.

- When asked about the viability of the process given the problems stated, Reviewer \#8 stated that the process was good given the constraints, but that it would be easier to be a critic than a defender. When asked to compare the output to that from the RSS, he stated that the source term information was clearly an advance, but that he didn't know if the probabilities were more viable.

Reviewer\#9. Reviewer \#9 had strong opinions on the characterization of the results:

- The activities of the LLH review team clearly do not define uncertainty. In fact, the request for input and the groundrules explicitly stated that it was not the goal to define uncertainty, and that was the reason for the approach using ranges and weighting factors selected for the study. There is no demonstration that this process has addressed uncertainty; rather, the sensitivity of key issues has been addressed within a framework that has severe limitations.

Reviewer \#10. Reviewer \#10 was not directly involved as an expert reviewer in the LLH process, but commented on the scope of the uncertainty analysis. He offered to supply additional written comments as well. 
- The LLH process cannot be characterized as an uncertainty analysis, since the range of input was limited. The LLH inputs do not really indicate the industry perspective, and it will be quite possible that results by other groups will fall outside the range allowed by the LLH study, making it difficult to call the LLH study an uncertainty analysis. Since issues tend to mix together, the actual risk calculations may be similar between the NRC analyses and those of the industry, and that may be sufficient. However, all of the inputs will likely be different, and there will be little agreement on the main issues. In some cases, the industry results were represented in the LLH ranges, but the weighting factors assigned caused the bounds of the range to be sampled too infrequently to be represented in the uncertainty. The LLH analysis is driven by the selection of the participants; hence, the output is no more than a sensitivity study. It was pointed out that the industry is doing its own analysis, and the result will be an examination of risk from two perspectives. The LLH study will have to be carefully described in terms of its limitations to ensure appropriate usage.

Reviewer \#11. Reviewer \#11 was unable to attend the meeting on April 3, 1986, but provided written comments at a later date. Those comments are reproduced here verbatim.

"In the SARRP report for Surry, I am listed as a member of the SARRP Containment Event Tree Review Group. Although I did participate and provide my numerical 'weighting factors,' additional discussion and explanations are required before my opinions can be properly evaluated. I believe that the study has provided many useful insights into risk analysis. Nothing that I write here should be construed to attack the excellent work done to diagnose many accident sequences, delineate the important phenomena, and to determine a defensible range for many parameters.

"My concern lies solely with the use of subjective probabilities/ weighting factors in narrowing ranges and providing numerical probability density functions (PDFs). The numbers and PDFs which I provided were, in most cases, merely guesses; the numerical 'weighting factors' cannot be defended in any scientific fashion in a quantitative sense, although they may have some value with respect to relative, but qualitative, comparisons. I believe that these qualifications also apply to the numerical probabilities supplied by others.

"I do not for a moment question the sincerity or integrity of the participants, organizers or sponsors of this work. I quarrel only with the use of Personal Opinion Probabilities (POPs) in the context of risk assessments.

"I believe that an important goal of the SARRP study is to provide estimates of risk uncertainties that are narrower than could be derived based on bounding analyses. In the absence of additional data, the.only way to achieve this goal is to poll experts for their opinions. However, I believe that uncertainty cannot be legitimately reduced by such a process.

"It is very difficult to arrive at the truth with a finite amount of data and a reasonable set of models. To provide numerical probabilities in the absence of data or validated models is an extremely risky proposition [1]. Although all the well-intentioned people involved in this process are as aware of its pitfalls as I am, they still participate. Why? 
"The primary justification is usually that decisions must be made now and we don't have time or money to do the necessary research. The statement, however, becomes a self-fulfilling prophecy. We may be spending more on POP polls today than we are on phenomenological research. Consider the following paradox. On one hand, the POP polls are justified because there is a need to make a decision now. On the other hand, to the best of my knowledge no decision based on POPs has ever been made; and if a decision were ever based on such a poll, the NRC would have difficulty defending it in any technical forum.

"I do not believe that regulatory decisions should be or need to be based on POP polls. If a decision must be made that requires additional knowledge, then that additional knowledge should be procured. Interim decisions can be based on any or all of the following:

(1) Existing regulations.

(2) Conservative calculations.

(3) Bounding calculations.

(4) POP values of 0.5 .

(5) Qualitative judgments of relative risk.

(6) Extrapolation of existing data bases, where appropriate.

(7) A strong desire to minimize high-consequence accidents, regardless of estimated probability.

(8) PRAs based on statistical data and validated models.

"The problem with using POPs is that they could be, and often are, completely wrong. That can lead to false complacency and increased risk, as may have happened with the Challenger and Chemobyl accidents.

"Because of the ambiguity of the definition of probability (or weighting factor), a POP poll does not distinguish between an expert's degree of belief that an event will (or will not) occur, or his guess at the frequency with which an event would occur if data were available. In the former case, the expert is providing his gambler's estimate of what will happen; in the latter case, he is providing his prediction or prophecy of the outcome of a future set of trials. Hence, a POP expert must assume either the role of gambler or prophet when he provides his subjective probability. In either of these roles, historical and scientific evidence [1] indicate that he will often greatly understate the true uncertainties. I believe that the SARRP study also underestimated uncertainties for this and the following reasons:

(1) Peer pressure. The subtle pressure of the group to force conformity. The effect of this force is evident in the obvious conformity in many POPs which are incompatible with the actual uncertainties.

(2) Latin Hypercube limitations. The limited staff time available limited the sample size, which would tend to narrow the uncertainty range. Also, for some issues, the range of levels was too wide, with the possible under- or over-stating of the importance of those issues. 
(3) Best-estimates. Experts were generally asked for their bestestimate points or PDFs, rather than uncertainty intervals or ranges of different PDFs. One of the objectives of the SARRP study was to 'provide a quantitative estimate of uncertainty that reflects a credible and realistic range in which the analysts have a reasonable confidence that the correct answer lies.' If I had been asked what my 'credible and realistic range' was, the numbers would have been much wider than the published numbers.

(4) Lack of a phenomenological understanding of some important processes.

(5) A wide variety of psychological factors such as subconscious desire to be perceived as a supporter of nuclear power, desire to appear knowledgeable, desire to maintain program funding, etc. [1].

"The SARRP investigators made a valiant attempt to produce a quantitative assessment of risk. However, I believe that that goal is impossible based on what we know today. The excellent work by the investigators, however, should not be wasted simply because of the reliance on indefensible POP polls. I would make the following suggestions to the investigators.

"In many cases, SARRP used existing data and models to establish technically defensible ranges for some parameters. The experts were then asked to narrow these ranges by providing point estimates or subjective PDFs. An alternative approach is possible. Assume that all PDFs are possible over the existing interval, ranging from a uniform distribution to a delta function at any point within the range. The first set of risk estimates could then be performed by assuming uniform distributions, or equal likelihoods. This would provide the central estimate (but not necessarily best estimate) of risk. Next, the study could explore the consequences of different assumptions. A sensitivity study could assume that parameters are forced to lie, e.g., within $1 / 3$ of the range (high, middle or low). The study would then determine the underlying assumptions or parameters which have the most influence on risk. This information would be highly valuable to the NRC in determining the research questions of greatest potential impact. Finally, bounding calculations (delta functions at the pessimistic and optimistic extremes) could be performed to see if these assumptions can dominate risk under certain conditions.

"The benefit of the above approach is that the space of all possible probabilities and PDFs would be explored. The study would be far less subjective since it would not involve either gambler's estimates or prophecies. The scientific validity of the study would depend only on the defense of the ranges employed, and the completeness of the analysis. Given a decent job on those two items, the study could not be shown to be wrong, nor could it mislead the NRC. The use of POP polls tends to reduce uncertainties, but without any technical justification. The suggested sensitivity study indicates where the major sensitivities lie, and where expert agreement should be achieved by generating solid evidence.

"I would like to leave the readers of the SARRP study with one final quote from Jean de la Bruyere (1645-1696): The exact contrary of what is generally believed is often the truth." " 


\section{Summary}

Most of the reviewers agreed that the uncertainty in the SARRP analyses riust be addressed and that the LLH method provided the best mechanism for doing this within the time and budget constraints of the program. Since the objective of the discussion was an airing of concerns about the approach, the specific comments are focused on potential problems in interpretation or implementation. Several reviewers also pointed out that the topic of uncertainty representation was so controversial that no method could be chosen that would not be subject to strong criticism.

As indicated above, there were two concerns most frequently voiced at the meeting:

(1) The LLH input process involved substantial guessing at parameters or outcomes that could have been avoided had more time been available.

(2) The results will need to be carefully characterized in terms of what they mean and what they do not mean. The semantics are very important. The group was split in their advice on this issue, however, since some wanted the results to be presented as a characterization of uncertainty, while others thought that they should be presented as the outcomes of sensitivity studies.

There were a number of specific concerns that were also raised as described in the comments of the individual reviewers. The group also endorsed a very careful presentation of the results that will help others understand the implications of the results on several levels.

\section{References for Appendix $F$}

1. Berman, M. "Vapor Explosions - Physics and Philosophy," presented at the Conference on High Temperature Chemistry, Wolfeboro, New Hampshire: July 23, 1986. 
-

-

•

-

- 
NUREG/CR-4551, VOL. 1: DRAFT REPORT FOR COMMENT (SEPTEMBER, 1986)

\section{Appendix G \\ DATA BASE FOR ESTIMATION OF COST AND PERSONNEL DOSE FOR PROPOSED MODIFICATIONS}


-

-

-

-

- 


\section{CONTENTS}

Section

G.1 COST INFORMATION NEEDS..........................................

G.2 SOURCES OF DATA AND THEIR GROUNDRULES........................G-3

G.2.1 Alternative Decay Heat Removal Study................................G-3

G.2.2 Filtered-Vented Containment Systems Study.............................G-3

G.2.3 Severe Accident Mitigation Systems Study...........................G-4

G.2.4 Plant-Specific Analyses in Support of the Decay Heat Removal Generic Issue Program......................................................

G.2.5 AIF Working Group.............................................6

G.2.6 EPRI Value/Impact Study.......................................... 6

G.2.7 Mitigation Systems for Mark II Reactors..............................G-7

G.2.8 Other Cost Data Sources................................................

G.3 USE OF THE DATA BASE FOR COST ESTIMATION.........................G-8

G.4 UNCERTAINTY IN COST ESTIMATION................................. G-8

G.5 RADIATION EXPOSURE ESTIMATION..................................G-11

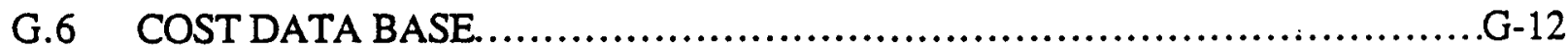

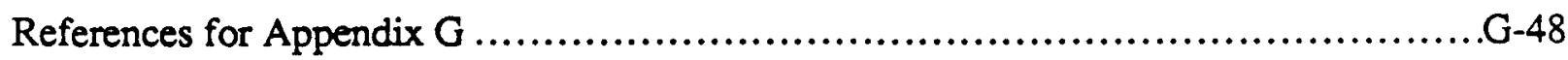


NUREG/CR-4551, VOL. 1: DRAFT REPORT FOR COMMENT (SEPTEMBER, 1986)

\section{LIST OF TABLES}

Table

Page

G-1 Radiation Exposure Data Base for Plant Modifications............................. 12

G-2 Summary of Cost Data Base.................................................... 14 


\section{Appendix G \\ DATA BASE FOR ESTIMATION OF COST AND PERSONNEL DOSE FOR PROPOSED MODIFICATIONS}

The analysis of risk reduction measures for the individual reference plants required the estimation of costs and personnel radiation exposure associated with proposed plant changes. Since the project schedule did not allow for detailed analysis of all the proposed modifications, previous studies that have generated this type of information were used as the source of information for options not specifically studied in SARRP. While this information is not precise due to the lack of specific design information for a specific plant, other uncertainties in the determination of value/impact measures are such that more accurate cost estimation would not change the overall conclusions. In any case, the costs and exposures developed for each option have been developed as a range, with upper, lower, and central estimates. Decisions regarding the viability and benefits of an option must consider the full range of costs as well as benefits.

\section{G.1 COST INFORMATION NEEDS}

Effective evaluation of the value/impact of proposed risk reduction measures requires as complete an estimation of total cost impact as possible. The total onetime cost associated with the option and the annual costs incurred as a result of operating or maintaining the plant after implementing the option are the two key parameters needed for comparison to averted costs associated with the risk reduction.

The total onetime cost estimate is intended to include the following:

- design and study costs

- components and materials

- owner's costs

- initial setup and testing

- taxes
- management and planning

- associated construction costs

- QA and other review

- regulatory interactions

- contingency

The sources of cost information generally treat the most significant of these cost elements, although format and assumptions vary.

The costs are also intended to account for the additional cost associated with backfit of existing facilities, since the reference plants are all operational units. Once again, the cost reference documents have information to help factor this element into the total. However, past 
experience indicates that there are also unforeseen consequences of a modification which can dramatically change the actual cost versus the original estimate. These circumstances are treated in the uncertainty estimation, as discussed in Section G.4.

Another important and potentially overriding cost consideration is that of replacement power if the modification involves additional downtime over the base case of an unmodified plant. This cost element is variable in magnitude depending on the exact circumstances at the utility at the time of the change. The state-regulated utility rate structure could also impact this element, since modifications that cause downtime may fall into a different category from those that do not. Many previous studies have not included this element because it is difficult to predict and because it is politically driven. In general, the schedule was reviewed to determine if the change could be completed within a normal outage. The possibility of extending the implementation schedule to prevent downtime specifically for the modification is also cited as a reason for not including downtime costs.

In spite of these factors, for many of the major modifications it is probably unreasonable to expect that the change could be made with no impact on the overall plant schedule. Even if a modification schedule indicates that it could be completed within a shutdown period, these estimates do not include the full perspective of the utility and its limitations in managing all other activities that are a part of a normal shutdown. Realistic consideration of the modification integrated with all other plant activities would probably result in the inclusion of some replacement power costs for the larger modifications.

The other cost that is difficult to generically assess is that related to financing and interest charges. The specific utility, the magnitude of the project, and the motivation for implementing a change could all impact the assessment of interest costs. Some of the cost reference sources have included some interest charges, but overall the costs associated with financing the modification are not clearly delineated.

In order to efficiently evaluate the value/impact of many proposed modifications, the estimates of costs were made on a fairly general basis without consideration of the details of implementation at the reference plant. The cost impacts were evaluated as follows:

1. The costs were estimated based on applicable information from other studies, excluding replacement power and specific calculation of interest expense and financing alternatives.

2. A range was developed by establishing high and low estimates as described in Section G.4.

3. The value/impact analysis was completed based on this information. 
NUREG/CR-4551, VOL 1: DRAFT REPORT FOR COMMENT (SEPTEMBER, 1986)

4. For any modifications that appeared to have a net benefit, the costs were reevaluated and the other costs such as replacement power and interest were considered. The specific cases requiring more detailed cost evaluation are documented in the individual appendices for each reference plant.

\section{G.2 SOURCES OF DATA AND THEIR GROUNDRULES}

Each of the references had specific methods for the development of cost information. When using the data from separate sources it is necessary to understand these methods and their limitations, particularly when trying to obtain estimates for individual cost elements rather than the total. This section outlines the important groundrules for each of the major references. The individual data listings in Section G.7 also include notes applicable to the specific entries.

\section{G.2.1 Alternative Decay Heat Removal Study}

NUREG/CR-2883 includes cost information for three potential alternative decay heat removal systems [1]. These concepts were evaluated in detail for a variety of different types of reactors, although the cost information extracted as part of this effort was based on one plant for each alternative, since the design differences between plants did not lead to significant cost differentials. The key elements of the cost estimates are summarized below:

- The costs are based on the Richardson Estimating Manual [2] and Burns and Roe in-house data. Vendor estimates were obtained for major equipment such as diesel generators.

- The estimates were normalized to fourth quarter 1981 dollars.

- A $25 \%$ contingency is included in all elements.

- Outage times were estimated based on rough schedules and estimates of 60 person crews working 40 -hour weeks.

- Site-to-site differences were included but were found to be negligible.

- Direct construction costs were estimated. Other costs, including escalation, interest during construction and owner's costs were figured roughly as a constant $45 \%$ of construction costs.

\section{G.2.2 Eiltered-Vented Containment Systems Study}

The information in NUREG/CR-4065 (draft) [3] was used as a primary source of information for costs associated with proposed concepts of accident mitigation through containment pressure control. This document includes previous estimates from other studies and also includes new cost information for alternative backfit options designed specifically for 
NUREG/CR-4551, VOL. 1: DRAFT REPORT FOR COMMENT (SEPTEMBER, 1986)

a Mark I containment. These specific estimates, by Holmes and Narver, have the following characteristics:

- Different seismic design levels were considered and it was found that the difference in costs between Seismic Categories I and II were negligible.

- A plant downtime of 5 to 15 days was assumed reasonable, at a cost of one million dollars per day. This is kept as a separate item for this study.

- The cost estimates apply for a retrofit or for a new plant.

- A $15 \%$ contingency is included.

- Owner's costs (management, QA, regulatory interaction, health physics, retraining, etc.) are not included and there is no allowance for first-of-a-kind costs.

\section{G.2.3 Severe_Accident_Mitigation_Systems_Study}

A number of proposed mitigation systems were evaluated specifically in conjunction with SARRP [4]. Three mitigation alternatives involving four different systems were evaluated for three different reactor types. For each option, estimates on only one plant were included in the data base since the geographic and plant-to-plant differences were small. The following methods were used in the estimation process:

- Costs were based on conceptual designs, and included evaluation of building construction, electrical loading and other details needed for a fairly accurate representation of costs.

- Labor estimates included consideration of location of work, access difficulty, temporary rigging, etc.

- Typical estimating contingencies were included in the totals.

- Owner's costs including administration, health physics, and others were included.

- No escalation was included due to the lack of a specific implementation schedule and the great degree of uncertainty in escalation rates.

- Interest is not directly included due to the many different options for financing. It was suggested that interest could be estimated as one year's interest on the total onetime cost at the existing interest rate.

- No replacement power costs are included. The work could be scheduled within one shutdown, but it should be recognized that this could require over 300 workers due to health physics requirements.

- The error band on costs, excluding the items that were not included such as replacement power costs, is estimated to be $33 \%$, plus or minus. 
NUREG/CR-4551, VOL 1: DRAFT REPORT FOR COMMENT (SEPTEMBER, 1986)

- Other costs such as equipment qualification could be incurred, depending on the exact regulatory requirements for these add-on systems. Qualification costs could be significant; for hydrogen igniters alone it is estimated to be $\$ 500,000$.

- Operation and maintenance costs were roughly estimated to be $\$ 150,000$ per year. This was judgmentally split among the systems within an alternative.

\section{G.2.4 Plant-Specific Analyses in Support of the Decay Heat Removal Generic Issue Program}

Preliminary estimates of plant impacts, including costs, have been developed in support of the Decay Heat Removal Program (TAP A-45) [5,6]. These cost estimates are for very specific modifications, grouped into alternatives, and then evaluated. Because the modifications are plant-specific and based on a review of accident sequence cut sets, this was one of the most useful sources of information for the the SARRP risk reduction evaluation.

The following characteristics apply to all of the cost information derived from this source:

- The design concepts were developed and supplemented with a site visit to determine plant-specific design requirements that could impact cost.

- The generic costs (rather than the costs adjusted for local labor and materials rates) were used in the data base.

- The estimates were developed with methods similar to those provided in the handbook for nuclear plant cost estimating [7]. The costs incurred by the government in implementing a regulatory requirement were not included.

- Owner's costs were estimated as $10 \%$ of the total for the modification. This is intended to include retraining, engineering support, interaction with regulatory agencies, health physics, contract management, and quality assurance activities. It is recognized that this is very dependent on the specific utility. The groundrule employed in this reference was that the entire modification would be contracted out and that the utility would therefore serve a minimal role as contract manager and in roles required by the regulator.

- A $25 \%$ contingency was included in the total estimate.

- The reference used an inflation rate of $6 \%$, a discount rate of $10.5 \%$, and a fixed charge rate of $17 \%$. None of these factors are included in the total onetime costs reported in the data base.

- The design and management allocation in the data base includes the costs associated with the contractors efforts, rather than the utility costs which are included in the owner's costs category.

- The category in the data base labeled "other" includes both the $25 \%$ contingency, and the temporary construction costs associated with the actual implementation of the modification. This category includes tests and startup costs. 
- The generic costs used in the data base are derived from the "Energy Economic Data Base"[8].

- The labor costs from Reference 8 were adjusted to reflect conditions associated with backfit costs.

- The operations and maintenance costs associated with an alternative were divided among the modifications within the alternative based on the qualitative information provided in the report. This was reported as the total recurring costs, based on 1985 dollars. A more accurate representation of the recurring costs would include a levelization factor, but this would be dependent on the individual plant and its remaining life.

- All costs are based on last quarter 1984 dollars.

\section{G.2.5 AIF Working Group}

In February of 1980 , a working group was formed under the auspices of the Atomic Industrial Forum (AIF) to estimate the total industry costs associated with the implementation of plant modifications associated with the draft of the post-Three Mile Island NRC Action Plan-NUREG-0660 [2]. The report of this working group included information relevant to the types of modifications included in SARRP [10]. The basic groundrules used in developing their estimates of cost are discussed below.

- The NRC-proposed modifications were defined more specifically and in some cases altered to reflect a more effective change. The brief description in the data base entry includes the basic scope of the modification being considered.

- The costs were estimated on a yearly basis for a schedule developed for each change. These were summed for the total onetime cost reported in the data base.

- Indirect costs, including owner's costs and Allowance for Funds Used During Construction (AFUDC) were also estimated. These were added to the total onetime costs in the data base. This results in a slight difference from most of the other sources of data since factors such as AFUDC were usually excluded. In this case these costs could not be separated from owner's costs. In any case, this indirect cost element is typically less that $10 \%$ of the total estimated cost.

- The costs were estimated on an industry-wide basis. The total cost had to be divided by the number of units affected to obtain the per-unit cost.

- The costs are based on the 1980 dollar.

\section{G.2.6 EPRI Value/Impact Study}

The Electric Power Research Institute (EPRD) sponsored a study of the value/impact of selected modifications as reported in Reference [11]. Each modification was defined in scope and evaluated using a form that detailed a cost breakdown. The approach used was intended to 
provide a onetime cost estimate, and did not include inflation, interest and other factors that have been extracted from other sources. The report includes study cost to refine the design concept, as well as the actual costs associated with the specific design activity. Specific owner's costs, including documentation, design review, quality assurance, and licensing approval were-also estimated and are reported in the data base under owner's costs. Plant outage time was also estimated, although this is not used directly in the first-cut SARRP analysis of value/impact. The reference also includes a listing of the assumptions associated with each of the specific modifications considered.

\section{G.2.7 Mitigation_Systems for Mark II_Reactors}

A series of reports by R \& D Associates summarizes research into options intended to reduce plant risk, principally through mitigation of accidents and limitation of the total containment loading. One of these reports, Reference 12, includes fairly detailed cost estimates. These estimates are included in the cost records and although the options were intended for a specific reactor design, they were only roughly designed and the estimates could therefore apply more broadly. The basic groundrules used in developing the costs are discussed below.

- The options were designed in modules such that some of the records describe pieces of systems that would have to be combined with others to fulfill the design objective.

- Inflation during construction was treated by escalating all costs by $4 \%$.

- A $25 \%$ contingency is included in all estimates.

- There was no factor to account for this being a nuclear plant project: standard construction cost factors were used.

- The costs are based on the mid-1984 dollar.

In general, a review of the costs from this source indicates that the estimates appear quite low for a number of the larger systems proposed. For example, the system listed in record 39 describes an independent and redundant heat rejection system in its own building. This option is estimated to cost very significantly less than the addition of an independent containment heat removal system or a single train of diesel cooling water (see records 49 and 50). Specific line-item comparisons with estimates from other sources also indicate similar differences. Several of the other options involve tunneling under an existing plant's basemat or extensive work in the reactor cavity: options industry spokespersons have refused to even consider based on lack of feasibility. It should be noted that Reference 12 does suggest that there might be feasibility problems with several of the options proposed. Finally, the fact that 
the costs do not include specific consideration of owner's costs and the cost premium associated with nuclear plant work also appears to be a severe limitation when the details of other estimates that include these costs are compared to those generated in this reference. The cost estimates derived from this source were used very carefully in this study due to these limitations.

\section{G.2.8 Qther Cost Data Sources}

There are other sources referenced in the specific data listings in Section G.6. In general, other sources were used to obtain cost information on a limited number of modifications, and a detailed understanding of the methodology used is not necessary. The cost data was extracted in a format similar to that used for the other sources, and the individual variation does not represent a major source of uncertainty.

\section{G.3 USE OF THE DATA BASE FOR COST ESTIMATION}

Several of the modifications considered are directly comparable to specific entries in the data base. For these modifications, the data in the cost data base was used directly as the central estimate of the cost for the option. For the cases where there was more than one data point that directly applied, engineering judgment was used to select the cost estimate that appeared to be most relevant, most complete, and from the best-documented source.

In many other cases, the modifications proposed for a specific risk reduction option were very plant specific and had not been evaluated in previous studies. These cases required more liberal use of engineering judgment, drawing from as many of the individual data entries as possible. The costs detailed in Section G.6 include estimates of line item costs for all sources that included that information. Thus there was available some cost information for most types of mechanical and electrical modifications that were proposed. There are also some entries in the data base for items such as procedural or staffing changes, or changes in information systems available to the operator.

The use of the data included in this appendix as a source of cost information is detailed in Appendix E. Appendix E references the individual data base entries provided here that were used in the estimates for Surry.

\section{G.4 UNCERTAINTY IN COST ESTIMATION}

The individual data entries only include point estimates of costs for the proposed modifications. The value/impact analysis performed for each reference plant includes consideration of the uncertainty associated with each measure of value and impact, although 
this consideration of uncertainty is not propagated in a rigorous statistical sense. The uncertainty associated with costs of modifications has been evaluated in a manner similar to the other inputs in the study: three values are used to characterize the range of costs that are associated with each option. As stated above, the central estimate was obtained by using appropriate data to obtain a realistic best estimate, or central estimate, for the proposed modification.

The estimates of the upper and lower estimates which represent a reasonable range of costs that might be expected in actual implementation of the option are also included, but these have been generated from the central estimate in the same manner for all options, rather than individually for each option. This was done because it was judged that the variation in costs would be expected to be driven by forces that are not specific to a particular option. Other factors such as regulatory interaction, utility-specific management capabilities and local variation in costs would apply similarly to all options. There is also uncertainty that would be specific to the options, for example, some options might result in costs over the estimate due to lack of room to efficiently place equipment, while other options such as a circuitry change would be less likely to have that type of uncertainty. However, the estimates used in this study are not detailed enough to allow a direct determination of these types of costs, and the overall uncertainty can be estimated using a set of rules that apply to all cost estimates.

Some of the references included cost uncertainty information. The uncertainty information derived from these sources is summarized below. Reference 4 reported an error band on costs of $+1-33 \%$, with no detailed supporting information.

Reference 10, the AIF study, included some uncertainty information in the form of histograms that were used to illustrate probabilities that the total cost fell between certain values. Most of the modifications examined were different from those proposed here and only limited uncertainty information was presented. Three uncertainty ranges that were presented appear to apply to the modifications considered in SARRP:

- $\$ 0.5 \mathrm{M}+/-\$ 0.2 \mathrm{M}$, with no definition of the confidence interval; a $10 \%$ to $90 \%$ spread would appear to reasonable;

- $\$ 5 \mathrm{M}$ with a $10 \%-90 \%$ confidence range of $\$ 3 \mathrm{M}$ to $\$ 11 \mathrm{M}$; and,

- $\$ 4 \mathrm{M}$ with a $95 \%$ confidence limit of $8 \mathrm{M}$

This limited information would seem to indicate that a reasonable upper estimate is approximately a factor of two above the central, while a lower estimate would be a factor of two below the central. 
The EPRI study [11] included upper and lower estimates for each cost element of each alternative, and these were summed to obtain upper and lower estimates for the alternative as a whole. There were no details given as to the statistical meaning of the estimates, but it would appear that they would be analogous to the upper and lower parameters used in the SARRP program. The uncertainty information from this source is summarized below:

$\begin{array}{ccc}\text { Central } & \text { Upper } & \text { Lower } \\ \$ 42,000 & \$ 60,000 & \$ 28,000 \\ \$ 37,000 & \$ 51,000 & \$ 24,000 \\ \$ 140,000 & \$ 180,000 & \$ 90,000 \\ \$ 100,000 & \$ 140,000 & \$ 70,000 \\ \$ 245,000 & \$ 360,000 & \$ 160,000\end{array}$

As illustrated in the information above, this reference would indicate that the upper estimate is roughly $35 \%$ above the central and the lower estimate is about $35 \%$ below the central estimate. Note that all of these options had relatively low total costs.

The only other source of information relative to uncertainty in costs is discussed in Reference 6. This source points out the weakness in the uncertainties reported in this section so far--they are not based on a before and after study of actual implementation at a utility. Although the data in Reference 6 is limited, it does appear to confirm conventional wisdom that it is unlikely that a nuclear plant retrofit will cost less than the original estimate and that there have been numerous cases in which the final cost was an order of magnitude or more greater than the original estimate. In many of the cases where the final costs were drastically different from the estimate, other factors, such as regulatory interaction or continuous scope increases relative to the original modification dominated the increase in cost. Nevertheless, it would appear to be unreasonable to expect the modifications proposed in this program to be immune from these typical problems encountered in nuclear plant backfit work. Thus it is believed that the upper estimates should reflect the character of actual backfit work, rather than just the uncertainty in estimating practices.

Engineering judgment based on all of the information presented above was used to develop the following uncertainty information for the cost estimates used in SARRP:

High Estimate--A factor of 2 times the central estimate

Low Estimate--A factor of 0.7 times the central estimate.

This formula was not always used, since some of the options were judged to have particular characteristics which would affect the uncertainty representation. 
NUREG/CR-4551, VOL 1: DRAFT REPORT FOR COMMENT (SEPTEMBER, 1986)

In order to clarify the use of uncertainty for the cost estimates, the parameters used are defined below:

- The central estimate is a best estimate of cost based on standard estimating practices

- The lower estimate reflects the variability in estimating techniques and the uncertainty associated with the use of the data in Section G.7 to represent different types of modifications

- The upper estimate reflects the uncertainty in estimating as well as the observation that a large history of nuclear plant backfit experience has shown that there have been large cost overruns associated with many backfit projects. As with the lower and central, the upper estimate does not include consideration of the either the replacement power costs or the interest charges that could accrue for modifications that were delayed or resulted in large plant downtime. The upper estimate is also intended to be concerned with the modification as defined, and not as altered to incorporate additional changes in scope.

As noted previously, the value/impact analysis is first carried out assuming that the project could be scheduled to avoid any downtime associated with the modification. It is known that this is optimistic for some of the large options, or those that involve significant containment work. A more accurate assessment of total cost was performed for all cases in which the range associated with the onetime estimate excluding replacement power and interest charges indicated a potentially beneficial status for the modification.

\section{G.5 RADIATION EXPOSURE ESTIMATION}

An accurate estimation of worker radiation exposure associated with each option is difficult without more specific planning of the proposed changes. The exposure is dependent on the nature of the modification, the location of the work within the individual plant, the scheduling of the entire job in terms of crew size and personnel availability, shielding measures taken during the construction phase and many other factors. Some of the references used for cost information did include estimates of radiation exposure. The insights derived from these sources are summarized in Table G-1.

As indicated in the table, there is limited data available, and the data that is available varies widely. This would be expected since the radiation exposure associated with the implementation of modifications would be expected to be entirely plant specific. In addition, the dose that the workers receive is determined by the actual day-to-day working environment, and probably cannot be estimated without detailed planning of the construction activity.

Radiation doses were estimated for the SARRP analysis based primarily on engineering judgment. The factors considered in this estimation are discussed in Appendix $\mathrm{E}$. A range of 
potential doses was estimated, using the same upper, central and low parameters that were used for the costs. The ranges are fairly wide due to the considerations discussed above. It should be noted that the costs associated with the impact of a radiation environment on task planning and performance were included in several of the cost estimates in the data base.

Table G-1

RADIATION EXPOSURE BASE FOR PLANT MODIFICATIONS

\begin{tabular}{lrl}
\hline \multicolumn{1}{c}{ Modification } & \multicolumn{2}{c}{$\begin{array}{l}\text { Radiation Dose } \\
\text { (Person-Rems) }\end{array}$} \\
\hline Add-on Containment Spray \& Recirculation & 360 & PWR \\
& 180 & Ice Condenser \\
Add-on Spray System, No Recirculation & 100 & BWR \\
& 150 & PWR \\
Addition of a Hydrogen Igniter System & 100 & BWR \\
& 560 & PWR \\
Backup Air Pressure for PORVs & 620 & Ice Condenser \\
Closed-Loop Containment Copling System & 450 & BWR \\
& 14 & \\
Independent Decay Heat Removal System [5] & 195 & PWR \\
Independent Decay Heat Removal System [6] & 150 & Ice Condenser \\
Redundant RHR Pump Cooler Outlet Valve & 120 & BWR \\
& 486 & \\
\hline
\end{tabular}

\section{G.6 COST DATA BASE}

The data from the various sources is summarized on the pages that follow. Table G-2 provides a summary of the more detailed data records that follow. Because this information is stored in a computerized data base for efficient retrieval and updating, each entry has the same format. Each data field was filled as completely as possible based on the information in the reference. The proposed modifications are divided into preventative options (those intended to reduce core melt frequency) and mitigative (those that reduce the consequences of an accident). 
These groupings are not always unique, particularly for the BWRs, since some options perform both functions. The comments on the data entries provide additional detail that can be used with the groundrules in Section G.2. The value reported as "Total Onetime Cost" includes all the onetime costs irrespective of interest and replacement power costs. As described earlier, these two items depend on the individual circumstances at the time of the modification and are not included in the first-cut cost estimate. 
Table G-2

SUMMARY OF COST DATA BASE

\begin{tabular}{|c|c|c|c|}
\hline Modification & Type & $\begin{array}{c}\text { Onetime } \\
\text { Cost }\end{array}$ & $\begin{array}{l}\text { Recurring } \\
\text { Costs }\end{array}$ \\
\hline 1. RWST Level Alarm Improvements & Prev. & $\$ 29,000$ & \\
\hline 2. Automate ADS Function & Prev. & $\$ 32,000$ & \\
\hline 3. Isolation Condenser Isolation Logic & Mit. & $\$ 35,300$ & \\
\hline 4. Auto Isolation of Isolation Condenser & Mit. & $\$ 36,480$ & \\
\hline 5. Install HPCI Time Delay & Prev. & $\$ 36,620$ & \\
\hline 6. Redundant RHR Pump Cooler Outlet Valve & Prev. & $\$ 38,000$ & \\
\hline 7. Install Diesel-Driven Service Water Pump & Prev. & $\$ 38,800$ & \\
\hline 8. Separation of RCIC/HPCI Actuation Levels & Prev. & $\$ 42,000$ & \\
\hline 9. Modify RCIC for Auto Restart & Prev. & $\$ 50,300$ & \\
\hline 10. Automatic Closure of PORV Block Valves & Prev. & $\$ 80,000$ & \\
\hline 11. Install Auto Restart of Injection Systems & Prev. & $\$ 81,060$ & \\
\hline 12. Diesel Intake/Exhaust Modification (106) & Prev. & $\$ 84,000$ & \\
\hline 13. AFW Reliability Study & Prev. & $\$ 100,000$ & \\
\hline 14. Connect Isolation Condenser Vent to Stack & Mit. & $\$ 135,000$ & \\
\hline 15. Wall Separating Service Water Pumps (109) & Prev. & $\$ 184,000$ & \\
\hline 16. Install New Service Water Train & Prev. & $\$ 246,000$ & \\
\hline 17. Backup Air Pressure for PORVs (508) & Prev. & $\$ 285,000$ & $\$ 3,000$ \\
\hline 18. Install Auto Transfer for ECCS Dc Bus (304) & Prev. & $\$ 330,000$ & $\$ 2,000$ \\
\hline 19. Electrical Equipment Seismic Anchorage (107) & Prev. & $\$ 334,800$ & \\
\hline 20. New Radiation Monitors & Mit. & $\$ 345,000$ & $\$ 10,000$ \\
\hline 21. Dedicated Battery for Diesel Starting (302) & Prev. & $\$ 361,000$ & $\$ 1,000$ \\
\hline 22. Add a Reactor Operator & Prev & $\$ 438,000$ & \\
\hline 23. Automatic Venting System & Mit. & $\$ 480,000$ & \\
\hline 24. New Drywell Spray System, Internal & Mit. & $\$ 515,000$ & \\
\hline 25. Increase Control Room Staff & Prev. & $\$ 600,000$ & \\
\hline 26. Connection of New Cooling to AFW pump & Prev. & $\$ 771,000$ & $\$ 8,000$ \\
\hline 27. Dedicated Diesel Generator Batteries (302) & Prev. & $\$ 786,000$ & $\$ 7,000$ \\
\hline 28. New Drywell Spray System, External & Mit. & $\$ 860,000$ & \\
\hline 29. Containment Vent Line & Mit. & $\$ 1,000,000$ & \\
\hline 30 Fire Detectors and Suppression Equipment & Prev. & $\$ 1,052,000$ & $\$ 6,500$ \\
\hline 31. Station Backup Batteries & Prev. & $\$ 1,180,000$ & $\$ 6,500$ \\
\hline
\end{tabular}


Table G-2 (Continued)

\begin{tabular}{|c|c|c|c|}
\hline Modification & Type & $\begin{array}{c}\text { Onetime } \\
\text { Cost }\end{array}$ & $\begin{array}{c}\text { Recurring } \\
\text { Costs }\end{array}$ \\
\hline 32. Separation of Dc Power Supplies (311) & Prev. & $\$ 1,287,000$ & \\
\hline 33. Improvement of Aux Building Ventilation & Mit. & $\$ 1,700,000$ & $\$ 10,000$ \\
\hline 34. Plant Stack Vent Anchorage (107) & Prev. & $\$ 1,714,000$ & \\
\hline 35. Containment Pressure Relief & Prev. & $\$ 1,728,000$ & \\
\hline 36. AFW Pump Room Fire Protection (810) & Prev. & $\$ 1,882,000$ & $\$ 7,000$ \\
\hline 37. Radiological Monitoring of Effluents & Mit. & $\$ 2,300,000$ & $\$ 35,000$ \\
\hline 38. Gas Combustor System & Mit. & $\$ 2,600,000$ & \\
\hline 39. Independent Containment Heat Removal & Prev/Mit & $\$ 2,770,000$ & \\
\hline 40. Low-Flow Vent Filter & Mit & $\$ 2,785,000$ & \\
\hline 41. Core Redistribution System & Mit & $\$ 3,330,000$ & \\
\hline 42. Core Retention and Cooling System & Mit & $\$ 3,445,000$ & \\
\hline 43. Addition of Two Hydrogen Recombiners & Mit & $\$ 3,572,000$ & \\
\hline 44. Add-on Spray System, No Recirculation & Mit. & $\$ 3,680,000$ & $\$ 50,000$ \\
\hline 45. Safety Parameter Display System & Prev. & $\$ 4,100,000$ & $\$ 25,000$ \\
\hline 46. High-Flow Vent Filter & Mit & $\$ 4,242,000$ & \\
\hline 47. Injection Suction Line From Fuel Pool (806) & Prev. & $\$ 4,870,000$ & $\$ 1,000$ \\
\hline 48. Safety System Status Monitor & Prev. & $\$ 6,000,000$ & $\$ 50,000$ \\
\hline 49. New Diesel Cooling Water Pump (803) & Prev. & $\$ 6,111,000$ & $\$ 6,000$ \\
\hline 50. Closed-Loop Containment Cooling System & Mit. & $\$ 7,360,000$ & $\$ 80,000$ \\
\hline 51. Addition of Hydrogen Igniter System & Mit. & $\$ 7,580,000$ & $\$ 50,000$ \\
\hline 52. Add-on Containment Spray \& Recirculation & Mit. & $\$ 9,130,000$ & $\$ 100,000$ \\
\hline 53. Filtered-Vent System & Mit. & $\$ 13,000,000$ & \\
\hline 54. Containment Vent and Filter & Mit. & $\$ 14,000,000$ & \\
\hline 55. Addition of a Fourth Diesel Generator (301) & Prev. & $\$ 16,000,000$ & $\$ 200,000$ \\
\hline 56. Add-on LPI and Suppression Pool Cooling & Prev. & $\$ 20,300,000$ & \\
\hline 57. Dry Crucible, Cooled Core Catcher & Mit & $\$ 20,500,000$ & \\
\hline 58. Add-on HPI for Feed and Bleed & Prev. & $\$ 26,800,000$ & \\
\hline 59. Add-on AFW and Primary Makeup & Prev. & $\$ 29,200,000$ & \\
\hline 60. High Efficiency Containment Vent \& Filter & Mit. & $\$ 33,000,000$ & \\
\hline 61. Independent Decay Heat Removal System & Prev. & $\$ 61,000,000$ & $\$ 585,000$ \\
\hline 62. Independent Decay Heat Removal System & Prev. & $\$ 65,000,000$ & $\$ 620,000$ \\
\hline 63. Independent DHR System (813) & Prev. & $\$ 77,550,000$ & $\$ 845,000$ \\
\hline
\end{tabular}


NUREG/CR-4551, VOL. 1: DRAFT REPORT FOR COMMENT (SEPTEMBER, 1986)

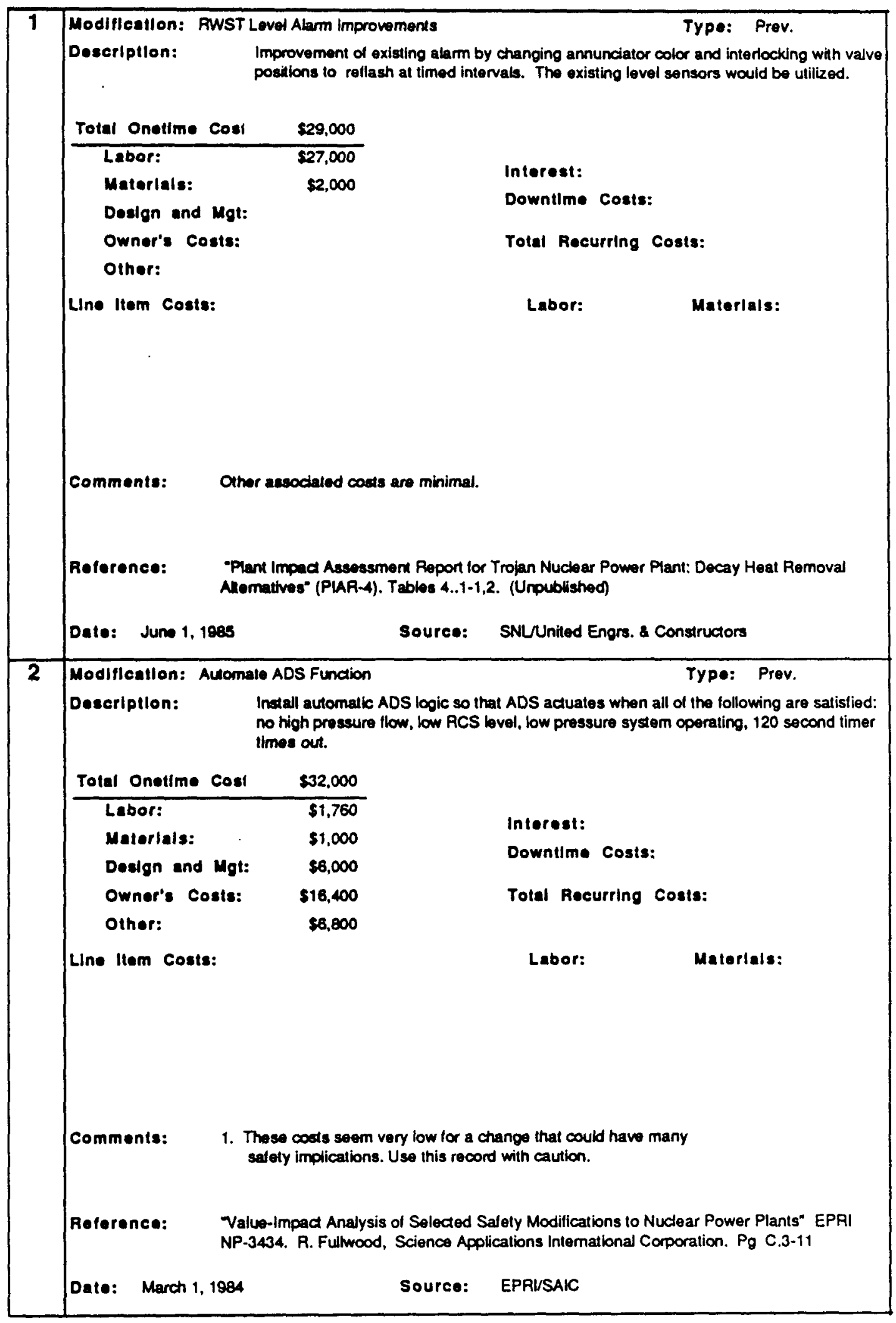




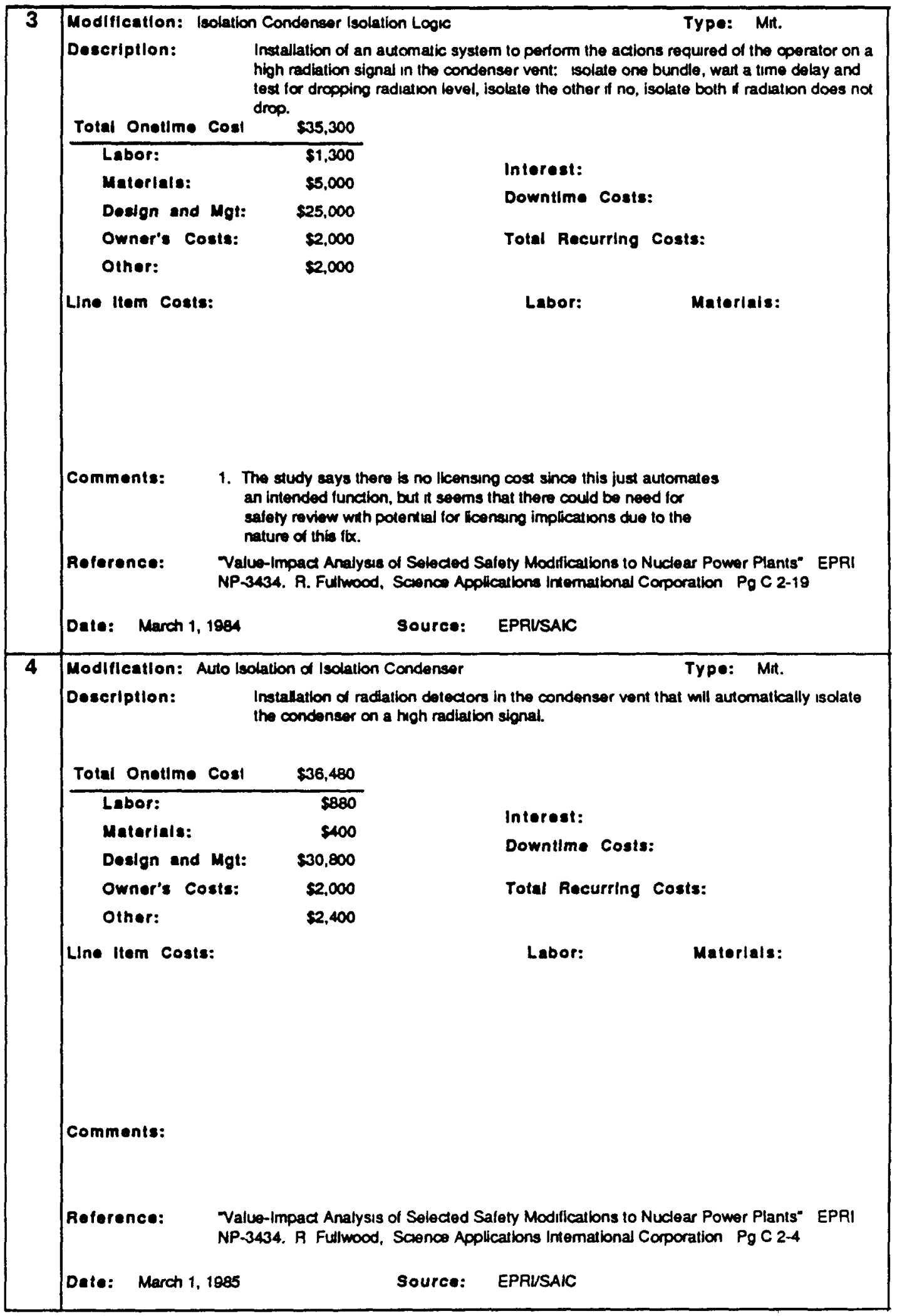


NUREG/CR-4551, VOL. 1: DRAFT REPORT FOR COMMENT (SEPTEMBER, 1986)

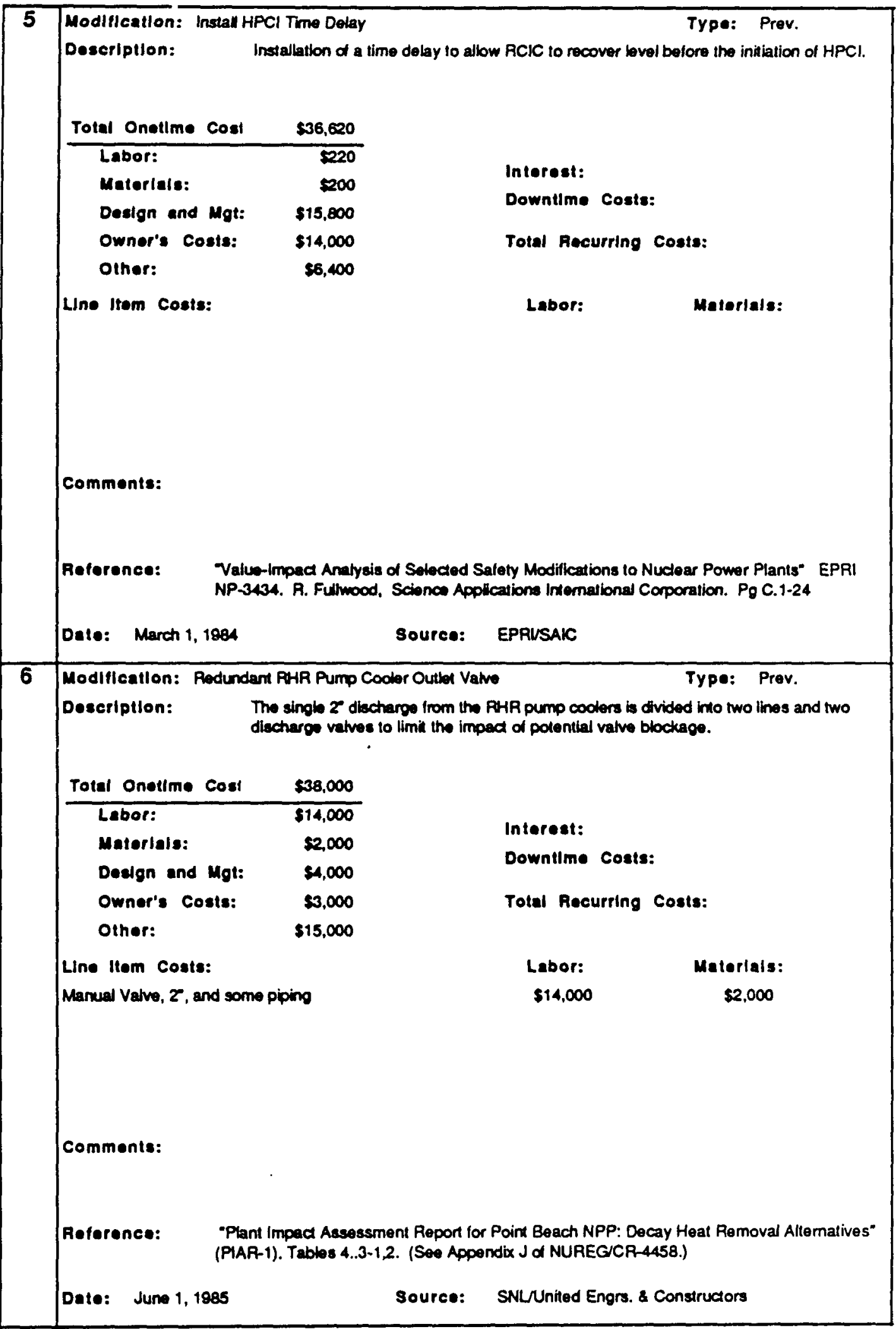


NUREG/CR-4551, VOL. 1: DRAFT REPORT FOR COMMENT (SEPTEMBER, 1986)

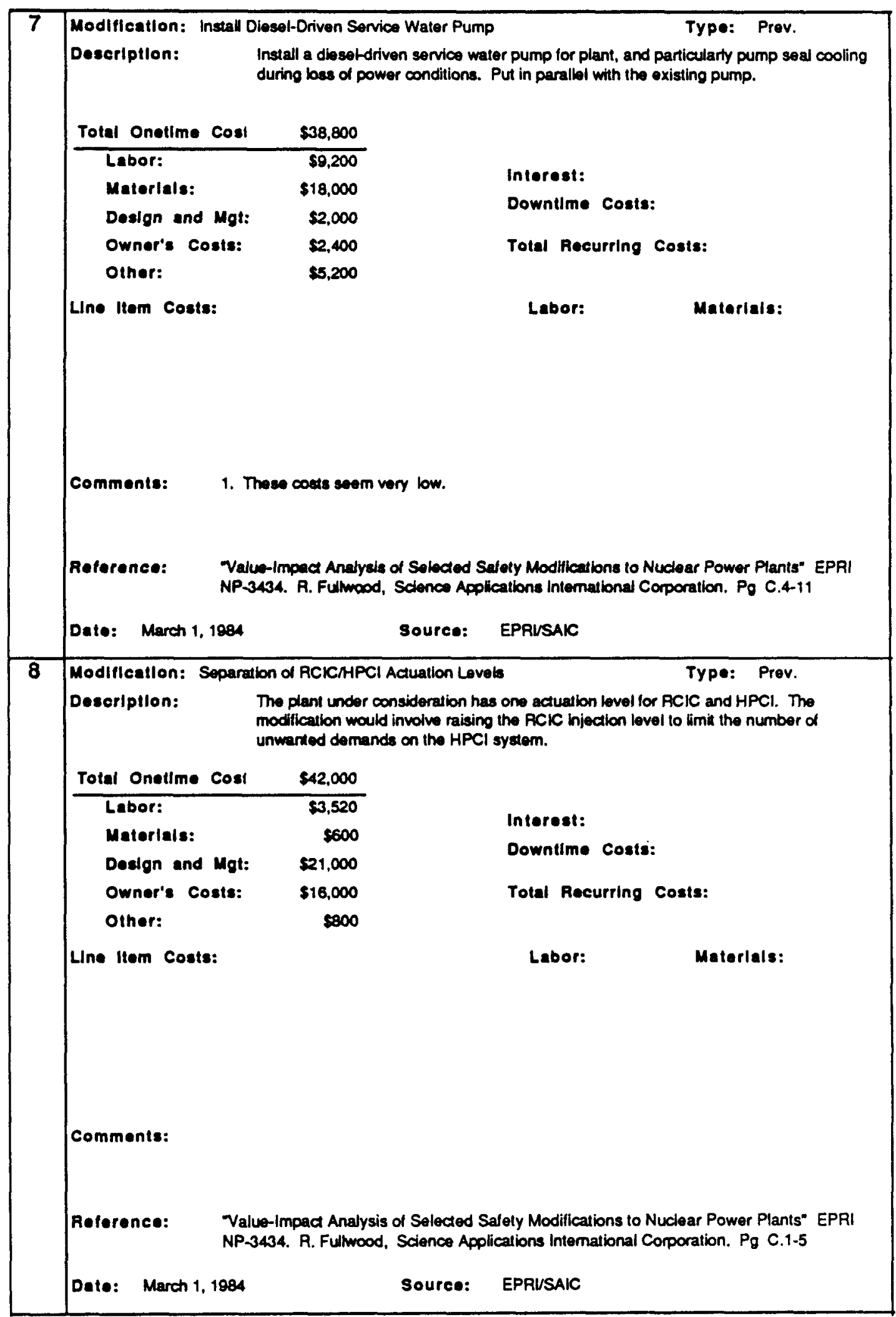


NUREG/CR-4551, VOL. 1: DRAFT REPORT FOR COMMENT (SEPTEMBER, 1986)

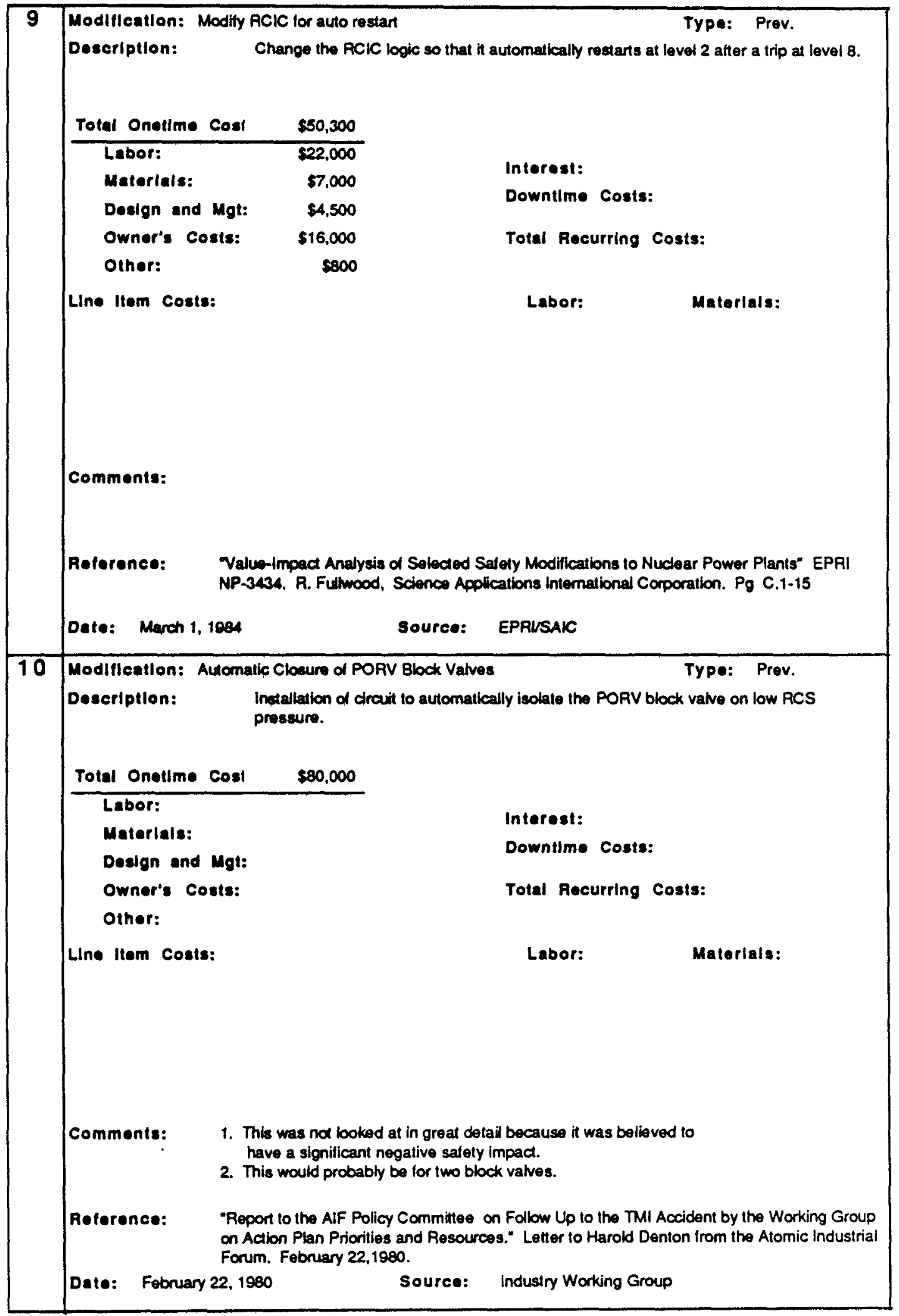




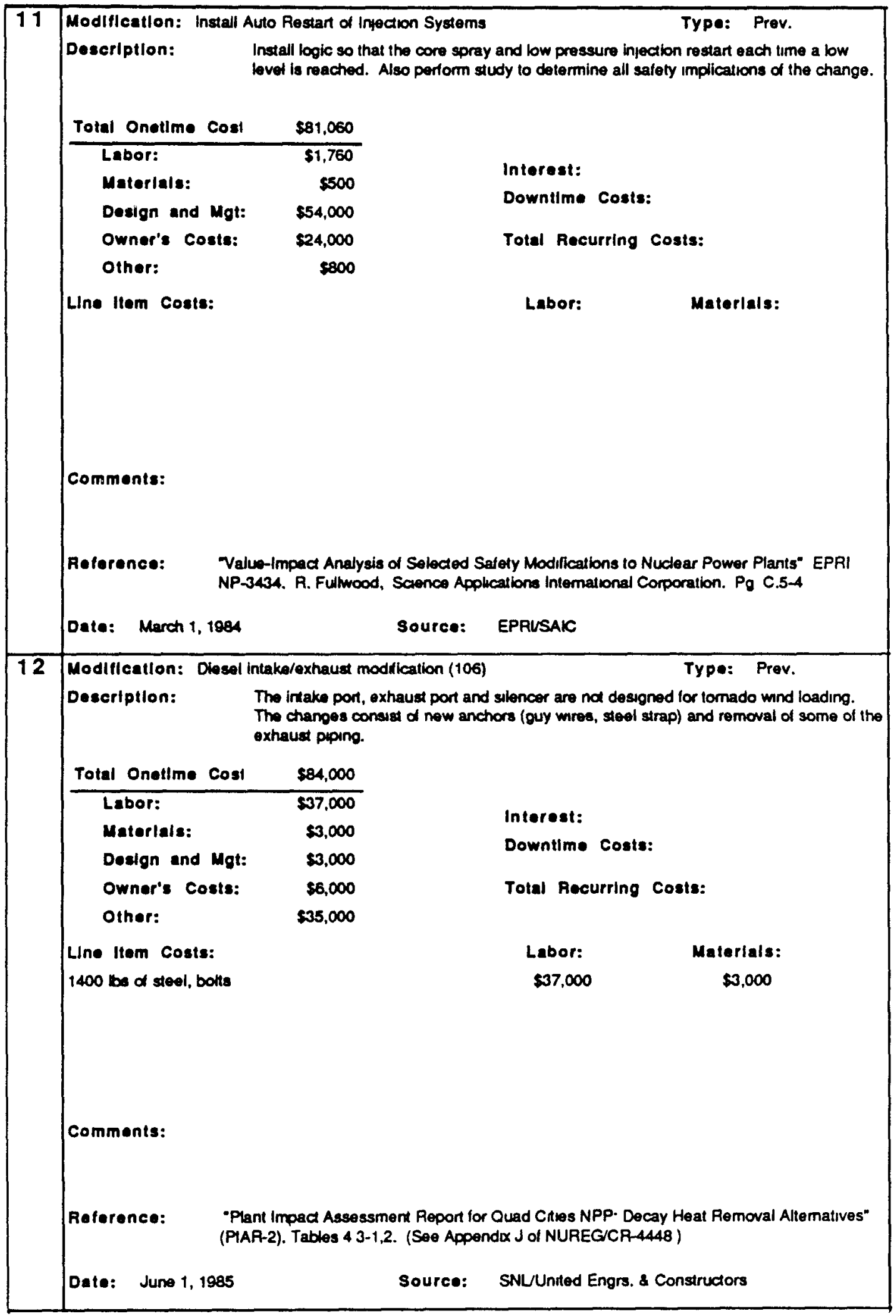


NUREG/CR-4551, VOL. 1: DRAFT REPORT FOR COMMENT (SEPTEMBER, 1986)

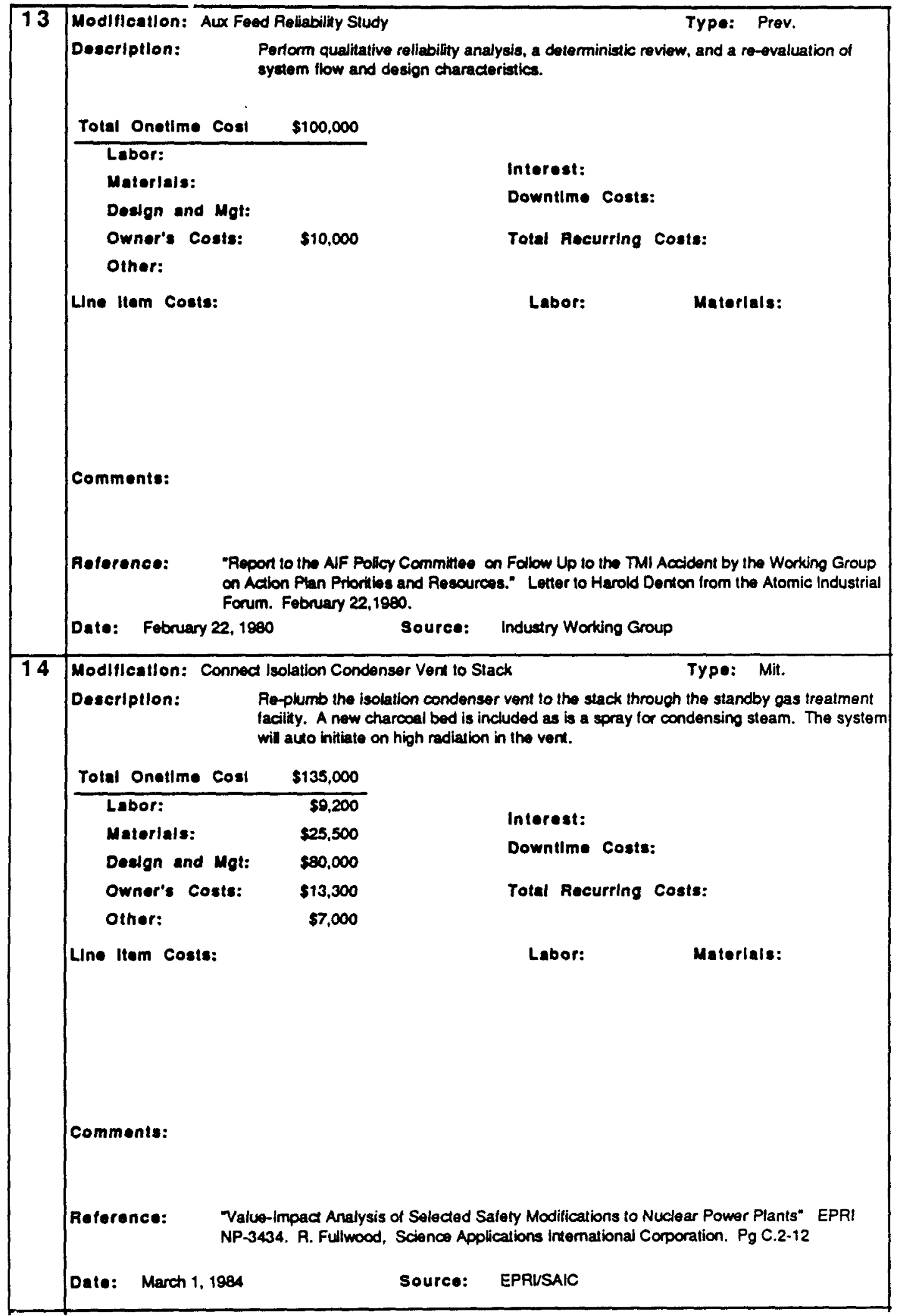




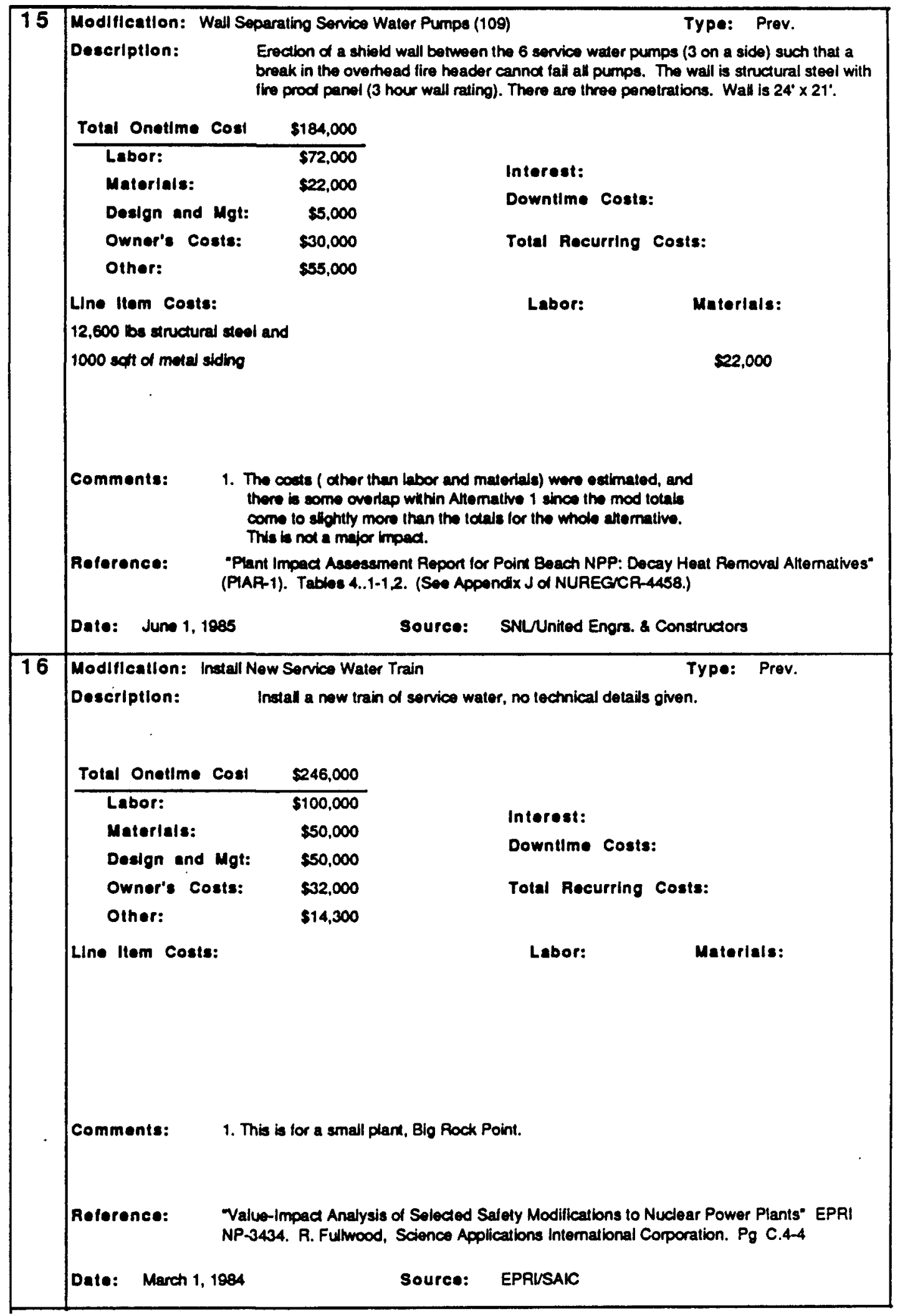




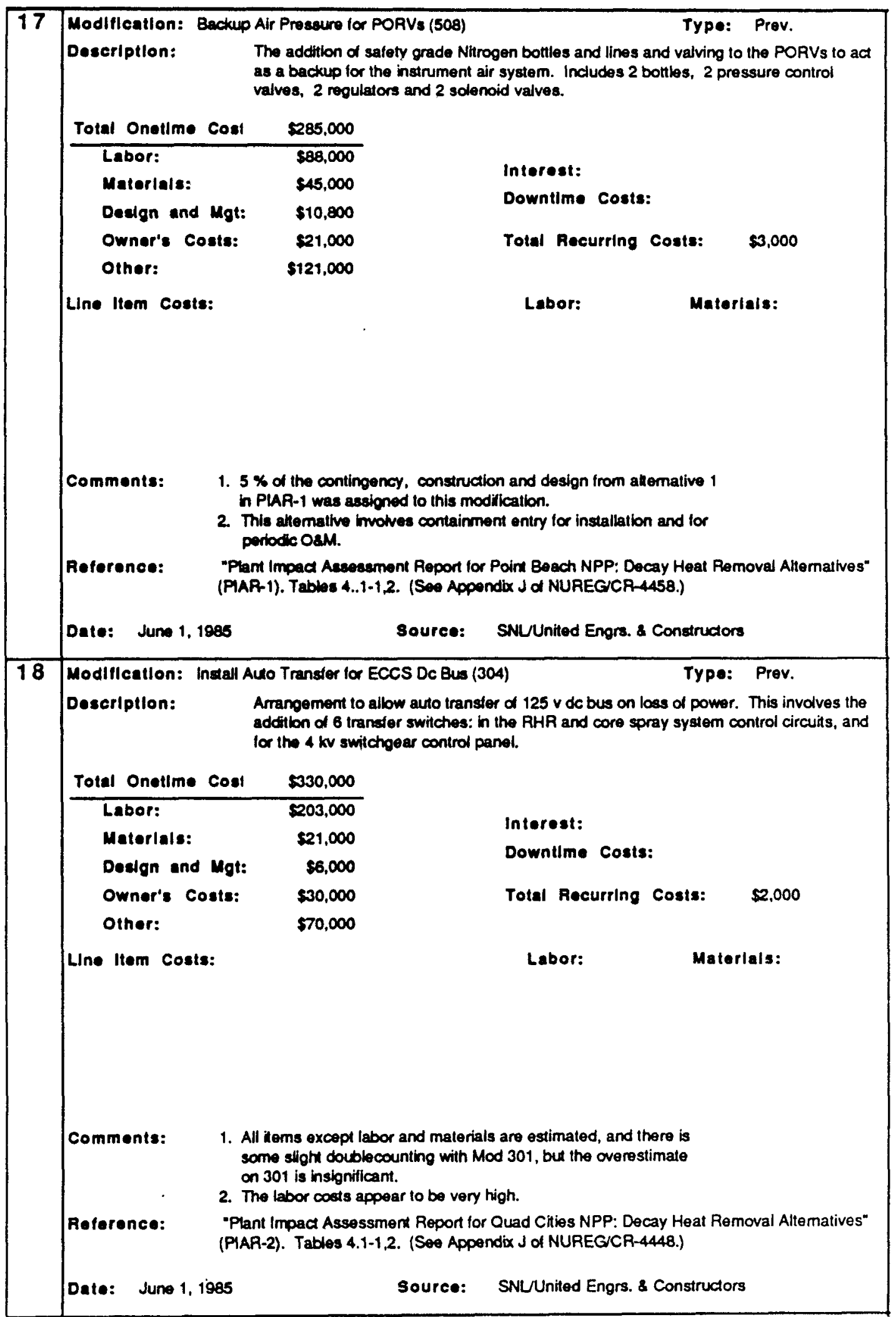




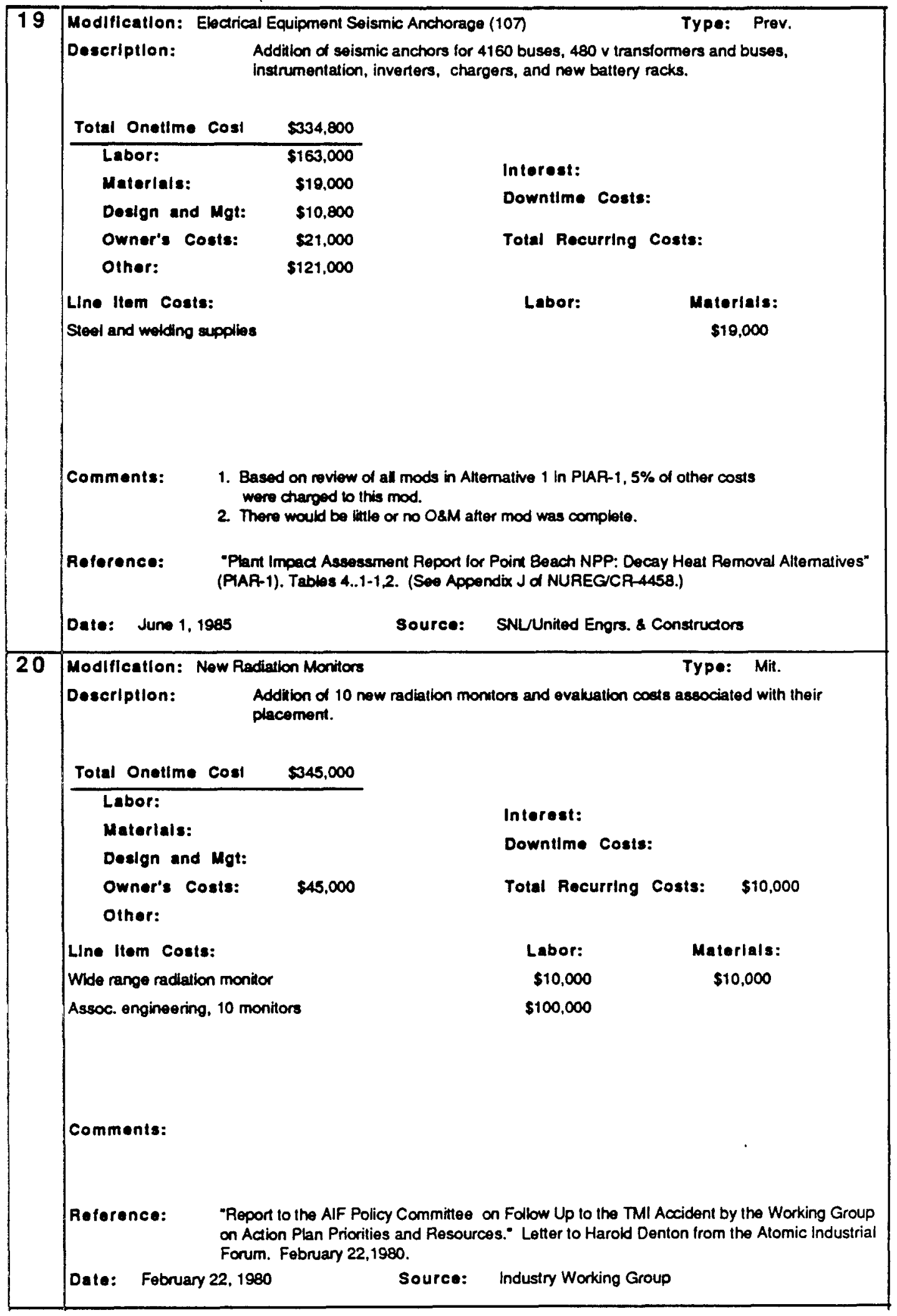


NUREG/CR-4551, VOL 1: DRAFT REPORT FOR COMMENT (SEPTEMBER, 1986)

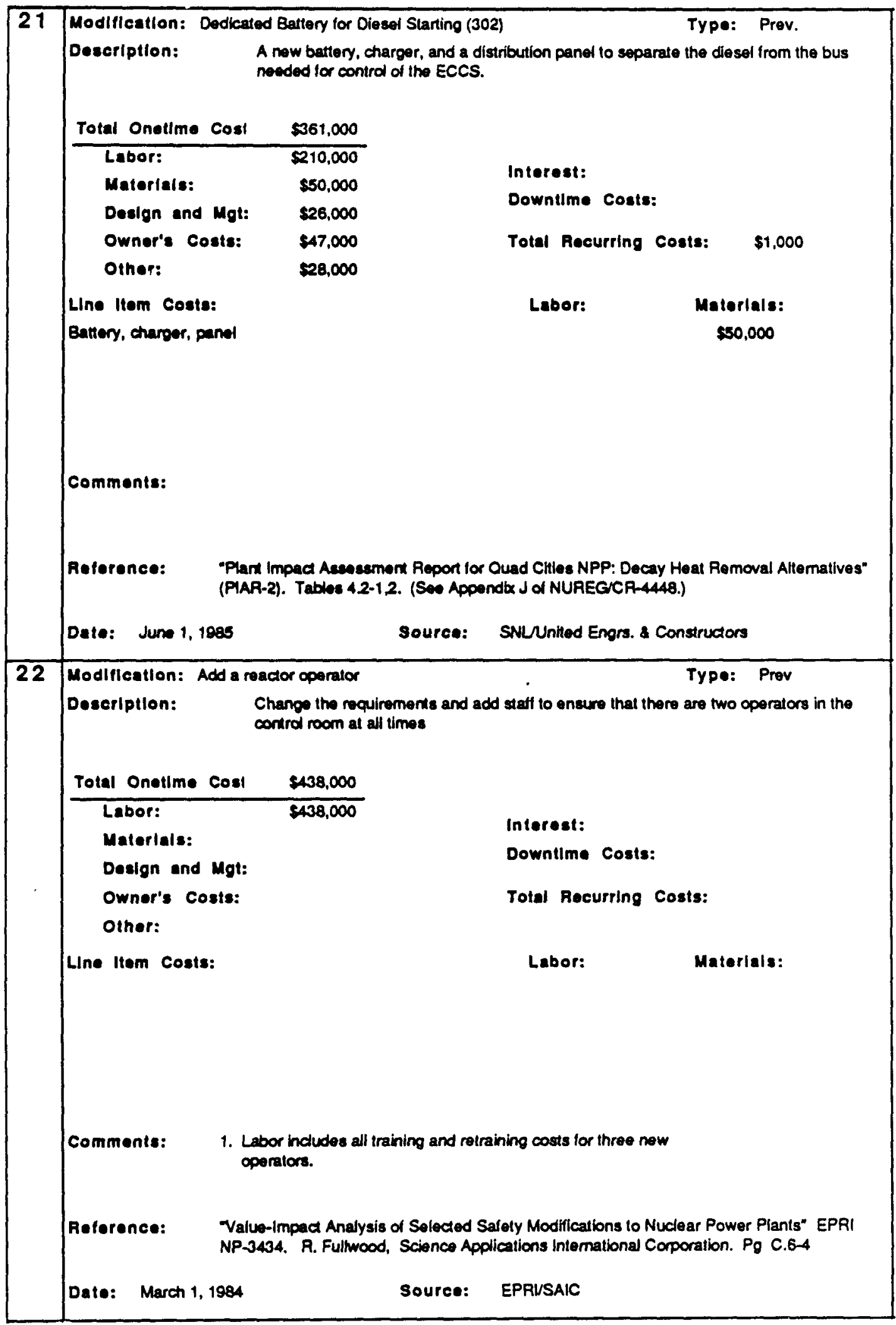




\begin{tabular}{|c|c|c|c|}
\hline 23 & 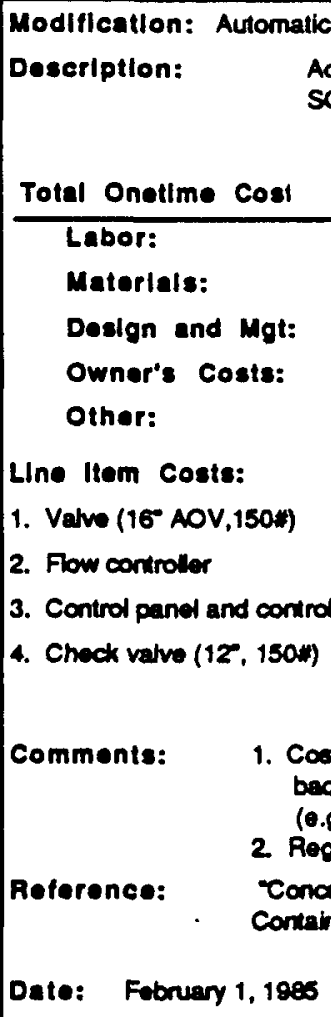 & 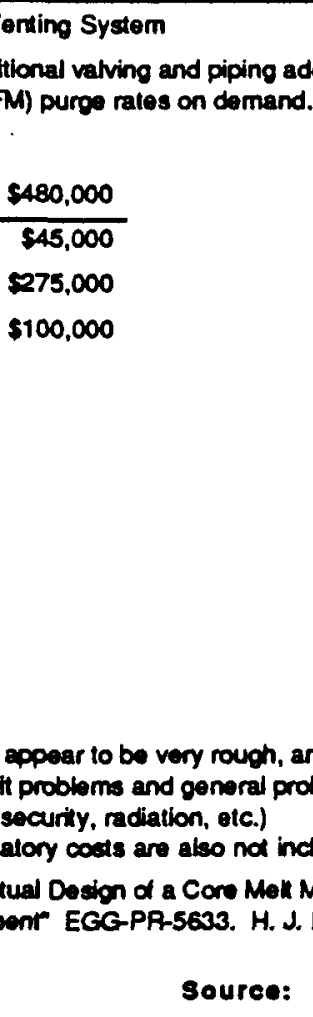 & 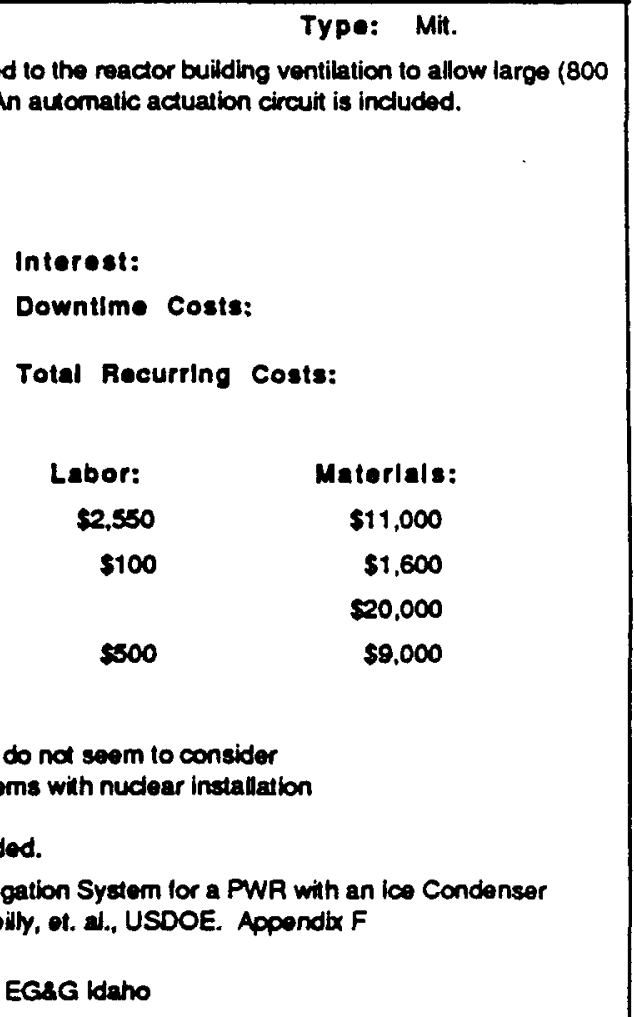 \\
\hline 24 & $\begin{array}{l}\text { Modiflestlon: Now Dryw } \\
\text { Description: } \\
\text { Total Onetime Cosl } \\
\text { Labor: } \\
\text { Materials: } \\
\text { Design and Mgt: } \\
\text { Owner's Costs: } \\
\text { Other: } \\
\text { Line Item Costs: } \\
\text { Radiation protection costs }\end{array}$ & $\begin{array}{l}\text { Spray Systom, Local ed in } \\
\text { r pipes deliver cooled water } \\
\text { iy hoaders. This option mus } \\
\text { is that descrbed in Recor } \\
\text { prern located directy beto } \\
\$ 15,000 \\
\$ 155,000 \\
\$ 60,000 \\
\$ 00,000 \\
\$ 100,000\end{array}$ & $\begin{array}{l}\text { a pumpingroooling system as in Record } 55 \text {. } \\
\text { ant. } \\
\text { d other indirect costs were given detailed consideration } \\
\text { be low. } \\
\text { s", Dooley, J. L., et al. R \& D Associales, Marina del } \\
\text { Table A-3 } \\
\text { A \& D Associates }\end{array}$ \\
\hline
\end{tabular}


NUREG/CR-4551, VOL. 1: DRAFT REPORT FOR COMMENT (SEPTEMBER, 1986)

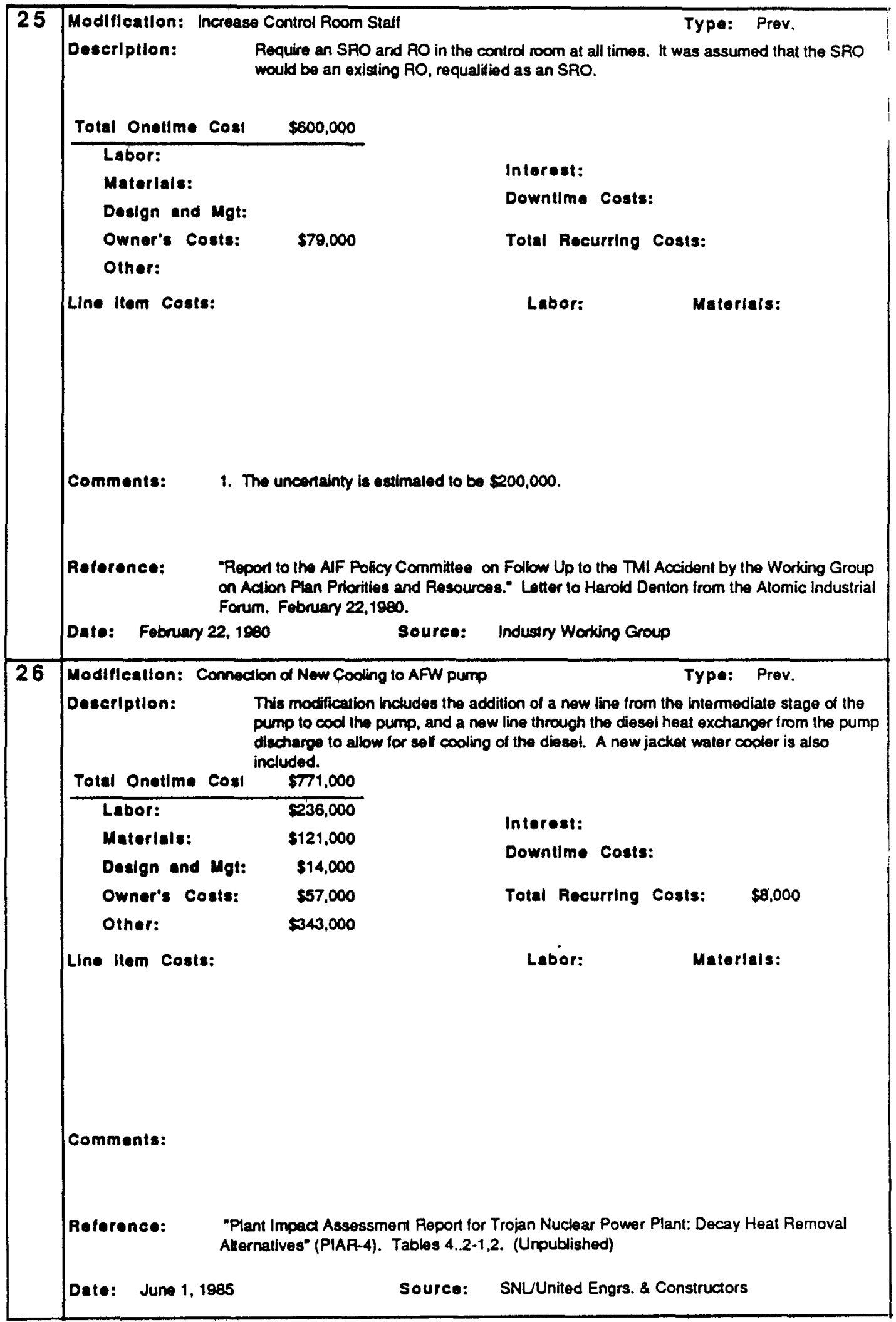




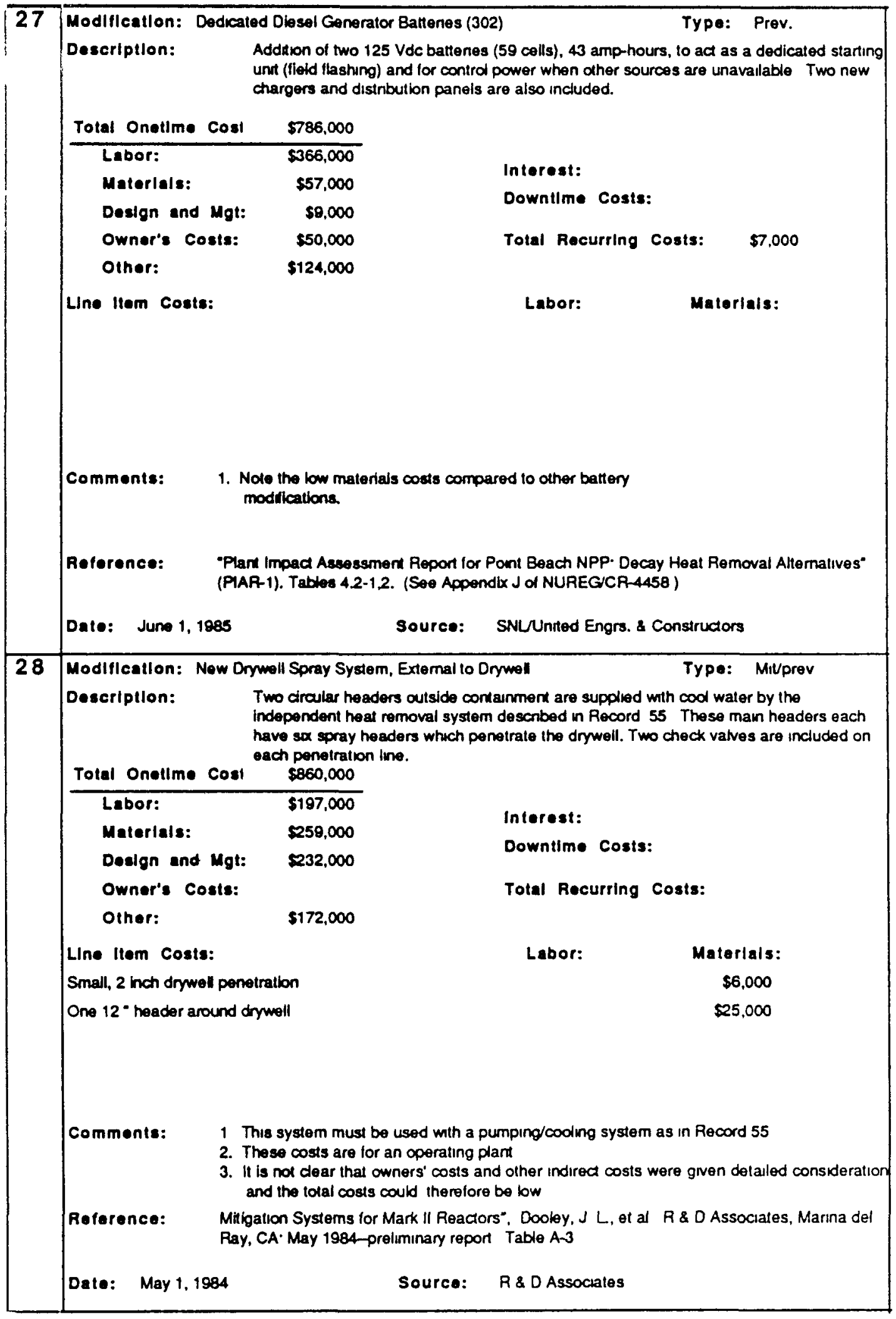




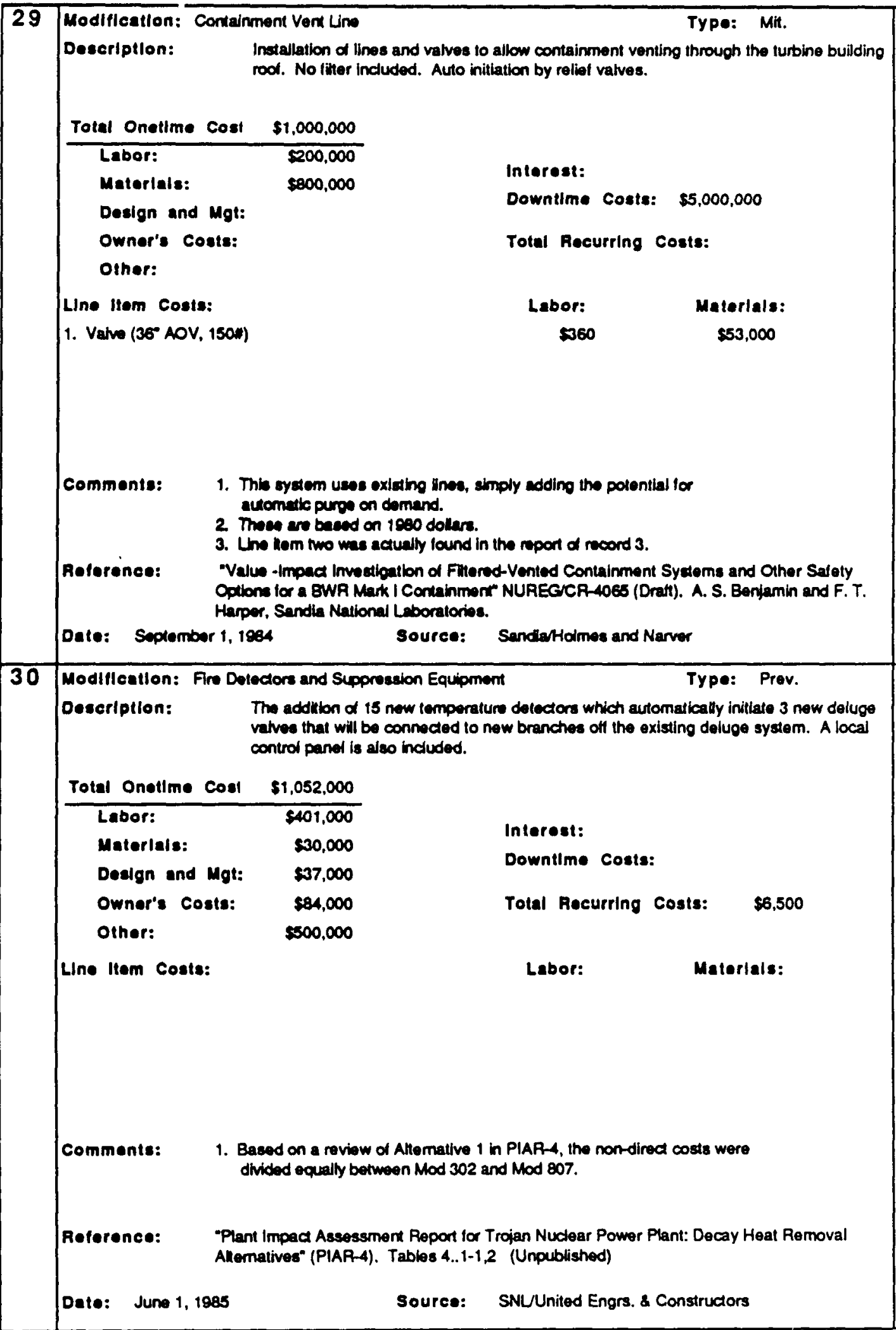




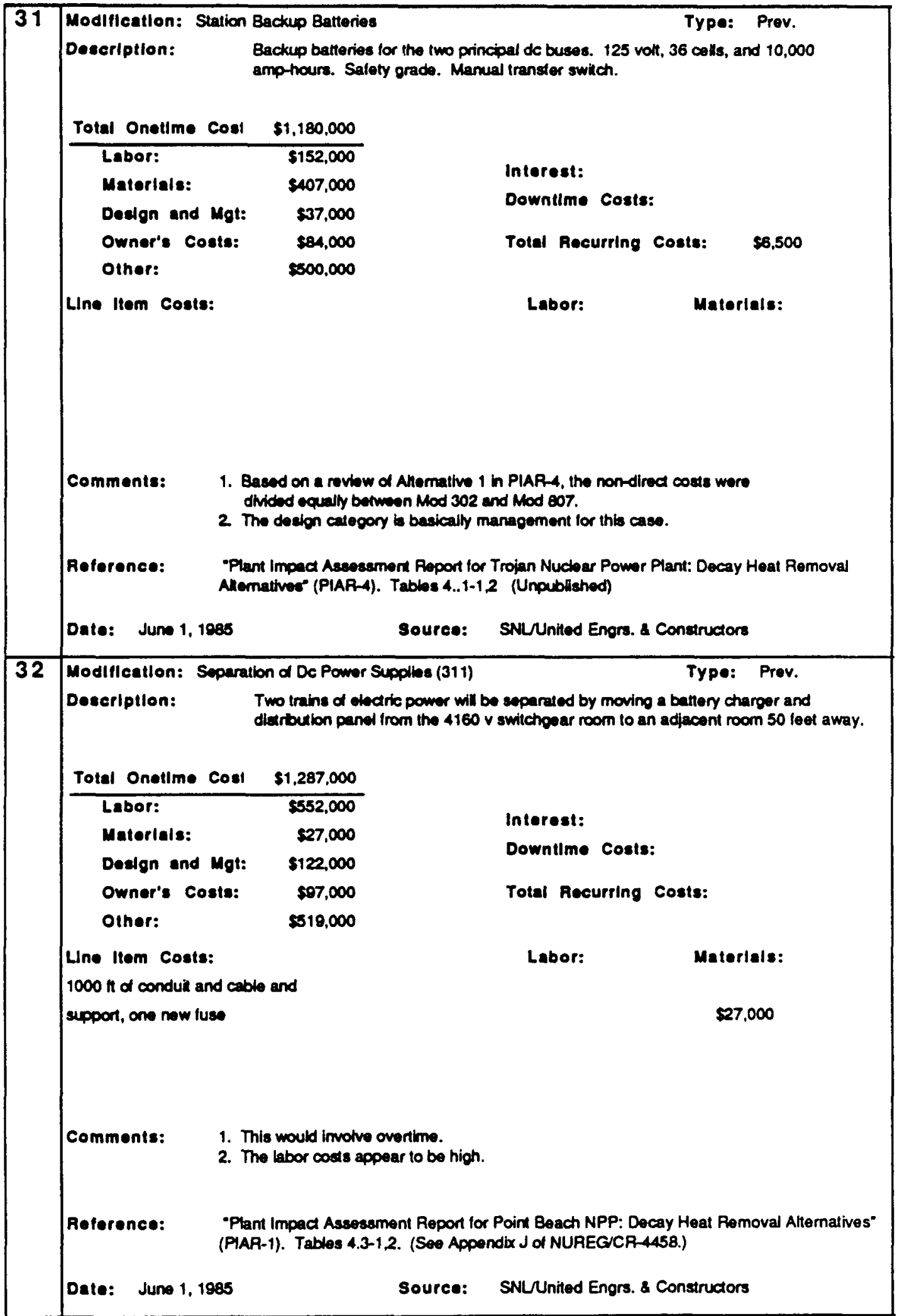




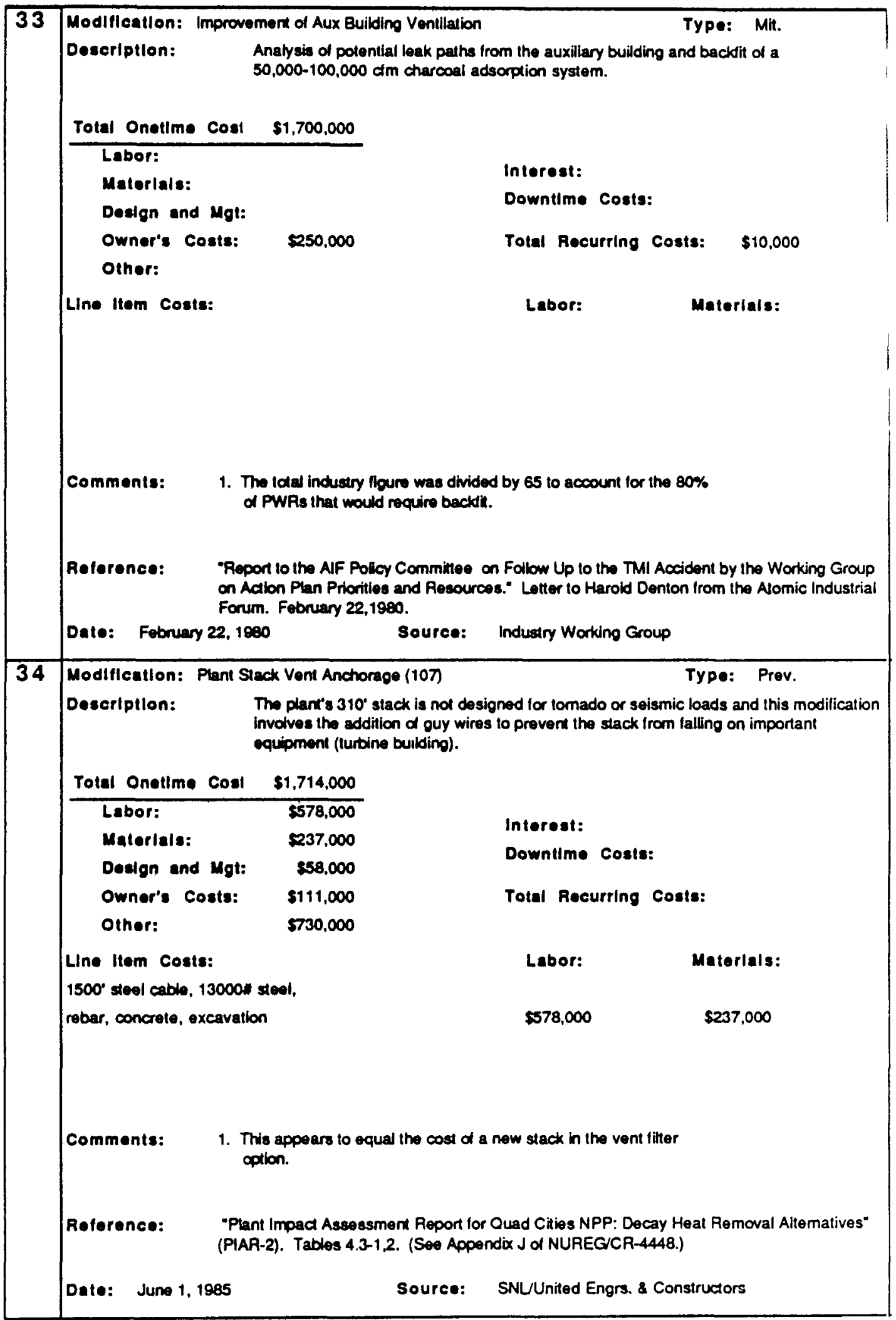




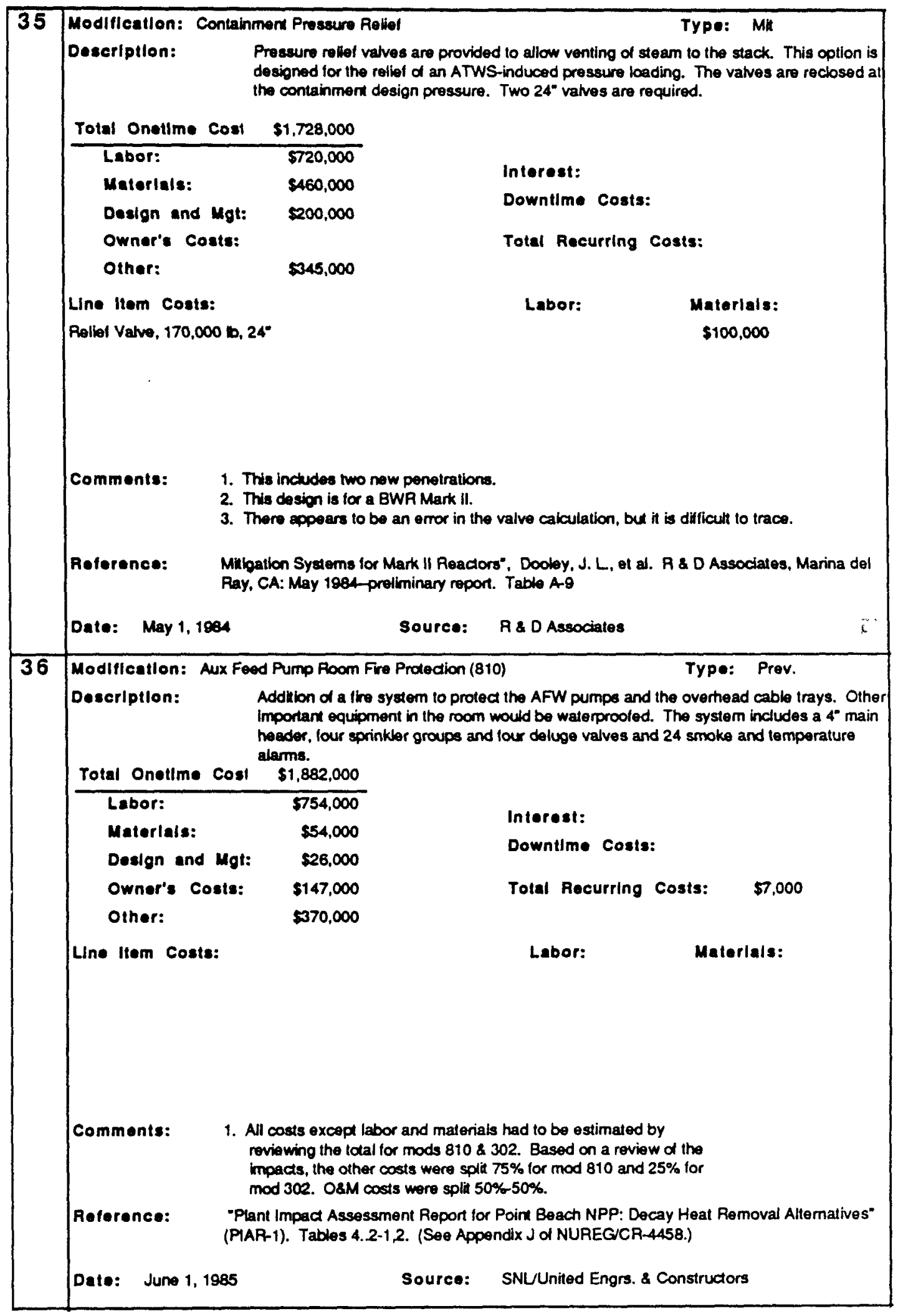




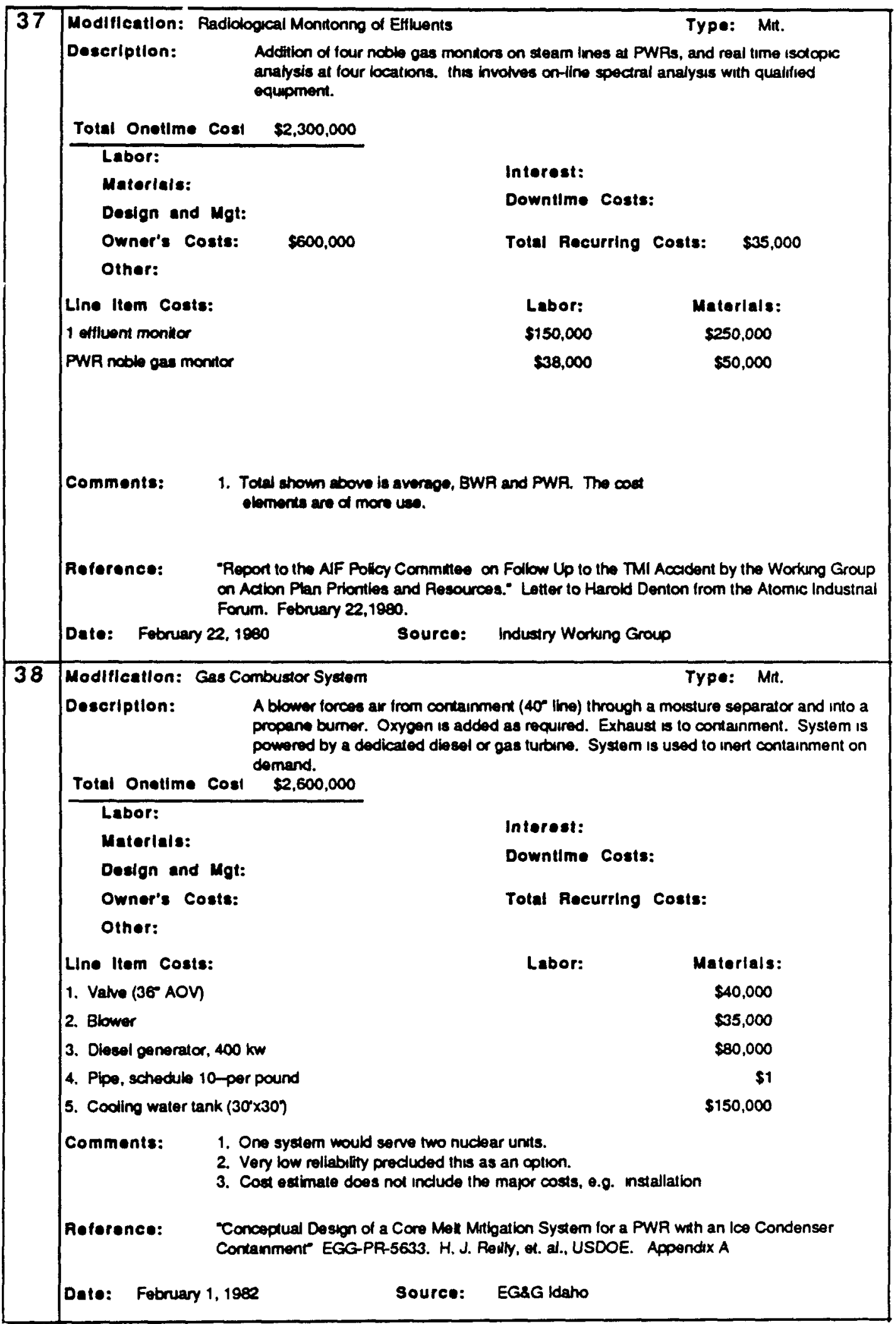




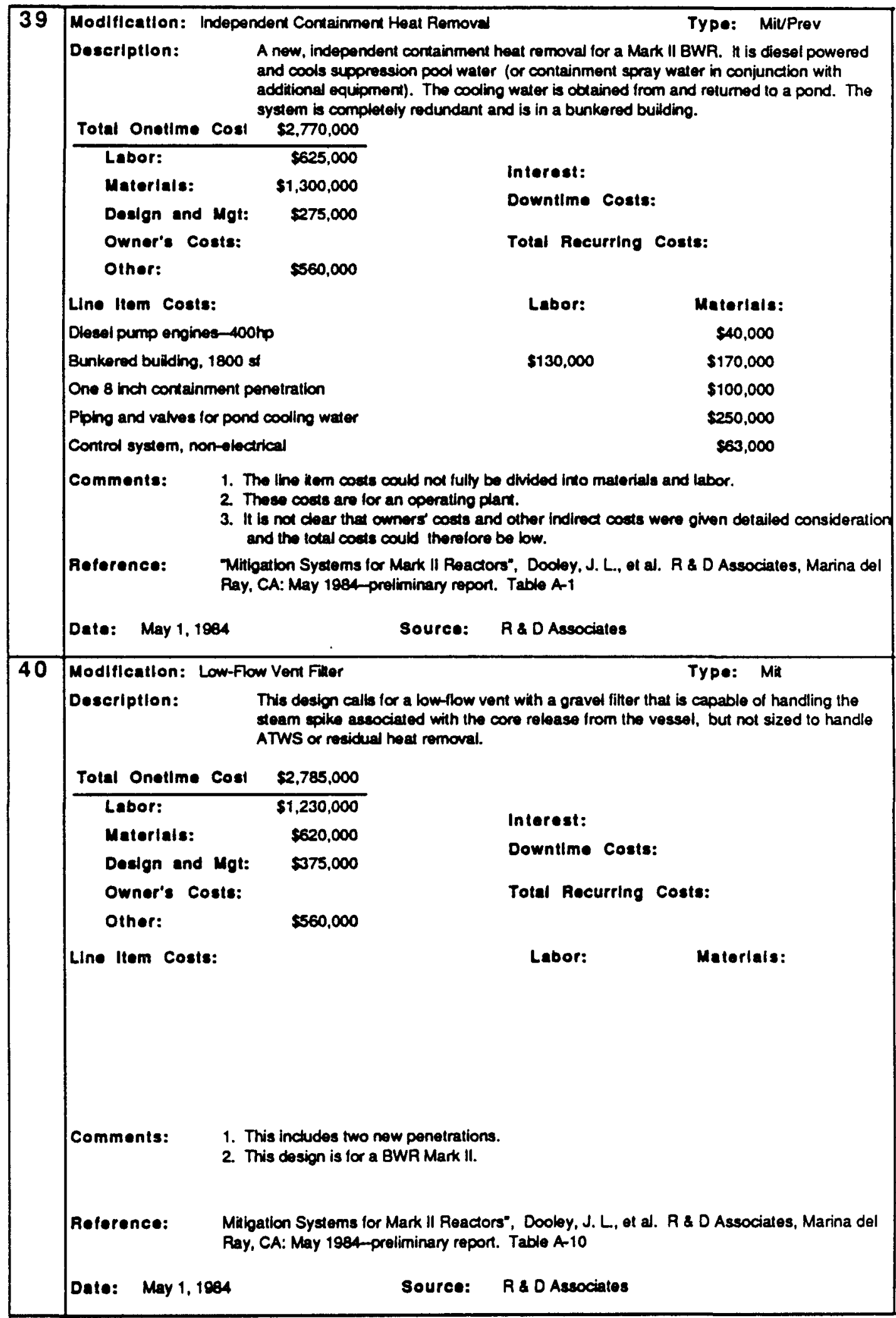




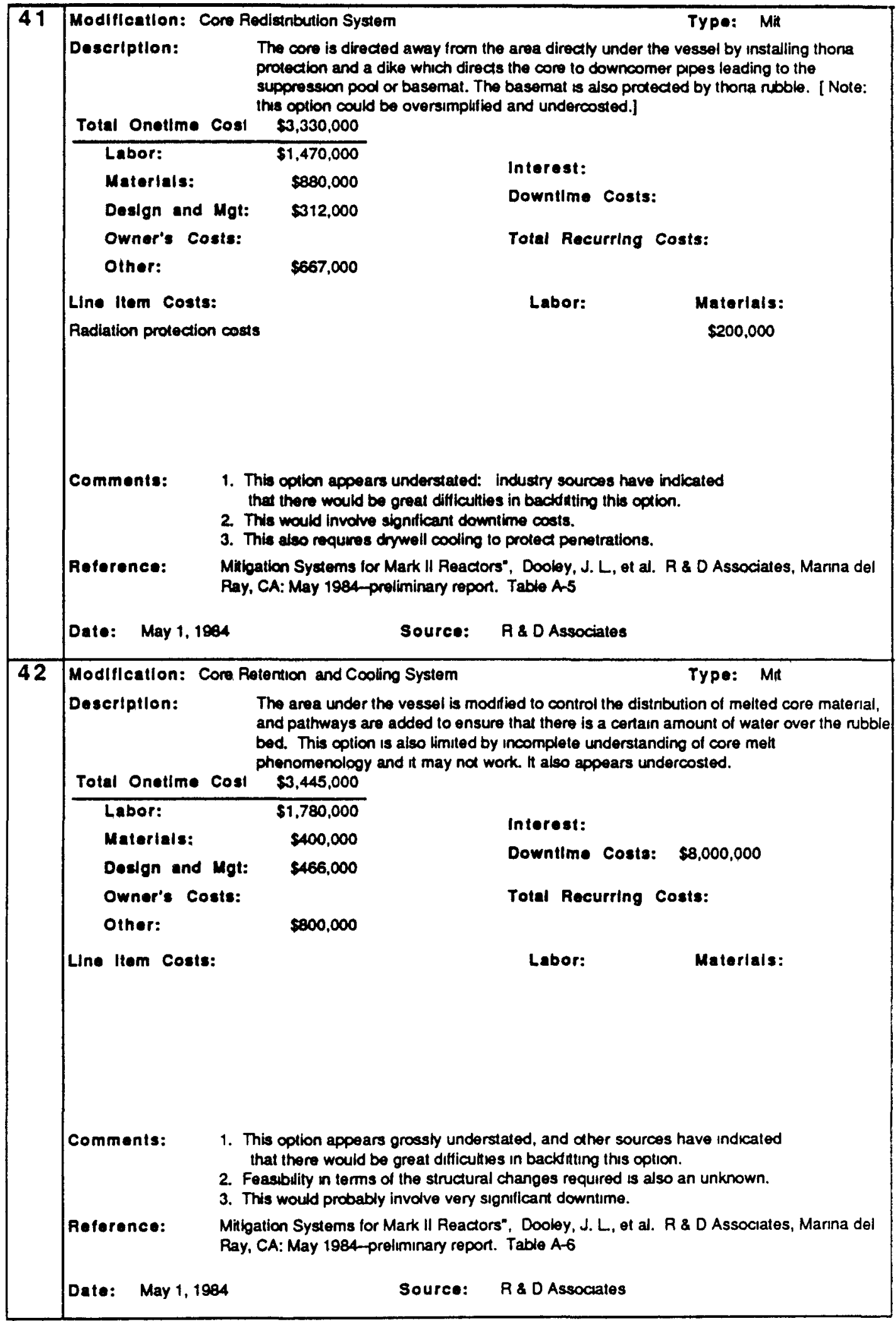




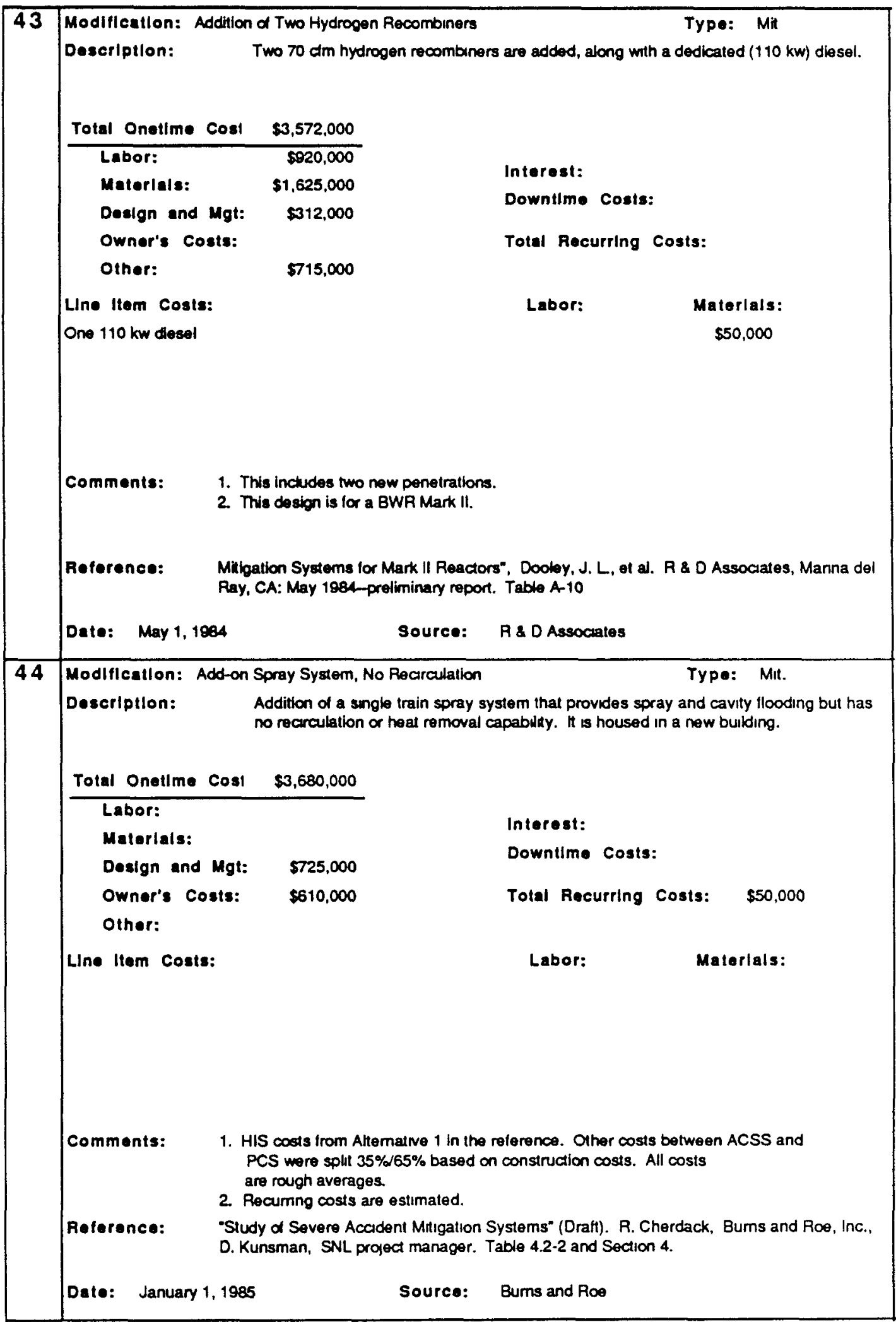


NUREG/CR-4551, VOL. 1: DRAFT REPORT FOR COMMENT (SEPTEMBER, 1986)

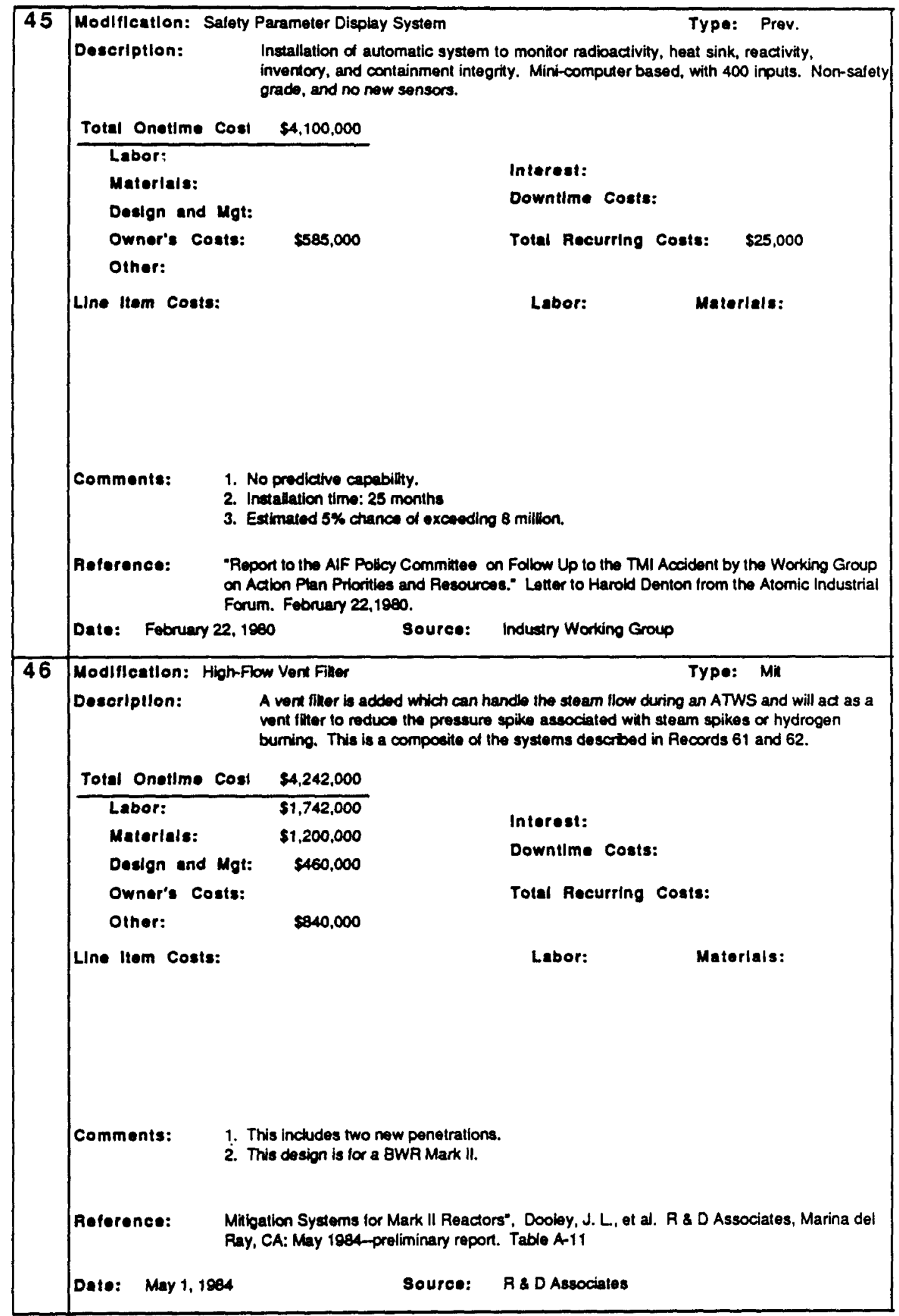




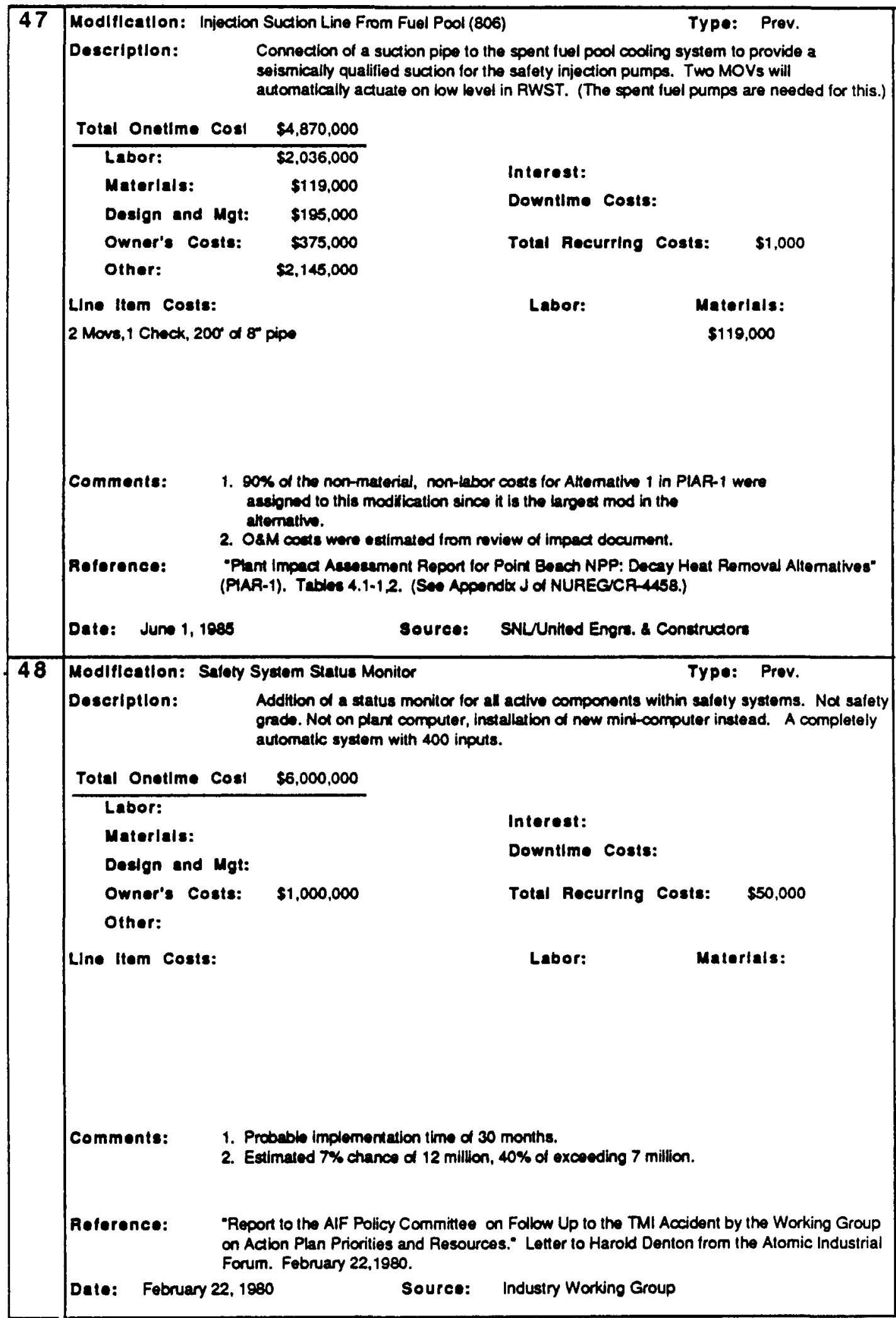




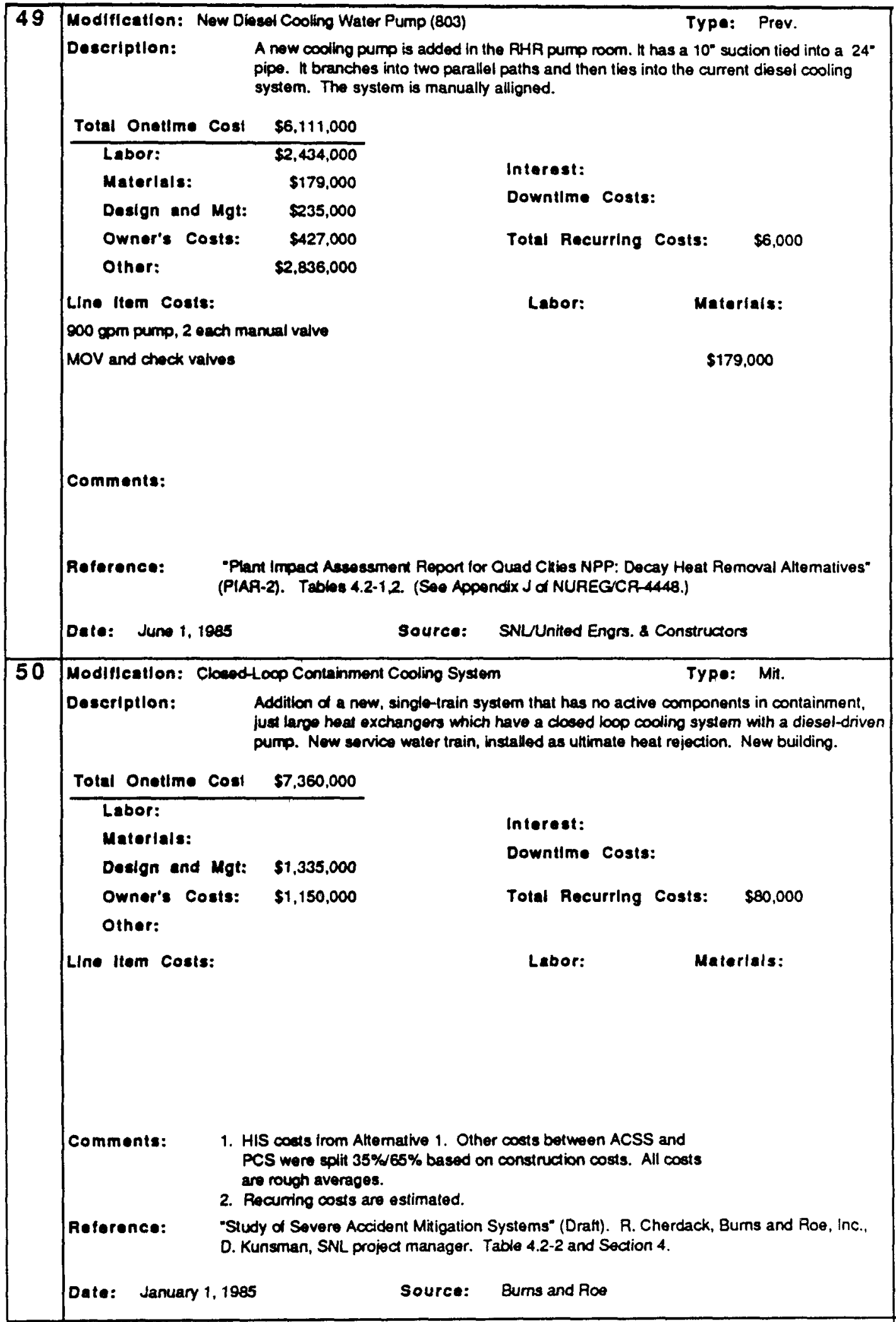




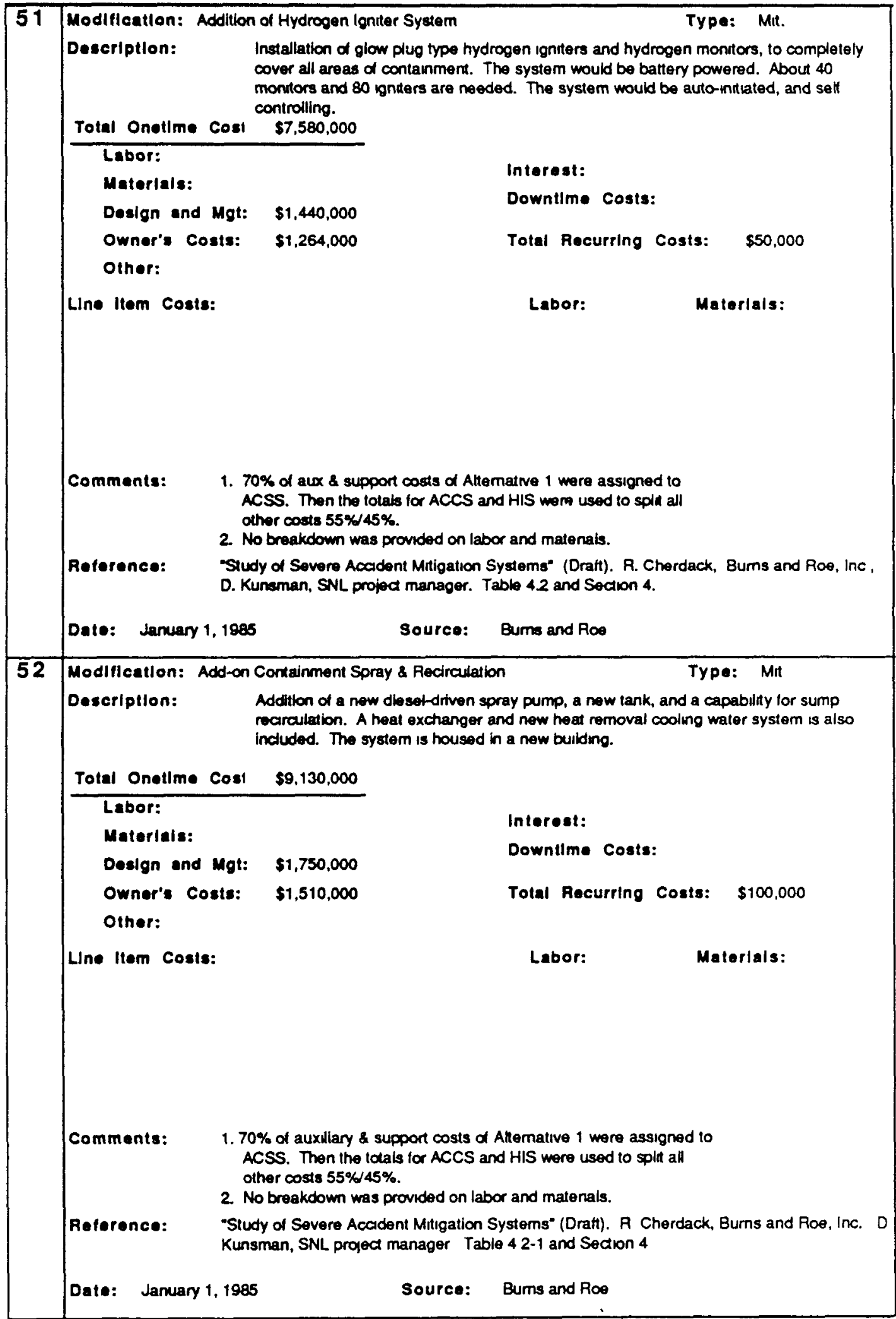


NUREG/CR-4551, VOL. 1: DRAFT REPORT FOR COMMENT (SEPTEMBER, 1986)

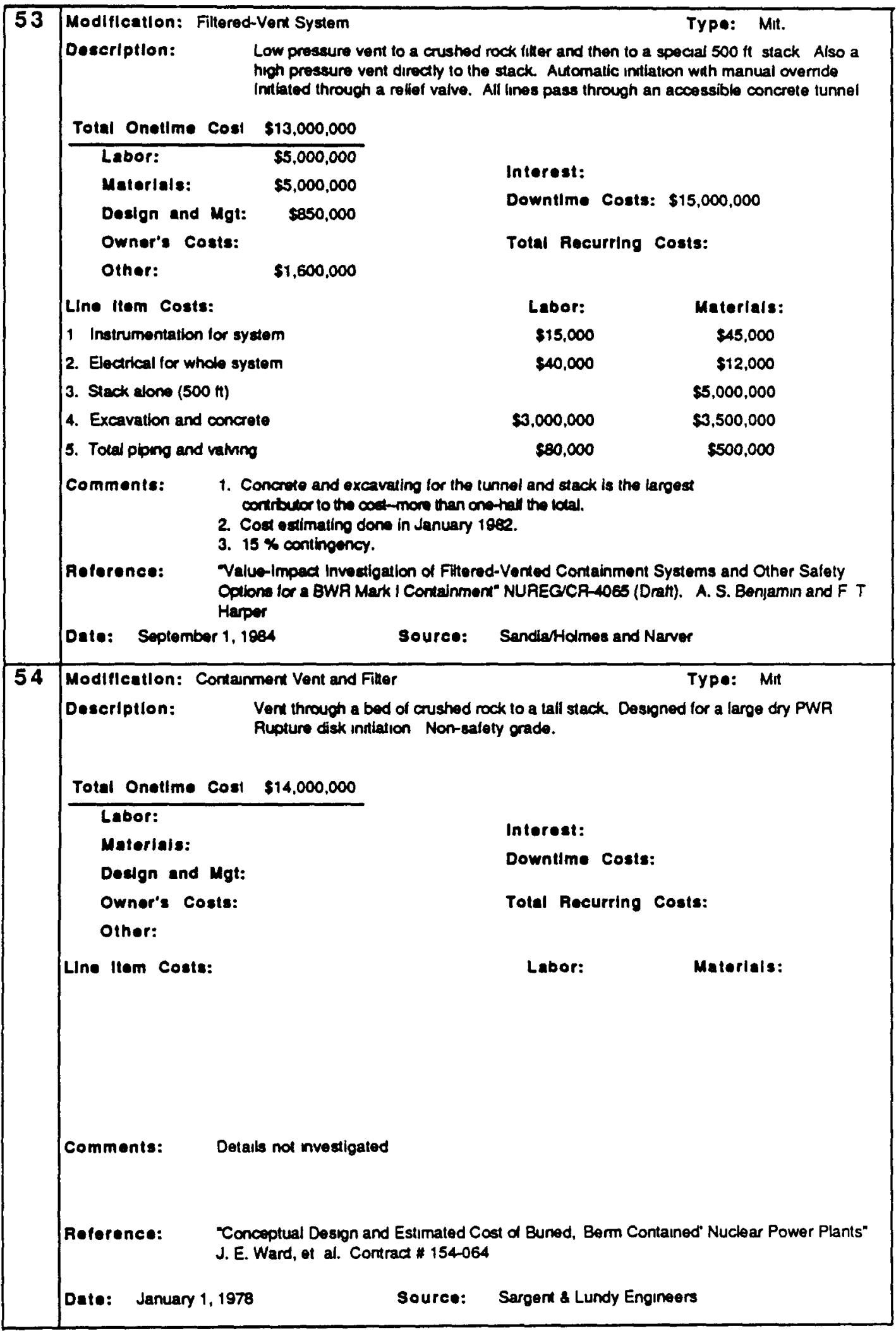




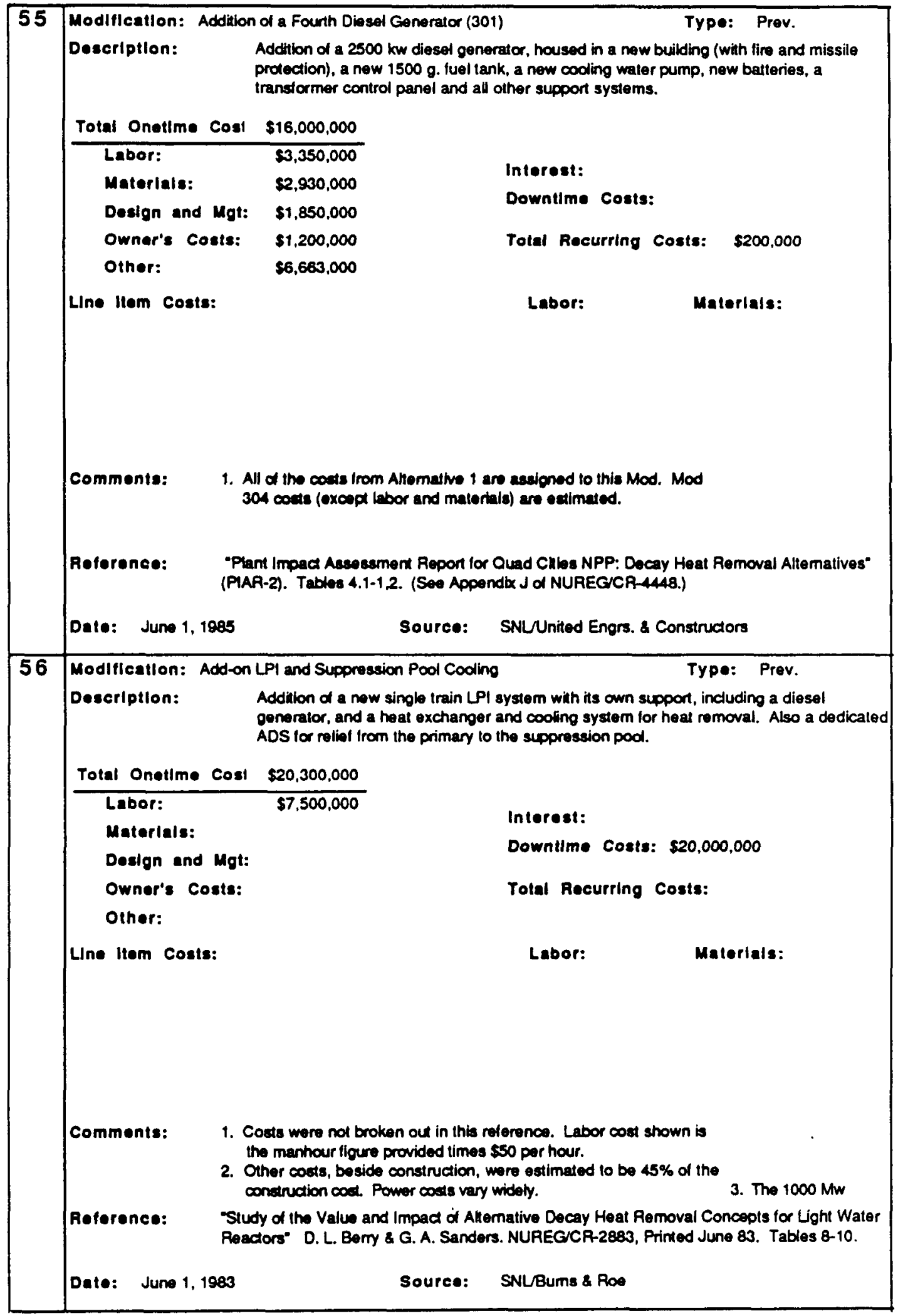




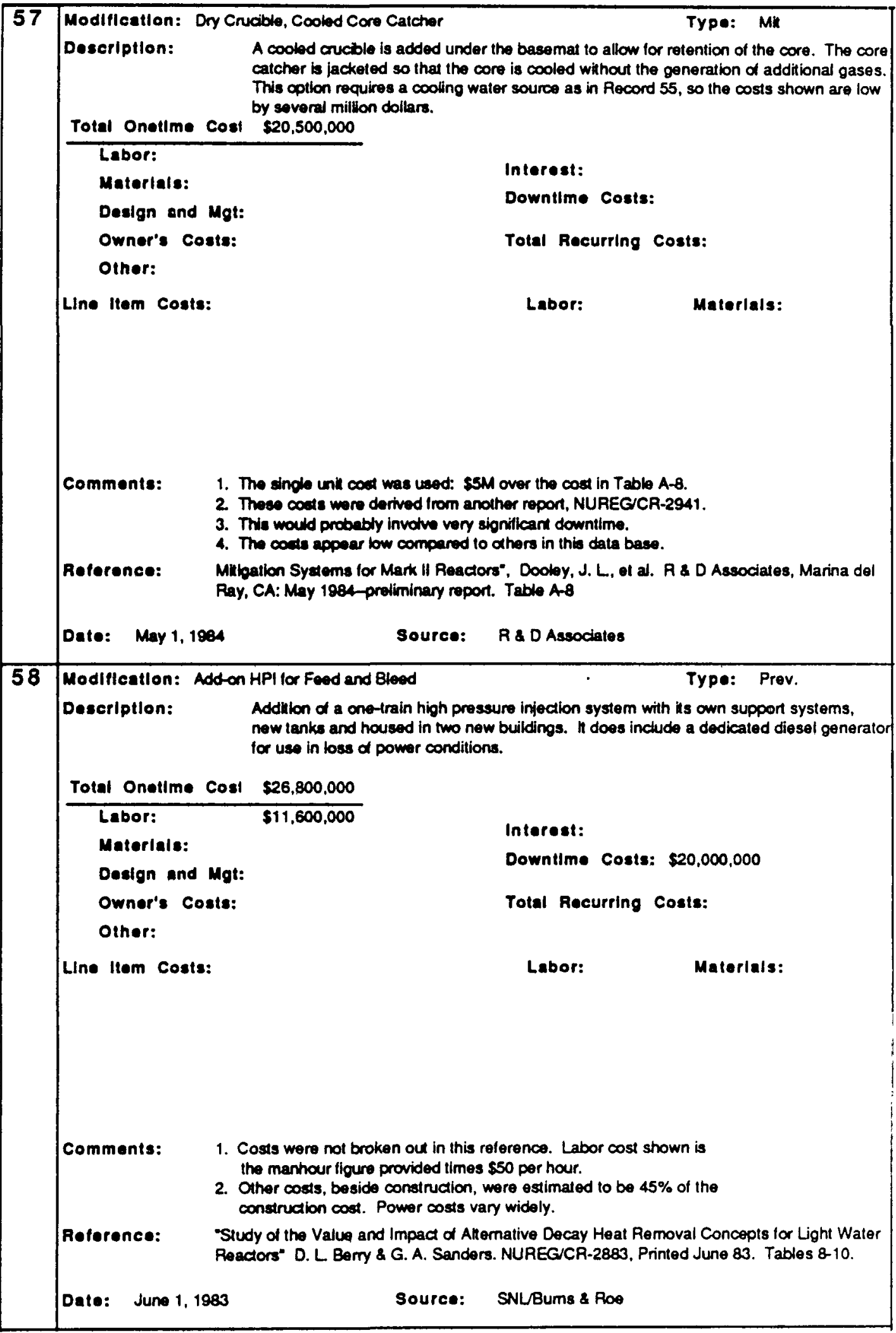




\begin{tabular}{|c|c|c|c|}
\hline \multirow[t]{3}{*}{59} & \multicolumn{3}{|c|}{ 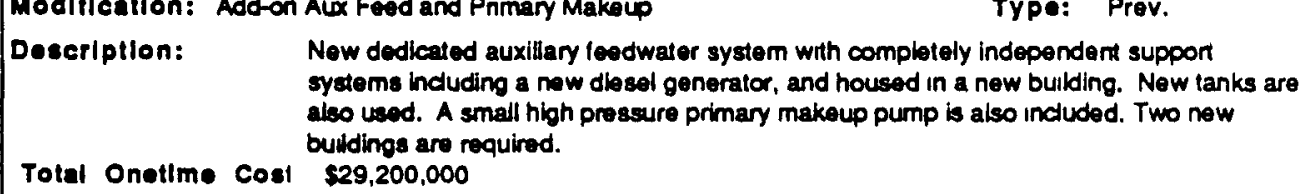 } \\
\hline & $\begin{array}{l}\text { Labor: } \\
\text { Materlals: } \\
\text { Deslgn and Mgt: } \\
\text { Owner's Costs: } \\
\text { Other: } \\
\text { Line Item Costs: }\end{array}$ & $\$ 12,850,000$ & $\begin{array}{l}\text { Interest: } \\
\text { Downtlme Costs: } \$ 20,000,000 \\
\text { Total Recurring Costs: } \\
\text { Labor: Materials: }\end{array}$ \\
\hline & 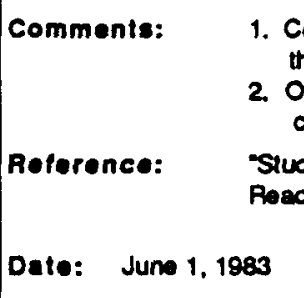 & $\begin{array}{l}\text { Costs were not broken out in this } \\
\text { the manhour figure pronded times } \\
\text { bher costs, beside construction, } \\
\text { construction cost. Power costs ve } \\
\text { dy of the Value and Impact of Alle } \\
\text { ctors" D. L. Berry \&. G. Sander } \\
\text { Source: }\end{array}$ & $\begin{array}{l}\text { Serence. Labor cost shown is } \\
\$ 50 \text { per hour. } \\
\text { vere estimated to be } 45 \% \text { of the } \\
\text { in widely. } \\
\text { mative Decay Heat Removal Concepts for Light Water } \\
\text { NUREGCR-2883. Printed June } 83 \text {. Tables } 8-10 \\
\text { SNLBUms \& Roe }\end{array}$ \\
\hline 60 & $\begin{array}{l}\text { Modification: High Ef } \\
\text { Description: } \\
\text { Total Onetime Cost } \\
\text { Lebor: } \\
\text { Materlals: } \\
\text { Deslgn and Mgt: } \\
\text { Owner's Costs: } \\
\text { Other: } \\
\text { Line Item Costs: }\end{array}$ & $\begin{array}{l}\text { filedency Conkanment Vent \& Fitte } \\
\text { Low and high pressure vent fiker } \\
\text { efficiency scrubbers and fliters. } \\
\$ 33,000,000\end{array}$ & $\begin{array}{l}\text { Interest: } \\
\text { Downtlme Costs: } \\
\text { Tolal Recurring Costs: } \\
\text { Labor: Materials: }\end{array}$ \\
\hline
\end{tabular}




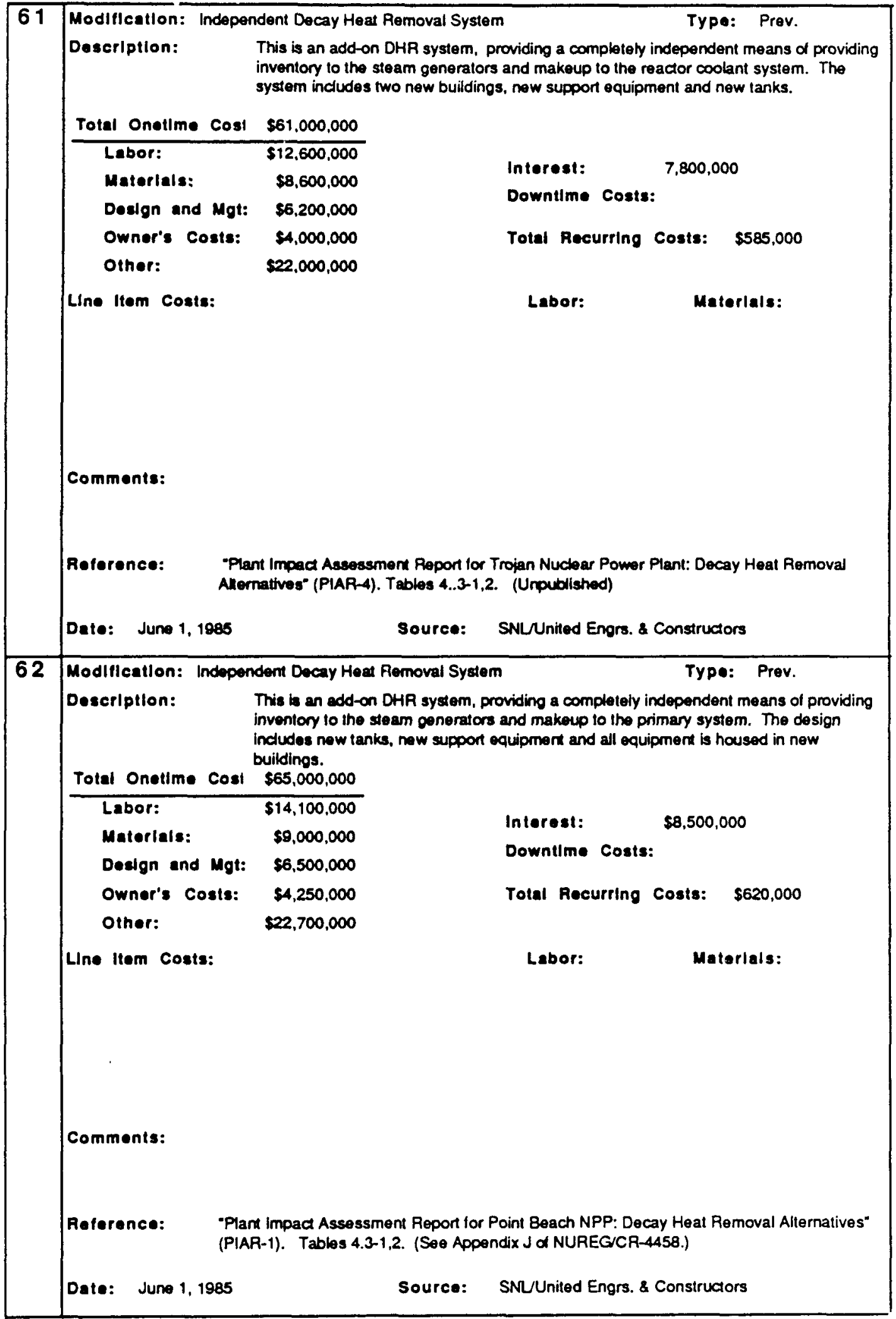


NUREG/CR-4551, VOL. 1: DRAFT REPORT FOR COMMENT (SEPTEMBER, 1986)

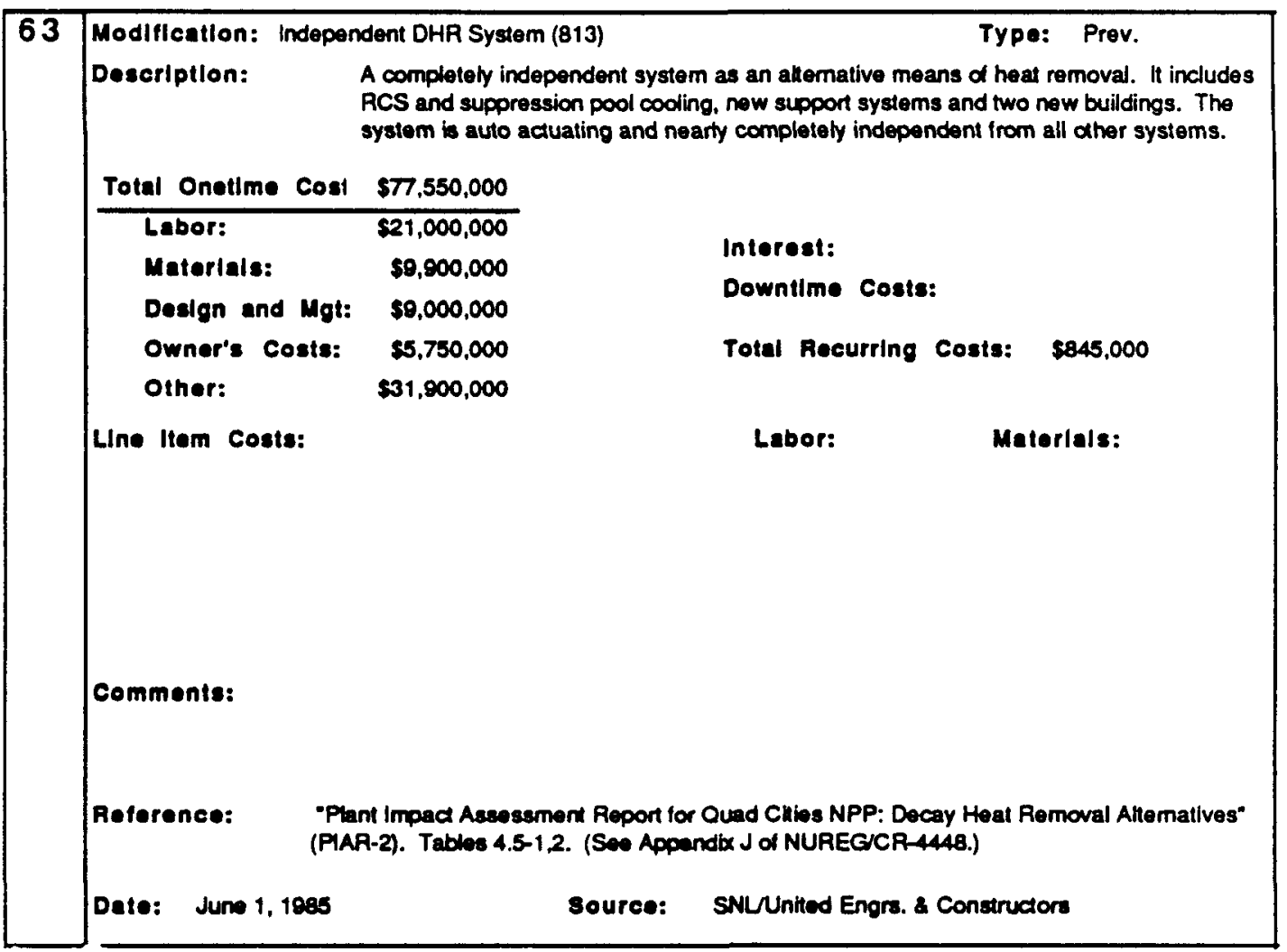




\section{References for Appendix G}

1. Berry, D. L., and G. A. Sanders. Study of the Value Impact of Alternative Decay Heat Removal Concepts for Light Water Reactors. U. S. Nuclear Regulatory Commission Report NUREG/CR-2883, Sandia National Laboratories, Albuquerque, NM: 1983.

2. Richardson Estimating Manual. R. S. Means Company, Inc. Kingston, MA: 1983.

3. Benjamin, A. S., and F. T. Harper. Value-Impact Investigation of Filtered-Vented Containment Systems and Other Safety Options for a BWR Mark I Containment . (Draft) NUREG/CR-4065, Sandia National Laboratories, Albuquerque, NM: September, 1984.

4. Cherdack, R. Study of Severe Accident Mitigation Systems. (Draft) Burns and Roe, Inc. Oradell, NJ: January, 1985.

5. "Plant Impact Assessment Report for the Point Beach Nuclear Power Plant" United Engineers and Constructors (June, 1985), See Appendix J of Shutdown Decay Heat Removal Analysis: Westinghouse Two Loop Plant Case Study, U. S. Nuclear Regulatory Commission Report NUREG/CR-4458, Sandia National Laboratories, Albuquerque, NM: (to be published).

6. "Plant Impact Assessment Report for the Quad Cities Nuclear Power Plant" United Engineers and Constructors (June, 1985), See Appendix J of Shutdown Decay Heat Removal Analysis: General Electric BWR3/Mark I Case Study. U. S. Nuclear Regulatory Commission Report NUREG/CR-4448, Sandia National Laboratories, Albuquerque, NM: (to be published).

7. Ball, J. R., S. Cohen, and E. J. Ziegler. A Handbook for Cost Estimating. NUREG/CR-3971, Argonne National Laboratories, Argonne, IL: October, 1984.

8. The Energy Economic Data Base, Phase IV. United Engineers and Constructors. Published periodically by the U. S. Department of Energy: 1983.

9. NRC Task Action Plan. (Draft) NUREG-0660. U.S. Nuclear Regulatory Commission Report, Washington, DC: 1979.

10. Lee, B. "Report to the AIF Policy Committee on the Follow-up to the Three Mile Island Accident by the Working Group on Action Plan Priorities and Resources." Letter to Harold Denton, U.S. Nuclear Regulatory Commission. Atomic Industrial Forum, Washington, DC: February 22,1980.

11. Fullwood, R. Value-Impact Analysis of Selected Safety Modifications to Nuclear Power Plants. EPRI NP-3434. Electric Power Research Institute, Palo Alto, CA: March, 1984.

12. Dooley, J. L. ,et al., Mitigation Systems for Mark II Reactors. Preliminary Report. RDATR-127303-001. R \& D Associates, Marina del Rey, CA: May 1984 


\section{DISTR I BUT ION :}

U. S. Government Printing office

Receiving Branch (Attn: NRC Stock)

8610 Cherry Lane

Laurel. MD 20707

$\begin{array}{ll}3141 & \text { S. A. Landenberger (5) } \\ 3151 & \text { W. L. Garner } \\ 6400 & \text { D. J. McCloskey } \\ 6410 & \text { N. R. Ortiz } \\ 6414 & \text { A. S. Benjamin (25) } \\ 6412 & \text { A. L. Camp } \\ 6412 & \text { F. T. Harper } \\ 6412 & \text { D. M. Kunsman } \\ 6512 & \text { D. M. Ericson. Jr. } \\ 6415 & \text { F. E. Haskin } \\ 6415 & \text { J. L. Sprung } \\ 6513 & \text { D. D. Carlson } \\ 6420 & \text { J. V. Walker } \\ 6422 & \text { D. A. Powers } \\ 6427 & \text { M. Berman } \\ 6440 & \text { D. A. Dahlgren } \\ 6442 & \text { W. A. Von Riesemann } \\ 6449 & \text { K. D. Bergeron } \\ 6449 & \text { D. C. Williams } \\ 8024 & \text { P. W. Dean }\end{array}$


Abbey, Frank

U.K. Atomic Energy Authority

Wigshaw Lane, Culcheth

Warrington, Chesire

United Kingdom, WA3 $4 \mathrm{NE}$

Aftergood, Steven

Committee to Bridge the Gap

1637 Butler Avenue. \#203

Los Angeles. CA 90025

Amos. C. N.

Technadyne. Inc.

300 Virginia SE

Albuquerque. NM 87108

Apostolakis, George

University of California, Los Angeles

Boelter Hall. Room 5532

Los Angeles, CA 90024

Ashkar, James $W$.

Boston Edison Company

800 Boylston street

Boston, MA 02199

Ashton, Donald H.

Bechtel Power Corporation

15740 Shady Grove Road

Gaithersburg. MD 20877

Averett . Mark

Florida Power Corporation

P.O. Box 14042

St. Petersburg, FL 33733

Aycock. Michael B.

TERA Corporation

7101 Wisconsin Avenue

Bethesda. MD 20814

Bagley. Raymond 0 .

Northeast Utilities

P.O. Box 270

Hartford. CT 06101

Bagues. J.

Consejo de Seguridad Nucleare

Sorangela de la Cruz 3

Madrid 38046

Spain 
Bairiot. H.

Belgonucleaire $S A$

Rue de Champ de Mars 25

B-1050 Brussels

Belgium

Balkovetz, Fred J.

EG\&G Idaho, Inc.

P.O. Box 1625

Idaho Falls, ID 83415

Baranowsky, Patrick

U.S. Nuclear Regulatory Commission

Office of Nuclear Regulatory Research

Washington, DC 20555

Bari. Robert A.

Brookhaven National Laboratories

Building 130

Upton. NY 11973

Barrett, Richard

U.S. Nuclear Regulatory Commission

Office of Nuclear Reactor Regulation

Washington, DC 20555

Baskin. Kenneth S.

S. California Edison Company

P.O. Box 800

Rosemead, CA 91770

Bastl, Werner

Geselischaft Fur Reaktorsicherheit

Forschungsgelande

8046 Garching

Federal Republic of Germany

Baybutt, Paul

Battelle Columbus Division

505 King Avenue

Columbus. OH 43201

Beckley. Bruce B.

Public Service Company

P.O. Box 330

Manchester. NH 03105

Bender, Myer

Querytech. Inc.

408 Cedar Bluff Road

Knoxville, TN 37923 


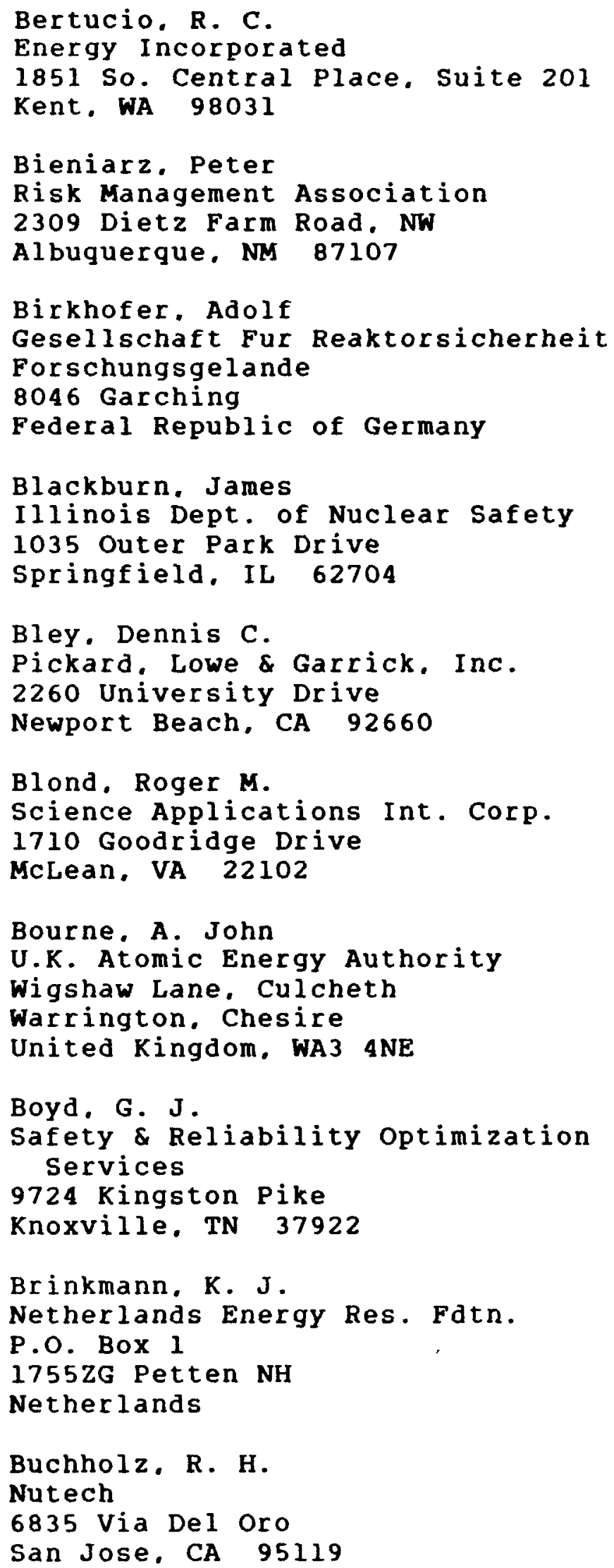

Boyd. G. J .

Safety \& Reliability optimization Services

Brinkmann, K. J. Netherlands Energy Res. Fdtn.

P.O. BoX 1

1755ZG Petten NH

Netherlands

Buchholz. R. H.

Nutech

6835 Via Del Oro

San Jose. CA 95119 


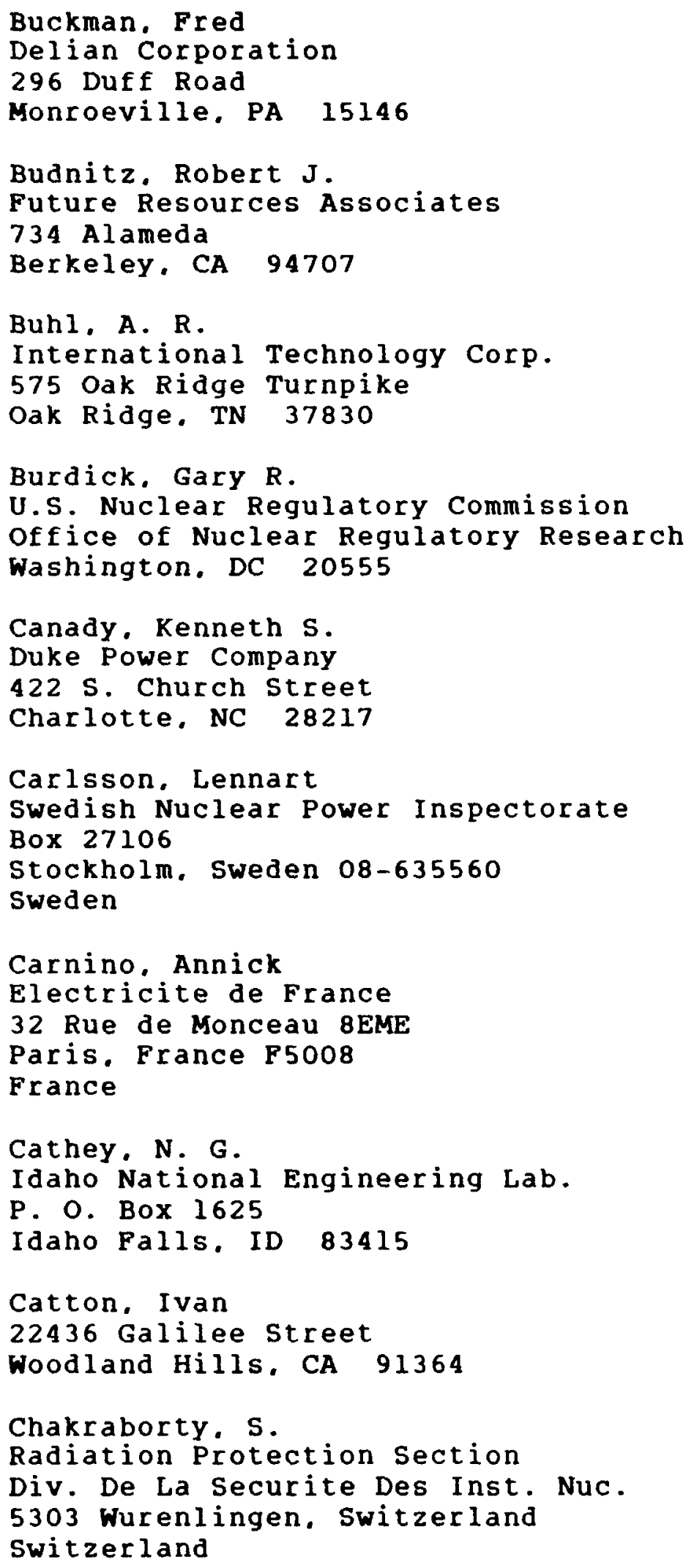


Chang. Sen-I

Institute of Nuclear Energy Research

P.O. Box 3

Lungtan. 325 Taiwan

Christie. Robert F.

Tennessee Valley Authority

400 W. Summit Hill Avenue. WlODl

90 Knoxville. TN 37902

Coffman. Frank

U.S. Nuclear Regulatory Commission

Office of Nuclear Reactor Regulation

washington. DC 20555

Conradi, Larry L.

Energy Incorporated

1851 South Central Place. Suite 201

Kent, WA 98031

Cooper, Peter

U.K. Atomic Energy Authority

Wigshaw Lane, Culcheth

Warrington, Chesire

United Kingdom, WA3 $4 \mathrm{NE}$

Corradini, Michael

University of Wisconsin

1500 Johnson Drive

Madison. WI 53706

Cullingford. Michael

IAEA

P.O. Box 200

A-1400 Vienna. Austria

Cummings. Garth

Lawrence Livermore Laboratory

L-91. Box 808

Livermore, CA 94526

Cunningham, Mark A.

U.S. Nuclear Regulatory Commission

Office of Nuclear Regulatory Research

Washington. DC 20555

Curry. James J.

7135 Salem Park Circle

Mechanicsburg. PA 17055

Cybulskis. Peter

Battelle Columbus Division

505 King Avenue

Columbus. OH $\$ 3201$ 
Davis, Peter R.

Intermountain Technologies. Inc.

P.O. Box 1604

Idaho Falls, ID 83401

DeCarlos. Jose

Sor Angela De la Cruz, No. 3, Planta 8

Madrid 28016. Spain

Deem. Richard

New York Power Authority

123 Main street

White Plains. NY 10601

Denning. Richard S.

Battelle Columbus Division

505 King Avenue

Columbus. OH 43201

Disalvo, Raymond

Battelle Columbus Division

$505 \mathrm{King}$ Avenue

Columbus, OH 43201

El-Bassioni, Adel A.

U.S. Nuclear Regulatory Commission

Office of Nuclear Reactor Regulation

Washington. DC 20555

Elliott, J. Mark

International Energy Associates. Ltd. 600 New Hampshire Ave. NW, Suite 600

Washington, DC 20037

Energy Issues Rep.

Sierra Club

1228 North street. \#31

Sacramento, CA 95814

Ernst, Malcolm L.

U.S. Nuclear Regulatory Commission

101 Marietta street. Suite 3100

Atlanta, GA 30323

Executive Director

Natural Resources Defense Council

122 East 42nd street

New York. NY 10168

Fehrenback, P.

Atomic Energy Canada. Ltd.

Chalk River

Ontario. Canada 
Fiege, A.

Kernforschungszentrum

Postfach 3640

7500 Karlsruhe

Federal Republic of Germany

Flanagan, George F.

Oak Ridge National Laboratories

P.O. BoX $Y$

Oak Ridge. TN 37831

Fontana, Mario

International Technology Corp.

575 Oak Ridge Turnpike

Oak Ridge. TN 37830

Fray, Ray R.

Pacific Gas and Electric Co.

215 Market street, Room 206

San Francisco, CA 94106

Fulford, James

NUS Corporation

910 Clopper Road

Gaithersburg. MD 20878

Fussell. J. B.

JBF Associates. Inc.

1630 Downtown West Boulevard

Knoxville, TN 37919

Gallucci. Raymond H. V.

Battelle Pacific Northwest Labs.

P.O. Box 999

Richland. WA 99352

Garrick. John

Pickard, Lowe \& Garrick. Inc.

2260 University Drive

Newport Beach, CA 92660

Gaunt. John

British Embassy

3100 Massachusetts Avenue. NW

Washington, DC 20008

Gieseke. J .

Battelle Columbus Division

$505 \mathrm{King}$ Avenue

Columbus, OH 43201 
Glynn, James C.

U.S. Nuclear Regulatory Commission

Office of Nuclear Regulatory Research

Washington, DC 20555

Hammar, L.

Statens Karnkraftinspektion

P.O. Box 27106

S-10252 stockholm. Sweden

Hayns, M. R.

U.K. Atomic Energy Authority

Wigshaw Lane, Culcheth

Warrington. Chesire

United Kingdom WA3 $4 \mathrm{NE}$

Henry, Robert E.

Fauske and Associates. Inc.

16 W070 West $83 \mathrm{rd}$ street

Burr Ridge, IL 60521

Hirsch, Daniel

University of California

A. Stevenson Prog. on Nuclear Policy

Santa Cruz, CA 95064

Hodge, Steven

Oak Ridge National Laboratories

P.O. BoX Y

Oak Ridge, TN 37831

Houghton. Thomas $C$.

KMC. Inc .

$174 \%$ Pennsylvania Avenue. NW

Washington, DC 20006

Houston, Dean

U.S. Nuclear Regulatory Commission

Advisory Comm. on Reactor Safeguards

washington. DC 20555

Hsia, Der Yu

Taiwan Atomic Energy Council

67. Lane 144. Keelung Rd.. Sec. 4

Taipei. Taiwan

Jaguith. R. E.

Combustion Engineering. Inc.

1000 Prospect Hill Road

M/C 9490-2405

windsor, CT 06095

Johannson. Kjell

Studsvik Energiteknik AB

S-611 82. Nykoping. Sweden 
Johnson, D. H.

Pickard, Lowe \& Garrick. Inc.

2260 University Drive

Newport Beach. CA 92660

Joksimovich. Vojin

NUS Corporation

16885 W. Bernardo Drive

San Diego. CA 92127

Jun, H, R.

Korea Adv. Energy Research Inst.

P.O. Box 7. Daeduk Danju

Chungnam 300-31. Korea

Kafka, Peter

Geselischaft Fur Reaktorsicherheit

Forschungsgelande 8046 Garching

Federal Republic of Germany

Kahatib-Rahbar, M.

Brookhaven National Laboratory

Associated Universities. Inc.

Upton. NY 11973

Kaiser, Geoffrey D.

NUS Corporation

910 Clopper Road

Gaithersburg. MD 20878

Kastenberg. $\boldsymbol{w}$.

UCLA

5532 Boelter Hall

Los Angeles. CA 90024

Kato, W.

Brookhaven National Laboratory

Associated Universities. Inc.

Upton. NY 11973

Kerr. William

University of Michigan. North Campus

Department of Nuclear Engineering

Ann Arbor. MI 48109

Klopp. George

Commonwealth Edison Company

P.O. Box 767. Room 35W

Chicago. IL 60690

Koberlein. Klaus

Gesellschaft Fur Reaktorsicherheit

Forschungsgelande 8046 Garching

Federal Republic of Germany 
Kohn. E.

Atomic Energy Canada Ltd.

Candu Operations. Mississauga

ontario. Canada

Kouts, Herbert J. C.

Brookhaven National Laboratories

Building 179C

Upton. L.I. NY 11973

Kress. Thomas

Oak Ridge National Laboratories

P.O. BoX $Y$

Oak Ridge, TN 37831

Lanore. Jeanne-Marie

CEA B.P. No. 6

92660 Fonenay-Au-Roses, France

Lee, John C.

University of Michigan. North Campus.

Dept. of Nuclear Engineering

Ann Arbor. MI 48109

Lewis, Harold $W$.

University of California

Physics Department

Santa Barbara, CA 93106

Librarian

Atomic Industrial Forum

7101 Wisconsin Avenue

Bethesda, MD 20814

Lin. Eng

Taiwan Power Company

242. Roosevelt Rd.. Sec. 3

Taipei. Taiwan

Longworth, J . P.

Central Electric Gen'Ting Brd.

Berkeley Gloucester

GL13 9PB United Kingdom

Luckas, William J.

Brookhaven National Laboratories

Building 130

Upton. L.I.. NY 11973

Ludewig, Hans

Brookhaven National Laboratories

Building 130

Upton. L.I.. NY 11973 
MacDonald. P.

EG\&G Idaho, Inc.

P.O. Box 1625

Idaho Falls. ID 83415

Mal inauskas. A. P.

Oak Ridge National Laboratories

P.O. BoX Y

Oak Ridge, TN 37831

Mancini, Giuseppe

Commission European Comm.

CEC-JRC Eraton

Ispra Varese. Italy

McGowan, Alan

Scientists Institute for Public Info. 355 Lexington Avenue

New York, NY 10017

Meyer. Ralph

U.S. Nuclear Regulatory Commission

Office of Nuclear Regulatory Research

washington. DC 20555

Mitchell. Joselyn

U.S. Nuclear Regulatory Commission

Office of Nuclear Regulatory Research

Washington. DC 20555

Murphy. Joseph A.

U.S. Nuclear Regulatory Commission

Office of Nuclear Regulatory Research

Washington. DC 20555

Niemczyk, Sue

$154518 \mathrm{th}$ St. NW. \#112

Washington. DC 20036

O'Donnell. Edward P.

Ebasco Services. Inc.

2 World Trade Center, 89th Floor

New York. NY 10048

Okrent, David

University of California. Los Angeles

Boelter Hall. Room 5532

Los Angeles. CA 90024

Parkinson, William

Delian Corporation

1340 Saratoga-Sunnyvale Road

Suite 206

San Jose, CA 95129 
Petrangeli, G.

ENEA Nuc1. Energ. ALT Disp

Via V Brancati 48

00144 Roma. Italy

Pollard, Robert D.

Union of Concerned Scientists

1616 P street. NW, Suite 310

Washington, DC 20036

Potter, R.

UK Atomic Energy Authority

Winfrith Dorchester Dorset

DT2 BDH United Kingdom

Pratt, William T.

Brookhaven National Laboratories

Building 130

Upton. NY 11973

Pyatt. David

U.S. Nuclear Regulatory Commission

Office of Nuclear Regulatory Research

Washington, DC 20555

Rasmussen, Ingvard

Riso National Laboratory

Postbox 49 DK-4000

Roskilde. Denmark

Rasmussen. Norman C.

Massachusetts Institute of Technology 77 Massachusetts Avenue

Cambridge, MA 02139

Richardson, Dennis

Westinghouse Electric Corporation

P. O. Box 355

Pittsburg. PA 15230

Ritzman, Robert

Electric Power Research Institute

3412 Hillview Avenue

Palo Alto. CA 94304

Ross. Denwood F.

U.S. Nuclear Regulatory Commission

office of Nuclear Regulatory Research

Washington. DC 20555

Rowsome, Frank

U.S. Nuclear Regulatory Commission office of Nuclear Reactor Regulation Washington. DC 20555 
Siegal, John

Atomic Industrial Forum

7101 Wisconsin Avenue

Bethesda. MD 20814

Siess. Chester P.

Advisory Comm. on Reactor Safeguards

805 Hamilton Drive

Champaign. IL 61820

Silberberg. Melvin

U.S. Nuclear Regulatory Commission

Office of Nuclear Regulatory Research

Washington. DC 20555

Soda, K.

Japan Atomic Energy Res. Inst.

Tokai-Mura Naka-Gun

I BARAKI-KEN 319-11, Japan

Soffer, Leonard

U.S. Nuclear Regulatory Commission

Office of Nuclear Reactor Regulation

Washington. DC 20555

Speis. Themis $P$.

U.S. Nuclear Regulatory Commission

Office of Nuclear Reactor Regulation

Washington. DC 20555

Stadie. Klaus $B$.

OECD-NEA, 38 BId. Suchet

75016 Paris, France

Stiede, Wayne L.

Commonwealth Edison Company

P.O. Box 767

Chicago, IL 60690

Taylor, John

Electric Power Research Institute

3412 Hillview Avenue

Palo Alto. CA 94303

Taylor. Merrill A.

U.S. Nuclear Regulatory Commission

Office of the EDO

Washington, DC 20555

Technical Library

Electric Power Research Institute P.O. Box 10412

Palo Alto, CA 94304 
Temme, Mark I.

General Electric. Inc.

P.O. Box 3508

Sunnyvale. CA 94088

Theofanous. T. G.

University of California. S.B.

Dept. of Chemical and Nuclear Eng.

Santa Barbara, CA 93106

Thompson. Gordon

Inst. for Resource \& Security

studies

27 Elsworth Avenue

Cambridge. MA 02139

Tong. Long sun

9733 Lookout Place

Gaithersburg. MD 20879

Torri. A. F.

Pickard. Lowe \& Garrick. Inc.

191 Calle Magdalena. Suite 290

Encinitas. CA 92024

Tung, Chao-Chin

Atomic Energy Council

67 Lane 144, Sec 4, Keelung Rd.

Taipei. Taiwan

Wal1. Ian B.

Electric Power Research Institute

P.O. BoX 10412

Palo Alto, CA 94303

Ward. David

Savannah River Laboratories

Building 773-A, A-219

Aiken. SC 29808

Warman. Edward

Stone \& Webster Engineering Corp.

P.O. Box 2325

Boston. MA 02107

Weber, Norman

Sargent \& Lundy Co.

55 E. Monroe street

Chicago. IL 60603

Webster, Lois

American Nuclear Society

$555 \mathrm{~N}$. Kensington Avenue

La Grange Park. IL 60525 
Wood, Paul

Delian Corporation

296 Duff Road

Monroeville. PA 15146

Wreathall. John

Battelle Columbus Division 505 King Avenue

Columbus. OH 43201

Wyrick, Roger

Inst. for Nuclear Power Operations

1100 Circle 75 Parkway. Suite 1500

Atlanta. GA 30339

Young. Jonathan

Energy. Inc.

1851 South Central Place

Suite 201

Kent. WA 98031 


\begin{tabular}{|c|c|}
\hline $\begin{array}{l}\text { MAC FOAM 336 } \\
\text { LA } 2841 \\
\text { NACM } 1102 \\
32013202 \\
\text { SEE INSTRUCTIONS ON THE REVERSE }\end{array}$ & $\begin{array}{l}\text { REPORT NUMBER (Astigned by TIOC ado Vol No if any) } \\
\text { NUREG/CR }-4551 \text {, Vol. } 1 \\
\text { SAND } 86-1309 \\
\text { (Draft for Comment) }\end{array}$ \\
\hline \multirow{2}{*}{$\begin{array}{l}\text { 2 TITLEAND SUBTITLE } \\
\text { EVALUATION OF SEVERE ACCIDENT RISKS AND } \\
\text { THE POTENTIAL FOR RISK REDUCTION: } \\
\text { SURRY POWER STATION, UNIT } 1\end{array}$} & 3 LEAVE BLANK \\
\hline & 4 DATE AEPORT COMPLETED \\
\hline \multirow{2}{*}{$\begin{array}{l}\text { SAUThor(S) } \\
\text { A. S. Benjamin, G. J. Boyd, D. M. Kunsman, } \\
\text { W. B. Murfin, D. C. Williams }\end{array}$} & September $\quad 1986$ \\
\hline & \begin{tabular}{c|c}
\multicolumn{2}{c}{ 6 DATE REPORT ISSUED } \\
MONTM & YEAR \\
February & 1987
\end{tabular} \\
\hline 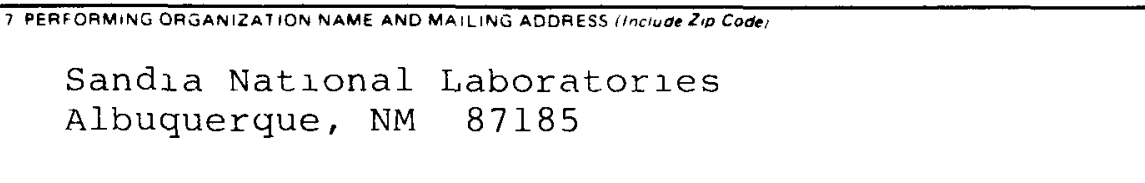 & $\begin{array}{l}8 \text { PROJECT/TASK WORK UNIT NUMBER } \\
\text { A FIN OR GRANT NUMBEA } \\
\text { A1 } 322\end{array}$ \\
\hline $\begin{array}{l}\text { 10 SPONSORING ORGANIZATION NAMEAND MAILING ADDRESS Inciud Zip Codel } \\
\text { Division of Reactor System Safety } \\
\text { Office of Nuclear Regulatory Research } \\
\text { U. S. Nuclear Regulatory Commission } \\
\text { Washington. DC } 20555\end{array}$ & $\begin{array}{l}\text { 11. TYPE OF REPOAT } \\
\text { DRAF'T FOR COMMENT }\end{array}$ \\
\hline \multicolumn{2}{|l|}{12 SUPPLEMENTARY NOTES } \\
\hline \multicolumn{2}{|c|}{ 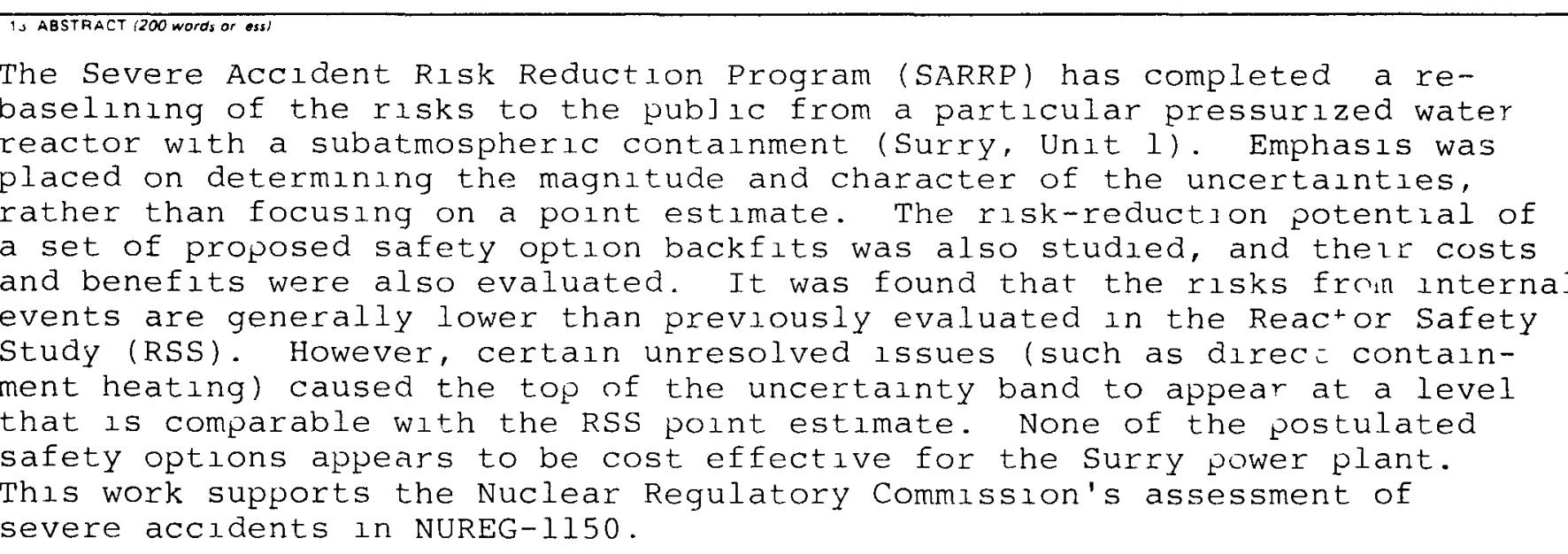 } \\
\hline \multirow[t]{3}{*}{14 DOCUMENT ANALYSIS - KEYWORDS DESCAIPTOAS } & $\quad \begin{array}{l}\text { ISAUALABLTY } \\
\text { STATEMENT } \\
\text { UnIImIted }\end{array}$ \\
\hline & 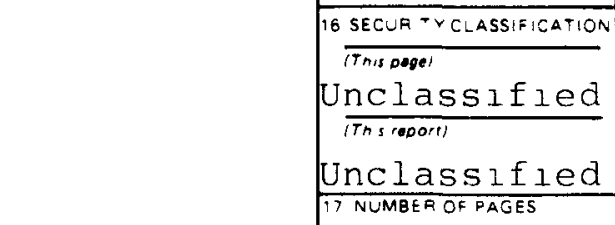 \\
\hline & 18 PAICE \\
\hline
\end{tabular}

